\title{
Radioactive Waste Management
}

\section{A Series of Bibliographies}

\section{High-Level Radioactive Wastes}

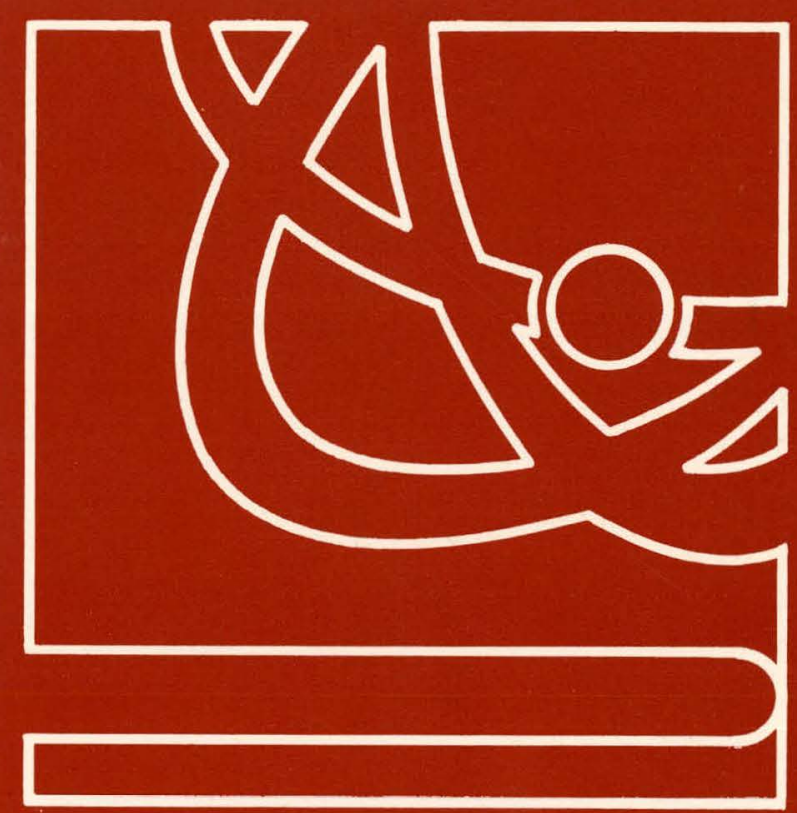




\section{DISCLAIMER}

This report was prepared as an account of work sponsored by an agency of the United States Government. Neither the United States Government nor any agency Thereof, nor any of their employees, makes any warranty, express or implied, or assumes any legal liability or responsibility for the accuracy, completeness, or usefulness of any information, apparatus, product, or process disclosed, or represents that its use would not infringe privately owned rights. Reference herein to any specific commercial product, process, or service by trade name, trademark, manufacturer, or otherwise does not necessarily constitute or imply its endorsement, recommendation, or favoring by the United States Government or any agency thereof. The views and opinions of authors expressed herein do not necessarily state or reflect those of the United States Government or any agency thereof. 


\section{DISCLAIMER}

Portions of this document may be illegible in electronic image products. Images are produced from the best available original document. 


\section{BIBLIOGRAPHIES PUBLISHED BY THE OFFICE OF SCIENTIFIC AND TECHNICAL INFORMATION}

The bibliographies listed below are available from the National Technical Information Service, U. S. Department of Commerce, Springfield, Virginia 22161. Requestors are urged to use the DE order number where provided.

Acid Precipitation: A Bibliography. April 1983. 3197 refs, DOE/TIC-3399 (DE83008750). \$30.00.

Appropriate Technology: A Bibliography. March 1981. 191 refs. DOE/TIC-3385. \$10.00.

Carbon Dioxide and Climate: A Bibliography. September 1981. 667 refs. covering July 1, 1976, through June 15, 1981. DOE/TIC3382-R1 (DE83009154). \$12.25.

Coal Desulfurization: A Bibliography. October 1983. 2139 refs. DOE/TIC-3400 (DE83017757). \$24.75.

Coal Production Expansion: A Selected Bibliography. July 1980. 138 refs. DOE/TIC-3381. $\$ 10.00$.

Computer Codes: A Bibliography. May 1981. 3916 refs. DOE/TIC3386. $\$ 25.00$.

Cooling Towers: A Bibliography. February 1979. 382 refs. covering January 1978 through December 1978. TID-3360(Suppl. 3). $\$ 1300$.

Cooling Towers: A Bibliography. February 1980. 426 refs. covering January 1979 through December 1979. DOE/TIC-3360(Suppl. 4). $\$ 13.00$.

Cooling Towers: A Bibliography. February 1981. 300 refs. DOE/TIC-3360(Suppl. 5). \$11.50.

DOE Patents Available for Licensing: A Bibliography. June 1982. 1344 refs. covering January 1974 through December 1980. DOE/TIC-3398 (DE82012479). \$17.00.

DOE Patents Available for Licensing: A Bibliography for the Period 1966-1974. October 1983. 510 refs. DOE/TIC-3398(Suppl. 1) (DE83017340). \$9.25.

Electric Batteries: A Bibliography. April 1978. 5422 refs. TID-3361. $\$ 31.50$.

Energy: Social and Economic Aspects. A Bibliography. July 1980. 3832 refs. DOE/TIC-3383, \$36.00.

Engineering Materials: A Bibliography. June 1982. 322 refs. DOE/TIC-4628 (DE82014444). \$9.50.

Fuel Cells: A Bibliography. July 1980. 1067 refs. covering June 1977 through June 1980. DOE/TIC-3359(Suppl. 1). \$16.00

Gas-Cooled Reactor Technology: A Bibliography. September 1981. 3358 refs. covering January 1978 through June 1981. DOE/TIC3339(Suppl. 3) (DE83009129). \$31.00.

Hydrogen Fuels: A Bibliography. February 1981. 3298 refs. DOE/TIC-3358(Suppl. 1). \$29.50.

Inventory of Federal Energy-Related Environment and Safety Research for FY 1979. December 1980. DOE/EV-0057/2. Vol. 1. Executive Summary. $\$ 10.00$. Vol. 2. Project Listings and Indexes. 3506 refs. $\$ 29.50$.

Liquid Metal Fast Breeder Reactors: A Bibliography. November 1980. 5465 refs. covering January 1978 through August 1980. DOE/TIC-3333(Suppl. 6), Vols. 1 and 2. \$42.00.

Low-Level Radiation: Biological Interactions, Risks, and Benefits. A Eibliography. September 1978. 3294 refs. covering July 1976 through September 1978. TID-3373. \$30.25.

Nuclear Medicine: A Bibliography. September 1978. TID3319-S8-P1. 4421 refs. \$29.00. TID-3319-S8-P2. 1787 refs. plus indexes. $\$ 28.75$.

Nuclear Quality Assurance: A Bibliography. September 1978. 375 refs. covering January 1976 through June 1978. TID-3374. $\$ 11.50$.

Oil Shales: A Selected Bibliography of DOE Sponsored Research. January 1980. 436 refs. DOE/TIC-3378. \$14.50.

Oil Shales and Tar Sands: A Bibliography. April 1981. 5142 refs. DOE/TIC-3367(Suppl. 1). \$36.25.
Oil Shales and Tar Sands: A Bibliography. July 1984. 4715 refs. DOE/TIC-3367(Suppl. 2)(Pts. 1 \& 2) (DE83018001). \$38.25.

Radioactive Waste Processing and Disposal: A Bibliography. January 1980. 4144 refs. covering June 1976 through Aug. 1978. NUREG-0643 (TID-3311-S8). \$35.50.

Radioactive Waste Processing and Disposal: A Bibliography. January 1980. 3598 refs. covering September 1978 through November 1979. NUREG-0644 (TID-3311-S9). \$34.50.

Radioactive Waste Processing and Disposal: A Bibliography. April 1981. 3700 refs. covering November 1979 through December 1980. DOE/TIC-3311-S10. \$34.00.

Radioactive Waste Processing and Disposal: A Bibliography. May 1982. 3382 refs. covering January 1981 through December 1981 . DOE/TIC-3311-S11 (DE82012480). \$33.50.

Radioactive Waste Processing and Disposal: A Bibliography. April 1983. 4314 refs. covering January 1982 through December 1982. DOE/TIC-3311-S12 (DE83007280). \$40.00.

Reactor Safety: A Bibliography. November 1980. 3082 refs. covering June 1977 through June 1980. DOE/TIC-3525(Rev. 5) (Suppl. 12). $\$ 30.50$.

Wood and Energy: A Bibliography. 1980. 941 refs. DOE/TIC-3380 (DE83005504). \$15.25.

\section{Radioactive Waste Management: A Series of Bibliographies}

Airborne Radioactive Effluents: Releases and Processing. October 1982. 870 refs. DOE/TIC-3397 (DE82012264). \$16.00.

Decontamination and Decommissioning. November 1982. 486 iefs. DOE/TIC-3391 (DE82012270). \$13.00.

Formerly Utilized Sites: Remedial Action. November 1982. 115 refs. DOE/TIC-3392 (DE82012269). \$8.50.

High-Level Radioactive Wastes. October 1982. 812 refs. DOE/TIC-3389 (DE82012272). \$16.25

High-Level Radioactive Wastes. September 1984. 1452 refs. DOE/TIC-3389(Suppl. 1) (DE84013656). \$19.75.

Low-Level Radioactive Waste: A Bibliography. December 1981. 215 refs. DOE/TIC-3387 (DE82004645). $\$ 10.00$

Low-Level Radioactive Waste. March 1983. 492 refs. DOE/TIC-3387(Suppl. 1) (DE83007212). \$14.50.

Low-Level Radioactive Waste. May 1984. 636 refs. DOE/TIC-3387(Suppl. 2) (DE84005533). \$12.50.

Nuclear Fuel Cycle: Reprocessing. December 1982. 1061 refs. DOE/TIC-3396 (DE82012265). \$16.25.

Nuclear Fuel Cycle: Reprocessing. September 1984. 555 refs. DOE/TIC-3396(Suppl.1) (DE84013561). \$11.50.

Radioactive Waste Inventories and Projections. November 1982. 25 refs. DOE/TIC-3394 (DE82012267). \$7.00.

Spent Fuel Storage. October 1982. 609 refs. DOE/TIC-3395 (DE82012266) \$12.00.

Spent Fuel Storage. August 1984. 580 refs. DOE/TIC-3395-S1 (DE84005534). \$11.75.

Transuranic Wastes. October 1982. 449 refs. DOE/TIC-3390 (DE82012271). \$14.50.

Uranium Mill Tailings. November 1982. 335 refs. DOE/TIC 3393 (DE82012268). \$13.00.

Waste Isolation. December 1982. 1024 refs. DOE/TIC-3388 (DE82012273). \$17.50.

This publication is available as DE84013656 from the National Technical Information Service,

U. S. Department of Commerce, Springfield, VA 22161.

Price: Printed Copy $\$ 19.75$

Microfiche A01 


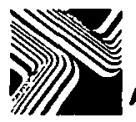

ABOUT THE TECHNICAL INFORMATION CENTER

The Office of Scientific and Technical Information, Technical Information Center in Oak Ridge, Tennessee, has been the national center for scientific and technical information for the Department of Energy (DOE) and its predecessor agencies since 1946. In developing and managing DOE's technical information program, the Center places under bibliographic control not only DOEoriginated information but also worldwide literature on scientific and technical advances in the energy field and announces the source and availability of this information. Whereas the literature of science is emphasized, coverage is extended to DOE programmatic, socioeconomic, environmental, legislative/regulatory, energy analysis, and policy-related areas. To accomplish this mission, the Center builds and maintains computerized energy: information data bases and disseminates this information via computerized retrieval systems and announcement publications such as abstracting journals, bibliographies, and update journals. Direct access to the Center's most comprehensive data base, the Energy Data Base, is available to the public through commercial on-line bibliographic retrieval systems. The Energy Data Base and many of the Center's energy-related data bases are available to DOE offices and contractors and to other government agencies via DOE/RECON, the Department's online information retrieval system. The Center has developed and maintains systems to record and communicate energy-related research-in-progress information, to maintain a register of $\mathrm{DOE}$ public communications publications, to track research report deliverables from DOE contractors, and to test and make available DOE-funded computer software programs with scientific ańd management applications. The Center also maintains a full-scale publishing capability to serve special publication needs of the Department. To effectively manage DOE's technical information resources, the Center's program is one of continual development and evaluation of new information products, systems, and technologies.

UNTED STATES DEPAATMENT OF ENERGY

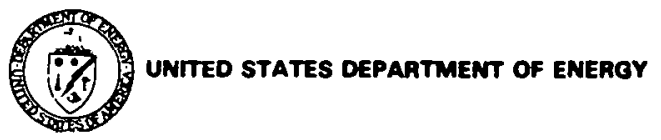

\section{Donald Paul Hodel Secretary}

Martha Hesse Dolan

Assistant Secretary

Management and Administration

William S. Heffelfinger

Director of Administration

Joseph G. Coyne

Manager

Office of Scientific and Technical Information

Lynda H. McLarẹn

Editor

\section{HIGH-LEVEL RADIOACTIVE WASTËS}

\section{ABOUT THIS BIBLIOGRAPHY}

This bibliography contains information on bigh-level radioactive wastes included in the Department of Energy's Energy Data Base from August 1982 through December 1983. The abstracts are grouped by subject category as shown in the table of contents. Entries in the subject index also facilitate access by subject, e.g., Radioactive Waste Disposal/Salt Deposits. Within each category the arrangement is by report number for reports, followed by nonreports in reverse chronological order. These citations are to research reports, journal articles, books, patents, theses, and conference papers from worldwide sources. Five indexes, each preceded by a brief description, are provided: Corporate Author, Personal Author, Subject, Contract Number, and Report Number.

Beginning in 1958, the Offnce of Scientific and Technical Information, Technical Information Center has, periodically issued general bibliographies containing 29,446 citations on radioactive wastes. Previously issued Radioactive Waste Processing and Disposal Bibliographies, including the number of citations and date issued, are:

TID-3311, 698 citations, 1958

TID-3555, 696 citations, 1960

TID-3555-S1, 874 citations, 1962

TID-331I-S1, 989 citations, 1964

TID-3311-S2, 1012 citations, 1966

TID-3311-S3, 2249 citations, 1972

TID-3311-S4. 464 citations. 1973

TID-3311-S5, 345 citations, 1974

TID-3311-S6, 2140 citations, 1975

TID-3311-S7, 1841 citations, 1976

TID-3311-S8, 4144 citations, 1978 (NUREG-0643)

TID-3311-S9, 3598 citations, 1980 (NUREG-0644)

DOE/TIC-3311-S10, 3700 citations, 1981

DOE/TIC-3311-S11, 3382 citations, 1982

DOE/TIC-3311-S 12,4314 citations, 1983

These bibliographies are available from the National Technical Information Service, 5285 Port Royal Road, Springfield, Virginia 22161.

Specialized bibliographies on radioactive waste management at Hanford (TID-3340), Savannah River (TID-3341), National Reactor Testing Station (TID-3342), and Oak Ridge (TID-3343) were produced in 1973.

The following publications begin a series of annual bibliographies covering the various subsets of radioactive waste information: Airborne Radioactive Effluents: Releases and Processing (DOE/TIC-3397), Decontamination and Decommissioning (DOE/TIC-3391), Formerly Utilized Sites: Remedial Action (DOE/TIC-3392), High-Level Radioactive Wastes (DOE/TIC-3389), Radioactive Waste Inventories and Prujections (DOE/TIC-3394), Low-Level Radioactive Waste (DOE/TIC-3387), Nuclear Fuel Cycle: Reprocessing (DOE/TIC-3396), Spent Fuel Storage (DOE/TIC-3395), Transuranic Wastes (DOE/TIC-3390), Uranium Mill Tailings (DOE/TIC-3393), and Waste Isolarion (DOE/TIC-3388).

A semimonthly abstract bulletin, Radioactive Waste Manogement. was begun in 1981 to announce current citations on radioactive waste which have been added to the Energy Data Base. This bulletin is available to the Department and its contractors from the Ofrice of Scientific and Technical Information and to others on subscription from the National Technical Information Service as PB84-902900; the annual rate is $\$ 40$ (domestic) and $\$ 80$ (outside the North American continent).

The citations in this publication and in those publications listed above, as well as other citations to information on radioactive waste back to July 1948, are available for on-line searching and retrieval using the DOE/RECON system. Citations from June 1976 to the present are available on the Energy Data Base, which may also be searched using the commercial on-line retrieval systems. 


\section{HIGH-LEVEL RADIOACTIVE WASTES}

\section{NUCLEAR FUELS}

\begin{abstract}
$1 \quad$ Uranium and nuclear energy: proceedings of the international symposium, 4th, 1979. Murray, J.; Casteel, K.D. (eds.). London, Engl.; Min J Books, Ltd (1980). 313p. (CONF7909192-).

From 4. international symposium on uranium and nuclear energy; London, UK (10 Sep 1979).

The conference proceedings consists of 21 papers. The following topics were discussed: aspects of supply and demand (such as uranium supply and demand to 1990; extraction of uranium from sea water); and nuclear issues (such as solidification and storage of high-activity nuclear waste; biological bases of radio protection; and the assessment of risk).
\end{abstract}

\section{By-products} for recovery and utilization of nuclear byproducts from defense wastes. Volume 1. Executive summary. (USDOE Assistant Secretary for Defense Programs, Washington, DC. Defense Waste and Byproducts Management Div.). Aug 1983. 21p. NTIS, PC A02/MF A01. Order Number DE83017049.

Portions are illegible in microfiche products. Original copy available until stock is exhausted.

Nuclear byproducts are a major national resource that has yet to be incorporated into the economy. The current Defense Byproducts Program is designed to match specific military and commercial needs with the availability of valuable products which are currently treated as waste at considerable expense in waste management costs. This program plan focuses on a few specific areas with the greatest potential for near-term development and application. It also recognizes the need for a continuing effort to develop new applications for byproducts and to continue to assess the impacts on waste management. The entire program has been, and will continue to be structured so as to ensure the safety of the public and maintain the purity of the environment. Social and institutional concerns have been recognized and will be handled appropriately. A significant effort will be undertaken to inform the public of the benefits of byproduct use and of the care being taken to ensure safe, efficient operation.

\section{Fuels Production And Properties}

(PNCT-831-81-01) Tokai Works annual progress report, April 1980-March 1981. (Power Reactor and Nuclcar Fuel Development Corp., Tokai, Ibaraki (Japan). Tokai Works). Sep 1981. 131p. NTIS (US Sales Only), PC A07/MF A01. Order Number DE83900415.

The main activities of Tokai Works at present are the development and fabrication of plutonium-bearing fuels, research and development of centifugal uranium enrichment technology, test operations of the Tokai Reprocessing Plant, and development of vitrification and FBR spent fuel reprocessing proccsses. This progress report contains those accomplishments in the activities of Tokai Works during the period from January 1980 to March 1981.

4 Chemistry of the nuclear fuel cycle. Wilkinson, W.L. (British Nuclear Fuels Ltd., Risley). pp 164-186 of Energy and chemistry. Energy and chemistry special publication no. 41. Thompson, R. (ed.). London, England; Royal Society of Chemistry (1981). (CONF-8104113-).

From Royal Society of Chemistry annual chemical congress; Guildford, Surrey, UK (7 Apr 1981).

The purpose of this paper is first briefly to review the basic chemistry underlying the processes used on an industrial scale for the manufacture of fuel elements for thermal nuclear power reactors and then to consider in more detail the chemistry involved in the reprocessing of this fuel after irradiation, paying particular attention to developments which have been made to allow the newer enriched oxide fuels to be processed. Section headings are: nuclear fuel manufacture (U metal fuel; $\mathrm{UO}_{2}$ ceramic fuel); nuclear fuel reprocessing; irradiated fuel dissolution; solvent extraction chemistry (chemistry and kinetics of Pu reduction; the behaviour of $\mathrm{Np}$ and $\mathrm{Tc}$ ); treatment of highly active raffinates (evaporation and storage of highly active liquors; highly active waste vitrification; thermal decomposition of wastes).

6 Chemistry in the development of nuclear power. Marples, J.A.C.; Potter, P.E. (UKAEA Atomic Energy Research Establishment, Harwell. Chemistry Div.); Nelson, R.L. (UKAEA Atomic Energy Research Establishment, Harwell. Chemical Technology Div.); Roberts, L.E.J. (UKAEA Atomic Energy Research Establishment, Harwell. Directorate). pp 131-163 of Energy and chemistry. Energy and chemistry special publication no. 41. Thompson, R. (ed.). London, England; Royal Society of Chemistry (1981): (CONF-8104113-).

From Royal Society of Chemistry annual chemical congress; Guildford, Surrey, UK (7 Apr 1981).

The subject is reviewed under the following headings: phase equilibria and thermodynamic properties of oxide fuels; gel precipitation process for preparing a mixed oxide $(\mathrm{U}, \mathrm{Pu}) \mathrm{O}_{2}$; glasses for the solidification of high level waste.

\section{Spent Fuels Reprocessing}

- (DOE/NE/00952-7) Reprocessing of nuclear fuel. Schneider, A. (Georgia Inst. of Tech., Atlanta (USA)). 20 Apr 1983. Contract AS09-76SR00952. 90p. NTIS, PC A05/MF A01. Order Number DE83012394.

Portions are illegible in microfiche products.

Chapters are devoted to the following: introduction; power reactor fuels; reprocessing schemes; the PUREX process; product conversion; waste management; equipment and facilities; and special reprocessing schemes.

7 (INIS-mf-7132, pp vp) Radioactive waste processing and disposal. Kyrs, M. 1980. (In Czech). NTIS (US Sales Only), PC A09/MF A01. (CONF-8006241-).

From Nuclear Research Institute scientific and technical conference; Prague, Czechoslovakia (5 Jun 1980).

The fluoride reprocessing of fast reactor fuels, the separation of selected valuable and biological emitters from radioactive wastes, and the problems of zircunium and uranium hydrometallurgy are discussed. The flow chart of the process uranium branch for spent fuel reprocessing for reactor BOR-60, developed by UJV in co-operation with Soviet institutions consists of several operations, viz., the removal of the stainless steel fuel element cladding by melting, the oxidation of $\mathrm{UO}_{2}$ to $\mathrm{U}_{0} \mathrm{O}_{8}$, fluorination and condensation, the separation of Pu from uranium, distillation purification of $\mathrm{UF}_{6}$, sorption-desorption purification of $\mathrm{UF}_{6}$, waste gas treatment. The development is described of the two-stage phosphate process of radioactive waste treatment (stage one: denitration and calcination, stage two:melting). (H.S.).

8 (KFK-3290, pp 33-51) Main activities and achievements of the DWK within the joint development programme. Kuhn, K.D.; Sahm, A. Mar 1982. (In German). NTIS (US Sales Only), PC A13/MF A01.

In Proceedings of the 4th status report of the Reprocessing and Waste Management Project (PWA) of November 5th, 1981.

In a coordinated development programme for the construction of fuel cycle centres, the DWK (Deutsche Gesellschaft fuer Wiederaufarbeitung) has the task to apply the experience gained by KfK and WAK to the planning and finally to the operation of industrial plants. In addition, the work carried out by DWK is aimed at finding solutions for safety engineering and economy problems in order to be able to cope with the requirements of the next decade. The main efforts thus are 
studies on spent fuel storage, especially head-end problems and detailed problems in the first coextraction cycle of the PUREX-process with components testing, development work for improving the safety of the reprocessing plant (instrumentation, $\mathrm{H}_{2}$ radiolysis), and finally the vitrification of high-level radioactive waste (PAMELA-plant).

9 (RHO-PB-SR-10-B-SUM-JUN-83) Rockwell Hanford Operations monthly report summary, June 1983. (Rockwell International Corp., Richland, WA (USA). Rockwell Hanford Operations). 1983. Contract AC06-77RL01030. 41p. NTIS, PC A03/MF A01. Order Number DE83016171.

Portions are illegible in microfiche products.

Shipment of the initial stable material from Pacific Northwest Laboratory 303-C Building in support of the Hanford Plutonium Scrap Processing Plan was initiated. Six shipments have been completed on schedule per the 303-C Shipping and Receiving Plan. Another PUREX Phase ID enhanced readiness test run of two weeks was successfully completed. The task to modify nine railroad well cars for PUREX fuel transportation support was completed one month ahead of schedule. The performance specification for the Helicupter Hangar/Support Facility was approved by Kaiser Englneers Hanlord and Kockweil Hunford Operations on schedule. Combined jet pump production for TX and BY Tank Farms in the month of June was approximately 123,000 gal. The fiscal year-to-date volume pumped is about 315,000 gal versus 350,000 gal planned. The first shipments of $\mathrm{N}$-Reactor decontamination waste were received from UNC via railroad tank cars in the first week of the month. Two to three shipments per week are being received and a total of thirty-three loads are expected to be received through the middle of August (about $500,000 \mathrm{gal}$ of waste). Twenty-three cesium capsules were produced in June for a fiscal total of 100 . A technical plan for the Transportable Grout Facility was approved, and submitted to DOERichland. This technical plan addresses the need and background, as well as the objective, strategy, work breakdown structure, schedule, and milestones. Completion of this technical plan represents a significant accomplishment in the grouting of defense nuclear wastes. Both PNL and ORNL participated by contributing to this plan. The final draft of Waste Form Acceptance Requirements for Spent Fuel, Commercial and Defense High-Level Waste, and Commercial Transuranic Waste for a Nuclear Waste Repository in Basalt was completed and transmitted to DOE-Richland.

10

Land Baden-Wuerttemberg: public riotice of the Ministry of Economics, Medium-size Industry and Transport concerning a permit under the Atomic Energy Law. - 3rd Supplement to the 9th partial licence for the construction of Karlsruhe reprocessing plant - System for storage and evaporation of highactivity liquid waste (LAVA). Bundesanzeiger; 35: No. 17, 729(26 Jan 1983). (In German).

Published in summary form only.

WAK; CONSTRUCTION PERMITS; HIGH-LEVEL RADIOACTIVE WASTES; RADIOACTIVE WASTE STORAGE; RADIOACTIVE WASTE PROCESSING; EVAPORATORS

11 Formation of inflammable radiolytic gases in the PUREX puroes. Stieglitz, L,i Becker, R.; Wuertz, R. KFK-Nachrichten; 14: No. 3, 137-142(1982). (În German).

The results on the radiation-chemical formation of hydrogen and other combustible gases in the high-level radioactive process streams of reprocessing have been presented and comparcd with values of previous investigations on idealized systems. For the hydrogen formation it showed that the release is not only due to the chemical compound of the solutions, but that physical and apparative parameters such as kinetic condition and geometry of the irradiated solution play a considerable part. Taking these values into account, release values are obtained that fall below the values, assumed so far by several orders of magnitude. They have been confirmed by measurements on the containment exhaust gas of the WAK. As for the formation of hydrogen and other combustible gases in the HA-column it could equally be shown that the actual concentrations are below the low limit of inflammability by at least one order of magnitude. Slightly volatile radiolysis products are thus - if ever at all - of minor importance with respect to safety during reprocessing of nuclear fuels.
12 Ruthenium nitrosyl complexes in radioactive waste solutions of reprocessing plants. Pt. 1. Blasius, E.; Glatz, J.P.; Neumann, W. Radiochimica Acta; 29: No. 2/3, 159-166(1981). Preparative isolation of the calionic ruthenium nitrosyl nitrato complexes succeeds by counter-pressure column electrophoresis on Lichroprep $\mathrm{Si} 60$ as supporting medium. The composition of the separated complexes is elucidated by the ionic charge determined via the electrophoretic migration distance and by the content of $\mathrm{Ru}$ and $\mathrm{NO}_{0}{ }^{-}$. The amounts of the single complexes in simulated HAW and MAW solutions are practically equal to those in the corresponding pure nitric solutions. By denitration using formic acid or formaldehyde, formato complexes are formed, the composition of which is revealed by means of a new formate determination method analogous to that for the nitrato complexes. From the mixture of the complexes the neutral complexes can be extracted well, the cationic ones only very slightly. In order to determine the extraction behavior of the simple cationic complexes with TBP-Dodecane, they are separated by column electrophoresis at $0^{\circ} \mathrm{C}$. The low temperature not only prevents the complexes from transformation, but also improves the extraction in the same way as does the electrolyte, necessarily contained in the eluent. In the case of the cationic complexes, the extraction coefficients increase with decreasing ionic charge and increasing number of nitrato ligands.

13 Policy and development of reprocessing of spent nuclear fuel and high-level radioactive waste management in the F.R. Fermany. Salander. C. (Deutsche Gesellschaft fuer Wiederaufarbeitung von Kernbrennstoffen m.b.H. (DWK), Hannover (Germany, F.R.)). ; No. 14, 189-193, 214-221(1981). (In Japanese).

From 14. annual conference of the Japan Atomic Industrial Forum; Tokyo (Japan) (10 - 12 Mar 1981).

The original German concept of a comprehensive center for the back-end (Entsorgungs-center) was scrapped for political reasons. In its place, "Entsorgungs-concept", namely the design and construction of many small facilities in various parts of the country proceed. A smallscale demonstration reprocessing facility is planned by DWK in Hesse, after the sites were examined. For low and intermediate level radioactive wastes, West Germany has successfully demonstrated the disposal technology; the wastes are currently disposed of at in the Asse 2 rock salt mine. For high level wastes, vitrification process is developed; experimental research has been conducted in Asse. Gorleben is the planned site for high level waste storage, where the geological survey of the salt dome was conducted. Spent fuel is stored for the time being in transportation casks.

\section{Transport And Storage}

14 (CONF-830528-13) Economic evaluation of nuclear waste transportation casks. Schneringer, P.E.; Joy, D.S.; Cashwell, J.W. (GA Technologies, Inc., San Diego, CA (USA); Oak Ridge National Lab., TN (USA); Sandia National Labs., Albuquerque, NM (USA)). 1983. Contract W-7405-ENG-26. 3p. NTIS, PC A02/MF A01. Order Number DE83012650.

From 7. international symposium on packaging and transportation of radioactive materials; New Orleans, LA, USA (15 May 1983).

A method is described which allows the systematic economic evaluation of transportation cask designs which meet the requirements of the Test and Evaluation Facility (TEF) program. The heart of the method described is the Waste Management Transportation Model. This model uses a set of computer-based algorithms to a33omble epociffe case information input, combine this input with the data base of transportation information maintained within the model, and calculate the cask types and quantities necessary, the cask utilization factors, and the total costs for each transport line specified. The model is capable of handling a large variety of transportation problems given the specific input related to each type. Three combinations of waste packaging facilities were examined. The first assumes all consolidation and packaging occurs at an existing hot cell. The second assumes all consolidation and packaging is donc at the TEF site. The third combination assumes that spent fuels are consolidated at an existing hot cell while waste packaging occurs at the TEF site. Some of the general findings are: (1) defense high-level waste (DHLW) is generally luwer in cost than SF as the prime waste form because of the fewer number of shipments required prior to the waste consolidation activity; (2) when DHLW is the prime waste form, it is beneficial to locate the packaging facility (PF) close to the TEF site because the packaged waste form is heavier, more costly to 
transport; (3) when SF is the prime waste form, it is beneficial to locate the PF close to the waste source to reduce the length of the transport links containing unconsolidated spent fuel assemblies; and (4) truck casks, and legal weight truck casks in particular, are generally superior to the rail casks on an economic basis.

15 (CONF-830528-Summ., pp IV.16-IV.17) Storage and transportation of spent fuel and high-level waste using dry storage casks. Eggers, P.E. (Ridihalgh, Eggers \& Associates, Inc., Columbus, OH). 1983. NTIS, PC A21/MF A01.

From 7. international symposium on packaging and transportation of radioactive materials; New Orleans, LA, USA (15 May 1983).

SPENT FUEL CASKS; HIGH-LEVEL RADIOACTIVE WASTES; RADIOACTIVE WASTE STORAGE; TRANSPORT; SPENT FUELS

16 (CONF-830528-Summ., pp D.II.23-D.II.24) Combined storage for LWR spent fuel and high-level waste. Baxter, B.J.; Ganley, J.T. (GA Technologies Inc., San Diego, CA). 1983. NTIS, PC A21/MF A01.

From 7. international symposium on packaging and transportation of radioactive materials; New Orleans, LA, USA (15 May 1983). MOdular DRy EXpandable storage system. SPENT FUEL STORAGE; RADIOACTIVE WASTE STORAGE; HIGH-LEVEL RADIOACTIVE WASTES; STORAGE FACILITIES

17 (CONF-830528-Summ., pp XIX.5-XIX.6) Estimated transportation cost and risk for operation of a nuclear waste repository at candidate sites. Peterson, R.W. (Battelle Memorial Inst., Columbus, $\mathrm{OH}$ ); Varadrajan, R.V.; MCSweeney, T.I;; Cashwell, J.W.; Wilmot, E.L.; Joy, D.S. 1983. NTIS, PC A21/MF A01. Contract AC06-76RL01830;AC0476DP00789;W-7405-ENG-26.

From 7. international symposium on packaging and transportation of radioactive materials; New Orleans, LA, USA (15 May 1983). WASTE TRANSPORTATION; COST; RISK ASSESSMENT; HIGH-LEVEL RADIOACTIVE WASTES; HANFORD RESERVATION; NEVADA TEST SITE; UTAH; TEXAS; LOUISIANA; MISSISSIPPI; ROAD TRANSPORT; RAIL TRANSPORT

18 (CONF-830528-Summ., pp XXV.5) Construction and inservice inspection rules for nuclear spent fuel and high-level waste transport packagings. Goldmann, K. (Transnuclear, Inc., White Plains, NY); Brinkman, W.H.; Canonico, D.A.; Claverie, R.J.; Nickell, R.E. 1983. NTIS, PC A21/MF A01.

From 7. international symposium on packaging and transportation of radioactive materials; New Orleans, LA, USA (15 May 1983). TIVE WASTES; PACKAGING

19 (CONF-830528-Summ., pp XXV.8) Design rules for containment systems of nuclear spent fuel and high-level waste transport packagings. Nickell, R.E. (Electric Power Research Inst., Palo Alto, CA). 1983. NTIS, PC A21/MF A01. From 7. international symposium on packaging and transportation of radioactive materials; New Orleans, LA, USA (15 May 1983).

DESIGN; PACKAGING; SPENT FUELS; HIGH-LEVEL RADIOACTIVE WASTES; CONTAINMENT SYSTEMS

20 (CONF-830528-Summ., pp XXV.9-XXV.10) Transportation system preconceptual description for subseabed disposal of bigh-level waste. Shirley, C.G. (Sandia National Labs., Albuquerque, NM); McClure, J.D.; Miller, M.; Chryssostomidis, C.; Giovanetti, R.C.; Wee, C.S. 1983. NTIS, PC A21/MF A01. Contract AC04-76DP00789.

From 7. international symposium on packaging and transportation of radioactive materials; New Orleans, LA, USA (15 May 1983). HIGH-LEVEL RADIOACTIVE WASTES; WASTE TRANSPORTATION; LAND TRANSPORT; MARITIME TRANSPORT; SF.A BED; RADIOACTIVE WASTE DISPOSAL

21 (DOE/S-0020) Report on financing the disposal of commercial spent nuclear fuel and processed high-level radioactive waste. Benny, R.I.; Sprecher, W.M. (USDOE Nuclear
Waste Policy Act Project Office, Washington, DC). Jun 1983. 55p. NTIS, PC A04/MF A01. Order Number DE83014080.

Projected revenues generated from the 1.0 mill per $\mathrm{kWh}$ fee mandated by the Act are sufficient to cover the full range of reference case program costs, assuming 3\% annual inflation and nuclear installed capacity of 165 gigawatts-electric by the year 2000 . Total estimated costs of the reference waste disposal program, encompassing either spent nuclear fuel disposal or reprocessing waste disposal, range between $\$ 18$ to 20 billion in constant 1982 dollars. Sensitivity case analyses established upper and lower program cost bounds of \$28 billion and $\$ 16$ billion, respectively (in 1982 dollars). In terms of discounted levelized unit costs, the disposal of spent fuel equates to $\$ 122$ to 125 per kilogram (uranium) compared with $\$ 115$ to 119 per kilogram for the reprocessing waste equivalent. The levelized unit costs for reprocessing exclude the solidification of liquid wastes. Such costs are estimated to be $\$ 8$ per kilogram. Discounted levelized unit costs corresponding to the upper and lower limits of the sensitivity cases equate to $\$ 176$ per kilogram and $\$ 107$ per kilogram. The 1.0 mill per $\mathrm{kWh}$ fee will be reviewed annually and adjusted, if necessary, to accommodate changes in program costs due to inflation and program shifts. When adjustments are made for applicable discount rates, inflation, repository design changes, and other factors, levelized unit costs for the reference case presented in this analysis agree closely with the results of two previous Department of Energy studies concerning charges for spent fuel storage and disposal services provided by the Federal government. The cost estimates developed for the program were based on the best available data.

22 (DP-MS-82-60) Predicting transport requirements for radioactive-waste slurries. Motyka, T.; Randall, C.T. (Du Pont de Nemours (E.I.) and Co., Aiken, SC (USA). Savannah River Lab.). 1983. Contract AC09-76SR00001. 27p. (CONF830350-1). NTIS, PC A03/MF A01. Order Number DE83011377.

From 8. international technical conference on slurry transport; San Francisco, CA, USA (15 Mar 1983).

Portions are illegible in microfiche products.

A method for predicting the transport requirements of radioactive waste slurries was developed. This method involved preparing nonradioactive sludge slurries chemically similar to the actual high-level waste. The rheological and settling characteristics of these synthetic waste slurries were measured and found to compare favorably with data on actual defense waste slurries. Pressure drop versus flow rate data obtained fom a 2-in. slurry test loop confirmed the Bingham plastic behavior of the slurry observed during viscometry measurements. The pipeline tests, however, yielded friction factors 30 percent lower than those predicted from viscometry data. Differences between the sets of data were attributed to inherent problems in interpreting accurate yieldstress values of slurry suspensions with Couette-type viscometers. Equivalent lengths of fittings were also determined and found to be less than that of water at a specified flow rate.

23

(DPST-82-294) Comparative transportation risk assessment for borosilicate-glass and ceramic forms for immobilization of SRP Defense waste. Moyer, R.A. (Du Pont de Nemours (E.I.) and Co., Aiken, SC (USA). Savannah River Lab.). Apr 1982. Contract AC09-76SR00001. 26p. NTIS, PC A03/MF A01. Order Number DE82014168.

It is currently planned to immobilize the SRP high-level nuclear waste in solid form and then ship it from SRP to a federal repnsitnry. This report compared transportation operations and risks for SRP highlevel waste in a borosilicate glass form and in a ceramic form. Radiological and nonradiological impacts from normal transport and from potential accidents during transit were determined using the Defense Waste Process Facility Environmental Impact Statement (DWPF EIS) as the source of basic data. Applicable regulations and some current regulatory uncertainties are also discussed.

24 (GA-A-16631) System evaluation model for selecting spent nuclear fuel storage concepts. Finch, W.C.; Morissette, R.P.; Postula, F.D. (General Atnmic Co., San Diego, CA (USA)). Jan 1982. Contract AT03-81SF11528. 13p. (CONF820615-2). NTIS, PC A02/MF A01. Order Number DE82017574.

From 26. American Association of Cost Engineers annual meeting; Houston, TX, USA (27 Jun 1982). 
Portions of document are illegible.

President Reagan has challenged the nuclear community to develop a safe and economical system to handle spent fuel assemblies from commercial nuclear power generating stations and high level radioactive wastes from fuel reprocessing facilities. This paper will illustrate a management system approach used to identify and evaluate monitored, retrievable fuel storage concepts that fulfill ten key criteria for meeting the functional requirements and system objectives of the National Nuclear Waste Management Program. These selection criteria include: health and safety, schedule, costs, socio-conomic factors and environmental factors. An MRS (Monitored Retrievable Storage) System is designed to safety store spent fuel assemblies and high level waste in a dry, passively cooled environment for an indefinite period of time up to 100 years. Forth-five feasible MRS design concepts utilizing 20 basic storage methods were initially screened and reduced to 15 concepts with 7 methods. A system evaluation model was developed to evaluate the ability of each of the remaining 15 alternative concepts to meet the selection criteria. This model allows the ranking of each system according to MRS needs and life-cycle costs. The methodology used to establish the selection criteria, develop a weight of importance for each criterion and assess the relative merit of each MRS system will be described. The impact of cost relative to technical criteria will be discussed along with experience in obtaining relative merit data and its application in the model. This system evaluation model is universally applicahle when many concepts in various stages of design and cost development need to be evaluated. It provides a structured approach which allows the evaluators to make a rational, well-founded selection.

25 (INIS-mf-7471) Rail transport of vitrified high-level radioactive fission products - risk analysis of a model. Schneider, K. (Technische Hochschule Aachen (Germany, F.R.). Fakultaet fuer Maschinenwesen). 7 Nov 1980. 218p. (In German). NTIS (US Sales Only), PC A10/MF A01. Order Number DE83780211.

Thesis.

This report gives an outline of a transportation system for highlevel radioactive waste on an industrial scale and the risk which can be expected for the human populations involved. The known planningconceptions for reprocessing have extreme timelags for the intermediate storage of high-level radioactive waste. In general one is thinking of a minimum age for high-level radioactive waste - calculated from the time when it is taken out of the nuclear reactor - of five years up to a maximum age of fifty years when it is transported to a waste disposal site in geological formations. Thus the above-mentioned examinations are carried nut. for two corresponding reference ages of the high-level radioactive waste.

26 (ONWI-404) Southeastern Utah nuclear-wastetransportation study. (Bechtel Group, Inc., San Francisco, CA (USA)). Oct 1982. Contract AC06-76RL01830. 87p. NTIS, PC A05/MF A01. Order Number DE83002763.

Portions of document are illegible.

This report is published as a product of the National Waste Terminal Storage (NWTS) Program. The objective of this program is the development of terminal waste storage facilities in deep stable geologic formations for high-level nuclear waste, including spent fuel elements from commercial power reactors and transuranic nuclear waste for which the Federal Government is responsible. The initial purpose of this study was to analyze and compare possible transpurt mules and curridors connecting each of four candidate sites in Utah with existing rail lines of the Denver and Rio Grande Western Railroad (D \& RG) or the Atchison, Topeka, and Santa Fe Railway (ATSF). The primary use of these routes would be for shipping spent nuclear fuel and other nuclear wastes. During the study, the Gibson Dume area was recommended as the preferred location by the screening process evaluating the four Utah areas. Therefore, this report presents transportation routes to Gibson Dome examining order-of-magnitude capital costs of railroad and heavy haul routes, railroad and heavy haul truck operating costs and environmental factors. The scope of work did not include consideration of using public highways. Information on the other candidate sites and possible routes to them is also presented. 15 figures, 10 tables.

27 (PNL-3721) Defense waste transportation: cost and logistics studies. Andrews, W.B.; Cole, B.M.; Engel, R.L.; Oylear, J.M. (Battelle Pacific Northwest Labs., Richland, WA
(USA)). Aug 1982. Contract AC06-76RL01830. 106p. (TTC0191). NTIS, PC A06/MF A01. Order Number DE82021966. Transportation of nuclear wastes from defense programs is expected to significantly increase in the 1980 s and 1990 s as permancnt waste disposal facilities come into operation. This report uses models of the defense waste transportation system to quantify potential transportation requirements for treated and untreated contact-handled transuranic (CH-TRU) wastes and high-level defense wastes (HLDW). Alternative waste management strategies in repository siting, waste retrieval and treatment, treatment facility siting, waste packaging and transportation system configurations were examined to determine their effect on transportation cost and hardware requirements. All cost estimates used 1980 costs. No adjustments were made for future changes in these costs relative to inflation. All costs are reported in 1980 dollars. If a single repository is used for defense wastes, transportation costs for CH-TRU waste currently in surface storage and similar wastes expected to be generated by the year 2000 were estimated to be 109 million dollars. Recovery and transport of the larger buried volumes of CH-TRU waste will increase CH-TRU waste transportation costs by a factor of 70. Emphasis of truck transportation and siting of multiple repositories would reduce CH-TRU transportation costs. Transportation of HLDW to repositories for 25 years beginning in 1997 is estimated to cost $\$ 229$ $M$ in 1980 costs and dollars. HLDW transportation costs could either increase or decrease with the selection of a final canister configuration. HLDW transportation costs are reduced when multiple repositories exist and emphasis is placed on iruck transpurt.

28 (PNL-4032) Risk assessment for the transportation of radioactive zeolite liners. (Pacific Northwest Lab., Richland, WA (USA)). Jan 1982. Contract AC06-76RL01830. 155p. NTIS, PC A08/MF A01. Order Number DE82008034.

Portions of document are illegible.

The risk is estimated for the shipment of radioactive zeolite liners in support of the Zeolite Vitrification Demonstration Program currently underway at Pacific Northwest Laboratory under the sponsorship of the US Department of Energy. This program will establish the feasibility of zeolite vitrification as an effective means of immobilizing highspecific-activity wastes. In this risk assessment, it is assumed that two zeolite liners, each loaded around July 1,1981 to $60,000 \mathrm{Ci}$, will be shipped by truck around January 1,1982 . However, to provide a measure of conservatism, each liner is assumed to initially hole $70,000 \mathrm{Ci}$, with the major radioisotopes as follow: ${ }^{90} \mathrm{Sr}>3000 \mathrm{Ci}$, ${ }^{104} \mathrm{Cs}>7000$ $\mathrm{Ci}_{1}{ }^{107} \mathrm{Cs}>60,000 \mathrm{Ci}$. Should shipment take place with essentially no delay after initial loading (regardless of loading date), the shipment loading would be only $2.7 \%$ higher than that for the assumed six-month delny. Thin would negligibly affect the overall risk. As a result of this risk assessment, it is concluded that the transport of the radioactive zeolite liners from TMI to PNL by truck can be conducted at an insignificant level of risk to the public.

29

(SAND-81-2133) Transportation packagings for high-level wastes and unprocessed transuranlc wastes. Wilmot, E.L.; Romesberg, L.E. (Sandia National Labs., Albuquerque, NM (USA)). 1982. Contract AC04-76DP00789. 21p. (TTC0256; CONF-820705-1). NTIS, PC A02/MF A01. Order Number DE82005771.

From 2. joint ASME/ANS nuclear engineering conference; Portland, OR, USA (25 Jul 1982).

Packagings used for nuclear waste transport are varied in size, shape, and weight because they must accomnuclate a wide variety of waste forms and types. However, this paper will discuss the common characteristics among the packagings in order to provide a broad undcrstanding of packaging designs. The paper then discusses, in some detail, a design that has been under development recently at Sandia National Laboratories (SNL) for handling unprocessed, contact-handled transuranic (CHTRU) wastes as well as a cask design for defense high-level wastes (HLW). As presently conceived, the design of the transuranic package transporter (TRUPACT) calls for inner and outer boxes that are separated by a rigid polyurethane foam. The inner box has a steel frame with stainless steel surfaces; the outer box is similarly constructed except that carbon steel is used for the outside surfaces. The access to each box is through hinged doors that are sealed after loading. To mect another waste management need, a cask is bcing developed to transport defense HLW. The cask, which is at the preliminary design stage, is being developed by General Atomic under the direction of the TTC. The cask design relies heavily on state-of-the-art spent-fuel cask designs 
though it can be much simpler due to the characteristics of the HLW. A primary purpose of this paper is to show that CHTRU waste and defense HLW currently are and will be transported in packagings designed to meet the hazards of transportation that are present in general commerce.

30 (SAND-82-0510C) Review of the status of transportation activities in various waste management systems. Allen, G.C. Jr.; Pope, R.B. (Sandia National Labs., Albuquerque, NM (USA)). 1982. Contract AC04-76DP00789. 23p. (CONF820303-7). NTIS, PC A02/MF A01. Order Number DE82011469.

From Waste management conference (ANS); Tucson, AZ, USA (8 Mar 1982).

Portions of document are illegible.

Since the early 1970's, DOE and its predecessor agencies have recognized the importance of the safe transportation of radioactive materials. Initially, this interest took the form of a series of isolated pro grams, each directed at providing a solution to a specific transportation problem. By the mid 1970's this approach became inadequate to address the growing complexities of the problem, and in late FY 78 the Transportation Technology Center (TTC) was established together with a coherent program in the transportation of radioactive materials with the Albuquerque Operations Office as the lead DOE field office and Sandia National Laboratories (SNL) as the lead contractor. This paper reviews the current status of transportation activities being conducted by the TTC in support of DOE waste management programs. These activities are focused on the following radioactive wastes and spent fuels: (1) defense wastes (contract-handled transuranic wastes, special-handled transuranic wastes, processed transuranic wastes, high level wastes); (2) commercial wastes (terminal isolation wastes, West Valley wastes, Three Mile. Island wastes, low-level wastes, remedial action wastes); (3) spent fuels (LWR spent fuel, breeder reactor spent fuel, research reactor spent fuel); (4) miscellaneous DOE materials (beneficial-use materials, radioactive sodium waste)

31 (SAND-82-0664-Vol.2-App.-Pt.2, pp 359-391) Subseabed nuclear waste disposal: conceptual ship description. Miller, M.M. (Massachusetts Inst. of Tech., Cambridge); Chryssostomidis, C.; Shirley, C.G. Jul 1982. NTIS, PC A99/MF A01

In Subseabed disposal program annual report, JanuarySeptember 1981. Volume Il. Appendices (principal investigator progress reports). Part 2.

Concepts for transporting radioactive waste by ship from a specialized port facility to an ocean site for disposal in the subseabed bave been investigated to develop a reference transportation system, for risk analysis purposes. The investigation included ship design requirements, radioactive material storage methods, and methods of handling this material during loading at the port and in preparation for emplacement in the subseabed. In this phase of the work, emplacement of free-fall penetrators was assumed. The design concept has been developed from a review of existing and developing technologies for handling and storage of radioactive materials and from considerations of shipboard conditions. The concept incorporates transfer casks for loading the ship with assembled penetrators, a water pool for storage aboard ship while in transit, and individual drop stations in the pool for the penetrators. The concept is described at a level of detail which elucidates the main ideas; the direction of further analyses which would be required in order to carry concept development forward is also indicated.

(SAND-82-1994C) Experimental and analytical program to evaluate packaging and target hardness as related to nuclear waste transportation systems. Sutherland, S.H.; Allen, G.C.; Yoshimura, H.R.; Huerta, M. (Sandia National Labs., Albuquerque, NM (USA); Southwest Engineering Associates, El Paso, TX (USA)). 1983. Contract AC04-76DP00789. 8p. (CONF-830528-28). NTIS, PC A02/MF A01. Order Number DE83013471.

From 7, international symposium on packaging and transportation of radioactive materials; New Orleans, LA, USA (15 May 1983). Portions are illegible in microfiche products.

This paper describes a portion of an analytical/experimental program being conducted to determine the effects of target and packaging characteristics on the structural response of containers subjected to impact tests. As described, the experimental portion of the program will utilize half-scale models of a generic high-level radioactive materia shipping cask. The models will be impacted at different velocities, in an end-on orientation, into a steel faced target, a concrete target, a typical soil and other materials as needed to envelope other potential target effects. The analytical studies are being conducted with a dynamic finite-element computer program which calculates the detailed response of the container under the different impact conditions. The results of the analyses indicate that a slight amount of yielding in the target can substantially reduce the amount of damage that a container sustains compared to using a very rigid target. Results of the analytical studies are to be compared to the experimental results from tests which are currently being conducted.

33 (SAND-82-1997C) Transportation system preconceptual description for subseabed disposal of highlevel waste. Shirley, C.G.; McClure, J.D.; Miller, M.; Chryssostomidis, C.; Giovanetti, R.C.; Wee, C.S. (Sandia National Labs., Albuquerque, NM (USA); Massachusetts Inst. of Tech., Cambridge (USA)). 1983. Contract AC04-76DP00789. 9p. (CONF830528-29). NTIS, PC A02/MF A01. Order Number DE83013684.

From 7.. international symposium on packaging and transportation of radioactive materials; New Orleans, LA, USA (15 May 1983).

In support of an environmental assessment of the subseabed concept for nuclear waste disposal, which is being evaluated in the US Department of Energy (DOE) Subseabed Disposal Program (SDP), a preconceptual description has been developed for land and sea transportation systems and for a port facility where the land and sea systems interface. The complete system has a reference capacity for shipping and disposing of the commercial high-level waste (CHLW) generated from reprocessing $3000 \mathrm{MTHM} / \mathrm{yr}$ of spent reactor fuel elements ( 1950 canisters/yr). Two scenarios are considered: (1) A 1500 MTHM/yr reprocessing plant located in the Southeast US shipping to an East coast port 500 miles away from which the waste is transported to an Atlantic disposal site; and a $1500 \mathrm{MTHM} / \mathrm{yr}$ reprocessing plant located in the Northwest US shipping to a West coast port 500 miles away from which the waste is transported to a Pacific disposal site. (2) A $3000 \mathrm{MTHM} / \mathrm{yr}$ reprocessing plant located in the Southeast US shipping $50 \%$ of the CHLW produced to an East coast port 500 miles away for disposal in the Atlantic and shipping the other $50 \%$ to a West coast port $\mathbf{2 0 0 0}$ miles away for disposal in the Pacific.

34 (SAND-82-7202) High-Level Waste TransportationSystem Development Program design report for Fiscal Year 1980. (General Atomic Co., San Diego, CA (USA)). Jan 1982. Contract AC04-76DP00789. 230p. (GA-A-16124; TTC-0202). NTIS, PC A11/MF A01. Order Number DE83002965.

This report summarizes the work done on the high-level defense waste (HLDW) transportation system at General Atomic in FY 80. This system will transport HLDW from the Defense Waste Processing Facility (DWPF) at Savannah River to a federal repository. A design goal of the HLDW transportation system is also to be able to transport solidified HLDW generated at the Hanford and Idaho sites and solidified commercial high-level waste generated at West Valley. Design concepts have been generated on the basis of the Savannah River DWPF reference waste form, which is borosilicate glass placed in a $610-\mathrm{mm}$ (24-in.) diameter by $3-\mathrm{m}$ ( $9 \mathrm{ft}-10 \mathrm{in}$.) long stainless steel canister. Work was directed toward establishment of a system design basis and consolidated waste criteria as well as selection of a reference design. The reference design is an $81,650-\mathrm{kg}$ (90-ton), solid-steel-body rail cask with a convertible basket and a five- to eight-canister capacity. The cask is carried on a standard four-axle rail car with a maximum gross vehicle weight of $119,300 \mathrm{~kg}(263,000 \mathrm{lb})$. $56 \cdot$ figures, 56 tables.

36 (SAND-83-0867) Preliminary analysis of the cost and risk of transporting nuclear waste to potential candidate commercial repository sites. Wilmot, E.L.; Madsen, M.M.; Cashwell, J.W.; Joy, D.S. (Sandia National Labs., Albuquerque, NM (USA); Oak Ridge National Lab., TN (USA)). Jun 1983. Contract AC04-76DP00789. 59p. (CONF-830528-54; SAND-83-0867C). NTIS, PC A04/MF A01. Order Number DE83016348.

From 7. international symposium on packaging and transportation of radioactive materials; New Orleans, LA, USA (15 May 1983).

This report documents preliminary cost and risk analyses that were performed in support of the Nuclear Waste Terminal Storage 
(NWTS) program. The analyses compare the costs and hazards of transporting wastes to each of five regions that contain potential candidate nuclear waste repository sites being considered by the NWTS program. These regions are: the Gulf Interior Region, the Permian Basin, the Paradox Basin, Yucca Mountain, and Hanford. Two fuel-cycle scenarios were analyzed: once-through and reprocessing. Transportation was assumed to be either entirely by truck or entirely by rail for each of the scenarios. The results from the risk analyses include those attributable to nonradiological causes and those attributable to the radioactive character of the wastes being transported. 17 references.

36 (UCRL-53154) Swedish nuclear waste efforts. Rydberg, J. (Lawrence Livermore National Lab., CA (USA)). Sep 1981. Contract W-7405-ENG-48. 149p. NTIS, PC A07/MF A01. Order Number DE82016139.

Portions of document are illegible.

After the introduction of a law prohibiting the start-up of any new nuclear power plant until the utility had shown that the waste produced by the plant could be taken care of in an absolutely safe way, the Swedish nuclear utilities in December 1976 embarked on the Nuclear Fuel Safety Proiect. which in November 1977 presented a first report, Handling of Spent Nuclear Fuel and Final Storage of Vitrified Waste (KBS-I), and in November 1978 a second report, Handling and Final Storage of Unreprocessed Spent Nuclear Fuel (KBS II). These summary reports were supported by 120 technical reports prepared by 450 experts. The project engaged 70 private and governmental institutions at a total cost of US \$15 million. The KBS-I and KBS-II reports are summarized in this document, as are also continued waste research efforts carried out by KBS, SKBF, PRAV, ASEA and other Swedish organizations. The KBS reports describe all steps (except reprocessing) in handling chain from removal from a reactor of spent fuel elements until their radioactive waste products are finally disposed of, in canisters, in an underground granite depository. The KBS concept relies on engineered multibarrier systems in combination with final storage in thoroughly investigated stable geologic formations. This report also briefly describes other activities carried out by the nuclear industry, namely, the construction of a central storage facility for spent fuel elements (to be in operation by 1985), a repository for reactor waste (to be in operation by 1988), and an intermediate storage facility for vitrified high-level waste (to be in operation by 1990). The $R$ and $D$ activities are updated to September 1981.

37

(UCRL-53248) Instrumentation report 1: specification, design, calibration, and installation of instrumentation for an experimental, high-level, nuclear waste storage facility. Brough, W.G.; Patrick, W.C. (Lawrence Livermore National Lab., CA (USA)). Jan 1982. Contract W-7405-ENG-48. 101p. NTIS, PC A06/MF A01. Order Number DE82016313.

Portions of document are illegible.

The Spent Fuel Test-Climax (SFT-C) is being conducted $420 \mathrm{~m}$ underground at the Nevada Test Site under the auspices of the US Department of Energy. The test facility houses 11 spent fuel assemblies from an operating commercial nuclear reactor and numerous other thermal sources used to simulate the near-field effects of a large repository. We developed a large-scale instrumentation plan to ensure that a sufficient quality and quantity of data were acquired during the three- to five-year test. These data help satisfy scientific, operational, and radiation safety objectives. Over $\mathbf{8 0 0}$ data channels are being scanned to measure temperature, electrical power, radiatiun, ait now, dew point, stress, displacement, and equipment operation status (on/off). This document details the criteria, design, specifications, installation, calibration, and current performance of the entire instrumentation package.

38 Background and historical perspectives for monitored retrievable storage. Vieth, D.L.; Stein, R. (U.S. Department of Energy, Washington, DC 20545). Transactions of the American Nuclear Society; 41: 126(Jun 1982). (CONF-820609-).

From American Nuclear Society annual meeting; Los Angeles, CA, USA (6 Jun 1982).

ECONOMICS; HIGH-LEVEL RADIOACTIVE WASTES; PUBLIC OPINION; RADIOACTIVE WASTE MANAGEMENT; RETRIEVAL SYSTEMS; SAFETY; SPENT FUEL STORAGE; RADIOACTIVE WASTE STOR $\wedge$ GE locations. Kirkwood, C.W.; Ko, S.C. (Woodward-Clyde Consul- tants, Three Embarcadero Center, Suite 700, San Francisco, CA 94111). Transactions of the American Nuclear Society; 41: 126-127(Jun 1982). (CONF-820609-).

From American Nuclear Society annual meeting; Los Angeles, CA, USA (6 Jun 1982).

ECONOMIC ANALYSIS; FUEL CYCLE; HIGH-LEVEL RADIOACTIVE WASTES; RADIOACTIVE WASTE MANAGEMENT; RETRIEVAL SYSTEMS; SENSITIVITY ANALYSIS; SITE SELECTION; SPENT FUEL STORAGE; RADIOACTIVE WASTE STORAGE; RADIOACTIVE WASTE FACILITIES

40 Economics of monitored retrievable storage of nuclear wastes. Morissette, R.P. (General Atomic Company, P.O. Box 81608, San Diego, CA 92138). Transactions of the American Nuclear Society; 41: 127-129(Jun 1982). (CONF820609-).

From American Nuclear Society annual meeting; Los Angeles, CA, USA (6 Jun 1982).

ECONOMIC ANALYSIS; FUEL CYCLE; HIGH-LEVEL RADIOACTIVE WASTES; RADIOACTIVE WASTE MANAGEMENT; RETRIEVAL SYSTEMS; SPENT FUEL STORAGE; RADIOACTIVE WASTE STURAGE

41 Conceptual design of monitored retrievable storage faclities. Wilde, R.T. (Rockwell Hanford Operations, P.O. Box 800, Richland, WA 99352). Transactions of the Americun Nuclear Society; 41: 129-130(Jun 1982). (CONF-820609-). From American Nuclear Society annual meeting; Los Angeles, CA, USA (6 Jun 1982).

COST; DESIGN; DRY STORAGE; FUEL RACKS; HIGHLEVEL RADIOACTIVE WASTES; RADIOACTIVE WASTE STORAGE; RETRIEVAL SYSTEMS; RADIOACTIVE WASTE FACILITIES

42 Risk assessment for the transportation of radioactive zeolite liners. Gallucci, R.H.V. (Pacific Northwest Lab., Richland, WA). pp 530-534 of Proceedings of the ANS topical meeting on the treatment and handling of radioactive wastes. Columbus, OH; Battelle Press (1982). (CONF-820424-).

From ANS topical meeting on treatment and handling of radioactive wastes; Richland, WA, USA (19 Apr 1982).

The accident risk is estimated for the shipment of two zeolite liners containing radioactive cesium and strontium. Each liner, assumed to hold $68,200 \mathrm{Ci}$ and sealed inside a CNS 1 to $13 \mathrm{C}$, type-B shipping cask, is transported by truck over a $4200-\mathrm{km}$ route. The risk to the population along the route is calculated for potential transportation accidents involving fire, impact, and puncture forces. The total risk is 5.3E-7 manrem (50-year inhalation dose) and the maximum dose (from the leastlikely accident) is 0.7 man-rem. Both estimates are less than $0.1 \%$ of comparable risk measures for natural background radiation and spent fuel shipment accidents.

43 Economical dry storage of large amounts of spent fuel and vitrified high level radioactive waste. Bokelmann, R.F.; Kuehnel, R.R.; Leidinger, B.J.G. (Kraftwerk Union AG, Offenbach (Germany, F.R.)). pp 79-88 of Proceedings of an NEA specialist workshop on dry storage of spent fuel elements. Paris (France); OECD (1982), (CONF-820578-).

From OECD specialist workshop on techniques for the dry storage of spent fuel elements; Madrid, Spain (11 May 1982).

The economical dry storage of spent fuel assemblies and vitrified high-level radioactive waste demands facilities that fulfill, besides safety requirements, especially the following criteria: minimization of the specific space required for the storage of one fuel assembly or glass cylinder, minimization of the number of active systems and components to allevia te operation and maintenance, application of current materials. Comprehensive thermodynamical calculations on the single and dual cycle dry storage facilities, developed by KWU, have shown that the flow and temperature distributions within the storage rack and the other parts of the facility determine the feasibility of such dry storage systems. The parameters are mainly governed by the orientation and the pitch of the storage racks.

44 Evaluation of proposed methods of radioactive material container tie-down design for truck transport. Lamoreaux, 
G.H. (Sandia, Albuquerque, NM 87185). Transactions of the American Nuclear Society; 39: 192(1981). (CONF-811103-). 1981).

From ANS winter meeting; San Francisco, CA, USA (29 Nov

DESIGN; EVALUATION; HIGH-LEVEL RADIOACTIVE WASTES; LAND TRANSPORT; SPENT FUEL CASKS; TRUCKS; RESTRAINTS; CASKS; RADIOACTIVE MATERIALS; WASTE TRANSPORTATION

45 Transport of highly radioactive materials. Salmon, A. (Pacific Nuclear Transport Ltd., Risley, Warrington, England). Transactions of the American Nuclear Society; 35: 8(1980). (CONF-801107-). Nov 1980)

From ANS international conference; Washington, DC, USA (17

HIGH-LEVEL RADIOACTIVE WASTES; IAEA SAFEGUARDS; INTERNATIONAL COOPERATION; NUCLEAR FUELS; RADIOACTIVE MATERIALS; SAFETY STANDARDS; TRANSPORT; WASTE TRANSPORTATION

\section{Marketing And Economics}

46 (AAEC/E-526) Use of thorium as an alternative nuclear fuel. Wilson, D.J. (Australian Atomic Energy Commission Research Establishment, Lucas Heights). Apr 1982. 137p. NTIS (US Sales Only), PC A07/MF A01. Order Number DE82703266. tion37 refs.

Microfiche only, copy does not permit paper copy reproduc-

The use of thorium as an alternative or supplementary nuclear fuel is examined and compared with uranium. A description of various reactor types and their suitability to thorium fuel, and a description of various aspects of the fuel cycle from mining to waste disposal, are included. Comments are made on the safety and economics of each aspect of the fuel cycle and the extension of the lifetime of nuclear fuel.

47

(ONWI-212, pp 50-61) Socioeconomic etaluntions: 8 Dec 1980. NTIS, PC A17/MF A01. (CONF-801209-).

From 2. annual National Waste Terminal Storage (NWTS) information meeting; Columbus, OH, USA ( 9 Dec 1980).

The session on Socioeconomic Evaluations consisted of the forlowing seven papers: (1) Socioeconomic Considerations in Nuclear Waste Management; (2) High-Level Radioactive Waste - the Social Decision; (3) Role of Impact Assessment in Program Planning - A Social Science Perspective; (4) Social and Demographic Impacts Associated with Large-Scale Resource Developments - Implications for Nuclear Waste Repositories; (5) Economic and Fiscal Impacts of Largescale Development Projects - Implications for Nuclear Waste Repositories; (6) Socioeconomic Analyses of the Proposed Waste Isolation Pilot Plant Project; and (7) Existing Institutional Arrangements and Fiscal Incentives for Siting Publicly Sensitive Facilities. (ATT)

\section{Waste Management}

(ANL-81-35) Fuel-cycle programs, Quarterly prosress report, January-March 1981 . Steindler, M.J.; Vogler, S.; Vandegrift, G.F. (Argonne National Lab., IL (USA)). Mar 1982. Contract W-31-109-ENG-38. 116p. NTIS, PC A06/MF A01. Order Number DE82014373.

A program continues for the development of an interim waste form that can be transported from facilities where waste is generated to terminal waste processing. Measurements of rates of leaching from simulated nuclear waste forms ar continuing. The data suggest that leaching from SRL glasses is inhibited by the formation of a Si/ $\mathrm{Ca} / \mathrm{Fe}$; Al-rich surface layer. Impact tests of solid alternative waste forms (glass and ceramic) are being performed to assess source terms of airborne release (from particle size distributions) and to assess the increases in source terms of leach rates (from increases in surface area). Logging techniques are being developed to measure the relative amount of residual oil in a depleted oil reservoir. A simple technique for the elution of pore fluid (groundwater) from igneous rocks is described, using a special coreholder, as is the elution of brine (in the same apparatus) from a core of Precambrian granite. Testing and development of equipment for the destructive analysis of full-length irradiated fuel rods from the LWBR is in progress.
(ANL-81-53) Fud-cycle programs. Quarterly progress report, Aprit-June 1981. Steindler, M.J.; Vogler, S.; Vandegrift, G.F. (Argonne National Lab., IL (USA)). Mar 1982. Contract W-31-109-ENG-38. 110p. NTIS, PC A06/MF A01. Order Number DE82014374.

Portions of document are illegible.

A program continues for the development of an interim waste form capable of being transported from facilities where waste is generated to terminal waste processing. Measurements of rates of leaching from simulated waste glasses are continuing. Laboratory-scale impact teats of solid alternative waste forms are being performed to assess source terms of airborne release and to assess the increases in source terms of leach rates. A review was made of the methodology under development for the characterization of the impact behavior of brittle materials. Logging techniques are being developed to measure the relative amount of residual oil in a depleted oil reservoir by injecting gamma-active solution into it. Analyses of samples from the Notch Peak (House Range, Utah) intrusive body suggest that groundwater flow was through fractures and not through the rock matrix, indicating that no new processes of migration need be considered in the design of a nuclear waste repository. Testing and development of equipment for the destruc. tive analysis of full-length irradiated fuel rods from the LWBR is in progrese.

50 (ANL-81-82) Fod cycle programs, Quarterly progress report, Jely-Sejtember 1981. Steindler, M.J.; Bates, J.K.; Bowers, D.L. (Argonne National Lab., IL (USA)). May 1982. Contract W-31-109-ENG-38. 132p. NTIS, PC A07/MF A01. Order Number DE83000921.

Portions of document are illegible.

A program continues for the development of an interim waste form for transport. Work on processes for converting high-level liquid waste to solids included the preparation of $\mathrm{NaNO} / \mathrm{NaNO}_{2}$ salt monoliths, phosphate-immobilized product, and silicate-agglomerated $\mathrm{Fe}_{2} \mathrm{O}_{0}$ pellets. Rates of dissolution of various interim waste forms in quiescent water were measured; the pellets were found to be exceptionally stable. A method of measuring rates of leaching from simulated waste glasses using ridicactive spiking has been demonstrated. Leaching tests of glass samples irradiated with $3.5-\mathrm{MeV}{ }^{4} \mathrm{He}^{2+}$ ions indicated that the leach rates of irradiated samples were as little as one-fourth of these of nonirradinted glass. Laboratory-scale impact tests of solid alternative waste forms are being performed to measure the BET surface areas of the fragments. The surface areas increased smoothly with increasing impact enerey. A revieiw of the methodology for the impact bebavior of brittle materials included the following: correlation of the values of several parameters obtained in standard impact tests on Pyrex and SRL 131 glass with energy denisities, comparison of lognormal parameters for fragments of SRL 131 glass obtained by six measurement methods, and comparison of the effects on lognormal parameters of axial vs. diametral impect of 25-mm-OD $\times 25-\mathrm{mm}$ specimens.

81

(AARC-1136) Thermal and radiation stability of simalated lift-lerel vitififed raste products. Rastogi, R.C. (ed.). (Bhabha Atomic Research Centre, Bombay (India)). 1981. 41p. NTIS (US Sales Only), PC A03/MF A01. Order Number DE82904604.

Portions of document are illegible.

Twelve different simulated wastes contained waste oxide, $\mathrm{SiO}_{2}$, $\mathrm{B}_{2} \mathrm{O}_{2} \mathrm{Na} 2 \mathrm{O}$, and others. Thermal analysis was carried out up to $1000^{\circ} \mathrm{C}$, using DTA, differential scanning calorimetry, thermogravimetry, and thermal expansion. One of the 12 wastes was irradiated in a reactor to simulate $10^{19}$ alpha decays per gram of glass. No crystallization of new ir absorption bands could be detected in the irradiated sample. 17 figures, 3 tables. (DLC)

62 (CONF-820609-10) Integrated data base for spent focland radweste: inventories. Notz, K.J.; Carter, W.L.; Kibbey, A.H. (Oak Ridge National Lab., TN (USA)). 1982. Contract W-7405-ENG-26. 7p. NTIS, PC A02/MF A01. Order Number DE82017409.

From American Nuclear Soxiety annual meeting; Los Angelos, CA, USA (6 Jun 1982).

The Integrated Data Base (IDB) program provides and maintains current, integrated data on spent reactor fuel and radwaste, including historical dats, current inventories, projected inventories, and material characteristics. The IDB program collects, organizes, integrates, 
and - where necessary - reconciles inventory and projection (I/P) and characteristics information to provide a coherent, self-consistent data base on spent fuel and radwaste.

53 (CONF-820845-, pp 17-29) Historical overview. Perge, A. (Dept. of Energy, Washington, DC). Dec 1982. NTIS, PC A25/MF A01.

From Alpha-contaminated waste management meeting; Gaithersburg, MD, USA (10 Aug 1982).

An historical review is presented of radioactive waste manageent in the United States from the 1940's to the present. It covers waste generation, accidents, clean up operations, criteria for acceptable levels for materials released to the environment and focuses on the storage and disposal aspects of high-level and transuranic wastes.

64 (CONF-820845-, pp 117-142) Alpha-contaminated waste from reprocessing of nuclear fuel. Sumner, W. (Allied General Nuclear Services, Barnwell, SC). Dec 1982. NTIS, PC A25/MF AÓ1.

From Alpha-contaminated waste management meeting; Gaithersburg, MD, USA (10 Aug 1982).

The anticipated alpha-waste production rates from the Barnwell Nuclear Fuel Reprocessing plant is discussed. The estimated alphawaste production rate from the 1500 metric ton/year plant is about $85,000 \mathrm{ft}^{0} /$ year at the $10 \mathrm{nCi} / \mathrm{g}$ limit. Most of this waste is estimated to come from the separation facility, and the major waste sources were cladding, which was $27 \%$, and low-level contact-handled general process trash, which was estimated at $32 \%$ of the total. It was estimated that $45 \%$ of the waste was combustible and $72 \%$ of the waste was compactible. These characteristics could have a significant impact on the final volumes as disposed. Changing the alpha-waste limit from $10 \mathrm{nCi} / \mathrm{g}$ to $100 \mathrm{nCi} / \mathrm{g}$ was estimated to reduce the amount of alpha waste produced by about $20 \%$. Again, the uncertainty in this value obviously has to be substantial. One has to recognize that these estimates were just that; thcy were not based on any operating experience. The total plutonium losses to waste, including the high-level waste, was estimated to be $1.5 \%$ The cladding waste was estimated to be contaminated with alpha emitters to the extent of $10^{4}$ to $10^{5} \mathrm{nCi} / \mathrm{g}$.

65 (DOE/EA-0179) Environmental assessment: wasteform selection for SRP high-level waste. (Department of Energy, Washington, DC (USA). Assistant Secretary for Defense Programs; Department of Energy, Aiken, SC (USA). Savannah River Operations Office). Jul 1982. 92p. NTIS, PC A05/MF A01. OIder Number DE82019365.

DOE has recently decided to construct and operate a Defense Wastc Processing Facility (DWPF) at the Savannah River Plant (SRP) to immobilize the high-level radioactive waste generated and stored pending disposal in a federal geologic repository. In the immobilization process the high-activity fraction of the SRP high-level waste is mixed with glass frit to form the feed for the melter. I he glass is cust frum an electric-heated, ceramic-lined melter into canisters. Crystalline ceramic, the leading alternative to borosilicate glass, also appears to be an acceptable form for immobilizing the SRP high-level waste. Both are expected to meet regulations and repository acceptance criteria. The assessment also shows that the environmental effects of disposing of SRP high-level waste as a crystalline ceramic form would not differ significantly from the projected effects for disposal of the borosilicate glass form. A comprehensive evaluation program led to the recommendation of borosilicate glass as the preferred waste form. 10 figures, 19 tables. (DLC)

56 (DOE/ET/40110-1) Information base for commercial radioactive-waste management. (Mitre Corp., McLean, VA (USA). METREK Div.). Jul 1982. Contract ACO180ET40110. 286p. NTIS, PC A13/MF A01. Order Number DE83001240.

Portions of document are illegiblc.

This document provides an overview of the Department of Energy's responsibilities and plans for the management of radioactive wastes generated as part of the commercial fuel cycle, as well as that from industry, research, and medical institutions of the nation. The report also highlights the federal interagency cooperation and integration of programs needed to assure a national effort to isolate existing and future radioactive wastes from the biosphere, so that these wastes pose no significant threat to the environment or to public health and safety.
This Information Base provides a status report as of December 1981, and outlines a strategy from that point and on into the future, when all of the wastes types will be safely and permanently disposed. The information provided herein is organized as follows: high-level waste; spent nuclear fuel; repository development; low-level waste; uranium mill tailings; and wastes from decommissioning and decontamination. The Department of Energy will continue to provide the commercial sector and states with the results of years of experimentation and technology development in the radioactive waste field. The Department will also continue to provide coordination and the needed information transfer, such as this Information Base, to assure that the waste management plans and programs, outlined in this document, will move forward in a timely fashion.

67 (DOE/NE-0017-1) Spent fuel and radioactive-waste inventories, projections, and characteristics. (Oak Ridge National Lab., TN (USA)). Oct 1982. Contract W-7405-ENG26. 350p. NTIS, PC A15/MF A01. Order Number DF.83001800.

Portions of document are illegible.

Current inventories and characteristics of commercial spent fuels and both commercial and US Department of Energy radioactive wastes were compiled, based on the most reliable information available from government sources and the open literature, technical reports, and direct contacts. Future waste and spent fuel to be generated over the next $\mathbf{4 0}$ years, and characteristics of these materials are also presented, based on a present DOE/EIA projection of US commercial nuclear power growth and expected defense-related and industrial and institu. tional activities. Materials considered, on a chapter-by-chapter basis, are: spent fuel, high-level waste, transuranic waste, low-level waste, remedial action waste, active uranium mill tailings, airborne waste, and decommissioning. For each category, current and projected inventories are given through the year 2020 . The land usage requirements are given for storage/disposal of low-level and transuranic wastes, and for the present inventories of inactive uranium mill tailings. For each waste category the radioactivity and thermal power arc calculated. Isotopic compositions and cost data are given for each waste type and for spent fuel.

68 (DOE/NE/44146-T1) Building the institutional capacity for managing commerclal high-level radioactive waste. (National Academy of Public Administration, Washington, DC (USA)). May 1982. Contract AC01-81NE44146. 61p. NTIS, PC A04/MF A01. Order Number DE82019468.

Portions of document are illegible.

This report presents the findings and recommendations of an Academy pancl formed to look into the inajor instltutiuntul issues associated with the commercial radioactive waste management program. The two major areas examined by the Panel wcrc: (1) intergovernmental relationships, how federal, state, local and Indian tribal council governments relate to each other in the planning and implementation of a waste managemont program; and (2) interagency relationships, how the federal agencies with major responsibilities in this public policy arena interact with each other. The objective of the study was to apply the perspectives of public administration to a difficult and controversial question - how to devise and execute an effective waste management program workable within the constraints of the federal system.

59 (DOE/NE/44146-T2) Building the institutional capacity for managing commercial high-lovel radionctive waste. (National Academy of Public Administration, Washington, DC (USA)). May 1982. Contract AC01-81NE44146. 61p. NTIS, PC A04/MF A01. Order Number DE83001125.

Portions of document are illegible.

In July 1981, the Office of Nuclear Waste Management of the Department of Energy contracted with the National Academy of Public Administration for a study of institutional issues assoclated with the commercial radioactive waste management program. The two major sets of issues which the Academy was asked to investigate were (1) intergovernmental relationships, how federal, state, local and Indlan tribal council governments relate to each other in the planning and implementation of a waste management program, and (2) interagency relationships, how the fedcral agencies with major responsibilities in this public policy arena interact with each other. The objective of the study was to apply the perspectives of public administration to a difficult and controversial question - how to devise and execute an effective waste management program workable within the constraints of the federal system. To 
carry out this task, the Academy appointed a panel composed of individuals whose background and experience would provide the several types of knowledge essential to the effort. The findings of this panel are presented along with the executive summary. The report consists of a discussion of the search for a radioactive waste management strategy, and an analysis of the two major groups of institutional issues: (1) intergovernmental, the relationship between the three major levels of government; and (2) interagency, the relationships between the major federal agencies having responsibility for the waste management program.

60 (DOE/NV/10250-1) Commercial nuclear waste research and development program. Quarterly report, OctoberDecember 1981. Hakl, A.R. (Westinghouse Electric Corp., Mercury, NV (USA). Advanced Energy Systems Div.). Jan 1982. Contract AC08-82NV10250. 20p. NTIS, PC A02/MF A01. Order Number DE82016026.

This document is a report of activities performed by AESDNevada Operations in meeting task objectives of the Nevada Nuclear Waste Storage Investigations (NNWSI) planning documentation for Fiscal Year (FY) 1982. Significant activities were: Completion of a fuel assembly exchange was successfully in support of the Spent Fuel TestClimax (SFT-C) Program; input was provided, in response to FY 1982 guidance, to DOE/NV and the Office of Nuclear Waste Isolation (ONWI) for a proposed co-located Generic Packaging Facility (GPF); in response to FY 1982 guidance, drafts were prepared of facility layouts, material and process flow charts, equipment requirements, costs, and schedules for packaging (at E-MAD) of 28 alternative configurations of defense high-level waste, disassembled spent fuel, and commercial high-level waste in support of a proposed Test and Evaluation Facility (TEF); and quarterly dry run operations for canister emplacement in a drywell and in the Hot Bay Transfer Pit using the Emplacement/ Installation Vehicle (EIV).

61 (DOE/NV/10250-2) Commercial nuclear waste research and development program. Quarterly report, JanuaryMarch 1982. Hakl, A.R. (Westinghouse Electric Corp., Mercury, NV (USA). Advanced Energy Systems Div.). Apr 1982. Contract AC08-82NV10250. 21 p. NTIS, PC A02/MF A01. Order Number DE82016041.

The Westinghouse Advanced Energy Systems Division Nevada Operations, under contract to the US Department of Energy, provides trained personnel and services at the E-MAD Facility required for nuclear materials handling, packaging, data gathering, and other related activities in support of the Commercial Nuclear Waste Research and Development Programs. Support is provided to research programs, including: Waste packaging experiments, spent fuel test - Climax (SFTC), Hanford Near Surface Test Facility (NSTF), Tests of spent reactor fuel interim handling and storage, tests to assess safety of transport systems for spent reactor fuel and solidified high level nuclear waste, and other special waste package development and handling tests as requested by the DOE. Activities reported have been performed to meet subtask objectives of the Nevada Nuclear Waste Storage Investigations (NNWSI) planning documentation for Fiscal Year 1982: Preliminary facility designs, process flow charts, and recommendations for equipment and services which would be required in a Generic Packaging Facility (GPF) co-located with the proposed Test and Evaluation Facility (TEF); E-MAD facility descriptions, logic diagrams, and process flow charts for E-MAD packaging of nuclear waste for the proposed TEF, if the TEF were not located on the Nevada Test Site; Preliminary cost estimates and schedules for a proposed large scale fuel storage demonstration, as requested by DOE/Headquarters; a remote source integrity check of the Cobalt 60 source; calibration and recertification of the Astro Arc automatic welding system. Installation of illustrated alarin panel for the E-MAD Remote Area Monitor (RAM) system in the E-MAD gatehouse; and installation of the RAM/CAM (Constant Air Monitoring) system computer interface hardware.

62 (DOE/TIC-3394) Radioactive waste inventories and projections. McLaren, L.H. (ed.). (Department of Energy, Oak Ridge, TN (USA). Technical Information Center). Nov 1982. 17p. NTIS \$7.00. Order Number DE82012267.

Portions of document are illegible. Printed copy available until stock is exhausted.

This bibliography contains information on radioactive waste inventories and projections included in the Department of Energy's Energy Data Base from January 1981 through September 1982. The arrangement is by report number for reports, followed by nonreports in reverse chronological order. These citations are to research reports, journal articlcs, books, patents, theses, and conference papers from worldwide sources. Five indexes, each preceded by a brief description, are provided: Corporate Author, Personal Author, Subject, Contract Number, and Report Number. (25 abstracts).

63 (DP-80-125-3) Waste-management program technical progress report, July-September 1980. (Du Pont de Nemours (E.I.) and Co., Aiken, SC (USA). Savannah River Plant; Du Pont de Nemours (E.I.) and Co., Aiken, SC (USA). Savannah River Lab.). Dec 1981. Contract AC09-76SR00001. 208p. NTIS, PC A10/MF A01. Order Number DE82008953.

Portions of document are illegible.

This quarterly report provides current information on operations and development programs for the management of radioactive wastes from operation of the Savannah River Plant. The studies on the Defense Waste Processing Facility Environmental Impact Statement, environmental and safety assessments, separation and concentration, waste form development and characterization, process and equipment development, the Defense Waste Processing Facility, transuranic waste, and low-level waste R \& D are a part of the Long-Term Waste Managcment Technology Program. The following studies are reported for the SR Interim Waste Operations Program: surveillance and maintenance, waste concentration, low-level effluent waste, waste tank evaluation, tank replacement/waste transfer, and solid waste storage. Work for the Office of Nuclear Waste Isolation is described.

64 (DP-81-125-3) Waste-management program. Technical progress report, July-September 1981. (Du Pont de Nemours (E.I.) and Co., Aiken, SC (USA). Savannah River Lab.). Jun 1982. Contract AC09-76SR00001. 122p. NTIS, PC A06/MF A01. Order Number DE82019745.

This quarterly report provides current information on operations and development programs for the management of radioactive wastes from operation of the Savannah River Plant. The studies on environmental and safety assessments, waste form development and characterization, and process and equipment development are a part of the LongTerm Waste Management Technology Program. The following studies are reported for the SR Interim Waste Operations Program: surveillance and maintenance, waste concentration, low-level effluent waste, tank replacement/waste transfer, process development, and solid waste storage and related activities.

65

(DP-82-125-4) Waste Management Program. Technical progress report, October-December 1982. (Du Pont de Nemours (E.I.) and Co., Aiken, SC (USA)). Jul 1983. Contract AC09-76SR00001. 205p. NTIS, PC A10/MF A01. Order Number DE83017050.

Portions are illegible in microfiche products. Original copy available until stock is exhausted.

This quarterly report provides current information on operations and development programs for the management of radioactive wastes from operation of the Savannah River Plant and offplant participants. The studies on environmental and safety assessments, in situ storage or disposal, waste from development and characterization, process and equipment development, and low-level waste management are a part of the Long-Term Waste Management Technology Program. The following studies are reported for the SR Interim Waste Operations Program: survcillance and maintenance, waste concentration, low-level effluent waste, tank replacement/waste transfer, and solid waste storage and related activities.

66 (DP-82-157-1) Composite quarterly technical report: long-term high-level-waste technology, January-March 1982. Cornman, W.R. (comp.). (Du Pont de Nemours (E.I.) and Co., Aiken, SC (USA). Savannah River Lab.). Aug 1982. Contract AC09-76SR00001. 72p. NTIS, PC A04/MF A01. Order Number DE83000531.

Progress is reported in the following areas: program management and support, waste preparation (sulidification and precipitation), waste fixation (in glass, concrete, or tailored ceramics), and final handling. Leaching data are given. (DLC)

67 (DP-MS-81-57) National long-term high-level waste-technology program. Gray, P.L. (Du Pont de Nemours 
(E.I.) and Co. Aiken, SC (USA). Savaninah River Lab.). 1981. Contract ACO9-76SR00001. 12p. (CONF-811103-120). NTIS, PC A02/MF A01. Order Number DE82008186. 1981).

From ANS winter meetins; San Francisco, CA, USA (29 Nov

The national program for lonsterm management of biph-tovel waste (HLW) from nuclear fuels reprocenting is discussed. This cover only DOE defense wastes. Current emphasis is on colidification of waste into a form that, along with additional barrien, may be permanently stored in a repository. An integrated national plan incoiporates all the elements of such'an overall HLW dispoeal system. Interim stomie is in near-aurface tanks at the Hanford and Savanati River sitea. At the Idabo site, waste is stored in biss after beine calcined. Some Idalio wate is liquid and is alno stored in tanibs before calcination. Retrieval ind immobilization of HLW -into colid, low-release form represent the major elements for which our longterm proyram has responsibility. Once solidified, the waste will temporarily remain onsite until the final disposal site is prepared for receipt of waste. Trumiportation, if neces sary, to the ultimate dispoeal site is the responsibility of the Trunsporta tion Technology Center at Sandia National Laboratory. Diapoial in a location remote from the human environment is the responibility of the Office of NWTS Integration (ONI) and Office of Nuclear Wate inola. tion (ONWI) operated by Battelle Memorial Institute, Columbus, Ohio. Currently, a geologic repository is favored for ultimate dispacal, although other poesibilitie such se seibed, icecap, spice, and nearsurface disposal are also being considered. The HLW technolosy por thon of the program (which involve immobilizing the HLW) includen (1) development of technolosy to support i realistic choice of in isolation system for each HLW site, (2) development of the candidate formi and proceseses with sufficient cale-up to provide desipn date for fuilscale facilities, and (3) construction and operation of these facilities. (ATT)

68 (DP-MS-83-4) Antyes of meclear fod and Mitr

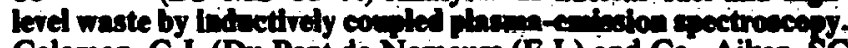
Coleman, C.J. (Du Pont de Nemours (E.I.) and Co., Aiken, SC (USA). Savannah River Lab.). 1983. Contract ACO976SR00001. 8p. (CONF-831025-10). NTIS, PC A02/MF. A01. Order Number DE84001592.

From 26. ORNL-DOE conference on analytical chemistry in energy technology; Knoxville, TN, USA (11 Oct 1983).

Containment of the inductivoly coupled plasm (ICP) iource has increased the application of ICP emisston spectroscopy at the Savannab River Laboratory (SRL). Nuclear materials are analyzed for up to 41 elements simultaneously with an ICP polychromitor detection aystem. This technique is used for determining trace impurities is unnivim that can adversely affect nuclear fuel processing. Uranium amples, after solvent extraction removal of unnium to reduce spectril interferences are satisfactorily analyzed for impurity elements. ICP emission speo troccopy is also effective for analyzing aludge from tho Savannah River Plant (SRP) high-level waste tants. Methods were developed for inilyzing a wide range of sludge compositions for major and minor elements. ICP analyses of waste tank sludge are required for SRP defenee wate solidification programs. Method development and anilyoes of typical samples will be presented.

68 (DP-MS-83-55) Saivinal Nher Fiant everitew. Morris, O.M. (Du Pont de Nemours (E.I.) and Co., Aiken, SC (USA). Savannah River Lab.). 1983. Contract ACO9 76SR00001. 16p. (CONF-830650-1). NIIS, PC A02/MF A01. Order Number DE83013340.

From FRG/US workshop on size reduction and decontamination facilities; Karlsruhe, F.R. Germany (27 Jun 1983).

Portions are illegible in microfiche products.

The Savannah River Plant (SRP) is located in the state of Sointh Carolina. SRP's primary function is the production of plutonium, tritium, and other special nuclear materials for the national defenive, for other governmental uses, and for some civilian purposes. The liguid high-Hevel wastes produced from these operntions are shown. Fiscion products from the separations plants are sent to underground waste storage tanks where the volume and mobility of the wastes are decreased by evaporation. Solid wastes generated by the facilities are cent to a 195 . acre burial ground. The beta-gamma and low-tevel alphe wastes are buried in trenches. TRU wastes extimated to contain > $10 \mathrm{nCi} / \mathrm{s}$ are stored retrievably on pads. The long-range plan for managing TRU waste at SRP is shown graphically.

70 (IDO-1010S) Environmental evaluation of alternatives for locis-term management of Defense high-level radioactive wastes at te Idibo Cremical Processing Plant. (Idaho National Engineering Lab., Idaho Falls (USA)). Sep 1982. Contract AC07-79ID01675. 665p. NTIS, PC A99/MF A01. Order Number. DE83002504.

Portions of document are illegible .

The U.S. Department of Energy (DOE) is considering the selection of a strategy for the long-term management of the defense highlevel wistes at the Idaho Chemical Processing Plant (ICP.P). This report describes the environmental impacts of alternative strategies. These altemative strategies include leaving the calcine in its present form at the Idabo National Engineering Laboratory (INEL), or retrieving and modifying the calcine to a more durable waste form and disposing of it either at the INEL or in an offsite repository. This report addresses only the alternatives for a program to manage the high-level waste generated at the ICPP. 24 figures, 60 tables.

71 (INIS-inf-6924) Manigement of nuclear fuel waste. Find rejort. (Select Committee on Ontario Hydro Affairs, Toronto, Ontario (Canada)). Jun 1980. 81p. NTIS (US Sales Only), PC A05/MF A01. Order Number DE82780586.

A Select Committee of the Legislature of Ontario was established to examine the affairs of Ontario Hydro, the provincial electrical utility. The Committee's terms of reference included examination of the waste management program being carried out jointly by the Ontario provincial government and the Canadian federal government. Public bearine were held which included private citizens as well as officials of organizations in the nuclear field and independent experts. Recommendations were made concerning the future direction of the Canadian fuel waste management program. (O.T.).

72

(NKA/A0-81-5) Enlanged Nordic cooperative prorai on Ecclear safety. Nordic study on reactor waste: main repart. (Nordisk Kontaktorgan for Atomenergispoergsmaal, Risoe (Denmark)). Aug 1981. 116p. (INIS-mf-7403). NTIS (US Sales Only), PC A06/MF AO1. Order Number DE82903434.

Portions of document are illegible.

In 1981, 14 nuclear power reactors in operation and 2 under construction in the Nordic countries. So far, the reactor waste originating from day-today operation of these plants has been stored in solidified form at the reactor sites. Within a few years a satisfactory disposal pro codure needs to be established. While the main $R$ and $D$ efforts in the waste field have earlier been devoted to the question of irradiated fuel and waste from reprocessing, there is therefore now an increased interest in reactor waste with its much lower radioactivity but somewhat larger volumes. Many of the methods developed to evaluate the safety of high lovel wiste can also be applied to reactor waste. Furthermore, the principles used in nuclear reactor safety analysis are essentially applicable. The main difference is the difficulty in defining possible accident sce narios in the case of reactor waste management systems. Since 1977 , efforts have been made in a joint Nordic study to examine which facts noed to be known in order to perform a compreliensive safoty assecsment of e roapter waste management system, and how the results can be interpreted. It turns out that even in the absence of well-defined requirements from the regulating authorities it is quite feasible to evaluate the afety of a proposed waste management arrangement and to ensure a balanced and reasonable safety level in all sub-systems. In the present atudy a reference system related to the waste generated over 30 years from aix $500 \mathrm{MW}$-reactors is examined. In the report it is demonstrated bow a safety assessment can be carried out in detail and how the resultin doeses can be presented together with their probabilities of occurrence. In come cases, especially in connection with the repository, probabilities are 20 low or 80 difficult to quantify that a probabilistic risk ascesoment is not meaningful. nent of meclear-waste-form criteria for the NRC. Task 3. Waste Grentory revlew. Majumdar, D.; Indusi, J.P.; Manaktala, H.K. (Brookhaven National Lab., Upton, NY (USA)). Feb 1982. 
Contract AC02-76CH00016. 267p. (BNL-NUREG-51458Vol.3). NTIS, PC A12/MF A01. Order Number DE82009883. Portions of document are illegible.

This inventory of high level nuclear waste in the US was prepared by the Nuclear Waste Management Division of Brookhaven National Laboratory for the Office of Nuclear Materials Safety and Safeguards of the US Nuclear Regulatory Commission. Existing and projected inventories of high level waste (HLW), transuranic (TRU) waste, and spent fuel will be required in making prudent decisions in the licensing of high level waste repositories. This report describes the quantity and characteristics of existing and projected HLW and TRU waste from the government nuclear programs, the existing HLW and TRU waste from the commercial power industry, and the existing and projected spent fuel from the commercial nuclear power industry.

74 (ORNL/NFW-81/34, pp 75-100) EPRI's programs in low-level waste management. Williams, R.F. (Electric Power Research Inst., Palo Alto, CA). Dec 1981. NTIS, PC A25/MF A01. (CONF-811130-).

From 3. annual DOE participants information meeting on lowlevel waste management; New Orleans, LA, USA (4 Nov 1981).

The programs at the Electric Power Research Institute (EPRI) are conducted in two departments. Those rclated to low-level waste siting technology and safety analysis are within the Systems and Materials Department. Those that relate to process technology for low-level waste disposal, equipment that would be at a power plant, are in the Engineering and Operations Department. The EPRI program objectives in waste disposal are: (1) help establish technically sound disposal regulations and licensing procedures; (2) focus $R \& D$ programs on developing essential information; (3) help implement safe disposal in a reasonable time frame; (4) provide input to needed legislation; (5) provide input to public understanding. In the area of low-level waste disposal, EPRI's objective is to provide a technology package, including the analysis of tradeoffs between waste form package and site characteristics to open a new low-level waste disposal site. To accomplish the low-level waste siting technology objective, the focus is on preparing a handbook illustrating the necessary analysis to license a new low-level waste disposal site. EPRI's accomplishments in 1982 are: retention quotient methodology for high-level waste extended to low-level waste; and development of low-level waste siting technology. Programs that are ongoing in the areas of process technology are: assessment of volume reduction. technologies; assessment of incinerator performance; identification and reduction of low-level radioactive waste source; use of microwave technology in solidifying resins.

76 (ORNL/NFW-81/34, pp 101-104) Role of utility nuclear waste management group. Stanford, R.E.L. (Edison Electric Inst., Washington, DC). Dec 1981. NTIS, PC A25/MF A01. (CONF-811130-).

From 3. annual DOE participants information meeting on lowlevel waste management; New Orleans, LA, USA (4 Nov 1981).

The Utility Nuclear Waste Management Group (UNWMG) is a group of 39 nuclear utilities which support a general program which is under the administration of Edison Electric Institute (EEI), but which actually operates quite separately. The function of the group is primarily to interact with Congress in the development of desired legislation, interact with NRC and EPA in the development of regulations, and interact with DOE in the carrying out of programs in which they are interested. The group is organized into four working groups. One devotes its efforts to high-level waste activities, one to low-level waste interests, one to spent fuel storage and reprocessing, and a fourth to public information. Each of these working groups has a number of task forces that are organized to respond to a particular need at the time. The activities of each group are discussed briefly.

76 (ORNL/TM-8061) Graphical and tabular summaries of decay characteristics for once-through PWR, LMFBR, and FFTF fuel cycle materials. Croff, A.G.; Liberman, M.S.; Morrison, G.W. (Oak Ridge National Lab., TN (USA)). Jan 1982. Contract W-7405-ENG-26. 144p. D. Order Number DE82007128.

Purtions of document are illegible.

Based on the results of ORIGEN2 and a newly developed code called ORMANG, graphical and summary tabular characteristics of spent fuel, high-level waste, and fuel assembly structural material (cladding) waste are presented for a generic pressurized-water reactor
(PWR), a liquid-metal fast breeder reactor (LMFBR), and the Fast Flux Test Facility (FFTF). The characteristics include radioactivity, thermal power, and toxicity (water dilution volume). Given are graphs and summary tables containing characteristic totals and the principal nuclide contributors as well as graphs comparing the three reactors for a single material and the three materials for a single reactor.

77 (ORNL/TM-8322) Radwaste inventories and projections: an overview. Notz, K.J. (ed.). (Oak Ridge National Lab., TN (USA)). Jul 1982. Contract W-7405-ENG-26. 32p. NTIS, PC A03/MF A01. Order Number DE82018208.

The Integrated Data Base program was set up to provide fully integrated and reconciled inventories, characteristics, and projections for spent nuclear fuel and all categories of radioactive waste. Eight summary papers, six of which were presented at an ANS special session in Los Angeles in June 1982, are included in this report: data base needs and functions: national planning; integrated data base for spent fuel and radwaste: inventories; integrated data base projections; RAWSYM: radioactive waste management system; NWTS program waste projection data needs; low-level waste management data base system; waste sludge composition the Savannah River Plant; and summary of characteristics of transuranic waste found at DOE sites. (DLC)

78 (OTA-0-172) Managing commercial high-level radioactive waste: summary. (Office of Technology Assessment (U.S. Congress), Washington, DC). Apr 1982. 61p. Office of Technology Assessment (US Congress), Washington, DC 20510.

This summary presents the findings and conclusions of OTA's analysis of Federal policy for the management of commercial high-level radioactive waste - an issue that has been debated over the last decade and that now appears to be moving toward major congressional action. After more than 20 years of commercial nuclear power, the Federal Government has yet to develop a broadly supported policy for fulfilling its legal responsibility for the final isolation of high-level radioactive waste. OTA's study concludes that until such a policy is adopted in law, there is a substantial risk that the false starts, shifts of policy, and fluctuating support that have plagued the final isolation program in the past will continue. The continued lack of final isolation facilities has raised two key problems that underlie debates about radioactive waste policy. First, some question the continued use of nuclear power until it is shown that safe final isolation for the resulting wastes can and will be accomplished, and argue that the failure to develop final isolation facilities is evidence that it may be an insoluble problem. Second, because there are no reprocessing facilities or federal waste isolation facilities to accept spent fuel, existing reactors are running out of spent fuel storage space, and by 1986 some may face a risk of shutting down for some period. Most of the 72,000 metric tons of spent fuel expected to be generated by the year 2000 will still be in temporary storage at that time. While it is possible that utilities could provide all necessary additional storage at reactor sites before existing basins are filled, some supplemental storage may be needed if there are delays in their efforts.

79 (PNL-3570) Understanding radioactive waste. Murray, R.L. (Pacific Northwest Lab., Richland, WA (USA)). Dec 1981. Contract AC06-76RL01830. 116p. NTIS, PC A06/MF A01. Order Number DE82007628.

Portions of document are illegible.

This document contains information on all aspects of radioactive wastes. Facts are presented about radioactive wastes simply, clearly and in an unbiased manner which makes the information readily accessible to the interested public. The contents are as follows: questions and concerns about wastes; atoms and chemistry; radioactivity; kinds of radiation; biological effects of radiation; radiation standards and protection; fission and fission products; the Manhattan Project; defense and development; uses of isotopes and radiation; classification of wastes; spent fuels from nuclear reactors; storage of spent fuel; reprocessing, recycling, and resources; uranium mill tailings; low-level wastes; transportation; methods of handling high-level nuclear wastes; project salt vault; multiple barrier approach; research on waste isolation; legal requirem$\mathrm{nts}$; the national waste management prugram; societal aspects of radioactive wastes; perspectives; glossary; appendix A (scientific American articles); appendix B (reference material on wastes). (ATT)

80 (PNL-4121) Materials Characterization Center. Second workshop on irradiation effects in nuclear waste forms. 
Summary report. Weber, W.J.; Turcotte, R.P. (Pacific Northwest Lab., Richland, WA (USA)). Jan 1982. Contract ACO676RL01830. 58p. NTIS, PC A04/MF A01. Order Number DE82007587.

The purpose of this second workshop on irradiations effects was to continue the discussions initiated at the first workshop and to obtain guidance for the Materials Characterization Center in developing test methods. The following major conclusions were reached: Ion or neutron irradiations are not substitutes for the actinide-doping technique, as described by the MCC-6 Method for Preparation and Characterization of Actinide-Doped Waste Forms, in the final evaluation of any waste form with respect to the radiation effects from actinide decay. Ion or neutron irradiations may be useful for screening tests or more fundamental studies. The use of these simulation techniques as screening tests for actinide decay requires that a correlation between ion or neutron irradiations and actinide decay be established. Such a correlation has not yet been established and experimental programs in this area are highly recommended! There is a need for more fundamental studies on dose-rate effects, temperature dependence, and the nature and importance of alpha-particle effects relative to the recoil nucleus in actinide decay. There are insufficient data presently available to evaluate the potential for damage from ionizing radiation in nuclear waste forms. No additional test methods were recommended for using ion or neutron irradiations to simulate actinide decay or for testing ionization damage in nuclear waste formis. It was recognized that additional test methods may be required and déveloped as more data become available. An American Society for Testing and Materials (ASTM) Task Group on the Simulation of Radiation Effects in Nuclear Waste Forms (E 10.08.03) was organized to act as a continuing vehicle for discussions and development of procedures, particularly with regard to ion irradiations.

81 (PNL-4228) Workshop on the leaching mechanisms of nuclear-waste forms, Octobei 27-28, 1981. Summary report. Mendel, J.E. (comp.). (Pacific Northwest Lab., Richland, WA (USA)). Apr 1982. Contract AC06-76RL01830. 66p. NTIS, PC A04/MF A01. Order Number DE82013193.

The purpose of this informal workshop was to initiate the program and achieve the following goals: (1) acquaint laboratory investigators (data generators) with the needs of the mathematical modelers (data users). Session I was devoted to a tutorial by D.D. Jackson, mathematical modeler for the leaching mechanisms program, on PROTOCOL, a general case waste form leaching model; (2) define important testing parameters. based on the present state of knowledge. To achicve this, a number of impertant testing parumeters were identified for apecial discussion in Session II; (3) develop an understanding of the interrelationships between the activities of leaching mechanisms program participants, and begin definition of the specific role of each participant in the overall program; and (4) establish good communication between the leaching mechanisms program and related programs, particularly the waste form leaching program at the Savannah River Laboratory (SRL) and the various Nuclear Waste Terminal Storage (NWTS) waste package programs. The agenda for the workshop is attached as Appendix A; a list of attendees is in Appendix B. Because this workshop was devoted to preliminary planning for the leaching mechanisms program, the presentations and discussions were purposely kept informal. This report represents a synopsis of the proceedings that has been prepared by the leaching mechanisms coordinator and reviewed by the workshop participants.

82 - (PNL-4382) Materials Characterization Center workshop on leaching mechanisms of nuclear waste forms, May 19-21, 1982, Gaithersburg, Maryland. Summary report. Mendel, J.E. (comp.). (Pacific Northwest Lab., Richland, WA (USA)). Aug 1982. Contract AC06-76RL01830. 275p. NTIS, PC A12/MF A01. Order Number DE83000261.

Portions of document are illegible. Printed copy available until stock is exhausted.

This is a report of the second workshop on the leaching mechanism of nuclear waste forms, which was held at Geithersburg, Maryland, May 19-21, 1982. The first session of the workshop was devoted to progress reports by participants in the leaching mechanisms program. These progress reports, as prepared by the participants, are given in Section 3.0. The goal of the remainder of the workshop was to exchange information on the development of repository-relevant leach testing techniques, often called interactions testing. To this end, a wide spectrum of investigators, many of whose work is sponsored by DOEffis
Nuclear Waste Terminal Storage (NWTS) project, made presentations at the workshop. These presentations were a significant and beneficial part of the workshop and are summarized in Sections 4.0, 5.0 and 6.0 according to the workshop agenda topies. In many cases, the presenters provided a written version of their presentation which has been included verbatim; in the other cases, the workshop chairman has supplied a brief synopsis. Twenty-one papers have been abstracted and indexed for inclusion in the data base.

83

(PNL-4405) Summary of non-US national and International fuel cycle and radioactive waste management programs 1982. Harmion, K.M.; Kelman, J.A. (Pacific Northwest Lab. Richland, WA (USA)). Aug 1982. Contract AC06-76RL01830. 106p. NTIS, PC A06/MF A01. Order Number DE82022520. Brief program overviews of fuel cycle, spent fuel, and waste management activities in the following countries are provided: Argentina, Australia, Austria, Belgium, Brazil, Canada, Chins, Denmark, Finland, France, German-Federal Republic, India, Italy, Japan, Republic of Korea, Mexico, Netherlands, Pakistan, South Africa, Spain, Sweden, Switzerland, Taiwan, USSR, and the United Kingdom. International nonproliferation activitios, multilateral agreements and projects, and the iulernational agenoios opecifically involved in the nuclear fuel cycle are also described.

8 ry, January 1978 through July 1982. (Pacific Northwest Lab. Richland, WA (USA)). Sep 1982. Contract AC06-76RL01830. 242p. NTIS MF A01. Order Number DE83007457.

Microfiche only, copy does not permit paper copy reproduction. This bibliography lists publications (831 abstracts) from the Pacific Northwest Laboratory's Department of Energy sponsored research and development programs from January 1978 through July of 1982. The abstracts are grouped in subject categories, as shown in the table of contents. Entries in the subject index also facilitate access by subject, e.g., High-Level Riadioactive' Wastes. Three indexes, each preceded by a brief description, are provided: personal author, subject, and report number. Cited are reseanch reports, journal articles, books, patents, theses, and conference papers. Excluded are technical progress reports. Since 1978 the Nuclear Waste Management Quarterly Progress Report has been published under the series number PNL-3000. Beginning in 1982, this publication has been issued semiannually, under the series number PNL-4250. This bibliography is the successor to two others, BNWL-2201 (covering the years 1965-1976) and PNL-4050 (1975-1978). It is intended to provide a useful reference to literature in waste management written or compiled by PNL staff.

86

(PNL-4637) International fuel-cycle and wasteminagement technolopy-exchange activities sponsored by the United States Department of Energy: FY 1982 evaluation report. Lakey, L.T.; Harmon, K.M. (Pacific Northwest Lab., Richland, WA (USA)). Feb 1983, Contract AC06-76RL01830. 78p. NTIS, P.C A05/MF A01. Order Number DE83008556.

In FY 1982, DOE and DOE contractor personnel attended 40 international symposia and conferences on fuel reprocessing and waste management subjects. The treatment of high-level waste was the topic most of ten covered in the visits, with geologic disposal and general waste management also being covered in numerous visits. Topics discussed lose frequently inlcudo TRU/LLW treatment, airbnrne waste treatment, $D$ and $D$, spent fuel handling, and transportation. The benefits accuring to the US from technology exchange activities with other countries are both tangible, e.g., design of equipment, and intangible, c.g., improved foreign relations. New concepts initiated in other countries, particularly those with sizable nuclear programs, are beginning to appear in US efforts in growing numbers. The spent fuel dry storage corcept originating in the FKG is being considered at numerous sites. Similarly, the German handling and draining concepts for the jouleheated ceramic melter used to vitrify wastes are being incorporated in US designs. Other foreigh technologies applicable in the US include the slagging incinerator (Belgium), the SYNROC waste form (Australia), the decontamination experience gained in decommissioning the Eurochemic reprocessing plant (Belgium), the engineered surface storage of low- and intermediate-level waste (Belgium, FRG, France), the aircooled storage of vitrified high-level waste (France, UK), waste packaging (Canada, FRG, Sweden), disposal in salt (FRG), disposal in granite (Canada, Sweden), and sea dumping (UK, Belgium, The Netherlands, 
Switzerland). These technologies did not necessarily originated or have been tried in the US but for various reasons are now being applied and extended in other countries. This growing nuclear technological base in other countires reduces the number of technology avenues the US need follow to develop a solid nuclear power program.

86 (PNL-SA-8916) Radiation damage in crystalline nuclear-waste solids. Turcotte, R.P.; Weber, W.J.; Roberts, F.P. (Battelle Pacific Northwest Labs., Richland, WA (USA)). Jun 1980. Contract AC06-76RL01830. 31p. (CONF-800609-19).

NTIS, PC A03/MF A01. Order Number DE82010732.

From 10. international symposium on effects of radiation on materials; Savannah, GA, USA (3 Jun 1980)

Portions of document are illegible.

Radiation damage in nuclear wastes is shown to be linked to decay of the actinide elements, particularly $\mathrm{Cm}$ and Am. Both alpharecoil damage in actinide-containing phases and alpha bombardment of all phases must be considered. Phases either remain crystalline, with small volume changes $(<2 \%)$, or become amorphous, of ten with large volume changes, when subjected to alpha-recoil or ion bombardment. Ionic compounds appear to fall mainly in the stable category and the stability can be estimated from the ionicity. Both alpha-recoil and alpha bombardment induced changes follow an exponential function of the dose to some saturation value. It is possible to establish some limits of general application in terms of total dose received and relate these to the corresponding nuclear waste storage times. A figure schematically summarizes this information showing lattice constant changes plotted against dose. The waste storage times required to achieve these doses are given for both defense and commercial HLW waste solids, assuming a $30 \%$ waste loading. This comparison shows that existing defense wastes can be converted to crystalline solids without concern for radiation-induced changes. If commercial nuclear fuels are reprocessed and wastes solidified, there is a real potential for large radiation-induced changes, even within the first hundred years.

87 (PNL-SA-10124) New look at forecasting. Petrie, G.M. (Pacific Northwest Lab., Richland, WA (USA)). Jun 1982. Contract AC06-76RL01830. 20p. (CONF-820678-2). NTIS, PC A02/MF A01. Order Number DE82019203.

From International symposium on hydrometerology; Denver, CO, USA (13 Jun 1982).

In an effort to aid the Department of Energy in the identification and characterization of future natural processes which may degrade the performance of a mined repository for high-level nuclear waste, a stochastic/deterministic computer model was developed for application to specific sites. This model was designed to consider long time periods over which relatively slow geologic processes can be of importance. Evaluation of the results of the first analyses suggests that this technique gives useful insight into identifying and characterizing the important processes, and appears to be particularly useful in characterizing synergistic effects. Moreover, analysis of the results suggests that applying the same technique, using a shorter time scale, to water management would be rewarding. Some of the suggested advantages of such a new application of existing technology include: (1) early detection of errors in either the data or conceptual model, (2) quantification of the probabilities for the timing and magnitude of future natural events, (3) simulation of synergistic interactions, (4) quantification of uncertainty through the use of probability density functions in a manner that is relatively easy for non-specialists to understand, (5) exchange of information among technical disciplines and across political boundaries, (6) rapid evaluation of the effects of changes in regulations, and (7) clear separation of objective and subjective inputs.

(PNL-SA-10184S) State-of-the-art in-situ determination of transuranics. Brodzinski, R.L. (Pacific Northwest Lab., Richland, WA (USA)). Jan 1982. Contract ACO676RL01830. 6p. (CONF-820609-60). NTIS, PC A02/MF A01. Order Number DE82018846.

From American Nuclear Society annual meeting; Los Angeles, CA, USA (6 Jun 1982)

Two techniques for in-situ analysis of transuranics are described. 'The first provides quantitative information in actinide-only environments by analyzing high energy gamma-rays with a high resolution germanium diode spectrometer. This techinique is simple, safe, fast, specific, economical, and can interrogate representative sample volumes for the simultaneous determination of six actinide isotopes. The disadvan tage lies in the inability to operate in high fission product environments. For high fission product concentrations, a method is described using exposure metal plates, usually copper, and subsequent analysis of the transuranic-generated-neutron induced activation products in the plate specifically developed for use in high gamma-ray environments. The technique is not isotope specific and requires a relatively long turnaround time, but has many desirable attributes not found in other techniques. One application of this technique where the plutonium concentration was measured as a function of depth in a high-level waste tank containing salt cake is presented in a table.

89 (SAND-82-1804) NWTS transportation interface technology development priority report. Shirley, C.G. (Sandia National Labs., Albuquerque, NM (USA)). Jul 1983. Contract AC04-76DP00789. 359p. NTIS, PC A16/MF A01. Order Number DE83017411.

Priority for the development of occupational radiation exposure control concepts have been evaluated and compared to current practices and technology for handling high-radiation-level nuclear material shipments in this report. Large-throughput and long-term receiving operations at nuclear waste repositories being developed by the Nationa Waste Terminal Storage (NWTS) program are unprecedented in terms of the frequency of handling shipments. The use of current practices and technology to perform these operations represents a major problem in operational exposure control at the repository. Methods and equipment for reducing exposures are examined and ranked to provide recommendations for repository and transportation system development activities in the NWTS program. 79 figures, 61 tables.

90 (TENRAC/ADV-79-003) Nuclear energy in Texas: major issues and policy recommendations. (Texas Energy and Natural Resources Advisory Council, Austin (USA)). 7 Dec 1979. 94p. NTIS, PC A05/MF A01. Order Number DE83902076.

On December 15, 1978 the Texas Energy Advisory Council (TEAC) adopted the Texas Energy Policy, 1978 Update. The Council recognized the great complexity of technical, economic, social, and political issues which have an impact on nuclear power, and therefore decided that a special committee was needed to examine in greater detail the nuclear energy issues facing Texas. This report summarizes the work of the Advisory Committee on Nuclear energy. Topics discussed include: biological affects of low levels of radiation; transportation of radioactive material in Texas; uranium mining and milling; severance $\operatorname{tax}$ on uranium mining; alternate reactor designs and fuel cycles; financing nuclear plant construction; cost of disposing of nuclear wastes and decommissioning nuclear plants; low-level wastes; disposal of highlevel wastes; commercial spent nuclear fuel reprocessing; and transmutation of radioactive wastes.

91 Managing commercial high-level radioactive waste. Radioactive Waste Management and the Nuclear Fuel Cycle, 3: No. 3-4, 279-345(Jun 1983).

The article is a summary of issues raised during US Congress deliberations on nuclear waste policy legislation. It is suggested that, if history is not to repeat itself, and the current stalemate on nuclear waste is not to continue, a comprehensive policy is needed that addresses the near-term problems of interim storage as part of an explicit and credible program for dealing with the longer term problem of developing a final isolation system. Such a policy must: 1) adequately address the concerns and win the support of all the major interested parties, and 2) adopt a conservative technical and institutional approach - one that places high priority on avoiding the problems that have repeatedly beset the program in the past. It is concluded that a broadly supported comprehensive policy would contain three major elements, each designed to address one of the key questions concerning Federal credibility: commitment in law to the goals of a comprehensive policy; credible institutional mechanisms for meeting goals; and credible measures for addressing the specific concerns of the states and the various publics. Such a policy is described in detail.

92 Nuclear waste management policy in France. Lefevre, J.F. (Centre d'Etudes Nucleaires, Boite Postale No. 6, F-92260 Fontenay-aux-Roses). Nuclear Technology; 61: No. 3, 455-459(Jun 1983).

The object of the nuclear waste management policy in France has always been to protect the worker and the public from unacceptable 
risks. The means and the structures developed to reach this objective, however, have evolved with time. One fact has come out ever more clearly over the years: Nuclear waste problems cannot be considered in a piecemeal fashion. The French nuclear waste management structure and policy aim at just this global approach. Responsibilities have been distributed between the main partners: the waste producers and conditioners, the research teams, the safety authorities, and the long-term waste manager, National Radioactive Waste Management Agency. The main technical options adopted for waste forms are embedding in hydraulic binders, bitumen, or thermosetting resins for low-level waste (LLW) and medium-level waste (MLW), and vitrification for highlevel, liquid wastes. One shallow land disposal site for LLW and MLW has been in operation since 1969, the Centre of La Manche. Alphabearing and high-level waste will be disposed of by deep geological storage, possibly in granite formations. Further R\&D aims mainly at improving present-day practices, developing more durable, long-term, alpha-bearing waste for all solid waste forms and going into all aspects of deep geological disposal characterization.

93 Results of instrument reliability study for high-levei nuclear waste repositories. Rogue, F.; Binnall, E.P. (Lawrence Livermore National Laboratory, P. O. Box 808, Livermore, CA 94550). g Technology; 30: No. 1, 565-570(Feb 1983).

Reliable instrumentation will be needed to monitor the performance of future high-level waste repository sites. A study has been made to assess instrument reliability at Department of Energy (DOE) waste repository related experiments. Though the study covers a wide variety of instrumentation, this paper concentrates on experiences with geotechnical instrumentation in hostile repository-type environments. Manufacturers have made some changes to improve the reliability of instruments for repositories. In this paper, the authors review the failure modes, rates, and mechanisms, along with manufacturer modifications and recommendations for additional improvements to enhance instrument performance.

94 Handling of nuclear waste in Great Britain. Gries, W. Atom + Strom; 29: No. 1, 11-14(Jan-Feb 1983). (In German).

With 31 refs.

The author describes the complete handling of nuclear waste in Great Britain according to the organisation, volume occurring and the current circumstances. The article is supplemented by details on research and development and by the current status of nuclear disposal methods in Great Britain. The report is backed-up by a comprehensive list of reference literature.

95 Conflicting views on a neutrality criterion for radioactive-waste management. Cochran, T.B. (Natural Resources Defense Council, Washington, DC); Bodde, D.L. pp 110-128 of Energy and the future. MacLean, D.; Brown, P.G. (eds.). Totowa, NJ; Rowman and Littlefield (1983).

Two essays are presented by authors who agree that risks imposed on future generations through the management of radioactive waste are acceptable if they meet a criterion of neutrality, but who disagree on the interpretation of the neutrality criterion. The first viewpoint argues that acceptable isolation of high-level radioactive waste is yet to be accomplished and that a fundamental criterion for radioactive waste disposal must include consideration of the intergenerational radiation effects. The second essay promotes balanced resource allocation, technological progress, and adequate problem-solving institutions as the solution to the problem. The debate illustrates the complexities involved in applying philosophical principles to public policy, even when the principles have been agreed upon. 23 references, 1 figure.

96 Predictive geology: with emphasis on nuclear-waste disposal. De Marsily, G.; Merriam, D.F. (Pergamon Press, Maxwell House, Fairview Park, Elmsford, New York 10523). Economic Geology and the Bulletin of the Society of Economic Geologists; 77: No. 7, 1771-1772(Nov 1982).

Reviews book which primarily discusses applications of earth science to the disposal of high-level radioactive wastes. Points out that very little is said regarding practical experience with, or the epistemological foundation of, prediction in the earth and geotechnical sciences. Suggests that an in-depth examination of the difficulties of retrodiction in the earth sciences might have provided the philosophical overview missing in a volume whose title stresses predictive geology.
97 Evaluation of an international, perpetual, and retrievable facility for storage of vitrified radioactive waste. Libby, L.M.; Whipple, C.G.; Wurtele, M.G. (University of California Environmental Science and Engineering Program, Los Angeles, California 90024). Nuclear Technology; 59: No. 1, 85-98(Oct 1982).

It is technically feasible to site a retrievable but permanent surface storage facility for vitrified radioactive wastes in the northwestern Egyptian desert. Present-day commercial vitrification plants are in England and France and produce glass cylinders in the shape of an annulus, about $9 \mathrm{ft}$ high, clad in a stainless steel can, containing about $25 \%$ of fission product and actinide oxides, weighing about 10 tonnes, having a volume of about $70 \mathrm{ft}^{0}$, releasing about $1.8 \times 10^{5} \mathrm{Btu}$ heat $/ \mathrm{h}$. The high-level waste (HLW) glass cylinders, in lead shipping casks, are to be shipped to European ports by truck, sent to Mersa Matruh on the Egyptian coast, about ten at a time in small barges, then offloaded and sent by train a short distance inland to the site. The storage facility envisaged at the site is a concrete-walled round house with a radial crane, equipped with recanning facilities in case of breakage of stainless steel canisters, with a shop for repair of the train as needed, and with a turntable for the engine. Cooling is provided by natural air draft resulting from the canister surface temperature of about $100^{\circ} \mathrm{C}$. If needed, backup cooling is provided by equipment for forced-air drafts and by tanks of water. The canister arrangement is that produced by coaxial vertical stacking; horizontal coaxial arrangements are yet to be analyzed. The site chosen is exposed hard rock close to the Mediterranean in the northwest corner of the Egyptian desert. Groundwater is found at about $100 \mathrm{~m}$. The rainfall is about $4 \mathrm{in}$./yr so that flash floods sometimes occur and surface drains are needed. Meteorology, seismicity, agriculture, and wildlife are all favorable factors, and plane studies show no thermal or radioactive threat.

98 Subsurface migration of radioactive waste materials by particulate transport. Eichholz, G.G.; Craft, T.F.; Powell, G.F.; Wahlig, B.G. (Georgia Institute of Technology, School of Nuclear Engineering, Atlanta, Georgia 30332). Nuclear Technology; 58: No. 3, 511-520(Sep 1982).

The role of suspended particles as carriers of dissolved nuclides from high-level radioactive waste repositories has been investigated. Depending on the concentrations of suspended particles and the nature of the invading water, it has been found that cationic nuclides may be competitively adsorbed on suspended clay particles, the partitioning being largely determined by $\mathrm{pH}$, temperature, and comparative surface areas of particulates and surrounding rocks. Column tests with activated particles have been conducted and showed that the clay particles pass resdily through porous mineral solumns and are increasingly retained if salinity is increased. Retention in basalt columns is stronger in the presence of high concentrations of sodium and calcium ions and has been explained in terms of van der Waals forces. The range of particulate migration then depends on the condition of the rock surfaces, the persistence of a clay coating, and the total dissolved ion concentration. For adsorbable waste ions, this may represent a pathway comparable in significance to ion-exchange-controlled migration. For some bed materials, the particulate movement displayed a prompt and a delayed component; the nature of the delay mechanism is not fully understood at present.

99 Radwaste panel, NRC head highlight 44th American Power Conference prugram. Sunuck, R.W., Minner, D.F. ; 60: No. 6, 13-14(Jun 1982).

DOE Secretary Edwards urged Congress to compromise on current bills dealing with radioactive waste management in a speech to the American Power Conference. Noting that the government is only responsible for the nuclear waste disposal, not temporary spent-fuel storage, Edwards suggested a government-private consortium for financing the Barnwell, SC reprocessing plant. Other speakers also called for congressional action on comprehensive legislation to deal with both highlevel and low-level wastes. (DCK)

100 DOE's commercial waste management and fuel cycle programs. Coffman, F.E. (U.S. Department of Energy, Washington, D.C. 20545). Transactions of the American Nuclear Society; 41: 226(Jun 1982). (CONF-820609-).

From.American Nuclear Society annual meeting; Los Angeles, CA, USA (6 Jun 1982). 
FUEL CYCLE; US DOE; RADIOACTIVE WASTE MANAGEMENT; HIGH-LEVEL RADIOACTIVE WASTES; SPENT FUEL STORAGE; REPROCESSING; SPENT FUELS; RADIOACTIVE WASTE DISPOSAL; RADIOACTIVE WASTE PROCESSING

101 Management of radioactive wastes. Saunders, P.A.H. (UKAEA Atomic Energy Research Establishment, Harwell. Environmental and Medical Sciences Div.). London, England; UKAEA (Jun 1982). 32p.

This booklet describes in simple terms and with many illustrations, current practices and the methods being developed to ensure that radioactive waste management in the UK can be carried out with no significant threat to current or future generations. Sections are headed: introduction; characteristics of radioactive wastes; sources and quantities; principles, safety criteria and legislation; management techniques and their radiological consequences; solidification and storage of highlevel wastes; disposal of highly active wastes.

102 Projections of high-level waste production in Brazil. Oliveria, L.F.; Hamacher, M.E. (COPPE/UFRJ (Nuclear), Ilha do Fundao, Rio de Janeiro, RJ). Transactions of the American Nuclear Society; 41: 88(Jun 1982). (CONF-820609-).

From American Nuclear Society annual meeting; Los Angeles, CA, USA (6 Jun 1982).

BRAZIL; HIGH-LEVEL RADIOACTIVE WASTES; INTERNATIONAL AGREEMENTS; NUCLEAR ENERGY; PROJECTION SERIES; RADIOACTIVE WASTE DISPOSAL; REPROCESSING; SPENT FUEL STORAGE; FORECASTING; NUCLEAR POWER PLANTS

103 Radiation damage from alpha decay in ceramic nuclear waste forms. Weber, W.J.; Turcotte, R.P.; Roberts, F.P. (Pacific Northwest Lab., Richland, WA (USA)). Radioactive Waste Management; 2: No. 3, 295-319(Mar 1982).

The effects of radiation damage on ceramic forms of nuclear waste solids have been studied by actinide-doping techniques and by external alpha irradiation. The results of several other investigations on simulated ceramic waste forms are reviewed, and the expected phase behavior, in general, is discussed based on rare-earth chemistry, X-ray diffraction data, and available information on both self damage in actinide compounds and irradiation damage in other ceramic materials.

104 Nuclear waste management. Journal of the Environmental Engineering Division (American Society of Civil Engineers); 108: No. EE1, 1-50(Feb 1982).

This Task Committee report presented reviews the current technology and many of the key technical issues concerned with the longterm storage and disposal of high-level and intermediate-level nuclear wastes from the commercial nuclear fuel cycle. The report reviews the origin of nuclear wastes from the total nuclear fuel cycle, the biological and environmental significance of the wastes, and technical options for waste repositories, both for shallow land burial and federal repositories. The report discusses issues on spent fuel reprocessing. 50 refs.

$105 \quad$ High-level nuclear waste: who governs. Smith, R.F.; Morris, F.A. Underground Space; 6: No. 4-5, 211-216(Jan 1982).

The existing framework for managing nuclear waste is described with some attention to the agencies, US Departments of Energy, Interior, and Transportation, US Nuclear Regulatory Commission, US Environmental Protection Agency, and state governments, which share responsibility for high-level waste isolation. Some of the unsolved problems connected with this existing framework are discussed, and some recommendations for necessary legislation and regulatory decisions are made. (BLM)

106 Disposal of radioactive wastes. Dlouhy, Z. Amsterdam, Netherlands; Elsevier Scientific Publishing Company (1982). 264p. Elsevier/North-Holland, Inc., 52 Vanderbilt Avenue, New York, NY 10017.

Radioactive wastes arise throughout the entire cycle from uranium mining and fuel fabrication to power production and the reprocessing of spent fuel. This book aims to provide information on the origin and characteristics of radioactive wastes, the processing of liquid, solid, and gaseous wastes, the processing of wastes from spent fuel reprocess- ing, the fixation of radioactive concentrates, the solidification of highlevel radioactive wastes, the use of these wastes as raw material, radioactive waste disposal, transport of radioactive wastes, economic problems of radioactive waste disposal. Protection of the health of the population and of the environment are the most important criteria for any considerations on processing and discarding radioactive wastes. 41 figures, 37 tables.

107 Scientific basis for nuclear waste management. Topp, S.V. (ed.). New York, NY; Elsevier Science Publishing Company, Inc. (1982). 789p. (CONF-811122-Pt.1).

From Annual meeting of the Materials Research Society; Boston, MA, USA (16 Nov 1981).

This volume contains the proceedings of the fourth International Symposium on the Scientific Basis for Nuclear Waste Management, held in Boston, Massachusetts, on November 16-19, 1981, as part of the Annual Meeting of the Materials Research Society. The purpose of this Symposium was to provide an interdisciplinary forum for the discussion of scientific research dealing with all levels and types of radioactive wastes and their management. These symposia have been held annually since 1978. The proceedings of the first three meetings were published as Volumes 1, 2, and 3 in a series. With this, the fourth meeting, the volume numbering system is changed to coincide with the system used to number Materials Research Society Annual Meeting Proceedings. The reports presented here give the results of research and development activities from a large number of universities, government laboratories and private industry in nine countries. The 92 papers published in these proceedings have been divided into 92 chapters. These encompass various aspects of high-level and non-high-level radioactive waste management ranging from repository characterization and waste form production to product and performance assessment. All of the papers have been abstracted and indexed for the data base.

108 Management of radioactive wastes in Europe (EC) history, philosophy and plans for the next five years. Orlowski, S.; Simon, R.A. (Commission of the European Communities, Brussels, Belgium). pp 733-739 of Scientific basis for nuclear waste management. Topp, S.V. (ed.). New York, NY; Elsevier Science Publishing Company, Inc. (1982). (CONF-811122-Pt. 1).

From Annual meeting of the Materials Research Society; Boston, MA, USA (16 Nov 1981)

Since its first $R$ \& $D$ programme on radioactive waste management in 1973, the European Community has been contributing a steadily increasing share of the European research effort in this field. A major part of the programme is devoted to the investigation and evaluation of management concepts for high-level and TRU wastes from the backend of the fuel cycle. Risk analysis studies for the assessment of multibarrier terminal isolation systems currently have high priority. 2 figures.

109 Materials research for the Canadian Nuclear Fuel Waste Management Program. Cameron, D.J. (Atomic Energy of Canada Research Co., Ltd., Pinawa, Manitoba). pp 741-751 of Scientific basis for nuclear waste management. Topp, S.V. (ed.). New York, NY; Elsevier Science Publishing Company, Inc. (1982), (CONF-811122-Pt.1).

From Annual meeting of the Materials Research Society; Boston, MA, USA (16 Nov 1981).

Nuclear fuel waste disposal research in Canada is concentrating on hard-rock disposal. The research programs concerned with the manmade components of the disposal system are reviewed. Irradiated fuel and solidified reprocessing wastes are both being researched, as are durable containers, and buffer and backfill materials. This review concentrates mainly on the more scientific aspects of the research, which contribute to the selection of preferred options for the various components of the system, and which support directly or indirectly the safety analysis of the disposal concept. Some technology development is included in the program now, and this is expected to expand as confidence in the acceptability of the disposal concept grows.

110 High-Level Nuclear-Waste Management. Oversight Hearing before the Subcommittee on Energy and the Environment of the Committee on Interior and Insular Affairs, House of Representatives, Ninety-Seventh Congress, Second Session, June 
17, 1982. Washington, DC; Government Printing Office (1982). $151 \mathrm{p}$.

A review of federal programs to develop and regulate permanent high-level nuclear-waste repositories focused on the relevance of technical target dates and the importance of compiling appropriate information. Three witnesses (from the Environmental Protection Agency, DOE, and the Nuclear Regulatory Commission) as well as a panel of four technical experts reported on the progress made under current regulations and ocnstraints. Their testimony is followed by additional material submitted for the record. (DCK)

111 Radioactive waste management 1980-81. Supplement to AIRAC No. 6. Canberra, Australia; AGPS (1982). 21 p.

Parliamentary paper no. 223/1981.

In June 1979 AIRAC submitted to the Australian Minister for Science and the Environment a report on radioactive waste management (AIRAC No 6). Developments since 1979 have been reviewed. It was felt that there was no need to modify the original conclusions.

112 Relations of equivalence of conditioned radioactive waste. Kumer, L.; Szeless, A.; Oszuszky, F. (Oesterreichische Elektrizitaetswirtschafts AG, Vienna, Austria). pp 476-480 of Proceedings of the ANS topical meeting on the treatment and handling of radioactive wastes. Columbus, $\mathrm{OH}$; Battelle Press (1982). (CONF.820424).

From ANS topical meeting on treatment and handling of radioactive wastes; Richland, WA, USA (19 Apr 1982).

A compensation for the wastes remaining with the operator of a waste management center, to be given by the agent having caused the waste, may be assured by effecting a financial valuation (equivalence) of wastes. Technically and logically, this equivalence between wastes (or specifically between different waste categories) and financial valuation has been established as reasonable. In this paper, the possibility of establishing such equivalences are developed, and their suitability for waste management concepts is quantitatively expressed.

113 Radioactive waste management. Response by the Town and Country Planning Association to the white paper 'Radioactive Waste Management' - Cmnd 8607. London, England; Town and Country Planning Association (1982). 9p. Town and Country Planning Assoc., London, England.

Technical, ethical, social, political and organizational aspects of the management of low-, intermediate-, and high-level radioactive wastes arising from operations in the United Kingdom are discussed. Recommendations are made to provide scope for public discussion, to consult the appropriate local water and other authorities, and to take other specified actions relevant to town and country planning.

114 Nuclear waste, the nation, and South Carolina: an update on the major issues. Kearney, R.C.; Jos, P.H. Columbia, SC; University of South Carolina (1982). 12p. Bureau of Governmental Research and Service, University of South Carolina, Columbia, SC $29208 \$ 2.50$.

This report provides an update on the most-salient nuclear-wastemanagement issues affecting South Carolina and the nation. High-level waste developments are considered, including DOE's National Waste Terminal Storage Program, pending congressional legislation on highlevel wastes, and the stumbling blocks which continue to make a statutory solution difficult. The status of low-level waste management is examined with particular reference to the Low-Level Radioactive Waste Policy Act of December 1980. The author concludes with a brief look at future problems and prospects related to the radioactive waste management in South Carolina and in the United States.

115 Waste management and transportation. Manion, W.J. pp 19p, Paper 9 of Nuclear commerce in the 80 's. New York, NY; McGraw-Hill Publications Co. (1982). (CONF820498-).

From NRC conference on nuclear commerce in the 80 's; St. Charles, IL, USA (28 Apr 1982).

Information on the sources and types of radioactive wastes, spent fuel, high-level waste, transuranic waste, low-level waste, remedial action waste, mill tailings, and airborne waste, was given. The varieties of wet and dry storage for spent fuel were detailed. Statistics on waste volumes and volume reduction systems were included. (JMT)
116 Radiation effects in high-level radioactive waste forms. Turcotte, R.P. (Pacific Northwest Lab., Richland, WA (USA)). Radioactive Waste Management; 2: No. 2, 169177(Dec 1981).

Radiation from decay of actinide elements is shown to produce saturation changes in bulk properties of waste forms near $10^{19}$ alpha decays $/ \mathrm{cm}^{0}$ (<approximately $1 \mathrm{dpa}$ ). Accelerated actinide doping experiments in this dose range show only slight radiation induced changes in leach rates of nuclear waste glasses.

117 Second annual report made to the Secretary of State for the Environment, Secretary of State for Scotland, Secretary of State for Wales. London, England; HMSO (May 1981). 61p. Also available from HMSO, price Pound $\mathbf{\$ 3} .90$.

Recent developments that affect waste management, the evolution of overall management strategy and management of wastes from the defence programme are reviewed. Storage and disposal of solidified high-level wastes are considered and the options compared. Annexes on research and public information and the perception of risk and appendices or a guide to further reading and areas for geological investigation in the U.K. are included.

118 Policy and some problems on the return waste. Shimoda, H. (Tokyo Electric Power Co., Inc. (Japan)). ticky a Kartograficky Obzor; 27: No. 2, 42-48(Feb 1981). (In Japanese).

The radioactive wastes generated at the time of reprocessing the spent fuel from the nuclear power stations in Japan in the reprocessing companies in Great Britain and France are expected to be returned to Japan in 1990 and later. The specification of returned wastes will be presented by the reprocessing companies in 1981, but in order to approve it, it is necessary to obtain the perspective whether the transport, acceptance and handling, and storage of the wastes can be carried out safely or not. First, the plan for the reprocessing of spent fuel from the nuclear power stations in Japan is shown. Next, the features in the new contracts on reprocessing with BNFL and COGEMA are explained. Electric power companies are jointly examining the concept about the quantity to be generated, properties, transport, storage and disposal of the returned wastes, based on the informations from COGEMA. The facilities in the reprocessing plants of BNFL and COGEMA, and the kinds and quantities of the wastes to be returned are outlined. The long term scenario of returned waste management. the transport and storage of returned wastes, and the examination on the rate of waste content and the soundness of high level glass solidified bodies, the stability of glass solidified bodies, the material of canisters and low and medium level wastes, and the final disposal of returned wastes are described.

119 Nuclear waste management. Draper, E.L. Jr. (Gulf States Util, Beaumont, TX 77704). Transactions of the American Nuclear Society; 38: 21-22(1981). (CONF-810606-).

From American Nuclear Society's annual meeting; Miami Beach, FL, USA (7 Jun 1981).

DIAGNOSIS; HIGH-LEVEL RADIOACTIVE WASTES; INDUSTRIAL RADIOGRAPHY; LOW-LEVEL RADIOACTIVE WASTES; NUCLEAR MEDICINE; POWER GENERATION; RADIOACTIVE WASTE MANAGEMENT; RADIOISOTOPES; THERAPY; URANIUM ORES; AI.PHA-BEARING WASTES; MILL TAILINGS; USA

120 Heating effects on groundwater flow through a repository fracture system. Maxwell, D.E.; Ross-Brown, D.M.; Trent, B.C. (SAI, San Leandro, San Leandro, CA 94577). Transactions of the American Nuclear Society; 39: 180-182(1981). (CONF-811103-). 1981).

From ANS winter meeting; San Francisco, CA, USA (29 Nov

EPRI; GROUND WATER; HIGH-LEVEL RADIOACTIVE WASTES; HYDRAULIC FRACTURING; RADIONUCLIDE MIGRATION; TEMPERATURE EFFECTS; RADIOACTIVE WASTE STORAGE

121 The national long-term high-level waste technology program. Gray, P.L. (SRL, Aiken, SC 29808). Transactions of 
the American Nuclear Society; 39: 224-225(1981). (CONF$811103-)$ 1981).

From ANS winter meeting; San Francisco, CA, USA (29 Nov

APPROPRIATE TECHNOLOGY; HANFORD RESERVATION; HIGH-LEVEL RADIOACTIVE WASTES; IDAHO NATIONAL ENGINEERING LABORATORY; NATIONAL ENVIRONMENTAL POLICY ACT; SAVANNAH RIVER PLANT; SOLIDIFICATION; US DOE; RADIOACTIVE WASTE MANAGEMENT

122 Technology for long-term management of ICPP Defense Waste. Berreth, J.R. (Exxon Nuclear Idaho, Idaho Falls, ID 83401). Transactions of the American Nuclear Society; 39: 225-226(1981). (CONF-811103-).

From ANS winter meeting; San Francisco, CA, USA (29 Nov

APPROPRIATE TECHNOLOGY; CALCINATION; HIGHLEVEL RADIOACTIVE WASTES; IDAHO CHEMICAL PRO CESSING PLANT; SOLIDIFICATION; RADIOACTIVE WASTE MANAGEMENT; LEACHING

123 Speech before the Atomic Industrial Forum Conference. Davis, J.G. (US Nucl Regul Comm). pp 1-16 of Atomic Industry Forum, fuel cycle conference ' 81,1981 . Washington, DC; Atomic Industry Forum (1981). (CONF-810324-). 1981).

From Fuel cycle conference; Los Angeles, CA, USA (15 Mar

Nuclear Regulatory Commission's (NRC) programs for highlevel and low-level radioactive waste management are discussed. The topics considered are: NRC statutory responsibilities; impact on administration program; major NRC rules; and coordination with DOE.

124 Conflicting views on a neutrality criterion for radioactive-waste management. Bodde, D.L.; Cochran, T.B. College Park, MD; Univ. of Maryland (1981). 26p. \$2.00.

Public debate over the management of radioactive wastes illustrates the moral dilemma of intergenerational justice. Because of low priority, there has been no permanent disposal of high-level radioactive wastes or decontamination and decommisioning of reactors. The problem is now receiving public attention because of the near depletion of temporary storage capacity, the deferral of reprocessing, and concerns for the safe transport and disposal of hazardous materials. Two authors examine the criterion of neutrality in which the risks of radioactive wastes can be balanced by the risks future generations would face without the opportunity for nuclear power. They disagree, however, in whether the model can possibly represent the real world and whether that risk is a significant consideration. 27 references, 1 figure. (DCK)

125 Seminaire sur les dechets radioactifs. (Meeting on radioactive wastes). Fontenay-aux-Roses, France; Societe Francaise de Radioprotection (1981). 244p. (In French). (CONF810273-). 1981).

From Meeting on radioactive wastes; Cadarache, France (2 Feb

The main topics of these papers about radioactive waste management are dealing with the French and International management, characteristics of wastes (alpha, beta + gamma, high-level, vitrified), processing storage, underground disposal and marine disposal, storage site and briefly the sociological, economic and legal aspects.

126 Effects of cracks on glass leaching. Perez, J.M. Jr.; Westsik, J.H. Jr. (Battelle, Pac Northwest Lab, Richland, Wash). Nuclear and Chemical Waste Management; 2: No. 2, 165-168(1980).

The effects of cracks on glass leaching have been studied to determine to what degree the increase in the surface area of the glass caused by cracks might contribute to the overall leachability of the glass. Bnrnsilicate glass in the shape of cylindrical dioks (pollets) has been used in a static leach test. The pellets were leached in teionized water at $90 / \mathrm{degree} / \mathrm{C}$. Parameters included crack widths of nominally zero and $0.038 \mathrm{~cm}$, and the ratio of crack surface area to total surface area. Leaching from the cracks with $0.038 \mathrm{~cm}$ crack widths are found to be two to five times less than leaching from external surfaces. No apparent leaching from crack surfaces with nominally zero crack widths was observed.

127 (ANL-81-34) Application of neutron-activation analysis to the determination of leach rates of simulated nuclearwaste forms. Bates, J.K.; Jardine, L.J.; Flynn, K.F.; Steindler, M.J. (Argonne National Lab., IL (USA)). Feb 1982. Contract W-31-109-ENG-38. 50p. NTIS, PC A03/MF A01. Order Number DE82008709.

Portions of document are illegible.

The application of neutron activation analysis to the determination of element release from simulated nuclear waste forms during leaching is described for several different glasses. Potential neutron irradiation effects are discussed, and it is shown, by a series of leach tests on activated and nonactivated glass samples, that neutron irradiation has no discernible effect on the release of silicon and cesium during leaching. The radioisotopes best suited for analysis with this method and their associated detection limits are identified, and the method's applicability to waste forms other than glass is discussed.

128 (ANL-82-10) Measurement of leaching from simulated nuclear-waste glass using radiotracers. Bates, J.K.; Jardine, L.J.; Steindler, M.J. (Argonne National Lab., IL (USA)). Sep 1982. Contract W-31-109-ENG-38. 72p. NTIS, PC A04/MF A01. Order Number DE83003340.

Portions of document are illegible.

The use of radiotracer spiking as a method of measuring the leaching from simulated nuclear-waste glass is shown to give results comparable with other analytical detection methods. The leaching behavior of ${ }^{85} \mathrm{Sr},{ }^{106} \mathrm{Ru},{ }^{100} \mathrm{Ba},{ }^{107} \mathrm{Cs},{ }^{141} \mathrm{Ce},{ }^{152} \mathrm{Eu}$, and other isotopes is measured for several defense waste glasses. These tests show that radiotracer spiking is a sensitive, multielement technique that can pro vide leaching data, for actual waste elements, that are difficult to obtain by other methods. Additionally, a detailed procedure is described that allows spiked glass to be prepared with a suitable distribution of radionuclides.

129 (ANL-82-39) Final report of experimental laboratory-scale brittle fracture studies of glasses and ceramics. Jardine, L.J.; Mecham, W.J.; Reedy, G.T.; Steindler, M.J. (Argonne National Lab., IL (USA)). Oct 1982. Contract W-31-109-ENG38. 141p. NTIS, PC A07/MF A01. Order Number DE83005939.

An experimental program was conducted to characterize the fragments generated when brittle glasses and ceramics are impacted. The direct application of the results is to radioactive waste forms for which the effects of accidental impacts must be known or predictable. Two major measurable experimental responses used for characterization of these effects are (1) the size distribution of the fragments, including the sizes that are respirable, and (2) the increase in surface area of the brittle test specimen. This report describes the glass and ceramic materials characterized, the procedures and techniques used for the characterization of size distributions and surface areas, and the results of the two key responses of the impact tests. Five alternative methods of determining size distributions were compared. Also examined were the effects of diametral and axial specimen impact configurations and the use of mechanical stops to eliminate secondary crushing during testing. Microscopic characterizations of Pyrex and SRL 131 simulated waste glass and SYNROC fragments were also performed. Preliminary correlations of impact energy with key size-distribution parameters, fragment surface areas, and respirable fines were proposed as useful for future verification and for use with modeling and scale-up studies of brittle fracture of larger realistic waste forms. The impact fragments of all specimens could be described by lognormal size distributions.

130 (BLG-546) Preparation and microstructural characterization of silicate and borosilicate glasses containing cerium or plutonium. Izahara, S.; Peters, L.; van Iseghem, P. (Centre d'Etude de l'Energie Nucleaire, Mol (Belgium)). Apr 1981. 28p. NTIS (US Sales Only), PC A03/MF A01. Order Number DE82700674.

The preparation of Pu-containing silicate and borosilicate glasses, referring to the conditioning of alpha contaminated and of high leve waste, respectively, has been studied. The preparation of a typical borosilicate glass did not give rise to problems regarding to pouring and final homogeneity of the glass. The preparation of silicate glasses, however, 
was much more difficult, since at the melting temperatures available these glasses could not be poured, and showed pronounced heterogeneities. Different melting parameters, such as melting time and physicochemical preparation of the melting batch were investigated in order to improve the homogeneity. None of these parameters had a marked beneficial influence upon the homogeneity. Chemical attack of the alumina crucible by the glass was found to be the main reason for the heterogeneities observed. Preliminary observations suggest that $\mathrm{PuO}_{2}$ has been dissolved in the glass matrix.

131 (BNL-18978) Savannah River Laboratory long-term waste storage program. Progress report No. 5, March-April 1974. (Brookhaven National Lab., Upton, NY (USA)). Jun 1974. Contract AC02-76CH00016. 25p. NTIS, PC A02/MF A01. Order Number DE82014639.

A program has been implemented at Brookhaven National Laboratory (BNL) in support of Savannah River Laboratory's (SRL) development program for long-term engineered storage of solidified highlevel radioactive wastes. The FY 1974 BNL program objectives are: (1) the preparation of appropriate formulations of cements with synthetic sludges, synthetic slurries and zeolites, and (2) an evaluation of the properties of the radwaste-concrete composite materials.

132 (BNL-19109) Savannah River Laborntory long-term waste storage program. Progress report No. 6, May-Jun 1974. (Brookhaven National Lab., Upton, NY (USA)). Jul 1974. Contract ACO2-76CH00016. 15p. NTIS, PC A02/MF A01. Order Number DE82014642.

A program has been implemented at Brookhaven National Laboratory (BNL) in support of Savannah River Laboratory's (SRL) development program for long-term engineered storage of solidified highlevel radioactive wastes. The FY 1974 BNL program objectives are: (1) the preparation of appropriate formulations of cements with synthetic sludges, synthetic slurries and zeolites; and (2) an evaluation of the properties of the radwaste-concrete composite materials.

133 (BNL-19323) Savannah River Laboratory long-term waste storage program. Progress report No. 7, July-September 1974. (Brookhaven National Lab., Upton, NY (USA)). Oct 1974. Contract AC02-76CH00016. 16p. NTIS, PC A02/MF A01. Order Number DE82014637.

The information given in this report is part of a study at Brookhaven National Laboratory (BNL) in support of the Savannah River Laboratory (SRL) development program for long-term engineered storage of solidified high-level radioactive wastes. The FY 1975 BNL program objectives are: (1) the preparation of appropriate formulations of cements with synthetic sludges, synthetic slurries and zeolites and; (2) an evaluation of the properties of the radwaste-concrete composite materials. While the emphasis is now being placed on the development of cement-concrete composites containing cesium loaded zeolites and slurries, a major portion of the BNL study on the development of cement-concrete composites containing synthetic sludges has been completed.

134 (BNWL-80) Solidification of high level wastes. Part IV. Phosphate melts for fixation of radioactive residues from Purex type wastes: three to fifty percent fission product oxides. Barton, G.B. (Battelle Pacific Northwest Labs., Richland, WA (USA)). Jul 1965. 39p. NTIS.

The possible composition range for the high-level radioactive wastes generated from various power reactor fuels processed through a Purex-type process is very broad. A partial survey of this wide range was conducted in an effort to establish the composition limits that bound the operable range of the phosphate melt system. Two screening criteria were used: (a) drip temperature, an empirical measure of flow tempera. ture at which a container could be filled, and (b) solubility. Measurements indicated the occurrence of two (or possibly more) optimum phosphate additions near $\mathrm{M}_{2} \mathrm{O} / \mathrm{P}_{2} \mathrm{O}_{5}$ ratios of 2.1 and 1.0. It was found that fission products equivalent to at least $50 \%$ of the weight of oxides in the plant waste can be successfully incorporated in a phosphate melt that can be transferred as a fluid into containers at less than $900^{\circ} \mathrm{C}$.

135 (CEA-CONF-6241) French status of high level radioactive wastes solidification. Sombret, $C$. (CEA Etablissement de la Vallee du Rhone, 30 - Bagnols-sur-Ceze (France)). Apr 1982.
11 p. (CONF-820424-34). NTIS (US Sales Only), PC A02/MF A01. Order Number DE82703060.

From ANS topical meeting on trcatment and handling of radioactive wastes; Richland, WA, USA (19 Apr 1982).

Research and development carried out in France for a long time about solidification of high level radioactive wastes have been mainly directed toward the glassy state. Investigations about borosilicates lead up to choose glass as a final form for the fission products solutions stored in both reprocessing plants. Works related to various techniques to make use of this type of material started also a long time ago. A two steps continuous process was selected owing to satisfactory results rised from a long term prototype running with respects to the glass manufacturing as well as the remotely handling ability. AVM, the Marcoule Vitrification Plant has been running since 1978. Further Vitrification Plants related to the La Hague reprocessing plant are under design.

138 (CEA-CONF-6278) Actual point about fission products vitrification. Bonniaud, $R$. (CEA Etablissement de la Vallec du Rhone, 30 Bagnols-sur-Ceze (France)), May 198?. 18p. (In French). (CONF-8205164-1). NTIS (US Sales Only), PC A02/MF A01. Order Number DE83701825.

From French Nuclear Energy Society meeting on nuclear waste; Paris, France (13 May 1982).

The main characteristics concerning the continuous vitrification process for the confinement of fission product solutions operated at AVM are summarized. The general principle of a vitrification plant is described. The AVM plant efficiency as also its conception of consumable parts interchangeability are satisfying. The evolution of the process and its application developed in two ways: a more spaced installation conception and the improvement of the weak points remarked at AVM, as also the capacity of output. Two industrial units are designed at $\mathrm{La}$ Hague. The future evolution of the process aims at manufacturing glass at higher temperatures about $1400^{\circ} \mathrm{C}$. Some problems remain to be resolved for using ceramic melters associated with a calcination unit. The studies provide for a satisfying behaviour for the material to longterm. The risks of damage by crystallisation, leaching and effects of alpha emission are analysed.

137 (CEA-CONF-6307) Encapsulation of tritiated wastes in hot laboratories. Hayet, L.; Bourdinaud, P. (CEA Centre d'Etudes Nucleaires de Saclay, 91 - Gif-sur-Yvette (France)). Jun 1982. 27p. (In French). (CONF-8206149-3). NTIS (US Sales Only), PC A03/MF A01. Order Number DE83701042.

From 21. Euratom workshop on hot laboratories and remote handling; Mol, Belgium (10 Jun 1982).

From 1962 to 1976, one of the laboratories called "Cellule 4", was equipped to produce large quantities of tritium. The decisions taken in June 1976 to redirect the activities of the Radioisotopes Department included the suspension of the tritium activities of "Cellule 4" at the Saclay Nuclear Research Center. From 1976 to 1981, many CEA departments contributed to the design and implementation of a packaging procedure for tritiated wastes resulting from the dismantling of "Cellule 4". A olassification into three groups was arinpted for this purpose. (1) Packaging in a welded stainless steel container. (2) Packaging in a reinforced concrete shell lined internally with a thick coat of epoxy resin charged with sand. (3) Packaging in a reinforced concrete shell lined internally with a thin coat of epoxy resin. The dismantling operations were carried out in three phases. (1) $(21 / 2$ months): the T activity remained unchanged during this period and waste selection was carried out. (2) (2 1/2 months): waste dismantling and packaging of groups (2) and (3) were carried out in this phase. Activity decayed rapidly. (3) (2 months): the work performed included the loosening and cutting of the general structures.

138 (CONF-810528-16) Coating crystalline nuclear waste forms to improve inertness. Stinton, D.P.; Angelini, P.; Caputo, A.J.; Lackey, W.J. (Oak Ridge National Lab., TN (USA)). 1981. Contract W-7405-ENG-26. 27p. NTIS, PC A03/MF A01. Order Number DE82015675.

From 83. American Ceramic Society symposium on nucleation and crystallization in glasses; Washington, DC, USA (3 May 1981).

Crystalline waste forms of high simulated waste loading were successfully coated with layers of pyrolytic carbon and silicon carbide. Sol-gel technology was used to produce microspheres that contained simulated waste. A separate process for cesium immobilization was 
developed, which loads 5 wt \% Cs onto zeolite particles for subsequent coating. The chemical vapor deposition process was developed for depnsiting thin layers of carbon and silicon carbide onto particles in a fluidized-bed coater. Pyrolytic carbon-coated particles were extremely inert in numerous leach tests. Aqueous leach test results of coated waste forms were below detection limits of such sensitive analytical techniques as atomic absorption and inductively coupled plasma atomic emission.

139 (CONF-811088-7) Incorporation of high-level nuclear waste in gel spheres. Robinson, S.M.; Arnold, W.D.; Bond, W.D.; Angelini, P.; Stinton, D.P. (Oak Ridge National Lab., TN (USA)). 1981. Contract W-7405-ENG-26. 24p. NTIS, PC A02. Order Number DE82022271.

From American Ceramic Society conference; Newport Beach, CA, USA (25 Oct 1981).

Paper copy only, copy does not permit microfiche production. Waste sludge is incorporated in gel spheres by the method of internal gelation. Gel spheres containing up to $90 \mathrm{wt} \%$ waste have been produced from defense and commercial wastes. A generic cesiumbearing waste form has been developed. Pyrolytic carbon and $\mathrm{SiC}$ coatings reduce the leachability of all tested arlicles to the detection limits.

140 (CONF-820636-4) Near-surface leaching studies of Pb-implanted Savannah River waste glass. Arnold, G.W.; Northrup, C.J.M.; Bibler, N.E. (Sandia National Labs., Albuquerque, NM (USA); Du Pont de Nemours (E.I.) and Co., Aiken, SC (USA). Savannah River Lab.). 1982. Contract AC0476DP00789. 22p. NTIS, PC A02/MF A01. Order Number DE82017022.

From 5. international symposium on the scientific basis for radio active waste management; Berlin, F.R. Germany (7 Jun 1982).

Portions of document are illegible.

The present experiments with Savannah River Plant simulated nuclear waste glass implanted with $\mathrm{Pb}$ ions, used Rutherford backscattering spectrometry and elastic recoil detection to follow in detail the changes in composition which occur in the near-surface region upon leaching in deionized water at $90^{\circ} \mathrm{C}$. Analyses of the leach solutions were made in an attempt to correlate the actual leach rates with the observed near-surface compositional changes. These experiments show that radiation damage can cause changes in the composition of the nearsurface of the leached glass. We also find that a critical fluence is reached where abrupt changes of the surface elemental composition occur as a result of leaching. This fluence is near the value observed by both Dran, et al. and Primak. Solution analyses were not made for all the leaching experiments. However, those analyses which were made indicate that the amount of material actually leaving the glass is not significantly increased as a result of the radiation damage.

141 (CONF-820833-Vol.1, pp 371-380) Volatilization and trapping of ruthenium in high temperature processes. Klein, M.; Weyers, C.; Goossens, W.R.A. (SCK/CEN, Nuclear Energy Center, Mol, Belgium). Feb 1983. NTIS, PC A99/MF A01.

From 17. DOE nuclear air cleaning conference; Denver, $\mathrm{CO}$ USA (1 Aug 1982).

This experimental study has indicated the importance of moisture and $\mathrm{NO}_{\mathrm{x}}$ vapors on the volatility and trapping conditions of ruthenium in high temperature processes. Also the process operating conditions have a great influence on the ruthenium behavior in the off-gas purification units. Of particular interest is the observation that the ruthenium release during direct vitrification of simulated high-level liquid waste is a factor of about 5 smaller than the ruthenium release during calcination of this type of waste. Moreover, in the direct vitrification case the ruthenium escapes mostly in the form of an aerosol whereas in the calcination case a volatile ruthenium compound is dominating. Consequently, a specific ruthenium filter is not needed in the off-gas line of a direct vitrifier simplifying in this way the number of units in this off-gas line and avoiding the handling and controlling problems of such a ruthenium filter. In the future, a similar program will be started on the volatility of cesium and antimony in a liquid fed melter and on the technical reliability of the liquid fed melter and its associated gas purification units on a semi-pilote scale under simulated conditions.

142 (CONF-821005pp

VII.85-VII.98) Decommissioning alternatives for Savannah River Plant highlevel waste tank 16. Prendergast, J.T. Jr. (E.I. du Pont de
Nemours \& Co., Aiken, SC). 1982. NTIS, PC A99/MF A01. Contract AC09-76SR00001.

From DOE international decommissioning symposium; Seattle, WA, USA (10 Oct 1982).

As part of the waste management operations at Savannah River Plant (SRP), 24 waste tanks will be removed from service in the next 10 years. These tanks stored high-activity fission-product waste from nuclear fuel reprocessing operations at SRP. Waste Tank 16, in H Area at SRP, had earlier been removed from service in 1972 due to containment problems and later emptied. This report, using Tank 16 as a basis, assesses costs and radiological doses for four methods of decommissioning: 30-year layaway, immediate entombment, immediate dismantlement, and 30-year delayed dismantlement. The 30-year layaway alternative would cost about $\$ 0.9$ million with a workforce dose of about 2 manrem. Immediate entombment would cost about $\$ 4$ million with an occupational dose of 10 man-rem. Immediate dismantlement would cost about $\$ 36$ million. The workforce dose would be approximately 93 manrem. The 30-year delayed dismantlement alternative would cost about $\$ 32$ million with an associated workforce dose of 47 man-rem. All costs are in 1982 dollars and are not discounted. 4 figures, 5 tables.

143 (CONF-821107-50) Mechanical stability of a Cmdoped celsian glass-ceramic. Roubort, J.L.; Offermann, P. Matzke, H. (Commission of the European Communities, Karlsruhe (Germany, F.R.). European Inst. for Transuranium Elements; Hahn-Meitner-Institut fuer Kernforschung Berlin G.m. b.H. (Germany, F.R.)). Oct 1982. Contract W-31-109-ENG-38. 9p. NTIS, PC A02. Order Number DE83007862.

From 6. international symposium on the scientific basis for radioactive waste management (Materials Research Society); Boston, MA, USA (1 Nov 1982)

Paper copy only, copy does not permit microfiche production.

The Hertzian indentation technique has been used to measure the fracture properties of a celsian glass-ceramic (B1-3) developed for high-level waste storage. For spherical glass indentors of radii, $R \leq 5$ $\mathrm{mm}$ the load required to form ring cracks is proportional to $R$ and intersects the origin, confirming Auerbach's law. The measurements are analyzed and result in a fracture toughness, $\mathrm{K}_{\mathrm{IC}}>1.3 \mathrm{MNm}^{-3} 2 /$. For $\mathbf{R}$ $>5 \mathrm{~mm}$ flaw statistics cause a deviation from the ideal behavior of Auerbach's law. Spontaneous failure due to radiation was not observed, on the contrary, the self-radiation damage caused by recoiling $\mathrm{Pu}$ atoms resulting from $\alpha$-decay of a ${ }^{244} \mathrm{Cm}$ doped sample to a dose of about 1.5 $\times 10^{19} \alpha$-decays $/ \mathrm{cm}^{0}$ increases $K_{I C}$ by at least $25 \%$. This increase in toughness is probably caused by the internal stresses which result from disordering due to the radiation damage. This is confirmed by the broadening of the x-ray spectra as the result of self-radiation. Annealing a damaged sample at high temperatures produces fracture presumably by the coalescence of helium bubbles.

144 (CONF-830205-33) Ceramic process and plant design for high-level nuclear waste immobilization. Grantham, L.F.; McKisson, R.L.; De Wames, R.E.; Guon, J.; Flintoff, J.F.; McKenzie, D.E. (Rockwell International Corp., Canoga Park, CA (USA). Energy Systems Group). 1983. Contract AT0381SF11572. 8p. NTIS, PC A02/MF A01. Order Number DE83016080. Feb 1983)

From Waste management conference; Tucson, AZ, USA (27

Portions are illegible in microfiche products.

In the last 3 years, significant advances in ceramic technology for high-level nuclear waste solidification have been made. Product quality in terms of leach-resistance, compositional uniformity, structural integrity, and thermal stability promises to be superior to borosilicate glass. This paper addresses the process effectiveness and preliminary designs for glass and ceramic immobilization plants. The reference two-step ceramic process utilizes fluid-bed calcination (FBC) and hot isostatic press (HIP) consolidation. Full-scale demonstration of these well-developed processing steps has been established at DOE and/or commercial facilities for processing radioactive materials. Based on Savannah River-type waste, our model predicts that the capital and operating cost for the solidification of high-level nuclear waste is about the same for the ceramic and glass options. However, when repository costs are included, the ceramic option potentially offers significantly better economics due to its high waste loading and volume reduction. Volume reduction impacts several figures of merit in addition to cost such as system logistics, storage, transportation, and risk. The study con- 
cludes that the ceramic product/process has many potential advantages, and rapid deployment of the technology could be realized due to fullscale demonstrations of FBC and HIP technology in radioactive environments. Based on our finding and those of others, the ceramic innovation not only offers a viable backup to the glass reference process but promises to be a viable future option for new high-level nuclear waste management opportunities.

145 (CONF-830451-18) Effect of composition and radiation on the Hertzian indentation behavior of nuclear waste glasses. Matzke, H.; Kahl, L.; Routbort, J.L.; Saidl, J. (Commission of the European Communities, Karlsruhe (Germany, F.R.). European Inst. for Transuranium Elements; Kernforschungszentrum Karlsruhe G.m.b.H. (Germany, F.R.). Inst. fuer Nukleare Entsorgungstechnik; Argonne National Lab., IL (USA)). 1983. Contract W-31-109-ENG-38. 25p. NTIS, PC A02/MF A01. Order Number DE83014346.

From 85. American Ceramic Society annual meeting; Chicago, IL, USA (25 Apr 1983).

The Hertzian indentation technique has been used to determine the fracture toughness, $K_{I c}$ of two borosilicate glasses developed to contain high-level nuclear waste. For the product VG 98/12, adding selected groups of fission products leaves $K_{I c}$ unchanged, but addition of $\mathrm{Pb}$ lowers $\mathrm{K}_{\mathrm{lo}}$ by $\sim 20 \%$. Radiation with $77 \mathrm{MeV} \alpha$-particles to a dose of $\sim 10^{15} \alpha / \mathrm{cm}^{2}$ increases $K_{\text {Ic }}$ by $\sim 75 \%$. For the product SM $58 \mathrm{LW}$ 11 , the fracture toughness was measured on pieces taken from different parts of a large cylinder to investigate the effects of segregation phenomena and of partial crystallization and formation of small cristobalite inclusions which decrease $\mathrm{K}_{\mathrm{Ic}}$ by $\sim 25 \%$.

\section{6 (CONF-830808-5)}

Octyl(phenyl)-N,N-diisobutylcarbamoylmethylphosphine oxide as an extractant for actinides from nitric acid waste. Horwitz, E.P.; Diamond, H.; Kalina, D.G.; Kaplan, L.; Mason, G.W. (Argonne National Lab., IL (USA)). 1983. Contract W-31-109ENG-38. 9p. NTIS, PC A02/MF A01. Order Number DE83010774.

From International solvent extraction conference; Denver, $\mathrm{CO}$, USA (26 Aug 1983).

The ability of neutral bifunctional organophosphorus compounds to extract trivalent actinides, specifically Am(III), from nitric acid solution has stimulated investigations into the processing of PUREX high level liquid waste. The authors' work in this area has focused primarily on derivatives of carbamoylmethylphosphine oxide (CMPO). The authors of this paper have found that the best extractant of this type is octyl(phenyl)-N,N-diisobutylmethylphosphine oxide (abbrev. O phi D [IB]CMPO). This extractant has a unique combination of substituent groups which impart to the resulting molecule substantially improved ability to extract actinides from acidic nitrate media and to withstand hydrolytic and radiolytic degradation. At the same time good selectivity of Am(III) actinides over fission products and favorable solubility properties on actinide loading are maintained. This paper describes the application of $O$ phi D [IB] CMPO to the extraction of transuranium elements (Np, Pu, and Am) and fission product rare earths (F.P.R.E.) from evaporated highly acidic ( $5 \mathrm{M} \mathrm{HNO}_{0}$ ) PUREX waste. Additional information on the influence of phenyl substitution in CMPO's and mixed CMPO-TBP extractant solutions on $\mathrm{D}_{\mathrm{Am}}$ is also presented. 3 figures, 2 tables.

147 (DOE/ET/41900-12) Preliminary assessment of key process steps for converting Savannah Kiver Plant high-level wastes to tailored ceramics. Guon, J. (Rockwell International Corp., Canoga Park, CA (USA). Energy Systems Group). 30 Sep 1981. Contract AC09-79ET41900. 56p. (ESG-DOE13360). NTIS, PC A04/MF A01. Order Number DE82012506.

The status of the process design description and of the processing studies for the preparation of the Tailored Ceramics waste form from Savannah River Plant high-level wastes is prescnted. The topics included are the (1) general requirements for the process; (2) design, analytical, and experimental work to verify that the process can be scaled to plant-size operation; and (3) suggested improvements in processing, equipment selection, and plant layout. Suggestions are also given for the direction of future work.
148 (DOE/ET/41900-14) CsAlSi, $\mathrm{O}_{12:}$ a possible host for ${ }^{107}$ Cs immobilization. Adl, T.; Vance, E.R. (Rockwell International Corp., Canoga Park, CA (USA). Energy Systems Group; Pennsylvania State Univ., University Park (USA). Materials Research Lab.). 31 Mar 1982. Contract AC09-79ET41900. 21p. (ESG-DOE-13369). NTIS, PC A02/MF A01. Order Number DE82013246.

CsAlSis $\mathrm{O}_{12}$ exhibits more acid resistance than pollucite $\left(\mathrm{Cs} \mathrm{AlSi}_{2} \mathrm{O}_{6}\right)$. At $\mathrm{pH}$ values of 1.02 and 1.40 , the extraction of $\mathrm{Cs}$ from $\mathrm{CsAlSis}_{12}$ at $25^{\circ} \mathrm{C}$ was approximately proportional to the square root of leach time. The Cs extraction at $25^{\circ} \mathrm{C}$ varied as $\left[\mathrm{H}^{+}\right]^{006}$ over the $\mathrm{pH}$ range of 1 to 6 . Also, the Cs extraction in various brines at $300^{\circ} \mathrm{C} / 30$ $\mathrm{MPa}$ was comparable with that for pollucite. $\mathrm{CsAlSi}_{5} \mathrm{O}_{12}$ can be crystallized at about $1000^{\circ} \mathrm{C}$ from calcines if a small amount of $\mathrm{CaO}$ is present, but in the absence of such sintering acids, crystallization temperatures of about $1400^{\circ} \mathrm{C}$ are necessary. Compatibility data were also obtained with respect to several other phases with which $\mathrm{CsAlSi}_{5} \mathrm{O}_{12}$ might be expected to coexist in tailored ceramics designed for high-level defense waste.

149 (DOE/ET/41900-16) Process description and plant design for preparing ceramic high-level waste forms. Grantham, L.F.; McKisson, R.L.; Guon, J.; Flintoff, J.F.; McKenzie, D.E. (Rockwell International Corp., Canoga Park, CA (USA). Energy Systems Group). 25 Feb 1983. Contract AC09. 79ET41900. 72p. (ESG-DOE-13397). NTIS, PC A04/MF A01. Order Number DE83007885.

Portions are illegible in microfiche products. Original copy available until stock is exhausted.

The ccramics process flow diagram has been simplified and upgraded to utilize only two major processing steps - fluid-bed calcination and hot isostatic press consolidating. Full-scale fluid-bed calcinatlon has been used at INEL to calcine high-level waste for $18 \mathrm{y}$; and a second-generation calciner, a fully remotely operated and maintained calciner that meets ALARA guidelines, started calcining high-level waste in 1982. Full-scale hot isostatic consolidation has been used by DOE and commercial enterprises to consolidate radioactive components and to encapsulate spent fuel elements for several years. With further development aimed at process integration and parametric optimization, the operating knowledge of full-scale demonstration of the key process steps should be rapidly adaptable to scale-up of the ceramic process to full plant size. Process flowsheets used to prepare ceramic and glass waste forms from defense and commercial high-level liquid waste are descrihed. Preliminary layouts of process flow diagrams in a high-level processing canyon were prepared and used to estimate the preliminary cost of the plant to fabricate both waste forms. The estimated costs for using both options were compared for total waste management costs of SRP high-level liquid waste. Using our design, for both the ceramic and glass plant, capital and operating costs are essentially the same for both defense and commercial wastes, but total waste management costs are caloulated to be signifirantly less for defense wastes usine the ceramic option. It is concluded from this and other studies that the ceramic form may offer important advantages over glass in leach resistance, waste loading, density, and process flexibility. Preliminary economic calculations indicate that ceramics must be considered a leading candidate for the form to immobilize high-level wastes.

160 (DOE/ET/41900-17) Commercial high-level-waste management: options and econnmics. A comparative analysis of the ceramic and glass waste forms. McKisson, R.L.; Grantham, L.F.; Guon, J.; Recht, H.L. (Rockwell International Corp., Canoga Park, CA (USA). Energy Systems Group). 25 Feb 1983. Contract AC09-79ET41900. 133p. (ESG=DOE-13391). NIIS, PC A07/MF A01. Order Number DE83006235.

Results of an estimate of the waste management costs of the commercial high-level waste from a $\mathbf{3 0 0 0}$ metric ton per year reprocessing plant show that the judicious use of the ceramic waste form can save about $\$ 2$ billion during a 20-year operating campaign relative to the use of the glass waste form. This assumes PWR fuel is processed and the waste is encapsulated in 0.305 -m-diam canisters with ultimate emplacement in a BWIP-type horizantal-borehole repository. The estimated total cost (capital and operating) of the management in the ceramic form is $\$ 2.0$ billion, and that of the glass form is $\$ 4.0$ billion. Waste loading and waste form density are the driving factors in that the low-waste loading (25\%) and relatively low density $\left(3.1 \mathrm{~g} / \mathrm{cm}^{0}\right)$ characteristic of the glass form require several times as many canisters to handle a given 
waste throughput than is needed for the ceramic waste form whose waste loading capability exceeds $60 \%$ and whose waste density is nominally $5.2 \mathrm{~g} / \mathrm{cm}^{0}$ ) characteristic of the glass form requires several timcs as many canisters to handle a given waste throughput than is needed for the ceramic waste form whose waste loading capability exceeds $60 \%$ and whose waste density is nominally $5.2 \mathrm{~g} / \mathrm{cm}^{0}$. The minimum-cost ceramic waste form has a $60 \mathrm{wt}$. \% waste loading of commercial high-level waste and requires 25 years storage before emplacement in basalt with delayed backfill. Because of the process flexibility allowed by the availability of the high-waste loading of the ceramic form, the intermediatelevel liquid waste stream can be mixed with the high-level liquid waste stream and economically processed and emplaced. The cost is greater by $\$ 0.3$ billion than that of the best high-level liquid waste handling process sequence ( $\$ 2.3$ billion vs $\$ 2.0$ billion), but this difference is less than the cost of the separate disposal of the intermediate-level liquid waste.

151 (DOE/ET/41900-18) Development of polyphase ceramics for the immobilization of high-level Defense nuclear waste. Morgan, P.E.D.; Harker, A.B.; Clarke, D.R.; Flintoff, J.J.; Shaw, T.M. (Rockwcll International Corp., Thousand Oaks, CA (USA). Science Center). 25 Feb 1983. Contract AC09-79ET41900. 108p. (ESG-DOE-13400). NTIS, PC A06/MF A01. Order Number DE83007892.

The report contains two major sections: Section I - An Improved Polyphase Ceramic for High-Level Defense Nucleation Waste reports the work conducted on titanium-silica based ceramics for immobilizing Savannah River Plant waste. Section II - Formulation and Processing of Alumina Based Ceramic Nuclear Waste Forms describes the work conducted on developing a generic alumina and alumina-silica based ceramic waste form capable of immobilizing any nuclear waste with a high aluminum content. Such wastes include the Savannah River Plant wastes, Hanford neutralized purex wastes, and Hanford N-Reactor acid wastes. The design approach and process technology in the two reports demonstrate how the generic high waste loaded ceramic form can be applied to a broad range of nuclear waste compositions. The individual sections are abstracted and indexed separately.

152 (DOE/ET/41900-18, pp 5-46) Improved polyphase ceramic form for high-level defense nuclear waste. Harker, A.B.; Morgan, P.E.D.; Clarke, D.R.; Flintoff, J.J.; Shaw, T.M. 25 Feb 1983. NTIS, PC A06/MF A01. (ESG-DOE-13400).

In Development of polyphase ceramics for the immobilization of high-level Defense nuclear waste.

An improved ceramic nuclear waste form and fabrication process have been developed using simulated Savannah River Plant defense high-level waste compositions. The waste form provides flexibility with respect to processing conditions while exhibiting superior resistance to ground water leaching than other currently proposed forms. The ceramic, consolidated by hot-isostatic pressing at $1040^{\circ} \mathrm{C}$ and $10,000 \mathrm{psi}$, is composed of six major phases, nephelline, zirconolite, a murataite-type cubic phase, magnetite-type spinel, a magnetoplumbite solid solution, and perovskite. The waste form provides multiple crystal lattice sites for the waste elements, minimizes amorphous intergranular material, and can accommodate waste loadings in excess of $60 \mathrm{wt} \%$. The fabrication of the ceramic can be accomplished with existing manufacturing technology and eliminates the effects of radionuclide volatilization and offgas induced corrosion experienced with the molten processes for vitreous form production.

153 (DOE/ET/41900-18, pp 47-107) Formulation and processing of alumina based ceramic nuclear waste forms. Harker, A.B.; Morgan, P.E.D.; Clarke, D.R.; Flintoff, J.J.; Jantzen, C.M. 25 Feb 1983. NTIS, PC A06/MF A01. (ESG-DOE13400).

In Development of polyphase ceramics for the immobilization of high-level Defense nuclear waste.

A class of dense, high-alumina ceramics has been developed for the immobilization of Savannah River Plant defense nuclear waste. The ceramics, fabricated by hot isostatic pressing or reactive hot pressing, normally consist of four compatible crystalline phases - magnetoplumbite, spinel, uraninite, and corundum. A fifth compatible phase, nepheline, can be designed into the assemblage when high sodium levels are present in the waste. All radionuclides are incorporated into the magnetoplumbite and uraninite phases, with the nonradiophases providing microstructural isolation of the individual radiophase grains. The chemi- cal formulation of the ceramic waste form and the processing requirements is discussed.

164 (DOE/IG-0187) High-level nuclear-wasteimmobilization program. (Department of Energy, Washington, DC (USA). Office of Inspector General). 19 Nov 1982. 45p. TIC. Order Number DE83006930.

Report to the Secretary.

Savings that could be achieved in the Department's programs for immobilizing high-level defense nuclear waste (HLDW) and in the West Valley Demonstration Project, which is part of the Department's commercial nuclear waste program, are discussed. The programs are the Department's main effort for developing the technology to convert highly radioactive, liquid waste into more stable waste forms. We concluded that the potential exists for avoiding certain large, future expenditures. For example, the Department could avoid at least $\$ 173$ million in costs for additional HLDW storage facilities by proceeding expeditiously to immobilize current inventories. The Savannah River immobilization process produces a costly waste package that appears to exceed the requirements established by the Nuclear Regulatory Commission. In the commercial program, it appears that the cooperative agreement negotiated between the Department and the State of New York provided for more activities and involves greater costs than are required by the West Valley Demonstration Project Act, Public Law 96-368. The cost of the West Valley Project might also be reduced by immobilizing the relatively small amount of high-level commercial waste at West Valley into an interim waste form, instead of immobilizing it into borosilicate glass, the proposed final waste form. We concluded that two programs at the Savannah River Operations Office could be terminated, or substantially reduced in scope, without adverse impact on defense activities. In the first of these, concerning the development of alternative waste forms for immobilizing HLDW, the Department agreed with our conclusion and has terminated the program. The second program is to conduct extensive archaeological research at Savannah River. We believe that the degree of effort being pursued is not required.

165 (DOE/SR/00952-T7) Nuclear-fuel-cycle education: Module 8. Waste management. O'Hara, F.A.; Roberts, L.; Daglish, J. (Ohio State Univ., Columbus (USA); UKAEA Atomic Energy Research Establishment, Harwell; Kansas State Univ., Manhattan (USA)). Dec 1981. Contract AS09. 76SR00952. 123p. NTIS, PC A06/MF A01. Order Number DE83015187.

Portions are illegible in microfiche products.

This module has chapters devoted to: an international perspective on radioactive waste management; high-level waste management research; transuranic waste; high-level radioactive waste; and low-level waste in the light water reactor fuel cycle without reprocessing.

166 (DOE/SR-WM-79-3-Rev.2/83) Strategy document: long-term technclogy program for defense high-level waste. (USDOE Savannah River Operations Office, Aiken, SC). Feb 1983. 44p. NTIS, PC A03/MF A01. Order Number DE83010816.

This document describes the strategy of the Defense Waste Management Program in meeting the technology requirements for long-term management of high-level waste (HLW) at the individual DOE defense sites at the Savannah River Plant near Aiken, SC, the Hanford Plant near Richland, WA, and the Idaho Chemical Processing Plant near Idaho Falls, ID. The immediate program focus is on implementing waste processing strategy selected in compliance with the National Environmental Policy Act (NEPA) at Savannah River, while continuing development of alternative strategies at Hanford and Idaho until such time as the required documents are scheduled in compliance with NEPA.

167 (DOE/SR/WM-82-1) Sarannah River Waste Management Program Plan - FY 1982. (Du Pont de Nemours (E.I.) and Co., Aiken, SC (USA). Savannah River Plant). Dec 1981. Contract AC09-76SR00001. 124p. NTIS, PC A07/MF A01. Order Number DE82006070.

This document provides the program plan as requested by the Savannah River Operations Office of the Department of Energy. The plan was developed to provide a working knowledge of the nature and extent of the waste management programs being undertaken by Savannah River (SR) contractors for the Fiscal Year 1982. In addition, the document projects activities for several years beyond 1582 to ade- 
quately plan for safe handling and storage of radioactive wastes generated at Savannah River, for developing technology to immobilize highlevel radioactive wastes generated and stored at SR, and for developing technology for improved management of low-level solid wastes. A revised plan will be issued prior to the beginning of the first quarter of each fiscal year. In this document, work descriptions and milestone schedules are current as of the date of publication. Budgets are based on available information as of October $1,1981$.

168 (DOE/TIC-3311-S12) Radioactive waste processing and disposal: a bibliography. McLaren, L.H. (ed.). (Department of Energy, Oak Ridge, TN (USA). Technical Information Center). Apr 1983. 1122p. C. Order Number DE83007280.

This compilation contains 4314 citations of foreign and domestic research reports, journal articles, patents, conference proceedings, and books entered into the DOE Energy Data Base from January 1982 through December 1967. These references, as well as references from the period January 1967 through December 1981, are available for online searching and retrieval using the DOE/RECON system. The citations are arranged by subject category. Within a given category the arrangement is alphanumeric by report number. Citations of untinumbered documents are arranged in reverse chronological order after the reports. Each complete bibliographic record is followed by an abstract or a listing of the subject descriptors used to describe the contents of the reference for machine storage and retrieval. In many cases phrases or clauses that augment the title are also included. Five indexes, each preceded by a brief description, are provided: Corporate, Author, Subject, Contract Number, and Report Number.

169 (DOE/TIC-3389) High-level radioactive wastes. Grissom, M.C. (ed.). (Department of Energy, Oak Ridge, TN (USA). Technical Information Center). Oct 1982. 247p. NTIS \$16.25. Order Number DE82012272.

Portions of document are illegible.

This bibliography contains $\mathbf{8 1 2}$ citations on high-level radioactive wastes included in the Department of Energy's Energy Data Base from January 1981 through July 1982 . These citations are to research reports, journal articles, books, patents, theses, and conference papers from worldwide sources. Five indexes are provided: Corporate Author, Personal Author, Subject, Contract Number, and Report Number.

160 (DOE/TIC-11611) Evaluation and selection of candidate high-level waste forms. (Department of Energy, Aiken, SC (USA). Savannah River Operations Office). Mar 1982. 109p. NTIS, PC A06/MF A01. Order Number DE82014134. Seven candidate waste forms being developed under the direction of the Department of Energy's National High-Level Waste (HLW) Technology Program, were evaluated as potential media for the immobilization and geologic disposal of high-level nuclear wastes. The evaluation combined preliminary waste form evaluations conducted at DOE defense waste-sites and independent laboratories, peer review assessments, a product performance evaluation, and a processability analysis. Based on the combined results of these four inputs, two of the seven forms, borosilicate glass and a titanate based ceramic, SYNROC, were selected as the reference and alternative forms for continued development and evaluation in the National HLW Program. Both the glass and ceramic forms are viable candidates for use at each of the DOE defense waste-sites; they are also potential candidates for immobilization of commercial reprocessing wastes. This report describes the waste form screening process, and discusses each of the four major inputs considered in the selection of the two forms.

161 (DOE/TIC-11612) Method for product performance evaluation of candidate waste forms for immobilization of highlevel radioactive wastes. Interface working group on high-level waste form selection factors. (USDOE, Washington, DC). Mar 1982. 53p. NTIS, PC A04/MF A01. Order Number DE82014916.

An evaluation method to discriminate among candidate waste forms for immobilizing high-levell radioactive wastes for geologic disposal has been developed. The evaluation method provides a means to judge the relative merits of potential waste forms based on quantitative product performance data and to establish a comparative ranking.

162 (DP-80-125-4) Waste Management Program. Technical progress report, October-December 1980. (Du Pónt de
Nemours (E.I.) and Co., Aiken, SC (USA). Savannah River Lab.; Du Pont de Nemours (E.I.) and Co., Aiken, SC (USA). Savannah River Plant). May 1982. Contract AC0976SR00001. 213p. NTIS MF A01. Order Number DE82017564.

Portions of document are illegible.

This quarterly report provides information on operations and development programs for the management of radioactive wastes from operation of the Savannah River Plant. The studies on the environmental and safety assessments, waste form development and characterization, waste retrieval, process equipment and development, transuranic waste management, and low-level waste management are a part of the Long-Term Waste Management Technology Program. The following studies are reported for the SR Interim Waste Operations Program: surveillance and maintenance, waste concentration, low-level effluent waste, tank replacement/waste removal, process development, and solid waste storage operations. Studies for the Office of Nuclear Waste Isolation are reported.

183

(DP-81-157-3) Long-term high-level waste technology. Compusite quarterly techuical repor'h July-Stptember 1981. Cornman, W.R. Jr. (comp.). (Du Pont de Nemours (E.I.) and Co., Aiken, SC (USA). Savannah River Lab.). Mar 1982. Contract AC09-76SR00001. 106p. NTIS, PC A06/MF A01. Order Number DE82012374.

This report summarizes work performed at 17 participating sites to immobilize high-level radioactive wastes from fuel reprocessing. Fixdtion in glass, concrete, tailored ceramics (SYNROC), coated waste forms, and matrix waste forms are covered. (DLC)

164

(DP-81-157-4) Composite quarterly technical report long-term high-level-waste technology, October-December 1981. Cornman, W.R. (comp.). (Du Pont de Nemours (E.I.) and Co., Aiken, SC (USA). Savannah River Lab.). Jun 1982. Contract AC09-76SR00001. 80p. NTIS, PC A05/MF A01. Order Number DE82016240.

Portions of document are illegible.

This document summarizes work performed at participating sites on the immobilization of high-level wastes from the chemical reprocessing of reactor fuels. The plan is to develop waste form alternatives for each of the thrce DOE sites (SRP. ICPP, and Hanford). Progress is reported in the following areas: waste preparation; fixation in glass, concrete, tailored ceramics, and coated particles; process and equipment development; and final handling. 12 figures, 19 tables. (DLC)

165 (DP-82-157-2) Long-term high-level-waste technology. Composite quarterly technical report, April-June 1982. Comman, W.R. (comp.). (Du Pont de Nemours (E.I.) and Co., Aiken, SC (USA). Savannah River Lab.). Nov 1982. Contract AC09-76SR00001. 51p. NTIS, PC A04/MF A01. Order Number DE83003830.

Portions of document are illegible. Printed copy available until stock is exhausted.

This composite quarterly technical report summarizes work performed at participating sites to immobilize high-level radioactive wastes. ENICO is preparing a document to recommend strategy for the long-term management of high-level wastes generated at ICPP. Postclusure risks are compared for a borosilicate refercnce waste form and an alternative waste form for defense high-level waste at SRP. A Liquid Solidification Study at Hanford showed that solidification and disposal of non-TRU Hanford defense residual liquors into single-shell tanks is technically feasible and economically advantageous over double-shell tank disposal. In-tank drying and consolidation of wet synthetic salt is being demonstrated at Rockwell Hanford Operation (RHO) with a microwave drying system. Four-month corrosion tests of Hanford double-shell slurry on carbon-steel tanks were concluded. A series of experiments were completed that qualified the use of neutron activation analysis and radioactive tracers for measuring leach rates from simulated waste glasses. Glass frits 127 and 532 are being investigated for vitrification of new blends of ICPP calcined waste which have high sodium contents. The composition of the borosilicate glass waste form for SRP has been optimized. Development and evaluation of tailored cementitious waste hosts for Hanford TRU waste sludges is under way. SYNROC C samples containing both PW-4b- and PW-7a-type wastes were prepared and tested at LLNL. Static leach tests were completed at Rockwell Energy Systems Group (RES) on the RSC-S29 ceramic 
waste form for SRP defense waste. A ceramic waste form was designed at RES specifically for acid waste streams from processing of reactor fuel. Development work on the Large-Scale Glass Melter continued at SRL. Studies of the potential for steam explosions in the Defense Waste Processing Facility melter are under way at SRL.

166 (DP-82-157-3) Long-term high-level waste technology. Composite quarterly technical report, July-September 1982. Cornman, W.R. (comp.). (Du Pont de Nemours (E.I.) and Co., Aiken, SC (USA). Savannah River Lab.). Feb 1983. Contract AC09-76SR00001. 41p. NTIS, PC A02/MF A01. Order Number DE83010987.

This series of reports summarizes research and development studies on the immobilization of high-level wastes from the chemical reprocessing of nuclear reactor fuels. Immobilization of the wastes (defense and commercial) consists of placing them in a high-integrity form with a very low potential for radionuclide release. Immobilization of commercial wastes is being considered on a contingency basis in the event that reprocessing is resumed. The basic plan for meeting the goal of immobilization of the DOE high-level wastes is (1) to develop technology to support a realistic choice of waste form alternatives for each of the three (SRP, ICPP, and RHO) DOE sites, (2) to develop product and processing technology with sufficient scaleup to provide design data for full-scale facilities, and (3) to support $R$ and $D$ for construction and operation of immobilization facilities. Most of the existing high-level nuclear wastes in the United States have been generated in defense activities of DOE at the Savannah River Plant (SRP), Aiken, SC; at Hanford, WA; and at the Idaho Chemical Processing Plant. An inventory of approximately 90 million gallons of high-level defense waste is on hand with a continuous annual production rate of 0.5 to 1.0 million gallons.

167

(DP-1541) Physical and chemical characterization of synthetic calcined sludge. Slates, R.V.; Mosley, W.C. Jr.; Tiffany, B.; Stone, J.A. (Du Pont de Nemours (E.I.) and Co., Aiken, SC (USA). Savannah River Lab.). Mar 1982. Contract

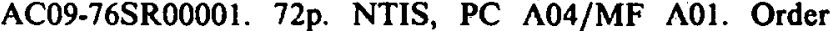
Number DE82012373.

Calcined synthetic sludge was chemically characterized in support of engineering studies to design a processing plant to solidify highly radioactive waste at the Savannah River Plant. An analytical technique is described which provides quantitative data by mass spectrometric analysis of gases evolved during thermogravimetric analysis without measurements of gas flow rates or mass spectrometer sensitivities. Scanning electron microprobe analysis, Mossbauer spectroscopy, and several other common analytical methods werc also used. Calcined sludge consists primarily of amorphous particles of hydrous oxides with iron, manganese, nickel, and calcium distributed fairly uniformly throughout the powder. Iron, manganese, nickel, and calcium exist in forms that are highly insnluble in water, hut aluminum, sulfate, nitrate, and sodium exhibit relative water solubilities that increase in the given order from $60 \%$ to $94 \%$. Evolved gas analysis in a helium atmosphere showed that calcined sludge is completely dehydrated by heating to $400^{\circ} \mathrm{C}$, carbon dioxide is evolved between 100 to $700^{\circ} \mathrm{C}$ with maximum evolution at $500^{\circ} \mathrm{C}$, and oxygen is evolved between 400 and $1000^{\circ} \mathrm{C}$. Evolved gas analyses are also reported for uncalcined sludge. A spinel-type oxide similar to $\mathrm{NiFe}_{2} \mathrm{O}_{4}$ was detected by $x$-ray diffraction analysis at very low-level in calcined sludge.

168 (DP-1606-Rev.1) Description of Defense Waste Processing Facility reference waste form and canister. Revision 1. Baxter, R.G. (Du Pont de Nemours (E.I.) and Co., Aiken, SC (USA). Savannah River Plant). Aug 1983. Contract AC09. 76SR00001. 69p. NTIS, PC A04/MF A01. Order Number DE84001627.

Portions are illegible in microfiche products. Original copy available until stock is exhausted.

The Defense Waste Processing Facility (DWPF) will be located at the Savannah River Plant in Aiken, SC, and is scheduled for construction authorization during FY-1984. The reference waste form is borosilicate glass containing $\sim 28$ wt \% sludge oxides, with the balance glass frit. Borosilicate glass was chosen because of its high resistance to leaching by water, its relatively high solubility for nuclides found in the sludge, and its reasonably low melting temperature. The glass frit contains about $58 \% \mathrm{SiO}_{2}$ and $15 \% \mathrm{~B}_{2} \mathrm{O}_{0}$. Leachabilities of SRP waste glasses are expected to approach $10^{-8} \mathrm{~g} / \mathrm{m}^{2}$-day based upon 1000 -day tests using glasses containing SRP radioactive waste. Tests were performed under a wide variety of conditions simulating repository environments. The canister is filled with $3260 \mathrm{lb}$ of glass which occupies about $85 \%$ of the free canister volume. The filled canister will generate $\sim \mathbf{4 7 0}$ watts when filled with oxides from 5-year-old sludge and 15-year-old supernate from the sludge and supernate processes. The radionuclide content of the canister is about $177,000 \mathrm{ci}$, with a radiation level of 5500 $\mathrm{rem} / \mathrm{h}$ at canister surface contact. The reference canister is fabricated of standard 24-in.-OD, Schedule 20,304L stainless steel pipe with a dished bottom, domed head, and a combined lifting and welding flange on the head neck. The overall canister length is $9 \mathrm{ft} 10$ in. with a 3/8-in. wall thickness. The 3-m canister length was selected to reduce equipment cell height in the DWPF to a practical size. The canister diameter was selected as an optimum size from glass quality considerations, a logical size for repository handling and to ensure that a filled canister with its double containment shipping cask could be accommodated on a legal-weight truck. The overall dimensions and weight appear to be compatible with preliminary assessments of repository requirements. 10 references.

169 (DP-1625) Assessment of processes, facilities, and costs for alternative solid forms for immobilization of SRP defense waste. Dunson, J.B. Jr.; Eisenberg, A.M.; Schuyler, R.L. III; Haight, H.G. Jr.; Mello, V.E.; Gould, T.H. Jr.; Butler, J.L.; Pickett, J.B. (Du Pont de Nemours (E.I.) and Co., Aiken, SC (USA). Savannah River Lab.). Mar 1982. Contract AC09. 76SR00001. 228p. NTIS, PC A11/MF A01. Order Number DE82014221.

Portions of document are illegible.

A quantitative merit evaluation which assesses the relative difficulty of remote processing of Savannah River Plant high-level wastes for seven alternative waste forms is presented. The reference borosilicate glass process is rated as the simplest, followed by FUETAP concrete. The other processes evaluated in order of increasing complexity were: glass marbles in a lead matrix, high-silica glass, crystalline ceramic (Synroc-D and tailored ceramic), and coated ceramic particles. Cost appraisals are summarized for the borosilicate glass, high-silica glass, and ceramic waste form processing facilities.

170 (DP-1626) Description of a ceramic waste form and canister for Savannah River Plant high-level waste. Butler, J.L.; Allender, J.S.; Gould, T.H. Jr. (Du Pont de Nemours (E.I.) and Co., Aiken, SC (USA). Savannah River Lab.). Apr 1982. Contract AC09-76SR00001. 30p. NTIS, PC A03/MF A01. Order Number DE82014350.

A canistered ceramic waste form for possible immobilization of Savannah River Plant (SRP) high-level radioactive wastes is described. Characteristics reported for the form include waste loading, chemical composition, heat content, isotope inventory, mechanical and thermal properties, and leach rates. A conceptual design of a potential production process for making this canistered form are also described. The ceramic form was selected in November 1981 as the primary alternative to the reference waste form, borosilicate glass, for making a final waste form decision for SRP waste by FY-1983. 11 tables.

171 (DP-1627) Comparison of properties of borosilicate glass and crystalline ceramic forms for immobilization of Savannah River Plant waste. Stone, J.A.; Allender, J.S.; Gould, T.H. Jr. (Du Pont de Nemours (E.I.) and Co., Aiken, SC (USA). Savannah River Lab.). Apr 1982. Contract AC09-76SR00001. 144p. NTIS, PC A07/MF A01. Order Number DE82014133. Properties of borosilicate glass and the crystalline ceramic Synroc-D waste forms for immobilization of Savannah River Plant (SRP) defense high-level waste are compared. Waste loading, leach resistance, thermal stability, mechanical stability, and radiation stability are the properties compared. Leaching data are from comparative tests performed independently at the Savannah River Laboratory (SRL) and at the Materials Characterization Center, supplemented by data on borosilicate glass from SRL and data on Synroc-D from Lawrence Livermore National Laboratory (LLNL). Mechanical stability data are from comparative impact tests at Argonne National Laborato ry. Data on other properties are primarily from tests at SRL and LLNL. The comparison shows that borosilicate glass is superior in some properties, and Synroc-D is superior in others, with neither material showing a clear advantage. Based on product properties alone, either material 
would be acceptable for SRP waste. Potential improvements in the quality of both waste forms are discussed.

172 (DP-1629) Assessment of Savannah River borosilicate glass in the repository environment. Plodinec, M.J.; Wicks, G.G.; Bibler, N.E. (Du Pont de Nemours (E.I.) and Co., Aiken, SC (USA). Savannah River Lab.). Apr 1982. Contract AC0976SR00001. 98p. NTIS, PC A05/MF A01. Order Number DE82014913.

Since 1973, borosilicate glass has been studied as a matrix for the immobilization of high-level radioactive waste generated at the Savannah River Plant (SRP). In 1977, efforts began to develop and test the large-scale equipment necessary to convert the alkaline waste slurries at SRP into a durable borosilicate glass. A process has now been developed for the proposed Defense Waste Processing Facility (DWPF) which will annually produce approximately 500 canisters of SRP waste glass which will be stored on an interim basis on the Savannah River site. Current national policy calls for the permanent disposal of high-level waste in deep geologic repositories. In the repository environment, SRP waste glass will eventually be exposed to such stresses as lithostatic or hydrostatic pressures, radiation fields, and self-heating due to radioactive decay. In addition, producing and handling each canister of glass will also expose the glass to thermal and mechanical stresses. An important objective of the extensive glass characterization and testing programs of the Savannah River Laboratory (SRL) has been to determine how these stresses affect the performance of SRP waste glass. The results of these programs indicate that: these stresses will not significantly affect the performance of borosilicate glass containing SRP waste; and SRP waste glass will effectively immobilize hazardous radionuclides in the repository environment.

173 (DP-1636) Precipitation process for supernate decontamination. Lee, L.M.; Kilpatrick, L.L. (Du Pont de Nemours (E.I.) and Co., Aiken, SC (USA). Savannah River Lab.). Nov 1982. Contract AC09-76SR00001. 35p. NTIS, PC A03/MF A01. Order Number DE83010815.

A precipitation and adsorption process has been developed to remove cesium, strontium, and plutonium from water-soluble, high-level radioactive waste. An existing waste tank serves as the reaction vessel and the process begins with the addition of a solution of sodium tetraphenylborate and a slurry of sodium titanate to the contained waste salt solution. Sodium tetraphenylborate precipitates the cesium and sodium titanate adsorbs the strontium and plutonium. The precipitate/ adsorbate is then separated from the decontaminated salt solution by crossflow filtration. This new process offers significant capital savings over an earlier ion exchange process for salt decontamination. Chemical and small-scale engineering studies with actual waste are reported. The effect of many variables on the decontamination factors and filter performance are defined.

174 (DP-MS-81-111) Processability analysis of candidate waste forms. Gould, T.H. Jr.; Dunson, J.B., Jr.; Eisenberg, A.M.; Haight, H.G. Jr.; Mello, V.E.; Schuyler, R.L. III. (Du Pont de Nemours (E.I.) and Co., Aiken, SC (USA). Savannah River Lab.; Du Pont de Nemours (E.I.) and Co., Newark, DE (USA). Engineering Dept.). 1982. Contract AC09-76SR00001. 20p. (CONF-820547-5). NTIS, PC A02/MF A01. Urder Number DE82014442.

From 84. annual meeting of the American Ceramic Society; Cincinnati, OH, USA (2 May 1982).

A quantitative merit evaluation, or processability analysis, was performed to assess the relative difficulty of remote processing of Savannah River Plant high-level wastes for seven alternative waste form candidates. The reference borosilicate glass process was rated as the simplest, followed by FUETAP concrete, glass marbles in a lead matrix, high-silica glass, crystalline ceramics (SYNROC-D and tailored ceramics), and coated ceramic particles. Cost estimates for the borosilicate glass, high-silica glass, and ceramic waste form processing facilities are also reported.

176 (DP-MS-81-112) Decontamination of Savannah River Plant waste glass canisters. Rankin, W.N. (Du Pont de Nemours (E.I.) and Co., Aiken, SC (USA). Savannah River Lab.). 1982. Contract AC09-76SR00001. 16p. (CONF-
820903-3). NTIS, PC A02/MF A01. Order Number DE82015510. 1982).

From ANS topical meeting; Niagara Falls, NY, USA (22 Sep

A Defense Waste Processing Facility (DWPF) is currently being designed to convert Savannah River Plant (SRP) liquid, high-level radioactive waste into a solid form, such as borosilicate glass. The outside of the canisters of waste glass must have very low levels of smearable radioactive contamination before they are removed from the DWPF to prevent the spread of radioactivity. Several techniques were considered for canister decontamination: high-pressure water spray, electropolishing, chemical dissolution, and abrasive blasting. An abrasive blasting technique using a glass frit slurry has been selected for use in the DWPF. No additional equipment is needed to process waste generated from decontamination. Frit used as the abrasive will be mixed with the waste and fed to the glass melter. In contrast, chemical and electrochemical techniques require more space in the DWPF, and produce large amounts of contaminated byproducts which are difficult to immobilize by vitrification.

178 (DP-MS-81-119) Develupment of a slurry-fed in-can melter for nuclear defense waste . d'Entremont, P.D.; Wolf, H.C. (Du Pont de Nemours (E.I.) and Co., Aiken, SC (USA). Savannah River Lab.). 1982. Contract AC09-76SR00001. 19p. (CONF-820547-7). NTIS, PC A02/MF A01. Order Number DE82015511.

From 84. annual meeting of the American Ceramic Society; Cincinnati, OH, USA (2 May 1982).

In-Can Melting is the backup vitrification process for Savannah River Plant waste. A full-scale, nonradioactive process has been studied in which a slurry of waste sludge and glass formers is fed to a heated can. Saturated steam is used to cool the off-gas. Initial results show the concept to be viable. 4 figures.

177 (DP-MS-81-120) Large-scale continuous process to vitrify nuclear defense waste: operating experience with nonradiouctive waste. Cosper, M.B.; Randall, C.T.; Traverso, G.M. (Du Pont de Nemours (E.I.) and Co., Aiken, SC (USA). Savannah River Lab.). 1982. Contract AC09-76SR00001. 28p. (CONF-820547-8). NTIS, PC A03/MF A01. Order Number DE82015512.

From 84. annual meeting of the American Ceramic Society; Cincinnati, OH, USA (2 May 1982).

Porituns of ducument are illegible.

The developmental program underway at SRL has demonstrated the vitrification process proposed for the sludge processing facility of the DWPF on a large scale. DWPF design criteria for production rate, equipment lifetime, and operability have all been met. The expected authorization and construction of the DWPF will result in the safe and permanent immobilization of a major quantity of existing high level waste. 11 figures, 4 tables.

178 (DP-MS-82-2) Alternative-waste-form evaluation for Savannah River Plant high-level waste. Gould, T.H. Jr.; Crandall, J.L. (Du Pont de Nemours (E.I.) and Co., Aiken, SC (USA). Savannah River Lab.). 1982. Contract AC0976Sk00001. 35p. (CONF-820609-56). NTIS, PC A03/MF A01. Order Number DE82016350.

From American Nuclear Society annual meeting; Los Angeles, CA, USA (6 Jun 1982).

Results of the waste form evaluation are summarized as: risks of human exposure are comparable and extremely small for either borosilicate glass or Synroc ceramic. Waste form properties are more than adequate for either form. The waste form decision can therefore be made on the basis of practicality and cost effectiveness. Synroc offers lower costs for transportation and emplacement. The borosilicate glass form offers the lowest total disposal cost, much simpler and less costly production, an established and proven process, lower future development costs, and an earlier startup of the DWPF.

179 (DP-MS-82-8) Characterization of borosillcate glass containing Savannah River Plant radioactive waste. Bibler, N.E.; Smith, P.K. (Du Pont de Nemours (E.I.) and Co., Aiken, SC (USA). Savannah River Lab.). 1982. Contract AC09- 
76SR00001. 15p. (CONF-820891-5). NTIS, PC A02/MF A01. Order Number DE83004931.

From 18. ceramic science conference on advances in materials characterization; Alfred, NY, USA (16 Aug 1982).

Portions of document are illegible.

Vitrification is the reference process for immobilization of radioactive waste from the Savannah River Plant. The waste, consisting mostly of hydrous oxides of $\mathrm{Fe}, \mathrm{Al}$, and $\mathrm{Mn}$ contaminated with fission products and alpha-emitting radionuclides, is mixed with glass-forming chemicals and melted at $1150^{\circ} \mathrm{C}$ to produce a durable borosilicate glass. In this paper, the results of studies characterizing glass containing actual radioactive waste are presented. The glass was produced in a small-scale joule heated melter in a shielded facility at the Savannah River Laboratory. Feed for the melter was a mixture of 35 wt \% waste (primarily $\mathrm{Fe}_{2} \mathrm{O}_{0}$ and $\mathrm{Al}_{2} \mathrm{O}_{0}$ ) and 65 wt \% frit (primarily $\mathrm{SiO}_{2}, \mathrm{Na}_{2} \mathrm{O}$, and $\mathrm{B}_{2} \mathrm{O}_{0}$ ). The melt was poured into $600 \mathrm{cc}$ stainless steel beakers and allowed to cool. The glass was intensely radioactive. Surface dose rates were approximately $10^{6} \mathrm{rad} / \mathrm{h}$. Specific activities (dpm/g glass) of the principle radionuclides were: $1.2 \times 10^{10}$ for ${ }^{107} \mathrm{Cs}, 7.9 \times 10^{9}$ for ${ }^{90} \mathrm{Sr}$, and $1.08 \times 10^{8}$ for alpha activity (primarily ${ }^{208} \mathrm{Pu}$ and ${ }^{244} \mathrm{Cm}$ ). Examination by optical and electron microscopy indicated the presence of 1 to $5 \mathrm{wt}$ $\%$ spinel crystals - primarily $\mathrm{NiFe}_{2} \mathrm{O}_{4}$. Leach tests indicated good durability in deionized water, brine, and silicate water. These latter two leachants simulate groundwater from possible geologic repositories. Release rates at $40^{\circ} \mathrm{C}$ based on gamma, beta, and alpha activity in a 28 day test were approximately $0.01 \mathrm{~g}$ glass $/ \mathrm{m}_{2}$-day. This result agrees with results of tests with glass containing simulated waste and indicates that the borosilicate glass effectively immobilizes SRP radioactive waste. During the leach tests, the $\mathrm{pH}$ of the leachates did not decrease even though they were exposed to the gamma, beta, and alpha radiation from this glass.

180 (DP-MS-82-26-Rev.) SRP radioactive glass studies: small-scale process development and product performance. Plodinec, M.J.; Soper, P.D.; Bibler, N.E.; Kessler, J.L. (Du Pont de Nemours (E.I.) and Co., Aiken, SC (USA). Savannah River Lab.). 1982. Contract AC09-76SR00001. 30p. (CONF820424-31-Rev.). NTIS, PC A03/MF A01. Order Number DE82019158.

From ANS topical meeting on treatment and handling of radioactive wastes; Richland, WA, USA (19 Apr 1982).

A small-scale joule-heated ceramic melter contained in the Shielded Cells Facility has demonstrated the vitrification process for actual Savannah River Plant radioactive waste. Losses of radionuclides due to volatility are low and easily treated, and the glass produced is of comparable quality to laboratory-prepared simulated glass. Future work will include studies with wastes from other tanks, using new frit compositions. Leaching tests will continue, with emphasis being placed on long-term tests under anticipated repository conditions.

181 (DP-MS-82-31) High-level-waste immobilization. Crandall, J.L. (Du Pont de Nemours (E.I.) and Co., Aiken, SC (USA). Savannah Rivcr Lab.). 1982. Contract AC0976SR00001. 25p. (CONF-820424-17). NTIS, PC A02/MF A01. Order Number DE82014172.

From ANS topical meeting on treatment and handling of radioactive wastes; Richland, WA, USA (19 Apr 1982).

Portions of docurnent arc illegible.

Analysis of risks, environmental effects, process feasibility, and costs for disposal of immobilized high-level wastes in geologic repositories indicates that the disposal system safety has a low sensitivity to the choice of the waste disposal form.

182 (DP-MS-82-40) Theoretical predictions for continuous-slurry feeding of a glass melter. Routt, K.R. (Du Pont de Nemours (E.I.) and Co., Aiken, SC (USA). Savannah River Lab.) 1982. Contract AC09-76SR00001. 9p. (CONF-82110742). NTIS, PC A02/MF A01. Order Number DE83004928.

From 6. international symposium on the scientific basis for radioactive waste management (Materials Research Society); Boston, MA, USA (1 Nov 1982).

Portions of document are illegible.

Radioactive defense waste currently stored at the Savannah River Plant in liquid form is to be immobilized by incorporation into a borosilicate glass. The glass melter for this process will consist of a refractory-lined tank enclosed in a water-cooled, steel vessel and fed by an aqueous slurry of glass frit plus radioactive waste. As an aid to understanding the melting process and scaling data from small melters, conservation principles were used to develop equations for predicting the melt rate for a feedpile of arbitrary radius plus additional information regarding feedpile behavior and energy consumption.

183 (DP-MS-82-42) Efficient particulate scrubber for glass melter off-gas. Wright, G.T. (Du Pont de Nemours (E.I.) and Co., Aiken, SC (USA). Savannah River Lab.). 1982. Contract AC09-76SR00001. 12p. (CONF-821107-44). NTIS, PC A02/MF A01. Order Number DE83002687.

From 6. international symposium on the scientific basis for radioactive waste management (Materials Research Society); Boston, MA USA (1 Nov 1982).

Operation of joule-heated, continuous slurry fed melters bas demonstrated that off-gas aerosols are generated by entrainment of feed slurry and vaporization of volatile species from the melt. Effective offgas stream decontamination for these aerosols can be obtained by utilizing a suitably designed and operated wet scrubber system. Results are presented for performance tests conducted with an air aspirating-type venturi scrubber processing a simulated melter off-gas aerosol. Mass removal efficiencies ranged from 99.5 to $99.8 \%$. Details of the testing program and applications for melter off-gas system design are discussed.

184 (DP-MS-82-46) Precipitation process for decontamination of water-soluble SRP radioactive waste. Lee, L.M.; Kilpatrick, L.L. (Du Pont de Nemours (E.I.) and Co., Aiken, SC (USA). Savannah River Lab.). 1982. Contract AC09. 76SR00001. 25p. (CONF-821103-89). NTIS, PC A02/MF A01. Order Number DE83011378.

From American Nuclear Society winter meeting; Washington, DC, USA (14 Nov 1982).

A precipitation and adsorption process has been developed to remove cesium, strontium, and plutonium from high-level radioactive waste salt solution. Sodium tetraphenyl borate (STPB) is used to precipitate cesium, and sodium titanate slurry is used to sorb strontium and plutonium. The precipitate/adsorbate is separated from the decontaminated salt solution by crossflow filtration. This process has the potential of being implemented in existing waste tanks and would result in significant capital savings compared to an ion-exchange process for salt decontamination. Chemical and small-scale engineering studies with actual waste are reported. The effect of many variables on the decontamination factors and filter performance is defined.

185 (DP-MS-82-47) Development, evaluation, and selection of candidate high-level waste forms. Bernadzikowski, T.A.; Allender, J.S.; Gordon, D.E.; Gould, T.H. Jr. (Du Pont de Nemours (E.I.) and Co., Aiken, SC (USA). Savannah River Lab.). 1982. Contract AC09-76SR00001. 45p. NTIS, PC A03/MF A01. Order Number DE82020155.

The seven candidate waste forms, evaluated as potential media for the immobilization and gelogic disposal of high-level nuclear wastes were borosilicate glass, SYNROC, tailored ceramic, high-silica glass, FUETAP concrete, coated sol-gel particles, and glass marbles in a lead matrix. The evaluation, completed on August 1, 1981, combined preliminary waste form evaluations conducted at Department of Energy (DOE) defense waste-sites and at independent laboratories, peer review assessments, a product performance evaluation, and a processability analysis. Based on the combined results of these four inputs, two of the seven forms, borosilicate glass and a titanate-based ceramic, SYNROC, were selected as the reference and alternative forms, respectively, for continued development and evaluation in the National HLW Program. The borosilicate glass and ceramic forms were further compared during FY-1982 on the basis of risk assessments, cost comparisons, properties comparisons, and conformance with proposed regulatory and repository criteria. Both the glass and ceramic forms are viable candidates for use at DOE defense HLW sites; they are also candidates for immobilization of commercial reprocessing wastes. This paper describes the waste form screening process, discusses each of the four major inputs considered in the selection of the two forms in 1981, and presents a brief summary of the comparisons of the two forms during 1982. and the selection process to determine the final form for SRP defense HLW.

186 (DP-MS-82-65) DWPF process control. Heckendorn, F.M. II. (Du Pont de Nemours (E.I.) and Co., Aiken, SC (USA). Savannah River Lab.). 1983. Contract 
AC09-76SR00001. 17p. (CONF-830205-26). NTIS, PC A02/MF A01. Order Number DE83011381.

From Waste management conference; Tucson, AZ, USA (27 Feb 1983).

Portions are illegible in microfiche products.

The Defense Waste Processing Facility (DWPF) for waste vitrification at the Savannah River Plant (SRP) is in the final design stage. Instrumentation to provide the parameter sensing required to assure the quality of the 2 -ft-diam, 10-ft-high waste canister is in the final stage of development. All steps of the process and instrumentation are now operating as nearly full-scale prototypes at SRP. Quality will be maintained by assuring that only the intended material enters the canisters, and by sensing the resultant condition of the filled canisters. Primary emphasis will be on instrumentation of the process.

187 (DP-MS-82-73) Precipitation process for decontaminating water-soluble SRP radioactive waste. Lee, L.M.; Kilpatrick, L.L.; Wright, W.V. (Du Pont de Nemours (E.I.) and Co., Aiken, SC (USA). Savannah River Lab.). 1983. Contract AC09-76SR00001. 15p. (CONF-830633-1-Summ.). NTIS, PC AO2./MF A01. Order Number DE83011446.

From 3. symposium on separation science and technology fur energy applications; Gatlinburg, TN, USA (27 Jun 1983).

A precipitation and adsorption process has been developed for the removal of cesium, strontium, and plutonium from high-level radioactive waste salt solution. Sodium tetraphenylborate (NaTPB) is used to precipitate cesium as the very insoluble cesium tetraphenylborate, and sodium titanate slurry is used to adsorb strontium and plutonium. The precipitate/adsorbate is then separated from the decontaminated salt solution by crossflow filtration. Chemical and small-scale engineering studies with actual waste are reported in which the effect of several variables on the decontamination factors and filter performance are defined. Implementation of this process using existing waste tanks avoids the building of a heavily shielded canyon building for salt processing with considerable savings in capital investment.

188 (DP-MS-82-91) Startup of a Joule-heated glass melter with a graphite slurry. Allen, T.L.; Routt, K.R.; Porter, M.A. (Du Pont de Nemours (E.I.) and Co., Aiken, SC (USA). Savannah River Lab.). 1983. Contract AC09-76SR00001. 18p. (CONF-830451-8). NTIS, PC A02/MF A01. Order Number DE83011451.

From 85. American Ceramic Society annual meeting; Chicago, ILL, USA (25 Apr 1983).

This paner discusses the thenretical equations and physical and electrical property data of various graphite slurries for starting up a glass melter. An application test is also included to demonstrate the graphite slurry startup technique.

189 (DP-MS-82-94) Evaluation of glass-contact materials for waste glass melters. Rankin, W.N. (Bu Pont de Nemours (E.I.) and Co., Aiken, SC (USA). Savannah River Lab.). 1983. Contract AC09-76SR00001. 15p. (CONF-830451-2). NTIS, PC A02/MF A01. Order Number DE83011452.

From 85. American Ceramic Society annual meeting; Chicago, IL, USA (25 Apr 1983).

Alternative refractory and electrode materfals have been evaluated as glase-contact materials for the vitrification of SRP waste. Monofrax $\mathrm{K}-3$ (The Carborundum $\mathrm{Co}$.) is the optimum refractory, based on corrosion and thermal-shock resistance. Inconel 690 (Huntington Alloys, Inc.) is the recommended electrode alloy. However, a potentially more corrosion-resistant alternative, UCAR Metal Ceramic, Grade LT-1 (Union Carbide Corp.), is being evaluated further. This material melts at a much higher temperature than Inconel 690 . In the event of an unexpected high-temperature excursion in a melter, this material may offer significantly greater protection.

190 (DP-MS-82-96) Physical modeling of a glass melter designed for vitrification of defense waste. Routt, K.R. (Du Pont de Nemours (E.I.) and Co., Aiken, SC (USA). Savannah River Lab.). 1983. Contract AC09-76SR00001. 29p. (CONF830451-3). NTIS, PC A03/MF A01. Order Number DE83011445.

From 85. American Ceramic Society annual meeting; Chicago, IL, USA (25 Apr 1983).
Portions are illegible in microfiche products.

Physical modeling principles applied to the simulation of a glass melter designed for processing of radioactive waste glass appear to be excellent tools for evaluating melter design and performance. Models can certainly provide information in greater detail regarding temperature and voltage profiles than is usually obtainable in an actual glass melter. Furthermore, the measurement of vclocity profiles in an actual melter is not possible but can be easily performed in a model. A model can not only be used to increase general understanding of the melting process, but it can also provide specific suggestions for improvement in melter design. As a result of the modeling results presented here, for example, it was recommended that the bottom heat transfer coefficient $U_{b}$ be decreased in future melter designs in order to raise the temperature of the glass near the bottom of the melt pool. It was also recom. mended that alternate electrode configurations be examined in order to provide flexibility in control of the electrical power density in the vertical direction. Future work with physical models will study these recommendations in detail.

191 (DP-MS-83-22) Treatment and conditioning of wastes from US nuclear fuels reprocessing plants. Crandall, J.L.; Oertel, G.; MuElroy, J. (Du.Pont de Nemnire (E.I.) and Co., Aiken, SC (USA). Savannah River Lab.; Department of Energy, Washington, DC (USA); Pacific Northwest Lab., Richland, WA (USA)). 1983. Contract AC09-76SR00001. 29p. (CONF-830523-20). NTIS, PC A03/MF A01. Order Number DE83016834.

From International conference on radioactive waste management; Seattle, WA, USA (16 May 1983).

Portions are illegible in microfiche products.

The US has operated nuclear fuels reprocessing plants at Hanford, Idaho Falls, and Savannah River in its definse programs and at West Valley in its commercial program. Other commercial plants have been proposed and some constructed but not operated. The liquid highlevel wastes at Hanford and Savannah River have, for nearly 40 years, been conditioned for storage by adding $\mathrm{NaOH}$, a process which precipitates most of the radionuclides except $\mathrm{Cs}$ in an insoluble sludge. Mechanical separation of this sludge coupled with decontamination of the supernate by ion exchange and/or precipitation gives a waste concentrate for immobilization. The Defense Waste Processing Facility at Savannah River is under construction to immobilize this concentrate in borosilicate glass. Idaho is just placing a new calciner in operation to treat their acid wastes directly. Techniques have also been devcloped to remove $\mathrm{Cs}_{\mathrm{s}} \mathrm{Sr}$, and noble motals from the liquid wastes for defense purposes and commercial uses. Except at West Valley, the US technique to daie has been to dissolve tho fuol ulud rather than ehrarelench it. Hnwever, because shear-leach is the expected future process, the US has also developed techniques both for immobilizing leached clad in concrete and for decontaminating it by melting. As regard to gaseous waste, the current US reprocessing plants treat their off-gas mainly to remove iodine and particulates. Idahn alsn has a small facility for recovery of ${ }^{85} \mathrm{Kr}$ for beneficial use. By US Environmental Protection Agency rules on the nuclear fuel cycle, it may be necessary to remove $T,{ }^{14} \mathrm{C}$, and $\mathrm{Kr}$ if a large commercial reprocessing industry matures. Oak Ridge has developed a voloxidation process as a first step in such removal. Finally, the US has the decontamination, assay, sorting, incineration and burial technologies and facilities for treating the low-level liquid and solid wastes from reprocessing.

192 (DP-MS-83-24) Electrical service and controls for Joule heating of a defense waste experimental glass melter. Erickson, C.J.; Haideri, A.Q. (Du Pont de Nemours (E.I.) and Co., Wilmington, DE (USA). Engineering Dept.). 1983. Contract AC09-76SR00001. 10p. (CONF-831070-1). NTIS, PC A02/MF A01. Order Number DE83012171.

From IEEE/IAS glass industry committee meeting; Mexico City, Mexico (3 Oct 1983).

Vitrification of radioactive liquid waste in a glass matrix is a leading candidate for long-term storage of high-level waste. This paper describes the electrical service and control system for an experimental electrically heated, nonradioactive glass melter installed at Savannah River Laboratory. Data accumulated, and design/operating experience acquired in operating this mclter, are being used to design a modified melter to be installed in a processing area for use with radioactive materials. 
193 (DP-MS-83-75) Solidification of ash from incineration of low-level radioactive waste. Roberson, W.A.; Albenesius, E.L.; Becker, G.W. (Du Pont de Nemours (E.I.) and Co., Aiken, SC (USA). Savannah River Lab.). 1983. Contract AC0976SR00001. 20p. (CONF-8310186-1). NTIS, PC A02/MF A01. Order Number DE84001530.

From Symposium on the GEM experience - an American education success stcry; Laurel, MD, USA (18 Oct 1983).

The safe disposal of both high-level and low-level radioactive waste is a problem of increasing national attention. A full-scale incineration and solidification process to dispose of suspect-level and low-level beta-gamma contaminated combustible waste is being demonstrated at the Savannah River Plant (SRP) and Savannah River Laboratory (SRL). The stabilized wasteform generated by the process will meet or exceed all future anticipated requirements for improved disposal of lowlevel waste. The incineration process has been evaluated at SRL using nonradioactive wastes, and is presently being started up in SRP to process suspect-level radioactive wastes. A cement solidification process for incineration products is currently being evaluated by SRL, and will be included with the incineration process in SRP during the winter of 1984. The GEM alumnus author conducted research in a related disposal solidification program during the GEM-sponsored summer internship, and upon completion of the Masters program, received full-time responsibility for developing the incineration products solidification process.

194 (DPSPU-60-11-34) Contamination of the hot gang valve corridor and first-level clean areas of Building 221-F - September 13, 1960. Bebbington, W.P. (Du Pont de Nemours (E.I.) and Co., Aiken, SC (USA). Savannah River Plant). Dec 1960. Contract AC09-76SR00001. 10p. NTIS MF A01. Order Number DE83016832.

Microfiche only, copy does not permit paper copy reproduction. On September 13, 1960, the hot gang valve corridor and some personnel areas at a lower elevation in Building 221-F were extensively contaminated by cooling water into which high activity waste from the Purex process had leaked through a defective waste evaporator reboiler. The escape and dispersal of the highly radioactive solution was permitted by a series of valve manipulations which nullified protective features of the equipment and opened a path for the escape. Approximately 5000 $\mathrm{Ci}$ was released. Most of this was contained within the building or was flushed to waste handling facilities. Minor amounts were released to sepage basins. Decontamination and cleanup within the building will be costly and time-consuming. The incident was due to an improper sequence of valve operations and could have been prevented by thorough planning and use of procedures together with better organization of and communication among the operators and supervisors involved.

195 (DPSPU-81-30-16) Defense Waste Processing Facility: a remote process for solidification of Savannah River Plant high level waste. Maher, R.; Shafranek, L.F.; Kelley, J.A.; Zeyfang, R.W.; Lethco, A.J. (Du Pont de Nemours (E.I.) and Co., Aiken, SC (USA). Savannah River Plant). Mar 1982. Contract AC09-76SR00001. 18p. (CONF-820424-26). NTIS, PC A02/MF A01. Order Number DE82017676.

From ANS topical meeting on treatment and handling of radioactive wastes; Richland, WA, USA (19 Apr 1982).

The Department of Energy is proposing that a Defense Waste Processing Facility be built at the Savannah River Plant (SRP) to remotely process and immobilize high level radioactive waste produced at the site. Research, development, and design of the facility is being provided by a multidisciplined task force of personnel from the Du Pont Company which designed, built and has operated SRP since 1950. This remotely operated facility will immobilize 28 million gallons of high level waste now stored in tanks, plus the waste to be generated from continued reprocessing operations. Borosilicate glass has been selected as the reference waste form for the immobilization process.

196 (DPSPU-83-30-4-Rev.) Immobilizing defense waste at the Savannah River Project. Schneider, R.F.; Davis, J.C.; Mellen, I. B. (Rechtel National, Inc.. San Francisco, CA (USA); Du Pont de Nemours (E.I.) and Co., Aiken, SC (USA). Savannah River Plant). Mar 1983. Contract AC09-76SR00001. 22p. (CONF-830347-2). NTIS, PC A02/MF A01. Order Number DE83012866.
From Atomic industrial forum fuel cycle conference; Kansas City, MO, USA (20 Mar 1983).

The Department of Energy is proceeding with a project to construct and operate a Defense Waste Processing Facility at the Savannab River Plant. Because of the two phases in the waste, the project will consist of two distinct parts: the first to treat and immobilize the sludge portion of the waste, and the second to deal with the evaporated liquid portion or salt waste. Firm design for the sludge vitrification plant is now underway. The vitrification process using borosilicate glass as the matrix materials is discussed.

197 (DPST-81-914) Hazards analysis of TNX Large Melter-Off-Gas System. Randall, C.T. (Du Pont de Nemours (E.I.) and Co., Aiken, SC (USA). Savannah River Lab.). Mar 1982. Contract AC09-76SR00001. 66p. NTIS, PC A04/MF A01. Order Number DE83012206.

Portions are illegible in microfiche products.

Analysis of the potential safety hazards and an evaluation of the engineered safety features and administrative controls indicate that the LMOG System can be operated without undue hazard to employees or the public, or damage to equipment. The safety features provided in the facility design coupled with the planned procedural and administrative controls make the occurrence of serious accidents very improbable. A set of recommendations evolved during this analysis that was judged potentially capable of further reducing the probability of personnel injury or further mitigating the consequences of potential accidents. These recommendations concerned areas such as formic acid vapor hazards, hazard of feeding water to the melter at an uncontrolled rate, prevention of uncontrolled glass pours due to melter pressure excursions and additional interlocks. These specific suggestions were reviewed with operational and technical personnel and are being incorporated into the process. The safeguards provided by these recommendations are discussed in this report.

198 (DPST-82-410) Assessment of high-level waste form conformance with proposed regulatory and repository criteria. Gordon, D.E.; Gray, P.L.; Jennings, A.S.; Permar, P.H. (Du Pont de Nemours (E.I.) and Co., Aiken, SC (USA). Savannah River Lab.). Apr 1982. Contract AC09-76SR00001. 37p. NTIS, PC A03/MF A01. Order Number DE82015753.

Federal regulatory criteria for geologic disposal of high-level waste are under development. Also, interim performance specifications for high-level waste forms in geologic isolation are being developed within the Federal program responsible for repository selection and operation. Two high-level waste forms, borosilicate glass and crystalline ceramic, have been selected as candidate immobilization forms for the Defense Waste Processing Facility (DWPF) which is to immobilize high-level wastes at the Savannah River Plant (SRP). An assessment of how these two waste forms conform with the proposed regulatory criteria and repository specifications was performed. Both forms were determined to be in conformance with postulated rules for radionuclide releases and radiation exposures throughout the entire waste disposal system, as well as with proposed repository operation requirements.

199 (EGG-FM-5758) Iron-enriched basalt as a waste form for high-level nuclear wastes. Welch, J.M.; Schuman, R.P.; Henslee, S.P.; Tallman, R.L.; Flinn, J.E. (Idaho National Engineering Lab., Idaho Falls (USA)). Oct 1982. Contract AC0776ID01570. 44p. NTIS, PC A03/MF A01. Order Number DE83005698.

Simulated high-level waste calcines from the Savannah River and Idaho Chemical Processing Plants as well as an ion-exchange zeolite commonly used for decontamination of reactor coolant water were incorporated via melting into iron-enriched basalt waste forms. After cooling from the melts, specimens were examined for microstructure and tested for durability, i.e., leaching and in some cases mechanical response. The leaching tests were performed in $90^{\circ} \mathrm{C}$ deionized water for $28 \mathrm{~d}$. The bulk leach rates for the entire series of specimens ranged from 1.2 to $18 \mu \mathrm{g} / \mathrm{cm}^{2} \mathrm{~d}$.

200 (EGG-FM-6045) Evaluation of forms for the immobilization of high-level and transuranic wastes. Schuman, R.P.; Cox, N.D.; Gibson, G.W.; Kelsey, P.V. Jr. (EG and G Idaho, Inc., Idaho Falls (USA)). Aug 1982. Contract AC07- 
76ID01570. 95p. NTIS, PC A05/MF A01. Order Number DE84002613.

A figure-of-merit (FOM) analysis has been made of a number of waste forms for solidifying both defense and commercial high-level reprocessing waste (HLW) and transuranic (TRU) wastes. The evaluation includes iron-enriched basalt (IEB), a fusion-produced glassceramic, which has not been included in other assessments. For HLW, concrete receives the highest FOM, but may not meet regulatory requirements; IEB and glass are the best choices of the materials that should easily meet regulatory requirements. Concrete waste forms are the best choice for TRU wastes, with IEB a close contender. 116 references, 3 figures, 112 tables.

201 (EGG-M-07881) Preparation and leaching of radioactive INEL waste forms. Schuman, R.P.; Welch, J.M.; Staples, B.A. (EG and G Idaho, Inc., Idaho Falls (USA); Exxon Nuclear Idaho Co., Inc., Idaho Falls (USA)). 1982. Contract AC07. 76ID01570. 16p. (CONF-820303-39). NTIS MF A01. Order Number DE82016342.

From Waste management conference (ANS); Tucson, AZ, USA (8 Mar 1982).

Portions of document are illegible.

The purpose of this study is to prepare and leach test ceramic and glass waste form specimens produced from actual transuranic waste sludges and high-level waste calcines, respectively. Description of wastes, specimen fabrication, leaching procedure, analysis of leachates and results are discussed. The conclusion is that radioactive waste stored at INEL can be readily incorporated in fused ceramic and glass forms. Initial leach testing results indicate that these forms show great promise for safe long-term containment of radioactive wastes.

202 (ENICO-1123) Attrition, elutriation, and growth of particles produced in nuidized-bed waste calciners. McDonald, F.N. (Exxon Nuclear Idaho Co., Inc., Idaho Falls (USA)). Sep 1982. Contract AC07-79ID01675. 57p. NTIS, PC A04/MF A01. Order Number DE83002731.

The Idaho Chemical Processing Plant reduces the volume of high-level liquid radioactive wastes in a fluidized bed to produce a granular calcine product. In the past, difficulties have been experienced in controlling the product's particle size when processing certain blends of sodium-bearing waste. Therefore, experiments in attrition, elutriation, and particle growth were done to characterize how best to control these three parameters. 15 figures, 16 tables.

203

(ENICO-1131) Evaluation of stainless steel tank corrosion in ICPP high-level radioactive waste service. Hoffman, T.L.; Norby, B.C.; Mizia, R.E.; Zimmerman, C.A. (Exxon Nuclear Idaho Co., Inc., Idaho Falls (USA)). Apr 1983. Contract AC07-79ID01675. 42p. NTIS, PC A03/MF A01. Order Number DE83011033.

At the Idaho Chemical Processing Plant, Types $304 \mathrm{~L}$ and 348 stainless steels were corrosion tested in raffinates from reprocessing spent nuclear fuels. After exposure to the raffinate solutions for periods up to 28.6 years total service time, maximum penetration rates observed on these stainless steels were 0.059 mil per year in aluminum-zirconium process first-cycle raffinate, and 0.0004 mil per year in second- and third-cycle raffinates.

204

(ENICO-1139) Alternative fuels and heating methods for calciner process heat. McCray, J.A. (Exxon Nuclear Idaho Co., Inc., Idaho Falls (USA)). Aug 1983. Contract AC0779ID01675. 39p. NTIS, PC A03/MF A01. Order Number DE83017393.

Since 1963, the Idaho Chemical Processing Plant (ICPP) has been solidifying radioactive wastes using a fluidized-bed calcination process. The facility had been using a recirculating sodium-potassium eutectic (NaK) method as a process heat source from 1963 to 1970 , and an in-bed combustion method using kerosene as a fuel from 1970 to 1981. The New Waste Calcining Facility (NWCF) has been in operation since 1982 and also uses in-bed combustion with kerosene for process heat. This report documents an evaluation of potential alternative fuels and different heating methods for supplying process heat during the calcination process. nuclide release source-term in granite: dissolution of the waste form. Savage, D.; Chapman, N.A. (SEE CODE- 9050311 Institute of Geological Sciences, Harwell (UK). Environmental Protection Unit). Jul 1980. 30p. Environmental Protection Unit, Institure of Geological Sciences, Building 151, Harwell Laboratory, Oxfordshire OX11 ORA, England.

Portions of document are illegible.

This study was designed to provide information on the behavior of the glass waste form at realistic repository conditions of temperature, pressure, and fluid content. Particular attention has been paid to the mechanisms of glass corrosion and the nature of the products of glass dissolution. Alkali dissolution from glasses 189 and 209 at $100^{\circ} \mathrm{C}$ and $150^{\circ} \mathrm{C}$ is controlled by diffusion through a surface alteration layer. This means that the rate at which alkalis are dissolved from the glass decreases with time and it would appear that the surface layer is protective against dissolution. Dissolution is temperature dependent, increasing two orders of magnitude for glass 209 between $100^{\circ} \mathrm{C}$ and $350^{\circ} \mathrm{C}$. Increasing total pressure from $60 \mathrm{MPa}$ to $100 \mathrm{MPa}$ increased dissolution by a factor of two at $150^{\circ} \mathrm{C}$, but had little effect at $250^{\circ} \mathrm{C}$. SEM and XRD examination of the surface layer on the glasses indicates that at lemperatures of $100^{\circ} \mathrm{C}$ and $150^{\circ} \mathrm{C}$ this layer is amorphous with trace amounts of crystalling kaolinite. At temperatures of $200^{\circ} \mathrm{C}$ and above this surface layer is covered with a $\mathrm{Na}-\mathrm{Cs}-\mathrm{Zr}$ smectite mineral, and at $350^{\circ} \mathrm{C}$ a sodic feldspar is also present. (DMC)

206 (EPRI-NP-3225) Assessment of power reactor waste immobilization by vitrification. Final report. Larson, D.E.; Buelt, J.L.; Heath, W.O.; Partain, W.L. (Battelle Pacific Northwest Labs., Richland, WA (USA)). Aug 1983. Contract AC0676RL01830. 159p. NTIS, PC A08/MF A01. Order Number DE84001893.

A study was performed to determine the technical and economic viability of applying vitrification to volume reduction and immobilization of nuclear power plant radioactive waste (radwaste). Vitrification technology has been extensively developed in the United States and abroad for immobilization of high-level radioactive waste. A conceptual design of a facility to vitrify light-water reactor radwaste (except noncompactible/noncombustible wastes) was developed. Technical, economic, and safety evaluations were performed for waste vitrification. Technical and economic comparisons were made with available technologies for radwaste volume reduction and immobilization. It was concluded that vitrification is a viable and competitively attractive approach; however, some additional process verification should be conducted prior to plant application.

207 (ETR-310) Separation of mercury from HEWC solutions. Laboratory assay. Hendricks, J.P.; Humblet, L.; van Geel, J. (European Company for the Chemical Processing of Irradiated Fuels, Mol (Belgium)). May 1982. 42p. (In French). NTIS (US Sales Only), PC A03/MF A01. Order Number DE83701312.

Before conditioning of the HEWC. (high enriched waste concentrate) solution containing as main elements aluminium nitrate nitric acid, mercuric ions, ion and fission products, the mercuric ions must be eliminated from the solution for safety reasons. A method was investigated on bench scale consisting in a reduction of mercuric ions by the formaldehyde. The report presents the laboratory results and considers the efficiency of the formaldehyde as reducing agent to precipitate the mercury. The four results were obtained concerning the recycling, the wasting solutions, the decontamination factors and the concentration of the solution.

208 (EUR-7426) Actinide recycling in light water reactors: results of reactor physics calculations. Guardini, S.; Smith, B.G.R. (Commission of the European Communities, Ispra (Italy). Joint Research Centre). 1981. 80p. NTIS (US Sales Only), PC A05/MF A01. Order Number DE82701948.

For the management of high level nuclear waste a new concept was explored involving the possibility of partitioning the by-product actinides and transmuting them into fission products. Previous reports gave the description of LWR (Light Water Reactor) codes used and preliminary results of the assessment studies for transmutation of byproduct actinides in LWR's. Scope of this paper is now to give final results of the LWR calculations and the conclusions reached by the overall light water reactor physics study. Two different recycling 
schemes are investigated, involving recycling of only transplutonium isotopes or respectively all actinides, $\mathrm{U}, \mathrm{Pu}, \mathrm{Am}$, and $\mathrm{Cm}$.

209 (HW-66091) Solidification of high level wastes. Part III. Solubility, melting point, flow temperature and thermal conductivity of some calcined, synthetic purex-type wastes. (General Electric Co., Richland, WA (USA). Hanford Atomic Products Operation). 15 Jul 1960. 31 p. NTIS.

The melting behavior of a number of compositions that approximate high-level Purex waste was observed and the solubility measured on the melted material. The ternary sodium, aluminum, iron phosphate composition was studied over a wide range of typical plant wastes to determine the variation of melting point with composition. The addition of phosphate to wastes appeared most suitable for the production of a meltable material with a low solubility.

210 (HW-66571) Research and development activities: fixation of radioactive residues. Quarterly progress report, AprilJune 1960. Pearce, D.W. (ed.). (General Electric Co., Richland, WA (USA). Hanford Atomic Products Operation). 15 Jul 1960. 30p. NTIS.

Progress is reported in research and development work in the field of fixation of residues from radioactive wastes. The areas of investigation include studies of various waste-treatment processes, such as calcination, fixation in silicates, and utilization of certain minerals having selective retentivity for certain isotopes or groups of isotopes, as well as waste-disposal studies and economic aspects of both treatment and disposal. Waste calcination studies were continued in the fluid-bed calciner using simulated high acid Purex waste as feed. A batch calcination facility capable of handling pots 54-in. high and 14-in. in diameter was built. Studies were continued on methods of improving the thermal conductivity of calcined powder. Data are included on the effect of feed rate, steam pressure, and sugar concentration on powder holdup on the column walls and on the extent of calcination of the powder formed from Purex waste. Neutralized Zirflex waste was successfully calcincd. Corrosion tests were continued. Laboratory experiments were conducted to study the effect of $\gamma$ irradiation on the ability of clinoptilolite to absorb cesium. Studies were also made on the influence of solution $\mathrm{pH}$ on anion replacement reactions in minerals. Laboratory studies to investigate the possibility of direct decontamination of some types of high-level wastes gave promising results. The effectiveness of clinoptilolite columns was tested with aged Redox high-salt waste,aluminum coated wastes, and Purex high-salt wastes. Data are tabulated from tests on cesium removal by clinoptilolite. The arrangement of the micro pilot plant used in the studies is illustrated photographically. Several metals were examined to determine their ability to decontaminate ruthenium.

211 (HW-66796) Solid fixation of high-level radioactive waste by sorption on clinoptilolity-scouting studies. Nelson, J.L.; Mercer, B.W.; Haney, W.A. (General Electric Co., Richland, WA (USA). Hanfurd Alumic Products Operation). 4 Oct 1960 . 25p. NTIS.

Combinations of actual and synthetic waste supernatant solutions of four types were decontaminated with mineral beds. The wastes were: neutralized Purex $1 \mathrm{ww}$, neutralized formaldehyde-killed $1 \mathrm{ww}$, Redox D-8, and decladding waste. All major radioisotopes, except ${ }^{106} \mathrm{Ru}$, were reduced below MPC or detection limit for each of the wastes. Since most of the inert salts passed through the columns, volume reduction factors were large, ranging from 27 to 55 . Discussions are presented on possible applications, problems, and estimated costs.

212 (HW-67334) Research and development activities: fixation of radioactive residues. Quarterly progress report, JulySeptember 1960. Pearce, D.W. (ed.). (General Electric Co., Richland, WA (USA). Hanford Atomic Products Operation). 15 Oct 1960. 26p. NTIS.

Waste calcination studits in the fluid bed calciner were continued using simulated high acid Purex wastes. Studies with the fluid bed waste calciner were continued using a feed of simulated high-acid Purex waste; results are described. Results of laboratory-scale-batch calcination studies of simulated Purex-type high-level waste are also discussed. Data from tests to find the effects of variables on calcination of caustic neutralized Purex waste are tabulated; run conditions and resulting powder characterisitics are given. Laboratory studies of fixation of highand low-level wastes were extended to include examination of other types of waste and experiments with new varieties of absorption media.
High-level studies conducted with clear supernatant fluids indicate the possibility of a simple fixation process that may be applicable to many types of waste. Cation exchange properties of clinoptilolite and natural zeolites were compared for cesium selectivity. Micro plant tests made by passing Purex tank-farm condensate waste through ion-exchange materials are reported. Results are listed for clinoptilolite, synthetic apatite, and alumino-silicate ion exchangers.

213 (HW-69176) Fixation of radioactive residues. Quarterly progress report, January-March 1961. Pearce, D.W. (ed.). (General Electric Co., Richland, WA (USA). Hanford Atomic Products Operation). 15 Apr 1961. 43p. NTIS.

Research and development activities in the field of fixation of radioactive wastes are reported. Calcination studies of simulated Purex high-level waste solutions by the batch method were continued on the bench scale. Melting of the calcine was studied with emphasis on the effects of the relative concentrations of metal ions in the waste solution. Batch calcination in annular pots using induction heating was demonstrated as was a procedure for removing the calcines for rework. A general equation was developed relating temperatures in proposed cylind rical and annular cylindrical containers full of high-activity-level calcined waste with other parameters of vessel size, heat generation rates, coolant temperatures, calcine thermal conductivity, and vessel-coolant heat transfer rates. Laboratory investigations of mineral reactions for the solid fixation of radioisotopes from wastes were continued. Studies were made of adsorbers, elution of mineral beds, evaluation of adsorption variables, and new mineral reactions. The decontamination abilities of clinoptilolite and organic ion exchange resins were explored.

214 (HW-70998) Recovery and purification of multikilocurie quantities of fission product strontium by cation exchange. Bray, L.A.; Lust, L.F.; Moore, R.L.; Roberts, F.P.; Smith, F.M.; Van Tuyl, H.H.; Wheelwright, E.J. (General Electric Co., Richland, WA (USA). Hanford Atomic Products Operation). 8 Sep 1961. 35p. NTIS.

In seven production runs, 75,000 curies of ${ }^{90} \mathrm{Sr}$ were isolated and purified in the ion-exchange equipment of Hanford Laboratories High Level Cells. The production goal $\left(60,000\right.$ curies of purified $\left.{ }^{90} \mathrm{Sr}\right)$ and the time schedule were met or exceeded and the product exceeded the customer's purity requirements. The hot-cell-purified strontium made possible the completion on time of the Weather Bureau power source and the Martin 10 watt SNAP VII-A and VII-C units. The isotopic purity of the strontium product was $56 \%{ }^{90} \mathrm{Sr}$. The chemical purity was greater than $95 \%$ strontium. The $\mathrm{Zr}-{ }^{95} \mathrm{Nb}$ contamination of the product was less than $5 \times 10^{-5}$ curies per curie of ${ }^{90} \mathrm{Sr}$ and the $\mathrm{Ce}^{144} \mathrm{Pr}$ contamination was less than $1 \times 10^{-5}$ curies per curie of ${ }^{90} \mathrm{Sr}$. The final run yielded 16,500 curies of ${ }^{90} \mathrm{Sr}$ in an eight liter product solution. The product was $>98 \%$ strontium and contained less than one curie of $\mathrm{Zr}{ }^{95} \mathrm{Nb}$ or $\mathrm{Ce}-{ }^{144} \mathrm{Pr}$. Eleven days of continuous operation were required to complete the run.

215 (HW-81829) Research and development activities [on] solidification of radioactive wastes. Progress report. Platt, A.M. (ed.). (General Electric Co., Richland, WA (USA). Hanford Atomic Products Operation). 15 Apr 1964. Contract AT(45-1)-1350. 26p. NTIS.

Research and development activities on the solidification of radioactive residues are reported. Work is reported on the following activities: small-scale spray and pot calcination studies with actual highlevel radioactive wastes, large-scale spray solidification studies with non-radioactive simulated wastes, and design and fabrication of an engineering-scale prototype for demonstration of three promising processes (pot calcination, spray solidification, and phosphate glass solidification) with actual high-level radioactive wastes.

216 (HW-83610) Progress in radioactive residue process development. A summary of Hanford laboratories achievements in this program under General Electric, 1958-1964. Platt, A.M. Cooley, C.R. (General Electric Co., Richland, WA (USA). Hanford Atomic Products Operation). Nov 1964. 36p. NTIS.

Results are summarized for studies on high-level waste calcination, hot cell pilot plants, waste solidification, and methods for intermediate-level waste treatment. fuel reprocessing wastes by spray and pot calcination. Hot-cell 
pilot plant studies. Allemann, R.T.; Roberts, F.P.; Upson, U.L. (General Electric Co., Richland, WA (USA). Hanford Atomic Products Operation). Sep 1964. 97p. NTIS.

When excess sodium was present in the waste, the loss of cesium was increased during pot calcination. The calcined waste containers did not develop any excess pressure upon storage except when excess sodium nitrate was present in the waste. Fission product distribution in the melted wastes was fairly uniform and in the same ratio as in the liquid waste except for ruthenium losses.

218 (HW-SA-3279) Management of high-level radioactive wastes. Platt, A.M. (General Electric Co., Richland, WA (USA). Hanford Atomic Products Operation). 14 Nov 1963. 19p. General Electric Co., Richland, WA, Hanford Atomic Products Operation.

A survey is presented of current practices of USAEC and its contractors for managing high-level radioactive wastes. The future waste problems associated with a growing nuclear power industry are discussed, and the progress of research and development activities on solidification of wastes is summarized.

219 (IDO-14620) ICPP waste calcining facility safety analysis report. Lakey, L.T.; Bower, J.R. (eds.). (Phillips Petro leum Co., Idaho Falls, ID (USA). Atomic Energy Div.). 1 Dec 1963. Contract AT(10-1)-205. 342p. NTIS.

A study was made of the radiological hazards associated with operation of the ICPP Waste Calcining Facility. In addition to the normal release of small amounts of radioactivity with the process offgas, possible accidental releases of larger amounts of radioactivity through operational error or equipment failure were examined. Bypassing of off-gas cleaning elements, uncontrolled chemical reactions, overheating by fission product heat, corrosion, abrasion, vibration, overpressure, utility failures, and major catastrophes were studied as possible causes of accidental radioactivity releases. No major safety deficiencies were found, and the facility appeared satisfactory for processing of highlevel radioactive wastes. Details of the safety analysis and information on the process, equipment, and operation procedures are furnished.

220 (INIS-mf-8084, pp vp) Glass as a high level waste matrix: a review of the variables. Lewis, R.A.; Myhra, S.; Segall, R.L.; Smart, R. St. C.; Turner, P.S. (Griffith Univ., Brisbane (Australia). School of Science). 1982. NTIS (US Sales Only), PC A04/MF A01. (CONF-821187-Absts.).

From 11. AINSE radiation chemistry conference; Lucas Heights, Australia (10 Nov 1982).

GLASS; HIGH-LEVEL RADIOACTIVE WASTES; RADIOACTIVE WASTE PROCESSING; STABILITY; STRESSES; TEMPERATURE DEPENDENCE; MATRIX MATERIALS

221 (INIS-mf-8084, pp vp) Structural chemistry of Synrnc. Cousens, D.R.; Penrose, J.; Segall, R.L.; Smart, R. St. C.; Turner, P.S.; White, T.J. (Griffith Univ., Brisbane (Australia). School of Science). 1982. NTIS (US Sales Only), PC A04/MF A01. (CONF-821187-Absts.).

From 11. AINSE radiation chemistry conference; Lucas Heights, Australia (10 Nov 1982).

HIGH-LEVEL RADIOACTIVE WASTES; HOLLANDITE; MICROSTRUCTURE; PEROVSKITE; STRUCTURAL CHEMICAL ANALYSIS; SYNTHETIC ROCKS; IWINNING; ZIRCONOLITE

222 (INIS-mf-8524) Vitrification of high active waste. Vitrification techniques. Sombret, $C$. (Kernforschungszentrum Karlsruhe G.m.b.H. (Germany, F.R.). Schule fuer Kerntechnik). 1982. 81 p. (CONF-8209134-18). NTIS (US Sales Only), PC A05/MF A01. Order Number DE83703510.

From IAEA interregional training course on waste management in nuclear facilities; Karlsruhe, F.R. Germany (6 Sep 1982).

The main stream of high level radioactive materials generated as wastes by the reprocessing of spent nuclear fuel is composed of fission products solutions. The storage of these liquids has been operated until now in stainless steel tanks almost everywhere. From a general slandpoint, it is now assumed that these solutions, in which long-life actinides are also present, must be solidified owing to the hazards related to a long term disposal. Vitrification has generally been taken more into consideration than other processes owing to a relatively simple implementation and mainly because glass is a medium flexible enough to house the various elements bound to be present in the radioactive liquids without inducing drastic changes in the physicochemical properties. Among these properties particular attention is paid to the ones connected to the fabrication: corrosiveness, volatilization, viscosity as well as to the interim storage and long term disposal: thermal stability, chemical stability versus $\beta$ ff and 3 irradiation including the subsequent effects of helium build up. Investigations in those fields have been carried out principally on borosilicate and aluminoborosilicate glasses. The manufacture techniques under development are numerous. Some of them are continuous processes, other are batch ones. Both can involve either a one stage or a two stage fabrication. The most advanced technique is a 2 stages continuous process involving calcination in a rotary tube and vitrification in a metallic melter. (orig./HW).

223 (JAERI-M-9378) Effects of various waste contents on properties of borosilicate glasses for high-level waste solidification in several countries. Furuya, T.; Senoo, M.; Banba, T. (Japan Atomic Energy Research Inst., Tokyo). Feb 1981. 29p. (In Japanese). NTIS (US Sales Only), PC A03/MF A01. Order Number DE82701324.

The Borosilicate glass is the most developed of the various solidified products of high-level waste (HLW) from reprocessing. The products have been studied in Japan and there is the possibility of the HLW glass from overseas fuel reprocessing being returned to Japan. The previous report issued the results of a study of the properties of glass. Waste (14\%) content glass has the same chemical composition those as developed in several countries. In this examination, the following HLW glass properties, with 20 - $30 \%$ waste contents, were obtained: visible observation, density, softening temperature and leach rate etc. Effects of various waste contents on properties of HLW glasses were shown. Using the obtained results, an upper limit of acceptable waste content of HLW glasses was discussed with the relationship between softening temperature and calculated centerline temp. of HLW glasses in a given air cooling system. The time reached for Maximum Permissible Concentration in an assumed accident in a water cooling system was calculated using the obtained leach rate of $\mathrm{HLW}$ glasses.

228 (JAERI-M-9386) Introduction of studles on highlevel radioactive waste immobilization in synthetic minerals. Supercalcine and SYNROC. Murakami, T.; Tashiro, S.; Araki, K. (Japan Atomic Energy Research Inst., Tokyo). Mar 1981. 28p. (In Japanese). NTIS (US Sales Only), PC A03/MF A01. Order Number DE82701325.

It is proposed that high-level radioactive wastes be immobilized in vitrified products and then isolated in gevlugival media. On the othet hand, various alternative waste forms have been developed to find better solidified products. Of these, Supercalcine and SYNROC are characteristic. Waste elements are distributed over crystal structurcs as host elements and are retained in the solidified products by strong chemical bonding. The products themselves consist of synthetic minerals. Supercalcine and SYNROC were introduced with the emphasis on composition, preparation, thermodynamic stability, leachability and radiation damage.

225 (JAERI-M-9627) Development of partitioning method - cold and semihot experimental works with partitioning testing apparatus. Kubota, M.; Yamaguchi, I.; Nakamura, H.; Tachimori, S.; Sato, A. (Japan Atomic Enorgy Research Inst., Tokyo). Aug 1981. 29p. (In Japanese). NTIS (US Sales Only), PC A03/MF A01. Order Number DF.83701520.

The partitioning has been developed under the concepts of separating transuranium elements, ${ }^{90} \mathrm{Sr}$ and ${ }^{107} \mathrm{Cs}$ from a high-level fucl reprocessing waste, and then transmuting transuranium elements to fission products with fast neutron and fixing ${ }^{90} \mathrm{Sr}$ and ${ }^{107} \mathrm{Cs}$ as mineral like compound more stable than glass. The present paper describes the results of mainly 2 times semihot experimental works and of 2 times cold experimental works on the partitioning method consisting of solvent extraction and ion exchange by using the partitioning testing apparatus constructed in July of 1979 . In these works a few troubles such as emulsification in the solvent extraction apparatus and foaming in the denitration vessel werc cvolved and solutions to these troubles could be found out individually. Even though there is a problem on designing an inorganic ion exchanger column for recovering ${ }^{90} \mathrm{Sr}$ and ${ }^{107} \mathrm{Cs}$, transuranium elements which is the first objective in the partitioning could be separated in the yield over $99.9 \%$. Complete provision of the cask for trans- 
lorting a high-level liquid waste from PNC and performance tests for he partitioning testing apparatus will be continued and the partitioning nethod will be examined with $1 \mathrm{kCi}$ of high-level liquid waste from PNC in the fiscal year of 1981 .

226 (Juel-Conf-42-Vol.1) Proceedings of the international seminar on chemistry and process engineering for highlevel liquid waste solidification. Odoj, R.; Merz, E: (eds.). (Kernforschungsanlage Juelich G.m.b.H. (Germany, F.R.). Inst. fuer Chemische Technologie der Nuklearen Entsorgung; Gesellschaft Deutscher Chemiker, Frankfurt am Main (Germany, F.R.). Fachgruppe Nuklearchemie). Jun 1981. 550p. (In several languages). (CONF-810650-Vol.1). NTIS (US Sales Only), PC A24/MF A01. Order Number DE82905136.

From International seminar on chemistry and process engineering for high-level waste solidification; Julich, F.R. Germany (1 Jun 1981).

Portions of document are illegible.

Fifty one papers were presented at the International Symposium on Chemistry and Process Engineering for High-Level Liquid Waste Solidification. Volume I of this Proceedings has 28 papers, of which 19 have been abstracted and indexed and have been title listed. The seminar covered four topics, solidification, process and product chemistry, waste product forms, and waste containment requirements. (ATT)

227 (Juel-Conf-42-Vol.1, pp 1-11) High level waste solidification: the future. Grover, J.R. (AFRE, Harwell, England). Jun 1981. NTIS (US Sales Only), PC A24/MF A01. (CONF810650-Vol.1).

From International seminar on chemistry and process engineering for high-level waste solidification; Julich, F.R. Germany (1 Jun 1981).

The paper reviews the developments in solidification technology since the compilation and publication in 1977 of the IAEA Technical Reports Series No. 176 Techniques for the solidification of High Level Wastes. New processes and concepts which have been proposed since 1977 are discussed. The paper then considers the future, and the developments which will be required in solidifcation technology as the arisings of high level wastes increase significantly during the next twenty years. The need for an overall systems approach to high level waste management is stressed, linking togeter the reprocessing, solidification, storage, transport and disposal. Particular attention is focussed on the requirements for the final waste form in relation to the geological disposal environment and the implications of this on the choice of solidification process.

228 (Juel-Conf-42-Vol.1, pp 12-34) Marcoule vitrification shop: 30 months service. Moncouyoux, J.P.; Hugony, P.; Pieraggi, A. Jun 1981. (In French). NTIS (US Sales Only), PC A24/MF A01. (CONF-810650-Vol.1).

From International seminar on chemistry and process engineering for high-level waste solidification; Julich, F.R. Germany (1 Jun 1981).

The Marcoule Vitrification Shop (A.V.M.) allows solidification of high activity wastes, which are fission products solutions, by the technique of vitrification which is continually improved by the C.E.A. This Shop is managed by the COGEMA, it is intended for teatment of solutions produced by the reprocessing at Marcoule of the spent fuel from primarily natural uranium type reactors. Since being put into service in June 1978, it has completed through December 31, 1980, 39 months of active service.

229 (Juel-Conf-42-Vol.1, pp 35-51) Fission product solidification: FIPS. Dix, S.; Halaszovich, S.; Mirschinka, V. Jun 1981. (In German). NTIS (US Sales Only), PC A24/MF A01. (CONF-810650-Vol.1).

From International seminar on chemistry and process engineering. for high-level waste solidification; Julich, F.R. Germany (1 Jun 1981).

In German. RADIOACTIVE WASTE PROCESSING; HIGH-
LEVEL RADIOACTIVE WASTES; SOLIDIFICATION;
DENITRATION; CALCINATION; VITRIFICATION; FISSION PRODUCTS

230 (Juel-Conf-42-Vol.1, pp 52-67) Vitrification of high level wastes using microwave power. Hardwick, W.H.; Gayler,
R.; Murphy, V. (AERE, Harwell, England). Jun 1981. NTIS (US Sales Only), PC A24/MF A01. (CONF-810650-Vol.1). From International seminar on chemistry and process engineering for high-level waste solidification; Julich, F.R. Germany (1 Jun 1981).

A process for radioactive waste vitrification which exploits advantages peculiar to microwave heating is under development. The advantages claimed are the removal of the heat source from the radioactive environment, the elimination of heat transfer barriers by direct coupling of the energy with the process materials, and the ability to evaporate liquors absorbed in a glass fibre matrix which constitutes the glass forming additive. This glass fibre is also used to filter off-gases and give a condensate free of solids. The fibre loaded with dried waste is converted to a homogeneous glass by melting using microwave power. Pilot scale equipment has been built and operated to demonstrate the feasibility of the concept using $2450 \mathrm{MHz}$ radiation. Experimental results are reported. It is proposed to scale up the process to commercial throughout using $896 \mathrm{MHz}$ in the evaporation stage and the first tests of an 896 $\mathrm{MHz}$ powered evaporator are described.

231 (Juel-Conf-42-Vol.1, pp 68-82) Design of Harwell Joule Ceramic Melter. Robinson, K.S.; Fowler, G. (UKAEA, Harwell, England). Jun 1981. NTIS (US Sales Only), PC A24/MF A01. (CONF-810650-Vol.1).

From International seminar on chemistry and process engineering for high-level waste solidification; Julich, F.R. Germany (1 Jun 1981).

A large continuous glass making process has been selected at Harwell for development for the future generation of vitrification plant. The plant should fulfill the following requirements: (1) at least 5 to 10 years operational lifetime; (2) stable and easily controlled operation; (3) flexibility with respect to feed and precursor glass composition and final product form; (4) corrosion/erosion sensitive components to be replaceable. The Joule Ceramic Melter (JCM) has been demonstrated in the USA and continental Europe to offer a process capable of development to meet these requirements. The Harwell J.C.M. has been designed for a throughout of $1 / 2$ to 1 tonnes/day of glass-forming feedstock from a melt volume of approximately $0.2 \mathrm{~m}^{0}$, contained in a simple rectangular cavity. This paper discusses the design principles, melter wall heat losses, heat transfer equations, off-gas systems, and plant layout. It is expected that the pilot plant J.C.M. will be ready for commissioning in mid-1982.

232 (Juel-Conf-42-Vol.1, pp 103-112) Solidification of radioactive waste forms and waste containment by the $H$ I P $O$ W process. Larker, H.T. (High Pressure Lab., Robertsfors, Sweden). Jun 1981. NTIS (US Sales Only), PC A24/MF A01. (CONF-810650-Vol.1).

From International seminar on chemistry and process engineering for high-level waste solidification; Julich, F.R. Germany (1 Jun 1981).

The Hot Isotactic Pressing of Waste (HIPOW) process offers versatile and very powerful means for making dense bodies even of materials with high melting pint, a characteristic common for very stable and corrosion resistant materials. The high temperature processing is carried out with the material contained in a hermetically sealed container, thus avoiding contamination of the high temperature equipment. Tritium which readily passes through metals at temperatures above $400^{\circ} \mathrm{C}$ can because of the fact that the radioactive material is enclosed in a cold wall pressure vessel during the high temperature treatment be quantitatively confined in e.g. zircaloy blocks made from fuel hulls. A special, axially resilient metal container has been proposed and it enables most radioactive waste materials in powder or particulate form to be filled into such containers in an uncomplicated way, still giving reproducable shape of the final product. The process can also be used to manufacture a monolithic corrosion resistant containment of inactive material. Spent fuel rods could e.g. at a temperature of below $700^{\circ} \mathrm{C}$ be embedded in solid copper separating the rods from one another and with a thick wall copper encasement surrounding the array of fuel rods.

233 (Juel-Conf-42-Vol.1, pp 112-129) Laboratory activity in Italy in the field of vitrification of high-level wastes. Donato, A.; Grossi, G.; Cantale, C. (CNEN - CSN, Rome, 
Italy). Jun 1981. NTIS (US Sales Only), PC A24/MF A01. (CONF-810650-Vol.1).

From International seminar on chemistry and process cnginccring for high-level waste solidification; Julich, F.R. Germany (1 Jun 1981).

The laboratory activity concerning the vitrification of $\mathrm{HLW}$ is carried out at the CNEN-Casaccia Nuclear Center and is strictly connected to the operations of a small scale hot vitrification plant remotely operated for the vitrification of actual HLW and the production of real high-level radioactive glass blocks ( 2 divided by $3 \mathrm{Kg}$ ). This plant, whose name is ESTER, is placed in a hot cell of the ESSOR-ADECO facility at the EURATOM Center of Ispra and at present is tested with cold simulated HLW solutions. The first hot run is planned for next September. The choice of the best borosilicate glass formulations and the study of their characteristics are performed at Casaccia nuclear center. Several gass compositions have been studied and selected in connection with two HLW reference solutions.

234 (Jucl-Conf-42-Vol.1, pp 130-152) Prelimianry experience with vitrification inactive pilot plant in Italy. Cao, S. (Comitato Nazionale Energia Nucleare, Rome, Italy); Candelieri, T.; Mataloni, P.; Risoluti, P. Jun 1981. NTIS (US Sales Only), PC A24/MF A01. (CONF-810650-Vol.1).

From International seminar on chemistry and process engineering for high-level waste solidification; Julich, F.R. Germany (1 Jun 1981).

An inactive vitrification pilot plant (called IVET) has been installed at CNEN's TRISAIA Center, Southern Italy, for full scale testing of the HLW solidification process to be applied at EUREX and/or ITREC, the two reprocessing pilot plants owned by CNEN. The applied process is based on the pot vitrification concept. The simulated HLW solution is fed to the pot at $10 / 201 / \mathrm{h}$ of average feeding rate. A $250 \mathrm{~mm}$ in diameter and 1.70 meter long vessel is employed. With 1 meter filling height, fifty liters glass block can be produced per batch. The glass forming additives may be fed to the reaction vessel in liquid phase as well as in solid form. A five zone induction heated furnace is employed, providing net $30 \mathrm{kw} /$ zone, at $1000 \mathrm{HZ}$ frequency. The off gas cleanup system, which is also fully tested, includes condensation, scrubbing and Ru filtering. Four preliminary runs have so far been carried out, showing that a production of fifty liters glass block is achievable with liquid feeding rate ranging from 15 to 20 liters per hour. Feeding rate as high as $301 / \mathrm{h}$ can also be maintained with minor liquid hold up in the canister. A heating mode based on differential power delivery to the zones has been tested.

235 (Juel-Conf-42-Vol.1, pp 153-160) Status of HAWCeramic-Melting Technology at the Institute of Nuclear Affairs. Gruenewald, W.; Weisenburger, S. (Kernforschungszentrum, Karlsruhe GmbH, Germany). Jun 1981. (In German). NTIS (US Sales Only), PC A24/MF A01. (CONF-810650-Vol.1).

From International seminar on chemistry and process engineering for high-level waste solidification; Julich, F.R. Germany (1 Jun 1981).

The influence of relevant parameters on the process technology of a HAW-ceramic melter with fluid input of the fission product solution is explained. The design of the remotely controllable INE melter K-3 is described and its state of development is briefly outlined.

236 (Juel-Conf-42-Vol.1, pp 161-178) Electrodes for the direct heating of highly radioactive borosilicate glasses in $\mathrm{HAW}$ ceramic furnaces. Weisenburger, $S$. (Kernforschungszentrum, Karlsruhe GmbH, Germany). Jun 1981. (In German). NTIS (US Sales Only), PC A24/MF A01. (CONF-810650-Vol.1).

From International seminar on chemistry and process engineering for high-level waste solidification; Julich, F.R. Germany (1 Jun 1981).

in German; Kernforschungszentrum, Karlsruhe. RADIOACTIVE WASTE PROCESSING; HIGH-LEVEL RADIOACTIVE WASTES; SOLIDIFICATION; VITRIFICATION; BOROSILICATE GLASS; KERNFORSCHUNGSZENTRUM KARLSRUHE; ELECTRODES

237 (Juel-Conf-42-Vol.1, pp 179-204) Inactive vitrification in Mol. Ceramic melting, feed and off gas system, pouring equipment for glassmelting. Heimerl, H. Jun 1981. (In German).
NTIS (US Sales Only), PC A24/MF A01. (CONF-810650Vol.1).

From International seminar on chemistry and process engineering for high-level waste solidification; Julich, F.R. Germany (1 Jun 1981).

In German. BELGIUM; RADIOACTIVE WASTE PROCESSING; HIGH-LEVEL RADIOACTIVE WASTES; SOLIDIFICATION; VITRIFICATION; GLASS; OFF-GAS SYSTEMS

238 (Juel-Conf-42-Vol.1, pp 205-228) Inactive Pamela vitrification facility in Mol; manufacture and characteristics of Vitromet products. van Geel, J. (Eurochenic, Mol, Belgium). Jun 1981. (In German). NTIS (US Sales Only), PC A24/MF A01. (CONF-810650-Vol.1).

From International seminar on chemistry and process engineering for high-level waste solidification; Julich, F.R. Germany (1 Jun 1981).

in German. RADIOACTIVE WASTE PROCESSING; HIGHLEVEL RADIOACTIVE WASTES; SOLIDIFICATION; VITRIFICATION; BELGIUM

239 (Juel-Conf-42-Vol.1, pp 229-251) Influence of the reprocessing flow sheet on the HLW solidification technology. Bạetsle. L.H. Jun 1981. NTIS (US Sales Only), PC. A24/MF A01. (CONF-810650-Vol.1).

From International seminar on chemistry and process engineering for high-level waste solidification; Julich, F.R. Germany (1 Jun 1981).

Dissolution of $\mathrm{UO}_{2}$ fuel does not create special problems but the introduction of Pu recycled LWR and LMFBR fuel will require the addition of a second dissolution step to quantitatively recover plutonium and to transform the hulls and insoluble residues into a short term waste management problem. If process modifications can be brought to the head-end procedures it is advisable to remove quantitatively the insoluble fission product $(\mathrm{Ru}, \mathrm{Tc}, \mathrm{Mo}, \mathrm{Pd})$ by high performance centrifugation and to volatilize as much as possible soluble $\mathrm{RuNO}\left(\mathrm{NO}_{0}\right)_{2}$ by sparging with ozone. The conversion of high active waste (HAW) to the high active waste concentrate (HAWC) by evaporation is accompanied by some-volatilization of $\mathrm{Ru}$ and Cs. Addition of organic reductants e.g. formal dehyde, sugar and formic acid will further reduce the Ru volatilization. But the addition of organics increases the corrosiveness of the solutions. A removal of Ru from HAW prior to conversion into HAWC by evaporation is a commendable practice. The introduction of salt free rengents during feed adjustment steps will decrease the NA content in the HLW. But the main impact of the use of salt free reagent will have its bearing on the LAW and ILLW treatment and conditioning. If Ru can be removed during the head-end steps it may be expected that calcination and vitrification processes will not be complicated by very elaborate off-gas control equipment.

240 (Juel-Conf-42-Vol.1, pp 252-276) Immobilization of Idaho Chemical Processing Plant high-level wastes. Knecht, D.A.; Berreth, J.R. (Exxon Nuclear Idaho Company, Inc., Idaho Falls). Jun 1981. NTIS (US Sales Only), PC A24/MF A01. (CONF-810650-Vol.1). Contract AC07-79ID01675.

From International seminar on chemistry and process engineering tor high-level wastc solidificalion, Julicl, F.R. Oetılany (1 Jun 1981).

Solidifying high-level waste as a calcine and storig it in stainless steel bins has been a very safe and practical storage method at Idaho Chemical Processing Plant (ICPP). (Storing calcined commercial waste may not be practical because of grcatcr heat gencration.) In the future, it may be desirahle in immobilize ICPP calcined wastes for final disposal. Studies of a glass waste form show that glasses formed from ICPP calcines have properties comparable to high-level waste glasses produced elsewhere. Of the ceramic immobilization products being examined for ICPP high-level wastes, glass-ceramics appears to be the most promising based on relative leach resistance, waste loading, accommodation to varying calcine composition, and process simplicity. Process development work is most advanced for zirconia calcine vitrification, with laboratory and pilot-scale ceramic melter test at ICPP and pilotand full-scale in-can melting tests at PNL. Very little process development work has been performed with alternate ceramic waste forms. Further evaluation of waste forms and processes alternative to glass is 
planned before selecting a process to immobilize ICPP calcined waste for final disposal.

241 (Juel-Conf-42-Vol.1, pp 277-302) Denitration of simulated fast ractor highly active liquor waste. Saum, C.J.; Ford, L.H.; Blatts, N. Jun 1981. NTIS (US Sales Only), PC A24/MF A01. (CONF-810650-Vol.1).

From International seminar on chemistry and process engineering for high-level waste solidification; Julich, F.R. Germany (1 Jun 1981).

A series of tests have been made at the Springfields Nuclear Laboratories of UKAEA to demonstrate the advantages of denitration in the reduction of volatility of ruthenium during the calcination of simulated PFR HAL. In these tests a quantity of liquor, both standard simulated HALL and fully denitrated HAL, have been evaporated to dryness at low temperature, $90^{\circ} \mathrm{C}$. Samples of the dried residue were then heated in air at a range of temperatures and the percentage weight loss and ruthenium volatility were calculated relative to the initial dried material. The results of these calculations show that the ruthenium volatility of the standard simulate is significantly higher than that of the denitrated simulate. Furthermore, the weight loss, i.e. degree of calcination, is very much higher at low temperatures for the denitrated samples. This would lead to lower calcination temperatures with consequent advantages to the system.

242 (Juel-Conf-42-Vol.1, pp 303-320) Comparison of wet slurry and dry frit (crizzle) feeding of glass-formers in pot vitrification. Morris, J.B.; Chidley, B.E.; Walmsley, D. (AERE, Harwell, England). Jun 1981. NTIS (USS Sales Only), PC A24/MF A01. (CONF-810650-Vol.1).

From International seminar on chemistry and process engineering for high-level waste solidification; Julich, F.R. Germany (1 Jun 1981).

The HARVFST pot vitrification process has been investigated with the glass-formers fed either as a slurry of unreacted chemicals or as dry preformed glass frit (crizzle). The product quality is very good from both processes, with perhaps a slight advantage lying with the slurry system. This method might also be just superior in terms of cesium carry-over. However in all other major respects such as throughput, process stability and general off-gas behavior, the process entailig the crizzle feed is undoubtedly to be preferred. A single line of plant based on a $0.6 \mathrm{~m} \mathrm{GMV}$ and operating with a dead time of $33 \mathrm{~h}$ per cycle can make glass at a mean rate of $15 \mathrm{~kg} / \mathrm{h}$, equivalent to conditioning the waste arising from $8.5 \mathrm{Gw}(\mathrm{e})$ of installed generating capacity. It is confidently concluded that pot vitrification based on crizzle feed of glass-formers is a suitable process for a production plant dealing with high level waste.

243 (Juel-Conf-42-Vol.1, pp 321.337) Equipment, operation and some results from a hot test of the CTH actinide separation process. Liljenzin, J.O. (Chalmers Univ. of Tech., Goeteborg, Sweden); Persson, G.; Bonnevie-Svendsen, M.; Michelsen, O.B. Jun 1981. NTIS (US Sales Only), PC A24/MF A01. (CONF-810650-Vol.1).

From International seminar on chemistry and process engineering for high-level waste solidification; Julich, F.R. Germany (1 Jun 1981).

The CTH actinide separation process has been tested by treating 161 of 10 year old waste solution from PUREX reprocessing of metallic fuel. It was in general found to operate well and, in some respects, slightly better than design specifications The extraction process removed more than $99.995 \%$ of initial alpha activity. After the sorption steps $<150 \mathrm{~Bq} / 1 \alpha$ - and $<2.510^{5} \mathrm{~Bq} / 1 \beta$-activity remained in solution. The modified reversed TALSPEAK process used to separate Am and $\mathrm{Cm}$ from the lanthanides gave an $\mathrm{Am}-\mathrm{Cm}$ product with less than $0.7 \%$ of the lanthanides and vice versa. This result can probably be somewhat improved by continuous addition of lactic acid and closer $\mathrm{pH}$ control.

244 (Juel-Conf-42-Vol.1, pp 338-349) Solidification of high-level radioactive wastes and alpha-bearing wastes by a solgel process. Zimmer, E. (Kernforschungsanlage, Juelich GmbH, Germany). Jun 1981. NTIS (US Sales Only), PC A24/MF A01. (CONF-810650-Vol.1).
From International seminar on chemistry and process engineering for high-level waste solidification; Julich, F.R. Germany (1 Jun 1981).

in German. RADIOACTIVE WASTE PROCESSING; SOLIDIFICATION; HIGH-LEVEL RADIOACTIVE WASTES; ALPHA-BEARING WASTES; SOL-GEL PROCESS

245 (Juel-Conf-42-Vol.1, pp 350-367) Sol-gel-derived waste forms. Lackey, W.J.; Angelini, P.; Arondl, W.D.; Bond, W.D.; Caputo, A.J.; Stinton, D.P. (Oak Ridge National Lab., TN). Jun 1981. NTIS (US Sales Only), PC A24/MF A01. (CONF-810650-Vol.1). Contract W-7405-ENG-26.

From International seminar on chemistry and process engineering for high-level waste solidification; Julich, F.R. Germany (1 Jun 1981).

Coated waste forms of high waste loading have been successfully produced at Oak Ridge National Laboratory. Development has centered on producing particles usig simulated Savannah River Plant (SRP) waste. The chemical vapor deposition process is being developed for coating particles with either pyrolytic carbon or silicon carbide in fluidized bed coaters. The followig materials have been successfully produced and coated: Synroc-B, Synroc-D ( $70 \mathrm{wt} \%$ simulated SRP waste without aluminum removal plus $30 \mathrm{wt} \%$ additives), $70 / 30$ (70 wt \% simulated SRP waste with aluminum removal plus 30 wt \% Synroc additives), 90/10 (90 wt \% simulated SRP waste with aluminum removal plus $10 \mathrm{wt} \% \mathrm{ZrO}_{2}$ ), and a cesium waste form ( $5 \mathrm{wt} \% \mathrm{Cs}$ ). Pyrolytic-carbon-coated particles have shown no attack when exposed to gaseous chlorine for $6 \mathrm{~h}$ at $1000^{\circ} \mathrm{C}$. Aqueous leach test data of the coated waste forms have been below the detection limits of such sensitive analytical techniques as atomic absorption and inductively coupled plasma.

246 (Juel-Conf-42-Vol.1, pp 368-393) Steam explosions caused by te contact of molten glass and water. Robinson, C.H.; Fry, C.J. (Atomic Energy Establishment, Winfrith, England). Jun 1981. NTIS (US Sales Only), PC A24/MF A01. (CONF$810650-$ Vol.1).

From International seminar on chemistry and process engineering for high-level waste solidification; Julich, F.R. Germany (1 Jun 1981).

Steam explosions may occur when a hot liquid is brought into contact with water. This paper briefly describes the phenomena and identifies three stages, mixing, triggering and propagation, which are required to establish an energetic steam explosion. Experiments to investigate each of three stages, using molten glass and water, are described. The results indicate that the viscosity of the glass controls the triggering stage and that steam explosions cannot be triggered if the viscosity of the molten glass is greater than 0.3 Pas. The viscosity of the glasses used for the vitrification of highly active wastes is significantly above this threshold value, at all operational conditions. Consequently, in the vitrification of highly active wastes, contact between the molten glass and water will not cause a steam explosion.

247 (Juel-Conf-42-Vol.1, pp 394-405) Sodium titanate in solidification of radioactive wastes. Heinonen, $\mathrm{O} . J$.; Lehto, J.; Miettinen, J.K. (Univ. of Helsinki, Finland). Jun 1981. NTIS (US Sales Only), PC A24/MF A01. (CONF-810650-Vol.1).

From International seminar on chemistry and process engineering for high-level waste solidification; Julich, F.R. Germany (1 Jun 1981).

Investigations of borosilicate glasses as the primary nuclear waste form have shown that these materials are both chemically and physically unstable under certain hydrothermal conditions. An ion exchange-ceramic process has been developed as an alternative for the calcination-glass processes for the solidification and stabilization of high-level liquid streams. Most attention has been given to the sodium form of titanate, $\mathrm{NaTi}_{2} \mathrm{O}_{5}$ to increase the selectivity for certain radionuclides. A ceramic synthetic rock, SYNROC, composed of several minerals, e.g. zirconolite, perovskite and hollandite, is one of the interesting potential applications of ceramic waste forms. Both of the large scale sodium titanate methods developed rely on rather expensive chemicals. In this paper, the possibility of synthesizing a sodium titanate from hydrated titanium oxide, an intermediate produced in the sulfate process for pigments, was investigated. The synthesized product was then tested to see the effects of complex forming agents, salt $(\mathrm{NaCl})$ concentrations, and radiation on the sorption capacities. The sorption of strontium ions at $\mathrm{pH}_{5}$ and in the presence of complex forming agents, e.g. 
EDTA and sodium citrate, is sufficiently good. Sorption of actinides and other fission products seems to be satisfactory even at high salt concentrations. The effect of irradiation on the sorption properties of sodium titanate seems to be rather small.

248 (Juel-Conf-42-Vol.1, pp 406-429) Immobilization of reprocessing waste by means of titanate ion exchangers. Forberg, S.; Westermark, T. (Royal Inst. of Tech., Stockholm, Sweden). Jun 1981. NTIS (US Sales Only), PC A24/MF A01. (CONF-810650-Vol.1).

From International seminar on chemistry and process engineering for high-level waste solidification; Julich, F.R. Germany (1 Jun 1981).

The purpose of this work was to elaborate conditions for sorption in titanate ion exchangers of a liquid waste from reprocessing experiments in the 1960's on low burn up natural uranium fuel. The work was done at Kjeller, Norway, where a small amount of this medium level waste was available. The waste was $6 \mathrm{M}$ with respect to nitric acid and chemically similar to HLLw, although the concentration of iron and uranium was higher. Guided by tests on a $\mathrm{ml}$ scale, dimethylamine was chosen for neutralization and sodium titanate as the bed material. Precipitations occurring at the neutralization point were mastered by a device provoking intense turbulence; clogging of the columns was evaded by neutralization to a modest $\mathrm{pH}$. In the latest experiments, all with five 0.151 columns in series, 0.31 of the waste solution was neutralized to $\mathrm{pH}>1.0$ at a three-fold dilution. At a flow rate of $1.3 \mathrm{~cm} / \mathrm{min}$ or less, the decontamination factors were in many experiments higher than: $10^{8}$ for $\mathrm{Sr}, 10^{7}$ for $\mathrm{Cs}, 10^{5}$ for $\mathrm{Pu}$ and $10^{4}$ for $\mathrm{Am}, \mathrm{Co}, \mathrm{Sb}$ and $\mathrm{U}$. After an experiment, the influent in the head column was displaced by water, the bed was slurried and suctioned into a cotton bag. Caused by the displacing water, a transient pool of activity propagated forwards reducing the total decontamination. The transport water was contaminated. For this reason, a system of cartridges is outlined for the handling of titanates from filling the columns to drying and packing of canisters for hot isostatic pressing. A combination of experiences and suggestions given here might result, in a hygenic way, in MEGANNITE, a rutile body with an inactive outer layer, with a million year warranty against radioactive leaching.

249 (Juel-Conf-42-Vol.1, pp 430-494) Leaching of solidlfied highly radioactive decay products: overview. Malow, G. (Hahn-Meitner-Institut fuer Kernforschung, Berlin, Germany). Jun 1981. (In German). NTIS (US Sales Only), PC A24/MF A01. (CONF-810651-Vol,1).

From International seminar on chemistry and process engineering for high-level waste solidification; Julich, F.R. Germany (1 Jun 1981).

In German. RADIOACTIVE WASTE PROCESSING; HIGHLEVEL RADIOACTIVE WASTES; FEDERAL REPUBLIC OF GERMANY; SOLIDIFICATION; VITRIFICATION; LEACHING; GLASS

250 (Juel-Conf-42-Vol.1, pp 495-514) SYNROC: leaching performance and process technology. Ringwood, A.E.; Oversby, V.M.; Kesson, S.E. (Australian National Univ., Canberra). Jun 1981. NTIS (US Sales Only), PC A24/MF A01. (CONF810650-Vol.1)

From International seminar on chemistry and process engineering for high-level waste solidification; Jullch, F.R. Germany (1 Jun 1981).

The titanate ceramic wasteform SYNROC has been subjected to leaching in pure water at $95^{\circ} \mathrm{C}$ and $200^{\circ} \mathrm{C}$. Leach-rates for univalent and divalent elements ( $\mathrm{Cs}, \mathrm{Ca}, \mathrm{Sr}, \mathrm{Ba}$ ) are 500 to 2000 times smaller than from a typical borosilicate glass proposed for radwaste immobilization. Leach-rates for multivalent elements (Nd, $\mathrm{Zr}, \mathrm{Ti}, \mathrm{U}$ ) from SYNROC are about 10,000 times smaller than from borosilicate glass. SYNROC leach-rates remain essentially constant as waste-loading is increased from 9 to $20 \mathrm{wt} \%$. A conceptual flow-sheet for the full-scale production of SYNROC involves chemical precipitation of a highly surface-active precursor material which acts like an ion-exchanger, absorbing $\mathrm{HLW}$ cations from solution. The resultant slurry is dried at $130^{\circ} \mathrm{C}$ and then calcined in a controlled atmosphere within a vertical kiln. The powder is sealed in a bellows-shaped stainless steel container and subjected to isostatic or uniaxial hot-pressing at temperatures of 1150 to $1200^{\circ} \mathrm{C}$. The bellows collapse axially to contain the dense SYNROC ceramic monolith. These sealed cans are stacked in a large disposal can- ister for final geological disposal. The above technology may offer ecc nomic advantages and reduced radiological hazards when compare with borosilicate glass production.

251 (Juel-Conf-42-Vol.1, pp 515-532) Infuence of metal oxides on the leach rate and crystallisation behaviour of waste glasses. Dalton, J.T.; Boult, K.A.; Chamberlain, H.E.; Marples, J.A.C. (AERE, Harwell, England). Jun 1981. NTIS (US Sales Only), PC A24/MF A01. (CONF-810650-Vol.1).

From International seminar on chemistry and process engineering for high-level waste solidification; Julich, F.R. Germany (1 Jun 1981).

Time-Temperature-Transformation curves for the initial precipitation of the phases which occur in glasses suitable for vitrifying Magnox reactor wastes have been determined experimentally. The crystal phases developed in these glasses have been analyzed. The lithium sodium borosilicate precursors of the Magnox waste glasses have been melted with 2, 5, 10 and $20 \mathrm{wt} \%$ additions of various individual oxides, many typical of fission products and processig additions. Samples from these melts have been leached in a $100^{\circ} \mathrm{C}$ Soxhlet test and, although some additions resulted in improved leachability compared to the base glass, in general this advantage was lost if the glasses were crystallized by heat treatment. Alumina additions are exceptional in conferring improved chemical durability, even after cyrstallization has occurred. The UK candidate glasses containing the full simulate Magnox waste do not show any significant loss of leach resistance after any heat treatment.

262 (Juel-Conf-42-Vol.2, pp 540-557) Overview on product forms. Merz, E. (Kernforschungsanlage, Juelich, Germany). Jun 1981. (In German and English). NTIS (US Sales Only), PC A21/MF A01. (CONF-810650-Vol.2).

From International seminar on chemistry and process engineering for high-level waste solidification; Julich, F.R. Germany ( 1 Jun 1981).

In German. RADIOACTIVE WASTE PROCESSING; HIGHLEVEL RADIOACTIVE WASTES; SOLIDIFICATION; REVIEWS; BOROSILICATE GLASS; PHOSPHATE GLASS; SYNTHETIC ROCKS; SYNROC PROCESS; VITRIFICATION; CALCINED WASTES; CEMENTS; COATINGS; COMPOSITE MATERIALS

253 (Juel-Conf-42-Vol.2, pp 558-575) Development of borosilicate glass for the vitrification facility PAMELA. Lutzc, W. (Hahn-Meitner-Institut fuer Kernforschung, Berlin GmbH, Germany); Schiewer, E.; De, A.K. Jun 1981. (In German). NTIS (US Sales Only), PC A21/MF A01. (CONF-810650Vol.2).

From International seminar on chemistry and process engineering for high-level waste solidification; Julich, F.R. Germany (1 Jun 1981).

RADIOACTIVE WASTE PROCESSING; HIGH-LEVEL RADIOACTIVE WASTES; SOLIDIFICATION; VITRIFICATION; RADIOACTIVE WASTE FACILITIES; BOROSILICATE GLASS

254 (Juel-Conf-42-Vol.2, pp 576-602) Hydroxylated ceramic waste forms and the absurdity of leach tests. Roy, R. (Pennsylvania State Univ., Universtiy Park). Jun 1981. NTIS (US Sales Only), PC A21/MF A01. (CONF-810650-Vol.2).

From International seminar on chemistry and process engineering for high-level waste solidification; Julich, F.R. Germany (1 Jun 1981).

The repository pressure and temperature conditions during the thermal period projected in US repositories have been drastically lowered in the last year or two to new values of say $115 \pm 50^{\circ} \mathrm{K}$. Using the argument that the evidence from natural models indicates the most stable mineral ( $>$ ceramic) hosts for radionuclides, one finds that under these new repository conditions such crystalline assemblages would be micas, clays, zeolites and other hydrated minerals, plus the tetravalent anhydrous oxide families. A waste form consisting of specific hydroxylated candidate phases can be made via a simple in-can technology (demonstrated by Oak Ridge) by reacting liquid wastes with precursor gels or phyllo or tektosilicates at $<200^{\circ} \mathrm{C}$ under modest pressure within the final disposal canister. The data on the rate of reaction of typical oxide materials to yield hydroxylated phases under these conditions show that 
the typical leach test (at 25 to $100^{\circ} \mathrm{C}$ in deionized water) does not provide a simulation of the reactions which will occur. Hence such tests are not only totally meaningless with respect to qualifying a waste form for its role in a repository, they can be downright misleading.

255 (Juel-Conf-42-Vol.2, pp 621-642) Receptivity of borosilicate glass VG $\mathbf{9 8 / 1 2}$ for incorporating high-level radioactive wastes. Saidl, J.; Kahl, L. (Kernforschungszentrum, Karlsruhe GmbH, Germany). Jun 1981. (In German). NTIS (US Sales Only), PC A21/MF A01. (CONF-810650-Vol.2).

From International seminar on chemistry and process engineering for high-level waste solidification; Julich, F.R. Germany (1 Jun 1981).

In German. RADIOACTIVE WASTE PROCESSING; HIGHLEVEL RADIOACTIVE WASTES; SOLIDIFICATION; VITRIFICATION; KERNFORSCHUNGSZENTRUM . KARLSRUHE; BOROSILICATE GLASS

256 (Juel-Conf-42-Vol.2, pp 643-655) FUETAP concrete: an alternative radioactive waste host. Moore, J.G.; Rogers, G.C.; Dole, L.R.; Kessler, J.H.; Morgan, M.T.; Devaney, H.E. (Oak Ridge National Lab., TN). Jun 1981. NTIS (US Sales Only), PC A21/MF A01. (CONF-810650-Vol.2). Contract W-7405-ENG-26.

From International seminar on chemistry and process engineering for high-level waste solidification; Julich, F.R. Germany (1 Jun 1981).

Tailored autoclaves concretes (FUETAP concretes) offer a number of advantages as hosts for a wide vairety of radioactive wastes. They are formed at low temperatures and pressures $\left(100^{\circ} \mathrm{C}\right.$ and 0.1 $\mathrm{MPa}$ ) from readily available raw materials and require no new processing technology. The extreme latitude in concrete formulations ensures the acceptance of the gamut of waste materials. The leachability of nuclides from the resulting ceramic-like concretes is quite low with essentially no prospect of pressure build-up from long-term selfirradiation in the final storage containers. The solids are thermally stable up to at least $900^{\circ} \mathrm{C}$. Additional studies are in progress to verify that FUETAP concretes are acceptable alternative waste hosts for defense, TRU, and commercial high-level radioactive waste.

267 (Juel-Conf-42-Vol.2, pp 656-674) Compared quantitative determination of nuclear waste glass crystallization rate by light microscopy and $x$ ray diffraction. Morlevat, J.P. (C.E. N., Grenoble, France); Uny, G.; Jaquet-Francillon, N. Jun 1981. NTIS (US Sales Only), PC A21/MF A01. (CONF810650-Vol.2).

From International seminar on chemistry and process engineering for high-level waste solidification; Julich, F.R. Germany (1 Jun 1981).

Crystalline phases of heat treated borosilicate glass were investigated by means of $x$-ray diffraction and by electron micro-analysis (scanning electron microscopy) of a polished cross-section of the glass. These measurements were completed by the determination of the total volume content that could be apprehended by image analysis i.e. covering precipitates at least $1 \mu \mathrm{m}$ in size. The aim of this work was to set up a methodology to measure by different methods the corresponding volume fractions.

258 (Juel-Conf-42-Vol.2, pp 675-692) Diopside glassceramic material for the immobilization of radioactive wastes. Ninomiya, M.; Yamanaka, T.; Sakane, T.; Hora, M.; Nakamura, S.; Kawamura, S. Jun 1981. NTIS (US Sales Only), PC A21/MF A01. (CONF-810650-Vol.2).

From International seminar on chemistry and process engineering for high-level waste solidification; Julich, F.R. Germany (1 Jun 1981).

Diopside glass-ceramic produced by the crystallization of the glasses in $\mathrm{SiO}_{2}-\mathrm{Al}_{2} \mathrm{O}_{0}-\mathrm{CaO}-\mathrm{MgO}-\mathrm{Fe}_{2} \mathrm{O}_{0}-\mathrm{HLW}$ system has the improved properties as waste form. In its production, the homogeneous glass was made first and then it was crystallized. Crystallization was performed conventionally by cooling the glass melt in a thermally insulated canister without external heating equipment. The draining of glass in to canister was also performed as the same way in vitrification process. In conclusion Diopside glass-ceramic is material with the improved properties and easy production.
269 (Juel-Conf-42-Vol.2, pp 693-706) Ceramic nuclear waste forms. Jantzen, C.M.; Flintoff, J.; Morgan, P.E.D.; Harker, A.B.; Clarke, D.R. (Rockwell International Science Center, Thousand Oaks, CA). Jun 1981. NTIS (US Sales Only), PC A21/MF A01. (CONF-810650-Vol.2).

From International seminar on chemistry and process engineering for high-level waste solidification; Julich, F.R. Germany (1 Jun 1981).

Processing of radioactive wastes into a tailored ceramic monolith of the desired combination of mineralogic phases is dependent on the chemical additions, redox control and consolidation conditions. The waste composition controls the crystalline phases and determines the tailoring additives chosen. Minimization of additives enables high waste loading whereas excess additives produce inert phases which provide microstructural isolation of the radiophases.

260 (Juel-Conf-42-Vol.2, pp 713-732) SYNROC through melting: thermal analysis, thermogravimetry and crystal chemical characterization of phases. Pentinghaus, H. (Muenster Univ., Germany). Jun 1981. NTIS (US Sales Only), PC A21/MF A01. (CONF-810650-Vol.2).

From International seminar on chemistry and process engineering for high-level waste solidification; Julich, F.R. Germany (1 Jun 1981).

Synthetic rocks of different compositions have been prepared through melting in order to evaluate the technological potential of this process (in contrast with the favored hot-pressing technique working well below solidus temperatures). Thermal analyses guiding the cooling process and the optimization of the texture of synthetic rocks have been performed as well as thermal gravimetry has been applied to control mass losses due to incongruent evaporation. The crystalline phases formed have been characterized crystal-chemically by means of X-ray diffraction. So far all synthetic rocks prepared through melting contain smaller amounts of glass.

261 (Juel-Conf-42-Vol.2, pp 733-766) Critical review of radiation effects on borosilicate glasses. Lanza, F. (CEC/IRC, Ispra, Italy); Manara, A.; Antonini, M.; van Rutten, F. Jun 1981. NTIS (US Sales Only), PC A21/MF A01. (CONF810650-Vol.2).

From International seminar on chemistry and process engineering for high-level waste solidification; Julich, F.R. Germany (1 Jun 1981).

In order to compare the damage produced by the different types of radiation and to evaluate the usefulness of the different types of simulation it is necessary to use a common base of measurements. The number of displacements per atom (dpa) seems to be the most appropriate. To calculate the number of displacements due to a single event it is necessary to calculate the energy spent for each displacement (Ed). Normally a value of $25 \mathrm{ev}$ is assumed. It begins to appear, however, that such a value is overestimated and a lower one has to be assumed. With the usual value it appears that the most important source of displacements are the alpha-emitter recoils. An evaluation of the relative importance of beta rays in the case of a lower value of Ed is also presented. Most of the experimental values have been obtained by loading the glass with alpha emitters like $\mathrm{Cm} 244$ and $\mathrm{Pu} 238$. Some simulations have been performed using fission fragments or bombardment with accelerated ions. The data existing in literature on stored energy, and denstiy variation are presented and discussed. Particular attention is given to the variation of the leaching rate due to the radiation effect. Samples loaded with alpha emitters have given data up to $0.17 \mathrm{dpa}$. No large variations in the leaching rate have been detected. Samples bombarded with heavy ions show large effects which are probably due to dose rate effects. A study on defect formation has shown that under electrons irradiation, formation of bubbles is possible. Such a formation is dose rate dependent and seems to indicate a low Ed value.

262 (Juel-Conf-42-Vol.2, pp 829-843) Interaction of vitrified high level waste with clay environment. van Iseghem, P.; Timmermans, W.; de Batist, R. (S.C.K./C.E.N., Mol, Belgium). Jun 1981. NTIS (US Sales Only), PC A21/MF A01. (CONF-810650-Vol.2).

From International seminar on chemistry and process engineering for high-level waste solidification; Julich, F.R. Germany (1 Jun 1981). 
Preliminary results of corrosion experiments at different temperatures in different media with a number of simulated borosilicate waste glasses suggest marked differences in corrosion resistance between the different glasses. Higher corrosion stability is found in glasses with smaller $\mathrm{B}_{2} \mathrm{O}_{0}$ content. These differences in corrosion stability between the different glasses, which are most pronounced in contact with distilled water, persist at the different temperatures. This results in considerable weight losses, and mechanical disruption of the glasses with the higher $\mathrm{B}_{2} \mathrm{O}_{0}$ content after a one week's exposure at $250^{\circ} \mathrm{C}$. In both distilled water and clay-water the initial corrosion rate increases by a factor of 2 to 5 between 95 and $150^{\circ} \mathrm{C}$, and by a factor of 10 to 100 between 150 and $250^{\circ} \mathrm{C}$. Clay-water is found to corrode the glasses up to five times faster than distilled water. In general, however, corrosion rates are found to saturate after relatively short periods.

263 (Juel-Conf-42-Vol.2) Proceedings of the international seminar on chemistry and process engineering for highlevel liquid waste solidification. Odoj, R.; Merz, E. (eds.). (Kernforschungsanlage Juelich G.m.b.H. (Germany, F.R.). Inst. fuer Chemische Technologie der Nuklearen Entsorgung; Gesellschaft Deutscher Chemiker, Frankfurt am Main (Germany, F.R.). Fachgruppe Nuklearchemie). Jun 1981. 480p. (In German and English). (CONF-810650-Vol.1). NTIS (US Sales Only), PC A21 /MF A01. Order Number DE82905800.

From International seminar on chemistry and process engineering for high-level waste solidification; Julich, F.R. Germany (1 Jun 1981).

Portions of document are illegible.

Volume II contains 23 of the 51 papers which were presented at the International Seminar on Chemistry and Process Engineering for High-Level Liquid Waste Solidification. The seminar consisted of four topics, solidification, process and product chemistry, waste product forms, and waste containment requirements. Seventeen papers have been abstracted and indexed and the remaining 6 have been title listed. (ATT)

264 (Juel-Conf-42(Vol.2), pp 540-557) Overview on product forms. Merz, E. Jun 1981. (In German). NTIS (US Sales Only), PC A21/MF A01. (CONF-810650-Vol.2).

From International seminar on chemistry and process engineering for high-level waste solidification; Julich, F.R. Germany (1 Jun 1981).

For the future development and optimization of methods and products a better integration of the container barrier including migration-impending filler materials seems to be important. Borosilicate glasses belong to the appropriate product forms for the ultimate storage, whereas crystallized synthetic rocks are still in the test stage. The testing of the SYNROC-products is on.

265 (Juel-Conf-42(Vol.2), pp 558-575) Development of the borosilicate glass for the vitrification plant PAMELA. Lutze, W.; Schiewer, E.; De, A.K. Jun 1981. (In German). NTIS (US Sales Only), PC A21/MF A01. (CONF-810650Vol.2).

From International seminar on chemistry and process engineering for high-level waste solidification; Julich, F.R. Germany (1 Jun 1981).

The glass development is not finished yet. Therefore the glass constituents $\mathrm{SiO}_{2}, \mathrm{~B}_{2} \mathrm{O}_{0}, \mathrm{Al}_{2} \mathrm{O}_{0}$ as well as alkali- and alkaline earth oxides and $\mathrm{TiO}_{2}$ have not been quantified. 20 tons of glass have already been produced with simulated waste mixtures, and they have been tested in the HAW-ceramic-melter or in the inactive Pamelavitrification plant.

266 (Jucl-Conf-42(Vol.2), pp 576-602) Hydroxylated ceramic waste forms and the absurdity of 'leach tests'. Roy, R. Jun 1981. NTIS (US Sales Only), PC A21/MF A01. (CONF810650-Vol.2).

From International seminar on chemistry and process engineering for high-level waste solidification; Julich, F.R. Germany (1 Jun 1981).

The repository pressure and temperature conditions during the thermal period projected in U.S. repositories have been drastically lowered in the last year or two to new values of say $175 \pm 50 \mathrm{~K}$. Using the argument that the evidence from natural models indicates the most stable mineral ( $>$ ceramic) hosts for radionuclides, one finds that under these new repository conditions such crystalline assemblages would be micas, clays, zeolites, and other hydrated minerals, plus the tetravalent anhydrous oxide families. A waste form consisting of specific hydroxylated candidate phase can be made via a simple in-can technology (demonstrated by Oak Ridge) by reacting liquid wastes with precursor gels or phyllo or tektosilicates at $2200^{\circ} \mathrm{C}$ under modest pressure within the final disposal canister. The data on the rate of reaction of typical oxide materials to yield hydroxylated phases under these conditions show that the typical leach test (at $25-100^{\circ} \mathrm{C}$ in deionized water) does not provide a simulation of the reactions which will occur. Hence such tests are not only totally meaningless with respect to qualifying a waste form for its role in a repository, they can be downright misleading.

267 (Juel-Conf-42(Vol.2), pp 621-642) Capacity of the borosilicate glass VG $98 / 12$ for components of the high-level radioactive waste. Saidl, J.; Kahl, L. Jun 1981. (In German). NTIS (US Sales Only), PC A21/MF A01. (CONF-810650Vol.2).

From International seminar on chemistry and process engineering for high-level waste solidification; Julich, F.R. Germany (1 Jun 1981).

The maximum capacity of the borosilicate glass product GP $98 / 12$ and the gadolinium-containing GP $98 / 26$ has been studied for the $\mathrm{HAW}$-elements and element groups $\mathrm{Mo}, \mathrm{Cr}, \mathrm{Zr}, \mathrm{Ce}, \mathrm{U}, \mathrm{Np}, \mathrm{Pu}, \mathrm{SO}_{4}{ }^{2 *}$, $\mathrm{PO}_{4}{ }^{a}$ and $\mathrm{Cl}^{-}$. As the rules for a multi-component and variable system are unknown, only empiric investigations of the properties of the HAWcontaining glass products were possible. The concentration of parasitic substances determines the capacity of the glass matrix for the whole HAW-substances.

268 (Juel-Conf-42(Vol.2), pp 643-655) FUETAP concrete - an altemative radioactive waste host. Moore, J.G.; Rogers, G.C.; Dole, L.R.; Kessler, J.H.; Morgan, M.T.; Devaney, H.E. Jun 1981. NTIS (US Sales Only), PC A21/MF A01. (CONF-810650-Vol.2).

From International seminar on chemistry and process engineering for high-level waste solidification; Julich, F.R. Germany (1 Jun 1981).

These tailored autoclaved concretes (FUETAP concentrates) offer a number of advantages as hosts for a wide variety of radioactive wastes. They are formed at low temperatures and pressures $\left(100^{\circ} \mathrm{C}\right.$ and $0.1 \mathrm{MPa}$ ) from readily available raw materials and require no new processing technology. The extreme latitude in concrete formulations ensures the acceptance of the gamut of waste materials. The leachability of nuclides from the resulting ceramic-like concretes is quite low with essentially no prospect of pressure build-up from long-term selfirradiation in the final storage containers. The solids are thermally stable up to at least $900^{\circ} \mathrm{C}$. Additional studies are in progress to verify that FUETAP concretes are acceptable alternative waste hosts for defense, TRU, and commercial high-level radioactive waste.

269 (Juel-Conf-42(Vol.2), pp 656-674) Compared quantitative determination of nuclear waste glass crystallization rate by light microscopy and X-ray diffraction. Morlevat, J.P.; Uny, G.; Jacquet-Francillon, N. Jun 1981. NTIS (US Sales Only), PC A21/MF A01. (CONF-810650-Vol.2).

From International seminar on chemistry and process engineering for high-level waste solidification; Julich, F.R. Germany (1 Jun 1981).

The silic-borate base glass SON-58-30-24U2 contains 22 weight percent of fission products oxides. A methodology to measure by different methods the corresponding volume fractions is set up. The samples were heated at $760^{\circ} \mathrm{C}$ and different holding times.

270 (Juel-Conf-42(Vol.2), pp 675-692) Diopside glassceramic material for the immobilization of radioactive wastes. Ninomiya, M.; Yamanaka, T.; Sakane, T.; Hora, M.; Nakamura, S.; Kawamura, S. Jun 1981. NTIS (USS Sales Only), PC A21/MF A01. (CONF-810650-Vol.2).

From International seminar on chemistry and process engineering for high-level waste solidification; Julich, F.R. Germany (1 Jun 1981).

This study concerns with glass-ceramics in Diopside system in which main crystalline phase is Diopside $\left(\mathrm{CaO} \times \mathrm{MgO} \times 2 \mathrm{SiO}_{2}\right)$ and main components of the inert additives are $\mathrm{SiO}_{2}, \mathrm{Al}_{2} \mathrm{O}_{0}, \mathrm{CaO}, \mathrm{MgO}$ and $\mathrm{Fe}_{2} \mathrm{O}_{0}$. Simulated oxides of HLLW from the reprocessing plant of PNC 
Japan were incorporated. As this glass-ceramic is crystallized without the independent heat treatment step for crystallization, the simplification for manufacturing process is performed.

271 (Juel-Conf-42(Vol.2), pp 693-706) Ceramic nuclear waste forms. Jantzen, C.M.; Flintoff, J.; Morgan, P.E.D.; Harker, A.B.; Clarke, D.R. Jun 1981. NTIS (US Sales Only), PC A21/MF A01. (CONF-810650-Vol.2).

From International seminar on chemistry and process engineering for high-level waste solidification; Julich, F.R. Germany (1 Jun 1981).

Minimization of additives enables high waste loading whereas excess additives produce inert phases which provide microstructural isolation of the radiophases. Simple tailoring and processing can be used to accommodate all the radio-nuclides in most commercial and defense wastes into fully dense strong ceramics. Tailoring formulation can be readily understood in terms of the crystal chemistry and the interactions that occur between the crystallochemical groupings when processing consideration such as control of oxidation-reduction and the effects of processing temperature and pressure are considered.

272 (Juel-Conf - 42(Vol.2), pp 707-712) Review of multibarrier waste form development. Rusin, J.M. Jun 1981. NTIS (US Sales Only), PC A21/MF A01. (CONF-810650-Vol.2).

From International seminar on chemistry and process engineering for high-level waste solidification; Julich, F.R. Germany (1 Jun 1981).

The application of coatings and matrices as part of a multibarrier concept for the immobilization of nuclear wastes has been demonstrated by several investigators. The multibarrier concept provides an enhanced waste form by improvements in mechanical strength, leach rcsistance, and thermal stability. Process complexity is increased in the multibarrier process due to additional processing steps, high-coating temperatures, molten metal transfer, and flammable coating gas mixtures.

273 (Juel-Conf-42(Vol.2), pp 713-732) Thermal analysis, thermogravimetry and crystal chemical characterization of phases. Pentinghaus, H. Jun 1981. NTIS (US Sales Only), PC A21/MF A01. (CONF-810650-Vol.2).

From International seminar on chemistry and process engineering for high-level waste solidification; Julich, F.R. Germany (1 Jun 1981).

Synthetic rocks of different compositions have been prepared through melting in order to evaluate the technological potential of this process (in contrast with the favoured hot-pressing technique working well below solidus temperatures). Thermal analyses guiding the cooling process and the optimization of the texture of synthetic rocks have been performed as well as thermal gravimetry has been applied to control mass losses due to incongruent evaporation. The crystalline phases formed have been characterized crystal-chemically by means of $X$-ray diffraction. So far all synthetic rocks prepared through melting contain smaller amounts of glass.

274 (Juel-Conf-42(Vol.2), pp 829-843) Interaction of vitrified high level waste with clay environment. Iseghem, $P$. van; Timmermans, W.; Batist, R. de. Jun 1981. NTIS (US Sales Only), PC A21/MF A01. (CONF-810650-Vol.2).

From International seminar on chemistry and process engineering for high-level waste solidification; Julich, F.R. Germany (1 Jun 1981).

Preliminary results of corrosion experiments at different temperatures in different media with a number of simulated borosilicates waste glasses suggest marked differences in corrosion resistance between the different glasses. Higher corrosion stability is found in glasses with smaller $\mathrm{B}_{2} \mathrm{O}_{0}$ content. These differences in corrosion stability between the different glasses, which are most pronounced in contact with distilled water, persist at the different temperatures. This results in considerable weight losses, and mechanical disruption of the glasses with the higher $\mathrm{B}_{2} \mathrm{O}_{0}$ content after a one week's exposure at $250^{\circ} \mathrm{C}$. In both distilled water and clay-water the initial corrosion rate increases by a factor of 2 to 5 between 95 and $150^{\circ} \mathrm{C}$, and by a factor of 10 to 100 between 150 and $250^{\circ} \mathrm{C}$. Clay-water is found to corrode the glasses up to five times faster than distilled water. In general, however, corrosion rates are found to saturate after relatively short periods.
275 (Juel-Conf-42(Vol.2), pp 978-1002) Actinide sorption on rock minerals. Allard, B.; Andersson, K.; Rydberg, J. Jun 1981. NTIS (US Sales Only), PC A21/MF A01. (CONF810650-Vol.2).

From International seminar on chemistry and process engineering for high-level waste solidification; Julich, F.R. Germany (1 Jun 1981).

The distribution coefficient (Ksub(d))-values show little temperature and particle size dependence, but increase with time. The Ksub(d)-values for actinides highly depend on groundwater $\mathrm{pH}$ and redox conditions. All actinide are in the tri- and tetravalent oxidation state in typical Swedish deep bedrock groundwater. Ion exchange on clay, adsorption on quartz, chemisorption on apatite and precipitation of hydroxides can be identified.

278 (KFK-3126) Results report on research and development work 1980 of the Institut fuer Nukleare Entsorgungstechnik. (Kernforschungszentrum Karlsruhe G.m.b.H. (Germany, F.R.). Inst. fuer Nukleare Entsorgungstechnik). Feb 1981. 39p. (In German). NTIS (US Sales Only), PC A03/MF A01. Order Number DE82750083.

The Institut fuer Nukleare Entsorgungstechnik performs research and development work on the treatment, solidification and final storage of radioactive wastes. The main target of the work is to develop processes and concepts for a safe disposal of radioactive wastes from nuclear plants. The work on waste treatment is carried out within the framework of the reprocessing and waste treatment project. It comprises of the development and characterization of ultimate storable waste products as well as the development of processes for the treatment and solidification of radioactive wastes mainly from reprocessing irradiated nuclear fuels. The studies on the ultimate storage are carried out together with the 'Gesellschaft fuer Strahlen- und Umweltforschung'. Their main concern is the analysis of processes in the neighbourhood of ultimate storage (waste product, packing, filling and immediate surrounding storage medium) for normal and accident conditions and their safety technical optimization. The report is supplemented by a list of publications and lectures of the institute coworkers in 1980.

277 (KFK-3251e) Preparation and characterization of an improved borosilicate glass for the solidification of high level radioactive fission product solutions (HLW). Pt. 2. Kahl, L.; Ruiz-Lopez, M.C.; Saidl, J.; Dippel, T. (Kernforschungszentrum Karlsruhe G.m.b.H. (Germany, F.R.). Inst. fuer Nukleare Entsorgungstechnik; Kernforschungszentrum Karlsruhe G.m.b.H. (Germany, F.R.). Projekt Wiederaufarbeitung und Abfallbehandlung). Apr 1982. 53p. NTIS (US Sales Only), PC A04/MF A01. Order Number DE82750740.

In the 'Institut fuer Nuklare Entsorgungstechnik' the borosilicate glass VG $98 / 12$ has been developed for the solidification of the high level radioactive waste (HLW). This borosilicate glass can be used in a direct heated ceramic melter and forms together with the HLW the borosilicate glass product GP $98 / 12$. This borosilicate glass product has been examined in detail both in liquid and solid state. The elements contained in the HLW can be incorporated without problems. Only in a few exceptions the concentration must be kept below certain limits to exclude the formation of a second phase ('yellow phase') by separation. No spontaneous crystallization and no crystallization over a long time could be observed as long as the temperature of the borosilicate glass product is kept below its transformation area. Simulating accidental conditions in the final storage, samples had been leached at temperatures up to $200^{\circ} \mathrm{C}$ and pressures up to 130 bar with saturated rock salt brine and saturated quinary salt brine. The leaching process seems to be stopped by the formed 'leached layer' on the surface of the borosilicate glass product after a limited leaching time. Detailed investigations have been started to explain this phenomenon.

278 (LA-UR-83-307) Measurement of radioactive contaminated wastes. Caldwell, J.T.; Close, D.A.; Crane, T.W. (Los Alamos National Lab., NM (USA); EG and G, Inc., Goleta, CA (USA)). 1983. Contract W-7405-ENG-36. 13p. (CONF830205-11). NTIS, PC A02/MF A01. Order, Number DE83007541.

From Waste management conference; Tucson, AZ, USA (27 Feb 1983).

Portions are illegible in microfiche products. 
At Los Alamos, a comprehensive program is underway for the development of sensitive, practical, nondestructive assay techniques for the quantification of low-level transuranics in bulk solid wastes. The program encompasses a broad range of techniques, including sophisticated active and passive gamma-ray spectroscopy, passive neutron detection systems, pulsed portable neutron generator interrogation systems, and electron accelerator-based techniques. The techniques can be used with either low-level or high-level beta-gamma wastes in either low-density or high-density matrices. The techniques are quite sensitive $(<10 \mathrm{nCi} / \mathrm{g}$ detection) and, in many cases, isotopic specific. Waste packages range in size from small cardboard boxes to large metal or wooden crates. Considerable effort is being expended on waste matrix identification to improve assay accuracy.

279 (LALP-81-59-Rev.) Waste management bibliography, 1978-1983. Wheeler, K. (ed.). (Los Alamos National Lab., NM (USA)). 1983. Contract W-7405-ENG-36. 24p. NTIS, PC A02/MF A01. Order Number DE83016007.

The Los Alamos National Laboratory is conducting a variety of research and development to ensure the safety of storing and treating all types of radioactive wastes. These activities include the assay and sorting of waste, the interaction of waste with the earth, and the treatment of waste to reduce the volume and mobility of radionuclides in waste. The practical lessons learned from safety storing waste at Los Alamos since the mid 1940's are an ingredient in determining the direction of our research. National waste management programs are structured according to categories of waste, for example, high level, low level, mill tailings, and transuranic. This bibliography lists publications according to disciplines to show the relevance of work to more than one category of waste. The publications listed here were published between 1978 and mid 1983.

280 (NCSU-80-4) Subsolidus sintering of SYNROC: I Initial demonstrations. Solomah, A.G.; Hare, T.M.; Palmour, H. III. (North Carolina State Univ., Raleigh (USA)). Aug 1980. Contract AC09-80ET41902. 20p. NTIS, PC A02/MF A01. Order Number DE82019466.

Simulated High Level Radioactive Waste has been incorporated in sintered SYNROC-B Ceramic matrices prepared by subsolidus sintering techniques. Phase and compositional analyses have been carried out by $x$-ray diffraction techniques and scanning electron microscope equipped with energy dispersive analysis of x-ray (SEM/EDAX) analyses on fracture surfaces. Modified SNYROC-B compositions have been formulated based on computer-calculated ion substitutions relative to the stoichiometries of the major phases, [Hollandite $(H)$, Perovskite (P), Zirconolite $(Z)$ ] and their respective abilities to retain HLW ions in their lattice structures, either substitutionally or interstitially. When prepared and sintered, the Modified SYNROC-B compositions yield clean $H, P, Z$ assemblages, free of minor phases which might be questionable with respect to compatibility with major phases and HLW ions, and/or chemical and/or radiation stability under long-term disposal conditions. Chemical stability of the Modified SYNROC-B loaded with 10 and $15 \mathrm{wt} \%$ simulated HLW has been studied by leaching in pure distilled water at $\cdots 100^{\circ} \mathrm{C}$ and in simulated brines similar to those found in geologic disposal sites at their boiling temperatures $\left(\sim 110^{\circ} \mathrm{C}\right)$ at atmospheric pressure. High pressure, high temperature leaching in simulated brine has also been investigated at $\sim 50$ atm and $270^{\circ} \mathrm{C}$ for the Modified SYNROC-B Ceramic waste form loaded with 15 wt\% simulated PW-4b radwaste. In ail instances, the observed leach rates have been near or below (mostly below) present analytical detection limits (AAS or NAA).

281 (NCSU-81-3) Subsolidus sintering of SYNROC: II. Materials selections, process improvements, waste form evaluations. Palmour, H. III.; Hare, T.M.; Russ, J.C.; Boss, C.B.; Solomah, A.G.; Batchelor, A.D. (North Carolina State Univ., Raleigh (USA)). Jul 1981. Contract AC09-81SR10957. 100p. NTIS, PC A05/MF A01. Order Number DE82019467.

Portions of document are illegible.

The principal areas of research were related to materials selections and characterization, process optimizations, crystalline phase development, sinterability, resultant microstructures and evaluations of leaching behavior. With and without simulated radwaste doping, the Modified SYNROC.B formulation was found to be sinterable to technical density (D $>0.95$ in the CTS mode) at temperatures in the range $1195^{\circ} \mathrm{C}$ to $1285^{\circ} \mathrm{C}$, depending upon $\mathrm{TiO}_{2}$ and $\mathrm{CaCO}_{0}$ materials selec- tions, and upon powder processing methods employed prior to firing. Of the $16 \mathrm{TiO}_{2}$ raw materials evaluated in air-fired, undoped batches, 15 yielded technically dense compacts $(\mathrm{D}>0.95$ ). Three fine pigmentary grades of $\mathrm{TiO}_{2}$ were selected for further study in doped and undoped versions fired in $\mathrm{Ar}, 4 \% \mathrm{H}_{2}$. When intensively milled with other well chosen matrix constituents and $10 \%$ spray-calcined simulated waste, each of them yielded sintered densities of $\geq 4.2 \mathrm{~g} / \mathrm{cm}^{0}(D \geq 0.96)$ at $1260^{\circ} \mathrm{C}, 2 \mathrm{~h}$ in $\mathrm{Ar}, 4 \% \mathrm{H}_{2}$ atmosphere. Leachability studies have been carried out in triple distilled $\mathrm{H}_{2} \mathrm{O}$ according to $\mathrm{MCC}-1$ and $\mathrm{MCC}-2$ procedures at $25^{\circ}$ and $150^{\circ} \mathrm{C}$, respectively, and under $\gamma$-irradiation for dose rates of $2-5 \times 10^{5} \mathrm{rad} / \mathrm{h}$ at $\sim 25^{\circ} \mathrm{C}$. The results obtained showed that freshly exposed interions of sintered Modified SYNROC-B ceramics were highly stable in the leaching environment, and were very retentive of simulated waste ions, including the most leachable species, Cs. Depending on leaching conditions, the highest Cs leach rates (after 3 days) were on the order of $10^{-1} \mathrm{~g} \cdot \mathrm{m}^{-2}$.day , but diminished sharply for longer times (up to 92 days) to the range $10^{-2}-10^{-4}$ 8. $\mathrm{m}^{-2} \cdot \mathrm{day}^{-1}$.

282 (ND-R-658(S)) Denitration of simulated fast reactor highly active liquor waste. Saum, C.J.; Ford, L.H.; Platts, N. (UKAEA Risley Nuclear Power Development Establishment). Nov 1981. 25p. NTIS (US Sales Only), PC A02/MF A01. Order Number DE83702200.

Vitrification of the highly active arisings from PFR fuel reprocessing is proposed as the optimum long-term solution to the disposal problem. During vitrification ruthenium volatilizes as the tetroxide. Evidence is presented which indicates that a substantial reduction in volatility can be effected by denitration of the liquid feed by treatment with formic acid. The kinetics and stoichiometry of the reactions involved in denitration are examined and empirical rate equations developed. The predictions of the empirical rate equation have been confirmed using a one-tenth scale continuous denitrator, thus giving confidence for the design of full-scale units.

283 (NUREG/CR-2333-Vol.1) Nuclear waste management technical support in the development of nuclear waste form criteria for the NRC. Task 1. Waste package overview. Dayal, R.; Lee, B.S.; Wilke, R.J.; Swyler, K.J.; Soo, P.; Ahn, T.M.; McIntyre, N.S.; Veakis, E. (Brookhaven National Lab., Upton, NY (USA)). Feb 1982. Contract AC02-76CH00016. 426p. (BNL-NUREG-51458-Vol.1). NTIS, PC A19/MF A01. Order Number DE82009990.

Portions of document are illegible.

In this report the current state of waste package developiment for high level waste, transuranic waste, and spent fuel in the US and abroad has been assessed. Specifically, reviewed are recent and on-going research on various waste forms, container materials and backfills and tentatively identified those which are likely to perform most satisfactorily in the repository environment. Radiation effects on the waste package components have been reviewed and the magnitude of these effects has been identified. Areas requiring further research have been identified. The important variables affecting radionuclide release from the waste package have been described and an evaluation of regulatory criteria for high level waste and spent fuel is presented. Finally, for spent fuel, high level, and TRU waste, components which could be used to construct a waste package having potential to meet NRC performance requirements have been described and identified.

284 (NUKLG/CK-2333-Vol.4) Nuclear waste management technical support in the development of nuclear waste form criteria for the NRC. Task 4. Test development review. Ahn, T.M.; C.zyscinski, K.S.; Franz, E.M.; Klamut, C.J.; I Re, R.S.; McIntyre, N.S.; Swyler, K.J.; Wilke, R.J. (Brookhaven National Lab., Upton, NY (USA)). Feb 1982. Contract ACO276CH00016. 193p. (BNL-NUREG-51458-Vol.4). NTIS, PC A09/MF A01. Order Number DE82009884.

Portions of document are illegible.

This final report describes the development of testing methods by the Department of Energy for a high level nuclear waste package. Such methods serve several purposes: quality control of the materials being used, comparative testing of alternative components, and eventual qualification of the waste package to comply with Nuclear Regulatory Commission criteria in 10 CFR 60 . Since the issuance of the earlier Interim Report, a major effort has been spent reviewing the activities of the Materials Characterization Organization. To date this organization is dealing successfully with comparative testing aspects of the waste 
package, but compliance testing, in general, still remains to be addressed. Testing and characterization of each component are discussed in separate chapters. Testing of the waste form is considered in terms of chemically induced changes (leaching), mechanical effects and radiation effects. The waste container testing involves corrosion investigations, mechanical testing and studies of radiation effects. Testing of geologic backfill is discussed with the emphasis placed on the use of dynamic approaches to determine radionuclide migration. Backfill is chiefly investigated for its ability to retain radionuclides under different aqueous flow conditions. Finally, the different objectives for whole package testing are reviewed and compared to recent experiments started in DOE laboratories. Recommendations for further research are made in all the above areas and general types of testing for compliance purposes are identified.

285 (NUREG/CR-2333-Vol.5) Nuclear waste management technical support in the developmnt of nuclear waste form criteria for the NRC. Task 5 . National waste package program. Davis, M.S. (Brookhaven National Lab., Upton, NY (USA)). Feb 1982. Contract AC02-76CH00016. 55p. (BNL-NUREG51458). NTIS, PC A04/MF A01. Order Number DE82009513.

Portions of document are illegible.

This report assesses the need for a centrally organized waste package effort and whether the present national program meets those needs. It is the conclusion of the BNL staff that while the DOE has in principle organized a national effort to develop high-integrity waste packages for geologic disposal of high level waste, the effort has not yet produced data to demonstrate that a waste package will comply with NRC's criteria. The BNL staff feels, however, that such a package is achievable either by development of high integrity components which by themselves could comply with 1000 -year containment or by the development of new waste package designs that could comply with both the containment and the controlled release criteria in the 10CFR 60 performance objectives. In terms of waste forms, high-integrity components such as pyrolytic carbon coated waste and radioactive glass coated with non-radioactive glass offer higher potential than normal borosilicate waste glass. The existing container research program has yet to produce the data base on which to assess the potential of a container material to contain the waste for 1000 years. However, there may be the potential, based on Swedish calculations and work done on titanium in the DOE program, that Ti or its alloys may satisfy this criterion. Existing data on natural backfills will not be acceptable as the sole source for satisfying containment and the long-term release rate criteria. However, a synthetic zeolite system is an example of a backfill with a potential to satisfy both criteria. In this particular case, it is the BNL staff's opinion that existing technology and data for this system indicate that major development programs may not be required to qualify this material for licensing applications. The most likely means available for satisfying 10 CFR 60 with a single package component is through the performance of a discrete backfill.

286 (NUREG/CR-2482) Review of DOE waste package program. Subtask 1.1. National waste package program. Davis, M.S.; Schweitzer, D.G. (Brookhaven National Lab., Upton, NY (USA)). Feb 1982. Contract AC02-76CH00016. 138p. (BNLNUREG-51494-Vol.1). NTIS, PC A07/MF A01, Order Number DE82009915.

Portions of document are illegible.

The purpose of this report is to continue the assessment of the state of the DOE waste package development effort for high level waste. The report updates past reviews on waste forms, container materials, and backfill materials emphasizing evaluation of the materials with respect to their demonstrated or potential abilities to satisfy NRC performance objectives. The report also reviews whole waste package testing where such information exists. A major objective of this report is to begin describing the information that DOE must submit so that NRC can complete its licensing actions and other identified NRC objectives. Present information indicates that the DOE programs on waste forms will emphasize borosilicate glass and SYNROC. The information NRC will need to license a waste package in which the high level waste form borosilicate glass is given either partial or full credit for containment or for controlled release rates does not exist at present. After 1000 year containment, radionuclide release from a borosilicate glass waste form will be determined by how leaching of the long lived actinides is affected by variables in the waste form and in the environment surrounding the breached waste package. Almost all the existing information deals with leaching of the short lived fission products which become essentially innocuous after 1000 years. The report summarizes the information needed by NRC on TiCode- 12 if it is to be used for compliance with 1000 year containment. Some problems associated with prediction and accelerated testing are noted. The appendix contains a review paper on glass corrosion.

287 (NUREG/CR-2731) Evaluation of the safety aspects of the design and operation of temporary/mobile radioactivewaste solidification systems. McDonald, F.N.; McClure, L.W. (Exxon Nuclear Idaho Co., Inc., Idaho Falls (USA)). May 1982. 44p. NTIS - GPO \$4.75.

An evaluation of the safety aspects of the design and operation of temporary/mobile radioactive waste solidification systems in use at commercial nuclear power reactors was completed. The study was undertaken in response to a General Accounting Office report issued in August 1978 calling for more regulatory oversight of commercial lowlevel radioactive waste treatment. After reviewing the design and operation of three different vendor-operated waste solidification systems, it is clear that there are areas in which the vendors can improve their services. However, the vendors generally do a good job of solidifying waste in a manner that is well controlled and safe. 8 figures.

288 (ONWI-9-81-3, pp 35-81) Waste package. 1981. NTIS, PC A12/MF A01.

In Technical progress report, 1 April-30 June 1981

Summaries of quarterly highlights under the task entitled Waste Package are presented in this section. These reports consist of studies on performance measures, waste forms, barrier materials, design and stesting of waste packages, and performance evaluation through modeling demonstration testing, and natural experiments. A study on spent fuel disassembly alternatives is under way to assess the impacts of five alternative spent fuel waste forms for geologic disposal and to recommend the reference form. After assessments were made on technical, operating, safety, and economic bases, the results, when used with the figure-of-merit methodology developed at the initiation of the studies, resulted in the option of disassembly and close packing as the reference disposal waste form. Preparations continued for the shipment of the glass HLW $\log$ to NTS/EMAD. All of the cask-handling and shipping hardware has been fabricated and accepted. The cask-handling procedure is completed and approved. Phase stability studies in waste form and material studies continue to focus on the structural integrity of ceramic waste forms. Several ceramic samples of cesium hollandite and potassium hollandite were produced; their densities of 80 to 90 percent of theoretical value are favorable for evaluating the transport of cesium ions in these types of structures. For gas-liquid inclusions, the development of numerical algorithms for the spherical liquid inclusion with a centrally positioned spherical gas bubble is complete for the decoupled temperature effects. (DMC)

289 (ONWI-305) Reaction and devitrification of a prototype nuclear-waste-storage glass with hot magnesium-rich brine. Komarneni, S.; Freeborn, W.P.; Scheetz, B.E.; White, W.B.; McCarthy, G.J. (Pennsylvania State Univ., University Park (USA). Materials Research Lab.). Oct 1982. Contract AC0676RL01830. 67p. NTIS, PC A04/MF A01. Order Number DE83002639.

PNL 76-68, a prototype nuclear waste storage glass, was reacted under hydrothermal conditions at 100,200 , and $300 \mathrm{C}$ with NBT-6a (Ca-Mg-K-Na-Cl) brine. Reaction products were identified, the state of the residual glass determined, and the concentrations of various elements remaining in the solutions analyzed. Solid products formed by reaction of the glass and brine talc (bydrated magnesium silicate), powellite $\left(\mathrm{CaMoO}_{4}\right)$, hematite $\left(\mathrm{Fe}_{2} \mathrm{O}_{0}\right)$ and rarely an unidentified uranium-containing phase. Glass fragments were leached to depths of 300 to $500 \mu \mathrm{m}$, depending on time and temperature. Most elements were extracted, but the silicate framework remained intact. Distinct diffusion fronts due to $\mathrm{K} / \mathrm{Na}$ exchange and $\mathrm{Mg} / \mathrm{Zn}$ exchange were identified. A complex compositional layering develops in the outer reaction rind. The concentration of silica in brine solution was lower by an order of magnitude than the concentration of silica in deionized water reacted under similar conditions. The concentration of cesium, strontium, uranium, rare earths, and other alkali and alkaline earth elements in solution increases exponentially with temperature of reaction. Behavior of the transition metals is more complex. In general the extraction of elements 
from the glass by hydrothermal brine leads to concentrations in solution that are from 10 to 100 times higher than the concentrations obtained by deionized water extraction under similar conditions of temperature and pressure.

290 (ORNL-TM-603) Waste treatment and disposal. Quarterly progress report, February-April, 1963. Blanco, R.E.; Parker, F.L. (Oak Ridge National Lab., TN (USA)). 16 Dec 1963. Contract W-7405-ENG-26. 121 p. Oak Ridge National Lab., TN.

Progress made in the development of integrated procresses for the treatment and disposal of radioactive wastes resulting from reactor operations and reactor fuel processing is reported. Information is given on high-level waste pot calcination; fixation of high-level waste in phosphate glass; treatment of low-level waste by scavenging, ion exchange, and foam separation; waste disposal in deep wells and natural salt formations; mineral exchange studies; and Clinch River and White Oak Creek Basin studies.

291 (ORNL-TM-757) Waste treatment and disposal. Progress report, May-October 1963. Parker, F.L.; Blanco, R.E. (Oak Ridge National Lab., TN (USA)). Apr 1964. Contract W-7405-ENG-26. 192p. NTIS.

Progress is reported on developments in waste treatment and disposal in terms of: high-level-waste calcination; low-level-waste treatment; engineering, economics, and safety evaluation; disposal in deep wells; disposal in natural salt formations; Clinch River study; fundamental studies of minerals; and White Oak Creek basin study.

292 (ORNL/TM-8124) Incorporation of simulated highlevel nuclear waste in gel spheres. Arnold, W.D.; Bond, W.D.; Robinson, S.M. (Oak Ridge National Lab., TN (USA)). Dec 1982. Contract W-7405-ENG-26. 38p. NTIS, PC A03/MF A01. Order Number DE83004171.

Gel sphere technology developed for reactor fuel fabrication was applied to the fixation of simulated high-level radioactive waste in crystalline ceramic form for permanent disposal. Gel spheres containing simulated alkaline defense waste sludges and ceramic matrix materials were prepared by internal gelation at waste loadings as high as $90 \%$. The gel spheres were amenable to subsequent drying, sintering, and coating procedures to produce crystalline waste forms with extremely high leach resistances. Potential application of this technique to the processing of commercial power reactor waste was demonstrated by incorporating simulated Purex solvent extraction waste in gel spheres with up to $20 \%$ waste loading. Cesium present in the simulated waste was adsorbed on zeolite and immobilized by coating with carbon.

293. (ORNL/TM-8579) Cement-based radioactive waste hosts formed under elevated temperatures and pressures (FUETAP concretes) for Savannah River Plant high-level defense waste. Dole, L.R.; Rogers, G.C.; Morgan, M.T.; Stinton, D.P.; Kessler, J.H.; Robinson, S.M.; Moore, J.G. (Oak Ridge National Lab., TN (USA)). Mar 1983. Contract W-7405-ENG26. 88p. NTIS, PC A05/MF A01. Order Number DE83009878.

Concretes that are formed under elevated temperatures and pressures (called FUETAP) are effective hosts for high-level radioactive defense wastes. Tailored concretes developed at the Oak Ridge National Laboratory (ORNL) have been prepared from common Portland cements, fly ash, sand, clays, and waste products. These concretes are produced by accelerated curing under mild autoclave conditions ( 85 to $200^{\circ} \mathrm{C}, 0.1$ to $1.5 \mathrm{MPa}$ ) for $24 \mathrm{~h}$. The solids are subsequently dewatered (to remove unbound water) at $250^{\circ} \mathrm{C}$ for $24 \mathrm{~h}$. The resulting products are strong (compressive strength, 40 to $100 \mathrm{MPa}$ ), leach resistant [plutonium leaches at the rate of $\left.10 \mathrm{pg} /\left(\mathrm{cm}^{2} . \mathrm{d}\right)\right]$, and radiolytically stable, monolithic waste forms (total gas value $>0.005$ molecule $/ 100 \mathrm{eV}$ ). This report summarizes the results of a 4year FUETAP development program for Savannah River Plant (SRP) high-level defense wastes. It addresses the major questions concerning the performance of concretes as radioactive waste forms. These include leachability, radiation stability, thermal stability, thermal conductivity, impact strength, permeability, phase complexity, and effect of waste composition.

294 (PB-82-204983) Actinide recycling in light water reactors: results of reactor physics calculations. Guardini, S.; Smith, B.G.R. (Commission of the European Communities,
Ispra (Italy). Joint Research Centre). [nd]. 83p. NTIS, PC E05/MF E05.

A new concept for the management of high level nuclear waste has been explored involving the possibility of partitioning the by-product actinides and transmuting them into fission products. Previous reports described the LWR codes and preliminary results. Final results of the LWR calculations can now be given as well as the conclusions reached by the overall light water reactor physics study, based on: (a) zero, one, and two dimension calculations performed to evaluate isotope vectors, reactivity values and spatial flux and power perturbations, caused by the recycling of by-product actinides and, (b) sensitivity studies carried out to evaluate the influence of various parameters between cycle cooling time such as power density, fuel temperature and coolant density. Two different recycling schemes are investigated, involving recycling of transplutonium isotopes only or of all actinides, respectively, viz, U, Pu, Am and $\mathrm{Cm}$.

296 (PNC-N-141-81-06) Current technics and management strategy for Pu-contaminated wastes at PNC. (Power Reactor and Nuclear Fuel Development Corp., Tokyo (Japan)). Feb 1981. 25p. NTIS (US Sales Only), PC A02/MF A01. Order Number DE83700540.

Power Reactor and Nuclear Fuel Development Corporation (PNC) was designated as a leading organization for the Pucontaminated waste technology program in Japan. For this purpose, number of efforts in the research and development are proceeding. That is, Pu-contaminated waste technology including volume reduction system and the immobilization of wastes is being developed. The design of a Pu-contaminated waste treatment facility (PWTF) is being made for the demonstration of the technology developed. Studies are in progress to find the criteria for waste products in disposal. The current procedures and strategy for the management of Pu-contaminated wastes at PNC are described as follows: current and future management; technology development including controlled air incineration, acid digestion, immobilization melting, dismantling, and liquid waste treatment; the Pu-contaminated waste treatment facility.

290

(PNL-3000-8) Nuclear waste management. Quarterly progress report, October through December 1980. Chikalla, T.D.; Powell, J.A. (comps.). (Pacific Northwest Lab., Richland, WA (USA)). Mar 1981. Contract AC06-76RL01830. 132p. NTIS, PC A07/MF A01. Order Number DE83009153. Portions are illegible in microfiche products. Original copy available until stock is exhausted.

Progress reports and summaries are presented under the following headings: high-level waste process development; alternative waste forms; nuclear waste materials characterization center; TRU waste immobilization; TRU waste decontamination; krypton solidification; thermal outgassing; iodine-129 fixation; monitoring and physical characterization of unsaturated zone transport; well-logging instrumentation development; mobility of organic complexes of radionuclides in soils; waste management system studies; waste management safety studies; assessment of effectiveness of geologic isolation systems; waste/rock interactions technology; high level waste form preparation; development of backfill material; development of structural engineered barriers; ONWI disposal charge analysis; spent fuel and fuel component integrity program; analysis of spent fuel policy implementation; analysis of postulated criticality events in a storage array of spent LWR fuel; asphalt emulsion sealing of uranium tailings; liner evaluation for uranium mill tailings; multilayer barriers for sealing of uranium tailings; application of long-term chemical biobarriers for uranium tailings; revegetation of inactive uranium tailing sites; verification instrument development.

297 (PNL-4098) Development and testing of matrices for the encapsulation of glass and ceramic nuclear waste forms. Wald, J.W.; Brite, D.W.; Gurwell, W.E.; Buckwalter, C.Q.; Bunnell, L.R.; Gray, W.J.; Blair, H.T.; Rusin, J.M. (Pacific Northwest Lab., Richland, WA (USA)). Feb 1982. Contract AC06-76RL01830. 116p. NTIS, PC A06/MF A01. Order Number DE82008774.

This report details the results of research on the matrix encapsulation of high level wastes at PML over the past few years. The demonstrations and tests described were designed to illustrate how the waste materials are effected when encapsulated in an inert matrix. Candidate materials evaluated for potential use as matrices for encapslation of pelletized ceramics or glass marbles were categorized into four groups: 
metals, glasses, ceramics, and graphite. Two processing techniques, casting and hot pressing, were investigated as the most promising methods of formation or densification of the matrices. The major results reported deal with the development aspects. However, chemical durability tests (leach tests) of the matrix materials themselves and matrixwaste form composites are also reported. Matrix waste forms can provide a low porosity, waste-free barrier resulting in increased leach pro tection, higher impact strength and improved thermal conductivity compared to unencapsulated glass or ceramic waste materials. Glass marbles encapsulated in a lead matrix offer the most significant improvement in waste form stability of all combinations evaluated. This form represents a readily demonstrable process that provides high thermal conductivity, mechanical shock resistance, radiation shielding and increased chemical durability through both a chemical passivation mechanism and as a physical barrier. Other durable matrix waste forms evaluated, applicable primarily to ceramic pellets, involved hot-pressed titanium or $\mathrm{TiO}_{2}$ materials. In the processing of these forms, near $100 \%$ dense matrices were obtained. The matrix materials had excellent compatibility with the waste materials and superior potential chemical durability. Cracking of the hot-pressed ceramic matrix forms, in general, prevented the realization of their optimum properties.

298

(PNL-4145) Radiation damage studies related to nuclear waste forms. Gray, W.J.; Wald, J.W.; Turcotte, R.P. (Battelle Pacific Northwest Labs., Richland, WA (USA)). Dec 1981. Contract AC06-76RL01830. 59p. NTIS, PC A04/MF A01. Order Number DE82010028.

Much of the previously reported work on alpha radiation effects on crystalline phases of importance to nuclear waste forms has been derived from radiation effects studies of composite waste forms. In the present work, two single-phase crystalline materials, $\mathrm{Gd}_{2} \mathrm{Ti}_{2} \mathrm{O}_{7}$ (pyrochlore) and $\mathrm{CaZrTi}_{2} \mathrm{O}_{7}$ (zirconolite), of relative importance to current waste forms were studied independently by doping with ${ }^{244} \mathrm{Cm}$ at the $3 \mathrm{wt} \%$ level. Changes in the crystalline structure measured by $\mathrm{x}$-ray diffraction as a function of dose show that damage ingrowth follows an expected exponential relationship of the form $\Delta V / V_{0}>A[1-\exp (-B D)]$. In both cases, the materials became $x$-ray amorphous before the estimated saturation value was reached. The predicted magnitudes of the unit cell volume changes at saturation are $5.4 \%$ and $3.5 \%$, respectively, for $\mathrm{Gd}_{2} \mathrm{Ti}_{2} \mathrm{O}_{7}$ and $\mathrm{CaZrTi}_{2} \mathrm{O}_{7}$. The later material exhibited anisotropic behavior in which the expansion of the monoclinic cell in the co direction was over five times that of the $a_{0}$ direction. The effects of transmutations on the properties of high-level waste solids have not been studied until now because of the long half-lives of the important fission products. This problem was circumvented in the present study by preparing materials containing natural cesium and then irradiating them with neutrons to produce ${ }^{104} \mathrm{Cs}$, which has only a $2 y$ half-life. The properties monitored at about one year intervals following irradiation have been density, leach rate and microstructure. A small amount of $x$-ray diffraction work has also been done. Small changes in density and leach rate have been observed for some of the materials, but they were not large enough to be of any consequence for the final disposal of high level wastes.

299 (PNL-4146) Consolidated waste forms: glass marbles and ceramic pellets. Treat, R.L.; Rusin, J.M. (Pacific Northwest Lab., Richland, WA (USA)). May 1982. Contract AC0676RL01830. 77p. NiIS, PC A05/MF A01. Order Number DE82015521.

Glass marbles and ceramic pellets have been developed at Pacific Northwest Laboratory as part of the multibarrier concept for immobilizing high-level radioactive waste. These consolidated waste forms served as substrates for the application of various Inert coatings and as ideal-sized particles for encapsulation in protective matrices. Marble and pellet formulations were based on existing defense wastes at Savannah River Plant and proposed commercial wastes. To produce marbles, glass is poured from a melter in a continuous stream into a marble-making device. Marbles were produced at PNL on a vibratory marble machine at rates as high as $60 \mathrm{~kg} / \mathrm{h}$. Other marble-making concepts were also investigated. The marble process, including a leadencapsulation step, was judged as one of the more feasible processes for immobilizing high-level wastes. To produce ceramic pellets, a series of processing steps are required, which include: spray calcining - to dry liquid wastes to a powder; disc pelletizing - to convert waste powders to spherical pellets; sintering - to densify pellets and cause desired crystal formation. These processing steps are quite complex, and thereby render the ceramic pellet process as one of the least feasible processes for immobilizing high-level wastes.

300 (PNL-4249) Summary report for the interlaboratory round robin on the MCC-1 static leach test method. Johnston, J.W.; Daniel, J.L. (Pacific Northwest Lab., Richland, WA (USA)). Mar 1982. Contract AC06-76RL01830. 171p. NTIS, PC A08/MF A01. Order Number DE82015491.

Portions of document are illegible.

The MCC-1 Static Leach Test Method Round Robbin (RR) was conducted by the Materials Characterization Center (MCC) over a period of 15 months, 1980-82. A total of 25 laboratories provided data in connection with the RR. All together these laboratories tested $\mathbf{7 6 9}$ waste form specimens using the 1980 draft of MCC-1. The specimens tested were approximately one gram wafers cut by the laboratory from samples provided by MCC: NBS borosilicate glass; core dillings of basalt from the Hanford Reservation; and MCC Type 76-68 simulated waste glass. These specimens were tested for $3,7,14,28$ days in ovens at $90^{\circ} \mathrm{C}$ using three different leachants. The resulting leachates were analyzed for elemental concentrations, and elemental mass losses were calculated. The specimens were weighed before and after leaching to determine specimen mass loss. This summary report on the round robin has two major parts: Part I provides plots of the data (which are listed in an Appendix) and summary statistics so that the participating laboratories can compare performance with other laboratories. From these plots and summaries, each laboratory also can identify its problem areas. Part II discusses the statistical analysis of the data, and characterizes the precision of the MCC-1 Static Leach Test Method at the time the RR was conducted. The precision characterization indicates that the MCC and waste form community need to institute tighter control of MCC-1 and similar testing practices to reduce the impact of betweenlaboratory differences on statistical comparisons.

301 (PNL-4250-1) Nuclear-waste-management. Semiannual progress report, October 1981-March 1982. Chikalla, T.D.; Powell, J.A. (comps.). (Pacific Northwest Lab., Richland, WA (USA)). Jun 1982. Contract AC06-76RL01830. 153p. NTIS, PC A08/MF A01. Order Number DE82018547.

Portions of document are illegible.

Progress reports are grouped under the following headings: waste treatment; nuclear materials characterization center; airborne waste management; low-level waste management; waste isolation; remedial actions; Hanford support; supporting studies. Some of the reports under these headings are: high-level waste process development; alternate waste forms; high-level waste container development; in-situ vitrification of TRU wastes; nuclear waste materials handbook; waste form test method development; leaching mechanisms program; krypton implantation; iodine-129 fixation; well-logging instrumentation development mobility of organic complexes of radionuclides in soils; ground-water transport modeling documentation and evaluation guidelines; waste package program; geochemical modeling and nuclide/rock groundwater interaction studies; revegetation/rock cover for stabilization of inactive uranium tailings sites; radon barrier systems for uranium mill tailings; nuclear resource recovery assessment; waste management system studies; nuclear materials transportation technology studies.

302 (PNL-4250-2) Nuclear-waste management semiannual progress report, April 1982-September 1982. Chikalla, T.D.: Powell, J.A. (Pacific Northwest Lab., Richland, WA (USA)). Dec 1982. Contract AC06-76RL01830. 134p. NTIS, PC A07/MF A01. Order Number DE83005125.

This document is one of a series of technical progress reports designed to report on radioactive waste management programs at Pacific Northwest Laboratory. Accomplishments in the following programs are reported: waste treatment; nuclear waste Materials Characterization Center (MCC); airborne waste management; low-level waste management; waste isolation; remedial actions; and supporting studies.

303 (PNL-4250-3) Nuclear waste management. Semiannual progress report, October 1982-March 1983. Chikalla, T.D. Powell, J.A. (comps.). (Pacific Northwest Lab., Richland, WA (USA)). Jun 1983. Contract AC06-76RL01830. 160p. NTIS PC A08/MF A01. Order Number DE83013900.

Portions are illegible in microfiche products.

This document is one of a series of technical progress reports designed to report radioactive waste management programs at the 
Pacific Northwest Laboratory. Accomplishments in the following programs are reported: waste stabilization; Materials Characterization Center; waste isolation; low-level waste management; remedial action; and supporting studies.

304 (PNL-4299) Deposition of volatile fission products during commercial high-level waste vitrification. Hanson, M.S.; Carter, J.G. (Pacific Northwest Lab., Richland, WA (USA)). Sep 1982. Contract AC06-76RL01830. 82p. NTIS, PC A05/MF A01. Order Number DE83000661.

High-level waste from spent commercial PWR fuel was vitrified during the Nuclear Waste Vitrification Project (NWVP), conducted at Pacific Northwest Laboratory in 1978 and 1979. The solidification equipment employed sintered metal filters and a conventional wet offgas cleanup system to decontaminate the gases generated during waste processing. This report is a summary of a study completed under contract with U.S. Department of Energy (DOE) to determine the fate of volatile fission products in the filters and off-gas system. The study objectives were to determine (1) the extent of volatile fission product deposition within the sinterer metal filters. (2) the distribution of fission products in the off-gas system, and (3) the factors that control the distribution. Such information is needed for the design and operation of future waste immobilization systems. The scope of the study included identification, preparation. and microprobe analyses of suitable filter samples and radiochemical analyses of off-gas system samples. The report describes the methods of sample selection and gathering, the analytical methods and results, and the interpretations of the results. Relationships to vitrification system operations are defined and operating conditions to minimize problems recommended. 37 figures, 14 tables.

305 (PNL-4382, pp 52-73) Dissolution mechanisms in high-level nuclear waste consolidation forms. Harker, A.B.; Clarke, D.R. (Rockwell International, Thousand Oaks, CA). Aug 1982. NTIS, PC A12/MF A01.

In Materials Characterization Center workshop on leaching mechanisms of nuclear waste forms, May 19-21, 1982, Gaithersburg, Maryland. Summary report.

This report summarizes the Science Center results from the first six months of study on the MCC/DOE sponsored Nuclear Waste Form Dissolution Mechanisms program. During this initial period the bulk of the Science Center effort has focused upon polycrystalline ceramic nuclear waste forms as no samples of defense waste borosilicate glass were available. The polycrystalline ceramics studied were those develnped at Lawrence Livermore National Laboratory (LLNL) and the Rockwell Science Center specifically lor the immobilization of Savannah River Plant nuclear defense waste. This report contains results of studies upon solution-solids interface phenomena and the role of cracking in determining the overall performance of a waste form undergoing ground water leaching. 9 figures, 7 tables.

306 (PNL-4423) Design and performance of feed-delivery systems for simulated radioactive waste slurries. Perez, J.M. Jr. (Pacific Northwest Lab., Richland, WA (USA)). Feb 1983. Contract AC06-76RL01830. 72p. NTIS, PC A04/MF A01. Order Number DE83008721.

Processes for vitrifying simulated high-level radioactive waste have been developed at the Pacific Northwest Laboratory (PNL) over the last several years. Paralleling this efforh, several fecd systems used to deliver the simulated waste slurry to the melter have been tested. Because there had been little industrial experience in delivering abrasive shurries at feed rates of less than $10 \mathrm{~L} / \mathrm{min}$, early experience helped direct the design of more-dependable systems. Also, as feed delivery requirements changed, the feed system was modified to meet these new requirements. The various feed systems discussed in this document are part of this evolutionary process, so they have not been ranked against each other. The four slurry feed systems discussed are: (1) verticalcantilevered centrifugal pump system; (2) airlift feed systems; (3) pressurized-loop systems; and (4) positive-displacement pump system. 20 figures, 11 tables.

307 (PNL-4442) In situ vitrification: application analysis for stabilization of transuranic waste. Oma, K.H.; Farnsworth, R.K.; Rusin, J.M. (Pacific Northwest Lab., Richland, WA (USA)). Sep 1982. Contract AC06-76RL01830. 120p. NTIS, PC A06/MF A01. Order Number DE83002889.
The in situ vitrification process builds upon the electric melter technology previously developed for high-level waste immobilization. In situ vitrification converts buried wastes and contaminated soil to an extremely durable glass and crystalline waste form by melting the materials, in place, using joule heating. Once the waste materials have been solidified, the high integrity waste form should not cause future ground subsidence. Environmental transport of the waste due to water or wind erosion, and plant or animal intrusion, is minimized. Environmental studies are currently being conducted to determine whether additional stabilization is required for certain in-ground transuranic waste sites. An applications analysis has been performed to identify several in situ vitrification process limitations which may exist at transuranic waste sites. Based on the process limit analysis, in situ vitrification is well suited for solidification of most in-ground transuranic wastes. The process is best suited for liquid disposal sites. A site-specific performance analysis, based on safety, health, environmental, and economic assessments, will be required to determine for which sites in situ vitrification is an acceptable disposal technique. Process economics of in situ vitrification compare favorably with other in-situ snlidification processes and are an order of magnitude less than the costs for exhumation and disposal in a reposliury. Leachability of thic vitrified produot oomparoc olosely with that of Pyrex glass and is significantly bettcr than granite, marble, or bottle glass. Total release to the environment from a vitrified waste site is estimated to be less than $10^{-5}$ parts per year. 32 figures, 30 tables.

308 (PNL-4530-Vol.1) Systems study of the feasibility of high-level nuclear-waste fractionation for thermal-stress control in a geologic repository: main report. McKee, R.W.; Elder, H.K.; McCallum, R.F.; Silviera, D.J.; Swanson, J.L.; Wiles, L.E. (Pacific Northwest Lab., Richland, WA (USA)). Jun 1983. Contract AC06-76RL01830. 170p. NTIS, PC A08/MF A01. Order Number DE83016402.

This study assesses the benefits and costs of fractionating the cesium and strontium $(\mathrm{Cs} / \mathrm{Sr}$ ) components in commercial high-level wastc (HLW) to a separate waste stream for the purpose of reducing geologic-repository thermal stresses in the region of the HLW. System costs are developed for a broad range of conditions comparing the $\mathrm{Cs} / \mathrm{Sr}$ fractionation concept with disposal of 10-ycar-old vitrified HLW and virffled HLW aged to achieve (through decay) the came heat output as the fractionated high-level waste (FHLW). All comparisons are based on a 50,000 metric ton equivalent (MTE) system. The FHLW and the $\mathrm{Cs} / \mathrm{Sr}$ waste are both disposed of as vitrified waste but emplaced in separate areas of a basalt repository. The FHLW is emplaced in highintegrity packages at relatively high waste loading but low heat loading, whlle the $\mathrm{Cs}_{3} / \mathrm{Sr}$ wuste is enpluced in minimum-integrity pankagar at relatively high heat loading in a separate region of the repository. System cost comparisons are based on minimum cost combinations of canister diameter, waste concentration, and canister spacing in a basalt repository. The effects on both long- and near-term safety considerations are also addressed. The major conclusion is that the $\mathrm{Cs} / \mathrm{Sr}$ fractionation concept offers the prospect of a substantial total system cost advantage for HLW disposal if reduced HLW package temperatures in a basalt repository are desired. However, there is no cost advantage if currently designated maximum design temperatures are acceptable. Aging the HLW for 50 to 100 years can accomplish similar results at equivalent or lower costs. 37 figures, 58 tables.

309 (PNL-4530-Vol.2) Bystons study of the feasibility of high-level nuclear waste fractionation for thermal stress control in a geologic repository: appendices. McKee, R.W.; Elder, H.K.; McCallum, R.F.; Silviera, D.J.; Swanson, J.L.; Wiles, L.E. (Pacific Northwest Lab., Richland, WA (USA)). Jun 1983. Contract AC06-76RL01830. 184p. NTIS, PC A09/MF A01. Order Number DE83016468.

This study assesses the benefits and costs of fractionating the cesium and strontium (Cs/Sr) components in commercial high-level waste (HLW) to a separate waste stream for the purpose of reducing geologic-repository thermal stresses in the region of the HLW. The major conclusion is that the $\mathrm{Cs} / \mathrm{Sr}$ fractionation concept offers the prospect of a substantial total system cost advantage for HLW disposal if reduced HLW package temperatures in a basalt repository aro desired. However there is no cost advantage if currently designated maximum design temperatures are acceptable. Aging the HLW for 50 to 100 years can accomplish similar results at equivalent or lower costs. Volume II contains appendices for: (1) thermal analysis supplement; (2) fractiona- 
tion process experimental results supplement; (3) cost analysis supplement; and (4) radiological risk analysis supplement.

310 (PNL-4819) Off-gas characteristics of defense wastc vitrification using liquid-fed Joule-heated ceramic melters. Goles, R.W.; Sevigny, G.J. (Pacific Northwest Lab., Richland, WA (USA)). Sep 1983. Contract AC06-76RL01830. 237p. NTIS, PC Al1/MF A01. Order Number DE84001019.

Portions are illegible in microfiche products. Original copy available until stock is exhausted.

Off-gas and effluent characterization studies have been established as part of a PNL Liquid-Fed Ceramic Melter development program supporting the Savannah River Laboratory Defense Waste Processing Facility (SRL-DWPF). The objectives of these studies were to characterize the gaseous and airborne emission properties of liquid-fed joule-heated melters as a function of melter operational parameters and feed composition. All areas of off-gas interest and concern including effluent characterization, emission control, flow rate behavior and corrosion effects have been studied using alkaline and formic-acid based feed compositions. In addition, the behavioral patterns of gaseous emissions, the characteristics of melter-generated aerosols and the nature and magnitude of melter effluent losses have becn established under a variety of feeding conditions with and without the use of auxiliary plenum heaters. The results of these studies have shown that particulate emissions are responsible for most radiologically important melter effluent losses. Melter-generated gases have been found to be potentially flammable as well as corrosive. Hydrogen and carbon monoxide present the greatest flammability hazard of the combustibles produced. Melter emissions of acidic volatile compounds of sulfur and the halogens have been responsible for extensive corrosion observed in melter plenums and in associated off-gas lines and processing equipment. The use of auxiliary plenum heating has had little effect upon melter off-gas characteristics other than reducing the concentrations of combustibles.

311

(PNL-SA-8529) Waste-management R and D for the nuclear fuel cycle. McElroy, J.L. (Pacific Northwest Lab., Richland, WA (USA)). Apr 1980. Contract AC06-76RL01830. 5p. (CONF-800943-21). NTIS, PC A02/MF A01. Order Number DE83002679.

From National topical meeting of fuel cycles for the 80's; Gatlinburg, TN, USA (29 Sep 1980).

The $R$ and $D$ being done in the US to manage spent fuel, highlevel waste, low-level waste, remedial-action wastes, and transuranic waste is covered briefly. It is suggested that the programs would benefit greatly from well-defined and substantial criteria that would provide goals for the technology developers.

312 (PNL-SA-9045) Initial operations in the remote laboratory-scale waste-treatment facility. Knox, C.A. (Pacific Northwest Lab., Richland, WA (USA)). Nov 1980. Contract AC06-76RL01830. 12p. (CONF-801107-76). NTIS, PC A02/MF A01. Order Number DE83002702.

From ANS international conference; Washington, DC, US $\wedge$ (17 Nov 1980)

The Remote Laboratory-Scale Waste-Treatment Facility (RLSWTF) is a system in which small volumes of actual radioactive liquid wastes can be processed into solidified waste forms. The objectives of these radioactive operations are to: evaluate process effluents as a function of equipment operating conditions and radioactive waste compositions; test and improve effluent decontamination systems; and produce representative waste solidification products (glass and alternative forms) for evaluation. The data from the radioactive runs in the RLSWTF are needed to verify the process development work being done with nonradioactive simulated wastes and to provide guidance in developing full-scale waste-treatment equipment. These radioactive operations will identify processing problems that are encountered with radioactive materials and equipment problems when equipment is exposed to a radioactive atmosphere over long-term operations. The runs in the RLSWTF will use several different radioactive, simulated-waste compositions, which will be made by blending nonradioactive chemicals with high-level wastc that is already in storage at Pacific Northwest Laboratory (PNL). The nonradinactive nperations that have already been completed at PNL with laboratory-scale, pilot-scale, and full-scale equipment indicate that the small size of the RLSWTF will not be a major factor in interpreting and using the data from these radioactive runs. Effluents investigations will concentrate on the following for each waste composition: particulate distribution and control; volatile fissionproduct distribution and control; and nitrogen oxides control.

313 (PNL-SA-9545) Characterization and testing of basalt glass and glass ceramic waste forms. Lokken, R.O.; Chick, L.A. (Pacific Northwest Lab., Richland, WA (USA)). Oct 1981. Contract AC06-76RL01830. 24p. (CONF-8110885). NTIS, PC A02/MF A01. Order Number DE83002666.

From American Ceramic Society conference; Newport Beach, CA, USA (25 Oct 1981).

Basalt was used to solidify three simulated high-level waste calcines and a transuranic blend waste. The materials were produced by melting basalt powder with simulated waste at $1300^{\circ} \mathrm{C}$ for $2 \mathrm{hr}$ in platinum crucibles and pouring into bars which were annealed for at least $2 \mathrm{hr}$ at $550^{\circ} \mathrm{C}$. Crystallization of the glasses was affected by nucleating at temperatures from $670^{\circ} \mathrm{C}$ to $850^{\circ} \mathrm{C}$, followed by further treatment at temperatures from $900^{\circ} \mathrm{C}$ to $1100^{\circ} \mathrm{C}$. Heat treatment and leaching studies were performed on these waste forms. Based on the results from this study, the following conclusions can be drawn. Natural basalt can be used to solidify simulated high-level and transuranic waste in either a basalt glass or a basalt glass ceramic. Recrystallization of basalt glass containing simulated high-level and transuranic waste improves the short-term leachability. Different waste compositions and heat treatment have a minimal effect on the chemical durability of basalt glass ceramics. Chemical durability of basalt glass and glass ceramic waste forms is comparable to or better than the durability of a reference highlevel borosilicate waste glass under the same conditions.

314 (PNL-SA-9811) Operating characteristics of a direct liquid-fed vitrification process. Bjorklund, W.J.; Brouns, R.A. (Pacific Northwest Lab., Richland, WA (USA)). Jan 1982. Contract AC06-76RL01830. 12p. (CONF-820424-9). NTIS, PC 102/MF A01. Order Number DE82012854.

From ANS topical meeting on treatment and handling of radio active wastes; Richland, WA, USA (19 Apr 1982).

Portions of document are illegible.

For several years, The Pacific Northwest Laboratory (PNL) has studied the process of immobilizing simulated high-level radioactive waste by directly feeding it to an electric melter as a slurry containing glass-forming chemicals. Over $65,000 \mathrm{~L}$ of simulated waste has been processed in more than 1000 hours of operation in various-sized melters. A feed system using an air-operated positive-displacement diaphragm pump has been shown to be reliable. Melting rates ranging from $\mathbf{4 0}$ to $60 \mathrm{~kg} / \mathrm{h}-\mathrm{m}^{2}$ (kilograms of total oxides per hour per square meter of melter surface area) have been demonstrated. The off gas exiting the melter has been characterized as to volatile, semivolatile, and particulate behavior. Particle entrainment has been shown to be the most significant source of emission, averaging about $0.20 \mathrm{wt} \%$ of the feed oxides. Large-scale tests have shown the process to be stable, safe and capable of capacities meeting the needs of both commercial and defense waste immobilization programs now under study in the United States.

316 (PNLSA-10199) High-level waste solidification system for the Western New York Nuclear Service Center. Carrell, J.R.; Holton, L.K.; Siemens, D.H. (Pacific Northwest Lab., Richland, WA (USA)). Jan 1982. Contract AC0676RL01830. 14p. (CONF-820424-38). NTIS, PC A02/MF A01. Order Number DE83002723.

From ANS topical meeting on treatment and handling of radio active wastes; Richland, WA, USA (19 Apr 1982).

A preconceptual design for a waste conditioning and solidification system for the immobilization of the high-level liquid wastes (HLLW) stored at the Western New York Nuclear Service Center (WNYNSC), West Valley, New York was completed in 1981. The preconceptual design was conducted as part of the Department of Energy's (DOE) West Valley Demonstration Project, which requires a waste management demonstration at the WNYNSC. This paper summarizes the bases, assumptions, results and conclusions of the preconceptual design study.

310 (PNL-SA-10249) Full-scale impact tests of simulated high-level waste canisters. Slate, S.C. (Pacific Northwest Lab., Richland, WA (USA)). Feb 1982. Contract AC0676RL01830. 4p. (CONF-820609-58). NTIS, PC A02/MF A01. Order Number DE82018773. 
From American Nuclear Society annual meeting; Los Angeles, CA, USA (6 Jun 1982).

Full-scalc impact tests of simulated high-level waste canisters at PNL were carried out in 1977 and 1981 . In the first series of tests, cannisters were dropped from heights ranging from $6 \mathrm{~m}$ to $32 \mathrm{~m}$ from a crane onto a specially constructed test pad of steel plate set into a reinforced concrete mass. The canister impacts were recorded with both video and a high speed camera. The purpose of the tests was to determine the postimpact integrity of various canister designs. In the second series of tests, 6 canisters were dropped from a $9 \mathrm{~m}$ height to determine the performance of the PNL Twist-Lock fill closure design and SRL fill/closure design. Five of the canisters were glass filled while the sixth contained glass marbles in a lead matrix. Impacted-glass data has led to empirical correlations useful in predicting glass fragmentation for evaluating the consequences of possible accidents.

317 (PNL-SA-10261) Waste vitrification: a historical perspective. McElroy, J.L.; Bjorklund, W.J.; Bonner, W.F. (Pacific Northwest Lab., Richland, WA (USA)). Feb 1982. Contract AC06-76RL01830. 15p. (CONF-820424-21). NTIS MF A01. Order Pumber DE82014817.

From ANS topical meeting on treatment and handling of radio active wastes; Richland, WA, USA (19 Apr 1982).

Portions of document are illegible.

The possibility of converting high-level wastes (HLW) to glass was first pursued in Canada and England at a tinte wlien utlici countries. were evaluating many other alternatives. By 1966, the British had completed radioactive demonstrations of the FINGAL pol process, converting HLW to borosilicate glass. By this time other countries, including France and the United States, had begun using the glass waste form. Beginning in 1966, several processes, including phosphate and borosilicate glass, were demonstrated by the US in the Waste Solidification Engineering Prototypes (WSEP) program at the Pacific Northwest Laboratory (PNL). Most of the current vitrification processes are adaptations of the FINGAL pot process or the continuous metallic melter used in the WSEP program. One notable exception is the joule-heated ceramic melter, which was adapted from commercial glass technology for HLW by PNL in the mid-1970's. Both batch and continuous processes have been developed to an advanced stage of readiness. These processes are described and compared in this paper.

318

(PNL-SA-10269) Feasibility of incinerating and vitrifying organic resins in a single step. Buelt, J.L. (Pacific Northwest Lab., Richland, WA (USA)). Mar 1981. Contract AC0676RL01830. 14p. (CONF-820424-22). NTIS MF A01. Order Number DE82014856.

From ANS topical meeting on treatment and handling of radioactive wastes; Richland, WA, USA (19 Apr 1982).

Portions of document are illegible.

Pacific Northwest Laboratory has completed a feasibility testing program for a process designed to incinerate and vitrify the inorganic and radionuclide components of EPICOR II resins in a single step. The process tests utilized pilot-scale, high-level waste vitrification equipment with a special feeding technique that forces contact of the combustible resins with molten glass. This technique demonstrated high retention of nonradioactive forms of cesium and strontium in the glass product. Other performance evaluations of the process were also favorable.

310 (PNLSA-10290) Off-gas phameteristios of liquidfed joule-heated ceramic melters. Goles, R.W.; Sevigny, G.J. (Pacific Northwest Lab., Richland, WA (USA)). Jun 1982. Contract AC06-76RL01830. 30p. (CONF-820833-15). NTIS, PC A03/MF A01. Order Number DE83002072.

From 17. DOE nuclear air cleaning conference; Denver, CO, USA (1 Aug 1982).

The off-gas characteristics of liquid-fed joule-heated ceramic melters have been investigated as a function of melter operational condition and simulated waste feed composition. The results of these studies have established the identity and behavior patterns of gaseous emissions, the characteristics of melter-generated aerosols, the nature and magnitude of melter effluent losses and the factors affecting melter operational performance. 8 figures, 16 tables

320 (PNL-SA-10303) Solubility limits of metal ions and mechanism of leaching nuclear-waste glasses. Grambow, B. (Pacific Northwest Lab., Richland, WA (USA)). Mar 1982. Con- tract AC06-76RL01830. 19p. NTIS MF A01. Order Number DE82014851.

Portions of document are illegible.

A number of studies have qualitatively considcred solubility limits to be important in the leaching of nuclear waste forms with respect to reaction layer formation and the prediction of long-term behavior under accidental conditions in a repository. These considerations are quantified in this paper by the application of data from soil chemistry to the conditions of leach tests with PNL 76-68 glass. The conclusions of this study are: (1) leaching mechanism for PNL 76-68 glass involves congruent matrix dissolution and formation of insoluble reaction products; (2) the solubility of the reaction products is similar to $\mathrm{Fe}(\mathrm{OH})_{0}, \mathrm{Zn}(\mathrm{OH})_{2}, \mathrm{Nd}(\mathrm{OH})_{0}, \mathrm{SrCO}_{0}, \mathrm{CaCO}_{0}$ or $\beta-\mathrm{CaSiO}_{0}$; and (3) the conditions for layer formation and the composition can be predicted.

321 (PNL-SA-10316) Corrosion experience with a slurry-fed ceramic melter. Barnes, S.M.; Sevigny, G.J.; Goles, R.W. (Pacific Northwest Lab., Richland, WA (USA)). Mar 1982. Contract AC06-76RL01830. 10p. (CONF-820424-13). NTIS, PC A02/MF A01. Order Number DE82013725.

Frnm ANS topical meeting on treatment and handling of radio. active wastes; Richland, WA, USA (19 Apr 1982).

This report presents the findings of an investigation into the longterm performance of construction materials for joule-heated, ceramiclined melters. The materials investigated include: the glass contact refiacturics, the meltor olcotrodes, and the proress nff-gas containment materials. The corrosion rate and mechanism for these materials are presented. The report also includes the initial corrosion data from testing of improved effluent containment materials.

322 (PNL-SA-10365) Waste glasses: requirements and characteristics. Mendel, J.E. (Pacific Northwest Lab., Richland, WA (USA)). Apr 1982. Contract AC06-76RL01830. 16p. (CONF-820424-23). NTIS, PC A02/MF A01. Order Number DE82014855.

From ANS topical meeting on treatment and handling of radioactive wastes; Richland, WA, USA (19 Apr 1982).

The regulatory requirements relating to waste glass that have been presented recently in draft form by the US Environmental Protcction Agency and Nuclear Regulatory Commission are reviewed. These requirements assume that waste glass will be considered as a subcomponent of a waste package. Current waste package concepts are described and the important characteristics of waste glass are summarized, with emphasis on the present status of standardizing methods for measuring the characteristics of waste glass.

323 (PNL-SA-10489) Commercial waste treatment $R$ and $D$ needs in the United States. Burkholder, H.C. (Pacific Northwest Lab., Richland, WA (USA)). May 1982. Contract AC06-76RL01830. 6p. (CONF-820636-8). NTIS, PC A.02/MF A01. Order Number DE83002727.

From 5 . international symposium on the scientific basis for radioactive waste management; Berlin, F.R. Germany (7 Jun 1982).

The mission of the commercial waste treatment program is to establish treatment technology for safe and efficient management of high-level and transuranic wastes from reprocessing and fuel fabrication and special wastes from other fuel cycle activities. The four functional objectives that must be achieved to fulfill the mission are: (1) define wuste product and troatment process performance requiromente; (2) specify adequately safe waste products and verify their performance; (3) specify adequately cfficient treatment processes and equipment and verify their performance; (4) solve existing waste treatment problems using verified products and processes. Although cummercial waste treatment technology is in many respects highly advanced, there remains a number of areas where significant research and development is needed. These are: (1) technically-based performance requirements for both waste products and treatment processes; (2) pilot-scale radioactive demonstration of liquid-fed ceramic melting process and equipment for borosilicate glass; (3) non-glass TRU waste product and treatment process development; (4) waste product performance testing and predictive modeling; (5) quality verification for treatment processes.

324 (PNL-SA-10521) Recovery of noble metals from fission products. Jenson, G.A.; Platt, A.M.; Mellinger, G.B.; Bjorklund, W.J. (Pacific Northwest Lab., Richland, WA (USA)). 
Nov 1982. Contract AC06-76RL01830. 7p. (CONF-82110333). NTIS, PC A02/MF A01. Order Number DE83003458.

From American Nuclear Society winter meeting; Washington, DC, USA (14 Nov 1982).

Scoping studies were started in 1979 to develop a cost-effective, waste-management-compatible process to extract noble metals from fission products. The process, involving the reaction with glassmelting chemicals, a metal oxide ( $\mathrm{PbO}$ ), and a reducing agent (charcoal), was demonstrated for recovering noble metals from simulated high-level waste oxides. The process has now been demonstrated on a laboratory scale $(100 \mathrm{~g})$ using irradiated fuels. Recoveries in the recovered lead averaged $80 \%$ for $\mathrm{Pd}, 60 \%$ for $\mathrm{Rh}$, and $14 \% \mathrm{Ru}$. The resulting glass product was homogeneous in appearance, and the chemical durability was comparable to other waste oxides.

325 (PNL-SA-10528) Vitrification of highly-loaded SDS zeolites. Siemens, D.H.; Bryan, G.H.; Knowlton, D.E.; Knox, C.A. (Pacific Northwest Lab., Richland, WA (USA)). Nov 1982. Contract AC06-76RL01830. 16p. (CONF-821103-92). NTIS, PC A02/MF A01. Order Number DE83006198.

From American Nuclear Society winter meeting; Washington, DC, USA (14 Nov 1982).

Pacific Northwest Laboratory (PNL) is demonstrating a vitrification system designed for immobilization of highly loaded SDS zeolites. The Zeolite Vitrification Demonstration Project (ZVDP) utilizes an in-can melting process. All steps of the process have been demonstrated, from receipt of the liners through characterization of the vitrified product. The system has been tested with both nonradioactive and radioactive zeolite material. Additional high-radioactivity demonstrations are scheduled to begin in FY-83. 5 figures, 4 tables.

326 (PNL-SA-10712) Evaluation of conditioned highlevel waste forms. Mendel, J.E.; Turcotte, R.P.; Chikalla, T.D.; Hench, L.L. (Pacific Northwest Lab., Richland, WA (USA); Florida Univ., Gainesville (USA)). 1983. Contract AC0676RL01830. 21p. (CONF-830523-1\%). N1IS, PC A02/MF A01. Order Number DE83013368.

From International conference on radioactive waste management; Seattle, WA, USA (16 May 1983).

The evaluation of conditioned high-level waste forms requires an understanding of radiation and thermal effects, mechanical properties, volatility, and chemical durability. As a result of nuclear waste research and development programs in many countries, a good understanding of these factors is available for borosilicate glass containing high-level waste. The IAEA through its coordinated research program has contributed to this understanding. Methods used in the evaluation of conditioned high-level waste forms are reviewed. In the US, this evaluation has been facilitated by the definition of standard test methods by the Materials Characterization Center (MCC), which was established by the Department of Energy (DOE) in 1979. The DOE has also established a 20-member Materials Review Board to peer-review the activities of the MCC. In addition to comparing waste forms, testing must be done to evaluate the behavior of waste forms in geologic repositories. Such testing is complex; accelerated tests are required to predict expected behavior for thousands of years. The tests must be multicomponent tests to ensure that all potential interactions between waste form, canister/overpack and corrosion products, backfill, intruding ground water and the repository rock, are accounted for. An overview of the status of such multicomponent testing is presented.

327 (PNLSA-10777) Cesium and strontium fractionation from HLW for thermal-stress reduction in a geologic repository. McKee, R.W. (Pacific Northwest Lab., Richland, WA (USA)). Feb 1983. Contract AC06-76RL01830. 20p. (CONF830205-15). NTIS, PC A02/MF A01. Order Number DE83008238.

\section{Feb 1983).}

From Waste management conference; Tucson, AZ, USA (27

Results are described for a study to assess the benefits and costs of fractionating the cesium and strontium components in commercial high-level waste (HLW) to a separate waste stream for the purpose of reducing geologic repository thermal stresses. System costs are developed for a broad range of conditions comparing the $\mathrm{Cs} / \mathrm{Sr}$ fractionation concept with disposal of 10-year old vitrified HLW and vitrified HLW aged to achieve (through decay) the same heat output as the fractionated high-level waste (FHLW). All comparisons are based on a 50,000 metric ton equivalent (MTE) system. The FHLW and the Cs/Sr waste are both disposed of a vitrified waste but emplaced in separate areas of a basalt repository. The FHLW is emplaced in high-integrity packages at relatively high waste loading but low heat loading, while the $\mathrm{Cs} / \mathrm{Sr}$ waste is emplaced in minimum integrity packages at relatively high heat loading. System cost comparisons are based on minimum cost combinations of canister diameter, waste concentration, and canister spacing in a basalt repository for each waste type. The effects on both long- and near-term safety considerations are also addressed. The major conclusion is that the Cs/Sr fractionation concept offers, potentially, a substantial total system cost advantage for HLW disposal if reduced HLW package temperatures in a basalt repository are desired. However, there is no cost advantage if currently designated maximum design temperatures are acceptable. Aging the HLW for 50 to 100 years can accomplish similar results at equivalent or loser costs.

328 (PNL-SA-10829) Using process instrumentation to obviate destructive examination of canisters of $H L W$ glass. Kuhn, W.L.; Slate, S.C. (Pacific Northwest Lab., Richland, WA (USA)). Jan 1983. Contract AC06-76RL01830. 7p. (CONF-830205-20). NTIS, PC A02/MF A01. Order Number DE83007876.

From Waste management conference; Tucson, AZ, USA (27 Feb 1983).

An important concern of a manufacturer of packages of solidified high-level waste (HLW) is quality assurance of the waste form. The vitrification of HLW as a borosilicate glass is considered, and, based on a reference vitrification process, it is proposed that information from process instrumentation may be used to assure quality without the need for additional information obtained by destructive examining (core drilling) canisters of glass. This follows mainly because models of product performance and process behavior must be previously established in order to confidently select the desired glass formulation, and to have confidence that the process is well enough developed to be installed and operated in a nuclear facility.

329 (PNL-SA-10934) Remote design features for the West Valley slurry-fed ceramic melter system. Siemens, D.H.; Chapman, C.C. (Pacific Northwest Lab., Richland, WA (USA)). Feb 1983. Contract AC06-76RL01830. 10p. (CONF830451-5). NTIS, PC A02/MF A01. Order Number DE83011950.

From 85. American Ceramic Society annual meeting; Chicago, IL, USA (25 Apr 1983).

This paper discusses features that have been identified as needing additional development to make the melter system remotely operable for use at West Valley, and the planned approach for developing the identified items. Each of the following features are discussed: melter electrode power supply; in-melter viewing; thermocouple connectors; overflow section heaters; nozzle cleaning; replaceable inserts in the melter overflow section; canister installation and removal; melter overflow to turntable tank connection; and detection glass level in the storage canister during filling.

330 (PNL-SA-11136) Status, direction, and critical issues of waste treatment technology. Knowlton, D.E.; Bonner, W.F. (Pacific Northwest Lab., Richland, WA (USA)). Jun 1983. Contract AC06-76RL01830. 15p. (CONF-830812-58). NTIS, PC A02/MF A01. Order Number DE84001342.

From 18. intersociety energy conversion engineering conference; Orlando, FL, USA (21 Aug 1983).

Nuclear power production and related activities generate radioactive wastes that must be safely managed to protect workers and the general public. The liquid-fed ceramic melting (LFCM) vitrification process is the reference process for vitrifying high-level nuclear waste in the US as well as in Japan and India. The French are currently using a rotary kiln calciner/metallic melter system at their reprocessing facility. Compaction or controlled-air incineration are the currently preferred options for low-level waste solids, followed by immobilization in an appropriate matrix. The Nuclear Waste Policy Act of 1982 is a significant step in proceeding with waste treatment and disposal. Programs can now build on past work to assure that public safety and regulations atre met in a cost-effective manner. 7 references, 2 figures, 3 tables.

331 (PNL-SA-11199) Vitrification of simulated West Valley waste using a liquid-fed ceramic melter. Bjorklund, W.J.; 
Mellinger, G.B.; Pope, J.M. (Pacific Northwest Lab., Richland, WA (USA)). Feb 1983. Contract AC06-76RL01830. 16p. (CONF-830451-10). NTIS, PC A02/MF A01. Order Number DE83012514.

From 85. American Ceramic Society annual meeting; Chicago, IL, USA (25 Apr 1983).

Simulated high-level waste representing the waste stored at West Valley, New York, was vitrified to a borosilicate glass and poured into canisters using a liquid-fed ceramic melter at Battelle Memorial Institute's Pacific Northwest Laboratory. This was the first large-scale demonstration of the vitrification of simulated West Valley high-level waste. Results of the experiment and glass analyses are presented.

332

(PRAV-3-28) Research programme on the conditioning of nuclear power waste. Progress report June 1981. Hultgren, A. (ed.). (Programraadet foer Radioaktivt Avfall, Stockholm (Sweden)). 1981. 26p. NTIS (US Sales Only), PC A03/MF A01. Order Number DE82701328.

Main parts of this programme have included the use of zeolites and titanates to improve reactor waste treatment, the fractionation of high level reprocessing waste by extraction, and a study of liquid partition chromotngraphic technique for the removal of impurities from reactor cooling circuits. Preparation of large crystal zeolites has been continued and refined. For titanate production new routes are tried to produce material of a form suitable for use in a sorption process. The possibility of lithium-7 recovery from spent PWR resins in the elution process is under study. Final products from different routes of heat treatment of loaded zeolites and titanates are characterized and compared. In paral- lell to this work a full-scale system is under study including transport, system design, integrated process flowsheets and cost estimates. The aim is to have basis early in 1982 to decide on the merits of a plant at the planned repository for low and medium level waste (SFR), to be commissioned around 1990. In the high level waste fractionation project, a demonstration of the developed process has been performed on a fission product solution from the reprocessing of low burn-up fuel. Disregarding some equipment malfunction the design goal of better than $99.99 \%$ actinide removal from the high level waste waste solution was reached. The basic chemistry of the process seems to be quite tolerant against reasonable flow rate deviations in the extraction cycles. Also the concluding sorption step on mordenite-titanate worked quite well. The small scale experiments on liquid partition chromatographic techniques have included studies of the capacity of various carrier materials treated with $\mathrm{NH}_{4}$ DEHP or Aliquat-336 to sorb radioactive impurities from reactor water, both in the laboratory and at the Ringhals-1 BWR.

\section{3}

(RFP-3282) Commercial and Defense nitrate wastes and processing alternatives. Johnson, A.J.; Meile, L.J.; Jaskot, G.F. (Rockwell International Corp., Golden, CO (USA). Rocky Flats Plant). 25 Aug 1982. Contract AC04-76DP03533. 20p. NTIS, PC A02/MF A01. Order Number DE83000027.

Nitric acid is extensively used at commercial and defense facilities engaged in nuclear fuel reprocessing and/or actinide recovery and purification. The high volume usage of nitric acid results in the generation of large amounts of nitrate wastes. Commercial facilities in the nuclear fuel cycle report that they are able to handle most of their nitrate waste under current discharge limits but could have problems if these limits are lowered. The defense nuclear facilities generate by far the greater amount of waste nitrates. A separation system now planned for HLW at SRP, will convert large volumes of stored waste into a concentrated volume of HLW and large volumes of LLW nitrates. No processing systems are now operating that will produce a desirable final waste form. Several methods have been identified that could be used to destroy or convert the nitrate compounds to a waste more amenable to conversion into an acceptable waste form for final disposal.

334 (RHO-C-47) Ozone mass transfer and kinetics experiments. Bollyky, L.J.; Beary, M.M. (Rockwell International Corp., Richland, WA (USA). Rockwell Hanford Operations; Bollyky Associates, Stamford, CT (USA)). Dec 1981. Contract AC06-77RL01030. 75p. NTIS, PC A04/MF A01. Order Number DE82007158.

Experiments were conducted at the Hanford Site to determine the most efficient $\mathrm{pH}$ and temperature levels for the destruction of complexants in Hanford high-level defense waste. These complexants enhance migration of radionuclides in the soil and inhibit the growth of crystals in the evaporator-crystallizer. Ozone mass transfer and kinetics tests have been outlined for the determination of critical mass transfer and kinetics parameters of the ozone-complexant reaction.

336 (RHO-PB-SR-10-B-WM-JUN-83) Rockwell Hanford Operations. Defense waste and byproducts management monthly report. (Rockwell International Corp., Richland, WA (USA). Rockwell Hanford Operations). Jun 1983. Contract AC06-77RL01030. 112p. NTIS, PC A06/MF A01. Order Number DE83016173.

Portions are illegible in microfiche products.

Combined jet pump production for TX and BY Tank Farms in the month of June was approximately 132,000 gallons. The fiscal yearto-date volume pumped is approximately 324,000 gallons versus 350 , $\mathbf{0 0 0}$ gallons planned. The first shipments of $\mathrm{N}$-Reactor decontamination waste were received from UNC via railroad tank cars in the first week of the month. Two to three shipments per week are being received and a total of thirty-three loads are expected to be received through the middle of August (approximately 500,000 gallons of waste). Twentythree cesium capsules were produced in June for a fiscal year-to-date total of 100. A techinical plan for the Tiansportable Grout Facility was compiled, approved, and submitted to DOE-RL. This technical plan addresses the need and background, as well as the ubjecilive, stiategy, work breakdown structure, schedule, and milestones. Completion of this technical plan represents a significant accomplishment in the grouting of defense nuclear wastes. Both PNL and ORNL participated by contributing to this plan. The B-461 Functional Design Criterla and Preliminary Conceptual Design Report were completed and submitted to the Department of Energy, Richland Operations Office for approval.

336 monthly report. (Rockwell International Coducts management (USA). Rockwell Hanford Operations). Sep 1983. Contract AC06-77RL01030. 109p. NTIS, PC A06/MF A01. Order Number DE84002697.

Portions are illegible in microfiche products.

Scheduled saltwell jet pumping in TX and TY Tank Farms was completed in FY 1983, and 18 single-shell tanks in these farms were declared interim-stabilizied, surpassing the fiscal year milestone of 2 stabilized tanks by 16 tanks. Isolation Projects B-145 and B-181 were completed, and effort in Isolation Projects B-222 and B-231 was initiated. A total of twenty-three single-shell tanks were declared interimisolated, which exceeds the fiscal year milestone of 15 stabilized tanks by 8 tanks. The milestone for completing 125 strontium capsules was met July 30, two months ahead of schedule. Nine strontium capsules were completed in September for a record total of 150 strontium capsules in FY 1983. A total of 172 acres of outdoor radiation areas, including trenches, inactive burial grounds, small contaminated surface areas, and the Gable Mountain Pond peripheral area was surface stabilized. Seeding and mulching of the stabilized areas will be completed during the first quaner of FY 1984. Waste Managentul favilities weite certified as ready to receive PUKEX Waste. A total of 818,210 gailons was jet pumped from the $\mathrm{IX}$, "IY and $\mathrm{BY}$ Tank Farms versus a productlon goal of 800,000 . The milestone for completing encapsulation of the cesium backlog stured in B Plant was met during fiscal year 1983. In September 38 cesium capsules were produced as a result of the yield from cesium process equipment clean up. A total of 176 capsules were produced during the fiscal year. A tulal of $6.3 \mathrm{MCi}$ of strontium feed was purified in FY 1983. A total of $1.3 \mathrm{MCi}$ of cesium was purified in September for a fiscal year-to-date total of $20.7 \mathrm{MCi}$. The planned $\mathrm{FY}$ 1983 evaporator campaign was completed in February. Total evaporator throughput was approximately 9.6 million gallons with a waste volume reduction of about 3.5 million gallons.

337

(RHO-WM-SR-82-1-2Q-P) Long-Term High-Level Defense Waste technology progress report, January-March 1982. (Rockwell International Corp., Richland, WA (USA). Energy Systems Group). May 1982. Contract AC0677RL01030. 27p. NTIS, PC A03/MF A01. Order Number DE82020954.

This report summarizes the work accomplished on the LongTerm High-Level Defense Waste end function during the second quarter of Fiscal Year 1982. Two major technology tasks are supportcd within Rockwell Hanford Operations (Rockwell): rcsidual liquid solidification and in situ drying. In addition, Rockwell is providing guidance for pass through funded waste tank and cesium capsule corrosion tests 
reing performed at Battelle Pacific Northwest Laboratory (PNL) and soncrete grout bulk waste immobilization studies at Oak Ridge National Laboratory (ORNL). In the residual liquid solidification effort, the primary focus of the alternatives study this year is on the wiped film evaporator approach to drying salt well pumped liquids and returning the dried material to single-shell tanks. Both systems analysis and experimental work are proceeding to evaluate this approach. The study of in situ drying of in-tank wastes is focusing on microwave drying of wet salt cake. Experimental work has been successfully conducted on a 1/12 scale tank containing wet synthetic salt cake. Related systems analysis of a full-scale system has been initiated. A smaller support task also being funded at Rockwell under this program is to provide liaison to PNL and ORNL on waste immobilization studies. For the PNL vitrification work, Rockwell has provided Hanford defense waste feed stream formulations, flowsheets and a revised vitrification system study. For the ORNL concrete grout work, Rockwell has provided simulated Hanford waste and grout performance objectives. The waste tank and cesium capsule corrosion testing efforts at PNL have focused on test planning and testing preparation. Waste tank corrosion tests have begun, and capsule corrosion electrical mock-up tests have been conducted.

338 (RHO-WM-SR-82-1-3QP) Long-Term High-Level Defense-Waste technology. Progress report, April-June 1982. (Rockwell International Corp., Richland, WA (USA). Energy Systems Group). Jul 1982. Contract AC06-77RL01030. 19p. NTIS, PC A02/MF A01. Order Number DE83000375.

This report summarizes the work accomplished during the third quarter of Fiscal Year 1982. Two major technology tasks are supported within Rockwell Hanford Operations (Rockwell): residual liquid solidification and in situ drying. In addition, Rockwell is providing technical guidance for waste tank and cesium capsule corrosion tests being performed at Battelle Pacific Northwest Laboratory (PNL) and passthrough funded concrete grout bulk waste immobilization studies at Oak Ridge National Laboratory (ORNL). In the residual liquid solidification effort, the primary alternative studied this year is the wiped-film evaporator (WFE) approach to solidifying salt well pumped liquids and returning the molten material to single-shell tanks for microwave final stabilization to a hard dry product. Both systems analysis and experimental work are proceeding to evaluate this approach. The primary alternative for in situ stabilization of in-tank wastes is microwave drying of wet salt cake and unpumped sludges. Experimental work has been successfully conducted on a $1 / 12$ scale tank containing wet synthetic salt cake. Related systems analysis of a full-scale system has been initiated.

339 (SAND-82-1045C) Radiation-damage effects in nuclear-waste glasses. Arnold, G.W. (Sandia National Labs., Albuquerque, NM (USA)). 1982. Contract AC04-76DP00789. 17p. (CONF-820864-3). NTIS, PC A02/MF A01. Order Number DE82021726.

From 4. europhysical topical conference on lattice defects in ionic crystals; Dublin, Ireland (30 Aug 1982).

Portions of document are illegible.

Ion implantation is a convenient means of simulating $\alpha$-decay damage in nuclear waste glasses. Rutherford backscattering spectrometry and elastic recoil detection show that significant near-surface compositional changes can occur in leached $\mathrm{Pb}$-ion implanted borosilicate glasses. In addition, the rates and mechanisms for hydration, ion exchange, and network dissolution depend upon the glass composition. A critical ion energy deposition value has been noted at which major changes concur in near-surface elemental profiles of some leached implanted glasses. An interpretation of this latter effect, based on iontrack overlap and consequent plastic flow, is advanced.

340 (SAND-82-2980) Processing, microstructure, leaching, and long-term stability studies related to titanate high-level waste forms. Dosch, R.G.; Headley, T.J.; Northrup, C.J.; Hlava, P.F. (Sandia National Labs., Albuquerque, NM (USA)). May 1983. Contract AC04-76DP00789. 82p. NTIS, PC A05/MF A01. Order Number DE83015637.

Portions are illegible in microfiche products. Original copy available until stock is exhausted.

A process leading to titanate-based waste forms for commercial high-level nuclear waste is described. Radionuclides are fixed on hydrous caloium titanate by ion exchange/sorption reartinns and the material is converted to a dense, ceramic form by hot pressing. Transmission electron microscopy-electron microprobe characterization was done to determine the effects of compositional changes and process variations on microstructure. Leaching studies in the range of 22 to $150^{\circ} \mathrm{C}$ and $\mathrm{pH} 2$ to 12 were done to assess the effects of the same variables on leaching behavior. Samples of a reference borosilicate glass waste form were leached under the same conditions to provide a direct comparison between the two waste forms. Lead-ion implantation was used to simulate long-term $\alpha$-recoil damage in the crystalline titanate phases. Comparison of $\alpha$-recoil damage in natural minerals with damage induced in synthesized samples of the same mineral suggest that $\mathrm{Pb}$-ion implantation is a valid technique for simulating $\alpha$-recoil effects. All the titanate phases sustained significant lattice damage at equivalent $\alpha$-doses of 1 $\times 10^{19} / \mathrm{cm}^{0}$; however, Rutherford backscattering and transmission electron microscopy studies showed that the damage did not result in significant matrix dissolution in these leaching tests.

341 (SKBF/KBS-TR-81-15) Effect of $\mathrm{Fe}_{2} \mathrm{O}_{0} / \mathrm{ZnO}$ on two glass compositions for solidification on Swedish nuclear wastes. Nogues, J.L.; Hench, L.L. (SEE CODE- 6029620 Swedish Nuclear Fuel Supply Co., Stockholm). Nov 1981. 30p. NTIS (US Sales Only), PC A03/MF A01. Order Number DE82702957.

Low melting alkaliborosilicate glasses have been considered for use in the immobilization of high level radioactive wastes for years. A recent study comparing the surface behavior of two nuclear waste glasses concluded that "Addition of $\mathrm{Fe}_{2} \mathrm{O}_{0}$ to a soda borosilicate nuclear waste glass significantly reduces damage by water attack because of a Fe-rich film that forms on the glass surface". However, in the previous study there were significant differences in the concentration of $\mathrm{SiO}_{2}$, $\mathrm{B}_{2} \mathrm{O}_{0}, \mathrm{CaO}$ and simulated fission products in the glasses which made it impossible to ascribe the improved leach resistance solely to $\mathrm{Fe}_{2} \mathrm{O}_{0}$ content. Thus, the objective of the present investigation is to compare the leaching and surface behavior of two nuclear waste glasses which differ only by the substitution of $\mathrm{Fe}_{2} \mathrm{O}_{0}$ for some of the $\mathrm{ZnO}$ in the glass. By this comparison the authors hope to establish whether $\mathrm{Fe}_{2} \mathrm{O}_{0}$ provides a unique contribution to improvements in the leach resistance of these complex glasses. Both glass compositions studied are compatible with the low melting temperature, $<1150^{\circ} \mathrm{C}$, required for the French AVM Process. The quantity of simulated waste products is $9 \%$, characteristic of the Swedish nuclear waste program.

342 (TID-7550) Report of working meeting on fixation of radioactivity in stable, solid media at the Johns Hopkins University, June 19-21, 1957. Morgan, J.M. Jr. (USAEC Division of Reactor Development, Washington, DC). 1957. 112p. NTIS.

Papers were presented at the meeting on the following subjects: waste characteristics governing fixation in soils; chemical processing of the alumina fluid calciner product into alumina silicates; fixation of fission products in stable compounds; resume of ceramic section of Oak Ridge National Laboratory; fixation of fission products in ceramic glazes; fission product disposal in glass; leaching studies on fired clays containing radionuclides; evaluation of experimental clinkers; fixation of active wastes in solid form using natural soils as media for glass formation; separation of cesium and strontium from calcined metal oxides as a process in disposal of high level wastes; fixation of radioisotopes on Hanford type soils; tests of stability; calcination of aqueous reactor fuel wastes in a fluidized bed; experiments on fluidized bed calcination of Idaho Chemical Processing Plant wastes using radioactive feed material; ball kiln calcination of alloy fuel element wastes; incorporation of fission products into glass; ultimate disposal of long-lived fission products; high-temperature gas cleaning problems; heat evolution and dissipation; problems associated with waste calcination schemes; sintering of reactor wastes; problems associated with the formation of ceramic clinkers; and storage of reactor fuel wastes in salt formations.

343 (UCID-19404) Sintering of Synroc D. Robinson, G. (Lawrence Livermore National Lab., CA (USA)). 1 Jun 1982. Contract W-7405-ENG-48. 30p. NTIS, PC A03/MF A01. Order Number DE82017118.

Portions of document are illegible.

Sintering has been investigated as a method for the mineralization and densification of high-level nuclear defense waste powder. Studies have been conducted on Synroc D composite powder LS04. Optimal densification has been found to be highly dependent on the characteristics of the starting material. Powder subjected to milling, 
which was believed to reduce the level of agglomeration and possibly particle size, was found to densify better than powder not subjected to this milling. Densities of greater than 95\% of theoretical could be achieved for samples sintered at 1150 to $1200^{\circ} \mathrm{C}$. Mineralogy was found to be as expected for Synroc D for samples sintered in a $\mathrm{CO}_{2} / \mathrm{CO}$ atmosphere where the $\mathrm{Fe}^{+2} / \mathrm{Fe}^{+0}$ ratio was maintained at 1.0 to 5.75 . In a more oxidizing, pure $\mathrm{CO}_{2}$ atmosphere a new phase, not previously identified in Synroc D, was found.

344 (UCID-19492) Leaching study of PNL 76-68 glass beads using the LLNL continuous-flow method and the PNL modified IAEA method. Final report. Buddemeier, R.W.; Coles, D.G.; Mensing, R.W.; Rego, J.; Weed, H.C. (Lawrence Livermore National Lab., CA (USA)). 20 Aug 1982. Contract W-7405-ENG-48. 257p. NTIS, PC A12/MF A01. Order Number DE82021539.

Portions of document are illegible.

Lawrence Livermore National Laboratory (LLNL) has conducted a long-term single-pass continuous-flow (SPCF) leaching test of the glass waste form PNL 76-68. Leaching rates of $\mathrm{Np}, \mathrm{Pu}$, and various stable elements were measured at $25^{\circ}$ and $75^{\circ} \mathrm{C}$ with three different solutions and three different flow rates. The purposes of the study were: (1) to compare SPCF leaching results with the results of a modified IAEA leach test performed by Pacific Northwest Laboratories (PNL); (2) to establish elemental leach rates and their variation with temperature, flow rate and solution composition; and (3) to gain insight into the leaching mechanisms. The LLNL and PNL leach tests yielded results which appear to agree within experimental uncertainties. The magnitude of the leach rates determined for $\mathrm{Np}$ and the glass matrix elements is $10^{-5}$ grams of glass $/ \mathrm{cm}^{2}$ geometric solid surface area/day. The rates increase with temperature and with solution flow rate, and are similar in brine and distilled water but higher in a bicarbonate solution. Other cations exhibit somewhat different behavior, and $\mathrm{Pu}$ in particular yields a much lower apparent leach rate, probably because of sorption or precipitation effects after release from the glass matrix. After the initial few days, most elements are leached at a constant rate. Matrix dissolution appears to be the most probable rate controlling step for the leaching of most elements.

345

(UCID-19598) SYNROC powder preparation: preliminary fluid bed tests in a cold-flow unit. Peters, P.E.; Ackerman, F.J.; Grens, J.Z. (Lawrence Livermore National Lab., CA (USA)). 28 Oct 1982. Contract W-7405-ENG-48. 32p. NTIS, PC A03/MF A01. Order Number DE83003150.

SYNROC is a titanate-based material which is bcing developed as a medium for immobilizing high-level nuclear waste. The use of a fluid bed unit for production of SYNROC powder has a precedent in the use of a fluid-bed calciner for high-level waste processing at the Idaho Chemical Processing Plant (Idaho Falls, ID). In order to facilitate the design of a fluid bed and demonstrate its use for SYNROC production, two small units have been constructed, one for low temperature use and a second for actual SYNROC production at temperatures up to $800^{\circ} \mathrm{C}$. The low-temperature unit is constructed with glass walls to allow observation of the fluidization process, including the onset of fluidization, bed and gas bubble behavior and mixing phenomena. Disturbances caused by side streams entering the bed have been examined. Side streams may represent fuel and oxidizer admission or slurry feed with subsequent flash vaporization of the carrier liquid. This report is a summary of the initial tests made with the low-temperature, glass-walled fluid bed. The tests described include: (1) the measurement of basic fluidization parameters; (2) measurement of the effect of distribution-plate design on bed fluidization; (3) observation of jet penetration, bubble formation and coalescence, and surface behavior; and (4) studies of mixing within the bed. (DMC)

346 (UCRL-15480) Immobilization of sodium and phosphorus-bearing PW-7a waste in SYNROC. Progress report. Ringwood, A.E. (Australian National Univ., Canberra. Research School of Earth Sciences). 9 Aug 1982. Contract W-7405-ENG-48. 22p. NTIS, PC A02/MF A01. Order Number DE83000734.

The phosphorus, sodium and gadolinium-rich PW-7a waste can be successfully incorporated in SYNROC-C. However, a new accessory phase, a $\mathrm{Ca}, \mathrm{Na}, \mathrm{Ba}$ phosphate isostructural with $\mathrm{Ca}_{5} \mathrm{Na}_{2}\left(\mathrm{PO}_{4}\right)_{4}$ apppears in the SYNROC mineralogy. There is no evidence for the partition of key radionuclides (e.g. Sr, REE and hence actinides) into this phos- phate. Its poor resistance to groundwater dissolution, whilst hardly desirable, may therefore not have a serious effect on the leaching performance of SYNROC containing PW-7a. 9 tables.

347 (UCRL-53237) Formulation of SYNROC-D additives for Savannah River Plant high-level radioactive waste. Ryerson, F.J.; Burr, K.; Rozsa, R. (Lawrence Livermore National Lab., CA (USA)). Dec 1981. Contract W-7405-ENG48. 41p. NTIS, PC A03/MF A01. Order Number DE82012495.

Portions of document are illegible.

SYNROC-D is a multiphase ceramic waste form consisting of nepheline, zirconolite, perovskite, and spinel. It has been formulated for the immobilization of high-level radioactive wastes now stored at Savannah River Plant (SRP) near Aiken, South Carolina. This report utilizes existing experimental data to develop a method for calculating additives to these waste products. This method calculates additions based on variations of mineral compusitions as a function of sludge composition and radionuclide partitioning among the SYNROC phases. Based on these calculations, a FORTRAN program called ADSYN has been developed to determine the proper reagent proportions to be added to the SRP sludges.

348 (UCRL-53392) Densification studies of Synroc D for high-level defense waste. Hoenig, $C_{\text {. }}$; Otto, $R_{\text {; }}$ Campbell, J. (Lawrence Livermnre National I.ah., C.A (ISSA)) 17 Jan 1983. Contract W-7405-ENG-48. 57p. NTIS, PC A04/MF A01. Order Number DE83011564.

Small- to medium-scale densification experiments were conducted on Synroc D using graphite dies and metal canisters. Pressures at elevated temperatures were applied both isostatically (HIP) and unidirectionally (HUP). Spray-dried/calcined powders formulated for composite or average sludge compositions exhibited initial packing densities of about 25\% theoretical. Final densities were in the range of 95 to $99 \%$ theoretical, depending on applied pressure and temperature. In final-stage HUP densification, we have found that porosity varies exponentially with time acording to the well-known expression $P+P_{0}$ $\exp \left(-K_{0} t\right)$. The rate constant $\left(K_{0}\right)$ has the Arrhenius form $K_{0}>A \sigma \operatorname{cxp}(-$ E/RT) which includes a stress or pressure term. Rate constants are calculated from approximately 20 densification experiments conducted under a wide range of conditions; activation energies in the range of 20 to $35 \mathrm{kcal} / \mathrm{mole}$ were calculated for the densification process. HIP densification and leaching results are reported for experiments with a wide range of variahles: pressure ( 3 in $3 n$ ksi), temperature $\left(900\right.$ to $\left.1300^{\circ} \mathrm{G}\right)$, redox calcination method, powder fill density and metal canister material. The results support the conclusion that HIIP nnd HIP densification parameters are very similar and that Synroc-D leaching behavior is essentially independent of density in the range of 90 to $100 \%$ theoretical. The densification of Synroc D in a collapsible metal-bellows canister has been simulated by means of modeling calculations. Radial buckling tendencies were also evaluated. Results from large-scale HIP experiments are also reported. Up to $50 \mathrm{~kg}$ of Synroc D was densified to greater than $99 \%$ thenretical density in a metal-bellowe canister $36 \mathrm{~cm}$ diamctcr by $24 \mathrm{~cm}$ in height. These data were used as a guide to make recommendations for the full-scale HIP densification of Synroc D using metalbellows canisters.

349 (UCRL-53405) Fluid-bed process for SYNROC production. Ackerman, F.J.; Grens, J.Z.; Ryerson, F.J.; Hoenig, C.L.; Bazan, F.; Peters, P.E.; Smith, R.; Campbell, J.H. (Lawrence Livermore National Lab., CA (USA)). 30 Mar 1983. Contract W-7405-ENG-48. 36p. NTIS, PC A03/MF A01. Order Number DE83016873.

Portions are illegible in microfiche products. Original copy available until stock is exhausted.

SYNROC is a titanate-based ceramio waste devcloped for the immobilization of high-level nuclear reactor waste. Lawrence Livermore National Laboratory (LLNL) has investigated a fluid-bed technique for the large-scale production of SYNROC precursor powders. Making SYNROC in a fluid bed permits slurry drying, calcination and reduction-oxidation reactions to be carried out in a single unit. We present the results of SYNROC fluid-bed studies from two fluid-bed units $10 \mathrm{~cm}$ in diameter: an internally heated fluid-bed unit developed by Exxon Idaho and an externally heated unit constructed at LLNL. Bed operation over a range of temperatures, feed rates, fluidizing rates, and redox conditions indicate that SYNROC powders of a bigh density and 
a uniform particle size can be produced. These powders facilitate the densification step and yield dense ceramics (greater than $95 \%$ theoretical density) with well-developed phases and low leaching rates.

350 (UCRL-53425) Plutonium doping of SYNROC-D. Van Konynenburg, R.A.; Guinan, M.W. (Lawrence Livermore National Lab., CA (USA)). 27 May 1983. Contract W-7405ENG-48. 24p. NTIS, PC A02/MF A01. Order Number DE84000987.

The purpose of this work was to perform an experimental simulation of the radiation effects that SYNROC-D (a ceramic waste form and the alternate to borosilicate glass for US defense high-level nuclear waste) will experience during the first million years of storage. Technology was developed for doping SYNROC-D with ${ }^{208} \mathrm{Pu}$ and performing external gamma irradiation to simulate both actinide and fission product decay. The doping technique was tested using both $\mathrm{Ce}$ and $\mathrm{U}$ as stand-ins to simulate the +3 and +4 oxidation states of $\mathrm{Pu}$, respectively. Samples wcre characterized by ceramography, density measurements, $x$-ray diffraction, scanning electron microscope-energy dispersive $x$-ray analysis, electron microprobe, scanning transmission electron microscope, gamma ray spectrometry, and leaching; equipment was fabricated for dilatation measurements. An early decision by the Department of Energy (DOE) to select borosilicate glass and terminate SYNROC-D development prevented doping with ${ }^{208} \mathrm{Pu}$ or external gamma irradiation. However, a sample was doped with ${ }^{209} \mathrm{Pu}$ in order to study the $\mathrm{Pu}$ distribution, and characterization of this sample was completed. Although conclusive proof was not developed, all indications from this work are that $\mathrm{Pu}$ will go into the zirconolite and perovskite phases in SYNROC-D, favoring perovskite under the redox conditions prevailing in a graphite die. Technology development and results of $\mathrm{Ce}, \mathrm{U}$, and ${ }^{209} \mathrm{Pu}$ doping studies are described in this report.

351 (UCRL-86558) Immobilization of high-level defense wastes in SYNROC-D: recent research and development results on process scale-up. Campbell, J.H.; Rozsa, R.B.; Hoenig, C.L. (Lawrence Livermore National Lab., CA (USA)). 15 Mar 1982. Contract W-7405-ENG-48. 14p. (CONF-820424-4). NTIS, PC A02/MF A01. Order Number DE82012263.

From ANS topical meeting on treatment and handling of radioactive wastes; Richland, WA, USA (19 Apr 1982).

Portions of document are illegible.

SYNROC is a titanate-based ceramic waste form being developed to immobilize high-level nuclear reactor wastes. SYNROC-D is a unique variation of SYNROC designed to contain high-level defense wastes, particularly those in storage at the Savannah River Plant (SRP). We review recent research and development on SYNROC-D processing options and report on work in progress on various unit operations. The overall immobilization process can be divided into three general parts: (1) slurry preparation (formulation, reactant addition and blending); (2) powder processing (spray drying, calcination/redox control); and (3) mineralization (densification). Powder processing research is directed toward development of a slurry-fed, fluidized-bed calciner based on the ICPP design. Densification research is focused on use of hot isostatic pressing (HIP) or hot uniaxial pressing (HUP). The successful use of both have been demonstrated.

352 (UCRL-87065) Incorporation of high-level wastes in SYNROC: results from recent process-engineering studies at Lawrence Livermore National Laboratory. Campbell, J.H.; Hoenig, C.L.; Ackerman, F.J.; Peters, P.E.; Grens, J.Z. (Lawrence Livermore National Lab., CA (USA)). 15 Apr 1982. Contract W-7405-ENG-48. 11p. (CONF-820636-2). NTIS, PC A02/MF A01. Order Number DE82013402.

From 5. international symposium on the scientific basis for radioactive waste management; Berlin, F.R. Germany (7 Jun 1982).

Portions of document are illegible.

In this paper, highlights from recent engineering research and development, in particular, results from fluidized bed calcination studies of SYNROC slurry are summarized. A schematic diagram of the envisioned SYNROC process (at this stage of development) is also presented. It shows the use of a fluidized bed calciner to prepare SYNROC powder that is then fed to a storage hopper. Bellows-type canisters are filled, evacuated, sealed and preheated. The preheated canisters are loaded into a hot isotactic pressing unit where they are densified, then removed and cooled and finally loaded into a waste storage container. After sealing, this container is decontaminated and transferred to the interim storage facility and then, ultimately, to an underground repository.

363 (UCRL-87742) SYNROC production using a fluid bed calciner. Ackerman, F.J.; Grens, J.Z.; Ryerson, F.J.; Hoenig, C.L.; Bazan, F.; Campbell, J.H. (Lawrence Livermore National Lab., CA (USA)). 27 Sep 1982. Contract W-7405ENG-48. 9p. (CONF-821107-11). NTIS, PC A02/MF A01. Order Number DE83001181.

From 6. international symposium on the scientific basis for radioactive waste management (Materials Research Society); Boston, MA, USA (1 Nov 1982).

SYNROC is a titanate-based ceramic developed for immobilization of high-level nuclear reactor wastes in solid form. Fluid-bed SYNROC production permits slurry drying, calcining and redox to be carried out in a single unit. We present results of studies from two fluid beds; the Idaho Exxon internally-heated unit and the externally-heated unit constructed at Lawrence Livermore National laboratory. Bed operation over a range of temperature, feed rate, fluidizing rate and redox conditions indicate that high density, uniform particle-size SYNROC powders are produced which facilitate the densification step and give HUP parts with dense, well-developed phases and good leaching characteristics. 3 figures, 3 tables.

364 (UJV-5734-Ch) Phosphate system as medium for radioactive waste fixation. Simplex experiment. Part I. Santarova, M.; Vojtech, O.; Suessmilch, J.; Stejskal, J. (Ustav Jaderneho Vyzkumu CSKAE, Rez (Czechoslovakia)). Jun 1981. 16p. (In Czech). NTIS (US Sales Only), PC A02/MF A01. Order Number DE82700735.

Results are reported of laboratory experiments with solidification of HLW using polyphosphates as a matrix. Before heat treatment, phosphoric acid and ferric nitrate were used as the only two admixtures to HLW solution. Physico-chemical properties of the final solidification products were measured; corresponding data characterizing the heat treatment were also obtained. The preparation of solidification products and the results of hydrolytic resistance measurements are dealt with in detail. The present study serves as a basis for future pilot plant experiments.

365 (UJV-5927-CH) Phosphate system as medium for radioactive waste fixation - simplex experiment. Part II. Vojtech, O.; Voldan, J.; Suessmilch, J.; Santarova, M. (Ustav Jaderneho Vyzkumu CSKAE, Rez (Czechoslovakia)). Dec 1981. 19p. (In Czech). NTIS (US Sales Only), PC A02/MF A01. Order Number DE82702759.

For Part I see UJV-5734-Ch (Jun 1981).

The properties are described of the solidification product of the system: fission product oxides- $\mathrm{Na}_{2} \mathrm{O}-\mathrm{Fe}_{2} \mathrm{O}_{0}-\mathrm{P}_{2} \mathrm{O}_{5}$. Sample preparation and analytical methods used are described. The results are shown of calcinate behaviour during melting, of viscosity measurement, of the system crystal forming capacity, of differential thermal analysis, of density measurement, transformation temperature and other dilatometric constants measurement, and microhardness data determination. Specimens containing $30 \mathrm{wt} . \%$ of fission product oxides, $60 \mathrm{wt} . \%$ of $\mathrm{P}_{2} \mathrm{O}_{5}$ and 10 wt.\% of $\mathrm{Fe}_{2} \mathrm{O}_{0}$ were found to be most suitable for technological experiments. Depending on the composition of actual high-level radioactive wastes, especially on the presence of corrosion products, the amount of $\mathrm{Fe}_{2} \mathrm{O}_{0}$ used as an additive could further be reduced. Thus, the vitrification process would be simplified and would only require proportioning a single additive, ie., phosphoric acid.

356 Synroc solution. Ringwood, A.E. (Australian National Univ., Canberra. Research School of Earth Sciences). Nuclear Active; No. 29, 16-20(Jul 1983).

This article deals with the storage of high-level radioactive wastes, which must be isolated from the biosphere for extremely long periods, because of their high-level of radioactivity. Thus the wasteform itself should function as an 'immobilisation barrier' fully capable of preventing any significant release of radionuclides to the biosphere for $10^{5}-10^{6}$ years. Synroc is a wasteform that posseses a great resistance to leaching. The paper deals with the composition of synroc, the comparative leaching behaviour of synroc and borosilicate glass, the effect of radiation damage, and the functioning of a synroc precursor. 
357 netsov, Yu V. Shashukov, E A. Aloi, A S. Kuznetsov, B.S (Rockwell International Corp., Golden, CO (USA). Rocky Flats Plant). Jun 1983. Contract AC04-76DP03533. Translation of IAEA-CN-43/473. 11p. NTIS, PC A02/MF A01. Order Number DE83013308.

Portions are illegible in microfiche products.

This report presents data on further development of means of inclusion of highly radioactive wastes and concentrates of long-lived radionuclides in glass-metal compounds and ceramic materials. It also contains data on the properties of the materials being used to produce the glass-metal compounds and the properties of ceramics based on claylike materials.

358 Highly dense graphite matrix: a new material for the conditioning of radioactive wastes. Hrovat, M.; Hackstein, K.G.; Huschka, H.; Pirk, H.A.; Schmidt-Hansberg, T. (NUKEM GmbH, Postfach 110080 D-6450 Hanau 11). Nuclear Technology; 61: Nu. 3, 460-464(Jun 1983).

In continuation of $20 \mathrm{yr}$ of experience with high-temperature gascooled reactor graphite fuel element fabrication (molded fuel spheres and monolithic block fuel elements), NUKEM introduced a new graphite-based material with an inorganic binder produced by molding. A mixture of nickel and sulfur is used as the inorganic binder for natural graphite powder. The fabrication is facilitated by the low temperature of $500^{\circ} \mathrm{C}$ in generating a chemically and thermally stable nickel sulfide. The newly developed material is suitable as a matrix for the fixation of high-active waste and as a corrosion-resistant layer of the steel supporting tube for final disposal of spent fuel elements. The material of natural graphite/nickel sulfide is distinguished by high density, high corrosion resistance, a low leaching rate, good thermal conductivity, an appropriate coefficient of thermal expansion, and high thermal stability due to the high melting point of nickel sulfide $\left(790^{\circ} \mathrm{C}\right)$. Currently, a pilot plant for fabrication of containers for spent fuel elements is being erected. Additionally, development work is in progress to obtain basic design data for a high-level waste fixation facility.

359 Commercial waste treatment technology and programs. Burkholder, H.C.; McElroy, J.L. (Battelle-Pacific Northwest Laboratory, P.O. Box 999, Richland, WA 99352). Transactions of the American Nuclear Society; 44: 100-101(Jun 1983). (CONF-830609-).

From ANS annual meeting; Detroit, MI, USA (12 Jun 1983). COMPACTING; FINANCIAL INCENTIVES; HIGHLEVEL RADIOACTIVE WASTES; LOW-LEVEL RADIOACTIVE WASTES; RADIOACTIVE WASTE PROCESSING; TECHNOLOGY ASSESSMENT; UNDERGROUND DISPOSAL; VITRIFICATION; SOLIDIFICATION; ALPHA-BEARING WASTES

360 Method for producing molded bodies containing highly active radioactive wastes from glass granules embedded in a metallic matrix. Ondracek, $G$. (to Kernforschungszentrum Karlsruhe Gmbh (Germany, Federal Republic Of)). US Patent 4,383,944. 17 May 1983. Priority date 28 Dec 1978, Germany, Federal Republic of (F.R. Germany), vp.

PAT-APPL-108166.

A method is claimed for producing a molded body containing highly radioactive wastes. The wastes are mixed with molten glass or are melted together with glass formers, and converted into glass granules or glass powder. These granules or powder are mixed with a metal powder containing at least one of the metals lead, iron, silver, cobalt, nickel and tin, and the mixture is condensed at pressures of 25 to 500 newtons $/ \mathrm{mm} 2$ to form a molded body.

301 Calcination of liquid radioactive wastcs. Part I. Tcehnology of radioactive waste calcination. Napravnik, J.; Neumann, L. (Ustav Jaderneho Vyzkumu CSKAE, Rez (Czechoslovakia)); Ditl, P. (Ceske Vysoke Uceni Technicke, Prague (Czechoslovakia)). JADEA; 29: No. 3, 86-92(Mar 1983). (In Czech).

The results are summed up of nuclear waste disposal research at the Nuclear Research Institute at Rez. It was decided on the basis of experience to consider denitration by formic acid to be the basic denitration method. Further research was carried out of the effect of catalysts on denitration. In case the temperature at which waste fixation takes place is higher than $300 \mathrm{degC}$, decomposition of nitrates is necessary Calcination temperature has to be chosen such as to secure the decom position of nitrates already during calcination. The chosen method o fixation influences the choice of the technology of denitration and calcination.

362 Phosphate systems - a medium for high level waste solidification. Vojtech, O.; Santarova, M.; Suessmilch, J.; Stejskal, J. (Ustav Jaderneho Vyzkumu CSKAE, Rez (Czechoslovakia)). JADEA; 29: No. 2, 45-49(Feb 1983). (In Czech).

The results are summed up of experiments with the systems fission products- $\mathrm{Fe}_{2} \mathrm{O}_{0}-\mathrm{P}_{2} \mathrm{O}_{5}$ and fission products- $\mathrm{Na}_{2} \mathrm{O}-\mathrm{Fe}_{2} \mathrm{O}_{0}-\mathrm{P}_{2} \mathrm{O}_{5}$ which by their composition correspond to the mixture of actual wastes and proposed additives. The process was studied of solidification using thermal analysis and the properties of the resulting products were studied as were the data related to the rheological properties and crystallization capabilities of melts. The possibility was shown of using phosphate technology for a two-stage solidification process with continuous calcination and melting. The product quality characterized by the rate of $\mathrm{Cs}$ and $\mathrm{Sr}$ leaching and the rate of the decuay of the whole skeletun of polyphosphates corresponded to glass or minerals with a good chemical resistance.

363 Neutron scattering studies of vitrified radioactive waste. Sinclair, R.N.; Desa, J.A.E.; Wright, A.C. (Materials Physics Division, AERE Harwell, Didcot, Oxon, OXII ORA). $S . / T A ; 66$ : No. 1, 72-77(Jan 1983).

The short-range atomic structure of borosilicate glasses has not been widely studied by diffraction techniques. The HARVEST glass contains the modifiers $\mathrm{Na}_{2} \mathrm{O}$ and $\mathrm{Li}_{2} \mathrm{O}$ and so is considerably more complicated than a simple mixture of $\mathrm{B}_{2} \mathrm{O}_{0}$ and $\mathrm{SiO}_{2}$. However, the experiments with $0.1 \mathrm{~nm}$ neutrons showed that the structure of the HARVEST glass can be understood as a network of $\mathrm{SiO}_{4}$ tetrahedra and $\mathrm{BO}_{0}$ triangles. The effect of the $25 \%$ by weight of waste oxides does not seriously perturb the network of the glass matrix. Small-angle scattering is observed from the glass plus waste. Since small-angle scattering arises from variations in scattering length density, its presence implies that the material is to some degree inhomogeneous. Causes may include phase immiscibility, complexes, clusters, voids, etc., the neutron experiments being sensitive to inhomogeneities with sizes up to $100 \mathrm{~nm}$. The average radius of gyration of the inhomogeneous regions is found to be $12.1 \mathrm{~nm}$, although this is probably lower than the true average value since the present experiment is not sensitive to much larger regions. An increased intensity of small-angle scattering is observed from a partially devitrified sample but the derived radius of gyration remains at $12.2 \mathrm{~nm}$. The simplest interpretation of the increase in scattering per gram of sample (by a factor of 3.7) is that more regions of the same mean dimension appeared on annealing. Small-angle scattering studies of glasses prepared in different ways might be useful if they could be correlated with devitrification and leaching results.

364 Conditioning of radioactive wastes for storage and disposal. Vienna, Austria; International Atomic Energy Agency (1983). 526p. (CONF-820651-).

From International symposium on the conditioning of radioactive wastes for storage and disposal; Utrecht, Netherlands (21 Jun 1982).

Thirty-five papers were presented in eight sessions covering the following main topics: the technical bases for conditioning, the immobilization processes, packaging methods and materials, evaluation of conditioned wastes, quality assurance and cost/benefit considerations. Individual papers were indexed.

365 Analysis of thin leaching layers and fracture mechanics of irradiated borosilicate glass for solidincatlon of high-level fission product solutions (HAW). Matzke, H.; Pickering, S.; Linker, G. pp 488-491 of Jahrestagung Kerntechnik ' 83 - Reaktortagung 1983. Tagungsberichte. Sektion 4: Brennstoffkreislauf und Abfallbehandlung. Eggenstein-Leopoldshafen, Germany, F.R.; Fachinformationszentrum Energie, Physik, Mathematik (1983). (In German)(CONF-830636-).

From Annual meeting on nuclear technology '83; Berlin, F.R Germany (14 Jun 1983).

Published in summary form only.

HIGH-LEVEL RADIOACTIVE WASTES; SOLIDIFICATION; VITRIFICATION; LEACHING; PERFORMANCE TEST- 
ING; BOROSILICATE GLASS; FRACTURE MECHANICS; FRACTURE PROPERTIES; SIMULATION; PHYSICAL RADIATION EFFECTS; CHEMICAL REACTION KINETICS; ION BEAMS; HEAVY IONS; RADIOACTIVE WASTE PROCESSING; FISSION PRODUCTS

366 Scientific basis for nuclear waste management VI. Brookins, D.G. (ed.). New York, NY; North-Holland (1983). 810p. (CONF-821107-Pt.1). Elsevier Science Publishing Co., Inc., 52 Vanderbilt Avenue, New York, NY 10017.

From 6. international symposium on the scientific basis for radioactive waste management (Materials Research Society); Boston, MA, USA (1 Nov 1982).

The papers presented here give the results of research and development activities from a large number of universities, government laboratories, and private industry in twelve countries. The 95 papers published in these proceedings have been divided into 8 sections. These encompass various aspects of high-level and non-high-level radioactive waste management ranging from repository characterization and waste form production to product and performance assessment. Sixty of the papers are indexed separately; the remaining 35 have been indexed previously.

367 Fixation of high level solid waste (HLSW) in a TiO, base ceramic matrix provided with an integrated overpack. Bauer, C.; Ondracek, G. (Kernforschungszentrum, Karlsruhe, Germany). Materials Research Society Symposia Proceedings; 15: 71-76(1983). (CONF-821107-Pt.1).

From 6. international symposium on the scientific basis for radio active waste management (Materials Research Society); Boston, MA, USA (1 Nov 1982).

Compared with glass resulting from ceramic melter, the product described has the following advantages: significantly higher leaching resistance, lower losses of radioactive isotopes during processing in the off-gas, and possibility of integrating a ceramic overpack into the waste form. Compared to other crystalline waste forms, the advantages are: no necessity of very intimate mixing of HLW and matrix materials and potentially lower effects of transmutation (i.e., $\alpha$-recoil ageing) on the stability of the product as they should be confined to a very small area around each waste particle. Obviously, the process development can by no means be compared with the glass melting technology which, however, should not lead to the conclusion that in the long rung glass will keep its leading position as a primary candidate for HLW fixation.

$368 \quad$ Nuclear waste management in France. Lefevre, J.; Sugier, A. Materials Research Society Symposia Proceedings; 15: 391-397(1983). (CONF-821107-Pt.1).

From 6. international symposium on the scientific basis for radio active waste management (Materials Research Society); Boston, MA, USA (1 Nov 1982).

A General Waste Management and Research and Development Program has been presented for approval by CEA to the government indicating the short and long-term objectives. This report entails: objectives related to long-term waste management and objectives related to $\mathbf{R}$ and $\mathbf{D}$ on treatment, conditioning, and storage processes. As far as the near and mean terms are concerned, i.e., the conditioning and disposal of low and intermediate-level wastes, and the primary conditioning and the intermediate storage of alpha-bearing and high-level waste, the processes are available and give every wished for quality and safety assurances. Improvements are always possible, such as better performing, simpler, or cheaper processes, or safer from the point of view of lowering personnel exposure or long-term behavior, and work is therefore still going on. A shallow land final disposal site will have to be started soon, to reserve the use of LA MANCHE to LA HAGUE. As far as the long term is concerned, solutions exist and work is underway to select the most suitable geological formations and sites which entails sustained research efforts in order to identify and quantify the leading parameters. This type of research effort is particularly suited to international cooperation, which can also cover site exploration, transfer modelling and conditioned waste qualification. However, the results will only be proved through actual demonstration sites. The sinall volume of the long term wastes, and the more than satisfactory safety of their intermediate storage may seem to make their long term disposal less than urgent: this is a fallacy. The highest standard of safety at the lowest cost - i.e., optimization - will only be attained through pursuing the effort to the end, which is, the commissioning and running of a disposal site. The time has come - not to rush blindly headlong, certainly, but to get on with it.

369 United Kingdom regulatory procedures for radioactive wastes. Feates, F.S.; Richards, H.J. (Department of the Environment, London, England). Materials Research Society Symposia Proceedings; 15: 399-405(1983). (CONF-821 107-Pt. 1).

From 6. international symposium on the scientific basis for radioactive waste management (Materials Research Society); Boston, MA, USA (1 Nov 1982).

In the United Kingdom the responsibility for radioactive waste management policy lies with the environmental Ministers. The Department of the Environment is also responsible in England for authorization of all radioactive waste disposal activities and has its own Radiochemical Inspectorate to ensure that government policy is implemented. An independent Radioactive Waste Management Advisory Committee reports directly to the Secretary of State for the Environment. Low-level wastes are currently disposed of by shallow burial or to the deep ocean. Sites for the disposal of intermediate-level wastes are being sought as a matter of urgency and heat-mitting, reprocessing wastes will be stored on the surface for at least fifty years. The rationale of this policy is explained.

370 Supernatant treatment considerations for the neutralized waste at West Valley. Gockley, G.B. (Westinghouse R and D Center, Pittsburgh, PA); Lahoda, E.J.; Pope, J.M. Materials Research Society Symposia Proceedings; 15: 663-668(1983). (CONF-821107-Pt.1). Contract AC07-81NE44139.

From 6. international symposium on the scientific basis for radioactive waste management (Materials Research Society); Boston, MA, USA (1 Nov 1982).

The neutralized high-level waste, stored at the Western New York Nuclear Service Center in West Valley, New York, was produced during the operation of the Nuclear Fuel Service, Inc. commercial nuclear fuel reprocessing plant. The supernatant is a highly concentrated salt solution ( $\mathrm{NaNO}_{0}, \mathrm{NaOH}, \mathrm{Na}_{2} \mathrm{SO}_{4}$ and $\mathrm{NaCl}$ ) contained essentially all of the dissolved cesium as the primary radioactive component. The sludge is primarily iron and aluminum hydroxides and contains strontium and the bulk of the long-lived isotopes. The supernatant will be treated to remove essentially all of the radioactivity and then be concentrated and disposed of as low level nuclear waste. The following supernatant treatment considerations have been evaluated on a laboratory scale using simulated West Valley waste: (1) organic ion exchange resins; (2) inorganic ion exchange media; (3) in-tank processing. These processes are described and preliminary laboratory data will be presented.

371 Investigations of suitable metallic container materials for HAW solidification. Mirschinka, V. (Kernforschungsanlage Juelich GmbH, Germany); Halaszovich, S.; Odoj, R.; Forch, U.; Forch, K. Materials Research Society Symposia Proceedings; 15: 695-702(1983). (CONF-821107-Pt.1).

From 6. international symposium on the scientific basis for radioactive waste management (Materials Research Society); Boston, MA, USA (1 Nov 1982).

The influence of the main alloying elements of six different steels and high-temperature-resistant alloys for crucibles containing HAW glass on the homogeneity of the waste glass was investigated. The distributions of iron, chromium, nickel, and cobalt were studied by an electron beam microprobe. The results are given and discussed.

372 Review of the current status of radiation effects in solid nuclear waste forms. Weber, W.J. (Pacific Northwest Lab., Richland, WA). Materials Research Society Symposia Proceedings; 15: 407-414(1983). (CONF-821107-Pt.1). Contract AC06-76RL01830.

From 6. international symposium on the scientific basis for radioactive waste management (Materials Research Society); Boston, MA, USA (1 Nov 1982).

The current status of radiation effects in solid nuclear waste forms is reviewed. The general nature of radiation effects and the simulation techniques used are briefly discussed. Recently reported data on radiation effects are presented and discussed, and those areas requiring further theoretical or experimental study are identified. 
373

Discharge of high activity and long half-life wastes arising from a nuclear power programme. Dejonghe, P.; Hermans, R. (Centre d'Etude de l'Energie Nucleaire, Mol (Belgium)). n); No. 176, 3-11(1983). (In Frcnch).

After a review of types and quantities of wastes, the article considers the storage of very low activity, of high activity and alpha emitters of long period contaminated wastes. The situation in Belgium is analyzed in view of the storage of high activity wastes in clay formations. The preliminary works, the studies of the local clay properties, the type of artificial barriers, the hydrological monitoring of the surface and underground waters as well as the underground laboratories are presented.

374 New SYNROC plant offers $\mathbf{N}$-waste disposal solution. Science Technology (Canberra City); 2: No. 12, 1-2, 19(Dec 1982).

Work will begin soon at Lucas Heights on the design and construction of a commercial demonstration plant to prove the feasibility of using Synroc for the safe disposal of high-level wastes. The plant wil use only simulated non-radioactive waste and will be capable of handling $20 \mathrm{~kg}$ of Synroc, containing $4 \mathrm{~kg}$ of waste, per hour.

375 Analysis for silicon in solution in high level waste glass durability studies. Lewis, R.A.; Smart, R.St.C. (Griffith Univ., Nathan (Australia)); Dale, L.S.; Levins, D.M. (Australian Atomic Energy Commission Research Establishment, Private Mail Bag, Sutherland, New South Wales). Radioactive Waste Management and the Nuclear Fuel Cycle; 3: No. 2, 191198(Dec 1982).

In comparative studies of the durability of HLW glasses, the measurement of the dissolution of the silicate network, in terms of both rate and extent, is of prime importance. To achieve this, analytical techniques such as colorimetry, flame atomic absorption spectrometry and inductively-coupled plasma emission spectrometry are used. The reliability of these analytical techniques for determination of silicon concentration in dissolution of HLW glasses, is examined. At high concentrations both FAA and ICP are accurate but colorimetry, even with HF pretreatment or $\mathrm{NaOH}$ digestion, does not give agreement with ICP. At concentrations below $40 \mathrm{mg} \mathrm{l}^{-1}$ all three methods are reliable.

376 Final conditioning of high-level fission product solutions. Krause, K. ATW, Atomwirtschaft. Atomtechnik; 27: No. 12, 618-621(Dec 1982). (In Germán).

The vitrification of high-level fission product solutions is, among other things, also planned as part of the German concept for nuclear waste management. From the large number of the glass types initially taken into account a global evaluation of method and product proved borosilicate glass to be the most suitable. As to the methods, the French AVM-method which has already been technically tested is facing a development of the KfK which is also pursued in a similar way in the USA. Contrary to the use of a metal melter with the French method the glass is produced in electrode-heated ceramic melters.

377 Radiation damage in nuclear waste ceramics. Turcotte, R.P.; Roberts, F.P.; Rusin, J.M.; Wald, J.W. (Pacific Northwest Laboratory, Richland, Washington 99352). S.ITA; 65: No. 12, 589-593(Dec 1982). Contract AC06-76RL01830.

The tcxt contains a numbor of opeoifio obcervations about the radiation-induced changes in glass, glass-ceramic, and supercalcine nuclear waste forms. Other, more general conclusions can be summarized: Radiation-induced property changes follow an exponential ingrowth curve to saturation. Actinide host phases in both crystalline waste forms become X-ray amorphous. The magnitudes of the wasteform density changes observed could not be directly related to observed changes in the primary actinide phases. Although large crystal-structure changes occur in the materials studied, obvious physical degradation was not observed.

378 Microwave energy for post-calcination treatment of high-level nuclear wastes. Gombert, D.; Berreth, J.R.; Priebe, S.J. (Exxon Nuclear Idaho Co. P.O. Box 2800, Idaho Fulls, ID 83401). al; 18: No. 6, 755-759(Nov 1982).

Heating by microwave energy is explored in processing of two nuclear waste forms: drying of a pelleted form and vitrification. It is shown that residence time can be greatly reduced compared to conventional heating sources, without affecting product quality.

379 Method for solidifying aqueous radioactive wastes for noncontaminating storage. Koster, R.; Riege, U.; Scheffler, K. (to Kernforschungszentrum Karlsruhe Gmbh (Germany, Federal Republic Of)). US Patent 4,354,954. 19 Oct 1982. Priority date 29 Apr 1978, Germany, Federal Republic of (F.R. Germany), vp.

PAT-APPL-034534.

Method for solidifying high and medium radioactivity and/or actinide containing aqueous waste concentrates or fine-grained solid wastes suspended in water for final noncontaminating storage. The waste concentrates or suspensions are set, by evaporation, to a water content in the range between $\mathbf{4 0}$ and 80 percent by weight, and a solid content whose metal ion and/or metal oxide component lies between 10 and 30 percent by weight of the evaporate being formed. The ph of the evaporate is set to between 5 and 10 . The resulting evaporate is then pelletized with the aid of a clay-like substance. The pellets are then heat treated. including drying at temperatures between room temperature and about $150^{\circ} \mathrm{C}$, then calcining at room temperatures up to about $800^{\circ} \mathrm{C}$ And subsequently firing at temperatures between $800^{\circ} \mathrm{C}$. And $1400^{\circ} \mathrm{C}$ To form practically undissolvable mineral phases. The pellets are then enclosed on all sides in a dense, continuous ceramic or metallic matrix.

380 Fixation of radioactive materials in a glass matrix. Macedo, P.B.; Litovitz, 'I.A.; Simmons, J.H. Australian Patent 1978/34,388/B/. 9 Sep 1982. Priority date 4 Apr 1977, United States of America (USA), vp.

A non-porous glass composition containing radioctive materials chemically incorporated in the glass matrix is claimed. It contains up to $5 \mathrm{~mol}$ percent $\mathrm{B}_{2} \mathrm{O}_{0}$, at least $75 \mathrm{~mol}$ percent $\mathrm{SiO}_{2}$ and immobilized oxides of the radio-active material chemically incorporated into the silica structure. Furthermore, it has a radiation activity above one millicurie per cubic centimeter, and a high chemical durability to aqueous solution.

381 Preparation of gel spheres containing simulated highlevel radioactive waste. Arnold, W.D.; Bond, W.D.; Robinson, S.M. (Oak Ridge National Lab., IN (USA)). Radioactive Waste Management; 3: No. 1, 5\%-74(Sep 1982).

Supersedes Radioactive Waste Management.

Gel-derived microspheres containing simulated high-level radioactive waste sludge and ceramic additive material were prepared by internal gelation at waste loadings as high as $90 \%$. The gel spheres were amenable to subsequent drying, sintering, and coating procedures producing highly leach-resistant crystalline waste forms suitable for permanent disposal. Potential application of this technique to commercial power reactor waste processing was demonstrated by incorporating simulated Purex solvent extraction waste in gel spheres at $20 \%$ waste loading.

382 Radioactive wastes. Grass, F. (Atominstitut der Oesterreichischen Universitaeten, Vienna); Krejsa, P. (Oesterrcichisches Forschungszentrum Seibersdorf G.m.b.H. Hauptabtcilung Abfallverarbeitung); Stueger, R. (Gesellschaft fuer neue Technologien in der E-Wirtschaft (Austria)). Oesterreichische Chemie-Zeitschrift: 83: No. 9, 211-216(Sep 1982). (In German).

Following a definition of the ler 'I' 'I adinactive waste', including a discussion of possible criteria allowing a delimitation of low-level radioactive against inactive wastes, present techniques of handling highlevel, intermediate-level and low-level wastes are described. The factors relevant for the establishment of definitive disposals for high-level wastes are discussed in some detail. Finally, the waste management organization currently operative in Austria is described.

383 IAEA report on actinide partitioning and transmutation. White, I.F. (National Radiological Protection Board, Harwell (UK)). Radiological Protection Bulletin; No. 48, 15 18(Sep 1982).

A summary is given of an IAEA report on the partitioning and transmutation of actinides from high level reprocessing wastes. The objective of the IAEA Co-ordinated Rsearch Programme was to examine critically the technical feasibility of the partitioning-transmutation (P-T) concept and its potential effect on the radiological impact of high 
level waste disposal. It was concluded that the implementation of P-T would be an immense undertaking, involving a large proportion of a country's nuclear power programme, but providing at best a rather small reduction in potential long-term radiological hazards.

384 Vitrifying Britain's waste. Clelland, D.W.; Corbet, A.D.W. (British Nuclear Fuels Ltd., Risley). Nuclear Engineering International; 27: No. 331, 33-35(Aug 1982).

A description is given of the Windscale Vitrification Plant which is to be built at British Nuclear Fuel's Sellafield Site. The plant, which should be operational in the late 1980s at a cost of some Pound 200 million, will use the French AVM (Atelier de Vitrification de Marcoule) process.

385 Generation and vitrification of high-level light water reactor liquid waste. Wheelwright, E.J.; Bjorklund, W.J.; Browne, L.M.; Bryan, G.H.; Holton, L.K.; Irish, E.R.; Siemens, D.H. (Pacific Northwest Laboratory, P.O. Box 999, Washington 99352). Nuclear Technology; 58: No. 2, 271-293(Aug 1982).

The Nuclear Waste Vitrification Project was conducted to demonstrate the vitrification of high-level liquid waste (HLLW) generated during the reprocessing of spent fuel discharged from an operating light water reactor. Six pressurized water reactor fuel assemblies, containing $2.3 \mathrm{tU}$, were processed for the generation at HLLW. A conventional Purex-type process was used for the first cycle so that the HLLW generated would be typical of the nitric acid, fission product waste stream from the first extraction cycle of a commercial plant. Uranium and nonradioactive chemicals, normally added to the HLLW by back-cycling of waste from second and third solvent-extraction cycles, were added to the dilute HLLW to produce a waste composition typical of the HLLW from a commercial plant. The waste was then concentrated tenfold to provide feed for solidification by the spray calciner/in-can melting pro cess. During calcination, the liquid waste was pumped to the calciner vessel, which was heated to $750^{\circ} \mathrm{C}$. The powdered calcine fell into a stainless steel canister, which was maintained at $1050^{\circ} \mathrm{C}$; this canister was attached directly to the bottom of the calciner. Glass-forming chemicals were metered into the canister simultaneously with the calcine. After the materials melted, the canister was cooled to produce glass. Two canisters containing glass were produced.

$386 \quad$ Coating of crystalline nuclear waste forms to improve inertness. Stinton, D.P.; Angelini, P.; Caputo, A.J.; Lackey, W.J. (Metals and Ceramics Division, Oak Ridge National Laboratory, Oak Ridge, Tennessee 37830). American Ceramic Society Bulletin; 65: No. 8, 394-398(Aug 1982). Contract W-7405ENG-26.

Microspheres of a crystalline waste form prepared by sol-gel processing were successfully coated with layers of pyrolytic carbon and silicon carbide to isolate the radioactive wastes from the biosphere. A separate process for cesium immobilization was developed which loads 5 wt\% Cs onto zeolite particles for subsequent coating. Pyrolytic carboncoated particles showed leach rates $\approx 2$ to 4 orders of magnitude less than the candidate reference borosilicate glass waste form. Aqueous leach-test results of coated waste forms were below detection limits of such sensitive analytical techniques as atomic absorption and inductively coupled plasma atomic emission. Significant progress was made in applying sol-gel and fluidized bed coating technology to the solidification of high-level nuclear wastes. The authors successfully prepared microspheres containing simulated wastes of Synroc-B, Synroc-D, a modified Synroc-D where alumina was previously removed from the waste, and a very high waste-loading composition containing $90 \%$ waste and $10 \% \mathrm{ZrO}_{2}$. Each of these compositions was sintered at $1000^{\circ} \mathrm{C}$ and coated with pyrocarbon at temperatures as low as $1000^{\circ} \mathrm{C}$. Silicon carbide can be applied to these pyrocarbon-coated particles at temperatures as low as $900^{\circ} \mathrm{C}$ for additional inertness or for oxidation resistance. Cesium was isolated by pyrocarbon-coating cesium-loaded zeolite. Pyrocarbon and $\mathrm{SiC}$ coatings successfully reduced the leach rates to below detection limits.

$387 \quad$ Coating of crystalline nuclear waste forms to improve inertness. Stinton, D.P.; Angelini, P.; Caputo, A.J.; Lackey, W.J. (Metals and Ceramics Division, Oak Ridge National Laboratory, Oak Ridge, Tennessee 37830). S.JTA; 65: No. 8, 394399(Aug 1982).
Microspheres of a crystalline waste form prepared by sol-gel processing were successfully coated with layers of pyrolytic carbon and silicon carbide to isolate the radioactive wastes from the biosphere. A separate process for cesium immobilization was developed, which loads 5 wt\% Cs onto zeolite particles for subsequent coating. Pyrolytic carboncoated particles showed leach rates $\approx 2$ to 4 orders of magnitude less than the candidate reference borosilicate glass waste form. Aqueous leach-test results of coated waste forms were below detection limits of such sensitive analytical techniques as atomic absorption and inductively coupled plasma atomic emission.

388 Treatment of high level nuclear waste. Ringwood, A.E. (to Australian National Univ., Canberra). Australian Patent 1979/48,708/B/. 29 Jul 1982. Filed date 14 Jul 1978. vp.

A process is claimed for immobilising high level radioactive waste calcine. It comprises: mixing the HLW calcine in a minor proportion with a mixture of oxides. When the mixture is heated and then cooled it crystallises to produce a mineral assemblage containing crystals capable of providing lattice sites in which elements of the HLW are securely bound. The crystals possess structures closely related to crystals belonging to mineral classes which are resistant to leaching and also closely related to at least two of the titanate mineral classes selected from perovskite, zirconolite and hollandite-type mineral classes.

389 Method of treating high level radioactive solid waste. Abe, T.; Tsukumo, K.; Yasufuku, K.; Oosaki, T.; Iwata, A. (to Kawasaki Heavy Industries Ltd., Kobe (Japan)). Japanese Patent 57-116,299/A/. 20 Jul 1982. 4p. (In Japanese).

A radioactive substance which has adhered to an article is treated so as not to allow the substance to be scattered. This method treats high level radioactive waste exhausted from an atomic power plant or the like. The waste to be treated and thrown into a cooler by a transport vehicle is cooled to a temperature lower than embrittling temperature with the cooling medium supplied from a cooling medium supplying device, and is thrown into and pulverized by a pulverizing machine or a press via a conveyor. The waste to be treated and thus finely pulverized is removed by a removing device, filled, solidified and sealed by a filling, solidifying and sealing unit, and is conveyed and treated by the conveyor.

390 Equipment for radioactive waste solidification. Nachmilner, L.; Skaba, V. (Ustav Jaderneho Vyzkumu CSKAE, Rez (Czechoslovakia)). JADEA; 28: No. 7, 246248(Jul 1982). (In Czech).

The pilot plant consists of an induction furnace, a heat source, a programmable temperature controller, and a part for trapping and decontamination of gaseous products and the inlet air. The induction furnace consists of an inner steel crucible placed in a protective ceramic crucible surrounded with an inductor, and of a head which closes the inner crucible. A modified melting unit of the ISTS X/0.1-2 type is used as a power supply. It is designed for the maximum power of $50-100 \mathrm{~kW}$ at a frequency of $2-3.5 \mathrm{kHz}$ at an output voltage of $100-400 \mathrm{~V}$. The programmable temperature controller is in-built in a frequency converter. 5 steps can be programmed, each of the maximum duration of 10 hours. A Pt-PtRh 10 thermocouple, linearized within 90 and $1200 \mathrm{degC}$ is used for temperature sensing. The equipment is suitable for testing solidification processes at medium and high temperatures.

$391 \quad$ Method of preparing nuclear wastes for transportation and interim storage. Bandyopadhyay, G.; Galvin, T.M. US Patent Application 6-383,880. 1 Jun 1982. 15p. Contract W-31109-ENG-38.

Nuclear waste is formed into a substantially water-insoluble solid for temporary storage and transportation by mixing the calcined waste with at least 10 weight percent powdered anhydrous sodium silicate to form a mixture and subjecting the mixture to a high humidity environment for a period of time sufficient to form cementitious bonds by chemical reaction. The method is suitable for preparing an interim waste form from dried high-level radioactive wastes.

392 Developing technology of microwave vitrification. Allen, P.L.; Ford, L.H. (UKAEA Springfields Nuclear Power Development Labs.). Nuclear Energy (Institution of Nuclear Engineers); 21: No. 3, 195-200(Jun 1982). 
The development of a vitrification process utilizing microwave energy is described. The vitrification equipment evolved during the development programme has been operated continuously for extended periods of time and at throughputs which are relevant to the UK's fast reactor programme. This process uses microwave energy to evaporate simulated waste highly active liquors within a moving column of glass fibre plugs contained in a stainless steel tube. The off-gases are filtered as they pass through the fibre plugs before exiting to the final clean-up system. The plugs with their burden of waste solids are then vitrified in a continuous melter. The development of the microwave power transmission system is described, particularly the microwave windows which allow power transmission while preventing the corrosive process atmosphere from diffusing along the waveguides into the power source (magnetron).

393 Process improvements in the NWCF. Fortsch, M.; Smith, R.R. (Fluor Engineers and Constructors, 2801 Kelvin Avenue, Irvine, CA 92714). Transactions of the American Nuclear Society; 41: 87(Jun 1982). (CONF-820609-).

From American Nuclear Society annual meeting; Los Angeles, r.A, ISA (K Jun 198?).

CALCINED WASTES; IDAHO CHEMICAL PROCESSING PLANT; OFF-GAS SYSTEMS; PROCESS CONTROL; RADIOACTIVE WASTE PROCESSING; RUTHENIUM; SAFETY; HIGH-LEVEL RADIOACTIVE WASTES; SOLIDIFICATION; CALCINATION; ADSORPTION; FLUIDIZED BED; SILICA GEL; FILTERS; SORPTIVE PROPERTIES; SCRUBBERS

394

Highly dense graphite matrix - a new material for the conditioning of radioactive wastes. Hrovat, M.; Huschka, $\mathbf{H}$.; Pirk, H.; Schmidt-Hansberg, T. (Nukem GmbH, Postfach 110080, D-6450 Hanau 11). Transactions of the American Nuclear Society; 41: 157-158(Jun 1982). (CONF-820609-).

From American Nuclear Society annual meeting; Los Angeles, CA, USA (6 Jun 1982)

GRAPIITE; HIGH-LEVEL RADIOACTIVE WASTES; MATRIX MATERIALS; PHYSICAL PROPERTIES; SOLIDIFICATION; RADIOACTIVE WASTE PROCESSING

396 Crystalline ceramics as a high-level nuclear waste form. Harker, A.B.; Clarke, D.R.; Morgan, P.E.D. (Rockwell International, Science Center, P.O. Box 1085, 1049 Camino Dos Rios, Thousand Oaks, CA 91360). Transactions of the American Nuclear Society; 41: 279-280(Jun 1982). (CONF820609-).

From American Nuclear Society annual meeting; Los Angeles, CA, USA (6 Jun 1982).

CERAMICS; CRYSTALLIZATION; HIGH-LEVEL RADIOACTIVE WASTES; SAVANNAH RIVER PLANT; STABILITY; THERMODYNAMICS; RADIOACTIVE WASTE PROCESSING; SOLIDIFICATION

396 SYNROC for containment of high-level nuclear waste. Reeve, K.D.; Levins, D.M.; Ramm, E.J.; Woolfrey, J.L. (Australian Atomic Energy Commission, Lucas Heights Research Laboratories, Sutherland, N.S.W. 2232). Transactions of the American Nuclear Society; 41: 278(Jun 1982). (CONF-820609-).

From American Nuclear Socicty annual meeting; Los Angeles, CA, USA (6 Jun 1982).

CALCINATION; CONTAINMENT; HIGH-LEVEL RADIOACTIVE WASTES; IRRADIATION; LEACHING; SYNROC PROCESS; SYNTIIETIC ROCKS

397 Precipitation-adsorption process for the decontamimation of nuclear waste supernates. Lee, L.M.; Kilpatrick, L.L. US Patent Application 6-379,800. 19 May 1982. 84p. Contract AC09-76SR00001.

High-level nuclear waste supernate is decontaminated of cesium by precipitation of the cesium and potassium with sodium tetraphenyl boron. Simultaneously, strontium-90 is removed from the waste supernate sorption of insoluble sodium titanate. The waste solution is then filtered to separate the solution decontaminated of cesium and strontium.

$398 \quad$ Process for the treatment of high level nuclear wastes. Ringwood, A.E. (to Australian National University The (Aus- tralia)). US Patent 4,329,248. 11 May 1982. Priority date 1 Mar 1979, Australia, vp.

PAT-APPL-124953.

A process is disclosed for immobilizing high level waste (Hlw) sludge containing aluminium and/or iron compounds. It comprises the steps of: (1) mixing the sludge with a mixture of oxides, the oxides in said mixture and the relative proportions thereof being selected so as to form a mixture when heated at temperatures between $800^{\circ}$ and $1400^{\circ}$ C. Crystallizes to produce a mineral assemblage containing (I) crystals capable of providing lattice sites in which the fission product and actinide elements of said hlw sludge are securely bound, and (II) crystals of at least one inert phase containing excess aluminium and/or iron, said crystals belonging to or possessing crystal structures closely related to crystals belonging to mineral classes which are resistant to leaching and alteration in appropriate geologic environments; and (2) heating and then cooling said mixture under reducing conditions so as to cause crystallization of the mixture to a mineral assemblage having the fission product and actinide elements of said hlw sludge incorporated as solid solutions within the crystals thereof, and the excess aluminium and/or iron crystallized in at least one inert phase. A mineral assemblage containine immobilized blw sludge containing aluminium and/or iron compounds incorporated within the crystals thereof is also disclosed.

399 Removal of ${ }^{90} \mathrm{Sr},{ }^{107} \mathrm{Cs}$, and ${ }^{106} \mathrm{Ru}$ from vent gases produced during solidification of high-level radioactive solutions. Kepak, F.; Uner, E.; Kanka, J.; Koutova, S. (Ustav Jaderneho Vyzkumu CSKAE, Rez (Czechoslovakia)). JADEA; 28: No. 5 , 177-180(May 1982).

Laboratory procedures and apparatus were developed for the sorption of ${ }^{106} \mathrm{RuO}_{4}$ and $/$ or ${ }^{107} \mathrm{Cs}_{2} \mathrm{O}$ vapours and for filtration of aerosols of ${ }^{106} \mathrm{Ru}$, ${ }^{107} \mathrm{Cs}$, and ${ }^{90} \mathrm{Sr}$ that were produced during denitration, calcination, and vitrification of high-level radioactive wastes. The ${ }^{106} \mathrm{RuO}_{4}$ and/or ${ }^{107} \mathrm{Cs}_{2} \mathrm{O}$ vapours were sorbed on inorganic sorbents, the aerosols of ${ }^{106} \mathrm{Ru},{ }^{107} \mathrm{Cs}$, and ${ }^{90} \mathrm{Sr}$ were filtered by prefiltration and highefficiency filtration materials of glass fibres. ${ }^{106} \mathrm{Ru}$ and ${ }^{107} \mathrm{Cs}$ concentration in the gas was reduced by more than four orders, ${ }^{90} \mathrm{Sr}$ concentration was reduced by three orders.

400 Synroc technology for immobilizing U.S. defense waste. Newkirk, H.W.; Hoenig, C.L.; Ryerson, F.J.; Tewhey, J.D.; Smith, G.S.; Rossington, C.S.; Brackmann, A.J.; Ringwood, A.E. (Lawrence Livermore Natl Lab, CA, USA). American Ceramic Society Bulletin; 61: No. 5, 559-566(May 1982).

Synroc technology has been applied to immobilize U.S. defense high level wastes similar to those stored at the Savannah River Plant (SRP). Titanate-based ceramic waste-form specimens (Synroc D), containing simulated SRP sludge of average composition, were synthesized by conventional hot-pressing techniques. The waste-form specimens were characterized by $x$-ray diffraction and scanning electron microscopy, and their stability assessed by leaching methods. Major phases present are zinconolite, perovskite, spinel solid solution, nepheline, and a Cshollandite analog. prepared separately and added to the calcined powder before densification. Normalized single-day static leach rates for the major matrix elements in Synroc $D$ using deionized water at $150^{\circ} \mathrm{C}$ are in the range $10^{-2}-10^{-5} \mathrm{~g} / \mathrm{m}^{2}$ day. Static leach rates for cesium were 1.85 $\mathrm{g} / \mathrm{m}^{2}$ day on the first day, decreasing to $1.7 \times 10^{-1} \mathrm{~b} / \mathrm{m}^{2}$ day after the third day. Dynamic leach rates for cesium in the Synroc-D waste form and in the $(\mathrm{Ba}, \mathrm{Cs})$-hollandite analog material using deionized water at $75^{\circ} \mathrm{C}$ were $5 \times 10^{-1} \mathrm{~g}$ Synroc $\mathrm{D} / \mathrm{m}^{2}$ day on the first day, decreasing to $1.2 \times 10^{-2}$ 8 Synroc $D / m^{2}$ day after the 12 th day, and $1 \times 10^{-1} \mathrm{~g}(\mathrm{Ba}, \mathrm{Cs})-$ hollandite $/ \mathrm{m}^{2}$ day on the first day, decreasing to $5 \times 10^{-4}$ $\mathrm{g}(\mathrm{Ba}, \mathrm{Cs})$-hollandite $/ \mathrm{m}^{2}$ day after 44 days, respectively. 45 refs.

401 Fission product transmutation effects on high-level radioactive waste forms. Gray, W.J. (Pacific Northwest Lab., Richland, WA (USA)). Nature (London); 296: No. 5857, 547549(8 Apr 1982).

The problem of self-radiation of high-level radioactive waste by the transmutation of fission products caused by their $\beta$-decay is being studied by using ${ }^{104} \mathrm{Cs}$ in waste forms and other solids to simulate the behaviour of the long-lived ${ }^{107} \mathrm{Cs}$ and ${ }^{90} \mathrm{Sr}$ isotopes. In this preliminary report, after $2 \mathrm{yr}$ of decay, the results indicate negligible changes in the properties of the materials studied. 
402 Containing waste material. Ramm, E.J.; Ringwood, A.E. (to Australian Atomic Energy Commission Research Establishment, Lucas Heights; Australian National Univ., Canberra). Australian Patent 1981/72,825/A/. 1 Apr 1982. Filed date 15 Jul 1980 . vp.

A method is claimed for forming solid blocks of synthetic rock in which nuclear reactor waste is immobilised. It comprises: (a) establishing a quantity of material for forming the synthetic rock and a small proportion of radioactive waste in a heat and corrosion resistant metal canister: (b) applying pressure and heat to cause densification and the formation of a block of synthetic rock containing the waste; and (c) either before or after the densification step sealing the canister with a metal cap. The sealed canister can then be removed and placed in a suitable long term storage location.

403 Method and device for solidifying high level liquid wastes from a re-processing plant. Toshima, 1. (to IshikawajimaHarima Heavy Industries Co. Ltd., Tokyo (Japan)). Japanese Patent 57-37,299/A/. 1 Mar 1982. 3p. (In Japanese).

The purpose of this patent is to decrease the production of chlorides and reduce the amount of secondary liquid wastes by using low concentration nitric acid at a low radioactive level obtained from a condensator as scrubber feeding liquid. In a solidification device for high level liquid wastes from a re-processing plant in which off-gases resulted from solidification of high level liquid wastes from a re-processing plant are cleaned with gas cleaning liquids and returned while being concentrated to a high level liquid waste solidification stage, the off gases produced in the concentration stage are condensed and the condensed liquids are fed as the gas cleaning liquids.

404 CsAISis ${ }_{5} \mathrm{O}_{12}$ a possible host for ${ }^{107} \mathrm{Cs}$ immobilization. Adl, T.; Vance, E.R. (Pennsylvania State Univ., University Park (USA). Materials Research Labs.). Journal of Materials Science: 17: No. 3, 849-855(Mar 1982).

$\mathrm{CsAlSis}_{12}$ exhibits more acid resistance than pollucite ( $\mathrm{CsAlSi}{ }_{2} \mathrm{O}_{6}$ ). At $\mathrm{pH}$ values of 1.02 and 1.40 , the extraction of $\mathrm{Cs}$ from $\mathrm{CsAlSi}_{5} \mathrm{O}_{12}$ at $25^{\circ} \mathrm{C}$ was approximately proportional to the square root of leach time. The Cs extraction at $25^{\circ} \mathrm{C}$ varied as $\left[\mathrm{H}^{+}\right] \sup (0.36)$ over the $\mathrm{pH}$ range of 1 to 6 . Also, the $\mathrm{Cs}$ extraction in various brines at $300^{\circ} \mathrm{C}$ and $30 \mathrm{MPa}$ was comparable with that for pollucite. $\mathrm{CsAlSi}_{5} \mathrm{O}_{12}$ can be crystallized at about $1000^{\circ} \mathrm{C}$ from calcines if a small amount of $\mathrm{CaO}$ is present, but, in the absence of such sintering aids, crystallization temperatures of about $1400^{\circ} \mathrm{C}$ are necessary. Compatibility data were also obtained with respect to several other phases with which $\mathrm{CsAlSis}_{12}$ might be expected to co-xist in tailored ceramics designed for highlevel defense waste.

405 Synroc programme progresses. Reeve, K.D.; Levins, D.M.; Ramm, E.J.; Woolfrey, J.L. (Australian Atomic Energy Commission Research Establishment, Lucas Heights). Nuclear Engineering International; 27: No. 324, 26-27(Mar 1982).

Preliminary results with the in-can hot pressing rig at the Lucas Heights Research Laboratories, have confirmed that Synroc is a promising concept for radioactive waste immobilization. Results of leach tests and radiation experiments are reported.

406 Method of processing off-gas condensate containing precipitations resulted upon solidification of high-level liquid wastes issued from a reprocessing plant. Toyoshima, I. (to Ishikawajima-Harima Heavy Industries Co. Ltd., Tokyo (Japan)). Japanese Patent 57-29,999/A/. 18 Feb 1982. 4p. (In Japanese).

A method is proposed to facilitate the operation and stabilize the denitration process of a denitrating concentration stage upon solidification of high-level liquid wastes issued from a reprocessing plant. Highlevel liquid wastes issued from a reprocessing plant are denitrated and concentrated, mixed with glass material, sintered and then melted to thereby solidify the high-level liquid wastes. Off-gases generated upon solidification are cooled and the resulted liquid condensates are returend to the denitrating concentration stage. The cooled off-gases are further cooled and the liquid condensates resulted thereby are concentrated and then returned to the denitrating concentration stage. This enables to decrease the amount of precipitations in the liquid wastes which have to be treated in a concentrator and increase the degree of concentration. Further, since the concentration of the nitric acid in the secondary liquid wastes to be recycled can be adjusted to the same level as that of the nitric acid in the high-level liquid wastes, the operation for the denitrating operation system can be simplified and the denitrating process be stabilized.

407 Vitrification of nuclear waste with low heat generation. Tennant, M.H.; Murphree, B.E. (Du Pont de Nemours (E.I.) and Co., Aiken, SC (USA). Savannah River Lab.). Nuclear Engineering and Design; 67: No. 3, 415-423(Feb 1982). Analyses of thermal processes in the glass melter and storage container are given for vitrification of defense waste.

408 Method of processing high level radioactive wastes by glass solidification. Urakawa, T. (to Nippon Electric Glass Co. Ltd., Otsu). Japanese Patent 57-2000/A/. 7 Jan 1982. 3p. (In Japanese).

A method is proposed to enable complete and rapid issue and interruption of molten glass, as well as enable safety and reliable supply of high-level-waste-containing glass or crystallized glass into canister with no aids of manual operation. High level radioactive wastescontaining glasses are melted in a glass melting furnace which has a downwardly projecting heat resistance metal nozzle disposed to the bottom of the furnace, then flown out by passing electrical current to the nozzle and then supplied to a container placed under the nozzle. When the molten glass reaches a predetermined amount in the container, the electrical supply to the nozzle is interrupted and the nozzle is cooled. When the nozzle temperature lowers to such a level that is capable of cutting the glass, the flown out glass is cut by a cutter disposed near the lower end of the nozzle and, simultaneously, the pressure in the melting furnace is reduced to suck the molten glass in the nozzle thereby interrupting flowing out of the glass.

409 Nuclear waste vitrification in a ceramic-lined electric glass melter. Weisenburger, S. (Inst fuer Nukl Entsorgungstech, Karlsruhe, Ger). al; IA-18: No. 1, 73-82(Jan-Feb 1982).

Vitrification of high-level liquid waste from the nuclear fuel cycle in a liquid-fed electric glass melter is described with particular reference to the present status of the melter technology developed at the Institut fuer Nukleare Entsorgungstechnik (INE), Karlsruhe, West Germany. Based on available experience with simulated waste solutions. It is shown that electric melting of waste glass is a promising technique that can substantially simplify nuclear waste vitrification. 4 refs.

410 National program for isolating high-level nuclear waste. Smedes, H. Underground Space; 6: No. 4-5, 220-228(Jan 1982).

From Underground space conference and exposition; Kansas City, MO, USA (10 Jun 1981).

For some time, the US program for managing high-level radioactive waste has focused on the method of deep geologic isolation. The national objectives for waste isolation in this manner are summarized herein, and the status and outlook of the program designed to carry out the objectives are described. Attention is given to the goals and plans of the US Department of Energy, the departmentffis work is described, and key issues in siting a geologic repository for high-level waste are pinpointed.

411 Solidification of high-level waste. Harrington, R.V. (Ferro Corp., Independence, OH); Apps, J.A. Underground Space; 6: No. 4-5, 259-263(Jan 1982).

Various solid waste forms suitable for solidification of high-level radioactive waste were reviewed, and the properties of these are tabulated. Solid forms considered were glass, glass-ceramic, calcine, supercalcine, ceramic analogues to minerals, and cement. Borosilicate glass, which has been widely studied, appears to be highly satisfactory, but some of the other types of materials hold promise as fixatants. The choice of final form may be dictated on the basis of economics, process ing safety, or repository interaction rather than just on the basis of leachability. (BLM)

412 Leachability study of modified Synroc-B waste form: effect of gamma irradiation. Solomah, A.G.; Zumwalt, L.R. (North Carolina State Univ., Raleigh (USA)). Radiation Effects; 60: No. 1-4, 245-248(1982).

The effects of gamma irradiation on the chemical stability and particularly the leaching behavior of Modified SYNROC-B ceramic waste form for high level radioactive waste immobilization are pres- 
ented. Experimentally obtained leach rates for uranium, iron and cerium from $\gamma$-irradiated and unirradiated SYNROC-B are given. The results of the study are discussed. A decrease in $\mathrm{pH}$ is found with $\gamma$-irradiation due to radiolytic nitric acid generation. Higher initial leach rates with a more pronounced subsequent drop in the case of iron and cerium was observed with $\gamma$-irradiation than without irradiation. The effect of a $\mathrm{TiO}_{2}$ film and uranium leach rate behavior are also discussed.

413 Disposal of radioactive wastes. Dlouhy, Z. (Nuclear Research Institute, Rez, Czechoslovakia). Amsterdam, Netherlands; Elsevier Scientific (1982). 264p.

This book provides information on the origin, characteristics and methods of processing of radioactive wastes, as well as the philosophy and practice of their storage and disposal. Chapters are devoted to the following topics: radioactive wastes, characteristics of radioactive wastes, processing liquid and solid radioactive wastes, processing wastes from spent fuel reprocessing, processing gaseous radioactive wastes, fixation of radioactive concentrates, solidification of high-level radioactive wastes, use of radioactive wastes as raw material, radioactive waste disposal, transport of radioactive wastes and ewilumic problems of radioac tive wastes disposal.

414 Use of the drum dryer in radioactive waste solidification. Halaszovich, S.; Dix, S.; Merz, E. pp 455-457 of Jahrestagung Kerntechnik '82 - Reaktortagung 1982. Tagungsberichte. Sektion 4: Brennstotf kreislauf und Abfallbelıandluing. Eggenstein-Leopoldshafen, Germany, F.R.; Fachinformationszentrum Energie, Physik, Mathematik (1982). (In German)

From Annual meeting on nuclear technology '82; Mannheim, Germany, F.R. (4 - 6 May 1982).

Radioactive waste solutions with and without sintered glass powder were dried in a drum dryer of the common kind. The relations between drying temperature, content of solids in the suspension and drying performance have been studied with respect to an optimum fluid product. Two possible systems for the application of the suspensions on the drums have been developed.

415 Development and testing of SYNROC $\mathrm{C}$ as a high level nuclear waste form. Reeve, K.D.; Levins, D.M.; Ramm, E.J.; Woolfrey, J.L.; Buykx, W.J. (Australian Atomic Energy Commission Research Establishment, Lucas Heights). -881XJournal of the Australian Ceramic Society; 18: No. 1, 2-5(1982).

From Annual meeting of the Materials Research Society; Boston, MA, USA (16 - 19 Nov 1981).

The current status of SYNROC C research and development by the Australian Atomic Energy Commission is reviewed. A nonradioactive fabrication demonstration line designed to produce $10 \mathrm{~cm}$ o.d., $90 \mathrm{~cm}$ long, cylinders of SYNROC canned in stainless steel by the method of in-can hot pressing is being commissioned. Leach tests are proving the excellent leach resistance of SYNROC. Accelerated radiation damage testing using fast neutrons has simulated storage times of up to $6.7 \times 10^{5}$ years. Thermophysical properties of SYNROC have been measured over the temperature range 20 to $650 \mathrm{deg}$. $C$.

416 Iron-enriched basalt for containment of nuclear wastes. Welch, J.M.; Schuman, R.P.; Sill, C.W.; Kelsey, P.V. Jr.; Henslee, S.P.; Tallman, R.L.; Horton, R.M.; Owen, D.E.; Flinn, J.E. (EG and G Idaho, Inc.. Idaho Falls). pp 23-30 of Scientific basis for nuclear waste management. Tupp, S.V. (ed.). New York, NY; Elsevier Science Publishing Company, Inc. (1982). (CONF-811122-Pt.1). Contract AC0776ID01570。

From Annual meeting of the Materials Research Society; Boston, MA, USA (16 Nov 1981).

Iron-enriched basalt (IEB) has been investigated as a containment medium for defense transuranic (TRU) and defense high-level wastes (HLW). IEB samples have been fabricated incorporating simulated and actual defense TRU wastes generated at the Rocky Flats Plant (RFP), and simulated defense high-level wastes. These samples were tested for leaching resistance and fracture toughness. Cesium and strontium volatility from an IEB melt was also assessed, using radioaclively spiked samples. 2 figures, 4 tables.

417 XPS valence state determination of $\mathrm{Np}$ and $\mathrm{Pu}$ in multicomponent borosilicate glass and application to leached
76-68 waste glass surfaces. Karim, D.P. (Argonne National Lab., IL); Lam, D.J.; Diamond, H.; Friedman, A.M.; Coles, D.G.; Bazan, F.; McVay, G.L. pp 67-73 of Scientific basis for nuclear waste management. Topp, S.V. (ed.). New York, NY; Elsevier Science Publishing Company, Inc. (1982). (CONF811122-Pt.1).

From Annual meeting of the Materials Research Society; Boston, MA, USA (16 Nov 1981).

The present study involves the use of $x$-ray phutoemission spectroscopy (XPS) to examine the behavior of actinides at the surface of glasses. XPS is an inherently surface sensitive technique, probing the outermost 20 to $30 \mathrm{~A}$ of a sample. The technique is routinely used to provide quantitative compositional information by monitoring the intensity of photoemitted electrons from core levels characteristic of specific elements as a sample is illuminated with monochromatic $x$-rays. In some cases, small changes in the binding energies of these core levels (chemical shifts) can be used to get specific information on the environments and chemical states of ions in a solid. The results of the study show that chemical shifts in actinide $4 \mathrm{f}$ core levels can be related to valence state. In 76 to 101 glass, neptunium is present primarily in a single valence stated while plutonium is present in two distinct valence states (probably +3 and +4 ). The Ti $2 p_{3} 2 /$ core line seems to be a good internal reference for $\mathbf{4 f}$ chemical shift (and consequently valence state) determination. The valence band photoemission spectrum of the neptunium glass shows a quite narrow $\mathrm{Np5f}$ peak indicating a $\mathrm{f}^{0} \rightarrow \mathrm{f}^{2}$ transition resulting from the presenoo of $\mathrm{Np}^{+4} .4$ figures.

418 Development and testing of SYNROC. C. as a high level nuclear waste form. Reeve, K.D.; Levins, D.M.; Ramm, E.J.; Woolfrey, J.L.; Buykx, W.J. (Australian Atomic Energy Commission Research Establishment, Sutherland, Australia) pp 99-106 of Scientific basis for nuclear waste management. Topp, S.V. (ed.). New York, NY; Elsevier Science Publishing Company, Inc. (1982). (CONF-811122-Pt.1).

From Annual meeting of the Materials Research Society; Boston, MA, USA (16 Nov 1981).

The current status of SYNROC C research and devclopment by the Australian Atomic Energy Commission is reviewed. A nonradioactive fabrication demonstration line designed to produce $10 \mathrm{~cm}$ $0 . d ., 90 \mathrm{~cm}$ long, cylinders of SYNROC canned in stainless steel by the method of in-can hot pressing is being commissioned. Leach tests are proving the excellent leach resistance of SYNROC. Accelerated radiation damage testing using fast neutrons has simulated storage times of up to $6.7 \times 10^{5}$ years. Thermophysical properties of SYNROC have been measured over the temperature range 20 to $650^{\circ} \mathrm{C}$. 3 flgures, 1 table.

419 Respirable fines produced by impacts of simulated alternative high-level waste materials. Jardine, L.J.; Reedy, G.T.; Mecham, W.J. (Argonne National Lab., IL). pp 115-123 of Scientific basis for nuclear waste management. Topp, S.V. (ed.). New York, NY; Elsevier Science Publishing Company, Inc. (1982). (CONF-811122-Pt.1).

Fiuni Annual mecting of the Materials Research Socicty: Boston, MA, USA (16 Nov 1981).

Standardized comparative drop weight impact tests were conducted on solid alternative waste forms under consideration for immobilizing Sayannah River Laboratory (SRL) defense wastes. The fragment size distributions were theasured in the size ranges of $\sim 5$ to $8000 \mu \mathrm{m}$. All waste form fragment size distributions could be described accurately by lognormal plots. Respirable sizes $(\leq 10 \mu \mathrm{m}$ ) were measured. Borosilicate glass and SYNROC specimens yielded the same mass fractions of respirable sizes; FUETAP concrete, high silica and alkoxide glass specimens yielded $\sim 2$ to 3 times morc mass fractions of particles of respirable sizes, whcreas tailored (Spinel) ceramic specimens yielded the smallest mass fractions of respirable sizes, $\sim 2-1 / 2$ times less. 1 figure, 3 tables.

420 Methodology for characterizing brittle fracture of solid waste forms in accidental impacts. Mecham, W.J.; Jardine, L.J.; Steindler, M.J. (Argonne National Lab., IL). pp 125-131 of Scientific basis for nuclear waste management. Topp, S.V. (ed.). New York, NY; Elsevier Science Publishing Company, Inc. (1982). (CONF-811122-Pt.1). 
From Annual meeting of the Materials Research Society; Boston, MA, USA (16 Nov 1981).

A general method for characterizing the major practical effects of accidental impacts on waste packages and for evaluation of scalemodel tests has been partially developed. Impact fracture of brittle waste forms has been shown elsewhere to produce particulates whose size distributions are described by the lognormal probability distribution. The model proposed for fragment generation involves the transformation of impact (kinetic) energy into elastic strain energy which is followed by fracture and energy dissipation into heat by the fracture particulates. The peak stresses developing during compressions are approximated as a function of time using elastic theory for a wide range of practical impact conditions for typical (glass) waste forms. The proposed methodology requires experimental validation in terms of correlation of stress and energy parameters with particulate parameters describing the results of fracture. Two kinds of preliminary correlations are presented: (1) calculations of stress parameters for a range of impact conditions; and (2) averages of particulate parameters obtained in standard impact tests. 1 figure, 1 table.

421 Formulation and processing of polyphase ceramics for high level nuclear waste. Harker, A.B.; Morgan, P.E.D.; Clarke, D.R.; Flintoff, J.F. (Rockwell International Science Center, Thousand Oaks, CA). pp 567-573 of Scientific basis for nuclear waste management. Topp, S.V. (ed.). New York, NY; Elsevier Science Publishing Company, Inc. (1982). (CONF811122-Pt.1). Contract AC09-79ET41900.

From Annual meeting of the Materials Research Society; Boston, MA, USA (16 Nov 1981).

Two basic crystalline phase assemblages have been developed for incorporating the full range of Savannah River Plant waste compositions into polyphase ceramic forms. Both phase assemblages provide crystalline host phases, with stable mineral analogues, for all radionuclides in the waste. The first, an alumina based assemblage, immobilizes the radioactive elements in solid solutions of magnetoplumbite and uraninite with the bulk non-radioactive waste elements being present in spinel and nepheline. The second assemblage uses the titanate based zirconolite type fluorite structure and the alumina/iron based magnetoplumbite phases to host the radioactive nuclei with spinel and nepheline, again providing crystalline hosts for the non-radioactive elements. Both phase assemblages can be consolidated to a fine grain ceramic by hot isostatic pressing at $1040^{\circ} \mathrm{C}$ pressures from 20,000 to 30,000 psi. Redox control during processing, just sufficient to reduce uranium to the tetravalent state, is used. 3 figures, 1 table.

422 On-line measurements of the volatilization of ruthenium in a vitrification process. Odoj, R.; Schwitz, R. (Kernforschungsanlage, Juelich, Germany). pp $575-583$ of Scientific basis for nuclear waste management. Topp, S.V. (ed.). New York, NY; Elsevier Science Publishing Company, Inc. (1982). (CONF-811122-Pt.1).

From Annual meeting of the Materials Research Society; Boston, MA, USA (16 Nov 1981).

The studies were performed using Ru-103 as a tracer. The volatilization of $\mathrm{Ru}$ from ruthenium containing liquids, like HAW-solutions, is about $15 \%$. A chemical denitration of these solutions with $\mathrm{HCHO}$ or $\mathrm{HCOOH}$ reduces the volatilization to about $2 \%$. Additional catalytic reduction with $\mathrm{Fe}$-powder lowers the volatilization to about $4 \%$. The carry over of Ru seems to depend on the aerosol production rate and not the vapor pressure. Only $\mathrm{RuO}_{2}$ could be detected in the off-gas. 9 figures.

423 Leaching behaviour of ion-implanted simulated HLW glasses and tentative prediction of their alpha-recoil aging. Dran, J.C.; Langevin, Y.; Maurette, M.; Petit, J.C.; Vassent, B. (Laboratoire Rene Bernas, Orsay, France). pp 651-659 of Scientific basis for nuclear waste management. Topp, S.V. (ed.). New York, NY; Elsevier Science Publishing Company, Inc. (1982). (CONF-811122-Pt.1).

From Annual meeting of the Materials Research Society; Boston, MA, USA (16 Nov 1981).

The iun implantation-based simulation of $\alpha$-recoil aging in radwaste glasses has been applied to several simulated HLW glasses. The results are qualitatively described by a new model of leaching for the implanited glasses although several specific features of irradiated surfaces are nnt clearly understood. This model suggests that for the most likely case of diffusion-controlled corrosion, where the reaction scheme involves several steps (hydration, dissolution of hydrated silica gel, etc.. .), the kinetics-controlling one would not necessarily be radiation sensitive. Indeed for these more representative glasses, no evidence for a drastic increase in the dissolution rate of the silica gel, induced by ion implantation has been so far detected, whereas the hydration rate seems in some cases more significantly enhanced. However the implications of these observations for the durability of the glasses remain to be assessed. 3 figures, 1 table.

424 Influence of gamma irradiation on the leaching behavior of a modified SYNROC-B ceramic waste form. Solomah, A.G. (North Carolina State Univ., Raleigh). pp 727732 of Scientific basis for nuclear waste management. Topp, S.V. (ed.). New York, NY; Elsevier Science Publishing Company, Inc. (1982). (CONF-811122-Pt.1). Contract AC0980ET41902;AC09-81SR10957.

From Annual meeting of the Materials Research Society; Boston, MA, USA (16 Nov 1981).

The leaching behavior of a sintered Modified SYNROC-B ceramic waste form containing $10 \mathrm{wt} \%$ simulated high level radioactive waste, under the influence of $\gamma$-irradiation, has been investigated. Similar leaching experiments have been carried out in the absence of $\gamma$-irradiation, and the leach rates of $\mathrm{Ba}$ and $\mathrm{Cs}$ are presented for comparison studies. Acid/Base formation has been monitored through $\mathrm{pH}$ measurements of the leachants. A decrease in $\mathrm{pH}$ is found with $\gamma$-irradiation due to radiolytic nitric acid generation. Higher initial leach rates with a more pronounced subsequent drop in the case of $\mathrm{Ba}$ and $\mathrm{Cs}$ were observed with $\gamma$-irradiation than without irradiation. The results of the study are presented and discussed. 1 figure, 1 table.

426 Proceedings of the ANS topical meeting on the treatment and handling of radioactive wastes. Columbus, $\mathrm{OH}$; Battelle Press (1982). 681p. (CONF-820424-). NTIS, PC A99/MF A01. Order Number DE83007269.

From ANS topical meeting on treatment and handling of radioactive wastes; Richland, WA, USA (19 Apr 1982).

The objective of the meeting was to provide a thorough assessment of the status of technology. There were 123 papers presented in 17 sessions covering the treatment and associated handling of high-level, transuranic, and low-level liquid and solid wastes. Of the 123 papers, 80 were indexed separately, 39 have been previously processed, and the 4 papers from the plenary session on summary and trends were not indexed separately. (LM)

426 European Communities' $R$ and $D$ activities on treatment and handling of radioactive waste. Orlowski, S.; Simon, R. (Commission of the European Communities, Brussels, Belgium). pp 14-18 of Proceedings of the ANS topical meeting on the treatment and handling of radioactive wastes. Columbus, OH; Battelle Press (1982). (CONF-820424-).

From ANS topical meeting on treatment and handling of radioactive wastes; Richland, WA, USA (19 Apr 1982).

Since 1973 the Commission of the European Communities has been conducting research and development on the management and disposal of radioactive waste. The activities on treatment and handling presently concentrate on the processing and conditioning of hulls, ILLW, TRU waste and certain gaseous wastes. The important aspects of characterization and evaluation of final waste forms is being investigated under two separate parts of the CEC program.

427 National and cooperative program for waste management in France. Sousselier, Y. pp 22-26 of Proceedings of the ANS topical meeting on the treatment and handling of radioactive wastes. Columbus, OH; Battelle Press (1982). (CONF820424-).

From ANS topical meeting on treatment and handling of radioactive wastes; Richland, WA, USA (19 Apr 1982).

Conditioning processes developed in France are: vitrification of high-level radioactive wastes; embedding medium-level radioactive wastes into bitumen or thermnsettine resins; and incorporation into concrete. The French disposal policy and long-term industrial management policy are discussed briefly.

428 Status of high level and alpha bearing waste management in PNC. Uematsu, K. (Power Reactor and Nuclear Fuel 
Development Corp., Tokyo, Japan). pp 27-33 of Proceedings of the ANS topical meeting on the treatment and handling of radioactive wastes. Columbus, $\mathrm{OH}$; Battelle Press (1982). (CONF-820424-).

From ANS topical meeting on treatment and handling of radioactive wastes; Richland, WA, USA (19 Apr 1982).

High-level and alpha-bearing waste management in PNC is summarized. It is indispensible to find the solution of managing the radioactive waste in completing the nuclear fuel cycle in Japan. Thus, Japanese government, especially PNC in accordance with the decision of JAEC, is working hard for seeking the solution. Although progress in research and development in waste management has been remarkable, it is believed that the international cooperative efforts in waste management are necessary to achieve the goal of completing the nuclear fuel cycle.

429 Treatment and solidification of high active fission product solutions. Kuhn, K.D.; Bahr, W.B. (Wiederaufarbeitungsanlage Karlsruhe Betriebsgesellschaft mbH, Germany). pp 54-58 of Proceedings of the ANS topical meeting on the treatment and handling of radioactive wastes. Columbus, $\mathrm{OH}$; Battelle Press (1982). (CONF-820424-).

From ANS topical meeting on treatment and handling of radioactive wastes; Richland, WA, USA (19 Apr 1982).

On reprocessing spent fuel elements, $>97 \%$ of the fission products are found in the high active waste (HAW) solution. In order to avoid large amounts of sludge formation arising from phosphates produced by TBP degradation during evaporation and storage of these high level wastes, the suspended and dissolved TBP must be removed immediately from the HAW. It is proposed to separate the TBP by steamstripping. The the HAW will be concentrated in an evaporator, the concentration factor depending on the amount of sludge formation and the heat content of the concentrate. These concentrates may be stored for short periods in stainless steel tanks. Acid concentration and waste volume may be further reduced by in-tank denitration and evaporation. For vitrification of the HAW liquid feed, ceramic melters are being developed universally. The first active plant to use a liquid feed ceramic melter is the German plant PAMELA, which is being built at Mol in Belgium, with an operational date of 1985.

430 Selection and application of a processing system for the treatment of high level liquid waste in the UK. Clelland, D.W.; Corbet, A.D.W. (British Nuclear Fuels Ltd., Risley, England). pp 59-63 of Proceedings of the ANS topical meeting on the treatment and handling of radioactive wastes. Columbus, OH; Battelle Press (1982). (CONF-820424-).

From ANS topical meeting on treatment and handling of radioactive wastes; Richland, WA, USA (19 Apr 1982).

The historical background and the present status of technology in this field are reviewed and progress following the decision to implement vitrification at Windscale is reported. The choice of glass as a matrix is discussed. The process which has been selected for industrial scale operation and the characteristics of the vitrified waste are described. The design and handling of containers are indicated. Some of the principles relating to the storage of vitrified high level waste are discussed related to the method of storage to be employed in the UK. The rate of high level wastes arising in the UK together with present inventories and future conditioning rates are presented and the impact of vitrification on the future role of tank storage is discussed.

431 Development of high-level liquid waste solidification in PNC. Okamoto, H.; Yamamoto, M.; Nagai, H.; Ohnishi, N. (Power Reactor and Nuclear Fuel Development Corp., Tokyo, Japan). pp 64-71 of Proceedings of the ANS topical meeting on the treatment and handling of radioactive wastes. Columbus, OH; Battelle Press (1982). (CONF-820424-).

From ANS topical meeting on treatment and handling of radioactive wastes; Richland, WA, USA (19 Apr 1982).

$R$ and D activities on the solidification of HLLW and related technologies are being performed with the aim of designing and constructing the HLLW Solidification Pilot Plant at Tokai which is planned to start operations in 1987. Solidified waste forms and compositions have been studied with the aid of characterizing solidified products and evaluating solidification technologies, and two typical series of borosilicate glass products have been found to be feasible for the solidification of HLLW generated by the Tokai reprocessing plant. The HLLW Engineering Test Facility (ETF) with denitration and concentration equip- ment, two different types of Joule-heated ceramic melters, off-gas treatment equipment and a canister handling system, has been in operation since February 1980 in order to obtain various kinds of engineering data and perspectives. Air-cooling tests are also in progress to characterize natural or forced convection heat transfer in the storage of the HLLW solidified waste.

432 Industrial operating experience at the Marcoule vitrification facility. Chotin, M.M.; Hugony, P.; Pieraggi, A. (COGEMA, Marcoule, France). pp 72-75 of Proceedings of the ANS topical meeting on the treatment and handling of radioactive wastes. Columbus, OH; Battelle Press (1982). (CONF820424-).

From ANS topical meeting on treatment and handling of radioactive wastes; Richland, WA, USA (19 Apr 1982).

The Vitrification Facility (AVM) located at Marcoule is designed to vitrify fission product solutions from the Marcoule fuel reprocessing plant and has been in operation on an industrial basis since June 28, 1978. Extensive design precautions and sophisticated technological solutions have resulted in simplified operation. The facility has operated with a good duty factor. Nevertheless, the unit is an industrial prototype facility in which a number of problems encountered are discussed in this paper.

433 Reprocessing, decontamination and decommissioning wasle mauagement at Eurochomic: current and planned activities. Detillcux, E.; Hild, W.; van Geel, J. (Eurochemic, Mol, Belgium). pp 76-83 of Proceedings of the ANS topical meeting on the treatment and handling of radioactive wastes. Columbus, OH; Battelle Press (1982). (CONF-820424-).

From ANS topical meeting on treatment and handling of radio active wastes; Richland, WA, USA (19 Apr 1982).

Since the shutdown of its reprocessing activities, Eurochemic has undertaken the decontamination of the reprocessing plant and the management of the generated wastes. The decontamination has been satisfactorily completed, allowing access to all process cells. The waste management program, still going on, includes intermediate, and high-level liquid waste treatment by bituminization and vitrification, respectively. Other activities involved spent solvent destruction, the digestion into acids of alpha-bearing solid wastes, elc. The paper outlines the main features of the program.

434 West Valley Demonstration Project: strategy for an achievable but challenging project. Hamric, J.P. (Dept. of Energy, Idaho Falls, ID). pp 555-557 of Proceedings of the ANS topical mecting on the treatment and handling of radinactive wastes. Columbus, OH; Battelle Press (1982). (CONF820424-)

From ANS topical meeting on treatment and handling of radioactive wastes; Richland, WA, USA (19 Apr 1982).

The current status of the West Valley facilities is summarized. The spent fuel receiving and storage facility is currently operating with pool filled to about two-thirds capacity with 750 spent fuel assemblies (164 MTU). The reprocessing plant $\left(80,000 \mathrm{ft}^{2}\right)$ is maintained in a shutdown conditin (alpha: $200 \mathrm{Ci}$; beta-gamma: 11,700 Ci). In high level liquid waste storage there are two 750,000 -gal neutralized waste tanks. One tank holds about $560,000 \mathrm{gal}$ of waste; the other is a spare. There are two 15,000-gal acid waste tanks. One tank holds about $12,000 \mathrm{gal}$ of wastei the other is a spare. The NRC.-licensed facility burial ground contains $139,000 \mathrm{ft}^{0}$ of wastes buried up to $50 \mathrm{ft}$ deep. 'I'he burnal groünd contains ruptured spent fuel encased in concrete and is currently being used to dispose of plant-generated waste only. The NYS-licensed burial ground contains 2.4 million $\mathrm{ft}^{0}$ of burial waste. Operation of this burial ground ceased in 1975 due to trench water overflow. Corrective measures were taken.

435 Present status of the West Valley Demonstration Project. Knabenschuh, J.L. pp 558-560 of Proceedings of the ANS topical meeting on the treatment and handling of radioactive wastes. Columbus, OH; Battelle Press (1982). (CONF820424-).

From ANS topical meeting on treatment and handling of radioactive wastes; Richland, WA, USA (19 Apr 1982).

The West Valley Demonstration Project is a reality. The WVNS staff is essentially up to the full strength anticipated for FY 82. The West Valley Nuclear Service, $\mathrm{CO}_{0}$, in intimate cooperation with the 
Department of Energy, is actively and intensely engaged in the front end engineering, as well as the planning scheduling and budgeting for the West Valley Demonstration Project to achieve a cost-effective and early demonstration of commercial high-lcvel waste immobilization to assist in demonstrating in this country that the back end of the nuclear fuel cycle can be safely and successfully closed.

\section{$436 \quad$ Evaluation of process alternatives for solidification of} the West Valley high-level liquid wastes. Holton, L.K.; Larson, D.E. (Pacific Northwest Lab., Richland, WA). pp 561-565 of Proceedings of the ANS topical meeting on the treatment and handling of radioactive wastes. Columbus, $\mathrm{OH}$; Battelle Press (1982). (CONF-820424-). Contract AC06-76RL01830.

From ANS topical meeting on treatment and handling of radioactive wastes; Richland, WA, USA (19 Apr 1982).

The Department of Energy (DOE) established the West Valley Solidification Project (WVSP) in 1980. The project purpose is to demonstrate removal and solidification of the high-level liquid wastes (HLLW) presently stored in tanks at the Western New York Nuclear Service Center (WNYNSC), West Valley, New York. As part of this effort, the Pacific Northwest Laboratory (PNL) conducted a study to evaluate process alternatives for solidifcation of the WNYNSC wastes. Two process approaches for waste handling before solidification, together with solidification processes for four terminal and four interim waste forms, were considered. The first waste-handling approach, designated the salt/sludge separation process, involves separating the bulk of the nonradioactive nuclear waste constituents from the radioactive waste constituents, and the second waste-handling approach, designated the combined-waste process, involves no waste segregation prior to solidification. The processes were evaluated on the bases of their (1) readiness for plant startup by 1987, (2) relative technical merits, and (3) process cost. The study has shown that, based on these criteria, the salt/ sludge separation process with a borosilicate glass waste form is prefcrred when producing a terminal waste form. It was also concluded that if an interim waste form is to be used, the preferred approach would be the combined waste process with a fused-salt waste form.

437 Evaluation of the AVM process and its applicability to West Valley. Cameron, W.A.; Merlin, S. pp 566-570 of Proceedings of the ANS topical meeting on the treatment and handling of radioactive wastes. Columbus, $\mathrm{OH}$; Battelle Press (1982). (CONF-820424-).

From ANS topical meeting on treatment and handling of radioactive wastes; Richland, WA, USA (19 Apr 1982).

The CEA, the French Atomic Energy Commission, has adopted borosilicate glass as a safe, practical, reasonably economic, and adequate method for solidifying high level nuclear wastes. Based upon the information available to us we believe this is an appropriate technology for WVDP. Further, the CEA is most interested in supporting the efforts of DOE to demonstrate the capability for safe disposal of such waste. We recognize that a great deal of very significant work has been done on pluctess development in the country, and that work has been directed toward the specific US problem of neutralized waste. It is clear that in the normal course of events there is a strong and proper predisposition to utilize the technology developed in this country. It is possible, however, that under constraints dictated by time and/or budget, orlicensing (or quasi-licensing) proceedings, it should be advantageous to evaluate the alternate possibility of utilizing cxisting technology with a strong data base, available test facilities, man years of available technical experience, etc. In the final analysis the question can be resolved hy reviewing estimatos of total time and cust and the projected end product.

438 Synthesis of alkoxide glass formers for nuclear waste immobilization. Lahoda, E.J.; Pope, J.M. (Westinghouse Research and Development Center, Pittsburgh, PA). pP 577580 of Proceedings of the ANS topical meeting on the treatment and handling of radioactive wastes. Columbus, $\mathrm{OH}$; Battelle Press (1982). (CONF-820424-).

From ANS topical meeting on treatment and handling of radioactive wastes; Richland, WA, USA (19 Apr 1982).

Westinghnuse has developed a novel method which utilizes alkoxide-derived compounds instead of glass frit to vitrify high level waste. A conceptual design for synthesizing the starting alkoxide compounds is presented in terms of mass balances, flow sheets, equipment requirements, investment and operating costs. Based on the PROVES computer program, the cost of the alkoxide-derived glass formers is about $\$ 3.92$ per kilogram. The capilal cost of a plant producing 318 kilograms of glass formers per hour is about \$11.5 million.

439 High-level waste vitrification technique in a full-scale pilot plant. Weisenburger, S. (Karlsruhe Nuclear Research Centre, Germany). pp 190-196 of Proceedings of the ANS topical meeting on the treatment and handling of radioactive wastes. Columbus, OH; Battelle Press (1982). (CONF820424-).

From ANS topical meeting on treatment and handling of radioactive wastes; Richland, WA, USA (19 Apr 1982).

The liquid-fed ceramic melter process is reviewed and evaluated. Process application in a full-scale cold pilot plant, currently under construction in the Institute fuer Nukleare Entsorgungstechnik (INE), is described. It is concluded that the process has high potential for use in future industrial vitrification plants.

440 Utilization of a drum drier for the conditioning of radioactive wastes. Halaszovich, S.; Dix, S.; Merz, E. (Inst. for Chemical Technology, Kernforschungsanlage, Juelich $\mathbf{G m b H}$, Germany). pp 214-217 of Proceedings of the ANS topical meeting on the treatment and handling of radioactive wastes. Columbus, OH; Battelle Press (1982). (CONF-820424-).

From ANS topical meeting on treatment and handling of radioactive wastes; Richland, WA, USA (19 Apr 1982).

Arguments are presented for the preference of a separate drying step in the solidification process of liquid wastes. In particular, the possibilities and advantages of the utilization of a drum drier for the conditioning of high- and medium-level wastes are described, referring to the experience gained in its conventional utilization underlined by practical use and experiments. Main aspects discussed are handling and safety, variety of possible products, throughput and economy. Finally, the future work is outlined.

441 Microstructures and crystallization in simulated waste glasses. Skokan, A.; Schauer, V. (Kernforschungszentrum, Karlsruhe, Germany). pp 218-223 of Proceedings of the ANS topical meeting on the treatment and handling of radioactive wastes. Columbus, OH; Battelle Press (1982). (CONF-820424-).

From ANS topical meeting on treatment and handling of radioactive wastes; Richland, WA, USA (19 Apr 1982).

A borosilicate glass has been developed as a solidification matrix for high level radioactive waste solution (HAW) at the Karlsruhe Nuclear Research Centre. As a part of product characterization, the microstructures and the crystallization behavior of the glass products were investigated in the range from 350 to $750^{\circ} \mathrm{C}$ with annealing times up to 10,000 hours. In addition to ruthenium oxide and palladium alloys that are insoluble in the melt, two major silicate phases were observed after thermal treatment. The muximum degree of crystallinity measured by $x$-ray diffraction analysis was between 20 and $30 \%$ at $650^{\circ} \mathrm{C}$. Heat treatment below the transition temperature of the glass led to no appreciable crystallization.

442 Low level and intermediate level wastes management practice in France. Faussat, A. pp 243-248 of Proceedings of the ANS topical meeting on the treatment and handling of radioactive wastes. Columbus, $\mathrm{OH}$; Battelle Press (1982). (CONF820424-).

From ANS topical meeting on treatment and handling of radioactive wastes; Richland, WA, USA (19 Apr 1982).

Having been directly in charge of waste disposal since 1979, ANDRA has already drawn some conclusions from the past experience obtained in shallow-land repositories. The regulations which exist in France for creating and operating a Basic Nuclear Installation provide a good framework for managing a shallow-land repository safely. Almost all short-lived wastes produced in the french nuclear program may be disposed of in shallow-land repositories and only alpha wastes and high level waste need a geological repository. Some special kinds of wastes might be better disposed of by sea dumping (tritium-bearing wastes, for instance) or in geological formations (high-level activated materials). Shallow-land repositories do not need a large surface of land enven if little dedicated to reducing volume. For instance, only 30 to 40 hectares are needed to dispose of the overall production of short-lived wastes in the year 2000. The use of shielded transportation casks instead of con- 
crete integrated drums will reduce the volume of waste to be stored from the new electronuclear plants. The cost of disposal charged to ANDRA's customers is not very high. The average disposal costs for standardized package in 1981 were about: $\$ 300 / \mathrm{m}^{0}$ for wastes disposed in tumuli; $\$ 500 / \mathrm{m}^{0}$ for contact wastes disposed in normal concrete; and $\$ 1000 / \mathrm{m}^{0}$ for remote handled wastes disposed of in special trenches. Although all the technical aspects of shallow-land repositories are satisfactory, and while few problems arise with the population living around a repository already operational for a long time, some public opposition still remains to the creation of new disposal facilities - just as with any new nuclear installation. Basically, public option acceptance remains the only true bottleneck in the radwaste disposal.

443 Department of Energy's selection of high level waste forms. Walton, R.D. Jr. (Dept. of Energy, Washington, DC); Wilson, W.B.; Gordon, D.E. pp 313-317 of Proceedings of the ANS topical meeting on the treatment and handling of radioactive wastes. Columbus, OH; Battelle Press (1982). (CONF. 820424-).

From ANS topical meeting on treatment and handling of radioactive wastes; Richláno, WA, USA (19 Apir 1982).

Several candidate waste forms have been investiguled under US Department of Energy (DOE) programs as putential media for the immobilization and geologic disposal of high-level radioactive waste (HI,W) resulting from chemical processing nuclear reactor fuels and targets. Two of these HLW forms wete selected at tlit end of flsoul year (FY) 1981 for further development. Borosilicate glass was continued as the reference waste form and a crystalline ceramic material, Synroc; was selected as the alternative form. The selection of one of thes two forms for production in the Defense Waste Processing Facility (DWPF) at the Savannah River Plant site will be made before the end of FY 1983. This paper focuses on the selection of these two waste forms, including discussions on the historical perspectives of HLW treatment and disposal, the Materials Characterization organization, and the evaluation method used in the selection process.

444 Demonstration of SYNROC fabrication scaleup on a non-radioactive hasis. Ramm, E.J.; Reeve, K.D. (AAEC Research Establishment, Sutherland, Australia). pp 318-323 of Proceedings of the ANS topical meeting on the treatment and handling of radioactive wastes. Columbus, OH; Battelle Press (1982). (CONF-820424-).

From ANS topical meeting on treatment and handling of radioactive wastes; Richland, WA; USA (19 Apr 1982)

SYNROC fabrication development at Lucas Heights is based on hot uniaxial pressing, and a method known as in-can hut pressing was devised for the first scaleup demonstration. The present in-can hot press consists of a 1-m-long induction furnace into which a 115-mm-diam stainless steel tube can be fitted and provided with lateral support, together with associated hydrauics. Five $30-\mathrm{kg}$ canisters of dense SYNROC-B have been produced and the method is considered suitable for further development. Current plans for continuing SYNROC process development at Lucas Heights are outlined.

445 Leaching behavior of sintered modified SYNROC-B waste forms: effect of HLW addition. Solomah, A.G. (North Carolina State Univ., Raleigh). pp 331-334 of Proceedings of the ANS topical meeting on the treatment and handling of radioactive wastes. Columbus, $\mathrm{OH}$; Battclle Press (1982). (CONF-820424-).

From ANS topical meeting on treatment and handling of radioactive wastes; Richland, WA, USA (19 Apr 1982).

I eachability studies for sintered Modifled SYNROC-B wasto forms containing up to $20 \mathrm{wt} \%$ simulated high-level radioactive waste have been investigated. Significant decreases in the leach rates of $\mathrm{Ba}$, $\mathrm{Ca}$, and $\mathrm{Cs}$ are noticed with the increase in waste loadings. The values of leach rates for $\mathrm{Cs}$ in deionized $\mathrm{H}_{2} \mathrm{O}$ at $25^{\circ}$ Care 0.007 and 0.001 $\mathrm{g}-\mathrm{m}^{2}-\mathrm{d} \mathrm{a}$ ! for 10 and $20 \mathrm{wt} \%$ simulated radioactive waste respectively. Barium and calcium showed similar leaching behavior. The results are presented and discussed.

446 Improved polyphase ceramic for high-level defense waste. Harker, A.B.; Morgan, P.E.D.; Flintoff, J.; Clarke, D.R. (Rockwell International Science Center, Thousand Oaks, CA). pp 335-340 of Proceedings of the ANS topical meeting on the treatment and handling of radioactive wastes. Columbus, $\mathbf{O H}$;
Battelle Press (1982). (CONF-820424-). Contract AT03$81 S F 11572$.

From ANS topical meeting on treatment and handling of radioactive wastes; Richland, WA, USA (19 Apr 1982).

Modifications of the chemical formulation and processing of the Synroc-D polyphase ceramic for defense waste have been studied to provide greater flexibility with respect to compositional variations in the waste and to improve leach resistance. It has been demonstrated that by applying only that amount of reduction to the waste required to produce uranium in the $4+$ state and by using lower consolidation temperatures, an improved ceramic can be formed. The resulting ceramic consolidated at $1040^{\circ} \mathrm{C}$ and 10,000 psi maintanis the Synroc-D zirconolite, perovskite and nepheline phases; however, the two Synroc-D spinel phases are replaced with a single magnetite-type spinel and two additional radiophases, magnetoplumbite, and a cubic murataite-type phase. This modified phase assemblage provides crystalline ost sites for all radionuclides and trace elements in SRP waste, minmizes amorphoous intergranular material, and shows superior leach resistance.

447 High level waste forms: glass marbles aud thermal spray coutinge. Treat, R I, ; Oma. K.H,; Slate, S.C. (Pacific Northwest Lab., Richland, WA). pp 341-345 of Proceedings of the ANS topical meeting on the treatment and handling of radioactive wastes. Columbus, $\mathrm{OH}$; Battelle Press (1982). (CONF.820424-).

Fišn ANE topicul ureetliug on trantment and bandling of radio active wastes; Richland, WA, USA (19 Apr 1982).

A process that converts high-lcvel waste to glass marbles and then coats the marbles has been developed at Pacific Northwest Laboratory (PNL) under sponsorship of the US Department of Energy. The process consists of a joule-heated glass melter, a marble-making device based on a patent issued to Corning Glass Works, and a coating system that includes a plasma spray coater and a marble tumbler. The process was developed under the Alternative Waste Forms Program which strived to improve upon monolithic glass for immobilizing high-level wastes. Coated glass marbles wcrc found to be more leach-resistant, and the marbles, before coating were found to be very homogeneous, highly impact resistant, and conductive to encapsulation in a metal matric for improved heat transfer and containment. Marbles are also ideally suited for quality assurance and recycling. However, the marble process is more complex, and marbles require a larger number of canisters for waste containment and have a higher surface area than do glass monoliths.

448 Fraluation of coated particle waste forms. Gulden T.D.; Erlandson, O.D.; Kaae, J.L.; Kovacs, W.J. (Goneral Atomic Co., San Diego, CA). pp 346-350 of Proceedings of the ANS topical meeting on the treatment and handling of radionctive wastes. Columbus, OH; Battelle Press (1982). (CONF820424-).

From ANS topical meeting on treatment and handling of radioactive wastes; Richland, WA, USA (19 Apr 1982).

An in-depth evaluation of the technical merits and engineering practicality of coater particles. based on sol gel processes, as a candidate waste form has been performed. The results of the study showed that coated particle waste forms, because of the exceptional chemical inertness of the carbon and silicon carbide coatings, have the potentional for nearly absolute containment of radionuclides for geologic time pervids. The proccssos, although sumewhat complex, are amenable th remote operation, and have considerable potential for further development and-simplification. Coated particle fuels have inherent advantages for quality control sampling and are readily recycled for rework.

449 Remote aspects of treating and handling radioactive wastes. Wegncr, K. (NUKEM GmbH, Hanau, Germany). pp 423-427 of Proceedings of the ANS topical meeting on the treatment and handling of radioactive wastes. Columbus, $\mathrm{OH}$; Battelle Press (1982). (CONF-820424-).

From ANS topical meeting on treatment and handling of radioactive wastes; Richland, WA, USA (19 Apr 1982).

Carrying out detailed handling analysis on process components is the only way to get a fundamental basis for the choice of handling concepts. It is necessury to set up methods for the procedure of handling anlaysis in a systematic, uniform and checkable way. Large hot cell concepts promise a lot of advantages, but these concepts live and die with 
the availability of suitable handling equipment. High emphasis has to be put into the development of this special equipment.

450 Spray calcination of SRP waste compositions for ceramic waste forms. Grantham, L.F.; Flintoff, J.F. (Rockwell International Corp., Canoga Park, CA). pp 445-448 of Proceedings of the ANS topical meeting on the treatment and handling of radioactive wastes. Columbus, $\mathrm{OH}$; Battelle Press (1982). (CONF-820424-).

From ANS topical meeting on treatment and handling of radioactive wastes; Richland, WA, USA (19 Apr 1982).

A heated-gas calciner produces a reactive powder suitable for ceramic immobilization of nuclear waste. This spray calciner has the added advantages of simultaneously destroying nitrates and organics in the waste. In addition, chemical tailoring as well as valence control were also accomplished during the calcination step. Though spray calcination remains the reference calcination method. Rockwell and LLNL are investigating alternate calcination methods for ceramic immobilization of nuclear waste.

$451 \quad$ High level waste management at the Idaho Chemical Processing Plant. Berreth, J.R.; Dickey, B.R. (Exxon Nuclear Idaho Co., Idaho Falls, ID). pp 449-454 of Proceedings of the ANS topical meeting on the treatment and handling of radioactive wastes. Columbus, OH; Battelle Press (1982). (CONF820424-).

From ANS topical meeting on treatment and handling of radioactive wastes; Richland, WA, USA (19 Apr 1982).

This paper summarizes past and current high-level waste management practices at the Idaho Chemical Processing Plant (ICPP); options for long-term waste management of existing and future waste calcine are also emphasized.

452 New waste calcining facility at ICPP. Smith, R.R.; Bingham, G.E.; Bench, F.D. (Exxon Nuclear Idaho Co., Idaho Falls, ID). pp 455-458 of Proceedings of the ANS topical meeting on the treatment and handling of radioactive wastes. Columbus, OH; Battelle Press (1982). (CONF-820424-).

From ANS topical meeting on treatment and handling of radioactive wastes; Richland, WA, USA (19 Apr 1982).

A New Waste calcining Facility (NWCF) has been constructed at the Idaho Chemical Processing Plant (ICPP) to replace the Waste Calcining Facility (WCF) that was built to demonstrate fluidized-bed solidification of highly radioactive wastes. The NWCF is designed to provide capability for processing a variety of waste comositions at a higher throughput, more corrosion-resistant materials of construction, more effective cleanup of effluent streams, and extensive remote maintenance and equipment replacement capability. The NWCF also incorporates extensive decontamination capability in the event contact maintenance becomes necessary. Construction was completed in December 1980 and testing is approximately $95 \%$ complete, with completion in the spring of 1982. Hot operation is scheduled to begin in mid-year 1982.

453 Control of semivolatile radionuclides in gaseous effuents at nuclear facilities. Vienna, Austria; IAEA (1982). 56p.

An up-to-date review is presented of the subject, combining the results of laboratory studies on control of the most important semivolatile radionuclides in gaseous effluents at nuclear facilities and the results of operating experience in that area. Ruthenium is the most significant semivolatile contaminant in gaseous effluents at nuclear facilities. Volatilization of ruthenium can be reduced by various means, in particular by adding reductants. Volatilized ruthenium can be retained by adsorbents such as silica gel and ferric-oxide-based materials. Decontamination factors in the order of $10^{\circ}$ have been obtained with these adsorbents under optimum conditions. Volatilized ruthenium can also be removed by other equipment such as condensers and scrubbers. Experience with high-level liquid waste solidification plants has shown that, in general, ruthenium volatilization is in the order of $10 \%$ or more unless special treatment is undertaken. There is little experience with ruthenium adsorbers in plants. Silica gel seems to have performed best, with ruthenium decontamination factors of about $10^{2}$ to $10^{n}$. Huwever, feed-tostack ruthenium decontamination factors of $10^{9}$ or more have been obtained even without ruthenium adsorbers. Other semivolatiles are relatively insignificant under normal conditions because of a low level of volatilization potential or mass or activity in the inventory. Moreover, owing to particulate formation, they can be easily removed without specific equipment.

464 Microstructure of Synroc. Cousens, D.R.; Myhra, S.; Penrose, J.; Segall, R.L.; Smart, R.St.C.; Turner, P.S. (Griffith Univ., Queensland, Australia). pp 309-318 of Scientific basis for nuclear waste management V. Lutze, W. (ed.). New York, NY; North-Holland (1982). (CONF-820636-).

From 5. international symposium on the scientific basis for radio active waste management; Berlin, F.R. Germany (7 Jun 1982).

An investigation of the microstructure of Synroc B prepared by the oxide route has shown that minor phases are present to a significant extent. Among these is a modified form of zirconolite which consists of an intergrowth of normal zirconolite with a derivative structure of the same phase. This provides an additional mechanism for the flexible uptake of foreign elements into zirconolite. Synroc $\mathrm{C}$ is a complex multiphase material and radwaste additions significantly affect the final form of the titanate ceramic. These modifications of Synroc B may be beneficial, the finer grain and phase size seem advantageous, on the other hand if there were segregation of a critical waste element into a soluble miner phase this could be a serious problem. In Synroc B and Synroc C the distribution of phases shows systematic minor variation on the scale of tens of microns, probably as the result of initial local compositional variations in the mixture of oxide starting materials.

466 SIMS depth profiling studies of sphene-based ceramics and glass ceramics leached in synthetic groundwater. Hayward, P.J.; Hocking, W.H.; Doern, F.E.; Cecchetto, E.V. (Atomic Energy of Canada Limited, Pinawa, Manitoba). pp 319-328 of Scientific basis for nuclear waste management $V$. Lutze, W. (ed.). New York, NY; Horth-Holland (1982). (CONF-820636-).

From 5. international symposium on the scientific basis for radioactive waste management; Berlin, F.R. Germany (7 Jun 1982).

Glass ceramics and ceramics based on the mineral sphene (CaTiSiO $)_{5}$ are being developed to host the wastes arising from possible future CANDU fuel reprocessing. Results from leaching tests in deionized water and in synthetic groundwater indicate that these materials are highly durable. Secondary Ion Mass Spectrometry (SIMS) depth profiling of leached specimens suggests that leaching in the glass ceramics is predominantly confined to the glass phase. The high ionic strength and composition of the groundwater have a significant passivating effect on leaching and surface alteration phenomena, and encourage the precipitation of new phases on the ceramic surface. Leaching results, scanning electron microscope (SEM) observations and SIMS depth profile measurements are compared and discussed.

456 Leaching of natural and synthetic sphene and perovskite. Metson, J.B. (Univ. of Western Ontario, London); Bancroft, G.M.; Kanetkar, S.M.; Nesbitt, H.W.; Fyfe, W.S.; Hayward, P.J. pp 329-338 of Scientific basis for nuclear waste management V. Lutze, W. (ed.). New York, NY; Horth-Holland (1982). (CONF-820636-).

From 5 . international symposium on the scientific basis for radioactive waste management; Berlin, F.R. Germany (7 Jun 1982).

The leach rates of natural and synthetic sphenes in deionized water are initially between $10^{-8}$ and $10^{-9} \mathrm{~kg} \mathrm{~m}^{-2} \mathrm{~s}^{-1}$ and fall by an order of magnitude over 28 days. Natural crystalline and ceramic, and $\mathrm{CaTiSiO}_{5}$ glass show maximum leach rate differences of less than an order of magnitude over the 60-day time interval considered here. Perovskite leach rates from synthetic and naturally occurring single crystals, show similar initial rates to the more resistant sphenes and a parrallel decline in leach rate with time. Small but consistent weight gains are observed for ceramic samples leached in quartz-saturated/500 ppM $\mathrm{Ca}^{2+}$ solutions. Weight gains in sphene leaching experiments in a synthetic saline groundwater were observed. Surface studies on a leached CaTiSiOs glass show rapid loss of $\mathrm{Ca}^{2+}$ and $\mathrm{Si}^{4+}$ from the surface of the glass. After 8 days in deionized water at $90^{\circ} \mathrm{C}$ the zone of calcium and silica depletion extends to a depth of at least $200 \mathrm{~nm}$. There is evidence that the initial selectively leached layer, as observed after 1 to 24 hours leaching, has been replaced by a reprecipitated $\mathrm{TiO}_{2}$ layer of much greater depth after 8 days leaching. The leaching of crystalline sphenes in deionized water could be expected to follow the same path, as similar bulk leach rates results are observed. For natural crystalline materials however, our experiments indicate that the problem of obtaining a consistent surface on polished discs makes it difficult to resolve 
whether the same behavior occurs. Fractured surfaces, used extensively in surface studies of glass leaching, minimize surface modification and offer better prospects for characterization by surface techniques.

457 Attempt to assess the long-term crystallization rate of nuclcar waste glasses. Jacquet-Francillon, N.; Pacaud, F.; Queille, P. (CEA Valrho, Bagnols sur Ceze, France). pp 249259 of Scientific basis for nuclear waste management $V$. Lutze, W. (ed.). New York, NY; North-Holland (1982). (CONF820636-).

From 5. international symposium on the scientific basis for radioactive waste management; Berlin, F.R. Germany (7 Jun 1982).

There exists an empirical Arrhenius type relationship between time and temperature at maximum crystallization for temperatures above $T_{g}$; the apparent activation energy is lowered by an increase in the number of crystalline phases forming in the glass. A maximum crystallized volume fraction can be calculated by measuring the devitrification heat output either by isothermal calorimetry or by slow DSC. The volumetric devitrification heat is largely independent of the nature of the crystals formed. However, the microcalorimetric sensitivity limits this method to glasses which devitrify hy more than 2 to $3 \%$ of their volume in a short time. The utilization of these empirically calculated relationships for the determination of a crystallization rate below the glass transformation temperature, $T_{g}$, should be done with great caution, because at this temperature other phenomena control changes of the glass structure such as devitrification.

458 Quantitative determination of crystalline phases in nuclear waste glasses. Feld, R.H.; Stammler, M. (BattelleInstitut e.V., Frankfurt, Germany). pp 262-271 of Scientific basis for nuclear waste management $V$. Lutze, W. (ed.). New York, NY; North-Holland (1982). (CONF-820636-).

From 5 . international symposium on the scientific basis for radioactive waste management; Berlin, F.R. Germany (7 Jun 1982).

It could be shown that it is possible to determine the weight fractions of crystalline substances dispersed in a glass matrix. The error is about $10 \%$ of the actual content for weight fractions between 4 and 10 wt $\%$. It is about $20 \%$ for weight fractions between 1 and 4 wt $\%$ and up to $100 \%$ for weight-fractions less than $1 \mathrm{wt} \%$. Stable phases like ruthenium, were in fact determined with the expected weight fractions. Chemical reactions which may occur during sintering can be followed quantitatively. This method appears to be well suited for determining the recrystallization behavior of nuclear waste glasses. We learned during the conference that a similar procedure is applied by the CEA in characterize French HLW-glasses.

$459 \quad$ Fracture appraisal of large scale glass blocks under realistic thermal conditlons. Laude, F.; Vcrnaz, E.; SaintGaudens, M. (CEA Valrho, Bagnols sur Ceze, France). pp 239247 of Scientific basis for nuclear waste management V. Lutze, W. (ed.). New York, NY; North-Holland (1982). (CONF820636-).

From 5. international symposium on the scientific basis for radioactive waste management; Berlin, F.R. Germany (7 Jun 1982).

The theoretical studies show that it is possible to calculate the stresses created for different cooling histories, but it is difficult to evaluate quantitatively the state of fracture of a glass, because this depends on the presence and distribution of inclusions and faults. The experimental studics ooncerning latge glass blocks allow order of magnitude estimates of fracturing. It is difficult to avoid such fracturing, especially during the cooling period after casting in a hot cell. Short periods of reheating may cause the physical reconstitution of the glass. The heat created by fission product decay may contribute to this process.

460 Investigation on the oxidation state and the behavior of molybdenum in silicate glass. Horneber, A. (Univeı sitaet Erlangen-Nuernberg, Germany); Camara, B.; Lutze, W. pp 279-288 of Scientific basis for nuclear waste management $V$. Lutze, W. (ed.). New York, NY; North-Holland (1982). (CONF-820636-).

From 5. international symposium on the scientific basis for radio active waste management; Berlin, F.R. Germany (7 Jun 1982).

This paper deals with the following: (1) Oxidation state of Mo when organic reducing agents, i.e., formic acid, formaldehyde, tannic acid, are added to the batch. (2) Dependence of Mo solubility on the melting atmosphere. (3) Behavior of the different oxidation states of Mo depending on the temperature and the basicity of the glass. (4) Redox interaction of Mo with $\mathrm{Fe}, \mathrm{Cr}$ and $\mathrm{Ti}$.

461 Effect of $\mathrm{Fe}_{2} \mathrm{O}_{0} / \mathrm{ZnO}$ on two glass compositions for solidification of Swedish nuclear wastes. Nogues, J.L. (Universite des Sciences et Techniques du Languedoc, Cedex, France); Hench, L.L. pp 273-278 of Scientific basis for nuclear waste management $V$. Lutze, W. (ed.). New York, NY; NorthHolland (1982). (CONF-820636-).

From 5. international symposium on the scientific basis for radioactive waste management; Berlin, F.R. Germany (7 Jun 1982).

Replacement of half of the $\mathrm{ZnO}$ in an alkali-zinc-borosilicate nuclear waste glass with $\mathrm{Fe}_{2} \mathrm{O}_{0}$ degrades leach resistance by approximately a factor of 3 . Leach rates for the higher $\mathrm{ZnO}$ containing glass after 28 days are generally in the range of $5.10^{-5} \mathrm{~g} \cdot \mathrm{cm}^{-2} \cdot \mathrm{d}^{-1}$ for $\mathrm{B}^{0+}, \mathrm{Al}^{0+}$, $\mathrm{Mo}^{6+} ; 2.10^{-5} \mathrm{~g} \cdot \mathrm{cm}^{-2} \cdot \mathrm{d}^{-1}$ for $\mathrm{Na}^{+}, \mathrm{Si}^{4+}$; and 2 to $7.10^{-6} \mathrm{~g} \cdot \mathrm{cm}^{-2} \cdot \mathrm{d}^{-1}$ for $\mathrm{Fe}^{0+}$ and $\mathrm{Zn}^{2+}$. The surface of both types of glass appears to be protected by dual protective layers, one rich in $\mathrm{SiO}_{2}$ and a second very thin film rich in multivalent species. The second film that contains a mixture of $\mathrm{Zn}$ and $F e$ is less effectivo ao a diffusion barrier and is less resistant to network breakdown than the film without the $\mathrm{Fe}^{0+}$. This apparently is because a critical concentration of multivalence species is necessary to stabilize the second protective film. Differences in surface arca to solution volume ratios affect the formation of the protective films and the rates of surface damage of the glass.

462 Materials balance: scientific fundamentals for the quality assurance of vitrified waste. Schiewer, E. (HahnMeitner-Institut fuer Kernforschung Berlin, Germany); Rabe, H.; Weisenburger, S. pp 289-297 of Scientific basis for nuclear waste management V. Lutze, W. (ed.). New York, NY; NorthHolland (1982). (CONF-820636-).

From 5 . international symposium on the scientific basis for radioactive waste management; Berlin, F.R. Germany (7 Jun 1982).

For a 10 day vitrificatoin experiment with simulated HLLWQ an almost complete materials balance could be achieved. The dissolution of a molybdenum protecting tube could easily be recognized in the materials balance. The corrosion product input can be calculated from the materials balance, and the corrosion rates for the corresponding materials can also be obtained. The corrosion rates for the 10 day experiment do not show any interference inthe vitrification of the LEWC. The maximum $\mathrm{SO}_{0}$ content of the melt is $\sim 0.70 \%$. Changes in the composition of the glass frit only slightly influence the solubility of $\mathrm{SO}_{0}$ in the borosilicate glass. The saturation with $\mathrm{SO}_{0}$ occurs as soon as the LEWC content in the glass reachcs $14 \%$. In future experiments the HLW contants nhould be adapted th the greatest possible $\mathrm{SO}_{0}$ should be observed. If the solubility of the $\mathrm{SO}_{0}$ in the glass has been cxceeded, a secund phase forms in the melter, which must increase in amount when the evaporation products are completely recycled, and as the duration of vitrification is extended. It cannot, therefore, be excluded that the degree of the corrosion of other individual materials will not be affected.

463 Effects of radiation damage and radiolysis on the leaching of vitrified waste. Burns, W.G.; Hughes, A.E.; Marples, J.A.C.; Nelson, R.S.; Stoneham, A.M. (Atomic Energy Research Establishment, Harwell, England). pp 339-348 of Scientific basis for nuclear waste management V. Lutze, $W$. (ed.). Ncw York, NY; Horth-Holland (1982). (CONF820636-).

From 5. interualivinal symposium on the scientific: hasis for radioactive waste management; Berlin, F.R. Germany (7 Jun 1982).

Two sets of leach testing expcriments were carried out. The first was restricted to the glass composition UK189 doped with about 5 wt $\%{ }^{208} \mathrm{Pu}$. Frnm the results it can be seen that only modest increases in leach rate have been obscrved even after $5.6 \times 10^{18} \alpha$-decays per gram, equivalent to about 1.4 millinn years for vitrified Magnox waste. In the second series of experiments, samples of four glasses and a glass ceramic were doped with $2.5 \mathrm{wt} \%{ }^{208} \mathrm{PuO}_{2}$. Only relatively small changes in leach rate were found. Studies on recovery during irradiation indicate that recovery must be taken into consideration in assessing the relevance of simulation experiments to the real situation in vitrified wastes. In the radiolysis of leachant studies, three cases were considered: (1) 2.4 Mrad/h $\gamma$-radiation; (2) $4 \mathrm{Mrad} / \mathrm{h} \alpha$-radiation, which is the dose to theleachant when the ${ }^{208} \mathrm{PuO}_{2}$-doped samples are leach tested. Here the water is assumed to be saturated with dissolved air; (3) radiation conditions that could occur in a repository assuming the leachant contacts the 
ass at all times. The conclusion was reached that, in the absence of air, idiolysis of the leachant will not have a large effect on the leach rate i a real repository.

4os Structural effects of radiation damage in silica based glasses. Manara, A. (Joint Research Center, Ispra, Italy); Gibson, P.N.; Antonini, M. pp 349-346 of Scientific basis for nuclear waste management $V$. Lutze, W. (ed.). New York, NY; Horth-Holland (1982). (CONF-820636-).

From 5. international symposium on the scientific basis for radioactive waste management; Berlin, F.R. Germany (7 Jun 1982).

The results of recent measurements of optical absorption, etching rate and transmission electron microscopy in pure silica and borosilicate glasses are reported and discussed. At dose saturation conditions, the dependence of the optical density associated with the production of single atomic defects from the mass of the impinging particle shows a marked saturation at masses $\geq 20$ amu. The corresponding etching rates increase by about 4 times with respect to unirradiated samples. In borosilicate glasses, the temperature dependence of the threshold dose rate of electrons to initiate the nucleation of bubbles shows a marked increase from about $300^{\circ}$ to $600^{\circ} \mathrm{K}$.

405 Decomposition of oxalic acid with nitric acid. Kubota, M. (Japan Atomic Energy Research Inst., Tokai, Ibaraki. Nuclear Code Committee of Japan). Journal of Radioanalytical Chemistry; 75: No. 1-2, 39-49(1982).

8 refs.

Oxalic acid or oxalate is widely used as a precipitant and a detergent in the field of nuclear energy for developing a method of partitioning transuranium elements, ${ }^{90} \mathrm{Sr}$ and ${ }^{107} \mathrm{Cs}$ from a high-level liquid waste generated from fuel reprocessing. A method of decomposing oxalic acid with $\mathrm{HNO}_{0}$ in the presence of $\mathrm{Mn}^{2+}$ ion is developed. The use of $\mathrm{Mn}^{2+}$ ion as low as $10^{-0} \mathrm{~mol} / 1$ facilitated the complete decomposition of oxalic acid, and the acidity of the resulting solution became as low as $0.1 \mathrm{eq} / 1$ under the optimum conditions. The decomposition of oxalic acid is a first order reaction and proceeds at temperatures above $80 \mathrm{deg}$ C, the activation energy of the reaction is $18.6 \mathrm{kcal} / \mathrm{mol}$. This decomposition method is applicable to the dissolution of an oxalate precipitate.

468 Management of vitrified high level radioactive waste. Martin, A.; Kempe, T.F. (Associated Nuclear Services, Epsom (UK)); Browning, R. (Department of the Environment, London (UK)). pp 570-575 of Radiological protection - advances in theory and practice. Proceedings of the 3. international symposium beld in Inverness, Scotland, 6-11 June 1982. Berkeley, England; Society for Radiological Protection (1982). (CONF820613-).

From 3. international symposium of the Society for Radiological Protection; Inverncss, UK (6 Jun 1982).

The UK Government has recently announced that the strategy for management of HLW will be based on vitrification followed by an extended period of storage. The paper discusses some implications of this policy, outlines the characteristics of the waste and identifies the main options as to the mode of storage. The radiological impact of storage is likely to be very low. Broad indications of cost are given.

467 Inorganic sorbents in nuclear power plants. Dolezal, J.; Tympl, M. (Ustav Jaderneho Vyzkumu CSKAE, Rez (Czechoslovakia)). Nukleon; No. 1, 3-6(1982). (In Czech).

Inorganic ion exchangers show the following advantages: resistance to high radiation intensities, thermal stability, selectivity, possibility of use in media with higher salt contents, and very efficient capture of radionuclides. They can thus be used in nuclear power plants, e.g., in high level waste processing and in waste water treatment.

468 Precipitation process for decontamination of water soluble SRP radioactive waste. Lee, L.M.; Kilpatrick, L.L. (E. I. du Pont de Nemours \& Company, Savannah River Laboratory, Aiken, SC 29808). Transactions of the American Nuclear Society: 43: 124-125(1982). (CONF-821103-).

From American Nuclear Society winter meeting; Washington, DC, USA (14 Nov 1982).

DECONTAMINATION; HIGH-LEVEL RADIOACTIVE WASTES; LIQUID WASTES; PRECIPITATION; RADIOACTIVE
WASTE PROCESSING; SAVANNAH RIVER PLANT; SEPARATION EQUIPMENT; VITRIFICATION

469 Comparison of candidate elasses to vitrify ICPP calcined alumina waste. Cole, H.S.; Colton, E.L. (Exxon Nuclear Idaho Co., Inc., P.O. Box 2800, Idaho Falls, ID 83401). Transactions of the American Nuclear Society: 43: 109(1982). (CONF-821103-).

From American Nuclear Society winter meeting; Washington, DC, USA (14 Nov 1982).

ALUMINIUM OXIDES; CALCINED WASTES; COMPARATIVE EVALUATIONS; BOROSILICATE GLASS; HIGHLEVEL RADIOACTIVE WASTES; MATERIALS TESTING; RADIOACTIVE WASTE PROCESSING; VITRIFICATION; SOLIDIFICATION

470 Immobilization of radioactive liquid waste using water glass. Uetake, N.; Fukazawa, T.; Kikuchi, M. (Hitachi Ltd., Energy Research Laboratory, 1168 Moriyama-cho, Hitachi-shi, Ibaraki, 316). Transactions of the American Nuclear Society; 43: 110-111(1982). (CONF-821103-).

From American Nuclear Society winter meeting; Washington, DC, USA (14 Nov 1982).

BENCH-SCALE EXPERIMENTS; BOROSILICATE GLASS; CALCINATION; DRYING; HIGH-LEVEL RADIOACTIVE WASTES; LIQUID WASTES; RADIOACTIVE WASTE PROCESSING; SINTERING; VITRIFICATION; SOLIDIFICATION

471 Nondestructive techniques for evaluating the integrity of vitrified solids. Sano, A.; Uchida, K.; Murata, T. (Nippon Atomic Industry Group Co. Ltd., Tokyo); Matsumoto, K. NAIG Annual Review; No. 1981, 64(1982).

Published in summary form only.

CRACKS; HIGH-LEVEL RADIOACTIVE WASTES; NONDESTRUCTIVE TESTING; SOLID WASTES; VITRIFICATION; RADIOACTIVE WASTE PROCESSING

472 Fabication and properties of SYNROC. Reeve, K.D. Levins, D.M.; Ramm, E.J.; Woolfrey, J.L. (Australian Atomic Energy Commission Research Establishment, Lucas Heights). pp 143-148 of Austceram 82. Melbourne, Australia; Australian Ceramic Society (1982). (CONF-8208144-).

From 10. Australian ceramic conference; Melbourne, Australia (24 Aug 1982).

The first SYNROC fabrication method chosen for a scale-up demonstration at the AAEC was in-can hot pressing. Two other methods, the hot pressing of SYNROC in unsupported thin-walled bellows and a hybrid method using bellows-type cans within a canister which could be unsupported during hot pressing are being compared with the original concept. Attention has been given to the evolution of a simplified process for SYNROC C powder preparation involving flash drying, denitration and calcination in a rotary calciner. Leach tests show that SYNROC is more leach resistant than borosilicate glasses over the temperature range $45-300 \mathrm{deg}$. C, that the SYNROC leach rate does not increase greatly with temperature and decreases rapidly with time and that caesium is the most leachable HLW element.

473 Sintering of SYNROC: case history for phase formation and densification of complex oxide systems. Palmour, $\mathbf{H}$. III Hare, T.M. (North Carolina State Univ., Raleigh (USA)). pp 185-192 of Sintering - theory and practice. Kolar, D.; Pejovnik, S. (Institut Jozef Stefan, Ljubljana (Yugoslavia)); Ristic, M.M. (Institute of Technical Sciences of the Serbian Academy of Sciences and Arts, Belgrade (Yugoslavia)) (eds.). Amsterdam, Netherlands; Elsevier (1982). (CONF-8109227-).

From 5. international round table conference on sintering; Portoroz, Yugoslavia (7 Sep 1981).

This paper addresses several closely interwoven topics relating to the sintering of SYNROC, a potential ceramic waste form for disposal of high level radioactive waste (HLW). SYNROC is an acronym (SYNthetic ROCk) first used in 1978 by Ringwood to describe his thenemergent concept of very durable, and hence very disposable, manmade crystalline ceramic waste forms for stabilization of HLW. SYNROC compositions are based on naturally-occurring titanate/ zirconate minerals which are known to accomodate within their crystal 
structures a variety of naturally occurring radioactive species and to have endured within the deep earth environment for geologically long times $(0.2-0.6$ billion years).

474 Thermophysical properties of SYNROC and its constituent mineral phases. Buykx, W.J. (Australian Atomic Energy Commission Research Establishment, Lucas Heights. Materials Div.). pp 166-170 of Austceram 82. Melbourne, Australia; Australian Ceramic Society (1982). (CONF-8208144-). From 10. Australian ceramic conference; Melbourne, Australia (24 Aug 1982).

The specific heat, thermal diffusivity and thermal conductivity of cold pressed and sintered perovskite, barium hollandite, zirconolite, SYNROC-B and SYNROC-C were determined in the temperature ranges $20-700 \mathrm{deg}$. $C$ (thermal diffusivity) and 20-500 deg. $C$ (specific heat and thermal conductivity). SYNROC-B is the phase assemblage consisting of $25 \mathrm{wt} \%$ perovskite, $40 \mathrm{wt} \%$ barium hollandite and $35 \mathrm{wt} \%$ ziconolite, and not containing any waste. SYNROC-C consists of $85 \%$ SYNROC-B $+10 \%$ simulated radioactive waste $+4 \% \mathrm{TiO}_{2}$ and $1 \%$ $\mathrm{Al}_{2} \mathrm{O}_{0}$. The room temperature thermal diffusivity of hot pressed specimens of the same materials has also been determined.

$475 \quad$ Fixation of radiosctive materials in a glass matrix. Macedo, P.B.; Litovitz, T.A.; Simmons, J.H. Canadian Patent $1,110,051 / \mathrm{A} / .6$ Oct 1981. 35p.

A process is given for the encapsulation of high-level reprocessing wastes. The pores of a porous glass preform are impregnated with radioactive material, either in the gaseous state or as a solution, and the preform is sintered to decompose the radioactive material to its oxide and to collapse the porous structure. The preform may be treated with a dopant before impregnation to increase its surface area, and after impregnation steps may be taken to reduce the concentration of radioactive material near the surface of the preform. The final product is a nonporous glass composition containing at least $75 \mathrm{~mol}$ percent $\mathrm{SiO}_{2}$ and having at least one millicurie per cubic centimeter of immobilized radioactive oxides chemically incorporated into the silica structure. The product may be used as a radiation source, in particular for radiosterilization.

$476 \quad$ High temperature corrosion of heat-resisting alloys by borosilicate melts containing simulated high level nuclear wastes. Wakabayashi, H.; Terai, R. (Government Industrial Research Inst., Osaka, Ikeda (Japan)). Nippon Genshiryoku Gakkaishi, 23: No. 10, 772-781(Oct 1981). (In Japanese).

In connection with the development of vitrified solidification process of high level liquid wastes (HLLW) by metallic vessel, the high temperature corrosion of some heat-resisting alloys, such as stainless steel, Incoloy and Inconel, were investigated with the molten borosilicate glass containing simulated HLLW in the temperature range of $1,000-1,200^{\circ} \mathrm{C}$ for $3-50$ days. The concentration distributions of various constituents in the vicinity of the interface between the glass and the various metals were determined by means of EPMA. In the case of stainless steel, the general corrosion of alloys was observed without a protective layer. But in the case of both of Incoloy and Inconel, a chromium oxide layer was consequently formed between the glass and the metal, giving a protective film against general corrosion of alloys. When a $\mathrm{MgCr}_{2} \mathrm{O}_{4}$ layer on the chromium oxide layer and the alumina in alloy were, furthermore, formed, the corrosion of alloys became extremely depressed. The growth rate of the layer was controlled by chromium diffusion in alloy. The values of the effective diffusion coefficients $D$ tilde $\operatorname{sub}(\mathrm{Cr})$ were approximately $1 \times 10^{-10} \mathrm{~cm}^{2} / \mathrm{s}$ at $1,105^{0} \mathrm{C}$.

477 Alternative waste forms: process feasibility. Nesbitt, J.F.; Treat, R.L. (Battelle, Pac Northwest Lab, Richland, Wash, USA). American Ceramic Society Bulletin; 60: No. 9, 943-946, 954(Sep 1981).

The feasibility of solidifying high level nuclear waste on a production scale in a remotely operated and maintained facility was evaluated. Nine processes for solidifying liquid nuclear waste were compared on several process-related factors: process complexity, state of development, process demands and limitations, and safety concerns. The processes for making glass monoliths and ceramics were the most feasible, followed by the concrete and marbles-in-lead processes. 4 refs.
478 (KFK-tr-670) Method to encapsulate radioactive material. Larker, H. Sep 1981. Translation source information not available . 15p. (In German). NTIS (US Sales Only).

A method to encapsulate highly radioactive material is proposed. Pre-formed solid elements of radioactive material (e.g. glass, preferably pressure-sintered silicates) are cladded with corrosion-resistant metallic material, which can hardly be penetrated by radiation, encapsulated and are subjected to isostatic pressing. Pressure (preferably at least $50 \mathrm{MPa}$ ) and temperature (at least $500^{\circ} \mathrm{C}$ ) must be sufficient to form a compact dense cladding.

479. Preparation of glass containing high-level radioactive waste (simulated) - copper composite compacts by a continuous pressure sintering method. Kinoshita, M.; Terai, R. (Government Industrial Research Inst., Osaka, Ikeda (Japan)); Nagata, I. les Only); 28: No. 6, 231-236(Sep 1981). (In Japanese).

Glass-copper composite compacts were studied concerning solidification of radioactive wastes. The powder mixtures of the glass, which was a borosilicate glass containing about $27 \mathrm{wt} \%$ high-level radioactive wastes (simulated) offered by PNC, and electrolyzed, fine copper mwder were suhjected to pressure sintering. At first. sintering hehavinr of the mixtures and thermal properties of the compacts were investigated. The mixtures sintered almost to full densities at the temperatures above the yield point of the glass, when the copper content was not so much. The thermal conductivity of the compact containing $30 \mathrm{wt} \%$ copper was about 4.5 times higher than that of original glass. In order to obtain long size composite compacts, a continuous pressure sintering apparatus was built as trial. Using the mixture containing 30wt\% copper powder, the compacts of the diameter of $5 \mathrm{~cm}$ and of the length exceeding $30 \mathrm{~cm}$ were successfully prepared at the sintering conditions of $560^{\circ} \mathrm{C}$ and $150 \mathrm{~kg} / \mathrm{cm}^{2}$.

480

Melting of glass by direct induction heating in ceramic contriner. Ooka, K.; Oguino, N.; Kawanishi, N. (Toshiba Corp., Kawasaki, Kanagawa (Japan). Research and Development Center). Yogyo Kyokai Shi; 89: No. 1033, 516523(Sep 1981). (In Japanese).

The direct induction melting, a process of glass melting by high frequency induction heating, were founded to be the effective way of glass melting, especially desirable for the vitrification of High Level Radioactive Liquid Wastes, HLLW. A test instrument in the cold level was equipped with a high frequency oscillator of $65 \mathrm{~kW}$ anode output. The direct induction melting was successfully performed with two frequencles of $400 \mathrm{kHz}$ and $3 \mathrm{MHz}$, and the operation conditions were determined in the five cases of ceramic pot inner diameters of 170,200 , 230,280 and $325 \mathrm{~mm}$. The start-up of the direct induction melting was carried out by induction heating using a silicon carbide rod which was inserted in raw material powders in the ceramic pot. After the raw material powders partly melted down and the direct induction in the melt began, the start-up rod was removed out of the melt. At this stage, the direct induction melting was successively performed by adjusting the output power of the oscillator and by supplying the raw materials. It was also found that the capacity of this type of melting was reasonably large and the operation could be remotely controlled. Both applied frequencies of $400 \mathrm{kHz}$ and $3 \mathrm{MHz}$ was found to be successful with this melting system, especially in the case of lower frequency which proved more preferable for the incell work.

48i Some glass-ceramles for speclal appllcatlons. Wada, M.; Kawamura, S. (Nippon Electric Glass Co. Ltd., Otsu). Bulletin of the Institute for Chemical Research, Kyoto University; 59: No. 3, 256-265(Sep 1981).

Manufacturing process and characteristics of various glassceramics utilized as thermal shock resistant materials and as exterior/ interior wall panels of buildinge and for solidification of high level radioactive wastes are described.

482 Change of electrical properties of the borosilicate glasses containing $\mathrm{MoO}_{0}$ by phase separation. Terai, R.; Yamanaka, H.; Wakabayashi, H. (Industrial Research Inst., Osaka Prefecture (Japan)); Fukumoto, S.; Yamashita, K: Osaka Kogyo Gijutsu Shikenjo Kiho; 32: No. 3, 152-162(Sep 1981). (In Japanese).

The relation between the phase separation of borosilicate glasses containing $\mathrm{MoO}_{0}$ and the electrical properties has been investigated with the aid of the electron microscope. The borosilicate glasses contain- 
ing $10-20 \mathrm{~mol} \% \mathrm{Na}_{2} \mathrm{O}$ have brought about the increase of electrical conductivity by the phase separation. Meanwhile, the glass of 2.5 $\mathrm{Na}_{2} \mathrm{O} .27 .5 \mathrm{~B}_{2} \mathrm{O}_{0} .70 \mathrm{SiO}_{2}$ (mol\%) resulted in the decrease of conductivity by the phase separation annealing. In the former the dispersion phase mainly consists of $\mathrm{SiO}_{2}$ rich phase and the matrix is formed by the higher conductive $\mathrm{Na}_{2} \mathrm{O}-\mathrm{B}_{2} \mathrm{O}_{0}$ rich phase. In the latter case, on the other hand, the dispersion and matrix phases are $\mathrm{Na}_{2} \mathrm{O}-\mathrm{B}_{2} \mathrm{O}_{0}$ rich and $\mathrm{SiO}_{2}$ rich phases, respectively, coinciding with the electron microscopic observation. It was found that the addition of $\mathrm{MoO}_{0}$ into borosilicate glass led to the remarkable great tendency of phase separation and the loss peaks of dielectric relaxation shifted to slightly higher frequency side whenever any phase separation developed.

$483 \quad$ Method of solidifying radioactive wastes. Mizuno, F.; Someno, K.; Sato, T.; Miyazaki, K.; Sato, T. (Mitsui Mining and Smelting Co. Ltd., Tokyo (Japan)). (to Mitsui Mining and Smelting Co. Ltd., Tokyo (Japan); Japan Atomic Energy Research Inst., Tokyo). Japanese Patent 1956-94,299/A/. 30 Jul 1981. 4p. (In Japanese).

The purpose of this patent is to enable safety damping treatment for radioactive wastes by solidifying the filled portions within a corro sion resistant column filled with Cs-137, Sr-90 and the like by using solidifying substances such as metal and glass. Zeolite particles are filled in a stainless column, in which metal gauges are provided at the upper and the bottom portions. Radioactive liquid wastes containing Cs137, Sr-90 and the like are passed through a stainless pipe into the column to adsorb radioactive nuclides. Then, the filled layers are dried and sintered. Then, the column is cooled and incorporated to a system comprising an electric furance for molten lead, a cooling water tank and a vacuum pump. While evacuating the inside of the system by the vacuum pump, heated molten lead is gradually sucked and filled through a stainless pipe into the column. The molten lead is injected from the metal gauge at the bottom of the column, gradually rises through the gaps in the filled layers and permeates through the upper metal gauge. The column thus filled with the molten lead is cooled and detached from the system. Thus, the gaps in the upper, lower and bottom portions and the peripheral wall of the column, as well as in the zeolite filled layers are densely settled by lead.

484 Apparatus and method for immobilizing waste material. Ramm, E.J.; Reeve, K.D.; Woolfrey, J.L. (to Australian Atomic Energy Commission Research Establishment, Lucas Heights). Australian Patent 80/65,176/A/. 2 Jul 1981. Filed date 6 Dec 1979. vp.

An apparatus for forming blocks of synthetic rock in which radioactive waste is immobilized is claimed. The apparatus comprises a graphite die block with a cylindrical bnre, a graphite sleeve with a sliding fit in the bore and a cylindrical cavity and cylindrical graphite plunger members. A quantity of the supply material is placed between these members whereby it may be compacted by pressure applied to the members. The apparatus is adapted to be placed in a heated zone. The material forms a synthetic rock under the influence of heat and pressure with the nuclear waste immobilized in the crystal lattice. A disposable element containing radioactive waste in a form suitable for long-term storage is claimed. It is formed under heat and pressure. The element comprises a core of compacted synthetic rock including a minor proportion of the radioactive waste material, a surrounding buffer cover of lower density material adapted to absorb any radiation-induced expansion of the core during long-term storage, and a shroud of compacted synthetic rock free of radioactive waste providing an outer layer.

485 Report of the Ad Hoc Committee on Reprocessing and Wastes of the Sub-Committee on Nuclear Power of Committee on Overall Energy Study. tin, Geological Survey Wyoming; No. 128, 75-88(Jul 1981). (In Japanese).

In order for the nuclear power generation in Japan to be a stable energy source, it is important also to secure the long-term supply of nuclear fuels and to utilize them efficiently. In this connection, the proper treatment and disposal of wastes not only from nuclear power plants but also from fuel reprocessing plants are necessary. Under the situation, the Ad Hoc Committee on Reprocessing and Wastes has made the studies on the basic ideas of low-level waste treatment and disposal, the procedures of sea and ground disposals of wastes, the development of volume reduction techniques, the definite grouping of radioactive wastes, the measures of treatment and disposal of high-level wastes, the mcasurcs for wastes returned from overseas reprocessing, the siting of fuel reprocessing plants, and the burden of reprocessing and waste management costs. The results by the ad hoc committee are described for these items.

486 Treating nuclear waste. Marriott, R.; Henyey, F.S.; Hochstim. (to Perm, Inc., La Jolla, CA (USA)). Australian Patent 80/64353/A/. 11 Jun 1981. Priority date 5 Dec 1979, United States of America (USA), vp.

A method is claimed of decreasing the amount of long lived fission products of radioactive waste materials by producing relatively short lived radioactive nuclides and stable nuclides from the long lived products. It comprises the steps of: a) separating the fission products into (1) a plurality of components, each component having at least one long lived fission product nuclide and (2) short lived radioactive nuclides and stable nuclides, b) storing at least some of the short lived radioactive nuclides and stable nuclides, c) exposing at least some of the components to a high thermal neutron flux for inducing transformation of at least some of the long lived fission product nuclides to produce short lived radioactive nuclides and stable nuclides, d) removing each exposed component and the produced short lived radioactive nuclides and stable nuclides from the high thermal neutral flux, e) separating the components from at least some of the produced short lived radioactive nuclides and stable nuclides, and f) repeating steps b) - e) at least one time.

487 Development and investigation of the accepting system for solidified bodies returned from foreign countries after reprocessing. Sato, H. (Agency of Natural Resources and Energy, Tokyo (Japan)). Denki Kyokai Zasshi; No. 692, 9-14(Jun 1981). (In Japanese).

The Ministry of International Trade and Industry is to carry out from 1981 the development and investigation of the accepting system for high level radioactive wastes returned from foreign countries accompanying the execution of reprocessing contract. In Japan, all spent fuel from nuclear power stations is to be reprocessed to recover uranium and plutonium and to control radioactive wastes safely and in compact form. Until a new commercial reprocessing plant will be in operation around 1990 , reprocessing is made by the commission contract with Great Britain and France. The presentation of the specifications of glass solidification of wastes will be made by the reprocessing contractors by January, 1982, and the power companies in Japan must reply on the possibility of acceptance within two years. The high level radioactive wastes arising from reprocessing are stored in tanks for several years to decay and cool, and thereafter, solidified with glass in canisters. The Power Reactor and Nuclear Fuel Development Corp. is forwarding the design and construction of a pilot plant for solidifying and storing high level radioactive wastes, the operation of which is scheduled in 1987. The development and investigation of the accepting system for returned solidified bodies are outlined.

488 Flame-resistant radioactive waste processing method. Shinohara, T.; Anzai, T. (to Toyo Engineering Corp., Tokyo (Japan)). Japanese Patent 56-50,000/A/. 6 May 1981. 3p. (In Japanese).

Proposed is a container for dry distilling flame-resistant radioactive waste utilizing the excellent corrosion resistant properties of a silicon carbide heater. A container formed of silicon carbide is filled with flame resistant radioactive wastes containing high level radioactive wastes, the container is directly energized to be heated, or heating silicon carbide provided in the container is energized, and it is heated to be distilled.

489 Problem of reprocessing and of radioactive wastes in France. Gauvenet, A. (CEA Centre d'Etudes Nucleaires de Fontenay-aux-Roses, 92 (France). Inst. de Protection et de Surete Nucleaire). Notes d'Informations CEA (Commissariat a l'Energie Atomique); No. 5, 3-8(May 1981). (In French).

From Annual meeting on nuclear technology '81; Duesseldorf, Germany, F.R. (24 - 26 Mar 1981).

Advantages and disadvantages of reprocessing or not are compared. Long term problems concerning reprocessing wastes are examined. Owing to reprocessing wastes are without plutonium. French policy and studies concerning storage of the different kind of radioactive wastes are given, high level wastes are vitrified before storage.

490 Comparison of borosilicate glass and synthetic minerals as media for the immobilization of high-level radioactive 
waste. Tempest, P.A. (CEGB, Berkeley, Gloucestershire, Engl). Nuclear Technology; 52: No. 3, 415-425(Mar 1981).

In this paper, the structure and properties of the different solid forms currently being developed for high-level radioactive waste disposal are compared. Good capacity to accept all the elements in the waste and flexibility of composition range to accommodate variations in the waste, are primarily discussed, 13 refs.

491 Promotion of the research and development of high level radioactive waste treatment and disposal. $G E I G B ; 25$ : No. 12, 32-43(Mar 1981). (In Japanese).

The Subcommittee on Countermeasures to Radioactive Wastes, set up by the Atomic Energy Commission, for the deliberation on the treatment and disposal of low and high level radioactive wastes, has formulated the plans of research and development for the treatment and disposal of high level radioactive wastes. The basic ideas for the management are the temporary storage of liquid wastes from fuel reprocessing and others in tanks, the solidification as glass products, the storage of the glass products for a certain period, and their disposal mainly underground. The following matters are described: basic ideas, the technological development for glass solidification (procedures and the respective techniques), the research and development for the grnund dispusal (procedures and the respective techniques), and others such as overall management system.

492 Present status of techniques for the solidification of high level wastes. Tsunoda, N. (Power Reactor and Nuclear Fuel Development Corp., Tokai, Ibaraki (Japan). Tokai Works). ticky a Kartograficky Obzor; 27: No. 2, 37-41(Feb 1981). (In Japanese).

In the high level radioactive liquid wastes generated in the reprocessing plants for spent fuel, actinides with very long half-value period, plutonium of a minute quantity and others are contained in addition to FPs, accordingly in case of the storage and disposal, it is required to convert to solidified bodies, and the conversion to borosilicate glass solidified bodies is the worldwide main process in view of the properties of solidified bodies required to be stable for long period, the reliability of solidifying process, the achievement of technical development and the applicability of conventional techniques. Metallic composites and crystalline solidified bodies are also studied, and the conversion of liquid wastes to oxide powder is carried out in the U.S. The present status of the technical development in glass solidification in France, West Germany, Great Britain, the U.S.A. and the U.S.S.R., and the tendency in Japan are described. The technological test facility with 9451 treatment capacity per day was completed in the Power Reactor and Nuclear Fuel Development Corp. in February, 1980. The acceptance of liquid wastes, denitration, concentration, glass melting, filling into canisters, cap welding, inspection, decontamination and the cooling characteristic test in storage are systematized in a series of apparatuses. The problems hereafter are pointed out.

493 Development and testing of SYNROC for high-level radioactive waste fixation. Reeve, K.D.; Levins, D.M.; Ramm, E.J.; Woolfrey, J.L.; Buykx, W.J.; Ryan, R.K.; Chapman, J.F. (Australian Atomic Energy Commission Research Establishment, Lucas Heights). Atomic Energy (Sydney); 24: No. 1, 2-12(Jan-Apr 1981).

Leaching tests on SYNROC have confirmed its excellent resistance to the release of radionuclides by groundwater attacis over the temperature range $45-300 \mathrm{deg}$. C. A non-radioactive $30 \mathrm{~kg}$-scale SYNROC fabrication line incorporating in-can hot pressing as the fabrication step is being built at Lucas Heights as a first step in showing that SYNROC fabrication need not be expensive. In accelerated radiation damage tcsts, SYNROC has remained intact after a simulated $10^{5}$ years of disposal life and has expanded to only a modest extent. In supporting research, minera! phase development, impact friability and thermophysical properties of SYNROC are being studied.

494 Compound refining system for separation of gaseous fission products incorporated in a reprocessing pilot plant for spent fuel from neclear power stations. pp 46-54 of Saishori, hoshasei haikibutsu shori shobun; Berugi, Nippon, Oranda, Soren yon-kakoku godo senmonka semina hokokusho. Tokyo, Japan; Japan Atomic Industrial Forum, Inc. (Jan 1981). (In Japanese)(CONF-8011169-).
From Tripartite symposium on reprocessing of spent nuclear fu and treatment of associated radioactive waste; Leningrad, USSR (1 Nov 1980).

In the V. G. Khlopin Radium Institute, a gas treatment experimental apparatus was installed to the SU-2 type experimental facility. The purpose is to solve variety of problems in the separation, collection and subsequent treatment for gaseous fission products and highly volatile fission products in spent fuel reprocessing. The experimental apparatus has the functions as follows: the measurement of air flow such as flow rate, pressure, total $\gamma$ activity and krypton- 85 content, preliminary air flow cleaning and drying removing aerosol, hydrogen fluoride and nitrogen oxide, and the trapping and analysis of gaseous fission products and highly volatile fission products in air flow. For the collection of these two types of fission products, a liquid absorbent and a solid adsorbent are used in series arrangement.

496 Research in the field of high level waste solidification. pp 55-61 of Saishori, hoshasei haikibutsu shori shobun; Berugi, Nippon, Oranda, Soren yon-kakoku godo senmonka semina hokokusho. Tokyo, Japan; Japan Atomic Industrial Forum, Inc. (Jan 1981). (In Japanese)(CONF-801 1 169-).

From Tripartite sympnsium on reproressing of spent nuolear fuel and treatment of associated radioactive waste; Leningrad, USSR (17 Nov 1980).

In the V.G. Khlopin Radium Institute, researches have been forwarded on the fixation of high level radioactive wastes in glass/metal compounds, glassy materials, and ceramics. Some results obtained so far in this field are described. (1) Inclusion of high level radioactive wastes in glass/metal compound. Same as in other countries, the process of containing high level wastes in glass particles and then fixing them in metallic matrix has been studied. (2) Inclusion of wastes in inorganic porous material and subsequent conversion. The use of inorganic porous materials for the fixation of high level wastes is interesting because dehydration and calcination become easy. (3) Glass solidification of high level radioactive wastes with single-stage crucible apparatus. With the apparatus, experiment has been made on the solidification of high level wastes in the reprocessing of spent fuel from VVER reactors.

$496 \quad$ Results of the development of a single-stage glass solidification method. Kulichenko, V.V. (Vsesoyuznyj NauchnoIssledovatel'skij Inst. Neorganicheskikh Materialov, Moscow (USSR)). pp 62-68 of Saishori, hoshasei haikibutsu shori shobun; Berugi, Nippon, Oranda. Soren yon-kakoku godo senmonka semina hokokusho. Tokyo, Japan; Japan Atomic Industrial Forum, Inc. (Jan 1981). (In Japanese)(CONF-8011169-). From Tripartite symposium on reprocessing of spent nuclear fuel and treatment of associated radioactive waste; Leningrad, USSR (17 Nov 1980).

In the U.S.S.R., the development of the single-stage continuous solidification process of high level radioactive wastes is proceeding. According to the mietliud, the glass solidification process comprising dehydration and calcination is done in a single apparatus. The heating necessary in the process is made by applying A.C. to molten glass, whereby the process efficiency can be raised, and the melt in furnaces can be heated uniformly. In the research of the process, the model solutions of sodium nitrate and aluminum nitrate were used. To the solution, further the nitrates of iron, calcium, nickel, chromium, manganese, ocrium and zirconiuni, and radiuactive strontium-90, cesium-137 and ruthenium-106 were added. As the flux to make phosphate glass, orthophuspluric acid was used, and to make borosilicate glass, datolite and silicon dioxide were used.

497 Preparation of the high-level waste form for the nearsurface test facility. Berger, D.N. (PNL, Richland, WA 99352). Transactions of the American Nuclear Society; 38: 130-131(1981). (CONF-810606-).

From American Nuclear Society's annual meeting; Miami Beach, FL, USA (7 Jun 1981).

BWR TYPE REACTORS; CALORIMETRY; DATA ANALYSIS; HIGH-LEVEL RADIOACTIVE WASTES; RADIOACTIVE WASTE PROCESSING; REACTOR CORES; SAMPLING; TEST. ING; VITRIFICATION; SOLIDIFICATION; RADIOACTIVE WASTE FACILITIES; CONTAINERS; PACKAGING

498 . Assessment of rationale for development of improved waste forms. Musgrave, B.C.; Cohen, J.J.; Smith, C.F. (LLNL, 
Livermore, CA 94550). Transactions of the American Nuclear Society; 38: 131(1981). (CONF-810606-).

From American Nuclear Society's annual meeting; Miami Beach, FL, USA (7 Jun 1981).

COMPARATIVE EVALUATIONS; EFFICIENCY; PERFORMANCE; HIGH-LEVEL RADIOACTIVE WASTES; RADIOACTIVE WASTE PROCESSING; SOLIDIFICATION; SOLID WASTES; LEACHING; STABILITY; GROUND WATER

499 Engineering scale multibarrier waste form demonstrations. Bonner, W.F.; Treat, R.L.; Rusin, J.M. (PNL, Richland, WA 99352). Transactions of the American Nuclear Society; 38: 132-133(1981). (CONF-810606-).

From American Nuclear Society's annual meeting; Miami Beach, FL, USA (7 Jun 1981).

CRYSTALS; DEMONSTRATION PROGRAMS; GLASS; HIGH-LEVEL RADIOACTIVE WASTES; LEACHING; PELLETS; RADIOACTIVE WASTE PROCESSING; SOLIDIFICATION; ENCAPSULATION; CONTAINERS

$500 \quad$ West Vallcy high-level waste solidification program. McGoff, D.J.; Turi, J.A. (US Dept. of Energy, Washington, V.C. 20545). Transactions of the Americun Nuclear Society; 38: 133-134(1981). (CONF-810606-).

From American Nuclear Society's annual meeting; Miami Beach, FL, USA (7 Jun 1981).

DEMONSTRATION PROGRAMS; HIGH-LEVEL RADIOACTIVE WASTES; SOLIDIFICATION; US DOE; WEST VALLEY PROCESSING PLANT; RADIOACTIVE WASTE PROCESSING; THERMOX PROCESS

$501 \quad$ Evaluation of process alternatives for solidification of existing commercial high-level waste. Holton, L.K.; Larson, D.E. (PNL, Richland, WA 99352). Transactions of the American Nuclear Socicty; 38: 134(1981). (CONF-810606-).

From American Nuclear Society's annual meeting; Miami Beach, FL, USA (7 Jun 1981).

CALCINATION; DESIGN; HIGH-LEVEL RADIOACTIVE WASTES; LIQUID WASTES; MOLTEN SALTS; PUREX PROCESS; SOLIDIFICATION; THOREX PROCESS; US DOE; VITRIFICATION; RADIOACTIVE WASTE PROCESSING; EVALUATION

502 Materials studies of simulated interim waste forms for high-level radiactive wastes. Bandyopadhyay, G.; Gehl, S.M.; Poeppel, R.B. (ANL, Argonne, IL 60439). Transactions of the American Nuclear Society; 38: 170-171(1981). (CONF810606-).

From American Nuclear Society's annual meeting; Miami Beach, FL, USA (7 Jun 1981).

CALCINATION; FRACTURES; HIGH-LEVEL RADIOACTIVE WASTES; MOLTEN SALTS; WASTE PELLETS; RADIOACTIVE WASTE PROCESSING; SILICATES; SLUDGES; SLURRIES; SOLIDIFICATION

503 Small-scale processing of actual Savannah River high-level waste. Lee, L.M.; Papouchado, L.M.; Woolsey, G.B. (SRL, Aiken, SC 29808). Transactions of the American Nuclear Society; 39: 171(1981). (CONF-811103-). 1981).

From ANS winter meeting; San Francisco, CA, USA (29 Nov

CESIUM 137; HIGH-LEVEL RADIOACTIVE WASTES; SAVANNAH RIVER PLANT; SLUDGES; VITRIFICATION; RADIOACTIVE WASTE PROCESSING

$504 \quad$ Recent developments in defense waste vitrification. Bjorklund, W.J. (PNL, Richland, WA 99352). Transactions of the American Nuclear Society; 39: 173-174(1981). (CONF811103-). 1981).

From ANS winter meeting; San Francisco, CA, USA (29 Nov

HIGH-LEVEL RADIOACTIVE WASTES; RESEARCH PROGRAMS; SIMULATION; VITRIFICATION; RADIOACTIVE WASTE PROCESSING; SAVANNAH RIVER PLANT; SOLIDIFIEATIOIN
605 Development and demonstration of a process for vitrification of TMI zeolite. Bryan, G.H.; Siemens, D.H. (PNL, Richland, WA 99352). Transactions of the American Nuclear Society; 39: 174-176(1981). (CONF-811103-). 1981).

From ANS winter meeting; San Francisco, CA, USA (29 Nov

BENCH-SCALE EXPERIMENTS; DECONTAMINATION; DEMONSTRATION PROGRAMS; DESIGN; HIGH-LEVEL RADIOACTIVE WASTES; THREE MILE ISLAND-2 REACTOR; VITRIFICATION; ZEOLITES; DEMINERALIZERS; RADIOACTIVE WASTE PROCESSING

606 Solidification of Savannah River plant high-level waste. Kelley, J.A.; Maher, R.; Shafranek, L.F.; Zeyfang, R.W. (SRL, Aiken, SC 29808). Transactions of the American Nuclear Society; 39: 228(1981). (CONF-811103-). 1981).

From ANS winter meeting; San Francisco, CA, USA (29 Nov

APPROPRIATE TECHNOLOGY; HIGH-LEVEL RADIOACTIVE WASTES; SAVANNAH RIVER PLANT; SOLIDIFICATION; US DOE; RADIOACTIVE WASTE STORAGE; RADIOACTIVE WASTE PROCESSING

607 High-level waste solidification system for the Western New York State Service Center. Holton, L.K.; Siemens, D.H. (PNL, Richland, WA 99352). Transactions of the American Nuclear Society; 38: 715-716(1981). (CONF-810606-).

From American Nuclear Society's annual meeting; Miami Beach, FL, USA (7 Jun 1981).

CALCINATION; FEASIBILITY STUDIES; HIGH-LEVEL RADIOACTIVE WASTES; MELTING; PH VALUE; REMOTE HANDLING; SOLIDIFICATION; WEST VALLEY PROCESSING PLANT; RADIOACTIVE WASTE PROCESSING; VITRIFICATION

$508 \quad$ Model for predicting leaching behavior of high-level radioactive waste forms. Solomah, A.G. (North Carolina State Univ, Raleigh, NC 27650). Transactions of the American Nuclear Society; 39: 354-355(1981). (CONF-811103-). 1981).

From ANS winter meeting; San Francisco, CA, USA (29 Nov

FORECASTING; LEACHING; MATHEMATICAL MODELS; RADIOACTIVE WASTE DISPOSAL; HIGH-LEVEL RADIOACTIVE WASTES; RADIOACTIVE WASTE PROCESSING; SYNROC PROCESS; SOLIDIFICATION; GLASS; VITRIFICATION; SYNTHETIC ROCKS; MEDIUM TEMPERATURE; CESIUM 134; DIFFUSION; RADIONUCLIDE MIGRATION

509 Rethinking the management of high-level nuclear waste: the need for fractionation. Platt, A.M.; Eschbach, E.A. (Battelle, Pac Northwest Lab, Richland, Wash, USA). Proceedings of the Symposium on Waste Management; 1: 365-373(1981). (CONF-810217-).

From ANS waste management conference; Tucson, AZ, USA (23. Feb 1981).

Most of the technical concerns about disposal in mined cavities are associated with the difficulty of predicting the long-term behavior of the waste package, repository, and geology in view of the elevated temperature and high-radiation fields. An alternative meriting investigation is the fractionation of waste to achieve the following aims: greatly reduce the heat and radiation of packages containing the long-lived radioisotopes constituting the 'long-term' concerns; reclaim the fission products that have near-immediate commercial use; package and dispose of the short-lived, high-heat/radiation waste separately so as not to jeopardize the disposal of the long-lived waste. This paper discusses the technology for fractionating waste and the benefits it offers.

510 Development and testing of SYNROC for high level radioactive waste fixation. Reeve, K.D.; Levins, D.M.; Ramm, E.J.; Woolfrey, J.L.; Buykx, W.J.; Ryan, R.K.; Chapman, J.F. (Aust At Energy Comm, Sydney). Proceedings of the Symposium on Waste Management; 1: 249-266(1981). (CONF810217-).

From ANS waste management conference; Tucson, AZ, USA (23 Feb 1981) 
Research and development on the SYNROC concept for high level radioactive waste fixation commenced at the Australian Atomic Energy Commission Research Establishment, Lucas Heights, in March 1979 , in collaboration with a complementary program at The Australian National University (ANU). The present paper reports progress in the project's second year and reviews its current status. An inactive $30 \mathrm{~kg}$ scale SYNROC fabrication line incorporating in-can hot pressing as the fabrication step has been built for operation in mid-1981. Atmospheric pressure and hydrothermal leach tests are demonstrating the excellent leach resistance of SYNROC. Accelerated radiation damage tests using fast neutrons are simulating damage in SYNROC for periods of close to $10^{6}$ years. In supporting research, mineral phase development, impact friability and thermophysical properties of SYNROC are being studied. 21 refs.

511 Alternative waste form development - lowtemperature pyrolytic carbon coatings. Oma, K.H.; Rusin, J.M.; Kidd, R.W.; Browning, M.F. (Battelle, Pac Northwest Lab, Richland, Wash, USA). Proceedings of the Symposium on Waste Management; 1: 277-292(1981). (CONF-810217-). (23 Feb 1981)

From ANS waste management conference; Tucson, AZ, USA

Although several chemical vapor deposition (CVD) - coated waste forms have been successfully produced, some major disadvantages associated with the high-temperature fluidized-bed CVD coating process exist. To overcome these disadvantages, the Pacific Northwest Laboratory has initiated the development of a pyrolytic carbon CVD coating system to coat large waste-form particles at temperatures ranging from 400 to $500 /$ degret $/ C$. This relatively simple system has been used to coat kilogram quantities of simulated waste-glass marbles. Further development of this system could result in a viable process to coat bulk quantities of both glass and ceramic waste forms. This paper discusses various aspects of the development work, including coating techniques, parametric study, and coater equipment. 10 refs.

512 Borosilicate glass as a matrix for immobilization of SRP high-level waste. Wicks, G.G. (DuPont, Aiken, SC, USA). Proceedings of the Symposium on Waste Management; 1: 321-335(1981). (CONF-810217-).

From ANS waste management conference; Tucson, AZ, USA (23 Feb 1981).

The technology for producing waste glass forms is well developed and has been demonstrated on various scales using simulated as well as radioactive Savannah River Plant (SRP) waste. Recently, full-scale prototype equipment has been made operational at SRP. This insludes hinth a joule-heated ceramic melter and an in-can melter. Over the past five Years, the Savannah River Laboratory (SRL) has been investigating the properties of SRP waste glass with special emphasis on the quality and durability of the product. In progress at SRL are programs aimed at minimizing thermally induced cracking by carefully controlling cooling cycles and using ceramic liners or coatings. The leachability of SRP waste glass has been studied under many different conditions and consistently found to be low. The effects of other geological media including granite, basalt, shale, and tuft are also being studied as part of the multibarrier isolation system. 17 refs.

513 Status of development of the tailored ceramic highlevel nuclear waste form. Recht, H.L.; Martin. A.B. (Rnckwell Int, Canoga Park, Calif, USA). Proceedings of the Symposium on Waste Management: 2; 825-836(1981). (CONF-810217-).

From ANS waste management conference; Tucson, AZ, USA (23 Feb 1981).

The 'Tailored Ceramics project' of the Department of Energy investigates the immobilization of nuclear wastes in a dense ceramic, with emphasis on working with high-aluminum-content wastes of the Savannah River plant. Phase studies, the possible loss of volatile waste species, radiation damage, and the feasibility of production by largescale remote operation are discussed. 11 refs.

514 Incineration/vitrification of simulated low-level institutional wastes in a joule-heated glass melter. Buelt, J.L.; Oma, K.H. (Battelle, Pac Northwest Lab, Richland, Wash). Nuclear and Chemical Waste Management; 2: No. 3, 175-182(1981).

The purpose of this study was to determine the feasibility of reducing the volume of low-level institutional wastes by incineration and then converting the residual solids into glass with a single-step process.
Experimental test results with simulated waste show that a joule-heated ceramic melter originally developed for high-level waste vitrification can be used for the low-level waste volume reduction process. Off-gas analysis from the experiment shuws that incineration of combustible solids was essentially complete. This is attributable to high combustion temperatures ( $<950 /$ degree/C) and the introduction of excess combustion air or oxygen at the mcltcr's glass surface. Results of the incineration experiment indicate that process scale-up for higher capacities is quite feasible. Further tests aimed at adapting high-level waste vitrification technology to the management of both institutional wastes and lowlevel wastes generated from the nuclear fuel cycle are recommended.

616 Immobilization of high-level nuclear reactor wastes in SYNROC: a current appraisal. Ringwood, A.E.; Oversby, V.M.; Kesson, S.E.; Sinclair, W.; Ware, N.; Hibberson, W.; Major, A. (Aust Natl Univ, Canberra, ACT). Nuclear and Chemical Waste Management; 2: No. 4, 287-305(1981).

A detailed investigation has been made of the comparative ability of SYNROC and borosilicate glasses to immobilize high level nuclear wastes for extremely long periods of time when buried in geulogical repositories. Under dynamic leaching conditions glasses tend to dissolve quasicongruently whereas the dissolution of SYNROC minerals is highly incongruent. Loss of low valence cations (e.g., $\mathrm{Ca}, \mathrm{Ba}, \mathrm{Cs}$ ) from the near-surface regions of SYNROC minerals is highly incongruent. Loss of low valence cations (e.g., $\mathrm{Ca}, \mathrm{Ba}, \mathrm{Cs}$ ) from the near-surface regions of SYNROC titanate miuerals results in surface films consisting dominantly of titanium dioxide. Because of the extreme insolubility of $\mathrm{SiO}_{2}$ in water, these films tend to protect the surface from further dissolution and hence the leach rates of SYNROC minerals fall off drastically with time, commonly by 2 orders-of-magnitude after 10-30 days of dynamic (undersaturated) leaching. The results demonstrate that SYNROC is more resistant to leaching than borosilicate glass. Experimental studies demonstrate low leachability of SYNROC minerals despite their exposure to large radiation doses. The superior leach resistance of SYNROC at elevated temperatures will make it possible to bury it in geological repositories within a few years after reprocessing. thereby avoiding the necessity for prolonged storage and supcrvision. 41 refs.

616 Uranium hexafluoride actinide transmutation reactors-preliminary design and analysis. Wan, P.T. Atlanta, GA; Georgia Inst. of Tech. (1981). 145p. University Microfilms Order No. 82-08,803.

Thesis (Ph.D.).

The long term hazard potential of high-level wastes from fission reactors can be reduced significantly by partitioning waste actinides from the bulk wastes and transmuting them to short-lived or stable fission product nuclides by neutron bombardment. The objectives of the present work are to establish the waste actinide transmutation performance of a uranium hexafluoride actinide transmutation reactor (UHATR) and to ascertain if it has any special advantages over light water reactors (LWRs). Using present day and near term technology, a preliminary UHATR design was iefined. The reactor core consist 3 of a cylindrical cavity filled with a mixture of $\mathrm{U}^{200} \mathrm{~F}_{6}$ and helium, surrounded on all sides by graphite. An annular actinide blanket is enibedded in the graphite. Each actinide element is stainless steel clad, helium cooled and graphite moderated. Very high thermal neutron fluxes, from $10^{14}$ to $10^{13} \mathrm{n} / \mathrm{cm}^{2}-800$, wore obtaincd in the actinide tegiun. Fur one UHATR design, calculations showed that out of $250 \mathrm{~kg}$ of waste actinides oharged, about $75 \mathrm{~kg}$ net is converted to fission products after 220 days of irradiation. This is equivalent to an average waste actinide fission rate of $50 \%$ calendar year of irradiation. Compared with an LWR, the average blanket thermal flux of this UHATR is about ten to thirty times higher, leading a fifteen fold improvement in the actinide fission rate.

617 Alpha spectrum profiling of plutonium in leached simulated high-level radioactive waste-glass. Diamond, $H$.; Friedman, A.M. (Argonne National Lab., IL). Nuclear and Chemical Waste Management; 2: 207-212(1981).

Low-geometry x-ray spectra from ${ }^{209} \mathrm{Pu}$ and ${ }^{207} \mathrm{~Np}$, incorporated into simulated high-level radiuactive waste-glass, were transformed into depth distributions for these elements. Changes in the depth profiles were observed for a series of static leachings in $75^{\circ} \mathrm{C}$ water. Radiochemical assay of the leach solutions revealed that little neptunium or plutonium was leached, and that the amount leached was independent of 
leaching time. The depth profiles of the leached specimens showed that there was selective leaching of nonradioactive components of the glass, concentrating the remaining neptunium and plutonium in a broad zone near (but not at) the glass surface. Eventual redeposition of nonradioactive material onto the glass surface inhibited further leaching. Since the depth profiles exhibit uniform concentrations of plutonium and neptunium in undisturbed simulated waste-glass, the depth of attack by the solution can be inferred by departures from the uniform concentrations. A different mechanism was observed in leaching the simulated wasteglass by room temperature $0.03 \mathrm{M} \mathrm{NaHCO}$. There was no evidence for selective leaching or invasion of the glass by the leaching solution. There was, however, a steady buildup of plutonium (but not neptunium) upon the surface of the glass. 4 figures, 3 tables.

518 Alpha-recoil damage in natural zirconolite and perovskite. Sinclair, W.; Ringwood, A.E. (Australian National Univ., Canberra. Research School of Earth Sciences). Nuclear and Chemical Waste Management; 15: No. 5, 229-243(1981).

Zirconolite $\left(\mathrm{CaZrTi}_{2} \mathrm{O}_{7}\right)$ and perovskite $\left(\mathrm{CaTiO}_{0}\right)$ are the key minerals in SYNROC, a ceramic material developed for the immobilization of high level nuclear reactor wastes. When these minerals are incorporated in the SYNROC, long-lived radioactive actinide elements are preferentially partitioned into zirconolite and perovskite, consequently these are subjected to the effect of alpha-recoil resulting from the decay of the elements. This effect has been studied by X-ray and electron diffraction investigations of the natural samples of zirconolite and perovskite of varying ages and varying uranium and thorium contents. The samples studied have received the cumulative alpha dose ranging from $1.0 \times 10^{18}$ to $1.1 \times 10^{20} \alpha / \mathrm{g}$. These studies showed that zirconolite has remained to be crystalline up to and beyond the alpha dose of $2 \times 10^{19} \alpha / \mathrm{g}$. The density change produced by the irradiation was as small as up to $3 \%$. The density of perovskite samples decreased by $1.8 \%$, and their $X$-ray powder patterns were essentially unaffected. The results demonstrated that zirconolite and perovskite were extremely resistant to the effect of nuclear radiation, and provide stable crystal structures for the containment of radioactive waste elements during the time required for the decay of the radioactivity to safe level (typically $10^{5}-10^{6}$ years)

519 Process for producing biologically noxious waste, particularly radioactive waste, wich can be stored. Neumann, $\mathbf{W}$. (to Oesterreichisches Forschungszentrum Seibersdorf Ges.m.b. H., Vienna). German(FRG) Patent 2,855,738/C/. 13 Nov 1980. 3p. (In German).

After calcination, the granulate produced is introduced into a cylindrical wire cage. The wire cage is then coated with a liquid aluminium silicon alloy or bitumen, where the wire cage is centrifuged. Centrifuging produces penetration of the coating substance into the pores of the mass of granulate.

$520 \quad$ Process and facility for continuous urelting and dosed filling in casks of high-activity waste. Gattys, F.J. (to Gattys (F.J.) Ingenieurbuero fuer Chemische Maschinen- und Apparatebau, Neu-Isenburg (Germany, F.R.)). German(FRG) Patent 2,915,273/A/. 16 Oct 1980. 16p. (In German).

The melting furnace for the wastes is combined with a portioning tank installed at a somewhat higher level which is connected to a vacuum set. By means of connecting pipes so much of the melted mass from the melting furnace can be taken up until one of the pipes in the furnace becomes uncuvered, an ingress of air into the portioning tank takes place and the flow of melted mass will be interrupted. An interlocking bar valve is installed at the portioning tank for dropping off the melted mass.

521 Plant for solidification and storage of high-level radioactive waste. Ooka, K.; Oguino, N.; Matsumoto, T.E. (Toshiba Corp., Kawasaki, Kanagawa (Japan). Research and Development Center); Tarutani, K.; Matsumoto, K. Toshiba Rebyu; 35: No. 11, 943-947(Oct 1980). (In Japanese).

High-level radioactive liquid waste (HLLW) from reprocessing plants of fuel should be solidified and stored in solid form for long-term safeguard. Solidification and storage of HLLW is the key problem to be solved for establishing the nuclear fuel cycle. The solid form now considered the most promising is glass, in which the constituents of HLLW arc incorporated into structure. Toshiba has developed a cold test plant for HLLW, which is installed in Tokai plant of PNC. The main process in the plant is glass melting.

522 Combustion in the reactor of long-lived transuranium elements as a means of reducing the toxicity of HAW. Sacharov, V.N. Archiv fuer Energiewirtschaft; 34: No. 9, 752-764(Sep 1980). (In German).

The published data of detailed calculations of zero-dimensional models simulating modern thermal and fast breeding reactors confirm the expediency of a combustion of the actinides by action of neutron beams. Best qualified for that purpose are LMFBRs which can compete with the conception of a depositing of the $\alpha$-actine wastes. The actinides applied in such a reactor have sufficient burn-up velocity, while their presence excerts only a minor influence upon the neutron-physical parameters of the plant. As proved by thorough investigations, the actinide nuclides in a LWR indeed burn out faster (by transmutation to heavier isotopes), but if the respective advantages to transcurium elements and the influence of the actinides applied instead of fuel upon the physical data of the reactor are taken into account, preference should yet be given to FBRs.

623 Process for producing formed parts containing highactivity waste and consisting of glass granules in a metal matrix. Ondracek, G. (to Kernforschungszentrum Karlsruhe G.m.b.H., Germany, F.R.). German(FRG) Patent 2,856,466/A/. $10 \mathrm{Jul}$ 1980. 8p. (In German).

Glass balls are mixed with lead powder until a macroscopically homogeneous distribution of the two powders in the suspension is achieved. Then the mixing fluid is evaporated, the mixture is pressed into steel matrices and resintered.

624 Hydrolytic durability of two high-level waste oxide borosilicate glasses (gp 98/12 and gp 98/26). Kahl, L. (Kernforschungszent Karlstruhe, Ger). Nuclear and Chemical Waste Management; 2: No. 2, 143-146(1980).

Two glasses containing 15 wt.\% simulated high-level liquid waste (HLLW) oxides were leached several times in distilled water, Asse rock salt brine, and carnallite-containing quinary solution at normal pressure (Soxhlet: 100 /degree/C) and at 130 bars (autoclave: 100, 150 and 200/ degree/C). The leach rates increase with temperature and show only a slight difference between water and brine but a decrease in the case of quinary solution. Leaching at elevated pressure and $100 /$ degree $/ C$ is less than at normal pressure and the same temperature. The crystallization of the glass products (annealing time 100 days at $750 /$ degree/C) has no effect on the leach resistance as determined in Soxhlet tests.

626 Progress of the vitrification of $\mathrm{HLW}$ in France. Mamelle, J.; Sombret, M. (SGN-France, Yvelines, Cedex). Transactions of the American Nuclear Society; 35: 68-69(1980). (CONF-801107-).

From ANS international conference; Washington, DC, USA (17 Nov 1980).

FRANCE; HIGH-LEVEL RADIOACTIVE WASTES; RADIOACTIVE WASTE PROCESSING; VITRIFICATION; SOLIDIFICATION; RADIOACTIVE WASTE FACILITIES

626 HLW fixation in sintered modified SYNROC-B ceramics: chemical stability evaluation. Solomah, A.G.; Zumwalt, L.R. (NC State Univ, Raleigh, NC 27650). Transactions of the American Nuclear Society; 35: 191-193(1980). (CONF$801107-$ ). Nov 1980).

From ANS international conference; Washington, DC, USA (17

CERAMICS; EVALUATION; LEACHING; MATRIX ISOLATION; NORTH CAROLINA; SPECTROSCOPY; RADIOACTIVE WASTE PROCESSING; SOLIDIFICATION; HIGH-LEVEL RADIOACTIVE WASTES; SYNROC PROCESS; SYNTHETIC ROCKS

627 Radioactive waste management. Blomek, D. (Oak Ridge National Lab., TN (USA)). pp 97-106 of Nuclear power safety. Rast, D.: Viver, L. (eds.). Moscow, USSR; Atomizdat (1980). (In Russian)

The prospects of nuclear power development in the USA up to 2000 and the problems of the fuel cycle high-level radioactive waste processing and storage are considered. The problems of liquid and solidified 
radioactive waste transportation and their disposal in salt deposits and other geologic formations are discussed. It is pointed out that the main part of the high-level radioactive wastes are produced at spent fuel reprocessing plants in the form of complex aqueous mixtures. These mixtures contain the decay products of about 35 isotopes which are the nuclear fuel fission products, about 18 actinides and their daughter products as well as currusion products of fuel cans and structural materials and chemical reagents added in the process of fuel reprocessing. The high-level radioactive waste management includes the liquid waste coof ing which is necessary for the short and middle living isotope decay, separation of some most dangerous components from the waste mixture, waste solidification, their storage and disposal. The conclusion is drawn that the successful solution of the high-level radioactive waste manage ment problem will permit solving the problem of the fuel cycle radioactive waste management as a whole. The salt deposits, shales and clays are the most suitable for radioactive waste disposal.

628 Borosilicate glass and glass ceramics with real highly radioactive waste. Guber, W.; Mueller, W.; Saidl, J. (KFZ Karlsruhe-Germany). Transactions of the American Nuclear Society; 31: 528-530(Nov 1979). (CONF-790519-). May 1979).

From European nuclear conference; Hamburg. F.R. Germany (6

BOROSILICATE GLASS; CERAMICS; FISSION PROD UCTS; HIGH-LEVEL RADIOACTIVE WASTES; REPROCESSING; SOLIDIFICATION; RADIOACTIVE WASTE. PROC.RSSING

629 Solidification for final storage of high-level radioactive fission product solutions from spent fuel elements. Halaszovich, S. Aachen, Germany, F.R.; Technische Hochschule (1979). 118p. (In German).

Thesis.

As a contribution to solve the waste problem in the outer fuel cycle, a process for solidification of high-level radioactive fission product solutions in boron silica glass has been developed. The operations of concentration and denitration - adding of products for vitrification of glass - drying - melting of glass, which are more or less complex in every process, are carried out in steps which are more or less uncoupled. It is shown in four hot experiments with real radioactive waste from LWR fuel element reprocessing that this process is suitable in principle. These experiments, their findings and the disturbances which occurred during the experiments are described. Basic considerations show the possibility of an industrial-scale application.

630 (DP-tr-20) Development of borosilicate glass for the vitrification plant PAMELA. Lutze, W.; Schiewer, E.; De, A.K. (Du Pont de Nemours (E.I.) and Co., Aiken, SC (USA)). [nd]. Contract AC09-76SR00001. Translation source information not available . 18p. NTIS, PC A02/MF A01. Order Number DE82000531.

Portions oi dócument are illegible.

The Federated Republic of Germany established in the Eurochemic in Mol, Belgium, a plant, PAMELA, for solidification of socalled LEWC (low enriched waste concentrate). The concern there is with $65 \mathrm{~m}^{0}$ of high radioactivity waste inherited from reworking of fuel elements in the years 1967 to 1974 . In this plant the HLW will be fused in borosilicate glass and then equal amounts will be either drawn off into molds or imbedded as glass beads in lead (as so-called Vitromets). The vituification will be carried out in a melting pot with direct electrical heat. Nothing is reported here about the technical layout of the solidification plant. The state of development of the borosilicate glass at the Hahn-Meitner Institute, the relationship between glass formation, technical limiting conditions, and composition of the waste are discussed.

631 (DP-tr-22) Chemical denitration of radioactivewaste solutions with formic acid. Kelm, M.; Oser, B.; Drobnik, S.; Deckwer, W.D. (Du Pont de Nemours (E.I.) and Co., Aiken, SC (USA). Savannah River Lab.). [nd]. Contract AC09. 76SR00001. Translation of KFK-2940, March 1980. 12p. NTIS, PC A02/MF A01. Order Number DE82018764.

Portions of document are illegible.

The denitration process with formic acid as reducing agent has been investigated under various aspects in the course of several years. All work - especially such as on the safety of the process - will soon be concluded. This includes dying out and recovery behavior of the reaction on feed interruption, a fault-tree analysis, determination of the explosio behavior of formic acid vapor-air mixtures, as well as tests on furthe reduction of nitrogen oxide content of the reaction gas by washing th gas with hot formic acid. Altogether, denitration is an effective preliminary step for removal of nitric acid from radioactive waste solutions.

532 (AAEC/E-550) Assessment of overseas developments in methods for treatment and disposal of high-level radioactive wastes. Costello, J.M.; Wilson, E.G. (Australian Atomic Energy Commission Research Establishment, Lucas Heights). Oct 1982. 74p. NTIS (US Sales Only), PC A04/MF A01. Order Number DE83703468.

The principles of management and disposal of highly radioactive wastes contained in spent fuel from nuclear power generation are described. The status of developments in spent fuel reprocessing, highlevel waste solidification and geologic isolation is reviewed. Some generic studies on the possible range of annual radiological doses to individuals from waste repositories are discussed and compared with doses from some existing nuclear power and fuel cycle operations, and with the dose received annually from an average background of naturally occurring radiatinn

633 (AD-A-106573/9) Is spent fuel or waste from reprocessed spent fuel simpler to dispose of. (General Accounting Office, Washington, DC (USA). Energy and Minerals Div.). 12 Jun 1981. 67p NTIS, PC $\Lambda 04 / M F$ A01.

Currently, the Department of Energy plans to permanently dispose of spent fuel without reprocessing, but some experts believe that this will waste a valuable source of uranium and plutonium and pose special waste isolation problems. Until the Congress makes a decision on the future of nuclear power, DOE has no option but to plan for any eventuality-including the potential geological disposal of spent fuel. This report concludes that spent fuel; is more difficult to isolate from the biosphere than high-level waste reprocessed from spent fuel and discusses the status of DOE's efforts to provide a manmade barrier system which, when placed around the waste in a repository, will contain the radioactivity for at least the first 1,000 years.

634

(AERE-R-10222) Surface storage of vitrified highlevel radioactive waste in reinforced-concrete casks. Beale, $H$. George, M.W.; Robertson, T.J.M. (UKAEA Atomic Energy Research Establishment, Harwcll. Research Reactors Div.). Jun 1982. 31p. NTIS (US Sales Only), PC A03/MF A01. Oider Number DE83700874.

The feasibility of storing canisters containing vitrified high level radicactive waste in reinforced concrete casks is examined. This preliminary study identifies the limitations and probable cost of such a store and leads to the cunclusion that the concept is feasible.

635 (AERE-TP-944) Coupled heat and groundwater flow in pornus mrt. Rạe, J.; Robinson, P.C.; Wiokens, L.M (UKAEA Atomic Energy Research Establishment, Harwell. Theoretical Physics Div.). Jun 1982. 40p. NTIS (US Sales Only), PC A03/MF A01. Order Number DE83700876.

The report falls under the headings: introduction (reference to application to the possible disposal underground of high-level radioactive waste); the flow equation (mass conservation; Darcy's equation; energy conservation; thermodynamics; boundary conditions); the finite element formulation; the tirur stepping method; the responsc of clay pore water to a heat source; the response of granite pore water to a heat source.

630 (ANL-82-58) Fuel cycle programs. Quarterly progreśs report, April-June 1982 . Stcindler, M.J.; Bates, J.K.; Cannon, T.F. (Argonne National Lab., IL (USA)). Dec 1982. Contract W-31-109-ENG-38. 143p. NTIS, PC A07/MF A01. Order Number DE83011014.

Logging techniques are being developed to measure the relative amount of residual oil in a depleted oil reservoir by injecting gammaactive solution into it. Laboratory analog experiments are being done to examine the extent to which radioactivity might move from a nuclear waste repository because of flowing groundwater. The composition and transport of saline pore fluids in granite cores from northern lllinois and from the Canadian Shield were studied. Testing and development of equipment for the destructive analysis of full-length irradiated fuel rods from the Light Water Breeder Reactor is in progress. Work has.been 
done on: (1) the full-scale shear, (2) the dual dissolver system, (3) scrap and waste disposal, (4) the data management system, and (5) analytical systems. Contributions to an information base that will be used in an analysis of the status of the disposal of airborne wastes from the nuclear fuel cycle include (1) development of packaging for deep geologic disposal, (2) review of practices in the packaging of LLW, (3) review of ocean disposal of LLW and HLW, (4) comparison of regulations and criteria from several sources for deep geologic disposal, (5) shallow-land burial and its regulation, and (6) regulations on packaging for transportation of radioactive materials. Simulated-flow and true-flow leach tests were compared to develop an understanding of their differing characteristics, with emphasis on leach rates and concentrations of leached species in the effluent leachates. Two leach rate models were analyzed: (1) constant leach rate independent of concentrations of leached species in the leachates and (2) variable leach rate dependent on such concentrations.

537 (ANL-82-62, pp 540-547) Hydrogeologic testing of the E.J. Kubat borehole, San Juan County, Utah: utilization of a high pressure instrumented flow control system. Hoexter, D.F. (Woodward-Clyde Consultants, San Francisco, CA). Sep 1982. NTIS, PC A99/MF A01. (CONF-820612-).

From Symposium on instrumentation and control for fossil energy processes; Houston, TX, USA (7 Jun 1982).

The E.J. Kubat borehole, located in San Juan County, Utah, in the SW 1/4, Section 23, T37S, R19E, was drilled in 1958 as a petroleum exploration (wildcat) well. Drilling records and geophysical logs were examined as a part of the Paradox Basin Project, a feasibility and siting study for a high-level nuclear waste repository to be located in bedded salt deposits in the Utah portion of the Paradox Basin. This project is part of the Department of Energy National Waste Terminal Storage Program, and is being conducted by Woodward-Clyde Consultants under subcontract to Battelle Memorial Institute, Office of Nuclear Waste Isolation. The geophysical logs of the Kubat and nearby wells showed that salt cycles potentially suitable as a possible repository horizon existed within the Pennsylvanian-age Paradox Formation in the area. Thus, it was decided to re-enter the well and extend it deeper into the Mississippian Leadville Limestone, the major water-bearing formation underlying the repository horizon. A series of hydrogeologic tests was conducted to evaluate hydrogeologic properties of the Leadville Limestone. These tests provided data on potentiometric level, transmissivity and permeability of the test interval. Although testing of relatively highly permeable formations is common, obtaining reliable data from formations of low permeability is more difficult, and is a special aspect of the paradox Basin Project.

538 (ANL-82-78) Fuel cycle programs. Quarterly progress report, July-September 1982. Steindler, M.J.; Bates, J.K.; Cannon, T.F. (Argonne National Lab., IL (USA)). Mar 1983. Contract W-31-109-ENG-38. 165p. NTIS, PC A08/MF A01. Order Number DE83011920.

Portions are illegible in microfiche products. Original copy available until stock is exhausted.

The effect of slight differences in composition on the leachability of simulated nuclear waste (SRL 131 glass specimens) is being studied. In other work, contacting of simulated nuclear waste glass with a humid atmosphere has been found to be a method of accelerating aging reactions while simulating conditions that may exist in a nuclear waste repository. Nuclear logging methods are being considered for determining the oil content of depleted wells. Results indicated the desirability of measuring the integrated spectrum from a radioisotope in an injected solution. The behavior of radionuclides leached from proposed nuclear waste repositories is being studied in laboratory analog experiments, namely, flow-through experiments to examine the migration of radionuclides through backfill, the waste form, and basalt rock. The compositions of pore fluids in granites of the Canadian Shield are being determined to examine the origin and genesis of groundwater. Also being studied is the effect of dissolved organic matter (such as humic acid) in groundwater on the migration behavior of radionuclides. Testing and development of equipment for the destructive analysis of full-length irradiated fuel rods from the Light Water Breeder Reactor are in progress. An information base is being assembled that will be used in future analysis of the status of airborne waste disposal. The four radionuclides being studied are ${ }^{85} \mathrm{Kr},{ }^{129} \mathrm{I},{ }^{0} \mathrm{H}$, and ${ }^{14} \mathrm{C}$. The anticipated hydrologic, thermal, and hydrothermal conditions in high-level nuclear waste repositories and the resulting physical requirements for backfill materials are presented. A detailed analysis is presented on whether proposed mixtures of bentonite and crushed basalt will have adequately low permeability in the severe thermal, hydrothermal, and radiation environment expected in a repository.

639 (ANL-83-19) Fuel cycle programs. Quarterly progress report, October-December 1982. Steindler, M.J.; Bates, J.K.; Cannon, T.F.; Couture, R.A.; Deeken, P.G.; Fagan, J.E.; Gerding, T.J.; Hoh, J.C.; Kincinas, J.E.; Kolba, V.M. (Argonne National Lab., IL (USA)). Aug 1983. Contract W-31-109ENG-38. 182p. NTIS, PC A09/MF A01. Order Number DE84000191.

An information base has been assembled for the future analysis of concepts for disposing of ${ }^{85} \mathrm{Kr},{ }^{129} \mathrm{I},{ }^{0} \mathrm{H}$, and ${ }^{14} \mathrm{C}$ from the nuclear fuel cycle. A blanket processing program emphasizing tritium recovery and effective heat transfer is in progress. The current experiment designated TRIO-01 is designed to test the performance of a miniaturized solid breeder blanket assembly with continuous in situ recovery of tritium. The thermal-hydraulic response of solid breeder blankets and the tritium system problems associated with liquid metal blankets are being analyzed. Thermal-hydraulic analysis is also being supplied for the First Wall/Blanket/Shield Program. Tritium needs and systems required for both tokamak and mirror fusion reactors are being supplied. Work is being done on (1) the sealing properties of fresh and altered proposed backfill materials for high-level nuclear waste in the expected hydrothermal, high-radiation environments of repositories, (2) the extent to which radioactivity might move from a nuclear waste repository because of flowing groundwater, (3) the effects of dissolved organic acids on the partitioning of ${ }^{241} \mathrm{Am}$ and ${ }^{207} \mathrm{~Np}$ between synthetic groundwater and crushed basalt, and (4) the partitioning of a small group of toxic phenol compounds between dolomite and water. Development and testing of equipment for the destructive analysis of full-length irradiated fuel rods from the Light Water Breeder Reactor are in progress. Work has been done on: (1) the full-scale shear, (2) the dual dissolver system, (3) scrap and waste disposal, (4) the process control and data management system, and (5) analytical systems. Testing of simulated nuclear waste glasses is being done by exposing preleached Savannah River Laboratory type 131 glass to a second static leach test and by hydrating tektite glass meteorites and Pacific Northwest Laboratories type 76-68 glass in water vapor at elevated temperatures.

640 (BMI-OCRD-1) National survey of crystalline rocks and recommendations of regions to be explored for high-level radioactive waste repository sites. Smedes, H.W. (Battelle Memorial Inst., Columbus, OH (USA). Office of Crystalline Repository Development). Apr 1983. Contract ACO283CH10140. 120p. NTIS, PC A06/MF A01. Order Number DE83010252.

Portions are illegible in microfiche products.

A reconnaissance of the geological literature on large regions of exposed crystalline rocks in the United States provides the basis for evaluating if any of those regions warrant further exploration toward identifying potential sites for development of a high-level radioactive waste repository. The reconnaissance does not serve as a detailed evaluation of regions or of any smaller subunits within the regions. Site performance criteria were selected and applied insofar as a national data base exists, and guidelines were adopted that relate the data to those criteria. The criteria include consideration of size, vertical movements, faulting, earthquakes, seismically induced ground motion, Quaternary volcanic rocks, mineral deposits, high-temperature convective ground-water systems, hydraulic gradients, and erosion. Brief summaries of each major region of exposed crystalline rock, and national maps of relevant data provided the means for applying the guidelines and for recommending regions for further study. It is concluded that there is a reasonable likelihood that geologically suitable repository sites exist in each of the major regions of crystalline rocks. The recommendation is made that further studies first be conducted of the Lake Superior, Northern Appalachian and Adirondack, and the Southern Appalachian Regions. It is believed that those regions could be explored more effectively and suitable sites. probably could be found, characterized, verified, and licensed more readily there than in the other regions.

641 (BMI/OCRD-9) Expected repository environments in granite: assessment of rock stability of the container drill bole region. Brandshaug, T. (RE/SPEC, Inc., Rapid City, SD (USA)). Oct 1983. Contract AC06-76RL01830;AC02- 
83CH10140. 47p. NTIS, PC A03/MF A01. Order Number DE84002553.

This report was prepared as part of a study on Expected Repository Environments in Granite (ERE-G). The repository regions considered in this report were the disposal room, the pillar between rooms, and the waste container drillhole. Three-dimensional analyses were performed to predict temperatures in the rock and to investigate the thermoelastic response and its effect on rock stability. Calculated factors-ofsafety of intact rock identify zones of potential rock instability. The analyses were performed for three different types of waste: spent fuel (SF), commercial high-level waste (CHLW), and defense high-level waste (DHLW). The highest temperatures were predicted in the rock when considering CHLW and the lowest when considering DHLW. The analyses did not predict any rock instability as a consequence of excavating the room and the container drillhole. In all cases, relatively high tensile stresses develop in the upper part of the drillhole. The analyses give evidence of potential stability problems of the rock around the drillhole later in time for both the SF and the CHLW. In the case of DHLW, no instability of the rock was predicted during the time period considered in thesc analyses. 7 references, 24 figures.

642 (BNL-32669) High-level radioactive wste management: a means to social consensus. Pierce, B.; Hill, D.; Haefele, E.T. (Brookhaven National Lab., Upton, NY (USA); Pennsylvania Univ., Philadelphia (USA)). 1983. Contract AC0276CH00016. 14p. (CONF-830523-4). NTIS, PC A02/MF A01. Order Number DE83008592.

From International conference on radioactive waste management; Seattle, WA, USA (16 May 1983).

The problem of safely disposing of high-level radioactive waste is not new, but it is becoming more pressing as the temporary storage facilities of public utilities run out. The technical questions of how best to immobilize these wastes for many centuries have been studied for years and many feel that these problems are solved, or nearly so. Many states have set up roadblocks to the federal waste management program, however, and it is clear that social consensus must be reached for any waste disposal program to be successful. The Nuclear Waste Policy Act of 1982 provides a long-needed framework for reaching this consensus, giving the states unprecedented access to federal decision-making. The rights of the states in a process of cooperation and consultation are clearly defined by the Act, but the means by which the states exercise those rights are left entirely to them. We examine the structures, methods, and goals open to the states, and recommend a rationale for the state decision process defining the roles of the governor and legislature.

543 (BNL-33451) Radiation induced F-center and colloid formation in synthetic $\mathrm{NaCl}$ and natural rock salt: applications to radioactive waste repositories. Levy, P.W.; I.oman, J.M.; Kierstead, J.A. (Brookhaven National Lab., Upton, NY (USA)). 1983. Contract AC02-76CH00016. 28p. (CONF830575-5). NTIS, PC A03/MF A01. Order Number DE83016732.

From Spring meeting of the Japan Society of Corrosion Engineers; Tokyo, Japan (18 May 1983).

Radiation damage, particularly $\mathrm{Na}$ metal colloid formation, has been studied in synthetic $\mathrm{NaCl}$ and natural rock salt using unique equipment for making optical absurption, luminescence and other measurements during irradiation with 1 to $3 \mathrm{MeV}$ electrons. Previous studies have established the F-center and colloid growth phenomenology. At temperatures where colloids form most rapidly, 100 to $250 \mathrm{C}$. F-centers appear when the irradiation is initiated and increase at a decreasing rate to a plateau, reached at doses of $10^{6}$ to $10^{7} \mathrm{rad}$. Concomitant colloid growth is described by classical nucleation and growth curves with the transition to rapid growth occurring at $10^{6}$ to $10^{7} \mathrm{rad}$. The colloid growth rate is low at $100 \mathrm{C}$, increases markedly to a maximum at 150 to 175 $\mathrm{C}$ and decreases to a negligible rate at $225 \mathrm{C}$. At $1.2 \times 10^{8} \mathrm{rad} / \mathrm{h}$ the induction period is $>10^{4} \mathrm{sec}$ at $100 \mathrm{C},<3000 \mathrm{sec}$ at 150 to $175 \mathrm{C}$ and $>10^{4} \mathrm{sec}$ at $275 \mathrm{C}$. The colloid growth in salt from 14 localities is well described by $\mathrm{C}\left(\right.$ dose) ${ }^{n}$ relations. Data on WIPP site salt (Los Medanos, NM, USA) has been used to estimate roughly the colloid expected in radioactive waste repositories. Doses of 1 to $2 \times 10^{10} \mathrm{rad}$, which will accumulate in salt adjacent to lightly shielded high level canisters in 200 to 500 years, will convert between 1 and $100 \%$ of the salt to Na colloids (and $\mathrm{Cl}$ ) if back reactions or other limiting reactions do not occur. Each high level lightly shielded canister may ultimately be surrounded by 200 to $300 \mathrm{~kg}$ of colloid sodium. Low level or heavily shielded canisters may produce as little as $1 \mathbf{~ k g}$ sodium.

644 (BNL-NUREG-31329) High-level-waste containment for a thousand years: unique technical and research problems. Davis, M.S. (Brookhaven National Lab., Upton, NY (USA)). 1982. Contract AC02-76CH00016. 8p. (CONF8206106-1). NTIS, PC A02/MF A01. 1982).

From 14. conference on nuclear safety; Tokyo, Japan (4 Jun

In the United States the present policy for disposal of high level nuclear wastes is focused on isolation of solidified wastes in a mined geologic repository. Safe isolation is to be achieved by utilizing both natural and man-made barriers which will act in concert to assure the overall conservative performance of the disposal system. The incorporation of predictable man-made barriers into the waste disposal strategy has generated some new and unique problems for the scientific community.

645 (BNL-NUREG-32107) Identification of crevice corrosion in the titanium alloy TiCode-12 in simulated rock salt brine at $150^{6} \mathrm{C}$. Ahn, T.M.; Lee, B.S.; Soo, P. (Brookhaven National Lab., Upton, NY (USA)). 1982. Contract AC0276CH00016. 20p. (CONF-820955-2). NTIS, PC A02/MF A01. Order Number DE83002819.

From ASTM conference on titanium and zirconium in industrial applications; New Orleans, LA, USA (21 Sep 1982).

TiCode-12 (Ti-0.3Mo-0.8 Ni) is a prime corrosion-resistant material for high-level nuclear-waste containers which will be emplaced in mined geologic repositories such as those in rock salt. The crevice corrosion behavior of this alloy was investigated in simulated rock salt brine solutions at a temperature of $150^{\circ} \mathrm{C}$. A distinct corrosion product with a range of interference colors was observed in a mechanically simulated crevice after two to four-weeks' exposure. Low pH accelerated the reaction rate and deaerated solutions give less corrosion than aerated ones. Also, increasing specimen size, decreasing crevice gap, and preoxidation of the cathodic area gave more voluminous corrosion products inside the crevice. High temperature did not necessarily accelerate crevice corrosion. These results are consistent with those expected from macroscopic concentration cell formation accompanied by oxygen depletion, potential drop, and acidification inside the crevice. TEM and SEM techniques were extensively utilized to identify the film formed inside the crevice at each stage of the corrosion process. Based on the study, and pH and potential measurements inside the crevice of commercially pure titanium done by other workers, a mechanism for crevice corrosion in Ticulo 12 has been developed. It involves the initial furmation of compact anatase crystals inside the crevice.As the macroscopic cell develops further, it is postulated that cither the anatase form of $\mathrm{TiO}_{2}$ will transform to the lower oxide $\mathrm{Ti}_{0} \mathrm{O}_{3}$ and to the rutile form of $\mathrm{TiO}_{2}$, ur titanium dissolves into the solution after the breakdown of the protective film and subsequent hydrolysis takes place to form the lower oxide and the rutile form of $\mathrm{TiO}_{2}$. The role of alloying elements (Mo and $\mathrm{Ni}$ ) and dissolved solutes are discussed with respect to these postulations. 10 figures, 3 tables.

646 (BNL-NUREG-33192) Draft technical position on waste-package reliability. Sastre, C.; Prince, A.; Pescatore, C. (Brookhaven National Lab., Upton, NY (USA)). May 1983. Contraot $\Lambda$ C02.76CH00016. 71p. NTIS, PC A04/MF A01. Order Number DE84000540.

A method for the evaluation of reliability of a high-level waste package to comply with the requirements of 10 CFR 60 is being proposed. The method is based on the repetitive usage of a performance model for values of the model parameters which span their range of uncertainty. The techniques for selecting values for the input parameters, viewed as random variables, and for generating empirical correlations among experimental data are described and illustrated with an example of a simplified waste package analysis.

647 dynamics of basalt-oxygen and basalt-water reactions. Schweitzer, D.G.; Davis, M.S. (Brookhaven National Lab., Upton, NY (USA)). Aug 1983. Contract AC02-76CH00016. 10p. (CONF-8308126-2). NTIS, PC A02/MF A01 - GPO. Order Number DE84001458. 
From NRC research annual review meeting of nuclear waste management research on geochemistry of HLW disposal; Reston, VA, USA (30 Aug 1983).

A knowledge of basalt-oxygen equilibria and basalt-water equilibria are required to predict the performance of a high-level waste package in a basalt repository. In this report we have evaluated uncertainties in these equilibria using thermodynamic data from two sources, the JANAF Thermochemical Tables (1971) and from Kubaschewski (1974). Our analysis indicates that the uncertainties in the basic thermodynamic data lead to 30 orders of magnitude in uncertainty in the oxygen fugacity for the magnetite-hematite reaction $\left(10^{-57}\right.$ to $\left.10^{-86} \mathrm{~atm}\right)$ and about 15 orders of magnitude uncertainty in the hydrogen equilibrium pressure $\left(10^{-12}\right.$ to $\left.10^{+0} \mathrm{~atm}\right)$. A vast volume of literature exists on reactions involving magnetite and hematite in water at temperatures pertinent to basalt repositories $\left(50^{\circ}\right.$ to $\left.350^{\circ} \mathrm{C}\right)$. These data show that $\mathrm{Fe}_{0} \mathrm{O}_{4}$ and $\mathrm{Fe}_{2} \mathrm{O}_{0}$ can coexist in water with oxygen fugacities from about $10^{-4}$ to 1 rather than the calculated oxygen fugacities of $\sim 10^{-60}$ to $10^{-00}$ assumed in the past. 5 references.

548 (CEA-CONF-6223) Elimination of used sources. Taking over and storage. Desroches, J. (CEA Centre d'Etudes Nucleaires de Saclay, 91 - Gif-sur-Yvette (France)). Feb 1982. 11 . (In French). (CONF-820270 3). NTIS (US Sales Only), PC A02/MF A01. Order Number DE83700877. Feb 1982).

From SFRP meeting on radioactive sources; Paris, France (16

The waste disposal of sealed radioactive sources used in medicine and industry poses technical problems for high activity sources and economic problems for small sources. Some cases of large radioactive sources elimination are reviewed and the formalities to be completed for the waste disposal of sources in general are briefly described.

$549 \quad$ (CF-60-8-72) Analysis of waste disposal practice and control at ORNL. Feige, Y.; Parker, F.L.; Struxness, E.G. (Oak Ridge National Lab., TN (USA)). 4 Oct 1960. Contract W-7405-ENG-26. 50p. NTIS.

An analysis of liquid-waste-disposal methods employed at ORNL is made. High-level wastes arc stored in tanks and allowed to decay; medium-level wastes are pumped to seepage pits; and low-level wastes are passed to a nearby creek with or without treatment, depending upon the level of radioactivity. Methods of monitoring and control are discussed.

550 (CNEN-RT/ING-81-12) Laboratory determination of the thermal properties of large-scale samples of natural clays. Bocola, W.; Girolimetti, G.; Giulianelli, G.; Tassoni, E.; Testa, L. (Comitato Nazionale per l'Energia Nucleare, Casaccia (Italy). Dipartimento Radiazioni e Ricerche di Sicurezza e Protezione). Jul 1981. 52p. (In Italian). NTIS (US Sales Only), PC A04/MF A01. Order Number DE82902983.

Portions of document are illegible.

The determination of the thermal properties is important in the evaluation of argillaceous formations as potential host rock for the geologic disposal of high level radioactive wastes. The present paper reports the heating tests, carried out on a large cubic block of clay $(1>80 \mathrm{~cm})$ by means of 3 small heaters, whose thermal power has been varied from 20 to 40 watt. The experimental results, which have been processed by the curve fitting method, show that the thermal diffusivity is about 20 $\mathrm{cm}^{2} / \mathrm{h}$, while the thermal conductivity range from 0.014 to 0.016 watt $/ \mathrm{cm}{ }^{0} \mathrm{C}$.

(CONF-820544-1) Remote automatic plasma arcclosure welding of a dry-storage canister for spent nuclear fuel and high-level radioactive waste. Sprecace, R.P.; Blankenship, W.P. (Westinghouse Electric Corp., Pittsburgh, PA (USA). Advanced Energy Systems Div.). 1982. Contract AC0881NV10171. 48p. NTIS, PC A03/MF A01. Order Number DE82013963.

From International conference on welding technology for energy applications; Gatlinburg, TN, USA (16 May 1982).

Portions of document are illegible.

A carbon steel storage canister has been designed for the dry encapsulation of spent nuclear fuel assemblies or of logs of vitrified high level radioactive waste. The canister design is in conformance with the requirements of the ASME Code, Section III, Division I for a Class 3 vessel. The canisters will be loaded and sealed as part of a completely remote process sequence to be performed in the hot bay of an experimental encapsulation facility at the Nevada Test Site. The final closure to be made is a full penetration butt weld between the canister body, a 12.75-in O.D. $x$ 0.25-in wall pipe, and a mating semiellipsoidal closure lid. Due to a combination of design, application and facility constraints, the closure weld must be made in the $2 \mathrm{G}$ position (canister vertical). The plasma arc welding system is described, and the final welding procedure is described and discussed in detail. Several aspects and results of the procedure development activity, which are of both specific and general interest, are highlighted; these include: The critical welding torch features which must be exactly controlled to permit reproducible energy input to, and gas stream interaction with, the weld puddle. A comparison of results using automatic arc voltage control with those obtained using a mechanically fixed initial arc gap. The optimization of a keyhole initiation procedure. A comparison of results using an autogenous keyhole closure procedure with those obtained using a filler metal addition. The sensitivity of the welding process and procedure to variations in joint configuration and dimensions and to variations in base metal chemistry. Finally, the advantages and disadvantages of the plasma arc process for this application are summarized from the current viewpoint, and the applicability of this process to other similar applications is briefly indicated.

662 (CONF-821053-2) Oxidation potential (E) and pH control during experimentation. Seitz, M.G. (Argonne National Lab., IL (USA)). 1982. Contract W-31-109-ENG-38. 11p. NTIS, PC A02/MF A01. Order Number DE83014287.

From Workshop on standardization of methods for measuring migration of radionuclides in geomedia; Berlin, F.R. Germany (25 Oct 1982).

Purpose of this statement is to introduce the subject of oxidation potential, $E_{b}$, and to discuss its control in experiments. After the concept of $E_{h}$ is reviewed, the range of oxidation potentials expected to be associated with a repository for high level nuclear waste will be addressed. Finally, three laboratory methods of controlling $E_{b}$ will be described, along with some perspective that has been derived from experience given for each method.

653 (CONF-821107-32) Technetium behavior in sulfide and ferrous iron solutions. Lee, S.Y.; Bondietti, E.A. (Oak Ridge National Lab., TN (USA)). 1982. Contract W-7405ENG-26. 11 . NTIS, PC A02/MF A01. Order Number DE83003418.

From 6. international symposium on the scientific basis for radioactive waste management (Materials Research Society); Boston, MA, USA (1 Nov 1982).

Pertechnetate oxyanion $\left({ }^{99} \mathrm{TcO}_{4}{ }^{-}\right)$, a potentially mobile species in leachate from a breached radioactive waste repository, was removed from a brine solution by precipitation with sulfide, iron, and ferrous sulfide at environmental pH's. Maghemite $\left(\gamma-\mathrm{Fe}_{2} \mathrm{O}_{0}\right)$ and geothite $(\alpha-\mathrm{FeOOH})$ were the dominant minerals in the precipitate obtained from the $\mathrm{TcO}_{4}$-ferrous iron reaction. The observation of small particle size and poor crystallinity of the minerals formed in the presence of Tc suggested that the $\mathrm{Tc}$ was incorporated into the mineral structure after reduction to a lower valence state. Amorphous ferrous sulfide, an initial phase precipitating in the $\mathrm{TcO}_{4}$-ferrous iron-sulfide reaction, was transformed to goethite and hematite $\left(\alpha-\mathrm{Fe}_{2} \mathrm{O}_{0}\right)$ on aging. The black precipitate obtained from the $\mathrm{TcO}_{4}$-sulfide reaction was poorly crystallized technetium sulfide $\left(\mathrm{Tc}_{2} \mathrm{~S}_{7}\right)$ which was insoluble in both acid and alkaline solution in the absence of strong oxidents. The results suggested that ferrous- and/or sulfide-bearing groundwaters and minerals in host rocks or backfill barriers could reduce the mobility of $T c$ through the formation of less-soluble Tc-bearing iron and/or sulfide minerals.

664 (CONF-821107-33) Electrical-conductivity mensurements of leachates for the rapid assessment of wasteform corrosion resistance. Sales, B.C.; Petek, M.; Boatner, L.A. (Oak Ridge National Lab., TN (USA)). 1982. Contract W-7405ENG-26. 8p. NTIS, PC A02/MF A01. Order Number DE83003419.

From 6. international symposium on the scientific basis for radio active waste management (Materials Research Society); Boston, MA USA (1 Nov 1982).

Measurements of the electrical conductivity of leachate solutions as a function of time can be used as an efficient, informative means of evaluation and comparison in the development of nuclear waste forms 
and in the preliminary analysis of their corrosion resistance in distilled water. Three separate applications of this technique are described in this work. These are: (1) its use in the optimization of the corrosion resistance of a crystalline wasteform (monazite); (2) a study of the protective ability of the surface layer (gel layer) which forms on the nuclear waste glass Frit $21+20$ wt \% SRW in distilled water; and (3) making comparisons of the overall corrosion resistance of three different nuclear wasteforms (i.e., monazite, SYNROC, and borosilicate glass). A complete solution analysis of the borosilicate glass leachate and a straightforward analysis of the conductivity results agree to within $\pm 20 \%$. In the absence of a complete, time consuming solution analysis, conductivity measurements can be used to estimate reliably the total ionic concentration in the leachate to within a factor of 2 .

555 (CONF-830205-7) Equipment for the emplacement of heat-producing waste in long horizontal boreholes. Young, K.D.; Scully, L.W.; Fisk, A.; deBakker, P.; Friant, J.; Anderson, A. (Sandia National Labs., Albuquerque, NM (USA); FosterMiller Associates, Inc., Waltham, MA (USA); Robbins Co., Kent, WA (USA)). 1983. Contract AC04-76DP00789. 5p. NTIS, PC A02/MF A01. Order Number DE83006399. Feb 1983)

From Waste management conference; Tucson, AZ, USA (27)

Emplacement of heat-producing waste in long horizontal holes may offer several technical and economic advantages over shallow vertical hole emplacement. Less of the host rock suffers damage as a result of drift construction; the heat from the waste can be isolated from the access drifts for long periods of time; and the amount of rock which must be excavated is much less than in traditional disposal scenarios. One of the major reasons that has been used to reject the long hole concept in the past and adhere to the shallow vertical hole concept is the equipment required to drill. the holes and to emplace and retrieve the waste. Such equipment does not currently exist. It clearly is more difficult to drill a 600 to 1000 foot horizontal hole, possibly 3 to 4 feet in diameter, and place a canister of waste at the end of it than to drill a 30 foot vertical hole and lower the waste to the bottom. A liner, for emplacement hole stabilization, appears to be feasible by adapting existing technology for concrete slip forming or jacking in a steel liner. The conceptual design of the equipment to drill long horizontal holes, emplace waste and retrieve waste will be discussed. Various options in concept will be presented as well as their advantages and disadvantages. The operating scenario of the selected concept will be described as well as solutions to potential problems encountered.

656 (CONF-830528-18) Impacts of transportation on a test and evaluation facility for nuclear waste disposal: a systems analysis. Varadarajan, R.V.; Peterson, R.W.; Joy, D.S.; Gibson, S.M. (Battelle Columbus Labs., OH (USA); Oak Ridge National Lab., TN (USA)). 1983. Contract ACO676RL01830;W-7405-ENG-26. 3p. NTIS, PC A02/MF A01. Order Number DE83012651.

From 7. international symposium on packaging and transportation of radioactive materials; New Orleans, LA, USA (15 May 1983). An essential element of the Test and Evaluation Facility (TEF) is a waste packaging facility capable of producing a small number Test and Evaluation Facility of packages consisting of several different waste forms. The study envisions three scenarios for such a packaging facility: (1) modify an existing hot cell facility such as the Engine Maintenance Assembly and Disassembly (EMAD) facility at the Nevada Test Site so that it can serve as a packaging facility for the TEF. This scenario is referred to as the EMAD Option. (2) Build a new generic packaging facility (GPF) at the site of the TEF. In other words, colocate the GPF and the TEF. This scenario is referred to as the GPF Option, and (3) utilize the EMAD facility in conjunction with a colocated GPF (of minimal size and scope) at the TEF. This scenario is referred to as the Split Option. The results of the system study clearly bring out the fact that transportation has a significant impact on the selection and siting of the waste packaging facility. Preliminary conclusions, subject to the assumptions of the study, include the following: (1) regardless of the waste form, the GPF option is preferable to the other two in minimizing both transportation costs and logistical problems, (2) for any given scenario and choice of waste forms, there exists a candidate TEF location for which the transportation costs are at a minimum compared to the other locations, (3) in spite of the increased transportation costs and logistical complexity, the study shows that the overall system costs favor modification of an existing hot cell facility for the particular case considered.

667 (CONF-830528-Summ., pp D.I.13-D.I.14) Development of waste package designs for disposal in a salt repository. Balmert, M.E. (Battelle Project Management Division, Columbus, OH). 1983. NTIS, PC A21/MF A01.

From 7. international symposium on packaging and transportation of radioactive materials; New Orleans, LA, USA (15 May 1983).

HIGH-LEVEL RADIOACTIVE WASTES; PACKAGING; SALT DEPOSITS; CONTAINERS; DESIGN; RADIOACTIVE WASTE DISPOSAL

658 (CONF-830528-Summ, pp XX.10-XX.11) Twoand three-dimensional dynamic analyses of DHLW casks. Osborne, D.M. (GA Technologies Inc., San Diego, CA); Grenier, R.M.; Rasmusson, P.C.; Nickell, R.E. 1983. NTIS, PC A21/MF AO1.

From 7 . international symposium on packaging and transportation of radioactive materials; New Orleans, LA, USA (15 May 1983).

CASKS; RAIL TRANSPORT; ROAD TRANSPORT; IMPACT TESTG; HIOII-LEVEL RADIOACTIVE WA3TES; TWO: DIMENSIONAL CALCULATIONS; THREE-DIMENSIONAL CALCULATIONS

Eoj (CONF-831047-49) Beiefit-cust-risk analysis of alternatives for greatcr-confinement disposal of radioactive waste. Gilbert, T.L.; Luner, C.; Peterson, J.M. (Argonne National Lab., IL (USA)). 1983. Contract W-31-109-ENG-38. 6p. NTIS, PC A02/MF A01. Order Number DE83014694.

From American Nuclear Society winter meeting; San Francisco, CA, USA (30 Oct 1983).

Seven alternatives are included in the analysis: near-surface disposal; improved waste form; below-ground engineered structure; augered shaft; shale fracturing; shallow geologic repository; and highlevel waste repository. These alternatives are representative generic facilities that span the range from low-level waste disposal practice to high-level waste disposal practice, tentatively ordered according to an expected increasing cost and/or effectiveness of confinement. They have been chosen to enable an assessment of the degree of confinement that represents an appropriate balance between public health and safety requirements and costs rather than identification of a specific preferred facility design. The objective of the analysis is to provide a comparative ranking of the alternatives un the basis of benefit-cost-risk considerations.

560

(CONF-8110162-1) Nuclear waste/nuclear power: their futures are linked. Skoblar, L.T. (Atomic Industrial Forum, Inc., Washington, DC (USA)). 1981. 13p. NTIS, PC A02/MF A01. Order Number DE82902176.

From 52. annual executive conference; Palm Springs, CA, USA (19 Oct 1981).

Portions of this report are illegible.

This paper briefly reviews current aspects of radioactive waste disposal techniques and transportation. Addressed are high-level and low-level radioactive wastes, interim spent fuel storage and transportation. The waste options being explored by DOE are listed. Problems of public acceptance will be more difficult to overcome than technical problems. (DMC)

661 (CONF-8308111-2) Handling and disposing of radioactive waste. Trauger, D.B. (Oak Ridge National Lab., TN (USA)). 1983. Contract W-7405-ENG-26. 7p. NTIS, PC A02/MF A01. Order Number DE83017221. (22 Aug 1983).

Radioactive waste has been separated by definition into six categories. These are: commercial spent fuel; high-level wastes; transuranium waste; low-level wastes; decommissioning and decontamination wastes; and mill tailings and mine wastes. Handling and disposing of these various types of radioactive wastes are discussed briefly.

562 (DOE/AL/10752-22) EEG review comments on the geotechnical reports provided by DOE to EEG under the stipulated agreement through March 1, 1983. (New Mexico Health and Environment Dept., Santa Fe (USA). Environmental 
'mprovement Div.). Apr 1983. Contract AC04-78AL10752. 83p. NTIS, PC A13/MF A01. Order Number DE83011783. Portions are illegible in microfiche products.

The purpose of the Environmental Evaluation Group (EEG) is to conduct an independent technical evaluation of the potential radiation exposure to people from the proposed federal radioactive Waste Isolation Pilot Plant (WIPP) near Carlsbad, in order to protect the public health and safety and ensure that there is minimal environmental degradation. Analyses are conducted of available data concerning the pro posed site, the design of the repository, its planned operation, and its long-term stability. These analyses include assessments of reports issúed by the US Department of Energy (DOE) and its contractors, other federal agencies and organizations, as they relate to the potential health, safety and environmental impacts from WIPP. This publication is a compilation of EEG's written comments on each of the following reports: Deep Dissolution; Breccia Pipes; DMG Hydrology; Natural Resources; Plans for Site and Preliminary Design Validation; Plans for Simulated Waste; Brine Reservoir Report; Disturbed Zone Exploration; and Fracture Flow in the Rustler Aquifers.

563 (DOE/DP-0015) Defense waste management plan. (USDOE Assistant Secretary for Defense Programs, Washington, DC). Jun 1983. 48p. NTIS, PC A03/MF A01. Order Number DE83013816.

Defense high-level waste (HLW) and defense transuranic (TRU) waste are in interim storage at three sites, namely: at the Savannah River Plant, in South Carolina; at the Hanford Reservation, in Washington; and at the Idaho National Engineering Laboratory, in Idaho. Defense TRU waste is also in interim storage at the Oak Ridge National Laboratory, in Tennessee; at the Los Alamos National Laboratory, in New Mexico; and at the Nevada Test Site, in Nevada. (Figure E-2). This document describes a workable approach for the permanent disposal of high-level and transuranic waste from atomic energy defense activities. The plan does not address the disposal of suspect waste which has been conservatively considered to be high-level or transuranic waste but which can be shown to be low-level waste. This material will be processed and disposed of in accordance with low-level waste practices. The primary goal of this program is to utilize or dispose of high-level and transuranic waste routinely, safely, and effectively. This goal will include the disposal of the backlog of stored defense waste. A Reference Plan for each of the sites describes the sequence of steps leading to permanent disposal. No technological breakthroughs are required to implement the reference plan. Not all final decisions concerning the activities described in this document have been made. These decisions will depend on: completion of the National Environmental Policy Act process, authorization and appropriation of funds, agreements with states as appropriate, and in some cases, the results of pilot plant experiments and operational experience. The major elements of the reference plan for permanent disposal of defense high-level and transuranic waste are summarized.

664 (DOE/ET/41900-13) Stability of ceramic waste forms in potential repository environments: a review. Johnston, R.J.; Palmer, R.A. (Rockwell International Corp., Canoga Park, CA (USA). Energy Systems Group). 31 Mar 1982. Contract AC09-79ET41900. 50p. (ESG-DOE-13364). NTIS, PC A03/MF A01. Order Number DE82013288.

Most scenarios for geologic disposal of high-level nuclear waste include the eventual intrusion of groundwater into the repository. Reactions in the system and eventual release of the radionuclides, if any, will be controlled by the chemistry of the groundwater, the surrounding rock, the waste form, and any engineered barrier materials that are present, as well as by the temperature and pressure of the system. This report is a compilation and evaluation of the work completed to date on interactions within the waste-form/host-rock/groundwater system at various points in its lifetime. General results from leaching experiments are presented as a basis for comparison. The factors involved in studying the complete system are discussed so that future research may avoid some of the oversights of past research. Although relatively little hard lata on prototype waste-form/repository-system -interactions exist at his time, the available data and their implications are discussed. Sorpion studies and models for predicting radionuclide migration are also presented, again with a study of the factors involved. with it. Academy forum. (National Academy of Sciences, Wash- ington, DC (USA)). 19 Nov 1979. Contract AC01-80ET44112. 45p. NTIS, PC A03/MF A01. Order Number DE84001 181. ented.

The dialogue from the Forum on nuclear waste disposal is pres-

668 (DOE/ET/44605-T1) Statistical analysis of lineaments and their relation to fracturing, faulting, and halokinesis In the East Texas Basin. Report of investigations No. 110. Dix, O.R.; Jackson, M.P.A. (Texas Univ., Austin (USA). Bureau of Economic Geology). 1981. Contract AC97-79ET44605;AC9780ET46617. 33p. (DOE/ET/46617-T1). NTIS, PC A03/MF A01. Order Number DE82013791.

Portions of document are illegible.

Lineament analysis is part of a broad spectrum of structural studies employed to determine the tectonic stability of the East Texas Basin. Such information is necessary to assess the suitability of East Texas salt domes as possible repository sites for the storage of high-level nuclear wastes. A sequence of statistical operations was designed to identify and assess the significance of lineament preferred orientation by means of a variety of statistical tests or parameters. Black-and-white aerial photographs, and band-5 Landsat imagery were analyzed. Welldefined, northeast-trending and northwest-trending lineament populations are present throughout the East Texas Basin. The northeast trend, comprising two peaks oriented at $045^{\circ}$ and $055^{\circ}$, corresponds to the orientation of the Mexia-Talco peripheral fault zone, to subsurface faults in the center of the basin, and to some lithologic contacts. The northwest trend comprises two peaks oriented at $310^{\circ}$ and $325^{\circ}$. Both the northeast and northwest trends are thought to result from preferential directions of fracture induced by interference folding at depth. Areas above shallow salt domes, particularly those in the southern part of the basin, are associated with higher lineament densities and lower preferred orientation of lineaments that are non-dome areas or areas above deep salt diapirs; this probably reflects radial and concentric fault and fracture patterns above the shallow domes. Analysis of computer-generated, geologically meaningless sets of lineaments strongly suggests that confidence levels of 99 percent are necessary to exclude randomly generated peaks, and that the significance of orthogonal pairsets has been exaggerated in the literature.

687 (DOE/ET/44802-T3) Interpreted resistivity and IP section line W1: Wahmonie Area, Nevada Test Site, Nevada. Smith, C.; Ross, H.P.; Edquist, R. (Geological Survey, Washington, DC (USA)). 1981. Contract AI08-78ET44802. 24p. (USGS-OFR-81-1350). NTIS, PC A02/MF A01. Order Number DE82008518.

The US Geological Survey, working under a memorandum of understanding EW-78-A-08-1543, with the Department of Energy, is engaged in a broad program to assess and identify potential repositories for high level nuclear waste on the Nevada Test Site (NTS). The USGS program consists of integrated geologic, hydrologic and geophysical studies of regional to site specific nature. This report discusses work done at the proposed Wahmonie site at which active work has been suspended in part due to structural complexity, faulting and potential mineralization. This work provides the principal geophysical basis on which an assessment of potential mineralization was made. The Wahmonie site was originally selected for study as a potential nuclear waste repository because of an inferred intrusive body at shallow depth in the area. Studies were initiated to determine the nature and extent of the intrusive mass in order to assess its potential as a repository. This report covers the two dimensional modeling and interpretation of a dipoledipole induced polarization (IP) line run across the center of the inferred mass. The modeling was done by the University of Utah Research Institute (UURI) under purchase order 84868 of the US Geological Survey. A brief discussion of the geology is given in the introduction because the results show significant potential for mineralization in the intrusive.

688 (DOE/ET/46634-1) State of Wisconsin Ad Hoc committee on radioactive waste management. Final report. (Wisconsin State Government, Madison (USA)). 18 Feb 1983. Contract FG02-81ET46634. 164p. NTIS, PC A08/MF A01. Order Number DE83008373.

Portions are illegible in microfiche products.

Part I describes the activities undertaken under the Grant with respect to Wisconsin's role in radioactive waste management. 
569

(DOE/LLW-18T-Rev.1) Radioactive waste management: a summary of state laws and administration. National Low-Level Radioactive Waste Mangement Program. (EG and G Idaho, Inc., Idaho Falls (USA)). May 1983. Contract AC0776ID01570. 495p. NTIS, PC A21/MF A01. Order Number DE83013155.

This is the first quarterly update of Radioactive Waste Management: A Summary of State Laws and Administration. Because states have been very active on waste management issues, the whole report is being reissued in this update. It covers the administration, the legislature and the laws in the 50 states related to radioactive waste. The report for each state is divided into four sections: Cover Page; Administrative; Legislative; and Applicable Legislation. The cover page indicates whether or not it is an Agreement State, the low-level waste compacts in which the state is listed as an eligible state, and the high-level waste repository site screening regions in which the state or a portion of it is located. The administrative section provides information on the governor, lead agencies, other involved administrative agencies, relevant commissions, boards and councils and various contacts. The Legislative section provides general information on the legislature and lists legislative leaders, the relevant committees and their chairs and a legislative contact. In the section covering Applicable Legislation. laws related to radiation protection, low-level waste and high-level waste have been summarized. Hazardous waste siting laws are included for states that do not have a siting law covering radioactive waste. The section also contains summaries of relevant bills introduced in 1982 and 1983 legislative sessions and their disposition. In general, the information in this report is accurate as of 15 April 1983.

570

(DOE/LLW-18T-Rev.2) Radioactive waste management: a summary of state laws and administration. (EG and $G$ Idaho, Inc., Idaho Falls (USA)). Sep 1983. Contract AC0776ID01570. 494p. NTIS, PC A21/MF A01. Order Number DE83017655.

This is the second update of Radioactive Waste Management: A Summary of State Laws and Administration. It completely replaces the first update (15 April 1983). The updated report covers the administration, the legislature and the laws in the 50 states related to radioactive waste. The report for each state is divided into four sections: Cover Page; Administrative; Legislative; and Applicable Legislation. The cover page indicates whether or not it is an Agreement State, the lowlevel waste compacts in which the state is listed as an eligible state, and the high-level waste repository site screening regions in which the state or a portion of it is located. Included under the compacts is a description of what the state has done or currently plans to do, as well as the compact status of other eligible states in the region. The Administrative section provides information on the governor, lead agencies, other involved administrative agencies, relevant commissions, boards and councils and various contacts. In a number of states, Boards of Health or similar boards are lead agencies, so they have been listed in that section. Each board's administrative agency is listed under it. The Legislative section provides general information on the legislature and lists legislative leaders, the relevant committees and their chairs, and a legislative contact. Many legislatures do not set a date for session adjournment, so the date listed represents a combination of information provided by the states and by the history of past sessions. In the section covering Applicable Legislation, laws related to radiation protection, low-level waste and high-level waste have been summarized. Hazardous waste siting laws are included for states that do not have a siting law covering radioactive waste. The section also contains summaries of relevant bills introduced in 1982 and 1983 legislative sessions and this disposition. In general, the information in this report is accurate as of July 15, 1983.

571 (DOE/NBM-3004280) Investigation of the utility of Gulf Coast salt domes for the storage or disposal of radionctive wastes. Martinez, J.D.; Thoms, R.L.; Kolb, C.R.; Kumar, M.B.; Wilcox, R.E.; Newchurch, E.J. (Louisiana State Univ., Baton Rouge (USA). Inst. for Environmental Studies). 31 Dec 1979. Contract AC02-76CH00016. 577p. NTIS, PC A25/MF A01. Order Number DE83004280.

Portions are illegible in microfiche products.

This study has the objective of determining tectonic and hydrologic stability of salt domes. Tectonic stability has been evaluated on the basis of studies focussed on three intervals of time. The Mesozoic and Tertiary record was examined for evidence of decreasing movement with time which would suggest a current state approximating domal qui- escence. Pleistocene and Recent sediments are being studied for possible indications of warping resulting from vertical domal movement. Hydrologic stability has also been assessed through the use of both historical and modern data. Chapters are devoted to geomechanics, geohydrology, regional geology, and quaternary studies.

672 (DOE/NBM-3011069) Nevada nuclear waste storage investigations: briefing book. (Department of Energy, Las Vegas, NV (USA). Nevada Operations Office). Mar 1983. 15p. NTIS, PC A02/MF A01. Order Number DE83011069.

The Nevada Nuclear Waste Storage Investigations (NNWSI) are discussed briefly. The tuff in Yucca mountains being investigated as a possible repository host for radioactive wastes. The Spent Fuel TestClimax began in the spring of 1980 in the northeastern Nevada Test Site about $1400 \mathrm{ft}$ below the desert surface. The test has provided significant scientific and technical contributions in the following areas: heat impact on a large underground facility in a hard, brittle rock, impact of ventilation designs on repository heat removal, suitability and operational characteristics of instrumentation in a repository, impact of the mining procedures on underground openings and the surrounding rock. and heat and radiation effects on the physical, mechanical. and chemical properties of granite.

673 (DOE/NBM-3012262) Research and development needs in the Department of Energy. Interim report. (USDOE Office of Energy Research, Washington, DC. Energy Research Advisory Board). 5 Sep 1980. 113p. NTIS, PC A06/MF A01. Order Number DE8301 2262.

In April 1980, the Deputy Secretary requested that the Board participate in the Department's review of the technology base component of DOE's R and D programs and that the Board address the following broad concerns: (1) The adequacy of the research underpinning for technology development programs; (2) Possible gaps or duplications of effort; (3) The balance among research performers (universities, laboratories, industry); (4) Significant $R$ and D opportunities that DOE's programs may be missing. The Board offered the following recommendations to the Secretary: (1) Place greater research emphasis on environmental and health issues to ensure the success of the national synfuels program. (2) Provide more research in energy use and productivity projects. (3) Increase the level of effort in basic research. (4) Place higher priority for high-level radioactive waste disposal $R$ and $D$. (5) Evaluate the various energy technology options on a common comparison basis to clearly identify the costs, benefits and risks of each option. (6) Develop more effective DOE procurement practices. Additional recommendations were directed to the Under Secretafy and Assistant Secretaries of Energy reviewing specific issues in conservation, fossil, nuclear and solar energy, resource applications, environment, and energy research.

$674 \quad(\mathrm{DOE} / \mathrm{NE}-0017 / 2)$ Spent fuel and radioactive waste inventories, projections, and characteristics. (Oak Ridge National Lab., TN (USA)). Sep 1983. Contract W-7405-ENG26. 312p. NTIS, PC A14/MF A01. Order Number DE84001618.

Portions are illegible in microfiche products.

Current inventories and characteristics of commercial spent fuels and both commercial and US Dopartmont of Energy radiosetive wastes were compiled through December 31, 1982, based on the most reliable information available from government sources and the open literature, technical reports, and direct contacts. Future waste and spent fuel to be generated over the next 40 ycars and characteristics of these materials are also presented, based on the latest DOE/EIA projection of US commercial nuclear power growth and expected defense-related and industrial and institutional activities. Materials considered, on a chapter-by-chapter bases, are: spent fuel, high-level waste, transuranic waste, low-level waste, active uranium mill tailings, airborne waste, remedial action waste, and decommissioning waste. For each category, current and projected inventories are given through the year 2020, and the radioactivity and thermal power are calculated, based on reported or calculated isotopic compositions. One chapter gives broad, summary data on the costs of spent fuel and radioactive waste management and disposal to provide an economic perspective. This chapter is not intended as a definitive guide, but it is a source of reasonable, order-ofmagnitude costs and also provides references to more-detailed and sce- 
nario-specific studies. An appendix on generic flowsheets and source terms used for the projections is also included.

575 (DOE/NE-0039) Program summary. Nuclear waste management and fuel cycle programs. (USDOE Assistant Secretary for Nuclear Energy, Washington, DC). Jul 1982. 79p. NTIS, PC A05/MF A01. Order Number DE82021692.

This Program Summary Document describes the US Department of Energy (DOE) Nuclear Waste Management and Fuel Cycle Programs. Particular emphasis is given to near-term, specifically Fiscal Year (FY) 1982, activities. The overall objective of these programs will be achieved by the demonstration of: (1) safe radioactive waste management practices for storage and disposal of high-level waste and (2) advanced technologies necessary to close the nuclear fuel cycle on a schedule which would assure a healthy future for the development of nuclear power in this country.

576 (DOE/NE/46640-7) Consultation and concurrence for NWTS in crystalline rocks. Quarterly progress report, October 1, 1982-December 31, 1982. (Maine Dept. of Conservation, Augusta (USA). Maine Geological Survey). 12 Jan 1983. Contract FG02-81NE46640. 3p. NTIS, PC A02/MF A01. Order Number DE83007142.

The last of the 1:250,000 draft compilations for the bedrock map were received, and a second draft of 1:250,000 and 1:500,000 mockups of the state bedrock map was prepared. Input of information for the Bibliography of Maine Geology continued; the job is approximately $80 \%$ complete. Software for coding the information for geographic location was completed.

577 (DOE/NE/93031-T1) Study of the isolation system for geologic disposal of radioactive wastes. (National Academy of Sciences, Washington, DC. (USA)). 1983. Contract AT0180NE93031. 356p. NTIS, PC A16/MF A01. Order Number DE84001084.

Portions are illegible in microfiche products.

This study was conducted for the US Department of Energy by a Waste Isolation System Panel of the Board on Radioactive Waste Management under the National Research Council's Commission on Physical Sciences, Mathematics, and Resources. The panel was charged to review the alternative technologies available for the isolation of radioactive waste in mined geologic repositories, evaluate the need for and possible performance benefits from these technologies as potential elements of the isolation system, and identify appropriate technical criteria for choosing among them to achieve satisfactory overall performance of a geologic repository. Information has been acquired through examination of a large body of technical literature, briefings by representatives of government agencies and their industrial and university contractors, in-depth discussions with individual experts in the field, site visits, and calculations by panel members and staff, with deliberations extending nver a perion of apprnximately twn years. The panel's principal findings are given. Chapters are devoted to: the geologic waste-disposal system; waste characteristics; waste package; conceptual design of repositories; geologic hydrologic, and geochemical properties of geologic wastedisposal systems; overall performance criterion for geologic waste disposal; performance analysis of the geologic waste-disposal system; and natural analogs relevant to geologic disposal. 336 references.

578 (DOE/NV/10250-4) Commercial nuclear-waste research and development program. Quarterly report, JulySeptember 1982. Hakl, A.R. (Westinghouse Electric Corp., Mercury, NV (USA). Advanced Energy Systems Div.). Oct 1982. Contract AC08-82NV10250. 34p. NTIS, PC A03/MF A01. Order Number DE83004754.

This document is a report of activities during the fourth quarter of FY-1982 to meet task objectives of the Nevada Nuclear Waste Storage Investigations FY-1982 Project Plan. Noteworthy accomplishments were: The second Climas fuel exchange of FY 1982 was completed. Source evaluation of the bidders was completed. The contract for glass refurbishment and window fabrication for the East Process Cell is being negotiated. All canister gas samples scheduled for FY 1982 were taken and analyzed; no ${ }^{85} \mathrm{~K}$ was detected. Fuel assembly B-02 was removed from the Surface Storage Cask (SSC) in preparation for fuel and canister contamination surveys and subsequent placement in the fuel temperature test. This event terminated a four year demonstration of dry interim storage of a sealed canister containing spent nuclear fuel in an above ground surface storage cask. The data acquisition systems which were damaged by lightning were repaired and returned to operation. The draft report for Task 13, Documentation of E-MAD Spent Fuel Assembly Histories and Predictions of Peak Clad Temperatures, was completed and transmitted to DOE and PNL for review. Twenty-one tours were conducted for 312 visitors to the E-MAD Facility, including the governor of Utah and his staff.

679 (DOE/NV/10250-5) Commercial nuclear waste research and development program. Annual report, fiscal year 1982. Hakl, A.R. (Westinghouse Electric Corp., Mercury, NV (USA). Advanced Energy Systems Div.). Oct 1982. Contract AC08-82NV10250. 55p. NTIS, PC A04/MF A01. Order Number DE83004770.

This document is a report of FY 1982 activities performed to meet task objectives of the Nevada Nuclear Waste Storage Investigations (NNWSI) FY-1982 Project Plan. Noteworthy accomplishments were: Fuel assembly B-02 was removed from the surface Storage Cask (SSC) after four years of testing, and placed in lag storage in preparation for contamination surveys and the subsequent Fuel Temperature Test. A draft report was prepared which documents histories of all fuel assemblies while in the custody of AESD-Nevada Operations. Canister gas samples were acquired from seven fueled canisters to determine the presence of ${ }^{85} \mathrm{Kr}$, which would indicate fuel pin leakage. No ${ }^{85} \mathrm{Kr}$ was detected in any of the samples. Two spent fuel assembly exchange operations were completed in support of the Spent Fuel Test - Climas. Fixtures have been designed and fabricated for handling canisters during fuel assembly/canister contamination examinations, and for holding and vibrating canisters to remove loose debris. Draft input was provided to ONWI for E-MAD nuclear waste package production for a proposed Test and Evaluation Facility. Process flow diagrams and supporting documentation were developed for eight alternate waste package configurations to be performed at a Generic Package Facility (GPF). A draft report, assessing requirements for a GPF, was completed and transmitted to DOE/NV and ONWI. AESD-Nevada Operations provided Quality Assurance support for the NNWSI drilling operations.E-MAD tours were conducted for approximately 1400 people. Two enclosures for E-MAD Stack Monitor detectors were constructed. Specifications and purchase requisitions were prepared for refurbishment of radiation shield windows to be installed in the E-MAD East Process Cell.

580 (DOE/NV/10250-7) Commercial Nuclear Waste Research and Development Program. Quarterly report, OctoberDecember 1982. Hakl, A.R. (Westinghouse Electric Corp., Mercury, NV (USA). Advanced Energy Systems Div.). Jan 1983. Contract AC08-82NV10250. 27p. NTIS, PC A03/MF A01. Order Number DE83009732.

Noteworthy accomplishments during the first quarter of FY1983 were: Schedules reflecting current plans for FY-1983 equipment maintenance, technical and administrative training, and anticipated task activities were prepared and transmitted to DOE/NV as Contract Deliverables. Cost and schedule estimates were prepared, at DOE/NV request, for cask testing and emergency storage of spent fuel at E-MAD, performance of a PWR cask characterization test at E-MAD, and AESD-Nevada Operations support of Climax Spent Fuel Test decommissioning. For the first time, full volume filtered gas samples were acquired from a sealed canister containing a spent nuclear fuel assembly for analysis to determine canister atmosphere composition. Another significant first: A sealed canister containing a spent nuclear fuel assembly was cut open and the fuel assembly removed for examination of the fuel and the canister interior. Maintenance was performed on Engine Maintenance, Assembly and Disassembly Facility (E-MAD) railroad tracks and vehicles including the Manned Control Car, EmplacementInstallation Vehicle, locomotives, and flatcars.Comments to the first draft of the report, Histories of Spent Nuclear Fuel Assemblies While at the E-MAD Facility, were resolved and a revised draft was issued for review. Comments received to this draft are being resolved and the document is being revised. The annual DOE audit of the AESD-Nevada Operations radiological safety program was performed, with no findings reported. A computer, to provide interface between the E-MAD Stack Air Monitor and the Remote Air Monitor console, was received and installed. Vendor recommendations were received for electrical modifications to the Plasma Arc Welder. A modified torch was received, installed on the welder, and satisfactorily tested. A procedure for Qual- 
ity Assurance surveillance and monitoring of the NNWSI drilling program was prepared and implemented.

B81 (DOE/NV/10250-8) Commercial nuclear waste research and development program. Quarterly report, JanuaryMarch 1983. (Westinghouse Electric Corp., Mercury, NV (USA). Waste Technology Services Div.). Apr 1983. Contract AC08-82NV10250. 27p. NTIS, PC A03/MF A01. Order Number DE83014384.

A second more detailed examination was performed on fuel assembly B02 which was utilized in the four-year Surface Storage Cask test. The examination included photo mosiacs of all four sides of the fuel assembly, closeup photographs using the newly installed periscope photography lighting system, and swipe sampling with improved equipment and techniques. Two fuel rods were removed from fuel assembly $\mathrm{BO2}$. The rods were placed in a storage container and stored in a Hot Bay lag storage pit pending a detailed examination, which will be performed later to document fuel rod cladding condition and crud adherence prior to the Fuel Temperature Test. Fuel assembly $\mathrm{BO2}$ was emplaced in the Fuel Temperature Test apparatus on February 25 and transferred from the Hot Bay to the West Process Cell. Power was turned on March 3 to initiate the test. The temperature profile stabilized March 10. Eight of the eleven canisterized fuel assemblies which were utilized in the Climax Spent Fuel Test have been transferred from the test facility to E-MAD. Gas samples were taken from seven of the canisters. All eight were placed in Hot Bay lag storage. All heater assemblies used in the Climax Spent Fuel Test were returned to E-MAD and receiving inspecthon was completed. The calorimeter was modified by installation of a filter system on the water fill line. Installation of isokinetic air sampling nozzles in the two E-MAD exchust stacks was completed. Modifications were completed to the Master Control Room communications system. A report, Histories of Spent Nuclear Fuel Assemblies While at the E-MAD Facility, was completed.

682 (DOE/NV/10250-9) Commercial nuclear waste research and development program. Quarterly report, April-June 1983. Hakl, A.R. (Westinghouse Electric Corp., Mercury, NV (USA). Waste Technology Services Div.). Jul 1983. Contract AC08-82NV10250. 29p. NTIS, PC A03/MF A01. Order Number DE83017654.

The last three of the eleven canisterized fuel assemblies from the Climax Spent Fuel Test facility were received and placed in Hot Bay lag storage pits. The canister containing fuel assembly D06 was removed from lag storage, gas and full volume filtration samples were acquired, and the canister was cut open. The fuel assembly was removed from the canister, examined, photographed, videotaped, installed in a new canister, and returned to lag storage. Gas samples were obtained from the Fuel Temperature Test (FTT) atmosphere which, when analyzzed, indicated the presence of $\mathbf{K r}$. Gas samples were acquired from the canisters containing fuel assemblies D09 and D18, which were used in the Climax Spent Fuel Test. The storage canisters containing the two fuel rods removed from fuel assembly $\mathrm{B02}$, prior to installation of the fuel assembly into the FTT assembly, were evacuated and backfilled with helium. Final installation and checkout of the Stack Air Monitor (SAM) system was completed and the Remote Area Monitor (RAM)/SAM system integration program was successfully executed. The air monitoring system is operational. Quality Assurance personnel completed an assessment regarding the implementation of ASME Quality Assurance Program Requirements for Nuclear Facilities (NQA-1) requirements by participants in the NNWSI drilling activities. The shield window glass which had been removed from the Reactor Maintenance, Assembly and Disassembly building was loaded on a truck for transport to the vendor for refurbishment of the windows for future installation in the E-MAD facility. A major change in the method for development of nuclear materials handling procedures was devised, approved and successfully implemented to improve safety and productivity during fuel handling operations. By using applicable sections of approved procedures, a Consolidated Procedure is now prepared and issued for each remote Hot Bay operation.

683 (DOE/NWTS-4) National plan for siting high-level radioactive waste repositories and environmental assessment. Public draft. (Department of Energy, Washington, DC (USA). Assistant Secretary for Nuclear Energy). Feb 1982. 205p.
(DOE/EA-151). NTIS, PC A10/MF A01. Order Number DE82009290.

Portions of document are illegible.

The National Plan for Siting High-Level Radioactive Waste Repositories describes the process the Department of Energy (DOE) is using to find sites suitable for disposal of high-level radioactive waste. Potential environmental impacts of implementing the Plan are included in an attached Environmental Assessment. The Plan is one element of the National Waste Terminal Storage (NWTS) Program being conducted by DOE to develop the necessary technology and to qualify sites to establish mined geologic repositories for these wastes. The Plan describes existing and planned activities for screening successively smaller portions of land within the United States to identify suitable candidate sites, and for subsequently selecting one or more of these sites, for permanent disposal of radioctive wastes. Environmental effects of the proposed action, including the anticipated range of field studies to characterize various land areas and reasonable alternative siting strategies, are assessed. The Environmental Assessment provides the basis for a finding of whether or not implementation of this plan will result in signficant environmental impacts.

684 (DOE/NWTS-30, pp 15-16) Repository and engineering harriers design. Scully, L.W.; Rothman, A.J. Dec 1982. NTIS, PC A10/MF A01. (CÓNF-821205-Absts.).

From NWTS information meeting on basalt waste isolation project; Las Vegas, NV, USA (14 Dec 1982).

RADIOACTIVE WASTE DISPOSAL; ENGINEERED SAFETY SYSTEMS; DESIGN; UNDERGROUND DISPOSAL; CLOSURES; HIGH-LEVEL RADIOACTIVE WASTES; RADIOACTIVE WASTE FACILITIES; GEOLOGIC DEPOSITS

685 (DOE/NWTS-30, pp 41-44) Waste package conceptual designs for a repository located in basalt. Smith, M.J. Dec 1982. NTIS, PC A10/MF A01. (CONF-821205-Absts.). From NWTS information meeting on basalt waste isolation project; Las Vegas, NV, USA (14 Dec 1982).

BASALT; RADIOACTIVE WASTE FACILITIES; HANFORD RESERVATION; HIGH-LEVEL RADIOACTIVE WASTES; DESIGN; SITE SURVEYS; ENGINEERED SAFETY SYSTEMS; RADIOACTIVE WASTE DISPOSAL; PACKAGING

686 (DOE/NWTS-30, pp 45-48) Testing of candidate maste package backfill and canister materials for besalt. Wood, M.I.; Anderson, W.J.; Aden, G.D. Dec 1982. NTIS, PC A10/MF A01. (CONF-821205-Absts.).

From NWTS information meeting on basalt waste isolation project; Las Vegas, NV, USA (14 Dec 1982).

HANFORD RESERVATION; RADIOACTIVE WASTE FACILITIES; BASALT; TESTING; BACKFILLING; HYDROTIILRMAL ALTERATION, RADIOACTIVE WA3TE DIBPOSAL; HIGH-LEVEL RADIOACTIVE WASTES; PERMEABILITY; ENGINEERED SAFETY SYSTEMS; BENTONITE; CONTAINERS; MATERIALS TESTING; PACKAGING

687 (DOE/NWTS-30, pp 70-76) Systems overview of the subseabed disposal program. Klett, R.D.; Brush, L.H.; Lipkin, J.; Percival, C.M. Dec 1982. NTIS, PC A10/MF A01. (CONF-821205-Absts.).

From NWTS information meeting on basalt waste isolation project; Las Vegas, NV, USA (14 Dec 1982).

SEA BED; RADIOACTIVE WASTE DISPOSAL; HIGHLEVEL RADIOACTIVE WASTES; TEMPERATURE EFFECTS; MARINE DISPOSAL

688 (DOE/NWTS-30, pp 81-82) Impact of NRC licensing requirements on salt site selection. Hewitt, W.M.; Chen, C.P. Dec 1982. NTIS, PC A10/MF A01. (CONF-821205-Absts.). From NWTS information meeting on basalt waste isolation project; Las Vegas, NV, USA (14 Dec 1982).

HIGH-LEVEL RADIOACTIVE WASTES; RADIOACTIVE WASTE DISPOSAL; SITE SELECTION; SALT DEPOSITS; REGULATIONS; RADIOACTIVE WASTE FACILITIES; LICENSING; RADIOACTIVE WASTE MANAGEMENT; US NRC 
89 (DOE/NWTS-30, pp 83-86) Salt repository proram: compliance with NEPA. Brown, M.L.; McIntosh, W.H. lec 1982. NTIS, PC A10/MF A01. (CONF-821205-Absts.).

From NWTS information meeting on basalt waste isolation project; Las Vegas, NV, USA (14 Dec 1982).

RADIOACTIVE WASTE FACILITIES; RADIOACTIVE WASTE DISPOSAL; SITE SELECTION; HIGH-LEVEL RADIO ACTIVE WASTES; ENVIRONMENTAL IMPACTS; SALT DEPOSITS; COMPLIANCE

590 (DOE/NWTS-30, pp 91-92) Status of site characterization in the Permian Basin. Newcomb, W.E. Dec 1982. NTIS, PC A10/MF A01. (CONF-821205-Absts.).

From NWTS information meeting on basalt waste isolation project; Las Vegas, NV, USA (14 Dec 1982).

SITE SELECTION; RADIOACTIVE WASTE DISPOSAL; HIGH-LEVEL RADIOACTIVE WASTES; SALT DEPOSITS; GEOLOGIC STRUCTURES; HYDROLOGY; GEOLOGY; SOCIO ECONOMIC FACTORS; CONSTRUCTION; RADIOACTIVE WASTE FACILITIES; FEASIBILITY STUDIES; SITE SURVEYS; TEXAS

591 (DOE/NWTS-30, pp 136-137) OECD Nuclear Energy Agency activities in high-level waste disposal. Olivier, J.P.; Johnston, P.D. (Nuclear Energy Agency, Paris, France). Dec 1982. NTIS, PC A10/MF A01. (CONF-821205-Absts.). From NWTS information meeting on basalt waste isolation project; Las Vegas, NV, USA (14 Dec 1982).

HIGH-LEVEL RADIOACTIVE WASTES; NEA; OECD; RADIOACTIVE WASTE DISPOSAL; GEOLOGIC DEPOSITS; RADIOACTIVE WASTE MANAGEMENT

592 (DOE/NWTS-30, pp 140-141) Canadian geoscience research for nuclear fuel waste management. Dormuth, K.W.; Simmons, G.R. Dec 1982. NTIS, PC A10/MF A01. (CONF-821205-Absts.).

From NWTS information meeting on basalt waste isolation project; Las Vegas, NV, USA (14 Dec 1982).

CANADA; RADIOACTIVE WASTE DISPOSAL; HIGHLEVEL RADIOACTIVE WASTES; GEOLOGIC DEPOSITS; RADIOACTIVE WASTE MANAGEMENT; SEA BED; INTERNATIONAL COOPERATION; IGNEOUS ROCKS; MARINE DISPOSAL

693 (DOE/NWTS-30, pp 205-210) Waste package conceptual designs for salt repositories. Basham, S.J.; Carr, J.A. Dec 1982. NTIS, PC A10/MF A01. (CONF-821205-Absts.). From NWTS information meeting on basalt waste isolation project; Las Vegas, NV, USA (14 Dec 1982).

RADIOACTIVE WASTE DISPOSAL; SALT DEPOSITS; PACKAGING; HIGH-LEVEL RADINACTIVE WASTES: SPENT FUELS; DESIGN; RADIOACTIVE WASTE FACILITIES; SPECIFICATIONS; CONTAINERS; PERFORMANCE TESTING; CORROSION

694 (DOE/NWTS-30, pp 216-217) Information ofifice concept. Pendleton, M. Dec 1982. NTIS, PC A10/MF A01. (CONF-821205-Absts.).

From NWTS information meeting on basalt waste isolation project; Las Vegas, NV, USA (14 Dec 1982).

PUBLIC INFORMATION; RADIOACTIVE WASTE FACILITIES; UTAH; INFORMATION DISSEMINATION; HIGH-LEVEL RADIOACTIVE WASTES; RADIOACTIVE WASTE DISPOSAL; US DOE

695 (DOE/NWTS-33(1)(Final)) NWTS program criteria for mined geologic disposal of nuclear waste: program objectives, functional requirements, and system performance criteria. (Battelle Memorial Inst., Columbus, OH (USA). Office of National Waste Terminal Storage Integration). Mar 1982. Contract AC06-76RL01830. 52p. NTIS, PC A04/MF A01. Order Number DE82014259.

The NWTS-33 series, of which this document is a part, provides guidance for the National Waste Terminal Storage (NWTS) program in the development and implementation of licensed mined geologic disposal systems for solidified high-level and TRU wastes. Program objec- tives, functional requirements, and system performance criteria are found in this document. At the present time final criteria have not been issued by the Nuclear Regulatory Commission (NRC) and Environmental Protection Agency (EPA). The criteria in these documents have been developed on the basis of DOE's judgment of what is required to protect the health and safety of the public and the quality of the environment. It is expected that these criteria will be consistent with regulatory standards. The criteria will be re-evaluated on a periodic basis to ensure that they remain consistent with national waste management policy and regulatory requirements. A reevaluation will be made when final criteria are promulgated by the NRC and EPA. A background section that briefly describes the mined geologic disposal system and explains the hierarchy and application of the NWTS criteria is included in Section 2.0. Secton 3.0 presents the program objectives, Section 4.0 functional requirements, Secton 5.0 the system performance criteria, and Section 6.0 quality assurance and standards. A draft of this document was issued for public comment in April 1981. Appendix A contains the DOE responses to the comments received. Appendix B is a glossary.

598 (DOE/NWTS-33-3) NWTS program criteria for mined geologic disposal of nuclear waste: repository performance and development criteria. Public draft. (Battelle Memorial Inst., Columbus, OH (USA). Office of National Waste Terminal Storage Integration). Jul 1982. Contract AC0676RL01830. 44p. NTIS, PC A03/MF A01. Order Number DE82021021.

This document, DOE/NWTS-33(3) is one of a series of documents to establish the National Waste Terminal Storage (NWTS) program criteria for mined geologic disposal of high-level radioactive waste. For both repository performance and repository development it delineates the criteria for design performance, radiological safety, mining safety, long-term containment and isolation, operations, and decommissioning. The US Department of Energy will use these criteria to guide the development of repositories to assist in achieving performance and will reevaluate their use when the US Nuclear Regulatory Commission issues radioactive waste repository rules.

697 (DOE/NWTS-33(4a)) NWTS program criteria for mined geologic disposal of auclear waste: functional requirements and performance criteria for waste packages for solidified high-level waste and spent fuel. (Battelle Memorial Inst., Columbus, OH (USA). Office of National Waste Terminal Storage Integration). Jul 1982. Contract AC06-76RL01830. 30p. NTIS, PC A03/MF A01. Order Number DE83004323.

The Department of Energy (DOE) has primary federal responsibility for the development and implementation of safe and environmentally acceptable nuclear waste disposal methods. Currently, the principal emphasis in the program is on emplacement of nuclear wastes in mined geologic repositories well beneath the earth's surface. A brief description of the mined geologic disposal system is provided. The National Waste Terminal Storage (NWTS) program was established under DOE's predecessor, the Energy Kesearch and Development Administration, to provide facilities for the mined geologic disposal of radioactive wastes. The NWTS program includes both the development and the implementation of the technology necessary for designing, constructing, licensing, and operating repositories. The program does not include the management of processing radioactive wastes or of transporting the wastes to repositories. The NWTS-33 series, of which this document is a part, provides guidance for the NWTS program in the development and implementation of licensed mined geologic disposal systems for solidified high-level and transuranic (TRU) wastes. This document presents the functional requirements and performance criteria for waste packages for solidified high-level waste and spent fuel. A separate document to be developed, NWTS-33(4b), will present the requirements and criteria for waste packages for TRU wastes. The hierarchy and application of these requirements and criteria are discussed in Section 2.2 .

688 (DOE/NWTS-96-Vol.1) NWTS waste package program plan. Volume I. Program strategy, description, and schedule. (Battelle Memorial Inst., Columbus, OH (USA). Office of National Waste Terminal Storage Integration). Oct 1981. Contract AC06-76RL01830. 235p. NTIS, PC A11/MF A01. Order Number DE82010888.

This document describes the work planned for developing the technology to design, test and produce packages used for the long-term 
isolation of nuclear waste in deep geologic repositories. Waste forms considered include spent fuel and high-level waste. The testing and selection effort for barrier materials for radionuclide containment is described. The NWTS waste package program is a design-driven effort; waste package conceptual designs are used as input for preliminary designs, which are upgraded to a final design as materials and testing data become available. Performance assessment models are developed and validated. Milestones and a detailed schedule are given for the waste package development effort. Program logic networks defining work flow, interfaces among the NWTS Projects, and interrelationships of specific activities are presented. Detailed work elements are provided for the Waste Package Program Plan subtasks - design and development, waste form, barrier materials, and performance evaluation - for salt and basalt, host rocks for which the state of waste package knowledge and the corresponding data base are advanced.

599 (DOE/NWTS-96-Vol.2) NWTS waste package program plan. Volume II. Program logic networks. (Battelle Memorial Inst., Columbus, OH (USA). Office of National Waste Terminal Storage Integration). Uct 1981. Contract AC0676RL01830. 72p. NTIS, PC A04/MF A01. Order Number DE82010862.

This document describes the work planned for developing the technology to design, test and produce packages used for the long-term isolation of nuclear waste in deep geologic repositories. Waste forms considered include spent fuel and high-level waste. The testing and selection effort for barrier materials for radionuclide containment is described. The NWTS waste package program is a design-driven effort; waste package conceptual designs are used as input for preliminary designs, which are upgraded to a final design as materials and testing data become available. Performance assessment models are developed and validated. Milestones and a detailed schedule are given for the waste package development effort. Program logic networks defining work flow, interfaces among the NWTS Projects, and interrelationships of specific activities are presented. Detailed work elements are provided for the Waste Package Program Plan subtasks - design and development, waste form, barrier materials, and performance evaluation - for salt and basalt, host rocks for which the state of waste package knowledge and the corresponding data base are advanced.

600

(DOE/NWTS-147(1)) Implementation of technical conservatism in the development of nuclear waste repositories. (Battelle Memorial Inst., Columbus, OH (USA). Office of National Waste Terminal Storage Integration). Mar 1981. Contract AC06-76RL01830. 18p. NTIS, PC A02/MF A01. Order Number DE83011111.

The Department of Energy (DOE) waste management program is committed to assuring the safe disposal of radioactive waste. It is recognized that lung-term disposal concepts will contain inherent uncertainties in predictive techniques and scientific information. Accordingly, conservative approaches ae being followed to enhance levels of confidence that the disposal system will perform in such a manner that the established performance requirements will be met. Limiting values of critical parameters will be established for each site based on its inherent characteristics prior to beginning site development. The performance limits will be established for each geometric region of the repository system and be applied simultaneously to assure that no single limit is violated over the repository life cycle. A site-specific set of specifications will be determined when the site is fully characterized, establishing a conservative design basis to increase confidence in safe system performance. The implementation of the NWTS program policy on technical conservatism, as discussed in this document, takes two forms-(1) conservatism in the conduct of the program and (2) conservatism in the performance of the disposal system. The first is achieved by a stepwise approach to system development and operation. the systems viewpoint, the retrievability requirement, and the extensive use of peer reviews throughout the conduct of the program. The second is achieved by the proper selection and application of conservative design and operational limits.

601 (DOE/RL-82-3-Vol.1) Site characterization report for the basall wuste isolation project. Volume 1 . (Rockwell International Corp., Richland, WA (USA). Rockwell Hanford Operations). Nov 1982. Contract AC06-77RL01030. 636p. NTIS, PC A99/MF A01. Order Number DE83017084.
Portions are illegible in microfiche products.

The reference location for a repository in basalt for the terminal storage of nuclear wastes on the Hanford Site and the candidate horizons within this reference repository location have been identified and the preliminary characterization work in support of the site screening process has been completed. Fifteen technical questions regarding the qualification of the site were identified to be addressed during the detailed site characterization phase of the US Department of EnergyNational Waste Terminal Storage Program site selection process. Resolution of these questions will be provided in the final site characterization progress report, currently planned to be issued in 1987, and in the safety analysis report to be submitted with the License Application. The additional information needed to resolve these questions and the plans for obtaining the information have been identified. This Site Characterization Report documents the results of the site screening process, the preliminary site characterization data, the technical issues that need to be addressed, and the plans for resolving these issues. Volume 1 contains chapters 1 through 5 : introduction; decision process for choosing a reference repository location and an alternate repository location: geologic description of the reference repository location and the surrounding area; geoengineering; and hydrogeology;

602 (DOE/RL-82-3-Vol.2) Site characterization report for the basalt waste isolation project. Volume II. (Rockwcll International Corp., Richland, WA (USA). Rockwell Hanford Operations). Nov 1982. Contract AC06-77RL01030. 599p. NTIS, PC A25/MF A01. Order Number DE83017044.

Portions are illegible in microfiche products.

The reference location for a repository in basalt for the terminal storage of nuclear wastes on the Hanford Site and the candidate horizons within this reference repository location have been identified and the preliminary characterization work in support of the site screening process has been completed. Fifteen technical questions regarding the qualification of the site were identified to be addressed during the detailed site characterization phase of the US Department of EnergyNational Waste Terminal Storage Program site selection proccss. Resolution of these questions will be provided in the final site characterization progress report, currently planned to be issued in 1987, and in the safety analysis report to be submitter with the I icense Application. The additional information needed to resolve these questions and the plans for obtaining the information have been identified. This Site Characterization Report documents the results of the site screening process, the preliminary site characterization data, the technical issues that need to be addressed, and the plans for resolving these issues. Volume 2 contains chapters 6 through 12: genchemistry; surface hydrology; climatology; meteorology, and air quality; environmental, land-use, and socioeconomic characteristics; repository design; waste package; and performance assessment.

603 (DOE/RL-82-3-Vol.3) Site characterization report for the basalt waste isolation project. Volume III. (Rockwell International Colp., Richland, WA (USA). Rockwell Hanford Operations). Nov 1982. Contract AC06-77RL01030. 611p. NTIS, PC A99/MF A01. Order Number DE83017077.

Portions are illegible in microfiche products.

The reference location for a repository in basalt for the terminal storage of nuclear wastes on the Hanford Site and the candidate horizons within this reference repository location have been identified and the preliminary characterization work in support of the site screening process has been completed. Fifteen technical questions regarding the qualification of the site were identified to be addressed during the detailed site characterization phase of the US Department of EnergyNational Waste Terminal Storage Program site selection process. Resolution of these questions will be provided in the final site characterization progress repor, curfently planned to be issued in 1987, and in the safety analysis report to be submitted with the License Application. The additional information needed to resolve these questions and the plans for ubtaining the information have been identified. This Site Characterization Report documents the results of the site screening process, the preliminary site characterization data, the technical issues that need to be addressed, and the plans for resolving these issues. Volume 3 contains chapters 13 through 19: site issues and plans; geoengineering and repository design issues and plans; waste package and site geochemistry issues and plans; performance-assessment issues and plans; site characterization program; quality assurance; and identification of alternate sites. 
604

(DOE/S-0020/1) Report on financing the disposal of commercial spent nuclear fuel and processed high-level radioactive waste. Benny, R.I.; Sprecher, W.M. (USDOE Nuclear Waste Policy Act Project Office, Washington, DC). Jul 1983. 55p. NTIS, PC A04/MF A01. Order Number DE83014886.

The major conclusions and observations which emerge from an extensive analysis of the cost of implementing a nuclear waste disposal program are: Projected revenues generated from the $1.0 \mathrm{mill}$ per kwhr fee mandated by the Act are sufficient to cover the full range of reference case program costs, assuming $3 \%$ annual inflation and nuclear installed capacity of 165 gigawatts-electric by the year 2000 . Total estimated costs of the reference waste disposal program, encompassing either spent nuclear fuel disposal or reprocessing waste disposal, range between $\$ 18$ to 20 billion in constant 1982 dollars. Sensitivity case analyses established upper and lower program cost bounds of $\$ 28$ billion and $\$ 16$ billion, respectively (in 1982 dollars). In terms of discounted levelized unit costs, the disposal of spent fuel equates to $\$ 122$ to 125 per kilogram (uranium) compared with $\$ 115$ to 119 per kilogram for the reprocessing waste equivalent. The levelized unit costs for reprocessing exclude the solidification of liquid wastes. Such costs are estimated to be $\$ 8$ per kilogram. Discounted levelized unit costs corresponding to the upper and lower limits of the sensitivity cases equate to $\$ 176$ per kilogram and \$107 per kilogram. The 1.0 mill per kwhr fee will be reviewed annually and adjusted, if necessary, to accommodate changes in program costs due to inflation and program shifts. When adjustments are made for applicable discount rates, inflation, repository design changes, and other factors, levelized unit costs for the reference case presented in this analysis agree closely with the results of two previous Department of Energy (DOE) studies concerning charges for spent fuel storage and disposal services provided by the federal government.

605 (DOE/S-0021) Monitored Retrievable Storage proposal research and development research and development report. (USDOE Nuclear Waste Policy Act Project Office, Washington, DC). Jun 1983. 23p. NTIS, PC A02/MF A01. Order Number DE83014567.

Past Monitored Retrieval Storage (MRS) studies indicate that passive dry storage technologics, which do not require any external power to provide cooling, are the preferred technologies for long-term MRS applications. In developing the MRS program, the Department of Energy has continued to pursue these passive dry storage technologies in order to minimize the dependence of the operation and safety of the facilities on active components. There have been almost forty years of actual operating experience with dry storage and handling of spent fuel and twelve years of research in passive dry storage technology in the United States and abroad. Based on an analysis of this experience and data, nu furthèi research and development activitics are considered necessary to develop a MRS proposal as required by the Nuclear Waste Policy Act.

606 (DOE/SR-WM-84-1) Savannal River interim waste management program plan, FY-1984. (Du Pont de Nemours (E.I.) and Co., Aiken, SC (USA). Savannah River Plant). Sep 1983. Contract AC09-76SR00001. 190p. NTIS, PC A09/MF A01. Order Number DE84000579.

Portions are illegible in microfiche products. Original copy available until stock is exhausted.

This document provides the program plan as requested by the Savannah River Operations Office of the Department of Energy. The plan was developed to provide a working knowledge of the nature and extent of the interim waste management programs being undertaken by Savannah River contractors for the Fiscal year 1984. In addition, the document projects activities for several years beyond 1984 to adequately plan for safe handling and storage of radioactive wastes generated at Savannah River and for developing technology for improved management of low-level solid wastes. A revised plan will be issued prior to the beginning of the first quarter of each fiscal year. In this document, work descriptions and milestone sahedules are current as of the date of publication. Budgets are based on available information as of June 1983.

(DOE/TIC-4621-Vol.1) Technology of high-level nuclear waste disposal. Advances in the science and engineering of the management of high-level nuclear wastes. Volume 1 . Hofmann, P.L.; Breslin, J.J. (eds.). (Department of Energy, Oak Ridge, TN (USA). Technical Information Center). 1981. Con- tract AC06-76RL01830. 402p. NTIS \$18.00. Order Number DE82009594.

The papers in this volume cover the following subjects: waste isolation and the natural geohydrologic system; repository perturbations of the natural system; radionuclide migration through the natural system; and repository design technology. Individual papers are abstracted.

608 (DOE/TIC-4621-Vol.1, pp 3-15) Earth science developments in support of waste isolation. Duguid, J.O. (Battelle, Washington, DC). 1981. NTIS $\$ 18.00$.

In Technology of high-level nuclear waste disposal. Advances in the science and engineering of the management of high-level nuclear wastes. Volume 1.

Earth science issues in geologic waste isolation can be subdivided into smaller questions that are resolvable. This approach provides a mechanism for focusing research on topics of definable priority and monitoring progress through the status of issue resolution. The status of resolution of major issues in borehole sealing, interpretation of groundwater hydrology, geochemistry, and repository performance assessment is presented. The Waste Terminal Storage Program has reached a point where the selection of sites, underground testing, and emplacement of waste can proceed on a well-defined schedule.

609 (DOE/TIC-4621-Vol.1, pp 16-41) Preliminary assessment of shales and other argillaceous rocks in the United States. Gonzales, S. (Earth Resource Associates, Inc., Athens, GA); Johnson, K.S. 1981. NTIS \$18.00.

In Technology of high-level nuclear waste disposal. Advances in the science and engineering of the management of high-level nuclear wastes. Volume 1 .

Shales and related clay-rich rock types throughout the conterminous United States are geologically characterized and evaluated on a regional basis relative to their promise as possible candidate rock sequences for the repository disposal of high-level radioactive wastes. Only stratigraphic intervals or parts of them that are laterally persistent, consist of $75 \mathrm{~m}$ or more of shale, mudstone, or argillite, and lie at depths of 305 to $915 \mathrm{~m}$ below the land surface are included. The general properties of clay-rich rocks, as well as the several desirable characteristics that make them potentially attractive as disposal host candidates, are reviewed. Also discussed are the geologic factors that dictate the potential acceptability of any shale sequence relative to the regional subsurface distribution of units that meet the basic criteria of extent, thickness, and depth. Included in this context are the tectonic setting, geologic structure, seismicity, groundwater hydrology, mineralogy and content of organic matter, mineral resource potential of both the shales and the enclosing geologic basins, and any construction experience on underground openings, such as hydrocarbon-storage facilities. The clay-rich strata that appear to be promising on the basis of this evaluation are inventoried according to their occurrence and distribution within nine geologic-geomorphic regions throughout the country. Also considered are several, more localized occurrences of Precambrian argillites whose mineralogies make them a related yet separate group in comparison with the sedimentary strata already summarized. Topics for which data are insufficient or on which inadequate study has been conducted to date are also identified.

610 (DOE/TIC-4621-Vol.1, pp 136-167) Radiation damage studies on synthetic $\mathrm{NaCl}$ crystals and natural rock salt for radioactive waste disposal applications. Levy, P.W.; Loman, J.M.; Swyler, K.J.; Klaffky, R.W. (Brookhaven National Lab., Upton, NY). 1981. NTIS \$18.00. Contract AC06-76RL01830. In Technology of high-level nuclear waste disposal. Advances in the science and engineering of the management of high-level nuclear wastes. Volume 1.

Radiation damage, particularly F-center and sodium metal colloid particle formation, was studied at 100 to $300^{\circ} \mathrm{C}$ in synthetic meltgrown $\mathrm{NaCl}$ crystals and natural rock salt from different geological localities. Optical absorption measurements were made at controlled temperatures during irradiation with $1.5-\mathrm{MeV}$ electrons in a unique facility for making optical absorption and other measurements during irradiation. The electron irradiations produced the same damage as do gamma rays of similar energy. The Jain-Lidiard theory for radiationinduced colloid formation in $\mathrm{NaCl}$ accounts for some but not all of the observations. Overall the theory appears to apply better to natural than to synthetic rock salt. The measurements made to date have been confined to total doses of 2 to $4 \times 10^{8}$ rads. Extrapolating the (irradiation 
time $)^{n}$ or (dose) $)^{n}$ relations to doses expected at the surface of the waste form in a radioactive waste repository indicated that doses of $10^{10} \mathrm{rads}$ will convert 0.1 to $10 \%$ and $2 \times 10^{10}$ rads will convert 1 to $50 \%$ of the adjacent rock salt to sodium metal colloid particles. The salt adjacent to the canisters will reach these doses in 50 to $400 \mathrm{yr}$. Since this large variation in damage formation results from differences in the radiationinduced colloid formation rates between samples from different locations, radioactive waste repositories are best placed in localities and/or horizons where the damage rates are low. Also, the properties of rock salt subjected to doses of $10^{9}$ to $10^{10}$ rads differ markedly from those of unirradiated salt. Irradiated salt is brittle and friable, contains chlorine, and reacts with water to form $\mathrm{H}_{2}$ gas and other products. Thus waste form canisters, which will be subject to heat, radiation, and pressure, must be designed to withstand a variety of physical and chemical interactions with irradiated rock salt.

611 (DOE/TIC-4621-Vol.1, pp 255-283) Oklo reactors: natural analogs to nuclear waste repositories. Curtis, D.B.; Benjamin, T.M.; Gancarz, A.J. (Los Alamos National Lab., NM). 1981. NTIS $\$ 18.00$.

In Technology of high-level nuclear waste disposal. Advances in the science and engineering of the management of high-level nuclear wastes. Volume 1.

The 2-billion-year-old fossil reactors at $O$ klo are ancient natural nuclear waste sites. Isotope dilution mass spectrometric analyses of the fission products in the reactor core uraninite and the peripheral pelitic sandstone provide data for calculating the reactor operating parameters, the quantities of fissiogenic isotopes produced, the fraction of these isotopes retained in the cores, and the location in the peripheral rocks of the fissiogenic fraction lost from the cores. For a duration of criticality of $3 \times 10^{5} \mathrm{yrs}$, the thermal plus resonance neutron fluence ranged between $10^{26}$ and $10^{21}$ neutrons $/ \mathrm{cm}^{2}$. The fraction of technetium (60 to $85 \%$ ), ruthenium (75 to $90 \%$ ), and neodymium ( 85 to $100 \%$ ) retained is negatively correlated with fluence. The lost fission products are contained within a few tens of meters of their source, the reactor cores. The systematics of the decay of ${ }^{99} \mathrm{Tc}\left(\mathrm{t}, 2 />2.13 \times 10^{5} \mathrm{yr}\right)$ to ${ }^{99} \mathrm{Ru}$ limits the period of fissiogenic element migration to approximately 1 million yr at a time 2 billion yr ago. Thermodynamic calculations of the temperature-dependent solubilities indicate that the loss of fissiogenic elements is diffusion controlled, whereas retention in the surrounding rocks is a result of temperature-dependent deposition from an aqueous solution. These results concerning the geochemistry of technetium, ruthenium, and neodymium at a natural waste site support the concept of geologic burial of man-made radioactive wastes.

612 (DOE/TIC-4621-Vol.1, pp 287-309) National waste terminal storage conceptual reference repository description. Odgers, I.L. (Bechtel Group, Inc., San Francisco, CA); Collings, J.L. 1981. NTIS $\$ 18.00$.

In Technology of high-level nuclear waste disposal. Advances in the science and engineering of the management of high-level nuclear wastes. Volume 1 .

The conceptual reference repository description (CRRD) discusses, from a conceptual engineering standpoint, the structures, systems, equipment, and operations necessary to: (1) receive unreprocessed spent fuel assemblies in standard casks; (2) unload these assemblies; (3) place them in canisters; (4) transport the canisters to underground storage locations in the salt dome; and (5) place the canisters in terminal storage. The CRRD also elaborates on the concepts for retrieval and recovery of the spent fuel after burial and describes the development of the shafts and the underground areas, as well as the supporting operational utility and administrative features of the repository.

613 (DOE/TIC-4621-Vol.1, pp 310-337) Mining technology development in crystalline rock. Hustrulid, W.A.; Chitombo, G.P.; El Rabaa, A.W.; King, R.H.; Montazer, P.M.; Rosasco, P.V. (Colorado School of Mines, Golden). 1981. NTIS $\$ 18.00$.

In Technology of high-level nuclear waste disposal. Advances in the science and engineering of the management of high-level nuclear wastes. Volume 1.

The Colorado School of Mines (CSM), under sponsorship of the Department of Energy through the Office of Nuclear Waste Isolation (ONWI), has established a hard-rock research facility at its experimental mine. Even through this site will not become a nuclear waste repository, the CSM has established and maintains an underground test room for use by its own personnel and ONWI and its contractors to condur in situ investigations. Furthermore, CSM is designing, conducting, an reporting on a series of field research programs to develop site evalu: tion procedures, excavation techniques, and instrumentation required for nuclear waste repository siting, construction, and monitoring (Hustrulid, 1981). This facility is presently being used to: evaluate and develop techniques for careful excavation of hard rock; develop the mapping techniques required to describe adequately the structural geology; evaluate the structural continuity in the granitic gneiss at the CSM site; evaluate the structural damage done to the rock mass by blasting; develop techniques for evaluating fracture permeability; evaluate permeability changes in the rock mass as a result of blasting. Although specifically oriented toward nuclear waste storage and disposal, the techniques and procedures being developed and evaluated have wide applicability to all underground excavations in hard rock.

614 (DOE/TIC-4621-Vol.1, pp 338-353) Geochemical factors in borehole-shaft plug longevity. Roy, D.M. (Pennsylvania State Univ., University Park). 1981. NTIS \$18.00. Contract AC06.76RL01830.

In Technology of high-level nuclear waste disposal. Advances in the science and engineering of the management of high-level nuclear wastes. Volume 1.

Geochemical investigations that address factors controlling the longevity of repository sealing materials in a genchemical environment are discussed. Studies are being made of cement-based materials as major candidates for seals for borehole plugging, and shaft and tunnel sealing in certain potential repository environments. Factors controlling the extent of attainment of equilibrium of the plug components with time and the rate of approach to a state of stable equilibrium of the plug component chemical subsystem within the total system are discussed. The effect of these factors on changes in physical, mechanical and thermal properties of a seal system, and the consequent effectiveness of the seal in preventing transport of radioactive waste species are the dominant features to be determined. Laboratory experiments on the effects of anticipated temperature, pressure, and environmental factors (including chemical composition and specific rock type) are described. Thermodynamic studies are used to determine the potentially stable reaction products under conditions similar to those anticipated for the repository boreholes, shafts, and tunnels during and after the operating stage. Multitemperature reaction series are studied, and reaction kinetics are investigated for the purpose of predicting the course of likely reactions. Detailed studies of permeability, diffusion, and interfacial properties and chemical and microphase characterization of the products of experiments are carried out. Characterization studies of old and ancient cements, mortars, and concretes and prototype man-made seal materials are performed to further assess the factors associated with longevity.

616 (DOE/TIC-4621-Vol.1, pp 354-369) Field-test programs of borehole plugs in southeastern New Mexico. Christensen, C.L.; (Sandia National Lab., Albuquerque, NM); Peterson, E.W. 1981. NTIS \$18.00.

In Technology of high-level nuclear waste disposal. Advances in the science and engineering of the management of high-level nuclear wastes. Volume 1.

This paper gives a general overview of the repository-sealing field test effort being conducted by Sandia National Laboratories in support of the Waste Isolation Pilot Plant in southeast New Mexico. Summary descriptions of supporting activities, such as performance assessment and plugging materials development, are included to create the connection between modeling and laboratory activities as they relate to field results. Results of tests on a portion of a 17-year-old plug (Plug 217) recovered from a mine horizon and the Bell Canyon Test, in which a cement plug was emplaced to isnlate a naturally pressurized aquifer, are given. Conclusions from these field plugging tests are included.

616 (DOE/TIC-4621-Vol.1, pp 370-386) Overview of nuclear waste disposal in space. Rice, E.E. (Battelle Columbus Labs., OH); Priest, C.C. 1981. NTIS \$18.00.

In Technology of high-level nuclear waste disposal. Advances ir the science and engineering of the management of high-level nucleal wastes. Volume 1.

One option receiving consideration by the Department of Energy (DOE) is the space disposal of certain high-level nuclear wastes. The National Aeronautics and Space Administration is assessing the space disposal option in support of DOE studies on alternatives for nuclear 
waste management. The space disposal option is viewed as a complement, since total disposal of fuel rods from commercial power plants is not considered to be economically practical with Space Shuttle technology. The space disposal of certain high-level wastes may, however, pro vide reduced calculated and perceived risks. The space disposal option in conjunction with terrestrial disposal may offer a more flexible and lower risk overall waste management system. For the space disposal option to be viable, it must be demonstrated that the overall long-term risks associated with this activity, as a complement to the mined geologic repository, would be significantly less than the long-term risk associated with disposing of all the high-level waste. The long-term risk benefit must be achieved within an acceptable short-term and overall program cost. This paper briefly describes space disposal alternatives, the space disposal destination, possible waste mixes and forms, systems and typical operations, and the energy and cost analysis.

617 (DOE/TIC-4621-Vol.1, pp 42-64) Use of radiogenic noble gases for dating groundwater. Marine, I.W. (Savannah River Lab., Aiken, SC). 1981. NTIS \$18.00. Contract AC0976SR00001.

In Technology of high-level nuclear wastc disposal. Advances in the science and engineering of the management of high-level nuclear wastes. Volume 1.

The accumulation in groundwater of products from the radioactive decay of elements naturally found in rocks offers a potential for measuring the time that the groundwater has been in contact with the rock. This method of dating groundwater has an advantage over using decay products from atmospheric radionuclides in that the amount of decay product becomes greater with increasing age rather than less. Different decay products accumulate at different rates, however, and, thus, have a different potential usefulness in age determinations. The most useful decay product is helium, which is produced from uranium and thorium. The use of argon-40 produced from potasslum is limited because it is abundant in meteoric water. Neon, xenon, and krypton can be uscd, but only with great difficulty because they are produced in extremely small quantities. In general, the potential for error increases when a long time is required to produce a small quantity of the dating nuclide. An example is given of the use of helium dissolved in groundwater to obtain an age of 840,000 y for water in crystalline metamorphic rock beneath the Savannah River Plant near Aiken, SC. Combined with other information, this water age can be used to measure the hydraulic conductivity of a large mass of rock (tens of kilometers in length). The hydraulic conductivity so calculated compares favorably with that obtained from hydraulic tests.

618 (DOE/TIC-4621-Vol.1, pp 65-83) Theoretical and laboratory investigations of flow through fractures in crystalline rock. Witherspoon, P.A.; Watkins, D.J.; Tsang, Y.W. (Lawrence Berkeley Lab., CA). 1981. NTIS \$18.00. Contract W-7405-ENG-48.

In Technology of high-level nuclear waste disposal. Advances in the science and engineering of the management of high-level nuclear wastes. Volume 1 .

A theoretical model developed for flow through a deformable fracture subject to stresses was successfully tested against laboratory experiments. The model contains no arbitrary parameters and can be used to predict flow rates through a single fracture if the fractional fracture contact area can be estimated and if stress-deformation data are available. These data can be obtained from laboratory or in situ tests. The model has considerable potential for practical application. The permeability of ultralarge samples of fractured crystalline rock as a function of stresses was measured. Results from tests on a pervasively fractured 1-m-diameter specimen of granitic rock showed that drastically simplifying assumptions must be used to apply theoretical models to this type of rock mass. Simple models successfully reproduce the trend of reduced permeability as stress is applied in a direction normal to the fracture plane. The tests also demonstrated how fracture conductivity increases as a result of dilatancy associated with shear displacements. The effect of specimen size on the hydraulic properties of fractured rock was also investigated. Permeability tests were performed on specimens of charcoal black granite containing a single fracture subjected to normal stress. Results are presented for tests performed on a $0.914-\mathrm{m}$ diameter specimen and on the same specimen after it had been reduced

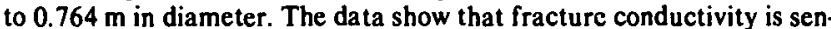
sitive to stress history and sample disturbance.
$619 \quad$ (DOE/TIC-4621-Vol.1 pp Thermomechanical studies in granite at Stripa, Sweden. Cook N.G.W. (Univ. of California, Berkeley); Myer, L.R. 1981. NTIS \$18.00. Contract W-7405-ENG-48.

In Technology of high-level nuclear waste disposal. Advances in the science and engineering of the management of high-level nuclear wastes. Volume 1.

Media other than rock salt are being considered for the deep, geologic disposal of nuclear wastes. The disposal of high-level nuclear waste in a deep, underground repository will subject the rock to a thermal pulse that will induce displacements, strains, and stresses in the rock. Thermomechanical experiments, with electrical heaters simulating the thermal output of waste canisters, were carried out in granite at a depth of $340 \mathrm{~m}$ below surface adjacent to a defunct iron ore mine at Stripa, Sweden. Changes in temperature, displacement, and stress in the rock around these heaters were measured, and the measurements were compared with predictions calculated from the theory of linear thermoelasticity. Measured temperature changes agreed well with predictions, but measured displacements and stresses were consistently less than those predicted with constant values for the coefficient of thermal expansion and elastic properties of the rock. A laboratory test program to measure these coefficients over ranges of stress and temperature representing those in the field experiment has been initiated. Test specimens were taken from cores recovered from the instrumentation holes in the Stripa experiments. Preliminary results from laboratory tests on specimens free of joints indicate that the values of Young's modulus and Poisson's ratio increase from about 60 to $80 \mathrm{MPa}$ and from 0.15 to 0.22 , respectively, as the confining stress is increased from 2 to $55 \mathrm{MPa}$; these values decrease with increasing temperature, more so at $2 \mathrm{MPa}$ than at $55 \mathrm{MPa}$. The linear coefficient of thermal expansion at a confining stress of $30 \mathrm{MPa}$ increases from about $10 \times 10^{-6} /{ }^{\circ} \mathrm{C}$ at $40^{\circ} \mathrm{C}$ to about $14 \times$ $10^{-6} /{ }^{\circ} \mathrm{C}$. The magnitudes of these changes are not sufficient to resolve the disparity between measured and predicted results. Perhaps the properties of test specimens containing joints will show greater variations in the values of the thermomechanical coefficients with temperature and pressure.

620 (DOE/TIC-4621-Vol.1, pp 100-109) Dome-salt thermomechanical experiments at Avery Island, Louisiana. Van Sambeek, L.L. (RE/SPEC Inc., Rapid City, SD). 1981. NTIS $\$ 18.00$.

In Technology of high-level nuclear waste disposal. Advances in the science and engineering of the management of high-level nuclear wastes. Volume 1.

An overview is given of three heater tests at Avery Island, Louisiana. The design considerations and operating conditions are described relative to the stated objective of each of the tests, and the data obtained to date are presented. An analysis of the results will be presented in the future. Measurements of temperature, heat flux, and displacement are presented. These three categories of measured responses were the most reliable and also are readily comparable with calculated responses. Determinations of induced stress in the salt and backfill pressure measurements in the heater borehole were not considered successful. Results are not presented for these measurements. The information presented is important for understanding the response of salt to an imposed heat source.

621 (DOE/TIC-4621-Vol.1, pp 110-135) Domal salt brine migration experiments at Avery Island, Louisiana. Krause, W.B.; Gnirk, P.F. (RE/SPEC Inc., Rapid City, SD). 1981. NTIS $\$ 18.00$

In Technology of high-level nuclear waste disposal. Advances in the science and engineering of the management of high-level nuclear wastes. Volume 1 .

Three in-situ brine migration experiments were performed in domal salt in the Avery Island mine located in southwestern Louisiana. The primary measurements included temperature, moisture collection, and pre- and post-test permeability at the experimental sites. Experimental data are discussed and compared with calculations based on the single-crystal brine migration theory. Comparisons indicate reasonable agreement between experiment and thenry.

622

(DP-81-125-1) Waste-management program. Technical progress report, January-March 1981. (Du Pont de Nemours (E.I.) and Co., Aiken, SC (USA). Savannah River 
Lab.). Jun 1982. Contract AC09-76SR00001. 222p. NTIS, PC A10/MF A01. Order Number DE82018213.

This quarterly report provides current information on operations and development programs for the management of radioactive wastes from operation of the Savannah River Plant. The studies on environmental and safety assessments, other support, waste form development and characterization, process and equipment development, and the Defense Waste Processing Facility are a part of the Long-Term Waste Management Technology Program. The following studies are reported for the SR Interim Waste Operations Program: surveillance and maintenance, waste concentration, low-level effluent waste, waste tank evaluation, tank replacement/waste transfer, process development, and solid waste storage. Work for the Office of Nuclear Waste Isolation is described.

(DP-83-125-1) Waste management program technical progress report, January-March 1983. (Du Pont de Nemours (E.I.) and Co., Aiken, SC (USA). Savannah River Lab.). Sep 1983. Contract AC09-76SR00001. 165p. NTIS, PC A08/MF A01. Order Number DE84001210.

Portions are illegible in microfiche products. Original copy available until stock is exhausted.

This quarterly report provides current information on operations and development programs for the management of radioactive wastes from operation of the Savannah River Plant and offplant participants. The studies on environmental and safety assessments, other support, in situ storage or disposal, waste form development and characterization, process and equipment development, airborne nuclear waste dispersion, and the Defense Waste Processing Facility are a part of the Long-Term Waste Management Technology Program. The following studies are reported for the Savannah River Interim Waste Operations: surveillance and maintenance, waste concentration, low-level effluent waste, tank replacement/waste transfer, waste engineering, and solid waste storage and related activities.

624 (DP-1631) Coordination meeting of high-level waste technology and national waste terminal storage programs, October 21-22, 1981. Permar, P.H. (Du Pont de Nemours (E.I.) and Co., Aiken, SC (USA). Savannah River Lab.). Jan 1982. Contract AC09-76SR00001. 162p. NTIS, PC A08/MF A01. Order Number DE83001657.

Portions of document are illegible.

The purpose of the first National Waste Terminal Storage/HighLevel Waste (NWTS/HLW) Coordination Meeting was to foster an exchange of information and to agree to a schedule of events to ensure the coordination of the two programs. Thirty-six participants, representing 11 contractors and two DOE offices, discussed the current status and planned programs under the following topics: The NWTS/HLW Coordination Schedule; Interim Performance Specifications and Data Requirements for Waste Forms; Interim Product Specifications for Defense High-Level Waste (DHLW) Forms; Description of Canistered Waste (Defense and Commercial); Reference Repository Conditions; Waste Package Conceptual Designs; Plans for Characterization of Defense and Commercial HLW Forms; Waste Package Modeling; Status of Materials Characterization Organization [Materials Charactèrization Center (MCC) and Materials Review Board (MRB)].

625 (DP-MS-81-92) Disposal of Savannah River Plant waste salt. Dukes, M.D. (Du Pont de Nemours (E.I,) and Co. Alken, SC (USA). Savannah River Lab.). 1982. Contract AC09-76SR00001. 11p. (CONF-820303-22). NTIS, PC A02/MF A01. Order Number DE82008917.

From Waste management conference (ANS); Tucson, AZ, USA (8 Mar 1982)

Approximately 26-million gallons of soluble low-level waste salts will be produced during solidification of 6-million gallons of high-level defense waste in the proposed Defense Waste Processing Facility (DWPF) at the Savannah Kiver Plant (SRP). Soluble wastes (primarily $\mathrm{NaNO}_{0}, \mathrm{NaNO}_{2}$, and $\mathrm{NaOH}$ ) stored in the waste tanks will be decontaminated by ion exchange and solidified in concrete. The resulting saltconcrete mixture, saltcrete, will be placed in a landfill on the plantsite such that all applicable federal and state disposal criteria are met. Proposed NRC guidelines for the disposal of waste with the radionuclide content of SRP salt would permit shallow land burial. Federal and state rules require that potentially hazardous chemical wastes (mainly nitrate-nitrate salts in the saltcrete) be contained to the degree necessary to meet drinking water standards in the ground water beneath the land- fill boundary. This paper describes the proposed saltcrete landfill and tests under way to ensure that the landfill will meet these criteria. The work includes laboratory and field tests of the saltcrete itself, a field test of a one-tenth linear scalc model of the entire landfill system, and a numerical model of the system.

626 (DP-MS-81-109) Comparative lcach testing of highlevel waste forms. Stone, J.A. (Du Pont de Nemours (E.I.) and Co., Aiken, SC (USA). Savannah River Lab.). 1982. Contract AC09-76SR00001. 20p. (CONF-820547-1). NTIS, PC A02/MF A01. Order Number DE82014176.

From 84. annual meeting of the American Ceramic Society; Cincinnati, OH, USA (2 May 1982).

Four candidate waste forms (borosilicate glass, high-silica glass, tailored ceramic, and Synroc) for Savannah River Plant high-level nuclear waste were compared in MCC-1 standard static leach tests. Relative leaching behavior differed for each element, and none of the waste forms was clearly superior to the others.

627 (DP-MS-81-114) High-level waste-form-product performance evaluation. Bernadzikowski, T.A.; Allender, J.S.; Stone, J.A.; Gordon, D.E.; Gould T.H. Jr.; Westberry, C.F. III. (Du Pont de Nemours (E.I.) and Co., Aiken, SC (USA). Savannah River Lab.). 1982. Contract AC09-76SR00001. 16p. (CONF-820547-2). NTIS, PC A02/MF A01. Order Number DE82013897.

From 84. annual meeting of the American Ceramic Society; Cincinnati, OH, USA (2 May 1982).

Seven candidate waste forms were evaluated for immobilization and geologic disposal of high-level radioactive wastes. The waste forms were compared on the basis of leach resistance, mechanical stability, and waste loading. All forms performed well at leaching temperatures of 40,90 , and $150^{\circ} \mathrm{C}$. Ceramic forms ranked highest, followed by glasses, a metal matrix form, and concrete. 11 tables.

628 (DP-MS-81-127) Research priorities for isolation of defense high-level and transuranic wastes in the United States. Gordon, D.E. (Du Pont de Nemours (E.I.) and Co., Aiken, SC (USA). Savannah River Lab.). 1982. Contract AC0976SkuvuU1. 24p. (CONF-820636-7). NTIS, PC A02/MF A01. Order Number DE82019159.

From 5. international symposium on the scientific basis for radioactive waste management; Berlin, F.R. Germany (7 Jun 1982).

Several research priorities relating to the isolation of HLW and TRU waste have been identified. For HLW, the rescarch needs center around the improvement of waste immobilization technology, a better understanding of leaching mechanisms and the interactions among waste system variables, and establishment of risk assessments as the technical bases for regulatory criteria. For TRU waste, additional work on classification techniques and methods to immobilize particulates is suggested.

629

(DP-MS-82-81) Alternatives for definse waste-salt disposal. Benjamin, R.W.; McDonell, W.R. (Du Pont de Nemours (E.I.) and .n, Aiken, SC (USA). Savannah River Lab.). 1983. Contract AC09-76SR00001. 31p. (CONF830205-28). NTIS, PC A03/MF A01. Order Number DER3011608

Feb 1983)

From Waste management conference; Tucson, AZ, USA (27

Alternatives for disposal of decontaminated high-level waste salt at Savannah River werc reviewed to estimate costs and potential environmental impact for several processes. In this review, the reference process utilizing intermediate-depth burial of salt-concrete (saltcrete) monoliths was compared with alternatives including land application of the decontaminated salt as fertilizer for SRP pine stands, ocean disposal with and without containment, and terminal storage as saitcake in existing SRP waste tanks. Discounted total costs for the reference process and its modifications were in the same range as those for most of the alternative processes; uncontained ocean disposal with truck transport to Savannah River barges and storage as saltcake in SRP tanks had lower costs, but presented other difficulties. Envirunmental impacts could generally be maintained within acceptable limits for all processes except retention of saltcake in waste tanks, which could result in chemical contamination of surrounding areas on tank collapse. Land application would require additional salt decontamination to meet radioactive 
waste disposal standards, and ocean disposal without containment is not permitted in existing US practice. The reference process was judged to be the only salt disposal option studied which would meet all current requirements at an acceptable cost.

630 (DP-MS-83-18) Prevention of stress corrosion cracking in nuclear waste storage tanks. Ondrejcin, R.S. (Du Pont de Nemours (E.I.) and Co., Aiken, SC (USA). Savannah River Lab.). 1983. Contract AC09-76SR00001. 32p. (CONF830467-1). NTIS, PC A03/MF A01. Order Number DE83012006.

From Multivariable regression symposium; Nashville, TN, USA (27 Apr 1983)

Portions are illegible in microfiche products.

At the Savannah River Plant, stress corrosion of carbon steel storage tanks containing alkaline nitrate radioactive waste is prevented by stress relief and specification of limits on waste composition and temperature. Actual cases of cracking have occurred in the primary steel shell of tanks designed and built before 1960 and were attributed to a combination of high residual stresses from fabrication welding and aggressiveness of fresh wastes from the reactor fuel reprocessing plants. The fresh wastes have the highest concentration of nitrate, which has been shown to be the cracking agent. Also, as the waste solutions age and are reduced in volume by evaporation of water, nitrite and hydroxide ions become more concentrated and inhibit stress corrosion. Thus, by providing a heel of aged evaporated waste in tanks that receive fresh wastes, concentrations of the inhibitor ions are maintained within specific ranges to protect against nitrate cracking. The concentration and temperature range limits to prevent cracking were determined by a series of statistically designed experiments.

631 (DP-MS-83-66) Preliminary report on a glass burial experiment in granite. Clark, D.E.; Zhu, B.F.; Robinson, R.S.; Wicks, G.G. (Florida Univ., Gainesville (USA). Dept. of Materials Science and Engineering; Du Pont de Nemours (E.I.) and Co., Aiken, SC (USA). Savannah River Lab.). 1983. Contract AC09-76SR00001. 23p. (CONF-830451-23). NTIS, PC A02/MF A01. Order Number DE83017426.

From 85. American Ceramic Society annual meeting; Chicago, IL, USA (25 Apr 1983).

Preliminary results of a two-year burial experiment in granite are discussed. Three compositions of simulated alkali borosilicate waste glasses were placed in boreholes approximately 350 meters deep. The glass sample configurations include mini-cans (stainless steel rings into which glass has been cast) and pineapple slices (thin sections from cylindrical blocks). Assemblies of these glass samples were prepared by stacking them together with granite, compacted bentonite and metal rings to provide several types of interfaces that are expected to occur in the repository. The assemblies were maintained at either ambient mine temperature $\left(8\right.$ to $\left.10^{\circ} \mathrm{C}\right)$ or $90^{\circ} \mathrm{C}$. The glasses were analyzed before burial and after one month storage at $90^{\circ} \mathrm{C}$. The most extensive surface degradation occurred on the glasses interfaced with bentonite. In general, very little attack was observed on glass surfaces in contact with the other materials. The limited field and laboratory data are compared.

632 (DP-MS-83-107) High-level waste description, inventory and hazard. Crandall, J.; Hennelly, E.J.; McElroy, J.L. (Du Pont de Nemours (E.I.) and Co., Aiken, SC (USA). Savannah River Lab.; Pacific Northwest Lab., Richland, WA (USA)). 1983. Contract AC09-76SR00001. 26p. (CONF831063-5). NTIS, PC A03/MF A01. Order Number DE84002429.

From American Institute of Chemical Engineers fall annual meeting; Washington, DC, USA (30 Oct 1983).

High-level nuclear waste (HLW), including its origin, is described and the current differences in definitions discussed. Quantities of defense and commercial radioactive HLW, both volume and curie content, are given. Current waste handling, which is interimin nature, is described for the several sites. The HLW hazard is defined by the times during which various radionuclides are the dominant contributors. The hazard is also compared to that of the ore. Using ICRP-2, which is the legal reference in the US, the hazard of the waste reduces to a level equal to the ore in about 300 years. The disposal plans are summarized and it is shown that regulatory requirements will probably govern disposal operations in such a conservative manner that the risk (product of hazard times probability of release) may well be lower than for any other wastes in existence or perhaps lower than those for any other human endeavor.

633 (DPSP-79-1003(Del.)) Savannah River Plant wastetank inspection manual. McNatt, F.G. (Du Pont de Nemours (E.I.) and Co., Aiken, SC (USA). Savannah River Plant). Jan 1979. Contract AC09-76SR00001. 84p. NTIS, PC A05/MF A01. Order Number DE83004821.

Portions of document are illegible.

This manual is provided as an aid in making visual and photographic inspections and steel thickness measurements of Building 241-F and $-\mathrm{H}$ underground waste storage tanks. It describes the inspection program, the storage tanks, the equipment and techniques used and the results of their application, and the inspection recordkeeping methods.

634 (DPSPU-81-30-7) Sampling and analysis of high level waste tank supernatant: an overview. Goergen, C.R. (Du Pont de Nemours (E.I.) and Co., Aiken, SC (USA). Savannah River Plant). 1981. Contract AC09-76SR00001. 7p. (CONF811025-28-Draft). NTIS, PC A02/MF A01. Order Number DE83000680.

From 25. conference on analytical chemistry and nuclear technology; Gatlinburg, TN, USA (6 Oct 1981).

The Savannah River Plant routinely samples its high level radioactive waste tank supernatants for analysis of major components. These results are important in maintaining proper levels of corrosion inhibiters for protection of the tank walls. Because the tank ambient temperature is elevated, the sample is heated to $70^{\circ} \mathrm{C}$ prior to removing aliquots for use in a variety of analytical methods. Typical analyses include density, $\mathrm{pH}, \mathrm{OH}^{*}, \mathrm{NO}_{0}^{-}$, and $\mathrm{NO}_{2}^{-}$, with occasional requests for $\mathrm{Al}(\mathrm{OH})_{4}^{-}$, $\mathrm{CO}_{0}>, \mathrm{PO}_{4}^{>}, \mathrm{SO}_{4}^{>}$, and various radionuclides.

635 (DPST-82-346) Comparison of SRP high-level waste disposal costs for borosilicate glass and crystalline ceramic waste forms. McDonell, W.R. (Du Pont de Nemours (E.I.) and Co., Aiken, SC (USA). Savannah River Lab.). Apr 1982. Contract AC09-76SR00001. 38p. NTIS, PC A03/MF A01. Order Number DE82015011.

An evaluation of costs for the immobilization and repository disposal of SRP high-level wastes indicates that the borosilicate glass waste form is less costly than the crystalline ceramic waste form. The wastes were assumed immobilized as glass with $28 \%$ waste loading in 10,300 reference 24-in.-diameter canisters or as crystalline ceramic with $65 \%$ waste loading in either 340024 -in.-diameter canisters or 590018 -in.diameter canisters. After an interim period of onsite storage, the canisters would be transported to the federal repository for burial. Total costs in undiscounted 1981 dollars of the waste disposal operations, excluding salt processing for which costs are not yet well defined, were about $\$ 2500$ million for the borosilicate glass form in reference 24-in.diameter canisters, compared to about $\$ 2900$ million for the crystalline ceramic form in 24-in.-diameter canisters and about $\$ 3100$ million for the crystalline ceramic form in 18-in.-diameter canisters. No large differences in salt processing costs for the borosilicate glass and crystalline ceramic forms are expected. Discounting to present values, because of a projected 2-year delay in startup of the DWPF for the crystalline ceramic form, preserved the overall cost advantage of the borosilicate glass form. The waste immobilization operations for the glass form were much less costly than for the crystalline ceramic form. The waste disposal operations, in contrast, were less costly for the crystalline ceramic form, due to fewer canisters requiring disposal; however, this advantage was not sufficient to offset the higher development and processing costs of the crystalline ceramic form. Changes in proposed Nuclear Regulatory Commission regulations to permit lower cost repository packages for defense high-level wastes would decrease the waste disposal costs of the more numerous borosilicate glass forms relative to the crystalline ceramic forms.

636 (DPSTD-82-65-Rev.1) Decontaminated salt disposal as saltcrete in a landfill. Technical data summary. (Du Pont de Nemours (E.I.) and Co., Aiken, SC (USA). Savannah River Lab.). 6 Aug 1982. Contract AC09-76SR00001. 34p. NTIS, PC A03/MF A01. Order Number DE83012204.

Portions are illegible in microfiche products.

This technical data summary presents a reference process for immobilizing decontaminated salt solution from the 200-Area waste storage tanks with cement, and disposing of the final waste material 
(called saltcrete) by burial in trenches. The saltcrete will be protected from leaching by clay and will be placed at least 3 meters above the historic high water table and beneath at least 5 meters of soil overburden. The decontaminated salt solution is a waste material which remains after the bulk of the radionuclides have been removed from waste tank supernate. This removal is effected by contacting the waste supernate with sodium tetraphenyl boron (Na-TPB) and sodium titanate $\left(\mathrm{NaTi}_{2} \mathrm{O}_{5} \mathrm{H}\right)$. These materials remove (by precipitation) most of the ${ }^{107} \mathrm{Cs}$ and ${ }^{90} \mathrm{Sr}$ as well as many other radioactive and non-radioactive elements. These precipitates, along with many other sludges which reside in the HLW tanks will be incorporated in borosilicate glass for eventual disposal in a geologic repository. An ion exchange process will also be used for removal of ${ }^{99} \mathrm{Tc}$. The decontaminated salt solution has sufficiently low levels of radioactivity that it can be disposed of on-site. The scope of the curent effort is to describe a process for blending decontaminated salt solution with cement to form a saltcrete product which has dimensional stability and relatively low leachability. The process is to be capable of solidifying $10 \mathrm{gpm}$ of supernate. About 100 million gallons of salt solution is to be solidified.

637 (EMD-82-64) Department of Energy does not plan to use an abandoned salt mine at Lyons, Kansas, for nuclear highlevel waste disposal. (G'eneral Accounting Office, Washington, DC (USA). Energy and Minerals Div.). 23 Mar 1982. 4p. General Accounting Office, Washington, DC 20548.

DOE's current high-level waste disposal program is aimed at opening an underground repository by the end of this century. The sites under consideration are in the States of Washington, Nevada, Texas, Mississippi, Utah, and Louisiana. Based on many years of tracking and reporting on DOE's high-level nuclear waste isolation program, the Lyons, Kansas salt mine is no longer being considered for high-level nuclear waste disposal. In addition, in recent conversations among officials from DOE, the Nuclear Regulatory Commission, the Kansas State Government, and the Rickano Corporation, it was confirmed that the Lyons mine is not part of the Federal high-level waste program.

638 (ENPU-80-11) Fracture analysis of crystalline rocks: field measurements and field geomechanical techniques. McEwen, T.J. (SEE CODE- 905031 1 Institute of Geological Sciences, Harwell (UK). Environmental Protection Unit). Jul 1980. 75p. Environmental Protection Unit, Institure of Geological Sciences, Building 151, Harwell Laboratory, Oxfordshire OX11 ORA, England.

The natural fractures occurring in crystalline rocks are described in terms of their occurrence in granite masses and in metamorphic rocks. Their orientations are shown to be related to the palen or present stresses which acted or act on the rocks, to the tectonic setting of the rock mass, and in some cases to any igneous banding that might exist. The analysis of fractures in surface outcrops and in boreholes is discussed in detail, and it is shown how estimates of fracture length, fracture area and fracture interconnectivity can be made from relatively simple measurements. The biases inevitably incurred during sampling are discussed in terms of their influence on the analyzed data, and methods for compensating for their effects are also included. The influence of the fracture surfaces on the mechanical properties of rock masses is described, and methods for measuring the shear strengths of fracture surfaces is discussed. The importance of the shear strength of rocks is analyzed in terms of their effects on determining the development of the fracture system. Finally the use of fracture orientations to determine the geotectonic stress field is discussed with examples from different tectonic environments. This report is in no sense a complete literature review of all available information on fractures in rocks and their influence on the response of rock masses to applied stresses and on its hydrogeolngical properties. It is designed to illustrate the types of analysis which area available for studying fractured rock, and which are specifically relevant to the programme of research into the feasibility of the disposal of high level radioactive waste in geological formations.

639 (ER-81-2E, pp 219-233) Radioactive waste management and disposal. 1981. NTIS (US Sales Only), PC A 16/MF A01.

In Nuclear policy review background papers.

The radioactive wastes in Canada are presently in storage. The objectives of the research being conducted by Atomic Energy of Canada Ltd. into their final disposal are to manage these wastes so that hazards are negligible and so that trouble and concern to future generations will be minimized or eliminated. Wastes may be disposed of either by cor tainment that achieves the required degree of isolation from man's env ronment, or by dispersion and dilution through the careful release $c$ effluents. Wastes are generated at all stages of the nuclear fuel cycle, and present storage methods range from surface tailings deposits to underwater storage of irradiated fuel. Most research is being conducted into underground storage of high-level wastes in hard rock formations, and volume reduction and immobilization of low- and medium- level wastes.

640 (EUR-7340) Storage of HLW in engineered structures: air-cooled and water-cooled concepts. Final report. Ahner, S.; Dekais, J.J.; Puttke, B.; Staner, P. (Commission of the European Communities, Brussels (Belgium)). 1981. 149p. NTIS (US Sales Only), PC A07/MF A01. Order Number DE82701148. A comparative study on an air-cooled and a water-cooled intermediate storage of vitrified, highly radioactive waste (HLW) in overground installations has been performed by Nukem and Belgonucleaire respectively. In the air-coled storage concept the desay heat from the storage area will be removed using natural convection. In the watercooled storage concept the decay heat is carried off by a primary and secondary foroed-cuoling systcm with reduitdant and diverse devices. The safety study carried out by Nukem used a fault tree method. It shows that the reliability of the designed water-cooled system is very high and comparable to the inherent, safe, air-cooled system. The impact for both concepts on the environment is determined by the release route, but even during accident conditions the release is far below permissible limits. The economic analysis carried out by Belgonucleaire shows that the construction costs for both systems do not differ very much, but the operation and maintenance costs for the watercooled facility are higher than for the air cooled facility. The result of the safety and economic analysis and the discussions with the members of the working group have shown some possible significant modifications for both systems, which are included in this report. The whole study has been carried out using certain national criteria which, in certain Member States at least, would lead to a higher standard of safety than can be justified on any social, political or economic grounds.

641 (GA-A-16971) Comparison of cask and dry well concepts for retrievable storage of high-level waste. Morissette R.P.; Ganley, J.T.; Ketchen, D.W.; Rasmussen, D.E. (GA Technologies, Inc., San Diego, CA (USA); Pacific Northwest Lab. Richland, WA (USA)). Feb 1983. Contract AT03-81SF11528. 6p. (CONF-830205-10). NTIS, PC A02/MF A01, Order Number DE83006802. Feb 1983).

From Waste management conference; Tucson, AZ, USA (27

This paper describes and compares two proposed concepts for storing high-level waste on the basis of their technical merits, technical status, research and development requirements, safety and environmental impact, and life cycle costs. This comparison indicates that although metal storage casks have technical advantages over dry wells, they are considerably more expensive and require large commitments of valuable resources.

642 (GA-A-17053) Monitored retrievable storage of nuclear waste in the US. Ganley, J.T.; Morissette, R.P.; Ketchen, D.W. (GA Technologies, Inc., San Diego, CA (USA)). 1983. Contract AT03-81SF11528. 9p. (CONF-830446-1). NTIS, PC A02/MF A01. Order Number DE83010809. 1983).

From American Power conference; Chicago, IL, USA (18 Apr

A monitored retrievable storage (MRS) facility is a long-term nuclear waste storage system. Monitoring ensures detection of waste package containment failures that could lead to release of radioactive materials. This protects facility personnel as well as the surrounding population and environment. Through basic design and monitoring of the stored material, easy retrievability is assured. This facilitates preparation of the stored material for later shipment to a reprocessing plan or repository. Monitored retrievable storage provides flexibility in th national nuclear waste management system. It can accommodate delay in the startup of the final repository or store spent fuel until questions regarding reprocessing are resolved. It also offers the capability to age spent fuel and HLW to ease the thermal loading on the repository, or conversely, to increase the capacity of a given size repository. The size 
and handling rate required in an MRS facility are highly dependent on the fuel cycle scenario proposed.

643 (GSF-T-133) Deep underground storage of [radioactive waste]. Annual report, 1981. (Gesellschaft fuer Strahlenund Umweltforschung m.b.H. Muenchen, Neuherberg (Germany, F.R.); Kernforschungszentrum Karlsruhe G.m.b.H. (Germany, F.R.)). 1981. 42p. (In German). (KFK-3321). NTIS (US Sales Only), PC A03/MF A01. Order Number DE83902467. Portions are illegible in microfiche products.

Information is presented on research and developmental activities sponsored by the Association and Research Center on the deep underground disposition of radioactive waste that varies in degree of activity. Data are also given on the seismic monitoring of burial sites.

644 (HMI-PSE-82/4-B) INTRACOIN, Level 1. Benchmark calculations with code SWIFT. Guelker, M.; Buetow, E.; Heredia, L. (Hahn-Meitner-Institut fuer Kernforschung Berlin G.m.b.H. (Germany, F.R.). Projekt Sicherheitsstudien Entsorgung (PSE)). Sep 1982. 94p. NTIS (US Sales Only), PC A05/MF A01. Order Number DE83750873.

For high-level waste, repositories in deep geologic media - either porous or fractured - are considered to be a reasonable disposal method. An important part of the long-term safety analysis of such repository concepts is an assessment of release and transport of long-lived radionuclides from the repository to the biosphere. In this assessment, mathematical models describing the mechanisms involved in the nuclide transport are essential tools to estimate the potential value of the geosphere as a safety barrier. To improve the understanding of the important phenomena of radionuclide transport and also of various strategies for their modelling, an international cooperation project has been set up. In this project, called INTRACOIN (International Nuclide TRAnsport COde INtercomparison study), comparisons are carried out between different mathematical models describing the far-field transport of radionuclides through geologic media. A comparison is performed at three levels aimed at examining: 1.) The numerical accuracy of the codes; 2 .) the ability of the codes to describe in-situ measurements; 3 .) the quantitative impact of choosing different modelling strategies on the nuclide transport calculations in a typical repository scenario assessment.

645 (ILR-MITT-119) Appraisal of a target-relevant evaluation of alternative strategies for global radioactive waste disposal. Koelle, H.H.; Apel, U.; Balzer, R.; Brieskorn, D.; Dorbandt, J.; Feuerstein, D.W.; Kill. H.; Koch, J.U.; Leukel, R.; Mueller, H. (Technische Univ. Berlin (Germany, F.R.). Inst. fuer Luft- und Raumfahrt). 15 Feb 1982. 54p. (In German). NTIS (US Sales Only), PC A04/MF A01. Order Number DE83751326.

This report results from a project within the course "Systems Engineering" during the winter semester 1982/83. The subject was to analyse alternatives for global nuclear waste disposal for the time period of 1980 through 2030 . Terrestrial and extraterrestrial alternatives, four each, were considered. 33 objectives, arranged in a formal hierarchical system, were developed to the purpose of valuation. The priorities for these objectives were simulated from the viewpoint of 16 different interest groups. - Individual valuations for low-, medium- and high-level radinactive waste were performed on the basis of the weighted objectives. This led to a ranking of the alternatives for each of the three types of waste. It is shown that the extraterrestrial alternatives (e.g. backside of the moon) are quite attractive particularly for the high-level radioactive waste.

646 (INIS-mf-6783) Proposal for basic safety requirements regarding the disposal of high-level radioactive waste. (Miljoestyrelsen, Copenhagen (Denmark). Arbejdsgruppe om Radioaktivt Affald). Apr 1980. 64p. NTIS (US Sales Only), PC A04/MF A01. Order Number DE82780392.

A working group commissioned to prepare proposals for basic safety requirements for the storage and transport of radioactive waste prepared its report to the Danish Agency of Environmental Protection. The proposais include: radiation protection requirements, requirements concerning the properties of high-level waste units, the geological conditions of the waste disposal location, the supervision of waste disposal areas. The proposed primary requirements for safety evaluation of the disposal of high-level waste in deep geological formations are of a general nature, not being tied to specific assumptions regarding the waste itself, the geological and other conditions at the place of disposal, and the technical methods of disposal. It was impossible to test the proposals for requirements on a working repository. As no country has, to the knowledge of the working group, actually disposed of high-level radioactive waste or approved of plans for such disposal. Methods for evaluating the suitability of geological formations for waste disposal, and background material concerning the preparation of these proposals for basic safety requirements relating to radiation, waste handling and geological conditions are reviewed. Appended to the report is a description of the phases of the fuel cycle that are related to the storage of spent fuel and the disposal of high-level reprocessing waste in a salt formation. It should be noted that the proposals of the working group are not limited to the disposal of reprocessed fuel, but also include the direct disposal of spent fuel as well as disposal in geological formations other than salt. (EG).

647 (INIS-mf-6784) Disposal of high-level waste from nuclear power plants in Denmark. Salt dome investigations. V.1. Summary. (Jysk-Fynske Elsamarbejde (ELSAM), Fredericia (Denmark); ELKRAFT A.m.b.A., Hellerup (Denmark)). 1981. 92p. NTIS (US Sales Only), PC A05/MF A01. Order Number DE82780393.

A summary is presented of a report in five volumes on possible disposal of radioactive waste in Denmark. The investigation was made by the Danish electric utilities ELKRAFT and ELSAM at the request of the Danish Government. The investigation proved it possible to consider two alternative designs for a disposal facility, one based on the deposition of waste in individual, deep holes, the other on placing the waste in mine galleries. A safety analysis was completed with the Mors dome as example. The purpose of the analysis was to prove whether safe disposal of high-level waste in Denmark was feasible. The utilities concluded that the results of the analysis were satisfactory and the report is now being assessed by the authorities. (BP).

648 (INIS-mf-6785) Disposal of high-level waste from nuclear power plants in Denmark. Salt dome investigations. V.2. Geology, text. (Jysk-Fynske Elsamarbejde (ELSAM), Fredericia (Denmark); ELKRAFT A.m.b.A., Hellerup (Denmark)). 1981. 411 p. NTIS (US Sales Only), PC A11/MF A01. Order Number DE82780394.

The present report deals with the geological investigations performed for determining the feasibility of a repository for high-level waste in a salt dome. It is volume 2 of five volumes that together constitute the final report of the Danish utilities' salt dome investigations. The purpose of the work was to procure a more detailed knowledge of the geology of salt domes in North Jutland on example of Mors. The Mors dome is oval with the two axes of approx. $12.5 \mathrm{~km}$ and $8 \mathrm{~km}$ respectively. Two deep wells have been drilled into the salt. These wells reach 3400 $3500 \mathrm{~m}$ below surface. Until a depth of about $3200 \mathrm{~m}$ Erslev 2 passes through rock salt of Zechstein 1 which is the oldest evaporite series. However, it could also be interlayed with the slightly younger Zechstein 2. At about $3200 \mathrm{~m}$ a marker layer was met with Zechstein 2 salt below. Interpretation of cores and results of downhole electromagnetic and borehole gravimetric measurements show that there is a large area around Erslev 2 which consists of very pure sodium chloride with traces of anhydrite (calcium, sulphate) $1.3 \%$. This area is used for the repository design and safety evaluation. The hydrological conditions existing in the strata above the salt dome (caprock) have been investigated with the help of four hydrogeological wells, placed two each, on two different sites. The cores themselves were taken at various depths in all four holes. With these laboratory methods it has been possible to measure data relevant to hydrology - such as porosity and permeability - as well as geochemistry. (BP).

649 (INIS-mf-6786) Disposal of high-level nuclear waste from nuclear power plants in Denmark. Salt dome investigations. V.2. Geology, figures. (Jysk-Fynske Elsamarbejde (ELSAM), Fredericia (Denmark); ELKRAFT A.m.b.A., Hellerup (Denmark)). 1981. 109p. NTIS (US Sales Only), PC A06/MF A01. Order Number DE82780395.

This report contains figures relating to the geological investigations described in vol 2 (text) of the report on disposal of high-level waste from nuclear power plants in Denmark.

650 (INIS-mf-6787) Disposal of high-level waste from nuclear power plants in Denmark. V.3. Material testing. 
(Jysk-Fynske Elsamarbejde (ELSAM), Fredericia (Denmark); ELKRAFT A.m.b.A., Hellerup (Denmark)). 1981. 123p. NTIS (US Sales Only), PC A06/MF A01. Order Number DE82780396.

The present report deals with material testing as part of the investigations regarding a repository for high-level waste in a salt dome. It is volume 3 of five volumes that together constitute the final report of the Danish utilities' salt dome investigations. The material testing programme chiefly comprised laboratory investigations and analyses of material samples, partly obtained from the salt dome in question and partly from the overlying geological formation. The test programme was fully completed for Eslev salt dome on Mors. Chemical analyses and mineralogical investigations were carried out in order to determine the type and extent of the impurities confined in the rock salt. Moreover, a programme was carried out to investigate the water content of the salt. Temperature conditions around a repository, the strenght and creep properties of the salt were investigated in order to determine whether drill holes, shafts and mine galleries could be constructed and maintained intact within the period of time required to complete the disposal of high-level waste. Chemical analysis were carried out in order to determine which substances are found dissolved in the water contained by the geological formation overlying the salt dome, as well as chemical/ physical investigations regarding the water content, porosity, velocity of a possible leak of radioactive waste products, etc. Materials that would be introduced into a repository were studied with regard to their corrosion resistance in the salt environment. Concrete materials were investigated and characterized for their use in the final sealing of the access routes to the repository through the geological formation.

651 (INIS-mf-6788) Disposal of high-level waste from nuclear power plants in Denmark. Salt dome investigations. V.4. Repository design. (Jysk-Fynske Elsamarbejde (ELSAM), Fredericia (Denmark); ELKRAFT A.m.b.A., Hellerup (Denmark)). 1981. 80p. NTIS (US Sales Only), PC A05/MF A01. Order Number DE82780397.

The present report deals with construction, operation and sealing of disposal facilities for high-level waste in a salt dome. It is volume 4 of five volumes that together constitute the final report on the Danish utilities' salt dome investigations. The safety investigations were carried out for a deep-hole disposal facility located in the salt dome on Mors. In principle the results of the investigations also apply to a shaft/mine disposal facility. The facility is designed for the disposal of vitrified high-level waste in the shape of glass canisters. There is a low concentration of waste in each canister, approx. $10 \%$. Furthermore, it was selected to place the waste in an intermediate storage for about 40 years prior to its final disposal. Consequently, heat generation in the waste at the time of final disposal will be modest, resulting in low temperature increase in the salt. As an example, the highest temperature increase will be approx. $40 \mathrm{deg}$. C. and it will occur at the edge of the hole five years after disposal has taken place. Prior to disposal, the glass canisters are encased in steel casks with $15 \mathrm{~cm}$ thick walls. Three canisters are placed in each cask, and 215 casks are stacked on top on one another in each deep-hole from a depth of $1200 \mathrm{~m}$ to $2500 \mathrm{~m}$ underground. The additional encasing is designed to protect the glass from dissolution should any brine reach the disposal facility. Furthermore, the steel cask protects the glass canisters against pressure from the wall of the hole. The technical design of the disposal facility gives it a considerable safety margin against unexpected events. The investigations proved Cretaceous strata to constitute an effective secondary barrier that would prevent radioactive matter from travelling from the underlying disposal facility to the biosphere.

652 (INIS-mf-6789) Disposal of high-level waste from nuclear power plants in Denmark. Salt dome investigations. V.5. Safety evaluation. (Jysk-Fynske Elsamarbejde (ELSAM), Fredericia (Denmark); ELKRAFT A.m.b.A., Hellerup (Denmark)). 1981. 143p. NTIS (US Sales Only), PC A07/MF A01. Order Number DE82780398.

The present report deals with safety evaluation as part of the investigations regarding a repository for high-level waste in a salt dome. It is volume 5 of five volumes that together constitute the final report on the Danish utilities' salt dome investigations. Two characteristics of the waste are of special importance for the safety evaluation: the encasing of the waste in steel casks with $15 \mathrm{~cm}$ thick walls affording protection against corrosion, protecting the surroundings against radiation, and protecting the glass cylinders from mechanical damage resulting from the pressure at the bottom of the disposal hole, and the modest generation of heat in the waste at the time of disposal resulting in a maximum temperature increase in the salt close to the waste of approx. 40 deg. C. These characteristics proved to considerably improve the safety margin with respect to unforeseen circumstances. The character of the salt dome and of the salt in the proposed disposal area offers in itself good protection against contact with the ground water outside the dome. The relatively large depth of 1200 and $2500 \mathrm{~m}$ of the salt surface also means that neither dome nor disposal facility will be appreciably influenced by glaciations or earthquakes. The chalk above the proposed disposal area is very tight and to retain radioactive matter effectively even in the precence of high concentrations of $\mathrm{NaCL}$. The safety investigations included a number of natural processes and probable events such as the segregation of crystal water from overlooked salt minerals, faulty sealings of disposal holes, permeable fault zones in the chalk overlying the dome, the risk in connection with human penetration into the dome. Leaching of a cavern is the only situation which proved to result in a release of radioactive material to the biosphere, but the resulting doses was found to be small compared to the probability that such a situation should develop.

663 (INIS-mf-6974, pp vp) Temperature, pressure and time dependence of dissolution rates of high level radioactive waste glasses. Lewis, R.A.; Smart, R.St.C. (Griffith Univ., Brisbane (Australia). School of Science). Nov 1981. NTIS (US Sales Unly). PC' AU4/MF AOI. (CONF-811194-(Absts.)).

From 3. AINSE engineering conference; Lucas Heights, Australia (12 Nov 1981).

DISSOLUTION; GLASS; HIGH-LEVEL RADIOACTIVE WASTES; PRESSURE DEPENDENCE; TEMPERATURE DEPENDENCE; TIME DEPENDENCE

654 (INIS-mf-6974, pp vp) Simulation of radiation damage in HLW glasses accelerated with $(n, \alpha)$ and $(n, f)$ reactions. Cousens, D.; Myhra, S. (Griffith Univ. Brisbane (Australia). School of Science). Nov 1981. NTIS (US Sales Only), PC A04/MF A01. (CONF-811194-(Absts.)).

From 3. AINSE engineering conference; Lucas Heights, Australia (12 Nov 1981).

ALPHA PARTICLES; CAPTURE; DISSOLUTION; GLASS; HIGH-LEVEL RADIOACTIVE WASTES; NEUTRON REACTIONS; PHYSICAL RADIATION EFFECTS; SIMULATION

656 (INIS-mf-7049) Stripa project, quarterly report October through December 1981. (SEE CODE- 6029620 Swedish Nuclear Fuel Supply Co., Stockholm). Jan 1982. 31p. NTIS (US Sales Only), PC A03/MF A01. Order Number DE82780688.

The methodology development for hydrogeological investigations in boreholes is continuing in combination with hydraulic, chemical and isotopic characterization of granites and groundwaters. The migration investigation is continued by drilling sampling holes and designing the equipment. The first buffer mass tests have covered the measurement of temperature, swelling pressures and water migration in two deposition holes. The preparation of the bentonite/sand backfill has started.

656 (INIS-mf-7092, pp vp) Safe disposal of radioartive wastes from nuclear power plants. Malasek, E. (Ceskoslovenska Komise pro Atomovou Energii, Prague); Tittlova, E. (Vyskumny Ustav Jadrovych Elektrarni, Jaslovske Bohunice (Czechoslovakia)). 1979. (In Czech). NTIS (US Sales Only), PC A09/MF A01. (CONF-7904193-).

From Conference on nuclear power and the environment; Zdar nad Sazavou, Czechoslovakia (2 Apr 1979).

The principles are listed of the safe removal of radioactive wastes from nuclear power plants. The fuel cycle is represented with a detailed description of radioactive wastes and the typical annual amounts are given of wastes involved in the operation of a $1000 \mathrm{MWe}$ light water reactor. These include wastes from the mining and processing of uranium ores, from uranium treatment and fuel production, from the operation of nuclear power plants, from the storage and reprocessing of spent fuel and from the decommissioning of nuclear installations after shutdown. Also discussed are the possibilities of the safe storage of radioactive wastes and it is stated that the only feasible solution for Czechoslo- 
vakia is their burial several metres underground with adequate insulation against water and covered with an earth layer. High level radioactive wastes are planned to be deposited in deep geological formations. The future trend in this field is indicated. (B.S.).

657 (INIS-mf-7256) Investigations of salt domes for the purpose of high-level radioactive waste disposal. (Akademiet for de Tekniske Videnskaber, Lyngby (Denmark)). 1982. 110p. (In Danish). NTIS (US Sales Only), PC A06/MF A01. Order Number DE83780285.

Separate papers are indexed separately.

658 (INIS-mf-7256, pp 110) Geology of the Mors salt dome. Larsen, G. (Geologisk Institut, Aarhus Universitet, Denmark: 1982. (In Danish). NTIS (US Sales Only), PC A06/MF A01.

In Investigations of salt domes for the purpose of high-level radioactive waste disposal.

Stratigraphic survey of European salt deposits is given in general terms and Zcchstein formation at Mors salthorst is analysed in detail. Seismic measurements and borings were used to investigate Mors internal structure.

659 (INIS-mf-7256, pp 110) Hydrogeological conditions at Mors salt dome. Joshi, A.V. (Jysk-Fynske Elsamarbejde (ELSAM), Fredericia (Denmark)). 1982. (In Danish). NTIS (US Sales Only), PC A06/MF A01.

In Investigations of salt domes for the purpose of high-level radioactive waste disposal.

The cover layer of Mors salthorst gives good protection of salt layers. Ground water flow is small and retention properties of lime layers for transuranium wastes are good. Water velocity on $500 \mathrm{~m}$ depth is so low that only the immediate vicinity of the disposal room can be involved, therefore limited hydrological investigations are sufficient to evaluate the safety of radioactive waste disposal in the salthorst.

660 (INIS-mf-7256, pp 110) Technology of systems. Hasted, F. (ELKRAFT A.m.b.A., Hellerup (Denmark)). 1982. (In Danish). NTIS (US Sales Only), PC A06/MF A01.

In Investigations of salt domes for the purpose of high-level radioactive waste disposal.

High-radioactive waste is to be delivered to salt deposits in form of special steel containers. The disposal facility will have capacity of about 5000 such containers. Various types of shafts with horizontal tunnels and vertical storage facilities are considered. Deep-hole solution seems to be considerably cheaper than shaft-mine storage facility.

661 (INIS-mf-7256, pp 110) Evaluation of waste disposal safety. Pedersen, A. (Jysk-Fynske Elsamarbejde (ELSAM), Fredericia (Denmark)). 1982. (In Danish). NTIS (US Sales Only), PC A06/MF A01.

In Investigations of salt domes for the purpose of high-level radioactive waste disposal.

Requirements of the Environmental Agency for high-radioactive waste disposal are, that natural transport mechanisms must not contribute to biospheric pollution, that violent natural phenomena cannot release any biospheric pollution and that human activities will be very limited and health hazard insignificant compared to other man-created risks. Investigation of radioaactive waste disposal in Mors salt deposits has proved these requirements to be satisfied.

662 (INIS-mf-7416) Numerical calculation of threedimensional long-time temperature fields using a special finite element method: the final storage of highly radioactive waste in salt rocks. Ploumen, P. (Technische Hochschule Aachen (Germany, F.R.). Fakultaet fuer Maschinenwesen). 12 Jun 1980. 148p. (In German). NTIS (US Sales Only), PC A07/MF A01. Order Number DE83780482.

Thesis.

In the present case the spatial discretisation shows some differences compared with the current Galerkin method. The time discretisation is accomplished by the trapezoid rule. The systems of equations are linearized by the Newton-Raphson method and solved by iteration using the procedure of successive overrelaxation. The realiability of the numerical results has been tested for the present application of heating of salt rocks by radioactive thermal sources.

663 (INIS-mf-7475-Vol.A, pp vp) Considerations on the management of wastes expected to be generated by the operation of Brazilian nuclear reactors. Hamacher, M.E.; de Oliveira, L.F.S. (Rio de Janeiro Univ. (Brazil). Coordenacao dos Programas de Pos-graduacao de Engenharia). 1981. (In Portuguese). NTIS (US Sales Only), PC A19/MF A01. (CONF-8104136Vol.A). 1981).

From 2. Brazilian energy congress; Rio de Janeiro, Brazil (6 Apr

Some parameters which are essential to the formulation of a correct management strategy of radioactive waste in Brazil are evaluated. Among the most significant results are: a) the annual and cumulative inventory of high level products up to the year 2000 , and b) an estimate of the dates when it will be necessary to remove spent fuel from the plant sites. The results were obtained by assuming two distinct nuclear power plant introduction scenarios in Brazil.

664 (INIS-mf-7498, pp vp) Radioactive waste disposal options for the operation of nuclear power stations. Boger, $Z$. (Israel Atomic Energy Commission, Beersheba. Nuclear Research Center-Negev). 1982. NTIS (US Sales Only), PC A12/MF A01. (CONF-820268-Vol.9).

From Nuclear societies of Israel joint annual meeting; Haifa, Israel (16 Feb 1982).

FUEL CYCLE; HIGH-LEVEL RADIOACTIVE WASTES; ISRAEL; LOW-LEVEL RADIOACTIVE WASTES; NUCLEAR POWER PLANTS; RADIOACTIVE WASTE DISPOSAL; RADIO ACTIVE WASTE STORAGE

665 (INIS-mf-8525) Underground disposal of high active waste. Engelmann, H.J. (Kernforschungszentrum Karlsruhe G.m.b.H. (Germany, F.R.). Schule fuer Kerntechnik). 1982. 13p. (CONF-8209134-19). NTIS (US Sales Only), PC A02/MF A01. Order Number DE83703513.

From IAEA interregional training course on waste management in nuclear facilities; Karlsrube, F.R. Germany (6 Sep 1982).

This paper is concerned with the engineering aspects relating to the deep burial of high active waste in stable geological formations. The design of a repository depends upon a number of factors not least of which is the type of rock in which it is to be constructed. High level wastes must be isolated from man's environment for such periods that subsequent release will not result in an unacceptable hazard to human population. Design aspects of repositories are reviewed and conceptual designs are present in relation to the geological formations under consideration. Over long time periods the most probable mode of release of radionuclides is through groundwater contacting the waste. The proposed concepts therefore include the use of engineered and natural barriers to delay the eventual release of waterborne radionuclides into mans environment. In all cases the ultimate barrier will be the geological formation. Nevertheless, depending upon the type of host rock, use will be made of various additional engineered barriers to delay water contacting the high level waste for several hundreds of years. During this time the level of radiation and associated heat emitted by the waste, will fall by several orders of magnitude and the rock temperatures within a repository will be returning to ambient. Thereafter the residual activity will mainly arise from the actinides. Containment may be enhanced by surrounding the canisters with materials having high sorption capabilities for many of the radionuclides involved. The depth at which a repository is excavated must be sufficient to ensure that the overburden will withstand changes in environmental conditions. The depth of cover required in different rock types may vary. In clay excavating at depth of up to $-250 \mathrm{~m}$ appears feasible, while in hard rocks and salts working at depth of up to $-1000 \mathrm{~m}$ is entirely practicable. (orig./RW).

666 (JAERI-M-82-007) Corrosion resistance of metal materials for HLW canister. Furuya, T.; Muraoka, S.; Tashiro, S. (Japan Atomic Energy Research Inst., Tokyo). Feb 1982. 35p. (In Japanese). NTIS (US Sales Only), PC A03/MF A01. Order Number DE83702436.

In order to verify the materials as an important artificial barrier for canister of vitrified high-level waste from spent fuel reprocessing. data and reports were researched on corrosion resistance of the materials under conditions from glass form production to final disposal. Then, 
in this report, investigated subjects, improvement methods and future subjects are reviewed. It has become clear that there would be no problem on the inside and outside corrosion of the canister during glass production, but long term corrosion and radiation effect tests and the vitrification methods would be subjects in future on interim storage and final disposal conditions.

667 (JAERI-M-82-061) Study of $\gamma$-ray irradiation effects on corrosion resistance of alloys for storage of high-level waste packages, (1). Furuya, T.; Muraoka, S.; Tashiro, S.; Araki, K.; Tomari, H.; Fujiwara, K.; Fukuzuka, T. (Japan Atomic Energy Research Inst., Tokyo). Jun 1982. 32p. (In Japanese). NTIS (US Sales Only), PC A03/MF A01. Order Number DE83702400.

The effects of $\boldsymbol{\gamma}$-ray irradiation on corrosion resistance have been studied about candidate alloys as high-level waste canisters, overpacks and storage fasility materials. The purpose of this study is to evaluate integrity of alloys in the view of safety evaluation for interim storage systems such as water and air cooling system. Stress corrosion cracking (SCC) test has been carried out and the double U-bend type specimens were used. The alloys tested were Type 304 ss, Type 304L ss, Type $304 E L$ ss, Type 309s ss, Incoloy 825, Inconel 600, Inconel 625 and SMA 50. Sensitized Type 304 ss, Type 304L ss and Type 309s ss have been found to be susceptible to SCC because of radiolysis in boiling deionized water with $\gamma$-ray irradiation but not without irradiation. It was also confirmed that they were susceptible to SCC under the existence of a little chloride ion and dissolved oxygen even without $\gamma$-ray irradiation. Fractographic observation revealed that the cracking mode was completely intergranular. The other alloys were not susceptible to SCC, but SMA 50 rusted over its surface. On the other hand, no alloy was susceptible to SCC in atmosphere at room temperature even with $\gamma$-ray irradiation. This study was carried out in corporation at JAERI and Kobe Steel Ltd.

668 (Juel-Conf-24, pp 17-26) Underground storage of radioactive wastes in salt deposits in the Federal Republic of Germany. Albrecht, E. Mar 1981. (In German). NTIS (US Sales Only), PC A04/MF A01.

In Present topics of nuclear energy.

It can be assumed that by the time the federal final storage facility for radioactive wastes will be installed, reliable and acceptable solutions will also be available for the storage of high-level radioactive waste. Today there are already safe and reliable technologies for the storage of low-level and medium-level radioactive waste. As in any young technology, also in nuclear engineering and especially in the last link of the nuclear fuel cycle - the development is pushed forward with vigour, in order to Improve and optimize again the solutions, in particular with regard to safety.

669 (Juel-Conf-42-Vol.2, pp 767-783) Concept of final storage in geological formations. Roethemeyer, $\mathrm{H}$. (Physikalisch-Technische Bundesanstalt, Braunschweig, Germany). Jun 1981. (In German). NTIS (US Sales Only), PC A21/MF An1. (CoNF-81ubsu-vol.'2).

From International seminar on chemistry and process engineering for high-level waste solidification; Julich, F.R. Germany (1 Jun 1981).

RADIOACTIVE WASTE DISPOSAL: HIGH-LEVEL RADIOACTIVE WASTES; UNDERGROUND DISPOSAL; GEOLOGIC DEPOSITS

670 (Juel-Conf-42-Vol.2, pp 784-791) Backfill barriers for nuclear waste repositories in salt. Nowak, E.J. (Sandia National Labs., Albuquerque, NM). Jun 1981. NTIS (US Sales Only), PC A21/MF A01. (CONF-810650-Vol.2).

From International seminar on chemistry and process engineering for high-level waste solidification; Julich, F.R. Germany (1 Jun 1981).

Backfill materials were evaluated for containment of radionuclides, chemical modification of brine, and sensitivity to hydrothermal conditions. Experimental conditions were relevant to nuclear waste isolation in bedded salt. They were based on geolngic conditions at the site of the Waste Isolation Pilot Plant (WIPP) in southeastern New Mexico, USA. Conclusions are: backfill mixtures surrounding the waste form and canister can provide a neutral or slightly acidic, potentially reducing environment, prevent convective aqueous flow, and act as an effective radionuclide migration barrier; bentonite is likely to remain hydrother- mally stable but potentially sensitive to waste package interactions which could alter the $\mathrm{pH}$, the ratio of dissolved ions, or the sorption properties of radionuclide species; effects of irradiation from high level waste should be investigated.

671 (Juel-Conf-42-Vol.2, pp 792-815) Requirements for the disposal of high-level radioactive wastes. Warnecke, E.; Illi, H.; Ehrlich, D. (Physikalisch-Technische Bundesanstalt, Germany). Jun 1981. NTIS (US Sales Only), PC A21/MF A01. (CONF-810650-Vol.2).

From International seminar on chemistry and process engineering for high-level waste solidification; Julich, F.R. Germany (1 Jun 1981).

High-level radioactive wastes in the Federal Republic of Germany will be disposed of in salt deposits. Feasibility studies are presently being conducted in the salt dome at Gorleben. First results have already been obtained. Existing plans depend on model assumptions and there is a need to adapt the final layout of the repository to the geological situation at the end of the investigations. As the requirements for disposal of high-level radioactive wastes are dependent on the geological situation, it is necessary to have a realistic data base for the formulation of final requirements with a safety analysis. The following requirements are discussed: requirements for waste characterization; and requirements for disposal of high-level radioactive wastes and comparison with first rcsults of model calculatiuns. The latter takes into account direct radiation and air-borne releases, water-borne radionuclide releases, thermal aspects, and quality control.

672

(Juel-Conf-42-Vol.2, pṕ 816-828) Composition of rocks - criteria for the conditioning of high-level radioactive wastes. Herrmann, A.G. (Goettingen Univ., Germany). Jun 1981. (In German). NTIS (US Sales Only), PC A21/MF A01. (CONF-810650-Vol.2).

From International seminar on chemistry and process engineering for high-level waste solidification; Julich, F.R. Germany (1 Jun 1981).

Any discussion of safe burial of radwaste in rocks returns to the question of likely geochemical, mineralogical, and geological developments in the rock during the next $10^{0}$ to $10^{6}$ years. A scientifically well founded answer to this question will take account of the processes that combine to produce and to alter rocks and will offer more than a recital of stratigraphic data on the rock sequence. Every effort should be made to limit deformations and mineral reactions of the rocks to a possible minimum. The intervals for reprocessed waste and/ar fuel elements and the fission-product concentration in the radwaste canisters must be controlled by the limits for deformations and mineral reactions of the rocks.

673 (Juel-Conf-42-Vol.2, pp 844-872) Effects of product properties on the final salt storage medium and the conditions producing it. Gies, H.; Rothfuchs, T. (Gesellschaft fuer Strahlen= und Unweltfurschung mbH, Muenchen, Germany). Jun 1981. (In German). NTIS (US Sales Only), PC A21/MF A01. (CONF-810650-Vol.2).

From International seminar on chemistry and process engineering for high-level waste solidification; Julich, F.R. Germany (1 Jun 1981).

In the final storage of bigh-level radioactive waster. product properties occur especially heat production and radiation. In the following, the previously known or theoretical effects on the salt storage medium will be indicated. The conditions thereby resulting determine possibility and limitations for an optimium utilization of the storage medium as a geologic barrier.

674 (Juel-Conf-42-Vol.2, pp 873-890) Evaluation of barriers in a final storage site for radioactive wastes. Memmert, $G$. (Technische Univ., Berlin, Germany). Jun 1981. NTIS (US Sales Only), PC A21/MF A01. (CONF-810650-Vol.2).

From International seminar on chemistry and process engineering for high-level wastc solidification; Julich, F.R. Germany (1 Jun 1981).

RADIOACTIVE WASTE DISPOSAL; HIGH-LEVEL RADIOACTIVE WASTES; GEOLOGIC DEPOSITS; ENGINEERED SAFETY SYSTEMS; UNDERGROUND DISPOSAL; EVALUATION 
¿75 (Juel-Conf-42-Vol.2, pp 891-916) Canister materials roposed for final disposal of high level nuclear waste - a review ith respect to corrosion resistance. Mattsson, E. (Swedish Corrusion Inst., Stockholm). Jun 1981. NTIS (US Sales Only), PC A21/MF A01. (CONF-810650-Vol.2).

From International seminar on chemistry and process engineering for high-level waste solidification; Julich, F.R. Germany (1 Jun 1981).

Spent fuel from nuclear reactors bas to be disposed of either after reprocessing or without such treatment. Due to toxic radiation the nuclear waste has to be isolated from the biosphere for 300-1000 years, or in extreme cases for more than 100,000 years. The nuclear waste will be enclosed in corrosion resistant canisters. These will be deposited in repositories in geological formations, such as granite, basalt, clay, bedded or domed salt, or the sediments beneath the deep ocean floor. There the canisters will be exposed to groundwater, brine or seawater at an elevated temperature. Species formed by radiolysis may affect the corrosivity of the agent. The corrosion resistance of candidate canister materials is evaluated by corrosion tests and by thermodynamic and mass transport calculations. Examination of ancient metal objects after long exposure in nature may give additional information. On the basis of the work carried out so far, the principal candidate canister materials are titanium materials, copper and high purity alumina.

676 (Juel-Conf-42-Vol.2, pp 944-977) Corrosion of materials in a clay environment. Casteels, F. (S.C.K./C.E.N., Mol, Belgium); Tas, H.; Brabers, M.; Heremans, R. Jun 1981. NTIS (US Sales Only), PC A21/MF A01. (CONF-810650Vol.2).

From International seminar on chemistry and process engineering for high-level waste solidification; Julich, F.R. Germany (1 Jun 1981).

The candidate canister materials to be used for the storage of low, medium and high activity should be compatible with the different possible environments. These are direct contact with clay, interstitial clay water, humid atmospheres loaded with corrosive products and ground water. The corrosion rates and mechanisms of candidate canister materials have been studied after tests carried out in direct contact with clay, in synthetic clay atmospheres, in interstitial clay water, in ground water and in situ in clay formations. Corrosion tests have been carried out at $25^{\circ} \mathrm{C}, 50^{\circ} \mathrm{C}, 100^{\circ} \mathrm{C}, 150^{\circ} \mathrm{C}, 200^{\circ} \mathrm{C}$ and $300^{\circ} \mathrm{C}$ on the following materials: carbon and chromized steel, different grades of austenitic and ferritic stainless steels, nickel and super alloys, aluminiuum and its alloys, copper and copper alloys, $T i$ and its alloys. The alloys were tested in the annealed and heat-treated condition (determined by the conditioning process). Welded and stressed specimens were also inserted in the tests. The metallographic techniques have been used for the determination of the extent of localized attack. The selection of the most promising candidate canister materials has been based on their resistance to the localized forms of attack and stress corrosion cracking susceptibility it the different possible environmonto. Ti and its alloys, Hastelloy C, UHB $904 \mathrm{~L}$ and Inconel 625 possess the highest corrosion resistance.

677 (Juel-Conf-42-Vol.2, pp 978-1002) Actinide sorption on rock minerals. Allard, B.; Andersson, K.; Rydberg, J. (Chalmers Univ. of Tech., Gothenburg, Sweden). Jun 1981. NTIS (US Sales Only), PC A21/MF A01. (CONF-810650-Vol.2).

From International seminar on chemistry and process engineering for high-level waste solidification; Julich, F.R. Germany (1 Jun 1981).

Sorption studies have been carried out with about 20 fission elements and actinides on over 40 rock types and pure minerals with a variation of parameters such as: contact time, temperature, ground water $\mathrm{pH}$ and $\mathrm{Eh}$, concentration of complex formers, salt content, nuclide concentration, rock composition, mineral composition, and grain size. Conclusions are: (1) distribution coefficient measurements are essential for predicting radionuclide movements in an underground waste repository percolated by ground water; (2) the $K_{d}$-values show little temperature and particle size dependence (in the investigated range), but increase rastically with time: (3) the $\mathrm{K}_{d}$-values for actinides highly depend on roundwater $\mathrm{pH}$ and redox conditions; (4) all actinides will mainly be ..t the tri- and tetravalent oxidation state in typical Swedish deep bedrock groundwater; (5) for most minerals in Swedish bedrock, the $K_{d}$-values for the actinides will exceed 1 , i.e. show retention factors $\geq$ $10^{5}$; (6) several different sorption mechanisms can be identified, among them are ion exchange (e.g. on clay), adsorption (e.g. on quartz), chemisorption (e.g. on apatite), and precipitation (e.g. of hydroxides); and (7) $K_{d}+$ values will be specific for any selected site.

678 (Juel-Conf-42(Vol.2), pp 603-620) Criteria for high level waste disposal and characterization of barriers. Pottier, $\mathbf{P}$.; Sousselier, Y. Jun 1981. NTIS (US Sales Only), PC A21/MF A01. (CONF-810650-Vol.2).

From International seminar on chemistry and process engineering for high-level waste solidification; Julich, F.R. Germany (1 Jun 1981).

It is essential to characterize the four different barriers and their behaviour in normal and accident condition for ultimate disposal of HLW. For the characterization the normal parametric characterization and the characterization in the reference condition of a disposal site can be used. The four barriers are the unsolubilization matrix (waste form), the packing, the geochemical barrier and the host rock.

679 (Juel-Conf-42(Vol.2), pp 767-782) Concepts for the ultimate storage in geological formations. Roethemeyer, $\mathrm{H}$. Jun 1981. (In German). NTIS (US Sales Only), PC A21/MF A01. (CONF-810650-Vol.2).

From International seminar on chemistry and process engineering for high-level waste solidification; Julich, F.R. Germany (1 Jun 1981).

The protection aims for the ultimate storage have been presented quantitatively and qualitatively by national laws and regulations. The preliminary fundamentals layout data of ultimate storage mines for West-European countries and USA/Kanada are compared. The preliminary thermal layout data for ultimate storages in various host rocks concerning the USA are recorded.

680 (Juel-Conf-42(Vol.2), pp 785-791) Backfill barriers for nuclear waste repositories in salt. Nowak, E.J. Jun 1981. NTIS (US Sales Only), PC A21/MF A01. (CONF-810650Vol.2).

From International seminar on chemistry and process engineering for high-level waste solidification; Julich, F.R. Germany ( 1 Jun 1981).

Backfill mixtures surrounding the waste form and canister can provide a neutral or slightly acidic, potentially reducing environment, prevent convective aqueous flow, and act as an effective radionuclide migration barrier. Bentonite is likely to remain hydrothermally stable but potentially sensitive to waste package interactions which could alter the $\mathrm{pH}$, the ratio of dissolved wires, or the sorption properties of radionuclide species.

681 (Juel-Conf-42(Vol.2), pp 792-815) Requirements for the disposal of high-level radioactive wastes. Warnecke, E.; Illi, H.; Ehrlich, D. Jun 1981. NTIS (US Sales Only), PC A21/MF A01. (CONF-810650-Vol.2).

From International seminar on chemistry and process engineering for high-level waste solidification; Jullch, F.R. Germany (1 Jun 1981).

In the FRG it is planned to dispose HLW in the salt dome in Gorleben. As the requirements for the disposal are dependent on the geological situation, it is necessary to have a realistic data base for the formulation of final requirements with a safety analysis. Air-borne and waterborne radionuclide releases, thermal aspects, and quality control are some requirements which must be considered.

682 (Juel-Conf-42(Vol.2), pp 816-828) Composition of rocks - criteria for the conditioning of high-level radioactive wastes. Herrmann, A.G. Jun 1981. (In German). NTIS (US Sales Only), PC A21/MF A01. (CONF-810650-Vol.2).

From International seminar on chemistry and process engineering for high-level waste solidification; Julich, F.R. Germany (1 Jun 1981).

Any discussion of safe burial of radwaste in rocks returns to the question of likely geochemical, mineralogical, and geological developments in the rock during the next $10^{0}$ to $10^{6}$ years. A scientifically well founded answer to this question will take account of the prucesses that combine to produce and to alter rocks and will offer more than a recital of stratigraphic data on the rock sequence. Every effort should be made to limit deformations and mineral reactions of the rocks to a possible minimum. The intervals for reprocessing waste and/or fuel elements 
and the fission-product concentration in the radwaste canisters must be controlled by the limits for deformations and mineral reactions of the rocks.

683 (Juel-Conf-42(Vol.2), pp 844-872) Effects of the product properties upon the ultimate storage medium salt and resulting conditions. Gies, H.; Rothfuchs, T. Jun 1981. (In German). NTIS (US Sales Only), PC A21/MF A01. (CONF810650-Vol.2).

From International seminar on chemistry and process engineering for high-level waste solidification; Julich, F.R. Germany (1 Jun 1981).

During the storage of HAW in the Na2, e.g. the Stassfurtmineral salt of the Zechstein, the thermal release of water is of importance only if the hanging parts of the $\mathrm{Na} 2$ were heated up with polykalite and kieserite up to the releasing temperatures beginning at about $200^{\circ} \mathrm{C}$. The mechanical effects in the ultimate storage area lead to higher creep rates. The release of radionuclides as a result of container corrosion can thus he limited.

684 (Juel-Conf-42(Vol.2), pp 873-890) Evaluation of the barriers in an ultimate storage for radioactive waste. Memmert, G. Jun 1981. (In German). NTIS (US Sales Only), PC A21/MF A01. (CONF-810650-Vol.2)

From International seminar on chemistry and process engineering for high-level waste solidification; Julich, F.R. Germany (1 Jun 1981).

By means of a postulated intrusion of water in the mine building the reliability of individual and all barriers together is estimated. The shielding by the overlying rock seems to be extremely good for all shortlife isotopes. The future works on the barrier system in the ultimate storage should therefore more concentrate on the increase of the barrier effect for the long-life isotopes. Especially the chemical processes between the compounds of the long-life isotopes dissolved in the groundwater or alkaline solutions and the substances for filling of routes and pits have to be studied.

686 (Juel-Conf-42(Vol.2), pp 891-916) Canister materials proposed for final disposal of high level nuclear waste. Mattson, E. Jun 1981. NTIS (US Sales Only), PC A21/MF A01. (CONF-810650-Vol.2).

From International seminar on chemistry and process engineering for high-level waste solidification; Julich, F.R. Germany (1 Jun 1981).

The nuclear waste will be enclosed in corrosion resistant canisters. These will be deposited in repositories in geological formations, such as granite, basalt, clay, bedded or domed salt, or the sediments beneath the deep ocean floor. There the canisters will be exposed to groundwater, brine or seawater at an elevated temperature. Species formed by radiolysis may effect the corrosivity of the agent. The corrosion resistance of candidate canister materials is evaluated by corrosion tests and by thermodynamic and mass transport calculations. Examinations of ancient metal objects after long exposure in nature may give additional information. On the basis of the work carried out so far, the principal candidate canister materials are titanium materials, copper, and highpurity alumina.

686 (Juel-Conf-42(Vol.2). pp 944.977) Corrosion of materials in a clay environment. Casteels, F.; Tas, H.; Heremans, R.; Brabers, M. Jun 1981. NTIS (US Sales Only), PC A21/MF A01. (CONF-810650-Vol.2).

From International seminar on chemistry and process engineering for high-level waste solidification; Julich, F.R. Germany (1 Jun 1981).

Corrosion tests have been carried out at $25^{\circ} \mathrm{C}, 50^{\circ} \mathrm{C}, 100^{\circ} \mathrm{C}$, $150^{\circ} \mathrm{C}, 200^{\circ} \mathrm{C}$ and $300^{\circ} \mathrm{C}$ on the following materials: carbon and chromized steel, different grades of austenitic and ferritic stainless steels, nickel and super alloys, aluminium and its alloys, copper and copper alloys, $\mathrm{Ti}$ and its alloys. The alloys were tested in the annealed and heattreated condition (determined by the conditioning process). Welded and stressed specimens were also inserted in the tests. The metallographic techniques have been used for the determination of the extent of localized attack. The selection of the most promising candidate canister materials has been based on their resistance to the localized forms of attack and stress corrosion cracking susceptibility in the different possi- ble environments. Ti and its alloys, Hastelloy C, UHB 904L and Inconel 625 possesses the highest corrosion resistance.

687 (KFK-3221) Decay calculations on medium-level and actinide-containing wastes from the $\mathrm{LWR}$ fuel cycle. Pt. 1 . Basic data evaluation including activity and thermal decay power. Haug, H.O. (Kernforschungszentrum Karlsruhe G.m.b.H. (Germany, F.R.). Inst. fuer Heisse Chemie; Kernforschungszentrum Karlsruhe G.m.b.H. (Germany, F.R.). Projekt Wiederaufarbeitung und Abfallbehandlung). Oct 1981. 72p. (In German). NTIS (US Sales Only), PC A04/MF A01. Order Number DE82750338.

A number of basic data on medium-level and actinide-containing waste streams from the LWR fuel cycle were evaluated and the activity and thermal decay power were calculated for the nuclide inventories of cladding hulls and fuel assembly structural materials, for feed clarification sludge, medium-level aqueous process waste, low-level solid transuranium waste and for medium-level reactor operating waste. The activity as a function of decay time of the medium-level wastcs dccreases within 500 to 600 years by 1 to 3 orders of magnitude and is at the same time about 1 to 2 ofderis of magnilude lower than the activity of the highlevel waste. The thermal decay power of the medium-level wastes decreases after 10 to 100 years by about 3 orders of magnitude and is about a factor of 10 to 100 less than that of high-level waste. In the very long term the residual activity (and thermal power) decreases only slowly due to the long halflives of the dominant actinides. The activity after more that 1000 years is about 1 to 2 orders of magnitude lower than that of high-level waste, the low-level transuranium waste by a factor 10 to 4 , respectively. The activity per unit volume of the packaged waste of the medium-level and actinide-containing wastes because of the bigger volume of the conditioned wastes is lower by 2 to 4 orders of magnitude up to about 500 years. After more than 1000 years the activities per unit volume are lower by a factor of 20 to 200 than that of high-level waste.

688 (KFK-3222) Decay calculations on medium-level and actinide-containing wastes from the LWR fuel cycle. Pt. 2. Haug, H.O. (Kernforschungszentrum Karlsruhe G.m.b.H. (Germany, F.R.)). Dec 1981. 101p. (In German). NTIS (US Sales Only), MF A01. Order Number DE82750564.

1. The radiotoxicity index as inherent property of the radionuclide inventory was calculated for medium-lcvel and actinide-containing wastes. The calculations wcre based on the annual limits of intake of the German Radiation Protection Ordinance as wcll as the new values of annual limits of intake from ICRP-30. The latter imply a higher rating of the toxicity of Iransuranium nuclides and a lower rating of Sr-90, Tc-99, and $\mathrm{Ra}-226$. Thus, the annual radiotoxicity index is controlled by the transuranics after 10 to 100 years. 2. From the comparison of the radiotoxicity index of conditional and packed wastes with the same volume of uranium ore, it was evaluated that the relative radiotoxicity of the medium-level wastes decreases below the level of pitchblende after less than 100 years and below a $3 \%$ uranium ore after less than 2000 of decay. However, based on ICRP-30, the relative radiotoxicity index decreases below the level of pitchblende after 1000 years and decays to the level of the $3 \%$ uranium ore at about $10^{5}$ years. 3. The comparison of the radiotoxicity concentration of the total disposal layer with a uranium nre deposit shows that the radiotoxicity concentration based on ICRP-30 of the self-heating wastes placed in single boreholes decays within 2000 years (high level waste within 3000 years) below the level of a uranium ore deposit of $0.2 \%$ uranium. The radiotoxicity concentration of the medium-level proccss waste and the alpha-waste disposed off in disposal chambers decreases to the level of a uranium ore deposit with 0.4 to $6 \%$ uranium after about $10^{4}$ years, and $1 \%$ after about $10^{5}$ years.

689 (KFK-3230) Corrosion studies and mechanical tests on metallic materials for the design of packagings for vitrified high level wastes (HLW). Stichel, W.; Smailos, E.; Koester, R. (Kernforschungszentrum Karlsruhe G.m.b.H. (Germany, F.R.). Inst. fuer Nukleare Entsorgungstechnik). Dec 1981. 72p. (In German). NTIS (US Sales Only), PC A04/MF A01. Order Number DE82750586.

Selective corrosion studies and mechanical tests were performec on various metallic materials in order to find out appropriate materials for the design of HLW packagings serving as a barrier in the repository. Besides the $\mathrm{Cr}-\mathrm{Ni}$ steels to be used as the canister material according to the previous concept, three nickel-base alloys were investigated as 
well as a mild steel and the materials Ti 99.8-Pd and the steel $\mathrm{X} 1$ CrMoTi 182 (ELA-ferrite). Since within the framework of accident studies for a repository in a rock salt, salt solutions are being considered as a potential corrosion medium, the corrosion tests were made under selecting conditions in a quinary salt solution at $170^{\circ} \mathrm{C}$ and 1 bar. The mechanical tests have shown that the nickel-base alloys and most of the $\mathrm{Cr}-\mathrm{Ni}$ steels undergo slight changes of their mechanical properties as a result of welding and annealing treatment, respectively. This provided the evidence for the suitability of these materials as canister materials. Owing to their high susceptibility to local corrosion attacks, above all to stress corrosion, $\mathrm{Cr}$-Ni steels cannot take over the function of a barrier against brines. The materials Inconel 625, Incoloy 825, ELA ferrite and Ti-Pd resisted stress corrosion, but these materials are susceptible to pitting corrosion. High resistance to local corrosion attacks was exhibited by the materials Hastelloy $\mathrm{C} 4$ and mild steel. After a period of testing of $\mathbf{4 3 2}$ days Hastelloy $C 4$ showed a uniform corrosion rate of less than $0.03 \mathrm{~mm} / \mathrm{a}$. The corrosion rate for mild steel 1.0566 was less than 0.25 $\mathrm{mm} / \mathrm{a}$ in case of the initial material and less than $0.5 \mathrm{~mm} / \mathrm{a}$ in case of the annealed material.

690 (KFK-3411) Analysis of the growth of brine flow pathes in rock salt. Kienzler, B.; Korthaus, E. (Kernforschungszentrum Karlsruhe G.m.b.H. (Germany, F.R.). Inst. fuer Nukleare Entsorgungstechnik). Oct 1982. 49p. (In German). NTIS (US Sales Only), PC A03/MF A01. Order Number DE83750659.

In this paper a complemented and extended version of a mathematical model is presented, which was developed in the USA, to calculate the course of the enlargement of a fissure in rock salt by solution during water inflow. The calculated results are verified by laboratory experiments and measured values obtained after drowning of the Ronnenberg salt mine. In both cases a good agreement between calculations and experimental data was achieved. Application of the model to possible drowning of a HLW borehole and a mine were performed with different boundary conditions. The resulting diameter of a fissure is dependent on the inflowing volume of water. With the assumptions mentioned, HLW boreholes are drowned within a few hours, mines within a few years. In these calculations only dissolution processes were considered. Opposite effects like closure of fissures or the influence of $\mathrm{MgCl}_{2}$ containing brines on the dissolution of rock salt have not been considered up to now. Thus, the results cannot be transferred directly to the safety analysis of ultimate radioactive wastes disposal in real saltdomes.

691 (KFK-3438) Corrosion studies on non-metallic inorganic materials for HLW packaging design. Storch, W.; Engelke, H.; Smailos, E. (Kernforschungszentrum Karlsruhe G.m.b.H. (Germany, F.R.). Inst. fuer Nukleare Entsorgungstechnik). Feb 1983. 54p. (In German). NTIS (US Sales Only), PC A04/MF A01. Order Number DE83751275.

Within the framework of our studies to find out suitable materials for developing HLW packaging with a barrier function in a salt repository the corrosion hehavinr was investigated of non-metallic inorganic materials under specified hypothetical accident conditions. Moreover glass solders to be used as connecting materials between canister and lid as an alternative closing technique have been tested for their corrosion behavior. The corrosion studies on ceramic materials and glass solders are performed in a quinary salt brine (Q-brine) at temperatures between 80 and $200^{\circ} \mathrm{C}$ and a pressure of 130 bar. The investigations have confirmed that some materials show a high corrosion resistance. The highest corrosion resistance was exhibited by the $\mathrm{Al}_{2} \mathrm{O}_{0}$ material. It decisively depends on the purity, the structure and the surface finish of the material. The glasses investigated at $200^{\circ} \mathrm{C}$ showed that the chemically resistant glass $8330\left(19 \mu \mathrm{m} / \mathrm{a}\right.$ at $\left.200^{\circ} \mathrm{C}\right)$ and an $\mathrm{HLW}$ model glass with adapted dilatation coefficient $(86 \mu \mathrm{m} / \mathrm{a})$ exhibit a good resistance to corrosion. The results obtained so far have shown that development of HLW packaging with a barrier function in the repository can be generally achieved with the use of ceramic materials.

692 (LA-9095-PR) Research and development related to the Nevada Nuclear Waste Storage Investigations. Progress report, July 1-September 30, 1981. Daniels, W.R.; Wolfsherg, K.; Vaniman, D.T.; Erdal, B.R. (comps.). (Los Alamos National Lab., NM (USA)). Jan 1982. Contract W-7405-ENG-36. 84p. NTIS, PC A05/MF A01. Order Number DE82015029.

This report summarizes the contribution of the Los Alamos National Laboratory to the Nevada Nuclear Waste Storage Investiga- tions for the fourth quarter of FY-81. Progress reports are presented for the following tasks: waste package development; nuclide migration experiments in $\mathbf{G}$ tunnel-laboratory studies; geochemistry of tuff; mineralogy-petrology of tuff; volcanism studies; rock physics studies; exploratory shaft; and quality assurance.

693 (LA-9174-PR) Effects of long-term exposure of tuffs to high-level nuclear waste-repository conditions. Preliminary report. Blacic, J.; Carter, J.; Halleck, P.; Johnson, P.; Shankland, T.; Andersen, R.; Spicochi, K.; Heller, A. (Los Alamos National Lab., NM (USA)). Feb 1982. Contract W-7405-ENG36. 30p. NTIS, PC A03/MF A01. Order Number DE82009549.

Tests have been performed to explore the effects of extended exposure of tuffs from the southwestern portion of the Nevada Test Site to temperatures and pressures similar to those that will be encountered in a high-level nuclear waste repository. Tuff samples ranging from highly welded, nonzeolitized to unwelded, highly zeolitized varieties were subjected to temperatures of 80,120 , and $180^{\circ} \mathrm{C}$; confining pressures of 9.7 and $19.7 \mathrm{MPa}$; and water-pore pressures of 0.5 to $19.7 \mathrm{MPa}$ for durations of 2 to 6 months. The following basic properties were measured before and after exposure and compared: tensile strength, uniaxial compressive strength, grain density, porosity, mineralogy, permeability, thermal expansion, and thermal conductivity. Depending on rock type and exposure conditions, significant changes in ambient tensile strength, compressive strength, grain density, and porosity were measured. Mineralogic examination, permeability, and thermal property measurements remain to be completed.

694 (LA-9179-MS) Nevada Nuclear Waste Storage Investigations: exploratory shaft. Phase I. Conceptual design report. Nelson, D.C.; Merson, T.J.; McGuire, P.L.; Sibbitt, W.L. (Los Alamos National Lab., NM (USA)). Jun 1982. Contract W-7405-ENG-36. 230p. NTIS, PC Al1/MF A01. Order Number DE82019496.

Portions of document are illegible.

It is proposed that an Exploratory Shaft (ES) be constructed in Yucca Mountain on or near the southwest portion of the Nevada Test Site (NTS) as part of the Nevada Nuclear Waste Storage Investigations. This document describes a conceptual design for an ES and a cost estimate based on a set of construction assumptions. Included in this document are appendixes consisting of supporting studies done at NTS by Fenix and Scisson, Inc. and Holmes and Narver, Inc. These appendixes constitute a history of the development of the design and are included as part of the record.

695 (LA-9207-MS) Preliminary identification of interfaces for certification and transfer of TRU waste to WIPP. Whitty, W.J.; Ostenak, C.A.; Pillay, K.K.S. (Los Alamos National Lab., NM (USA)). Feb 1982. Contract W-7405-ENG36. 24p. NTIS, PC A02/MF A01. Order Number DE82009081.

This study complements the national program to certify that newly generated and stored, unclassified defense transuranic (TRU) wastes meet the Waste Isolation Pilot Plant (WIPP) Waste Acceptance Criteria. The objectives of this study were to identify (1) the existing organizational structure at each of the major waste-generating and shipping sites and (2) the necessary interfaces between the waste shippers and WIPP. The interface investigations considered existing waste management organizations at the shipping sites and the proposed WIPP organization. An effort was made to identify the potential wastecertifying authorities and the lines of communication within these organizations. The long-range goal of this effort is to develop practicable interfaces between waste shippers and WIPP to enable the continued generation, interim storage, and eventual shipment of certified TRU wastes to WIPP. Some specific needs identified in this study include: organizational responsibility for certification procedures and quality assurance (QA) program; simple QA procedures; and specification and standardization of reporting forms and procedures, waste containers, and container labeling, color coding, and code location.

696 (LA-9255-MS) Petrologic studies of drill cores USW-G2 and UE25b-1H, Yucca Mountain, Nevada. Caporuscio, F.; Vaniman, D.; Bish, D.; Broxton, D.; Arney, B.; Heiken, G.; Byers, F.; Gooley, R.; Semarge, E. (Los Alamos National Lab., NM (USA)). Jul 1982. Contract W-7405-ENG- 
36. 114p. NTIS, PC A06/MF A01. Order Number DE82021846.

The tuffs of the Nevada Test Site are currently under investigation as a possible deep geologic site for high-level radioactive waste disposal. This report characterizes tuff retrieved in core from two drill holes, USW-G2 and UE25b-1H, at the Yucca Mountain block. The USW-G2 drill core is from the northernmost extent of the block, whereas UE25b-1H is adjacent to an earlier drill hole, UE25a-1. The drill cores USW-G2 and UE25b-1H bottomed at 6000 and $4200 \mathrm{ft}$, respectively. Petrographic and $x$-ray diffraction studies of the two drill cores are presented in this report and indicate that tuffs (composed primarily of variably welded ash flows) are partially recrystallized to secondary minerals. Correlations of stratigraphy are also made with previous drill cores from Yucca Mountain.

697 (LA-9322-MS) Methodology for determining timedependent mechanical properties of tuff subjected to near-field repository conditions. Blacic, J.D.; Andersen, R. (Los Alamos National Lab., NM (USA)). Jan 1983. Contract W-7405-ENG36. 51p. NTIS, PC A04/MF A01. Order Number DE83007054.

Portions are illegible in microfiche products. Original copy available until stock is exhausted.

We have established a methodology to determine the time dependence of strength and transport properties of tuff under conditions appropriate to a nuclear waste repository. Exploratory tests to determine the approximate magnitudes of thermomechanical property changes are nearly complete. In this report we describe the capabilities of an apparatus designed to precisely measure the time-dependent deformation and permeability of tuff at simulated repository conditions. Preliminary tests with this new apparatus indicate that microclastic creep failure of tuff occurs over a narrow strain range with little precursory Tertiary creep behavior. In one test, deformation under conditions of slowly decreasing effective pressure resulted in failure, whereas some strain indicators showed a decreasing rate of strain

698 (LA-9325-MS) Status of volcanic hazard studies for the Nevada Nuclear Waste Storage Investigations. Crowe, B.M.; Vaniman, D.T.; Carr, W.J. (Los Alamos National Lab., NM (USA)). Mar 1983. Contract W-7405-ENG-36. 54p. NTIS, PC A04/MF A01. Order Number DE83015596.

Portions are illegible in microfiche products. Original copy available until stock is exhausted.

Volcanism studies of the Nevada Test Site (NTS) region are concerned with hazards of future volcanism with respect to underground disposal of high-level radioactive waste. The hazards of silicic volcanism are judged to be negligible; hazards of basaltic volcanism are judged through research approaches combining hazard appraisal and risk assessment. The NTS region is cut obliquely by a N-NE trending belt of volcanism. This belt developed about $8 \mathrm{Myr}$ ago following cessation of silicic volcanism and contemporaneous with migration of basaltic activity toward the southwest margin of the Great Basin. Two types of fields are present in the belt: (1) large-volume, long-lived basalt and local rhyolite fields with numerous eruptive centers and (2) smallvolume fields formed by scattered basaltic scoria cones. Late Cenozoic basalts of the NTS region belong to the second field type. Monogenetic basalt centers of this region were formed mostly by Strombolian eruptions; Surtseyean activity has been recognized at three centers. Geochemically, the basalts of the NTS region are classified as straddle A-type basalts of the alkalic suite. Petrological studies indicate a volumetric dominance of evolved hawaiite magmas. Trace- and rare-earth element abundances of younger basalt ( $<4 \mathrm{Myr}$ ) of the NTS region and southern Death Valley area, California, indicate an enrichment in incompatible elements, with the exception of rubidium. The conditional probability of recurring basaltic volcanism and disruption of a repository by that event is bounded by the range of $10^{-8}$ to $10^{-10}$ as calculated for a 1-yr period. Potential disruptive and dispersal effects of magmatic penetration of a repository are controlled primarily by the geometry of basalt feeder systems, the mechanism of waste incorporation in magma, and Strombolian eruption processes.

699 (LA-9326-MS) Aspects of possible magmatic disruption of a high-level radioactive waste repository in southern Nevada. Crowe, B.; Amos, R.; Perry, F.; Self, S.; Vaniman, D. (Los Alamos National Lab., NM (USA)). Oct 1982. Contract
W-7405-ENG-36. 43p. NTIS, PC A03/MF A01. Order Number DE83003990.

The Nevada Test Site (NTS) region is located within the central section of a north-northeast-trending basaltic volcanic belt of late Cenozoic age, a part of the Quaternary volcanic province of the Great Basin. Future volcanism within the belt represents a potential hazard to storage of high-level radioactive waste within a buried repository located in the southwestern NTS. The hazards of future volcanism in the region are being characterized through a combination of volcanic hazards studies, probability determinations, and consequence analyses. Basaltic activity within the NTS regions is divided into two age groups consisting of relatively large-volume silicic cycle basalts ( 8 to $10 \mathrm{Myr}$ ) and rift basalts ( $<8$ to $0.3 \mathrm{Myr}$ ). This paper describes the processes of basaltic magmatism ranging from derivation of basalt melts at depth, through ascent through the upper mantle and crust, to surface eruption. Each stage in the evolution and dispersal of basaltic magma is described, and the disruption and potential dispersal of stored radioactive waste is evaluated. These data document areas of knowns and unknowns in the processes of basaltic volcanisms and provide background data necessary to assist calculations of radiation release levels due to disruption of a repository. 9 figures, 11 tables.

700 (LA-9328-MS) Summary report on the geochemistry of Yucca Mountain and environs. Daniels, W.R.; Wolfsberg, K.; Ruindbirg, R.S. (Los Alamos National Lab., NM (USA)). Dec 1982. Contraot W.7405-ENG-36. 367p. NTIS, PC A16/MF A01. Order Number DE83013357.

Portions are illegible in microfiche products. Original copy available until stock is exhausted.

This report gives a detailed description of work at Los Alamos that will help resolve geochemical issues pertinent to siting a high-level nuclear waste repository in tuff at Yucca Mountain, Nevada. It is necessary to understand the properties and setting of the host tuff because this rock provides the first natural barrier to migration of waste elements from a repository. The geochemistry of tuff is being investigated with particular emphasis on retardation processes. This report addresses the various aspects of sorption by tuff, physical and chemical makeup of tuff, diffusion processes, tuff/groundwater chemistry, waste element chemistry under expected repository conditions, transport processes involved in porous and fracture flow, and geochemical and transport modeling.

701 (LA-9396-MS) Evaluation of research and development for terminal isolation of nuclear wastes. Burton, B.W. (Los Alamos National Lab., NM (USA)). Aug 1982. Contract W-7405-ENG-36. 26p. NTIS. PC A03/MF A01. Order Number DE82021265.

The National Waste Terminal Storage program is responsible for identifying and constructing a geologic repository for spent reactor fuel, high-level waste, and transuranic waste. Extensive research and development work is in progress in the areas of site selection, waste treatment and waste form development, model development and validation, and long-term repository performance assessment. Many potential technologies are under investigation, but specific technologies cannot be identified until a repository site is selected. It is too early in the program to assess the adequacy of environmental control technologies for deep geologic disposal.

702 (I,A-9481-MS) Sentinel Fap hasalt reacted in a temperature gradient. Charles, R.W.; Bayhurst, G.K. (Los Alamos National Lab., NM (USA)). Jan 1983. Contract W-7405-ENG36. 31p. NTIS, PC A03/MF A01. Order Number DE83008281.

Six basalt prisms were reacted in a controlled temperature gradient hydrothermal circulation system for two months. The prisms were centered at $72,119,161,209,270$, and $310^{\circ} \mathrm{C}$. Total pressure was $1 / 3$ kbar. All prisms showed large weight loss: $5.5 \%$ to $14.9 \%$. The matrix micropegmatite and natural nontronitic alteration reacted readily to clays at all temperatures. The first four prisms were coated with a calcium smectite, and the last two prisms were covered with discrete patches of potassium-rich phengite and alkali feldspar. The results indicated that clays may act as adsorbers of various ions.

703 (LA-9577-PR) Research and development related to the Nevada Nuclear Waste Storage Investigations. Progress report, July 1-September 30, 1982. Daniels, W.R.; Erdal, B.R.; 
"animan, D.T. (comps.). (Los Alamos National Lab., NM JSA)). Mar 1983. Contract W-7405-ENG-36. 76p. NTIS, PC .05/MF A01. Order Number DE83016352.

Portions are illegible in microfiche products. Original copy available until stock is exhausted.

This report summarizes the contribution of the Los Alamos National Laboratory to the Nevada Nuclear Waste Storage Investigations for the fourth quarter of FY 1982. Progress reports are presented for the following: (1) field experiments; (2) geochemistry of tuff; (3) mineralogy-petrology of tuff; (4) volcanism studies; (5) shaft and borehole sealing; (6) rock physics studies; (7) exploratory shaft; and (8) quality assurance.

704 (LA-9666-PR) Research and development related to the Nevada Nuclear Waste Storage Investigations. Progress report, October 1-December 31, 1982. Ogard, A.E.; Daniels, W.R.; Vaniman, D.T. (comps.). (Los Alamos National Lab., NM (USA)). May 1983. Contract W-7405-ENG-36.'55p. NTIS, PC A04/MF A01. Order Number DE83014805.

This report summarizes the contribution of the Los Alamos National Laboratory to the Nevada Nuclear Waste Storage Investigations for the first quarter of FY 1983.

705 (LA-9793-PR) Research and development related to the Nevada Nuclear Waste Storage Investigations. Progress report, January 1-March 31, 1983. Wolfsberg, K.; Vaniman, D.T.; Ogard, A.E. (comps.). (Los Alamos National Lab., NM (USA)). Jun 1983. Contract W-7405-ENG-36. 54p. NTIS, PC A04/MF A01. Order Number DE84002271.

This report summarizes the contribution of the Los Alamos National Laboratory to the Nevada Nuclear Waste Storage Investigations for the second quarter of FY 1983. The status of the following studies is reported: field experiments on nuclide migration in tuff; geochemistry of tuff; mineralogy-petrology of tuff; volcanism; rock physics; shaft and borehole sealing; exploratory shaft; and quality assurance.

706 (LA-UR-83-1304) Some geochemical considerations for a potential repository site in tuff at Yucca Mountain, Nevada. Erdal, B.R.; Bish, D.L.; Crowe, B.M.; Daniels, W.R.; Ogard, A.E.; Rundberg, R.S.; Vaniman, D.T.; Wolfsberg, K. (Los Alamos National Lab., NM (USA)). 1982. Contract W-7405ENG-36. 21p. (CONF-821052-2). NTIS, PC A02/MF A01. Order Number DE83012682.

From 4. international workshop on immune-deficient animals in experimental research; Chexbres, Switzerland (31 Oct 1982).

Portions are illegible in microfiche products.

The Nevada Nuclear Waste Storage Investigations, which is evaluating potential locations for a high-level waste repository at the Nevada Test Site and environs, is currently focusing its investigations on tuff, principally in Yucca Mountain, as a host rock. This paper discusses sume of the geucliemical investigations. Particular emphasis is placed on definition of some basic elements and necessary technical approaches for the geochemistry data acquisition and modeling program. Some site-specific tuff geochemical information that is important for site selection and repository performance will be identified and the current status of knowledge will then be discussed.

707 (LBL-13341) Thermal impact of waste emplacement and surface cooling associated with geologic disposal of nuclear waste. Wang, J.S.Y.; Mangold, D.C.; Spencer, R.K.; Tsang, C.F. (Lawrence Berkeley Lab., CA (USA)). Aug 1982. Contract AC03-76SF00098. 237p. (NUREG/CR-2910). NTIS, PC A11/MF A01. Order Number DE83007314.

The thermal effects associated with the emplacement of aged radioactive wastes in a geologic repository were studied, with emphasis on the following subjects: the waste characteristics, repository structure, and rock properties controlling the thermally induced effects; the current knowledge of the thermal, thermomechanical, and thermohydrologic impacts, determined mainly on the basis of previous studies that assume 10-year-old wastes; the thermal criteria used to determine he repository waste loading densities; and the technical advantages and disadvantages of surface cooling of the wastes prior to disposal as a means of mitigating the thermal impacts. The waste loading densities determined by repository designs for 10-year-old wastes are extended to older wastes using the near-field thermomechanical criteria based on room stability considerations. Also discussed are the effects of long sur- face cooling periods determined on the basis of far-field thermomechanical and thermohydrologic considerations. The extension of the surface cooling period from 10 years to longer periods can lower the nearfield thermal impact but have only modest long-term effects for spent fuel. More significant long-term effects can be achieved by surface cooling of reprocessed high-level waste.

708 (LBL-13600, pp 8-11) Iron-nickel alloys as canister material for radioactive waste disposal in underground repositories. Apps, J.A. Sep 1982. NTIS, PC A09/MF A01.

Portions are illegible in microfiche products. Original copy available until stock is exhausted.

In Earth Sciences Division annual report 1981.

Canisters containing high-level radioactive waste must retain their integrity in an underground waste repository for at least one thousand years after burial (Nuclear Regulatory Commission, 1981). Since no direct means of verifying canister integrity is plausible over such a long period, indirect methods must be chosen. A persuasive approach is to examine the natural environment and find a suitable material which is thermodynamically compatible with the host rock under the environmental conditions with the host rock under the environmental conditions expected in a waste repository. Several candidates have been proposed, among them being iron-nickel alloys that are known to occur naturally in altered ultramafic rocks. The following review of stability relations among iron-nickel alloys below $350^{\circ} \mathrm{C}$ is the initial phase of a more detailed evaluation of these alloys as suitable canister materials.

709 (LBL-13600, pp 48-49) Sorptive diffusion in clay gels. Apps, J.A.; Radke, C.J. Sep 1982. NTIS, PC A09/MF A01.

Portions are illegible in microfiche products. Original copy available until stock is exhausted.

In Earth Sciences Division annual report 1981.

Additional protection for the underground storage of high-level nuclear wastes can be provided by an engineered barrier or backfill material. If properly designed, such a barrier can significantly retard the migration of toxic ions even after failure of the waste canister. The backfill material should be inexpensive, relatively impermeable, thermally stable, highly sorptive to toxic ions, compatible with the host rock, easily applied, and, if possible, a fissure sealant. One material that apparently meets most of these qualifications is a swelling clay known as smectite or montmorillonite. The purpose of this research is to investigate experimentally the migration of representative nuclides in swollen clay gels and to formulate a quantitative transport model allowing reliable design of such an engineered barrier.

710 (LBL-13600, pp 61-64) Analysis of temperature data from the full-scale heater experiments at Stripa. Javandel, I.; Witherspoon, P.A. Sep 1982. NTIS, PC A09/MF A01.

Portions are illegible in microfiche products. Original copy available until stock is exhausted.

In Earth Sciences Division annual report 1980.

In order to examine the thermomechanical behavior of deeply fractured crystalline rock when it is used for the isolation of high-level nuclear waste, a series of experiments was conducted in deep granitic rock at Stripa, Sweden, in a site adjacent to an abandoned iron mine. Among these experiments were two full-scale heating experiments carried out at a depth of approximately $340 \mathrm{~m}$. These experiments were designed to study the near-field effects of heating the rock at two different thermal power levels, with electrical heaters substituting for nuclear waste canisters. Later, one of these experiments was supplemented by eight peripheral heaters to account for the interaction of surrounding waste canisters.

711 (LBL-14080) Thermal-gradient migration of brine inclusions in salt. Yagnik, S.K. (Lawrence Berkeley Lab., CA (USA); California Univ., Berkeley (USA). Dept. of Nuclear Engineering). Feb 1982. Contract AC03-76SF00098. 10p. (CONF-820359-1). NTIS, PC A02/MF A01. Order Number DE82013007.

From Western regional American Nuclear Society student conference; Corvallis, OR, USA (28 Mar 1982).

Portions of document are illegible.

It has been proposed that the high level nuclear waste be buried deep underground in a suitable geologic formation. Natural salt deposits have been under active consideration as one of the geologic formations 
where a nuclear waste repository may be built in future. The salt deposits, however, are known to contain a small amount (about $0.5 \mathrm{vol} . \%$ ) of water in the form of brine inclusions which are dispersed throughout the medium. The temperature gradients imposed by the heat generating nuclear waste will mobilize these brine inclusions. It is important to know the rate and the amount of brine accumulating at the waste packages to properly evaluate the performance of a nuclear waste repository. An extensive experimental investigation of the migration velocities of brine inclusions in synthetic single crystals of $\mathrm{NaCl}$ and in polycrystalline natural salt crystals has been conducted. The results show that in a salt repository the brine inclusions within a grain would move with the diffusion controlled velocities. The brine reaching a grain boundary may be swept across, if the thermal gradient is high enough. Grain boundaries in polycrystalline rock salt are apparently quite weak and open up due to drilling the hole for a waste canister and to the thermal stresses which accompany the thermal gradient produced by the heat generating waste. The enhanced porosity allows the water reaching the grain boundary to escape by a vapor transport process.

712 (LBL-14082) Radioactive waste disposal in granite. Witherspoon, P.A.; Watkins, D.J. (Lawrence Berkeley Lab., CA (USA)). Fcb 1982. Contract AC03-76SF00098. 5p. (CONF-820422-2). NTIS, PC A02/MF A01. Order Number DE82008873.

From American Society of Civil Engineers conference; Las Vegas, NV, USA ('26 Ap! 198'2).

The principal geotechnical problems in selecting a repository site for radioactive waste disposal in granite are to evaluate the suitability of the rock mass in terms of: (1) fracture characteristics, (2) thermomechanical effects, and (3) fracture hydrology. Underground experiments in a mine in Sweden have provided an opportunity to study these problems. The research has demonstrated the importance of hydrogeology and the need to improve predictions of the thermomechanical behavior of fractured rocks. To characterize a site, measurements made from the surface must be supplemented by extensive subsurface experiments. Much effort is needed to generate the technology required for the development of waste repositories.

713 (LBL-14752) Thermal-gradient migration of brine inclusions in salt crystals. Yagnik, S.K. (Lawrence Berkeley Lab., CA (USA)). Sep 1982. Contract AC03-76SF00098. 135p. NTIS, PC A07/MF A01. Order Number DE83004180.

Thesis.

It has been proposed that high-level nuclear waste be disposed in a geologic repository. Natural-salt deposits, which are being considered for this purpose, contain a small volume fraction of water in the form of brine inclusions distributed throughout the salt. Radioactivedecay heating of the nuclear wastes will impose a temperature gradient on the surrounding salt which mobilizes the brine inclusions. Inclusions filled completely with brine migrate up the temperature gradient and eventually accumulate brine near the buried waste forms. The brine may slowly corrode or degrade the waste forms which is undesirable. In this work, thermal gradient migration of both all-liquid and gas-liquid inclusions was experimentally studied in synthetic single crystals of $\mathrm{NaCl}$ and $\mathrm{KCl}$ using a hot-stage attachment to an optical microscope which was capable of imposing temperature gradients and axial compressive loads on the crystals. The migration velocities of the inclusions were found to be dependent on temperature, temperature gradient, and inclusion shape and size. The velocities were also dictated by the interfacial mass transfer resistance at brine/solid interface. This interfacial resistance depends on the dislocation density in the crystal, which in turn, depends on the axial compressive loading of the crystal. At low axial loads, the dependence between the velocity and temperature gradient is non-linear. At high axial loads, however, the interfacial resistance is reduced and the migration velocity depends linearly on the temperature gradient. All-liquid inclusions filled with mixed brines were also studied. For gas-liquid inclusions, three different gas phases (helium, air and argon) were compared. Migration studies were also conducted on single crystallites of natural salt as well as in polycrystalline natural salt samples. The behavior of the inclusions at large angle grain boundaries was observed. 35 figures, 3 tables.

714 (LBL-15010) Appraisal of nuclear waste isolation in the vadose zone in arid and semiarid regions (with emphasis on the Nevada Test Site). Wollenberg, H.A.; Wang, J.S.Y.; Korbin, G. (Lawrence Berkeley Lab., CA (USA)). May 1983. Contract
AC03-76SF00098. 135p. NTIS, PC A07/MF A01. Order Number DE84001066.

Portions are illegible in microfiche products. Original copy available until stock is exhausted.

An appraisal was made of the concept of isolating high-level radioactive waste in the vadose zone of alluvial-filled valleys and tuffaceous rocks of the Basin and Range geomorphic province. Principal attributes of these terranes are: (1) low population density, (2) low moisture influx, (3) a deep water table, (4) the presence of sorptive rocks, and (5) relative ease of construction. Concerns about heat effects of waste on unsaturated rocks of relatively low thermal conductivity are considered. Calculations show that a standard 2000-acre repository with a thermal loading of $40 \mathrm{~kW} /$ acre in partially saturated alluvium or tuff would experience an average temperature rise of less than $100^{\circ} \mathrm{C}$ above the initial temperature. The actual maximum temperature would depend strongly on the emplacement geometry. Concerns about seismicity, volcanism, and future climatic change are also mitigated. The conclusion reached in this appraisal is that unsaturated zones in alluvium and tuff of arid regions should be investigated as comprehensively as other geologic settings considered to be potential repository sites.

715 (LBL-15029) Selected hydrologic and geochemical issues in site characterization for nuclear waste disposal: flood basalts at the Hanford Reservation. Carnahan, C.I.; Delany, J.M.; Long, J.C.S.; Silva, R.J.; Watkins, D.J.; White, A.F.; Wilson, C.R. (Lawrence Berkeley Lab., CA (USA)). Sep i 982. Contract AC03-76SF00098. 179p. NTIS, PC A09/MF A01. Order Number DE83007204.

Portions are illegible in microfiche products. Original copy available until stock is exhausted.

Four issues are considered that must be addressed by a site characterization program designed to evaluate the suitability of the flood basalts of the Pasco Basin in central Washington as a site for the construction of a repository for the disposal of high-level nuclear waste. The four issues are (1) identification of hydrostratigraphic units within a sequence of flood basalts, (2) mechanisms and points of groundwater recharge and discharge, (3) solubility of radionuclides, and (4) phase transformation of fracture filling materials. Each issue is discussed in terms of its significance to waste isolation. Available approaches for resolving the issues are presented and their limitations identified. Where appropriate, research programs for overcoming these limitations are indicated.

716 (N-8229145) US Geological Survey research in radioactive waste disposal, fiscal year 1979. Schneider, R.; Roseboom, E.H. Jr.; Robertson, J.B.; Stevens, P.R. (Geological Survey, Washington, DC (USA)). 1982. 79p. (USGS-CIRC847). NTIS, PC A0S/MF A01.

Geologic and hydrologic research related to the disposal of radioactive wastes is reported. The categories are described as they relate most directly to: (1) high level and transuranic wastes (2) low level wastes or (3) uranium mill tailings. The identification and geohydrologic characterization of waste disposal sites, investigations of specific sites where wastes have been stored, and regions or environments where waste disposal sites might be located are studied. Techniques and methods for characterizing disposal sites and studies of geologic and hydrologic processes related to the transport and (or) retention of waste radionuclides are presented.

717 (N-8229146) Monitoring technologies for ocean disposal of radioactive waste. (RAND Corp., Santa Monica, CA (USA)). Jan 1982. 109p. NTIS, PC A06/MF A01.

The feasibility of using carefully selected subseabed locations to permanently isolate high level radioactive wastes at ocean depths greater than 4000 meters is discussed. Disposal at several candidate subseabed areas is being studied because of the long term geologic stability of the sediments, remoteness from human activity, and lack of useful natural resources. While the deep sea environment is remote, it also poses some significant challenges for the technology required to survey and monitor these sites, to identify and pinpoint container leakage should it occur, and to provide the cnvironmental information and data base essential to determining the probable impacts of any such occurrence. Objectives and technical approaches to aid in the selective development of advanced technologies for the future monitoring of nuclear low level and high level waste disposal in the deep seabed are presented. Detailed recommendations for measurement and sampling technology 
development needed for deep seabed nuclear waste monitoring are also presented.

718

(NASA/CR-161991) Analysis of space systems for the space disposal of nuclear waste follow-on study. Volume 1. Executive summary. (Boeing Aerospace Co., Seattle, WA (USA)). 1982. Contract AI97-79ET46603. 21p. NTIS, PC A02/MF A01. Order Number DE83006640.

Portions are illegible in microfiche products. Original copy available until stock is exhausted.

The following major conclusions resulted from this study: Parameters for the reference cermet waste form are available only by analogy. Detail design of the waste payload would require determination of actual waste form properties. Billet configuration constraints for the cermet waste form limit waste payload packing efficiency to slightly under $75 \%$ net volume, resulting in a $20 \%$ increase in the number of flights and subsequent increases in both cost and risk. Alternative systems for waste mixes requiring low launch rates (technetium-99, iodine129) can make effective use of the existing $65 \mathrm{~K}$ space transportation system in either single- or dual-launch scenarios. A trade study involving a comprehensive comparison of life cycle costs would be required to select the optimum orbit transfer system for low-launch-rate systems. This was not a part of the present effort due to selection of the cermet waste form as the reference for the study. The reference space system offers the best combination of cost, risk, and alignment with ongoing NASA technology development for disposal of the reference cermet waste form within specified system safety guidelines.

719 (NASA/CR-161992) Analysis of space systems for the space disposal of nuclear waste follow-on study. Volume 2. Technical report. (Boeing Aerospace Co., Seattle, WA (USA)). 1982. Contract AI97-79ET46603. 190p. NTIS, PC A09/MF A01. Order Number DE83006639.

Portions are illegible in microfiche products. Original copy available until stock is exhausted.

Some of the conclusions reached as a result of this study are summarized. Waste form parameters for the reference cermet waste form are available only by analogy. Detail design of the waste payload would require determination of actual waste form properties. The billet configuration constraints for the cermet waste form limit the packing efficiency to slightly under $75 \%$ net volume. The effect of this packing inefficiency in reducing the net waste form per waste payload can be seen graphically. The cermet waste form mass per unit mass of waste payload is lower than that of the iodine waste form even though the cermet has a higher density (6.5 versus 5.5 ). This is because the lead iodide is cast achieving almost $100 \%$ efficiency in packing. This inefficiency in the packing of the cermet results in a $20 \%$ increase in number of flights which increases both cost and risk. Alternative systems for waste mixes requiring low flight rates (technetium-99, iodine-129) can make effective use of the existing $65 \mathrm{~K}$ space transportation system in either singleor dual-launch scenarios. A comprehensive trade study would be required to select the optimum orbit transfer system for low-launch-rate systems. This study was not conducted as part of the present effort due to selection of the cermet waste form as the reference for the study. Several candidates look attractive for both single- and dual-launch systems (see sec. 4.4), but due to the relatively small number of missions, a comprehensive comparison of life cycle costs including DDT and E would be required to select the best system. The reference system described in sections 5.0,6.0, 7.0, and 8.0 offers the best combination of cost, risk, and alignment with ongoing NASA technology development efforts for disposal of the reference cermet waste form.

720

(NASA/CR-162028) Preliminary risk assessment for nuclear waste disposal in space. Volume I. Executive summary of technical report. Rice, E.E.; Denning, R.S.; Friedlander, A.L. (Battelle Columbus Labs., OH (USA)). 28 Feb 1982. Contract AI97-79ET46603. 40p. NTIS, PC A03/MF A01. Order Number DE83006637.

Portions are illegible in microfiche products. Original copy available until stock is exhausted.

Three major conclusions come from this preliminary risk assessment of nuclear waste disposal in space. Preliminary estimates of space disposal risk are low, even with the estimated uncertainty bounds. If calculated mined geologic repository (MGR) release risks remain low, and the EPA requirements continue to be met, then no additional space disposal study effort is warranted. If risks perceived by the public are sig- nificant in the acceptance of mined geologic repositories, then consideration of space disposal as an MGR complement is warranted. As a result of this study, the following recommendations are made to NASA and the US DOE: During the continued evaluation of the mined geologic repository risk over the years ahead by DOE, if any significant increase in the calculated health risk is predicted for the MGR, then space disposal should be reevaluated at that time. The risks perceived by the public for the MGR should be evaluated on a broad basis by an independent organization to evaluate acceptance. If, in the future, MGR risks are found to be significant due to some presently unknown technical or social factor, and space disposal is selected as an alternative that may be useful in mitigating the risks, then the following space disposal study activities are recommended: improvement in chemical processing technology for wastes; payload accident response analysis; risk uncertainty analysis for both MGR and space disposal; health risk modeling that includes pathway and dose estimates; space disposal cost modeling; assessment of space disposal perceived (by public) risk benefit; and space systems analysis supporting risk and cost modeling.

721 (NASA/CR-162029) Preliminary risk assessment for nuclear waste disposal in space. Volume II. Technical report. Rice, E.E.; Denning, R.S.; Friedlander, A.L. (Battelle Columbus Labs., OH (USA)). 28 Feb 1982. Contract AI9779ET46603. 261p. NTIS, PC A12/MF A01. Order Number DE83006638.

Portions are illegible in microfiche products. Original copy available until stock is exhausted.

Three major conclusions came from this preliminary risk assessment of nuclear waste disposal in space. Preliminary estimates of space disposal risk are low, even with the estimated uncertainty bounds. If calculated mined geologic repository (MGR) release risks remain low, and the EPA requirements continue to be met, then no additional space disposal study effort is warranted. If risks perceived by the public are significant in the acceptance of mined geologic repositories, then consideration of space disposal as an MGR complement is warranted. As a result of this study, the following recommendations are made to NASA and the US DOE: During the continued evaluation of the mined geologic repository risk over the years ahead by DOE, if any significant increase in the calculated health risk is predicted for the MGR, then space disposal should be reevaluated at that time. The risks perceived by the public for MGR should be evaluated on a broad basis by an independent organization to evaluate acceptance. If, in the future, MGR risks are found to be significant due to some presently unknown technical or social factor, and space disposal is selected as an alternative that may be useful in mitigating the risks, then the following space disposal study activities are recommended: improvement in chemical processing technology for wastes; payload accident response analysis; risk uncertainty analysis for both MGR and space disposal; health risk modeling that includes pathway and dose estimates; space disposal cost modeling; assessment of space disposal perceived (by public) risk benefit; and space systems analysis supporting risk and cost modeling.

722 (NP-3901071) Geologic aspects of disposal of highly radioactive nuclear waste. Open-File Report No. 15. Spoljaric, N. (Delaware Geological Survey, Newark (USA)). May 1981. 45p. NTIS, PC A03/MF A01. Order Number DE83901071. Portions of document are illegible.

This report provides a simple but comprehensive overview of pro grams and concepts of highly radioactive waste disposal. It is not based on original research, but was prepared from data and information reported in voluminous publications of the US Department of Energy, the Nuclear Regulatory Commission, the US Environmental Protection Agency, and the US Geological Survey. All these projects involve many different fields of science. It is important that the data generated in these studies are readily available to all the scientists involved in the nuclear waste disposal program. In addition, contacts are maintained with the Association of American State Geologists and with several foreign countries having similar programs underway. Particularly close consultations are carried out with the Federal Republic of Germany, Canada, and Sweden. The Delaware Geological Survey does not anticipate disposal of highly radioactive waste in Delaware. Nevertheless, this report was prepared because Delaware citizens have expressed general concern about nuclear power issues and we seek to clarify one geologic aspect of these issues. 
723

(NUREG-0856) Final technical position on documentation of computer codes for high-level waste management. Silling, S.A. (Nuclear Regulatory Commission, Washington, DC (USA). Office of Nuclear Material Safety and Safeguards). Jun 1983. 19p. NTIS, PC A02/MF A01 - GPO \$3.00. Order Number DE83902637.

Guidance is given for the content of documentation of computer codes which are used in support of a license application for high-level waste disposal. The guidelines cover theoretical basis, programming, and instructions for use of the code.

724 (NUREG-0960-Vol.1) Draft site characterization analysis of the site characterization report for the Basalt Waste Isolation Project, Hanford, Washington Site. Main report and Appendices A through D. (Nuclear Regulatory Commission, Washington, DC (USA). Office of Nuclear Material Safety and Safeguards). Mar 1983. 420p. NTIS, PC A18/MF A01 - GPO. Order Number DE83901600.

Pnrtinns are illegible in microfiche products.

On November 12, 1982, the US Department of Energy submitted to the US Nuclear Regulatory Commission the Site Characterization Report for the Basalt Waste Isolation Project (DOË/RL 82-3). The Basalt Waste Isolation Project is located on DOE's Hanford Reservation in the State of Washington. NUREG-0960 contains the detailed analysis, by the NRC staff, of the site characterization report. Supporting technical material is contained in Appendices A through W.

725 (NUREG-0960-Vol.2) Draft site characterization analysis of the site characterization report for the Basalt Waste Isolation Project, Hanford, Washington site. Appendices E through W. (Nuclear Regulatory Commission, Washington, DC (USA). Office of Nuclear Material Safety and Safeguards). Mar 1983. 429p. NTIS, PC A19/MF A01 - GPO. Order Number DE83901642

Portions are illegible in microfiche products.

Volume 2 contains Appendices $E$ through W: potential for largescale pump tests in the Grande Ronde; review of hydrochemical characterization related to flow system interpretation in Hanford basalts; limi: tations of packer-testing for head evaluation in Hanford basalts; hydrogeologic data integration for conceptual groundwater flow models; drilling mud effects on hydrogeologic testing; site issue analyses related to the nature at the present groundwater system at the Hanford site, Washington; structural and stratigraphic characteristics related to groundwater flow at the Hanford site, Washington; seismic hazard and some examples of hazard studies at Hanford; earthquake swarms in the Columbia Plateau; seismic ground motion at depth; failure modes for the metallic waste package component; degradation mechanisms of borosilicate glass; transport and retardation of radionuclides in the waste package; determination and interpretation of redox conditions and changes in underground high-level repositories; determination and interpretation of sorption data applied to radionuclide migration in underground repositories; solubility of radionuclide compounds presented in the BWIP site characterization report; and release rate from engineered system.

726 (NUREG/CP-0022) Proceedings of the symposium on uncertainties associated with the regulation of the geologic disposal of high-level radioactive waste. Kocher, D.C. (ed.). (Oak Ridge National Lab., TN (USA)). Mar 1982. Contract W-7405-ENG-26. 488p. (CONF-810372-). NTIS, PC A21/MF A01. Order Number DE82008883.

From Symposium on uncertainties associated with the regulation of the geologic disposal of high-level radioactive waste; Gatlinburg, TN, USA ( 9 Mar 1981)

Portions of document are illegible

The primary purpose of this symposium was to provide a forum for wide-ranging discussions on (1) technical aspects related to the development of standards for regulating geologic disposal of high-level radioactive waste, with particular emphasis on the sources and magnitudes of uncertainties associated with current methods for predicting post-closure repository performance and potential health risks to future genteratiuns, (2) important licensing and regulatory issues involved in geologic waste disposal, and (3) the current social and political climate in which issues of high-level waste management are being debated. Significant contributions to these discussions were provided by representatives from the US Nuclear Regulatory Commission (NRC), US Depart- ment of Energy, US Environmental Protection Agency (EPA), variol contractors of these three agencies, and other interested parties not aff: iated with the Federal Government or its contractors. The symposiu: was timed to coincide with the development and publication by the NRC of the proposed technical criteria for regulating the disposal of high-level radioactive wastes in geulugic repositories. An additional subject of considerable interest at the symposium was the development of environmental radiation protection standards for high-level radioactive waste by the EPA and the relationship of these standards to the NRC's proposed technical criteria. Separate abstracts have been prepared for individual articles for inclusion in the Energy Data Base. (DMC)

727 (NUREG/CR-1672-Vol.4) Risk-assessment methodology development for waste isolation in geologic media. Stevens, C.A.; Fullwood, R.R.; Amirijafari, B.; Basin, S.L.; Cohen, J. (Science Applications, Inc., Palo Alto, CA (USA)). Dec 1982 66p. (SAI-324-82-PA-Vol.4). NTIS, PC A04/MF A01 - GPO $\$ 5.00$. Order Number DE83901048.

A review of three doeuments prepuat for tlie USNRC by Sandia National Laboratories (SNL) is presented. These are NUREG/ CR-1634, Volume 4 concerned with the effects of variahle, hydrningy $n$ waste migration; NUREG/CR-2324, a user's manual for SWIFT; and NUREG/2343, a user's manual for DNET. This review completes Task 4 of the detailed technical review of the SNL program for Risk Assessment Methodology Development for Waste, Isnlation in Genlogic Media. In general, these reports exhibit high technical quality that characterizes the SNL work. They are tersely written with little condescension to the non-expert reader for understanding the physical situation being modeled. Indeed, the emphasis is on the mathematical procedures rather than the repository physics, leaving the adequacy of the results presented in many computer plots, pretty much to the interpretation of the reader. Other general comments have been presented previously, such as the data conservatisms, need for data that cannot be measured without disturbing the geometry, and the overall plan for use of the many codes developed in the program.

728 (NUREG/CR-2317-Vol.1-No.3) Container assessment: corrosion study of HLW container materials. Quarterly progress report, July-September 1981. Ahn, T.M.; Soo, P. (Brookhaven National Lab., Upton, NY (USA)). Jan 1982. Contract AC02-76CH00016. 34p. (BNL-NUREG-51449-Vol. 1-No.3). NTIS, PC A03/MF A01. Order Number DE82015088.

Work has been started on the corrosion and hydrogen embrittlement behavior of commercially pure titanium (ASTM Frade 2), TiCode-12 (ASTM Grade 12), and OFHC copper, which are primary candidate materials for high level waste containers. The test environment used is a simulated brine solution typical of bedded salt at $150^{\circ} \mathrm{C}$ or room temperature. The immersion test results for these materials are in reasonable agreement with previous screening test results of Sandia National I aboratory; electron beam welded titanium and TiCode-12 samples show higher corrosion rates than the non-welded samples. In the immersion tests on mechanically-creviced titanium, a major crevice corrosion product is observed which has various coloris and was identified by Transmission electron Microscopy to be a mixture of $\mathrm{TiO}_{2}$ and other low oxides such as $\mathrm{Ti}_{2} \mathrm{O}_{0}$ and $\mathrm{TiO}$. The crevice region of TiCode-12 shows a color change at $150^{\circ} \mathrm{C}$ with a thicker film forming at $200^{\circ} \mathrm{C}$. The correlation of electrochemistry and immersion tests is under study. Sulfur introduced in a crevice simulation test on copper has been shown to cause attack. Copper, in the absence of sulfur, however, shows no visible crevice corrosion. As an aid to understanding uniform and crevice corrosion results, potential-pH diagrams are being constructer. Slow strain rate embrittlement and impact embrittlement have been observed in cathodically hydrogen charged commercially pure titanium and TiCode-12 in tension and buckling tests. $C$-ring and U-bend specimens of titanium and TiCode- 12 have been designed and a slow strain rate test machine is being built to study stress corrosion cracking. Alternating Current Impedance and Scanning Reference Electrode Techniques are ready for pitting studies. For the study of radiation-induced corrosion, an irradiation cell was set up in the gamma pool to estimate quantit: tively the oxidants as well as hydrogen produced by the gamma radi: tion in the brine solutions. terly progress report, April-June 1981. Ahn, T.M.; Lee, B.S.; 
Soo, P. (Brookhaven National Lab., Upton, NY (USA)). Dec 1981. Contract AC02-76CH00016. 13p. (BNL-NUREG51449). NTIS, PC A02/MF A01. Order Number DE82008455.

A research program on container assessment has been initiated to determine the general and localized corrosion mechanisms in high level waste container materials such as titanium, copper, and lead. In this quarter, our efforts have been on the initiation of a program establishing specific experiments pertaining to uniform corrosion, crevice corrosion, pitting corrosion, stress corrusion, hydrogen embrittlement, radiation effects, vapor-solution-container wall interaction, and an analytical study. Preliminary tests have been performed for the uniform and crevice corrosion of copper and titanium in brine at $150^{\circ} \mathrm{C}$.

730 (NUREG/CR-2317-Vol.2-No.1) Container assessment: corrosion study of HLW containcr materials. Quarterly progress report, January-March 1982. Ahn, T.M.; Soo, P (Brookhaven National Lab., Upton, NY (USA)). Jun 1982. Contract ACO2-76CH00016. 28p. (BNL-NUREG-51449-Vol. 2-No.1). NTIS, PC A03/MF A01 - GPO. Order Number DE83002110.

Crevice corrosion products formed on titanium base materials exposed to WIPP Brine $A$ at $150^{\circ} \mathrm{C}$ have been analyzed by obtaining electron diffraction patterns of oxide films which were selected from various positions on the crevice surface. The crevice corrosion products for $\mathrm{CP}$ titanium and TiCode-12 are mainly an anatase form of $\mathrm{TiO}_{2}$. Both materials also showed trace amounts of $\mathrm{Ti}_{0} \mathrm{O}_{5}$. The intensity of the $\mathrm{Ti}_{0} \mathrm{O}_{5}$ peak in the diffraction pattern is stronger in CP titanium than in TiCode-12. More $\mathrm{Ti}_{0} \mathrm{O}_{5}$ is formed in the center of the crevice. From the study, it seems that the color differences of oxides inside the crevice are mainly due to optical interference colors caused by varying film thickness. The open-circuit corrosion potential behavior of $\mathrm{CP}$ titanium and TiCode-12 has been examined in acidified Brine $A$ at $80^{\circ} \mathrm{C}$. To reduce the potential effect of oxidizing impurities in test media these solutions were pre-electrolyzed. Both materials show breakdown of the passive film. TiCode- 12 reaches a quasi steady state potential more rapidly than CP titanium. Breakdown of the passive film is attributed to the high chloride concentration with $\mathrm{Ni}$ being preferentially dissolved. Singleedged-notched tensile specimens have been used to obtain the apparent stress intensity factors at $2 \%$ crack extension in hydrogenated TiCode-12 with hydrogen concentrations up to $10,900 \mathrm{ppM}$. For high hydrogen levels the apparent stress intensity factor dropped roughly by a factor of 10 compared to nonhydrogenated TiCode-12. Fractographs show both alpha phase crystallographic fracture and alpha-beta interface cracking. These fractographs indicate that the formation of hydride is responsible for crack initiation for hydrogen concentrations above $5000 \mathrm{ppM}$.

731

(NUREG/CR-2402) Risk-analysis methodology for spent-fuel repositories in bedded salt. Final report. Pepping, R.E.; Chu, M.S.Y.; Wahi, K.K.; Ortiz, N.R. (Sandia National Labs., Albuquerque, NM (USA)). Jun 1983. Contract AC04 76DP00789. 102p. (SAND-81-2409). NTIS, PC A06/MF A01

- GPO. Order Number DE83016132.

Portions are illegible in microfiche products.

A previously developed risk assessment methodology was modified and applied in the analysis of risks from the geologic disposal of spent unreprocessed fuel (SURF). The methodology had initially been developed for application to the risk assessment from the geologic disposal of reprocessed, high-level waste (HLW). A set of disruptive scenarios, chosen from a larger set of scenarios in the previous study, was analyzed in this study to demonstrate the methodology as applied to a conceptual SURF repository in bedded salt. The results are expressed in terms of integrated discharges and conditional cancer risk. A comparison with the proposed EPA Standard is also presented for demonstration purposes only. The results of individual SURF scenario analyses are compared with the corresponding HLW results. Based on the present analysis, it appears that over a period of 100,000 years the risk associated with the disposal of SURF are higher thài those for HLW for the assumed repository configuration at a hypothetical bedded salt site. The study also demonstrates how the degree and order of importance of various parameters and radionuclides can change when the basis for estimating the risk is changed. Whereas, the methodology could be extended to other media, one is cautioned against generalizing the con- clusions of this study to repositories in media other than bedded salt. 43 figures, 22 tables.

732 (NUREG/CR-2482-Vol.2) Review of DOE wastepackage program. Subtask 1.1. National waste-package program, September 1981-March 1982. Volume 2. Soo, P. (comp.). (Brookhaven National Lab., Upton, NY (USA)). Apr 1983. Contract AC02-76CH00016. 127p. (BNL-NUREG-51494Vol.2). NTIS, PC A07/MF A01 - GPO \$6.00. Order Number DE83011735.

Parts 1 and 2 are indexed separately.

733 (NUREG/CR-2482-Vol.2, pp 59p, Paper 1) Near-field repository conditions in basalt and salt. Siskind, B.; Hsieh, D. Apr 1983. NTIS, PC A07/MF A01 - GPO \$6.00. (BNL-NUREG-51494-Vol.2).

In Review of DOE waste-package program. Subtask 1.1. National waste-package program, September 1981-March 1982. Volume 2.

The characteristics and properties of the near-field repository environment in basalt and salt host rock which are potentially important in the consideration of chemical and mechanical failure modes for the waste package and which provide necessary input for the evaluation of the performance of the individual waste package components and for the test evaluation of the waste package are summarized. Data are presented on the ranges of conditions found in potential basalt and salt host rocks; groundwater and brine parameters and host rock characteristics as well as perturbations in these variables caused by the heat and radiation from the waste package are all addressed. Based on the available information, in particular, the reference repository conditions selected for DOE by the Reference Repository Conditions Interface Working Group, a description is provided of the chemical and physical environment as it is seen or experienced by the waste package. For basalt, the reference values in the undistubed host rock at $1100-\mathrm{m}$ depth for the temperature, hydrostatic and lithostatic pressures, $\mathrm{pH}$ and $\mathrm{Eh}$ are $50^{\circ} \mathrm{C}$, $11 \mathrm{MPa}$ and $33 \mathrm{MPa}, 9.45$ to 9.74 , and $<-0.3 \mathrm{~V}$, respectively. After emplacement of the waste package, the near-field temperature will attain a projected maximum value of $300^{\circ} \mathrm{C}$ before cooling. The reference values for undisturbed salt at $600-\mathrm{m}$ depth for temperature, lithostatic pressure, and $\mathrm{pH}$ are $34^{\circ} \mathrm{C}, 18$ to $35 \mathrm{MPa}$, and 4 to 6.5 , respectively. The maximum near-field temperature after emplacement of the waste package is projected to reach $156^{\circ} \mathrm{C}$ before cooling. The additional information about the near-field environment needed for a complete waste package licensing review are summarized.

734 (NUREG/CR-2482-Vol.2, pp 53p, Paper 2) Uniform- and pitting-corrosion data requirements for TiCode-12 high-level-waste containers. Shao, J.; Soo, P. Apr 1983. NTIS, PC A07/MF A01 - GPO \$6.00. (BNL-NUREG-51494-Vol.2).

In Review of DOE waste-package program. Subtask 1.1. National waste-package program, September 1981-March 1982. Volume 2.

The uniform and pitting corrosion behavior of TiCode- 12 in salt and basalt repository environments is addressed. The available corrosion data are presented and reviewed. It is concluded that insufficient data exist to fully characterize TiCode- 12 corrosion behavior under repository conditions and to determine its capability to contribute to the NRC 1000-year containment criterion. The additional data needs and testing requirements are specified. They are, however, for a generic waste package and may be modified as detailed waste package designs are specified by DOE. A summary of the needed information is presented.

736 (NUREG/CR-2482-Vol.3) Review of DOE waste package program. Subtask 1.1. National waste package program, April-September 1982. Soo, P. (ed.). (Brookhaven National Lab., Upton, NY (USA)). Mar 1983. Contract AC0276CH00016. 287p. (BNL-NUREG-51494-Vol.3). NTIS, PC A13/MF A01 - GPO \$8.00. Order Number DE83010102.

Portions are illegible in microfiche products.

The current effort is part of an ongoing task to evaluate the national bigh-level waste package effort. It includes evaluations of reference waste form, container, and packing material components with respect to determining how they may contribute to the containment and controlled release of radionuclides after waste packages have been emplaced in salt and basalt repositories. Chemical and mechanical failure/degradation modes for the waste package have been reviewed and 
the licensing data requirements to demonstrate compliance with NRC performance objectives specified.

736 (NUREG/CR-2482-Vol.4) Review of DOE waste package program. Subtask 1.1. National waste package program, October 1982-March 1983. Vol. 4. Sö, P. (ed.). (Brookhaven National Lab., Upton, NY (USA)). Sep 1983. Contract AC02-76CH00016. 180p. (BNL-NUREG-51494Vol.4). NTIS, PC A09/MF A01 - GPO \$6.00. Order Number DE83018273.

Portions are illegible in microfiche products.

The current effort is part of an ongoing task to review the national high-level waste package effort. It includes evaluations of reference : waste form, container, and packing material components with respect to determining how they may contribute to the containment and controlled release of radionuclides after waste packages have been emplaced in salt, basalt, and tuff repositories. In the current Biannual Report a section on tuff repository conditions has been included to complement prior work for salt and basalt. Failure/degradation modes for borosilicate glass waste forms, carbon steel containers, and bentonitebased and zeolite-based packing materials (discrete backfills) have been reviewed attd the licensing data requirements to demonstrate the degree of compliance with NRC' performance objectives specified

737 (NURFG/CR-2506) Uncertainties in gcologic disposal of high-level wastes: groundwater transport of radionuclides and radiological consequences. Kocher, D.C.; Sjoreen, A.L.; Bard, C.S. (Oak Ridge National Lab., TN (USA)). Jul 1983. Contract W-7405-ENG-26. 222p. (ORNL-5838). NTIS, PC A10/MF A01 - GPO. Order Number DE83014484.

The analysis for radionuclide transport in groundwater considers models and methods for characterizing (1) the present geologic environment and its future evolution due to natural geologic processes and to repository development and waste emplacement, (2) groundwater hydrology, (3) radionuclide geochemistry, and (4) the interactions among these phenomena. The discussion of groundwater transport focuses on the nature of the sources of uncertainty rather than on quantitative estimates of their magnitude, because of the lack of evidence that current models can provide realistic quantitative predictions of radionuclide transport in groundwater for expected repository environments. The analysis for the long-term health risk to man following releases of long-lived radionuclides to the biosphere is more quantitative and involves estimates of uncertainties in (1) radionuclide concentrations in man's exposure environment, (2) radionuclide intake by exposed individuals per unit concentration in the environment, (3) the dose per unit intake, (4) the number of exposed individuals, and (5) the health risk per unit dose. For the important long-lived radionuclides in high-level waste, uncertainties in most of the different components of a calculation of individual and collective dose per unit release appear to be no more than two or three orders of magnitude; these uncertainties are certainly much less than uncertainties in predicting groundwater transport of radionuclides between a repository and the biosphere. Several limitations in current models for predicting the health risk to man per unit release to the biosphere are discussed.

738 (NUREG/CR-2547) Evaluation of geotechnical surveillance techniques for monitoring high-level waste repository performance. Final report 19 Sep 81-31 Dec 82. St. John, C.M.; Aggson, J.R.; Hardy, M.P.; Hocking, G. (Agapito (J.F.T.) and Associates, Grand Junction, CO (USA)). Mar 1982. 382p. NTIS, PC A17/MF A01.

This report recomunends geotechnical monitoring programs for evaluation of high-level nuclear waste repository performance. It is based upon assessments of conditions and responses of the geologic media to the site of a repository. The state of technology in geotechnical monitoring was reviewed for applicability to repository monitoring. The recommended geotechnical monitoring program utilizes existing geotechnical instrumentation. The proposed activities are delineated in each phase of repository development: the proposed monitoring is detailed for various dimensional scales. Only geotechnical instrumentation presently available or easily within the state of the art is considered and it was concluded that it is possible to define an integrated geotechnical monitoring program that would meet the proposed licensing requirements.

739 (NUREG/CR-2755) Packing material testing required to demonstrate compliance with 1000 -year radionuclide containment. Semiannual report on waste package verification tests. Bida, G.; Eastwood, D. (Brookhaven National Lab., Upton, NY (USA)). Jan 1983. Contract AC.02-76CH00016. 35p. (BNL-NUREG-51544). NTIS, PC A03/MF A01 - GPO $\$ 4.50$. Order Number DE83007326.

This semiannual report is an evaluation of the testing requirements for bentonite and zeolite-based packing materials (discrete backfills) to assess their ability to contribute to the NRC's 1000-year radionuclide containment requirement for the waste package. The status of current test mcthodologies is described and suggestions for additional evaluation are outlined.

740 (NUREG/CR-2782) Summary of repository siting models. Final report. Thomas, S.D.; Ross, B.; Mercer, J.W. (Teknekron Research, Inc., Bethesda, MD (USA); Gilbert/ Commonwealth, Reading, PA (USA)). Jul 1982. 242p. GPO $\$ 7.50$.

This report is the first in a series of reports that will provide critical reviews and summaries of computer programs that can be used to analyze the potential performance of a high-level radioactive waste repnsitory. The computer programo identified address thit fulluwing phenomenal aaturnted and unsaturaled subsurfuue fluw, heat transpoil, solute transport, surface water runoff, geomcchanical interactions, and geochemical interactions. The report identifies 183 computer programs that can be used to analyze a repository site and provides a summary description of 31 computer programs. The summary descriptions can be used: to assist in code evaluation, to facilitate code comparison, to determine applicability of codes to specific problems, to identify code deficiencies, and to provide a screening mechanism for code selection.

741 (NUREG/CR-2854-813-1164D) Evaluation of alternative shaft-sinking techniques for high-level nuclear waste (HLW) deep geologic repositories. Final report (Task 3), June 1981-July 1982. Gonano, L.; Findley, D.; Wildanger, W.; Gates, R.; Phillips, S. (Golder Associates, Inc., Bellevue, WA (USA)). Mar 1983. 187p. NTIS, PC A09/MF A01 - GPO \$7.00. Order Number DE83901896.

Portions are illegible in microfiche products.

This report represents the results of Task 3 of US Nuclear Regulatory Commission (NRC) Contract, Technical Assistance for Repository Design. The purpose of the complete project is to provide NRC with technical assistance for the following reasons: To enable the focused, adequate review by NRC of aspeots related to design and construction of an in situ test facility and final geologic rcpository, as presented in US Department of Energy (DOE) Site Characterization Reports (SRC), To ascertain that the DOE site characterization program will provide, as far as possible, all the information necessary to permit a review to be conducted by NRC of a license application for construction authorization. It is assumed that the Site Characterization Report and License Application will describe the exploratory shaft and concept designs for the repository shafts. This report provides a comparative evaluation of various shaft sinking techniques for production shafts for a repository. The primary comparative evaluation has been conducted for 14-ft internal diameter shafts developed in two compositc media using four different methods of sinking/lining. The technical, cost and schedule comparisons draw a major distinction between shafts sunk blind and those which utilize bottom access. Based on the system of ranking introduced to grade the significant attributes of each method and the resulting design, it is conoludod that for applicaliun tu repusitury access, no one particular method of sinking exhibits a clear overall superiority. When a specific site is made available for a study of the most suitable shaft sinking methods, it will be necessary to establish actual geological conditions and technological capabilities and the comparisons prescnted herein reviewed accordingly.

742 (NUREG/CR-2959-813-1165) Relationship of an insitu test facility to a deep geologic repository for high-level nuclear waste. Final report (Task 4), June 1981-October 1982. Gates, R.; Bauhof, F.; Gonano, L. (Golder Associates, Inc., Bellevue, WA (USA)). Mar 1983. 57p. NTIS, PC A04/MF A01 - GPO \$5.00. Order Number DE\$3901818.

Portions are illegible in microfiche products.

An in-situ test facility has been proposed for site characterization and design data collection for high-level nuclear waste deep geologic repositories. This report evaluates and presents recommendations on the relationship between an in-situ test facility (exploratory shaft and under- 
ground test facility) and the repository. Technical and construction issues are addressed in generic terms for an in-situ test facility which forms an integral part of the repository. Facility requirements defined and justified include location and size of the test facility, the design, construction and operational features of the exploratory shaft and underground test facilities, and the nature and duration of in situ testing. Media/site specific considerations are also presented. It is recommended that the exploratory shaft allow for adequate characterization of the strata surrounding the repository horizon. The underground test facility must be of a size and at a location which allow direct and explicit full-scale sampling of the engineering behavior of the materials of the repository site. Construction of the exploratory shaft and test facility should utilize the same methods as planned for the proposed corresponding repository structures.

743 (NUREG/CR-3065-813-1163-Vol.1) In-situ test programs related to design and construction of high-level nuclear waste (HLW) deep geologic repositories. Final report (Task 2) June 1981-November 1982. Roberds, W.; Bauhof, F.; Gonano, L. (Golder Associates, Inc., Bellevue, WA (USA)). Mar 1983. 220p. NTIS, PC A10/MF A01 - GPO \$7.50. Order Number DE83901897.

Portions are illegible in microfiche products.

The media and sites considered include (1) basalt at Hanford, Washington; (2) tuff at Yucca Mountain, Nevada Test Site; (3) domal salt at specific Gulf Coast sites; (4) bedded salt at an unspecified site and (5) granite at an unspecified site. A licensing perspective is outlined and a defensible rationale developed and utilized for the test selection process. This rationale essentially consists of: establishing the information needs for construction authorization; assessing the relevant capabilities of available tests; and matching the capabilities of specific tests to the perceived information needs. The information needs at any time consist of the additional information (if any) needed in order to predict satisfactory repository system performance with the required level of confidence, and thus are a function of: the significance of the repository engineered components and site characteristics to system performance; the currently available information, which may be supplemented with time; and the acceptable level of confidence in satisfactory performance for each licensing step. Determination of the acceptable levels of confidence and the significance of repository system components is outside the scope of this report. Suitable assumptions have thus been made regarding the development of information needs for construction authorization by the time of initial site submittals. Tests which are available and respond to the perceived media/site specific information needs, either by simulation or assessment of site characteristics, are identified and their capabilities assessed. Specific in situ tests are investigated and described in detail. Research and development which might be effective in improving test capabilities have been recommended.

744 (NUREG/CR-3065-813-1163-Vol.2) In-situ test programs related to design and construction of high-level nuclear waste (HLW) deep geologic repositories. Appendices. Final report (Task 2), June 1981-November 1982. Roberds, W.; Bauhof, F.; Gonano, L. (Golder Associates, Inc., Bellevue, WA (USA)). Mar 1983. 694p. NTIS, PC A99/MF A01 - GPO \$13. 00. Order Number DE83901895.

Portions are illegible in microfiche products.

The media and sites considered include: (1) basalt at Hanford, Washington; (2) tuff at Yucca Mountain, Nevada Test Site; (3) domal salt at specific Gulf Coast sites; (4) bedded salt at an unspecified site; and (5) granite at an unspecified site. A licensing perspective is outlined and a defensible rationale developed and utilized for the test selection process. This rationale essentially consists of: establishing the information needs for construction authorization; assessing the relevant capabilities of available tests; and matching the capabilities of specific tests to the perceived information needs. The information needs at any time consist of the additional information (if any) needed in order to predict satisfactory repository system performance with the required level of confidence, and thus are a function of: the significance of the repository engineered components and site characteristics to system performance; the currently available information, which may be supplemented with time; and the acceptable level of confidence in satisfactory performance for each licensing step. Determination of the acceptable levels of confidence and the significance of repository system components is outside the scope of this report. Suitable assumptions have thus been made regarding the development of information needs for construction autho- rization by the time of initial site characterization reports submittals. Tests which are available and respond to the perceived media/site specific information needs, either by simulation or assessment of site characteristics, are identified and their capabilities assessed. Specific in situ tests are investigated and described in detail. Research and development which might be effective in improving tests capabilities have been recommended.

745 (NUREG/CR-3066) Parameters and variables appearing in repository-siting models. Mercer, J.W.; Thomas, S.D.; Ross, B. (GeoTrans, Inc., Reston, VA (USA)). Dec 1982. 258p. NTIS, PC A12/MF A01 - GPO \$8.00. Order Number DE83900701.

Portions of document are illegible.

Included in this report is a summary of data characterizing the parameters and variables appearing in repository siting models. These data cover the processes of saturates flow, unsaturated flow, surface water flow, geochemistry, beat transport, solute transport, and geomechanical response. Definitions and ranges of values are provided for equation parameters, source terms, dependent variables, boundary conditions, and initial conditions for the equations that are solved in the repository siting models. The data were compiled to help guide the selection of values of parameters and variables to be used in benchmark problems.

746 (NUREG/CR-3097) Benchmark problems for repository siting models. Ross, B.; Mercer, J.W.; Thomas, S.D.; Lester, B.H. (GeoTrans, Inc., Reston, VA (USA)). Dec 1982. 147p. NTIS, PC A07/MF A01 - GPO \$6.50. Order Number DE83900956.

Portions are illegible in microfiche products.

This report describes benchmark problems to test computer codes used in siting nuclear waste repositories. Analytical solutions, field problems, and hypothetical problems are included. Problems are included for the following types of codes: ground-water flow in saturated porous media, heat transport in saturated media, ground-water flow in saturated fractured media, heat and solute transport in saturated porous media, solute transport in saturated porous media, solute transport in saturated fractured media, and solute transport in unsaturated porous media.

747

(NUREG/CR-3111-Vol.1) Assessment of the proposed rule (10CFR60) for disposal of high-level radioactive wastes in geologic repositories. Volume I. Chu, M.S.; Ortiz, N.R.; Wahi, K.K.; Pepping, R.E.; Campbell, J.E. (Sandia National Labs., Albuquerque, NM (USA)). Jun 1983. Contract AC04-76DP00789. 188p. (SAND-82-2969-Vol.1). NTIS, PC A09/MF A01 - GPO. Order Number DE83016862.

This report summarizes the work performed for the Nuclear Regulatory Commission (NRC) to assess its proposed Rule (10CFR60) for disposal of high-level radioactive wastes in geologic repositories. The objectives of this project were to provide information that NRC could use to evaluate the rationale for the technical requirements in the Rule, to respond to public comments on the proposed Rule, and to analyze the benefits of alternative criteria for the final rule. Three of the numerical criteria of the Performance Objectives of the Rule were analyzed in detail in this study. The three criteria pertain to: a containment period, a controlled release rate, and a pre-waste emplacement ground-water travel time. The Rule requires geologic repositories to comply with the draft EPA Standard (40CFR191); accordingly, this study assesses the relevance of the three numerical criteria to the draft EPA Standard. A series of parametric analyses were performed on the potential releases of radionuclides to the accessible environment in order to determine the impact of the three criteria on compliance with the draft EPA Standard. The analyses were performed for three geologic media: basalt, bedded salt, and tuff. The study also examined the achievability of the three numerical criteria based on an assessment of the existing technology. Parameters and techniques that could be used to assess compliance were identified. In addition to the three numerical criteria, certain other requirements were also addressed in varying degrees of detail. The structure and content of the Rule were analyzed for completeness, consistency, and redundancy. As part of this study, a panel of experts was formed to address and debate the issues relating to the requirements contained in the proposed Rule. A number of recommendations have 
been made in the report based on the analyses performed and the outcome of the panel discussions.

748 (NUREG/CR-3206) Unsaturated flow and transport through fractured rock - related to high-level waste repositories. Final report. Phase I. Evans, D.D. (Arizona Univ., Tucson (USA). Dept. of Hydrology and Water Resources). Mar 1983. 242p. NTIS, PC A11/MF A01 - GPO \$7.50. Order Number DE83901845.

Portions are illegible in microfiche products.

The principal objective of this study was to assess the state-ofthe-art of characterizing unsaturated fractured rock geologic settings for possible high-level radioactive waste isolation. The primary approach has been to evaluate existing methods and computer models developed for unsaturated granular material and saturated fractured rock for application to unsaturated rock. The experimental methods explored relate to: (1) rock matrix characterization, (2) fracture characterization, (3) rock moisture status, and (4) water movement on a mesoscale. A model was developed to demonstrate the decrease in water conductance of fractures with decrease in water potential. Another model was developed to show vapor transport in the nonisothermal zone surrounding a repository, thus causing a drying and a wetting zone. For macroscale modeling of a repository setting, three fields are considered: (1) near-field, the zone surrounding the repository which is influenced by the heat generated by the radioactive waste, (2) meso-field, the unsaturated zone other than the near-field and to the regional water table, and (3) far-field, the zone beneath the regional water table. Clearly the nonisothermal unsaturated near-field is most complex and no existing model is available to realistically model this zone. Models are available for the meso-field and far-field but parameter estimation is a serious problem.

749 (NUREG/CR-3219-Vol.1) Draft Technical Position Subtask 1.1: waste package performance after repository closure. Volume 1. Davis, M.S.; Schweitzer, D.G. (Brookhaven National Lab., Upton, NY (USA)). Aug 1983. Contract ACO276CH00016. 51 p. (BNL-NUREG-51658-Vol.1). NTIS, PC A04/MF A01 - GPO. Order Number DE83017108.

This document provides guidance to the DOE on the issues and information necessary for the NRC to evaluate waste package performance after repository closure. Minimal performance objectives of the waste package are required by proposed 10 CFR 60 . This Draft Technical Position describes the various options available to the DOE for compliance and discusses advantages and disadvantages of various choices. Examples are discussed dealing with demonstrability, predictability and reasonable assurance. The types of performance are considered. The document summarizes presently identified high priority issues needed to evaluate waste package performance after repository closure. 20 references, 7 tables.

760 (NUREG/CR-3235-Vol.1) Technical assistance for regulatory development: review and evaluation of the EPA standard 40 CFR191 for disposal of high-level waste. Vol. 1. Ortiz, N.R.; Wahi, K.K. (Sandia National Labs., Albuquerque, NM (USA)). Apr 1983. Contract AC0476DP00789. 21 p. (SAND82-1557-Vol.1). NTIS, PC A02/MF A01. Order Number DE83011884.

Portions are illegible in microfiche products.

The Environmental Protection Agency (EPA) has prepared a draft Standard (40CFR 191, Draft 19) which, when finalized, will provide the overall system requirements for the geologic disposal of radioactive waste. This document (Vol. 1) provides an Executive Summary of the work performed at Sandia National Laboratories, Albuquerque, NM, under contract to the US Nuclear Regulatory Commission to analyze certain aspects of the draft Standard. The issues of radionuclide release limits, interpretation, uncertainty, achievability, and assessment of compliance with respect to the requirements of the draft Standard are addressed based on the detailed analyses presented in five companion volumes to this report.

761 (NUREG/CR-3405-Vol.1) Long-term performance of materials used for high-level waste packaging. Annual report, March 1982-April 1983. Vol.1. Stahl, D.; Miller, N.E. (Battelle Columbus Labs., OH (USA)). Jul 1983. 196p. (BMI-2105-Vol. 1). NTIS, PC A09/MF A01 - GPO \$6.00. Order Number DE83902820.
The Nuclear Regulatory Commission's Office of Nuclear Reg latory Research is developing predictive methodologies to assist in ev: uating the Department of Energy's application for construction al.operation of deep-mined geologic repositories for high-level radioactive waste disposal. As part of the NRC's effort, Battelle's Columbus Laboratories is investigating the long-term performance of materials used for high-level waste packages. This report documents the results of our first year's investigations. The topics addressed are internal and external corrosion and hydrogen ombrittlement of the waste container, waste form degradation, accelerated testing and statistics, and model development both for separate effects and for multiple effects on the integrity of the waste package. Experimental work has been initiated to address modeling needs, either to fill in missing data, to confirm existing data at protoypic conditions, or to evaluate mechanisms at accelerated test conditions. A first-generation system code using simple separate-effects correlations is described. 149 references.

762 (NUREG/CR-3427-Vol.1) Long-term performance of materials used for high-level waste packaging. Quarterly report, April-June 1983. Stahl, D.; Miller, N.E. (comps.). (Battelle Columbus Labs., OH (USA)). Aug 1983. 54p. NTIS, PC A04/MF A01 - GPO \$4.25. Order Number DE84900307.

The Nuclear Regulatory Commission is responsible for the licensing of deep-mined geologic repositories for high-level radioactive waste disposal. The NRC's Office of Nuclear Regulatory Research is developing the technical capability to assess the Department of Energy's compliance with applicable regulations. As part of the NRC's effort, Battelle's Columbus Laboratories is investigating the long-term performance of materials used for high-level waste packages. The experimental program for investigating waste form degradation is in preparation, and the mathematical description of waste glass dissolution has been refined. Groundwater corrosion studies have been nearly completed for titanium and are under way for steel container materials. Internal glassstainless steel corrosion tests have been initiated and pitting chemical interactions are being evaluated. A first-cut physical description of general corrosion of container materials in contact with groundwaters is near completion. An improved description of fluid flow through a nuclear waste package was developed, along with a comprehensive method for tracking release of specific radioisotopes. Efforts continue in water-chemistry studies, accelerated testing, and procedure development. This report documents investigations performed during AprilJune, 1983. 7 references.

763 (NUREG/CR-3472-Vol.1) Surface properties and performance prediction of alternative waste forms. Annual report, October 1, 1981-September 30, 1982. Volume 1. Hench, L.L.; Clark, D.E. (Florida Univ., Gainesville (USA). Coll. of Engineering). Sep 1983. 102p. NTIS, PC A06/MF A01 - GPO \$4.75. Order Number DE84900049.

Leaching mechanisms of simulated HLW-borosilicate glasses are studied by various surface spectroscopic measurement and solution chemistry analysis. The variables included in this annual report are: degree of glass fracture, glass composition, devitrification, and wet-dry cyclic effect.

764 (NVO-196-24-Rev.2) Bibliography of the published reports, papers, and articles on the Nevada Nuclear Waste Storage Investigations. (USDOE Nevada Operations Office, Las Vegas). Jul 1982. 64p. NTIS, PC A04/MF A01. Order Number DE82019280.

This document is a bibliography of the published reports, papers, and articles on the Nevada Nuclear Waste Storage Investigations (NNWSI). A brief history is included to familarize the reader with the general direction and activity highlights of the NNWSI and to give the reader some insight into the kinds of bibliographic references to be found in this document. The bibliography is categorized by principal NNWSI participant organization; participant-sponsored subcontractor reports, papers, and articles are included in the sponsoring organization's bibliography list. The principal participant bibliography listin! are arranged in chronological order by title. An author index is provide after the bibliography. Copies of most NNWSI reports and other docL ments published by the DOE and the principal participant organizations are available at nominal cost from, National Technical Information Service, US Department of Commerce, 5285 Port Royal Road, Springfield, VA 22161. 
755 (NVO-196-24(Rev.3)) Bibliography of the published reports, papers, and articles on the Nevada nuclear waste storage investigations. (USDOE Nevada Operations Office, Las Vegas). Apr 1983. 84p. NTIS, PC A05/MF A01. Order Number DE83012511.

This document is a bibliography of the published reports, papers, and articles on the Nevada Nuclear Waste Storage Investigations (NNWSI). A brief history is included to familiarize the reader with the general direction and activity highlights of the NNWSI and to give the reader some insight into the kinds of bibliographic references to be found in this document. The bibliography is categorized by principal NNWSI participant organization. Participant-sponsored subcontractor reports, papers, and articles are included in the sponsoring organization's bibliography list. The principal participant bibliography listings are arranged in chronological order by title. An author index is provided after the bibliography.

766 (NV0-196-30) Nevada nuclear waste-storage investigations. Quarterly report, January-March 1982. (Department of Energy, Las Vegas, NV (USA). Nevada Operations Office). Jun 1982. 73p. NTIS, PC A04/MF A01. Order Number DE82016400.

Purpose of the investigations is to determine whether specific underground rock masses are suitable for the permanent disposal of high-level wastes, and whether the Nevada Test Site would qualify as a suitable repository site. The work is broken into the following areas: systems, waste package, site, repository, regulatory and institutional, test facilities, land acquisition, and program management. Emphasis is on studying the tuffs under Yucca Mountain: backfill, diffusion, permeability, geology, hydrology, etc. (DLC)

767 (NVO-196-31) Nevada Nuclear-Waste-Storage Investigations. Quarterly report, April-June 1982. (Department of Energy, Las Vegas, NV (USA). Nevada Operations Office). Sep 1982. 94p. NTIS, PC A05/MF A01. Order Number DE82021099.

The Nevada Nuclear Waste Storage Investigations (NNWSI) are studying the Nevada Test Site (NTS) area to establish whether it would qualify as a licensable location for a commercial nuclear waste repository; determining whether specific underground rock masses in the NTS area are technically acceptable for permanently disposing of highly radioactive solid wastes; and developing and demonstrating the capability to safely handle and store commercial spent reactor fuel and high-level waste. Progress reports for the following eight tasks are presented: systems; waste package; site; repository; regulatory and institutional; test facilities; land acquisition; and program management. Some of the highlights are: A code library was established to provide a central location for documentation of repository performance assessment codes. A two-dimensional finite element code, SAGUARO, was developed for modeling saturated/unsaturated groundwater flow. The results of an initial experiment to determine canister penetration rates due to corrosion indicate the expected strong effect of toxic environmental conditions on the corrosion rate of carbon steel in tuff-conditioned water. Wells USW-H3 and USW-H4 at Yucca Mountain have been sampled for groundwater analysis. A summary characterizing and relating the mineralogy and petrology of Yucca Mountain tuffs was compiled from the findings of studies of core samples from five drill holes.

758 (NVO-196-32) Nevada nuclear waste storage investigations. Quarterly report, July-September 1982. (USDOE Nevada Operations Office, Las Vegas). Aug 1983. 85p. NTIS, PC A05/MF A01. Order Number DE83017610.

The purpose of the DOE National Waste Terminal Storage (NWTS) Program is to safely dispose of spent reactor fuel and the highlevel and transuranic wastes associated with commercial nuclear reactor fuel cycles. The Nevada Nuclear Waste Storage Investigations (NNWSI), which are a part of the NWTS program, are studiying the Nevada Test Site (NTS) area to establish whether it would qualify as a licensable location for a commercial nuclear waste repository; determining whether specific underground rock masses in the NTS area are technically acceptable for permanently disposing of highly radioactive solid wastes; and developing and demonstrating the capability to safely handle and store commercial spent reactor fuel and high-level waste. High-precision analyses of tuff samples have been carried out using neutron activation analysis. X-ray diffraction analyses of samples from the für tuffaccous horizons of interest at Yurca Mnuntains were complet- ed. Hydrologic testing was conducted in Well UE25b-1 to determine the chemistry of the groundwater in Drill Hole Wash at Yucca Mountain. Laboratory single-fracture tracer experiments were performed on rocks containing both a natural and an artificial fracture. Two types of hydrothermal experiments were conducted to examine tuff mineral stability and fluid composition in equilibrium with tuff at elevated temperatures. Calculations made using current rainfall conditions showed water travel time from the surface to the water table at Yucca Mountain to exceed the 10 CFR 60 requirements. The first phase of the small-diameter heater experiments in nonwelded tuff was initiated; the preliminary findings of this experiment are different than for the experiment conducted in welded tuff. Initial mechanical testing of both jointed and intact samples of nonwelded zeolitized tuffs was completed.

769 (NVO-196-33) Nevada Nuclear Waste Storage Investigations. Quarterly report, October-December 1982. (USDOE Nevada Operations Office, Las Vegas). Aug 1983. 74p. NTIS, PC A04/MF A01. Order Number DE83017611.

This document is a compilation of the technical progress of the principal Nevada Nuclear Waste Storage Investigations (NNWSI) participants during the first quarter of FY 1983. The NNWSI Project Work Breakdown Structure for FY 1983 is comprised of nine tasks: systems; waste package; site; repository; regulatory and institutional; exploratory shaft; test facilities; land acquisition; and program management. The progress in each task is reported.

760 (NVO-196-34) Nevada Nuclear Waste Storage Investigations. Quarterly report, January-March, 1983. (USDŌE Nevada Operations Office, Las Vegas). Sep 1983. 75p. NTIS, PC A04/MF A01. Order Number DE84001834.

The Nevada Nuclear Waste Storage Investigations (NNWSI), include site-exploration and regional studies of the Nevada Test Site (NTS) area to establish whether a technically acceptable site exists for consideration as a potential location for one of the commercial nuclear waste repositories to be established. Yucca Mountain is located on and adjacent to the southwestern boundary of the NTS about 100 miles northwest of Las Vegas. The mountain is a long narrow ridge generally running north and south with a steep western slope and a gentler eastern slope. The underlying strata are inclined at three to eight degrees to the east. The ridge stands from about 1000 to 1200 feet above the surrounding alluvial flats. One of the characteristics that makes Yucca Mountain attractive is the presence of a potentially suitable repository host rock known as tuff; tuff is a highly sorptive rock composed of solidified and welded volcanic ash and detritus (rock fragments and other loose material). Tuff has good physical, mechanical, and chemical characteristics for nuclear waste containment and for preventing waste from reaching the biosphere. Each layer of tuff was formed separately millions of years ago by volcanic activity. Yucca Mountain also has an extremely deep static water table - more than $\mathbf{1 7 0 0}$ feet below land surface. This feature makes it possible to choose the most favorable repository horizon in the unsaturated zone where water would not be expected to fill a repository because of very low water infiltration rates. The horizon selected for detailed at-depth characterization is a unit of the Topopah Spring tuff about 1200 feet below land surface and about 550 feet above the static water table.

761 (NVO-196-36) Nevada Nuclear Waste Storage Investigations: FY 1981 project plan and FY 1982 forecast. (USDOE Nevada Operations Office, Las Vegas). Aug 1983. 86p. NTIS, PC A05/MF A01. Order Number DE83016456.

The project consists of systems studies, waste packaging, siting studies, repository studies, and licensing strategies for the development of a repository for high-level radioactive wastes and spent fuels at the Nevada Test Site. This document presents the FY 1981 project plans, as well as the history, organization, management and planned activities for FY 1982. The 1981 project guidance statement from ONWM is included. (DMC)

762 (NVO-196-37) Nevada Nuclear Waste Storage Investigations: FY 1982 project plan. (USDOE Nevada Operations Office, Las Vegas). Aug 1983. 79p. NTIS, PC A05/MF A01. Order Number DE83016187.

Since late FY 1977 , the Nevada Nuclear Waste Storage Investigations (NNWSI) Project has been investigating the Nevada Test Site (NTS) area to determine whether underground rock masses on or near the NTS are suitable for the permanent isolation of spent reactor fuel 
or high-level radioactive wastes. During FY 1981, the Project developed, evaluated, and began exercising a method for screening the southwest NTS and contiguous lands for nuclear waste repository locations. The Project continued to concentrate on evaluating the technical acceptability of Yucca Mountain as a potential repository location. The drilling program was accelerated to meet the new Administration's decision-making schedule. In addition, media characterization and evaluation work, including studies of thermal-mechanical response and thermal degradation of minerals, continued. Engineering studies leading toward the Exploratory Shaft and repository conceptual designs were initiated. Tuff-speciffc waste package assessments and evaluations and far-field radionuclide release scenario analyses were also initiated. Existing environmental data inventories will be used to plan FY 1982 environmental studies in selected topical areas where additional information is needed. In addition, three other related activities are in progress within the NNWSI. The spent fuel storage test being conducted 1400 feet underground at the NTS in the Climax granite has been under way since April 1980. Work continued on two developmental field radionuclide migration experiments, one near the Climax experiment area and one in G-Tunnel at the NTS. The third major related aotivity is focused on the development and demonstration of the capability to safely handle, encapsulate, and store commercial spent reactor fuel in a pusslve illcrim storage made at or near the earth's surface.

763 (NVO-250) Overview of energy and mineral resonuces for the Nevada nuclear-waste-storage investigations, Nevada Test Site, Nye County, Nevada. Bell, E.J.; Larson, L.T. (Department of Energy, Las Vegas, NV (USA). Nevada Operations Office). Sep 1982. 73p. NTIS, PC A04/MF A01. Order Number DE83001418

This report was prepared for US Department of Energy as part of the Environmental Area Characterization for the Nevada Nuclear Waste Storage Investigations (NNWSI) at the Nevada Test Site (NTS). The characterization addresses energy resources including hydrocarbons, geothermal and radioactive fuel materials, mineral resources including base and precious metals and associated minerals, and industrial minerals and rock materials which occur in the vicinity of the NNWSI area. A generalized commentary is provided on past and present mining and exploration activity, with discussions of resource potential in a context of the regional resource base and projected future demands for these resources. The purpose of the study was to document known energy and mineral resources, to identify areas of potential resources, and to delineate areas for which additional data should be obtained if a site is to be developed for high-level radioactive waste storage in the vicinity of the southwest part of the Nevada Test Site. The characterization of energy and mineral resources will be integrated by Sandia National Laboratory (SNL) personnel with a variety of other studies to develop an Environmental Area Characterization report and to plan environmental analyses as well as further resource analyses.

764 (NVO-255) Project management plan for explorutory shaft at Yucca Mountain. (USDOE Nevada Operations Office, Las Vegas). Aug 1983. 89p. NTIS, PC A05/MF A01. Order Number DE83017146.

Portions are illegible in microfiche products.

This Project Management Plan (PMP) provides the basic guidance and describes the organizational structure and procedures for the design, construction, and testing of a large-diameter Exploratory Shaft (ES) in tufraceous media as a major element within the Nevada Nuclear Waste Storage Investigations (NNWSI) project, which is a part of the National Waste Terminal Storage (NWTS) Program, US Department of Energy (DOE). The PMP encompasses activities identified as construction phase and in situ phase testing to be conducted from the ES through September 30, 1986. Specific topics addressed are the ES project objectives, the management organization and responsibilities, functional support requirements, work plan (including quality assurance aspects), work breakdown structure, milestone schedule, logic diagram, performance criteria, cost estimates, management control systems, procurement plan, test plan, and environmental, health and safety plans.

765 (NVO-258) Nevada nuclear waste storage investigations site-characterization activities: preliminary atmospheric assessment of a nuclear waste repository. Bowen, J.L.; Egami, R.T. (Nevada Univ., Las Vegas (USA). Desert Research Inst.).
May 1983. 50p. NTIS, PC A03/MF A01. Order Number DE83011476.

A preliminary assessment was made of nonradiological air pollution caused by a high level nuclear waste repository in the southwest part of NTS. Basic assumptions about repository design are made from information concerning a repository for spent nuclear fuel in granite. Emissions and emission rates are determined for construction and operations while decommissioning is also discussed. Emissions include suspended soil particles from surface facility construction waste rock transport, and wind erosion and gaseous and particulate emissions from the burning of fossil fuel. The maximum 24-hour concentrations are estimated using the Valley model because of the complex terrain features surrounding the repository. These concentrations show high values for fugitive dust near the site exceeding NAAQS and PSD increments during construction. Regulatory requirements by federal and State of Nevada agencies are presented. PSD is described and some attempt to determine its applicability is made, although the actual determination has to be made by the proper regulatory agency. State permits and their possible requirements for meteorological and air quality monitoring are briefly discussed. It is expected that at least meteorological and TSP measurements would be required. Again the actual requirements have to be made by the. regulatory agency.

768 (NVO-263) Public hearings panel report: a summary of public concerns regarding the characterization of a repository site In Nevada. (USDOE Nevada Operations Office, Las Vegas). Nov 1983. 196p. NTIS, PC A09/MF A01. Order Number DE84002240.

This report summarizes testimony given during public hearings on March 30,1983, in Las Vegas, Nevada, and March 31, 1983, in Reno, Nevada, regarding the proposed nomination of Yucca Mountain as a candidate for intensive site characterization to determine whether it is suitable for a high-level radioactive waste repository. It also summarizes the written comments received by the Department of Energy through April 25, 1983, an announced deadline for written submissions.

767 (NVO-266) Cultural resources overview for the Nevada Nuclear Waste Storage Investigations, Nevada Test Site, Nye County, Nevada. Pippin, L.C.; Zerga, D.L. (Department of Energy, Las Vegas, NV (USA). Nevada Operations Office). Nov 1983. Contract AC08-81NV10162. 122p. NTIS, PC A06/MF A01. Order Number DE84002824.

Chapters are devoted to: the natural setting; history of archaeological research on the Nevada Test Site; the cultural setting; and known hisloric and prehistotic cultural resources within the Nevada Nuclear Waste Storage Investigations project area.

768 (NVO-267) Annotated bibliography of cultural resources literature for the Nevada Nuclear Waste Storage Investigations. (Department of Energy, Las Vegas, NV (USA). Nevada Operations Office). Nov 1983. Contract AC0881NV10162. 63p. NTIS, PC A04/MF A01. Order Number DE84002825.

This annotated bibliography of the cultural resources literature pertinent for the Nevada Nuclear Waste Storage Investigations was assembled in order to (1) identify and evaluate the prehistoric and historic properties previously recorded in the Nevada Nuclear Waste Storage Investigations Project Area of southern Nye County, Nevada, (2) identify and develop research problems that have been and/or oould be addressed by the cultural resources of this area, (3) isolate factors that might be important in the selection of a potential locality for a high level nuclear waste repository in the project area, and (4) critically evaluate the adequacy and current status of cultural recouroes knowledge in the project area. 195 references.

769 (ONI-3) Projected costs for mined geologic repositories for dispoal of commercial nuclear wastes. Waddell, J.D.; Dippold, D.G.; McSweeney, T.I. (Battelle Memorial Inst., Columbus, OH (USA). Office of National Waste Terminal Storage Integration). Dec 1982. Contract AC06-76RL01830. 57p. NTIS, PC A04/MF A01. Order Number DE83004872.

Portions are illegible in microfiche products.

This documen reports cost estimates for: (1) the exploration and development activities preceding the final design of terminal isolation facilities for disposal of commercial high-level waste; and (2) the design, construction, operation, and decommissioning of such facilities. Explo- 
ration and evelopment costs also include a separate cost category for related programs such as subseabed research, activities of the Transportation Technology Center, and waste disposal impact mitigation activities.

770 (ONWI-2-Suppl.3) ONWI library reports list. (Battelle Memorial Inst., Columbus, OH (USA). Office of Nuclear Waste Isolation). Sep 1982. Contract ACO676RL01830. 108p. NTIS, PC A06/MF A01. Order Number DE82022248.

This document is the fourth in a series listing reports on nuclear waste isolation received by the Library of the Office of Nuclear Waste Isolation (ONWI).

771 A12/MF A01.

(ONWI-9-81-3, pp 14-34) Systems. 1981. NTIS, PC

In Technical progress report, 1 April-30 June 1981.

Summarics of quarterly highlights under the task entitled Systems are included in this section. Topics include: socio-conomic assessment, systems analysis, alternative disposal concepts, and performance assessment for the permanent isolation of nuclear wastes. A computer model was developed to assist in preparing preliminary estimates of repository costs and the cost impact of design changes. The model uses cost data from numerous detailed architectural and engineering estimates as a basis. Significant progress was made in the laboratory research on the closure of fractures under conditions of high temperature and pressure. New methods of measuring the extent of fracture closure are now being developed. The two-dimensional analytical transport code, UCBNE 25, which predicts radionuclide concentration in steady ground-water flows, was used to predict the far-field migration of radionuclides released from waste packages at WIPP and BWIP. An additional code for radionuclide transport through a fractured rock has been modified to include decay chains. A method was developed which yields physical insight into leach rate trends while minimizing the

- required mathematical effort. A necessary condition, at this time, is the time-invariance of the parameters used to represent the mechanisms controlling waste form leaching. (DMC)

(ONWI-9-81-3, pp 82-144) Site. 1981. NTIS, PC A12/MF A01.

In Technical progress report, 1 April-30 June 1981.

Summaries of quarterly highlights under the task entitled Site, are presented in this section. These reports deal with the earth sciences of geophysics, geochemistry, and transport properties, and with geologic, hydrologic, and environmental characterization of sites that provide natural barriers in salt, granite, and shale. In the Permian Basin, portions of the study area meeting preliminary screening specifications were identified and recommended for further investigation. The Texas Bureau of Economic Geology completed detailed stratigraphic cross sections of the Upper Permian, Triassic, and Tertiary strata in the Palo Duro Basin. The salt dome site characterization planning effort centered on meetings in Texas, Louisiana, and Mississippi to discuss the key issues of conccrn. In the Paradox Basin, primary effort was directed toward preparing the five-volume area characterization report, laboratory analysis of core samples from the Salt Valley and Gibson Dome boreholes, and re-entry of the existing Kubat hole. Work is being conducted in the Lake Superior region to identify potentially suitable intrusions through a study of existing literature. Pacific Northwest Laboratory continued gathering baseline environmental data in the Northeast region. Meetings were held with Argonne National Laboratory (performing the Northeast geologic characterization), wherein agreement was reached on the boundaries of the study region.Oak Ridge National Laboratory completed gathering about 95 percent of the required environmental baseline data for the Southeast region. Laboratory observations of diagenetic alterations of the Conasauga shale suggest that in a HLW repository in shale, the increased temperature may cause the crystallization of chlorite, quartz, and/or calcite in the small tension fracture caused by the excavation, producing a self-healing process that will decrease fracture porosity and permeability. (DMC)

773 (ONWI-9-81-3, pp 145-245) Repository. 1981. NTIS, PC $\Lambda 12 / \mathrm{MF}$ A01.

In Technical progress report, 1 April-30 June 1981.

Summaries of quarterly highlights under the task entitled Repository arc presented in this section. The repository reports involve studies to build a data base; equipment, instrument, and seal development; repository engineering and conceptual design; and performance evaluation. The salt model pillar studies project tested a series of model pillars under temperature and pressure conditions that would be expected in an actual disposal operation. The results indicate that for each sample there is a high rate of deformation that decreases with time. This decrease in deformation rates is shown to continue to 600 hours. The convective heat transfer in room and pillar geometries project made significant progress in obtaining a correlation for fully developed Nusselt numbers in a rectangular duct with aspect ratio 1.0 . Reynolds numbers ranged from 98,300 to 32,000 . The main activity of the analysis of flowthrough fractured crystalline rock project was to develop the basic statistical parameters needed to characterize the fracture system at Stripa. In the project on rock behavior under elevated pressure and temperature, calibration of the thermal conductivity measurement was achieved with the completion of the run on the reference standard of GE 124 fused silica. A draft report on numerical simulations of earthquake effects on tunnels for generic nuclear waste repositories, by Science Applications, was completed. The objectives of this generic study are to use numerical modeling techniques to determine under what conditions seismic waves generated by an earthquake might cause instability to an underground opening,or cause fracturing and joint movement that would lead to an increase in the permeability of the rock mass. In repository sealing work, the results from the plug flow test have been analyzed and are in good agreement with the results obtained from the Bell Canyon borehole plug test.

774 (ONWI-9-81-3, pp 247-250) Test facilities and excavations. 1981. NTIS, PC A12/MF A01.

In Technical progress report, 1 April-30 June 1981.

Summaries of quarterly highlights under the task entitled Test Facilities and Excavations are presented in this section. This area is concerned with facilities in both salt and alternative media which will eventually be used for laboratory and in situ testing of the relationships among the package, repository, and site. In the alternative geology test facility mine screening project, computer programs for screening and ranking were completed. A list of 66 mines meeting the general identification criteria was developed. The screened data base was supplemented by additional data from various sources. (DMC)

775 (ONWI-9-81-3) Technical progress report, 1 April-30 June 1981. (Battelle Memorial Inst., Columbus, $\mathrm{OH}$ (USA). Office of Nuclear Waste Isolation). 1981. Contract AC06-76RL01830. 264p. NTIS, PC A12/MF A01. Order Number DE82015780.

Portions of document are illegible.

This report describes the technical accomplishments during the quarter ending June 1981, on the commercial nuclear waste management programs under the direction of the Office of Nuclear Waste Isolation (ONWI). The ONWI program is organized into 8 tasks entitled: systems, waste package, site, repository, regulatory and institutional, test facilities and excavations, land acquisition, and program management. Principal investigators in each of these areas have submitted summaries of quarterly highlights for inclusion in this report. Separate abstracts have been prepared for 5 of these tasks for inclusion in the Energy Data Base. (DMC)

776 (ONWI-9(81-4)) Technical progress report for the quarter, 1 July-30 September 1981. (Battelle Memorial Inst., Columbus, $\mathrm{OH}$ (USA). Office of Nuclear Waste Isolation). 1981. Contract AC06-76RL01830. 244p. NTIS, PC A11/MF A01. Order Number DE83005479.

Portions of document are illegible.

This report describes the technical accomplishments during the period from July through September 1981 on the commercial nuclear waste management programs under the direction of the Office of Nuclear Waste Isolation (ONWI). This office has lead responsibilities in the National Waste Terminal Storage program structure for oversight and for development of the general technology, and for geologic exploration of non-DOE land. A project management group, ONWI directs and evaluates studies, research, and development performed by subcontractors on repository sites and designs. The scope of ONWI activities Includes providing the technology and facilities for the terminal isola. tion of these wastes by disposal in mined repositories deep underground in stable geologic formations. The ONWI program is organized into eight tasks: Systems; Waste Package; Site; Repository; Regulatory and Institutional; Test Facilities and Excavations; Land Acquisition; and 
Program Management. The accomplishments in each of these tasks are reported.

777 (ONWI-9(82-1)) Technical progress report for the quarter, 1 October-31 December, 1981. (Battelle Memorial Inst., Columbus, OH (USA). Office of Nuclear Waste Isolation). 1981. Contract AC06-76RL01830. 179p. NTIS, PC A09/MF A01. Order Number DE83005478.

Portions of document are illegible.

This report describes the technical accomplishments during the period from October through December 1981 on the commercial nuclear waste management programs under the direction of the Office of Nuclear Waste Isolation (ONWI). This offce has lead responsibilities in the National Waste Terminal Storage program structure for oversight and for development of the general technology, and for geologic exploration of non-DOE land. A project management group, ONWI directs and evaluates studies, research, and development performed by subcontractors on repository sites and designs. The scope of ONWI activities includes providing the technology and facilities for the terminal isolation of these wastes by disposal in mined repositories deep undergruund in stahle genlngis formations. The ONWI program is organized into eight tasks: Systems; Waste Package: Site; Repository; Regulatory and Institutional; Test Facilities and Excavations; Land Acquisition; and Program Management. The accomplishments in each of these tasks are reported.

778

(ONWI-9(82-2)) Technical progress report for the quarter 1 January-31 March 1982. (Battelle Memorial Inst., Columbus, $\mathrm{OH}$ (USA). Office of Nuclear Waste Isolation). 1982. Contract AC06-76RL01830. 197p. NTIS, PC A09/MF A01. Order Number DE83008477.

This report describes the technical accomplishments on the commercial nuclear waste management programs under the direction of the Office of Nuclear Waste Isolation (ONWI). The scope of ONWI activities includes providing the technology and facilities for the terminal isolation of nuclear wastes by disposal in mined repositories deep underground in stable geologic formations. The ONWI program is organized into the following eight tasks: (1) systems; (2) waste package; (3) site; (4) repository; (5) regulatory and institutional; (6) test facilities and excavations; (7) land acquisition; and (8) program management. Progress reports and summaries are presented for the first 6 tasks.

779 (ONWI-9(82-3)) Technical progress report for the quarter, 1 April-30 June 1982. (Battelle Memorial Inst., Columbus, OH (USA). Office of Nuclear Waste Isolation). 1982. Contract ACO6-76RL01830. 202p. NTIS, PC A10/MF A01. Order Number DE83007304.

Portions are illegible in microfiche products. Original copy availablc until stock is exhausted.

This report describes the technical accomplishments during the perion from April through June 1982 on the commercial nuclear waste management programs under the direction of the Office of Nuclear Waste Isolation (ONWI). This office has lead responsibilities in the National Waste Terminal Storage program structure for oversight and for development of the general technology, and for geologic exploration of non-DOE land. A project management group, ONWI directs and evaluates studies, research, and development performed by subcontractors on repository sites and designs. The scope of ONWI activities includes providing the technology and facilities for the terminal isolation of these wastes by disposal in mined repositories deep underground in stable geologic formations. The ONWI program is organized into eight tasks: Systems; Waste Package; Site; Repository; Regulatory and Institutional; Test Facilities and Excavations; Land Acquisition; and Program Management. The accomplishments in each of these tasks are reported.

780 (ONWI-9(82-4)) National Waste Terminal Storage Program. Technical progress report for the quarter 1 July-30 September 1982. (Battelle Memorial Inst., Columbus, OH (USA). Office of Nuclear Waste Isolation). 1982. Contract AC06-76RL01830. 191p. NTIS, PC A09/MF A01. Order Number DE83012117.

Portions are illegible in microfiche products.

The National Waste Terminal Storage (NWTS) program was established in 1976 and given the responsibility of developing a system for the permanent isolation of nuclear wastes. NWTS is part of the
Office of Nuclear Waste Management in the US Department of Energy. The establishment of NWTS signaled the acceleration of the national program. The purpose of the NWTS program is to develop the technology and facilities required for the disposal of commercial spent nuclear fuel, defense high-level waste, and transuranic waste, ensuring public health and safety and protection of the environment. The program includes facilities for receiving, encapsulation, and disposal of wastes. The scope of the NWTS repository development effort includes comprehensive geologic exploration, technology development, equipment testing, and model verification activities, as well as the design, licensing, construction, operation, and decommissioning activities required to provide geologic repositories and spent fuel handling and packaging facilities. Development of the technology, methodology, and facilities for interim spent fuel storage, waste treatment, and waste transportation, which are interfacing activities with the terminal isolation activity in the national waste-management program, are being carried out in other DOE efforts.

781 (ONWI-9(83-1)) Technical progress report for the quarter 1 Octobcr-31 December 1982. (Battelle Memnnrial Inst., Columbus, $\mathrm{OH}$ (USA). Office of Nuclear Waste Isolation). 1982. Contract ACO6-76RL01830,AC02-03CII10140. 125p. N'IIS, PC A06/MF A01. Order Number DE84001687.

Portions are illegible in microfiche products. Original copy available until stork is exhausted.

This report describes the technical accomplishments during the period from October through December 1982 on the commercial nuclear waste management programs under the direction of the Office of Nuclear Waste Isolation (ONWI). The ONWI program is organized into eight tasks: systems, waste package, site, repository, regulatory and institutional, test facilities and excavations, land, and program management. The progress in each of these tasks is reported.

782 (ONWI-102-Vol.1) Area environmental characterization report of the Dalhart and Palo Duro basins in the Texas Panhandle. Volume I. Dalhart Basin. (NUS Corp., Gaithersburg, MD (USA)). Sep 1982. Contract AC06-76RL01830. 193p. NTIS, PC A09/MF A01. Order Number DE83007219.

Portions are illegible in microfiche products. Original copy available until stock is exhausted.

This area report describes the environmental characteristics of the Dalhart and Palo Duro basins of the Texas Panhandle portion of the Permian basin. Both basins are rather sparsely populated, and the overall population is decreasing. The economic base is centered on agribusiness and manufacturing. Most of the potentially conflicting land uses in both basins (i.e., parks, historic sites) occupy small land areas, with the exception of a national grassland in the Dalhart and military air training routes in both basins. Ground transportation in the Dalhart basin is adequate, and it is well developed in the Palo Duro basin. In both basins irrigation constitutes the principal water use, and groundwater is the principal source. However, the dominant aquifer, the Ogallala, is being depleted. Both basins conslst primarily of grasslands, rangelands, and agricultural areas. No critical terrestrial or aquatic habitats have been identified in the basins, though several endangered, threatened, or rare terrestrial species occur in or near the basins. Aquatic resources in both basins are limited because of the intermittent availability of water and the high salt content of some water bodies. Playa lakes are common, though usually seasonal or rain dependent. The climate of the area is semiarid, with low humidity, relatively high wind speeds, and highly variable preipitation. Restriotive dispersion condi. tions are infrequent. National ambient secondary air quality standards for particulates are being exceeded in the area, largely because of fug:tive dust, although there are some particulate point sources.

783 (ONWI-102-Vol.2) Area environmental characterization report of the Dalhart and Palo Duro basins in the Texas Panhandle. Volume II. Palo Duro basin. (NUS Corp., Gaithersburg, MD (USA)). Sep 1982. Contract AC06-76RL01830. 303p. NTIS, PC A14/MF A01. Order Number DE83007215.

Portions are illegible in microfiche products. Original copy available until stock is exhausted.

This area report describes the environmental characteristics of the Dalhart and Palo Duro basins of the Texas Panhandlc portion of the Permian basin. Both basins are rather sparsely populated, and the overall population is decreasing. The economic base is centered on agribusiness and manufacturing. Most of the potentially conflicting land uses 
in both basins (i.e., parks, historic sites) occupy small land areas, with Ie exception of a national grassland in the Dalhart and military air aining routes in both basins. Ground transportation in the Dalhart oasin is adequate, and it is well developed in the Palo Duro basin. In both basins irrigation constitutes the principal water use, and groundwater is the principal source. However, the dominant aquifer, the Ogallala, is being depleted. Both basins consist primarily of grasslands, rangelands, and agricultural areas. No critical terrestrial or aquatic habitats have been identified in the basins, though several endangered, threatened, or rare terrestrial species occur in or near the basins. Aquatic resources in both basins are limited because of the intermittent availability of water and the high salt content of some water bodies. Playa lakes are common, though usually seasonal or rain dependent. The climate of the area is semiarid, with low humidity, relatively high wind speeds, and high variable precipitation. Restrictive dispersion conditions are infrequent. National ambient secondary air quality standards for particulates are being exceeded in the area, largely because of fugitive dust, although there are some particulate point sources.

784 (ONWI-106) Geological evaluation of Gulf Coast salt domes: overall assessment of the Gulf Interior Region. (Battelle Memorial Inst., Columbus, OH (USA). Office of Nuclear Waste Isolation; Law Engineering Testing Co., Atlanta, GA (USA)). Oct 1981. Contract AC06-76RL01830. 165p. NTIS, PC A08/MF A01. Order Number DE82015755.

Portions of document are illegible.

The three major phases in site characterization and selection are regional studies, area studies, and location studies. This report characterizes regional geologic aspects of the Gulf Coast salt dome basins. It includes general information from published sources on the regional geology; the tectonic, domal, and hydrologic stability; and a brief description the salt domes to be investigated. After a screening exercise, eight domes were chosen for further characterization: Keechi, Oakwood, and Palestine Domes in Texas; Vacherie and Rayburn's domes in North Louisiana; and Cypress Creek and Richton domes in Mississippi. A general description of each, maps of the location, property ownership, and surface geology, and a geologic cross section were presented for each dome.

786 (ONWI-109) Evaluation of area studies of the US Gulf Coast Salt Dome Basins: location recommendation report. (Battelle Memorial Inst., Columbus, OH (USA). Office of Nuclear Waste Isolation). Feb 1982. Contract AC0676RL01830. 206p. NTIS, PC A10/MF A01. Order Number DE82013192.

This document presents the evaluation methodology used for the area characterization studies in salt domes of the interior basins of the Gulf Coast region along with background information on salt domes, the site qualification process, criteria used for dome evaluation, and the technical approach applied. The geologic and environmental data are briefly summarized with reference to pertinent documents that contain details of the area characterization studies. Three recommendations are made as a result of the evaluaton: (1) to eliminate one dome from further study because of a safety flaw; (2) to eliminate three domes from further study for failure to meet site performance criteria; and (3) to continue further studies at the four domes which meet minimum standards for further consideration.

786 (ONWI-139) Preliminary investigation of the thermal and structural influence of ventilation drifts on repository rooms. Wagner, R.A. (RE/SPEC, Inc., Rapid City, SD (USA)). Jul 1983. Contract AC06-76RL01830;AC0283CH10140. 33p. NTIS, PC A03/MF A01. Order Number DE83016917.

The presence of ventilation drifts within a design of a National Waste Terminal Storage (NWTS) repository in domal salt is investigated for the thermal and structural influence on the corridors and disposal rooms. The structural influence of the ventilation drifts is emphasized since the heat and fluid flow aspects (e.g., air flow rate, air temperature, and air pathways) are beyond the scope of this study. The twoimensional numerical analyses considered two representative cross:ctions of the design perturbation of approximately $10^{\circ} \mathrm{C}$ near the venulation drift. The remaining portion of the repository should experience negligible temperature variation because of the physical presence of the ventilation drift. The mechanical results indicated a noticeable change in stress only near the region of the excavated ventilation drift. Addi- tional room and corridor deformation due to the presence of the ventilation drift was small. The ventilation drifts considered in this study do not appear to have a significant thermal or structural influence beyond the immediate vicinity of the ventilation drift. Therefore, the corridors and disposal rooms should be unaffected by the presence of the ventilation drifts discussed herein.

787 (ONWI-212) Proceedings of the 1980 National Waste Terminal Storage Program information meeting. (Battelle Memorial Inst., Columbus, $\mathrm{OH}$ (USA). Office of Nuclear Waste Isolation). 8 Dec 1980 . Contract AC0676RL01830. 386p. (CONF-801209-). NTIS, PC A17/MF A01. Order Number DE83001317.

From 2. annual National Waste Terminal Storage (NWTS) information meeting; Columbus, OH, USA (9 Dec 1980).

Portions of document are illegible.

Separate abstracts were prepared for each of the following sixteen sections in this Proceedings: overview of the National Waste Terminal Storage Program; earth sciences; socioeconomic evaluations; systems; repository design and equipment; package materials, waste form; far-field performance evaluations; repository sealing; regulatory and institutional issues; repository data base, field tests; package design; status of geologic/environmental characterization; issues in repository siting; quality assurance; near-field performance evaluations; repository data base development.

788 (ONWI-212, pp 1-26) Overview of the National Waste Terminal Storage Program. 8 Dec 1980. NTIS, PC A17/MF A01. (CONF-801209-).

From 2. annual National Waste Terminal Storage (NWTS) information meeting; Columbus, OH, USA (9 Dec 1980).

Section I on Overview of the National Waste Terminal Storage Program consists of the following 12 papers: (1) 1980-The Year of the Plan; (2) United States National Waste Terminal Storage Program; Status and Future Plans; (3) USGS Participation in the National Waste Terminal Storage Program; (4) Waste Management Institutional/ Regulatory Status; (5) Progress in Consultation and Concurrence; (6) Status of Nuclear Waste Package Development; (7) Site Selection Process for Nuclear Waste Isolation; (8) Status of geologic activities in the NWTS Program; (9) Status of geologic activities in the NWTS program: BWIP; (10) Status of activities in the Nevada Nuclear Waste Storage Investigations; (11) Status of Repository Development; and (12) Public Oversight of the NWTS Program.

789 (ONWI-212, pp 27-49) Earth Sciences. 8 Dec 1980. NTIS, PC A17/MF A01. (CONF-801209-).

From 2. annual National Waste Terminal Storage (NWTS) information meeting; Columbus, OH, USA (9 Dec 1980).

The following papers were presented at the earth science session: earth science developments in support of water isolation; development of models and parameters for ground-water flow in fractured rock masses; isotope geochemistry as a tool for determining regional groundwater flow; natural analogs of radionuclide migration; nuclide retardation data: its use in the NWTS program; and ground-water geochemistry and interaction with basalt at Hanford.

790 (ONWI-212, pp 62-76) Systems. 8 Dec 1980. NTIS, PC A17/MF A01. (CONF-801209-).

From 2. annual National Waste Terminal Storage (NWTS) information meeting; Columbus, OH, USA (9 Dec 1980).

Papers in this session describe the concept of mined geologic disposal system and methods for ensuring that the system, when developed, will meet all technical requirements. Also presented in the session are analyses of system parameters, such as cost and nuclear criticality potential, as well as a technical analysis of a requirement that the system permit retrieval of the waste for some period of time. The final paper discusses studies under way to investigate technical alternatives or complements to the mined geologic disposal system. Titles of the presented papers are: (1) Waste Isolation System; (2) Waste Isolation Economics; (3) BWIP Technical Baseline; (4) Criticality Considerations in Geologic Disposal of High-Level Waste; (5) Retrieving Nuclear Wastes from Repository; (6) NWTS Programs for the Evaluation of Technical Alternatives or Complements to Mined Geologic Repositories - Purpose and Objectives. 
791 (ONWI-212, pp 77-104) Repository design and equipment. 8 Dec 1980. NTIS, PC A17/MF A01. (CONF801209-).

From 2. annual National Waste Terminal Storage (NWTS) information meeting; Columbus, OH, USA (9 Dec 1980).

The following papers were presented at this session: (1) Implementing Technical Conservatism in Repository Design; (2) Development of General Design Criteria for Repositories; (3) Conceptual Design of a Nuclear Waste Repository in Basalt; (4) Engineering Feasibility Studies for Seven Candidate Salt Domes; (5) Seismic Effects on Underground Openings; (6) Repository Equipment Technology Development; (7) Repository Instrumentation Technology Development; (8) Status of Waste Isolation Pilot Project Design Activities; and (9) Waste Isolation Pilot Plant. The first two papers dealt with generic subjects of design and performance criteria for repositories, and how technical conservatism is implemented in design. Summaries are then presented for three major design activities conducted in 1980, a repository in basalt, engineering feasibility studies for candidate salt domes, and a review and update of the Waste Isolation Pilot Plant design activities. The remaining papers dealt with effects of seismic events on underground openings, and the results of equipment and instrumentation development planning notivition. (ATT)

792 (ONWI-212, pp 105-132) Package materials, waste form. 8 Dec 1980. NTIS, PC A17/MF A01. (CONF-801209-). From 2. annual National Waste Terminal Storage (NWTS) information meeting; Columbus, OH, USA (9 Dec 1980).

The schedules for waste package devclopment for the various host rocks were presented. The waste form subtask activities were reviewed, with the papers focusing on high-level waste, transuranic waste, and spent fuel. The following ten papers were presented: (1) Waste Package Development Approach; (2) Borosilicate Glass as a Matrix for Savannah River Plant Waste; (3) Development of Alternative High-Level Waste Forms; (4) Overview of the Transuranic Waste Management Program; (5) Assessment of the Impacts of Spent Fuel Disassembly - Alternatives on the Nuclear Waste Isolation System; (6) Reactions of Spent Fuel and Reprocessing Waste Forms with Water in the Presence of Basalt; (7) Spent Fuel Stabilizer Screening Studies; (8) Chemical Interactions of Shale Rock, Prototype Waste Forms, and Prototype Canister Metals in a Simulated Wet Repository Environment; (9) Impact of Fission Gas and Volatiles on Spent Fuel During Geologic Disposal; and (10) Spent Fuel Assembly Decay Heat Measurement and Analysis. (ATT)

793 (UNW1-212, pp 133-157) Far-field performance evaluations. (CONF-801209-). 8 Dec 1980. NTIS, PC A17/MF A01.

In Proceedings of the 1980 National Waste Terminal Storage Program information meeting.

The following seven papers were presented at the session on FarField Performance Evaluations: (1) Waste Isolation Performance Assessment; (2) Computerized Simulation of the Long-Term Geology and Hydrology of Regions Containing Nuclear Waste Repositories; (3) Supplements to the Release Scenario Analyses for the Waste Isolation Pilot Plant; (4) Radionuclide Release Scenario Selection Process for a Possible Basalt Repository; (5) Analytical Models of Radionuclide Transport; (6) Isolation System Sensitivity Analysis; (7) Radionuclide Transport Sensitivity Studies.

794 (ONWI-212, pp 158-186) Kepository sealing. 8 Dec 1980. NTIS, PC A17/MF A01. (CONF-801209-).

From 2. annual National Waste Terminal Storage (NWTS) information meeting; Columbus, OH, USA (9 Dec 1980).

Laboratory and field programs in progress are evaluating the properties of several materials over a range of environmental conditions. These studies include the examination of factors affecting the geochemical longevity of seals. In situ studies in bedded salt have included the recovery and laboratory evaluation of cement seals used in potashexploration drill holes. Observations revealed that no serious degradation had occurred after 17 years. Investigations will continue to develop various materials to operate in the environments that seals will encounter. These will include the geochemical characterization of specific sites and the evaluation of materials under the same conditions. In addition, techniques for efficient seal emplacement methods, quality assurance techniques, and in situ characterization of seals will be developed. Significant advances in sealing technology are expected before final sealing of the repository is required. The following papers were presented at this session: (1) Goals and Accomplishments of ONWI's Repository Sealing Program; (2) Design of Repository Sealing Systems - 1980; (3) Receni Developments in Seal Materials; (4) Preconceptual Design of a Borehole Plugging System in a Repository in Basalt; (5) Borehole Plugging Test Activities in southeastern New Mexico; (6) Results from the Bell Canyon Borehole Plugging Tests; (7) Analysis of Borehole Plug Performance; (8) Borehole Simulation Program.

795. (ONWI-212, pp 203-227) Repository data base, freld tests. 8 Dec 1980. NTIS, PC A17/MF A01. (CONF801209-).

From 2. annual National Waste Terminal Storage (NWTS) information meeting; Columbus, OH, USA (9 Dec 1980).

Development of appropriate data that can be used to solidify the base of technology for repository development frequently requires field testing. This field testing is performed in a variety of test facilities both within the US and abroad. Papers presented in this session describe a variety of field test programs currently being conducted or planned as part of the National Waste Terminal Storage (NWTS) program, including several geologic media. A subsequent companion session emphasizes penults of hnth lobarntary and fiold teatn; ns they relnto to davolnping tha repository data base. Titles of the papers presented are: (1) NearSurface Test Facility - Update on Field Testing in Basalt; (2) Field Testing at the Climax Stock on the Nevada Test Site: Spent Fuel and Radionuclide Migration Experiments; (3) Field Experiment Program for Tuff in G Tunnel; (4) Stripa Project: An International Project in the Management of Radioactive Waste Storage; (5) Brinc Migration Ficld Test Plans for Asse, West Germany; (6) Field Testing Activities in Dome Salt at Avery Island, Louisiana; (7) Experimental Studies at the Colorado School of Mines Hard Rock Test Site; (8) NWTS Field Testing - Forward Planning.

796 (ONWI-212, pp 228-256) Package design. $8 \mathrm{Dec}$ 1980. NTIS, PC A17/MF A01. (CONF-801209-).

From 2. annual National Waste Terminal Storage (NWTS) information meeting; Columbus, OH, USA ( 9 Dec 1980).

In this session the National Waste Terminal Storage program approach to waste package development was highlighted. The subtasks for the waste package development program were identified. These include the waste form, engineered barriers, design and development, performance evaluation, and baseline subtasks. In this session, engineered barriers and design and development subtask activities were reviewed. The following nine papers were presented: (1) Status of Sandia Backfill-Getter Development Studies; (2) Status of Sandia HLW Canister/Overpack Program Studies; (3) Joint PNL-WIPP HighLevel Waste Total System Test; (4) Experimental Demonstration Package Program; (5) Waste Package Development for a Nuclear Waste Repository in Basalt; (6) Status of Subseabed Repository Design Concepts and Radionuclide Migration Studies; (7) Repository Waste Package Design Studies; (8) Derivation of Waste Package Requirements Via Functional Analysis; (9) Nevada Nuclear Waste Storage Investigations: Waste Package/Tuff Studies. (ATT)

797 (ONWI-212, pp 257-283) Status of geologic/ environmental characterizations. 8 Dec 1980. NTIS, PC A17/MF A01. (CONF-801209-).

rrom 2. annual National Waste Terminal Storage (NW1S) information meeting; Columbus, OH, IJSA (9 nec 1980).

Studies within the NWTS program directed ultimately at siting one or more radioactive waste repositories consist principally of geologic and environmental characterizations. A draft criteria document has been developed which addresses both geologic and environmental characteristics of any potcntial sitc. Several media have becn identified as having generic features that could make them acceptable as host media for geologic repositories. Evaluations of these media are being carried out by relating them to the draft criteria document and are currently in various stages of the screening process. The end result will be the identification of at least four sites within the contiguous United States which would be suitable for construction of a repository. The following nin presentations were made: (1) Status of Geologic/Environmental Chat acterization Studies of Gulf Coast Interior Salt Dome Basins; (2) Statu of Geological/Environmental Characterization Studies in the Parodox Basin; (3) Nuclear Waste Isolation Studies of the Palo Duro and Dalhart Basins, Texas Panhandle, FY 1980; (4) Geologic Investigations for Nuclear Waste Repository Sites in Columbia River Basalt - Hanford 
Site; (5) Method for Screening for Repository Locations on and Contiguous to the Nevada Test Site; (6) Nationwide Review of Shales and Other Argillaceous Strata; (7) Geological Survey Program for Identification of Suitable Geohydrologic Environments for the Disposal of High-Level Radioactive Waste; (8) National Geologic/Environmental Screening for Repositories; (9) States of Characterizations.

\section{8 (ONWI-212, pp 284-297) Issues in repository siting.} 8 Dec 1980. NTIS, PC A17/MF A01. (CONF-801209-).

From 2. annual National Waste Terminal Storage (NWTS) information meeting; Columbus, OH, USA (9 Dec 1980).

Beginning this year and continuing at future information meetings of the National Waste Terminal Storage (NWTS) program, the Site Program Office hopes to sponsor a session which will explicitly address key issues related to siting or characterization of sites for geologic repositories. The intent of these sessions is twofold: (1) to provide timely and forthright discussion of issues in which the American public may have both interest and concern, and (2) to foster discussion among NWTS program technical participants working on different projects. This year the following issues were discussed: hydrology, volcanism, and seismicity; decision process in repository siting; and nontechnical and institutional considerations in the high-level waste isolation program. The titles of the papers presented are: (1) Hydrologic Issues in Repository Siting; (2) Hydrologic Testing Within the Culumbia River Basalts Beneath the Hanford Site, Washington - Recent Developments in the Basalt Waste Isolation Project; (3) Decision Process in Repository Siting; (4) Nontechnical and Institutional Considerations in the HLW Repository Program; (5) Status of Geologic Investigations at NTS; (6) Tectonics of the Southern Great Basin - Studies in Support of Nevada Nuclear Waste Storage Investigations; (7) Current Geologic Investigations in the Southwest Quadrant of the Nevada Test Site in Support of the Nuclear Waste Program; (8) Status of Geohydrologic Investigations That Are Part of the Nevada Nuclear Waste Storage Investigations program.

799 (ONWI-212, pp 298-309) Quality assurance. 8 Dec 1980. NTIS, PC A17/MF A01. (CONF-801 209-).

From 2. annual National Waste Terminal Storage (NWTS) information meeting; Columbus, OH, USA (9 Dec 1980).

The purpose of this session is to give an overview of the US Department of Energy quality assurance (QA) policy for nuclear energy and to describe the QA programs found at National Terminal Storage (NWTS) projects. The following papers were presented: (1) Assistant Secretary for Nuclear Energy - Quality Assurance Policy; (2) Need for Supplementary Quality Assurance Requirements - National Waste Terminal Storage Program; (3) National Waste Terminal Storage Program Quality Assurance Program; (4) Basalt Waste Isolation Project Quality Assurance Program; (5) Nevada Nuclear Waste Storage Investigations Quality Assurance Program; (6) Site Characterization Quality Assurance Concerns. (ATT)

\section{0 (ONWI-212, pp 310-338) Near-field performance} evaluations. 8 Dec 1980. NTIS, PC A17/MF A01. (CONF801209-).

From 2. annual National Waste Terminal Storage (NWTS) information meeting; Columbus, OH, USA (9 Dec 1980).

This session is primarily concerned with analyses of repository and waste package performance. The papers presented here, together with the papers concerned with site performance in Session II-F, provide examples from the complete spectrum of performance assessment activities under way in the National Waste Terminal Storage program. The following six papers were presented: (1) Development of Reference Repository Conditions; (2) Thermal/Mechanical Analysis of Tuff for Nuclear Waste Emplacement; (3) Comparison of Solutions to Benchmark Problems in Salt Using Different Numerical Methods; (4) Numerical Modeling in Support of Repository Designs in Basalt; (5) Waste Package Performance Analysis; (6) Estimation of Waste Package Performance Requirements for a Nuclear Waste Repository in Basalt. The first four papers are concerned with repository performance. The fifth paper describes waste package performance analysis. The last paper discusses the development of waste package performance requirements.

801 (ONWI-212, pp 339-363) Repository data base development. 8 Dec 1980. NTIS, PC A17/MF A01. (CONF801209-).
From 2. annual National Waste Terminal Storage (NWTS) information meeting; Columbus, OH, USA (9 Dec 1980).

Developing a data base for repository design requires the planning, design, execution, and interpretation of tests and experiments. This is a complex set of activities involving a variety of scientific and engineering disciplines. In the limited time available in this session, only a small sampling of this set of activities can be presented. The subjects chosen for presentation in this session include: a discussion of rock mechanics planning for multiple geologic media, results of ongoing rock mechanics investigations in salt and basalt, results from crystalline rock investigations in Sweden, development of basic rock properties for tuff, and fluid behavior in salt and tuff. The titles of the eight papers presented are: (1) Rock Mechanics in the National Waste Terminal Storage Program; (2) Creep of Rock Salt; (3) Rock Mechanics Strategies to Support Repository Design in Basalt; (4) Status of Test Results from Stripa Experiments; (5) Thermal and Mechanical Properties of Silicic Tuffs; (6) In Situ Tuff Water Migration/Heater Experiment; (7) Fluid Migration Studies in Salt; (8) Migration of Fluid Inclusions in Polycrystalline Salt Under Thermal Gradients in the Laboratory and in Salt Block II.

802 (ONWI-217) NWTS Field Testing In-Situ SiteConfirmation Tests Workshop, September 8-9, 1980. (D'Appolonia Consulting Engineers, Inc., Pittsburgh, PA (USA)). Jun 1983. Contract AC06-76RL01830;ACO283CH10140. 199p. (CONF-8009314-). NTIS, PC A09/MF A01. Order Number DE83012618.

From Nuclear Waste Terminal Storage (NWTS) field testing in situ site confirmation tests workshop; Columbus, OH, USA (8 Sep 1980).

Portions are illegible in microfiche products.

This document reports the proceedings of the In Situ Site Confirmation Tests Workshop held on September 8 and 9, 1980 in Columbus, Ohio. It was sponsored by Battelle's Project Management Division, Office of Nuclear Waste Isolation (ONWI), with assistance provided by D'Appolonia Consulting Engineers, Inc. Approximately 85 participants attended to discuss in situ field testing needs as appropriate for any or all candidate geologic media considered for mined geologic repositories. ONWI opened the workshop by presenting an overview of the National Waste Terminal Storage program, and outlining the scope and objectives of this workshop. The plenary session then divided into four Task Groups: site characterization; repository design, construction, and sealing; waste package and engineered barriers, and licensing. These individual groups then discussed issues pertinent to their topic and summarized their deliberations. The task groups met for a total of eight hours. The conclusions and recommendations of the individual groups were then presented to the plenary session and discussed. This document summarizes the activities, discussions, and conclusions of the workshop.

803 (ONWI-235) Evaluation of the thermomechanical behavior about a waste container/sleeve in salt. Waldman, $H$. (RE/SPEC, Inc., Rapid City, SD (USA)). Apr 1983. Contract AC06-76RL01830. 85p. NTIS, PC A05/MF A01. Order Number DE83011133.

This report deals with the very-near-field aspects of waste disposal in conceptual repositories within a salt medium and concentrates on the thermomechanical behavior around a drillhole containing a nuclear waste canister. Specifically, this includes an investigation of: (1) the expected closure of an unlined drillhole, and (2) the pressure buildup on a protective sleeve initially in direct contact with the drillhole wall. The results of the current study based on the disposal of unreprocessed spent fuel (SF) and a review of previous near-field studies based on the emplacement of high-level waste (HLW) are included. The current SF study uses a thermo/viscoelastic mechanical model involving an empirical creep law. Previous HLW studies were based on either a thermoelastic/plastic mechanical model or a thermo/viscoelastic model that did not include temperature dependence in the creep law. Several repository designs were considered with drillholes ranging from $50.8 \mathrm{~cm}$ to $61.0 \mathrm{~cm}$ in diameter. The thermomechanical behavior was modeled over a 25 year period after which retrieval was not expected to be a major concern. Sleeve pressures for the lined drillhole did not exceed $21 \mathrm{MPa}$ and would not warrant specially designed sleeves. Unlined drillholes $53.3 \mathrm{~cm}$ in diameter can accommodate HLW packages up to $45 \mathrm{~cm}$ in diameter for at least 10 years while a $61.0-\mathrm{cm}$-diameter drillhole will accommodate SF waste packages up to $43 \mathrm{~cm}$ in diameter for at least 25 years. 
804 (ONWI-251) Annotated bibliography for the design of waste packages for geologic disposal of spent fuel and highlevel waste. Wurm, K.J.; Miller, N.E. (Battelle Columbus Labs., OH (USA)). Nov 1982. Contract AC06-76RL01830. 365p. NTIS, PC A 16/MF A01. Order Number DE83004362.

This bibliography identifies documents that are pertinent to the design of waste packages for geologic disposal of nuclear waste. The bibliography is divided into fourteen subject categories so that anyone wishing to review the subject of leaching, for example, can turn to the leaching section and review the abstracts of reports which are concerned primarily with leaching. Abstracts are also cross referenced according to secondary subject matter so that one can get a complete list of abstracts for any of the fourteen subject categories. All documents which by their title alone appear to deal with the design of waste packages for the geologic disposal of spent fuel or high-level waste were obtained and reviewed. Only those documents which truly appear to be of interest to a waste package designer were abstracted. The documents not abstracted are listed in a separate section. There was no beginning date for consideration of a document for review. About 1100 documents were reviewed and about 450 documents were abstracted.

805 (ONWI-269) Possible approaches to community development for nuclear waste isolation. Voth, D.E.; Herrington, B.E. (Arkansas Univ., Fayetteville (USA). Dept. of Agricultural Economics and Rural Sociology). Oct 1982. Contract AC06-76RL01830. 54p. NTIS, PC A04/MF A01. Order Number DE83005334.

Community development is a process whereby communities assess their needs, determine their priorities, make plans, carry out these plans, and evaluate the results. This approach is applied in evaluating four possible decision-making scenarios that DOE could use in the site selection process and in the development of compensation/mitigation mechanisms. In the first scenario, DOE would select the repository site purely on geologic and technological grounds. In the second scenario, DOE would select the repository site using geologic and technological information, detailed socioeconomic information, and an understanding of potential socioeconomic impacts of a nuclear repository upon the community. In the third scenario DOE would select a repository site using the same information available in the second scenario; however, in this case, DOE would provide the local community with professionals who would act as advocates for the local community and argue their position within DOE's decision-making process. In the final scenario, DOE would invite potential repository site communities to engage in an auctioning process, whereby each community would bid on an acceptable compensation package (in their eyes) to be paid by DOE. Based on an evaluation of these scenarios, the report makes the following recommendations: (1) DOE should consider the auctioning process; (2) in the event that no communities are willing to present bids, DOE should provide communities with advocates; (3) compensation should be a collective package rather than in the form of individual cash payments; (4) a third party (other than DOE), with a heavy reliance on local organizations, should be specifically created for implementing the compensation/mitigation package; and (5) in order to approach the siting of additional repositories with greater understanding, longitudinal research at the first repository should be undertaken as a part of the monitoring phase.

806

(ONWI-302) Waste package performance evaluation. Lester, D.H.; Stula, R.T.; Kirstein, B.E. (Science Applications, Inc., La Jolla, CA (USA)). Mar 1983. Contract AC0676RL01830. 162p. NTIS, PC E08/MF A01. Order Number DE83011935.

Portions are illegible in microfiche products. Original copy available until stock is exhausted; Includes 3 sheets of $48 \times$ reduction microfiche.

This report describes continuing work to obtain performance assessments of waste package concepts being considered in the NWTS program for salt and generic hard rock using the BARIER code. The BARIER model simulates package behavior through a deterministic history of sure, slow degradation in a flooded, post-closure repository. The output consists of times at which individual barriers begin leaking, the failure modes responsible, and the rates of discharge of ${ }^{90} \mathrm{Sr}^{99} \mathrm{Tc}$, ${ }^{226} \mathrm{Ra},{ }^{207} \mathrm{~Np}$, and ${ }^{208} \mathrm{U}$ after contact of the waste form with water. During the degradation phase, successive calculations of corrosion rates followed by checks of stresses are carried out for each wetted barrier. As the barriers fail, water moves inward and pressure is transmitted inward. A release model calculates the diffusion and equilibrium sorption of radionuclides in the backfill after water contacts the waste and leaching begins. Calculated package lifetimes, defined by the point in time when water contacts the waste, vary from 0 to 2500 years. However, regardless of these failures, packages with backfill barriers delayed peak discharge of long-lived radionuclides for up to $10,000,000$ years for sand-bentonite mixtures and up to 10,000 years for crushed rock.

807 (ONWI-311) Laboratory rock mechanics testing manual. Public draft. Shuri, F.S.; Cooper, J.D.; Hamill, M.L. (Battelle Memorial Inst., Columbus, OH (USA). Office of Nuclear Waste Isolation; Foundation Sciences, Inc., Portland, OR (USA)). Oct 1981. Contract AC06-76RL01830. 309p. NTIS, PC A14/MF A01. Order Number DE82015763.

Standardized laboratory rock mechanics testing procedures have been prepared for use in the National Terminal Waste Storage Program. The procedures emphasize equipment performance specifications, documentation and reporting, and Quality Assurance acceptance criteria. Sufficient theoretical background is included to allow the user to perform the necessary data reduction. These procedures incorporate existing standards when possible, otherwise they represent the current stateof-the-art. Maximum flexibility in equipment design has been incorporated to allow use of this manual by existing groups and to encourage future improvements.

808 (ONWI-334-Vol.2-App.) Guidebook for reliability, availability, and maintainability analysis of NWTS repository equipment. Volume II. Appendices. Orvis, D.D.; Frank, M.V.; Jacobsen, F.K.; Clarke, W.M. (General Atomic Co., San Diego, CA (USA)). Apr 1981. Contract AC06-76RL01830. 243p. NTIS, PC A11.MF A01. Order Number DE83005213.

Portions of document are illegible.

The purpose of this Guidebook is to provide the background and methodology for developing and incorporating reliability, availability, and maintainability (RAM) requirements into the National Waste Terminal Storage program. The need for operational RAM requirements stems from two main considerations: (1) to assure the effectiveness of a repository over its lifetime, and (2) to reduce the likelihood and amount of radiation exposure to the repository operating and maintenance staff. The contents of the Guidebook are presented in two volumes. Volume 1 consists of seven chapters that are arranged so that the programmatic and general RAM aspects are presented first (Chapters 1, 2 and 3). These are followed by qualitative methodology (Chapters 4 and 5). An introduction to quantitative methods is presented in the last two chapters (Chapters 6 and 7). In addition, Chapter 3 presents a description of a reference repository and general RAM concerns in order to provide perspective for the examples in the Guidebook. Volume 2 expands the methods discussed in Volume 1 but concentrates on the quantitative methods (Appendices A-C) including the use of recommended computer programs (Appendix D). Appendix E provides copies of blank worksheets for applying the methodology that can be reproduced by the user. Finally, Appendix F presents a Glossary of terms used in the Guidebook.

809 (UNWI-352-Vol.1-Summ.) Úncertainties and sensitivities in the performance of geologic nuclear waste isolation systems. Volume I. Summary. Sutcliffe, W.G.; Feller, K.G.; Madsen, N.K.; Pollak, G.D. (Battelle Memorial Inst., Columbus, OH (USA). Office of Nuclear Waste Isolation; Lawrence Livermore National Lab., CA (USA)). Apr 1981. Contract W-7405-ENG-48;AC06-76RL01830. 34p. (UCRL-53142-Vol. 1-Summ.). NTIS, PC A03/MF A01. Order Number DE82017176.

This report presents an analysis of the long-term safety of geologic nuclear waste isolation systems. The study focuses on the effect of uncertainty about engineered and natural systems. We identify the subsystems and parameters that dominate the system performance under various conditions. We also consider the potential differences between waste forms, host media, and site characteristics, and how these differences affect the systemffis performance. The scope of this study is limited to probabilistic analysis of nuclear waste migration via groundwater flow in assumed pathways. The primary measure of performance is the peak rate of activity release $(\mathrm{Ci} / \mathrm{y})$ at the end point of a pathway. As well as demonstrating methods for uncertainty analysis, we present specific results and conclusions. Deterministic and probabilistic parametric analyses cover a range of parameter values large enough to 
compass significantly different waste forms, packages, and sites (in rious media). The results are useful for the assessment of geologic $n$ aste isolation systems in general. We also analyze specific hypothetical systems using generic data. The following are some general observations: A global, parametric analysis is more informative than a local sensitivity analysis. Individual contributions of multiple barriers (subsystems) are sometimes insignificant. Some uncertainties have little effect on system performance.

810

(ONWI-356) Permeability of selected sediments in the vicinity of five salt domes in the Gulf Interior Region. Slaughter, G.M.; White, R.M.; Alger, R.P. (Law Engineering Testing Co., Marietta, GA (USA)). Feb 1983. Contract AC0676RL01830. 76p. NTIS, PC AO5/MF A01. Order Number DE83008126.

Portions are illegible in microfiche products. Original copy available until stock is exhausted.

The purposc of this investigation was to determine what relationships might exist between permeability and parameters available from geophysical logs of sediments within the Gulf Interior Region. The parameters that have received the most attention in the literature are formation resistivity factor and porosity. Empirical relationships between permeability and porosity, gamma-ray bulk density, the fraction of intergranular pore space occupied by clay or silt, and porosity differences were investigated. Porosity appears to be the log parameter that best correlates with permeability.

811 (ONWI-383) Forced ventilation analysis of a commercial high-level nuclear repository in salt. Svalstad, D.K. (RE/SPEC, Inc., Rapid City, SD (USA)). Jul 1983. Contract AC06-76RL01830;AC02-83CH10140. 121p. NTIS, PC A06/MF A01. Order Number DE83016098.

Portions are illegible in microfiche products. Original copy available until stock is exhausted.

This report addresses the feasibility of using forced ventilation (blast cooling) for rapid drift cooling before a nuclear waste retrieval operation. A coupled conduction-convection analysis is used to examine the various parameters and conditions which control the blast cooling process for the purpose of assessing their relative significance and potential impact on developing a blast cooling scenario for a nuclear waste repository in salt.

812

(ONWI-399) Thermodynamic properties of chemical species in nuclear waste. Edelstein, N.; Bucher, J.; Silva, R.; Nitsche, H. (Lawrence Berkeley Lab., CA (USA)). Jan 1983. Contract AC06-76RL01830. 121p. (LBL-14325). NTIS, PC A04/MF A01. Order Number DE83005249.

Thermochemical data on the solubilities of compounds and solution complexes of the waste radionuclides likely to form in the natural systems are required to adequately assess and predict the ability of the storage site to meet established site characteristics and standards for rates of release of radioactive materials set by the US Nuclear Regulatory Commission. Taken as a group, the actinides $\mathrm{U}, \mathrm{Np}, \mathrm{Pu}, \mathrm{Am}$, and $\mathrm{Cm}$ represent the largest potential long-term hazard. However, with the exception of $\mathrm{U}$, reliable thermochemical data for even the simple compounds and solution complexes that the important actinides might form with the common ground-water anions is rather incomplete. In particular, very little data exist for the anions, hydroxide, carbonate, and phosphate. Investigations of the hydrolysis of $\mathrm{Nd}^{0+}$ (a natural analog for $\mathrm{Am}$ and $\mathrm{Cm}$ ) and $\mathrm{Cm}^{0+}$, the carbonate complexation of $\mathrm{NpO}_{2}{ }^{+}$, and the solubilities of $\mathrm{Nd}^{0+}$ and $\mathrm{Am}^{0+}$ in near neutral $\mathrm{pH}$ solutions have been conducted. A value of $\log K>-7.7 \pm 0.3$ was obtained for the first hydrolysis constant of both $\mathrm{Nd}^{0+}$ and $\mathrm{Cm}^{0+}$. For the carbonate complexes of $\mathrm{NpO}_{2}{ }^{+}$, values of $5.9 \pm 0.5$ for $\log \beta_{1}$ and $16.3 \pm 0.5$ for $\log \beta_{0}$ were determined for the formation constants of $\mathrm{NpO}_{2} \mathrm{CO}_{0}^{-}$and $\mathrm{NpO}_{2}\left(\mathrm{CO}_{0}\right)_{0}{ }^{5-}$, respectively. From measurements of solution Am concentrations as a function of $\mathrm{pH}$, a value of 17.5 was estimated for the 'ogarithm of the solubility product of amorphous $\mathrm{Am}(\mathrm{OH})_{0}$, i.e., $\mathrm{Am}(\mathrm{OH})_{0}$ (solid) $+3 \mathrm{H}^{+}>\mathrm{Am}^{0+}+3 \mathrm{H}_{2} \mathrm{O}$.

813 (ONWI-400) In situ and laboratory geotechnical test results from borehole GD-1 in southeast Utah. Nelson, R.A.; Kocherhaus, J.G.; Schnapp, M.R. (Woodward-Clyde Consultants, San Francisco, CA (USA)). Nov 1982. Contract AC06-
76RL01830. 202p. NTIS, PC A10/MF A01. Order Number DE83004904.

Portions of document are illegible.

In-situ testing was conducted in the Gibson Dome No. 1 (GD-1) borehole in southeastern Utah at depths ranging from 954 to $1507 \mathrm{~m}$ ( 3130 to $4945 \mathrm{ft}$ ). These tests were made in a number of salt strata to measure: (1) stress-strain behavior of the borehole (hole squeeze); (2) short-term borehole creep; and (3) in situ stresses in salt strata. Laboratory triaxial strength and creep tests were conducted on core removed from GD-1 to provide engineering data under a variety of test conditions (stress state, strain rate, and temperature. Results of in situ and laboratory tests will be used to assist in estimating the maximum feasible depth for a repository in the Gibson Dome Area. Stress-strain data reflected test zone behavior devoid of previous non-geologic stress influences. Radial strain creep rates were generally consistent with other laboratory tests and other direct measurements of borehole creep. Hydraulic fracture data indicated a non-lithostatic stress state at depths shallower than $1220 \mathrm{~m}(4000 \mathrm{ft})$. Laboratory triaxial strength tests and Young's modulus results displayed trends similar to those from salt in other locations, showing that Paradox salt has higher strengths than many other salts in similar test conditions.

814 (ONWI-416) Quaternary geology of Rayburn's salt dome: North Louisiana Salt Dome Basin. Kolb, C.R.; Holmes, J.C. (Louisiana State Univ., Baton Rouge (USA). Inst. for Environmental Studies). Feb 1983. Contract AC0676RL01830;AC02-83CH10140. 176p. NTIS, PC A09/MF A01. Order Number DE83007232.

Portions are illegible in microfiche products. Original copy available until stock is exhausted.

A 20 - to $100-\mathrm{ft}$ sequence of fine sediments with increasing amounts of sand and chert gravel with depth underlies a topographic low above the salt dome. These deposits are of Quaternary (specifically late Quaternary) age. A boulder zone consisting of water-worn calcite boulders and cobbles in a matrix of dark gray and tan sandy clay and clayey sand is present beneath these gravel-bearing Quaternary deposits and above the anhydrite-gypsum caprock. Isotopic studies have identified the boulder zone as the remnant of a once continuous calcite caprock cover that was subjected to subaerial erosion in the geologic past. Generally speaking, the anhydrite-gypsum caprock is closer to the surface near the center of the topographic low over the salt dome and slopes down toward the outer limits of this low. However, the caprock surface rises again very abruptly and very steeply at the extreme edges of the low, suggesting that an underground ridge of caprock, sometimes only $15 \mathrm{ft}$ below the surface, completely encircles the outer boundaries of the low. The only outlet to the central topographic low that could have drained it during Quaternary time is to the south along Fouse Creek, now filled with Quaternary alluvium only 10 to $12 \mathrm{ft}$ deep. Thus, the $100-\mathrm{ft}$ thickness of gravel-bearing Quaternary that underlies the low can be accounted for only by dissolution of the underlying salt and subsidence of the Quaternary. Radiocarbon dates from samples of wood found in the gravel-bearing (the surficial) Quaternary above the dome range from about 13,500 to 19,500 years BP. If radiocarbon dates from two organic samples from the boulder zone are correct (about 19,000 to 21,000 years BP), dissolution and subsidence during the past 20,000 years range between 1.5 and $5.0 \mathrm{ft}$ per $1000 \mathrm{yr}$.

816 (ONWI-422) Waste-Mixes Study for space disposal. McCallum, R.F.; Blair, H.T.; McKee, R.W.; Silviera, D.J.; Swanson, J.L. (Pacific Northwest Lab., Richland, WA (USA)). Jan 1983. Contract AC06-76RL01830. 211p. NTIS, PC A10/MF A01. Order Number DE83006935.

Portions are illegible in microfiche products. Original copy available until stock is exhausted.

The Wastes Mixes Study is a component of Cy-1981 and 1982 research activities to determine if space disposal could be a feasible complement to geologic disposal for certain high-level (HLW) and transuranic wastes (TRU). The objectives of the study are: to determine if removal of radionuclides from HLW and TRU significantly reduces the long-term radiological risks of geologic disposal; to determine if chemical partitioning of the waste for space disposal is technically feasible; to identify acceptable waste forms for space disposal; and to compare improvements in geologic disposal system performance to impacts of additional treatment, storage, and transportation necessary for space disposal. To compare radiological effects, five system alternatives are defined: Reference case - All HLW and TRU to a repository. Alterna- 
tive A - Iodine to space, the balance to a repository. Alternative B - Technetium to space, the balance to a repository. Alternative C - $95 \%$ of cesium and strontium to a repository; the balance of HLW aged first, then to space; plutonium separated from TRU for recycle; the balance of the TRU to a repository. Alternative D - HLW aged first, then to space, plutonium separated from TRU for recycle; the balance of the TRU to a repository. The conclusions of this study are: the incentive for space disposal is that it offers a perception of reduced risks rather than significant reduction. Suitable waste forms for space disposal are cermet for HLW, metallic technetium, and lead iodide. Space disposal of HLW appears to offer insignificant safety enhancements when compared to geologic disposal; the disposal of iodine and technetium wastes in space does not offer risk advantages. Increases in short-term doses for the alternatives are minimal; however, incremental costs of treating, storing and transporting wastes for space disposal are substantial.

816 (ONWI-423) Engineered waste-package-system design specification. (Westinghouse Electric Corp., Pittsburgh, PA (USA). Advanced Energy Systems Div.). May 1983. Contract AC06-76RL01830;AC02-83CH10140. 99p. NTIS, PC A04/MF A01. Order Number DE83014061.

Portions are illegible in microfiche products. Uriginal copy available until stock is exhausted.

This report documents the waste package performance requirements and geologic and waste form data bases used in developing the conceptual designs for waste packages for salt, tuff, and basalt geologies. The data base reflects the latest geotechnical information on the geologic media of interest. The parameters or characteristics specified primarily cover spent fuel, defense high-level waste, and commercial high-level waste forms. The specification documents the direction taken during the conceptual design activity. A separate design specification will be developed prior to the start of the preliminary design activity.

817 (ONWI-438) Engineered waste-package conceptual design: defense high-level waste (Form 1), commercial high-level waste (Form 1), and spent fuel (Form 2) disposal in salt. (Westinghouse" Electric Corp., Pittsburgh, PA (USA). Advanced Energy Systems Div.). Apr 1983. Contract AC0676RL 01830;AC02-83CH10140. 499p. NTIS, PC A21/MF A01. Order Number DE83012767.

Portions are illegible in microfiche products. Original copy available until stock is exhausted.

This report provides conceptual waste package designs for use by the Office of Nuclear Waste Isolation (ONWI) in preparing a repository conceptual design in salt. Included are designs for the current reference waste form configurations of Defense High Level Waste, which consists of Savannah River Laboratory wastes immobilized in borosilicate glass, Commercial High Level Waste, which is a borosilicate glass waste form that results from the immobilization of commercial spent fuel reprocessing wastes, and Spent Fuel - Form 2, which consists of consolidated spent fuel rods from PWR or BWR assemblies. Reference designs are presented which are used as a baseline to evaluate design alternatives resulting from variations in the waste form configuration, the design approach, and the design data base. This broad spectrum of conceptual designs for salt has been included to provide ONWI a basis for concept comparison, an indication as to which of several package and repository design approaches are more cost effective, and guidance as to which approaches should be pursued in future design efforts. Based on available data and the analyses performed, all the concepts in this report offer technically viable approaches to the containment of the waste form for at least 1000 years and for adequate isolation of radionuclides thereafter. The reference borehole-type design is one which provides containment through the use of a titanium alloy corrosion-resistant overpack, backed with a carbon steel structural reinforcing member. An alternate borehole design is one which provides containment with an allsteel overpack which is sufficiently thick to withstand expected crushing loads and to accommodate expected corrosion. The self-shielded package approach provides containment through use of a thick section of moderately corrosion resistant ferrous alloy material. Development programs are identified that will be required to support designs during licensing. Pittsburgh, PA (USA). Advanced Energy Systems Div.). Apr
1983. Contract AC06-76RL01830. 462p. NTIS, PC A20/MF A01. Order Number DE83013112.

Portions are illegible in microfiche products. Original copy available until stock is exhausted.

This report provides conceptual waste package designs for use by the Nevada Nuclear Waste Storage Investigations (NNWSI) project in preparing a repository conceptual design in tuff at the Nevada Test Site. Included are designs for the current reference waste form configurations of Defense High Level Waste - Form 1, which consists of Savannah River Plant wastes immobilized in borosilicate glass, Defense High Level Waste - Form 2, which is a ceramic form of the same Savannah River waste, Spent Fuel - Form 2, which consists of consolidated spent fuel rods from PWR and BWR assemblies, and Commercial High Level Waste, which is a borosilicate glass waste form that results from the immobilization of commercial spent fuel reprocessing wastes. The reference designs are used as a baseline to evaluate design alternatives in the waste form size, the design approach, the emplacement configuration, the design data base uncertainty including overpack material corrosion rate data and waste form leaching data. This broad spectrum of conceptual designs for tuff has been included to provide NNWSI a basis for concept comparison, an indication as to which of several package and repository design approaches are morc cost effective, and guidance as to which approaches should be pursued in future design efforts. Based on available data and the analyses performed, all the concepts in this report offer technically viable approaches to the containment of the waste form for at least 1000 years and for adequate isolation of radionuclides thereafter for a repository either below or above the water table. Development programs have been identified which are needed to substantiate design assumptions or to resolve feasibility questions. 91 figures, 98 tables.

819 (ONWI-452) WAPPA: a Waste Package Performance Assessment code. Technical report. (INTERA Environmental Consultants, Inc., Houston, TX (USA)). Apr 1983. Contract AC06-76RL01830. 364p. NTIS (US Sales Only), PC E13/MF A01. Order Number DE83011665.

Portions are illegible in microfiche products. Original copy available until stock is exhausted; Includes 5 sheets of $24 x$ reduction microfiche.

The Waste Package Performance Assessment (WAPPA) code is a barrier degradation code for a nuclear waste package in a geologic repository. The model includes five distinct, coupled degradation process models (radiation, thermal, mechanlcal, corrosion, leaching) which are driven internally by waste decay heat and externally by repository stresses and fluids. The penetration of water into the waste package is tracked radially inwards. The output quantifies the residual state of integrity of the barriers as a function of time and also the heat and radionuclide fluxes to the repository.

820 (ONWI-455) Functional design criteria for the exploratory shaft in salt. (Battelle Memorial Inst., Columbus, OH (USA). Office of Nuclear Waste Isolation). Jul 1983. Contract AC06-76RL01830;AC02-83CH10140. 44p. NTIS, PC A03/MF A01. Order Number DE84001218.

The purpose of the Functional Design Criteria is to provide technical direction for the development of detailed design criteria for the exploratory shaft facility. This will assure that the completed exploratory shaft facility design is in accordance with the ONWI mission as well as the National Waste Terminal Storage program directives. The functional design criteria are not intended to limit or constrain the designer's flexibility to design. However, the following design philosophies will be incorporated in the designs: The exploratory shaft will be designed to fulfill its intended purpose and mission. Sound engineering principles and practices will be consistently applied. The health and safety of the public and the workers will be an essential factor in the design. The exploratory shaft will be designed to achieve economy in its construction, operation, and maintenance. All applicable federal, state, and local laws and regulations, as well as all applicable national consensus codes and standards, will be satisfied. The adverse impact to the environment will be minimized.

821 (UNWI-462) Conceptual waste package interim performance specifications for waste forms for geologic isolation in salt repositories. (Battelle Memorial Inst., Columbus, $\mathrm{OH}$ (USA). Office of Nuclear Waste Isolation). Jun 1983. Contract 
AC06-76RL01830. 31p. NTIS, PC A03/MF A01. Order Number DE83013814.

The interim performance specifications and data requirements presented are applicable to all types of radioactive wastes prepared for disposal in salt geologic repositories. In this document, an interim numerical value is provided for only one waste-form parameter - the waste-form release rate. This is the key waste-form parameter affecting waste isolation, and is defined here for spent fuel, defense high-level waste (HLW), and commercial HLW. The waste-form performance specifications and data requirements respond to the waste package performance criteria. Subject areas treated include: containment and controlled release, operational period safety, criticality control, identification, and waste-package performance testing requirements. This document was generated for use in the development of conceptual wastepackage designs in salt. It will be revised as additional data, analyses, and regulatory requirements become available.

822 (ONWI-463) Interim performance specifications for conceptual waste-package designs for geologic isolation in salt repositories. (Battelle Memorial Inst., Columbus, OH (USA). Office of Nuclear Waste Isolation). Jun 1983. Contract ACO283CH10140;AC06-76RL01830. 48p. NTIS, PC A03/MF A01. Order Number DE83014372.

The interim performance specifications and data requirements presented apply to conceptual waste package designs for all waste forms which will be isolated in salt geologic repositories. The waste package performance specifications and data requirements respond to the waste package performance criteria. Subject areas treated include: containment and controlled release, operational period safety, criticality control, identification, and waste package performance testing requirements. This document was generated for use in the development of conceptual waste package designs in salt. It will be revised as additional data, analyses, and regulatory requirements become available.

823 (ONWI-464) Conceptual waste package interim product specifications and data requirements for disposal of borosilicate glass defense high-level waste forms in salt geologic repositories. (Battelle Memorial Inst., Columbus, OH (USA). Office of Nuclear Waste Isolation). Jun 1983. Contract ACO676RL01830;AC02-83CH10140. 42p. NTIS, PC A03/MF A01. Order Number DE83013902.

The conceptual waste package interim product specifications and data requirements presented are applicable specifically to the normal borosilicate glass product of the Defense Waste Processing Facility (DWPF). They provide preliminary numerical values for the defense high-level waste form parameters and properties identified in the waste form performance specification for geologic isolation in salt repositories. Subject areas treated include containment and isolation, operational period safety, criticality control, waste form/production canister identification, and waste package performance testing requiremonts. This document was generated for use in the development of con ceptual waste package designs in salt. It will be revised as additional data, analyses, and regulatory requirements become available.

824 (ONWI-483) Results of repository conditions study for commercial and defense high-level nuclear waste and spent fuel repositories in salt. (Battelle Memorial Inst., Columbus, OH (USA). Office of Nuclear Waste Isolation). Jul 1983. Contract AC06-76RL01830;AC02-83CH10140. 42p. NTIS, PC A03/MF A01. Order Number DE83016536.

This report summarizes activities to determine repository conditions for temperatures, pressure, fluid, chemical, and radiation environments that are expected to exist in commercial and defense high-level nuclear waste and spent fuel repositories in salt. These conditions were generated by the Reference Repository Conditions Interface Working Group (RRC-IWG), an ad hoc IWG established by the National Waste Terminal Storage Program's (NWTS) Isolation Interface Control Board (I-ICB). These repository conditions are based on the standard roomand-pillar mined repository concept with waste emplaced in vertical holes drilled in the room floor. Some important results obtained are given below for selected local areal thermal loadings of 25,25 , and 11.6 $\mathrm{W} / \mathrm{m}^{2}$ for spent fuel (SF), commercial high-level waste (CHLW) and defense high-level waste (DHLW), respectively. In all cases, the results below are given in order for SF, CHLW, and DHLW. Some thermal results are: maximum waste temperature $-190,320$, and $100 \mathrm{C}$; maximum canister surface temperature $-160,260$, and $90 \mathrm{C}$; and maximum rock temperature $-150,160$, and $80 \mathrm{C}$. The length of significant thermal exposure is greater for $\mathbf{S F}$ than the other wastes. Thermal histories are given in the report. Vapor phase pressures are not expected to rise significantly above atmospheric pressure until the repository is sealed. After sealing, the pressure will gradually increase to approach lithostatic equilibrium. Estimated volumes of accumulation of brine from thermal migration for 1000 years are 3 to 4 liters, 8 liters, and 0.5 liter. Reference brine compositions are given, although actual brine compositions are strongly site dependent. The maximum absorbed gamma radiation dose delivered to the salt after $10^{4}$ year is $1.6 \times 10^{10}$ rads, and $9.6 \times 10^{8}$ rads for the CHLW and SF canisters, respectively. 12 figures, 5 tables.

825 (ONWI-490) Waste package materials testing for a salt repository: 1982 status report. Kircher, J.F.; Moak, D.P.; Clark, D.E. (Battelle Memorial Inst., Columbus, OH (USA). Office of Nuclear Waste Isolation). Aug 1983. Contract ACO676RL01830;AC02-83CH10140. 143p. NTIS, PC A07/MF A01. Order Number DE83016811.

The US plans to safely dispose of nuclear waste in deep, stable geologic formations. As part of these plans, the US Department of Energy (DOE) is sponsoring research on the designing and testing of waste packages and waste package materials. This report summarizes recent results of waste package materials testing in a salt environment. The results from these tests will be used by waste package designers and performance assessment experts. Release characteristic data are available on two waste forms (spent fuel and waste embedded in glass) which were exposed to leaching tests at various radiation levels, temperatures, $\mathrm{pH}$, volumes, and brine solution and oxygen concentrations simulating expected salt repository conditions. Candidate materials tested or soon to be tested for corrosion resistance and other properties include iron alloys; TiCode-12, the most promising titanium alloy for containment; nickel alloys; and stainless steel. Testing of fluid movement and thermal conductivity through various backfill mixtures shows that these materials can perform satisfactorily. In component interaction testing, no synergistic effects have ruled out any candidate material. 77 references, 37 figures, 30 tables.

826 (ONWL-493) Conceptual test plan for site confirmation testing at an exploratory shaft in salt. Ubbes, W.F. (Battelle Memorial Inst., Columbus, $\mathrm{OH}$ (USA). Office of Nuclear Waste Isolation). Sep 1983. Contract ACO6-76RL01830;ACO283CH10140. 39p. NTIS, PC A03/MF A01. Order Number DE84001008.

This document is developed to discuss, from a conceptual standpoint, the types of information that may be needed from an exploratory shaft facility and to describe the kinds of in situ tests that could be conducted to obtain that information during site confirmation testing. Site confirmation testing is designed to provide information to satisfy the stated objectives by supplying input parameters for performance assessment models and by direct observation of the host formation.

827 (ONWI-494) Performance assessment of a shaft seals system in a HLW repository in the Gibson Dome area. Gureghian, A.B.; Scott, L.A.; Raines, G.E. (Battelle Memorial Inst., Columbus, OH (USA). Office of Nuclear Waste Isolation). Aug 1983. Contract AC02-83CH10140;ACO676RL01830. 98p. NTIS, PC A05/MF A01. Order Number DE84000527.

Portions are illegible in microfiche products. Original copy available until stock is exhausted.

The performance assessment of a shaft seals system in a highlevel waste repository in the Gibson Dome area in southeastern Utah is presented. The analysis of the movement of the permeant in the shaft seals system under partially saturated flow conditions was based on the Green and Ampt theory of infiltration. The analysis of the transport of radionuclides leaching out of the repository was performed using two analytical solutions: a two-dimensional solution which could handle single decaying species in homogeneous soils and a one dimensional solution designed to handle a three-member decay chain in a layered stream tube. Various conservative scenarios of water intrusion into the repository were considered and the impact of these on the travel time of the wetting front in the shaft seals, as well as the potential migration of the radionuclides released from the two types of waste form, are reported. At our present knowledge of field data, the methodology has proved useful in reasonable estimations of some important aspects of the shaft seals performance, as well as in assessments of the potential radiological 
impact of the release of radionuclides from the repository into the underlying aquifer. The methodology described is not meant to compete with large-scale computer models; however, it is designed to assist the prospective investigator in economical analyses.

828 (ORNL-5703) Regional geological assessment of the Devonian-Mississippian shale sequence of the Appalachian, Illinois, and Michigan basins relative to potential storage/disposal of radioactive wastes. Lomenick, T.F.; Gonzales, S.; Johnson, K.S.; Byerly, D. (Oak Ridge National Lab., TN (USA)). Jan 1983. Contract W-7405-ENG-26. 166p. NTIS, PC A08/MF A01. Order Number DE83005178.

Portions of document are illegible. Printed copy available until stock is exhausted.

The thick and regionally extensive sequence of shales and associated clastic sedimentary rocks of Late Devonian and Early Mississippian age has been considered among the nonsalt geologies for deep subsurface containment of high-level radioactive wastes. This report examines some of the regional and basin-specific characteristics of the black and associated nonblack shales of this sequence within the Appalachian, Illinois, and Michigan basins of the north-central and eastern United States. Principal areas where the thickness and depth of this shale sequence are sufficient to warrant further evaluation are identified, but no attempt is made to identify specific storage/disposal sites. Also identified are other areas with less promise for further study because of known potential conflicts such as geologic-hydrologic factors, competing subsurface priorities involving mineral resources and groundwater, or other parameters. Data have been compiled for each basin in an effort to indicate thickness, distribution, and depth relationships for the entire shale sequence as well as individual shale units in the sequence. Included as parts of this geologic assessment are isopach, depth information, structure contour, tectonic elements, and energy-resource maps covering the three basins. Summary evaluations are given for each basin as well as an overall general evaluation of the waste storage/disposal potential of the Devonian-Mississippian shale sequence,including recommendations for future studies to more fully characterize the shale sequence for that purpose. Based on data compiled in this cursory investigation, certain rock units have reasonable promise for radioactive waste storage/disposal and do warrant additional study.

829 (ORNL-5818) Brine migration in salt and its implications in the geologic disposal of nuclear waste. Jenks, G.H.; Claiborne, H.C. (Oak Ridge National Lab., TN (USA)). Dec 1981. Contract W-7405-ENG-26. 187p. NTIS, PC A05/MF A01. Order Number DEszUU6119.

This report respresents a comprehensive review and analysis of available information relating to brine migration in salt surrounding radioactive waste in a salt repository. The topics covered relate to (1) the characteristics of salt formations and waste packages pertinent to considerations of rates, amounts, and effects of brine migration, (2) experimental and theoretical information on brine migration, and (3) means of designing to minimize any adverse effects of brine migration. Flooding, brine pockets, and other topics were not considered, since these features will presumably be eliminated by appropriate site selection and repository design. 115 references.

830 (ORNL/TM-8083) Expected near-field thermal environments in a sequentially loaded spent-fuel or high-level waste repository in salt. Rickertsen, L.D.; Arbital, J.G.; Claiborne, H.C. (Oak Ridge National Lab., TN (USA)). Jan 1982. Contract W-7405-ENG-26. 63p. NTIS, PC A04/MF A01. Order Number DE82007129.

This report describes the effect of realistic waste emplacement schedules on repository thermal environments. Virtually all estimates to date have been based on instantaneous loading of wastes having uniform properties throughout the repository. However, more realistic scenarios involving sequential emplacement of wastes reflect the gradual filling of the repository over its lifetime. These cases provide temperatures that can be less extreme than with the simple approximation. At isolated locations in the repository, the temperatures approach the instantaneous-loading limit. However, for most of the repository, temperature rises in the near-field are 10 to 40 years behind the conservative estimates depending on the waste type and the location in the repository. Results are presented for both spent-fuel and high-level reprocessing waste repositories in salt, for a regional repository concept, and for a single national repository concept. The national repository is filled sooner and therefore more closely approximates the instantaneou ${ }^{\text {l.. }}$ loaded repository. However, temperatures in the near-field are still 20 or more below the values in the simple model for $\mathbf{4 0}$ years after start of repository emplacement operations. The results suggest that current repository design concepts based on the instantaneous-loading predictions are very conservative. Therefore, experiments to monitor temperatures in a test and evaluation facility, for example, will need to take into account the reduced temperatures in order to provide data used in predicting repository performance.

831 (ORNL/TM-8109) Expected very-near-field thermal environments for advanced spent-fuel and defense high-level waste packages. Rickertsen, L.D.; Misplon, M.A.; Claiborne, H.C. (Oak Ridge National Lab., TN (USA)). Mar 1982. Contract W-7405-ENG-26. 54p. NTIS, PC A04/MF A01. Order Number DE82009607.

The very-near-field thermal environments expected in a nuclear waste repository in a salt formation have been evaluated for the Westinghouse Form I advanced waste package concepts. The repository descriptions used to supplement the waste package designs in these analyses are realistic and take into account decign constraints to acsure con " servatism. As a result, areal loadings are well below the acceptable values established for salt repositories. Predicted temperatures are generally well below any temperature limits which have been discussed for waste packages in a salt formation. These lnw temperatures result from the conservative repository designs. Investigations are also made of the sensitivity of these temperatures to areal loading, canister separation, and other design features.

832 (ORNL/TM-8491) Studies of waste-canister compatibility. McCoy, H.E. (Oak Ridge National Lab., TN (USA)). Jan 1983. Contract W-7405-ENG-26. 92p. NTIS, PC A05/MF A01. Order Number DE83005917.

Compatibility studies were conducted between 7 waste forms and 15 potential canister structural materials. The waste forms were $\mathrm{Al}-\mathrm{Si}$ and $\mathrm{Pb}-\mathrm{Sn}$ matrix alloys, FUETAP, glass, Synroc D, and waste particles coated with carbon or carbon plus silicon carbide. The canister materials included carbon steel (bare and with chromium or nickel coatings), copper, Monel, $\mathrm{Cu}-35 \% \mathrm{Ni}$, titanium (grades 2 and 12), several Inconels, aluminum alloy 5052 , and two stainless steels. Tests of either 6888 or $8821 \mathrm{~h}$ were conducted at 100 and $300^{\circ} \mathrm{C}$, which bracket the low and high limits expected during storage. Glass and FUETAP evolved sulfur, which reacted preferentially with copper, nickel, and alloys of these metale. The Pb Sn matrix alloy stuck to all camples and the carbon-coated particles to most samples at $300^{\circ} \mathrm{C}$, but the extent of chemical reaction was not determined. Testing for $0.5 \mathrm{~h}$ at $800^{\circ} \mathrm{C}$ was included because it is representative of a transportation accident and is required of casks containing nuclear materials. During these tests (1) glass and FUETAP evolved sulfur, (2) FUETAP evolved large amounts of gas, (3) Synroc stuck to titanium alloys, (4) glass was molten, and (5) both matiix alloys wcre multun mith cunsiduablic clicunical inturacliuns with many of the canister samples. If this test condition were imposed on waste canisters, it would be design limiting in many waste storage concepts.

833

(PB-82-190950) Storage of HLW in engineered structures: air-cooled and water-cooled concepts. Final report. Ahner, S.; Dekais, J.J.; Puttke, B.; Staner, P. (Commission of the European Communities, Luxembourg). [nd]. 149p. (EUR7340-EN). NTIS, PC E06/MF E06.

The conceptual designs of air-cooled (1) and water-cooled (2) intermediate storage facilities for vitrified, high-level radioactive waste in overground installations were studied. In (1) the decay heat from the storage area would be removed by natural convection, in (2) by a primary and secondary forced cooling system with redundant and diverse devices. A safety analysis and an economic analysis was applied to both concepts. Construction costs for both systems differ little, but operation and maintenance costs for (2) are higher than for (1). The study used criteria which in certain Member States of the European Communitv could result in a higher standard of safety than might be justified c social, political, or economic grounds.

834 (PB-83-102087) Impurities in rock-salt: consequences for the temperature increases at the disposal of highlevel nuclear waste. van den Broek, W.M.G.T. (Technische 
Hogeschool Delft (Netherlands)). Jun 1982. 53p. NTIS, PC A04/MF A01.

In part A the thermal properties of halite and the other materials occurring in rock-salt (the 'impurities') are collected. Except for sylvite (the specific heat of this salt is about $70 \%$ of the value for halite) all specific heats are larger than the specific heat of halite. The consequences for the temperature increases at the disposal of high-level nuclear waste in rock-salt are discussed for impurity concentrations of 1, 5, 10 and $15 \%$. If the presence and distribution of the impurities are not taken into account, then - under the most unfavorable conditions - extra temperature increases of about $3 \%$ per cent impurity may occur. If, however, the geological composition and its geometry for the disposal region are known, the temperature increases can be calculated more accurately: they may or may not differ from those for pure halite. In part B the measurement of the thermal conductivity for four salts (kainite, kieserite, carnallite, and polyhalite) is described.

836 (PB-83-165100) Disposal of high-level nuclear waste in rock-salt. A comparison of temperature increases calculated at ECN (Energieonderzoek Centrum Nederland)-Petten, RWTH (Rheinisch-Westfaelische Technische Hochschule Aachen)Aachen and THD (Delft University of Technology)-Delft. van den Broek, W.M.G.T. (Technische Hogeschool Delft (Netherlands)). Nov 1982. 49p. NTIS, PC A03/MF A01.

Disposal of high-level nuclear waste in rock-salt formations will cause temperature increases. These temperature increases have been calculated with different computer programs. In this report temperature increases, calculated with computer programs of ECN (Netherlands Energy Research Foundation) and RWTH (Rheinisch-Westfalische Technische Hochschule) are compared with temperature increases, calculated with the computer program of THD (Delft University of Technology). The three computer programs differ on subjects such as mathematical procedure, correction for the temperature dependencies of the thermal properties of rock-salt, physical description of the disposal region, disposal pattern. Because of these differences between the computer programs a comparison of results is useful.

836

(PB-83-169953) Population risks from disposal of high-level radioactive wastes in geologic repositories. Draft report. Smith, C.B.; Egan, D.J. Jr.; Williams, W.A.; Grunlke, J.M.; Hung, C. (Office of Radiation Programs, Washington, DC (USA)). Dec 1982. 326p. NTIS, PC A15/MF A01.

This technical report presents these assessments of long-term repository performance. It describes the models that the Agency developed specifically for this project, reviews the various assumptions made, and identifies the data used in these models. In general a relatively simple analytical methodology was formulated that should tend to overestimate the long-term risks from geologic repositories. Because much of this methodology is new, and because these risk assessments are a key part of our rulemaking, the Agency is publishing this as a draft report. During the public comment period on 40 CFR 191, a Subcommittee of the Agency's Science Advisory Board will conduct an independent technical review of our risk assessments (48 FR 509). All meetings of this Subcommittee will be announced in the Federal Register and will be open to the public.

837 (PB-83-170217) Draft regulatory impact analysis for 40 CFR 191: environmental standards for management and disposal of spent nuclear fuel, high-level and transuranic radioactive wastes. Draft report. (Office of Radiation Programs, Washington, DC (USA)). 1982. 88p. NTIS, PC A05/MF A01.

This Draft Regulatory Impact Analysis (RIA) addresses the requirements of Section 2 of Executive Order 12291. It reviews the projected costs associated with management and disposal of high-level radioactive waste, and it evaluates the potential effects of our environmental standards for disposal of these wastes (40 CFR Part 191)-as proposed for public review and comment on December 29, 1982 (47 FR 58196). The proposed standards are presented in the Appendix of this report, and they are explained in detail in the Draft Environmental Impact Statement (EIS) prepared for this action (EPA 82).

838 (PB-83-171157) Draft environmental impact statement for 40 CFR part 191: environmental standards for management and disposal of spent nuclear fuel, high-level and transuranic radioactive wastes. Final report. (Office of Radiation Pro- grams, Washington, DC (USA)). 1982. 239p. NTIS, PC A11/MF A01.

The Environmental Protection Agency is proposing environmental standards for the management and disposal of spent nuclear reactor fuel and high-level and transuranic radioactive wastes. Subpart $A$ of the standards would limit the radiation exposure of members of the public from management and storage of spent fuel and of waste prior to disposal. Subpart B would establish both quantitative containment requirements for disposal systems and qualitative requirements to assure that these containment requirements will be met. The containment requirements would limit the amount of radioactivity that may enter the environment for 10,000 years after disposal. The assurance requirements provide seven principles necessary for developing confidence that these long-term containment requirements will be complied with. These principles call for well-designed, multiple-barrier disposal systems that would not rely upon future generations for maintenance and would not be located near potentially valuable resources. They also require that future generations be provided information about the location and dangers of the wastes and an option to recover the wastes if they need to. In addition, Subpart B, contains procedural requirements to ensure that the containment requirements are properly applied.

839 (PB-83-184184) Backfilling and sealing a repository for high-level radioactive waste - a review. Butlin, R.N.; Hills, D.L. (Building Research Station, Watford (England)). [nd]. 38p. (CP-6/82). NTIS, PC E04/MF E04.

During a study of the disposal of high-level radioactive wastes, the authors examined the backfilling and sealing of tunnels, shafts and boreholes. This aspect of the construction of a repository for such waste material assumed that the waste is vitrified in borosilicate glass blocks, which are sealed and protected in containers. From a consideration of possible repository geometries, methods of construction and operation, rock characteristics, container types, and also of backfill and sealing systems, it was concluded that a repository could be backfilled and sealed using current engineering practice. A number of considerations were suggested for further investigation, development and design analysis so that the performance of a repository in the short and long term could be ensured.

840 (PB-83-187658) Technical support of standards for high-level radioactive waste management. Addendum to volumes C and D. Final report. (Little (Arthur D.), Inc., Cambridge, MA (USA)). Mar 1982. 97p. NTIS, PC A05/MF A01.

A major Federal effort is underway to develop methods for disposal of high-level radioactive waste in deep geologic repositories. An important element of this program is the development and promulgation by the U.S. Environmental Protection Agency (EPA) of environmental standards for the management of these wastes. The purpose of this report is to examine several issues relevant to release mechanisms and migration pathways.

841 (PNL-3172) High-temperature leaching of an actinide-bearing, simulated high-level waste glass. Westsik, J.H. Jr.; Harvey, C.O.; Kuhn, W.L. (Pacific Northwest Lab., Richland, WA (USA)). Mar 1983. Contract AC06-76RL01830. 59p. NTIS, PC A04/MF A01. Order Number DE83008755.

Portions are illegible in microfiche products. Original copy available until stock is exhausted.

The chemical durability of a simulated high-level waste glass when exposed to high-temperature geologic solutions was investigated. In this study, simulated high-level waste glass-beads ( 76 to 68 glass) doped with technetium, uranium, neptunium, plutonium, curium and americium were leached in deionized water, Waste Isolation Pilot Plant salt brine $B$, and $0.03 \mathrm{M}$ sodium bicarbonate solution at 150 and $250^{\circ} \mathrm{C}$ for $2,4,8,16$, and 32 days. The resulting solutions were analyzed for several nonradioactive glass components and for the radioactive dopants. The glass exhibited incongruent leaching behavior, i.e., the normalized releases $\left(\mathrm{g}-\mathrm{glass} / \mathrm{m}^{2}\right)$ based on the different elements spanned four orders of magnitude. Normalized releases based on boron, molybdenum, sodium, cesium, silicon, and technetium were the same within a factor of three. Most of the nonradioactive components of the glass were released more to the salt brine than to the other two solutions. However, silicon, boron, molybdenum, technetium, and the actinides had their lowest releases in the salt brine. Reaction-layer thickness on the glass surface and weight losses of the glass beads were also smallest in the brine solution. Actinide releases were highest in the sodium bicar- 
bonate solution. Calcium, strontium and barium releases decreased with time and temperature; the releases of most other elements increased with time and temperature. Solubility appears to be limiting the release of most elements. The leachate $\mathrm{pH}$ is controlled by chemical species within the original leachant and by species released as the glass leached. Carbonate ion complexes with some elements including uranium, effectively increasing their release. The more soluble elements including sodium, boron, molybdenum and technetium provide an indication of the actual rate of reaction between the glass and water.

842 (PNL-4266) Feasibility of using a high-level waste canister as an engineered barrier in disposal. Slate, S.C.; Pitman, S.G.; Nesbitt, J.F.; Partain, W.L. (Pacific Northwest Lab., Richland, WA (USA)). Aug 1982. Contract AC06-76RL01830. 54p. NTIS, PC A04/MF A01. Order Number DE82021361.

The objective of this report is to evaluate the feasibility of designing a process canister that could also serve as a barrier canister. To do this a general set of performance criteria is assumed and several metal alloys having a high probability of demonstrating high corrosion resistance under repository conditions are evaluated in a qualitative design assessment. This assessment encompasses canister manufacture, the glass-filling process, interim storage, transportation, and to a limited extent, disposal in a repository. A series of scoping tests were carried out on two titanium alloys and Inconel 625 to determine if the high temperature inherent in the glass-fill processing would seriously affect either the strength or corrosion resistance of these metals. This is a process-related concern unique to the barrier canister concept. The material properties were affected by the heat treatments which simulated both the jouleheated glass melter process (titanium alloys and Inconel 625 ) and the in-can melter (ICM) process (Inconel 625). However, changes in the material properties were generally within $20 \%$ of the original specimens. Accelerated corrosion testing of the heat treated coupons in a highly oxygenated brine showed basic corrosion resistance of titanium grade 12 and Inconel 625 to compare favorably with that of the untreated coupons. The titanium grade 2 coupons experienced severe corrosion pitting. These corrosion tests were of a scoping nature and suitable primarily for the detection of gross sensitivity to the heat treatment inherent in the glass-fill process. They are only suggstive of repository performance since the tests do not adequately model the wide range of repository conditions that could conceivably occur.

843 (PNL-4364) General corrosion, irradiationcorrosion, and environmental-mechanical evaluation of nuclearwaste-package structural-barrier materials. Progress report. Westerman, R.E.; Pitman, S.G.; Nelson, J.L. (Pacific Northwest Lab., Richland, WA (USA)). Sep 1982. Contract AC0676RL01830. 52p. NTIS, PC A04/MF A01. Order Number DE83004164.

Pacific Northwest Laboratory is studying the general corrosion, Irradiation-corrosion, and environmentally enhanced crack propagation of five candidate materials in high-temperature aqueous environments simulating those expected in basalt and tuff repositories. The materials include three cast ferrous materials (ductile cast iron and two low-alloy Cr-Mo cast steels) and two titanium alloys, titanium Grade 2 (commercial purity) and Grade 12 (a Ti-Ni-Mo alloy). The general corrosion results are being obtained by autoclave exposure of specimens to slowly replenished simulated ground water flowing upward through a hed of the appropriate crushed rock (basalt or tuff), which is maintained at the desired test temperature (usually $250^{\circ} \mathrm{C}$ ). In addition, tests are being performed in deionized water. Metal penetration rates of iron-base alloys are being derived by stripping off the corrosion product film and weighing the specimen after the appropriate exposure time. The corrosion of titanium alloy specimens is being determined by weight gain methods. The irradiation-corrosion studies are similar to the general corrosion tests, except that the specimen-bearing autoclaves are held in a ${ }^{60} \mathrm{Co}$ gamma radiation field at dose rates up to $2 \times 10^{6} \mathrm{rad} / \mathrm{h}$. For evaluating the resistance of the candidate materials to environmentally enhanced crack propagation, three methods are being used: U-bend and fracture toughness specimens exposed in autoclaves; slow strain rate studies in repository-relevant environments to $300^{\circ} \mathrm{C}$; and fatigue crack growth rate studies at ambient pressure and $90^{\circ} \mathrm{C}$. The preliminary data suggest a 1 -in. corrosion allowance for iron-base barrier elements intended for 1000-yr service in basalt or tuff repositories. No evidence has yet been found that titanium Grade 2 or Grade 12 is susceptible to environmentally induced crack propagation or, by extension, to stress corrosion cracking.

844 (PNL-4379) Investigation of environmentally assisted fracture of metallic nuclear-waste-package barrier materials in simulated basalt repository environments. Pitman, S.G. (Pacific Northwest Lab., Richland, WA (USA)). Nov 1982. Contract AC06-76RL01830. 34p. NTIS, PC A03/MF A01. Order Number DE83005126.

Statically loaded corrosion tests, slow strain rate (SSR) tests, and fatigue crack growth rate (FCGR) tests were conducted to evaluate the relative susceptibility of two titanium-base nuclear waste package candidate structural barrier materials Ti-grade 2 and Ti-grade 12-to environmentally enhanced cracking in a simulated repository environment. Statically loaded corrosion tests were done in oxic basalt ground water at $250^{\circ} \mathrm{C}$; SSR tests were done in oxic basalt ground water at 150,250 , and $300^{\circ} \mathrm{C}$ and in air at 20 and $250^{\circ} \mathrm{C}$; and FCGR tests were done in basalt ground water, fluoride-ion-enhanced basalt ground water, highpurity water, and air at $90^{\circ} \mathrm{C}$. The following conclusions can be drawn: the general corrosion rate of statically loaded corrosion coupons was very low in a 3-mo test, and no pitting or cracking of the specimene was ohserver. Ti-grade 2 and Ti-grade 12 exhibited strain rate dependent ductility diminution in SSR tests. The ductility diminution was most severe in Ti-grade 2 at $300^{\circ} \mathrm{C}$ and in Ti-grade 12 at $250^{\circ} \mathrm{C}$. For of Tigrade 12 it was found to be highly orientation dependent. The ductility diminution was also found in tests conducted in air as well as in those conducted in the basalt ground water environment; however, the extent of the degradation was less in air. The ductility diminution cannot be attributed to stress corrosion cracking because the fracture mode was microvoid coalescence in all tests. Evidence obtained in the current study and correlation of the present results with results obtained by other researchers indicate that dynamic strain aging is responsible for the loss of ductility. The FCGR of Ti-grade 2 and Ti-grade 12 was not affected by any of the environmental conditions used in this study, which indicates that no environmental cracking mechanism is operative under the conditions tested $\left(90^{\circ} \mathrm{C}\right.$, oxic ground water, and frequencies from 0.01 to $5 \mathrm{~Hz}$ ).

846 (PNL-4382, pp 5-19) Effects of composition, solutions, and burial on nuclear waste glasses. Hench, L.L.; Clark, D.E. (Univ. of Florida, Gainesville). Aug 1982. NTIS, PC A12/MF A01.

In Materials Characterization Center workshop on leaching mechanisms of nuclear waste forms, May 19-21, 1982, Gaithersburg, Maryland. Summary report.

During the last six months efforts in our laboratory have concentrated upon identifying the relative importance of a number of systems variables on the mechanisms and rates of surface attack of nuclear waste glasses. These studies have been conducted in collaboration with the Savannah River Laboratory and Pacific Northwest Laboratory in the US: SKBF/Project KBS and Studsvik Nuclear I ahnratory in Sweden; Marcoule Nuclear Laboratory and the University of Montpellier in France. A preliminary investigation of a nuclear waste ceramic has recently been initiated as well in collaboration with Rockwell International. The systems variables under consideration in our studies include: (1) glass composition; (2) waste percentage; (3) waste type; (4) leachant composition; (5) cyclic corrosion; (6) flow rate; (7) simulated geologic environment; (8) in-situ burial tests; (9) effects of overpacks and backfill materials; and (10) quality control test procedures. Prngress in rach of these areas are briefly reviewed with references as to the title, authors, and location of more lengthy papers on reports presenting details of the studies. 16 figures, 2 tables.

846 (PNL-4382, pp 74-83) Review of recent PNL research activities related to glass leaching mechanisms. McVay, G.L. (Pacific Northwest Lab., Richland, WA). Aug 1982. NTIS, PC A12/MF A01.

In Materials Characterization Center workshop on leaching mechanisms of nuclear waste forms, May 19-21, 1982, Gaithersburg. Maryland. Summary report.

Development of a defensible predictive model for elemental remnval from the repository necessitates the understanding, on a mechanistic level, of waste form leaching characteristics in a realistic repository environment. Borosilicate glass is the primary candidate for a highlevel waste form and thus has received the largest amount of leaching mechanism-related research activity. Research areas which are impor- 
tant to the general understanding of glass-water interactions are: identification of water reaction species; $\mathrm{pH}$; temperature; surface charge; solubility; back reactions; and surface area to solution volume ratio effects. For research areas specific to nuclear waste, the following areas are studied: radiation/radiolysis; interactions; alteration products; and accelerated testing. All of these research areas are being investigated to some degree by nuclear waste-related programs. The areas discussed in this paper are glass-metal interactions and surface-area-to-solutionvolume-ratio effects. All of this research was conducted on PNL 76-68 glass; the exact composition is presented in a table. 6 figures, 2 tables.

847 (PNL-4382, pp 84-96) HLW leaching mechanisms program. Semiannual status report, May 1982. Jackson, D.D. (Lawrence Livermore National Lab., CA). Aug 1982. NTIS, PC A12/MF A01.

In Materials Characterization Center workshop on leaching mechanisms of nuclear waste forms, May 19-21, 1982, Gaithersburg, Maryland. Summary report.

Development has been undertaken of a general-purpose numerical model (PROTOCOL) for the dissolution reactions of nuclear waste forms in water. It is intended to improve our predictive capability for leach rates by bringing together and applying all the known factors that affect them, including especially chemical properties and processes. PROTOCOL is evolving into an integrated software package. It has been provided with an elevated temperature capability, including an expanded data file and I more sophisticated file manager program. Checkout of PROTOCOL functioning was continued with dissolution exercises for amorphous silica. So far, the results seem very reasonable, and it is concluded that the major functions are working as intended. Predictions of chemical events can be made as a result of the interactions of both kinetic and equilibrium processes. Modeling exercises are in progress with experimental dissolution data. It is expected that this will be very effective in identifying areas where improvements are desirable. An analysis of data on the leaching of natural nepheline as a function of both $\mathrm{pH}$ and temperature predicts the formation of several solid prases, especially of aluminous materials. PROTOCOL is still under intensive development, and many extensions are planned or considered. It promises to be a very useful device for the interpretation and extrapolation of leaching data for nuclear waste form materials.

848 (PNL-4382, pp 161-181) Overview of hydrothermal testing of waste package barrier materials at the Basalt Waste Isolation Project. Apted, M.J. (Rockwell Hanford Operations, Richland, WA). Aug 1982. NTIS, PC A12/MF A01.

In Materials Characterization Center workshop on leaching mechanisms of nuclear waste forms, May 19-21, 1982, Gaithersburg, Maryland. Summary report.

The current Waste Package Department (WPD) hydrothermal testing program for the Basalt Waste Isolation Project (BWIP) has followed a systematic approach for the testing of waste-barrier-basalt interactions based on sequential penetration of barriers by intruding groundwaters. Present test activities in the WPD program have focused on determining radionuclide solubility limits (or steady-state conditions) of simulated waste forms and the long-term stability of waste package barriers under site-specific hydrothermal conditions. The resulting data on solution compositions and solid alteration products have been used to evaluate waste form degradation under conditions specific to a nuclear waste repository located in basalt (NWRB). Isothermal, time-invariant compositional data on sampled solutions have been coupled with realistic hydrologic flow data for near-field and far-field modeling for the calculation of meaningful radionuclide release rates. Radionuclides that are not strongly sorbed or precipitated from solution and that, therefore, may require special attention to ensure their isolation within the waste package have been identified. Taken together, these hydrothermal test data have been used to establish design requirements for waste packages located in basalt. Hydrothermal testing with flowthrough autoclave systems and use of tracer-loaded/fully-loaded radioactive waste forms (hotcell testing) are in progress. Results from these tests will be used to confirm performance assessment models based on test results of simulated waste forms under static hydrothermal conditions. 3 figures. stituents in backfill barriers. Nowak, E.J. (Sandia Labs., Albuquerque, NM). Aug 1982. NTIS, PC A12/MF A01. Contract AC04-76DP00789.
In Materials Characterization Center workshop on leaching mechanisms of nuclear waste forms, May 19-21, 1982, Gaithersburg, Maryland. Summary report.

An experiment has been planned to measure mass transport rates in backfill barrier materials for actinide species that have been leached from simulated glass waste forms under simulated salt repository conditions.

860 (PNL-4382, pp 227-237) Waste glass - seabed interactions: observations of the Soret effect. Brush, L.H. (Sandia Labs., Albuquerque, NM). Aug 1982. NTIS, PC A12/MF A01.

In Materials Characterization Center workshop on leaching mechanisms of nuclear waste forms, May 19-21, 1982, Gaithersburg, Maryland. Summary report.

Both constant temperature $\left(200^{\circ}, 300^{\circ} \mathrm{C}\right)$ and temperature gradient experiments were conducted to evaluate the physicochemical effects that would occur to a waste package in a seawater-saturated sedimentary medium, and to estimate corrosion rates for the waste package and radionuclide mobility in case of early failure of the engineered barriers. These experiments examined interactions of seawater with sediments from the study area $1000 \mathrm{~km}$ north of Hawaii and permitted sampling of solutions at experimental temperatures and pressures (500 or 600 bars). The results of the experimental studies illustrate that the chemistry of the near field environment is determined by a combination of water-rock interaction and thermal diffusion effects. Most important with respect to a thermal gradient is the thermal diffusion effect which results in large scale fluxes of $\mathrm{Na}$ and $\mathrm{Cl}$ from the hot zone towards the cool zone. This phenomenon serves to markedly decrease the ionic strength of the hot zone. Other elements, however, appear to be influenced by water-rock interaction processes as well as thermal diffusion. The behavior of $\mathbf{M g}$ is particularly important in this regard because of its potential of generating an acidic environment in the near field region. Resolution of the relative importance of these processes is necessary to determine the suitability of the subseabed environment as a repository for high level radioactive waste. 5 figures.

851 (PNL-4382, pp 247-255) Role of oxidation potential, Eh, in the dissolution of nuclear waste materials. White, W.B.; Freeborn, W.P. (Pennsylvania State Univ., University Park). Aug 1982. NTIS, PC A12/MF A01.

In Materials Characterization Center workshop on leaching mechanisms of nuclear waste forms, May 19-21, 1982, Gaithersburg, Maryland. Summary report.

In a complex system such as a nuclear waste, there are many reactions underway simultaneously. Since most waste forms are oxides, the reactions that occur can be described by oxidation potentials. If the system is to be at equilibrium, all of these reactions must be adjusted to a single oxidation potential which may be regarded as a system variable. This paper discusses how to measure the oxidation potential in a complex system and how to control it. Prior to this discussion, the definition of $\mathrm{Eh}$ is presented mathematically. The connection between $\mathrm{Eh}$, $\mathrm{pH}$, and oxygen fugacity is shown in the final derived equation. 4 figures. -

862 (PNL-4382, pp 182-191) Semi-unified approach to leach testing. Harvey, K.B. (Whiteshell Nuclear Research Establishment, Pinawa, Manitoba). Aug 1982. NTIS, PC A12/MF A01.

In Materials Characterization Center workshop on leaching mechanisms of nuclear waste forms, May 19-21, 1982, Gaithersburg, Maryland. Summary report.

The experimental work described suggests that the leach rates of major matrix ions of sodium borosilicates glasses under a variety of static and replenished leaching conditions are the same when expressed as a function of concentration dissolved into the leachant, and are independent of the geometry of the test. This finding is intuitively reasonable, and it may then be capable of extension to other matrices and also to minority ions diffusing from the matrix. However, since this treatment does not take into account either of changing $\mathrm{pH}$ or of changing temperature, it can only be termed a semi-unified approach. The results suggest that leaching numbers obtained from static tests can be applied to flowing conditions, particularly to the very low-flow conditions ( $~$ $10^{-5} \%$ volume turnover yearly) that might be anticipated under hardrock burial conditions, which are difficult to simulate experimentally. It follows that a static test is more economical since it gives a series of 
leach rate/solution concentration readings, rather than the single result obtainable from a flow test. With the use of active isotopes and counting techniques, this opens the way to completely sealed and maintenancefree leaching systems that could be monitored over a period of years. Leaching models that are appropriate to static leaching conditions are being developed. 5 figures.

853 (PNL-4450) Comparison of cask and drywell storage concepts for a monitored retrievable storage/interim storage system. Rasmussen, D.E. (Pacific Northwest Lab., Richland, WA (USA)). Dec 1982. Contract AC06-76RL01830. 291p. NTIS, PC A13/MF A01. Order Number DE83006550.

Portions are illegible in microfiche products. Original copy available until stock is exhausted.

The Department of Energy, through its Richland Operations Office is evaluating the feasibility, timing, and cost of providing a federal capability for storing the spent fuel, high-level wastes, and transuranic wastes that DOE may be obligated by law to manage until permanent waste disposal facilities are available. Three concepts utilizing a monitored retrievable storage/interim storage (MRS/IS) facility have been developed and analyzed. The first concept, co-location with a reprocessing plant, has been developed by staff of Allied General Nuclear Services. the second concept, a stand-alone facility, has been developed by staff of the General Atomic Company. The third concept, co-location with a deep geologic repository, has been developed by the Pacific Northwest Laboratory with the assistance of the Westinghouse Hanford Company and Kaiser Engineers. The objectives of this study are: to develop preconceptual designs for MRS/IS facilities: to examine various issues such as transportation of wastes, licensing of the facilities, and environmental concerns associated with operation of such facilities; and to estimate the life-cycle costs of the facilities when operated in response to a set of scenarios that define the quantities and types of waste requiring storage in specific time periods, generally spanning the years 1989 to 2037. Three scenarios are examined to develop estimates of life-cycle costs for the MRS/IS facilities. In the first scenario, the reprocessing plant is placed in service in 1989 and HLW canisters are stored until a repository is opened in the year 1998. Additional reprocessing plants and repositories are placed in service at intervals as needed to meet the demand. In the second scenario, the reprocessing plants are delayed in starting operations by 10 years, but the repositories open on schedule. In the third scenario, the repositories are delayed 10 years, but the reprocessing plants open on schedule.

(PNL-4452) Nuclear waste package materials testing report: basaltic and tuffaceous environments. Bradley, D.J.; Coles, D.G.; Hodges, F.N.; McVay, G.L.; Westerman, R.E. (Pacific Northwest Lab., Richland, WA (USA)). Mar 1983. Contract AC06-76RL01830. 139p. NTIS, PC A07/MF A01. Order Number DE83010270.

The disposal of high-level nuclear wastes in underground repositories in the continental United States requires the development of a waste package that will contain radionuclides for a time period commensurate with performance criteria, which may be up to 1000 years. This report addresses materials testing in support of a waste package for a basalt (Hanford, Washington) or a tuff (Nevada Test Site) repository. The materials investigated in this testing effort were: sodium and calcium bentonites and mixtures with sand or basalt as a backfill; iron and titanium-based alloys as structural barriers; and borosilicate waste glass PNL 76-68 as a waste form. The testing also incorporated site-specitic rock media and ground waters: Reference Umtanum Entablature-1 basalt and reference basalt ground water, Bullfrog tuff and NTS J-13 well water. The results of the testing are discussed in four major categories: Backfill Materials: emphasizing water migration, radionuclide migration, physical property and long-term stability studies. Structural Barriers: emphasizing uniform corrosion, irradiation-corrosion, and environmental-mechanical testing. Waste Form Release Characteristics: emphasizing ground water, sample surface area/solution volume ratio, and gamma radiolysis effects. Component Compatibility: emphasizing solution/rock, glass/rock, glass/structural barrier, and glass/ backfill interaction tests. This area also includes sensitivity testing to determine primary parameters to be studied, and the results of systems tests where more than two waste package components were combined during a single test.

865 (PNL-4556) Cesium chloride compatibility testing program. Annual report, FY 1982. Fullam, H.T. (Pacific North- west Lab., Richland, WA (USA)). Dec 1982. Contract ACOF 76RL01830. 45p. NTIS, PC A03/MF A01. Order Numbi DE83006919.

A program was started in FY 1982 to evaluate the compatibility of WESF-produced $\mathrm{CsCl}$ with $316 \mathrm{~L}$ stainless steel under the thermal conditions that would be encountered in a geologic repository. The program is funded through the Long-Term High-Level Defense Waste Program of the Department of Energy. The major part of the program involves compatibility testing of six standard WESF $\mathrm{CsCl}$ capsules at a maximum $\mathrm{CsCl} /$ metal interface temperature of $450^{\circ} \mathrm{C}$ : The capsules are allowed to self-heat to the test temperature in insulated containers and then held at temperature for 2200 to $32,000 \mathrm{~h}$. After thermal aging, the capsules are destructively examined to determine the extent of the metal attack by the $\mathrm{CsCl}$. This report describes the testing procedure and summarizes the activities completed during the first year of the program.

858

(PNL-4576) Glass leaching performance. Chick, L.A.; Turcotte, R.P. (Pacific Northwest Lab., Richland, WA (USA)). May 1983. Contract AC06-76RL01830. 59p. NTIS, PC A04/MF A01. Order Number DE83012465.

Portions are illegible in microfiche products. Uriginal copy available until stock is exhausted.

Current understanding of the leaching performance of high-level nuclear waste (HLW) glass is summarized. The empirical model of waste glass leaching behavior developed shows that at high water flow rates the glass leach rate is kinetically limited to a maximum value. At intermediate water flow rates, leaching is limited by the solution concentration of silica and decreases with decreasing water flow rates. Release of soluble elements is controlled by silica dissolution because silica forms the binding network of the glass. At low water flow rates, mass loss rates reach values controlled by formation rates of alteration minerals, or by diffusion of dissolution products through essentially stagnant water. The parameters reviewed with respect to their quantifiable influence on leaching behavior include temperature, $\mathrm{pH}$, leachant composition, glass composition, thermal history, and radiation. Of these, temperature is most important since the rate of mass loss approximately doubles with each $10^{\circ} \mathrm{C}$ increase in dilute solutions. The $\mathrm{pH}$ has small effects within the 4 to 10 range. The chemical composition of the leachant is most important with regard to its influence on alteration product formation. Glass composition exhibits the largest effects at high flow rates where improved glasses leach from ten to thirty times slower than glass 76 to 68 . The effects of the thermal history (devitrification) of the glass are not likely to be significant. Radiation effects are important primarily in that radiolysis can potentially drive $\mathrm{pH}$ values to less than 4 . Radiation damage to the glass causes insignificant changes in leaching performance.

867 (PNL-4625) MRS/IS facility co-located with a repository: preconceptual design and life-cycle cost estimates. Smith, R.I.; Nesbitt, J.F. (Pacific Northwest Lab., Richland, WA (USA)). Nov 1982. Contract AC06-76RL01830. 257p. NTIS, PC A12/MF A01. Order Number DE83008719.

Portions are illegible in microfiche products. Original copy available until stock is exhausted.

A program is described to examine the various alternatives for monitored retrievable storage (MRS) and interim storage (IS) of spent nuclear fuel, solidified high-level waste (HLW), and transuranic (TRU) waste until appropriate geologic repository/repositories are available. The objectives of this study are: (1) to develop a preconceptual design for an MRS/IS facility that would become the principal surface facility for a deep geologic repository when the repository is opened, (2) to examine various issues such as transportation of wastes, licensing of the facility, and environmental concerns associated with operation of such a facility, and (3) to estimate the life cycle costs of the facility when operated in response to a set of scenarios which define the quantities and types of waste requiring storage in specific time periods, which generally span the years from 1990 until 2016. The life cycle costs estimated in this study include: the capital expenditures for structures, casks and/or drywells, storage areas and pads, and transfer equipment; the cost staff labor, supplies, and services; and the incremental cost of transpol ing the waste materials from the site of origin to the MRS/IS facilit Three scenarios are examined to develop estimates of life cycle costs of the MRS/IS facility. In the first scenario, HLW canisters are stored, starting in 1990, until the co-located repository is opened in the year 1998. Additional reprocessing plants and repositories are placed in ser- 
vice at various intervals. In the second scenario, spent fuel is stored, starting in 1990, because the reprocessing plants are delayed in starting operations by 10 years, but no HLW is stored because the repositories open on schedule. In the third scenario, HLW is stored, starting in 1990, because the repositories are delayed 10 years, but the reprocessing plants open on schedule.

858 (PNL-SA-10375) Enviromechanical testing of Tigrade 2 and Ti-grade 12 in basalt ground water. Pitman, S.G. (Pacific Northwest Lab., Richland, WA (USA)). Dec 1982. Contract AC06-76RL01830. 26p. (CONF-820955-3). NTIS, PC A03/MF A01. Order Number DE83006202.

From ASTM conference on titanium and zirconium in industrial applications; New Orleans, LA, USA (21 Sep 1982).

In earlier corrosion screening studies, Ti-grade 2 and Ti-grade 12 were found to have exceptional resistance to uniform corrosion in basalt ground water. Further rigorous tests were then applied to characterize the resistance of these materials to degradation induced by groundwater radiolysis and by application of stress in the presence of the ground-water environment. These tests included irradiation-corrosion tests (using corrosion coupons, U-bend specimens, Charpy V-notch impact specimens, and bolt-loaded fracture specimens), slow strain rate (SSR) tests, and fatigue crack growth rate (FCGR) tests. Results of the current study suggest that Ti-grade 2 and Ti-grade 12 could be suitable for use as long-lived engineered barriers in a basalt repository. Further studies are needed, however, including a detailed analysis of the tendency of the materials to adsorb hydrogen. This paper describes the testing of Ti-grade 2 and Ti-grade 12 by corrosion and irradiation-corrosion tests, SSR tests, and FCGR tests.

859 (PNL-SA-10649) Overview of the Monitored Retrievable Storage (MRS) program. Rasmussen, D.E. (Pacific Northwest Lab., Richland, WA (USA)). Aug 1982. Contract AC06-76RL01830. 7p. (CONF-820943-7). NTIS, PC A02/MF A01. Order Number DE83002738.

From ANS topical meeting - spent fuel storage; Savannah, GA, USA (26 Sep 1982).

The current ubjeclive of the DOE MRS/Interim Storage (IS) program is to ensure that the Government has the capability to accept and store high-level waste and spent fuel in the amounts and on a schedule determined by DOE goals for nuclear energy, legislation and regulation. Preliminary studies are complete and development of the concepts, with commercial sector involvement, is about to start. Spent fuel and high-level waste storage requirements for the reference case indicate minimum interim storage requirements if start of commercial reprocessing and repository availability remain on schedule.

860

(PNL-SA-10913) Waste glass/metal interactions in brines. Shade, J.W.; Pederson, L.R.; McVay, G.L. (Pacific Northwest Lab., Richland, WA (USA)). May 1983. Contract $\Lambda$ C06 76RL01830. 21p. (CONF-830451-22). NTIS, PC A02/MF A01. Order Number DE83016786.

From 85. American Ceramic Society annual meeting; Chicago, IL, USA (25 Apr 1983).

Leaching studies of MCC 76-68 glass in synthetic brines high in $\mathrm{NaCl}$ were performed from 50 to $150^{\circ} \mathrm{C}$ and included interactive testing with ductile iron and titanium. Hydrolysis of the glass matrix was generally slower in saturated brines than in deionized water, due to a lower solubility of silica in the brines. Inclusion of ductile iron in the tests resulted in accelerated leach rates because irion-silica reactions occurred which reduced the silica saturation fraction. At $150^{\circ} \mathrm{C}$, iron also accelerated the rate of crystalline reaction product formation which were primarily Fe-bearing sepiolite and talc. 16 references.

861 (PNL-SA-11273) Engineered components for radioactive waste disposal systems - are they technically justified. Burkholder, H.C. (Pacific Northwest Lab., Richland, WA (USA)). May 1983. Contract AC06-76RL01830. 20p. (CONF830523-15; IAEA-CN-43/254). NTIS, PC A02/MF A01. Order Number DE83013259.

From International conference on radioactive waste management; Seattle, WA, USA (16 May 1983).

The technical justification for the development and use of sophisticated engineered components in radioactive waste disposal systems was evaluated. The evaluation applied information from many studies in the nuclear waste disposal literature and focused their combined results on determining the relative contribution of a number of subsystem functions to overall system performance. A wide range of spent fuel, commercial high-level, and Savannah River defense high-level waste disposal systems in dome salt, bedded salt, basalt, and granite geologic media were investigated. The results showed that the site portion of the disposal system so dominated overall system performance that even relatively poor sites were more than adequate to isolate the waste without the use of any engineered components. The performancedominating nuclides were so long-lived that even extremely sophisticated waste containers could not increase overall system performance above that required for adequacy. Even relatively poor sites are effective in controlling the release of nucides with short half-lives, like ${ }^{90} \mathrm{Sr}$, and even exceptional waste containers are ineffective in controlling the release of nuclides with long half-lives, like ${ }^{207} \mathrm{~Np}$. Based on the results of this study and other studies in the nuclear waste disposal literature, the use of long-lived waste containers and very low transport rate manmade subsystems seems unjustified from a technical standpoint and a geologic disposal point of view. The design objective for the waste container lifetime should not be greater than that desired for retrievability ( 0 to 100 years), and the design objective for the man-made subsystem transport rate should not be less than the $10^{-2}$ to $10^{-4}$ per year range.

862

(PNL-SA-11297) Effect of gamma radiolysis on waste glass leaching on brines. Pederson, L.R.; McVay, G.L. (Pacific Northwest Lab., Richland, WA (USA)). Apr 1983. Contract AC06-76RL01830. 18p. (CONF-830451-19). NTIS, PC A02/MF A01. Order Number DE83016177.

From 85. American Ceramic Society annual meeting; Chicago, IL, USA (25 Apr 1983).

The effect of gamma radiolysis of a deaerated, saturated, $\mathrm{NaCl}$ rich brine on leaching of a simulated nuclear waste glass was evaluated at 50 and $90^{\circ} \mathrm{C}$ and included interactive tests with ductile iron. Leach rate enhancements due to radiolysis were greatest at $50^{\circ} \mathrm{C}$. Inclusion of iron at this temperature, known to accelerate glass at $50^{\circ} \mathrm{C}$. Inclusion of iron at this temperature, known to accelerate glass leaching in the absence of radiolysis, gave leach rates greater than for glass alone plus radiolysis or for glass plus ductile iron in the absence of radiolysis. However, at $90^{\circ} \mathrm{C}$, no enhancement in leaching due to radiolysis was evident with or without iron. It is concluded that reaction of the glass with transient radiolysis products is responsible for the leach rate increase at $50^{\circ} \mathrm{C}$.

863 (PNL-SA-11308) Economic comparison of crystalline ceramic and glass waste forms for HLW disposal. McKee, R.W.; Daling, P.M.; Wiles, L.E. (Pacific Northwest Lab., Richland, WA (USA)). May 1983. Contract AC06-76RL01830. 21p. (CONF-830451-14). NTIS, PC A02/MF A01. Order Number DE83013251

From 85. American Ceramic Society annual meeting; Chicago, IL, USA (25 Ap1 1983).

A titanate-based, crystalline ceramic produced by hot isostatic pressing has been proposed as a potentially more stable and improved waste form for high-level nuclear waste disposal compared to the currently favored borosilicate glass waste form. This paper describes the results of a study to evaluate the relative costs for disposal of high-level waste from a 70,000 metric ton equivalent (MTE) system. The entire waste management system, including waste processing and encapsulation, transportation, and final repository disposal, was included in this analysis. The repository concept is based on the current basalt waste isolation project (BWIP) reference design. A range of design basis alternatives is considered to determine if this would influence the relative economics of the two waste forms. A thermal analysis procedure was utilized to define optimum canister sizes to assure that each waste form was compared under favorable conditions. Repository costs are found to favor the borosilicate glass waste form while transportation costs greatly favor the crystalline ceramic waste form. The determining component in the cost comparison is the waste processing cost, which strongly favors the borosilicate glass process because of its relative simplicity. A net cost advantage on the order of $12 \%$ to $15 \%$ on a waste management system basis is indicated for the glass waste form.

864 (PNL-SA-11579) Monitored retrievable storage: a waste management option for spent fuel and high-level wastes. Fletcher, J.F. (Pacific Northwest Lab., Richland, WA (USA)). 
Aug 1983. Contract AC06-76RL01830. 18p. NTIS, PC A02/MF A01. Order Number DE83018286.

One option for managing spent fuel and high-level wastes is the Monitored Retrievable Storage (MRS) concept. This concept will provide for long-term storage of spent fuel and nuclear wastes, under controlled and monitored conditions, in surface or near-surface facilities. The MRS concept is viewed primarily as a means for providing storage of spent fuel and nuclear wastes if mined geologic disposal is not available when needed. Eight concepts are under consideration for the storage of spent fuel and high-level wastes: metal casks, concrete silo, silo-intrench, field drywells, tunnel drywells, open-cycle vault, closed-cycle vaults, and tunnel rack. All eight concepts for MRS storage are now undergoing engineering evaluation. From this evaluation, two leading concepts will be selected for further design and development. A proposal for facility construction based on these leading designs is scheduled to be delivered to the US Congress in June 1985.

866 (PNLSA-11648) Monitoring land and water uses in the Columbia Plateau using remote-sensing computer analysis and integration techniques. Leonhart, L.S.; Wukelic, G.E. Foote, H.P.; Blair, S.C. (Pacific Northwest Lab., Richland, WA (USA)). Sep 1983. Contract AC06-76RL01830. 16p. (CÓNF830847-4). NTIS, PC A02/MF A01. Order Number DE84001415.

From Energy resource management '83; San Francisco, CA, U3A (39 Aug 1989).

Portions are illegible in microfiche products.

This study successfully utilized advanced, remote-sensing computer-analysis techniques to quantify and map land- and water-use trends potentially relevant to siting, developing, and operating a highlevel national, nuclear waste repository on the US Department of Energy's Hanford Site in eastern Washington State. Specifically, using a variety of digital data bases (primarily multidate LANDSAT data) and digital analysis programs, the study produced unique numerical data and integrated data reference maps relevant to regional (Columbia Plateau) and localized (Pasco Basin) hydrologic considerations associated with developing such a facility. Because all study data developed are in digital form, they can be called upon to contribute to future reference repository location monitoring and reporting efforts, as well as to be utilized in other US Department of Energy programmatic areas having technical and/or environmental interest in the Columbia Plateau region. The results obtained indicate that multidate digital LANDSAT data provide an inexpensive, up-to-date, and accurate data base and reference map of natural and cultural features existing in any region. These data can be (1) computer enhanced to highlight selected surface features of interest; (2) processed/analyzed to provide regional land cover/ use information and trend data; and (3) combined with other line and point data files to accommodate interactive, correlative analyses and integrated colorgraphic displays to aid interpretation and modeling efforts. Once the digital base is established, selected site information can be assessed immediately, various forms of data can be accessed concurrently or separately, and data sets may be displayed or mapped at any scale. Available editing software provides the opportunity to generate credible scenarios for a site while preserving the actual data base. 6 references.

866 (PTB-FMRB-86) SALTMP - a FORTRAN program for fast salt temperature calculations in HRW final storage. Ramthun, H. (Physikalisch-Technische Bundesanstalt, Braunschweig (Germany, F.R.). Forschungs- und Messreaktor). Jul 1981. 17p. (In German). NTIS (US Sales Only), MF A01. Order Number DE82750525.

The computer code SALTMP is the transformation of Calculations concerning the temperature increase in salt rock for a given concept of waste disposal into a FORTRAN program. It starts from an analytical solution of the heat conduction diffential equation and requires relatively short computing times. The disadvantage of less flexibility (assumption of an idealized model, of constant heat production and of temperature independent heat conductivity in salt rock) is compensated for by additional approximation calculations. As a consequence detailed temperature curves are obtained in a fraction of one hour also when long distances or long disposal times are requested and when numerical procedures fail because of too long computing times. As an example the task was chosen to evaluate the temperature curves for four disposal times, the loci for the calculation being given by a set of 48 coordinates in the median plane of the disposal and by a special choice of the routine, computing the distances from the 54 disposal positions. As in this case the way of computing is crossing three areas of heat production, each curve is subdivided into three sections, sparing these areas. This makes evident, that within the framework of these calculations the temperatures in the disposal positions remain undefined.

$86 \overline{7}$ (PTB-FMRB-88) Radiant energy dissipation during final storage of high-level radioactive waste in rock salt. Ramthun, H. (Physikalisch-Technische Bundesanstalt, Braunschweig (Germany, F.R.). Forschungs- und Messreaktor). Aug 1981. 34p. (In German). NTIS (US Sales Only), MF A01. Order Number DE82750523.

A final disposal concept is assumed where the high-active waste from $1400 \mathrm{t}$ of uranium, remaining after conditioning, is solidified in borosilicate glass and distributed in 1.760 waste casks. These containers $1.2 \mathrm{~m}$ in height and $0.3 \mathrm{~m}$ in diameter are to be buried 10 years after the fuel is removed from the reactor in the $300 \mathrm{~m}$ deep boreholes of a salt dome. For this design the mean absorbed dose rates are calculated in the glass die $(3.9 \mathrm{ry} / \mathrm{s})$, the stepl mantle $(0.26 \mathrm{~Gy} / \mathrm{s})$ and in the salt rock $(0.12 \mathrm{~Gy} / \mathrm{s}$ at a distance of $1 \mathrm{~cm}$ and $0.034 \mathrm{~Gy} / \mathrm{s}$ at a distance of $9 \mathrm{~cm}$ from the container surface) valid at the beginning of disposal. The risk involved with these amounts of stored lattice energy is shortly discussed.

868 (RHO-BW-CR-127-P) Basalt-radionuclide reacdons: FT-1Y8I. Anmual repurt. Ames, L.L.; McGarrah, J.E.; Walker, B.A. (Pacific Northwest Lab., Richland, WA (USA)). Aug 1981. Contract AC06-77RL01030. 139p. (PNL-3992). NTIS, PC A07/MF A01. Order Number DE83005224.

Sorption isotherms were obtained with $\mathrm{Na}, \mathrm{Ca}, \mathrm{Sr}, \mathrm{Pu}, \mathrm{U}, \mathrm{Cs}, \mathrm{Se}$, $\mathrm{Np}, \mathrm{Ra}$, and $\mathrm{Tc}$ and $60^{\circ} \mathrm{C}$, with two synthetic groundwaters on Columbia River basalts and associated secondary minerals. Where appropriate ( $\mathrm{U}, \mathrm{Se}, \mathrm{Np}$, and $\mathrm{Tc})$, isotherms were obtained under low $\mathrm{Eh}(-50 \mathrm{mV})$ conditions as well as oxidizing conditions. Straight line relationships between equilibrium radionuclide concentration on the basalt or secondary minerals and in the solution were obtained by use of the linearized Freundlich equation. Two exceptions were the uranium and cesium sorption isotherms. These were described by the Dubinin-Radushkevich equation. Isotherms determined under anoxic conditions (U. Se. Np) showed the effects of radionuclide precipitation and the generation of colloids that remained in the solution yielding relatively low sorption results. Radium distribution coefficients increased with increasing radium concentration, a good indication of precipitation as a removal mechanism. Neptunium, changing from $N p(V)$ to $N p(I V)$ also gave indications of precipitation occurring. In ion exchange, tracer level systems, cesium and strontium were used to show that the presence of increasing amounts of competing sodium and calcium caused the strontium and cesium sorption to follow simple linear regressions with negative regression coefficients. Uranium was quantitatively removed from solution at $150^{\circ} \mathrm{C}$ in the presence of basalt. Initial strontium and plutonium removal from solution was low but tended to steadily increase with up to 60 days solution-basalt contact. Pu, U, Cs, and Ra sorption and desorption were studied on the same basalt wafers of known surface area and weight. There was no apparent relationship between wafer surface area and radionuclide sorption rates or amounts. Radionuclides, sorbed and desorbed under the same conditions, always desorbed more slowly. There were indications that a percentage of the sorbed radionuclide was firmly fixed on the basalt.

869 (RHO-BW-CR-129P) Assessment of the effects of surficial geologic processes in the Pasco Basin. (Woodward-Clyde Consultants, San Francisco, CA (USA)). Jul 1980. Contract AC06-77RL01030. 119p. NTIS, PC A06/MF A01. Order Number DE83005495.

Based on a review of available geologic data regarding surficial geologic processes that shaped the landscape in the Pasco Basin during Quaternary time, an estimate has been made of rates and the net effect of similar processes for the next million years. I andscape modification of the Pasco Basin during the past million years has been dominated by degradational processes. During catastrophic floods, rates of both aggradation and erosion were briefly accelerated. However, long-term rates of denudation and aggradation have been relatively low. These rates probably reflect dry climate and, perhaps more importantly, relatively low rates of uplift and subsidence. The net effect of surficial geologic processs during the next million years probably will not be significantly different. Data on rates of surficial geologic processes, particularly rates 
of uplift and subsidence, are insufficient to accurately predict where the land surface will be in one million years. However, based on long-term rates of uplift, denudation, and channel incision, it is unlikely that more than $100 \mathrm{~m}$ of downcutting will occur anywhere within the Pasco Basin during the next million years. Rates of tectonic processes are the dominant factor affecting the evolution of the landscape in the Pasco Basin. More data are needed regarding distribution and ages of quaternary deposits to accurately assess tectonic rates during the past million years.

870 (RHO-BW-CR-132-P) 1981 Geodolite observations near Hanford, Washington. (Geological Survey, Menlo Park, CA (USA). Office of Earthquake Studies; Rockwell International Corp., Richland, WA (USA). Rockwell Hanford Operations). Oct 1981. Contract AC06-77RL01030. 53p. NTIS MF A01. Order Number DE83005227.

Microfiche only, copy does not permit paper copy reproduction. Repeated measures of strain accumulation at the Hanford Site in southeastern Washington over a 9-yr period provide a quantitative measure of deformation. The observations indicate a low rate of strain accumulation. In 1981, the existing geodetic network was expanded to provide more detailed strain accumulation for the Snively Basin portion of the Rattlesnake Hills anticline and for the Wallula Fault system south and east of the Hanford Site. The observed direction of compression, $\mathbf{N}$. $85^{\circ} \mathrm{W} . \pm 14^{0}$, is inconsistent with most focal mechanism solutions in the area. The direction is consistent with a model for subduction of the Juan de Fuca Plate, but the observed rates are higher than predicted by such a model.

871 (RHO-BW-CR-133P) Report on geologic remote sensing of the Columbia Plateau. Sandness, G.A.; Kimball, C.S.; Schmierer, K.E.; Lindberg, J.W. (Pacific Northwest Lab., Richland, WA (USA)). May 1982. Contract ACO677RL01030. 360p. (PNL-3140). NTIS, PC A16/MF A01. Order Number DE83010201.

Portions are illegible in microfiche products.

The purpose of this remote sensing study is to identify faults or other geologic features which may have a significant bearing on the structural and tectonic character of the Hanford Site and the surrounding region. Landsat imagery, Skylab photographs, and U-2 photographs were analyzed to identify and map geologic photolineaments in the Columbia Plateau. The Landsat and Skylab imagery provided a regional perspective and allowed the identification of large-scale linear features. The U-2 photography provided much greater spatial resolution as well as a stereoscopic viewing capability. This allowed identification of smaller structural or geologic features and the identification of many cultural and nongeologic lineaments detected in the Landsat and Skylab imagery. The area studied totals, approximately 85,000 square miles, and encompasses virtually all exposures of Columbia River Basalt in the states of Washington, Oregon, and Idaho. It also includes an area bordering the Columbia River Basalt outcrop. This border area was studied in order to identify significant structures that may extend into the plateau. Included are a description of the procedures used for image analysis, 20 lineament maps at a scale of 1:250,000, geological summaries for the areas covered by the lineament maps, and discussions of many of the lineaments shown on the maps. Comparisons of the lineament maps with available geologic maps showed that the number of detected lineaments was much greater than the number of known faults and other linear features. Approximately $70 \%$ of the faults shown on the geologic maps were detected and are characterized as lineaments. Lineament trends in the northwest-southeast and northeast-southwest directions were found to predominate throughout the study area.

872 (RHO-BW-CR-136-P) Waste package conceptual designs for a nuclear repository in basalt. (Westinghouse Electric Corp., Pittsburgh, PA (USA). Advanced Energy Systems Div.). Oct 1982. Contract AC06-77RL01030. 198p. (AESDTME-3142). NTIS, PC A09/MF A01. Order Number DE83006520.

The scope of conceptual waste package design activities reported was limited to include only waste packages for the disposal of the reference forms of Defense High Level Waste (DHLW) and Commercial High Level Waste (CHLW) and a form of spent fuel which consists of canisterized circular bundles of fuel rods from 3 Pressurized Water Reactor (PWR) assemblies or 7 Boiling Water Reactor assemblies. The reference waste packages in this report utilize low carbon steel for the containment. The steel is sufficiently thick to provide corrosion resis- tance for 1,000 years and to resist in situ crushing loads during this time period. These packages are designed to be emplaced in long horizontal boreholes. A pneumatically emplaced bentonite-basalt backfill is included to assist the waste form in satisfying the current long-term radionuclide release criterion becauce of lack of sufficient data at this time to conclude that the waste form itself can satisfy this requirement. In the event that delayed pneumatic backfill emplacement is not desirable or feasible with the reference design, an alternate borehole type design is included. This alternate utilizes a carbon steel containment barrier with the backfill placed inside this overpack to facilitate emplacement in the long horizontal holes. A second alternate design, which has certain potential repository and overall system advantages, utilizes cast ferrous material to provide containment. Material thicknesses are such that the overpack provides shielding to keep radiation levels low enough to permit limited hands-on operations. Preliminary performance evaluations, based on available data, indicate that the designs presented in this report meet applicable requiremaents.

873 (RHO-BW-SA-187P) Uplift and subsidence rates in the central Columbia Plateau and their relation to siting a waste repository at Hanford, Washington. Reidel, S.P.; Fecht, K.R. (Rockwell International Corp., Canoga Park, CA (USA). Energy Systems Group). May 1982. Contract AC0677RL01030. 9p. (CONF-820550-5). NTIS, PC A02/MF A01. Order Number DE82016062.

From Geological Society of America Rocky Mountain section meeting; Bozeman, MT, USA (7 May 1982).

The Hanford Site is located in the Pasco Basin, one of several structural basins on the Columbia Plateau. The Cold Creek syncline on the Hanford Site is being considered as a potential repository location for the storage of high-level nuclear waste. Important criteria for siting a nuclear waste repository are potentially adverse conditions that could affect the long-term stability of a mined repository. One such condition is structural deformation as it relates to uplift and subsidence in a repository area. The purpose of this talk is to discuss those areas that have undergone uplift and subsidence, with special reference to the Cold Creek syncline, and the rates at which this movement occurred. Also considered is the relation of this movement to siting a nuclear waste repository at Hanford.

87 (RHO-BW-SA-203-P) Engineered waste package concepts for high-level waste isolation in basalt. Smith, M.J. (Rockwell International Corp., Richland, WA (USA). Rockwell Hanford Operations). Jun 1982. Contract AC06-77RL01030. 4p. (CONF-820609-17). NTIS, PC A02/MF A01. Order Number DE82011003.

From American Nuclear Society annual meeting; Los Angeles, CA, USA (6 Jun 1982).

Portions of document are illegible.

The Basalt Waste Isolation Project (BWIP) is charged with determining the feasibility of permanently storing commercial highlevel radioactive wastes in a geologic repository located in the basalt underlying the Hanford Site. The BWIP Waste Package Program has been developed as a part of the National Waste Terminal Storage (NWTS) Program to provide a focal point for the selection of materials and the development of design specifications for site-specific, mutliple barrier assemblages for spent fuel and processed high-level wastes in this medium. While the concept of a multiple barrier waste package system designed to provide redundancy in waste isolation bas received wide support, the design limits, performance requirements, and functions of such a system have been described only in general terms. This paper presents the results of the BWIP efforts to more clearly define the environmental, regulatory, and performance factors that influence the development of waste package design criteria for a basalt medium. A technique for developing performance and design criteria based on the favorable environmental characteristics (low Eh, moderate $\mathrm{pH}$, low ionic strength groundwater) of the basalts beneath the Hanford Site are discussed. These site-specific considerations have been utilized to greatly reduce the complexity and hence the cost of waste packages for basalt. The techniques employed in this analysis are applicable to other systems for which sufficient site-specific data exists.

876 (RHO-BW-SA-204-P) Radionuclide solubillty and transport in basalt. Jacobs, G.K. (Rockwell International Corp., Richland, WA (USA). Rockwell Hanford Operations). 1982. 
Contract AC06-77RL01030. 12p. (CONF-820609-20). NTIS, PC A02/MF A01. Order Number DE82010778.

From American Nuclear Society annual meeting; Los Angeles, CA, USA (6 Jun 1982).

A performance assessment model to estimate maximum possible radionuclide releases to the accessible environment using solubility and transport characteristics for radionulclides is briefly described. Analyses under conditions relevant to a repository located in basalt at the Hanford Site indicate that the site will dominate containment of high-level wastes even under disruptive conditions. This performance by the site greatly reduces the requirements on the repository and waste package engineered systems. (DMC)

876 (RHO-BW-SA-205A-P) Thermomechanical simulation of a nuclear-waste canister in a repository in basalt. Topcubasi, A.F.; Lehnhoff, T.L.; Thirumalai, K. (Rockwell International Corp., Richland, WA (USA). Rockwell Hanford Operations). Aug 1982. Contract AC06-77RL01030. 6p. NTIS, PC A02/MF A01. Order Number DE83010608.

This paper discusses how a linear axisymmetric numerical thermo-elastic analysis, using average material properties, was determined to provide adequate accuracy for most near-yield predictions of temperatures, displacements, and stresses.

877 (RHO-BW-SA-209-P) Overview of waste-handling requirements for a nuclear-waste repository in basalt. Gimera, R.J.; Davis, J.M. (Rockwell International Corp., Richland, WA (USA). Rockwell Hanford Operations). 1982. Contract ACO677RL01030. 22p. (CONF-820424-35). NTIS, PC A02/MF A01. Order Number DE82021716.

From ANS topical meeting on treatment and handling of radioactive wastes; Richland, WA, USA (19 Apr 1982).

Portions of document are illegible.

The conceptual design for a nuclear waste repository in basalt (NWRB) for the permanent storage of commercial spent fuel was completed in September 1981. This design is now in the process of being modified to reflect the present administration's decision to restore the reprocessing of commercial spent fuel in the nuclear fuel cycle. The new conceptual design of the NWRB will accommodate both spent fuel and vitrified high-level waste, use a simplified waste package, and be designed to accommodate more conservative rock stresses at depth. The modified design is scheduled for completion in September 1982. The NWRB will have the capacity to store commercial spent fuel and commercial high-level waste (CHLW) to the equivalent of 64,000 tons ( 58 , $200 \mathrm{t}$ ) of heavy metal. This is expected to be approximately one-half of the national total at the year 2000 , and it is estimated that the NWRB receipts will be roughly a 50/50 division between spent fuel and $C H L W$. Storage capacity will also be provided for 32,000 drums of low-leveltransuranic (LL-TRU) waste received from offsite, in addition to any in-plant generated waste. The NWRB waste-handling system is described here as it was designed for handling canisters of spent fuel only. The current design modifications are not far enough along to describe. The waste-handling facilities on the surface (waste receiving, hot-cell operations, transfer cask, hoist, and hoist unloading station underground) will not be significantly altered by the new conceptualdesign modifications. Emplacement of waste in the storage panels may, however, be different, since the higher horizontal to vertical rock-stress ratio being used in the design upgrade may result in lower and wider tunnels, precluding vertical waste emplacement. In this paper the following waste-handling requirements for an NWRB are discussed: wastehandling building; receiving facilities; hot cell; transfer cask; transfercask transporter; and radwaste management.

878 (RHO-BW-SA-216P) Site-screening process on the Hanford Site: a summary report. Brown, D.J. (Rockwell International Corp., Richland, WA (USA). Rockwell Hanford Operations). Jun 1980. Contract AC06-77RL01030. 28p. (CONF821205-1). NTIS, PC A03/MF A01. Order Number DE82020779.

From NWTS information meeting on basalt waste isolation project: Las Vegas, NV, USA (14 Dec 1982).

A site-screening study was initiated to identify locations within the Hanford site for a nuclear waste repository. This report summarizes progress made in the following areas: identification of objectives and development of guidelines for application to the study area; a multi-step screening process that permits the application of guidelines to smaller and smaller areas until the site localities have been identified; and development of a data base of appropriate scope and detail that could be utilized for defining the conditions within the areas defined in each substep of the screening process. On the basis of the site characterization work and the engineering studies, the optimal areas for siting the principal borehole for an exploratory shaft has been identified. (DMC)

879 (RHO-BW-SA-219-P) Experimental investigation of sodium bentonite stability in Hanford basalt. Wood, M.I. (Rockwell International Corp., Richland, WA (USA). Rockwell Hanford Operations). Feb 1983. Contract AC06-77RL01030. 10p. (CONF-821107-52). NTIS, PC A02/MF A01. Order Number DE83008328.

From 6. international symposium on the scientific basis for radioactive waste management (Materials Research Society); Boston, MA, USA (1 Nov 1982).

Sodium bentonite is a candidate material for the waste package backfill component in a repository in basalt at the Hanford Sitc. Preliminary hydrothermal experiments have been conducted under near-field geveliemical conditious expected to vecur in the reference repusitury location in Grande Ronde Basalt. Experiments have been conducted in the basalt/groundwater, bentonite/groundwater, and basalt/bentonite/ gmindwater systems The experiments have, heen condisted at $3 m^{\circ} \mathrm{C}$. using a simulated Grande Ronde groundwater, reference Umtanum basalt, and sodium bentonite. Key data generated by the experiments include experimental solution analyses as a function of time and preliminary solids analysis by scanning transmission electron microscopy and $x$-ray diffraction. Solution trends of the major aqueous species were similar in the three systems and are characterized by: (1) the gradual reduction of the $\mathrm{pH}$ value from $\sim 9.75$ to a steady-state value of $\sim 6$, (2) an initial rapid increase followed by a gradual decreasein silica concentration, and (3) a slight or negligible increase in sodium, sulfate, and chloride concentrations. In the bentonite/groundwater experiment, small amounts $(<1 \%)$ of an albite reaction product were observed. Conserversely, the formation of illite, a common bentonite alteration product, was not observed. These results indicate tjhat sodium bentonite will remain sufficiently stablee at $300^{\circ} \mathrm{C}$ under hydrothermal conditions in basalt to permit its use as a backfill material.

880 (RHO-BW-SA-220P) Groundwater flow and transport characteristics of flood basalts as determined from tracer experiments. I.ennhart, I.S.; Jackson, R.I.; Graham, D.L.; Thompson, G.M.; Gelhar, L.W. (Rockwell International Corp., Richland, WA (USA). Energy Systems Group; Arizona Univ., Tucson (USA); Massachusetts Inst. of Tech., Cambridge (USA)). 15 Sep 1982. Contract AC06-77RL01030. 15p. (CONF-820964-2). NTIS, PC A02/MF A01. Order Number DE83000718.

From Joint Pacific Northwest American Geophysical Union and American Association for the Advancement of Science meeting; Fairbanks, AK, USA (14 Sep 1982).

Work described in this paper contributes to the understanding of hydrologic performance of a proposed repository at the Hanford Site. Specifically, this paper describes the conduct and analysis of a two-well (injection/withdrawal) tracer test performed on a basalt interflow horizon (McCoy Canyon) beneath the Hanford Site. The overall purpose of the test was to initiate a phase of hydrologir testing that is directed primarily at obtaining estimates of parameters essential for contaminant transport modeling. Associated with this purpose were the following objectives: (1) to develop techniques and procedures for conducting groundwater tracer tests in the fleld and attendant laboratory analytical support; (2) to provide field data useful for evaluating available analytical methodologies to obtain estimates of dispersivity and porosity parameters; (3) to obtain estimates of longitudinal dispersivity and effective thickness for the test horizon that could be utilized in contaminant transport models; and (4) to provide a set of data that could be used to compare the test results obtained earlier, using an alternative technique, for the same horizon. The test was conducted in January 1982. The earlier testing was done in December 1979.

881 (RHO-BW-SA-229P) Determination of rock mass deformation modulus in closely jointed Columbia River basalt. Lanigan, D.C.; Cramer, M.L.; Kim, K. (Rockwell International Corp., Richland, WA (USA). Rockwell Hanford Operations). 
182. Contract AC06-77RL01030. 25p. (CONF-820964-4). TIS, PC A02/MF A01. Order Number DE82021719.

From Joint Pacific Northwest American Geophysical Union and American Association for the Advancement of Science meeting; Fairbanks, AK, USA (14 Sep 1982).

Portions are illegible in microfiche products.

The basalts delineated for the repository feasibility study are located on the Hanford Site in southeastern Washington. One of the rock properties of interest is the deformation modulus. In order to properly apply a deformation modulus value to construction design or numerical models, it is necessary to understand the range of value the modulus can exhibit. The modulus values can vary as functions of sample size, stress level, location, and orientation. No single test method allows an evaluation of all these variables, so several different types of modulus tests must be performed. The three types of tests discussed are: laboratory testing of recovered core; borehole jacking tests using the Goodman Jack; and borehole deformation measurements during a flat jack test. The following conclusions can be inferred from data collected in tests in the closely jointed Pomona basalt: deformation modulus decreases with increasing specimen size; laboratory tests indicate a deformation modulus of approximately 87 gigapascals; borehole deformation measurements in a flatjack test indicate a deformation modulus of approximately $\mathbf{4 0}$ gigapascals; borehole jacking test with a Goodman Jack indicate a deformation modulus of approximately 20 gigapascals; and there is no significant temperature effect on modulus values (up to $100^{\circ} \mathrm{C}$ ).

882

(RHO-BW-SA-231A-P) Status report on the fullscale electric-heater tests at the Hanford Near-Surface Test Facility. Baxter, J.T.; Cunningham, J.P.; Gregory, E.C.; Jimenez, R.F.; Topcubasi, A.F. (Rockwell International Corp., Richland, WA (USA). Rockwell Hanford Operations; SEE CODE9516255 Kaiser Engineers, Oakland, CA (USA); Parsons, Brinckerhoff, Quade and Douglas, Inc., San Francisco, CA (USA)). [nd]. Contract AC06-77RL01030. 26p. NTIS, PC A03/MF A01. Order Number DE82019256.

Portions of document are illegible.

Purpose of the NSTF was to evaluate the thermomechanical response of basalt subjected to thermal loads, such as from high-level radioactive wastes in canisters. Prior to test startup, the response was modeled using DAMSWEL and a bilinear model. It was concluded that the response can be modeled satisfactorily on the canister or very nearfield scale. The agreement for horizontal displacements is not as good as for vertical displacements. Rock temperature was $>400^{\circ} \mathrm{C}$ at start of simulated cooldown, has been $>300^{\circ} \mathrm{C}$ for 30 days. No appreciable boreholes decrepitation or thermal cracking has been observed. 23 figures. (DLC)

883

(RHO-BW-SA-231-P) Status report on the full-scale electric heater tests at the Hanford Near-Surface Test Facility. Baxter, J.T.; Cunningham, J.P.; Gregory, E.C.; Jimenez, R.F.; Topcubasi, A.F. (Rockwell International Corp., Richland, WA (USA). Rockwell Hanford Operations; SEE CODE- 9516255 Kaiser Engineers, Oakland, CA (USA); Parsons, Brinckerhoff, Quade and Douglas, Inc., Oakland, CA (USA)). Aug 1982. Contract AC06-77RL01030. 27p. (CONF-820803-15). NTIS, PC A03/MF A01. Order Number DE83003716.

From 23. US symposium on rock mechanics; Berkeley, CA, USA (25 Aug 1982)

Portions of document are illegible.

A Near-Surface Test Facility (NSTF) was constructed at the Hanford Site, in order to evaluate the thermomechanical response of basalt subjected to thermal loads. Two large-scale field tests using electric heaters to simulate nuclear waste canisters were started in July 1980 , and are scheduled for completion during 1982. These tests are part of the program to examine the feasibility and provide the technology needed to design and construct a geological repository for the emplacement of high-level radioactive waste in basalt formations. In both tests, electric heaters were placed in vertical boreholes in the Pomona basalt flow in the floor of the NSTF. Full-Scale Heater Test lo. 1 consists of a central heater canister surrounded by eight periphal heaters. Heater power increased progressively during the test. Rock

imperature at the borehole wall was in excess of $400^{\circ} \mathrm{C}$ after 2 years of operation. Full-Scale Heater Test No. 2 consists of a single heater canister. Heater power increased progressively for $1-1 / 2$ years followed by a 120-day cooldown period. The heater was restarted and power is currently being raised to the limits of the equipment. Rock temperature at the borehole wall just prior to cooldown was in excess of $400^{\circ} \mathrm{C}$. A visual comparison of a heater borehole wall was made from photographs taken prior to testing and during the cooldown. Rock temperature was in excess of $400^{\circ} \mathrm{C}$ at the start of cooldown and had been in excess of $300^{\circ} \mathrm{C}$ for about 300 days. No appreciable borehole decrepitation or thermally induced cracking was observed.

884 (RHO-BW-SA-254-P) Waste package conceptual designs for a repository located in basalt. Smith, M.J. (Rockwell International Corp., Richland, WA (USA). Energy Systems Group). Sep 1982. Contract AC06-77RL01030. 11p. (CONF821205-12). NTIS, PC A02/MF A01. Order Number DE83001750.

From NWTS information meeting on basalt waste isolation project; Las Vegas, NV, USA (14 Dec 1982).

Results are reported of a joint effort among the Basalt Waste Isolation Project (BWIP), Westinghouse-Advanced Energy Systems Division, the Office of Nuclear Waste Isolation, and Kaiser Engineers, Inc. and Parsons Brinckerhoff Quade and Douglas, Inc. to develop conceptual designs for waste packages to be emplaced in a repository located in basalt. During fiscal year 1982, practical reference designs for commercial high-level waste and circular bundles of spent fuel rods from three pressurized water reactor assemblies or seven boiling water reactor assemblies were developed on the basis of cost effectiveness. Each of the designs is based upon clearly defined environmental and regulatory performance factors that were used by the BWIP in developing package design criteria. The package design has been utilized by the BWIP in updating the conceptual design of the nuclear waste repository in basalt (NWRB) which was also completed in fiscal year 1982. A technique for developing performance and design criteria based on the favorable environmental characteristics (low-Eh, moderate-pH, low-ionicstrength groundwater) of the basalts beneath the Hanford Site are also discussed. These site-specific considerations have been utilized to greatly reduce the complexity and, hence, the cost of waste packages for basalt. The techniques employed in this analysis are applicable to other systems for which sufficient site-specific data exists. 3 figures, 1 table.

885 (RHO-BW-SA-255-P) Testing of candidate wastepackage backfill and canister materials for basalt. Wood, M.I.; Anderson, W.J.; Aden, G.D. (Rockwell International Corp., Richland, WA (USA). Energy Systems Group). Sep 1982. Contract AC06-77RL01030. 9p. (CONF-821205-23). NTIS, PC A02/MF A01. Order Number DE83001487.

From NWTS information meeting on basalt waste isolation project; Las Vegas, NV, USA (14 Dec 1982).

The Basalt Waste Isolation Project (BWIP) is developing a multiple-barrier waste package to contain high-level nuclear waste as part of an overall system (e.g., waste package, repository sealing system, and host rock) designed to isolate the waste in a repository located in basalt beneath the Hanford Site, Richland, Washington. The three basic components of the waste package are the waste form, the canister, and the backfill. An extensive testing program is under way to determine the chemical, physical, and mechanical properties of potential canister and backfill materials. The data derived from this testing program will be used to recommend those materials that most adequately perform the functions assigned to the canister and backfill.

886 (RHO-BW-SA-256-P) Summary of Near-Surface Test Facility results and their application to repository design. Gregory, E.C.; Cramer, M.L.; McCabe, W.M.; Kim, K. (Rockwell International Corp., Richland, WA (USA). Energy Systems Group). Sep 1982. Contract AC06-77RL01030. 16p. (CONF-821205-20). NTIS, PC A02/MF A01. Order Number DE83001755.

From NWTS information meeting on basalt waste isolation project; Las Vegas, NV, USA (14 Dec 1982).

Results from ongoing and newly initiated tests at the NearSurface Test Facility have contributed substantially to the store of knowledge of rock mass characterization of basalt at a variety of temperatures. Enhancements in monitoring instrumentation and testing techniques have likewise been achieved. These developments have already assisted in the determination of site suitability of Hanford basalt formations and have contributed to refinement of predictive numerical models for use in repository design. Most recently, the testing program has resulted in the following: an improved method of determining ther- 
mal expansion effects on the borehole deformation gage; an improved method of monitoring the stress relief process during the doorstopper overcoring process; the development of a high-resolution optical device for monitoring rock mass deformation in several directions simultaneously; a preliminary indication that the columnar structure of basalt is reflected by significant differences in deformational characteristics parallel and perpendicular to the columns; initial values of block test deformation modulus to establish a relationship between small- and large-scale modulus test results; and the observation that slabbing and decrepitation of borehole walls do not occur in basalt even at temperatures that are twice the design value (i.e., at $400^{\circ} \mathrm{C}$ ). 5 figures.

887 (RHO-BW-SA-258-P) Nuclear-waste repository in basalt: seal development studies. Anderson, W.J.; Clayton, L.G.; Cottam, A.E.; Jacobs, G.K.; Schulz, W.W.; Watrous, R.A. (Rockwell International Corp., Richland, WA (USA). Energy Systems Group). Sep 1982. Contract AC06-77RL01030. 9p. (CONF-821205-16). NTIS, PC A02/MF A01. Order Number DE83001490.

From NWTS information meeting on basalt waste isolation project; Las Vegas, NV, USA (14 Dec 1982).

Current efforts have concentrated on establishing performance requirements and design criteria for a seal system. To accomplish this objective, the repository seal system is considered as one component of the multiple barriers concept of waste isolation. During fiscal year 1982 . a simple calculational method to predict steady-state flow rates through a system of shaft seals and backfill zones as a function of plug dimensions, material permeability, and predicted hydraulic gradient has been developed. A Tektronix desk top computer has been programmed to assist in executing the calculations. A preliminary analysis of computerproduced data indicates disturbed rock zone permeability dominates calculated flow rates and travel times and, therefore as further studies proceed, greater emphasis will be given to the disturbed rock zone characterization. As model development continues, many of the assumptions will be removed and fracture flow conditions will be considered. The goal is to develop a model that is supported and verified by design development and laboratory, field, and in situ materials testing. 4 figures.

888 (RHO-BW-SA-259-P) Conceptual design of the nuclear-waste repository in basalt. Turner, D.A. (Rockwell International Corp., Richland, WA (USA). Energy Systems Group). Sep 1982. Contract AC06-77RL01030. 11p. (CONF821205-24). NTIS, PC A02/MF A01. Order Number DE83001486.

From NWTS information meeting on basalt waste isolation project; Las Vegas, NV, USA (14 Dec 1982).

The conceptual NWRB is sized to dispose of spent fuel and commercial high-level waste equivalent to $\sim 52,200$ tons of heavy metal, cooled for $10 \mathrm{y}$, and 32,000 drums of low-level transuranic waste. Retrieval capability shall be maintained for up to $50 \mathrm{y}$. The reference repository location is in the Hanford Site. The surface and subsurface facilities will occupy 40.5 ha (200 acres) and 588 ha (1455 acres), respectively. The design of the underground spaces is described. 6 figures. (DLC)

889 (RHO-BW-SA-267-P) Rock mass deformation properties of closely jointed basalt. Kim, K.; Cramer, M.L. (Rockwell International Corp., Richland, WA (USA). Energy Systems Group). Dec 1982. Contract AC06-77RL01030. 20p. NTIS, PC A02/MF A01. Order Number DE83006084.

Portions are illegible in microfiche products.

The deformational behavior of the Columbia River basalt is being investigated as part of a comprehensive site characterization program intended to determine the feasibility of constructing a nuclear waste repository in basalt at Hanford, Washington. Direct field measurements were conducted in a 2-m cube of basalt to obtain truly representative rock mass deformation properties. Load was applied to the test block in three orthogonal directions through the use of flat jacks in two perpendicular planes and a cable anchor system in the third. This configuration allowed the block to be placed in a simulated triaxial stress state at stress levels up to $12.5 \mathrm{MPa}$. The deformation at the center of the test block was monitored through the use of an optical measurement system developed for this project. The results indicate that the vertically oriented columnar joints have a significant influence on the deformation behavior of the basalt. The modulus in the direction parallel to the column axis was $\sim 30 \mathrm{GPa}$, while the modulus value perpendicular to the columns was $\sim 20 \mathrm{GPa}$. Laboratory measurements of intact speci mens taken from this area yielded a value of $80 \mathrm{GPa}$ with no indicatio of anisotropy. Hysteresis was observed in all loading cycles, but was distinctly more pronounced perpendicular to the column axis, indicative of significant joint displacement in this direction. The results of this test represent the first true rock mass modulus data obtained in closely jointed rock on a large scale. These measurement methods have eliminated many of the ambiguities associated with borehole jacking and surface measurement techniques.

890 (RHO-BW-SA-271P) Performance allocation traceable to regulatory criteria as applied to site-characterization work at the Basalt Waste Isolation Project. Deju, R.A.; Babad, H.; Jacobs, G.K.; Bensky, M.S. (Rockwell International Corp., Richland, WA (USA). Rockwell Hanford Operations). Feb 1983. Contract AC06-77RL01030. 9p. (CONF-830205-32). NTIS MF A01. Order Number DE83015079. Feb 1983)

From Waste management conference: Tucson, AZ, USA (27

Microfiche only, copy does not permit paper copy reproduction.

The Basalt Waste Isulation Project has developed a method for defining in detail the work required to demonstrate the feasibility of emplacing and providing for the safe isolation of nuclear wastes in a repository in the deep basalts at the Ilanford Site near Richland, Washinglun. Criter la analysls ullows the identificlation of areas of significant technical uncertainty or controversy that can be highlighted as issues. A preliminary analysis has been conducted, which, by identifying key radionuclides and allocating performance among the multiple barriers in a repository constructed in a basalt, allows the design and develupment testing activities at the Basalt Waste Isolation Project to be put into perspective. Application of sophisticated uncertainty analysis techniques will allow refinements in the analysis to be made and to further guide characterization and testing activities. Preliminary results suggest that a repository constructed in basalt will provide for the safe isolation of nuclear wastes in a cust-effective and reliable manner with a high degree of confidence.

891

(RHO-BW-SA-277P) Geohydrologic characterization and qualification of a high-level waste site in basalts. Gephart, R.E.; Price, S.M. (Rockwell International Corp., Richland, WA (USA). Rockwell Hanford Operations). Feb 1983. Contract AC06-77RL01030. 10p. (CONF-830205-24). NTIS, PC A02/MF A01. Order Number DE83009655. Feb 1983)

From Waste management conference; Tucson, AZ, USA (27

Portions are illegible in microfiche products.

Geohydrologic studies are under way on the Hanford Site, Washington, to characterize the Columbia River basalt as a possible geologic medium for disposal of nuclear wastes. Findings indicate that the deformation of the Pasco asin and reference repository location has occurred slowly (averaging $<0.1 \mathrm{~mm} / \mathrm{y}$ ) and is expected to continue at a slow rate along existing geologic structues. Most groundwater in the deep basalts moves laterally through portions of basalt flow tops under low $\left(\sim 10^{-4} \mathrm{~m} / \mathrm{m}\right)$ hydraulic gradients. These flow tops are separated by basalt flow interiors having hydraulic conductives in the range of $10^{-11}$ to $10^{-10} \mathrm{~m} / \mathrm{s}$. Using site-specific characterization data, performance assessment modeling indicates that groundwater traveltimes to the accessible environment are $>10,000 \mathrm{y}$. Because of the limiting solubilities of major radionuclides and the sorptive capacity of basalt, most radionulcides are effectivcly immobilized in the basalt medium.

892 (RHO-BW-SA-281-P) Bibliography and documents issued list through fiscal year 1982. (Rockwell International Corp., Richland, WA (USA). Rockwell Hanford Operations). Dec 1982. Contract AC06-77RL01030. 59p. NTIS, PC A04/MF A01. Order Number DE83004853.

This document is a bibliography of the published reports, papers, and documents of the Basalt Waste Isolation Project (BWIP) that have been publicly released during fiscal years 1978 to 1982 . A brief histor. of the National Waste Terminal Storage Program and the BWIP $i$ included to provide the reader with the scope of activities involved $i$ : the technical endeavor to identify a potential geologic repository site for the permanent isolation of radioactive wastes. The bibliography is organized by scientific discipline and in chronological order. An author index is provided after the bibliography. 
893 (RHO-BW-SA-293P) Testimony of Dr. Raul A. Deju, Basalt Waste Isolation Project, before the Subcommittee on Energy Research and Production, Committee on Sceince and Technology, United States House of Representatives, March 2, 1983. (Rockwell International Corp., Richland, WA (USA). Rockwell Hanford Operations). 1983. Contract AC0677RL01030. 8p. NTIS, PC A02/MF A01. Order Number DE83009580.

Status of the Basalt Waste Isolation Project is given. Three key concerns have been identified that need to be resolved to either confirm or eliminate the basalts as a potential nuclear waste repository host medium. They are: A thorough understanding of the groundwater hydrology beneath the Hanford Site is needed to assure that a repository in basalt will not contribute unacceptable amounts of contaminants to the accessible environment. Our ability to construct a repository shaft and a network of underground tunnels needs to be fully demonstrated through an exploratory shaft program. Our ability to ultimately seal a repository, such that its integrity and the isolation of the waste are guaranteed, needs to be demonstrated.

894

(RHO-BW-SA-298A-P) Status of geologic and hydrologic characterization of a potential nuclear waste repository site in basalt. Price, S.M.; Gephart, R.E. (Rockwell International Corp., Richland, WA (USA). Rockwell Hanford Operations). Jul 1983. Contract AC06-77RL01030. 4p. (CONF8310192-1). NTIS, PC A02/MF A01. Order Number DE84001113.

From Geological Society of America annual meeting; Indianapolis, IN, USA (31 Oct 1983).

Within the Basalt Waste Isolation Project, geohydrologic studies are focusing on repository site characterization, particularly those factors that might influence groundwater transport of radionuclides to the accessible environment. Beneath the Hanford Site, the Columbia River basalt consists of at least 70 basalt flows extending to a depth of $>3 \mathbf{k m}$. Within the $180-\mathrm{km}^{2}$ central Cold Creek syncline, these flows appear to be nearly flat lying; at least four are thick $(>40 \mathrm{~m})$ and laterally continuous and are presently considered potential candidates for repository development. These flows lie 850 to $1150 \mathrm{~m}$ below ground level within a $47-\mathrm{km}^{2}$ area currently considered to be the prime candidate site. Tectonic studies appear to indicate that the site area was deforming at a low average rate of strain in the Miocene and that this rate has continued into the late Cenozoic. Available head and hydrologic data appear to indicate that groundwater within the deeper basalts generally moves laterally through zones of higher hydraulic conductivity (flow tops) and vertically through the fractured flow interiors. Hydraulic head gradients appear to be low $\left(\sim 10^{-4} \mathrm{~m} / \mathrm{m}\right)$. Horizontal hydraulic conductivity values of $10^{-11}$ to $10^{-14} \mathrm{~m} / \mathrm{s}$ have been measured within flow interiors; tests needed to obtain vertical hydraulic conductivity values are planned. An iterative approach involving numerical modeling and data collection and interpretation is being used to develop a preliminary geohydrologic conceptual model of the potential site and surrounding area.

895 (RHO-BW-SR-82-1-1Q-P) Basalt Waste Isolation Project. Quarterly report, October 1, 1981-December 31, 1981. (Rockwell International Corp., Canoga Park, CA (USA). Energy Systems Group). Jan 1982. Contract ACO677RL01030. 85p. NTIS, PC A05/MF A01. Order Number DE82018197.

This document reports progress made in the Basalt Waste Isolation Project during the first quarter of fiscal year 1982. Efforts are described for the following programs of the project work breakdown structure: systems; waste package; site; repository; regulatory and institutional; test facilities; and in situ test facilities.

896 (RHO-BW-SR-82-2-1Q-P) Basalt waste-isolation project drilling and testing. Quarterly report, January 1-March 31, 1982. (Rockwell International Corp., Richland, WA (USA). Rockwell Hanford Operations). 1 Apr 1982. Contract AC0677RL01030. 38p. NTIS, PC A03/MF A01. Order Number DE.83002810.

Rockwell Hanford Operations Drilling and Testing Group is responsible for all borehole drilling and testing conducted in support of the Basalt Waste Isolation Project. Geologic and hydrologic characterization data obtained by the group are an integral part of the studies required to assess the feasibility of using the deep basalts beneath the Hanford Site for the terminal disposal of nuclear waste. This document is a summary of drilling and testing results during the first calendar quarter (January through March) of 1982. The principal work during this period included the drilling and/or testing of boreholes RRL-2, DC16A, DC-7/8, DC-15, DH-25, DH-26, and BH-17. 12 figures, 8 tables.

897 (RHO-BW-SR-82-2-2Q-P) Basalt Waste Isolation Project drilling and testing. Quarterly report, April 1, 1982-June 30, 1982. (Rockwell International Corp., Richland, WA (USA). Energy Systems Group). 1 Jul 1982. Contract AC0677RL01030. 45p. NTIS, PC A03/MF A01. Order Number DE83007947.

This document is a summary of drilling and testing results during the second calendar quarter (April through June 1982). The principal work during this period included the drilling and/or testing of boreholes RRL-2, DC-16A, DC-7/8, DC-15, DH-24, DH-25, McGee and BH-17. Specific highlights of this work are summarized.

898 (RHO-BW-SR-82-2-3Q-P) Basalt Waste Isolation Project drilling and testing. Quarterly report, July 1, $1982-$ September 30, 1982. (Rockwell International Corp., Richland, WA (USA). Energy Systems Group). 1 Oct 1982. Contract AC06-77RL 01030. 51p. NTIS, PC A04/MF A01. Order Number DE83008195.

This document is a summary of drilling and testing results during the third calendar quarter (July through September 1982). The principal work during this period included the drilling and/or testing of boreholes RRL-2, RRL-6, RRL-14, DC-3, DC-16A, DC-16C, and the McGee Well. Specific highlights of this work are summarized.

899 (RHO-BW-SR-82-2-4QP) Basalt Waste Isolation Project: drilling and testing. Quarterly report, October $1,1982-$ December 31, 1982. (Rockwell International Corp., Richland, WA (USA). Rockwell Hanford Operations). 1 Jan 1983. Contract AC06-77RL01030. 48p. NTIS, PC A03/MF A01. Order Number DE83010644.

Rockwell Hanford Operations Drilling and Testing Group is responsible for all borehole drilling and testing conducted in support of the Basalt Waste Isolation Project. Geologic and hydrologic characterization data obtained by the group are an internal part of the studies required to assess the feasibility of using the deep basalts beneath the Hanford Site for the terminal disposal of nuclear waste. This document is a summary of drilling and testing results during the fourth calendar quarter (October through December 1982). The principal work during this period included the drilling and/or testing of boreholes RRL-2, RRL-6, RRL-14, DC-4/DC-5, DC-16A, DC-16B, and the McGee Well. Specific highlights of this work are summarized.

900 (RHO-BW-SR-83-1-1Q-P) Basalt Waste Isolation Project. Drilling and testing quarterly report, January 1, 1983March 31, 1983. (Rockwell International Corp., Richland, WA (USA). Rockwell Hanford Operations). 1 Apr 1983. Contract AC06-77RL01030. 46p. NTIS, PC A03/MF A01. Order Number DE83017483.

This document is a summary of dilling and testing results during the first calendar quarter (January through March 1983). The principal work during this period included the drilling and/or testing of boreholes RRL-2, RRL-6, RRL-14, DC-4/DC-5, DC-16A, DC-16B, and the McGee Well. Specific highlights of this work are summarized.

901 (RHO-BW-ST-19-P) Preliminary interpretation of the tectonic stability of the reference repository location, Cold Creek syncline, Hanford Site. Bergstrom, K.A.; Caggiano, J.A.; Cross, R.W.; Davis, J.D.; Duncan, D.W.; Fecht, K.R.; Mitchell, T.H.; Reidel, S.P.; Rohay, A.C. (Rockwell International Corp., Richland, WA (USA). Rockwell Hanford Operations). Mar 1983. Contract AC06-77RL01030. 246p. NTIS, PC A11/MF A01. Order Number DE83018233.

Portions are illegible in microfiche products. Original copy available until stock is exhausted.

This preliminary interpretation of available geologic, seismologic, geophysical, and geodetic data indicates that the references repository location in the Cold Creek syncline of the Pasco Basin meets the US Nuclear Regulatory Commission and Basalt Waste Isolation Project proposed criteria for tectonic stability. Uncertainties regarding the timing, sequence, and mechanics of fold and fault development will be addressed during the program of detailed site characterization. This pre- 
liminary assessment of tectonic stability does not appear to preclude development of a repository in the reference repository location. 230 references.

902 (RHO-BW-ST-21-P) Evaluation of sodium bentonite and crushed basalt as waste package backfill materials. Wood, M.I.; Aden, G.D.; Lane, D.L. (Rockwell International Corp., Richland, WA (USA). Rockwell Hanford Operations). Oct 1982. Contract AC06-77RL01030. 46p. NTIS, PC A03/MF A01. Order Number DE83007029.

Preliminary hydrothermal experiments were completed at $300^{\circ} \mathrm{C}, 300$ bars and $200^{\circ} \mathrm{C}, 300$ bars in the basalt/groundwater system to determine the chemical stability of crushed basalt. Analysis of solution data from the $300^{\circ} \mathrm{C}$ experiments as a function of time and reaction products indicated that the primary reaction was the alteration of the basalt glass phase to illite and/or smectite clays and quartz. The establishment of steady state $\mathrm{pH}$ values of about 6. the apparent rapid occurrence of a highly reducing environment in the system, and an insignificant increase in the solution concentration of potentially corrosive aqueous species were observed. These data indicate that a waste package backfill containing a significant amount of crushed basalt will provide a near-field geochemical environment favorable to the chemical stability of metal canister materials. A preliminary experiment was also completed in the sodium bentonite/groundwater system at $300^{\circ} \mathrm{C}, 300$ bars. Analyses of the reacted solutions and solids show that bentonite remains essentially stable with only minor alterations to albite. Sorption data were generated on crushed basalt, secondary minerals in basalt, and sodium bentonite at 60 to $65^{\circ} \mathrm{C}$ under oxic and anoxic conditions. These data indicate that $\mathrm{Cs}$ and $\mathrm{Sr}$ will be completely contained in a waste package backfill due to the formation of insoluble secondary minerals, ion exchange, and specific adsorption. Under reducing conditions, $\mathbf{N p}$ will be retained beyond $1000 \mathrm{yr}$ and $U$ and $P u$ will be retained under reducing conditions over $300 \mathrm{yr}$. An increase in retention time can be expected to occur with an increase in temperature. Theory, calculations, and a limited data base have been used to propose a reference waste package. The backfill component consisting of 25\% sodium bentonite and $75 \%$ crushed basalt with an initial density of $2.1 \mathrm{~g} / \mathrm{cm}^{0}$ and a thickness of $0.152 \mathrm{~m}$.

903 (RHO-BW-ST-25P) Conceptual design requirements for spent fuel, high-level waste, and transuranic waste packages. Anderson, W.J. (Rockwell International Corp., Richland, WA (USA). Rockwell Hanford Operations). Oct 1982. Contract AC06-77RL01030. 71p. NTIS, PC A04/MF A01. Order Number DER3Mn7458.

The responsibility for waste package conceptual designs for all geologic media has been assigned to Westinghouse-Advanced Energy Systems Division (W-AESD) by the National Waste Terminal Storage (NWTS) Waste Package Program Office. The responsibility for insuring that the waste package designs are compatible with each candidate repository medium lies with the respective site project office. For the Basalt Waste Isolation Project (BWIP), this responsibility has been assigned to the Waste Package Department. Studies are being conducted within the Waste Package Department for which focus on the development of data and information needed to define the design requirements for waste packages to be emplaced in a repository constructed in the basalt. Functional and performance requirements are defined, along with environmental conditions and materials properties used for design.

904

(RHO-BW-ST-26-P) Native copper deposits of the Portage Lake volcanics, Michigan: their implications with respect to canister stability for nuclear waste isolation in the Columbia River basalts beneath the Hanford Site, Washington. Crisman, D.P.; Jacobs, G.K. (Rockwell International Corp., Richland, WA (USA). Rockwell Hanford Operations). 1982. Contract AM06-76RL02225;AC06-77RL01030. 83p. NTIS, PC A05/MF A01. Order Number DE83003128.

Portions are illegible in microfiche products.

Copper deposits in the Portage Lake Volcanics of northern Michigan have been evaluated as a natural analogue for canister material to be emplaced in a nuclear waste repository located in the basalts beneath the Hanford Site near Richland, Washington. Te native copper, which precipitated from high temperature $\left(200^{\circ} \mathrm{C}\right.$ to $\left.300^{\circ} \mathrm{C}\right)$ hydrothermal solutions between 500 and 800 million yr ago, has remained relatively unaltered in the Portage lake basalt-groundwater system. The results of this study illustrate the stability of copper in solutions of moderate $\mathrm{pH}$, low to moderate Eh, and low total dissolved solids. Chemical trends and geochemical modeling of te near-surface waters suggestthat similarities (moderate pH, low E, low total dissolved solids) exist between the groundwater-basalt system of the Keweenaw Peninsula and the groundwater-basalt system at the Hanford Site. These similarities and the stability of copper in the Keweenswan basalts imply that copper alloys are adequate materials for nuclear waste canisters emplaced in a repository located in basalt.

905 (RHO-BW-ST-27-P) Characterization of reference materials for the Barrier Materials Test Program. Palmer, R.A.; Aden, G.D.; Johnston, R.G.; Jones, T.E.; Lane, D.L.; Noonan, A.F. (Rockwell International Corp., Richland, WA (USA). Rockwell Hanford Operations). Jun 1982. Contract AC0677RL01030. 109p. NTIS, PC A06/MF A01. Order Number DE8301 1092.

Initial characterization of the geologic and engineered barrier materials for a nuclear waste repository in basalt has been completed. Data have been obtained on the characteristics of the reference waste. forms which are being studied for eventual dispnsal in such a repository. Reference basalt entablature, colonnade, and flow top specimens have been selected from the Umtanum flow, which is the primary basalt flow under consideration for repository siting. Material from the Mabton Interbed Stratum. Pomona Flow basalt, smectite clay from the Pomona Flow, a potassium clinoptilolite, Beverly sandstone and tuff, and Grande Ronde groundwater are also included in the suite of reference geologic materials. Reference engineered barrier materials include sodium bentonite and canister metals such as carbon steel, cupronickel, Hastelloy and Inconel alloys. Spent fuel, borosilicate glass, and supercalcine ceramic comprise the reference waste forms. Analyses were made for physical, chemical, and morphological characteristics using techniques ranging from simple observations of color to sophisticated ultrastructural analysis in the electron microscope. Analyses of the elemental and phase chemistries for most of the reference materials have been completed on typical samples. Determinations of material homogeneity are currently being performed.

908 (RIO-BW-ST-33-P) Autumated data acquisition and analysis system used in the Basalt Waste Isolation Project's Near-Surface Test Facility. Starr, J.L. (Rockwell International Corp., Richland, WA (USA). Rockwell Hanford Operations). Dec 1982. Contract ACO6-77RL01030. 73p. NTIS, PC A04/MF A01. Order Number DE83007340.

A largo miniwmputer busod data acquisition and analysis system has been developed by the Basalt Waste Isolation project and is being applied to support research efforts on the response of rock (basalt) to thermal loads from simulated nuclear wastes. This system is believed to be the largest and most powerful system of its type in existence. It scans over 1000 different instruments. for three separate experiments. at a maximum frequency of once every 5 minutes. In addition to data acquisition, the system also performs the functions of data reduction, analog-to-digital conversion, computation of engineering units, data archiving, statistical analysis, and interactive graphics and reports. The system should be of general interest to those concerned with automated monitoring of instrumentation and computer graphics, as applied to large-scale engineering and scientific experimentation, especially in the fields of rock mechanics and nuclear waste disposal.

907 (RHO-BW-ST-36P) Technical conservatism in the design and analysis of a nuclear-waste repository in basalt. Jones, K.A. (Rockwell International Corp., Richland, WA (USA). Rockwell Hanford Operations). 30 Sep 1982. Contract AC06-77RL01030. 65p. NTIS, PC A04/MF A01. Order Number DE83011928.

Portions are illegible in microfiche products. Original copy available until stock is exhausted.

The US Department of Energy's National Waste Terminal Storage Program has adopted a policy of technical conservatism to guide the design and analysis of geologic disposal systems for commercial highlevel radioactive waste. Technical conservatism serves as the programmatic philosophy for managing uncertainty in the performance of the disposal system. The implementation of technical conservatism as applied to a nuclear waste repository in basalt is discussed. Preliminary assessments of the performance of the waste package, repository, and site subsystems are compared to key proposed regulatory criteria. The 
imparison shows that there are substantial safety margins in the prected performance of the nuclear waste repository in basalt.

908 (RHO-BWI-81-100-2Q) Basalt waste-isolation project. Quarterly report, January 1-March 31, 1981. (Rockwell International Corp., Richland, WA (USA). Rockwell Hanford Operations). May 1981. Contract AC06-77RL01030. 145p. NTIS, PC A07/MF A01. Order Number DE82012541.

Portions of document are illegible.

This document reports progress made in the Basalt Waste Isolation Project during the second quarter of fiscal year 1981. Efforts are described for the following End Functions of the project work breakdown structure: systems; waste package; site; repository; exploratory shaft test facility; regulatory and institutional; near-surface test facility Phase I; near-surface test facility Phase II; and support facilities.

909

(RHO-BWI-81-100-3Q) Basalt waste isolation project. Quarterly report, April 1, 1981-June 30, 1981. Deju, R.A. (Rockwell International Corp., Richland, WA (USA). Rockwell Hanford Operations). Aug 1981. Contract AC06-77RL01030. 104p. NTIS, PC A06/MF A01. Order Number DE82012355.

Portions of document are illegible.

This document reports progress made in the Basalt Waste Isolation Project during the third quarter of fiscal year 1981. Efforts are described for the following programs of the project work breakdown structure: systems; waste package; site; repository; regulatory and institutional; test facilities; in situ test facilities.

910 (RHO-BWI-81-100-4Q) Basalt Waste Isolation Project. Quarterly report, July 1, 1981-September 30, 1981. Deju, R.A. (Rockwell International Corp., Richland, WA (USA). Energy Systems Group). Nov 1981. Contract AC0677RL01030. 97p. NTIS, PC A05/MF A01. Order Number DE82012031.

Portions are illegible in microfiche products.

This document reports progress made in the Basalt Waste Isolation Project during the fourth quarter of fiscal year 1981. Efforts are described for the following programs of the project work breakdown structure: systems, waste package, site, repository, regulatory and institutional, test facilities, and in-situ test facilities.

911

(RHO-BWI-C-105) Geochemical behavior of supercalcine waste form: its stability in a basalt environment. Holloway, J.R.; Jenkins, D.M.; Kacoyannakis, J.F.; Apted, M.J. (Rockwell International Corp., Richland, WA (USA). Rockwell Hanford Operations). Jun 1981. Contract AC06-77RL01030. 43p. NTIS, PC A03/MF A01. Order Number DE82006849.

Portions of document are illegible.

This report presents the initial results of the quantitative phase of the hydrothermal testing program on the geochemical behavior of waste forms. Two investigations involving supercalcine have been performed and the results are recorded in this report. One experiment involves the interaction of supercalcine with groundwater while the second involves the interaction of a mixture of supercalcine plus basalt with groundwater. Both experiments have been carried out under the maximum conditions anticipated in the repository, namely $300^{\circ} \mathrm{C}$ and 300 bars $(30 \mathrm{MPa})$. Many unanswered questions remain, concerning the speciation of cations in solution, the full characterization of phases formed, and the prevailing Eh-pH conditions of reacted groundwater. Preliminary data have been obtained on the cations that were readily leached from unsintered supercalcine (SPC-4) and the general solubility properties of the groundwater buffered by SPC $-4 \pm$ basalt. In particular, it is observed that $\mathrm{Si}, \mathrm{Na}, \mathrm{Mo}, \mathrm{Rb}$, and $\mathrm{Cr}$ are readily leached from SPC-4 in the absence of basalt, and all of these except $\mathrm{Cr}$ are leached in the presence of basalt. Groundwater buffered by SPC-4 alone is thought to have in situ $\mathrm{pH}$ and $\mathrm{Eh}$ conditions of $\sim 9.5 \pm 0.5$ and 0.0 to $-0.4 \mathrm{~V}$, respectively. Four secondary phases have been identified with some degree of certainty: pollucite [(Cs, Na) $\mathrm{AlSi}_{2} \mathrm{O}_{6} \times \mathrm{xH}_{2} \mathrm{O}$ ], ulvospinel $\left(\mathrm{Fe}_{2} \mathrm{TiO}_{4}\right), \alpha-\mathrm{RuCl}_{0}$, and $(\mathrm{Sr}, \mathrm{Ca}) \mathrm{MoO}_{4}$. Phases have not been identified to house the insoluble elements $\mathrm{Zr}, \mathrm{U}, \mathrm{P}$, or $\mathrm{Ba}$ as well as the elements whose concentrations $<0.2 \mathrm{wt} \%$ of the oxide. Finally, it is pointed out that further attention must be directed to the elements that have radionuclides of high biological danger, even if they do not seem dangerous from the aspect of their low thermal output.
912 (RHO-BWI-C-118) Monitored Retrievable Storage Demonstration Facility and dry well storage field conceptual design study. (Rockwell International Corp., Richland, WA (USA). Energy Systems Group). Aug 1981. Contract AC0677RL01030. 316p. D. Order Number DE82008779.

Portions of document are illegible.

The Monitored Retrievable Storage (MRS) Demonstration Facility provides for the receipt by truck and packaging (encapsulation), and the dry well passive storage (DWS) of 1500 LWR spent-fuel assemblies and ten canisters of HLW accumulated as a result of commercial nuclear power plant operation. Packaging will occur at the rate of approximately two metric tons of uranium (MTU) per day (three pressurized water reactor (PWR) assemblies plus three boiling water reactor (BWR) assemblies). Functional and physical descriptions of the conceptual design for the MRS Demonstration Facility, sited adjacent to the 200 West Area of the Hanford Reservation, are given in Sections 3.1 through 3.9; similarly, descriptions of the DWS field, located near the receiving and packaging area of the MRS Demonstration Facility, are given in Section 3.10. The performance requirements (Section 4.0), and quality levels, codes and standards (Section 5.0), as defined for the Hanford Site, apply to the conceptual design of the MRS Demonstration Facility and DWS field. The schedule and methods of performance (Section 6.0) also apply to the conceptual design. Special requirements applicable to the facility security, safety, safeguards, and energy conservation are presented in Section 7.0. The overall construction period for the site will be about 33 months. The quality assurance (QA) program (Section 8.0) will apply to both the $R$ and $P$ building and the DWS field. The drawing list and specification outline (Section 9.0) for the on-site facilities include the additional support buildings and utilities described in Sections 3.2 through 3.10. Section 10.0 provides the estimated cost and activation schedule for the MRS Demonstration Facility.

913 (RHO-BWI-LD-32) Exploratory Shaft Test Facility in basalt. Project B-314 preconceptual design report. (Rockwell International Corp., Richland, WA (USA). Rockwell Hanford Operations). Sep 1980. Contract AC06-77RL01030. 341p. NTIS, PC A15/MF A01. Order Number DE82022289.

Portions of document are illegible.

The Basalt Waste Isolation Project (BWIP) has been chartered to assess the feasibility of disposing high-level nuclear waste in a repository within the Hanford Site and to identify and characterize a site for such a repository if feasibility is shown. The BWIP site-identification studies to date have shown that such storage appears to be feasible and have narrowed the site location to a current area of $70 \mathrm{mi}^{2}$ within the Hanford Site. A site characterization plan is scheduled to be submitted to the US Nuclear Regulatory Commission (NRC) in May 1981 defining the plans to further reduce this potential area to that of a reference repository site. Subsequent to completion of the work outlined in that plan, a detailed site characterization plan will be submitted to the NRC in early 1982 defining the effort required to fully characterize the candidate site, so that it may be considered by DOE for banking and for comparison with sites in other geologic media. This banking decision requires characterization of the host rock. Thus, an Exploratory Shaft Test Facility (ESTF) is proposed to provide access to the candidate rock horizon as part of the characterization process. The proposed test facility to provide the in situ test space needed will consist of a drilled shaft and underground test chambers at an expected depth of $3700 \mathrm{ft}$. The finished shaft inside diameter will be $6 \mathrm{ft}$. Approximately $460 \mathrm{ft}$ of chambers will be provided at the base of the shaft for testing space. The objectives of this preconceptual design effort were to: develop a feasible reference facility concept utilizing existing technology, provide the technical basis for functional design criteria (to be written later), assemble schedule and cost data for project planning, and recommend alternatives that should be considered in subsequent design.

914 (RHO-BWI-LD-50) Hydrology and Geology Overview Committee reports and responses from the Basalt Waste Isolation Project. (Rockwell International Corp., Golden, CO (USA). Rocky Flats Plant). Sep 1981. Contract AC0677RL01030. 208p. NTIS, PC A10/MF A01. Order Number DE82007071.

The Basalt Waste Isolation Project (BWIP) is one of the compo nents of the National Waste Terminal Storage Program (NWTS), and is specifically chartered with the responsibility of assessing the feasibility of using the deep basalts beneath the Hanford Site for the disposal of nuclear waste. In February 1980, a Geology Overview Committee 
and a Hydrology Overview Committee were formed with the objective to perform an independent peer group review of the findings and conclusions of the Rockwell geoscientists and consultants working on the BWIP. The committees keep abreast of geoscience investigations and studies through review of BWIP reports and attendance at technical meetings wherein Rockwell geologists and hydrologists present the progress and findings made in their various areas of responsibility. The Committee members have an opportunity to interface directly with Rockwell technical personnel during the meetings scheduled for the Overview Committee members. The Committee members consist of distinguished members from the academic community, the USGS, the State of Washington Department of Ecology. The Committee members were selected by, and report directly to the US Department of Energy. The Hydrology and Geology Overview Committee reports from meetings in September and December 1980, and the Rockwell responses are published in this report

915 (RHO-BWI-SA-158) Field guidebook association of engineering geologists. Chapters 1-10. Bielefeld, R.J. (Rockwell International Corp., Richland, WA (USA). Rockwell Hanford Operations). Sep 1981. Contract AC06-77RL01030. 69p. NTIS, PC A04/MF A01. Order Number DE82009847.

Portions of document are illegible.

The geologic conditions of the Hanford Site have been determined in support of the surface facilities established onsite. Since 1976, the subsurface geologic conditions have been explored for the purpose of determining the feasibility of siting a nuclear waste storage facility. This work is ongoing and some of the results are presented in the following chapters: introduction to Hanford geology; geology of the Columbia Plateau; tectonics and seismic studies; bedrock geology and repository siting studies, Cold Creek Syncline Area, Pasco Basin, Washington; lithologic studies of Grande Ronde basalt; groundwater hydrology of the Columbia River basalt beneath the Hanford Site; design, fabrication and installation of rock instrumentation for the near-surface test facility; rock mechanics field test results to date; Columbia River gorge.

916

(RHO-BWI-ST-16) Identifiction of a preferred site for the exploratory shaft within the reference repository location: Hanford Site. (Rockwell International Corp., Richland, WA (USA). Rockwell Hanford Operations). Jan 1982. 45p. NTIS, PC A03/MF A01. Order Number DE83000151.

Purtions are illegible in microfiche products.

The Basalt Waste Isolation Project is a multifaceted research and development project designed to provide technology for long-term geologic isolation of commercial nuclear waste. The objective of the BWIP is to identify potential geologic repository sites in basalt, associated facilities, and technology required for the permanent isolation of radioactive wastes in basalt formations. One of the efforts of the project is to choose a reference repository location and, specifically, a location for an exploratory shaft. This report identifies a specific location for the cxploratory shaft within the reference repository location, which is itself within the Hanford boundary. The reference repository location has been previously identfied through a series of siting and screening studies. The screening study reduced the area within the Pasco Basin to five site localities. Ten candidate sites were identified within the site localities. The ranking of the ten candidate sites resulted in the identification of the reference repository location and an alternate site that optimized siting criteria within the Hanford Site boundary. By applying a series of surface- and subsurface-engineering considerations, an optimum location for the exploratory shaft within the reference repository location was established. Although the entire reference repository location is considered geologically and hydrologically acceptable for the repository, it was necessary to optimize the location of the repository shaft-pillar area and, subsequently, the exploratory shaft, which is located within the shaft-pillar area, in marimize design flexibility. In addition, a deep borehole location was identified and will supply additional geotechnical data for site-specific information.

917 (RHO-BWI-ST-18) Waste package heat-transfer analysis: model development and temperature estimates for waste packages in a repository located in basalt. Altenhofen, M.K. (Rockwell International Corp., Richland, WA (USA). Rockwell Hanford Operations). Oct 1981. Contract AC06-77RL01030. 59p. NTIS, PC A04/MF A01. Order Number DE82014722.

The Office of Waste Package Studies of the Basalt Waste Isolation Project is conducting an ongoing study of waste package heat- transfer processes as part of the development of waste package-design requirements. The objective of these heat-transfer studies is to provide the temperature estimates necessary to predict the physical, chemical, and hydrothermal environment of the waste package during geologic isolation in a repository located in basalt. Heat-transfer analyses were performed for three nuclear waste types which exhibit different characteristics - commercial and defense high-level waste (both immobilized in borosilicate glass) and spent-fuel elements. The waste-isolation system parameters used in these analyses reflect the current conceptual designs for a high-level nuclear waste repository constructed in basalt. Details of the thermal properties of the host rock and waste package relevant to a repository in Columbia River basalt and the relevant properties of the waste forms themselves are presented and discussed. The physical layout of the nuclear waste repository was modeled using the heat-transfer computer code, HEATING5. The first step in this analysis was to evaluate repository temperatures using one-, two-, and threedimensional models with a homogenized heat source. Next, the results of the one-, two-, and three-dimensional models were integrated to produce an accurate estimate of waste package/host rock-interface temperatures as a function of time.

918 (RHO-RE-ST-1-P) Effects of Hanford high-level waste components on sorption of cobalt, strontium, neptunium, plutonium, and americium on Hanford sediments. Delegard, C.H.; Barney, G.S. (Rockwull International Corp., Richland, WA (USA). Rockwell Hanford Operations). Mar 1983. Contract AC06-77RL01030. 68p. NTIS, PC A04/MF A01. Order Number DE83013777.

To judge the feasibility of continued storage of high-level waste solutions in existing tanks, effects of chemical waste components on the sorption of hazardous radioelements were determined. Experiments identified the effects of 12 Hanford high-level waste-solution components on the sorption of cobalt, strontium, neptunium, plutonium, and americium on 3 Hanford 200 Area sediments. The degree of sorption of strontium, neptunium, plutonium, and americium on two Hanford sediments was then quantified in terms of the concentrations of the influential waste components. Preliminary information on the influence of the waste components on radioelement solubility was gathered. Of the 12 Hanford waste-solution components studied, the most influential on radicelement sorption were $\mathrm{NaOH}, \mathrm{NaAlO}_{2}$, HEDTA, and EDTA. The chelating complexants, HEDTA and EDTA, generally decrcased sorption by complexation of the radioelement metal ions. The components $\mathrm{NaOH}$ and $\mathrm{NaAlO}_{2}$ decreased neptunium and plutonium sorption and increased cobalt sorption. Americium sorption was increased by $\mathrm{NaOH}$. The three Hanford sedimonts' radioclement surption behavlors were similar, implying that their sorption reactions were also similar. Sorption prediction equations were gencrated for strontium, neptunium, plutoni$u m$, and americium sorption reactions on two Hanford sediments. The equations yielded values of the distribution coefficient, $K_{d}$, as quadratic functions of waste-component concentrations and showed that postulated radioclement migration rates through Hanford sediment could change by factors of 13 to 40 by changes in Hanford waste composition.

919 (RHO-RE-ST-4-P) Status of tank-assessment studies for continued in-tank storage of Hanford Defense waste. DeFigh-Price, C. (Rockwell International Corp., Richland, WA (USA). Energy Systems Group). Oct 1982. Contract AC0677RLn103n. 69p. NTIS, PC A01/MF A01. Ordar Number DE83001141.

Portions of document are illegible.

This report provides technical information on the status of an nngoing evaluation of underground waste tanks for cuntinued storage of radioactive waste at the Hanford Site. The initial status report was issued in September 1978, followed by an update in October 1980. The data are generic to all of the tinderground, reinforced-concrete waste storage tanks at Hanford and include study results, evaluation methods, and recommended future work related to long-term disposal. Though elevaled temperatures continue to be a dominant factor affecting concrete strength, the initial condition of the concrete appears to be most important in determining the present or future condition of the concrete. This document provides a summary of work completed in the past two years. This includes: (1) results of laboratory tests to determine concrete durability when exposed to various waste forms for use in establishing a technical data base for assessment studies; (2) properties of concrete removed from the haunch and wall of a single-shell tank; (3) computer analyses determining tank sensitivity to various loads; and (4) thermal 
heat transfer analyses of long-term disposal options. 35 figures, 16 tables.

920 (RHO-ST-34) Scientific basis for establishing drywell-monitoring frequencies. Isaacson, R.E.; Gasper, K.A (Rockwell International Corp., Richland, WA (USA). Rockwell Hanford Operations). Dec 1981. Contract AC06-77RL01030. 48p. NTIS, PC A03/MF A01. Order Number DE82014218.

A scientific basis has been developed for establishing the frequency of monitoring dry wells. Dry wells are used to detect radioactivity from a leaking underground high-level radioactive waste-storage tank. The frequency of monitoring a dry well is dependent on the response characteristics of the radiation-detection system periodically used to monitor dry wells for encroaching radioactivity. The response characteristics have been combined in the Dry Well Radioactivity Response Equation, which is derived from: variation in dose rate (roentgen per hour) as a function of source strength; variations in dose attenuation by the soil as the radioactive waste front approaches the dry well; response of radiation detector, in counts per second, as dose rate changes (instrument calibration); distance of dry well from tank leak source; leak rate; geometry of soil wetted by leaking waste; and hydrologic properties of the soil. These variables are used with the current status of tank contents and available liquid-level-monitoring system information to generate a monitoring schedule for individual dry wells and horizontal laterals associated with single-shell, high-level waste-storage tanks on the Hanford Site.

921 (RI-125) Thermal expansion as a criterion for the quantity of high-level wastes disposed into geological formations. Bryzgalova, R.V.; Krivokhatskii, A.S.; Rogozin, Yu.M.; Sinitsyna, G.S. (Radievyj Inst., Leningrad (USSR)). 1980. 7p. (In Russian). NTIS (US Sales Only), PC A02/MF A01. Order Number DE82701149.

The thermal expansion of rocks is considered as a criterion of permissible quantity of high-level washes disposed. As a criterion the complete compensation of thermal expansion of melted rocks by free volume of cavity is suggested. On the example of a rock-salt massif using the thermal balance equation, the permissible high-level waster charge into the cavity depending on its free volume and time of attaining maximum melting volume are determined. It follows from the above data that radioactive wastes disposal with the total heat-evolution power of 1.9 MW leads to 14-15th year after disposal to melting of 110 thousand $t$ rock salt, the thermal expansion compensation of which requires creation of approximately 20 thousand $\mathrm{m}^{0}$ free cavity. The conclusion is drawn that high-level wastes disposal into geological formations causes the necessity of creation of free volume in a mountain-massif the value of which limits the permissible quantity of charged wastes on energy release.

922 (RISO-I-63) Corrosion aspects of high-level waste disposal in salt domes. Roerbo, $K$. (Risoe National Lab., Roskilde (Denmark)). Dec 1979. 59p. NTIS (US Sales Only), PC A04/MF A01. Order Number DE82701848.

In the ELSAM/ELKRAT waste management project it is planned that the high-level waste is glassified, encapsuled in canisters and finally deposited in a deep hole drilled in a salt dome. In the present report corrosion aspects of the canisters after deposition are discussed. The chemical environment will probably be a limited amount of brine coming from brine inclusions in the surrounding salt and moving up against the temperature gradient, the temperature at the canister surface being in the range of 100 to $150^{\circ} \mathrm{C}$. The possible types of corrosion and the expected corrosion rates for a number of potential canister materials (mild steel, austenitic and ferritic stainless steels, Ni-base alloys, copper, titanium and a few combinations of materials) are discussed. Mild steel (possibly combined with an inner layer of copper or titanium) might possibly be an appropriate choice of material for the canister.

923 (SAND-79-0267) Basic data report for drillhole ERDA 6 (Waste Isolation Pilot Plant - WIPP). (Sandia National Labs., Albuquerque, NM (USA); Geological Survey, Albuquerque, NM (USA)). Jan 1983. Contract ACO476DP00789. 105p. NTIS, PC A06/MF A01. Order Number DE83006392.

Portions are illegible in microfiche products. Original copy available until stock is exhausted.
ERDA 6 was drilled in eastern Eddy County, New Mexico, to investigate a candidate site for a nuclear waste repository. The site was subsequently rejected on the basis of geological data. ERDA 6 was drilled in the NE 1/4 SE 1/4, section 35, T21S,R31E. The borehole encountered, from top to bottom, $17 \mathrm{ft}$ of Quaternary deposits, $55 \mathrm{ft}$ of the Triassic Santa Rosa Sandstone, $466 \mathrm{ft}$ of the Dewey Lake Red Beds, $273 \mathrm{ft}$ of the Rustler Formation, $1785.5 \mathrm{ft}$ of the Salado Formation and $374.5 \mathrm{ft}$ of the upper Castile Formation, all of Permian age. Cores or drill cuttings were taken throughout the hole. A suite of wireline geophysical logs was run to a depth of $883 \mathrm{ft}$ to facilitate the recognition and correlation of rock units, to assure identification of major lithologies and to provide depth determinations independent of drill-pipe measurements. The site at ERDA 6 was rejected because the structure of the lower Salado and the Castile is too severe to develop a repository along a single set of beds. The borehole also intersected a reservoir of pressurized brine and gas at about $2710^{\prime}$. The pore volume for the reservoir was estimated to be in the range from about 200,000 to about 2 million barrels. ERDA 6 was re-entered in 1981 by the Department of Energy (DOE) for the purpose of further testing the brine reservoir. Those tests are described in separate reports by the DOE and its contractors. The WIPP is a demonstration facility for the disposal of transuranic (TRU) waste from defense programs. The WIPP will also provide a research facility to investigate the interactions between bedded salt and high level wastes.

924 (SAND-79-0268) Basic data report for Drillhole ADC 7 (Waste Isolation Pilot Plant - WIPP). (Sandia National Labs., Albuquerque, NM (USA); D'Appolonia Consulting Engineers, Albuquerque, NM (USA)). Jan 1983. Contract AC04-76DP00789. 146p. NTIS, PC A07/MF A01. Order Number DE83005889.

Portions are illegible in microfiche products.

AEC 7 is a borehole drilled in western Lea County, New Mexico, in section 31, T.21S.,R.32E. AEC 7 was drilled to 3918 feet in 1974 by Oak Ridge National Laboratory; Sandia deepened the hole to $4732 \mathrm{ft}$ in 1979. The borehole provided stratigraphic and lithologic information in the initial and final drilling. The borehole was used extensively for tests of borehole plugs and plugging operations. AEC 7 penetrated, in descending order, Holocene sands and Mescalero caliche $(8 \mathrm{ft})$, Santa Rosa Sandstone (109 ft), Dewey Lake Red Beds ( $542 \mathrm{ft}$ ), Rustler Formation (325 ft), Salado Formation (2014 ft), Castile Formation (1521 $\mathrm{ft}$ ), and the upper Bell Canyon Formation (197 ft). Cores were obtained from much of the borehole. An extensive suite of geophysical logs provides information on stratigraphy, lithology, and structure. Beds were in normal stratigraphic sequence and without structural deformation except in the lower Castile. Anhydrite II and Halite II appear to be repeated in the borehole. This section was penetrated during deepening by Sandia; the structural complication is consistent with deformation found nearby in ERDA 6. The potential site on which AEC 7 is located was abandoned in 1976 after ERDA 6 was drilled. The WIPP is a demonstration facility for the disposal of transuranic (TRU) waste from defense programs. The WIPP will also provide a research facility to investigate the interactions between bedded salt and high level wastes.

925 (SAND-79-0269) Basic data report for drillhole AEC 8 (Waste Isolation Pilot Plant - WIPP). (Sandia National Labs., Albuquerque, NM (USA); D'Appolonia Consulting Engineers, Albuquerque, NM (USA)). Jan 1983. Contract AC0476DP00789. 154p. NTIS, PC A08/MF A01. Order Number DE83008481.

Portions are illegible in microfiche products. Original copy available until stock is exhausted.

AEC 8 was originally drilled in 1974 to a depth of $3028 \mathrm{ft}$ by Oak Ridge National Laboratory as part of the initial investigations of a site for radioactive waste disposal. In 1976, Sandia National Laboratories deepened the borehole from the top of the Castile Formation into the Bell Canyon Formation to test the hydraulic properties of the Bell Canyon. The borehole encountered in descending order Holocene sands (20 ft), Mescalero caliche (6 ft), Santa Rosa Sandstone (143 ft), Dewey Lake Redbeds (491 ft), Rustler Formation (322 ft), Salado Formation $(1990 \mathrm{ft})$, Castile Formation (1335 ft), and the upper Bell Canyon Formation (603 ft). The borehole stratigraphy is in normal order and there is no significant deformation. An extensive suite of geophysical logs provides information on the lithology and stratigraphy. The potentiometric surfaces of Bell Canyon fluid-bearing zones are $550 \mathrm{ft}$ (for the zone at $4821 \mathrm{ft}$ to $4827 \mathrm{ft}$ ) and $565 \mathrm{ft}$ below land surface (for the zone at 4844 
to $4860 \mathrm{ft}$ ). The WIPP is a demonstration facility for the disposal of transuranic (TRU) waste from defense programs. The WIPP will also provide a research facility to investigate the interactions between bedded salt and high level wastes.

926 (SAND-79-0270) Basic data report for drillhole ERDA 9 (Waste Isolation Pilot Plant WIPP). (Sandia National Labs., Albuquerque, NM (USA); Geological Survey, Denver, CO (USA)). Jan 1983. Contract AC04-76DP00789. 118p. NTIS, PC A06/MF A01. Order Number DE83006393.

Portions are illegible in microfiche products. Original copy available until stock is exhausted.

ERDA 9 was drilled in eastern Eddy County, New Mexico, to investigate and test salt beds for the disposal of nuclear wastes. The hole was placed near the SE corner of section 20, T22S,R31E. It was drilled between April 28 and June 4, 1976, to a depth of $2889 \mathrm{ft}$ (measured from a kelly bushing altitude of $3,420.4 \mathrm{ft} \mathrm{MSL}$ ). The borehole encountered, from top to bottom, Holocene deposits (including artificial fill) of $22 \mathrm{ft}$, the Pleistocene Mescalero Caliche ( $5 \mathrm{ft}$ ) and Gatuna Formation (27 ft), $9 \mathrm{ft}$ of the Triassic Santa Rosa Sandstone, and $487 \mathrm{ft}$ of the Dewey Lake Red Beds, $290 \mathrm{ft}$ of the Rustler Formation, $1976 \mathrm{ft}$ of the Salado Formation and $53 \mathrm{ft}$ of the Castile Formation, all of Permian age. Cuttings were collected at 5 -ft intervals for the land surface to a depth of $1090 \mathrm{ft}$, and consecutive cores were taken to a depth of $2876.6 \mathrm{ft}$. A suite of wireline geophysical logs was run the full length of the borehole to measure distribution of radioactive elements and hydrogen, and variations in rock density and elastic velocity. On the basis of the borehole findings and related hydrological and geophysical programs, the site was judged suitable to pursue the extensive geological characterization program which followed. The core from ERDA 9 provided a suite of samples extensively tested for rock mechanics, physical properties, and mineralogy. Drill-stem tests in ERDA 9 indicated no significant fluids or permeability in the Salado beds of interest. The WIPP is a demonstration facility for the disposal of transuranic (TRU) waste from defense programs. The WIPP will also provide a research facility to investigate the interactions between bedded salt and high level wastes.

927 (SAND-79-0271) Basic data report for Drillhole ERDA 10 (Waste Isolation Pilot Plant - WIPP). (Sandia National Labs., Albuquerque, NM (USA); D'Appolonia Consulting Engineers, Albuquerque, NM (USA)). Feb 1983. Contract AC04-76DP00789. 179p. NTIS, PC A09/MF A01. Order Number DE83010296.

Portions are illegible in microfiche products. Original copy available until stock is exhausted.

Borehole ERDA 10 was drilled to obtain information on possible dissolution of halite within the Castile Formation and on the hydrologic characteristics of the fluid-bearing zones in the Bell Canyon Formation. The borehole is located in Section 34, T23S, R3OE in southern Eddy County, New Mexico. ERDA 10 was drilled to a depth of $4431.5 \mathrm{ft}$. Cores from the Castile were taken to obtain direct information which was supplemented by geophysical logging. Based on preliminary analysis of the data, the ERDA 10 stratigraphic section is interpreted as a sequence of sandstones, siltstones, claystones, and evaporites normal for the area. No evidence of dissolution of significant amounts of halite was observed.

928 (SAND-79-0272) Basic data report for drillhole WIPP 11 (Waste Isolation Pilot Plant - WIPP). (Sandia National Labs., Albuquerque, NM (USA); Geological Survey, Denver, CO (USA)). Feb 1982. Contract AC04-76DP00789. 98p. NTIS, PC A05/MF A01. Order Number DE8201 2053.

Portions of document are illegible.

Seismic reflection data from petroleum industry sources showed anomalous reflectors in the Castile Formation over a small area about 3 miles north of the center of the Waste Isolation Pilot Plant (WIPP) site. Additional corroborative seismic reflection data were collected as part of WIPP investigations, and WIPP 11 was drilled to investigate the anomaly. WIPP 11 was drilled near the northwest corner of Section 9 , T.22.S., R.31E. it penetrated, in descending order, sand dune deposits and the Gatuna Formation (29'), Santa Rosa Sandstone (132'), Dewey Lake Red Beds (502'), Rustler Formation (288'), Salado Formation (1379'), and most of the Castile Formation (1240'). Beds within the lower part of the Salado, and the upper anhydrite of the Castile, are thinner than normal; these beds are displaced upward structurally by the upper Castile halite which is highly thickened (about 968'). The lowest halite is thin (51') and the basal anhydrite was not completelv penetrated. Subsequent seismic and borehole data has shown WIPP 1 to be in a structural complex now identified as the disturbed zone. Tl WIPP is a demonstration facility for the disposal of transuranic (TRL, waste from defense programs. The WIPP will also provide a research facility to investigate the interactions between bedded salt and high level waste, though there are no plans at this time to dispose of high level waste or spent fuel at WIPP.

929 (SAND-79-0406C) Near-field interactions. Krumhansl, J.L.; McVey, D.F. (Sandia National Labs., Albuquerque, NM (USA)). Feb 1979. Contract AC04-76DP00789. 12 p. NTIS, PC A02/MF A01. Order Number DE82011303.

To approach the subject of high level nuclear waste disposal in deep ocean sediments it is convenient to differentiate between processes occurring in a near field environment, that region arbitrarily defined as lying between the canister surface and the maximum extent of the $100^{\circ} \mathrm{C}$ isotherm, and those which occur at lower temperatures and beyond the influence of intense radiation. A variety of considerations related to the chemistry of seawater-sediment mixtures suggests that about $200^{\circ} \mathrm{C}$ is the maximum temperature advisable in the near field environment. Results of a coupled fluid flow thermal transport com. putcr model show the maximum convection rate adjacent to a canister having surface temperature of $200^{\circ} \mathrm{C}$ is $0.3 \mathrm{~m} / 100$ years, and that this velocity is halved with the passage of cach thermal half life of the assumed waste form ( 30 years). Based on this convective model, it follows that compounds formed in the near field environment during the first thousand years following emplacement would be restricted to a region lying within two meters of the canister surface.

930 (SAND-80-2639) Thermal analysis of nuclear waste emplacement in welded tuff. Langkopf, B.S. (Sandia National Labs., Albuquerque, NM (USA)). Dec 1981. Contract ACO476DP00789. 38p. NTIS, PC A03/MF A01. Order Number DE82009353.

Welded tuff is being evaluated as a possible medium in which to store nuclear waste. This report analyzes the heat effects of emplacing radioactive waste in welded tuff below the water table at Yucca Mountain on the Nevada Test Site (NTS). One-, two-, and threedimensional calculations were used to evaluate the heat effects of spent fuel (SF) and commercial high-level waste (CHLW) in three regions: the very-near field, the room and pillar, and the far field. It was assumed that the canistered waste was placed in a borehole with no additional waste packaging. As a result of the calculations, interim referencerepository conditions of a gross thermal loading (GTL) of $100 \mathrm{~kW} /$ acre and a $20 \%$ extraction ratio (ER) were defined for both SF and CHLW. For these conditions, far-field temperatures remain below $100^{\circ} \mathrm{C}$ and those in the room-and-pillar domain below $120^{\circ} \mathrm{C}$. In the very-near field, canister centerline temperatures are $195^{\circ} \mathrm{C}$ for $\mathrm{SF}$ and $295^{\circ} \mathrm{C}$ for $\mathrm{CHLW}$; borehole wall temperatures are $184^{\circ} \mathrm{C}$ for $\mathrm{SF}$ and $222^{\circ} \mathrm{C}$ for CHLW. (The room-and-pillar and far-field temperatures are recognized as upper limits.) Once a full waste package is defined, canister loading may have to be reduced to prevent excessively high temperature within the waste package.

931

(SAND-80-2813) Preliminary thermal analyses for a nuclear waste repository in tuff. Gartling, D.K.; Eaton, R.R. Thomas, R.K. (Sandia National Labs., Albuquerquc, NM (USA)). Apr 1981. Contract AC04-76DP00789. 159p. NTIS, PC A08/MF A01. Order Number DE82008799.

Portions of document are illegible.

Preliminary thermal calculations have been completed for a generic nuclear waste repository sited in welded tuff below the water table at the Nevada Test Site. Parametric studies were carried out for the thermal response of the repository for both high level and spent fuel nuclear waste. Computations were made on three geometric scales which included global repository, room and pillar and near field models. Results of the particular studies carried out at Sandia National Laboratories are reported; references are provided for results obtained by othe contractors.

932 (SAND-81-0215) Three-dimensional thermal analysis for a conceptual high-level waste repository in welded tuff. Sundberg, W.D.; Eaton, R.R. (Sandia National Labs., Albu- 
querque, NM (USA)). Apr 1982. Contract AC04-76DP00789. 30p. NTIS, PC A03/MF A01. Order Number DE82015503.

Portions of document are illegible.

A near-field, three-dimensional, thermal-conduction analysis was done for 2.16-kW high-level waste canisters emplaced in welded tuff. Initial thermal loadings of 75 and $100 \mathrm{~kW} /$ acre were investigated. The conductivities used were $2.4 \mathrm{~W} / \mathrm{m} .{ }^{\circ} \mathrm{C}$ for the saturated tuff and 1.55 $\mathrm{W} / \mathrm{m} .{ }^{0} \mathrm{C}$ for the dry tuff. The tuff was modeled as always saturated and with boiling of the interstitial water. Calculations were done with the ADINAT finite-element code and associated pre- and post processor codes. For the $100-\mathrm{kW} /$ acre initial loading, boiling of the interstitial water, and an initial temperature of $35^{\circ} \mathrm{C}$, the maximum canister temperature was $296^{\circ} \mathrm{C}$.

933 (SAND-81-0629) Thermo-mechanical scoping calculations for a high level nuclear waste repository in tuff. Johnson, R.L. (Sandia National Labs., Albuquerque, NM (USA)). Jun 1981. Contract AC04-76DP00789. 80p. NTIS, PC A05/MF A01. Order Number DE82007550.

Thermal and mechanical stress scoping calculations have been completed for a high level nuclear waste repository sited in welded tuff below the water table at the Nevada Test Site. Reported are the results of the studies performed at Sandia National Laboratories. A ubiquitous jointed rock model which allows slip along randomly oriented joints and subsequent stress redistribution due to slip and fracture of intact rock was used to model the rock mass. Computations were made for two power input levels and for conditions of boiling of the groundwater at $100^{\circ} \mathrm{C}$ and for no boiling of the groundwater.

934 (SAND-81-0709) Program criteria for subseabed disposal of radioactive waste: site qualification plan. Laine, E.P.; Anderson, D.R.; Hollister, C.D. (Sandia National Labs., Albuquerque, NM (USA)). May 1982. Contract AC04-76DP00789. 13p. NTIS, PC A02/MF A01. Order Number DE82019846.

This document describes the evolving methodology which is being used to identify, and study sites in subseabed geological formations which may be candidates for use as repositories for high-level nuclear waste. Two primary criteria guide all phases of this work: the stability and barrier criteria. The stability criterion defines areas of the seabed that are unlikely to be disturbed by tectonic forces and oceanographic changes durig the lifetime of a waste repository. The barrier criterion defines those subseabed geological formations most likely to form an effective barrier to the release of radionuclides. Because of the large area of the oceans, a phased approach has been adopted through which successively smallcr arcas of the sea floor are studied in ever greater detail. The lirst phase, which is complcte, has identified abyssal clay deposits that are remote from tectonic boundaries and continental margins as being the regions $\left(>10^{5} \mathrm{~km}^{2}\right)$ on the sea floor within which acceptable sites might be most readily identified. The second phase involves downgrading less desirable areas $\left(>10^{4} \mathrm{~km}^{2}\right)$ within these regions, using archived seismic reflection profiling, sediment cores, and oceanographic data. This winnowing process identifies locations about one degree square $\left(\geq 10^{4} \mathrm{~km}^{2}\right.$ ) for more detailed field studies during the first part of the third phase. From these locations candidate sites ( $\leq$ $10^{4} \mathrm{~km}^{2}$ ) will be chosen based on detailed geological and geophysical surveying. In the second part of the third phase, detailed monitoring of the candidate sites will begin to determine long-term baseline conditions. After monitoring is underway, a pilot repository will : be established using waste canisters. Based on this work, a site selection/ rejection report will be written. The fourth and last phase will involve extended monitoring of oceanographic conditions at each repository.

935

(SAND-81-1095) Subseabed disposal program Annual report, January-December 1980. Volume -1. Summary. Hinga, K.R. (ed.). (Sandia National Labs., Albuquerque, NM (USA)). Feb 1982. Contract AC04-76DP00789. 47p. NTIS, PC E04/MF E01. Order Number DE82012378.

Includes 21 sheets of $24 x$ reduction microfiche

This is the seventh annual report describing the results of the investigations of the Subseabed Dispnsal Program. The program, international in scope, is evaluating the feasibility of disposing of high-level radioactive wastes by burial in certain geologically stable and economically valueless sediments of the deep sea floor. Volume I is a summary of the work of many individuals associated with the program. Volume II of this publication contains the reports of the principal investigators, which are the appendices referred to in Volume $I$, and is on microfiche included with Volume I.

936 (SAND-81-1768) Conceptual design of field experiments for welded-tuff rock-mechanics program. Zimmerman, R.M. (Sandia National Labs., Albuquerque, NM (USA)). Oct 1982. Contract AC04-76DP00789. 22p. NTIS, PC A02/MF A01. Order Number DE83004252.

This report furnishes objectives, typical descriptions, and modeling requirements for the conceptual designs of five experiments proposed for testing in welded tuff in G-Tunnel at Nevada Test Site. Two experiments, the Small-Diameter Heater and Unit-Cell Canister Scale, will be designed for model evaluation. Three experiments designed to measure in situ geotechnical properties are planned: the Heated Block, Rocha Slot, and Thermal Probe.

937 (SAND-81-2169C) Application of a risk-assessment methodology to a hypothetical high-level waste repository in bedded salt. Cranwell, R.M.; Helton, J.C. (Sandia National Labs., Albuquerque, NM (USA)). 1982. Contract AC0476DP00789. 23p. (CONF-820303-28). NTIS, PC A02/MF A01. Order Number DE82011947.

From Waste management conference (ANS); Tucson, AZ, USA (8 Mar 1982).

Portions of document are illegible.

The Sandia/NRC Risk Assessment Methodology consists of a procedure for assessing the post-closure, long-term risk from the disposal of radioactive waste in deep geologic formations. This procedure contains: (1) methods for selecting and screening potentially disruptive events, features and processes (i.e., scenarios); (2) models for use in simulating the physical processes and estimating the potential health effects associated with the deep geologic disposal of radioactive waste (e.g., repository evolution, ground-water flow and nuclide transport, biosphere transport and human exposure, and dose commitment and dose response); and (3) probabilistic and statistical procedures for use in risk estimates and in sensitivity and uncertainty analyses. Results of the demonstration of this methodology in the analysis of a hypothetical high-level waste repository in bedded salt are presented for the following three scenarios: (1) a hydraulic communication (boreholes or shafts) connects the middle and lower sandstone aquifers allowing water to flow through the depository; (2) a hydraulic communication allows water to flow from the middle sandstone aquifer through the depository and back to middle sandstone aquifer, referred to as a U-tube scenario; and (3) withdrawl wells completed into the middle sandstone aquifer downgradient from the depository are coupled with the U-tube of Scenario 2.

938 (SAND-81-2195-Rev.) Sealing concepts for the Waste Isolation Pilot Plant (WIPP) site. Christensen, C.L.; Gulick, C.W.; Lambert, S.J. (Sandia National Labs., Albuquerque, NM (USA)). Feb 1983. Contract AC04-76DP00789. 25p. NTIS, PC A02/MF A01. Order Number DE83013136.

Portions are illegible in microfiche products. Original copy available until stock is exhausted.

This report presents the current intentions and directions of the WIPP Plugging and Sealing Program and provides a basic structure for the engineering activities that may be required for final WIPP decommissioning and abandonment. It is intended as a guide in determining further directions for the sealing program. Obvious issues that require further development and understanding are identified.

939 (SAND-81-2498) Proceedings of the 1981 subseabed disposal program. Annual workshop. (Sandia National Labs., Albuquerque, NM (USA)). Jan 1982. Contract AC0476DP00789. 431p. NTIS, PC A19/MF A01. Order Number DE82011689.

Portions of document are illegible.

The 1981 Annual Workshop was the twelfth meeting of the principal investigators and program management personnel participating in the Subseabed Disposal Program (SDP). The first workshop was held in June 1973, to address the development of a program (initially known as Ocean Basin Floors Program) to assess the deep sea disposal of nuclear wastes. Workshops were held semi-annually until late 1977. Since November 1977 , the workshops have been conducted following the end of each fiscal year so that the program participants could review and critique the total scope of work. This volume contains a synopsis, 
as given by each Technical Program Coordinator, abstracts of each of the talks, and copies of the visual materials, as presented by each of the principal investigators, for each of the technical elements of the SDP for the fiscal year 1981. The talks were grouped under the following categories; general topics; site studies; thermal response studies; emplacement studies; systems analysis; chemical response studies; biological oceanography studies; physical oceanographic studies; instrumentation development; transportation studies; social environment; and international seabed disposal.

940

(SAND-81-2628) Waste Isolation Pilot Plant (WIPP) research and development program: in situ testing plan, March 1982. Matalucci, R.V.; Christensen, C.L.; Hunter, T.O.; Molecke, M.A.; Munson, D.E. (Sandia National Labs., Albuquerque, NM (USA)). Dec 1982. Contract AC04-76DP00789.

128p. NTIS, PC A07/MF A01. Order Number DE83005905.

Portions are illegible in microfiche products. Original copy available until stock is exhausted.

The WIPP in southeast New Mexico is being developed as an $\mathbf{R}$ \& $\mathrm{D}$ facility to demonstrate the safe disposal of radioactive defense wastes in bedded salt. The tests are done first without radioactive materials and then with transuranic (TRU) waste and Defense High-Level Waste (UHLW). 'I'he thermal/structural itneraction experiments include (a) geomechanical evaluations of access drifts, vertical shafts, and isothermal TRU disposal rooms during the Site and Preliminary Validation Program, (b) tests that represent the reference DHLW room configuraton $(5.5 \mathrm{~m} \times 5.5 \mathrm{~m})$ and areal thermal loading of $12 \mathrm{~W} / \mathrm{m}^{2}$, (c) an overtest of the DHLW congfiguration heated to about four times the reference thermal loading; (d) geomechanical evaluations of various room widths up to $9.1 \mathrm{~m}$, variable pillar widths, and a long-drift intersection, (e) an 11-m-dia axisymmetric heated pillar test, and (f) miscellaneous tests to determine stress field and clay seam sliding resistance. The plugging and sealing experiments include (a) salt permeability tests, (b) tests to determine effects of size and scale on behavior of plugs and to determine backfill material behavior and emplacement techniques, and (c) a plug test matrix to evaluate candidate sealing materials. Waste package interaction experiments include (a) simulated-waste package tests that use several design options and engineered barrier materials under reference and accelerated DHLW environments, (b) confirmatory brine migration tests, (c) TRU drum durability tests in dry and wet conditions, (d) options for radiation-source tests using cesium capsules, and (e) actual DHLW tests using up to $\mathbf{4 0}$ canisters for technical demonstrations and for addressing concerns of wasteform chemistry, leaching, and near-field radionuclide migration.

941 (SAND-81-7206) Forced ventilation analysis of a commercial high-level nuclear waste repository in tuff. Topical report RSI-0175. Svalstad, D.K.; Brandshaug, T. (Sandia National Labs., Albuquerque, NM (USA)). Jun 1983. Contract AC04-76DP00789. 129p. NTIS, PC A07/MF A01. Order Number DE83014855.

Purtluns are lllegible in mlcrotiche products.

This report addresses the feasibility of using blast cooling for rapid drift and formation cooling prior to a nuclear waste retrieval operation. A coupled conduction/convection analysis is used to examine the various parameters and conditions which control the blast cooling process for the purpose of assessing their relative significance and potential impact on developing a blast cooling scenario for a nuclear waste repository in tuff. Formation stability is addressed briefly with regard to the potential for thermal spalling induced by the rapid cooling of the heated repository walls.

942 (SAND-81-7210) Far-field thermal analysis of a high level waste repository in tuff. Topical report RSI-0137. Klasi, M.L.; Russell, J.E.; McClain, W.C.; Brandshaug, T. (Sandia National Labs., Albuquerque, NM (USA)). Jul 1982. Contract AC04-76DP00789. 42p. NTIS, PC A03/MF A01. Order Number DE82019803.

Thermal loadings of $25,50,75,100$, and $150 \mathrm{~kW} /$ acre were used for thermal analyses of a high-level waste repository at a depth of 800 $m$ in tuff. Three methods of handling the presence of pore water were considered; the first assumed that pore water did not boil, the second that pore water boiled in the interval $90^{\circ} \mathrm{C}$ to $110^{\circ} \mathrm{C}$, and the third that pore water boiled at a temperature governed by hydrostatic pressure. The results of two modeling methods performed independently by RE/ SPEC and Texas $A$ and $M$ University are presented. The RE/SPEC model was a two-dimensional finite element model while the Texas A and $\mathbf{M}$ model was a one-dimensional finite element model. The results of the two modeling methods were nearly identical. It was found that hydrostatic boiling did not occur for any thermal load considered and that boiling in the interval $90^{\circ} \mathrm{C}$ to $110^{\circ} \mathrm{C}$ did not occur for thermal loads of 25 or $50 \mathrm{~kW} /$ acre. The most important result was the significant suppression of maximum temperatures because of energy consumed in boiling pore water. The peak temperature suppression ranged from zero at a thermal load of $25 \mathrm{~kW} /$ acre to nearly 22 percent at $150 \mathrm{~kW} /$ acre.

943 (SAND-82-0024) Effect of a random variation of rock salt creep on calculations of storage room closure for the WIPP project. Branstetter, L.J.; Krieg, R.D.; Stone, C.M. (Sandia National Labs., Albuquerque, NM (USA)). Oct 1982. Contract AC04-76DP00789. 23p. NTIS, PC A02/MF A01. Order Number DE83005814.

Portions are illegible in microfiche products. Original copies available until stock is exhausted.

Heterogeneity is characteristic of many geologic media. In particular, naturally occurring rock salt in southeastern New Mexico shows a wide variation of secondary creep rates between tested samples. It is important to know how this scatter in properties affects room closure predictions. This report presents the results of a study designed to quantify this effect for a particular isothermal storage room model. A series of calculations of room closure are made with both homogeneous and randomly distributioned creep properties. Calculations involving random property variations were divided into two sets: those in which variations occurred only in the vertical direction, and those in which both horizontal and vertical variations were present. The introduction of randomly distributed creep properties is shown to yield room closure predictions which, when averaged among several calculations, are reduced from the single prediction of an analogous homogeneous calculation. The analysis finds an average creep property value which could be used to achieve the same closure result as the average of several random calculations. Some statements concerning confidence intervals on room closure predictions are made.

944 (SAND-82-0127) Report of the first interim meeting of the Seabed Working Group Engineering Studies Task Group. Talbert, D.M. (Sandia National Labs., Albuquerque, NM (USA)). Feb 1982. Contract AC04-76DP00789. 27p. NTIS, PC A03/MF A01. Order Number DE82009356.

Portions of document are illegible.

The first interim meeting of the Engineering Studies Task Group (ESTG) was held at the Delft Soil Mechanics Laboratory, Delft, The Netherlands, nn 2.1-2.4 September 1981. The main business of the meeting was the development of a network analysis for the ESTG. Significant progress was made; however, substantial further development remains to be accomplished, Other items discussed were (1) progress relevant to engineering studies made in the various national programs since the sixth annual meeting of the Seabed Working Group (SWG) held in Paris, February, 1981; (2) the ESTG Boundary Conditions and Scope of Work as previously defined at the Paris meeting; (3) the Draft II SWG Five-Year Plan; (4) the deep ocean drilling proposal made by the Site Selection Task Group at the Paris meeting and expanded upon at their May, 1981, meeting; and (5) a recent compilation of data from the Nares Abyssal Plain arising from the US Program studies. Finally, consideration was given to a plan for continued work by the ESTG. A brief discussion of the principal items is given. The current state of the network analysis is shown.

945 (SAND-82-0158C) Alternate strategy for commercial high-level radioactive-waste management. Northrup, C.J.; Jardine, L.J.; Steindler, M.J. (Sandia National Labs., Albuquerque, NM (USA); Argonne National Lab., IL (USA)). 1982. Contract AC04-76DP00789. 15p. (CONF-820651-1). NTIS, PC A02/MF A01. Order Number DE82017027.

From International symposium on the conditioning of radioactive wastes for storage and disposal; Utrecht, Netherlands (21 Jun 1982).

Portions of document are illegible.

A current strategy of geologic disposal of immobilized commercial, high-level, nuclear wastes provides long-term storage (hundreds of thousands of years) for a wide spectrum of wastes from the Purex process which would be immobilized in a borosilicate glass. When implaced in a repository, the temperature increases and peaks within the geologic 
formations housing the waste repository during the first several hundred years after burial and then declines towards the initial temperature. During this thermal and radiolysis pulse period, the geologic formation and waste packages could be significantly perturbed unless the effects are controlled by some engineered approach. Many of the proposed solutions introduce new economic penalties and/or have serious impacts on how the volume of waste must be handled in production, transportation and final interment in the repository. It is noted that the majority of the thermal energy (has high as $\mathbf{9 8 \%}$ after 30 years) in commercial waste aged between 3 and 150 years is due to only two radioactive isotopes, ${ }^{90} \mathrm{Sr}$ and ${ }^{107} \mathrm{Cs}$ and their decay chains, which constitute $<10 \mathrm{wt}$. \% of the total elements in $\mathrm{HLW}$. Thus removal of cesium and strontium from all the other HLW components greatly reduces the geologic/waste package perturbations caused by the thermal/radiolysis pulse.

946 (SAND-82-0170) Thermal analyses for a nuclearwaste repository in tuff using USW-G1 borehole data. Johnson, R.L. (Sandia National Labs., Albuquerque, NM (USA)). Oct 1982. Contract AC04-76DP00789. 50p. NTIS, PC A03/MF A01. Order Number DE83002648.

Portions of document are illegible.

Thermal calculations using properties of tuffs obtained from the USW-G1 borehole, located near the SW margin of the Nevada Test Site (NTS), have been completed for a nuclear waste repository sited in welded tuff below the water table. The analyses considered two wasteforms, high level waste and spent fuel, emplaced at two different, gross thermal loadings, 50 and $75 \mathrm{~kW} /$ Acre $(20.24$ and $30.36 \mathrm{~kW} / \mathrm{ha})$. Calculations were made assuming that no boiling of the groundwater occurs; i.e., that the hydrostatic head potential was reestablished soon after waste emplacement. 23 figures, 2 tables.

947 (SAND-82-0312C) Issues related to field testing in tuff. Zimmerman, R.M. (Sandia National Labs., Albuquerque, NM (USA)). 1982. Contract AC0476DP00789. 8p. (CONF820803-4). NTIS, PC A02/MF A01. Order Number DE82013636.

From 23. US symposium on rock mechanics; Berkeley, CA, USA (25 Aug 1982).

This paper has brought out the unique properties of tuffs and related them to needs associated with their use as a host rock for a high level nuclear waste repository. Major issues of temperature, pore water joints, and depositional patterns have been identified and related responses and impacts outlined in Table 1. Planned experiments have been outlined and their relationships to the rock mechanics issues summarized in Table 2. The conclusions from this paper are: (1) tuff is a complex rock and basic phenomenological understanding is incomplete; and (2) available field test facilities will be used for a series of experiments designed to improve phenomenological understanding and support repository design efforts.

948 (SAND-82-0426) Preliminary scenarios for consequence assessments of radioactive-waste repositories at the Nevada Test Site. Hunter, R.L.; Barr, G.E.; Bingham, F.W. (Sandia National Labs., Albuquerque, NM (USA)). May 1982. Contract AC04-76DP00789. 149p. NTIS, PC A07/MF A01. Order Number DE82018252.

This report describes the construction and preliminary analysis of $\mathbf{4 3 0}$ scenarios for the release of radioactive waste from a hypothetical repository located in or near the Nevada Test Site. These scenarios will be used to guide future consequence analyses. The preliminary analysis of each scenario was carried out separately for each of ten different rock types. It suggests that the flow of ground water is of great importance to the long-term performance of the repository and that many of the scenarios are more likely in some of the rock types than in others.

949 (SAND-82-0429) Materials for high-level waste canister/overpacks in salt formations. Molecke, M.A.; Ruppen, J.A.; Diegle, R.B. (Sandia National Labs., Albuquerque, NM (USA)). Dec 1982. Contract AC04-76DP00789. 106p. NTIS, PC A06/MF A01. Order Number DE83006438.

Studies on the corrosion and mechanical behavior of TiCode-12 and other titanium alloys, for use as candidate cunister or ovcrpack barriers in a high-level waste repository or test facility in salt, are reported. The corrosion behavior of TiCode- 12 was evaluated as a function of: brine composition, temperature, time, $\mathrm{pH}$, oxygen concentration, and gamma radiolysis. Uniform corrosion rates are in the range of 0.1 to 10 $\mu \mathrm{m} / \mathrm{yr}$, pitting or crevice corrosion has not yet been observed. The highly adherent, passivating titanium oxide film that provides the corrosion protection is being evaluated via electrochemical polarization and surface analytical techniques to enable modeling of the corrosion mechanism(s). An increase in the corrosion rate by a factor of about 2 was observed for sensitized TiCode-12; changes in the alloy microstructure are being analyzed in order to model this phenomenon. Alterations in the chemistry and processing procedure of TiCode-12 are being evaluated to optimize corrosion, mecahnical, and mill-producibility properties for high-level waste package applications. Slow strain rate testing of TiCode-12 revealed no apparent susceptibility to stress corrosion cracking; no significant changes in tensile properties were observed, but alterations in fracture mode were determined to be caused by internal hydrogen content. Hydrogen effects on titanium alloy mechanical properties and crack susceptibility are being studied. Some hydrogen embrittlement occurs at hydrogen concentrations in the range of 200 to 300 PpM by weight, but the strength of TiCode-12 is not affected at concentrations up to 1100 wppM. A TiCode-12 HLW canister-package is proposed that could provide long-term containment integrity and significantly minimize total HLW isolation system costs when compared to other waste package design concepts.

950 (SAND-82-0525) Stresses near waste canisters buried in salt. Canfield, T.R. (Sandia National Labs., Albuquerque, NM (USA)). Mar 1983. Contract AC04-76DP00789. 36p. NTIS, PC A03/MF A01. Order Number DE83014793.

Portions are illegible in microfiche products. Original copy available until stock is exhausted.

Near field stresses and displacements were estimated for a heated canister buried in a natural salt formation. The results could be used to estimate hole closure and brine migration near the canister. In the event of hole closure, contact stress could be bracketed by calculation extremes.

951

(SAND-82-0621C) Isolation of radioactive wastes in salt repositories. Weart, W.D. (Sandia National Labs., Albuquerque, NM (USA)). 1982. Contract AC0476DP00789. 7p. (CONF-820422-1). NTIS, PC A02/MF A01. Order Number DE82008191.

From American Society of Civil Engineers conference; Las Vegas, NV, USA (26 Apr 1982).

Portions of document are illegible.

Salt formations have been considered for disposal of radioactive wastes since they were first suggested in 1957 by a committee of the National Academy of Science. Twenty-five years of investigation continue to support salt as a repository medium. Earlier unknowns about its behavior under repository conditions are now either understood or bounded. Practical waste packages can be designed to provide adequate containment in the most adverse of postulated repository environments. The Department of Energy (DOE) has selected a bedded salt site in southeast New Mexico to demonstrate disposal of defense transuranic waste and to conduct experiments with defense high-level waste (WIPP). Two are domes and two are bedded salt. Two shafts are drilled to the planned facility depth of 2150 feet. Waste acceptance is planned for 1989. The search for the first commerical waste repository salt site has narrowed to four locations. Construction of a commercial salt repository could start in 1993 and operation begin in 1999.

952 (SAND-82-0627C) Assessment of technical criteris of 10CFR60 for geologic disposal of high-level waste. Chu, M.S. Y.; Ortiz, N.R.; Pepping, R.E.; Siegel, M.D. (Sandia National Labs., Albuquerque, NM (USA)). 1982. Contract AC04 76DP00789. 15p. (CONF-820303-29). NTIS, PC A02/MF A01. Order Number DE82011948.

From Waste management conference (ANS); Tucson, AZ, USA (8 Mar 1982).

Portions of document are illegible.

The Environmental Protection Agency (EPA) has issued a draft standard (40CFR191) which specifies permissible radionuclide release limits from a repository for high-level waste to the accessible environment. The US Nuclear Regulatory Commission (NRC) has published a proposed rule (10C.FR60) which specifies technical criteria for geologic disposal of high-level waste designed to facilitate compliance with the EPA draft standard. This paper presents preliminary results and observations of evaluating the effect of the 10CFR60 numerical technical criteria on reducing the risk and/or uncertainties associated with 
meeting the EPA draft standard. The impacts of the following three numerical technical criteria were examined: (1) waste package containment of at least 1000 years; (2) control release rate of at most $10^{-5}$ part/ year from the underground facility; and (3) groundwater travel time of at least $\mathbf{1 0 0 0}$ years to the accessible environment. The analysis consists of three sets of parametric calculations: (1) generic parametric analysis; (2) parametric analysis including geochemical retardation for basalt; and (3) parametric analysis for a hypothetical basalt site.

963 (SAND-82-0664/1) Subseabed Disposal Program. Annual report, January-September 1981. Volume I: summary. Hinga, K.R. (ed.). (Sandia National Labs., Albuquerque, NM (USA)). Nov 1982. Contract AC04-76DP00789. 55p. NTIS, PC E06/MF \$8.20. Order Number DE83005119.

Portions of document are illegible. Printed copy available until stock is exhausted. Includes 21 sheets of $24 x$ reduction microfiche.

This is the eighth annual report describing the results of the investigations of the Subseabed Disposal Program. The program, international in scope, is evaluating the feasibility of disposing of high-level radioactive wastes by burial in certain geologically stable and economically valueless sediments of the deep sea floor.

954 (SAND-82-0664-Vol.2-App.-Pt.1) Subseabed disposal program annual report, January-September 1981. Volume II. Appendices (principal investigator progress reports). Part 1. Hinga, K.R. (ed.). (Sandia National Labs., Albuquerque, NM (USA)). Jul 1982. Contract AC04-76DP00789. 1026p. NTIS, PC A99/MF A01. Order Number DE82020855.

Portions of document are illegible.

Volume II of the eighth annual report describing the progress and evaluating the status of the Subseabed Disposal Program contains the appendices referred to in Volume I, Summary and Status. The annual report this year covers the period January to September 1981 because the program is shifting from a calendar year to a fiscal year reporting period. Because of the length of Volume II, it has been split into two parts for publication purposes. Part 1 contains Appendices A-Z; Part 2 contains Appendices AA-TT. Separate abstracts have been prepared for each appendix for inclusion in the energy data base.

956 (SAND-82-0664-Vol.2-App.-Pt.1,

Subseabed disposal safety analysis. Koplick, C.M.; Kabele, T.J. (Analytic Sciences Corp., Reading, MA). Jul 1982. NTIS, PC A99/MF A01.

In Subseabod disposal program annual rejurt, JanuarySeptember 1981. Volume II. Appendices (principal investigator progress reports). Part 1.

This report summarizes the status of work performed by Analytic Sciences Corporation (TASC) in FY'81 on subseabed disposal safety analysis. Safety analysis for subseabed disposal is divided into two phases: pre-emplacement which includes all transportation, handling, and emplacement activities; and long-term (post-emplacement), which is concerned with the potential hazard after waste is safely emplaced. Details of TASC work in these two areas are provided in two technical reports. The work to date, while preliminary, supports the technical and environmental feasibility of subseabed disposal of HLW.

966 (S $\Lambda$ ND-82-0664-Vol.2-App.-Pt.1, pp 61-106) Accident scenarios and probabilities for the SDP transportation system. Ensniinger, D.A. (Analytic Sciences Corp., Reading, MA). Jul 1982. NTIS, PC A99/MF A01.

In Subseabed disposal program annual report, JanuarySeptember 1981. Volume İI. Appendices (principal investigator progress reports). Part 1.

A preliminary analysis of the safety of preemplacement operations in the Subseabed Disposal Program is performed. Handling, storage, shipment, and emplacement facilities are described and potential accident scenarios and probabilities are determined, based in part on existing studies. This document is intended to serve as a report of progress. Subsequent efforts will complete the safety analysis outlined here.

967 (SAND-82-0664-Vol.2-App.-Pt.1, pp 191-206) Analysis of geological and geophysical data from study locations $C_{1}, B_{3}$, and $E_{2}$ in the northwest PAC 1 study region. Hayes, D.E.; Damuth, J.E.; Jacobi, R.D.; Flood, R.D. (Columbia Univ., Palisades, NY). Jul 1982. NTIS, PC A99/MF A01.
In Subseabed disposal program annual report, Januar? September 1981. Volume II. Appendices (principal investigator pros ress reports). Part 1.

Analysis of the geological and geophysical data collected aboard the R/V VEMA in June 1980 in three study areas (nominally $1^{\circ}$ latitude by $1^{0}$ longitude) is well underway. Emphasis has been on (1) mapping the detailed bathymetry and the variations in sedimentary layer thicknesses within the upper 60 meters of the section, (2) quantitatively relating the acoustic properties of the sediments as recorded on $3.5 \mathrm{kHz}$ echograms to measured mass physical properties of the uppermost sediments recovered from piston cores, and (3) using results of (1) and (2) in assessing the lateral continuity of reflecting horizons. Analysis of study area $E_{2}$ is nearly completed and appears to possess many desirable geological characteristics suitable for potential subseabed, high level waste disposal sites.

958 (SAND-82-0664-Vol.2-App.-Pt.1, pp 207-233) Site qualification activities. Laine, E.; Fisher, J.; Sopher, C.; Barton, C. (Univ, of Rhode Island, Kingston). Jul 1982. NTIS, PC A99/MF A01.

In Subseabed disposal program annual report, JanuarySeptember 1981. Volume II. Appendices (principal investigator progress reports). Part 1.

Thirteen study locations in the North Atlantic have been evaluated in accordance with the Site Qualification Program Plan. This preliminary evaluation has shown that the characteristics of the Madcap, Great Meteor West, and Northern Bermuda Rise study locations are sufficiently unsuitable to warrant downgrading for further study. Archived geological, geophysical and oceanographic data from Cape Verde III and the Gambia Abyssal Plain must be more completely evaluated before a decision for intensive field study can be reached. The remaining study locations are acceptable for intensive field study; however, in some instances possible detracting characteristics, which may require special analysis, have been noted.

969 (SAND-82-0664-Vol.2-App.-Pt.1, pp 235-334) Paleomagnetic study of areas B1, $\mathrm{C} 1$ and E2. Barton, C.; Sopher, C. (Univ. of Rhode Island, Narragansett). Jul 1982. NTIS, PC A99/MF A01.

In Subseabed disposal program annual report, JanuarySeptember 1981. Volume II. Appendices (principal investigator progress reports). Part 1.

Sediments from all three areas retain a stable primary remanence with a small viscous overprint which can be removed by AF cleanins. This marginally roduces the scatter in NRM dala and Improves the constraint"s on some reversal boundaries. Excellent reversal stratigraphies exist in all cores, particularly within area E2, with the exception of core B1-43P. This core is normally magnetized throughout and has a larger viscous component than other cores. Sedimentation rates are slower during the Brunbes epoch in all cores except C1-32P and C1-33P. Cores $\mathrm{C1}$-34P and E2-46P bave almost constant sedimentation rates throughout. The abnormally low average sedimentation rate during the Brunhes in core $\mathrm{Cl-35P}$ suggest a loss of up to $2 \mathrm{~m}$ of sediment, either during coring or by in situ erosion. Overall sedimentation rates are highest in area B1, lowest in area E2, and show least variation between cores in area E2. There is no general correlation between lithology and the paleomagnetic record. Ash layers and horizons with abnormally low water contents snmetimes coincide with spikes in the palesumaguetic records.

960 (SAND-82-0664-Vol.2-App.-Pt.1, 335-387) Pacific site assessment. Leinen, M. King, T.A. (Univ. of Rhode 'Island, Kingston). Jul 1982. NTIS, PC A99/MF A01.

In Subseabed disposal program annual report, JanuarySeptember 1981. Volume II. Appendices (principal investigator progress reports). Part 1.

The primary focus of the mineralogical analysis group at the University of Rhode Island is site assessment studies in the North Pacific. During FY 1981, our site assessment efforts focused on: (1) quantitative analysis of biogenic and eolian quartz in the VEMA 26-12 piston core tops (ADDENDUM 1), (2) quantitative clay mineralogical analysis of the VEMA core tops, (3) evaluation of regional sedimentation patterns in the northwest Pacific, (4) presentation of GLOMAR CHALLENGER site proposals to Deep Sea Drilling Project subject panels, and (5) participation in US task group meetings on site assessment. 
961

(SAND-82-0664-Vol.2-App.-Pt.1, pp 131-137) Effects of radiation on corrosion of titanium. Glass, R.S (Sandia National Labs., Albuquerque, NM). Jul 1982. NTIS, PC A99/MF A01.

In Subseabed disposal program annual report, JanuarySeptember 1981. Volume II. Appendices (principal investigator progress reports). Part 1.

A literature search has been conducted with the goal of identify. ing possible radiolysis products in the Subseabed repository. Effects of a radiation field on the corrosion of prospective canister materials has been considered. Suggestions for further work are given.

962 (SAND-82-0664-Vol.2.App.Pt.2) Subseabed disposal program annual report, January-September 1981. Volume II. Appendices (principal investigator progress reports). Part 2. Hinga, K.R. (ed.). (Sandia National Labs., Albuquerque, NM (USA)). Jul 1982. Contract AC04-76DP00789. 937p. NTIS, PC A99/MF A01. Order Number DE82020854.

Portions of document are illegible.

Volume II of the eighth annual report describing the progress and evaluating the status of the Subseabed Disposal Program contains the appendices referred to in Volume I, Summary and Status. The annual report this year covers the period January to September 1981 because the program is shifting from a calendar year to a fiscal year reporting period. Because of the length of Volume II, it has been split into two parts for publication purposes. Part 1 contains Appendices A-Z; Part 2 contains Appendices AA-TT. Separate abstracts have been prepared for each appendix for inclusion in the Energy Data Base.

963 (SAND-82-0664-Vol.2-App.-Pt.2, pp 11-97) Constitutive properties for undisturbed marine sediments in support of the subseabed disposal program. Akers, S.A. (Army Engineer Waterways Experiment Station, Vicksburg, MS). Jul 1982. NTIS, PC A99/MF A01.

In Subseabed disposal program annual report, JanuarySeptember 1981. Volume II. Appendices (principal investigator progress reports). Part 2.

A laboratory material property investigation was conducted on newly acquired undisturbed samples of Pacific illite in order to quantify the effective-stress, stress-strain and strength properties of the material when subjected to high-pressure static and dynamic loading conditions. The laboratory testing program consisted of triaxial shear (TX), hydrostatic compression, and uniaxial strain (UX) tests. Consolidatedundrained static TX tests with pore pressure measurements resulted in an effective-stress Mohr-Coulomb friction angle and cohesion intercept of $28.6^{0}$ and $0.004 \mathrm{MPa}$, respectively. Comparable static and dynamic tests indicated that the undrained shear strength of undisturbed Pacific illite increased nominally $200 \%$ for an increase in strain rate from $10^{-6} /$ second to $1 /$ second. In terms of total stresses (up to $35 \mathrm{MPa}$ ), Pacific illite was observed to behave as a phi $>0$ material in shear. Constrained modulus values derived from consolidated-undrained test results exhibited an expected dependence on specimen density. UX tests conducted to investigate loading rise time effects, however, produced divergent results. Two dynamic tests performed on virgin specimens exhibited negligible increases in undrained constrained modulus compared to static test results. In contrast, two multiply-loaded test specimens showed a modulus increase of $20 \%$ by the third load cycle. Drained UX tests were successfully accumplished to stress levels in excess of 50 $\mathrm{MPa}$ and strains in excess of $60 \%$. Representative static and dynamic laboratory test results were selected for future use by constitutive modellers in characterizing the response of undisturbed Pacific illite. At the request of the sponsoring agency, several drained hydrostatic creep tests were also conducted as part of this investigation.

964 (SAND-82-0664-Vol.2-App.-Pt.2, pp 99-224) Geotechnical studies for subseabed disposal: high level radioactive wastes. Silva, A.J.; Jordan, S.A.; Levy, W.P. (Univ. of Rhode Island, Narragansett). Jul 1982. NTIS, PC A99/MF A01.

In Subseabed disposal program annual report, JanuarySeptember 1981. Volume II. Appendices (principal investigator progress reports). Part 2.

This is the eighth annual progress report, for calendar year 1981, of the University of Rhode Island geotechnical program which is part of the Subseabed Disposal Program concerned with disposal of high level radivaitive wastec. Detailend data and results are presented for sev- eral activities. Sediment characterization results (used for site evaluation) are presented for three areas; MPG-I, MPG-IIIN, and MPG-IV. New results in the N. Central Pacific MPG-I area agree well with previous data which show that this site is feasible, from a geotechnical point of view, for waste disposal. However, the W. Atlantic MPG-IIIN area showed appreciable concentrations of sand size material below 10 meters which may have an adverse affect on dynamic penetration and fluid migration. The W. Pacific MPG-IV results completed on sites $B_{1}$, $C_{1}$ and $E_{2}$ indicate favorable conditions for waste disposal with $E_{2}$ as the most desirable. The triaxial program centered on assessing radiation, high pressure and overconsolidation effects on stress-strain properties and behavior. A creep program is continuing to study the long term stress-strain-time properties and behavior under both triaxial (at various temperatures) and uniaxial conditions. Compressibility tests are continuing in the three study areas; MPG-I, MPG-IIIN and MPG-IV. Permeability measurements in the MPG-I area agree well with those reported previously. Results from the MPG-IIIN permeability tests show values similar to MPG-I. However, MPG-IV tests reveal permeabilities consistently higher than in MPG-I. High temperatures (up to $220^{\circ} \mathrm{C}$ ) and/or high pressures (up to $60 \mathrm{MPa}$ ) do not seem to have any discernible effects on permeability except for predicted effects on physical properties of the constituents. Simple shear equipment has been assembled and calibrated and preliminary testing is underway.

966 (ȘAND-82-0664-Vol.2-App.-Pt.2, pp 803-941) Sociopolitical aspects of a subseabed disposal program for high level nuclear waste. Kildow, J.L. (Massachusetts Inst. of Tech., Cambridge). Jul 1982. NTIS, PC A99/MF A01.

In Subseabed disposal program annual report, JanuarySeptember 1981. Volume II. Appendices (principal investigator progress reports). Part 2.

The research included in this report provides data describing and assessing the basic national and international socio-political and legal factors which could influence the formation and implementation of a Subseabed Disposal Program for High Level Radioactive Waste and Spent Fuel. Using the particulars identified in this research effort, the report summarizes the basic socio-political constraints and aids which currently exist or might come about which would influence a Subseabed Disposal Program.

966 (SAND-82-0664-Vol.2-App.-Pt.2, pp 943-954) Public education and participation. Kelly, J.E. (Univ. of New Hampshire, Durham). Jul 1982. NTIS, PC A99/MF A01.

In Subseabed disposal program annual report, JanuarySeptember 1981. Volume II. Appendices (principal investigator progress reports). Part 2.

As prescribed in Step 1 of the Public Education and Participation Process (attachment 1), industry, public interest groups, and decision-makers were briefed about the Subseabed Disposal Program. In regard to public interest groups, Drs. Hollister and Kelly were invited to present the technical and policy aspects of the Subseabed Program at a public forum in Hawaii sponsored by the Hawaii League of Women Voters, the Health Physics Society, and the East-West Center. The sponsors videotaped the forum for a film, entitled Slowly Dying Embers: Radioactive Waste and the Pacific, which will be shown on television in Hawaii. In response to requests for information about the Subseabed Program, Congressional Staff, Representatives, and Senators (attachment 2) were briefed about the Subseabed Program as legislation related to the Program moved through Congress (attachment 3). Science oriented publications also were contacted about the Program (attachment 4).

967 (SAND-82-0664-Vol.2-App.-Pt.2, pp 955-969) Annual report of the management system design group. Susskind, L. (Massachusetts Inst. of Tech., Cambridge). Jul 1982. NTIS, PC A99/MF A01.

In Subseabed disposal program annual report, JanuarySeptember 1981. Volume II. Appendices (principal investigator progress reports). Part 2.

A management system for subseabed disposal of high-level radio active wastes must have at least nine components: (1) a land transportation component, (2) a storage and reprocessing component, (3) a port transfer component, (4) a sea transport, emplacement, and retrieval component, (5) a monitoring component, (6) a security component, (7) a safety and accident prevention component, (8) an emergency response component, and (9) a development and administration component. The 
investigators attempted to outline the key questions that must still be answered before the design of an effective management system can proceed.

988 (SAND-82-0793C) Natural convection experiments in a liquid-saturated porous medium bounded by vertical coaxial cylinders. Reda, D.C. (Sandia National Labs., Albuquerque, NM (USA)). 1983. Contract AC04-76DP00789. 34p. (CONF830301-1). NTIS, PC A03/MF A01. Order Number DE82017024.

From ASME-JSME thermal engineering joint conference; Honolulu, HI, USA (20 Mar 1983).

An experimental effort is presently underway to investigate natural convection phenomena in liquid-saturated porous media utilizing a geometry, and hydrodynamic/thermal boundary conditions, relevant to the problem of nuclear-waste isolation in geologic repositories. During the first phase of this research program, detailed measurements were made of the steady-state thermal field throughout an annular test region bounded by a vertical, constant-heat-flux, inner cylinder and a concentrically-placed, constant-temperature, outer cylinder. An overlying, constant-pressure, fluid layer was utilized to supply a permeable upper surface boundary condition. Results showed the heater surface temperature to increase with increasing vertical distance due to the presence of a buoyantly-dri\%en upflow. The measured temperature difference $(\Delta T)$ between the average heater surface temperature and the constant, outersurface, temperature was found to be progressively below the straightline/conduction-only solution for $\Delta \mathrm{T}$ vs power input as the latter was systematically increased. Comparisons between measured results and numerical predictions generated with the finite-element code MARIAH showed very good agreement, thereby contributing to the qualification of this code for repository-design applications.

989 (SAND-82-0962) Transient creep model for salt during stress loading and unloading. Munson, D.E.; Dawson, P.R. (Sandia National Labs., Albuquerque, NM (USA); Cornell Univ., Ithaca, NY (USA). Sibley School of Mechanical and Aerospace Engineering). Sep 1982. Contract ACO4 76DP00789. 57p. NTIS, PC A04/MF A01. Order Number DE83002137.

An empirical inodel is developed which accurately predicts the transient response of salt creep to incremental and decremental changes in stress and temperature. Even though the model is empirical, it is derived from a firm theoretical framework based upon the deformationmechanism map for the micromechanical deformation processs and treats both steady-state and transient creep In the modol, transient functions modify the steady-state creep behavior. The description considers bypothetical potential functions in order to obtain strain rate vs. strain functions for the workhardening and recovery that occurs during transient creep. Conventional creep tests or stress-drop tests are analyzed using new methods for the complete evaluation of all parameters of the model. Then, the model is applied to stress-drop experimental results for complete recovery. Parameters for the model are also developed for southeastern New Mexico salt and are used to describe experi. mental results frum tests that have complex loading and unloading histories. The model predictions compare well with the experimental results.

970 (SAND-82-1164) Effects of composition, porosity, bedding-plane orientation, water content and a joint on the thermal conductivity of tuff. Moss, M.; Koski, J.A.; Haseman, G.M.; Tormey, T.V. (Sandia National I a bis., Albuquefque, NM (USA)). Nov 1982. Contract AC04-76DP00789. 28p. NTIS, PC A03/MF A01. Order Number DE83005888.

Portions are illegible in microfiche products. Original copy available until stock is oxhausted.

This study deals with the effects of composition, porosity, bedding-plane orientation, water content and a joint on the thermal ennductivity of tuff from the Nevada Test Site, one medium being considered for nuclear waste burial. Over the temperature range of 310 to $423 \mathrm{~K}$, the thermal conductivity of dehydrated, $20 \%$ porous, welded, devitrified tuff (Grouse Canyon Member, Belted Range Tuff, G-tunnel), as measured by a linear heat-flow technique with the Dynatech comparator, increased from approximately 1.20 to $1.26 \mathrm{~W} / \mathrm{m}-\mathrm{K}$ on average, with only a small difference in temperature dependence of conductivity between samples in which heat fluxes were parallel and perpendicular to bedding. The same samples infiltrated with water to approximately $70 \%$ of pore volume displayed a $31 \%$ increase in conductivity at $310 \mathrm{~K}$, on average. The thermal resistance of two additional samples from G-tunnel, each $1.27 \mathrm{~cm}$ long, placed together to simulate a planar joint, was within one percent of the sum of the resistances of the pieces measured separately from 300 to $373 \mathrm{~K}$. This artificial joint displayed the same, essentially zero, resistance, under uniaxial pressures from 400 to $6900 \mathrm{kPa}$ (60 to $1000 \mathrm{psi}$ ) at $373 \mathrm{~K}$. Several dehydrated samples of tuff from the exploratory hole USW-Gl (Yucca Mountain) were measured from 309 to $424 \mathrm{~K}$ to determine the effects of composition, porosity and temperature on conductivity. Their conductivity increased several percent over this temperature range. The devitrified tuff was more conductive than the zeolitic tuff at all temperatures and conductivity declined with increasing porosity in all cases. Full water saturation produced approximately a $45 \%$ increase in conductivity in the devitrified tuff, and a 54 to $80 \%$ increase in the zeolitic at $310 \mathrm{~K}$.

971 (SAND-82-1199C) Constant temperature and thermal gradient investigations of the near field subseabed environment: chemical and thermodiffusional effects. Thornton, E.C.; Seyfried, W.E. Jr. (Sandia National Labs., Albuquerque, NM (USA); Minnesota Univ., Minneapolis (USA)). 1982. Contract AC0476DP00789. 15p. (CONF-820570-3). NTIS, PC A02/MF A01. Order Number DE82018589.

From Materials Characterization Center workshop on leaching; Gaithersburg, MD, USA (19 May 1982).

Both constant temperature $\left(200^{\circ}, 300^{\circ}\right)$ and temperature gradient experiments were conducted to examine interactions of seawater with sediment from study area $1000 \mathrm{~km}$ north of Hawaii. Solutions were sampled at experimental temperatures and pressure ( 500 or 600 bars). The results of the experimental studies presented illustrates that the chemistry of the near field environment is determined by a combination of water-rock interaction and thermal diffusion effects. Most important with respect to a thermal gradient is the thermal diffusion effect which results in large scale fluxes of $\mathrm{Na}$ and $\mathrm{Cl}$ from the hot zone towards the cool zone. This phenomenon serves to markedly decrease the ionic strength of the hot zone. Other elements, however, appear to be influenced by water-rock interaction processes as well as thermal diffusion. The behavior of $\mathrm{Mg}$ is particularly important in this regard because of its potential of generating an acidic environment in the near field region. Resolution of the relative importance of these processes is necessary to determine the suitability of the subseabed environment as a repository for high level radioactive waste.

872 (SAND-82-1274C) Thermal conductivity of bentonite/quartz high-level-waste-package harkfill. Moss, M.; Mulecke, M.A. (Sandia National Labs., Albuquerque, NM (USA)). 1982. Contract AC04-76DP00789. 17p. (CONF821107-4). NTIS, PC A02/MF A01. Order Number DE82022160.

From 6. international symposium on the scientific basis for radioactive waste management (Materials Research Society); Boston, MA, USA (1 Nov 1982).

A mixture of bentonite clay and quartz sand is being considered for use as a waste-package backfill, the material placed between a radioactive-waste canister and the repository host rock. Compacts of bentonitc/quartz with weight-percent ratios of $100 / 0,70 / 30,50 / 50$ and $30 / 70$ were made at room temperature under a pressure of $100 \mathrm{MPa}(15 \mathrm{ksi})$. Upon initial heating, the thermal conductivity of the 70/30 compact rose from $1.20 \mathrm{~W} / \mathrm{m} . \mathrm{K}$ at $298^{\circ} \mathrm{K}$ to $1.32 \mathrm{~W} / \mathrm{m} . \mathrm{K}$ at $373^{\circ} \mathrm{K}$. After further heating to $473^{\circ} \mathrm{K}$, it fell to $1.10 \mathrm{~W} / \mathrm{m} . \mathrm{K}$, renecting the loos of interlamellar water from the bentonite. The conductivity of the now-dehydrated compact was reproducible through several heating and cooling cycles, varying from $1.15 \mathrm{~W} / \mathrm{m} . \mathrm{K}$ at $573^{\circ} \mathrm{K}$ to $1.03 \mathrm{~W} / \mathrm{m} . \mathrm{K}$ at $298^{\circ} \mathrm{K}$. The other mixtures were qualitatively similar to the $70 / 30$ : the $100 / 0,50 / 50$ and 30/70 dehydrated compacts displayed conductivities of $0.59,1.06$ and $0.83 \mathrm{~W} / \mathrm{m} . \mathrm{K}$, respectively, at $298^{\circ} \mathrm{K}$. Measured densities ranged from 1.98 to $2.12 \mathrm{~g} / \mathrm{cc}$. Cothbined geometric-mean and Maxwell models for thermal conduction in composite systems predict the measured results reasonably well. An analysis of the impact of backfills on highlevel waste (HLW) package design indicates that no significant thermal penalty is imposed. 4 figures.

973 (SAND-82-1439C) Formulation of an integrated physicochemical-hydrologic model for predicting waste-nuclide retardation in geologic media. Muller, A.B.; Langmuir, D.; Duda, L.E. (Sandia National Labs., Albuquerque, NM (USA); Colorado School of Mines, Golden (USA)). 1982. Contract 
AC04-76DP00789. 18p. (CONF-821107-38). NTIS MF A01. Order Number DE83005006.

From 6. international symposium on the scientific basis for radio active waste management (Materials Research Society); Boston, MA, USA (1 Nov 1982).

Mf only; illegibility does not permit PC reproduction.

The inability of empirical single value elements $\boldsymbol{R}_{\boldsymbol{d}}$ values to model radionuclide retardation in nautral ground-water systems has demonstrated the need for developing a comprehensive integrated phenomenological model of the physicochemical and hydrologic mechanisms involved in retardation. The model must account for: (1) radioactive decay, (2) chemical precipitation/dissolution of bulk phases, (3) chemical substitution reactions, (4) isotopic exchange reactions, (5) cation and anion exchange, (6) specific adsorption, (7) diffusion into adjacent flow paths, (8) diffusion into fluid not involved in flow, (9) diffusion into the solid matrix and (10) ultrafiltration. The formulation of such a model is proposed, based on a reaction-path-simulation code with modifications for adsorption processes (by a surface-complexation sitebinding model) and diffusion processes. The coupling of the physicochemical portion of this model to a ground-water transport code can be achieved by alternately iterating through the chemical and physical portions of the code during each time step. Sensitivity analysis used to determine dominant species, dominant retardation mechanisms and temporal and spatial homogeneities in the flow system will allow the integrated model to be reduced to a manageable form.

974 (SAND-82-1783) Basic data report for Drillhole WIPP 14 (Waste Isolation Pilot Plant - WIPP). (Sandia National Labs., Albuquerque, NM (USA); D'Appolonia Consulting Engineers, Albuquerque, NM (USA)). Aug 1982. Contract AC04-76DP00789. 97p. NTIS, PC A05/MF A01. Order Number DE82022241.

Portions of document are illegible.

Borehole WIPP 14 is an exploratory well drilled in eastern Eddy County, New Mexico, in section 9, T22S,R31E. The borehole was drilled to a depth of $1000.0 \mathrm{ft}$ measured from ground level. It penetrated, from top to bottom, $15.4 \mathrm{ft}$ of Quaternary sands, $125.6 \mathrm{ft}$ of the Triassic Santa Rosa Sandstone, and in the Permian strata, $497.7 \mathrm{ft}$ of the Dewey Lake Red Beds, $312.9 \mathrm{ft}$ of the Rustler Formation and $48.4 \mathrm{ft}$ of the Upper Salado Formation. Seven hundred feet of the well were cored, at consecutive and nonconsecutive $10-\mathrm{ft}$ intervals to a depth of $925.5 \mathrm{ft}$. Cuttings were collected where core was not taken. Density, gamma ray neutron and caliper logs were run the full depth of the hole. The WIPP is a demonstration facility for the disposal of transuranic (TRU) waste from defense programs. The WIPP will also provide a research facility to investigate the interactions between bedded salt and high level wastes.

975

(SAND-82-1880) Basic data report for deepening of drillhole WIPP 13 (Waste Isolation Pilot Plant-WIPP). (Sandia National Labs., Albuquerque, NM (USA); D'Appolonia Consulting Engineers, Albuquerque, NM (USA)). Oct 1982. Contract AC0476DP00789. 110p. NTIS, PC A06/MF A01. Order Number DE83003782.

Portions of document are illegible. Printed copy available until stock is exhausted.

WIPP 13 is a torehole drilled in eastern Eddy County, New Mexico, in section 17, T22S, R31E, in order to investigate a subsurface seismic disturbed zone. The first 1035 ft of the borehole were drilled in July and August 1978. The deepening of WIPP 13 was performed in 1979 between August and October. This report documents the deepening of WIPP 13 to $3861.8 \mathrm{ft}$. Only rocks of the Permian, Salado and Castile Formations were penetrated in the deepening. Cores were obtained for some portions of the hole and cuttings were collected from some of the sections which were not cored (see Table 1). A suite of geophysical logs was run to provide information on lithology, structure and geochemistry. The WIPP is a demonstration facility for the disposal of transuranic (TRU) waste from defense programs. The WIPP will also provide a research facility to investigate the interactions between bedded salt and high level wastes. bed Disposal Program. Klett, R.D.; Brush, L.H.; Lipkin, J.; Percival, C.M. (Sandia National Labs., Albuquerque, NM (USA)).
1982. Contract AC0476DP00789. 14p. (CONF-821205-5). NTIS, PC A02/MF A01. Order Number DE83000091. From NWTS information meeting on basalt waste isolation project; Las Vegas, NV, USA (14 Dec 1982).

Portions of document are illegible.

The Subseabed Disposal Program (SDP) is considering highlevel waste (HLW) disposal in the oceanic geologic formations as a possible longer term complement to mined geologic repositories. The approach to safety assessment is to compute occupational exposure for all processes, predict the consequences and probabilities of preemplacement accidents and controlled release from the sediments, and analyze of all pathways to man and resulting bealth effects. Models are being developed to form a physical/mathematical computer description of each process; to measure as well as possible associated phenomena and properties in the laboratory; to make predictions and run confirming in-situ experiments; and to modify predictive methods if required. Models have been developed to describe heat transfer, fluid flow, mechanical response of the sediment, nuclide migration in the sediment, physical and biologic oceanography, land transport accidents, dose to man, and health effects. The largest systems effort in FY-82 has been in the area of sensitivity analyses for the purposes of eliminating unnecessary $\mathbf{R}$ and $\mathbf{D}$, quantifying general requirements to obtain specific criteria, defining model requirements, specifying significant variables, defining accuracy requirements for data acquisition, and providing input to the first round of safety assessments scheduled for FY-83.

977 (SAND-82-2147C) Preliminary technical constraints for a repository in tuff. Johnstone, J.K.; Gnirk, P.F. (Sandia National Labs., Albuquerque, NM (USA)). 1982. Contract AC04-76DP00789. 6p. (CONF-821205-4). NTIS, PC A02/MF A01. Order Number DE83000324.

From NWTS information meeting on basalt waste isolation project; Las Vegas, NV, USA (14 Dec 1982).

Portions of document are illegible.

The Nevada Nuclear Waste Storage Investigations (NNWSI) project has established preliminary technical constraints for a repository in tuff. The purpose of the technical constraints is to provide quantitative limits against which to assess the performance of a repository during the different phases of its existence. The constraints should also provide a link between individual repository component function and specifications to be used for conceptual and final repository design. The study focused on the very-near, near, and far field repository domains and time intervals of 0 to 110 years (operational period), 111 to 1000 years (containment period), and greater than 1000 years (isolation period). To date, only mined geologic disposal in tuff has been studied with emplacement of waste packages in vertical boreholes located in the floors of disposal rooms. A repository has been considered located in both saturated (below the static water level) and unsaturated (above the static water level) rock and waste packages with and without a backfill and containing spent fuel or reprocessed commercial high-level waste (borosilicate glass). Accidental or extraordinary events in the repository were not considered nor did we attempt to establish economic constraints.

978 (SAND-82-2198C) NNWSI regulatory status and plans. Neal, J.T.; Bertram, S.G. (Sandia National Labs., Albuquerque, NM (USA)). 1982. Contract AC04-76DP00789. 6p. (CONF-821205-6). NTIS, PC A02/MF A01. Order Number DE83001271.

From NWTS information meeting on basalt waste isolation project; Las Vegas, NV, USA (14 Dec 1982).

Portions of document are illegible.

Reporting requirements for the licensing of a high-level radioactive waste repository are reviewed. Major changes have been instituted in the proposed rules since last year and have particular relevance to Nevada Nuclear Waste Storage Investigations (NNWSI), especially regarding the use of the unsaturated zone, and the advocacy of an overall systems orientation in establishing technical criteria. Legislation pending at the time that this paper was written will have major policy impact on schedule direction, leading to submittal of a license application in 1988. The focus of site exploration has been on the volcanic tuff sequence underlying Yucca Mountain, on and adjacent to the Nevads Test Site. The major regulatory activities of NNWSI this past year have been the preparation of its initial Site Characterization Report (SCR) and an Environmental Assessment (EA) covering the Exploratory Shaft, both to be released in mid-1983. The final environmental docu. 
mentation, required at the time of the license application, will be an Environmental Report, which will be developed from the site-selection Environmental Impact Statement. (DMC)

979 (SAND-82-2238C) Nuclear-waste disposal: technical issues and status. Hunter, T.O. (Sandia National Labs., Albuquerque, NM (USA)). 1982. Contract AC04-76DP00789. 21p. (CONF-821090-1). NTIS, PC A02/MF A01. Order Number DE83002185.

From Institute for Nuclear Materials Management seminar on spent fuel management and waste disposal; Washington, DC, USA (20 Oct 1982).

Portions of document are illegible.

The nuclear waste from the defense programs of the United States and the commercial nuclear fuel cycle are planned for disposal in mined geologic repositories. High-level waste (HLW) will require the development of one to three repositories. A number of technical issuess are associated with the selection and characterization of a suitable site, the design of surface and underground facilities, development of an appropriate waste package, and methods to assess the performance of the system relative to regulatory requirements. These issues are being addressed by four major projects to evaluate salt, basalt, volcanic tuff, and other crystalline rocks such as granite. The key technical issues and the status of activities to resolve those issues are reviewed.

980 (SAND-82-2254C) Effects of wire rope in mitigating a waste-shaft accident. Ellett, D.M. (Sandia National Labs., Albuquerque, NM (USA)). 1983. Contract AC04-76DP00789. 4p. (CONF-830205-1). NTIS, PC A02/MF A01. Order Number DE83005830. Feb 1983)

From Waste management conference; Tucson, AZ, USA (27

Portions are illegible in microfiche products.

This report describes the empirical test program conducted on a 1/22-scale model of the WIPP facility. The model was designed to study the actions and effects of the wire rope used in the Koepe or friction-type hoist during various accident scenarios. It is assumed that the hoist cable breaks and the cab (or shaft conveyance) falls to the bottom of the shaft during such an accident. The report gives the results of the study and makes recommendations for a continuing program of testing and redesign of the shaft to mitigate the effects of such an accident. The wire rope is shown to act as a good shock-absorbing material.

981 (SAND-82-2259C) Analysis of waste-package behavior for high-level waste. Chu, M.S.; Campbell, J.E.; Stuckwisch, S.E.; Wahi, K.K.; Siegel, M.D. (Sandia National Labs., Albuquerque, NM (USA); INTERA Environmental Consultants, Lakewood, CO (USA); Auburn Univ., AL (USA); Science Applications, Inc., Albuquerque, NM (USA)). 1982. Contract AC04-76DP00789. 8p. (CONF-821107-40). NTIS, PC A02/MF A01. Order Number DE83005008.

From 6. international symposium on the scientific basis for radioactive waste management (Materials Research Society); Boston, MA, USA (1 Nov 1982).

A sensitivity analysis was performed to determine the impact of the containment time criterion on compliance with the Environmental Protection Agency Draft Standard when using temperature-dependent leach rates. The results of this analysis indicate that canister design life can be shown to be important when the effects of temperature on leach rates are taken into account.

982 (SAND-82-2260C) Compliance assessments of hypothetical geological nuclear waste isolation systems with the draft EPA standard. Siegel, M.D.; Chu, M.S.; Pepping, R.E. (Sandia National Labs., Aibuquerquiè, NM (USA)). 1982. Cuntract AC04-76DP00789. 10p. (CONF-821 107-6). NTIS, PC A02/MF A01. Order Number DE83001170.

From 6. international symposium on the scientific basis for radioactive waste management (Materials Research Society); Boston, MA, USA (1 Nov 1982).

Preliminary calculations of potential radionuclide discharges from hypothetical repositories in tuff and bedded salt have been completed. The theoretical approach and computational methods are illustrated in this paper with examples for a repository in volcanic tuff. Calculations suggest the following conclusions for the hypothetical tuff repository: 1) sorption of radionuclides by several thousand feet of zeoli- tized tuff is an effective barrier to migration of actinides even in the absence of solubility constraints. 2) All violations of the EPA Draft Standard in the base case are due to discharges of ${ }^{99} \mathrm{Tc}$ and ${ }^{14} \mathrm{C}$. Retardation due to matrix diffusion, however, may eliminate discharge of these nuclides for realistic groundwater flow rates. 3) If the radionuclides do not flow through several thousand feet of zeolitized tuff, discharges of $\mathrm{U}$ and $\mathrm{Np}$ under oxidizing conditions may be much larger than the EPA limits. Under reducing conditions, however, the low solubilities of these elements may provide an effective barrier to radionuclide release. For both the tuff and bedded salt sites the investigators have found that calculated radionuclide discharge is very sensitive to the source model chosen. Radionuclide solubility limits, waste form leach rate and mixing processes within the engineered facility must all be considered in modeling the compliance of hypothetical repositories with the EPA Draft Standard.

983 (SAND-82-2313C) Summary of research and development for disposal of US Defense wastes in the Waste Isolation Filot Plant (WIPP). Hunter, T.O. (Sandia National Labs., Albuquerque, NM (USA)). 1983. Contract AC04-76DP00789. 19p. (CONF-830523-2). NTIS, PC A02/MF A01. Order Number DE83007840.

From International conference on radioactive waste management; Seattle, WA, USA (16 May 1983).

Partinna are illogible in microfiche produrts

The Waste Isolation Pilot Plant (WIPP) is an R and D facility located in bedded rock salt in southeastern New Mexico to demonstrate the safe disposal of nuclear wastes from the US defense programs. Excavation is now in progress for the underground rooms in which in situ experiments will be performed. These experiments are a follow-on to site selection and characterization studies that began as early as 1972, and are based on an extensive amount of laboratory testing, analytical modeling, and field tests in boreholes and nearby mines. Results of experimental activities to date and the plans for remaining tests are presented, with emphasis on those to be completed in 1984 and 1985. The first phase of the experiments is performed without using radioactive material. These experiments began with the Site and Preliminary Design Validation (SPDV) program, which consisted of (1) two shafts, $12 \mathrm{ft}$ and 6 $\mathrm{ft}$ in diameter, drilled to $2150 \mathrm{ft}$; and (2) underground drifts and rooms monitored to determine the response of the rock salt under ambient conditions. The SPDV program will be followed by experiments in a series of underground rooms in which the response of the rock to heat emplacements with an areal loading of $12 \mathrm{~W} / \mathrm{m}^{2}$ will be determined. Other experiments in rooms include overtest configurations that will generate about four times that heat loading, and other well-defined geometric configurations to evaluate predictive models. Also planned are experiments addressing waste package interactions with rock salt and tests in other technical areas, such as repository sealing and operational considerations. In about 1989, a series of experiments and demonstrations will be performed with radioactive wastes. These include a full-scale storage demonstration using about 6 million $\mathrm{ft}^{0}$ of TRU wastes and tests using up to 40 canisters of defense high-level wastc.

984 (SAND-82-2336) Basic data report for drillhole WIPP 12 (Waste Isolation Pilot Plant-WIPP). (Sandia National Labs., Albuquerque, NM (USA); D'Appolonia Consulting Engineers, Albuquerque, NM (USA)). Oct 1982. Contract AC04-76DP00789.118p. NTIS, PC A06/MF A01. Order Number DE83003833.

Portions of document are illegible. Printed copy available until stock is exhausted.

WIPP 12 is a borehole drilled in eastern Eddy County, New Mexico, to investigate the stratigraphy, structure and lithology in the WIPP area. WIPP 12 was drilled in section 17, T22S,R31E, between Nuvember 9 and December 7, 1978. The hole was drillod to a depth of $2785.8 \mathrm{ft}$. It encountered from top to bottom, $16.2 \mathrm{ft}$ of sand, $3 \mathrm{ft}$ of Mescalero Caliche and $9.6 \mathrm{ft}$ of the Gatuna Formation, all of Quaternary age; $138.2 \mathrm{ft}$ of the Triassic Santa Rosa Formation, $483 \mathrm{ft}$ of the Dewey Lake Red Beds, $326 \mathrm{ft}$ of the Rustler Formation, $1771.5 \mathrm{ft}$ of the Salado Formation, and $48.3 \mathrm{ft}$ of the Castile Formation, all of Permian age. Cores or cuttings were obtained for the entire hole. A suite of geophysical logs, including neutron gamma and density curves, was run to the full depth of WIPP 12. The borehole demonstrated that the clcvation of the top of the Castile is about 160' above the same horizon in ERDA 9. The WIPP is a demonstration facility for the disposal of transuranic (TRU) waste from defense programs. The WIPP will also provide a 
search facility to investigate the interactions between bedded salt and igh level wastes.

985 (SAND-82-2413C) Approach to resolution of geologic uncertainty in the licensing of a high-level-waste repository in tuff. Neal, J.T. (Sandia National Labs., Albuquerque, NM (USA)). 1983. Contract AC04-76DP00789. 17p. (CONF830315-1). NTIS, PC A02/MF A01. Order Number DE83006204.

From GSA symposium on geological licensing topics in high- and low-level nuclear waste; College Station, TX, USA (3 Mar 1983).

Portions are illegible in microfiche products.

Resolution of uncertainty in geological information is an essential element in the licensing process for a geologic repository. Evaluation of these uncertainties within the licensing framework established by the Nuclear Regulatory Commission (NRC) is required. The Nevada Nuclear Waste Storage Investigations (NNWSI), in focusing its site characterization program on unsaturated tuff, has developed a logic hierarchy of technical issues, including key issues, issues, and information needs. Key issues are statements of major requirements whose lack could be disqualifying. An example of a key issue is the demonstration of radionuclide containment and isolation within the required release limits and transport time set by the EPA and NRC. Key issues are broken down into issues, such as the groundwater flow time to the accessible environment. Resolving uncertainty ultimately comes back to satisfying individual information needs that collectively form issues. Hydraulic conductivity is an example of an information need required to determine groundwater flow rate. Sources of uncertainty often arise in either amount, quality, or other limitations in geological data. The hierarchical structuring of geological information needs provides a perspective that allows proportionate attention to be placed on various site characterization activities, and to view them within the whole range of licensing issues that must be satisfied to ensure public bealth and safety. However, it may not prevent an issue from being contentious, as some geological questions are known to be emotion-laden. The mitigation of uncertainty in geological information ultimately will depend on the validity and credibility of the information presented during the licensing process.

986 (SAND-82-2497) Thermal response to emplacement of nuclear waste in long, horizontal boreholes. Peters, R.R. (Sandia National Labs., Albuquerque, NM (USA)). Apr 1983. Contract AC04-76DP00789. 36p. NTIS, PC A03/MF A01. Order Number DE83011870.

The Nevada Nuclear Waste Storage Investigations (NNWSI) Project, is cxamining the feasibility of siting a repository for high-level nuclear waste at Yucca Mountain on and adjacent to the Nevada Test Site. This work, intended to extend our understanding of repository design options and their impacts on expected temperatures at representative locations, was funded by the NNWSI Project. The emplacement of nuclear waste in long, horizontal boreholes appears to offer several advantages when compared to the mure conventional scheme of floor emplacement. The most important one is a significant reduction in the amount of material mined. The capability also exists for leaving a short distance (called the standoff distance) near both ends of the emplacement tube empty, which reduces the access drift temperature rise significantly in the first $\mathbf{3 0}$ years or so after nuclear wastc emplacement. This paper discusses the results of an investigation of the temperature field around horizontal emplacement repositories containing either spent fuel, commercial high-level waste, or defense high-level waste. The effects of variation of the Gross Thermal Loading (GTL), canister power, and rock type on the temperature field were investigated. The effect of standoff distance on access drift temperature rise was investigated with a reasonable distance, under most conditions, being about 10 $\mathrm{m}$. GTLs of $100 \mathrm{~kW} /$ acre or canister loadings above $2.2 \mathrm{~kW}$ appear to be too high, especially if the emplacement medium is a zeolitized tuff which has a low thermal conductivity and diffusivity. The computer code used in this investigation was, by necessity, simple and quick running. The limitation of this code was that because thermal properties tere constrained to be constant, the effects of boiling could not be scluded. This problem is nul severe if the boiling region is small s.nmared to the region of interest.

987 (SAND-82-2711) 1982 Subseabed Disposal Program. Annual report, site assessment October 1981 to September 1982. Shephard, L.E. (ed.). (Sandia National Labs., Albuquer- que, NM (USA)). Sep 1983. Contract AC04-76DP00789. 291p. NTIS, PC A13/MF A01. Order Number DE84000996.

Portions are illegible in microfiche products.

The Site Assessment Program is continuing its evaluation of marine geological formations in conjunction with Subseabed Disposal Program efforts to evaluate the technical, environmental, and engineering feasibility for disposing of high-level radioactive waste. Currently, site assessment efforts are focused on 3 candidate locations in the North Pacific and 4 locations in the North Atlantic. 1982 Site Assessment activities, summarized in this document, emphasized completing the analyses of data collected on Vema 36-12, preparing for and successfully completing drilling in the PAC 1 region, and continuing cooperative efforts with the international NEA/SWG Site Selection Task Group in the North Atlantic.

988 (SAND-82-2771) Code development in support of nuclear waste storage investigations for a repository in tuff. Eaton, R.R.; Martinez, M.J.; Wilson, R.K.; Nunziato, J.W. (Sandia National Labs., Albuquerque, NM (USA)). Mar 1983. Contract AC04-76DP00789. 129p. NTIS, PC A07/MF A01. Order Number DE83013140.

Portions are illegible in microfiche products. Original copy available until stock is exhausted.

A summary of the code development provided by the Fluid and Thermal Sciences Department in support of the design and performance evaluation of a nuclear waste repository in tuff (NNWSI) is documented herein. Various aspects of equation derivation, code development, code verification and scoping calculations for flow through partialy saturated media are presented.

989 (SAND-83-0178C) WIPP research and development program: providing the technical basis for defense waste disposal. Hunter, T.O. (Sandia National Labs., Albuquerque, NM (USA)). 1983. Contract AC04-76DP00789. 4p. (CONF830205-6). NTIS, PC A02/MF A01. Order Number DE83006480. Feb 1983).

From Waste management conference; Tucson, AZ, USA (27

The Waste Isolation Pilot Plant is a research and development facility that includes a phased program of site characterization, in-situ tests, and operations demonstrations. It addresses all the technical issues associated with transuranic and defense high-level wastes from US defense programs. Underground construction is in progress, and data collection from experiments without radioactivity will begin in 1983. Radioactive experiments can be performed in the late 1980's, thus providing a comprehensive technical basis for the disposal of defense wastes in bedded salt.

990 (SAND-83-0226C) Conclusions regarding geotechnical acceptability of the WIPP site. Weart, W.D. (Sandia National Labs., Albuquerque, NM (USA)). 1983. Contract AC04-76DP00789. 1lp. (CONF-830205-5). NTIS, PC A02/MF A01. Order Number DE83006483. Feb 1983).

From Waste management conference; Tucson, AZ, USA (27

The Waste Isolation Pilot Plant (WIPP) was authorized by Congress in 1980 as an unlicensed research and development (R \& D) facility to demonstrate the safe disposal of radioactive wastes arising from the defense activities and programs of the United States. WIPP is now being constructed in southeast New Mexico, using salt beds about 655 $\mathrm{m}$ below the surface of the ground. Construction of the full WIPP facility will not $r$ mmence until a preliminary underground excavation phase, called Site and Preliminary Design Validation (SPDV), is satisfactorily concluded in the summer of 1983. This SPDV program permits confirmation of subsurface geology, in drifts at planned facility depth that extend for $1555 \mathrm{~m}$ in a north-south direction, and in the two vertical shafts that provide access to these drifts. The subsurface studies are nearing completion, and it is therefore appropriate to draw conclusions regarding the geotechnical acceptability of the WIPP site. Four geotechnical elements are discussed: dissolution, deformation, hydrologic regime, and natural resources.

991 (SAND-83-0247C) Design considerations for occupational exposure for a potential repository at Yucen Mountain: high-level-waste handling operations. Dennis, A.W. (Sandia National Labs., Albuquerque, NM (USA)). 1983. Contract 
AC04-76DP00789. 13p. (CONF-830609-14). NTIS, PC A02/MF A01. Order Number DE83013472.

From ANS annual mceting; Detroit, MI, USA (12 Jun 1983). Portions are illegible in microfiche products.

The present status of the NNWSI program, with respect to planned worker radiation protection during high-level waste handling operations, is presented. Information on the types and quantities of highlevel waste to be sent to the facility, the casks in which this waste will be shipped to the facility, the proposed facility operating plan, and the facility design criteria as they relate to worker radiation protection are included in this paper.

992 (SAND-83-0280) Hydrology of sealing a repository in saturated tuff. Mondy, L.A. (Sandia National Labs., Albuquerque, NM (USA)). Mar 1983. Contract AC04-76DP00789. 66p. NTIS, PC A04/MF A01. Order Number DE83015633.

Portions are illegible in microfiche products. Original copy available until stock is exhausted.

In the hydrologic modeling studies it was assumed that the repositories were located in volcanic tuff such as is found in Yucca Mountain at the Nevada Test Site. This modeling study dealt only with repositnrics in saturâted iock and investlgated the effects on groundwater flow once drifts and shafts are filled with materials of various permeability. Temperature effects were not examined. Modeling was limited to two dimensions, necessitating simplifying assumptions in some cases. Groundwater flow for the drift model was assumed to be due primarily to a hydraulic head gradient caused by a dipping water table similar to that found in the Yucca Mountain region. In the shaft model a vertical pressure gradient was assumed so that comparisons of sealing designs could be made. Results indicated that observable deviations in the groundwater flow near a repository would occur unless the drifts and shafts were backfilled to a permeability approaching that of the native rock. A series of low permeability blocks in the drifts showed promise in reducing these deviations in groundwater flow, as long as the spacing between blocks remained relatively short $(<10 \mathrm{~m})$. Sealing only the intersections of drifts proved ineffective. The preferred orientation for the repository appeared to be one with the emplacement drifts perpendicular to the dominant flow direction. This orientation allowed less water to flow past the waste, and the access drifts could be used to provide a path of least resistance to draw more water away from the emplacement drifts. The most effective measure to prevent large flow rates through vertical shafts was to backfill the shaft to a permeability approaching that of the undisturbed rock. Bulkheads placed in the shaft had little influence on the flow. The effects of a highly permeable disturbed zone $1.37 \mathrm{~m}$ in thickness produced by drilling the shaft were also studied.

993 (SAND-83-0465C) Methodology for assessing the risk from the disposal of high-level radioactive wastes in deep geologic formations. Cranwell, R.M.; Ortiz, N.R.; Runkle, G.E. (Sandia National Labs., Albuquerque, NM (USA)). 1983. Contract AC04-76DP00789. 23p. (CONF-830523-5). NTIS, PC A02/MF A01. Order Number DE83009164.

From International conference on radioactive waste management; Seattle, WA, USA (16 May 1983).

The Fuel Cycle Risk Analysis Division of Sandia National Labo ratories, Albuquerque, New Mexico, has recently completed the development of a risk assessment methodology for use in assessing the postclosure, long-ter in risk frum the dlsposal of high-level radioactive wastes in deep geologic formations. Briefly, this methodology consists of: (1) techniques for selecting and screening scenarios, (2) models for use in simulating the physical processes and estimating the consequences associated with the occurrence of these scenarios, (3) probabilistic and statistical techniques for use in risk estimates and sensitivity and uncertainty analyses, (4) a procedure for utilizing these mndels and techniques to arrive at estimates of consequences and risk. The methodology has been demonstrated by applying it to the analysis of a hypothetical site containing a bedded salt formation as the hast medium for the waste repasitory. In this demonstration analysis, consequences resulting from the occurrence of several hypothetical scenarios were determined. These consequences were expressed in terms of radionuclide discharges to the biosphere and health effects resulting from these discharges. Health effects were expressed in terms of the probability of an individual developing a latent cancer. Sensitivity analyses were performed to determine the model input variables which are most important in influencing variation in predicted discharges and health effects. The result of the analysis was compared with the requirements of the US Environmental Protec tion Agency Standard for high-level waste disposal.

- 994 (SAND-83-0516) Comparison of brines relevant to - nuclear waste experimentation. Molecke, M.A. (Sandia National Labs., Albuquerque, NM (USA)). May 1983. Contract AC04-76DP00789. 61p. NTIS, PC A04/MF A01. Order Number DE83012968.

Portions are illegible in microfiche products.

The ionic compositions of 18 brines used in nuclear waste-related - "laboratory tests or obtained from field tests are described and compared. - Also described are the origin of each brine, its predominant use for laboratory testing, and its relavancy for future testing. The brines include Brines A and B (Waste Isolation Pilot Plant (WIPP)/generic), Office of Nuclear Waste Isolation (ONWI) Composite Permian Brine $P$ and - Equilibrated Permian P No. 2, Battelle Pacific Northwest Laboratory (PNL)-Sandia High-Level Waste (HLW) package interactions test - brines (flow and downhole), ERDA-6 brines (flow and downhole), WIPP Inclusions No. 1 and No. 2, Materials Characterization Center (MCC) brine, German quinare Brine Q, US Geological Survey bittern NRT-Ka saturated $\mathrm{NaCl}\left(20^{\circ}\right.$ and $\left.100^{\circ} \mathrm{C}\right)$, and standard seawater.

995 (SAND-83-0678) Comparison of wastc emplacement configurations for a nuclear waste repusitury in tuff. II. Ventlation analysis. Hickox, C.E. (Sandia National Labs., Albuquerque, NM (USA)). Aug 1983. Contract AC04-76DP00789. 34p. NTIS, PC A03/MF A01. Order Number DE84000976.

Ventilation requirements are assessed for three proposed nuclear waste emplacement schemes. The configurations investigated involve canisters of nuclear waste (1) placed periodically along the floor of a storage drift. (2) emplaced in vertical holes bored in the floor of a storage drift, and (3) emplaced in bored holes extending horizontally from the sides of an access drift. Preliminary analyses indicate that, for a drift wall temperature of $25^{\circ} \mathrm{C}$, the maximum required cooling air velocity is slightly less than $1 \mathrm{~m} / \mathrm{s}$ and occurs for configuration 1 . The other two configurations require approximately one-half as much cooling air velocity, the exact value depending on the drift length. The associated frictional pressure loss is shown to be small. Estimates are made of the power required to reduce the temperature of the ambient air to levels suitable for effective cooling of the drifts. Based on the arrangements required for the storage of 1000 canisters, it is shown that configuration 1 requires the most refrigeration and configuration 3 the least. Finally, brief consideration is given to the physiological environment associated with the thermal energy released. Initial results indicate that it should be relatively easy to maintain an acceptable working environment.

998 (SAND-83-0757) Comparison of waste emplacement configurations for a nuclear waste respository in tuff. IV. Thermo-hydrological analysis. Mondy, L.A.; Wilson, R.K.; Bixler, N.E. (Sandia National Labs., Albuquerque, NM (USA)). Aug 1983. Contract AC04-76DP00789. 121p. NTIS, PC A06/MF A01. Order Number DE84000968.

Portions are illegible in microfiche products. Original copy available until stock is exhausted.

This report summarizes the results of a hydrological analysis of two emplacement schemes being considered for the storage of commercial high-level nuclear waste at the Nevada Test Site. The analysis is two-dimensional, considers the flow of water in partially saturated tuff (the Topopah Springs member of the Paintbrush tuff in Yucca Mountain) and includes the effects of the heat source (waste canisters) on that flow. The results include measures of the heat flux entering the access and emplacement drifts, measures of the flow rates near the canisters and a comparison of the temperature fields. It was necessary in the analysis to approximate the boundary conditions at the walls of the access and emplacement drifts in order to simulate the ventilation process. As a result the analysis was done for several cases which were expected to bracket the actual situation. A discussion of this problem is also included in the report. It should be noted that these results are intende as a means of comparing emplacement schemes, not as a performanc assessment. 72 figurcs, 6 tables. braenslefoersoerjning AB, Stockholm). Mar 1982. 44p. NTIS 
'US Sales Only), PC A03/MF A01. Order Number JE83700169.

Various types of repositories for low-,medium-,and high level radioactive wastes require concrete for plugging or construction purposes, the concrete being in direct contact with clay buffer materials. In the case of $\mathrm{Na}$ montmorillonite, two main chemical processes can affect this type of clay, namely $\mathrm{pH}$-induced attack on the crystal lattices, and exchange of initially adsorbed $\mathrm{Na}$ to $\mathrm{Ca}$. The ion exchange is of minor importance while lattice destruction may be serious. Theoretically, it would lead to the formation of zeolites. Three experimental studies were conducted to find out the type and extent of chemical reaction. One concerned a close investigation of a 70 year old concrete/clay foundation, which showed that alterations of the chemical composition could not be found at larger distances than about $50 \mu \mathrm{m}$ from the interface. The second study was an accelerated 3-month test in a borehole in the Stripa Mine, which indicated no mineralogical alterations whatsoever, even at the interface, and no sign of a calcium diffusion profile into the clay. The third study involved percolation of a $\mathrm{Na}$ bentonite disc with $\mathrm{NaOH}$ solution for one year. No mineralogical alterations, such as zeolite formation, could be identified in this case either, but some disso lution of quartz and possibly feldspars was observed. The permeability was typical of that of untreated $\mathrm{Na}$ bentonite while the swelling pressure increased, which was interpreted as resulting from an improved $\mathrm{Na}$ adsorption. It was cocluded that, assuming all the bentonite to be ultimately converted into zeolites, this reaction will be confined to about $1 \mathrm{~cm}$ from the clay/concrete interface of the SFR silo walls in 500-1000 years. Ion exchange to calcium will extend deeper into the clay but with very slight effects only on the physical and mechanical properties.

998 (SKBF-KBS-TR-81-17) KBS annual report 1981. Including summaries of technical reports issued during 1981. (Svensk Kaernbraenslefoersoerjning AB, Stockholm). May 1982. 133p. NTIS (US Sales Only), PC A07/MF A01. Order Number DE83701474.

The nuclear power utilities have commissioned the jointly owned Swedish Nuclear Fuel Supply Company (SKBF) to assume responsibility for a safe handling of the waste and a safe final storage. KBS is the department within SKBF that is responsible for research and development within the area of radioactive waste management. The government agency of PRAV (the National Council for Radioactive Waste) was dissolved as of mid-year 1981 and its research activities were transferred to SKBF/KBS. Simultaneously, the National Board for Spent Nuclear Fuel, NAK, was created and charged with the duties of overseeing the work being conducted by SKBF within the nuclear waste field and administering the funds that are to be set up for the financing of future waste management activities. The present annual report describes activities within KBS during 1981. The work conducted during the year has been concentrated on three areas: 1) A systematic review has begun of geologically interesting areas in Sweden that might be suitable as sites for a final repository for high level waste or spent fuel. 10-20 areas are scheduled for study during the 1980 s. 2) The chemical research has been broadened in order to further understanding of the chemical interplay that exists in the repository between the canister material, the buffer, the waste matrix and the groundwater. The retardation effects associated with the transport of radioactive elements with the groundwater in the bedrock also constitute an important subject of these studies. 3) The preliminary planning and engineering of a final repository for low-and medium-level operating waste from the Swedish reactors is in progress. The aim is to submit an application during the spring of 1982 for permission to build the facility at the Forsmark Nuclear Power Station.

999

(SKBF-KBS-TR-82-02) Radiolysis of groundwater from HLW stored in copper canisters. Christensen, $H$; Bjergbakke, E. (Svensk Kaernbraenslefoersoerjning AB, Stockholm). 29 Jun 1982. 35p. (STUDSVIK-NW-82-273). NTIS (US Sales Only), PC A03/MF A01. Order Number DE83702345.

A large number of computer calculations of the radiolysis of ground water outside copper canisters have been carried out. At dose rates higher than $5 \times 10^{-2} \mathrm{rad} / \mathrm{s}$ the hydrogen concentration is constant, approximately $10^{-0} \mathrm{~mol} / \mathrm{dm}^{0}$, and independent of time and dnse rate. Therefore the rate of diffusion out of the system is constant, approximately $7 \times 10^{-4} \mathrm{~mol} /$ year. At dose rates lower than $5 \times 10^{-4} \mathrm{rad} / \mathrm{s}$ the logarithm of the hydrogen production is proportional to the logarithm of the dose rate. The hydrogen production depends on fuel type, burn-up and canister thickness only to the extent that the dose rate varies with these parameters. The hydrogen production is dependent of the volume of water in which the energy is assumed to be absorbed ( 5 or $15 \mathrm{~cm}$ layer outside the surface of the canister). The hydrogen production depends on the concentration of $\mathrm{Fe}^{2+}$ ions in the water. In the basic calculations the concentration was assumed to be $5 \mathrm{ppm}$. An increase to $50 \mathrm{ppm}$ increases the total hydrogen production after one million years outside a 1-cm-thick canister from 360 to $1000 \mathrm{~mol}$. A decrease to $0.5 \mathrm{ppm}$ decreases the same yield to $80 \mathrm{~mol}$.

1000 (TENRAC/ADV-80-002) Report of the Low-Level Nuclear Waste Subcommittee. (Texas Energy and Natural Resources Advisory Council, Austin (USA)). 5 Sep 1980. 95p. NTIS, PC A05/MF A01. Order Number DE83902062.

Portions are illegible in microfiche products.

The following recommendations are made. The disposal site should be established for disposal of low-level nuclear waste LLW generated in the State of Texas for the immediate benefit of residents of Texas. Provision should be made for accepting LLW from other states if the Texas Legislature approves, on a state-by-state basis, mutually beneficial agreements. The proposed LLW disposal site should be located on state-owned land or the site selected should be acquired by the state. The proposed disposal site should be operated and maintained by an authority of the State of Texas such as the Gulf Coast Waste Disposal Authority. The state authority so designated should be responsible for selecting the site, obtaining licenses from appropriate state agencies, and managing and directing site operations. The proposed site should be self-supported by disposal fees. The disposal fees are to be established by the disposal authority and should be based on volume and relative hazard. Waste materials disposed at the proposed site shall be limited to ${ }^{14} \mathrm{C}$ or material with a half-life of $100 \mathrm{y}$ or less, including waste from nuclear power plants but excluding irradiated nuclear reactor fuel and high-level waste as defined by federal regulations. Shipments of LLW will be transported in accordance with existing regulations and will comply with some additional requirements. A schedule of surcharges should be established by the disposal authority and assessed when improperly packaged or processed wastes are shipped to the site. The state authority responsible for managing the proposed disposal site must remain abreast of improvements in LLW technology and implement those developments which are appropriate.

1001 (TENRAC/EP-83-004) High-level radioactivewaste-disposal investigations in Texas. Smith, R.D. (Texas Energy and Natural Resources Advisory Council, Austin (USA)). Jan 1983. 71p. NTIS, PC A04/MF A01. Order Number DE83902061.

Portions are illegible in microfiche products.

The Texas Energy and Natural Resources Advisory Council (TENRAC) was designated in 1980 to coordinate the interaction between the State of Texas and the federal government relating to the high-level radioactive waste disposal issue. This report was prepared to summarize the many aspects of that issue with particular emphasis on the activities in Texas. The report is intended to provide a comprehensive introduction for individuals with little or no previous exposure to the issue and to provide a broader perspective for those individuals who have addressed specific aspects of the issue but have not had the opportunity to study it in a broader context. Following the introduction, contents of this report are as follows: (1) general status of major repository siting investigations in the US; (2) detailed review of Texas studies; (3) possible facilities to be sited in Texas; (4) current Texas policy; (5) federal regulations; and (6) federal legislation. 9 figures, 2 tables.

1002 (UCID-18946) Climax Granite, Nevada Test Site, as a host for a rock mechanics test facility related to the geologic disposal of high level nuclear wastes. Heuze, F.E. (Lawrence Livermore National Lab., CA (USA)). Feb 1981. Contract W-7405-ENG-48. 50p. NTIS, PC A03/MF A01. Order Number DE83013648.

Portions are illegible in microfiche products.

This document discusses the potential of the Climax pluton, at the Nevada Test Site, as the host for a granite. mechanics test facility related to the geologic disposal of high-level nuclear waste. The Climax granitic pluton has been the site of three nuclear weapons effects tests: Hard Hat, Tiny Tot, and Piledriver. Geologic exploration and mapping of the granite body were performed at the occasion of these tests. Currently, it is the site Spent Fuel Test (SFT-C) conducted in the vicinity of and at the same depth as that of the Piledriver drifts. Significant 
exploration, mapping, and rock mechanics work have been performed and continue at this Piledriver level - the 1400 (ft) level - in the context of SFT-C. Based on our technical discussions, and on the review of the significant geological and rock mechanics work already achieved in the Climax pluton, based also on the ongoing work and the existing access and support, it is concluded that the Climax site offers great opportunities for a rock mechanics test facility. It is not claimed, however, that Climax is the only possible site or the best possible site, since no case has been made for another granite test facility in the United States. 12 figures, 3 tables.

1003 (UCID-19672) Drilling-induced borehole-wall damage at spent fuel test-climax. Weed, H.C.; Durham, W.B. (Lawrence Livermore National Lab., CA (USA)). Dec 1982. Contract W-7405-ENG-48. 24p. NTIS, PC A02/MF A01. Order Number DE83006417.

Microcracks in a sample of quartz monzonite from the Spent Fuel Test-Climax were measured by means of a scanning electron microscope in order to estimate the background level of damage near the borehole-wall. It appears that the hammer-drilling operation used to create the borehole has caused some microfracturing in a region 10 to $30 \mathrm{~mm}$ wide around the borehole. Beyond $30 \mathrm{~mm}$, the level of microfracturing cannot be distinguished from background.

1004 (UCID-19764) Waste form/rock interaction leaching study using PNL 76-68 glass beads and Umtanum basalt. Part I. Bazan, F.; Rego, J.; Failor, R.; Coles, D. (Lawrence Livermore National Lab., CA (USA)). 31 Mar 1983. Contract W-7405-ENG-48. 197p. NTIS, PC A09/MF A01. Order . Number DE 83012449.

Portions are illegible in microfiche products. Original copy available until stock is exhausted.

A 440-day single-pass continuous-flow leaching experiment was conducted at LLNL from September 1980 to December 1981. The data obtained for only one-third of the experiment are presented. The laboratory and data analysis of the remaining portion is still in progress at this time and a second report will follow at the end of FY83. This report concerns itself with the study of PNL 76-68 glass beads interacting with crushed uranium flow basalt and a simulated basalt groundwater under controlled conditions of temperature $\left(25^{\circ} \mathrm{C}\right.$ and $\left.75^{\circ} \mathrm{C}\right)$ and flow rate $(1$, 10 , and $300 \mathrm{ml} /$ day). The main purpose of the experiment was to determine the absorption on basalt of $\mathrm{Pu}, \mathrm{Np}$, and some of the stable elements such as B, Mo, U, and Cs, as they were leached from the glass beads. Results are presented, as incremental and cumulative leach rates and sorption rates have been calculated for $\mathrm{Pu}, \mathrm{Np}, \mathrm{B}, \mathrm{Mo}$ and $\mathrm{U}$. also sorption profiles as a function of temperature and flow rate are graphically shown for $\mathrm{Pu}, \mathrm{Np}$, and $\mathrm{U}$.

1005 (UCID-19926) Initial specifications for nuclear waste package external dimensions and materials. Gregg, D.W.; O'Neal, W.C. (Lawrence Livermore National Lab., CA (USA)). Sep 1983. Contract W-7405-ENG-48, 27p. NTIS, PC A03/MF A01. Order Number DE84002110.

Portions are illegible in microfiche products. Original copy available until stock is exhausted.

Initial specifications of external dimensions and materials for waste package conceptual designs are given for Defense High Level Waste (DHLW), Commercial High Level Waste (CHLW) and Spent Fuel (SF). The designs have been developed for use in a high-level waste repository sited in a tuff media in the unsaturated zone. Drawings for reference and alternative package conceptual designs are presented for each waste form for both vertical and horizontal emplacement configurations. Four metal alloys: 304L SS, 321 SS, 316L SS and Incoloy 825 are considered for the canister or overpack; 1020 carbon steel was selected for horizontal borehole liners, and a preliminary packing material selection is either compressed tuff or compressed tuff containing iron bearing smectite clay as a binder.

1006 (UCRL-52000-79-6, pp 1-7) Leaching studies of radioactive glass. Jun 1979. NTIS, PC A03/MF A01.

In Energy and technology review.

The radioactive materials in an underground nuclearexplosion test site or a reactor-waste repository could present a potential risk to the environment if the materials were leached into the groundwater supply. To help develop a technical basis for assessing these risks, we are studying the leaching process in glassy materials, such as those developed for holding high-level reactor waste and those formed in underground nuclear tests. We use various water compositions, flow rates, and temperatures to determine the response of both nuclearexplosion melt glass and reactor-waste glass to a variety of repository conditions. With our method-single-pass leaching-we simulate underground conditions by continuously replenishing the leaching solution and passing it through the cell containing the waste only once.

1007 (UCRL-53188-81) Nuclear waste-form risk assessment for US Defense waste at Savannah River Plant. Annual report FY 1981. Cheung, H.; Edwards, L.L.; Harvey, T.F.; Jackson, D.D.; Revelli, M.A. (Lawrence Livermore National Lab., CA (USA)). Dec 1981. Contract W-7405-ENG-48. 78p. NTIS, PC A05/MF A01. Order Number DE82020575.

Savannah River Plant has been supporting the Lawrence Livermore National Laboratory in its present effort to perform risk assessments of alternative waste forms for defense waste. This effort relates to choosing a suitable combination of solid form and geologic medium on the hasis of risk of exposure to future generations; therofore, the focus is on post-closure considerations of deep geologic repositories. The waste forms baing investigated inolude borosilieate glass, SYNROC, and others. Geologic media under consideration are bedded salt, basalt, and tuff. The results of our work during FY 1981 are presented in this, our second annual report. The two complementary tasks that comprise our program, analysis of wastc-form dissolution and risk assessment, are described.

1008 (UCRL-53188-82) Nuclear waste-form risk assessment for US defense waste at Savannah River Plant. Annual report FY, 1982. Cheung, H.; Edwards, L.L.; Harvey, T.F. (Lawrence Livermore National Lab., CA (USA)). 9 Aug 1982. Contract W-7405-ENG-48. 107p. NTIS, PC A06/MF A01. Order Number DE83017548.

A network model was developed to simulate the hydrological flow and the transport of radionuclides from a deep geological repository to the biosphere subsequent to closure. By means of very efficient computational methods for solving the fundamental differential equations, a code was developed to treat in great detail the effects of waste form characteristics and of repository designs on the repository risks. It is possible to examine near field effects heretofore not attempted. Without sacrificing the essential details of description, the code can also be applied to perfurm probabilistic risk analyses to high confidence levels. Analytical results showed: (1) for waste form release rates greater than appruximately $5 \times 10^{-7} / \mathrm{yr}$, dose to man is insensitive to release rate and release rate uncertainty; (2) significant reduction in dose can be achieved through simple design modifications; (3) a basalt repository generally does not perform as well as a salt repository; and (4) disruptive events are relatively unimportant for repository safety. 82 references.

1009

(UCRL-53269) Postclosure risks of alternative SRP nuclear waste forms in geologic repositories. Cheung. H.; Edwards, L.; Harvey, T.; Revelli, M. (Lawrence Livermore National Lab., CA (USA)). May 1982. Contract W-7405-ENG48. 43p. NTIS, PC A03/MF A01. Order Number DE82017234.

The postclosure risk of REFERENCE and ALTERNATIVE waste forms for the defense high-level waste at the Savannah River Plant (SRP) were compared by analyses with a computer code, MISER, written to study the effects of repository features in a probabilistic framework. MISER traces radionuclide flows through a network of stream tubes from the repository to risk-sensitive points. Uncertainties in waste form, package properties, and geotechnical data are accounted for with Monte Carlo techniques. Our results show: (1) for generic layered-salt and basalt repositories, the difference in performance between the two waste forms is insignificant; (2) where the doses are sensitive to uncertainties in leaching rates, the doses are orders of magnitude below background; (3) disruptive events contribute only slightly to the risk of a layered-salt repository; (4) simple design alterations have strong effects on near field doses; (5) great care should be exercised in selecting the location at which repository risks are to be measured, calculated, or regulated.

1010

(UCRL-53315) Disposal costs for SRP high-level wastes in borosilicate glass and crystalline ceramic waste forms. Rozsa, R.B.; Campbell, J.H. (Lawrence Livermore National 
- b., CA (USA)). 25 Aug 1982. Contract W-7405-ENG-48.

p. NTIS, PC A02/MF A01. Order Number DE83003105.

Purpose of this document is to compare and contrast the overall burial costs of the glass and ceramic waste forms, including processing, storage, transportation, packaging, and emplacement in a repository. Amount of waste will require approximately 10,300 standard (24 in. i.d. $x$ 9-5/6 ft length) canisters of waste glass, each containing about 3260 $\mathrm{lb}$ of waste at $28 \%$ waste loading. The ceramic waste form requires about one-third the above number of standard canisters. Approximately \$2.5 billion is required to process and dispose of this waste, and the total cost is independent of waste form (glass or ceramic). The major cost items (about $80 \%$ of the total cost) for all cases are capital and operating expenses. The capital and 20-year operating costs for the processing facility are the same order of magnitude, and their sum ranges from about one-half of the total for the reference glass case to two-thirds of the total for the ceramic cases.

1011 (UCRL-53326) Properties of SYNROC C nuclearwaste form: a state-of-the-art review. Oversby, V.M. (Lawrence Livermore National Lab., CA (USA)). Sep 1982. Contract W-7405-ENG-48. 32p. NTIS, PC A03/MF A01. Order Number DE83002464.

SYNROC C is a titanate ceramic waste form designed to contain the waste generated by the reprocessing of commercial nuclear reactor fuel. The properties of SYNROC C are described with particular emphasis on the distribution of chemical elements in SYNROC, the fabrication of good quality specimens, and the chemical durability of SYNROC. Data obtained from testing of natural mineral analogues of SYNROC minerals are briefly discussed. The information available on radiation effects in SYNROC in relation to structural alteration and changes in chemical durability are summarized. 26 references, 2 figures, 18 tables.

1012 (UCRL-53394) Thermal and thermomechanical calculations of deep-rock nuclear waste disposal with the enhanced SANGRE code. Heuze, F.E. (Lawrence Livermore National Lab., CA (USA)). Mar 1983. Contract W-7405-ENG-48. 41p. NTIS, PC A03/MF A01. Order Number DE83011054.

An attempt to model the complex thermal and mechanical phenomena occurring in the disposal of high-level nuclear wastes in rock at high power loading is described. Such processes include melting of the rock, convection of the molten material, and very high stressing of the rock mass, leading to new fracturing. Because of the phase changes and the wide temperature ranges considered, realistic models must provide for coupling of the thermal and mechanical calculations, for large deformations, and for steady-state temperature-depenent creep of the rock mass. Explicit representation of convection would be desirable, as would the ability to show fracture development and migration of fluids in cracks. Enhancements to SNAGRE consisted of: array modifications to accommodate complex variations of thermal and mechanical properties with temperature; introduction of the ability of calculate thermally induced stresses; improved management of the minimum time step and minimum temperature step to increase code efficiency; introduction of a variable heat-generation algorithm to accommodate heat decay of the nuclear materials; streamlining of the code by general editing and extensive deletion of coding used in mesh generation; and updating of the program users' manual. The enhanced LLNL version of the code was renamed LSANGRE. Phase changes were handled by introducing sharp variations in the specific heat of the rock in a narrow range about the melting point. The accuracy of this procedure was tested successfully on a melting slab problem. LSANGRE replicated the results of both the analytical solution and calculations with the finite difference TRUMP code. Following enhancement and verification, a purely thermal calculation was carried to 105 years. It went beyond the extent of maximum melt and into the beginning of the cooling phase.

1013 (UCRL-86225) Instrument reliability for high-level nuclear-waste-repository applications. Rogue, F.; Binnall, E.P.; Irmantrout, G.A. (Lawrence Livermore National Lab., CA USA); I awrence Rerkeley Iab., CA (USA)). 31 Jan 1983. Sontract W-7405-ENG-48. 9p. (CONF-830205-2). NTIS, PC A02/MF A01. Order Number DE83006281.

From Waste management conference; Tucson, AZ. USA (27 Feb 1983).

Portions are illegible in microfiche products.
Reliable instrumentation will be needed to evaluate the characteristics of proposed high-level nuclear-wasted-repository sites and to monitor the performance of selected sites during the operational period and into repository closure. A study has been done to assess the reliability of instruments used in Department of Energy (DOE) waste repository related experiments and in other similar geological applications. The study included experiences with geotechnical, hydrological, geochemical, environmental, and radiological instrumentation and associated data acquisition equipment. Though this paper includes some findings on the reliability of instruments in each of these categories, the emphasis is on experiences with geotechnical instrumentation in hostile repository-type environments. We review the failure modes, rates, and mechanisms, along with manufacturers modifications and design changes to enhance and improve instrument performance; and include recommendations on areas where further improvements are needed.

1014 (UCRL-86582) Nuclear-waste disposal in geologic repositories. Isherwood, D. (Lawrence Livermore National Lab., CA (USA)). 2 Aug 1982. Contract W-7405-ENG-48. 17p. (CONF-820154-1). NTIS, PC A02/MF A01. Order Number DE82020176.

From Society of Civil Engineers conference on geotechnics of waste management; Philadelphia, PA, USA (19 Jan 1982).

Portions of document are illegible.

Deep geologic repositories are being widely studied as the most favored method of disposal of nuclear waste. Scientists search for repository sites in salt, basalt, tuff and granite that are geologically and hydrologically suitable. The systematic evaluation of the safety and reliability of deep geologic disposal centers around the concept of interacting multiple barriers. The simplest element to describe of the geologic barrier is the physical isolation of the waste in a remote region at some depth within the rock unit. Of greater complexity is the hydrologic barrier which is determined by the waste dilution factors and groundwater flow rates. The least understood is the geochemical barrier, identified as a series of waste/water/rock interactions involving sorption, membrane filtration, precipitation and complexing. In addition to the natural barriers are the engineered barriers, which include the waste form and waste package. The relative effectiveness of these barriers to provide longterm isolation of nuclear waste from the human environment is being assessed through the use of analytical and numerical models. The data used in the models is generally adequate for parameter sensitivity studies which bound the uncertainties in the release and transport predictions; however, much of the data comes from laboratory testing, and the problem of correlating laboratory and field measurements has not been resolved. Although safety assessments based on generic sites have been useful in the past for developing site selection criteria, site-specific studies are needed to judge the suitability of a particular host rock and its environment.

1016 (UCRL-87091) Nuclear-waste-package program for high-level isolation in Nevada tuff. Rothman, A.J. (Lawrence Livermore National Lab., CA (USA)). Jan 1982. Contract W-7405-ENG-48. 5p. (CONF-820609-75). NTIS, PC A02/MF A01. Order Number DE82020173.

From American Nuclear Society annual meeting; Los Angeles, CA, USA (6 Jun 1982)

The objective of the waste package program is to insure that a package is designed suitable for a repository in tuff that meets performance requirements of the NRC. In brief, the current (draft) regulation requires that the radionuclides be contained in the engineered system for 1000 years, and that, thereafter, no more than one part in $10^{5}$ of the nuclides per year leave the boundary of the system. Studies completed as of this writing are thermal modeling of waste packages in a tuff repository and analysis of sodium bentonite as a potential backfill material. Both studies will be presented. Thermal calculations coupled with analysis of the geochemical literature on bentonite indicate that extensive chemical and physical alteration of bentonite would result at the high power densities proposed (ca. $2 \mathrm{~kW} /$ package and an area density of 25 $\mathrm{W} / \mathrm{m}^{2}$ ), in part due to compacted bentonite's relatively low thermal conductivity when dehydrated $\left(\sim 0.6 \pm 0.2 \mathrm{~W} / \mathrm{m}^{0} \mathrm{C}\right)$. Because our groundwater contains $\mathrm{K}^{+}$, an upper hydrothermal temperature limit appears to be 120 to $150^{\circ} \mathrm{C}$. At much lower power densities (less than $1 \mathrm{~kW}$ per package and an areal density of $12 \mathrm{~W} / \mathrm{m}^{2}$ ), bentonite may be suitable.

1016 (UCRL-87510) Modeling of nuclear waste disposal by rock melting. Heuze, F.E. (Lawrence Livermore National 
Lab., CA (USA)). Apr 1982. Contract W-7405-ENG-48. 10p. (CONF-820803-3). NTIS, PC A02/MF A01. Order Number DE82012798.

From 23. US symposium on rock mechanics; Berkeley, CA, USA (25 Aug 1982).

Portions of document are illegible.

Today, the favored option for disposal of high-level nuclear wastes is their burial in mined caverns. As an alternative, the concept of deep disposal by rock melting (DRM) also has received some attention. DRM entails the injection of waste, in a cavity or borehole, 2 to 3 kilometers down in the earth crust. Granitic rocks are the prime candidate medium. The high thermal loading initially will melt the rock surrounding the waste. Following resolidification, a rock/waste matrix is formed, which should provide isolation for many years. The complex thermal, mechanical, and hydraulic aspects of DRM can be studied best by means of numerical models. The models must accommodate the coupling of the physical processes involved, and the temperature dependency of the granite properties, some of which are subject to abrupt discontinuities, during $\alpha-\beta$ phase transition and melting. This paper outlines a strategy for such complex modeling.

1017

(UCRL-87808) Results of instrument reliability study for high-level nuclear-waste repositories. Rogue, F.; Binnall, E.P. (Lawrence Livermore National Lab., CA (USA); Lawrence Berkeley Lab., CA (USA)). Oct 1982. Contract W-140S-ENG-48. \%p. (CONF-821011-11). NTIS, PC A02/MF A01. Order Number DE83001540.

From IEEE nuclear science symposium; Washington, DC, USA (20 Oct 1982).

Reliable instrumentation will be needed to monitor the performance of future high-level waste repository sites. A study has been made to assess instrument reliability at Department of Energy (DOE) waste repository related experiments. Though the study covers a wide variety of instrumentation, this paper concentrates on experiences with geotechnical instrumentation in hostile repository-type environments. Manufacturers have made some changes to improve the reliability of instruments for repositories. This paper reviews the failure modes, rates, and mechanisms, along with manufacturer modifications and recommendations for additional improvements to enhance instrument performance. 4 tables.

1018 (UCRL-88192) Design of a nuclear-waste package for emplacement in tuff. O'Neal, W.C.; Rothman, A.J.; Gregg, D.W.; Hockman, J.N.; Revelli, M.A.; Russell, E.W.; Schornhorst, J.R. (Lawrence Livermore National Lab., CA (USA); Westinghouse Electric Corp., Pittsburgh, PA (USA). Advanced Energy Systems Div.). 1 Feb 1983. Contract W-7405-ENG-48. 20p. (CONF-830205-8). NTIS, PC A02/MF A01. Order Number DE83006428. Feb 1983).

From Waste management conference; Tucson, AZ, USA (27

Portions are illegible in microfiche products.

Design, modeling, and testing activities are under way at LLNL in the development of high level nuclear waste package designs. We discuss the geological characteristics affecting design, the 10CFR60 design requirements, conceptual designs, metals for containment barriers, economic analysis, thermal modeling, and performance modeling.

1019 (UCRL-88648) Borehole-to-borehole geophysical methods applied to investigations of high level waste repository sites. Ramirez, A.L. (Lawrence Livermore National Lab., CA (USA)). 1983. Contract W-7405-ENG-48. 6p. (CONF-8302054). NTIS, PC A02/MF A01. Order Number DE83006410.

From Waste management conference; Tucson, AZ, USA (27 Feb 1983).

Portions are illegible in microfiche products.

This discussion focuses on the use of borehole to borehole geophysical measurements to detect geological discontinuities in High Level Waste (HLW) repository sites. The need for these techniques arises from: (a) the requirement that a HLW repository's characteristics and projected performance be known with a high degree of confidence: and (b) the inadequacy of other geophysical methods in mapping fractures. Probing configurations which can be used to characterize HLW sites are described. Results from experiments in which these techniques were applied to problems similar to those expected at repository sites are briefly discussed. The use of a procedure designed to reduce uncertainty associated with all geophysical exploration techniques is proposed; key components of the procedure are defined.

1020 (UCRL-89404) Selection of barrier metals for a waste package in tuff. Russell, E.W.; McCright, R.D.; O'Neal, W.C. (Lawrence Livermore National Lab., CA (USA)). Sep 1983. Contract W-7405-ENG-48. 11p. (CONF-83i174-3). NTIS, PC A02/MF A01. Order Number DE83018182.

From Materials Research Society annual meeting; Boston, MA, USA (14 Nov 1983)

The Nevada Nuclear Waste Storage Investigation (NNWSI) project under the Civilian Radioactive Waste Management Program is planning a repository at Yucca Mountain at the Nevada Test Site for isolation of high-level nuclear waste. LLNL is developing designs for an engineered barrier system containing several barriers such as the waste form, a canister and/or an overpack, packing, and near field host rock. The selection of metal containment barriers is addressed. 13 references.

1021 (UCRL-89656) Status report on dissolution model development. Jackson, D.D. (Lawrence Livermore National Lab., CA (USA)). Jul 1983. Contract W-7405-ENG-48. 16p. (CONF-83061 18-1). NTIS, PC A02/MF A01. Order Number DE83016194.

From 4. semiannual workshop on the leaching mechanisms of defense high-level waste forms; State College, PA, USA (20 Jun 1983).

The compuler prograin PROTOCOL nudels the dlssulutun reactions of chemical species in water. It is being developed particularly to study the dissolution of proposed nuclear waste forms and related phases. Experimentally derived leaching rate functions are coupled to thermochemical equilibrium calculations and water flow rates. The program has been developed over a period of years. This report describes improvements that have been done in the past year

1022 (USGS-OFR-82-409) Three-dimensional modeling of the Nevada Test Site and vicinity from teleseismic p-wave residuals. Monfort, M.E.; Evans, J.R. (Geological Survey, Denver, CO (USA)). 1982. Contract AI08-78ET44802. 71 p. NTIS, PC A04/MF A01. Order Number DE83006666.

Portions are illegible in microfiche products.

A teleseismic P-wave travel-time residual study is described which reveals the regional compressional-velocity structure of southern Nevada and neighboring parts of California to a depth of $280 \mathrm{~km}$. During 1980, 98 teleseismic events were recorded at 53 sites. P-wave residuals were calculated relative to a network-wide average residual for each event and are displayed on maps of the stations for each of four event-azimuth quadrants. Fluctuations in these map-patterns of residuals with approach azimuth combined with results of linear, threedimensional inversions of some $\mathbf{2 8 8 7}$ residuals indicate the following characteristics of the velocity structure of the southern Nevada region: (1) a low-velocity body exists in the upper crust $50 \mathrm{~km}$ northeast of Beatty, Nevada, near the Miocene Timber Mountain-Silent Canyon caldera complex. Another highly localized low-velocity annmaly occurs near the southwest corner of the Nevada Test Site (NTS). These two anomalies seem to be part of a low-velocity trough extending from Death Valley, California, to about $50 \mathrm{~km}$ north of NTS; (2) there is a highvelocity body in the mantle between 81 and $131 \mathrm{~km}$ deep centered about $10 \mathrm{~km}$ north of the edge of the Timber Mountain caldera; (3) a broad low-velocity body is delineated between 81 and $131 \mathrm{~km}$ deep centered about $30 \mathrm{~km}$ north of Las Vegas: (4) there is a monotonic increase in travel-time delays from west to east across the region, probably indicating an eastward decrease in velocity, and lower than average velocities in southeastern Nevada below $31 \mathrm{~km}$; and (5) considerable complexity in three-dimensional velocity structure exists in this part of the southern Great Basin. Inversions of teleseismic P-wave travel-time residuals were also performed on data from 12 seismometers in the immediate vicinity of the NTS to make good use of the closer station spacing in that area.

1023 (USGS-OFR-82-466) Electrical studies at the proposed Wahmonie and Calico Hills nuclear waste sites, Nevada Test Site, Nye Co., Nevada. Hoover, D.B.; Chornack, M.P.; Nervick, K.H.; Broker, M.M. (Geological Survey, Reston, VA (USA)). 1982. Contract AI08-78ET44802. 95p. NTIS, PC A05/MF A01. Order Number DE82019308.

Portions of document are illegible.

These two sites in the southwest quadrant of NTS were investigated as potential repositories for high-levei nuclear waste. The 
emplacement medium at both sites was to be an inferred intrusive body at shallow depth; the inference of the presence of the body was based on aeromagnetic and regional gravity data. This report summarizes results of Schlumberger VES, induced polarization dipole-dipole traverses and magnetotelluric soundings made in the vicinity of the sites in order to characterize the geoelectric section.

1024 (USGS-OFR-82-701) Preliminary results of gravity investigations at Yucca Mountain and vicinity, Southern Nye County, Nevada. Snyder, D.B.; Carr, W.J. (Geological Survey, Menlo Park, CA (USA)). 1982. Contract AI08-78ET44802. 56p. NTIS, PC A04/MF A01. Order Number DE83001725.

Exploration for a high-level-nuclear-waste-repository site in the Yucca Mountain area, Nevada, resulted in the addition of 423 new gravity stations during the past 2 years to the 934 existing stations to form the data base of this study. About 100 surface-rock samples, three borehole gamma-gamma logs, and one borehole gravity study provide excellent density control. A linear increase in density of $0.26 \mathrm{~g} / \mathrm{cm}^{0}$ per $\mathrm{km}$ is indicated in the tuff sequences makes the density contrast across the basal contact of the tuff the only strong source of gravity fluctuations. Isostatic and $2.0 \mathrm{~g} / \mathrm{cm}^{0}$ Bouguer corrections were applied to the observed gravity values to remove deep-crust-related regional gradients and topographic effects, respectively. The resulting residual-gravity plot shows significant gravity anomalies that correlate closely with the structures inferred from drill-hole and surface geologic studies. Gravity highs over the three Paleozoic rock outcrops within the study area - Bare Mountain, the Calico Hills, and the Striped Hills - served as reference points for the gravity models. At least $3000 \mathrm{~m}$ of tuff fills a large steepsided depression in the prevolcanic rocks beneath Yucca Mountain and Crater Flat. The gravity low and thick tuff section probably lie within a large collapse area comprising the Crater Flat-Timber MountainSilent Canyon caldera complexes. Gravity lows in Crater Flat itself are thought to coincide with the source areas of the Prow Pass Member, the Bullfrog Member, and the unnamed member of the Crater Flat Tuff. Southward extension of the broad gravity low associated with Crater Flat into the Amargosa Desert is evidence for sector graben-type collapse scgments related to the Timber Mountain caldera and superimposed on the other structures within Crater Flat. 13 figures, 4 tables.

1025 (USGS-OFR-82-931) Preliminary appraisal of gravity and magnetic data at Syncline Ridge, Western Yucca Flat, Nevada Test Site, Nye County, Nevada. Ponce, D.A.; Hanna, W.F. (Geological Survey, Menlo Park, CA (USA)). 1982. Contract AI08-78ET44802. 21 p. NTIS, PC A03/MF A01. Order Number DE83001806.

A gravity and magnetic study of the Syncline Ridge area was conducted as part of an investigation of argillite rocks of the Eleana Formation under consideration as a medium for the possible storage of highlevel radioactive waste. Bouguer gravity anomaly, low-level aeromagnetic anomaly, density, and magnetization data collectively indicate the following, relative to the Eleana Formation, the principal target of the investigation: (1) in an area extending northwestward from Mine Mountain, through Syncline Ridge, to the Eleana Range, the Eleana Formation, where not exposed, occurs at depths of less than $\sim 200 \mathrm{~m}$, except for a small region of exposed older Paleozoic rocks; (2) in the region of shallowly buried Eleana Formation, occurrences of volcanic rock cover are delineated by low-level aeromagnetic anomaly data, which also discriminate normally polarized from reversely polarized tuff units; and (3) selective detection of high-quartz argillite relative to low-quartz argillite using surface gravity data is not feasible if the high-quartz and lowquartz varieties are intimately interbedded, as observed in boreholes. 4 figures, 2 tables.

1026 (USGS-OFR-82-1041) Principal facts of gravity stations with gravity and magnetic profiles from the southwest Nevada Test Site, Nye County, Nevada, as of January 1982. Jansma, P.E.; Snyder, D.B.; Ponce, D.A. (Geological Survey, Menlo Park, CA (USA)). 1983. Contract AI08-78ET44802. 69p. NTIS, PC A04/MF A01. Order Number DE83010300.

Portions are illegible in microfiche products. Original copy available until stock is exhausted.

Three gravity profiles and principal facts of $\mathbf{2 6 0 4}$ gravity stations in the southwest quadrant of the Nevada Test Site are documented in this data report. The residual gravity profiles show the gravity measurements and the smoothed curves derived from these points that were used in geophysical interpretations. The principal facts include station label, latitude, longitude, elevation, observed gravity value, and terrain correction for each station as well as the derived complete Bouguer and isostatic anomalies, reduced at $2.67 \mathrm{~g} / \mathrm{cm}^{0}$. Accuracy codes, where available, further document the data.

1027 (USGS-OFR-82-1042) E-field ratio telluric traverses near Fortymile Wash, Nevada Test Site, Nevada. Hoover, D.B.; Chornack, M.P.; Broker, M.M. (Geological Survey, Denver, CO (USA); Fenix and Scisson, Inc., Mercury, NV (USA)). 1982. Contract AI08-78ET44802. 15p. NTIS, PC A02/MF A01. Order Number DE83006230.

Portions are illegible in microfiche products. Original copy available until stock is exhausted.

E-Field ratio telluric traverses have identified abrupt changes in resistivity at several places along the Fortymile Wash drainage. These resistivity changes have been interpreted to result from Basin and Range normal faulting along the wash. East of the Yucca Moutnain site four north-south trending faults have been identified. The central block between these faults comprises the graben through which Fortymile Wash flows. Further south the graben appears to be much broader and only the eastern boundary has been defined by telluric data near Lathrop Wells. Extrapolation of trends from either the northern two lines or the southern set does not give any correspondence. This suggests that cross structures may offset the Basin and Range faults between line 1 and line L-N. These lines are separated by $12 \mathrm{~km}$ ( 7.5 miles). In order to define the electrical structure in the intervening region additional work would be required.

1028 (USGS-OFR-82-1043) Resistivity sounding investigation by the Schlumberger method in the Yucca Mountain and Jackass Flats area, Nevada Test Site, Nevada. Senterfit, R.M.; Hoover, D.B.; Chornack, M. (Geological Survey, Denver, CÖ (USA); Fenix and Scisson, Inc., Mercury, NV (USA)). 1982. Contract AI08-78ET44802. 41p. NTIS, PC A03/MF A01. Order Number DE83006231.

Portions are illegible in microfiche products. Original copy available until stock is exhausted.

A Schlumberger resistivity survey was made in the west-central sector of the Nevada Test Site as part of an extensive program to assess and identify potential repositories for high-level nuclear waste. The survey area is located within the Topopah Spring 15-minute quadrangle. The intent of the survey was to determine the geoelectric characteristics of the area and to relate them to the thicknesses and horizontal continuity of lithologic units in the Yucca Mountain and Jackass Flats area, and to locate faulting within the survey area. A total of 29 soundings is included. The interpreted results of some of the 29 Schlumberger resistivity soundings indicate some lateral discontinuities which appear to be caused by vertical displacement due to faulting. Because the lithologic section in this survey area is composed primarily of ash-flow tuffs beneath alluvium, many of the lateral resistivity variations are probably caused by differences in amounts of clay and other fine-grained materials within the alluvium, variations of lithology within the volcanic rocks, and the effects of fracturing within the rock types.

1029 (USGS-OFR-82-1044) Study of surface and subsurface ground motions at Calico Hills, Nevada Test Site. King, K.W. (Geological Survey, Reston, VA (USA)). 1982. Contract A108-78ET44802. 22p. NTIS, PC A02/MF A01. Order Number DE83005245.

Portions are illegible in microfiche products. Original copy available until stock is exhausted.

A study of earthquake ground motions recorded at depth in a drill hole and at the ground surface has derived the surface to subsurface transfer functions such as might be expected at a potential nuclear waste repository in a similar setting. The site under investigation has small seismic velocity contrasts in the layers of rock between the surface and the subsurface seismometer location. The subsurface seismic motions were similar in spectral characteristics to the surface motions and were lower in amplitude across the recorded band-width by a factor of 1.5 .

1030 (USGS-OFR-83-141) Geohydrologic and drill-hole data for test well USW H-1, adjacent to Nevada Test Site, Nye County, Nevada. Rush, F.E.; Thordarson, W.; Bruckheimer, L. (Geological Survey, Reston, VA (USA)). 1983. Contract AI08- 
78ET44802. 43p. NTIS, PC A03/MF A01. Order Number DE83013550.

Portions are illegible in microfiche products.

This report presents data collected to determine the hydraulic characteristics of rocks penetrated in test well USW H-1. The well is one of a series of test wells drilled in and near the southwestern part of the Nevada Test Site, Nye County, Nevada, in a program conducted on behalf of the US Department of Energy. These investigations are part of the Nevada Nuclear Waste Storage Investigations to identify suitable sites for storage of high-level radioactive wastes. Data on drilling operations, lithology, borehole geophysics, hydrologic monitoring, core analysis, ground-water chemistry and pumping and injection tests for well USW $\mathrm{H}-1$ are in this report.

1031 (WIPP-DOE-154) Waste Isolation Pilot Planit. Project progress report, December 1, 1982-February 28, 1983. (USDOE Albuquerque Operations Office, NM. Waste Isolation Pilot Plant Project Office). 1983. 16p. NTIS, PC A02/MF A01. Order Number DE83009949.

The Waste Isolation Pilot Plant (WIPP) is a defense activity of the Department of Ënergy. Its express purpose is to provide a research and development facility to demonstrate the safe disposal of radioactive waste resulting from the defense activities and programs of the United States exempted from regulation by the Nuclear Regulatory Commission. Site construction progress, design and engineering progress, technical and institutional activities and scientific and experimental studies are discussed briefly.

1032 The movement of a redox front downstream from a repository for nuclear waste. Neretnieks, I. (Royal Institute of Technology (KTH) Department of Chemical Engineering, S-100 44 Stockholm). Nuclear Technology; 62: No. 1, 110 115(Jul 1983).

In a repository for high-level nuclear waste in bedrock that may carry water, the waste will eventually come in contact with water. Radionuclides will dissolve in the water and migrate away from the repository. In crystalline rock in Sweden, the waters at repository depths are reducing. Many of the important radionuclides, e.g., neptunium, uranium, and technetium, have very low solubilities under these conditions (parts per billion levels). The solubility will considerably limit the transport of these species. If by some means the conditions were to change from reducing to oxidizing, the solubility of these species would increase very much, in some cases by 4 to 6 orders of magnitude. Under such circumstances, these nuclides would escape much faster. The authors have investigated one possible way in which the redox conditions might change, i.e., spent fuel has a considerable alpha activity, which may radiolyze water and produce oxidizing agents such as hydrogen peroxide. The hydrogen peroxide will make the water oxidizing. The compensating factor is ferrous iron in the bedrock. In the investigation of the interaction of these two species, a conceptional and a mathematical model is developed describing the movement of the redox front downstream of a repository. A sample calculation based on minimum meaşured ferrous iron contents in the bedrock and computed (conservatively on the high side) hydrogen peroxide production shows that the redox front could move several tens of metres downstream in the million-year perspective. The rate of radiolysis would decrease considerably if the spent fuel is not wetted to the high degree assumed in the calculations. The results in the sample calculation should be seen as maximum values for the type of repository considered.

1033 Nuclear waste legislation: issues and impacts. Stein, R. (U.S. Department of Energy, Washington, DC 20545), Transactions of the American Nuclear Society; 44: 58(Jun 1983). (CONF-830609-w).

From ANS annual meeting; Detroit, MI, USA (12 Jun 1983). GOVERNMENT POLICIES; HIGH-LEVEL RADIOACTIVE WASTES; IMPLEMENTATION; LEGISLATION; RADIOACTIVE WASTE DISPOSAL; RADIOACTIVE WASTE FACILITIES; RADIOACTIVE WASTE MANAGEMENT; RADIOACTIVE WASTE STORAGE; SITE SELECTION; US DOE

1034 Groundwater flow modeling in support of salt site evaluations. Brandstetter, A.; Andrews, R.W. (Battelle, Office of NWTS Integration, 505 King Avenue, Columbus, OH
43201). Transactions of the American Nuclear Society; ; 58-59(Jun 1983). (CONF-830609-).

From ANS annual meeting; Detroit, MI, USA (12 Jun 198:

FLOW MODELS; GROUND WATER; HIGH-LEVEL RADIOACTIVE WASTES; HYDRAULIC CONDUCTIVITY; HYDROLOGY; RADIOACTIVE WASTE DISPOSAL; RADIONUCLIDE MIGRATION; S CODES; SALT DEPOSITS; SITE SURVEYS; STRATIGRAPHY

1035 Results of the area-to-location phase of repository siting in Nevada. Sinnock, S. (Sandia National Laboratories, P.O. Box 5800, Albuquerque, NM 87185). Transactions of the American Nuclear Society; 44: 60-61(Jun 1983). (CONF830609-).

From ANS annual meeting; Detroit, MI, USA (12 Jun 1983). COMPARATIVE EVALUATIONS; HIGH-LEVEL RADIOACTIVE WASTES; MAPS; NEVADA TEST SITE; RADIOACTIVE WASTE DISPOSAL; REGIONAL ANALYSIS; ROCKS; SATURATION; SITE SURVEYS; WEIGIITING FUNCTIONS; SITE SELECTION

1036 Present status of the Gorleben site investigations. Oesterle, F.P. (Physikalisch-Technische Bundesanstalt, Bundesallee 100, Postfach 3345, D-3300 Braunschweig). Transactions of the American Nucleur Suciety; 44: 61(Jun 1983). (CONF. 830609-).

From ANS annual meeting: Detroit, MI, USA (12 Jun 1983).

ENGINEERING GEOLOGY; GEOMORPHOLOGY; GEOPHYSICAL SURVEYS; HIGH-LEVEL RADIOACTIVE WASTES; MINING ENGINEERING; RADIOACTIVE WASTE DISPOSAL; FEDERAL REPUBLIC OF GERMANY; SITE SELECTION; SALT DEPOSITS; SITE SURVEYS; STRATIGRAPHY; WELL DRILLING; WELL LOGGING

1037 Development of a comparative analysis of potential repository salt sites. Pardue, W.M.; Duffy, M.A.; Pobereskin, M. (Battelle, Office of NWTS Integration, 505 King Avenue, Columbus, OH 43201). Transactions of the American Nuclear Society: 44: 61-62(Jun 1983). (CONF-830609-).

From ANS annual meeting; Detroit, MI, USA (12 Jun 1983). COMPARATIVE EVALUATIONS; SITE SELECTION; COST BENEFIT ANALYSIS; ENVIRONMENTAL IMPACTS; HIGH-LEVEL RADIOACTIVE WASTES; MATHFMATICAL MODELS; RADIOACTIVE WASTE DISPOSAL; RECOMMENDATIONS; REGIONAI. ANALYSIS: SALT DEPOSITS; SITE. SURVEYS

1038 Disposal of high-activity nuclear wastes. Hamilton, E.I. (Institute for Marine Environmental Research, Plymouth (UK)). Marine Pollution Bulletin; 14: No. 6, 238-239(Jun 1983).

(Environmental effects of ocean dumping).

A discussion is presented on the deep sea ocean disposal for highactivity nuclear wastes. The following topics are covered: effect of ionizing radiation on marine ecosystems; pathways by which radionuclides are transferred to man from the marine environment; information about releases of radioactivity to the sea; radiological protection; storage and disposal of radioactive wastes and information needs.

1039 Surface analysis features of Synroc B and C. Myhra, S.; Bishop, H.E.; Riviere, J.C. (UKÄEA Atomic Energy Research Establishment, Harwell. Materials Development Div.). Surface Technology; 19: No. 2, 145-160(Jun 1983).

Surface analysis features of Synroc B and C, proposed high level nuclear waste forms without (B), and with (C), incorporated simulated waste, were studied. It was shown that all species of the solid matrix as well as some important waste species can be detected reliably with X-ray photoelectron spectroscopy (XPS) and Auger electron spectroscopy. Fully quantified depth profiles for all major species were obtained by XPS analysis and ion beam sputtering; the inferred abundances are good agreement with the nominal stoichiometry. Information about 1 chemical states of all major species and some waste species was a inferred from details of XPS pcak positions and envelopes; there is good correlation, in general, with the valence states predicted for the phase assemblage. However, there were non-tetravalent contributions to the Ti 2 p spectral envelope, some anomalies related to the $\mathrm{Ca} 2 \mathrm{p}$ envelope 
and molybdenum was found mainly as a hexavalent species. The effect of ion bombardment (for profiling purposes) was to reduce most species to states of lower valency. Hollandite and zirconolite phase regions were particularly susceptible to ion beam modification. The Ti $2 p$ envelope was most severely affected, but molybdenum and iron XPS peaks also exhibited shifts with increasing ion dose.

1040 Methodology for a consequence analysis of a nuclear waste disposal facility. Buchheim, B.K.; Bunschi, H.; Fitzpatrick, F.J.; Hoop, F.J. (Electrowatt Engineering Services Ltd, P. O. Box 8022, Zurich). Nuclear Technology; 61: No. 3, 444449(Jun 1983).

One of the methods currently under consideration for the disposal of radioactive wastes is emplacement in a repository within deep, continental formations. A licensing requirement for such a facility will presumably be some form of safety analysis report on the radiological impact of the facility. A methodology has been developed to make an assessment of the radiological consequences both for normal operation and for possible accident situations for a specific repository design in a salt dome at Gorlehen in Germany, a repository which has been designed to accommodate all categories of radioactive waste (low, intermediate, and high level). Radionuclide release scenarios were derived from a systematic analysis of the facility design and proposed operational procedure. Where necessary, simple numerical models for such topics as direct radiation exposure from waste containers, release and transport of radionuclides, radiolysis, heat transfer, creep, and impact were developed to give a first estimate of the radiological consequences due to radionuclide releases. The results enabled sensitive areas of the design and the operational procedure to be identified, and thus possible modifications and/or precautionary measures could be recommended. The results also gave an indication of those parts of the analysis requiring morc sophisticated and specialized modeling techniques to yield a more detailed radiological consequence analysis in preparation for a safety analysis report.

1041 Projected occupational exposures during WIPP operations. Baer, W. (Westinghouse Electric Corp., Advanced Energy Systems, Waste Isolation Pilot Plant, P.O. Box 40039, Albuquerque, NM 87196). Transactions of the American Nuclear Society; 44: 78(Jun 1983). (CONF-830609-).

From ANS annual meeting; Detroit, MI, USA (12 Jun 1983).

ALARA; HIGH-LEVEL RADIOACTIVE WASTES; MATERIALS HANDLING; PILOT PLANTS; RADIATION DOSES; RADIATION PROTECTION; RADIOACTIVE WASTE FACILITIES; REGULATIONS; RISK ASSESSMENT; US DOE; PERSONNEL; RADIOACTIVE WASTE DISPOSAL

1042 High-level waste disposal. Vieth, D.L. (U.S. Department of Energy, Nevada Operations Office, P.O. Box 14110, Las Vegas, NV 89114). Transactions of the American Nuclear Society; 44: 101-102(Jun 1983). (CONF-830609-).

From ANS annual meeting; Detroit, MI, USA (12 Jun 1983).

ENVIRONMENTAL EFFECTS; GEOCHEMICAL SURVEYS; HIGH-LEVEL RADIOACTIVE WASTES; RADIATION HAZARDS; RADIOACTIVE WASTE DISPOSAL; RADIOACTIVE WASTE MANAGEMENT; SITE SURVEYS; UNDERGROUND DISPOSAL; GEOLOGIC DEPOSITS

1043 Waste packages for high-level waste repositories. Basham, S.J. Jr. (Battelle, Office of NWTS Integration, 505 King Avenue, Columbus, $\mathrm{OH}$ 43201). Transactions of the American Nuclear Society; 44: 102-103(Jun 1983). (CONF830609-).

From ANS annual meeting; Detroit, MI, USA (12 Jun 1983). CONTAINERS; CONTAINMENT; DESIGN; ENVIRONMENTAL EFFECTS; HIGH-LEVEL RADIOACTIVE WASTES; PACKAGING; RADIO'ACTIVE WASTE DISPOSAL; RADIOACTIVE WASTE MANAGEMENT; SALT DEPOSITS; UNDERGROUND DISPOSAL

1044 Leach testing of INEL waste forms in a gamma field. Schuman, R.P. (EG \& G Idaho Inc., P.O. Box 1625, Idaho Falls, ID 83415). Transactions of the American Nuclear Society; 44: 119-120(Jun 1983). (CONF-830609-).

From ANS annual meeting; Detroit, MI, USA (12 Jun 1983).
BASALT; BOROSILICATE GLASS; CALCINED WASTES; CERAMICS; GAMMA RADIATION; HIGH-LEVEL RADIOACTIVE WASTES; LEACHING; RADIOACTIVE WASTE MANAGEMENT; RADIOACTIVE WASTE DISPOSAL; SALT DEPOSITS

1045 Development of CT techniques for integrity evaluation of vitrified solids. Nagaki, H.; Murata, T.; Nakamura, Y.; Sano, A.; Uchida, K. (Power Reactor and Nuclear Fuel Development Corporation, 9-13, l-chome, Akasaka, Minato-ku, Tokyo). Transactions of the American Nuclear Society: 44: 120(Jun 1983). (CONF-830609-).

From ANS annual meeting; Detroit, MI, USA (12 Jun 1983).

COMPUTERIZED TOMOGRAPHY; GAMMA RADIATION; GE SEMICONDUCTOR DETECTORS; HIGH-LEVEL RADIOACTIVE WASTES; IMPLEMENTATION; NONDESTRUCTIVE TESTING; RADIATION DETECTION; RADIOACTIVE WASTE MANAGEMENT; SIMULATION; VITRIFICATION; GLASS; SOLIDIFICATION; RADIOACTIVE WASTE DISPOSAL

1046 Peak high-level waste package temperature versus annular air gap width. Burns, B.S.; Christensen, R.N. (The:Ohio State University, Columbus, $\mathrm{OH} 43210$ ). Transactions of the American Nuclear Society; 44: 121-122(Jun 1983). (CONF830609-).

From ANS annual meeting; Detroit, MI, USA (12 Jun 1983).

AIR; COMPUTER CODES; CREEP; HEAT TRANSFER; HIGH-LEVEL RADIOACTIVE WASTES; ONE-DIMENSIONAL CALCULATIONS; RADIOACTIVE WASTE DISPOSAL; SALT DEPOSITS; TEMPERATURE GRADIENTS; THERMAL INSULATION; WASTE-ROCK INTERACTIONS

1047 Pacing and logistics of high-level waste disposal. Kohn, H.W. (State of Ohio Power Siting Board, 580 S. High Street, Suite 300, Columbus, OH 43215). Transactions of the American Nuclear Society; 44: 122(Jun 1983). (CONF830609-).

From ANS annual meeting; Detroit, MI, USA (12 Jun 1983). HIGH-LEVEL RADIOACTIVE WASTES; INVENTORIES; ORGANIZING; PACKAGING; PLANNING; RADIOACTIVE WASTE DISPOSAL; RADIOACTIVE WASTE MANAGEMENT RADIOACTIVE WASTE PROCESSING; TECHNOLOGY ASSESSMENT; TIME MEASUREMENT

1048 Actinide transmutation with a UF 6 reactor. Wan, P.T.; Clement, J.D. (McMaster University, Hamilton, Ontario L8S 4M1). Transactions of the American Nuclear Society; 44: 123-124(Jun 1983). (CONF-830609-).

From ANS annual meeting; Detroit, MI, USA (12 Jun 1983),

ACTINIDES; BUILDUP; HIGH-LEVEL RADIOACTIVE WASTES; MULTIGROUP THEORY; NEUTRON LEAKAGE; PARTITION; RADIOACTIVE WASTE DISPOSAL; RISK ASSESSMENT; TRANSMUTATION; UNDERGROUND DISPOSAL; URANIUM HEXAFLUORIDE

1049 "Project Crystal" for ultimate storage of highly radioactive waste. Technica (Basel); 32: No. 7, 499-503(13 Apr 1983). (In German).

NAGRA (The National Association for storage of radioactive waste) in Baden has launched in North Switzerland an extensive geological research program. The current research program, under the title of "Project Crystal", aims at providing the scientific knowledge which is required for the assessment of the suitability of the crystalline sub-soil of North Switzerland for the ultimate storage of highly radioactive waste. Safety and feasibility of such ultimate storage are in the forefront of preoccupations. Scientific institutes of France, Germany, USA and Canada are cooperating more particularly on boring research and laboratory analyses. Technical data are given on the USA and German installations used.

1050 Disposal of high-activity nuclear wastes in the oceans. Baxter, M.S. (Glasgow Univ. (UK). Dept. of Chemistry). Marine Pollution Bulletin; 14: No. 4, 126-132(Apr 1983).

The potential for marine pollution inherent in a deep ocean disposal policy for high-activity nuclear wastes is reviewed. In discussing 
this disposal problem high-activity waste and natural marine inventories of radioactivity are compared and the hypothetical programme of highactivity waste disposal in the deep ocean is contrasted to the past and present practice of coastal marine discharges of low-level nuclear effluents.

$1051 \quad$ Nuclear Waste Policy Act of 1982; proposed general guidelines for recommendation of sites for nuclear waste repositories. ermany); 48: No. 26, 5670-5682(7 Feb 1983).

In accordance with the requirements of the Nuclear Waste Policy Act of 1982 (Pub. L. 97-425), hereinafter referred to as the Act, the Department of Energy is proposing general guidelines for the recommendation of sites for repositories for disposal of high-level radioactive waste and spent nuclear fuel in geologic formations. These guidelines are based on the criteria that the Department has used in its National Waste Terminal Storage program, the criteria proposed by the Nuclear Regulatory Commission (NRC), and the environmental standards proposed by the Environmental Protection Agency. These guidelines establish the performance requirements for a geologic repository system, specify how the Department will implement its site-selection program, and define the technical qualifications that candidate sites must meet in the various steps of the site-selection process mandated by the Act. After considering comments from the public; consulting with the Council on Environmental Quality, the Administrator of the Environmental Protection Agency, the Director of the Geological Survey, and interested Governors; and obtaining NRC concurrence, the Department will issue these guidelines in final form as a new Part 960 to Title 10 of the Code of Federal Regulations (10 CFR Part 960).

1052 (ORNL-tr-4980) Glasses for the solidification of high-level radioactive waste: their behavior in the presence of water. Grauer, R. (Eidgenoessisches Inst. fuer Reaktorforschung, Wuerenlingen (Switzerland); Oak Ridge National Lab., TN (USA)). Feb 1983. Contract W-7405-ENG-26. C; EIR 477. 141 p. NTIS, PC A07/MF A01. Order Number DE83013402.

Because of their amorphous structure, glasses are particularly suitable for the solidification of the mixture of high-level radioactive wastes resulting from reactor fuel reprocessing: they are not sensitive to variations in the compositions of waste oxides and are resistant to the damaging effects of radiation. The borosilicate glasses used for this purpose have been investigated for about 25 years, and waste vitrification techniques have been tested on a commercial scale. In view of possible accidents in a final waste repository, the chemical resistance of this type of glasses to attack by groundwaters is of special interest. The present report deals with the corrosion behaviour of glasses and discusses the most significant controlling parameters. The dissolution rates needed for safety analysis must be determined in relatively short-term experiments. Since the results can depend strongly on the type of test procedures used, a critical assessment of these techniques is necessary. Experimental results are illustrated by means of selected examples. Particular emphasis is placed upon the effects of increased temperatures and of nuclear radiation. The models which have been proposed for the estimation of the long-term behavior of vitrified waste are not yet fully complete and require improvement. Furthermore, the actual dissolution tates which are used in such models should be revised: to be desired are values which take into account the actual environmental conditions at the storage site. It should be noted, however, that even with current conservative input data on corrosion rates, a lifetime on the order of $10^{5}$ years can be expected for the glass blocks to be deposited. The report concludes with recommendations fo further investigations.

1053 Radiation effects in SYNROC-D. Konynfnburg, R.A.; Guinan, M.W. (University of California, Lawrence Livermore National Laboratory, P.O. Box 808, Livermore, California 94550). Nuclear Technology; 60: No. 2, 206-217(Feb 1983).

SYNROC-D is a ceramic material proposed as a waste form for defense high-level nuclear waste. During the first million years of storage, it would be subjected to about $8 \times 10^{24}$ alpha decay $/ \mathrm{m}^{0}$ of SYNROC-D and a total ionization dose of about $1 \times 10^{11}$ rad. There are several methods of simulating the resulting radiation effects, including external bombardment using gamma rays, electrons, light ions, heavy ions, or neutrons, and internal bombardment using short half-life actinide doping to bring about internal alpha decay, or doping with uranium, boron, or lithium, coupled with neutron irradiation, to induce internal fissions or (n. $\alpha$ ) reactions. The results show that dose rate effects are not important in determining the swelling and metamictization of the perovskite and zirconolite phases over a wide range of dose rate for lo temperatures and doses of 2 to $3 \times 10^{25}$ alpha $/ \mathrm{m}^{8}$ of each phase, corre sponding to expected million year doses in SYNROC-D. Based on thi observation and a consideration of the basic processes involved, the authors argue that the million-year radiation damage expected in SYNROC-D can be adequately simulated in a few months by doping samples with ${ }^{208} \mathrm{Pu}$, and simultaneously carrying out external gammaray bombardment. The ${ }^{208} \mathrm{Pu}$ will undergo alpha decay, producing the same type of damage in the same phases as would long-term actinide decay in actual waste. The gamma irradiation will simulate the ionization dose, which would result primarily from fission product decay in actual waste. SYNROC-D samples have been fabricated and characterized using cerium and uranium, respectively, as stand-ins for plutonium. These samples show good properties, and ${ }^{269} \mathrm{Pu}$ doping experiments are expected to take place soon to determine if plutonium will dissolve properly in SYNROC-D.

1054 Radwaste paradox. Carter, L.J. Science (Washington, D.C.): 219: 33-36(7 Jan 1983).

The Paradox Basin is one of the places where the US Department of Energy is looking for a site for a deep-mined repository for high-level radioactive waste. This seems appropriately symbolic because the geologic disposal problem has increasingly taken on the aspect of a political and technical conundrum, replete with real or seeming contradictions and paradoxes. A central paradox is that, while the concept of sequestering long-lived wastes in mined repositories is attractive intuitively, the very efforts made to confirm the suitability of particular rock formations give rise to further uncertainties. The new law contemplates repository construction will start as early as 1989 . Experience so far at the several sites suggests that the technical and political questions tend to proliferate rather than diminish as more becomes known about the geology and hydrology. The following sites were discussed: the Hanford basalt; the Nevada tuff; and salt beds and salt domes (Utah, Texas, Mississippi). (DP)

1056 Dumping nuclear waste into the sea: international control and the role of science and law. Boehmer-Christiansen, S.A. (Winzererster, Munich, Germany). Marine Policy; 7: No. 1, 25-36(Jan 1983).

A case study of international organization is presented, which illustrates the interdependence of political and scientific factors in the maintaining of decision-making powers by major nuclear states over the allocation of the assimilative capacity of the deep ocean and seabed for long-lived radionuclides. The controversy over the use of the seabed and ocean for the disposal of high-level radioactive waste is analyzed with reference to its scientific basis in relation to the national and international legal framework and the political and national-interest considerations of the various participants in the controversy. 85 references.

1056 Developments, recommendations and applications of tests for evaluating the chemical stability of high-level radioactive solid waste forms, Solomah, A.G. (North Carolina State Univ., Raleigh (USA). School of Engineering). tvennoi Akademii: 5: No. 1, 9-16(.Jan 1983)

The chemical stability of any proposed high-level radioactive waste form, and particularly its reaction with the groundwater during long-term deep geological disposal, is an important factor in its evaluation and characterisation. The two most important issues related to this field of study are: (1) the pH behaviour of the leaching medium; (2) the fractional release of radioactivity to the surrounding environment. The problems associuted with $\mathrm{pH}$ measurement are discussed. The advantages of the use of fractional releases of the radioactive species (instead of normalised leach rate) to describe the release mechanisms involved in the chemical behaviour of the waste form during long-term deep geological disposal periods are also emphasised.

1067 Dumping nuclear waste into the sea. International control and the role of science and law. Bnehmer-Christiansen, S.A. Marine Policy; 7: No. 1, 25-36(Jan 1983).

A case study of international organization is presented, whick illustrates the interdependence of political and scientific factors in the maintaining of decision-making powers by major nuclear states over the allocation of the 'assimilative capacity' of the deep ocean and seabed for long-lived radio-nuclides. The controversy over the use of the seabed and ocean for. the disposal of high-level radinactive waste is analysed with reference to its scientific basis in relation to the national and interna- 
tional legal framework and the political and national interest considerations of the various participants in the controversy.

1058 Limiting-individual dose from an extended-source high-level waste repository model. Edwards, L.L.; Harvey, T.F. (Lawrence Livermore National Laboratory, P.O. Box 808, Livermore, California 94550). Nuclear Technology; 60: No. 1, 84 96(Jan 1983).

This paper focuses on the effects of mine design features on postclosure risk associated with a nuclear waste repository. Under conditions that enhance the effects of mine features and where the waste package allows high release rates, it was found that point-source repository models can lead to a range of 'limiting-individual' doses covering several orders of magnitude. If engineered features are included in the models, these predicted doses are substantially lower.

1059 Correlation between dynamic leach test results and geochemical observations. Barkatt, A.; Macedo, P.B.; Sousanpour, W.; Barkatt, A.; Boroomand, M.A.; Szoke, P.; Rogers, V.L. (Catholic Univ. of America, Washington, DC). Materials Research Society Symposia Proceedings; 15: 227-234(1983). (CONF-821107-Pt.1).

From 6. international symposium on the scientific basis for radioactive waste management (Materials Research Society); Boston, MA, USA (1 Nov 1982).

A test method based on partial leachant exchange at constant intervals until leachate concentrations become stabilized has been used to obtain a data-base in evaluating long-term leaching patterns. Applied to basalt and granite specimens and compared with ground-water analyses, correlations which can be used in long-term leachability extrapolations have been established. They are based on the following principles: (1) control of solubilities of major components by combined aluminosilicate species; (2) continued increase in levels of soluble species, e.g. $\mathrm{Na}^{+}$ of $\mathrm{SO}_{4}{ }^{2-}$, along with $\mathrm{Ca}^{2+}$ and $\mathrm{K}^{+}$, followed by slowing down, probably due to the presence of selectivity leachable phases; (4) initial retardation of $\mathrm{Mg}$ and $\mathrm{Fe}$ leaching followed by regrowth as sorption sites become saturated.

1060 Suitability of natural geomedia for radwaste storage. Fyfe, W.S. (Univ. of Western Ontario, London). Materials Research Society Symposia Proceedings; 15: 281-289(1983). (CONF-821107-Pt.1)

From 6. international symposium on the scientific basis for radio active waste management (Materials Research Society); Boston, MA USA (1 Nov 1982)

Selection of the best rock types for radwaste disposal will depend on their having minimal permeability, maximal flow dispersion, minimal chance of forming new wide aperture fractures, maximal ion retention, and minimal thermal and mining disturbance. While no rock is perfect, thinly bedded complex sedimentary sequences may have good properties, either as repository rocks, or as cover to a repository. Long time prediction of such favorable properties of a rock at a given site may be best modelled from studies of in situ rock properties. Fracture flow, dispersion history, and geological stability can be derived from direct observations of rocks themselves, and can provide the parameters needed for convincing demonstration of repository security.for appropriate times.

1061 Natural analogues: a way to increase confidence in predictions of long-term performance of radioactive waste disposal. Birchard, G.F.; Alexander, D.H. (Nuciear Regulatory Commission, Washington, DC). Materials Research Society Symposia Proceedings; 15: 323-329(1983). (CONF-821 107-Pt. 1).

From 6. international symposium on the scientific basis for radioactive waste management (Materials Research Society); Boston, MA, USA (1 Nov 1982).

No exact analogue to a radioactive waste disposal system exists but by studying natural analogues of the most important components or subsystems of a waste repository, confidence in long-term predictions can be increased. The US Nuclear Regulatory Commission (NRC) is supporting research on igneous intrusions into proposed repository-type host rock (basalt, tuff, crystalline hard-rock, and salt), on uranium ore body analogues, on actinide solubility as a function of natural complexing agents in closed basin lakes and on field migration of radionuclide species at low-level waste and uranium mill tailings disposal sites. The NRC is studying analogues to ensure that important parameters which might be overlooked in laboratory tests and in modeling because of problems with scaling time, space and geologic complexity can be identified and their importance bounded.

1082 Radiolysis of groundwater from HLW stored in copper canisters. Christensen, $H$. (Studsvik Energiteknik AB, Nykoeping Sweden); Bjergbakke, E. Materials Research Society Symposia Proceedings; 15: 429-436(1983). (CONF$821107-$ Pt.1).

From 6. international symposium on the scientific basis for radio active waste management (Materials Research Society); Boston, MA, USA (1 Nov 1982).

A large number of computer calculations of the radiolysis of ground water outside copper canisters have been carried out within the Swedish Nuclear Fuel Safety Project. At dose rates higher than $5 \times 10^{-2}$ $\mathrm{rad} / \mathrm{s}$ the hydrogen concentration is constant, approximately $10^{-0}$ $\mathrm{mol} / \mathrm{dm}^{0}$, and independent of time and dose rate. Therefore the rate of diffusion out of the system is constant, approximately $7 \times 10^{-4} \mathrm{~mol} /$ year. At dose rates lower than $5 \times 10^{-4} \mathrm{rad} / \mathrm{s}$ the logarithm of the hydrogen production is proportional to the logarithm of the dose rate. The hydrogen production depends on fuel type, burn-up and canister thickness only to the extent that the dose rate varies with these parameters. The hydrogen production is independent of the volume of water in which the energy is assumed to be absorbed ( 5 or $15 \mathrm{~cm}$ layers outside the surface of the canister). The hydrogen production depends on the concentration of $\mathrm{Fe}^{2+}$ ions in the water. In the basic calculations the concentration was assumed to be $5 \mathrm{ppM}$ : An increase to $50 \mathrm{ppM}$ increases the total hydro gen production after one million years outside a $1 \mathrm{~cm}$ thick canister from 360 to $1000 \mathrm{~mol}$. A decrease to $0.5 \mathrm{ppM}$ decreases the same yield to 80 mol.

1083 Modeling of waste/near field interactions for a waste repository in bedded salt: the Dynamic Network (DNET) model. Cranwell, R.M. (Sandia National Labs., Albuquerque, NM). Materials Research Society Symposia Proceedings; 15: 507-514(1983). (CONF-821107-Pt.1).

From 6. international symposium on the scientific basis for radio active waste management (Materials Research Society); Boston, MA, USA (1 Nov 1982).

The Fuel Cycle Risk Analysis Division of Sandia National Laboratories has been funded by the US Nuclear Regulatory Commission to develop a methodology for use in assessing the long-term risk from the disposal of radioactive wastes in deep geologic formations. As part of this program, the Dynamic Network (DNET) model was developed to investigate waste/near field interactions associated with the disposal of radioactive wastes in bedded salt formations. The model is a quasimulti-dimensional network model with capabilities for simulating pro cesses such as fluid flow, heat transport, salt dissolution, salt creep, and the effects of thermal expansion and subsedence on the rock units surrounding the repository. The use of DNET has been demonstrated in the analysis of a hypothetical disposal site containing a bedded salt formation as the host medium for the repository. An example of this demonstration analysis is discussed. Furthermore, the outcome of sensitivity analyses performed on the DNET model are presented.

1064 Long-term thermomechanical and thermohydrological factors controlling the optimal design of a nuclear waste repository. Wang, J.S.Y.; Mangold, D.C.; Tsang, C.F. (Lawrence Berkeley Lab., CA). Materials Research Society Symposia Proceedings; 15: 531-538(1983). (CONF-821107-Pt.1). Contract AC03-76SF00098.

From 6. international symposium on the scientific basis for radio active waste management (Materials Research Society); Boston, MA, USA (1 Nov 1982).

Surface uplift and buoyancy flow are two of the major long-term, far-field perturbations to the geologic formations around a nuclear waste repository. The allowable surface uplift has been accepted in the literature as a criterion limiting the repository waste loading density. It has also been recognized in generic modeling studies that the buoyancy distortion of the ambient groundwater flow around a repository is a major mechanism for radionuclide transport. However, the buoyancy considerations have yet to be quantified for the purpose of optimal,design of a radioactive waste repository. We suggest the possibility of using the buoyancy gradient when compared with the ambient regional (horizontal) gradient as a measurable thermohydrologic factor that controls the design of waste loading. This study indicates that the buoyancy gradient 
could in some cases become the main controlling factor. To decrease the buoyancy effects, much lower values of waste loading density should be considered in the design of the waste repository.

1065 Thermal conductivity of bentonite/quartz high-level waste package backfill. Moss, M.; Molecke, M.A. (Sandia National Labs., Albuquerque, NM). Materials Research Society Symposia Proceedings; 15: 719-726(1983). (CONF821107-Pt.1). Contract AC0476DP00789.

From 6. international symposium on the scientific basis for radioactive waste management (Materials Research Society); Boston, MA, USA (1 Nov 1982).

A mixture of bentonite clay and quartz sand is being considered for use as a waste-package backfill, the material placed between a radioactive-waste canister and the repository host rock. Compacts of bentonite/quartz with weight-percent ratios of $100 / 0,70 / 30,50 / 50$ and 30/70 were made at room temperature under a pressure of $100 \mathrm{MPa}(15 \mathrm{ksi})$. Upon initial heating, the thermal conductivity of the 70/30 compact rose from $1.20 \mathrm{~W} / \mathrm{m} . \mathrm{K}$ at $298 \mathrm{~K}$ to $1.32 \mathrm{~W} / \mathrm{m} . \mathrm{K}$ at $373 \mathrm{~K}$. After further heating to $473 \mathrm{~K}$, it fell to $1.10 \mathrm{~W} / \mathrm{m} . \mathrm{K}$, reflecting the loss of interlamellar water from the bentonite. The conductivity of the now-dehydrated compacts displayed conductivities of $0.59,1.06$ and $0.83 \mathrm{~W} / \mathrm{m} . \mathrm{K}$, respectively, at $298 \mathrm{~K}$. Measured densities ranged from 1.98 to 2.12 $\mathrm{g} / \mathrm{cc}$. Combined geometric-mean and Maxwell models for thermal conduction in composite systems predict the measured results reasonably well. An analysis of the impact of backfills on high-level waste package design indicates that no significant thermal penalty is imposed.

1066 Corrosion of TiCode-12 in a simulated Waste Isolation Pilot Project (WIPP) brine. Ahn, T.M.; Lee, B.S.; Woodward, J.; Sabatini, R.L.; Soo, P. (Brookhaven National Lab., Upton, NY). Materials Research Society Symposia Proceedings; 15: 761-767(1983). (CONF-821107-Pt.1).

From 6. international symposium on the scientific basis for radioactive waste management (Materials Research Society); Boston, MA, USA (1 Nov 1982).

The corrosion behavior of TiCode-12 (Ti-0.3 Mo-0.8 Ni) highlevel nuclear waste container alloy has been studied for a simulated WIPP brine at a temperature of $150^{\circ} \mathrm{C}$ or below. Crevice corrosion was identified as a potentially important failure mode for this material. Within a mechanical crevice, a thick oxide film was found and shown to be the rutile form of $\mathrm{TiO}_{2}$, with a trace of lower oxide also present. Acidic conditions were found to cause a breakdown of the passive oxide layer. Solution aeration and increased acidity accelerate the corrosion rate. In hydrogen embrittlement studies, it was found that hydrogen causes a significant decrease in the apparent stress intensity level in fracture mechanics samples. Hydride formation is thought to be responsible for crack initiation. Stress corrosion cracking under static loads was not observed. Attention has also been given to methods for extrapolating short term uniform corrosion rate data to extended times.

1067 Functional dependence of leaching on the surface area-to-solution volume ratio. Machiels, A.J.; Pescatore, C. (Univ. of Illinois, Urbana). Materials Research Society Symposia Proceedings; 15: 209-216(1983). (CONF-821107-Pt.1).

From 6. international symposium on the scientific basis for radioactive waste management (Materials Research Society); Boston, MA, USA (1 Nuv 1982).

The effects of the surface area-to-solution volume ratio on waste glass leach rates are investigated from a theoretical point of view. Simple leach models are discussed first. Correlation variables to interpret the results of similar leaching experiments performed at different values of the surface area-to-solution volume ratio are obtained for static leach testing. For dynamic leaching conditions, the source term required for risk assessment is derived and its dependence on the leachant flow rate and leach specimen surface area is discussed. The findings are upheld by a more complex leach model, the mathematical formulation of which has been implemented in a computer code named LIX. When tested against actual PNL 76-68 glass leaching data, LIX shows excellent capabilities in reproducing the experimental evidence, in particular the effects of the surface area-to-solution volume ratio.

1068 Overview of nuclear waste management. Shemilt, L.W.; Sheng, G. (McMaster Univ., Hamilton, Ontario). ikeren; 2: No. 1, 41-56(1983).
The paper examines in detail the Canadian waste manageme and research programs and discusses various international projects nuclear waste disposal. The disposal and safe handling of high-leve radioactive wastes resulting from the generation of electricity by nuclear fission is a topic of great current concern and future importance. All types of nuclear fuel produce similar quantities of fission products per $\mathbf{G W}$ over time, and the most important factor in handling is whether the waste is used fuel or reprocessing waste. It is believed that deep terrestrial or sub-seabed disposal offers the most risk-free options, the former being the more immediate proposition. Developments in media for encapsulating reprocessed wastes may increase the ease with which they can be disposed of. All geologic systems of disposal require a multibarrier approach, but there are no significant technical problems to safe disposal in this way. Site investigations must study geologic formations, hydrogeology, geochemical properties of host rock and groundwater and long-term stability of the site. Risk assessment of the long-term safety of disposal sites is necessarily probabilistic, and is the subject of much study. The major problem of such assessments is the lack of a commonly-accepted concept of safe levels of radiation over geologic timescales.

1069 Application of systems analysis to the disposal of high level waste in deep ocean sediments. De Marsily, G. (Ecole des Mines de Paris, Fontainbleau, France); Hill, M.D.; Mobbs, S.F.; Webb, G.A.M. (National Radiological Protection Board, Chilton, Didcot, UK); Murray, C.N. (Commission of the European Communities; Ispra (Italy). Joint Research Centre); Talbert, D.M. (Sandia National Labs., Albuquerque, NM (USA)); van Dorp, F. (NAGRA, Baden, Switzerland). Radioactive Waste Management and the Nuclear Fuel Cycle; 3: No. 2, 199213(Dec 1982).

Emplacement in deep ocean sediments is one of the disposal options being considered for solidified high level radioactive waste. Task groups set up within the framework of the NEA Seabed Working Group have been studying many aspects of this option since 1976. The methods of systems analysis havc been applied to enable the various parts of the problem to be assessed within an integrated framework. This paper describes the progress made by the Systems Analysis Task Group lowards the development of an overall system model. The Task Group began by separating the problem into elements and defining the interfaces between these elements. A simple overall system model was then developed and used in both a preliminary assessment and a sensitivity analysis to identify the most important pafameters. These preliminary analyses used a very simple model of the overall system and therefore the results cannot be used to draw any conclusions as to the acceptability of the subseabed disposal option. Fiowever they served to show the utility of the systems analysis method. The work of the other task groups will focus on the important parameters so that improved results can be fed back into an improved systcm model. Subsequent iterations will eventually provide an input to an acceptability decision.

1070 Impact of thermal constraints on the optimal design of high-level waste repositories in geologic media. Malbrain, $\mathrm{C}$.; Lester, R.K. (Massachusetts Inst. of Tech., Cambridge (USA). Dept. of Nuclear Engineering). Nuclear Engineering and Design; 73: No. 3, 331-341(Dec 1982).

An approximate, semi-analytical heat conduction model for predicting the time-dependent temperature distribution in the region of a high-level waste repository hao boen doveloped. The model provides the. basis for a systematic, inexpensive examination of the impact of several independént thermal design constraints on key repository design parameters and for determining the optimal set of design parameters which satisfy these constraints. Illustrative calculations have been curried out for conceptual repository designs for spent pressurized water reactor (PWR) fuel and reprocessed PWR high-level waste in salt and granite media.

1071 Management of high-level waste repository siting. Hill, D.; Pierce, B.L.; Metz, W.C.; Rowe, M.D.; Haefele, E.T.; Bryant, F.C.; Tuthill, E.J. (Brookhaven National Lab., Uptor N.Y). Science (Washington, D.C.): 218: 859-864(26 Nov 1982

The selection of sites to store high-level radioactive waste wi require more than technical decisions; an acceptable site must gain widespread public support. Ad hoc approaches have recently served as a stimulus to overcome institutional inertia in radioactive waste management, as exemplified by the Interagency Review Group and the State Planning council for Radioactive Waste Management, but ad- hoc 
approaches have not characteristically succeeded in resolving intense conflicts. Acceptable sites can best be established through traditional processes of legal and scientific advocacy, and their ultimate legitmacy will depend on the proper use of established democratic processes. 1 figure.

1072 Geological aspects of the final disposal of radioactive waste. Hof richter, E. eeting of the German Society of Metallurgical and Mining Engineers; 35: No. 11, 570-575(Nov 1982). (In German). (CONF-8205132-).

From Annual meeting of the German Society of Metallurgical and Mining Engineers (Gesellschaft Deutscher Metallhuetten- und Bergleute); Freiburg/Breisgau, F.R. Germany (12 May 1982).

The author gives a brief comparative account of the various possible host rocks for the final disposal of radioactive waste. He comments on the deciding factors in the selection of a particular salt dome in the Federal Republic of Germany. Initial results of studies on the Gorleben salt dome are presented. Moreover, the author gives his opinion on problems that have often been the subject of controversial discussion, as for example the stability of a salt deposit and seismic hazards that endanger it, the influence of erosion of salt, and the safety of a 'final-disposal mine'. Some alternative methods of final disposal within the foreseen concept are given, for example the final disposal of high-level waste from the surface into deep drill-holes.

1073 Alpha-recoil damage and solution effects in minerals: uranium isotopic disequilibrium and radon release. Fleischer, R.L. (General Electric Research \& Development Center, Schenectady, NY). biologicheskii Zhurnal; 46: No. 11, 21912201(Nov 1982).

Study of five major minerals (a mica, a feldspar, a pyroxene, quartz, and a natural glass) and two abundant accessory minerals (apatite and sphene) shows that virtually any solution will remove a significant fraction of alpha recoil nuclei that enter a mineral, a fraction that increases with time of exposure to solutions. For waste solids that are rich in transuranic nuclides such effects could be of great importance. Similarly for natural ores and other rocks that are rich in uranium and its descendent radium the effects are likely to play a major role in the release of radon. (JMT)

$1074 \quad$ United States steps up waste isolation programme. Smedes, H.W. (Department of Energy, Germantown, MD (USA). Office of Waste Isolation); Carbiener, W.A. (Battelle Columbus Labs., OH (USA)). Nuclear Engineering International; 27: No. 334, 23-26(Nov 1982).

A description is given of the United States' waste isolation programme which now involves tests of specific sites. The US Department of Energy plans to build a system of mined geological repositories for the disposal of commercially generated high-level and transuranic radioactive waste. It is hoped that the first repository will be available by 1998. Studies of the geology and hydrulugy of the proposed 3itos, the waste packaging and the repository design are reported.

1075 Containing waste material. Ramm, E.J.; Ringwood, A.E. (to Australian Atomic Energy Commission Research Establishment; Lucas Heights). Australian Patent 81/72,825/ B/. \% Oct 1982. Filed date 15 Jul 1980. vp.

The supply material is compressed along an axis of the metal canister in which it is contained. Heat is applied to cause densification and the formation of a block of synthetic rock including the nuclear reactor waste. Either before or after the densification step the canister is sealed with a metal cap. The sealed canister can be placed in a suitable long term storage location.

1076 Molecular glasses for nuclear waste encapsulation. Ropp, R.C. (to Vitrex Corp). US Patent 4,351,749. 28 Sep 1982. Filed date 5 May 1980. vp.

PAT-APPL-146302.

A molecular glass based upon a phosphate of aluminum, or other trivalent metal, provides significant improvement over prior art glasses for encapeulation of high level radinactive nuclear waste. When containing a controlled amount of those elemental oxides found in a typical nuclear waste, the waste-glass would not devitrify under conditions which produced devitrification in the non-nuclear-waste-containing glass, exhibited hydrolysis losses lower by an order of magnitude, had high solvency power for those elemental oxides, exhibited little tendency for internal crystallite formation, and possessed other desirable physical characteristics, all in direct antithesis to the properties of the best priorknown glasses used for this application.

1077 Iodine-129 in high-activity nuclear wastes: an assessment of the deep-ocean disposal option. Baxter, M.S. (Glasgow Univ. (UK). Dept. of Chemistry); Aston, S.R. (International Laboratory of Marine Radioactivity, Musee Oceanographique, Principality of Monaco). Radioactive Waste Management; 3: No. 1, 47-55(Sep 1982).

Supersedes Radioactive Waste Management.

By 2000 A.D. a significant global inventory of ${ }^{129} \mathrm{I}$ will be present in high-activity nuclear wastes pending ultimate disposal. Not only does ${ }_{129}$ I have the longest half-life of any major radionuclide in these wastes but it also is very water-soluble, is difficult to contain in a solid matrix and has, in the oceans, a well-defined critical pathway through seaweeds to man. In this paper, the potential hazard inherent in a deep ocean program for high-activity ${ }^{129} \mathrm{I}$ disposal is assessed by considering the effect of the most pessimistic sequence of post-disposal events and nuclide transfer processes. The results show clearly that, by accepted international standards of safety, the ${ }^{129} \mathrm{I}$-derived hazard to man from such a program would be negligible.

1078 Nuclear trash. What do we do. Scientific Australian; 6: No. 7, 5-9(Aug-Sep 1982).

Alternative solutions to the waste disposal problem are outlined. Synroc, with its long-term stability, seems a more viable option than borosilicate glass. The advantages of burying wastes in deep drill holes are noted. This solution compares favourably with the suggestion of a mined repository in a suitable geological environment.

1079 Storage of high-level radioactive waste. Beale, $H$. (UKAEA Waste Management Executive Unit, Harwell). Nuclear Energy (Institution of Nuclear Engineers); 21: No. 4, 245-252(Aug 1982).

The role of storage in management of high-level radioactive waste is considered. The requirements of both short-term and long-term stores are outlined and store designs are reviewed. It is concluded that canisters containing vitrified high-level waste should be stored in aircooled vaults for at least a decade to allow the decay heat to diminish. They should then be transferred to sealed casks made of reinforced concrete where they can remain for an indefinite period.

1080 Materials for high-level waste containment. Marsh, G.P. (UKAEA Atomic Energy Research Establishment, Harwell. Materials Development Div.). Nuclear Energy (Institution of Nuclear Engineers); 21: No. 4, 253-265(Aug 1982). (AERER-10439).

The function of the high-level radioactive waste container in storage and of a container/overpack combination in disposal is cunsidered. The consequent properties required from potential fabrication materials are discussed. The strategy adopted in selecting containment materials and the experimental programme underway to evaluate them are described.

1081 Radiological impact of disposal of UK solid radioactive wastes, present and future. Hill, M.D.; White, I.F. (National Radiological Protection Board, Harwell (UK)). Nuclear Energy (Institution of Nuclear Engineers); 21: No. 4, 225-233(Aug 1982).

In the UK the more radioactive solid wastes are currently stored, awaiting treatment and disposal. Radiological assessments of solid waste management are therefore mainly concerned with options for the future, and the information they provide will form an input to decisions on treatment and disposal methods. Assessments performed so far by the NRPB are summarized; they have clarified research priorities, and have given preliminary indications of the radiological acceptability of some disposal options. Although such preliminary assessments will continue to be made, the current NRPB work programme is aimed at providing more comprehensive, site-specific assessments, and at, develuping criteria for judging the radiological acceptability of disposal options. Priority is now being given to intermediate-level wastes, because it has been recognized that these should be disposed of as soon as it is technically fcasible. 
1082 Repository engineering design for high-level waste. Griffin, J.R. (UKAEA Risley Nuclear Power Development Establishment). Nuclear Energy (Institution of Nuclear Engineers); 21: No. 4, 267-273(Aug 1982).

The broad principles involved in designing a repository for highlevel wastes are described. The vitrified waste and its characteristics are discussed and compared with other wastes. A design philosophy is developed which shows the influence of the waste characteristics, the host rock, time of emplacement and nuclide movement. Some conceptual engineering designs developed in the UK are illustrated and described, and the most significant engineering aspects commented upon. The problems of short- and long-term safety are briefly discussed. In conclusion it is suggested that there are no insurmountable problems in achieving designs which are acceptable from a safety point of view and at an acceptable cost.

1083 Thermal and thermomechanical effects in the repository storage of high level waste. Koester, R.; Bechthold, W. ATW, Atomwirtschaft, Atomtechnik; 27: No. 7, 386-388(Jul 1982). (In German).

With the aid of validated calculation methods and by means of extrapolation to a long period of time and large deposit geometries, it will be possible to find the limiting values which are necessary for interpreting the per,nissible thermal load on the waste disposal site and thus to assure the stability of the underground storage site and to eliminate processes such as thermal morphosis and non-permissible stress concentrations in the salt dome.

1084 Isolation of high-level nuclear wastes in a geologic repository. Donath, F.A. (CGS, Inc., Urbana, IL). Radiation Research; 91: No. 1, 22-33(Jul 1982).

From Radiation Research Society meeting; Minneapolis, MN, USA (31 May 1981).

Options seriously considered for isolation of high-level nuclear wastes that involve geologic media differ primarily in emplacement technique and location. Of these options, emplacement in a deep, excavated cavity, or geologic repository, is the currently favored method. The most likely means of returning radionuclides from a sealed geologic repository to the biosphere is by dissolution and transport of these by ground water. The potential hazard from deeply buried radionuclides therefore depends primarily upon three important aspects: the amount and rate of supply of radionuclides to the groundwater; the pathways and rate of groundwater movement; and the degree of geochemical retardation imposed by the geologic media. The assessment of sites possibly suitable for a geologic repository must involve a critical evaluation of: (1) the performance of the existing geologic system; (2) the probable future performance of the natural system, taking into consideration evolutionary change and potentially disruptive events; and (3) the disturbance to the natural system caused by excavation, waste emplacement, sealing, and the presence thereafter of the waste facility. The assessment of risk is possible only if reasonable predictions can be made regarding the repository environment. Because actual tests and demonstrations of repository system behavior cannot be conducted under various changing conditions over representative periods of time, mathematical models must be used to predict the long-term behavior of the system.

1085 CEC project to assess high level waste disposal options. Hill, M.W.; Webb, G.A.M. (National Radiological Protection Board, Harwell (UK)). Radiological Protection Bulletin; No. 47, 4-5(Jul 1982).

During 1981, the Commission of the European Communities (CEC) initiated a project the objectives of which are to carry out, at Community Level, assessments of disposal of high-level radioactive waste in geological formations such as salt, clay, granite and seabed sediments. The various phases of the project with their envisaged timescales are outlined together with information on the contributions to the project to be made by the different EC countries. Preliminary results have shown that the project will make a valuable contribution to harmonizing work on high-level waste disposal assessments within the European Community.

1086 NWTS program waste projection data needs. McSweeney, T.I.; Peterson, R.W. (Battelle Memorial Institute, 505 King Avenue, Columbus, OH 43201.). Transactions of the
American Nuclear Society: 41: 85(Jun 1982). (CONF 820609-).

From American Nuclear Society annual meeting; Los Angeles, CA, USA (6 Jun 1982).

ALPHA-BEARING WASTES; HIGH-LEVEL RADIOACTIVE WASTES; INVENTORIES; NATIONAL PROGRAM PLANS; PROJECTION SERIES; RADIOACTIVE WASTE DISPOSAL; RADIOACTIVE WASTES; SPENT FUELS; TECHNOLOGY ASSESSMENT

1087 High-level waste disposalitechnical and institutional siting factors remaining to be resolved. Newcomb, W.E. (Battelle Memorial Institute, Office of Nuclear Waste Isolation, 505 King Avenue, Columbus, $\mathrm{OH}$ 43201). Transactions of the American Nuclear Society; 41: 229(Jun 1982). (CONF820609-).

From American Nuclear Society annual meeting; Los Angeles, CA, USA (6 Jun 1982).

BASALT; HIGH-LEVEL RADIOACTIVE WASTES; HYDROLOGY; SALT CAVERNS; SITE SELECTION; TECTONICS; IHERMUCHEMICAL PROCESSES; RADIOACTIVE WASIE DISPOSAL; GEOLOGIC DEPOSITS; SALT DEPOSITS; TUFF

1088 Pullcy aspects of Fedèral-State relations. Brown, H.C. (National Governors' Association, 400 North Capitol Street, N.W., 205, Washington, D.C. 20001). Transactions of the American Nuclear Society; 41: 230(Jun 1982). (CONF820609-).

From American Nuclear Society annual meeting; Los Angeles, CA, USA (6 Jun 1982).

FINANCIAL ASSISTANCE; GOVERNMENT POLICIES; NATIONAL GOVERNMENT; SITE SELECTION; STATE GOVERNMENT; RADIOACTIVE WASTE DISPOSAL; HIGH-LEVEL RADIOACTIVE WASTES

1089 Licensing: technological implications for geologic dispasal of high-level radioactive wastes. Comella, P.A.; Arsenault, F.J. (U.S. Nuclear Regulatory Commission, Washington, D.C. 20555). Transactions of the American Nuclear Saciety: 41: 231(Jun 1982). (CONF-820609-).

From American Nuclear Society annual meeting; Los Angcles, CA. ISSA (6 Jun 198?)

GEOLOGIC DEPOSITS; HIGH-LEVEL RADIOACTIVE WASTES; LICENSING; TECHNOI OGY ASSESSMENT; US DOE; US NRC; RADIOACTIVE WASTE DISPOSAL; REGULATIONS

1090 Models and risk from high-level waste disposal-the missing pieces. Kocher, D.C. (Oak Ridge National Laboratory, Oak Ridge, TN 37830). Transactions of the American Nuclear Society; 41: 232(Jun 1982). (CONF-820609-).

From American Nuclear Society annual meeting; Los Angeles, CA, USA (6 Jun 1982).

ACTIVATION ANALYSIS; COMPTON SCATTERING TOMOGRAPHY; GEOLOGIC DEPOSITS; GROUND WATER; HIGH-LEVEL RADIOACTIVE WASTES; RADIOUNUCLIDE MIGRATION; RISK ASSESSMENT; SHIELDING; RADIOACTIVE WASTE DISPOSAL; MATHEMATICAL MODELS; EVALUATION

1091 Geological disposal of high-level radioactive waste a review of the Institute of Geological Sciences' research programme. Mather, J.D.; Chapman, N.A.; Black, J.H.; Lintern, B.C. (Institute of Geological Sciences, London (UK)). Nuclear Energy (Institution of Nuclear Engineers); 21: No. 3, 167 173(Jun 1982).

The Institute of Geological Sciences became responsible for the geological research relating to high-level waste disposal in 1980, and before this was a subcontractor to the UK Atomic Energy Authority Field-oriented studies have involved a drilling programme at Altnabreac in Caithness and reconnaissunce studies at a number of other defined research areas in the UK. Following a government announcement in December 1981 these studies have been curtailed. Complementary laboratory studies have provided data on radio-nuclide release and rock/ leachate interactions in the near field and radio-nuclide migration in the 
$f_{a r}$ field. These data have been combined with rock and hydraulic proprties derived from the field studies to produce a coherent model of raste mobilization and migration.

1092 NRC regulations for high-level nuclear waste management. Arsenault, F.J.; Comella, P.A. (U.S. Nuclear Regulatory Commission, Washington, DC 20555). Transactions of the American Nuclear Society; 41: 94(Jun 1982). (CONF820609-).

From American Nuclear Society annual meeting; Los Angeles, CA, USA (6 Jun 1982).

HIGH-LEVEL RADIOACTIVE WASTES; LICENSING; RADIOACTIVE WASTE DISPOSAL; REGULATIONS; STANDARDS; US DOE; US NRC

1093 DOE implementation of high-level waste criteria and standards. Cooley, C.R.; Dayem, N.; Newton, D.C. (Battelle Memorial Institute, Office of Nuclear Waste Isolation, 505 King Avenue, Columbus, $\mathrm{OH}$ 43201). Transactions of the American Nuclear Society; 41: 95(Jun 1982). (CONF820609-).

From American Nuclear Society annual meeting; Los Angeles, CA, USA (6 Jun 1982).

DESIGN; HIGH-LEVEL RADIOACTIVE WASTES; PUBLIC HEALTH; RADIOACTIVE WASTE DISPOSAL; SITE SELECTION; STANDARDS; UNDERGROUND DISPOSAL; US DOE; SPENT FUELS; US NRC; US EPA; SPECIFICATIONS; RADIOACTIVE WASTE MANAGEMENT

1094 Regulatory implementation considerations for deep geologic disposal of high-level radioactive wastes. Glora, M.A. (Battelle Memorial Institute, Office of Nuclear Waste Isolation, 505 King Avenue, Columbus, $\mathrm{OH}$ 43201). Transactions of the American Nuclear Society; 41: 95(Jun 1982). (CONF820609-).

From American Nuclear Society annual meeting; Los Angeles, CA, USA (6 Jun 1982).

HIGH-LEVEL RADIOACTIVE WASTES; LICENSING REGULATIONS; SITE SELECTION; UNDERGROUND DISPOSAL; US DOE; US NRC; RADIOACTIVE WASTE DISPOSAL; GEOLOGIC DEPOSITS; BASALT; TUFF; SALT DEPOSITS

1095 Marking a nuclear waste repository: an archaelogist's perspective. Kaplan, M.F. (TASC (The Analytic Sciences Corporation), One Jacob Way, Reading, MA 01867). Transactions of the American Nuclear Society; 41: 96(Jun 1982). (CONF-820609-).

From American Nuclear Society annual meeting; Los Angeles, CA, USA (6 Jun 1982).

ARCHAEOLOGY; CONSTRUCTION; DESIGN; HIGHLEVEL RADIOACTIVE WASTES; STORAGE; SITE SELECTION; STANDARDS; UNDERGROUND STORAGE; RADIOACTIVE WASTE FACILITIES

1096 Host State perspective to high-level waste management criteria and standards for siting, storage, and monitoring Christofferson, J. (Governor's Office, Room 203, State Capitol, Salt Lake City, UT 84114). Transactions of the American Nuclear Society; 41: 98(Jun 1982). (CONF-820609-).

From American Nuclear Society annual meeting; Los Angeles, CA, USA (6 Jun 1982).

HIGH-LEVEL RADIOACTIVF WASTFS; MONITORING RADIOACTIVE WASTE STORAGE; SITE SELECTION; STANDARDS; UNDERGROUND STORAGE; REGULATIONS

1097 Effects of high-level gamma irradiation on radioactivity release from waste disposal forms. Solomah, A.G. (North Carolina State University, Raleigh, NC 27650). Transactions of the American Nuclear Society; 41: 154-155(Jun 1982). (CONF-820609-).

From American Nuclear Society annual meeting; Los Angeles, CA, USA (6 .Jun 1982).

FISSION PRODUCT RELEASE; FORM FACTORS: GAMMA RADIATION; HIGH-LEVEL RADIOACTIVE WASTES PHYSICAL PROPERTIES; RADIOACTIVE WASTE DISPOSAL;
RADIOCHEMICAL ANALYSIS; STABILITY; SYNTHETIC ROCKS; LEACHING; PHYSICAL RADIATION EFFECTS

1098 Full-scale impact tests of simulated high-level waste canisters. Slate, S.C. (Battelle-Pacific Northwest Laboratory, P.O. Box 999, Richland, WA 99352). Transactions of the American Nuclear Society; 41: 160(Jun 1982). (CONF820609-)

From American Nuclear Society annual meeting; Los Angeles, CA, USA (6 Jun 1982).

CONTAINERS; GLASS; IMPACT TESTS; HIGH-LEVEL RADIOACTIVE WASTES

1099 Characterization of high-level waste forms. Mendel, J.E. Transactions of the American Nuclear Society; 41: 280(Jun 1982). (CONF-820609-).

From American Nuclear Society annual meeting; Los Angeles, CA, USA (6 Jun 1982).

ENVIRONMENTAL EFFECTS; MATERIALS; DATA RADIOACTIVE WASTES; TESTING; US ORGANIZATIONS

1100 Development of structural barriers for containment of nuclear waste in basalt. Westerman, R.E.; Nelson, J.L.; Pitman, S.G. (PNL). Transactions of the American Nuclear Society; 41: 283(Jun 1982). (CONF-820609-).

From American Nuclear Society annual meeting; Los Angeles, CA, USA (6 Jun 1982).

BASALT; CORROSION RESISTANT ALLOYS; MATERIALS TESTING; HIGH-LEVEL RADIOACTIVE WASTES; TESTING; RADIOACTIVE WASTE DISPOSAL; GEOLOGIC DEPOSITS; ENGINEERED SAFETY SYSTEMS

1101 Materials for high-level waste canister/overpacks in salt formations. Molecke, M.A.; Diegle, R.B.; Ruppen, J.A. (Sandia National Laboratories, Albuquerque, NM 87185). Transactions of the American Nuclear Society; 41: 284-285(Jun 1982). (CONF-820609-).

From American Nuclear Society annual meeting; Los Angeles, CA, USA (6 Jun 1982).

CORROSION RESISTANT ALLOYS; ENVIRONMENTAL EFFECTS; MATERIALS; HIGH-LEVEL RADIOACTIVE WASTES; SAFETY; TESTING; RADIOACTIVE WASTE DISPOSAL; SALT DEPOSITS; PACKAGING

1102 Nuclear waste package program for high-level isolation in Nevada tuff. Rothman, A.J. (Lawrence Livermore National Laboratory, P.O. Box 808, Livermore, CA 94550). Transactions of the American Nuclear Society; 41: 285(Jun 1982). (CONF-820609-).

From American Nuclear Society annual meeting; Los Angeles, CA, USA (6 Jun 1982).

NEVADA TEST SITE; HIGH-LEVEL RADIOACTIVE WASTES; TUFF; US ORGANIZATIONS; PACKAGING; BACK. FILLING; RADIOACTIVE WASTE DISPOSAL; GEOLOGIC DEPOSITS

1103 UK view of the management of high-level waste. Marshall, W. (UKAEA Headquarters, London). up) Annual Conference Proceedings; 24: No. 2, 25-29(Jun 1982).

The UK strategy for the management of high-level radioactive wastes from nuclear power stations and reprocessing plants is: to solidify the waste by turning it into glass blocks; to store the glass blocks until they have cooled sufficiently, perhaps 50 to 100 years and maybe more; to dispose of the blocks, possibly into deep geological formations, once the heat output has fallen to an acceptable level. Options other than disposal in deep geological formations include disposal on or disposal under the ocean bed; however, the knowledge of such other options is still at an earlier stage than geological disposal.

1104 Alternate waste form evaluation for Savannah Rlver Plant high-level waste. Gould, T.H. Jr. (E. I. du Pont de Nemours \& Company, Savannah River Laboratory, Aiken, SC 29808). Transactions of the American Nuclear Society: 41: 276-277(Jun 1982). (CONF-820609-). 
From American Nuclear Society annual meeting; Los Angeles, CA, USA (6 Jun 1982).

GLASS; SAVANNAH RIVER PLANT; SYNROC PROCESS; US DOE; RADIOACTIVE WASTE PROCESSING; SOLIDIFICATION; HIGH-LEVEL RADIOACTIVE WASTES

1105 Borosilicate glass for immobilization of high-level SRP waste. Kelley, J.A.; Papouchado, L.M. (E. I. du Font de Nemours \& Company, Savannah River Laboratory, Aiken, SC 29808). Transactions of the American Nuclear Society; 41: 277-278(Jun 1982). (CONF-820609-).

From American Nuclear Society annual meeting; Los Angeles, CA, USA (6 Jun 1982).

GAMMA RADIATION; BOROSILICATE GLASS; HIGHLEVEL RADIOACTIVE WASTES; IMPACT STRENGTH; LEACHING; SAVANNAH RIVER PLANT; STORAGE; RADIOACTIVE WASTE PROCESSING; SOLIDIFICATION

1106 Canister arrangement for storing radioactive waste. Lorenzo, D.K.; Van Cleve, J.E. Jr. (to Department Of Energy). US Patent 4,328,423. 4 May 1982. Filed date 23 Apr 1980. vp. PAT-A'PPL-143060.

The subject invention relates to a canister arrangement for jointly storing high level radioactive chemical waste and metallic waste resulting from the reprocessing of nuclear reactor fuel elements. A cylindrical steel canister is provided with an elongated centrally disposed billet of the metallic waste and the chemical waste in vitreous form is disposed in the annulus surrounding the billet.

1107 Geologic disposal of nuclear waste. Stahlkopf, K.; Williams, R.; Carson, A.B. Review (English Translation); 7: No. 4, 6-13(May 1982).

The natural and engineered barriers provided by geologic storage of nuclear wastes are the most likely choice of countries looking for a permanent solution. A review of the properties of nuclear wastes and the management strategies that will protect the public and the environment describes the isolation and disposal systems and their geologic requirements. These include a host-rock formation of adequate size, low-water content and movement, known physical and chemical characteristics, and geologic stability. Work in progress at DOE's test and evaluation facility at the Nevada Test Site and in other countries focuses on site selection, cannister design, and procedural requirements. Legal and institutional uncertainties must be resolved in addition to a successful demonstration of technology. 3 figuroo. (DCK)

1108 Nuclear waste disposal: a case of benign neglect>. Hileman, B. dnichestvo Stran-Chlenov SEV; 16: No. 5, 271A275A(May 1982).

Problems associated with safe disposal of spent fuel rods, uranium mill tailings, high and low-level nuclear waste are reviewed. The pros and cons of reprocessing, low-level sites, geologic repositories and use of borosilicate waste forms are discussed. (JMT)

1109 Geological disposal of radioactive waste. Research in the OECD area. Paris, France; OECD (May 1982). 55p.

Also published in French.

This report present brief descriptions of research activities in the OECD area, including those supported by the CEC and OECD Nuclear Energy Agency (NEA), which contribute to the development of safe options for the disposal of radioactive waste deep underground. It covers principally geological disposal options for high-level radioactive wastes from nuclear fuel reprocessing and spent nuclear fuel, but some form of geological disposal may also be appropriate for other radioactive wastes which may remain potentially hazardous for long periods of time.

1110 Characterization of drained and undrained response of thermally loaded repository rocks. Palciauskas, V.V.; Domenico, P.A. (Univ. of Illinois, Urbana-Champaign). Water Resources Research; 18: No. 2, 281-290(Apr 1982).

The fluid pressure and mechanical response of a potential repository rock to heating is shown to be characterized by the isothermal parameters of the classic stress-strain theory for a porous medium, in combination with some nonisothermal parameters describing the fluid, solid, and pore volume expansivities. The isothermal coefficients are described in terms of easily interpretable parameters by noting that the fluid response can be formulated within the limits of drained and und- rained behavior. The low permeability-high thermal conductivity environment generally considered to be ideal for nuclear waste storage would appear to favor an undrained response, at least within the isolate pores and cracks of a fractured rock medium. Several cases are presented that provide a qualitatively correct demonstration of the effects of beating in this environment. These include fluid pressure increases in excess of temperature-induced increases in in situ stress, elastic strain and the potential for inelastic crack propagation, and porositypermeability augmentation. If the rocks are dry, or of a high permeability such that fluid flow takes place at constant fluid pressure, similar rock material alterations are possible. This follows from the fact that when the temperature is raised to some high value, say $80^{\circ}$ or $90^{\circ} \mathrm{C}$, and then decreases to its ambient value, the final volume of a polycrystalline substance will generally be greater than the initial one. Hence the effect of temperature is irreversible because of the differential thermal expansion of the composite mineral grains and the generation of new grain boundaries. The increase in porosity during such a heating episode is calculable and empirically related to increases in permeability.

1111 Underground openings for in situ experiments. Wollenberg. H.A.: Korbin, F. (I awrence. Rerkeley I.ah, rA) Earth Sciences; 5: No. 1, 1, 4-7(Apr 1982).

In situ tests include a wide variety of heater experiments with single and multiple arrays at full and reduced scale, block tests, heated room and pillar tests, brine and water migration experiments, permeability tests, fracture hydrology and groundwater chemistry studies, instrumentation development and testing, and other investigations. This article describes the identification of underground openings to accommodate such tests and the concept of a coupled hydrologicthermomechanical experiment. The hydro/thermomechanical experimental program has five stages: 1) design and fabrication; 2) baseline studies; 3) chamber excavation; 4) test chamber experiment; and 5) data analysis and modeling. From the calculations presented, it was concluded that a large volume of rock (approximately 50 times that in the Stripa full-scale heater test) can be influenced within a reasonable time in the hydro/thermomechanical experiment, thereby bridging the gap between laboratory and repository-sized experiments. (JMT)

1112 Geochemistry research planning for underground repositories. Apps, J.A. (Lawrence Berkeley Lab., CA). Earth Sciences; 5: No. 1, 1-3, 7(Apr 1982).

In 1980 the Nuclear Regulatory Commission asked the Lawrence Berkeley Laboratory to lay out a plan for research on the geo cheminical yuestiuns pertaining to underground isolation of high-level radinartive waste. These questions were to relate to the pepnsitnry and surrounding host rocks, but not waste form, container, or overpack, which were to be examined separately. The plan was to identify projects that could resolve present uncertainties and contribute to an acceptable technology for waste disposal. These projects were compared with the current Department of Energy program in order to pinpoint areas for improvement. (IMT)

1113 Geological disposal of radioactive waste. Pigford, $T$. (Univ of Calif, Berkeley). Chemical Engineering Progress; 78: No. 3, 18-26(Mar 1982).

This paper is concerned with the disposal of high-level nuclear waste in deep geological formations which is less of a hazard than near surface uranium ores or uranium mill tailings. This type of disposal depends in part upon the ability of the natural rock to isolate radioactivity from the biosphere. Attractive geologic candidates are natural salt deposits, which are free of groundwater, or granites, shales, and other media which are subject to little or no movement of natural groundwater. The concentrated wastes will be cast into solids, such as glass, and encased in metal containers. this waste package is expected to last for hundreds and thousands of years underground without appreciable deterioration. Details of the system under considerations are given. 12 refs.

1114 Leaching studies on SYNROC at $95^{\circ} \mathrm{C}$ and $200^{\circ} \mathrm{C}$. Oversby, V.M.; Ringwood, A.E. (Australian National Univ., Canberra. Research School of Earth Sciences). Radioactive Waste Management; 2: No. 3, 223-237(Mar 1982).

Crushed samples of SYNROC containing $9 \%, 16 \%$ and $20 \%$ of simulated high-level nuclear waste were tested for leaching behavior in distilled water at $95^{\circ} \mathrm{C}$ and $200^{\circ}$. Leach solutions were analyzed for $\mathrm{Cs}$, $\mathrm{Ca}, \mathrm{Ba}, \mathrm{Sr}, \mathrm{Ti}, \mathrm{Zr}, \mathrm{Nd}$ and $\mathrm{U}$. Results showed that leach rates based on these elements did not change significantly as the waste loading was 
increased from 9 to 20\%. At both temperatures, leach rates showed a srease as leaching progressed until a plateau level was reached. Plau leach rates, which were between 10 and 100 times lower than initial .._ch rates, reflect the expected long term leaching behaviour of the samples. Plateau values of leach rates for SYNROC depend on the element being leached. Highest values are found for $\mathrm{Cs}$ and $\mathrm{Ba}(1$ to $2 \mathrm{x}$ $10^{-7} \mathrm{~g} / \mathrm{cm}^{2} \mathrm{~d}$ at $\left.95^{\circ} \mathrm{C}\right)$ and lowest values for $U\left(5 \times 10^{-10} \mathrm{~g} / \mathrm{cm}^{2} \mathrm{~d}\right.$ at $95^{\circ} \mathrm{C}$ ). Increasing leaching temperature to $200^{\circ} \mathrm{C}$ produces higher leach rates for all elements except $\mathrm{Nd}$. Comparison of SYNROC leach rate data with that for PNL 76-68 glass shows that at $200^{\circ} \mathrm{C}$ the leach rate for $\mathrm{U}$ from SYNROC is $\mathbf{3 0 0 0}$ times less than that from glass.

1115 Development of an assessment methodology for the disposal of high-level radioactive waste into deep ocean sediments. Murray, C.N.; Stanners, D.A. (Commission of the European Communities, Ispra (Italy). Joint Research Centre). Radioactive Waste Management; 2: No. 3, 239-293(Mar 1982).

This paper presents the results of a theoretical study concerning the option of disposal of vitrified high activity waste (HAW) into deep ocean sediments. The development of a preliminary methodology is presented which concerns the assessment of the possible effects of a release of radioactivity on the ecosystem and eventually on man. As the long-term hazard is considered basically to be due to transuranic elements (and daughter products) the period studied for the assessment is from $10^{0}$ to $10^{6}$ years. A simple ecosystem model is developed so that the transfer of activity between different compartments of the systems, e.g. the sediment column, sediment-water interface, deep sea water column, can be estimated. A critical pathway analysis is made for an imaginary critical group in order to complete the assessment. A sensitivity analysis is undertaken using the computed minimum-maximum credible values for the different parameters used in the calculations in order to obtain a minimum-maximum dose range for a critical group.

1116 Disposal of high-level radioactive wastes. Costello, J.M. (Australian Atomic Energy Commission Research Establishment, Lucas Heights). Radiation Protection in Australia; no. 1/82 : 157-188(Mar 1982).

From 6. Annual conference of ARPS; Sydney (Australia) (24 27 Aug 1981).

The aims and options for the management and disposal of highly radioactive wastes contained in spent fuel from the generation of nuclear power are outlined. The status of developments in reprocessing, waste solidification and geologic burial in major countries is reviewed. Some generic assessments of the potential radiological impacts from geologic repositories are discussed, and a perspective is suggested on risks from radiation.

1117 Fracturing of simulated high-level waste glass in canisters. Peters, R.D.; Slate, S.C. (Battelle Pacific Northwest Labs., Richland, WA (USA)). Nuclear Engineering and Design; 67: No. 3, 425-445(Feb 1982).

Waste-glass castings generated from engineering-scale developmental processes at the Pacific Northwest Laboratory are generally found to have significant levels of cracks. This report discusses the causes and extent of fracturing in full-scale canisters of waste glass as a result of cooling and accidental impact.

1118 Hcat transfer in high-level waste management. Dickey, B.R.; Hogg, G.W. (Allied Chemical Corp., Idaho Falls, ID (USA). Idaho Chemical Programs - Operations Office). Nuclear Engineering and Design; 67: No. 3, 473-487(Feb 1982).

Heat transfer in the storage of high-level liquid wastes, calcining of radioactive wastes, and storage of solidified wastes are discussed. Processing and storage experience at the Idaho Chemical Processing Plant are summarized for defense high-level wastes; heat transfer in power reactor high-level waste processing and storage is also discussed.

1119 Thermal criteria for terminal storage of spent nuclear fuel assemblies in bedded salt. Ritchie, J.S.; Busch, J.S.; Julien, H.L.; Watson, D.L. (Kaiser Engineers, Oakland, CA (USA)). uclear Engineering and Design; 67: No. 3, 307-316(Feb 1982).

Design considerations, particularly thermal design considerions, for terminal storage of spent nuclear fuel in a bedded salt repository are discussed in a set of four papers which address: thermal criteria, uncoupling thermal problems, near-field temperatures, and optimization of mine arrangement. This paper outlines the thermal criteria used in the conceptual design of a bedded salt repository and discusses the historical rationale behind developments of each criterion.

1120 Uncoupling thermal problems of terminal storage of spent nuclear fuel assemblies in bedded salt. Busch, J.S.; Julien, H.L.; Ritchie, J.S.; Watson, D.L. (Kaiser Engineers, Oakland, CA (USA)). Nuclear Engineering and Design; 67: No. 3, 317326(Feb 1982).

Spent nuclear fuel assemblies stored in bedded salt can be modeled with a large array of dimensioned decay heat sources (spent fuel assemblies) in an extended thermal conducting media. Although a finite-difference or finite-element representation of the total storage facility could be established, regions of the repository should be analyzed separately since a model of the total repository would require formidable digital computing capacity. This paper explains the basis for thermally analyzing the total storage facility with separate models for the stored fuel assembly package and the salt medium. In addition, the effect of fuel assembly packaging on the maximum fuel temperature, the related problems of fuel handling prior to storage, and uncoupling of the effects of mine ventilation and conduction in the salt medium are discussed.

1121 Near-field salt temperatures resulting from storage of nuclear fuel assemblies in bedded salt. Julien, H.L.; Busch, J.S.; Ritchie, J.S.; Watson, D.L. (Kaiser Engineers, Oakland, CA (USA)). Nuclear Engineering and Design; 67: No. 3, 327337(Feb 1982).

The calculation of near-field salt temperatures is necessary in the design of a bedded salt repository to evaluate alternative storage arrangements. Transient salt temperatures calculated for this purpose with a finite-difference conduction code are reported. An approximate but more efficient conduction code, which can be used in future design efforts for initial scanning of alternatives, is also described.

1122 Calculation of media temperatures for nuclear sources in geologic depositories by a finite-length line source superposition model (FLLSSM). Kays, W.M.; HossainiHashemi, F. (Stanford Univ., Palo Alto, CA (USA). Dept. of Mechanical Engineering); Busch, J.S. (Kaiser Engineers, Oakland, CA (USA)). Nuclear Engineering and Design; 67: No. 3, 339-347(Feb 1982).

A linearized transient thermal conduction model was developed to economically determine media temperatures in geologic repositories for nuclear wastes. Individual canisters containing either high-level waste or spent fuel assemblies are represented as finite-length line sources in a continuous medium. The combined effects of multiple canisters in a representative storage pattern can be established in the medium at selected point of interest by superposition of the temperature rises calculated for each canister. A mathematical solution of the calculation for each separate source is given in this article, permitting a slow hand calculation. The full report, ONWI-94, contains the details of the computer code FLLSSM and its use, yielding the total solution in one computer output.

1123 Optimization of mine layout for nuclear fuel assembly storage. Watson, D.L.; Busch, J.S.; Julien, H.L.; Ritchie, J.S. (Kaiser Engineers, Oakland, CA (USA)). Nuclear Engineering and Design; 67: No. 3, 349-356(Feb 1982).

The development of a mine layout for a nuclear waste repository in bedded salt is discussed. Optimizations of storage room arrangements and mine arrangements are addressed separately, and a conceptual repository design based on these optimizations is described.

1124 Sensitivity calculations for low-beat generating defense waste repository temperatures. Tennant, M.H. (Du Pont de Nemours (E.I.) and Co., Aiken, SC (USA). Savannah River Lab.). Nuclear Engineering and Design: 67: No. 3, 391-396(Feb 1982).

Repository temperatures were calculated for Savannah River wastes by using both two and three-dimensional numerical schemes. The error introduced by using the simpler and more efficient two dimensional models is less than the present uncertainties introduced by waste power generation and host rock properties. Waste canister temperatures were found to be relatively insensitive to geometric asymme. 
try and model detail outside the immediate vicinity of the waste canister.

1125 Realizing the geological final storage of radioactive wastes. Merz, E. Escher Wyss Mitteilungen; 32: No. 2, 156161(Feb 1982). (In German).

The consequent technical realization of the processes and concepts for the safe disposal of radioactive wastes developed on the research sector is now required.

1126 Thermomechanical effects of the final storage of high-level radioactive wastes in salt domes. Ehlert, C. Escher Wyss Mitteilungen; 32: No. 2, 162-168(Feb 1982). (In German).

The phenomena to be considered in the development of the calculation model are represented by the computer programme MAUS (Mechanical Analysis of Underground Storage). The temperature increases in salt domes and the related thermomechanical load last over a long period of time as to human standards, but over a short period as to geological standards, i.e. of 1000 resp. 10000 years. The maximum load for the main barrier, i.e. the mountains, but also for the barrier 'hlrw chills' are of such a kind that it is not very probable that integrity risks of the barriers must be expected.

1127 Deadly legacy. Cowan, R. (Town and Country Planning Association, London (UK)). Town and Country Planning: 51: No. 2, 31-33(Feb 1982).

Comments are made on the government announcement that it had been decided to abandon the programme of geological test drillings, intended to find out if highly radioactive waste could safely be disposed of deep underground. Reasons for the decision are discussed. The desirability of storing the high-level waste at the surface in solid form for a period of $\mathbf{5 0}$ years or more, postponing the decision about final disposal, is critically examined.

1128 Shielded containers for waste could prove beneficial. Burton, W.R. (Nu-Tech, Culcheth (UK)). Nuclear Engineering International; 27: No. 323, 41-43(Feb 1982).

A design study of long-term storage and underground disposal systems for highly active vitrified waste is reviewed. A thick, iron container for the glass block is described. The alternatives of a 'dry' repository above the water table and of a 'wet' repository below it are discussed. Safety and cost assessments of the various multi-barrier systems have been carried out.

1129 Disposal of high-level radioactive wastes by burial in the sea floor. Hinga, K.R. (Anachem, Inc., Albuquerque, NM); Heath, G.R.; Anderson, D.R.; Hollister, C.D. dnichestvo StranChlenov SEV: 16: No. 1, 28A-36A(Jan 1982).

The U.S. Subseabed Disposal program is discussed briefly. A short history of research work done on this disposal is given, along with how the disposal criteria were formulated. The authors conclude that the results to date indicate that the method is promising and that subseabed disposal may begin about the year 2010. (JMT)

1130 Regulating the development of a waste repository. Fehringer, D.J.; Knapp, M.R. Underground Space; 6: No. 4-5. 217-219(Jan 1982).

The three federal agencies, Department of Energy (DOE), Nuclear Regulatory Commission (NRC), and Environmental Protection Agency (EPA), responsible for regulating the development of a waste repository oversee various aspects of the operation. DOE is responsible for siting, designing, constructing, and operating the repository. EPA is to develop generally applicable environmental rediation protection standards to set the overall safety standards for high-level waste. NRC is responsible for developing technical criteria to implement the EPA standard and to ensure, by the licensing process, that the proposed or designed repository will meet the criteria. The function of the NRC is discussed in some detail as it relates to the technical rule - reduction or elimination of uncertainties to the extent practicable, and compensation for unavoidable uncertainties to the extent practicable, and compensation for unavoidable uncertainties by redundancy in the overall system of major barriers in the repository. A status report on the acceptance rule is given. (BLM)
1131 Complex geologic characterization of the repository environment. Harper, T.R. (British Petroleum Research Center, Sunberry, England); Szymanski, J.S. Underground Space; 6: No. 4-5, 229-233(Jan 1982).

The present basis for characterizing geological environments is identified in this paper, and the additional requirements imposed by the need to isolate high-level waste safely are discussed. Solutions to these additional requirements are proposed. The time scale of concern and the apparent complexity of the required multidisciplinary approach are identified. It is proposed that an increased use of the geologic record, together with a recognition that all geologic processes operate within an interdependent system, be a key feature in geologic characterization of deep repositories.

1132 Potential host media for a high-level waste repository. Hustrulid, W. (Colorado School of Mines, Golden). Underground Space; 6: No. 4-5, 234-240(Jan 1982).

Earlier studies of burial of radioactive wastes in geologic repositories had concentrated on salt formations for well-publicized reasons. However, under the Carter administration, significant changes were made in the US nuclear waste management program. Changes which were made were: (1) expansion of the number of rosk types under sonnsideration; (2) adoption of the multiple-barrier approach to waste containment; (3) additional requirements for waste retrieval; and (4) new crilerla propused by the INuclear Regulatury Cunmission for the lsolation of high-level waste in geologic repositories. Results of the studies of different types of rocks as repository sites are summarized herein. It is concluded that cach gencric rock type has certain advantages and disadvantages when considered from various aspects of the waste disposal problem and that characteristics of rocks are so varied that a most favorable or least favorable rock type cannot be easily identified. This lack of definitive characteristics of rocks makes site selection and good engineering barriers very important for containment of the wastes. (BLM)

1133 Selecting a repository site. Ghovanlou, A.H. (MITRE Corp., McLean, VA); Ettlinger, L.; Cotton, T.; Barnard, W.; Siever, R. Underground Space; 6: No. 4-5, 241246(Jan 1982).

Present knowledge about the regional and local geologic predictions as it relates to waste repository sites and some related knowledge gained from oil and mining explorations are summarized. The types of geologic testing involved in selecting a repository site are described, and a simple analytic scheme for estimating the costs of such a program is discussed. This scheme is based on the sequential accumulation of knowledge throughout the process of siting. It is concluded that several sites slivuld be invertiguted simultanevurly sinve there is a trade off to be made between the economic costs of carrying forward all the sites that pass the screening process and the political costs of selecting from a limited number of qualified sites. This would help to insure that a qualified site would be publicly acceptable at some point in the future. (BLM)

1134 Repository design. St. John, C.M. (Applied Mechanics, Inc., Grand Junction, CO). Underground Space; 6: No. 4-5, 247-258(Jan 1982).

Various technical issues of radioactive waste design are addressed in this paper. Two approaches to repository design considered herein are: (1) design to minimize the disturbance of the hot rock; and (2) designs that intentionally modify the hot rock to insure better containment of the wastes. The latter designs range from construction of a highly impermeable barrier around a spherical cavern to creating a matrix of tunnels and boreholes to form a cage within which the hydraulic pressure is nearly constant. Examples of these design alternatives are described in some detail. It is concluded that proposed designs for repositories illustrate that performance criteria considered acceptable for such facilities can be met by appropriate site selection and repository engineering. With these technically feasible design concepts, it is also felt that socioeconomic and institutional issues can be better resolved. (BLM)

1136 Proposal for broader participation in radioactiv waste management. Montague, K. (Environmental Researcl Foundation, Lawrenceville, NJ); Montague, P. Undergrounu Space; 6: No. 4-5, 280-288(Jan 1982).

The Department of Energy (DOE) has set the rather ambitious goals of sinking exploratory shafts for proposed high-level nuclear waste 
isolation by 1983 , selecting one site by 1985 , and having an isolation site fully operational by 2006 . This program must overcome many obstacles, some of which are described in this article. Several techniques that could help develop technical consensus on the difficult problems are suggested for resolving some of the difficult issues related to this program. The lack of public confidence is seen as a key element missing from the nationffis radioactive waste management program, and a program of public participation that ought to help revive public confidence in the agencies responsible for radioactive waste management is described. (BLM)

1136 Improving public understanding of nuclear waste management. Gnirk, P.F.; McClain, W.C. (RE/SPEC Inc., Rapid City, SD). Underground Space; 6: No. 4-5, 289-291(Jan 1982).

The timetable for high-level waste isolation in mined formations by 2000 (in salt formations somewhat sooner) is seen as technically feasible. However, public acceptance of this form of waste isolation is not now on schedule. The main issues of concern to the public are set forth, and some discussion of courses of action for the resolution of these issues is presented. The basic course of action calls for implementation of substantial programs to improve the technical understanding of the issues within the political and non-involved technical communities and to enhance the stature of those responsible for managing nuclear waste isolation. (BLM)

1137 Nuclear waste disposal: can we rely on bedrock. Lindblom, U.; Gnirk, P. Oxford, England; Pergamon Press (1982). 67p.

The disposal of high-level radioactive wastes in bedrock' is discussed in a non-technical manner. The chapters of the book are: (1) The form and final disposal of nuclear wastes; (2) the natural rock and ground water; (3) the disturbed rock and the groundwater; (4) long-term behavior of the rock and the groundwater; (5) nuclear waste leakage into the groundwater; and (5) a summary chapter entitled what does it all mean. (JMT)

1138 Solar-thermionic generator for nuclear wastes disposal in the space. Sahin, S. Atomkernenergie/Kerntechnik; 40: No. 3, 155-156(1982).

The main technical data of a space vehicle for nuclear waste disposal have been evaluated. The vehicle is layed out (1) for the transportation to a stable solar orbit and (2) for the injection into the sun, starting from a relatively low Earth orbit at approximately $300 \mathrm{~km}$. As the propulsion mechanism, (1) an arc-electrothermal engine, and (2) an ionelectrostatic engine have been considered. The main outlines of a solarthermionic generator for the supply of the primary electrical energy have been presented.

1139 Site investigations for repositories for solid radioactive wastes in deep continental geological formations. Vienna, Austria; IAEA (1982). 109p.

This report reviews the earth-science investigations and associated scientific studies that may be needed to select a repository site and confirm that its characteristics are such that it will provide a safe confinement for solidified high-level and alpha-bearing and certain other solid radioactive wastes. Site investigations, as used in this report, cover earth sciences and associated safety analyses. Other site-investigation activities are identified but not otherwise considered here. The repositories under consideration are those consisting of mined cavities in deep continental rocks for accepting wastes in the solid and packaged form. The term deep as used in this report is used solely to emphasize the distinction between the repositories discussed in this report and those for shallow-ground disposal. In general, depths under consideration here are greater than $\mathbf{2 0 0}$ metres. The term continental refers to those geological formations that occur either beneath present-day land masses and adjoining islands or beneath the shallow seas. One of the objectives of site investigations is to collect the site-specific data necessary for the different evaluations, such as modelling required to assess the long-term safety of an underground repository.

1140 Data base for site specific migration analysis of radioactive elements in repositories. Hadermann, J. Atomkernenergie/Kerntechnik; 40: No. 4, 247-253(1982). (In German).
Migration analysis is of considerable importance in long-term safety aspects of radioactive waste repositories. The present work gives the data base for a selected part of a comprehensive geosphere transport calculation. We restrict ourselves to a critical evaluation of parameters pertinent to the migration analysis of the ${ }^{245} \mathrm{Cm}$ chain. This includes the important nuclide ${ }^{207} \mathrm{~Np}$. With these we are able to perform a site specific calculation for repositories planned in deep geologic formations in Switzerland. It is shown that the granitic basement induces strong time delays in nuclide migration. In contrast to that, the overlaying sedimentary layers cause primarily a dilution of the radionuclide concentrations.

1141 Mechanical load on highly radioactive waste containers in final storage. Biurrun, E.; Ehlert, C. pp $405-408$ of Jahrestagung Kerntechnik '82 - Reaktortagung 1982. Tagungsberichte. Sektion 4: Brennstoffkreislauf und Abfallbehandlung. Eggenstein-Leopoldshafen, Germany, F.R.; Fachinformationszentrum Energie, Physik, Mathematik (1982). (In German)

From Annual meeting on nuclear technology '82; Mannheim, Germany, F.R. (4 - 6 May 1982).

HIGH-LEVEL RADIOACTIVE WASTES; CONTAINERS; CASKS; THERMAL STRESSES; STATIC LOADS; UNDERGROUND STORAGE; SALT DEPOSITS

1142 Preliminary design of a Danish high level waste disposal facility in the Mors dome. Hasted, F.; Jensen, P. (Elkraft Power Co-Denmark, DK-2750 Ballerup). Transactions of the American Nuclear Society; 40: 83-86(1982). (CONF-820420-).

From International ENS/ANS conference on nuclear energy with emphasis on fuel cycles; Brussels, Belgium (26 Apr 1982).

CONSTRUCTION; DENMARK; DESIGN; HIGH-LEVEL RADIOACTIVE WASTES; RADIOACTIVE WASTE DISPOSAL; RADIOACTIVE WASTE FACILITIES; SAFETY; GEOLOGY; HYDROLOGY; SALT DEPOSITS

1143 Effects of gamma irradiation on the leaching behavior of a synthetic mineral waste form (SYNROC-B). Solomah, A.G. (NC State Univ-U.S. Raleigh, NC 27650). Transactions of the American Nuclear Society; 40: 99-101(1982). (CONF820420-).

From International ENS/ANS conference on nuclear energy with emphasis on fuel cycles; Brussels, Belgium (26 Apr 1982).

HIGH-LEVEL RADIOACTIVE WASTES; LEACHING; SOLIDIFICATION; SPENT FUELS; SYNROC PROCESS; TECHNOLOGY ASSESSMENT; GAMMA RADIATION; RADIATION EFFECTS; SYNTHETIC ROCKS

1144 Hydrogeology of radioactive waste isolation: the challenge of a rational assessment. Davis, S.N. (Univ. of Arizona, Tucson). ety of America, Memoir; No. 189, 389-396(1982). (CONF-790284-).

From Symposium on recent trends in hydrogeology; Berkeley, CA, USA (8 Feb 1979).

Plans to isolate radioactive waste raise more questions than can be answered within the lifetime of today's researchers. The existence of a multitude of unanswered questions, however, is not a unique phenomenon related to waste isolation, but is a general characteristic of all science. Although most questions related to the isolation of radioactive waste are interesting scientifically, answers to only a small but critical fraction of the questions are vital to the practical aspects of the problem. Almost all hydrogeologic evaluations of repository sites convey an excessively negative view with respect to geologic processes. Repositories do not necessarily become more hazardous with time. Many slow, normal geologic processes will seal and cover repository sites, making radionuclide migration less likely in the future than at present. Furthermore, several more rapid geologic processes, which are viewed with great apprehension, will not always have negative effects. Faulting could block ground-water circulation near the repository and consequently slow the outward migration of radionuclides; volcanism could cover repository sites, with only a slight chance of a volcanic vent contracting the radioactive waste; and in most of the northern part of the Midwest, future glacial activity would probably plaster the ground surface with layers of till, thus helping to isolate and protect any potential deep repository. Safety of waste repositories will depend on multiple barriers to migration of radionuclides. Further research focused on each barrier, however, is required before repository safety is assured. 
1145 Processing effects on the behavior of titanate waste forms in aqueous solutions. Dosch, R.G. (Sandia National Labs., Albuquerque, NM). pp 15-22 of Scientific basis for nuclear waste management. Topp, S.V. (ed.). New York, NY; Elsevier Science Publishing Company, Inc. (1982). (CONF-811122-Pt. 1). Contract AC04-76DP00789.

From Annual meeting of the Materials Research Society; Boston, MA, USA (16 Nov 1981).

Titanate processing parameters including temperature, waste loading, redox conditions, and additives used to promote specific phases were evaluated with respect to their effect on leaching of $\mathrm{Cs}, \mathrm{Mo}, \mathrm{Ca}$, $\mathrm{Sr}, \mathrm{Ba}, \mathrm{U}, \mathrm{Gd}$, and $\mathrm{Ti}$ in the range of $22^{\circ}$ to $150^{\circ} \mathrm{C}$ in leachates which included deionized water, acidic ( $\mathrm{pH} 2)$, and basic $(\mathrm{pH} 12)$ solutions. Surface analyses and microstructural characterization were used to relate observed leaching behavior to processing parameters. Redox conditions were found to be very important with respect to Cs (and Mo) retention. Two Cs-bearing phases were found in titanate prepared under oxidizing conditions. One had a hollandite structure and the other contained $\mathrm{Cs}, \mathrm{Mo}, \mathrm{Ca}$, and $\mathrm{Fe}$ as major constituents. The latter phase, which was more susceptible to leaching, was not observed in titanates prepared using adequate reducing conditions. Where applicable, a reference glass (PNL 76 to 68) was included in the leaching tests. Comparative leach rates for elements common to both waste forms were generally one to four orders of magnitude lower for the titanates within the range of conditions used. 5 figures, 1 table.

1146 Predicting Pu concentrations in solutions contacting geologic materials. Strickert, R.G.; Rai, D. (Pacific Northwest Lab., Richland, WA). pp 215-221 of Scientific basis for nuclear waste management. Topp, S.V. (ed.). New York, NY; Elsevier Science Publishing Company, Inc. (1982). (CONF-811122-Pt. 1). Contract AC06-76RL01830.

From Annual meeting of the Materials Research Society; Boston, MA, USA (16 Nov 1981).

Knowledge of Pu solid phases present in nuclear wastes is important for predicting the geochemical behavior of Pu. Thermodynamic data and experimental measurements using discrete $\mathrm{Pu}$ compounds, $\mathrm{Pu}$ doped borosilicate glasses (simulating a high-level waste form), and Pu contaminated sediments suggest that $\mathrm{PuO}_{2}(\mathrm{c})$ is very stable and is expected to be present in the repository. The solubility of the stable phase, such as $\mathrm{PuO}_{2}$ (c), can be used to predict the maximum $\mathrm{Pu}$ concentration in solutions for long-term safety assessment of nuclear waste repositories. 2 figures.

1147 Transient hydraulic tests in granite: fissured porous medium analysis and results. Black, J.H.; Barker, J.A. (Harwell Lab., Oxfordshire, England). pp 223-230 of Scientific basis for nuclear waste management. Topp, S.V. (ed.). New York, NY; Elsevier Science Publishing Company, Inc. (1982). (CONF$811122-\mathrm{Pt} .1$ ).

From Annual meeting of the Materials Research Society; Boston, MA, USA (16 Nov 1981).

Slug and pulse tests have been used extensively to measure the hydraulic conductivity and specific storage of granitic rocks. After several hundred tests using an Institute of Geologic Sciences (IGS) design of straddle packer test equipment, it became clear that the conventional methods of test analysis were inadequate. The straddle packer equipment developed by IGS allows the time scale of a test to be altered without repositioning the packers so that a range of tests can be carried out on the same test interval. The new analysis procedure presented here uses a model of fissured rock which incorporates water movement in both the fissures and the rock matrix. A new variable is introduced which includes the parameters matrix hydraulic conductivity, matrix specific storage fissure specific storage and fissure hydraulic conductivity together with the controllable variables, effective casing radius and packer interval length. Examples of analysis using multiple tests in the same interval are presented together with the apparent relationship between measured hydraulic conductivity and specific storage. It would appear that a small number of fissures endow the rock mass with the bulk of its hydraulic conductivity and that many of the higher hydraulic conductivity tests are probably associated with localized pipe flow. 5 figures.

1148 Diffusion measurements in compacted bentonite. Torstenfelt, B.; Andersson, K.; Kipatsi, H.; Allard, B.; Olofsson, U. (Chalmers Univ. of Tech., Goeteborg, Sweden). pp 295-302 of Scientific basis for nuclear waste management. Topp, S.V. (ed.). New York, NY; Elsevier Science Publishing Company, Inc. (1982). (CONF-811122-Pt.1).

From Annual meeting of the Materials Research Society; Boston, MA, USA (16 Nov 1981).

The diffusion of $\mathrm{Cs}, \mathrm{Sr}, \mathrm{Am}$ and $\mathrm{Tc}$ in compacted bentonite clay has been studied, using a diffusion cell where diffusion takes place axially from the center of a cylinder of the clay. The effect of addition of potential radionuclide getters to the clay (apatite for Am, pyrite, olivine, and $\mathrm{Fe}(\mathrm{s})$ for $\mathrm{Tc}$ ) as well as using complexed (acetate) or colloidal Am has been studied. The diffusivities obtained for $\mathrm{Cs}$ and $\mathrm{Sr}$ were very high $-9.0 \times 10^{-10} \mathrm{~m}^{2} / \mathrm{s}$ and $2.6 \times 10^{-12} \mathrm{~m}^{2} / \mathrm{s}$, considering that if the effect of sorption on the clay is substrated the diffusivities would be $1.8 \times 10^{-9}$ $\mathrm{m}^{2} / \mathrm{s}$ and $3.1 \times 10^{-8} \mathrm{~m}^{2} / \mathrm{s}$. These diffusivities are, however, maximum values as the concentration profiles do not follow the theoretical form for these nuclides. A small amount of the ions seems to migrate very fast, while the rest moves slower. For Tc, that may be expected to be anionic and thus poorly sorbed, a diffusivity of $5.3 \times 10^{-11} \mathrm{~m}^{2} / \mathrm{s}$ was obtained. Addition of $\mathrm{Fe}(\mathrm{s})$ decreases the diffusivity by one order of magnitude while olivine and pyrite have no measurable influence during the present contact times ( 2 to 3 months). Americium is practically immobile. 6 figures, 3 tables.

1149 Migration rates of brine inclusions in single crystals of NaCl. Chou, I.M. (Geological Survey, Reston, VA). pp 303310 of Scientific basis for nuclear waste management. Topp, S.V. (ed.). New York, NY; Elsevier Science Publishing Company, Inc. (1982). (CONF-811122-Pt.1).

From Annual meeting of the Materials Research Society; Boston, MA, USA (16 Nov 1981).

Rock-salt deposits have been considered as a possible medium for the permanent storage of high-level radioactive wastes and spent fuel. Brine inclusions present in natural salt can migrate toward the waste if the temperature and the temperature gradients in the vicinity of the radioactive waste are large enough. This migration is due to the dissolution of salt at the hot side of the salt-brine interface, ion diffusion through the brine droplet, and the precipitation of salt at the cold side of the salt brine interface. In order to quantify the problem, the migration rate of these brine inclusions must be estimated under various repository conditions. This paper estimates migration rates for all-liquid brine inclusions in single crystals of $\mathrm{NaCl}$ by utilizing recent data for brines and the model of Anthony and Cline [T.R. Anthony and H.E. Cline, J. Appl. Phys., 42, pp. 3380-387 (1971)]. The predictions are compared with experimentally measured migration rates. 4 figures, 6 tables.

1160 Corrosion of construction materials in clay environments. Dresselaers, J.; Casteels, F.; Tas, H. (SCK/CEN Studiecentrum voor Kernenergie, Boeretang, Belgium). pp 311-319 of Scientific basis for nuclear waste management. Topp, S.V. (ed.). New York, NY; Elscvier Science Publishing Company, Inc. (1982). (CONF-811122-Pt.1).

From Annual meeting of the Materials Research Society; Boston, MA, USA (16 Nov 1981).

The corrosion rate and mechanism of construction materials of galleries and casings for boreholes (possibly to be used as consumable overpack of HLW canisters) have been evaluated after a first campaign of wisusiun expeninents in diral wilaut wilh clay, in a liunid clay atmosphere and in a gas corrosion chamber. The envisaged construction matrial is ductile iron (grade 60) and has been tested with and without metallic and organic coatings. The corrosion mechanism is governed by the formation of a rust scale composed of $\mathrm{Fe}_{0} \mathrm{O}_{4}$ and $\mathrm{C}$. Preferential attack underneath the surface reaction layer is associated with the progress of a reaction front at the interface between the graphic nodules or flakes and the ferritic or pearlitic structure. Post-corrosion analyses of old gray iron coal mine shafts exposed for 60 and 90 years in well defined conditions are included to validate the use of the results of the short-time tests for very long exposure times. 3 figures, 10 tables.

1161 Mechanisms of dissolution of radioactive waste storage glasses and cesium migration from a granite repository. Chapman, N.A.; McKinley, I.G.; Savage, D.; West, J.M. (Inst. of Geological Sciences, Environmental Protection Unit, Harwell, England). pp 347-354 of Scientific basis for nuclear waste management. Topp, S.V. (ed.). New York, NY; Elsevier Science Publishing Company, Inc. (1982). (CONF-811122-Pt.1). 
From Annual meeting of the Materials Research Society; sston, MA, USA (16 Nov 1981).

Experimental and theoretical data are used to compare the effect of three possible leach mechanisms for borosilicate glass waste buried in a granite host-rock on the release and subsequent migration of ${ }^{105} \mathrm{Cs}$. Protracted release episodes and variations of up to an order of magnitude in ground water transport times and five orders in output concentrations are possible. 4 figures.

1152 Strontium-basalt reactions under nuclear waste repository conditions. Komarneni, S. (Pennsylvania State Univ., University Park). pp 389-395 of Scientific basis for nuclear waste management. Topp, S.V. (ed.). New York, NY; Elsevier Science Publishing Company, Inc. (1982). (CONF-811122-Pt. 1).

From Annual meeting of the Materials Research Society; Boston, MA, USA (16 Nov 1981).

Hydrothermal interactions of $\mathrm{Sr}$ as strontium chloride or hydroxide with basalts or basalt phases were investigated at $300^{\circ} \mathrm{C}$ for 4 and/or 12 weeks under a confining pressure of $30 \mathrm{MPa}$. The nature and extent of $\mathrm{Sr}$ immobilization varied with the $\mathrm{Sr}$ source and with the basalt or basalt phase which immobilized up to $99.99 \%$ of the added Sr. The main mechanism of $\mathrm{Sr}$ fixation with basalts seems to be ion exchange when $\mathrm{SrCl}_{2}$ was the $\mathrm{Sr}$ source. Among the basalt phases, basalt glass was the most effective for $\mathrm{SrCl}_{2}$ immobilization by probably forming new strontium mineral phases. However, when $\operatorname{Sr}(\mathrm{OH})_{2}$ reacted with basalts or basalt phases $\mathrm{S}_{\mathrm{r}}$ was immobilized by the crystallization of strontium aluminosilicates such as $\mathrm{SrAl}_{2} \mathrm{Si}_{2} \mathrm{O}_{8}$. These results of Sr-near-field interactions in a basalt repository indicate that basalt can be a good barrier for Sr migration from nuclear wastes. 1 figure, 5 tables.

1153 Backfill-waste interactions in repository simulating tests. Sasaki, N. (Power Reactor and Nuclear Fuei Development Corp., Tokai-Ibaraki, Japan); Komarneni, S.; Scheetz, B.E.; Roy, R. pp 397-404 of Scientific basis for nuclear waste management. Topp, S.V. (ed.). New York, NY; Elsevier Science Publishing Company, Inc. (1982). (CONF-811122-Pt.1).

From Annual meeting of the Materials Research Society: Boston, MA, USA (16 Nov 1981).

Candidate backfill materials such as montmorillonite and clinoptilolite with and without the presence of simulated nuclear waste solids such as borosilicate glass, glass ceramic, sintered ceramic and supercalcine ceramic were investigated under repository simulating conditions $\left(>300^{\circ} \mathrm{C}\right.$ and $\left.30 \mathrm{MPa}\right)$. Experiments were conducted under semi-wet as well as wet repository conditions. Montmorillonite and clinoptilolite did not seem to alter under both semi-wet and wet repository conditions as determined by XRD. However, the above backfill materials reacted extensively with wastes under both conditions. They altered to feldspar (oligoclase) in the presence of borosilicate glass and to analcime in the presence of particular ceramics under both semi-wet and wet repository conditions. Alteration of montmorillonite could not be detected either in the presence of the glass ceramic or supercalcine ceramic under both repository conditions. However, clinoptilolite altered to analcime in the presence of glass ceramic or supercalcine ceramic under wet repository conditions. Reactions of backfill materials with simulated wastes immo bilized waste elements such as $\mathrm{Cs}$, Mo, ctc., by forming ncw phases such as analcime, oligoclase and powellite. In fact, analyses of product solutions from interaction runs indicated that the presence of backfill materials during the alteration of waste solids served to drastically reduce the concentration of some radionuclides in solutions. These results suggest that properly selected backfills can provide the simplest and most effective chemical (or thermodynamic) engineered barrier in an intelligently designed multibarrier system. Moreover, they indicate that the $\mu, P$, and $T$ in the usual leach tests are such that results of such tests cannot have any value in evaluating waste form behavior under repository conditions. 3 figures, 5 tables.

1154 Point defects and microstructural stability of glasses under irradiation. Antonini. M, (GNSM and Istituto di Fisica dell'Universita, Modena, Italy); Buckley, S.N.; Camagni, P.; Gibson, P.N.; Manara, A. pp 709-716 of Scientific basis for nuclear waste management. Topp, S.V. (ed.). New York, NY; Elsevier Science Publishing Company, Inc. (1982). (CONF811122-Pt.1).
From Annual meeting of the Materials Research Society; Boston, MA, USA (16 Nov 1981).

The results of previous optical absorption experiments aiming to investigate the behaviour of atomic defects in silica based glasses irradiated with various types of particles have been extended to examine the effects of multiple irradiations which take place during long term storage of glasses containing HLW. In addition, point defect clustering phenomena have been investigasted by means of high voltage transmission electron microscopy. It has been found that due to the large number of defects already present in amorphous silica prior to irradiations, the contributions of different bombarding particles to the total damage cannot be considered fully additive in terms of the relative number of displaced atoms, but some consideration has to be made of defect interactions and ionization damage. Clustering effects, while being absent in pure silica, are present in borosilicates at large dose rates $\left(\geq 10^{22}\right.$ electrons $\mathrm{m}^{-2}$ $\left.\mathrm{sec}^{-5}\right)$. At lower beam current densities and doses comparable to those achieved after 10 to 100 years of HLW storage, phase separation into a microcrystalline compound has been detected by transmission electron microscopy. 5 figures, 1 table.

1165 Uranium-lead radiometric age determinations of naturally occurring $U(V I)$ minerals: application to radwaste storage. Brookins, D.G. (Univ. of New Mexico, Albuquerque). pp 265 271 of Scientific basis for nuclear waste management. Topp, S.V. (ed.). New York, NY; Elsevier Science Publishing Company, Inc. (1982). (CONF-811122-Pt.1).

From Annual meeting of the Materials Research Society; Boston, MA, USA (16 Nov 1981).

U(VI) bearing species are commonly formed under oxidizing laboratory rock-water-HLW experimental conditions. The experimental stability of artifically produced $\mathrm{Na}-\mathrm{Cs}-\mathrm{Rb}$ weeksite (alkali uranyl hydrosilicate), and other U(VI) species, is uncertain. Naturally occurring U(VI) bearing minerals (uranophane and tyuyamunite) from the Grants mineral belt, New Mexico, have been studied for their U-Pb systematics as natural analogs to these experimentally produced phases. Samples from highly oxidized rocks and from chemically reducing environments have been studied. The samples from oxidized rocks are mixed with hematite-barite-gypsum-calcite gangue and are close to, but not in contact with, pyritiferous-organic carbon-uraniferous (U(IV))-chloritic ore. The reduced rock uranophane yields nearly concordant ${ }^{208} \mathrm{U}-{ }^{206} \mathrm{~Pb}$ and ${ }^{205} \mathrm{U}-207 \mathrm{~Pb}$ ages of 8.32 and $8.33 \mathrm{MYBP}$ (millions of years before the present); the oxidized rock uranophane and tyuyamunite yield more discordant ages $(n>8)$ between 7.4 and 2.7 MYBP. These data argue for long range stability of $\mathrm{U}(\mathrm{VI})$-rich phases in the geologic environment. 1 figure, 1 table.

1166 Leach characterization of cement encapsulated wastes. Roy, D.M.; Scheetz, B.E.; Wakeley, L.D.; Barnes, M.W. (Pa State Univ, University Park, USA). Nuclear and Chemical Waste Management; 3: No. 1, 35-42(1982).

Matrix encapsulation of defense nuclear waste as well as intermediate-level commercial wastes within a low-temperature cementitious composite were investigated. The cements for this study included both as-received and modified calcium silicate and calcium aluminate cements. Specimens were prepared following conventional formulation techniques designed to produce dense monoliths, followed by curing at $60^{\circ} \mathrm{C}$. An alternative preparation procedure is contrasted in which the specimens were 'warm' pressed in a uniaxial press at $150^{\circ} \mathrm{C}$ at 50,000 psi for $0.5 \mathrm{~h}$. Specimens of the waste/cement composites were leached in deionized water following three different procedures which span a wide range of temperatures and solution saturation conditions. Aluminate and compositionally adjusted silicate cements exhibited a better retentivity for $\mathrm{Cs}$ and $\mathrm{Sr}$ than did the as-received silicate cement. 15 refs.

1167 Intercomparison of leach-testing methods and the effects of waste-form composition on test type and duration. Harvey, K.B.; Jensen, C.D. (AECL, Pinawa, Manit, Can). Nuclear and Chemical Waste Management; 3: No. 1, 4350(1982).

Several leach-testing methods were evaluated for their relevance as scoping tests appropriate to proposed Canadian conditions for the disposal of high-level nuclear waste, and a static, terminated leach test was chosen. For a particular glass composition, methods in which the leachant was replenished gave apparent leach rates up to ten times less than did the static test. Under static leaching conditions, the leach rate of a 
number of sodium borosilicate glasses was observed to first rise and then fall with leaching time. This behavior is explained in terms of a pH change in the leachant, which is itself a function of the glass composition. The implications of these observations on glass compositions and on leach-testing methods that are relevant to the needs of final disposal are briefly discussed.

1158 Concept of a canister for high-level radioactive waste. McCoy, H.E. (Oak Ridge Natl Lab, TN, USA). Nuclear and Chemical Waste Management; 3: No. 1, 51-57(1982).

The specific problem of developing a canister for the multibarrier waste isolation concept was considered. A conceptual design was developed for a canister that is relatively easy to fabricate, inspect, and handle. The design has the option of storing the canister or adding an overpack before storage. The costs for canisters made of three materials were estimated. Sufficient resources exist to allow the use of several common metals without significant impact on expected availability. 17 refs.

1159 DOE's nuclear waste management and fuel cycle programs. Coffman, F.E. (Dept. of Energy, Washington, DC). pp 3-6 of Proceedings of the ANS topical meeting on the treatment and handling of radioactive wastes. Columbus, $\mathrm{OH}$; Battelle Press (1982). (CONF-820424-).

From ANS topical meeting on treatment and handling of radio active wastes; Richland, WA, USA (19 Apr 1982).

The Nuclear Waste management and Fuel Cycle Programs of the US Department of Energy are discussed including the West Valley Demonstration project and the commitment of DOE to Remedial Actions.

1160 Review of radioactive waste management programs in the United Kingdom. Keen, N.J. (United Kingdom Atomic Energy Authority, Harwell, England); Duncan, A.G. pp 34-39 of Proceedings of the ANS topical meeting on the treatment and handling of radioactive wastes. Columbus, $\mathrm{OH}$; Battelle Press (1982). (CONF-820424-).

From ANS topical meeting on treatment and handling of radioactive wastes; Richland, WA, USA (19 Apr 1982).

The paper reports progress by the Department of Environment and the nuclear industry in developing and implementing a strategy for the management of radioactive waste in the UK. It outlines the range of possible disposal facilities required to deal with all classes of waste arising from nuclear power generation. The present practices for waste management and the main lines of development by the main waste producers of the plant needed in future are outlined, together with a summary of some of the more generic $R$ and $D$ devoted to treatment, packaging and transport of wastes.

1161 Operational features of the Waste Isolation Pilot Plant (WIPP). Brown, R.K.; Likar, V.F. pp 657-661 of Proceedings of the ANS topical meeting on the treatment and handling of radioactive wastes. Columbus, $\mathrm{OH}$; Battelle Press (1982). (CONF-820424-).

From ANS topical meeting on treatment and handling of radioactive wastes; Richland, WA, USA (19 Apr 1982).

The Department of Energy plans to construct the Waste Isolation Pilot Plant (WIPP) in southeast New Mexico. The facility will retrievably store transuranic waste from defense activities of the United States and ocnduct experiments with Defense High Level Waste which would be retrieved at the end of the experiments. This paper describes the handling of the transuranic waste, both by contact and by remote handling methods. It also describes the arrangement of the shaft and the underground and the underground ventilation system, and the protection provided during surface handling of the waste provided during surface handling of the waste. Finally, the present status of construction at WIPP site is discussed.

1162 Dissolution of evaporites and its possible impact on the integrity of the Waste Isolation Pilot Plant (WIPP) New Mexico, USA. Zand, S.M. pp 439-448 of Scientific basis for nuclear waste management V. Lutze, W. (ed.). New York, NY; North-Holland (1982). (CONF-820636-).

From 5 . international symposium on the scientific basis for radioactive waste management; Berlin, F.R. Germany (7 Jun 1982).
The radiological consequences of a breach of the WIPP TRI waste repository, as a result of dissolution processes were shown to $b_{1}$ in two broad classes. Most of the possible scenarieos that could happeI as a result of the deep dissolution processes were shown to be addressed by the scenarios already presented elsewhere or are being investigated at the present. The remaining possible scenarios, except one, showed that the possible release of radionuclides through the geosphere to the biosphere would take periods of time orders-of-magnitude longer than the half-life of Plutonium-239. The one not specifically addressed, a postulated hydralogic pathway with the characteristics of the Salado/ Castile interface aquifer, appears unlikely. However, it should be quantified when more data become available. To obtain a better understanding of the radiological health effects of the deep dissolution-caused breach of the repository, emphasis must be placed on better evaluation and refinement of the existing mathematical models and associated parameters. In the majority of the existing scenarios the most significant pathway of the radionuclide through the geosphere to the biosphere is the dolomitic Magenta and Culebra aquifers. Because these formations are fractured, mathematical modeling of radionuclide transport is subject to a large degree of uncertainty. Other uncertainties that relate to this pathway which need further study are: (a) the possibility of having nouic pinmeable abanduned mined pulash cumirels as a part of the radionuclide flow path and (b) the outcrops of the Magenta and Culebra formations in Nash Draw, $11 \mathrm{~km}(7 \mathrm{mi})$ before the outlet to the Pecos River at Maiaga Bend. I' he problems associated with the quantifications of these uncertainties as well as the effects of lung-term climatic and hydrologic changes and hydrogeological data requirements of the models should be further addressed.

1163 Deformation-dissolution potential of bedded salt, Waste Isolation Pilot Plant site, Delaware Basin, New Mexico. Anderson, R.Y. (Univ. of New Mexico, Albuquerque). pp 449. 458 of Scientific basis for nuclear waste management $V$. Lutze, W. (ed.). New York, NY; North-Holland (1982). (CONF820636-).

From 5. international symposium on the scientific basis for radioactive waste management; Berlin, F.R. Germany (7 Jun 1982).

This report briefly discusses evidence for deep-seated dissolution considers teh realtionship between deformation, dissolution, and brine, and examines the potential for dissolution at the WIPP (Waste Isolation Pilot Plant) site with respect to stages in the deformation-dissolution process. The Delaware Basin, which contains the Upper Permian Castile and Salado evaporites, was uplifted, faulted on the western margin and tilted $19 \mathrm{~m} / \mathrm{km}$ to the east-northeast during the latter part of the Cenozoic, probably 4 to 6 my ago. This event exposed the tilted limestone (reen) and sandstone aquifers beneath the overlying anliydite and salt beds. Meteoric waters entered the exposed aquifers in the uplifted western and southern margins of the basin. The aquifers became charged and pressurized, with the pressure surface high within the evaporites; almost at the surface. Salt has been removed from beneath and within the evaporite body, apparently by means not related to ordinary nearsurface groundwater flow. This report considers how this may have happened.

1164 Mineralogical and geochemical factors influencing the final disposal of HLW in the Stassfurt halite. Gies, H. (Gesellschaft fuer Strahlen- und Umweltforschung mbH Muenchen, Germany). pp 459-465 of Scientific basis for nuclear waste management V. Lutze. W. (ed.). New York, NY; NorthHolland (1982). (CONF-820636-).

From 5. international symposium on the scientific basis for radioactive waste management; Berlin, F.R. Germany (7 Jun 1982).

Because of the water content of the Transition Zone which may potentially enter a borehole, it is recommended that his zone be excluded from HLW-disposal activities. It is also necessary to maintain a distance from the stratigraphically overlying carnallite seam, which guarantees that it is not heated to more than $135^{\circ} \mathrm{C}$ in order to avoid a thermal liberation of $\mathrm{H}_{2} \mathrm{O}$. With these requirements met it should be possible to maintain a maximum temperature of $200^{\circ} \mathrm{C}$ in the repository. This requires, a safety distance from the carnallite of $\geq 40 \mathrm{~m}^{6}$. This would also insure that the stratigraphic transition zone, assuming a max imum width of $4 n$ in $60 \mathrm{~m}$, would be excluded from the HLW-disposal region.

1165 Gas production and liberation from rock salt samples and potential consequences on the disposal of bigh-level radioac- 
tive waste in salt domes. Jockwer, N. (Gesellschaft fuer Strahlen- und Umweltforschung mbH, Braunschweig, Germany). pp 467-475 of Scientific basis for nuclear waste management V. Lutze, W. (ed.). New York, NY; North-Holland (1982). (CONF-820636-)

From 5 . international symposium on the scientific basis for radioactive waste management; Berlin, F.R. Germany (7 Jun 1982).

These preliminary results of gas generation at elevated temperature up to $200^{\circ} \mathrm{C}$ and radiation up to $10^{7}$ rad indicate that in disposing of high level radioactive waste in rock salt, the components $\mathrm{H}_{2} \mathrm{~S}, \mathrm{CO}_{2}$, $\mathrm{O}_{2}, \mathrm{H}_{2}$ and $\mathrm{CH}_{4}$ will be liberated into the emplacement borehole. This presumes that the in $>$ situ rock salt conditions will indeed be comparable to the conditions during the laboratory tests. The components $\mathrm{H}_{2} \mathrm{~S}, \mathrm{ECl}$ and $\mathrm{O}_{2}$ can corrode the waste container and might interact with the solidified waste products. Therefore it is necessary to investigate what will happen to the solidified waste products when exposed to these components. If the borehule $\mathrm{s}$ rinsed and is absolutely tight, the components $\mathrm{H}_{2} \mathrm{O}, \mathrm{CO}_{2}, \mathrm{H}_{2}$ and $\mathrm{CH}_{4}$ will generate pressure in the borchole, in the intergranular spaces and between the crystal boundaries. This elevated pressure may increase to the fracture pressure of the host rock which then may lead to an uncontrolled release of the gas components. To avoid destruction of the containment by corrosive gases and their interaction with the solidified waste products and to avoid fracturing of the host rock due to elevated pressure, rinsing the borehole until the majority of the gas components are liberated might be one technical solution. Afterwards the borehole can be sealed tight. It is not yet possible to calculate the time required to rinse the borehole.

1166 Thermomechanical in-situ experiments and finite element computations. Pudewills, A.; Mueller, R.; Korthaus, E.; Koester, R. (Kernforschungzentrum Karlsruhe, Germany). pp 477-483 of Scientific basis for nuclear waste management $V$. Lutze, W. (ed.). New York, NY; North-Holland (1982). (CONF-820636-).

From 5 . international symposium on the scientific basis for radioactive waste management; Berlin, F.R. Germany (7 Jun 1982).

The experiments on heat-induced borehole closure and their evaluation by model computations have shown that with the methods of computation and material laws used, a relatively good prediction can be made of the thermomechanical effects in the ultimate disposal of high level wastes in rock salt, at least with respect to near field and relatively short time spans. Regarding a verification of the creep law and the model, it would be necessary to make accompanying measurements of the initial stresses at the place of the experiment, as well as laboratory measurements on the creep behavior of the respective salt. Since the measurement of absolute stresses in salt has posed problems, at attempt should also be made to determine the initial stress condition at the place of the experiment by large-space computations.

1167 Investigation of diffusion of $\mathrm{UO}_{2} \mathrm{Cl}_{2}$ in saturated $\mathrm{NaCl}$ solutions at various temperatures. Murso, $\mathrm{H}$.; Plewinsky, B.; Leopold, D.; Marx, G. (Free Univ. of Berlin, Germany). pp 491-496 of Scientific basis for nuclear waste management $V$. Lutze, W. (ed.). New York, NY; North-Holland (1982). (CONF-820636-)

From 5 . international symposium on the scientific basis for radioactive waste management; Berlin, F.R. Germany (7 Jun 1982).

Diffusion measurements of actinides in saturated $\mathrm{NaCl}$ solutions have been made in order to determine which mechanism (diffusion or convection) is rate determining. The diffusion coefficients of $\mathrm{UO}_{2} \mathrm{Cl}_{2}$ in saturated $\mathrm{NaCl}$ solutions were measured by use of an ultracentrifuge at 25,40 and $50^{\circ} \mathrm{C}$. The results are compared with those determined for the binary system $\mathrm{UO}_{2} \mathrm{Cl}_{2}-\mathrm{H}_{2} \mathrm{O}$. Measurements of viscosity and molar mass were made in order to elucidate the difference in diffusion velocities in the systems studied.

1168 Diffusion in crystalline rocks. Skagius, K.; Neretnieks, I. (Royal Inst. of Tech., Stockholm, Sweden). pp 509-518 of Scientific basis for nuclear waste management V. Lutze, W. (ed.). New York, NY; North-Holland (1982). (CONF820636-).

From 5 . international symposium on the scientific basis for radioactive waste management; Berlin, F.R. Germany (7 Jun 1982).

Laboratory experiments to determine the sorption and the rate of diffusion of cesium and strontium in pieces of granite have been performed. The effective diffusivity, $D_{p} . \epsilon_{p}$ was found to be $1 \cdot 2 \cdot 10^{-12}$ $\mathrm{m}^{2} / \mathrm{s}$ for both cesium and strontium. The diffusion on non-sorbing species in granites and other rock materials have been studied in laboratory scale. The non-sorbing species were iodide, tritiated water, Cr-EDTA and Uranine. In granites the effective diffusivities were determined to be $0.7 \cdot 1.3 \cdot 10^{-18} \mathrm{~m}^{2} / \mathrm{s}$ for iodide and $1.3-1.8 \cdot 10^{-10} \mathrm{~m}^{2} / \mathrm{s}$ for tritiated water. Electrical resistivity measurements in salt water saturated rock cores have been performed. The resistivity is measured in the saturated core and in the salt solution with which the core has been saturated. The ratio between these two resistivities has a direct relation to the ratio of the effective diffusivity for a component in the rock material and the diffusivity in free water for the same component. The results from the electrical resistivity measurements and the experiments with diffusion of non-sorbing species are in fair agreement. The effective diffusivity for cesium and strontium (sorbing species) are, however, more than ten times higher than expected from the results of diffusion of non-sorbing species and the electrical resistivity measurements. This is interpreted as an effect of surface diffusion.

1169 Modeling approach to determine short- and longterm thermal and thermomechanical effects of waste emplacement in a repository in basalt. Lehnhoff, T.F. (Univ. of Missouri, Rolla); Thirumalai, K.; Krug, A.D. pp 597-609 of Scientific basis for nuclear waste management $V$. Lutze, $W$. (ed.). New York, NY; North-Holland (1982). (CONF-820636-).

From 5 . international symposium on the scientific basis for radioactive waste management; Berlin, F.R. Germany (7 Jun 1982).

Basalt, in general, is characteristicaly a jointed and fractured rock. Preliminary field measurements to date, however, indicate that major portions of the deep basalt flows are highly impermeable to groundwater flow because of mineral infilling and large lithostatic pressure. For near-field considerations, the intraflow structures in jointed basalt have a governing influence on the rock mass-property parameters and their response to the repository environment. Much of the early work was done with closed-form solutions or boundary-element methods. These techniques are seen as the only reasonable and practical appraoch to scoping studies. The large number of parameter variations necessary for conceptual design of a repository preclude the initial application of elaborate and detailed finite-element methods. The thermomechanical analysis completed at the BWIP has progressed through much of the scoping phase and is now entering the detailed analysis and design phase in some areas. Methods for detailed analysis are being demonstrated and many uncertainties are being clarified. Final repository-design studies will warrant the special effort necessary to produce extensive finiteelement analyses. The resulting finite-element models then permit analysis of design details and can expose various problem areas for inspection and final evaluation. Nonlinear effects of all types can be evaluated to determine if concerns exist as real problems. The detailed finiteelement modeling will contribute to the basis for making rational correct decisions that will result in a safe repository in basalt for storing nuclear waste.

1170 Combined analytical model for preformance assessment of the waste package/geologic medium systems. Ray, A.K. (Univ. of Kentucky, Lexington); Nair, S.; Nuttall, H.E. pp 611 618 of Scientific basis for nuclear waste management V. Lutze, W. (ed.). New York, NY; North-Holland (1982). (CONF820636-)

From 5. international symposium on the scientific basis for radioactive waste management; Berlin, F.R. Germany (7 Jun 1982).

For sparsely soluble isotopes leaching rates are probably controlled by solubility limits. For a situation like this backfill provides a significant resistance to escape of nuclides from the repository. Isotope ${ }^{129}$ I shows significant concentration level at a $10 \mathrm{~km}$ downstream distance. For this isotope the backfill thickness of $0.25 \mathrm{~m}$ used in the test case is not sufficient to reduce the concentration to an insignificant level. Materials for backfill and thickness of the backfill probably have to be chosen from the consideration of nuclides like ${ }^{129} \mathrm{I}$. The three dimensional transport model used here provides a relatively more accurate estiamtes of concentration levels and discharge rates than the one dimensional model.

1171 Engineered components for spent fuel radioactive waste isolation systems: are they technically justified. Burkholder, H.C. (Battelle Memorial Inst., Columbis, OH). pp 619-630 of Scientific basis for nuclear waste management $V$. 
Lutze, W. (ed.). New York, NY; North-Holland (1982). (CONF-820636-). Contract AC06-76RL01830.

From 5 . international symposium on the scientific basis for radioactive waste management; Berlin, F.R. Germany (7 Jun 1982).

The technical justification for development and use of sophisticated engineered components in radioactive waste isolation is evaluated. The evaluation requires selection of performance measures for the overall system and each of four subsystem functions and selection of an acceptability standard against which oversll system performance can be judged a population to which the performance measure is applied. The evaluation combines the results of waste isolation performance assessments from the literature and focuses those integrated results on the engineered component technical justification question. The results of this study are in substantial agreement with the results of other similar analyses in the nuclear waste isolation literature. Thus, the use of waste containers with long lifetimes and manmade subsystems with very low transport rates seems unjustified from a technical standpoint and a geologic isolation point of view. The design objective for the waste container lifetime should be not greater than that desired for retrievability $(0$ to 100 years), and the design objective for the man-made subsystem transport rate should not be less than the $10^{-2}$ to $10^{-4}$ per year range.

1172 Recent advances in repository seal materials. Roy, D.M.; Burns, F.L. (Pennsylvania State Univ., University Park). pp 63I-639 of Scientific basis for nuclear waste management $V$. Lutze, W. (ed.). New York, NY; North-Holland (1982). (CONF-820636-). Contract AC06-76RL01830.

From 5 . international symposium on the scientific basis for radioactive waste management; Berlin, F.R. Germany (7 Jun 1982).

Studies of radioactive waste repository seal materials have shown the apparent compatibility of some cementitious seal materials with the repository environments for which they were designed, both stratigraphic sequences from a bedded salt area, and plateau basalt flows. The accompanying mechanical and physical properties appear favorable. Accelerated test data on some clays and zeolitic materials have placed tentative limits on conditions for their adequate performance. Low permeability has been shown to be a key factor in assuring durability; however, chemical compatibility also is important for preventing slow changes from taking place in the seal materials, which over prolonged periods might have cumulative harmful effects on the materials' performance. Thermodynamic data and information from old and ancient analog materials are used as supplemental evaluatory data concerning long-term durability.

1173 Development of a backfill for containment of highlevel nuclear waste. Hodges, F.N, Westsik, J.H. Jr.; Bray, L.A. (Pacific Northwest Lab., Richland, WA). pp $641-648$ of Scientific basis for nuclear waste management V. Lutze, $W$. (cd.). New York, NY; North-Holland (1982). (CONF820636-). Contract AC06-76RL01830.

From 5 . international symposium on the scientific basis for radioactive waste management; Berlin, F.R. Germany (7 Jun 1982).

Sodium and calcium bentonites, pressed to densities between 1.9 and $2.2 \mathrm{~g} / \mathrm{cm}^{0}$, have hydraulic ratios $\left(R_{d}\right)$ indicate that $S r, C s$, and $A m$ are strongly sorbed on bentonites and zeolites, that $N p$ and $U$ are moderately sorbed on bentonites and zeolites, and that $\mathrm{Am}, \mathrm{Np}, \mathrm{U}, \mathrm{I}$, and $\mathrm{Tc}$ are strongly sorbed on charcoal. Sorption results with basalt and tuff ground waters are similar; however, iodine in tuff ground water sorbs more strongly on bentonites. Thermal diffusivity measurements for dry, compacted $\left(\mathrm{rho} \sim 2.1 \mathrm{~g} / \mathrm{cm}^{0}\right.$ ) sodium bentonite indicate that the thermal conductivity of a high density bentonite backfill should be roughly similar to that of silicate host rocks (basalt, granite, tuff). These results indicate that a bentonite backfill can significantly delay the first release of many radionuclides into the host rock and that by forming a diffusion barrier a bentonite backfill can significantly decrease the long-term release rate of radionuclides from the waste package.

1174 Ion/water migration phenomena in dense bentonites. Pusch, R. (Univ. of Lulea, Sweden); Eriksen, T.; Jacobsson, A. pp 649-658 of Scientific basis for nuclear waste management V. Lutze, W. (ed.). New York, NY; North-Holland (1982). (CONF-820636-).

From 5 . international symposium on the scientific basis for radioactive waste management; Berlin, F.R. Germany (7 Jun 1982).

The development of a suitable technique for isolating unreprocessed nuclear reactor wastes fromthe biosphere has led to the Swedish multibarrier concept KBS 2 with two engineered components, a thic walled copper canister and a clay body which confines the canister. T] clay consists of well fitting blocks of highly compacted $\mathrm{Na}$ bentoni made by isostatic compression of bentonite powder. They are not water saturated when placed in the deposition holes but take up water from the surrounding rock, swell and ultimately form a tight contact with the rock and the canisters. When the bentonite is in physical equilibrium with the surroundings it forms a medium with a number of valuable properties, such as self-healing and ion exchange capacities. the healing means that initial joints between blocks and voids, or local passages in the clay cased by slight rock or canister displacements, will be sealed by the swelling potential of the clay. The high ion exchange capacity retards the migration of radionuclides through the clay barrier after corrosion of the canisters. the most important feature is, however, the very low hydraulic conductivity and the low ion diffusivity. These properties are discussed.

1175 Transport of actinides through a bentonite backfill. Torstenfelt, B.; Kipatsi, H.; Andersson, R.; Allard, B.; Olofsson, U. (Chalmers Univ. of Tech., Goeteborg, Sweden). pp 659-668 of Soientifio hania for nunolear waste management $V$. Lulich, W. (ed.). New York, NY; North-Holland (1982). (CONF820636-).

From 5. international symposium on the scientific basis for radio active waste management; Berlin, F.R. Germany (7 Jun 1982).

It has been confirmed that $\mathrm{Sr}$ and to some extent $\mathrm{Cs}$ migrates faster than expected, considering measured sorption data. A fraction of these elements seems to migrate rapidly. The actinides $U$ and $N p$ in their higher oxidation state (V, VI) exhibit a mobility similar to but slightly lower than what is observed for $\mathrm{Cs}$, considering only the apparent diffusivities. For Th, Pu and Am which are in the lower oxidation states (III, IV), the apparent diffusivities are 1 to 3 orders of magnitude lower. For all of the actinides (with the exception of $\mathrm{Pa}$ ) similar diffusivities (without sorption) are obtained. These are significantly lower than for $\mathrm{Sr}$ and Cs. The dominating oxidation state for Pu is probably IV, while $U(V I)$ seems to be reduced to U(IV) by Fe in the clay. The presence of a high carbonate concentration or humic acid has little effect on the U(VI)-diffusivity. All of the nuclides ${ }^{107} \mathrm{Cs},{ }^{229} \mathrm{Th},{ }^{241} \mathrm{Am}$ and ${ }^{204} \mathrm{Am}$ would decay to insignificant concentration levels before breakthrough, assuming a $1-\mathrm{m}$ thick clay barrier. For ${ }^{90} \mathrm{Sr},{ }^{209} \mathrm{Pu}$ and ${ }^{240} \mathrm{Pu}$ a significant reduction of the concentration would be expected, while the concentration of ${ }^{207} \mathrm{~Np}$ would be very little reduced.

1176 Analysis of the corrosion products formed on $\mathrm{Ti}$ and a Ti-Pd alloy during exposure in hot water. Olefjord, I.; Mattsson, H. (Chalmers Üniv. of Tech., Goeteborg, Sweden). pp 669678 of Scientific basis for nuclear waste management $V$. Lutze, W. (ed.). New York, NY; North-Holland (1982). (CONF820636-).

From 5 . international symposium on the scientific basis for radioactive waste management; Berlin, F.R. Germany (7 Jun 1982).

This is a preliminary report dealing with the surface analysis of reaotion products formed on $\mathrm{Ti}$ and a Ti-Pd alloy during their exposure in hot water. The compositions of the aqueous media were varied with respect to the dissolved oxygen and the content of chloride ions. The temperature was $60^{n} \mathrm{C}$ and the expusure limes were $10 \mathrm{~min}$. and 6 months. Work is in progress in which samples are exposed at $80^{\circ} \mathrm{C}$ and $95^{\circ} \mathrm{C}$ in the aqueous solutions. Surface analysis was also performed on a sample which had been exposed in water-saturated bentonite. It appears from te ESCA spectra that the oxide products formed on the surface consist of $\mathrm{TiO}_{2}$. The results also indicate that the thickness of the film formed at $60^{\circ} \mathrm{C}$ in water is in the range $50 \mathrm{~A}$ to $100 \mathrm{~A}$. This is somewhat more than that obtained after exposure in water at room temperature: Exposure for 6 months incrcases the thickness of the oxide two to three times compared to that obtained during the short exposure at $60^{\circ} \mathrm{C}$. The analyses of the samples that had been embedded in bentonite indicate that the surface rcaction products are thinner than those found on the surface after exposure in an open vessel.

1177 Hydrothermal conditions around a radioactive was repository. Thunvik, R. (Royal Inst. of Tech., Stockholm, Sweden); Braester, C. pp 587-595 of Scientific basis for nuclear waste management V. Lutze, W. (ed.). New York, NY; NorthHolland (1982). (CONF-820636-). 
From 5. international symposium on the scientific basis for radioactive waste management; Berlin, F.R. Germany (7 Jun 1982).

The possibility of permanent burial of radioactive waste from nuclear power plants, is studied in Sweden at the KBS (Nuclear Fuel Safety) - project. Definite repository sites have not yet been selected, but the general principles of construction regarding the layout have been devised. The feasibility of a prospective site for radioactive waste disposal is highly dependent on the geohydrological conditions. Heat emitted by the decaying waste will increase the temperature of the rock, changing groundwater density gradients and creating convective currents. Under certain conditions water particles passing through the repository may reach the ground surface. It is therefore of significant interest in the safety analysis to predict pathlines and travel times of water particles, should any of the waste canisters be breached and the groundwater be contaminated. The solutions presented illustrate the effect of heat released from a hypothetical repository on the groundwater movements around the repository.

1978 Geologic predictions and radioactive-waste disposal: a time limit for the predictive requirements. Gera, F. (Nuclear Energy Agency, 75 -Paris (France)). pp 9-24 of Predictive geology with emphasis on nuclear-waste disposal. Marsily, G. de (Ecole des Mines de Paris, Fontainebleau, France. Centre d'Informatique Geologique); Merriam, D.F. (Syracuse Univ., NY (USA)) (eds.). Oxford, England; Pergamon Press (1982). (CONF-8007144-). 1980).

From 26. international geological congress; Paris, France (1 Jul

The long-term isolation of radioactive waste in geologic repositories relies on the capability of certain barriers to restrict the migration of radionuclides. The efficiency of the barriers, particularly the natural ones, could be decreased by a number of geological changes. For changes determined by discrete events, probabilities and consequences can be evaluated. For slow geologic processes, both most likely and maximum possible rates and effects on the isolation capability of the disposal system can be estimated. If it were possible to define a duration of time after which waste isolation is nn longer essential, disposal systems able to isolate the waste even in the situation of maximum predictable changes could be designed. The radiotoxicity of high-level radioactive waste after 50,000 to 100,000 years of decay becomes comparable to the radiotoxicity of the naturally radioactive elements which were destroyed in the process that generated the waste. A time period in that order of magnitude seems to be a reasonable target for a geologic isolation system.

1179 Salt domes - the past and present, keys to the future. Martinez, J.D. (Louisiana State Univ., Baton Rouge (USA)). pp 33-43 of Predictive geology with emphasis on nuclear-waste disposal. Marsily, G. de (Ecole des Mines de Paris, Fontainebleau, France. Centre d'Informatique Geologique); Merriam, D.F. (Syracuse Univ., NY (USA)) (eds.). Oxford, England; Pergamon Press (1982). (CONF-8007144-). 1980).

From 26. international geological congress; Paris, France (1 Jul

A familiar dictum in geology is: the present is the key to the past. The geologic analysis required to predict future impacts introduces a new concept: the present is a key to the future. Contemporary studies of the utility of salt domes for the isolation of radioactive wastes furnish an example of the application of this concept. Predictions for both shortrange and long-range hazards are made on the basis of conventional geologic studies of salt-dome growth, hydrologic conditions, caprock formation, and others. Both on-going and historical geologic processes are utilized in this exercise in predictive geology. The more difficult problem is predicting future cultural changes. Here again geology offers some promise of help in evaluation of facility integrity and the establishment of protective modes.

1180 Fault-tree analysis for probabilistic assessment of radioactive-waste segregation: an application to a plastic clay formation at a specific site. D'Alessandro, M.; Bonne, A. (Commission of the European Communities, Ispra (Italy). Joint Research Centre; Centre d'Etude de l'Energie Nucleaire, Brussels (Belgium)). pp 45-63 of Predictive geology with emphasis on nuclear-waste disposal. Marsily, G. de (Ecole des Mines de Paris, Fontainebleau, France. Centre d'Informatique Geologique); Merriam, D.F. (Syracuse Univ., NY (USA)) (eds.). Oxford, England; Pergamon Press (1982). (CONF8007144-). 1980).

From 26. international geological congress; Paris, France (1 Jul

This study concerns a probabilistic safety analysis of potential nuclear-waste repository which may be mined into a Tertiary clay formation underlying the Nuclear Research Centre at Mol (Belgium). The value of the geological barrier has been analyzed in probabilistic terms through the application of the Fault-Tree Analysis (FTA) which can answer two main questions: how can the barrier fail (query) and what is the failure probability (query). FTA has been applied to conceptual radioactive-waste disposal systems. In this paper this methodology has been applied to a specific clay formation, to test the applicability of the procedure to a potential site. With this aim, release probabilities to three different receptors (groundwater, land surface, and atmosphere) were estimated for four different time periods. Because of obvious uncertainties in geology predictive capabilities, a probability band has been obtained. Faulting phenomena are among the main mechanisms having the potential to cause release to groundwater, whereas direct releases to land surface may be linked to various glacial phenomena; on short term, different types of human actions may be important. The overall failure probabilities seem to be sufficiently low to offer a good safety margin.

1181 Predictive geology in nuclear-waste management. Brotzen, O. pp 65-81 of Predictive geology with emphasis on nuclear-waste disposal. de Marsily, G. (Ecole des Mines de Paris, Fontainebleau, France. Centre d'Informatique Geologique); Merriam, D.F. (Syracuse Univ., NY (USÄ)) (eds.). Oxford, England; Pergamon Press (1982). (CONF8007144-). 1980).

From 26. international geological congress; Paris, France (1 Jul

The present situation at a specific site on the Baltic Shield is viewed in the light of its geologic history. Prediction, at a given level of confidence and from a limited number of drillholes of the minimum average spacing of conductive zones in subsurface rocks of lowhydraulic conductivity, is based on a combination of the binomial and Poisson distributions, regarding the holes as a profile sampling and assuming a cubic pattern of fractures. The data provide an empirical basis for linking the nature and frequency of past geologic events to their local effects. Special attention is given to the preservation of tectonic blocks of large rock volumes with low-hydraulic conductivity throughout the present cratonic stage, whereas intermittent movement can be traced in marked fault zones bordering the Shield and three different orogenies affected the surrounding regions. Rock mechanical, stochastic, and deterministic approaches are utilized to predict future effects from this basis.

1182 Nuclear power on unstable ground. Morner, N.A. (Stockholm Univ. (Sweden). Geological Inst.). pp 83-99 of Predictive geology with emphasis on nuclear-waste disposal. Marsily, G. de (Ecole des Mines de Paris, Fontainebleau, France. Centre d'Informatique Geologique); Merriam, D.F. (Syracuse Univ., NY (USA)) (eds.). Oxford, England; Pergamon Press (1982). (CONF-8007144-). 1980).

From 26. international geological congress; Paris, France (1 Jul

It is unacceptable scientifically to say that the problem of nuclear-waste disposal is solved today. The possibilities for future progress remain a question of subjective evaluation. Overoptimistic and careless predictions are not only meaningless but also dangerous factors for future failures and accidents. The Swedish KBS project is in all respects overoptimistic, and the Swedish Stipulation Law in all respects is not fulfilled. The geodynamics of the Fennoscandian bedrock are different from those claimed by KBS. There is a complex of forces and movements (including both novel factor and factors of uncertain or unknown origin) where the amounts and rates of movement are larger than previously realized. Intensive faulting, fracturing, and paleoseismicity are recorded during glacial periods. In this highly active bedrock medium, it is difficult to see how a safe closed deposition could ever be determined. As an emergency action for the time being in order to keep control and freedom of action, an 'open deposition' is recommended, while studies continue. 
1183 Long-term thermohydrologic behaviour of nuclearwaste repositories. Wang, J.S.Y.; Tsang, C.F.; Cook, N.G.W.; Witherspoon, P.A. (California Univ., Berkeley (USA). Lawrence Berkeley Lab.). pp 101-115 of Predictive geology with emphasis on nuclear-waste disposal. Marsily, G. de (Ecole des Mines de Paris, Fontainebleau, France. Centre d'Informatique Geologique); Merriam, D.F. (Syracuse Univ., NY (USA)) (eds.). Oxford, England; Pergamon Press (1982). (CONF. 8007144-). 1980).

From 26. international geological congress; Paris, France ( $1 \mathrm{Jul}$

In the selection and assessment of geologically suitable sites as potential underground repositories for nuclear wastes, the impact of the heat generated by the wastes upon groundwater movement between the repository and the biosphere is an important consideration. The buoyancy flows are calculated in simple fracture models and porous medium models representing fractured rock masses. The buoyancy driving force is determined by the thermal disturbances which are dependent on the waste power characteristics, thermal properties of rocks. and depth of the repository. Long-term buoyancy convective flow is calculated as a function of these parameters, as well as the properties of geological formativis and recharge cunditlons.

1184 Influence of faulting on groundwater flow and contaminant transport. Schwartz, F.W. (Alberta Univ " Edmonton (Canada)); Donath, F.A. (IIllinois Univ., Úrbana (USA)). pp 117-136 of Predictive geology with emphasis on nuclear-waste disposal. Marsily, G. de (Ecole des Mines de Paris, Fontainebleau, France. Centre d'Informatique Geologique); Merriam, D.F. (Syracuse Univ., NY (USA)) (eds.). Oxford, England; Pergamon Press (1982). (CONF-8007144-). 1980).

From 26. international geological congress; Paris, France ( $1 \mathrm{Jul}$

It is possible through sensitivity analysis with a model to gain increased understanding of the factors influencing the isolation of highlevel radioactive wastes in the subsurface. Here, we evaluate the importance of geological setting and vertical disruptive features (faults) on the pattern of groundwater flow and contaminant transport. The twodimensional cross section considered in this study is representative of a layered sequence of sedimentary rocks in which a low-conductivity disposal unit occurs. Patterns of groundwater flow and contaminant spread are influenced differently depending upon whether a high- or lowconductivity disruptive zone is added to the downstream end of the flow system. In the situation of a high-conductivity zone, there is little change compared to the unfaulted situation. Only when a repository is located within or immediately adjacent to the disruptive zone is the confining character of the disposal horizon diminished. In the situation of the lowconductivity zone, there are marked changes in flow and transport. With a slight decrease in hydraulic conductivity of the disruptive zone, the confining character of the disposal unit is enhanced. However, as hydraulic conductivity is reduced further, the disposal unit has diminished confining capabilities.

1185 Influence of microfissures in crystalline rock on radionuclide migration. Neretnieks, I. (Royal Inst. of Tech., Stockholm (Sweden)). pp 137-152 of Predictive geology with emphasis on nuclear-waste disposal. Marsily, G. de (Ecole des Mines de Paris, Fontainebleau, France. Centre d'Informatique Geologique); Merriam, D.F. (Syracuse Univ., NY (USA)) (eds.). Oxford, England; Pergamon Press (1982). (CONF8007144-). 1980).

From 26. international geological congress; Paris, France (1 Jul

Escaping radionuclides from a repository for spent nuclear fuel will travel with the water moving through the bedrock. In lowpermeability rocks such as granites and gneisses, the water will move essentially in the fissures of the rock. Solved species however may migrate into the rock matrix by diffusion in the microfissures between grains. The species can migrate far into the rock during the times of interest and thus will be stronger retarded than if the sorption only occurs on the surface of the water-bearing fissures. On the other hand if fissures are far apart, the entire rock mass between fissures will not be reached by the diffusing species. A mathematical treatment of the problem is given as well as data on diffusivities in the matrix. The implications for a final repository are discussed.
$1186 \quad$ Long-term prediction of the fate of nuclear waste deeply buried in granite. Barbreau, A. (CEA Centre d'Etudes Nucleaires de Fontenay-aux-Roses, 92 (France)); Bonnet, M.: Margat, J.; Peaudecerf, P. (Bureau de Recherches Geologiques et Minieres (BRGM), 45 - Orleans (France)); Goblet, P.; Marsily, G. de (Ecole Nationale Superieure des Mines, 75. Paris (France)). pp 153-170 of Predictive geology with emphasis on nuclear-waste disposal. Marsily, G. de (Ecole des Mines de Paris, Fontainebleau, France. Centre d'Informatique Geologique); Merriam, D.F. (Syracuse Univ., NY (USA)) (eds.). Oxford, England; Pergamon Press (1982). (CONF8007144-). 1980).

From 26. international geological congress; Paris, France (1 Jul

The French program for the study of disposal of radioactive waste in deep geological formations is concerned above all with the study of crystalline formations within the framework of a shared expense contract with the Commission of the European Communities. Although the thenretical and experimental work is not finished yet, it has been suggested that a preliminary study be made of the efficiency of such a disposal site; using simplified but realistic simulations based on the results of the first series of measurements made In the field. Even if it is impossible to draw any definite conclusions as to the 'security' of the geological barrier, this method makes it possible to evaluate the importance of cach of the elementary mechanisms and parameters concerned, and these results will lead to a better definition of the work which remains to be done.

1187 Scientific basis for nuclear waste management $V$. Lutze, W. (ed.). New York, NY; North-Holland (1982). 910p. (CONF-820636-). Elsevier Science Publishing Co., Inc., 52 Vanderbilt Ave., New York, NY 10017.

From 5. international symposium on the scientific basis for radioactive waste management; Berlin, F.R. Germany (7 Jun 1982).

Ninety-one papers were presented in five sessions: properties of high-level radioactive waste forms; characterization of repositories; backfill materials and canister corrosion; migration of nuclides in the geosphere, and in-situ solidification of low- and medium-level radioactive waste. Research priorities for nuclear waste isolation are summarized. Eighty-five papers were indexed separately; six papers had already been indexed for the Energy Data Base.

1188 Effects of waste composition and loading on the chemical durability of a borosilicate glass. Clark, D.E.; Maurer, C.A.; Jurgensen, A.R.; Urwongse, L. (Univ. of Florida, Gainesville, FL). pp 1-13 of Scientific basis for nuclear waste management V. Lutze, W. (ed.). New York, NY; North-Holland (1982). (CONF-820636-).

From 5 . international symposium on the scientific basis for radioactive waste management; Berlin, F.R. Germany (7 Jun 1982).

The effects of waste cumpusition and percent loading in a borosilicate glass designed for US defense high level wastes (HLW) have been evaluated. Three types of simulated wastes were investigated; high alumina. high iron and a composite representative of an average waste comp [osition from Savannah River Plant (SRP) waste tanks. Corrosion resistance of the borosilicate glass is significantly enhanced by the presence of any of the three types of wastes. Additionally, corrosion resis. tance is improved as the \% waste loading is increased in the glass. The best corrosion performance was obtained with the high alumina waste in deionized water.

1189 Mechanisms for hydrothermal leaching of nuclear waste glasses: properties and evaluation of surface layers. Malow, G. (Ilahn-Meitner-Institut fuer Kernforschung, Berlin, Germany). pp 25-36 of Scientific basis for nuclear waste management V. Lutze, W. (ed.). New York, NY; North-Holland (1982). (CONF-820636-).

From 5. international symposium on the scientific basis for radioactive waste management; Berlin, F.R. Germany (7 Jun 1982).

The specific weight losses of the glass after leaching in rock salt and $\mathrm{MgCl}_{2}-\mathrm{MgSO}_{4}-\mathrm{NaCl}-\mathrm{KCl}$ solutions were measured and surface layers were investigated. Leaching with various leachants leads to the formation of surface layers. Their composition and structures are complex. From the relative concentrations of the elements in the layers it must be assumed that the layers do not represent a partially leached glass phase and are formed by instantaneous precipitation of insoluble 
compounds, formed upon leaching. The drop in the leach rates suggests that the surface layer protects the long-term stability of the layers are the most important properties which must be known to understand the corrosion kinetics and leaching mechanisms of the glass. The mass release seems to be best fitted by the equation $m_{(t)}>c . t^{n}$, but data are lacking for a precise calculation of the parameters $\mathrm{n}$ and $\mathrm{c}$ for all relevant conditions; $n$ was found to be well below 0.5 . Hence, congruent dissolution and diffusion controlled ion exchange, i.e., selective leaching, can be excluded as rate determining leaching mechanisms. Although the surface layers formed in the varous leachants are different in nature and composition, the protective property for the underlying glass can be assumed to be a common feature.

1190 Stability and alteration of naturally occurring lowsilica glasses: implications for the long term stability of waste form glasses. Allen, C.C. (Univ. of New Mexico, Albuquerque). pp 37-44 of Scientific basis for nuclear waste management $V$. Lutze, W. (ed.). New York, NY; North-Holland (1982). (CONF-820636-).

From 5 . international symposium on the scientific basis for radio active waste management; Berlin, F.R. Germany (7 Jun 1982).

Volcanic glass of basaltic composition $\left(\sim 50 \mathrm{wt} \% \mathrm{SiO}_{2}\right)$ was studied as a natural analugue to propused nuclear waste form glasses. Basaltic glass can resist devitrification and remain glassy for periods in excess of $10 \mathrm{~m} . \mathrm{y}$. Surface alteration of the glass occurs rapidly upon exposure to water or hydrnthermal fluids, but is essentially absent if the glass remains dry. Alteration is restricted to a rind of clay-like, cryptocrystalline palagonite, generally less than $100 \mu \mathrm{m}$ thick. Formation of this rind protects the underlying glass from further alteration. Similar rinds, or gel layers, have been produced in laboratory and field tests with borosilicate glasses. Major cations within the basaltic glass are selectively depleted or enriched relative to the glass in the alteration rind. Qualitatively-similar depletion and enrichment patterns charcterize the short-term leaching behavior of candidate waste form glasses.

1191 Long-term radioactivity release from solidified highlevel waste. Part I. An approach to evaluating experimental data. Altenhein, F.K. (Hahn-Meitner-Institut fuer Kernforschung, Berlin, Germany); Lutze, W.; Ewing, R.C. pp 45-56 of Scientific basis for nuclear waste management V. Lutze, $W$. (ed.). New York, NY; North-Holland (1982). (CONF820636-).

From 5 . international symposium on the scientific basis for radioactive waste management; Berlin, F.R. Germany (7 Jun 1982).

Safety and risk analyses for the isolation of radioactive waste in a repository must begin with a source term to quantify the amount of radioactivity released from the waste form under a specific set of conditions. The interaction of the waste form with aqueous solutions is the most important mechanism to consider, as any material released may be dissolved and reach the biosphere. In this regard the behavior of heat generating high-level waste is of particular importanco, booause reaotion rates are higher at elevated temperatures. A long-term leach rate was derived from previous and continuing experimental work. The purpose of this paper is not to describe the real case release but rather to provide guidelines for the design of leaching experiments and determine the required precisiond for the data. This can be derived from the relative sensitivity of extrapolated leach rates for various parameters measured in laboratory experiments.

1192 Flow model for the kinetics of dissolution of nuclear waste forms; a comparison of borosilicate glass, SYNROC and high-silica glass. Macebo, P.B.; Barkatt, A.; Simmons, J.H. (Catholic Univ. of Amcrica, Washington, DC). pp 57-69 of Scientific basis for nuclear waste management $V$. Lutze, $W$. (ed.). New York, NY; North-Holland (1982). (CONF820636-).

From 5 . international symposium on the scientific basis for radioactive waste management; Berlin, F.R. Germany (7 Jun 1982).

A model has been developed to predict the long-term leach or release rates of various waste-form materials under repository conditoins. The flow model has been applied to results of dynamic tests carried out over the range of flow rates between 0.5 to $300 \mathrm{~m} / \mathrm{yr}$ at a temperature of $70^{\circ} \mathrm{C}$ on three glassy and two crystlline waste-form materials. These materials include Battelle PNL 76-68 borosilicate glass, Savannah River Laboratory TDS-131 borosilicate glass, CU PGM (Porous Glass Matrix) high-silica glass, Rockwell International poly- phase ceramics (with nepheline as the major cesium host phase) supplied as representative of Tailored Ceramics, and Lawrence Livermore Laboratory Synroc-D. Quantitatively, the results indicate that the two borosilicate glasses, PNL 76-68 and SRL TDS-131, and the two ceramic waste-forms, Tailored Ceramics and Synroc-D, all exhibit approximately the same magnitudes of annual fractional loss $(\theta)$ over the entire range of flow rates. In the case of VU PGM (Porous Glass Matrix) highsilica glass, however, $\theta$ is lower by at least one order of magnitude, and at the higher flow rate region by two orders of magnitude, than the annual fractional low exhibited by all the other waste-forms. Most significantly, the results show that high-silica glass is the only material to fulfill the durability criterion required of high-level waste solids, i.e., a long-term value of $\theta$ smaller than $1 \times 10^{-5}(\mathrm{~g} / \mathrm{g} . \mathrm{yr})$ under repository conditions. Reults obtained with other components show that the loss rates based on $\mathrm{B}$ and $\mathrm{Na}$ show less decline than those based on $\mathrm{Si}$ or $\mathrm{Al}$ as the flow rates decrease, but in all cases the loss rates measured for CU PGM glass are lower by 1 to 2 orders of magnitude than those obtained with the other waste-form materials under the same flow conditions.

1193 Waste glass/repository interactions. Clark, D.E.; Maurer, C.A. (Univ. of Florida, Gainesville). pp 71-82 of Scientific basis for nuclear waste management $V$. Lutze, $W$. (ed.). New York, NY; North-Holland (1982). (CONF820636-).

From 5. international symposium on the scientific basis for radioactive waste management; Berlin, F.R. Germany (7 Jun 1982).

The effects of repository material and a tailored backfill or overpack on the leaching behavior of glass have been studied. Two types of glasses were used in this investigation: (1) model glasses comprised of $33 \mathrm{~mol} \%$ alkali oxide-67 mol \% $\mathrm{SiO}_{2}$, and (2) alkali borosilicate glasses with and without simulated wastes. Several types of repository material were placed in the same containers as the glasses to determine if their presence would alter the extent of leaching. A backfill material consisting of phosphate slime/sand mixtures was evaluated with the same procedure. The results indicate that the leaching behavior of the glass may be influenced by the presence of some materials. Preliminary results of a $161 / 2$ month burial experiment in Florida are also discussed.

1194 Leaching of vitrified high-level radioactive waste. Hall, A.R.; Hough, A.; Marples, J.A.C. (AERE Harwell, England). pp 83-92 of Scientific basis for nuclear waste management V. Lutze, W. (ed.). New York, NY; North-Holland (1982). (CONF-820636-).

From 5 . international symposium on the scientific basis for radioactive waste management; Berlin, F.R. Germany (7 Jun 1982).

The work reported here is part of a study of the leaching of individual elements from waste glasses. The first part of the paper reports on leaching of the major constituents at $30^{\circ} \mathrm{C}$, and leaching of the alkalis at $20^{\circ} \mathrm{C}$ from glass UK 189; the second part describes leaching of $\mathrm{Sr}, \mathrm{Cs}$, Tc and various actinides from glasses UK 189 and UK209.

1196 Relationship between glass leaching mechanism and geochemical transport of radionuclides. Avogadro, A.; Lanza, F. (Commission of the European Communities, Ispra, Italy). pp 103-112 of Scientific basis for nuclear waste management $V$. Lutze, W. (ed.). New York, NY; North-Holland (1982). (CONF-820636-).

From 5. international symposium on the scientific basis for radioactive waste management; Berlin, F.R. Germany (7 Jun 1982).

In this paper an attempt has been made to subdivide the different elements present in the vitrified waste into classes in order to simplify the description of the release of the radicelements in the various repository conditions. From the discussion of the different classes it appears clearly that the evaluation of the release of the different radionuclides depends strongly on the transport in the geological medium. In addition, to evaluate the transport it is necessary to know the physico-chemical species under which the various nuclides are leached. Consequently, there is a strong relationship between transport and leaching; any separation of the two phenomena risks being artificial.

1196 Leaching of actinides from simulated nuclear waste glass. Pickering, S. (Commission of the European Communities, Karlsruhe, Germany); Walker, C.T.; Offermann, P. pp 113-124 of Scientific basis for nuclear waste management $V$. Lutze, $W$. 
(ed.). New York, NY; North-Holland (1982). (CONF820636-).

From 5 . international symposium on the scientific basis for radioactive waste management; Berlin, F.R. Germany (7 Jun 1982).

Two types of simulated nuclear waste glass doped with actinides were leached at $200^{\circ} \mathrm{C}$ in distilled water and salt solutions. Am;-Np, Pu and $U$ were all preferentially retained in the surface layer on the glass. Leaching ratios of 0.1 to 0.2 for $\mathrm{Np}$ and $\sim 0.02$ for Am were measured. The losses of Am and Np to the leachant were proportional to the total weight loss of the glass and were larger at $10 \mathrm{ml}$ leachant $/ \mathrm{cm}^{2}$ glass than at $5 \mathrm{ml} / \mathrm{cm}^{2}$. Weight loss from the glass occurred only at the start of the experiments for periods ranging from $10 \mathrm{~h}$ to 10 days according to leachant composition and volume. Wt losses from the C31-3-EC glass were much greater in saturated $\mathrm{NaCl}$ solution than in distilled water. Enrichment in the outer surface layer of $\mathrm{Al}$ or $\mathrm{Ca}$ according to glass type could be correlated with leachant $\mathrm{pH}$, glass composition and weight loss measurements.

1197 Influence of a backfilling material on borosilicate glass leaching. Lanza, F.; Ronsecco, C. (Commission of the European Communities, Ispra, Italy). pp 125-133 of Scientific basis for nuclear waste management V. Lutze, W. (ed.). New York, NY; North-Holland (1982). (CONF-820636-).

From 5 . International symposium on the scientific basis for radio active waste management; Berlin, F.R. Germany (7 Jun 1982).

The results of the leaching tests in different types of paste have shown that the presence of an absorbing medium around the glass tends to eliminate the saturation conditions that are present in water or water solution. It is conceivable that such an effect is transitory. When the clay surrounding the glass is saturated, the composition of the leachate will begin to evolve toward saturation and will probably be diffusioncontrolled. However, at least for the first period, a model is dissolution in large amounts of water fits the experimental data better than a model based on satural effects. Tests performed with addition of $\mathrm{Fe}_{2} \mathrm{O}_{0}$ show that the influence of corrosion products is not negligible. Their presence partially saturates the absorptive center of the clay, altering the leaching conditions. The use of $\mathrm{Fe}_{2} \mathrm{O}_{0}$ to simulate corrosion products is probably a crude approximation. A more realistic approach is needed. If a similarity exists between leaching data tests in paste and in large amounts of water, the surface composition in the two cases is largely different. In the first case, in the external part of the film, silicon precominates while in the second case iron is prevalent. At $80^{\circ} \mathrm{C}$ the surface composition does not seem to affect the capacity of the surface layer of reducing the leaching rate with time. At $50^{\circ} \mathrm{C}$ tests with the highest iron content present lower values of weight losses.

$1198^{\circ}$ High level waste management and characterization at the Hanford plant site. Deichman, J.L. (Rockwell Hanford Operations, Richland, WA). pp 459-462 of Proceedings of the ANS topical meeting on the treatment and handling of radioactive wastes. Columbus, OH; Battelle Press (1982). (CONF820424-).

From ANS topical meeting on treatment and handling of radioactive wastes; Richland, WA, USA (19 Apr 1982).

Of the total 540-million curie fission product inventory at Hanford in 1981, 99\% was generated as high-level waste. Methods of storing this inventory and methods for reducing the volume of this inventory are discussed.

1199 Experience with the storage of high active waste at WAK. Wiese, H.; Schulte, B. Karisruhe, Germany; Fachinformationszentrum fuer Energie, Physik, Mathematik (1982). 20p. (CONF-8209134-).

From IAEA interregional training course on waste management in nuclear facilities; Karlsruhe, F.R. Germany (6 Sep 1982).

The WAK, a reprocessing plant located in the northern part of the Nuclear Research Center, Karlsruhe, is a pilot plant for reprocessing irradiated fuels, the throughput being about 35 tons of uranium per year. The maximum enrichment is $3.1 \%$ U-235 and the chemical reprocessing is carried out using the worldwide well known Purex method. Since the facility first operated in September 1971, fuel elements from 6 light and heavy water reactors containing 177 tons uranium and 540 $\mathrm{kg}$ plutonium have been reprocessed in the Karlsruhe reprocessing plant, WAK. In this way, a large amount of highly irradiated fuel ele- ments from light water reactors with average burn-up rates of $30,0 \mathrm{C}$ and maximum rates of $39,000 \mathrm{Mwd} / \mathrm{t}$ uranium were processed.

1200 Effect of overpack materials on glass leaching in geological burial. Werme, L. (KBS, Stockholm, Sweden); Hench, L.L.; Lodding, A. pp 135-144 of Scientific basis for nuclear waste management V. Lutze, W. (ed.). New York, NY; NorthHolland (1982). (CONF-820636-).

From 5 . international symposium on the scientific basis for radioactive waste management; Berlin, F.R. Germany (7 Jun 1982).

The metallic overpacks $(\mathrm{Pb}, \mathrm{Cu}, \mathrm{Ti})$ have little effect on the corrosion of borosilicate nuclear waste glasses under either $90^{\circ} \mathrm{C}$ or $8^{\circ} \mathrm{C}$ burial conditions in deep granite. Corrosion layers for metal-glassinterfaces are generally thinner than for glass-glass intrefaces. One possible reason for this is that the ductile metallic materials allow only a very thin water film between the glass and the metal. This thin water film becomes rapidly saturated with corrosion products and further corrosion in consequently slower.

1201 Interaction of borosilicate glass and granodiorite at $100^{\circ} \mathrm{C}$, $50 \mathrm{MPa}$ : implications for models of radionuclide release. Savage, D.; Robbins, J.E. (Harwell Lab., Oxfordshire, England). pp 145-152 of Scientific basis for nuclear waste management V. Lutzc, W. (cd.). Ncw York, NY; North-Holland (1982). (CONF-820636-).

From 5. international symposium on the scientific basis for radioactive waste management; Berlin, F.R. Germany (7 Jun 1982).

The interaction of a simulated borosilicate waste glass, granodiorite and deionized water at $100^{\circ} \mathrm{C}, 50 \mathrm{MPa}$ under closed system experimental conditions has revealed the rapid achievement of steady-state fluid concentrations for many chemical components of interest, (e.g., $\mathrm{SiO}_{2}, \mathrm{La}$ ) and their rates of release from the near-field would be most appropriately modelled by a function of solubility and groundwater flow-rate. The conversion of these solubilities into conventional leachrates has shown over five orders of magnitude range in relative release rates and emphasizes the need for source-term models to consider each radionuclide separately in terms of mechanisms of release.

1202 Burial effects on nuclear waste glass. Hench, L.L. (Univ. of Florida, Gainesville); Werme, L.; Lodding, A. pp 153162 of Scientific basis for nuclear waste management $V$. Lutze, W. (ed.). New York, NY; North-Holland (1982). (CONF820636-).

From S. international symposium on the scientific basis for radioactive waste management; Berlin, F.R. Germany (7 Jun 1982).

The purpose of this experiment was to cvaluate the effects of various components of the SKBF/KBS nuclear waste storage system on the leaching of the vitreous waste form. Two configurations of nuclear waste glasses, canisters, overpacks, and backfill material were inserted into 5.6 $\mathrm{cm} \times 3 \mathrm{~m}$ deep boreholes located at the $350 \mathrm{~m}$ level in the Stripa mine. Some were maintained at $90^{\circ} \mathrm{C}$. The others were allowed to equilibrate at the ambient temperature of the mine, about $8^{\circ} \mathrm{C}$. Two borosilicate nuclear waste glass compositions (ABS 39 and ABS 41) compatible with the French AVM process containing $9 \%$ by weight of simulated fission products were compared. The two compositions bracket the range of $\mathrm{SiO}_{2} / \mathrm{Na}_{2} \mathrm{O} / \mathrm{B}_{2} \mathrm{O}_{0}$ ratios likely to be selected for commercial vitrification operations at La. Hague. there arp only minor differenoes in the ion depletion levels at surfaces of glass ABS 39 and 41 during 28 days and $3 \mathrm{mo}$, 90 or $8^{\circ} \mathrm{C}$ burial in Stripa at $350 \mathrm{~m}$. In-depth compositional profiles show growth rates of layers with selective depletion of $\mathrm{Na}, \mathrm{B}$, at up to ca. $1.5 \times 10^{-7} \mathrm{~m} /$ day for glass 39 and up to ca. $2.5 \times 10^{-8} \mathrm{~m} /$ day for glass ABS 41. A thin film rich in $\mathrm{Si}, \mathrm{Fe}$, and $\mathrm{Al}$ forms on both glasses during burial. Moist or wet bentonite accelerates the rate of ion depletion for both glasses, with glass ABS 39 the worst, in both laboratory and Stripa burial experiments. The bentonite effect is much less in burial environments than in lab simulations. There is little difference in burial surface reactions between pineapple slice configurations and minicans.

1203 Chemical durability of some HLW glasses: effects o. hydrothermal conditions and ionizing radiation. Cousens, D.R.; Lewis, R.A.; Myhra, S.; Segall, R.L.; Smart, R.S.C.; Turner, P.S. (Griffith Univ., Queesland, Australia). pp 163-171 of Scientific basis for nuclear waste management $V$. Lutze, $W$. 
(ed.). New York, NY; North-Holland (1982). (CONF820636-).

From 5. international symposium on the scientific basis for radioactive waste management; Berlin, F.R. Germany (7 Jun 1982).

The time dependence of leaching and dissolution under hydrothermal conditions at $250^{\circ} \mathrm{C}$ has been investigated in an autoclave designed for continuous sampling; it is found that the rates are in accord with those obtained by the commonly used procedures. The effect of pressure by itself has been studied in an ultracentrifuge; ambient rates for $\mathrm{Si}$ and $\mathrm{Na}$ loss remain unchanged up to about $40 \mathrm{MPa}$ followed by a monotonic increase to about 1.5 times the ambient rates at $160 \mathrm{MPa}$ The surface layers formed as a result of chemical attack have been examined (XRD, IR, XPS and PIXE). It is found that $\mathrm{Zn}$ present in the glass or in the solution is retained in the siliceous layer which in some circumstances contains a crystalline zinc-hydroxy-silicate phase. A method for enhancing rates of displacement damage by a factor of $\sim$ $10^{6}$ has been developed. Powdered borosilicate glass and glass doped with $\mathrm{UO}_{2}$ have been neutron-irradiated in a high-flux reactor. Damage is thereby introduced in the bulk as a result of ${ }^{10} \mathrm{~B}(\mathrm{n}, \alpha)^{7} \mathrm{Li}$ and $(\mathrm{n}$, fission) events and subsequent slowing-down of energetic charged ions. Irradiation doses equivalent to $10^{6}$ years $\mathrm{HLW}$ storage have been achieved. The resultant stored energy of $\sim 10^{2} \mathrm{~kJ} \mathrm{~kg}^{-1}$ is released over the range 300 to $800 \mathrm{~K}$. The initial leaching/dissolution rates are found to be increased by a factor of about three by irradiation; the changes in longer-term rates are difficult to determine due to the effects of backreactions and changing particle size geometry and size distribution, but significant enhancement is found. Rates for fission and activation products are similar to those for bulk species.

1204 Determination of the corrosion mechanisms of highlevel waste containing glass. Scholze, H.; Conradt, R.; Engelke, H.; Roggendorf, H. (Fraunhofer-Institut fuer Silicatforschung, Wuerzburg, Germany). pp 173-180 of Scientific basis for nuclear waste management V. Lutze, W. (ed.). New York, NY; North-Holland (1982). (CONF-820636-).

From 5. international symposium on the scientific basis for radioactive waste management; Berlin, F.R. Germany (7 Jun 1982).

The observations and investigations of the corrosion behavior of two rather different model waste glasses in salt brines revealed the simultaneous action of several corrosion mechanisms. The differences between the two glasses refer to different extents of the same mechanisms. The glass matrix itself is involved in matrix dissolution (disintegration) and corrosion profile formation processes. The latter comprise silicon enrichment and alkali and alkaline earth depletion. A comparison between the matrix dissolution depths and the profile depths shows that the matrix is submitted to an almost congruent dissolution. Nevertheless the overall time law has an exponent between 0.5 and 1 . Taking into account the results by $G$. Malow one may assume that the reaction layers which are formed on the glass surfaces during corrosion enforce the time law. This assumption has to be modified by the fact that the profiles in the rest glass pass through a temporal development, too. I.e., the conditions for the reaction layer formation are continuously changed because of the surface concentration change of the underlying rest glass. The meaning of either processes for a long term behavior of waste glass till needs clarification.

1205 Leaching behavior of a simple borosilicate glass in a confined environment. Petit, J.C.; Langevin, Y.; Lameille, J.M.; Dran, J.C. pp 203-210 of Scientific basis for nuclear waste management V. Lutze, W. (ed.). New York, NY; North-Holland (1982). (CONF-820636-).

From 5 . international symposium on the scientific basis for radioactive waste management; Berlin, F.R. Germany (7 Jun 1982).

Centimeter-sized blocks of a simple borosilicate glass have been leached in hydrothermal conditions with various rates of water renewal Weight loss measurements reveal that restricted water access does not slow down the dissolution rate of this particular glass as would be expected from solubility limits, but instead accelerates its destruction and its transformation into secondary solid phase still ill-defined.

1206 Comparative study of seven glasses for solidification of nuclear wastes. Nogues, J.L. (Universite des Sciences et Techniques du Languedoc, Cedex, France); Hench, L.L.; Zarzycki, J. pp 211-218 of Scientific basis for nuclear waste man- agement V. Lutze, W. (ed.). New York, NY; North-Holland (1982). (CONF-820636-).

From 5 . international symposium on the scientific basis for radioactive waste management; Berlin, F.R. Germany (7 Jun 1982).

The relative leaching behavior of seven alkali borosilicate glasses considered for immobilization of high-level radioactive wastes was compared using a static $90^{\circ} \mathrm{C}$ leach test similar to $\mathrm{MCC}-1$. With the range of glass compositions studied, it was not possible to determine the effect of each element on leaching behavior, however some conclusions regarding the general influence of the glass network formers can be made: (1) The addition of $\mathrm{Al}_{2} \mathrm{O}_{0}$, between 2 and $5 \%$ (weight) results in a large increase in the chemical durability of the glass. (2) The presence of $\mathrm{Fe}_{2} \mathrm{O}_{0}$, between 1.5 and $3 \%$ (weight) is necessary to develop with $\mathrm{Al}_{2} \mathrm{O}_{0}$ a second protective layer on top of the silica-rich film that results from rapid dealkalization. (3) To obtain good durability the glass composition must contain more than $51 \%$ (weight) of $\left(\mathrm{SiO}_{2}+\mathrm{Al}_{2} \mathrm{O}_{0}\right)$ and more than $54 \%$ (weight) of $\left(\mathrm{SiO}_{2}+\mathrm{Al}_{2} \mathrm{O}_{0}+\mathrm{Fe}_{2} \mathrm{O}_{2}\right)$. (4) The best result was obtained with glass $\mathrm{M3}$ which contans $55.18 \%$ (weight) of $\left(\mathrm{SiO}_{2}+\right.$ $\left.\mathrm{Al}_{2} \mathrm{O}_{0}\right)$ and $56.78 \%$ (weight) of $\left(\mathrm{SiO}_{2}+\mathrm{Al}_{2} \mathrm{O}_{0}+\mathrm{Fe}_{2} \mathrm{O}_{0}\right)$. High leach resistance and good surface stability was present event though this glass contained the largest quantity of $\mathrm{Na}_{2} \mathrm{O}(13.19 \%)$ in the series. (5) the difference between the results obtained at $S a / V>1.0 \mathrm{~cm}^{-1}$ and $S A / V$ $>0.1 \mathrm{~cm}^{-1}$ shows the importance of understanding both the effects of glass composition and solution concentrations on the behavior of nuclear waste glasses.

1207 Chemical stability of simulated HLW forms in contact with clay media. Van Iseghem, P.; Timmermans, W.; De Batist, R. (SCK/CEN, Mol, Belgium). pp 219-227 of Scientific basis for nuclear waste management $V$. Lutze, $W$. (ed.). New York, NY; North-Holland (1982). (CONF-820636-).

From 5 . international symposium on the scientific basis for radioactive waste management; Berlin, F.R. Germany (7 Jun 1982).

The corrosion stability of six simulated HLW forms proposed by several European countries (five borosilicate glasses and one borosilicate glass ceramic) in contact with different media relating to the clay disposal has been investigated for periods up to 80 days and at a surface area to solution volume ratio of $1 \mathrm{~cm}^{-1}$ under non de-aerated conditions. In the reference medium distilled water the corrosion stabilities are largely determined by saturation effects for elements such as $\mathrm{Si}, \mathrm{Ca}$, $\mathrm{Mg}, \mathrm{Sr}, \mathrm{Fe}$ and $\mathrm{U}$. Si saturation is found to be enhanced by a large $\mathrm{Al}_{2} \mathrm{O}_{0}$ concentration in the glass. In the clay-water mixture these saturation effects are must less predominant. Wet clay attacks the waste forms faster than the clay-water mixture, although the corrosion rates tend to decrease with time in both clay media. In general, no simple Arrheniustype temperature dependence for the corrosion behavior is found for the interval between 40 and $200^{\circ} \mathrm{C}$.

1208 Isolation capability of a salt dome utilized for highlevel waste disposal. Hamstra, J. (Netherlands Energy Research Foundation, Petten). pp 419-428 of Scientific basis for nuclear waste management V. Lutze, W. (ed.). New York, NY; HorthHolland (1982). (CONF-820636-).

From 5 . international symposium on the scientific basis for radioactive waste management; Berlin, F.R. Germany (7 Jun 1982).

It can be concluded that a high isolation capability of a salt dome that is to be used for nuclear waste disposal will be dependant of the following prerequisites: the salt dome should have its top rock salt at about $200 \mathrm{~m}$ depth or more, should be sufficiently large in its dimensions and should have clearly reached its post-diapiric stage; the repository is to be developed carefully at greater depth and leaving a large isolation shield of at least $200 \mathrm{~m}$ thickness over and around the emplaced waste; and the abandonment and closure of the repository and the two shaft constructions is to be done carefully too. The conclusion then is that the only phenomenon that could be considered realistic in creating a future breach in the isolation shield will be a continuous subrosion process. Its low dissolutioning rate in the order of one meter every 100,000 years to one meter every million years is a good yardstick for the high isolation capability of the salt dome. The prediction then is that if the prerequisites can be fullfilled the isolation capability will fall outside the scale of human ehronology and appronch infinity.

1209 Dissolution rate of salt domes on the basis of interpretation of measured salinity profiles. Jenson, K.E.L. (ELSAM, Fredericia, Denmark). pp 429-438 of Scientific basis 
for nuclear waste management V. Lutze, W. (ed.). New York, NY; Horth-Holland (1982). (CONF-820636-).

From 5 . international symposium on the scientific basis for radioactive waste management; Berlin, F.R. Germany (7 Jun 1982).

Analytical solutions are described for calculation of salt concentrations in ground water corresponding to a certain dissolution rate of the dome. By using these solutions a method is described, which makes it possible to determine rate of dissolution, ground water velocity, and dispersivity in the strata just above cap rock on basis of measured salinity profiles above the dome. The method is applied on the Danish salt dome Mors located in the northern part of Jutland. The cap rock is located at a depth of $600 \mathrm{~m}$ with limestone strata above. Interpretation of salinity profiles in the limestone just above cap rock gives a transverse dispersivity of $20 \mathrm{~m}$ in the limestone. The pore water velocity, here determined entirely on basis of measured salinity profiles, is found to be about $0.3 \mathrm{~mm} / \mathrm{y}$, which corresponds largely to that derived from measured permeabilities in the limestone. The corresponding average dissolution rate for the dome is determined to $0.0004 \mathrm{~mm} / \mathrm{y}$.

1210 Nuclear waste management comprehensive legislation: Bouquard/Lujan Proposal. Hearings before the Subcommittee on Einergy Kesearch and Production of the Committee on Science and Technology, US House of Representatives, NinetySeventh Congress, First Session, June 17-18; Octoher 5, 7, 1981. Washington, DC; Government Printing Office (1982). 673p.

'l'estimony of witnesses from government, industry, and the environmental communities presented on 4 separate days of hearings on H.R. 5016 - Nuclear Waste Management Comprehensive Legislation is recorded herein. The bill provides for the temporary operation at sites to be selected by the Secretary of Energy of two demonstration facilities containing a minimum of $\mathbf{4 0}$ full-sized canisters of glassified high-level radioactive waste. The glassified wastes will be placed in additional engineered barriers. The wastes will be at sites that will meet all necessary requirements for isolation of the high-level radioactive waste. The first demonstration plant is to be in operation by the end of fiscal year 1987 and the second by the end of fiscal year 1988. This bill is designed to minimize the political barriers to radioactive waste disposal and to set in place a program to demonstrate that the technology exists for safe and permanent disposal. Data are also presented to support the design and construction of full-sized licensed repositories. (BLM)

1211 Study of radiation effects in curium-doped $\mathrm{Gd}_{2} \mathrm{Ti}_{2} \mathrm{O}_{7}$ (pyrochlore) and $\mathrm{CaZrTi}_{2} \mathrm{O}_{7}$ (zironolite). Wald, J.W. (Pacific Northwest Lab., Richland, WA); Offermann, P. pp 369-378 of Scientific basis for nuclear waste management $V$. Lutze, $W$. (ed.). New York, NY; Horth-Holland (1982). (CONF820636-). Contract AC06-76RL01830.

From $S$. international symposium on the scientific basis for radioactive waste management; Berlin, F.R. Germany (7 Jun 1982).

The present work shows that radiation damage in growth in $\mathrm{Gd}_{2} \mathrm{Ti}_{2} \mathrm{O}_{7}$ and $\mathrm{CaZrTi}{ }_{2} \mathrm{O}_{7}$ follows an expected exponential relationship of the form $\Delta V / V_{0}>A[1$-exp(-BD)] based on XRD lattice swelling, while in $\mathrm{CaZrTi}_{2} \mathrm{O}_{7}$, macroscopic swelling was about 1.5 times greater than lattice swelling at the last measurable levels. Estimated values of unit cell volume and density swelling atsaturation for $\mathrm{CaZrTi}_{2} \mathrm{O}_{7}$ are $7.6 \%$ and $10.3 \%$, respectively. The $\mathrm{CaZrTi}_{2} \mathrm{O}^{7}$ phase exhibits anisotropic behavior in which the expansion of the monoclinic cell in the c-direction is over five times that of the a-direction. Despite this relatively large anisotropic expansion rat 10 , no microcracking was observed. Microcracking was not observed in $\mathrm{Gd}_{2} \mathrm{Ti}_{2} \mathrm{O}_{7}$ either, and both materials maintained their physical integrity during the course of the study.

1212 Characterization of mechanical properties of nuclear waste glasses. Richter, H. (Frauhofer-Institut fuer Werkstoffmechanik, Freiberg. Germany); Offermann, P. pp 229-238 of Scientific basis for nuclear waste management $V$. Lutze, $W$. (ed.). New York, NY; North-Holland (1982). (CONF820636-).

From 5 . international symposium on the scientific basis for radioactive waste management; Berlin, F.R. Germany (7 Jun 1982).

In order to provide data for a mechanical stability assessment of nuclear waste forms, fracture mechanics crack growth parameters, strength values, Young's modulus (between room temperature and $500^{\circ} \mathrm{C}$ ) and the coefficient of thermal expansion (between 100 and $400^{\circ} \mathrm{C}$ ) were measured. As far as crack growth and strength is concerned the experimental results show that loading of borosilicate glass with waste oxides does not detrimentally influence the properties of the resulting glass; glass ceramics show an even improved behavior. Young's mudulus and coefficient of thermal expansion also lie in the range of data known for ordinary glasses. The results mean that the glasses investigated can be regarded to have a sufficient potential as a solidification product as far as their mechanical properties are concerned. However, in order to make a complete assessment of the mechnical stability of glassy nuclear waste forms the stress distribution in the glass form under consideration and its flaw size distribution must be analyzed as well.

1213 Leachability of zircon as a function of alpha dose. Ewing, R.C. (Univ. of New Mexico, Albuquerque); Haaker, R.F.; Lutze, W. pp 389-397 of Scientific basis for nuclear waste management V. Lutze, W. (ed.). New York, NY; HorthHolland (1982). (CONF-820636-). Contract AC0676RL01830.

From 5 . international symposium on the scientific basis for radioactive waste management; Berlin, F.R. Germany (7 Jun 1982).

The variation in the leachability of naturally occurring zircons $\left(\mathrm{ZrSiO}_{4}\right)$ as a function of total alpha dose has been measured at $87^{\circ} \mathrm{C}$. For caloulatod dooos in the range of $10^{16}$ to $10^{18}$ alplias/gui there is anl increase of one order of magnitude in the weight percent of zircon that is dissolved and an increase in the leach rate of the zircon $2.9 \times 10^{-8}$ to $2.3 \times 10^{-7} \mathrm{gm} / \mathrm{cm}^{2}$ day. Totally metamict specimens (dose $\geq 10^{19}$ alphas $/ \mathrm{gm}$ ) have leach ratcs as high as $1.8 \times 10^{-5} \mathrm{gIII} / \mathrm{cm}^{2}$ day.

1214 Isotopic fractionation of thorium and uranium upon leaching of monozite: alpha-recoil damage effects. Eyal, $Y$. (Israel Inst. of Tech., Haifa). pp 399-408 of Scientific basis for nuclear waste management V. Lutze, W. (ed.). New York, NY; Horth-Holland (1982). (CONF-820636-).

From 5 . international symposium on the scientific basis for radioactive waste management; Berlin, F.R. Germany (7 Jun 1982).

The present investigation includes a study of the relative dissolution rates of the actinide isotopes ${ }^{208} \mathrm{U},{ }^{204} \mathrm{U},{ }^{202} \mathrm{Th},{ }^{200} \mathrm{Th}$ and ${ }^{228} \mathrm{Th}$ upon leaching of monazite in a bicarbonate-carbonate solution. A preferential dissolution of the radiogenic isotopes ${ }^{204} \mathrm{U},{ }^{200} \mathrm{Th}$ and ${ }^{228} \mathrm{Th}$ relative to their corresponding structurally incorporated isotopes ${ }^{208} \mathrm{U}$ and ${ }^{204} \mathrm{U}$ has been observed in the early stages of the dissolution process as well as in an advanced stage of the leaching process. This isotopic fractionation may be attributed to radiation damage caused by alpha-recoil atoms. The leachability of thorium in the present monazite sample is considerably reduced after heat treatment. The heat treatment has presumably caused a partial annealing of the alpha-recoil damage. Evidence for the occurrence of lattice repair is provided by powder $x$-ray diffraction measurements. The present experiments may indicate that alpha-fecoil damage may endanger the integrity of any crystalline phase in groundwater environments. In particular, the damage may reduce the retention of nuclides formed by alpha transmutations and their decay products in teh solid phase relative to the retention of the corresponding isotopes bonded within the crystal structure. From thorium data reported [Eyal, Y. and Kaufman, A. (1982) Nuclear Technology (in press)] and in the present investigation, it may be inferred that in monozite-like phases the overall alpha-recoil damage may increase nearly in proportion to the alpha-particle dose in the time range $\left(\sim 10^{5} \mathrm{y}\right)$ which is required for the effective isolation of actinide wastes.

1216 Investigation of titanium in metamict $\mathrm{Nb}-\mathrm{Ta}-\mathrm{Ti}$ oxides using the extended $x$-ray absorption fine structure technique. Greegor, R.B. (Boeing Co., Seattle, WA); Lytle, F.W.; Ewing, R.C.; Haaker, R.F. pp 409-418 of Scientific hasis for nuclear waste management V. Lutze, W. (ed.). New York, NY; Horth-Holland (1982). (CONF-820636-).

From 5 . international symposium on the scientific basis for radioactive waste management; Berlin, F.R. Germany (7 Jun 1982).

A preliminary investigation of metamict orthorhombic $\mathrm{AB}_{2} \mathrm{O}_{6}$ complex Nb-Ta-Ti oxides has been conducted using the EXAFS/ XANES technique. This investigation has demonstrated the feasibility of the technique to study complex geologic or simulated radioactive waste samples. The element specificity of the technique has allowed a detailed structural analysis of the Ti site in complex metamict $\mathrm{Nb}-\mathrm{Ta}-\mathrm{Ti}$ oxides. Although preliminary, these results suggest that significant changes in Ti coordination number and geometry may take place during the metamictization process. This finding could have important implications for solid radioactive waste-forms. 
1216 Utilization of charged particle backscattering to itudy the near surface region of glasses. Application to depth profiling of lanthanium, cerium, throium and uranium induced by aqueous leaching. Trocellier, P.; Nens, B.; Engelmann, C. (CEN Saclay, France). pp 193-202 of Scientific basis for nuclear waste management V. Lutze, W. (ed.). New York, NY; NorthHolland (1982). (CONF-820636-).

From 5 . international symposium on the scientific basis for radioactive waste management; Berlin, F.R. Germany (7 Jun 1982).

The main advantage of this analytical method which enables the first micrometer of the glass surface to be studied is its nondestructive character. The results obtained clearly show that aqueous leaching of glass samples containing either lanthanum and uranium or cerium and thorium induces accumulation of these heavy elements in the hydrated layer developed on the sample surface. The effectis apparently less pronounced for the cerium thorium glass.

1217 Analytical models for thermal rock mechanics. Hodgkinson, D. Harwcll, England; UKAEA Atomic Energy Research Establishment (1982). 121p. (AERE-R-10341). H.M. Stationery Office, Price Pound 6.00.

Analytically soluble models are used to examine the consequences of burying a large heat source in hard rock, and in particular the thermal effects of high-level radioactive waste disposal. Models for the temperature, thermal stress and thermal convection of groundwater around buried heat sources are derived and discussed. In each case only the simplest physical models are considered namely heat conduction, linear thermo-elasticity and Darcy flow respectively. Elementary introductions are given to these topics and they are illustrated by a variety of simple analytical models. Finally, models that have been specially developed to assess the thermal impact of buried radioactive waste on its surroundings are described and discussed.

1218 Thermomechanical modeling and the selection of a salt repository site. Tammemagi, H.Y.; Loken, M.C.; Wagner, R.A.; Wigley, M.R. (RE/SPEC Ltd., 4614 Valient Drive, N.W., Suite 201, Calgary, Alberta T3A 0X9). Transactions of the American Nuclear Society; 43: 69-70(1982). (CONF821103-).

From American Nuclear Society winter meeting; Washington, DC, USA (14 Nov 1982).

HIGH-LEVEL RADIOACTIVE WASTES; MATHEMATICAL MODELS; MECHANICAL PROPERTIES; TEMPERATURE EFFECTS; DEFORMATION; HEAT TRANSFER; RADIOACTIVE WASTE DISPOSAL; SALT DEPOSITS; SITE SELECTION; THERMODYNAMIC PROPERTIES; WASTE-ROCK INTERACTIONS

1219 Post-closure performance assessment methodology for the Office of Nuclear Waste Isolation. Wilems, R.E.; Brandstetter, A. (INTERA Environmental Consultants, Inc., 11999 Katy Freeway, Suite 610, Houston, TX 77079). Transactions of the American Nuclear Society; 43: 70-71(1982). (CONF. 821103-).

From American Nuclear Society winter meeting; Washington, DC, USA (14 Nov 1982).

HIGH-LEVEL RADIOACTIVE WASTES; SHUTDOWNS; MATHEMATICAL MODELS; PERFORMANCE; RADIOACTIVE WASTE DISPOSAL; RADIOACTIVE WASTE FACILITIES; UNDERGROUND DISPOSAL

1220 WAPPA: an integrated waste package performance assessment code. Pearson, F.J.; Brecher, A. (INTERA Environmental Consultants, Inc., 11999 Katy Freeway, Suite 610, Houston, TX 77079). Transactions of the American Nuclear Society; 43: 71-72(1982). (CONF-821103-).

From American Nuclear Society winter meeting; Washington, DC, USA (14 Nov 1982).

CONTAINERS; DESIGN; HIGH-LEVEL RADIOACTIVE WASTES; MATHEMATICAL MODELS; PACKAGING; PERFORMANCE; RADIOACTIVE WASTE DISPOSAL; RADIOACTIVE WASTE FACILITIES

1221 Application of performance assessment models to evaluate uncertainty in site characterization. Sykes, J.F.; Andrews, R.W. (INTERA Environmental Consultants, Inc.,
11999 Katy Freeway, Suite 610, Houston, TX 77079). Transactions of the American Nuclear Society; 43: 72(1982). (CONF-821103-).

From American Nuclear Society winter meeting; Washington, DC, USA (14 Nov 1982).

DATA COVARIANCES; UNDERGROUND DISPOSAL; EVALUATION; GEOLOGY; GROUND WATER; HIGH-LEVEL RADIOACTIVE WASTES; MATHEMATICAL MODELS; RADIOACTIVE WASTE DISPOSAL; RADIOACTIVE WASTE FACILITIES; SITE SELECTION

1222 Satisfying performance assessment data needs. Baker, S.M.; Clifton, P.M.; Strait, S.R. (Rockwell Hanford Operations, P.O. Box 800, Richland, WA 99352). Transactions of the American Nuclear Society; 43: 73(1982). (CONF821103-).

From American Nuclear Society winter meeting; Washington, DC, USA (14 Nov 1982).

BASALT; DATA ACQUISITION; EVALUATION; HANFORD RESERVATION; HIGH-LEVEL RADIOACTIVE WASTES; RADIOACTIVE WASTE DISPOSAL; ROCK MECHANICS; GEOLOGIC DEPOSITS; RADIOACTIVE WASTE FACILITIES; PERFORMANCE; RISK ASSESSMENT; SITE SELECTION; UNDERGROUND DISPOSAL

1223 Waste package performance assessment: the importance of the very near-field physicochemical environment. Jacobs, G.K.; Altenhofen, M.K. (Rockwell Hanford Operations, P.O. Box 800, Richland, WA 99352). Transactions of the American Nuclear Society; 43: 73-75(1982). (CONF-821103-). From American Nuclear Society winter meeting; Washington, DC, USA (14 Nov 1982).

CONTAINERS;

GEOCHEMISTRY; HIGH-LEVEL RADIOACTIVE WASTES; MATHEMATICAL MODELS; PACKAGING; PARAMETRIC ANALYSIS; PERFORMANCE; RADIOACTIVE WASTE DISPOSAL; GEOLOGIC DEPOSITS; UNDERGROUND DISPOSAL; WASTE-ROCK INTERACTIONS

1224 Performance assessment in the Nevada Nuclear Waste Storage Investigations. Tyler, L.D.; Brannen, J.P.; Hayden, N.K.; Langkopf, B.S. (Sandia National Laboratories, P.O. Box 5800, Albuquerque, NM 87185). Transactions of the American Nuclear Society; 43: 75(1982). (CONF-821103-).

From American Nuclear Society winter meeting; Washington, DC, USA (14 Nov 1982).

DATA ACQUISITION; EVALUATION; FAULT TREE ANALYSIS; HIGH-LEVEL RADIOACTIVE WASTES; NEVADA TEST SITE; RADIOACTIVE WASTE DISPOSAL; RADIONUCLIDE MIGRATION; RISK ASSESSMENT; SEISMICITY; SITE SEI.ECTION; TUFF

1226 System study and performance constraints for repository horizon selection at the Nevada Test Site. Johnstone, J.K.; Lappin, A.R.; Peters, R.R. (Sandia National Laboratories, P.O. Box 5800, Albuquerque, NM 87185). Transactions of the American Nuclear Society; 43: 75-76(1982). (CONF-821 103-).

From American Nuclear Society winter meeting; Washington, DC, USA (14 Nov 1982).

COMPARATIVE EVALUATIONS; TUFF; RADIOACTIVE WASTE FACILITIES; PERFORMANCE; GEOCHEMISTRY; HIGH-LEVEL RADIOACTIVE WASTES; HYDROLOGY; NEVADA TEST SITE; RADIOACTIVE WASTE DISPOSAL; SITE SELECTION; UNDERGROUND DISPOSAL

1226 Status of the ONWI salt project. Madia, W.J.; Matthews, S.C. (Battelle, Office of NWTS Integration, 505 King Avenue, Columbus, OH 43201). Transactions of the American Nuclear Society; 43: 86(1982). (CONF-821103-).

From American Nuclear Society winter meeting; Washington, DC, USA (14 NoV 1982).

ENVIRONMENTAL IMPACTS; HIGH-LEVEL RADIOACTIVE WASTES; RADIOACTIVE WASTE DISPOSAL; RADIOACTIVE WASTE MANAGEMENT; SALT DEPOSITS; SITE SELECTION; SITE SURVEYS 
1227 Licensing considerations for the NWTS program. Stein, R.; Glora, M.A. (U.S. Department of Energy, Washington, DC 20545). Transactions of the American Nuclear Society; 43: 87(1982). (CONF-821103-).

From American Nuclear Society winter meeting; Washington, DC, USA (14 Nov 1982).

CONSTRUCTION; HIGH-LEVEL RADIOACTIVE WASTES; LICENSING; RADIOACTIVE WASTE DISPOSAL; GEOLOGIC DEPOSITS; RADIOACTIVE WASTE FACILITIES; REGULATIONS; SITE SELECTION; US DOE; US NRC

1228 Solubility-limited fractional dissolution rate of vitrified waste in groundwater. Chambre, P.L.; Pigford, T.H.; Zavoshy, S.J. (University of California, Berkeley, CA 94720). Transactions of the American Nuclear Society: 43: 111-112(1982). (CONF-821103-).

From American Nuclear Society winter meeting; Washington, DC, USA (14 Nov 1982).

BOROSILICATE GLASS; CALCULATION METHODS; DISSOLUTION; GROUND WATER; HIGH-LEVEL RADIOACTIVE WASTES; RADIOACTIVE WASTE DISPOSAL; RADIONUCLIDE MIGRATION; TIME DEPENDENCE; UNDERGROUND DISPOSAL; LEACHING; VITRIFICATION

1229 Effect of temperature on the leaching performance of SYNROC-B waste form. Solomah, A.G. (North Carolina State University, Raleigh, NC 27650). Transactions of the American Nuclear Society: 43: 112-113(1982). (CONF-821103-).

From American Nuclear Society winter meeting; Washington, DC, USA (14 Nov 1982).

EXPERIMENTAL DATA; HIGH-LEVEL RADIOACTIVE WASTES; LEACHING; RADIOACTIVE WASTE DISPOSAL; SOLVENT PROPERTIES; SYNROC PROCESS; SYNTHETIC ROCKS; TEMPERATURE EFFECTS; THERMAL TESTING; UNDERGROUND DISPOSAL

1230 Consequences of radiation effects on zeolites during interim storage. Palau, G.L.; Pillay, K.K.S. (The Pennsylvania State University, University Park, PA 16802). Transactions of the American Nuclear Society: 43: 113-114(1982). (CONF. 821103-).

From American Nuclear Society winter meeting; Washington, IC), USA (14 Nov 1982).

ASTM 1020. CHEMICAL RADIATION EFFECTS; CONTAINERS; CORROSION RESISTANT ALLOYS; CORROSIVE EFFECTS; HIGH-LEVEL RADIOACTIVE WASTES; ION EXCHANGE; RADIOACTIVE WASTE DISPOSAL; RADIOACTIVE WASTE PROCESSING; STAINLESS STEEL-304; STAINLESS STEEL-316; STEELS; CORROSION; ZEOLITES

1231 Laboratory studies of fluid flow through borehole seals. South, D.L.; Daemen, J.J.K. (University of Arizona, Tucson, AZ 85721). Transactions of the American Nuclear Society: 43: 114-115(1982). (CONF-821103-).

From American Nuclear Society winter meeting; Washington, DC, USA (14 Nov 1982).

BENCH-SCALE EXPERIMENTS; BOREHOLES; FLOW RATE; FLUID FLOW; HIGH-LEVEL RADIOACTIVE WASTES; PERFORMANCE TESTING; RADIOACTIVE WASTE DISPOSAL; ROCK-FLUID INTERACTIONS; SEALS; UNDERGROUND DISPOSAL

1232 Water-dilution volume for high-level wastes stored in deep geologic media. Choi, J.S.; Tosetti, R.J. (Bechtel National, Inc., P.O. Box 3965, San Francisco, CA 94119). Transactions of the American Nuclear Society; 43: 122-124(1982). (CONF821103-).

From American Nuclear Society winter meeting; Washington, DC, USA (14 Nov 1982).

DILUTION; DRINKING WATER; GROUND WATER; HIGH-LEVEL RADIOACTIVE WASTES; RADIOACTIVE WASTE DISPOSAL; RADIOECOLOGICAL CONCENTRATION; RADIONUCLIDE MIGRATION; TIME DEPENDENCE; UNDERGROUND DISPOSAL
1233 Monitoring an underground repository with modern seismological methods. Majer, E.L.; McEvilly, T.V.; Kings, M.S. (Univ of Calif, Berkeley). ndium; 18: No. 6, 517-527(Dec 1981).

An experiment utilizing microearthquake and acoustic emission techniques has been designed to investigate the discrete failure and behavior of hard rock in response to storage of high level nuclear waste. Data are presently being collected from a 15 -station, three-dimensional array of piezoelectric sensors surrounding 11 canisters of spent nuclear fuel and 6 electrically heated simulator canisters. The canisters are arranged in a linear array $420 \mathrm{~m}$ beneath the surface in the Climax Stock at the Nevada Test Site. The velocity and attenuation characteristics within the array imply that the rock is not homogeneous at the observed frequencies. The basis for the experiment's concept, the instrumentation emplaced, and preliminary results of the monitoring program are reviewed. 22 refs.

1234 Intermediate, long term and final storage of radioactive substances. Krejsa, P. (Oesterreichisches Forschungszentrum Seibersdorf Ges.m.b.H.). Kernkraftwerk; 22: No. 3, 9-15(Dec 1981). (In German).

The author discusses the problems of nuclear waste management, where radioactive materials may come from industrial, medical and other sources such as tritium from watch faces, as well as from power generaturs. He distingulshes between radioactive waste disjera sion and retention, indicates how waste can be vitrified and reviews the output of radioactive waste from nuclear power generation in Belgium, Canada, West Germany, France, Japan, Sweden, Switzerland and UK. Proposed underground waste storage in Switzerland is discussed.

1235 Human intrusion into geologic repositories for highlevel radioactive waste: potential and prevention. Cameron, F.X. (Nuclear Regulatory Commission, Washington, DC (USA). Office of Nuclear Regulatory Research). Radioactive Waste Management; 2: No. 2, 179-187(Dec 1981).

Isolation of high-level radioactive waste over long periods of time requires protection not only from natural events and processes, but also from the deliberate or inadvertent activities of future societies. This paper evaluates the likelihoud of inadvertent human intrusion due to the loss of societal memory of the repository site. In addition measures to prevent inadvertent intrusion, and to guide future societies in any decision to deliberately intrude into the repository are suggested.

1238 Issues in the use of payments in licu of taxes to provide nuclear waste facility siting incentives. Bjornstad, D.J. (Uak Ridge National Lab., TN (USA)); Guss, E. (Tennessee Univ., Knoxville (USA)). Radioactive Waste Management; 2: No. 2, 125-142(Dec 1981).

High-level, nuclear waste isolation facilities will be federally owned and therefore exempt from local taxation. Because local residents view tax payments as a major benefit of industrial development, the absence of these payments, coupled with the inherent undesirability of hazardous-materials-handling activities, may discourage communities from accepting a facility. One method to overcome this disincentive is for the facility to make payments in lieu of taxes to localities. This paper examines the concept of payments in lieu of taxes and presents statistics describing local fiscal characteristics. The range these payments might take under alternative-payment arrangements is calculated, and the impact of such payments on the facility's user-free schedule is estimated. It is concluded that a payment plan based on hypothetical propertytax liabilities for an identical, but taxable, facility would have a significant revenue impact on most localities but would not increase user fees significantly.

1237 Evaluating glasses for high-level radioactive waste immobilization. Cousens, D.R.; Lewis, R.A.; Myhra, S.; Segall, R.L.; Smart, R.St.C.; Turner, P.S. (Griffith Univ., Nathan (Australia)). Radioactive Waste Management (Oak Ridge, Tennessee); 2: No. 2, 143-168(Dec 1981).

The factors affecting the chemical durability of HLW glasses are identified and discussed. The science and technology underlying the development an optimization of such glasses are reviewed. Specific test: presently used to measure the leaching and dissolution rates for purposes of absolute rate determination and comparative evaluation of different glasses are shown to be less than satisfactory. The present criteria used for the evaluation of HLW glasses may be incomplete and unlikely 
- lead to the development of better materials. Arguments for specific ocedures and techniques which will lead to better understanding of echanisms and more meaningful evaluation of performance, are presented.

1238 Monitoring an underground repository with moderm seismological methods. Majer, E.L.; McEvilly, T.V.; King, M.S. (Lawrence Berkeley Lab., CA). ndium; 18: No. 6, 517-527(Dec 1981). Contract W-7405-ENG-48.

An experiment utilizing microearthquake and acoustic emission techniques has been designed to investigate the discrete failure and behavior of hard rock in response to storage of high level nuclear waste. Data are presently being collected from a 15-station, three-dimensional array of piezoelectric sensors surrounding 11 canisters of spent nuclear fuel and 6 electrically heated simulator canisters. The canisters are arranged in a linear array $420 \mathrm{~m}$ beneath the surface in the Climax Stock at the Nevada Test Site. Because of the unique scale of the experiment (array dimension - $50 \times 20 \times 10 \mathrm{~m}$ ), techniques employed are drawn from the fields of acoustic emission and earthquake analysis. The data are digitized and analyzed in-field via an Automated Seismic Processor (ASP) developed for earthquake studies. Although the discrete events are high frequency in nature (1-10 $\mathrm{kHz})$ their behavior and appearance are typical of shear failure observed in microearthquake sequences. Sought are not only the spatial and temporal characteristics of the events with respect to induced thermal, mechanical, or radiation stresses, but also a complete understanding of the source characteristics relative to these induced stresses. The velocity and attenuation characteristics within the array imply that the rock is not homogenous at the observed frequencies. This paper reviews the basis for the experiment's concept, the instrumentation emplaced, and preliminary results of the monitoring program.

$1239 \quad$ Natural draught centralized dry store for irradiated fuel and active waste. Bradley, N. (National Nuclear Corp. Ltd., London (UK)); Brown, G.A. (Central Electricity Generating Board, London (UK)). Nuclear Engineering International; 26: No. 320, 38-40(Nov 1981).

A modular design is described for the long term dry storage of irradiated fuel and vitrified fission products. The specification set by the Central Electricity Generating Board for the AGR fuel store was that the store should be capable of accommodating the lifetime discharge from 10 AGR reactors (7200 tonnes of irradiated fuel) and be cooled by natural convection. The fuel assemblies should be enclosed in individual steel containers. The store has an area for drying the AGR fuel and containering. The single dry cell storage capacities are, 5 years output from 1300 MWe station stored as fuel elements, or 14 year output from $1300 \mathrm{MWe}$ thermal reactors stored as vitrified fission products.

1240 Study of the migration of leached radionuclides in a natural fissure of granite rock. Seitz, M.G. (Argonne National Lab., IL (USA)); Seliga, M. (Ustav Radioekologie a Vyuzitia Jadrovej Techniky, Kosice (Czechoslovakia)). JADEA; 27: No. 11, 399-404(Nov 1981).

The results are presented of a leach-migration experiment in which radionuclides leached from a borosilicate glass by flowing water were carried downstream where they could react with the surface of a natural fissure of granite rock ( $4.5 \mathrm{~cm}$ long) and thus be captured or they could remain in solution and be carried further downstream. From analyses of the water, rock fissure surface and experimental tubing, $\mathrm{Pu}$ leached from the glass was seen to remain in the proximity of the vitrified waste. Most of the radiocesium was found to have reacted with the rock near the inlet to the fissure; some radiocesium, however, was transmitted through the fissure by the flowing water. Neptunium was the most mobile radioisotope identified with approximately $75 \%$ of the radionuclide leached from the glass being transmitted through the fissure. Inasmuch as the experiment simulates a hypothetical search of a waste repository, the results indicate the great difference in migration characteristics that can be expected for the different radionuclides.

1241 Regulation of geological disposal of high-level radioctive waste. White, L.A. (US Nucl Regul Comm). Bulletin of ie Association of Engineering Geologists; 18: No. 4, 349. s53(Nov 1981).

The Nuclear Regulatory Commission has been actively developing needed regulations over the last two years for the geological disposal of hight-level radioactive waste. Technical criteria are about to be pub- lished in the form of a proposed regulation. The waste packages, underground facility, and geologic setting form the major elements of any geologic repository and the basis of a multibarrier system. Performance objectives and supporting technical criteria have been developed for each of these repository elements to provide benchmarks for scientists and engineers working in each of these major areas. 9 refs.

1242 High level radioactive waste. Cohen, B.L. (Univ. of Pittsburg, PA). Natural Resources Journal; 21: No. 4, 703721(Oct 1981).

High-level radioactive wastes are discussed and compared with the wastes generated from coal-fired power plants. The quantity of waste generated by a large nuclear power plant in one year amounts to about 2-3 cubic yards,; a quantity five million times smaller by weight, and billions of times smaller by volume than the wastes from a coal burning plant. The author concludes that the burden placed on future generations for storing or disposing of nuclear waste will probably be far less than the burden resulting from the consumption of the earth's high grade mineral resources. (JMT)

1243 Subseabed disposal of nuclear wastes. Hollister, C.D. (Woods Hole Oceanographic Institution, MA); Anderson, D.R.; Heath, G.R. Science (Washington, D.C.); 213: No. 4514, 13211325(18 Sep 1981).

Fine-grained clay formations within stable (predictable) deepsea regions away from lithospheric plate boundaries and productive surface waters have properties that might serve to permanently isolate radioactive waste. The most important characteristics of such clays are their vertical and lateral uniformity, low permeability, very high cation retention capacity, and potential for self-healing when disturbed. The most attractive abyssal clay formation (oxidized red clay) covers nearly 30 percent of the sea floor and hence 20 percent of the earth's surface.

1244 Disposal of high level wastes. Masuda, S. (Power Reactor and Nuclear Fuel Development Corp., Tokyo (Japan)); Makino, M.; Ikeda, T. ticky a Kartograficky Obzor; 27: No. 9, 37-42(Sep 1981). (In Japanese).

In order to evaluate the system performance concerning the transfer of radioactivity to environment in the disposal of radio-nuclides in strata, it is necessary to make the model of the system for disposing in strata and to set up the scenario of the transfer of nuclides. The possibility that the disposed nuclides in wastes return to the biosphere is the elution and transport of the nuclides by underground water. The barriers against this possibility are technological barrier, solidification and strata. The transfer equation according to this model of disposal system is given. The computer code MGRAT-3 was used for the calculation of the transfer of nuclides, and the evaluation of system performance was carried out with Ingestion Toxicity Index. The behaviors of various nuclides are briefly explained. The time of starting exudation was used as the parameter showing the soundness of technological barrier. The effect of solidification as the barrier was represented with exuding time as the parameter. As the parameter showing the effect of stratd, the time of water reflux, which is the value dividing distance by underground water velocity, was used. The technological barrier is effective for reducing the effect of nuclides of short half life. Solidification is very useful for reducing the effect, and strata reduce the speed of transfer.

1245 Basic study on storage of tritium waste. Kiyose, R.; Suzuki, A.; Tanaka, S.; Okamoto, T.; Itsukaichi, T. (Tokyo Univ. (Japan). Faculty of Engineering). Tokyo Daigaku Kogakubu Sogo Shikenjo Nenpo; 40: 123-129(Sep 1981). (In Japanese).

Portland cement is probably the cheapest material which will be able to confine tritiated water and have a relatively low exchange rate. While the low rate of exchange is attributed to the monolithic nature of the solid, the hydrating water capacity and the leaching of tritium from cement paste blocks in an aqueous environment are not clear yet. The objective of this study is to measure these values and thereby to assess the engineering feasibility of application of cementation for tritiated water confinement. In this report, leach tests were performed in the following conditions: (1) from uncoated cement blocks immersed in water, (2) from uncoated cement blocks in wet air with humidity varied, (3) from uncoated cement blocks in water, tritium being absorbed in molecular sieve $4 \mathrm{~A}$ mixed uniformly in cement blocks, (4) from asphalt. 
coated cement blocks immersed in water, (5) from paraffin-coated cement blocks immersed in water.

1246 Environmental impact of nuclear power. Opening address. Lamont, N. Nuclear Energy (Institution of Nuclear Engineers): 20: No. 4, 279-281(Aug 1981).

From The environmental impact of nuclear power; London, UK (1. 2 Apr 1981)

The public acceptability of nuclear power is discussed. The UK government view on the waste management scene is presented. The dispusal of highly active wastes will be vitrified after which it will need to be stored for some time to cope with the significant amounts of heat produced. The subsequent method of disposal is yet to be decided upon, but four options are available: continued storage, disposal on the ocean bed, burial under the sea bed, and burial deep underground. Research is currently underway for a land-based facility for medium active waste disposal.

1247 Redox behavior, complexing, and adsorption of hexavalent actinides by humic acid and selected clays. Nash, K.; Fried, S.; Friedman, A.M.; Sullivan, J.C. (Argonne National Lab., IL). dnichestvo Stran-Chlenov SEV; 15: 834-837(Jul 1981).

The sediments smectite and illite clay strongly adsorb $\mathrm{Np}(\mathrm{VI})$ from deoxygenated artificial seawater solution. In addition the former reduces the $\mathrm{Np}(\mathrm{VI})$ to $\mathrm{Np}(\mathrm{V})$. Humic acid strongly adsorbs $\mathrm{U}(\mathrm{VI})$. INp(VI), and Pu(VI) from aqueous bicarbonate-carbonate media. The humic acid reduces $N p(V I)$ to $N p(V)$, and $P u(V I)$ to $P u(I V)$, but does not reduce $U(V I)$. Detailed spectrophotometric evidence for the complex-forming reactions is presented.

1248 Status of radioactive waste management: needs for reassessment. Eisenbud, M. (New York Univ., NY (USA). Inst. of Environmental Medicine). dnichestvo Stran-Chlenov SEV; 40: No. 4, 429-437(Apr 1981).

Three systems of radioactive waste management, land burial of wastes from biomedical laboratories, storage in mined cavities, and use of the oceans, are discussed briefly for the purpose of illustrating the need for re-examination of the basic approaches being taken at the present time. It is concluded that most of the low level wastes from biomedical institutions need not be shipped to burial grounds, but can be incinerated on site subject only to restrictions determined by the nonradioactive characteristics of the wastes. With respect to storage of high level wastes, it is suggested that studies of the mobilization rates of natural ore bodies may provide the best way of modeling the behavior of selected waste forms over long periods of time. The oceans, particularly the deep ocean sediments, should be more thoroughly investigated as a possible disposal option.

1249 Pussibility of high level waste underground disposal. Pigford, T.H. (California Univ., Berkeley (USA). Dept. of Nuclear Engineering); Masuda, S. ticky a Kartograficky Obzor; 27: No. 2, 31-36(Feb 1981). (In Japanese).

The possibility that the high level wastes disposed underground return to the biosphere again is the dissolution and transport of radioactive nuclides by underground water. As the strata suitable to underground disposal, rock salt strata without underground water, and granite or shale strata in which the movement of underground watcr is slight are enumerated as the candidates. Wastes are formed into solidified bodics like glass, unreuver the technical measures such as canisters and overpacks are applied, therefore even if underground water intrudes into the places of disposal, radioactive nuclides can be contained for considerable time. At the time of selecting the most suitable stratum and designing and evaluating the place of disposal to construct the underground disposal system with high potential for high level wastes, it is necessary to predict the movement of radioactive nuclides from the dissolution into underground water to the return to the biosphere. The potential danger of high level wastes, the danger of high level wastes disposed underground, the effect of isolation distance (the thickness of strata), and the comparison of the danger due to uranium ore and slag and the places of underground disposal are explained. The danger due to uranium ore and slag occurs early and lasts long, and is 1000 times as dangerous as the high level wastes disposed underground.

1260 Analysis of diffusional ion transport in ocean sediments: subseafloor disposal of radioactive waste. Nuttall, H.E.;
Ray, A.K. (Univ of NM, Albuquerque, USA). Nuclear Tech. nology; 52: No. 2, 260-272(Feb 1981).

Diffusion-controlled transport of radioactive ions through seabes sediments is analyzed to determine the rates of transport between a buried canister releasing radioactive waste and the ocean floor. The general solution of the unsteady-state diffusion problem is obtained for an arbitrary release rate from the canister, and analytical solutions are obtained for two special cases: instantaneous release of the ionic species, and a constant discharge rate. 14 refs.

1251 Ocean-bottom disposal of fission-product krypton85. Dalen, A. van (Netherlands Energy Research Foundation, Petten). pp 171-180 of Saishori, Hoshasei haikibutsu shori shobun; Berugi, Nippon, Oranda, Soren yon-kakoku godo senmonka semina hokokusho. Tokyo, Japan; Japan Atomic Industrial Forum, Inc. (Jan 1981). (CONF-8011169-).

From Tripartite symposium on reprocessing of spent nuclear fuel and treatment of associated radioactive waste; Leningrad, USSR (17 Nov 1980).

If the studies on the radiological consequences of unrestricted release of ${ }^{85} \mathrm{Kr}$ indicate a too high level of radioactivity in the atmoopherio envirnnment sturaye or dispusul becomes necessary. Several modes of temporary confinement for sufficient decay are under investigation. This study considers the disposal in double walled containers at the ncean bottom. Arrival in the atmospheric environment of the noble gas will bo delayed al least for a hundred years. For the concept of the container the IAEA recommendations for container types for transport of radioactive material were taken as guidelines. Actually no authorized description exists on containers of compressed gases, their transport and disposal at the ocean bettom.

1252 Radioactive waste legislation. Hearings before the Subcommittee on Energy and the Environment of the Committee on Interior and Insular Affairs, House of Representatives, Ninety-Seventh Congress, First Session on H.R. 1993; H.R. 2800; H.R. 2840; H.R. 2881; H.R. 3809, June 23 and 25; July 9, 1981. Washington, DC; Government Printing Office (1981). 675p. GPO.

This three-day hearing was held to consider five bills on radioactive waste, set priorities for a repository construction program, consider an administration proposal to develop a small-scale test facility for deep permanent storage of high-level wastes, and determine what role the public and private sectors should assume. The hearing record includes the text of H.R. 1993, H.R. 2800, H.R. 2840, H.R. 2881, and H.R. 3809; the statements of 10 witnesses and two panels; and three appendices with additional material submitted for the record. (DCK)

1263 Submarine geologic disposal of nuclear waste. Anderson, D.R.; Hollister, C.D. Transactions of the American Nuclear Society; 38: 87-88(1981). (CONF-810606-).

From American Nuclear Society's annual meeting; Miami Beach, FL, USA (7 Jun 1981)

ATLANTIC OCEAN; GEOLOGIC DEPOSITS; HEAT TRANSFER; MARINE DISPOSAL; PACIFIC OCEAN; RADIOACTIVE WASTE DISPOSAL; SEDIMENTS; SORPTION; SEA BED; HIGH-LEVEL RADIOACTIVE WASTES; FEASIBILITY STUDIES

1264 Rock-melt concept for nuclear waste disposal. Cohen, J.J.; Tewes, H.A. (SAI, Pleasanton). Transactions of the American Nuclear Society; 38: 89-90(1981). (CONF-810606-). From American Nuclear Snciety's annual mesting; Miami Beach, FL, USA (7 Jun 1981).

ENVIRONMENTAL IMPACTS; HEAT STORAGE; MELT. ING; RADIOACTIVE WASTE DISPOSAL; SOCIO-ECONOMIC FACTORS; SOLIDIFICATION; UNDERGROUND DISPOSAL; HIGH-LEVEL RADIOACTIVE WASTES; IGNEOUS ROCKS; CAVITIES; INTERMEDIATE-LEVEL RADIOACTIVE WAS'IES; LOW-LEVEL RADIOACTIVE WASTES

1255 Three aspects of reliability for a geological nucleal waste repository. Frank, M.V.; Orvis, D.D.; Fleming, K.N.; Henderson, N.C. (General Atomic, San Diego, CA 92138). Transactions of the American Nuclear Society; 38: 167-168(1981). (CONF-810606-). 
From American Nuclear Society's annual meeting; Miami leach, FL, USA (7 Jun 1981).

AVAILABILITY; DESIGN; HIGH-LEVEL RADIOACTIVE WASTES; MARKOV PROCESS; RADIOACTIVE WASTE FACILITIES; RADIOACTIVE WASTE DISPOSAL; RELIABILITY; SYSTEMS ANALYSIS; UNDERGROUND DISPOSAL; GEOLOGIC DEPOSITS

1256 Risk assessment for storage of high-level waste in single shell tanks. Wood, D.E. (Rockwell Hanford, Richland, WA 99352). Transactions of the American Nuclear Society; 38: 106-107(1981). (CONF-810606-).

From American Nuclear Society's annual meeting; Miami Beach, FL, USA (7 Jun 1981).

FAILURE MODE ANALYSIS; FAULT TREE ANALYSIS; GENETIC EFFECTS; HEALTH HAZARDS; HIGH-LEVEL RADIOACTIVE WASTES; NEOPLASMS; RADIATION HAZ ARDS; RISK ASSESSMENT; RADIOACTIVE WASTE STORAGE; TANKS

1257 Disposal of high-level radioactive wastes. Smart, R.S.C.; Myhra, S.; Segall, R.L.; Turner, P.S. (Griffith Univ., Brisbane (Australia). School of Science). pp 1-20 of A.N.Z.A.A.S. Congress papers: 51 st: 1981 . Sydney, Australia; University of New South Wales Libraries (1981). (CONF8105115-). 1981).

From 51. ANZAAS Congress; Brisbane, Australia (11 May

This paper considers wastes produced during the uranium fuel cycle with particular reference to the high level wastes from spent fuels. Methods for high level waste disposal are outlined. Glass and Synroc, as solid waste forms, are compared in terms of chemical, thermal, pressure and irradiation stability.

1258 Nuclear criticality safety analyses for the Waste Isolation Pilot Plant project. Lipner, M.H.; Raverts, J.M. (Westinghouse-NTD, Pittsburgh, PA 15230). Transactions of the American Nuclear Society; 38: 362-363(1981). (CONF810606-).

From American Nuclear Society's annual meeting; Miami Beach, FL, USA (7 Jun 1981).

CALCULATION METHODS; CRITICALITY; DISCRETE ORDINATE METHOD; PLUTONIUM; RADIOACTIVE WASTE DISPOSAL; SAFETY; TRANSPORT THEORY; TRANSURANIUM ELEMENTS; UNDERGROUND STORAGE; RADIOACTIVE WASTE FACILITIES; PILOT PLANTS; HIGH-LEVEL RADIOACTIVE WASTES

1259 Criticality safety of high-level waste containing unseparated plutonium. Christensen, R.N.; Yates, K.R. (Ohio State Univ, Columbus, OH 43210). Transactions of the American Nuclear Soclety; 38: 363-364(1981). (CONF-810606-).

From American Nuclear Society's annual meeting; Miami Beach, FL, USA (7 Jun 1981).

CALCINATION; CRITICALITY; FUEL CYCLE; HIGHLEVEL RADIOACTIVE WASTES; PLUTONIUM; PROLIFERATION; REPROCESSING; SAFETY; URANIUM

1260 Rapid estimation of the regional thermal impact of a nuclear waste repository. Blesch, C.J.; Christenden, R.N.; Kulacki, F.A. (Ohio State Univ, Columbus, OH 43210). Transartions of the American Nuclear Society: 39: 177-179(1981). (CONF-811103-). 1981).

From ANS winter meeting; San Francisco, CA, USA (29 Nov

DESIGN; ENVIRONMENTAL IMPACTS; HEAT TRANSFER; HIGH-LEVEL RADIOACTIVE WASTES; PARAMETRIC ANALYSIS; RADIOACTIVE WASTE FACILITIES; TEMPERATURE CONTROL; SPENT FUEL STORAGE; TEMPERATURE GRADIENTS; RADIOACTIVE WASTE DISPOSAL; SPENT FUELS

1261 Radiological risks for the alternatives for the longterm management of high-level wastes at West Valley. Peterson, J.M.; Rodger, W.A.; Yuan, Y.C. (ANL, Argonne, IL 60439).
Transactions of the American Nuclear Society; 39: 179-180(1981). (CONF-811103-). 1981).

From ANS winter meeting; San Francisco, CA, USA (29 Nov

ANL; DEMONSTRATION PROGRAMS; ENVIRONMENTAL IMPACT STATEMENTS; HEALTH HAZARDS; HIGHLEVEL RADIOACTIVE WASTES; RADIOACTIVE WASTE MANAGEMENT; RISK ASSESSMENT; SOLIDIFICATION; WEST VALLEY PROCESSING PLANT

1262 Radiation and transmutation effects on crystalline radioactive waste forms. Vance, E.R. (Pennsylvania State Univ, University Park, PA 16802). Transactions of the American Nuclear Society; 39: 353-354(1981). (CONF-811103-). 1981). From ANS winter meeting; San Francisco, CA, USA (29 Nov

CHEMICAL REACTIONS; FISSION PRODUCTS; PHYSICAL RADIATION EFFECTS; RADIOACTIVE WASTE DISPOSAL; TRANSMUTATION; RADIOACTIVE WASTE PROCESSING; SOLIDIFICATION; SIMULATION; URANIUM; DOPED MATERIALS; HIGH-LEVEL RADIOACTIVE WASTES; CESIUM; STRONTIUM; LITHIUM 6; BORON 10; PLUTONIUM 238; CURIUM 244

1263 Nuclear fuel waste disposal - status of the Canadian program. Rosinger, E.L.J.; Rummery, T.E. (At Energy of Can Res Co, Pinawa, Manit). Proceedings of the Symposium on Waste Management; 1: 87-108(1981). (CONF-810217-).

From ANS waste management conference; Tucson, AZ, USA (23 Feb 1981)

Canada has sufficient uranium reserves to fuel its nuclear power program well into the next century. To ensure a long-term future, the prospects of using the thorium cycle in Canada's reactors are being examined. Pending a decision on fuel recycling, two options for the management of nuclear fuel wastes are being studied: disposal of intact used fuel bundles or disposal of the wastes that would result from reprocessing. The research, development and demonstration program has three stages - concept assessment, site selection and the construction and operation of a demonstration disposal facility. These are discussed in the paper, along with the major program elements of environmental and safety assessment, geotechnical research, and immobilization studies. 38 refs.

1264 Retrievability: the NWTS position. Carbiener, W.A. (Battelle, Off of Nucl Waste Isol, Columbus, Ohio, USA). Proceedings of the Symposium on Waste Management; 1: 487-495(1981). (CONF-810217-). (23 Feb 1981).

From ANS waste management conference; Tucson, AZ, USA

The Nuclear Regulatory Commission (NRC) recently presented for public comment its draft regulations (10CFR60) which include a concept of retrievability for high-level nuclear wastes in geologic repositories. This paper describes the proposed National Waste Terminal Storage Program (NWTS) position related to the concept of retrievability by defining the position, discussing the basis for the position, and identifying the considerations and impacts on repository systems.

1265 Geological status of NWTS repository siting activities in the paradox basin. Frazier, N.A.; Conwell, F.R. (Battelle, Off of Nucl Waste Isol, Columbus, Ohio, USA). Proceedings of the Symposium on Waste Management; 2: 1135-1 145(1981). (CONF-810217-).

From ANS waste management conference; Tucson, AZ, USA (23 Feb 1981).

Emplacement of waste packages in mined geological repositories is one method being evaluated for isolating high-level nuclear wastes. Granite, dome salt, tuff, basalt and bedded salt are among the rock types being investigated. Described in this paper is the status of geological activities in the Paradox Basin of Utah and Colorado, one region being explored as a part of the National Waste Terminal Storage (NWTS) program to site a geological repository in bedded salt.

1266 DOE's program for high-level radioactive waste disposal. Heath, C.A. (US DOE). pp 1-17 of Atomic Industry 
Forum, fuel cycle conference ' 81,1981 . Washington, DC; Atomic Industry Forum (1981). (CONF-810324-). 1981).

From Fuel cycle conference; Los Angeles, CA, USA (15 Mar

There are a number of ways in which discharged spent fuel can be handled. These are, of course, a resumption of reprocessing as originally-planned, with recycle of mixed oxides in LWR's and disposal of vitrified waste; interim storage at central facilities with eventual reprocessing for use either in LWR's or breeders; or interim storage prior to direct disposal. In the fall of 1979 , there were a number of uncertainties concerning reprocessing. Consideration of the direct disposal of spent fuel as a reference case allowed a focus on the primary issues related only to waste disposal. In order to ensure that the NRC can make an appropriate rule concerning the future availability of waste disposal, it seems essential that the Department's program continue towards qualification of sites suitable for a system of large-scale regional repositories with adequate capacity to handle all the wastes that will be generated by current and future power plants within a reasonable time frame. The Nuclear Regulatory Commission has now passed into law procedural requirements for the characterization of sites leading up to an application for the construction of a full-scale waste repository. It is now required that exploratory shafts be developed and underground examination of sites take place prior to the consideration of any license application.

1267 Fuel Cycle Conference Atomic Industrial Forum. Incorporated. Knotts, J.B. Jr. (Debevoise \& Liberman, Washington, DC). pp 1-25 of Atomic Industry Forum, fuel cycle conference ' 81,1981 . Washington, DC; Atomic Industry Forum (1981). (CONF-810324-). 1981).

From Fuel cycle conference; Los Angeles, CA, USA (15 Mar

This rulemaking is a judicially-mandated effort by the Nuclear Regulatory Commission (NRC) to reassess its confidence that safe, permanent disposal of high-level nuclear waste will be available at the expiration of certain current reactor operating licenses. This article outlines the background of this rulemaking describes its status at the present time, and discusses some of the interactions between the rulemaking and reactor licensing, Congressional initiatives, the DOE waste management program, and new policy directions associated with the change of Administration.

1268 Phantom dissolution leach model. Richardson, G.L. (Hanford Eng Dev Lab, Richland, Wash). Nuclear and Chemical Waste Management; 2: No. 3, 237-241(1981).

A simplified method for analyzing leach data from monolithic solids is proposed that treats the combined leach mechanisms as a dissolution process. With this model, the leachant is assumed to remove the leached constituents as if they were dissolved from the surface of a phantom solid that decreases in size with time as leaching proceeds. The depth of penetration of the leachant to the phantom surface at any time is given by an equation. 14 refs.

1269 Monitoring an underground repository with modern seismological methods. Majer, E.L. (Lawrence Berkeley Lab., CA); McEvilly, T.V.; King, M.S. ndium; 18: 517-527(1981). Contract W-7405-ENG-48.

An experiment utilizing microearthquake and acoustic emission techniques has been designed to investigate the discrete failure and behavior of hard rock in response to storage of high level nuclear waste. Data are presently being collected from a 15 -station, three-dimensional array of piezoelectric sensors surrounding 11 canisters of spent nuclear fuel and 6 electrically heated simulator canisters. The canisters are arranged in a linear array $420 \mathrm{~m}$ beneath the surface in the Climax Stock at the Nevada Test Site. Because of the unique scale of the experiment, techniques employed are drawn from the fields of acoustic emission and earthquake analysis. The data are digitized and analyzed infield via an Automated Seismic Processor developed for eqrthquake studies. Although the discrete events are high frequency in nature their behavior and appearance are typical of shear failure observed in microearthquake sequences. Sought are not only the spatial and temporal characteristics of the events with respect to induced thermal, mechanical, or radialiun stresses, but also a compete understanding of the source characteristics relative to these induced stresses. The velocity and attenuation characteristics within the array imply that the rock is not homogenous at the observed frequencies. This paper reviews the basis for the experiment's concept, the instrumentation emplaced, and preliminary results of the monitoring program.

1270 Importance of creep failure of hard rock in the near field of a nuclear waste repository. Blacic, J.D. (Los Alamos National Laboratory, NM, (USA)). pp 121-131 of Proceedings of the workshop on near-field phenomena in geologic repositories for radioactive waste, Seattle, 31 Aug - 3 Sep 1981. Paris, France; OECD (1981). (CONF-810861-).

From OECD Nuclear Energy Agency workshop on near-field phenomena in geologic respositories for radioactive waste; Seattle, WA, USA (31 Aug 1981).

Potential damage resulting from slow creep deformation intuitively seems unlikely for a high-level nuclear waste repository excavated in hard rock. However, recent experimental and modeling results indicate that the processes of time-dependent microcracking and waterinduced stress corrosion can lead to significant reductions in strength and alteration of other key rock properties in the near-field region of a repository. We review the small data base supporting these conclusions and stress the need for an extensive laboratory program to obtain the new data that will be required for design of a repository.

1271 Parametric study of the effects of thermal environ= ment on a waste package for a tuff repository. Johnstone, J.K.; Sundberg, W.D.; Kr̈ümhänsl, J.L. (Sandia National Laboratolies Albuquerque, NM, (USA)). pp 219-229 of Proceedings of the workshop on near-field phenomena in geologic repositories for radioactive waste, Seattle, 31 Aug - 3 Sep 1981. Paris, France; OECD (1981). (CONF-810861-).

From OECD Nuclear Energy Agency workshop on near-field phenomena in geologic respositories for radioactive waste; Seattle, WA, USA (31 Aug 1981).

The thermal environment has been modeled in a simple reference waste package in a tuff repository for a variety of variables. The waste package was composed of the waste form, canister, overpack and backfill. The emplacement hole was $122 \mathrm{~cm}$ dia. Waste forms used in the calculations were commercial high level waste (CHLW) and spent fuel (SF). Canister loadings varied from 50 to $100 \mathrm{~kW} /$ acre. Primary attention was focused on the backfill behavior in the thermal and chemical environment. Results are related to the maximum temperature calculated for the backfill. These calculations raise serious concerns about the effectiveness of the backfill within the context of the total waste package.

1272 Transport phenomena of water and gas components within rock salt in the temperature field of disposed high level waste. Jockwer, Norbert (Gesellschaft fuer Strahlen- und Umweltforschung mbH, Braunschweig (F.R.Germany)). pp 269-277 of Proceedings of the workshop on near-field phenomena in geologic repositories for radioactive waste, Seattle, 31 Aug - 3 Sep 1981. Paris, France; OECD (1981). (CONF810861-).

From OECD Nuclear Energy Agency workshop on near-field phenomena in geologic respositories for radioactive waste; Seattle, WA, USA (31 Aug 1981).

Rock-salt formations, which will be used for the disposing of high level radioactive waste contain small amounts of water and gas components $\left(\mathrm{H}_{2} \mathrm{~S}, \mathrm{CO}_{2}\right.$ and hydrocarbons) which may interact with the waste containers or the solidified waste products and may be the transport media for radionuclides. These water- and gas components will be liberated in the elevated temperature field of the disposed high level waste and may migrate on the crystal boundary surfaces or intergranular pores as a result of the gradient of total pressure, partial pressure, concentration and temperature. In order to calculate the transport phenomena of these liberation products a diffusion and migration model has been developed.

1273 Assessment of barrier effects on isolation system performance of geologic disposal of high level waste. Masuda, S.; Mano, T. (Power Reactor and Nuclear Fuel Development Corporation, Tokyo, (Japan)); Makino, M.; Ikeda, T. (JGC Corporation, Yokohama, (Japan)). pp 345-360 of Proceedings of the workshop on near-field phenomena in geologic repositories for radioactive waste, Seattle, 31 Aug - 3 Sep 1981. Paris, France; OECD (1981). (CONF-810861-). 
From OECD Nuclear Energy Agency workshop on near-field enomena in geologic respositories for radioactive waste; Seattle, WA, iA (31 Aug 1981).

A sensitivity analysis was performed for assessing the effects of barriers, such as waste form, engineered barrier and geosphere, on the isolation system performance of the geologic disposal of high level wastes. For near-field phenomena, (1) time of initial release and (2) leach time were selected for assessing the sensitivity of the effects of waste form and engineered barrier, including waste package, buffer material and backfill. For far-fiel phenomena, following parameters were selected: (3) path length, (4) groundwater flow velocity, (5) retardation factor of radionuclides and (6) dispersion coeffecient of radionuclides. In this analysis, the values of parameters were selected in the wide range and for each target value of isolation system performance, acceptable combinations of each parameter values were derived.

1274 Waste package performance assessment. Lester, D.H. (Science Applications, Inc). pp 361-373 of Proceedings of the workshop on near-field phenomena in geologic repositories for radioactive waste, Seattle, 31 Aug - 3 Sep 1981. Paris, France; OECD (1981). (CONF-810861-).

From OECD Nuclear Energy Agency workshop on near-field phenomena in geologic respositories for radioactive waste; Seattle, WA, USA (31 Aug 1981).

This paper describes work undertaken to assess the lifeexpectancy and post-failure nuclide release behavior of high-level and waste packages in a geologic repository. The work involved integrating models of individual phenomena (such as heat transfer, corrosion, package deformation, and nuclide transport) and using existing data to make estimates of post-emplacement behavior of waste packages. A package performance assessment code was developed to predict time to package failure in a flooded repository and subsequent transport of nuclides out of the leaking package. The model has been used to evaluate preliminary package designs. The results indicate, that within the limitation of model assumptions and data base, packages lasting a few hundreds of years could be developed. Very long lived packages may be possible but more comprehensive data are needed to confirm this.

1275 High-level waste disposal. Rothemeyer, H.; Closs, K.D. (PTB-Germany, D-3300 Braunschweig). Transactions of the American Nuclear Society; 37: 165(1981). (CONF801107-).

From ANS international conference; Washington, DC, USA (17 Nov 1980).

HIGH-LEVEL RADIOACTIVE WASTES; LEGAL ASPECTS; LICENSING; RADIOACTIVE WASTE DISPOSAL; REGULATIONS; SITE SELECTION

1276 Valence states of actinides in synthetic monazites. Kelly, K.I.; Reall, F.W.; Young. J.P.; Boatner, L.A. (Oak Ridge National Lab, TN). pp 189-195 of Scientific basis for nuclear waste management. Volume 3. Moore, J.G. (ed.). New York, NY; Plenum Publishing Corporation (1981). Contract W-7405-ENG-26.

The valence states of various actinides doped into the lanthanide orthophosphates $\left(\mathrm{LnPO}_{4}\right)$ have been investigated. Actinide-doped $\mathrm{LnPO}_{4}$ single crystals were grown by means of a flux technique and the actinide valence states were determined by optical absorption spectrophotometry. Radiation damage effects were also studied in these systems. The lanthanide orthophosphates are found in nature in the form of the mineral monazite and synthetic analogs of this substance represent a promising primary containment medium for the isolation of highlevel nuclear wastes. 6 figures, 1 table.

1277 Low-gradient permeability testing of fine-grained marine sediments. Silva, A.J.; Hetherman, J.R.; Calnan, D.I. (Univ of RI, Kingston, USA). American Society for Testing and Materials, Special Technical Publication; 121-136(1981). CONF-7906121-).

From Permiability and groundwater containment transport symosium; Philadelphia, PA, USA (17 Jun 1979).

An apparatus is described which is used to determine the consolidation and permeability characteristics of a wide range of deep sea sediments. The unit can also be used to study temperature effects in the range of 2 to $200^{\circ} \mathrm{C}$. Rather accurate readings of fine-grained sediment are possible. Test procedures as well as some experimental results are also reported. 23 refs.

1278 Radiation effects in crystalline high-level nuclear waste solids. Weber, W.J.; Wald, J.W.; Gray, W.J. (Pacific Northwest Lab., Richland, WA). pp 441-448 of Scientific basis for nuclear waste management. Volume 3. Moore, J.G. (ed.). New York, NY; Plenum Publishing Corporation (1981). Contract AC06-76RL01830.

Glass, cement, and crystalline-ceramic waste forms are being considered as potential solid forms for incorporation of nuclear wastes. The solidified waste were subjected to high doses of many different radiations which may measurably alter physical properties and/or affect the durability of the solid waste form. The long-term stability of these waste forms is a subject of continuing research. General radiation damage problems and experimental results from studies of the effects of alpha decay, alpha bombardment, and transmutations on crystalline waste forms, related single-phase compounds, and some glass waste forms were included. Both alpha-recoil damage in actinide host phases and alpha damage to all other phases must be considered since significant structural changes may occur from either source. The ingrowth of damage follows experimental behavior for both alpha-recoil damage and alpha damage, leading to saturation effects in most materials. Preliminary results show no significant effect of transmutations on waste form stability. 2 figures, 3 tables. (DP)

1279 Backfilling and sealing a repository for high-level radioactive waste - a review. Butlin, R.N.; Hills, D.L. Watford, England; Building Research Establishment (1981). 29p. Building Research Establishment, Building Research Station, Garston, Watford WD2 7JR.

A review is presented of the non-site specific aspects of backfilling and sealing a high-level radioactive waste repository and the extent to which current civil engineering practice can meet the performance requirements. Areas requiring further study are indicated. The subject is examined assuming the high-level waste is vitrified in borosilicate glass blocks which are then sealed and protected in containers which are placed in an underground repository. Specific aspects reviewed include roles of backfill and seals, programme of repository construction, operation, backfilling and sealing, backfill and seal materials, equipment and placing techniques, design considerations, short term performance and instrumentation and assurance of long-term performance of backfill and seals.

1280 Underground storage of radioactive wastes. Barthoux, A.; Courtois, G. pp 160-181 of Seminaire sur les dechets radioactifs (Meeting on radioactive wastes). Fontenay-aux-Roses, France; Societe Francaise de Radioprotection (1981). (In French)(CONF-810273-). 1981).

From Meeting on radioactive wastes; Cadarache, France (2 Feb

Underground disposal in deep geologic formation is the more suitable for waste containing radionuclide of long half-life. Alpha bearing wastes are encapsulated in cement or bitumen and high level wastes from reprocessing and containing fission products and actinides are vitrified. Thermal power of glass is determined as a function of time and storage time vs cooling time. Potential risks for radionuclide migration are evaluated. Transmutation sub seabed and space are evoked for future disposal.

1281 (DOE-tr-246) R and D projects and studies: concerning the interim and terminal storage of radioactive wastes. Illi, H. (ed.). (Physikalisch-Technische Bundesanstalt, Braunschweig (Germany, F.R.)). Nov 1980. Translation source information not available . 302p. NTIS, PC A14/MF A01. Order Number DE8200106i.

The present compilation of $R$ and $D$ projects and studies dealing with the interim and terminal storage of radioactive wastes is intended to continue the $R$ and $D$ catalogue which was issued December 15, 1978, and bring it up to date, in accordance with the requirements of the licensing authorities. In contrast to the format selected by the RSK and SSK for their report of February 15, 1978, which involved a listing of problem areas on so-called leaves or sheets, the attempt is made here to classify the $R$ and $D$ projects and studies dealing with the federal repository so that they can be arranged according to the chapters of the planning inquiry documents. The table of contents lists the following: 
(1) federal repository at Corleben; (2) high level waste interim storage facility; (3) $\mathrm{Kr}-85$ interim storage facility; (4) safe storage of tritiumbearing water; (5) ocean dumping; and (6) appendices. Some of the items covered under the federal repository section are: site; naming of the site; site investigation; site investigation program; deep drilling program; geological-hydrogeological exploration program; status of the licensing process; construction and operation of federal facilities; planning inquiry procedure; operating plan; mining installations; radioactive waste; radiation protection measures; operation of mining installations; accident analyses; hazard potential and safety principles; barrier concept; and state of research.

1282 Method of measurement and borehole probe to measure heat-induced borehole convergence. Mueller, R.; Hofmann, B.; Korthaus, E.; Haferkamp, B. (to Kernforschungszentrum Karlsruhe G.m.b.H. (Germany, F.R.)). German(FRG) Patent 2,856,896/A/. 31 Jul 1980. 14p. (In German).

The borehole probe is introduced into boreholes of a salt deposit intended for storage of high-activity waste tanks. It essentially consists of a heating device for heating up the environment of the borehole and a measuring device for determining the borehole diameter by a capacitive method with a measuring capacitor.

1283 Thermodynamically stable glass ceramic product with radio-nuclides for the removal of radio-active waste and process for its manufacture. Guber, W.; Saidl, J.; Daruschy, P.; Hild, W. (to Kernforschungszentrum Karlsruhe G.m.b.H. (Germany, F.R.)). German(FRG) Patent 2,534,014/C/. 19 Jun 1980. 5p. (In German).

Up to $35 \%$ by weight of $\mathrm{Gd}_{2} \mathrm{O}_{0}$ and the highly radio-active waste are added to the glass ceramic product of the $\mathrm{SiO}_{2}-\mathrm{TiO}_{2}-\mathrm{Al}_{2} \mathrm{O}_{0}-\mathrm{B}_{2} \mathrm{O}_{0}-\mathrm{CaO}-\mathrm{Na}_{2} \mathrm{O}$ system, which increases the storage time. The manufacture of the glass is carried out by denitriding and calcining the waste solution, mixing with the constituents of the glass, melting and repeated cooling and heating.

1284 Seal for underground ultimate storage sites for radioactive waste, and method of installing the seal. Uerpmann, E.P. (to Gesellschaft fuer Strahlen- und Umweltforschung m.b.H. Muenchen, Neuherberg, Germany, F.R.). German(FRG) Patent 2,839,759/A/. 27 Mar 1980. 7p. (In German).

The high-level radioactive waste from reprocessing is melted into a mass of glass and sunk in moulds into the borings of a salt mine. The upper closure of the borings is formed by a conical plug which, for better adheston and seallng, has a rough surface. The plug is made of ceramics, cast steol, a Ph alloy or bitumen.

1285 Effects of radiation on the leaching behavior of nuclear waste forms. McVay, G.L.; Weber, W.J.; Pederson, L.R. (Battelle, Pac Northwest Lab, Richland, Wash). Nuclear and Chemical Waste Management; 2: No. 2, 103-108(1980).

Leaching tests conducted on several simulated waste glasses doped with ${ }^{244} \mathrm{Cm}$ show no significant change in leach rate as a function of increasing dose, even in the case of a partially devitrified waste glasses which exhibited both large volume changes (approximately $1 \%$ ) and amorphization of a crystalline phase. Significant increases in the leaching rate of PNL 76-68, a complex simulated nuclear waste glass, were observed to occur in the presence of gamma radiation. Some of the enhanced leaching is due to the generation of nitric acid from air radinlysis in the leach vessel. Increased rates were also found for samples irradiated while leaching, but with air excluded to eliminate nitric acid formation, indicating that water radiolysis products may also be important. 15 refs.

1286 Disposal of high-level radioactive wastes. Johnson, K.D.B. (UKAEA, Harwell, Oxfs, Engl). pp 201-213 of Energy in the balance. Guildford, Surrey, England; Westbury House (1980). (CONF-7909210-).

From Annual meeting of the British Association for the Advancement of Science; Edinburgh, UK (Sep 1979).

The nature and the decay of radioactive waste from a civil nuclear power program of the kind expected in Britain is discussed. Wastes from uranium mining are also of importance, but as that is a question addressed particularly by the countries which mine uranium, it is not discussed here. Three types of radioactive isotopes produced in a nuclear reactor as a result of the fission of uranium (and similar heavy metals such as plutonium) are analyzed. They are: fission product iso tope, actinide isotopes and isotopes produced by neutron activation. The quantities of fission product and actinides produced as high-level waste by nuclear reactors are assessed. Also discussed are the following subjects: the heat generated by high-level wastes, the physical form of wastes from a likely British reactor program, toxic potentials in the environment, etc.

1287 Can the oceans provide acceptable disposal sites for high-level radioactive waste. Laughton, A.S. (Inst of Oceanogr Sci, Wormley, Surrey, Engl). pp 221-234 of Energy in the balance. Guildford, Surrey, England; Westbury House (1980). (CONF-7909210-).

From Annual meeting of the British Association for the Advancement of Science; Edinburgh, UK (Sep 1979).

Two schemes for disposal of radioactive wastes are considered in this paper: disposal under the bed of the ocean, and disposal on the bed of the deep ocean. The assumptions are made about nature and quantity of waste generated by a global nuclear energy. Emplacement technolngy is reviewed. The criteria for the choice of an oceanic disposal site are described.

1288 Management of the DOE inventory of excess radioactively contaminated facilities. Kluk, A.F. (Off of Nucl Waste Manage. Washington, DC). pp 49.3-5n3 of Dccontamination and decommissioning of nuclear facilities. New York, NY; Plcnum Press (1980). (CONF-790923-).

From Decontamination and decommissioning of nuclear facilities conference; Sun Valley, ID, USA (16 Sep 1979).

Discussed is an updated version of the 'Plan for Decommissioning of DOE Radioactively Contaminated Surplus Facilities'. The plan was issued in 1978, while the updated version of it is in the state of preparation. The paper shows the various locations of the surplus facilities. All the facilities are categorized by their type. The reactors include training, research, production and demonstration reactors; the tanks include both steel and concrete types but not the large million gallon tanks used for high-level waste storage. By far the largest group is waste disposal facilities which consist primarily of contaminated soil locations. The current DOE organizational structure for surplus facilities management program is shown. Ongoing decommissioning projects are enumerated. Also listed are the major facilities awaiting decommissioning.

1289 High level waste disposal. Roethemeyer, H. (Physikalisch-Technische Bundesanstalt. Braunschweig, Germany). Transactions of the American Nuclear Society; 35: 9(1980). (CONF-801107-).

From ANS international conference; Washington, DC, USA (17 Nov 1980).

UNDERGROUND DISPOSAL; HIGH-LEVEL RADIOACTIVE WASTES; INTERNATIONAL REGULATIONS; PLANNING: RADIOACTIVE. WASTE. MANAGEMENT; SITE SELECTION; RADIOACTIVE WASTE DISPOSAL

1290 Illustrative nuclear waste disposal criteria and their application to high-level waste repository design. Williams, R.F.; Partlett, J.W.; Rodger, W.A.; Wilems, R.E. (Electric Power Research Inst., Palo Alto, CA). Transactions of the American Nuclear Society; 35: 63-65(1980). (CONF-801 107-). Nov 1980)

From ANS international conference; Wnshingtan, DC., IISA (17

CONSTRUCTION; DESIGN; EPRI; HIGH-LEVEL RADIOACTIVE WASTES; LICENSING; PARAMETRIC ANALYSIS; RADIOACTIVE WASTE DISPOSAL; RADIOACTIVE WASTE STORAGE; STORAGE FACILITIES; RADIOACTIVE WASTE FACILITIES

1291 Dumping of solid packaged radioactivity in the deep oceans. Forster, Wm. O.; Van As, D. (International Atomic Energy Agency, Vienna (Austria). Div. of Nuclear Safety). pp 134-141 of Management of environment [a felicitation volume presented to Dr A.K. Ganguly]. Patel, B. (ed.) (Bhabha Atomic Research Centre, Rombay (India). Health Physics Div.). New Delhi (India); Wiley Eastern (1980).

With the increasing use of nuclear energy, the quantity of radioactive wastes which are generated is also increasing. Their treatment and disposal is causing a concern in further development of nuclear 
energy. World's oceans are considered as a possible location for these wastes. A convention on the prevention of marine pollution caused by dumping of wastes and other matter into oceans was adopted at the Intergovernmental Conference held at London in November 1972. The convention prohibits dumping of high-level radioactive wastes in the oceans and has entrusted the IAEA the tasks of defining the high level radioactive wastes and providing recommendations for the issue of special permits for dumping of the radioactive materials which do not fall into the category of high-level wastes. A provisional definition and recommendations formulated by the IAEA and adopted by contracting parties in 1976 are outlined. On the basis of an oceanographic model developed by Shepherd (1976) and considered to be the best available. a revised definition and revised recommendations were formulated. Their salient features are mentioned. The key parameters for specific site assessments are mentioned. The Nuclear Energy Agency also formulated guidelines on sea-disposal packages for radioactive wastes in 1974 and revised them in 1978. Finally it is noted that criteria have not been established for dumping of non-radioactive wastes in the ocean, though such criteria are contained in the IAEA recommendations in case of radioactive wastes.

1292 Solidification for final storage of high-level radioactive fission product solutions from spent fuel elements. Halaszovich, S. Aachen, Germany; Technische Hochschule (20 Dec 1979). 118p. (In German).

\section{Thesis.}

As a contribution to solve the waste problem in the outer fuel cycle, a process for solidification of high-level radioactive fission product solutions in boron silica glass has been developed. The operations of concentration and denitration (adding of products for vitrification of glass - drying - melting of glass), which are more or less complex in every process, are carried out in steps which are more or less uncoupled. It is shown in four hot experiments with real radioactive waste from LWR fuel element reprocessing that this process is suitable in principle. These experiments, their findings and the disturbances which occurred during the experiments are described. Basic considerations show the possibility of an industrial-scale application.

1293 Stress corrosion cracking test with slow strain rate and constant current. Ondrejcin, R.S. (Savannah River Lab., Aiken, SC). pp 203-221 of Stress corrosion cracking: the slow strain-rate technique. Special Technical Publication 665. Ugiansky, G.M.; Payer, J.H. (eds.). Philadelphia, PA; American Society for Testing and Materials (1979).

A rapid electrochemical tension test was developed for evaluating stress corrosion crack initiation in carbon steel. Constant anodic current was imposed on smooth-bar tension specimens as the specimens were slowly strained to fracture at $1.3 \times 10^{-6} / \mathrm{s}$. Equivalent results were obtained for the following ductility properties measured: uniform elongation, total elongation, and reduction of area. Total elongation was chosen as the index for stress corrosion crack initiation. An equation was developed that allowed calculation of total elongation of specimens in electrolytes (test solutions) with composition ranges of 1.5 to $5.5 \mathrm{M}$ nitrate, 0 to $3.5 \mathrm{M}$ nitrite, and 0 to $5.0 \mathrm{M}$ hydroxide, and a temperature range of 50 to $100^{\circ} \mathrm{C}$. A minimum of $13 \%$ total elongation was selected to indicate the possible initiation of cracking in A 285-B steel alloy. The test was used to evaluate relative aggressiveness of synthetic nuclear wastes on A 285-B carbon steel and the relative resistances of several steels to given solution compositions. Test results formed one of the bases for setting temperature and concentration limits for several ions in nuclear wastes that are stored in carbon steel tanks at the Savannah River Plant.

1294 Thermomechanical impact around a borehole in rock salt after HAW disposal. Ehlert, C.; Schoen, R.; Winske, P. (RWTH Aachen-Germany,). Transactions of the American Nuclear Society; 31: 541-543(1979). (CONF-790519-).

From European nuclear conference; Hamburg, F.R. Germany (6 May 1979).

BOREHOLES; COMPUTER CALCULATIONS; COM PUTER CODES; FINITE ELEMENT METHOD; MECHANICAL PROPERTIES; PLASTICITY; RADIOACTIVE WASTE DISPOSAL; SALT DEPOSITS; STRESS INTENSITY FACTORS; THERMOELASTICITY; HIGHEI.FVF.I. RADIOACTIVE WASTES
1295

Far field temperature calculations for high-level waste disposal in salt formations. Ploumen, P.; Strickmann, G.; Winske, P. (RWTH Aachen). Transactions of the American Nuclear Society; 31: 539-541(1979). (CONF-790519-). May 1979).

From European nuclear conference; Hamburg, F.R. Germany (6

COMPUTER CODES; FEDERAL REPUBLIC OF GERMANY; FINITE ELEMENT METHOD; GEOLOGIC FORMATIONS; HEAT TRANSFER; RADIOACTIVE WASTE DISPOSAL; SALT DEPOSITS; TEMPERATURE GRADIENTS; THREEDIMENSIONAL CALCULATIONS

1296 High-level radioactive waste isolation by incorporation in silicate rock. Schwartz, L.L.; Cohen, J.J.; Lewis, A.E.; Braun, R.L. (California Univ., Livermore (USA). Lawrence Livermore Lab.). pp 57-71 of Peaceful nuclear explosions V. Proceedings of a technical committee on the peaceful uses of nuclear explosions organized by the IAEA and held in Vienna, 22-24 November 1976. Vienna, Austria; IAEA (1978). (IAEATC-81-5/4; CONF-7611126-).

From Technical committee on the peaceful use of nuclear explosions; Vienna, Austria (22 Nov 1976).

A number of technical possibilities for isolating high-level radioactive materials have been theoretically investigated at various times and places. Isolating such wastes deep underground to ensure long term removal from the biosphere is one such possibility. The present concept involves as a first step creating the necessary void space at considerable depth, say 2 to $5 \mathrm{~km}$, in a very-low-permeability silicate medium such as shale. Waste in dry, calcined or vitrified form is then lowered into the void space, and the access hole or shaft sealed. Energy released by the radioactive decay raises the temperature to a point where the surrounding rock begins to melt. The waste is then dissolved in it. The extent of this melt region grows until the heat generated is balanced by conduction away from the molten zone. Resolidification then begins, and ends when the radioactive decay has progressed to the point that the temperature falls below the melting point of the rock-waste solution. Calculations are presented showing the growth and resolidification process. A nuclear explosion is one way of creating the void space.

1297 Method for subsurface disposal of radioactive waste. Stogner, J.M. (to Halliburton Co.). US Patent 3,513,100. 19 May 1970. Filed date 25 Sep 1967. vp.

A method is described for disposing of high level, solid, radioactive waste material by delivering such material in a continuous, waterphase cement to a subterranean formation. The solid waste is used as a propping agent to hold formation fractures open as a slurry containing the solid, radioactive waste material is pumped into the formation. (7 claims)

1298 Answers to your questions about high-level nuclear waste isolation. Columbus, $\mathrm{OH}$; Office of Nuclear Waste Isolation ([nd]). 77p.

The primary objective of this US Department of Energy program is to isolate existing and future high-level radioactive waste from the environment so that radioactivity will not pose any significant threat to public health and safety. A need for long term storage facilities has been demonstrated. In 1981, ca 8000 tons of spent fuel assemblies from commercial power plants were in temporary storage facilities. Highlevel defense wastes in temporary storage totaled more than $10^{7} \mathrm{ft}^{0}$. A technical program that will meet all relevant radiological protection criteria as well as other applicable requirements is being developed to insure that nuclear waste management problems will not be deferred. Elements include site selection, the location, design and engineering of the repository, risks and safety, federal and state cooperation, environmental and regulatory aspects, international cooperation.

\section{Environmental Aspects}

1299 (AECL-6836) High-level waste glass field burial tests at CRNI: the effect of geochemical kinetics on the release and migration of fission products in a sandy aquifer. Melnyk, $\Gamma$. W.; Walton, F.B.; Johnson, L.H. (Atomic Energy of Canada Ltd., Pinawa, Manitoba. Whiteshell Nuclear Research Establishment). Jun 1983. 60p. Atomic Energy of Canada Ltd., White- 
shell Nuclear Research Establishment, Pinawa, Manitoba ROE 1 LO, Canada.

Work performed under the Canada/US Waste Management Information Fxchange Program.

In 1960 June, 25 nepheline syenite-based glass hemispheres containing the fission products ${ }^{107} \mathrm{Cs},{ }^{90} \mathrm{Sr},{ }^{144} \mathrm{Ce}$ and ${ }^{106} \mathrm{Ru}$ were buried below the water table in fluvial sand at the Chalk River Nuclear Laboratories of Atomic Energy of Canada Limited. Soil and groundwater concentrations of ${ }^{90} \mathrm{Sr}$ and ${ }^{107} \mathrm{Cs}$ have been determined since then and the data have been interpreted using kinetically limited migration models to deduce the leaching history of the glass for these burial conditions. The leaching history derived from the field data is compared to laboratory leaching of samples from a glass hemisphere retrieved in 1978, and also to pre-burial laboratory leaching of identical hemispheres. The time dependence of the leach rates observed for the buried specimens sug. gests that leaching is being inhibited by the formation of a protective surface layer, although no direct observation of this layer has been made. Using an average leach rate of $5.6 \times 10^{-14} \mathrm{~kg} /\left(\mathrm{m}^{2} . \mathrm{s}\right)$ derived from the field data for the period 1966 to 1977 , it is estimated that it would require about 20 million years to dissolve the glass hemispheres. The effect of the kinetic limitations of the fission-product/fluvial-sand interactions is discussed with respect to the migration of ${ }^{90} \mathrm{Sr}$ and ${ }^{107} \mathrm{Cs}$ over a 20-a time scale. It is concluded that kinetically limited sorption by oxyhydroxides rather than equilibrium ion exchange controls the long-term migration of ${ }^{90} \mathrm{Sr}$; the action of the oxyhydroxides immobilizes the ${ }^{90} \mathrm{Sr}$ on the longer time scale. Cesium is initially rapidly bound to the micacevus fraction of the sand. On a longer time scale, slow remobilization of ${ }^{107} \mathrm{Cs}$ in particulate form is observed and is believed to be related to bacterial action. 25 references, 16 figures.

1300 (AECL-6838) Diffusive transport of strontium-85 in sand-bentonite mixtures. Gillham, R.W.; Robin, M.J.L.; Dytynyshyn, D.J. (Atomic Energy of Canada Ltd., Pinawa, Manitoba. Whiteshell Nuclear Research Establishment; Waterloo Univ., Ontario (Canada). Dept. of Earth Sciences). Jun 1983. 41 p. Atomic Energy of Canada Ltd., Whiteshell Nuclear Research Establishment, Pinawa, Manitoba ROE. 1LO, Canada.

Work performed under the Canada/US Waste Management Information Exchange Program.

Diffusion experiments have been used to determine the transport of ${ }^{85} \mathrm{Sr}$ in sand-bentonite mixtures. The diffusion experiments were performed on one natural soil (Chalk River sand) and on seven mixtures of bentonite and silica sand, containing from $0 \%$ to $100 \%$ bentonite. Two non-reactive solutes $\left({ }^{06} \mathrm{Cl}\right.$ and $\left.{ }^{0} \mathrm{H}\right)$ and one reactive solute $\left({ }^{85} \mathrm{Sr}\right)$ were used in the study. The experiments with non-reactive solutes yielded estimates of tortuosity factors. Retardation factors were obtained from experimental porosities, experimental bulk densities, and from batch distribution coefficients $\left(K_{d}\right)$. These $K_{d}$ values are a simple way of describing the solute/medium reaction, and are based on the assumption that the cation-exchange reaction may be described by a linear adsorption isotherm passing through the origin. The results demonstrate that, for practical purposes and for our experimental conditions, the use of the distribution coefficient provides a convenient means of calculating the effective diffusion coefficient for ${ }^{85} \mathrm{Sr}$. The pornsity and bulk density were also found to have a considerable influence on the effective diffusion coefficient, through the retardation factor. Mixtures containing 5 to $10 \%$ bentonite were found to be more effective in retarding ${ }^{85} \mathrm{Sr}$ than either sand alone, or mixtures containing more bentonite. In the soils of higher bentonite content, the effect of increased cation-exchange capacity was balanced by a decreasing ratio of bulk density to porosity. 11 references, 7 figures, 4 tables.

1301 (BMI-X-698-Rev.) Preliminary environmental implications of alternatives for decommissioning and future use of the Western New York Nuclear Services Center. Reinhart, C... (ed.). (Battelle Columbus Labs., OH (USA)). Feb 1979. Contract W-7405-ENG-92-110. 254p. NTIS, PC A12/MF A01. Order Number DE83004726.

Portions of document are illegible.

This study describes in considerable detail the Western New York Nuclear Services Center (WNYNSC) site, the several facilities at the site and the pertinent features of the surrounding area. The geography, hydrology, geology, soils, seismology, meteorology, ecology, agriculture and population of the area and site are presented. The physical characteristics and present status of the nuclear fuel reprocessing facili- ty, the high-level liquid wastes and tanks, and the waste burial areas a described with emphasis on features which are important to the safe of the site in the present and near-term, on features which affect $t$ safety or expense of decontamination and decommissioning and on features which are important to future uses of the site. The current environmental trends at the WNYNSC are reviewed including a description of the continuing program of site monitoring. Five decommissioning alternatives: layaway, protective storage, dismantlement, deferred dismantlement, and entombment, and several possible future uses for the reprocessing plant are described and discussed. Six alternative plans for management of the high-level liquid wastes (HLLW) and three alternatives for disposing of the HLLW tanks are presented as being technically feasible but differing in effort required and risk to the public remaining after disposal. Several options are described for decommissioning or continued use of the two waste burial areas. These range from complete exhumation, repackaging and removal of the wastes to another site, to reopening for commercial waste disposal.

1302 (CONF-830329-1) Surveys for desert tortoise on the proposed site of a high-level nuclear waste repository at the Nevada Test Site. Collins, E.; Sauls, M.L.; O'Farrell, T.P. (EG and G, Inc., Goleta, CA (USA)). 1983. Contract $\Lambda$ CO883NV10282. 10p. NTIS, PC A02/MF A01. Order Number DE83008996.

From Desert Tortoise Council symposium; Lake Havasu, AZ, USA (26 Mar 1983).

Portions are illegible in microfiche products.

The National Waste Terminal Storage Program is a national search for suitable sites to isolate commercial spent nuclear fuel or highlevel radioactive waste. The Nevada Nuclear Waste Storage Investigation (NNWSI) managed by the U.S. Department of Energy (DOE), Nevada Operations Office, was initiated to study the suitability of a portion of Yucca Mountain on the DOE's Nevada Test Site (NTS) as a location for such a repository. EG and $G$ was contracted to pruvide information concerning the ecosystems encountered on the site. A comprehensive literature survey was conducted to evaluate the status and completeness of the existing biological information for the previously undisturbed area. Site specific studies were begun in 1981 when preliminary field surveys confirmed the presence of the desert tortoise (Gopherus agassizi) within the project area FY82 studios wero designed to ditic mine the overall distribution and abundance of the tortoise within the area likely to be impacted by NNWSI activities. The Yucca Mountain area of the Nevada Test Site is situated close to the northern range limit of the desert tortoise. Prior to the 1982 surveys, the desert tortoise was reported from only nine locations on NTS. A known population had been under study in Rosk Valley ahnut 25 miles southeast of the project urea. However, the distribution and population densities of tortoise in the southwest portion of NTS were virtually unknown. Results of our surveys indicate that desert tortoise can be expected, albeit in small numbers, in a wide range of Mojavean and Transitional habitats.

1303 (C.ONF-830375-1) Interaction of groundwater and fresh basalt fissure surfaces and its cffcet on the migration of actinides. Vandegrift, G.F.; Bowers, D.L.; Gerding, T.J.; Fried, S.M.; Wilbur, C.K.; Seitz, M.G. (Argome National Lab., IL (USA)). 1983. Contract W-31-109-ENG-38. 19p. NTIS, PC A02/MF A01. Order Number DE83014348.

From Conference on geochemical behavior of disposed radioactive waste; Seattle, WA, USA (20 Mar 1983).

Experiments are being performed at Argonne National Laboratory (ANL): (1) to identify interactions of radionuclides and repository components that effect nuclide migration, and (2) to assess changes in nuclide migration caused by modifications expected upon aging of the waste, clay backfill, and rock. The experiments are conducted with radioactive borosilicate glass, bentonite and mechanically fissured basalt rock in flowing water analogous to their configuration in a breach of a nuclear waste repository. Changes undergone by the groundwater as it passed through the fissure include: (1) drop in pH from 10 to 8 , (2) loss of suspended particulate, and (3) loss of dissolved/suspended U, $\mathrm{Np}$, and $\mathrm{Pu}$. These effects, also studied as functions of radiation do and of laboratory aging of the repository components, are related to $t$ ] predicted long-term performance of a nuclear waste repository.

1304 (CONF-8308126-1-Draft) Evaluation of DOE radionuclide solubility data and selected retardation parameters: description of calculational and confirmatory experimental 
activities. Kelmers, A.D.; Clark, R.J.; Cutshall, N.H.; Johnson, J.S.; Kessler, J.H. (Oak Ridge National Lab., TN (USA); Florida State Univ., Tallahassee (USA). Dept. of Chemistry). 1983. Contract W-7405-ENG-26. 15p. NTIS, PC A02/MF A01. Order Number DE84000731.

From NRC research annual review meeting of nuclear waste management research on geochemistry of HLW disposal; Reston, VA, USA (30 Aug 1983).

An experimentally oriented program has been initiated to support the NRC analysis and licensing activities related to high-level nuclear waste repositories. The program will allow the NRC to independently confirm key geochemical values used in the site performance assessments submitted by the DOE candidate repository site projects. Key radionuclide retardation factor values, particularly radionuclide solubility and sorption values under site specific geochemical conditions, are being confirmed. The initial efforts are being directed toward basalt rock/groundwater systems relevant to the BWIP candidate site in the Pasco Basin. Future work will consider tuff (NNWSI candidate site in Yucca Mountain) and salt (unspecified ONWI bedded or domal salt sites) rock/groundwater systems. Initial experimental results with technetium haye confirmed the BWIP values for basalt/groundwater systems under oxic redox conditions: high solubility and no sorption. Under reducing redox conditions, however, the experimental work did not confirm the proposed technetium values recommended by BWIP. In the presence of hydrazine to establish reducing conditions, an apparent solubility limit for technetium of about $5 E-7 \mathrm{~mol} / \mathrm{L}$ was encountered; BWIP recommended calculated values of $1 \mathrm{E}-12$ or greater than or equal to $1 \mathrm{E}-14 \mathrm{~mol} / \mathrm{L}$. Experimental evidence concerning sorption of reduced technetium species is incomplete at this time. Equilibrium speciation and saturation indices were calculated for well water data sets from BWIP using the computer code PHREEQUE. Oversaturation was indicated for hematite and quartz in all data sets. Near surface samples were undersaturated with respect to calcite, but most deep samples were oversaturated with respect to calcite and other carbonate minerals.

1305 (DOE/EIS-0081) Long-term management of liquid high-level radioactive wastes stored at the Western New York Nuclear Service Center, West Valley. Final environmental impact statement. (USDOE Assistant Secretary for Nuclear Energy, Washington, DC. Office of Terminal Waste Disposal and Remedial Action). Jun 1982. 535p. NTIS MF A01. Order Number DE82017565.

Portions of document are illegible.

The statement assesses and compares environmental implications of possible alternatives for long-term management of the liquid high-level radioactive wastes stored in underground tanks at the Western New York Nuclear Service Center in West Valley, New York. Four basic alternatives, as well as options within these alternatives, have been considered in the EIS: (1) onsite processing to a terminal waste form for shipment and disposal in a federal repository (the preferred alternative); (2) onsite converston to a solid interim form for shipmont to a federal waste facility for later processing to a terminal form and shipment and subsequent disposal in a federal repository; (3) mixing the liquid wastes with cement and other additives, pouring it back into the existing tanks, and leaving onsite; and (4) no action (continued storage of the wastes in liquid form in the underground tanks at West Valley). Mitigative measures for environmental impacts have becn considered for all alternatives. No significant stresses on supplies or irreversible and irretrievable resources are anticipated, and no scarce resource would be required.

1306 (DOE/EIS-0082-FINAL) Defense Waste Processing Facility: Savannah River Plant, Aiken, SC. Final environmental impact statement. (Department of Energy, Washington, DC (USA). Assistant Secretary for Defense Programs). Feb 1982. 542p. NTIS, PC A23/MF A01. Order Number DE82008157.

The purpose of this Environmental Impact Statement (EIS) is to provide environmental input into both the selection of an appropriate strategy for the permanent disposal of the high-level radioactive waste (HLW) currently stored at the Savannah River Plant (SRP) and the subsequent decision tn snnstruct and operate a Defense Waste Processing Facility (DWPF) at tlie SRP sitc. The SRP is a major US Department of Envgy (DOE) installation for the production of nuclear materials for national defense. Approximately $83 \times 10^{\circ} \mathrm{m}^{0}$ (22 million gal) of HLW currently are stored in tanks at the SRP site. The proposed DWPF would process the liquid HLW generated by SRP operations into a stable form for ultimate disposal. This EIS assesses the effects of the proposed immobilization project on land use, air quality, water quality, ecological systems, health risk, cultural resources, endangered species, wetlands protection, resource depletion, and regional social and economic systems. The radiological and nonradiological risks of transporting the immobilized wastes are assessed. The environmental impacts of disposal alternatives have recently been evaluated in a previous EIS and are therefore only summarized in this EIS.

1307 (DOE/TIC-4621-Vol.1, pp 171-202) Elemental release from glass and spent fuel. McVay, G.L. (Pacific Northwest Lab., Richland, WA); Bradley, D.J.; Kircher, J.F. 1981. NTIS $\$ 18.00$.

In Technology of high-level nuclear waste disposal. Advances in the science and engineering of the management of high-level nuclear wastes. Volume 1.

In the past several years, emphasis on interactions between waste forms and aqueous solutions has shifted from data gathering to understanding, and numerous mechanistic investigations have been initiated. These rely heavily on surface analytical techniques and control of many of the variables. Out of these efforts has come new insight into interactions between waste forms and water. Because of the fundamental differences in the interactions of simple silicate and complex borosilicate waste glasses with aqueous solutions, predictive models and/or results derived from simple silicate glasses generally cannot be used to predict the behavior of complex borosilicate glasses. In addition, it has been shown that realistic flow rates and groundwater differences do not alter elemental release from glass or spent fuel by amounts greater than one order of magnitude. The solubility limits for actinides contained in glasses have been shown to be identical to those observed for crystalline actinide oxide states themselves. Therefore thermodynamic arguments can be used to predict the upper limits of actinide isotopes in solution. Radiolysis effects in the absence of air have been shown to be important at lower temperatures but not significant at the elevated temperatures expected in a repository. If air (or perhaps just nitrogen) is present, however, nitric acid is generated as a radiolysis product; this greatly enhances elemental removal at all temperatures. Leaching of spent fuel is less sensitive to temperature change than is leaching of glass and, in some cases, shows a negative temperature dependence. As the oxygen content of the leachate decreases, actinide removal from both glass and spent fuel also decreases. In general, existing release models for glass and spent fuel are not adequate to predict long-term behavior in a meaningful and believable manner.

1308 (DOE/TIC-4621-Vol.1, pp 203-254) Status of radionuclide sorption-desorption studies performed by the WRIT program. Serne, R.J.; Relyea, J.F. (Pacific Northwest Lab., Richland, WA). 1981. NTIS \$18.00.

In Technology of high-level nuclear waste disposal. Advances in the science and engineering of the management of high-level nuclear wastes. Volume 1.

This paper focuses on interactions between dissolved radionuclides in groundwater and rocks and sediments away from the nearfield repository. Two approaches were used to study the primary mechanism, adsorption-desorption. Empirical studies rely on distribution coefficient measurements, and mechanism studies strive to identify, differentiate, and quantify the processes that control nuclide retardation. The status of sorption mechanism studies is discussed, with emphasis on delineating the usefulness of ideal ion-exchange, site-binding electrical doublelayer, and redox-controlled sorption constructs. Since studics to date show greater potential for site-binding electrical double-layer models, future efforts will concentrate on this construct. Laboratory studies are discussed which corroborate the importance of redox reactions in causing nuclide retardation for multivalent elements, such as $\mathrm{Tc}, \mathrm{Np}, \mathrm{Pu}$, and $U$. Results suggest that both solution-mediated reduction, such as the $\mathrm{Fe}$ (II)-Fe(III) couple, and solid-solution heterogeneous reduction reactions, such as reduction of solution $\mathrm{Pu}(\mathrm{VI})$ at the mineral surface by structural Fe(II), occur. Coupled microscopy, microprobe, and autoradiography studies have determined actual sorption sites for radionuclides on polymineralic rocks. The studies show that it is possible for minor phases to cumplelely dominate the mase of radionuclides adsorbed. The most active minerals are typically alternation products (clays and zeolites). Several exercises are discussed which rank radionuclides according to their potential dose hazards. In each of the analyses discussed, the top four radionuclides are I, Tc, Np, and $\mathrm{Ra}$. Other ele- 
ments that rank high in potential hazards are $\mathrm{Pu}, \mathrm{U}, \mathrm{Am}, \mathrm{Th}, \mathrm{Pb}, \mathrm{Sn}$, Pd, and Se.

1309 (EGG-1183-2419) Annotated bibliography for biologic overview for the Nevada Nuclear Waste Storage Investigations, Nevada Test Site, Nye County, Nevada. Collins, E.; O'Farrell, T.P.; Rhoads, W.A. (EG and G, Inc., Goleta, CA (USA). Energy Measurements Group). Dec 1981. Contract AC08-83NV10282. 48p. NTIS, PC A03/MF A01. Order Number DE84000864.

This annotated bibliography was compiled to accompany the Biologic Overview for the Nevada Nuclear Waste Storage Investigations, Nevada Test Site, Nye County, Nevada, EG and G, Santa Barbara Operations Report No. EGG 1183-2443, which documents and synthesizes important biotic information related to Nevada Nuclear Waste Storage Investigations (NNWSI). As such, it is an important part of the NNWSI screening process that was designed to include a systematic, traceable, defensible, and documented basis for a decision to proceed or not with site-specific phases on NTS. Included are all published, and available but unpublished, baseline information on life histories, habitat requirements, distributions, and ecological relationships of the flora and fauna of the region. Special effort was made to includo information on endangered, threatened, or sensitive species. 131 references.

1310 (EGG-1183-2460) Biologic nverview for the Nevada Nuclear Waste Storage Investigations, Nevada Test Site, Nye County, Nevada. Collins, E.; O'Farrell, T.P.; Rhoads, W.A. (EG and G, Inc., Goleta, CA (USA). Energy Measurements Group). Jan 1982. Contract AC08-83NV10282. 55p. NTIS, PC A04/MF A01. Order Number DE84000863.

Portions are illegible in microfiche products.

The Nevada Nuclear Waste Storage Investigations project study area includes five major vegetation associations characteristic of the transition between the northern extent of the Mojave Desert and the southern extent of the Great Basin Desert. A total of 32 species of reptiles, 66 species of birds, and 46 species of mammals are known to occur within these associations elsewhere on the Nevada Test Site. Ten species of plants, and the mule deer, wild horse, feral burro, and desert tortoise were defined as possible sensitive species because they are protected by federal and state regulations, or are being considered for such protection. The major agricultural resources of southern Nye County included 737,000 acres of public grazing land managed by the Bureau of Land Management, and 9500 acres of irrigated crop land located in the Beatty/Oasis valleys, the Amargosa Valley. and Ash Meadnws. Range lands are of poor quality. Alfalfa and cotton are the major crops along with small amounts of grains, Sudan grass, turf, fruits, and melons. The largest impacts to known ecosystems are expected to result from: extensive disturbances associated with construction of roads, seismic lines, drilling pads, and surface facilities; storage and leaching of mined spoils; disposal of water; off-road vehicle travel; and, over several hundred years, elevated soil temperatures. Significant impacts to off-site areas such as Ash Meadows are anticipated if new residential developments are bultt there to accommodate an increased work force. Several species of concern and their essential habitats are located at Ash Meadows. Avallable literature contained sufficient baseline information to assess potential impacts of the proposed project on an area-wide basis. It was inadequate to support analysis of potential impacts on specific lnsatinns selected for site characterization studies, mining an exploratory shaft, or the siting and operation of a repesitory.

1311

(EGG-10282-2004) 1982 biotic survey of Yucca Mountain, Nevada Test Site, Nye County, Nevada. O'Farrell, T.P.; Collins, E. (EG and G, Inc., Goleta, CA (USA). Energy Measurements Group). Feb 1983. Contract AC08-83NV10282. 47p. NTIS, PC A03/MF A01. Order Number DE84000865.

In 1981 an extensive literature review was conducted to determine the current state of knowledge about the ecological characteristics of the Yucca Mountain study area and to identify what site-specific information was lacking. Based on the findings of the review a field study was initiated in 1982 to gather site-specific information on the ecological characteristics of the project area. The biota observed were representative of either the Mojave or Transition deserts that are widely distributed in southern Nevada and the arid Southwest. No unusual vegetation associations or assemblages of animals were observed. Based on observations of tracks and scats it was concluded that low numbers of both mule deer and feral burros used the area seasonally, and that neither species should be severely threatened by the proposed activities The Mojave fishhook cactus and desert tortoise, both under considet ation for federal protection as threatened species, were found to occu, in the study area. The former was distributed in notable densities on the rocky ridgelines of Yucca Mountain in areas that should not be greatly disturbed by sitc characterization or future repository activities. Evidence of desert tortoise was observed throughout the project area to elevations of $5240 \mathrm{ft}$; however, relative densities were estimated to be low (less than 20 per square mile). Physical destruction of soils and native vegetation was determined to be the most significant negative effect associated with current and proposed characterization activities. Solution holes in exposed flat rock on ridgelines that served as passive collectors of precipitation and runoff were the only sources of free water observed. While these water supplies were not adequate to support riparian vegetation, there was evidence that they served as an important ephemeral source of water for wildlife.

1312 (EGG-FM-6202) Scientific data necessary to predict radionuclide migration within or near a mined nuclear repository. Downs, W.F. (EG and G Idaho, Inc., Idaho Falls (USA)). Mar 1983. Contract AC07-76ID01570. 59p. NTIS, PC A04/MF A01. Order Number DE83012102.

The National Waste Terminal Storage Program was created to develop a system to isolate radioactive wastes $\mathrm{frnm}$ the biospliere. It has been determined that the most reasonable means for accomplishing this task is to place the high-level and transuranic wastes in mined geologic repositories. Three geologic environments have been selected for further study and evaluation: (1) domed or bedded salt formations, (2) thick basalt flows fo the Columbia River Plateau and (3) alkali igneous rocks, both tuffs and granites, of the Nevada Test Site. Each of these candidate geologies will present a different physical-chemical environment to the waste package. The physical environments have been estimated based on depth of repository, radionuclide loading, and spacing of canisters. The chemical environments are based on initial host-rock mineralogy, native ground-water geochemistry, and likely alteration assemblages. The latter sections of this report discuss the mechanisms of radionuclide release, transport, and retention on the host rocks or their alteration products.

1313 (ENPU-80-7) Distribution coefficient (Kd) concept and its applicability to studies of radionuclide migration in geological media. McKinley, I.G.; Greenwood, P.B. (SEE CODE9050311 Institute of Ceological Sciences, Harwell (UK). Environmental Prntection I Init). Apr 1980. 21 p. Environmontal Protection Unit, Institure of Geological Sciences, Building 151, Harwell Laboratory, Oxfordshire OX11 ORA, England.

In this report the background of the IGS radionuclide migration project is described and the results of preliminary sorption studies reported. In particular, the limitations of the distribution coefficient (Kd) concept is discussed and illustrated for a degraded granite/ groundwater system spiked with ${ }^{107} \mathrm{Cs},{ }^{90} \mathrm{Sr}$ and ${ }^{109} \mathrm{Co}$. Proposed devel. opments of this project and future research aims are described.

1314 (LA-9217-MS) Analysis of the alleged Kyshtym disaster. Soran, D.M.; Stillman, D.B. (Los Alamos Natinnal Lab., NM (USA)). Jan 1982. Contract W-7405-ENG-36. 32p.

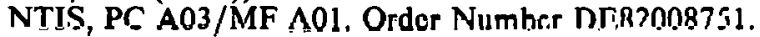

Portions of document are illegible.

The alleged Kyshtym disaster has been an intriguing intelligence puzzle for almost 25 years. Zhores Medvedev, a Soviet dissident, has written numerous journal articles as well as two books on the subject. He has argued that a vast contaminated area exists east of the city of Kyshtym in the southern Ural Mountains. Further, he has alleged that a nuclear waste disposal accident in 1957 to 1958 caused the contamination. The authors of this report are in partial disagreement with Medvedev's first allegation and in complete disagreement with his second. A contaminated area does exist east of Kyshtym, but Soviet carelessness coupled with general disregard for the citizenry and the environment ar the prime causative factors, not a nuclear waste accident.

1315 (LA-9329-MS) Elution of radionuclides through columns of crushed rock from the Nevada Test Site. Treher, E.N.; Raybold, N.A. (Los Alamos National Lab., NM (USA)). Oct 
1982. Contract W-7405-ENG-36. 136p. NTIS, PC A07/MF A01. Order Number DE83003891.

Results from the elution of radionuclides through columns of crushed tuff, granite, and argillite are presented. Good agreement between column and batch measurements on washed samples is generally observed for all three rock types. This is encouraging, since the results of batch measurements are often of value to show relative sorption under a variety of conditions, and their relevance to the migration of radionuclides under flowing conditions has been questioned. Column elution behavior depended upon sample mineralogy. For example, ${ }^{85} \mathrm{Sr}$ gave fairly sharp, symmetric peaks on granite, argillite, and devitrified tuffs; however, on a vitrophyre, the peak was 10 to 100 times broader. For ${ }^{107} \mathrm{Cs}$, the most unusual behavior was found on granites, where for columns run at $\sim 20 \mathrm{~m} /$ year, most of the cesium was retained at the load point. For all the radionuclides studied, except ${ }^{95 \mathrm{~m}} \mathrm{Tc}$, zeolitecontaining tuffs had to be run at flow rates of $10^{0}$ to $10^{4} \mathrm{~m} /$ year to obtain elutions, while samples composed primarily of alkali feldspar and $\mathrm{SiO}_{2}$ gave elutions of ${ }^{85} \mathrm{Sr},{ }^{107} \mathrm{Cs}$, and ${ }^{100} \mathrm{Ba}$ at flow rates of 10 to 100 $\mathrm{m} /$ year. Knowledge of the mineralogic compositions of tuffs and their ability to retard the migration of radionuclides is important - not only to identify the optimum horizon for a nuclear waste repository in Yucca Mountain, but also to estimate how well the repository will perform.

1316 (NUREG/CR-1636-Vol.1) Risk methodology for geologic disposal of radioactive waste: model description and user manual for Pathways model. Helton, J.C.; Kaestner, P.C. (Sandia National Labs., Albuquerque, NM (USA)). Mar 1981. Contract AC04-76DP00789. 154p. (SAND-78-1711-Vol.1). NTIS, PC A08/MF A01 - GPO.

Portions of document are illegible.

A model for the environmental movement and human uptake of radionuclides is presented. This model is designated the Pathways-toMan Model and was developed as part of a project funded by the Nuclear Regulatory Commission to design a methodology to assess the risk associated with the geologic disposal of high-level radioactive waste. The Pathways-to-Man Model is divided into two submodels. One of these, the Environmental Transport Model, represents the long-term distribution and accumulation of radionuclides in the environment. This model is based on a mixed-cell approach and describes radionuclide movement with a system of linear differential equations. The other, the Transport-to-Man Model, represents the movement of radionuclides from the environment to man. This model is based on concentration ratios. General descriptions of these models are provided in this report. Further, documentation is provided for the computer program which implements the Pathways Model.

1317 (NUREG/CR-2394) PATH1 self-teaching curriculum: example problems for Pathways-to-Man Model. Helton, J.C.; Finley, N.C. (Sandia National Labs., Albuquerque, NM (USA)). Oct 1982. Contract AC04-76DP00789. $116 \mathrm{p}$. (SAND-81-2377). NTIS, PC E06/MF \$4.75 - GPO \$7.00. Order Number DE83007228.

Portions are illegible in microfiche products; Includes 6 sheets of $48 x$ reduction microfiche.

The Pathways-to-Man Model was developed at Sandia National Laboratories to represent the environmental movement and human uptake of radionuclides. This model is implemented by the computer program PATH1. The purpose of this document is to present a sequence of examples of facilitate use of the model and the computer program which implements it. Each example consists of a brief description of the problem under consideration, a discussion of the data cards required to input the problem to PATH1, and the resultant program output. These examples are intended for use in conjunction with the technical report which describes the model and the computer progam which implements it (NUREG/CR-1636, Vol 1; SAND78-1711). In addition, a sequence of appendices provides the following: a description of a surface hydrologic system used in constructing several of the examples, a discussion of mixed-cell models, and a discussion of selected mathematical topics related to the Pathways Model. A copy of the program PATH1 is included with the report.

1318 (NUREG/CR-3062) Status of geochemical problems relating to the burial of high-level radioactive waste, 1982. Apps, J.A.; Carnahan, C.L.; Lichtner, P.C.; Michel, M.C.; Perry, D.; Silva, R.J.; Weres, O.; White, A.F. (Lawrence Berkeley Lab., CA (USA)). Mar 1983. Contract AC03-76SF00098. 380p.
(LBL-15103). NTIS, PC A17/MF A01 - GPO \$9.50. Order Number DE83010251.

Portions are illegible in microfiche products.

Geochemistry research supporting high-level radioactive waste burial in underground repositories is evaluated. General research covering the whole repository includes the bounding of physical and chemical conditions in the repository environment, estimation of toxic radionuclides present in spent fuel and high-level waste at various times after 1000 years, the forms in which radionuclides might be transported in groundwater, the mechanisms by which radionnuclides are retarded, and transport models incorporating chemical reactions. Specific issues relating to the backfill, near field, and far field are also examined. These include low-permeability backfills, diffusional radionuclide transport through backfills (both with and without sorption), the long-term criticality potential in a repository, near-field phase changes and their impact on rock geomechanical properties and radionuclide migration, the effect of temperature gradients on host rock permeability, and, finally, the assessment of groundwater dating to determine groundwater flow rates. Each evaluation results in a series of conclusions and recommendations. The recommendations are prioritized, and further research needed to resolve current uncertainties is suggested.

1319 (NUREG/CR-3219-Vol.2) Post-emplacement monitoring, Subtask 1.2. Draft Technical Position. Vol. 2. Panno, S.V. (Brookhaven National Lab., Upton, NY (USA)). May 1983. Contract AC02-76CH00016. 119p. (BNL-NUREG51658-Vol.2). NTIS, PC A06/MF A01 - GPO \$5.00. Order Number DE83013639.

Portions are illegible in microfiche products.

This describes the environmental conditions that should be monitored within a geologic high-level waste repository after the waste packages have been emplaced. Waste package monitoring and testing requirements are outlined and means by which the licensee may comply with these requirements are presented. Plans of Action, in the event that the waste package monitoring system signals the existence of out-ofdesign or hazardous conditions, are also discussed. Finally, a review has been included of state-of-the-art instrumentation available for in-situ waste package monitoring that may be used to evaluate waste package performance.

1320 (ONWI-27) Environmental characterization of bedded salt formations and overlying areas of the Permian Basin. (NUS Corp., Rockville, MD (USA)). Jul 1983. Contract AC0676RL01830;AC02-83CH10140. 428p. NTIS, PC A19/MF A01. Order Number DE83018271.

Portions are illegible in microfiche products. Original copy available until stock is exhausted.

This report constitutes one input to the first stage of site qualification studies: It presents a general environmental characterization of the region that is underlain by the Permian bedded salt formation. The formation covers portions of Colorado, Kansas, New Mexico, Oklaho$\mathrm{ma}$, and Texas. The Permian bedded salt formation is one of a number of deep, stable geologic formations being studied for potential locations for nuclear waste repositories. These studies will not necessarily lead to selection of a site. They are intended only to provide information necessary to evaluate the suitability of locations for repositories. The report is intended as a general characterization of the existing environmental setting of the Permian Region with emphasis on land, water, and air characteristics; resources; plant and animal life; and man's organizations and activities. The report provides background information about the role that this regional study will play in the overall plan for environmental impact assessments and statements deemed necessary as input to the decision-making process. Background information on the present concept of nuclear waste repository design and function is also included. The information presented in this report has been summarized from open literature readily accessible to the public. No field work was conducted nor new data used in developing the descriptions contained herein.

1321 (ONWI-192) Environmental characterization report for the Gulf Interior Region Louisiana study area. (Bechtel National, Inc., San Francisco, CA (USA)). Oct 1982. Contract AC06-76RL01830. 261p. NTIS, PC A12/MF A01. Order Number DE83010526. 
Portions are illegible in microfiche products. Original copy available until stock is exhausted.

This report is published as a product of the National Waste Terminal Storage (NWTS) Program. The objective of this program is the development of terminal waste storage facilities in deep, stable geologic formations for high-level nuclear waste, including spent fuel elements from commercial power reactors and transuranic nuclear waste for which the federal government is responsible. The report is part of the area study phase and contains environmental information for the Louisiana Study Area of the Gulf Interior Region acquired from federal, state, and regional agencies. The data in this report meet the requirements of predetermined survey plans and will be used in determining locations of approximately 80 square kilometers ( 30 square miles) that will be further characterized. Information on surface water, atmosphere, background radiation, natural ecosystems, agricultural systems, demography, socioeconomics, land use, and transportation is presented. The environmental characterization will ensure that data on environmental values required by the National Environmental Policy Act (NEPA) of 1969 are available.

1322

(ONWI-193) Environmental characterization report tor the Gulf Interior Kegion Mississippi study area. (Bechtel National, Inc., San Francisco, CA (USA)). Oct 1982. Contract AC06-76RL01830. 252p. NTIS MF A01. Order Number DE83010525.

Microfiche only, copy does not permit paper copy reproduction. Original copy available until stock is exhausted.

This report is published as a product of the National Waste Terminal Storage (NWTS) Program. The objective of this program is the development of terminal waste storage facilities in deep, stable geologic formations for high-level nuclear waste, including spent fuel elements from commercial power reactors and transuranic nuclear waste for which the federal government is responsible. The report is part of the area study phase and contains environmental information for the Mississippi Study Area of the Gulf Interior Region acquired from federal, state, and regional agencies. The data in this report meet the requirements of predetermined survey plans and will be used in determining locations of approximately 80 square kilometers ( 30 square miles) that will be further characterized. Information on surface water, atmosphere, background radiation, natural ecosystems, agricultural systems, demography, socioeconomics, land use, and transportation is presented. The environmental characterization will ensure that data on environmental values required by the National Environmental Policy Act (NEPA) of 1969 are available.

1323 (ONWI-194) Environmental characterization report for the Gulf Interior Region, Texas study area. (Bechtel National, Inc., San Francisco, CA (USA)). Oct 1982. Contract AC0676RL01830. 236p. NTIS, PC A11/MF A01. Order Number DE83010220.

Portions are illegible in microfiche products. Original copy available until stock is exhausted.

This report is published as a product of the National Waste Terminal Storage (NWTS) Program. The objective of this program is the development of terminal waste storage facilities in deep, stable geologic formations for high-level nuclear waste, including spent fuel elements from commercial power reactors and transuranic nuclear waste for which the federal government is responsible. The report is part of the area study phase and contains environmental information for the Texas Study Area of the Gulf Interior Region acquired from federal, state, and regional agencies. The data in this report meet the requirements of predetermined survey plans and will be used in determining locations of approximately 80 square kilometers ( 30 square miles) that will be further characterized. Information on surface water, atmosphere, background radiation, natural ecosystems, agricultural systems, demography, socioeconomics, land use, and transportation is presented. The environmental characterization will ensure that data on environmental values required by the National Environmental Policy Act (NEPA) of 1969 are available. ground water as radionuclide-mobilizing agents. Means, J.L. (Battelle Columbus Labs., OH (USA)). Dec 1982. Contract AC06-76RL01830. 69p. NTIS, PC A04/MF A01. Order Number DE83005335.
This report summarizes FY 1981 progress on Tasks 2 and 3 ONWI program E-516-01700, Radionuclide Mobilization by Organ Complexing Agents. The objective of Task 2: Radionuclide Partitionir Analyses is to investigate the effect of organic complexing agents on radionuclide adsorption from 25 to $200^{\circ} \mathrm{C}$. Preliminary experiments studying adsorption of $\mathrm{Co}, \mathrm{Sr}, \mathrm{Cs}$, and $\mathrm{U}$ by kaolinite, illite, and montmorillonite in the presence and absence of oxalic acid yielded two interesting results: (1) adsorption of $\mathrm{Co}, \mathrm{Sr}$, and $\mathrm{U}$ by all three clays was fairly constant from 25 to $100^{\circ} \mathrm{C}$, and then increased from 150 to $200^{\circ} \mathrm{C}$; (2) in numerous instances, adsorption in systems containing oxalic acid actually exceeded that in controls containing no oxalic acid. In Task 3, Humate Flocculation Experiments, the colloid characteristics of complexes of humic and fulvic acid with $\mathrm{Na}, \mathrm{Ca}, \mathrm{Al}, \mathrm{Fe}(\mathrm{II}), \mathrm{Fe}$ (III), ${ }^{208} \mathrm{U}$, ${ }^{202} \mathrm{Th},{ }^{241} \mathrm{Am},{ }^{207} \mathrm{~Np}$, and ${ }^{209} \mathrm{Pu}$ at $\mathrm{pH} 3.5$ to 9.0 were investigated. All metals tested had some effect on humic acid (HA) and fulvic acid (FA) solubility, with $\mathrm{Al}, \mathrm{Fe}$, and the actinides having the greatest effect. In general HA was flocculated to a greater extent than FA, and flocculation was $\mathrm{pH}$ dependent, low $\mathrm{pH}$ promoting $\mathrm{HA}$ or FA precipitation. While the metals affected HA and FA solubility, the HA and FA in turn altered inctal sulubility relative to Inorganlc blanks. In cases where extensive flocculation of HA or FA was evident at low pH, Fe(II). Th, and Am also precipitated from solution at low $\mathrm{pH}$. At near neutral $\mathrm{pH}$ HA and FA formed soluble complexes with $\mathrm{Fe}$ (II), $\mathrm{Fe}$ (III), U, Th, Am, and $\mathrm{Pu}$, increasing their solubility in solution relative to inorganic blanks.

1326 (ONWI-466) LAYFLO: a one-dimensional semianalytical model for the migration of a three-member decay chain in a multilayered geologic medium. Gureghian, A.B.; Jansen, G. (Battelle Memorial Inst., Columbus, OH (USA). Office of Nuclear Waste Isolation). May 1983. Contract ACO283CH10140;AC06-76RL01830. 94p. NTIS, PC A05/MF A01. Order Number DE83013890.

Portions are illegible in microfiche products.

A computer code LAYFLO designed to predict the onedimensional transport of a three-member decay chain in a stratified geological system is presented. The differential equations describing the migration process are solved by the Laplace transformation technique using a semi-analytical approach. The solution of the non-dispersive form of the transport equation is also provided. The model allows for two types of boundary conditions, i.e., a continuous source as well as a band release mode, respectively. The accuracy of LAYFLO was tested on a selected number of problems for which analytical, numerical solutions or experimental data were available. The solution of the non-dispersive furm of the mass transport equation can handle any number of layers, whereas these are restricted to a maximum of six for the gencral case. A graphics program using the DISSPLA package is provided with the LAYFLO code.

1326 (ONWI-470) Ecosystem studies, endangered species survey - Gibson Dome and Elk Ridge study areas, Paradox Basin, Utah. (Bechtel Group, Inc., San Francisco, CA (USA)). Apr 1983. Contract AC06-76RL01830. 63p. NTIS, PC A04/MF A01. Order Number DE83011126.

Portions are illegible in microfiche products.

This report is published as a product of the National Waste Terminal Storage (NWTS) Program. The objective of this program is the development of terminal waste storage facilities in deep stable geologic formations for high-level nuclear wastes, including spent fuel elements from commercial power reactors and transuranic nuclear waste for which the federal government is responsible. This report is part of the location and site characterization phase and contains threatened and endangered species information for the Gibson Dome and Elk Ridge study areas of the Paradox Region. The threatened and endangered species information was obtained through site surveys designed and implemented by arca cxperts. The site surveys were performed during the period late summer 1981 - spring 1982 in the Gibson Dome and Elk Ridge Study Areas. No threatened or endangered species were identified in cither Lavender or Davis canyons. Additional studies at the borehole locations in Beef Basin did identify the nearest occurrence of a sp cies proposed for endangered status (Astragalus monumentalis, a mon ment milkvetch, member of the legume family). The species was iden fied approximately 160 to $300 \mathrm{~m}$ (500 to $1000 \mathrm{ft}$ ) from a hydro testing drill site. Consequently, construction and operation activity should not cause any adverse impacts. This report will be used to satisfy Section 7 requirements of the Endangered Species Act (PL 93-205 as amended) 
and to allow the United States Fish and Wildlife Service to verify that no protected species are subject to disturbance as the result of project activities occurring in the Gibson Dome and Elk Ridge study areas.

1327 (RHO-BW-SA-188) Numerical modeling of groundwater flow and solute transport for a nuclear-waste repository in basalt. Clifton, P.M.; Arnett, R.C.; Baca, R.G. (Rockwell International Corp., Richland, WA (USA). Energy Systems Group). May 1982. Contract AC06-77RL01030. 27p. (CONF-8205501). NTIS, PC A03/MF A01. Order Number DE82014486.

From Geological Society of America Rocky Mountain section meeting; Bozeman, MT, USA (7 May 1982).

Near-field-modeling studies suggest that, over a 10,000-year period, a radionuclide plume leached from a high-level nuclear waste repository in the Umtanum flow under baseline conditions would not migrate more than about 200 meters vertically and a few kilometers horizontally from the repository. Far-field-modeling studies suggest that, under pre-waste-emplacement conditions, groundwater in the Grande Ronde Formation moves subhorizontally and to the southeast from the proposed repository location. Groundwater-travel times from the repository to the edge of the Pasco Basin are estimated to be on the order of 100,000 years.

1328 (RIIO-BW-ST-35P) Radionuclide sorption on basalt-interbed materials. Annual report FY 1981. Barney, G.S. (Rockwell International Corp., Richland, WA (USA). Energy Systems Group). Oct 1982. Contract AC06-77RL01030. 70p. NTIS, PC A04/MF A01. Order Number DE83008062.

Sorption and precipitation of key radionuclides in the groundwater/rock systems expected in Columbia River interbed zones were investigated. Sorption isotherms were defined for sorption of $\mathrm{Cs}, \mathrm{Sr}, \mathrm{Se}, \mathrm{Tc}$, $\mathrm{Ra}, \mathrm{U}, \mathrm{Np}$, and Am on an interbed sandstone standard from the Rattlesnake Ridge interbed. In addition, the effect of groundwater composition and redox potential on sorption and precipitation of these radionuclides on interbed sandstone and tuff were studied. Isotherms were measured at $23^{\circ} \mathrm{C}, 60^{\circ} \mathrm{C}$, and $85^{\circ} \mathrm{C}$. The Freundlich equation accurately fits the isotherm data when precipitation does not occur. Solubility limits were obtained for technetium and selenium using the isotherms. Cesium, selenium, technetium, uranium, and americium sorption are strongly affected by temperature over the $23^{\circ} \mathrm{C}$ to $85^{\circ} \mathrm{C}$ range. The major chemical components of Grande Ronde groundwater $\left(\mathrm{Na}^{+}, \mathrm{Ca}^{2+}\right.$, $\mathrm{K}^{+}, \mathrm{Mg}^{2+}, \mathrm{Cl}^{-}, \mathrm{F}, \mathrm{CO}_{0}{ }^{2-}, \mathrm{HCO}^{-}$, and $\mathrm{SO}_{4}{ }^{2-}$ ) were studied for their influence on sorption and precipitation. Statistically designed experiments identified significant groundwater components and measured their effects. The results gave evidence for selecting sorption mechanisms for each radionuclide (ion exchange, chemisorption, redox reactions, complex formation, or precipitation). Strontium, radium, and cesium are sorbed by ion exchange and compete for exchange sites with ions of similar size. Neptunium, plutonium, and technetium are reduced by hydrazine to their (IV) oxidation states and are sorbed by chemisorption. Selenium is not reduced to the metal by hydrazine, but appears to be precipitated as a calcium compound. The groundwater variables had little effect on americium sorption. A slightly soluble americium compound appears to form which is more soluble at low $\mathrm{pH}$.

1329 (RHO-BW-ST-39P) Geochemical controls on radionuclide releases from a nuclear waste repository in basalt: estimated solubilities for selected elements. Early, T.O.; Jacobs, G.K.; Drewes, D.R. (Atomics International Div., Richland, WA (USA). Rockwell Hanford Operations). Sep 1982. Contract AC06-77RL01030. 72p. NTIS, PC A04/MF A01. Order Number DE83013983.

Two basalt flows within the Grande Ronde formation at the Hanford Site in Southeastern Washington are candidates for a high-level nuclear waste repository. In order to determine the anticipated rates of release and migration of radionuclides from the repository, solubility controls must be determined for key elements. Solubilities, the solids controlling solubility and aqueous speciation for a variety of actinides and fission products in groundwater, have been determined at $25^{\circ} \mathrm{C}$ from available thermodynamic data over a range of Eh values. Groundwater compositions used include all available analyses from the Grande Ronde formation determined by the Basalt Waste Isolation Project. Solids predicted to control solubility for the selected radionuclides include hydroxides and hydrous oxides (palladium, antimony, samarium, europium, lead, americium), oxides (nickel, tin, thorium, neptunium, plutonium), elements (selenium, palladium) and silicates (zirconi- um, uranium). Dominant soluble species include hydroxy complexes (zirconium, palladium, tin, antimony, samarium, europium, thorium, uranium, neptunium) and carbonate species (nickel, samarium, europium, lead, uranium, neptunium, plutonium, americium). The effect on solubility of dissolved, reduced ( $\left.\mathbf{S}^{2-}, \mathrm{HS}^{-}\right)$sulfur species is large for nickel, palladium, antimony, and lead. However, available data suggest that essentially all sulfur in Grande Ronde groundwater is in the form of sulfate $\left(\mathrm{SO}_{4}{ }^{2-}\right)$. Selenium solubility appears to be a strong function of Eh and may reach very high levels where $-0.45>\mathrm{Eh}>-0.05 \mathrm{~V}$. This result suggests that future experimental work should include solubility determinations for selenium. In addition, other elements, notably technetium and radium, with large uncertainties in calculated solubility should also have solubilities determined experimentally.

1330 (SAND-82-0664-Vol.2-App.-Pt.2,pp 407-504) Deep sea biophysics. Yayanos, A.A. (Univ. of California, San Diego, La Jolla). Jul 1982. NTIS, PC A99/MF A01.

In Subseabed disposal program annual report, JanuarySeptember 1981. Volume II. Appendices (principal investigator progress reports). Part 2.

A collection of deep-sea bacterial cultures was completed. Procedures were instituted to shelter the culture collection from accidential warming. A substantial data base on the rates of reproduction of more than 100 strains of bacteria from that collection was obtained from experiments and the analysis of that data was begun. The data on the rates of reproduction were obtained under conditions of temperature and pressure found in the deep sea. The experiments were facilitated by inexpensively fabricated pressure vessels, by the streamlining of the methods for the study of kinetics at high pressures, and by computerassisted methods. A polybarothermostat was used to study the growth of bacteria along temperature gradients at eight distinct pressures. This device should allow for the study of microbial processes in the temperature field simulating the environment around buried HLW. It is small enough to allow placement in a radiation field in future studies. A flow fluorocytometer was fabricated. This device will be used to determine the DNA content per cell in bacteria grown in laboratory culture and in microorganisms in samples from the ocean. The technique will be tested for its rapidity in determining the concentration of cells (standing stock of microorganisms) in samples from the ocean.

1331 (SAND-82-7089) Solutions to radionuclide migration with nonlinear retardation mechanisms in backfill material. Interim progress report, June-December 1982. Anderson, H.M.; Pietz, J.; Smith, D. (New Mexico Univ., Albuquerque (USA). Dept. of Chemical and Nuclear Engineering). Aug 1983. Contract AC04-76DP00789. 73p. NTIS MF A01. Order Number DE83017647.

Microfiche only, copy does not permit paper copy reproduction. Original copy available until stock is exhausted.

Previous models of the backfill barrier have incorporated a linear isotherm with a single valued retardation term, Rd, for predicting the retardation effect of bentonite clays on radionuclide transport. The failure of this modeling approach to accurately predict radionuclide migration has prompted the development of more comprehensive phenomenological models for retardation. This paper advances a conceptual framework for modeling retardation in the backfill with any of a number of complex physiochemical processes including (1) sorption, (2) chemical precipitation/dissolution, (3) ion or isotope exchange, (4) chemical substitution reactions or (5) entrapment. These processes are accounted for by the inclusion of a general retardation sink term in a model for diffusion controlled flow through a porous medium. Nonlinearities in the retardation mechanism may render an analytic solution to the resultant differential equation unattainable. However, semi-analytic monotone iterative schemes described in this paper are shown to provide an effective technique for the necessary integrations. No attempt has been made to calibrate the model to reflect the backfill barrier's potential nor to provide quantitative estimates of effectiveness in an actual repository. Comparisons between models with different retardation mechanisms and estimates of potential delay time effectiveness in an actual repository will be the subject of future reports.

1332 (UCID-19729) Leaching study of nuclear melt glass: Part I. Failor, R.A.; Coles, D.G.; Rego, J.A.H. (Lawrence Livermore National Lab., CA (USA)). 23 Feb 1983. Contract 
W-7405-ENG-48. 76p. NTIS, PC A05/MF A01. Order Number DE83008312.

Portions are illegible in microfiche products. Original copy available until stock is exhausted.

Ground samples of three nuclear melt glasses from underground nuclear explosions at the Nevada Test Site (NTS) were leached at $25^{\circ} \mathrm{C}$ with natural ground water from NTS. Using our dynamic single-pass flow-through leaching system we monitored the release of radionuclides from the glasses during $\mathbf{4 2 0}$ days of leaching. We continually flowed the ground water over the melt glass at flow rates of $185 \mathrm{ml} /$ day for half of the samples and $34 \mathrm{ml} /$ day for the rest. Leachate solutions were collected continuously, and composite samples, collected on days $1,2,3$, $6,11,32,38,70,120,230$ and 420 , were analyzed using low-background $\mathrm{Ge}(\mathrm{Li})$ gamma spectrometers. For most of the radionuclides the leach rate decreased smoothly throughout the experiment. Except for ${ }^{95} \mathrm{Zr}$, ${ }^{144} \mathrm{Ce}$, and ${ }^{155} \mathrm{Eu}$, there was no difference between the fast ( $185 \mathrm{ml} /$ day) and slow ( $34 \mathrm{ml} /$ day) flow-rate leach rates. The measurable leach rates ranged from a high of $1 \times 10^{-2} \mathrm{~g}$-glass $/ \mathrm{m}^{2}$ day for ${ }^{22} \mathrm{Na}$ (slow flow-rate, day 1 in glass No. 2) to a low of $1 \times 6^{6}$ g-glass $/ \mathrm{m}^{2}$ day for ${ }^{54} \mathrm{Mn}$ (slow flow-rate, day 420 in glass No. 2). Most of the leach-rate values were about $5 \times 10^{-4} \mathrm{~g}$-glass $/ \mathrm{m}^{2}$ day initially, decreasing to $5 \times 10^{-6} \mathrm{~g}$-glass $/ \mathrm{m}^{2}$ day after $\mathbf{4 2 0}$ days of leaching. The leach rates had not leveled off by the end of the experiment and were, in general, continuing to decrease. From the activities in the leachate solutions, we determined the percent of the pre-leach activity leached from the melt glasses during the experiment. Only ${ }^{124} \mathrm{Sb}$, in one fast flow-rate channel of glass No. 2 , exceeded $3 \%$ of the initial activity leached. The majority of the samples released less than $1 \%$ of the pre-leach activity for a given radionuclide. The percent activity released from the samples leached at the fast and slow flow rates were nearly equal.

1333 Long-term radioactivity release from solidified highlevel waste. Part II. Parametric study of waste from properties, temperature and time. Altenhein, F.K. (Hahn-Meitner-Institut fuer Kernforschung Berlin, Germany); Lutze, W.; Ewing, R.C. Materials Research Society Symposia Proceedings; 15: 269-280(1983). (CONF-821107-Pt.1).

From 6. international symposium on the scientific basis for radioactive waste management (Materials Research Society); Boston, MA, USA (1 Nov 1982).

The computer code QTERM has been used to calculate the total released activity from a single glass block when in contact with brine in a salt dome repository as a function of: (1) waste form properties, (2) leaching mechanisms, (3) retention or precipitation of specific radionuclides in surface layers, (4) thermal history of the repository and (5) decreasing activity as a function of time.

1334 Radionuclide migration into natural fracture surfaces of granitic rock. Torstenfelt, B.; Eliasson, T.; Allard, B.; Andersson, K.; Hoeglund, S.; Ittner, T.; Olofsson, U. (Chalmers Univ. of Technology, Goeteborg, Sweden). Materials Research Society Symposia Proceedings; 15: 339-346(1983). (CONF821107-Pt.1).

From 6. international symposium on the scientific basis for radio active waste management (Materials Research Society); Boston, MA, USA (1 Nov 1982).

The update of cesium on natural fracture surfaces taken from granitic bedrock has been studied as well as the depth penetration into the underlying rock. The fracture minerals usually exhibit good sorptive propertles with respect to cesium (with the exception of calcite). The surface coatings do not act as physical diffusion barriers and do not prevent a migration into the underlying rock. A considerable depth penetration into the rock (several $\mathrm{mm}$ over a period of three months) was observed. The dominating pathways into the rock matrix were the highcapacity minerals in grains or in microfissures. The calculated diffusivity in the rook matrix was of the order of $10^{-10} \mathrm{~m}^{2} / \mathrm{s}$.

1335 Studies on speciation of americium, technetium and neptunium in simulated vitrified-waste leachates. Bidoglio, $G$.; de Plano, A.; Chatt, A. (Commission of the European Communities, Ispra, Italy). Materials Research Society Symposia Proceedings; 15: 373-382(1983). (CONF-821107-Pt.1).

From 6. international symposium on the scientific basis for radioactive waste management (Materials Research Society); Boston, MA, USA (1 Nov 1982).
Physico-chemical forms of several radionuclides are being inves tigated in order to characterize the migrating species in a deep geologi. repository. Under ground water bicarbonate and carbonate concentra tion levels, $\mathrm{Am}\left(\mathrm{CO}_{0}\right)_{2}^{-}$has been observed to be the most important complex while the formation of $\mathrm{Am}(\mathrm{OH})\left(\mathrm{CO}_{0}\right)_{2}{ }_{2}^{2-}$ has been indicated, stability constants of Am-complexes have been calculated, and a distribution diagram of $\mathrm{Am}$ (III) species as a function of $\mathrm{pH}$ is presented. Speciation of $\mathrm{Tc}$ has been studied using radiochemical and electromigration methods in oxic and anoxic environments; an effective diffusion coefficient of $1.37 \times 10^{-5} \mathrm{~cm}^{2} \mathrm{~S}^{-1}$ for $\mathrm{TcO}_{4}^{-}$has been obtained. Preliminary studies on $\mathrm{Np}(\mathrm{V})$ indicate that $\mathrm{NpO}_{2}{ }^{+}$does not form strong complexes with carbonate and bicarbonate ions at ground water concentrations.

1336 Neptunium concentrations in solutions contacting actinide-doped glass. Rai, D.; McVay, G.L.; Strickert, R.G. (Pacific Northwest Laboratory P.O. Box 999, Richland, Washington 99352). Nuclear Technology; 58: No. 1, 69-76(Jul 1982).

To help predict concentrations of neptunium leached from nuclear waste repositories in geologic environments, the solubility of neptunium in a neptunium-roper hmrnsilicate glass, which simulates a high-level waste glass, was investigated. The concentrations of neptunium in solutions contacting the crushed doped glass were found to be controlled by a neptunium solid phase that is similar to crystalline(c) $\mathrm{NpO}_{2}$ in solubility. Thus, the maximum concentration of the neptunium leached from this waste form can be predicted from the solubility of $\mathrm{NpO}_{2}$ (c). This conclusion is based on similar neptunium concentrations in solutions contacting neptunium-doped glass, neptunium-doped glass plus $\mathrm{NpO}_{2}(\mathrm{c})$, and $\mathrm{NpO}_{2}$ (c) alone, under controlled redox potentials and a range of $\mathrm{pH}$ values. The quinhydrone used in this study was found to be a very effective redox buffer (the approximate $\mathrm{pe}+\mathrm{pH}>11.8)$. The prediotions based on the thermodyinatic data and the sulvent exiraction tests showed $\mathrm{Np}(\mathrm{V})$ to be the primary oxidation state in solution.

1337 Review of radio-nuclide migration in geological formations. Lewis, J.B. (UKAEA Atomic Energy Research Establishment, Harwell. Chemical Technology Div.). Nuclear Energy (Institution of Nuclear Engineers): 21: No. 3, 175-180(Jun 1982).

The burial of suitably conditioned high-level radioactive wastes in deep geological strata, or in the sediments under the deep ocean. has been proposed as a safe method for the ultimate disposal of these wastes. Before any such burial can be carried out, the risk of subsequent transport of radioactivity back to man must be shown to be acceptably low. Nuclide migration through geological formations plays a key role in the transport mechanism, and the present position in understanding the factors involved is outlined. The role of nuclide migration in existing. shallow, burial sitcs for low-level wastes is also discussed.

1338 Solubility-limited dissolution rate in groundwater. Chambre, P.L.; Pigford, T.H.; Zavoshy, S. (University of California, Berkeley, CA 94720). Transactions of the American Nuclear Society; 41: 153-154(Jun 1982). (CONF-820609-).

From American Nuclear Society annual meeting; Los Angeles, CA, USA (6 Jun 1982).

BOROSILICATE GLASS; DISSOLUTION; FISSION PRODUCT RELEASE; GROUND WATER; HIGH-LEVEL RADIOACTIVE WASTES; PHYSICAL PROPERTIES; SOLUBILITY; RADIOACTIVE WASTE DISPOSAL; UNDERGROUND DISPOSAL; GEOLOGIC DEPOSITS

1339 Radionuclide migration in a two-dimensional flow field. Ting, D.K.; Chambre, P.L.; Pigford, T.H. (University of California, Berkeley, CA 94720). Transactions of the American Nuclear Society; 41: 156-157(Jun 1982). (CONF-820609-). From American Nuclear Society annual meeting; Los Angeles, CA, USA (6 Jun 1982).

ANALYTICAL SOLUTION; CALCULATION METHODS; FLUID FLOW; GROUND WATER; HIGH-LEVEL RADIOACTIVE WASTES; HYDROLOGY; RADIONUCLIDF MIGRATION; TWO-PHASE FLOW; AQUIFERS; HANFORD RESERVATION COLUMBIA RIVER

1340 Diffusional transport of radionuclide chains in subseabeds. Ray, A.K. (Kentucky Univ., Lexington (USA). Dept. of Chemical Engineering); Davis, E.J. (Institute of Paper Chemistry, Appleton, WI (USA)); Nuttall, H.E. (New Mexico 
Jniv., Albuquerque (USA)). Annals of Nuclear Energy; 9: No. 337-350(1982).

The diffusion-controlled migration of radionuclides released from a leaky canister located in a subseabed sedimentary layer, bounded at the top by the ocean and at the bottom by an impermeable basalt zone, is analyzed to determine the transport rate to the ocean floor.

1341 Diffusion in the matrix of granitic rock: field test in the Stripa mine. Birgersson, L.; Neretnieks, I. (Royal Inst. of Tech., Stockholm, Sweden). pp 519-528 of Scientific basis for nuclear waste management V. Lutze, W. (ed.). New York, NY; North-Holland (1982). (CONF-820636-).

From 5. international symposium on the scientific basis for radio active waste management; Berlin, F.R. Germany (7 Jun 1982).

A migration experiment with the objective to investigate the existence of a connected pore system in undisturbed rock has been performed in the Stripa mine at the $360 \mathrm{~m}$-level. Since all three tracers (C. EDTA $I^{-}$and Uranine) that were used in the experiment migrated at least $11 \mathrm{~cm}$ into the rock under a three-month period, this experiment indicates the existence of a connected pore system in undistubed rock.

1342 Migration in a single fracture. Abelin, $H$.; Gidlund, J.; Neretnieks, I. (Royal Inst. of Tech., Stockholm, Sweden). pp 529-538 of Scientific basis for nuclear waste management $V$. Lutze, W. (ed.). New York, NY; North-Holland (1982). (CONF-820636-).

From 5. international symposium on the scientific basis for radioactive waste management; Berlin, F.R. Germany (7 Jun 1982).

It has been decided to investigate flow and sorption in a readily identifiable fracture which can be excavated for a detailed examination of flow path and sorbtion sites. The investigation is performed in the Stripa mine, $360 \mathrm{~m}$ below ground, where there is a natural water flow towards the drift. The bedrock is granite. A method of tracer injection into a fracture, either as a step or a pulse, and of collection of water samples under anoxic atmosphere has been suggested and tested in a preparatory investigation. The introduction of tracers can be done either under natural pressure or by injection with over pressure. An injection of Rhodamine-WT, Na-iodide and Na-Fluorescein with over pressure has been performed. It as been found that Rhodamine-WT is influenced in some way along the flow path.

1343 Model for near field migration. Andersson, G.; Rasmuson, A.; Neretnieks, I. (Royal Inst. of Tech., Stockholm, Sweden). pp 539-548 of Scientific basis for nuclear waste management V. Lutze, W. (ed.). New York, NY; North-Holland (1982). (CONF-820636-).

From 5 . international symposium on the scientific basis for radioactive waste management; Berlin, F.R. Germany (7 Jun 1982).

A model is proposed which describes the transport to and from a waste canister in a repository. The model includes flow and diffusion in the fractures in the bedrock as well as diffusion in the backfill. Calculations have been made on the inward transport of corrosive agents to a buried canister from the surrounding bedrock. The oxidants are transported from the flowing groundwater thorugh the backfill to the surface of the canister, where :hey are assumed to react instantaneously with the canister material. Tine rock is modeled as a discrete fracture system. The fractures are assunied to be evenly spaced and the groundwatcr movement is described by potential flow. The model is three dimensional.

1344 Model for far field migration. Rasmuson, A.; Neretnieks, I. (Royal Inst. of Tech., Stockholm, Sweden). pp 549-558 of Scientific basis for nuclear waste management $V$. Lutze, $W$. (ed.). New York, NY; North-Holland (1982). (CONF820636-)

From 5 . international symposium on the scientific basis for radioactive waste management; Berlin, F.R. Germany (7 Jun 1982).

A transport model of radionuclide migration in fissured rock is resented. It includes advection and hydrodynamic dispersion in the fisures and sorption in the rock blocks. I and 2D analytical and 3D numercal solutions of the model have been developed. A large number of calculations for the approximate range of variation of the input parameters have been performed. A large impact of hydrodynamic dispersion and micropore diffusion on the amount of radioactive material reaching the biosphere is found.
1346 Leach rates of high level waste and spent fuel: limiting rates as determined by backfill and bedrock conditions. Neretnieks, I. (Royal Inst. of Tech., Stockholm, Sweden). pp 559-568 of Scientific basis for nuclear waste management V. Lutze, W. (ed.). New York, NY; North-Holland (1982). (CONF-820636-).

From 5 . international symposium on the scientific basis for radioactive waste management; Berlin, F.R. Germany (7 Jun 1982).

The release rate of radionuclides from a degraded canister containing radioactive waste is controlled by diffusion through the backfill and by diffusion into the water flowing past the canister. In low porosity low permeability bedrock like the Swedish granites the amount of water which can be contaminated may be very small. Two sample cases are calculated. One uses the conditions for a repository for vitrified high level waste, the other applies to a repository for spent fuel. The small amount of water which can carry the waste in combination with the low solubilities for the major long-lived actinides limit the release rate from the repositories to very small values.

1346 Permeability monitoring technique of the near-field and far-field interface openings. Jakubick, A.T. (Ontario Hydro, Toronto); De Korompay, V. pp 579-586 of Scientific basis for nuclear waste management V. Lutze, W. (ed.). New York, NY; North-Holland (1982). (CONF-820636-).

From 5. international symposium on the scientific basis for radioactive waste management; Berlin, F.R. Germany (7 Jun 1982).

In July/August 1981 and February/March 1982 we sampled water for isotope analysis from water-bearing fractures of two mines in Elliot Lake. The purpose of the investigation was to find out whether there is a relationship between the water present in some fractures in a deep mine and the shallow groundwater in the far-field of the mine. The investigation was done in co-operation with University of Waterloo. The results of the analyses for ${ }^{18} \mathrm{O}$ and ${ }^{2} \mathrm{H}$ contents are expressed as the delta ppM notation and refered to the Standard Mean Ocean Water. The ${ }^{18} \mathrm{O}$ and ${ }^{2} \mathrm{H}$ contents are in the same range as estimated for the Sudbury area. Compared to the global meteoric water line, the samples taken in the Denison Mine and Rio Algom Mine have an isotope composition close to precipitation and shallow groundwater. They ave, however, lower ${ }^{18} \mathrm{O}$ and ${ }^{2} \mathrm{H}$ content than the surface water samples of the area. Also the mine water is slightly to the left of the meteoric waterline. Thus it could be speculated that the water is a brackish, high ${ }^{2} \mathrm{H}$ content, deep groundwater which was highly diluted by the local meteoric water. The high salinity of mine water supports this theory. As the isotope composition of the water in the near excavation zone changed with time it was assumed that his is the place where the mixing occurs. Therefore, the conclusion can be made that shallow groundwater is entering the deeper workings of the mine. A hydrogeologically possible pathway is through the Quirke Lake overthrust which intersects Denison Mine between shaft No. 1 and No. 2. Below the fault the water is probably conducted by local fracture systems where mixing with deep groundwater occurs.

1347 Evaluation of solubility and speciation of actinides in natural groundwaters. Schweingruber, M.R. (Swiss Federal Inst. for Reactor Research, Wuerenlingen, Switzerland). Pp 679-688 of Scientific basis for nuclear waste management $V$. Lutze, W. (ed.). New York, NY; North-Holland (1982). (CONF-820636-).

From 5. international symposium on the scientific basis for radioactive waste management; Berlin, F.R. Germany (7 Jun 1982).

This paper summarizes first modeling efforts undertaken to evaluate the speciation and solubility of $\mathrm{U}, \mathrm{Th}, \mathrm{Np}$ and $\mathrm{Pu}$ in two selected Swiss groundwaters. Starting from a rather general box model, two simple case studies will be defined. The first could be visualized as pertaining to the far-field, the second to the near-field of a repository. For both cases the required model parameters are specified. Some emphasis is laid upon the effect of kinetically hindered solid phase precipitation.

1348 Study of radionuclide migration from deep-lying repository sites with over-lying sedimentary layers. Hadermann, J. (Swiss Federal Inst. for Reactor Research, Wuerenlingen); Roesel, F.; McCombie, C. pp 689-696 of Scientific basis for nuclear waste management V. Lutze, W. (ed.). New York, NY; North-Holland (1982). (CONF-820636-).

From 5 . international symposium on the scientific basis for radioactive waste management; Berlin, F.R. Germany (7 Jun 1982). 
A quick estiamte from the tables shows readily that for the large retention factors, concentrations at th. geosphere exit are reduced to total insignificance (less than $10^{-20} \mathrm{Ci} / \mathrm{m}^{0}$ ). This shows the well-known importance of determining the redox potential in great depths. Thus, only results from calculations with the low retention factors are discussed. The calculation with nuclide dependent leach rates do not differ too much from those with nuclide independent leach rates. The first one gives rise to somewhat higher values since most of the inventory is leached during the leaching period of $10^{5}$ years. Calculations with the time-dependent leach rates coincide with those resulting from constant rates since the effect of decreasing injection rate (for the first 500 years is smeared out by dispersion. One notices that for the upper repository site neptunium and uranium retardation times are shot compared to half-lives and essentially the full inventory exists the geosphere. Here, dilution by the groundwater bearing overlaying sediments is very important. In contrast, for the lower repository site retardation leads to a strong decay of these nuclides. Within the hydraulics model of flow through porous media our results are certainly very conservative for the following reasons. First, we have considered one-dimensional transport, only, with such long water transit times, transversal dispersion will contribute appreciably to a reduction of nuclide concentrations. Second, dilution might be two orders of magnitude larger. if the contaminated water does not exit in a localized spring, but is distributed over a larger geographical area. Third, the leach rates assumed seem to be unrealistically high in view of the low water supply at the repository.

\section{Comparison of in-situ radionuclide migration studies} in the Studsvik area and laboratory measurements. Landstroem, O. (Studsvik Energiteknik AB, Nykoeping, Sweden); Klockars, C.E.; Persson, O.; Andersson, K.; Torstenfelt, B.; Allard, B.; Larsson, S.A.; Tullborg, E.L. pp 697-706 of Scientific basis for nuclear waste management V. Lutze, W. (ed.). New York, NY; North-Holland (1982). (CONF-820636-).

From 5 . international symposium on the scientific basis for radioactive waste management; Berlin, F.R. Germany (7 Jun 1982).

A series of in-situ radionuclide migration tests are in progress in the Studsvik area on the Swedish east coast. Three well-defined flowpaths have been located and characterized using non-sorbing tracers (I-131 and H-3), and one of these pathways has been used for a study of the migration of sorbing elements (Sr-85). Laboratory sorption studies with $\mathrm{Sr}-85$ have been performed on materials (rock-water) from the same location as the field tests and with variation of parameters not easily varied in-situ (e.g., $\mathrm{pH}$ and nuclide concentration). A fairly good correlation was obtained between retention in natural fractures (field experiment) and laboratory column studies, although the latter were performed on crushed samples of whole rock (retention factors 15 to 30 ).

1350 Migration of radionuclides with ground water: a discussion of the relevance of the input parameters used in model calculations. Jensen, B.S. (Risoe National Lab., Roskilde, Denmark). pp 765-774 of Scientific basis for nuclear waste management V. Lutze, W. (ed.). New York, NY; North-Holland (1982). (CONF-820636-).

From 5 . international symposium on the scientific basis for radioactive waste management; Berlin, F.R. Germany (7 Jun 1982).

It is probably obvious to all, that establishing the scientific basis of geological waste disposal by going deeper and deeper in detail; may fill out the working hours of hundreds of scientists for hundreds of years. Such an endeavor is, however, impossible to attain, and we are forced to define some criteria telling us and others when knowledge and insight is sufficient. In thepresent case of geological disposal one need to be able to predict migration behavior of a series of radionuclides under diverse conditions to ascertain that unacceptable transfer to the biosphere never occurs. We have already collected a huge amount of data concerning migration phenomena, some very useful, oter less so, but we still need investigatoins departing from the simple ideal concepts, which most often have provided modellers with input data to their calculations. I therefore advocate that basic research is pursued to the point where it is possible to put limits on the effect of the lesser known factors on the migration behavior of radionuclides. When such limits have been established, it will be possible to make calculations on the worst cases, which may also occur. Although I personally believe, that these extra investigations will prove additional safety in geological disposal, this fact will convince nobody, only experimental facts will do.
1351 Sorption of actinides in well-defined oxidation states on geologic media. Allard, B.; Olofsson, U.; Torstenfelt, B.; Kipatsi, H.; Andersson, K. (Chalmers Univ. of Tech., Goeteborg, Sweden). pp 775-782 of Scientific basis for nuclear waste management V. Lutze, W. (ed.). New York, NY; NorthHolland (1982). (CONF-820636-).

From 5 . international symposium on the scientific basis for radioactive waste management; Berlin, F.R. Germany (7 Jun 1982).

The observed actinide sorption behavior in the present experiments indicate a predominantly physical adsorption mechanism, largely related to the degree of hydrolysis. The formation of anionic species would reduce the sorption. Under the present conditions Pu seems to exist largely as a mixture of various oxidation states, possibly III-V-IV, as has been suggested previously. The reduction of actinides from the penta- or hexavalent state (e.g., for $\mathrm{Np}(\mathrm{V})$ or $\mathrm{U}(\mathrm{VI})$ ) to the tetravalent state would give a higher distribution coefficient (by 2 to 3 orders of magnitude) in the environmental pH-range.

1362 Laboratory tests on the migration behavior of selected fission products in aquifer materials from a potential disposal site in northern Germany. Rehrens, H. (Institut fuer Radiohydrometrie der (Gesellschaft fuer Strahlen- und Umweltforschung mbH Muenchen, Neuherberg, Germany); Klotz, D.; Lang, H.; Moser, H.; Barke, G.; Bruehl, H.; Gehler, S.; Muehlenweg, U. pp 783-790 of Scientific basis for nuclear waste management $V$. Lutze, W. (ed.). New York, NY; NorthHolland (1982). (CONF-820636-).

From 5. intcrnational symposium on the scientific basis for radioactive waste management; Berlin, F.R. Germany (7 Jun 1982).

The migration behavior with respect to retardation by sorption of radioactive $\mathrm{Ce}, \mathrm{Cs}, \mathrm{I}, \mathrm{Nb}, \mathrm{Ru}, \mathrm{Sm}, \mathrm{Sr}, \mathrm{Tc}$ and $\mathrm{Zr}$ in fluviatile and aeolian sand aquifers has been examined in laboratory tests. In these tests it was attempted to simulate the natural conditions of the field. The tests were performed as batch and column experiments. The results of both types of experimental procedures agree within the error limits and show small sorption for $\mathrm{I}^{-}$and $\mathrm{TcO}_{4}^{-}$, medium sorption for $\mathrm{Sr}^{2+}$, and high sorption for $\mathrm{Cs}^{+}, \mathrm{Ce}^{0+}, \mathrm{Sm}^{0+}, \mathrm{Ru}^{4+}, \mathrm{Zr}^{4+}$ and $\mathrm{Nb}^{5+}$. The investigations will be continued with other geologic materials from the same site.

1353 Modelling of the migration of lanthanoids and actinoids in ground water; the medium dependence of equilibrium constants. Biedermann, G.; Bruno, J.; Ferri, D.; Grenthe, I.; Salvatore, F.; Spahiu, K. (Royal Inst. of Tech., Stockholm, Sweden). pp 791-799 of Scientific basis for nuclear waste management V. Lutze, W. (ed.). New York, NY; North-Holland (1982). (CONF-820636-).

From 5 . international symposium on the scientific basis for radioactive waste management; Berlin, F.R. Germany (7 Jun 1982).

The examples given in this communication indicate that it is possible to obtain a good estimate of the medium dependence of equilibrium constants by using the specific interaction thenry. The thenry is applicable both when extrapolating equilibrium constants to zero ionic strength and for the estimation of activity coefficients in mixtures of electrolytes. Many interaction coefficients are available in the literature, or can be calculated from published mean activity coefficient or isopiestic data. The magnitude of interaction coefficients can often be correlated with the charge and size of ions. This offers a possibility to estimate the coefficients for complexes, for which direct experimental information is difficult to get. The specific interaction theory is superior to the empirical equations of the Davies type. There is superior to the empirical equations of the Davies type. There is sufficient experimental information on interaction coefficients to warrant the implementation of the specific interaction approach in existing specifiation codes.

1364 Evaluation of radionuclide transport: effect of radionuclide sorption and solubility. Salter, P.F.; Jacobs, G.K. (Rockwell Hanford Operations. Richland, WA). pp 801-810 of Scientific basis for nuclear waste management V. Lutze, W. (ed.). New York, NY; North-Holland (1982). (CONF820636-).

From 5. international symposium on the scientific basis for radioactive waste management; Berlin. F.R. Germany (7 Jun 1982).

The sorption and solubility behavior of several potentially hazardous (key) radionuclides in the Columbia River Basalt geohydrologic system is discussed. Utilizing the transport characteristics of the site and the available sorption and solubility data, a preliminary site perfor- 
-ance analysis then is performed through comparison of the resultant edicted radionuclide transport behavior with proposed US environental regulations.

1356 Radionuclide retardation during transport through fractured granite. McKinley, I.G.; West, J.M. (Inst. of Geological Sciences, Oxfordshire, England). pp 811-820 of Scientific basis for nuclear waste management V. Lutze, W. (ed.). New York, NY; North-Holland (1982). (CONF-820636-).

From 5. international symposium on the scientific basis for radioactive waste management; Berlin, F.R. Germany (7 Jun 1982).

These results of laboratory sorption studies of particular radionuclides (isotopes of $\mathrm{Cs}, \mathrm{Sr}, \mathrm{Co}$, and $\mathrm{Ce}$ ) onto naturally weathered granite fracture infill are reported. From these results it is apparent that sorption of radionuclides onto weathered fracture infill is likely to be a complex process although data trends may be presented as empirical relationships (e.g., Freundlich isotherms). Detailed studies of reaction mechanisms are in progress and indicate that, for even simple chemical species, sorption can be a complex function of reaction time, temperature, elemental concentration, concentration of competing ions and reaction direction. By use of simple models of groundwater flow, sorption data can be placed in a realistic geological context and results indicate that terms of totally different retardation mechanisms. While various mechanisms might give similar retardation at short path lengths, more detailed information on mechanisms is required to justify extrapolation of data to the long pathlengths and timescales required in safety assessment and it is thus apparent that laboratory and in-situ studies are both essential and complementary in this field.

1366 Comparative analysis of fractured and porous medium radionuclide transport. Travis, B.J. (Los Alamos National Lab., NM); Nuttall, H.E. pp 821-830 of Scientific basis for nuclear waste management $V$. Lutze, $W$. (ed.). New York, NY; North-Holland (1982). (CONF-820636-).

From 5 . international symposium on the scientific basis for radioactive waste management; Berlin, F.R. Germany (7 Jun 1982).

This paper provides a comparative study of porous and fractured medium nuclide transport by modeling the far field $(1 \mathrm{~km})$ problem for three different types of geomedia, i.e., a homogeneous porous medium, a single fracture in the porous medium, and a multi-fracture medium. Analytical models were used to study the homogeneous and single fracture geometries while an advanced numerical code was used to model the multi-fractured medium. The following conclusions were reached. A set of models were developed and used to investigate the effects of fractures on nuclide migration rates in several types of geological media. A single uninterrupted fracture is in a sense the worst case for nuclide migration. (It provides early breakthrough of the nuclides.) The nonfractured and single fracture cases set lower and upper limits on the rate of nuclide migration with the multi-fracture cases giving intermediate results. A single fracture of multiple fractures lower the level of the nuclide concentration in the porous medium by spreading the material over a greater area. Fractures tend to accelerate nuclide transport and overall migration rates.

1357 Effect of microbial activity on the containment of radioactive waste in a deep geological repository. West, J.M.; McKinley, I.G.; Chapman, N.A. (Harwell Lab., Oxfordshire, England). pp 831-838 of Scientific basis for nuclear waste management V. Lutze, W. (ed.). New York, NY; North-Holland (1982). (CONF-820636-).

From 5 . international symposium on the scientific basis for radioactive waste management; Berlin, F.R. Germany (7 Jun 1982).

The sorption experiments described were intended as part of a pilot study and are consequently of a preliminary nature and difficult to interpret. However, they have shown very clearly that microorganisms play a significant quantitative role in the uptake and transport of radionuclides and that the topic requires serious onsideration. Several features have emerged that point to further studies and technique development. For example, sorption data are presented here in 7 rms of the total biomass in solution. Techniques are required for the parate quantification of each part of the biomass (viable, non-viable id dead organisms and organic and associated inorganic material) in both solution or on the rock phase. A realistic explanation of sorption processes where micro-organisms are involved is lacking at present and is likely to be very complex. Eventually partition coefficients could be calculated for each individual biogenic component. More data is required on the identification and quantification of microbial populations in relevant deep formations. Problems arise in such analysis due to the unusual metabolisms of such organisms and very slow growth in culture (expecially in the case of autotrophs). Information on the bioenergetics of these organisms and possible sources and inputs of energy into deep geplogical environments may allow the constraints of the population to be calculated and assessed. With such data the in-situ effect of micro-organisms on the containment of radioactive waste in a deep geological repository could be examined realistically.

1368 INTRACOIN: an international nuclide transport code intercomparison study. Andersson, K. (Swedish Nuclear Power Inspectorate, Stockholm); Grundfelt, B.; Hadermann, J. pp 839-847 of Scientific basis for nuclear waste management $V$. Lutze, W. (ed.). New York, NY; North-Holland (1982). (CONF-820636-).

From 5 . international symposium on the scientific basis for radioactive waste management; Berlin, F.R. Germany (7 Jun 1982).

To improve the understanding of various strategies for geospheric radionuclide tranport modelling an international cooperation project with ten participating organizations has been set up. In the project - INTRACOIN (Internatonal Nuclide Transport COde INtercomparison) study - a comparison between models is made on three levels with increasing complexity. Calculations are performed with some 20 computer codes representing different modelling strategies. The INTRACOIN study is limited in its scope to far-field radionuclide, geosphere transport models. Thus, near-field models, groundwater hydraulics and models for transort in the biosphere are not included. However, since the geological barrier is an important part of the safety system the study should contribute to the understanding of important phenomena related to long-term safety assessments. In addition it provides a forum for discussion and for direct exchange of experience between experts in the field.

1369 Aqueous phase diffusion in crystalline rock. Bradbury, M.H.; Lever, D.; Kinsey, D. (Harwell Labs., Oxfordshire, England). pp 569-578 of Scientific basis for nuclear waste management $V$. Lutze, W. (ed.). New York, NY; NorthHolland (1982): (CONF-820636-).

From 5 . international symposium on the scientific basis for radioactive waste management; Berlin, F.R. Germany (7 Jun 1982).

Diffusion coefficients have been measured in a number of granites, as a typical rock, using the very weakly sorbed iodide ion as tracer. In addition, measurements on weakly sorbed nuclides have yielded values of the sorption coefficient where other techniques are inadequate.

1360 Underground migration of long-lived radionuclides leached from a borosilicate glass matrix. Avogadro, A.; Murray, C.N.; De Plano, A.; Bidoglio, G. (Commission of the European Communities, Ispra (Italy). Joint Research Centre). pp 527-540 of Environmental migration of long-lived radionuclides. Proceedings of an international symposium on migration in the terrestrial environment of long-lived radionuclides from the nuclear fuel cycle organized by the IAEA, the CEC and the OECD NEA and held in Knoxville, USA, 27-31 July 1981. Vienna, Austria; International Atomic Energy Agency (1982). (CONF-810722-; IAEA-SM-257/73).

From International symposium on migration in the terrestrial environment of long-lived radionuclides from the nuclear fuel cycle; Knoxville, TN, USA (27 Jul 1981).

A programme on the safety analysis linked to the geological disposal of radioactive wastes is under study at the Joint Research Centre of the Commission of the European Communities at Ispra. In relation to the migration of radionuclides in the tertestrial environment following a possible release from the repository, specific experimental studies are being developed in order to provide the necessary input data for the risk assessment models. The present paper reports the results of studies performed with neptunium, plutonium, americium and technetium isotopes leached from a borosilicate glass simulating the vitrified high-level wastes. In order to simulate the expected conditions of glass leaching and underground transport in the laboratory, a water pathway is established which flows over the radioactive glass and then through columns containing typical soil samples. The columns are examined during the experiment by gamma scanning or cut into thin sections at the end of the run and the distribution profile of radioisotopes measured. Experimental results obtained on soil columns are compared with data 
obtained using filtering membranes and ion exchange resins. These experiments using borosilicate glass demonstrate the relative importance of colloidal filtration by the geological porous medium. Following fixation it has been shown that slow rate processes probably account for the continuous small release of the radioactivity observed in the laboratory system. This long-term behaviour of colloids during the continuous percolation of groundwater is interpreted, taking into account complex ion formation with inorganic ligands present in natural waters.

1361 Analytical solutions of radionuclide migration with one-dimensional flow in layered media. Gureghian, A.B.; Jansen, G.; Scott, L.A. (Battelle, Office of NWTS Integration, 505 King Avenue, Columbus, $O H$ 43201). Transactions of the American Nuclear Society; 43: 73(1982). (CONF-821103-).

From American Nuclear Society winter meeting; Washington, DC, USA (14 Nov 1982).

ANALYTICAL SOLUTION; FLUID FLOW; GEOLOGIC STRATA; HIGH-LEVEL RADIOACTIVE WASTES; LAYERS; ONE-DIMENSIONAL CALCULATIONS; RADIOACTIVE WASTE DISPOSAL; RADIONUCLIDE MIGRATION; UNDERGROUND DISPOSAL

1362 Migration of long actinides chains in geologic media. Ting, D.; Chambre, P.L.; Pigford, T.H. (Univ of California, Berkely, Berkeley, CA 94720). Transactions of the American Nuclear Society: 38: 163-164(1981). (CONF-810606-).

From American Nuclear Society's annual meeting; Miami Beach, FL, USA (7 Jun 1981).

ACTINIDES; BIOSPHERE; DECAY; FISSION PRODUCTS; GROUND WATER; HAZARDS; HIGH-LEVEL RADIO ACTIVE WASTES; LEACHING; RADIONUCLIDE MIGRATION; RADIOACTIVE WASTE DISPOSAL; ENVIRONMENTAL TRANSPORT

1363 Report of the Select Committee of the Legislative Council on uranium resources. Adelaide, Australia; Government Printer (1981). 206p.

This report contains results of an inquiry into developments since the completion of the Ranger Inquiry in 1977 which bave a bearing on the mining, processing and sale of South Australian uranium resources. The Committee was not unanimous in its conclusions and recommendations. Three members concluded that the benefits of mining deposits in the State would be considerable to the South Australian Community and that the hazards in connection with the mining, milling, transport, further treatment and storage of uranium could be overcome by the imposition of the stringent application of safeguards at all stages of the nuclear fuel cycle. Other conclusions and recommendations related to radiation protection, mine safety, contaiment of mill tailings, the nuclear fuel cycle, marketing, transport and safeguards. Two members recommended that uranium mining should not proceed in South Australia at this time and one member dissented to some matters in the report. Major concerns related to waste disposal and safeguards.

1364 Alpha spectrum profiling of plutonium in leached simulated high-level radioactive waste-glass. Diamond, H.; Friedman, A.M. (Argonne Natl Lab, Ill). Nuclear and Chemical Waste Management; 2: No. 3, 207-212(1981).

Low-geometry $\overline{\mathrm{X}}$-ray spectra from ${ }^{231} \mathrm{Pu}$ and ${ }^{237} \mathrm{~Np}$, incorporated into simulated high-level radioactive waste-glass, were transformed into depth distributions for these elements. Changes in the depth profiles were observed for a series of static leachings in 75/degree/C water. Radiochemical assay of the leach solutions revealed that little neptunium or plutonium was leached, and that the amount leached was independent of leaching time. The depth profiles of the leached specimens showed that there was selective leaching of nonradioactive components of the glass, concentrating the remaining neptunium and plutonium in a broad zone near (but not at) the glass surface. Eventual redeposition of nonradioactive material onto the glass surface inhibited further leaching.

1365 Waste migration by particulate carriers. Eicholz, G.G.; Craft, T.F.; Powell, G.F. (Georgia Inst. of Tech., Atlanta). Transactions of the American Nuclear Society; 35: 66-67(1980). (CONF-801107-).
From ANS international conference; Washington, DC, USA (17 Nov 1980).

HIGH-LEVEL RADIOACTIVE WASTES; RADIOACTIVE WASTE DISPOSAL; RADIONUCLIDE MIGRATION; UNDERGROUND DISPOSAL

1366 (ORNL-tr-4994) Data base for a site-specific analysis of the dispersion of radioactive elements from a final repository. Hadermann, J. (Oak Ridge National Lab., TN (USA); Eidgenoessisches Inst. fuer Reaktorforschung, Wuerenlingen (Switzerland)). [nd]. Contract W-7405-ENG-26. Translation of EIR Report No. 447, November 1981. 30p. NTIS, PC A03/MF A01. Order Number DE83015040.

Migration analysis is of considerable importance in long-term safety analysis of radioactive waste repositories. In a first step our plans are to calculate transport of radionuclides using data - as far as possible - for an undisturbed hydrogeology. Thereby a reference case is defined. In a later step, possible events and processes can be considered leading to a deviation from the reference case. The present work gives the data base for a selected part of a comprehensive geosphere transport calculation. We restrict ourselves to a critical evaluation of parameters pertinent to the migration analysis of the ${ }^{243} \mathrm{Cm}$ chain. This includes the important nuclide ${ }^{207} \mathrm{~Np}$. For the first time we are able to perform a site specific calculation for repositories planned in deep geologic formations in Switzerland. The well known faot that the data basis is extremely sparse is pointed out once more and concretized in detail.

\section{Health And Safety}

1367 (CONF-820303-27) Uncertainties associated with geologic disposal of high-level radioactive waste. Kocher, D.C.; Sjoreen, A.L.; Bard, C.S.; Olsen, C.R. (Oak Ridge National Lab., TN (USA)). 1982. Contract W-7405-ENG-26. 20p. NTIS, PC A02/MF A01. Order Number DE82008810.

From Waste management conference (ANS); Tucson, AZ, USA (8 Mar 1982).

This paper focuses on uncertainties associated with models for predicting: (1) groundwater transport of radionuclides between a repository and the biosphere; and (2) long-term collective dose and health effects following release of long-lived radionuclides to the biosphere. We do not present numerical estimates of uncertainties in such predictions. Rather, we emphasize the various sources of uncertainty and attempt to evaluate the extent to which current models and supporting data bases can realistically describe long-term repository performance and health risks. We do not consider uncertainties associated with the longterm performance of engineered barriers at a repository or with human intrusion.

1368 (CONF-830205-3) Comparison of risks due to HLW and SURF repositories in bedded salt. Chu, M.S.Y.; Ortiz, N.R.; Wahi, K.K. (Sandia National Labs., Albuquerque, NM (USA); Science Applications, Inc., Albuquerque, NM (USA)). 1983. Contract AC04-76DP00789. 8p. NTIS, PC A02/MF A01. Order Number DE83006807.

From Waste management conference; Tucson, AZ, USA (27 Feb 1983).

Portions are illegible in microfiche products.

A methodology was developed for use in the analysis of risks from geologie disposal of nuclear wastes. This methudology is applied to two conceptual nuclear waste repositories in bedded salt containing High-Level Waste (HLW) and Spent Un-Reprocessed Fuel (SURF), respectively. A comparison of the risk estimated from the HLW and SURF repusitories is presented.

1369 (CONF-830528-Summ., pp VI.6-VI.7) Risk analysis of transporting vitrified high level radioactive waste by train. Schneider, K.A. (Transnuklear GmbH, Hanau, Germany); Merz, E. 1983. NTIS, PC A21/MF A01.

From 7. international symposium on packaging and transportation of radioactive materials; New Orleans, LA, USA (15 May 1983)

Borosilicate glass waste form. RAIL TRANSPORT; HIGH LEVEL RADIOACTIVE WASTES; WASTE TRANSPORTATION, VITRIFICATION; RISK ASSESSMENT

1370 (DPST-82-327) Comparative risk assessments for the production and interim storage of glass and ceramic waste 
forms: defense waste processing facility. Huang, J.C.; Wright, V.V. (Du Pont de Nemours (E.I.) and Co., Aiken, SC (USA). javannah River Lab.). Apr 1982. Contract AC09-76SR00001. 145p. NTIS, PC A07/MF A01. Order Number DE82014169. Portions of document are illegible.

The Defense Waste Processing Facility (DWPF) for immobilizing nuclear high level waste (HLW) is scheduled to be built at the Savannah River Plant (SRP). High level waste is produced when SRP reactor components are subjected to chemical separation operations. Two candidates for immobilizing this HLW are borosilicate glass and crystalline ceramic, either being contained in weld-sealed stainless steel canisters. A number of technical analyses are being conducted to support a selection between these two waste forms. The present document compares the risks associated with the manufacture and interim storage of these two forms in the DWPF. Process information used in the risk analysis was taken primarily from a DWPF processibility analysis. The DWPF environmental analysis provided much of the necessary environmental information. To perform the comparative risk assessments, consequences of the postulated accidents are calculated in terms of: (1) the maximum dose to an off-site individual; and (2) the dose to off-site population within 80 kilometers of the DWPF, both taken in terms of the 50year inhalation dose commitment. The consequences are then multiplied by the estimated accident probabilities to obtain the risks. The analyses indicate that the maximum exposure risk to an individual resulting from the accidents postulated for both the production and interim storage of either waste form represents only an insignificant fraction of the natural background radiation of about 90 mrem per year per person in the local area. They also show that there is no disaster potential to the off-site population. Therefore, the risks from abnormal events in the production and the interim storage of the DWPF waste forms should not be considered as a dominant factor in the selection of the final waste form.

1371 (DPST-82-475) Relative hazard of DWPF reference glass canister ICRP 2 vs ICRP 30. Marter, W.L. (Du Pont de Nemours (E.I.) and Co., Aiken, SC (USA). Savannah River Lab.). 16 Jul 1982. Contract AC09-76SR00001. 20p. NTIS, PC A02/MF A01. Order Number DE83012457.

The hazard of SRP high-level radioactive waste (HLW) in a glass canister was calculated relative to uranium ore with BEIR III dose-effect estimates and with dose constants from ICRP Publication 2 and ICRP Publication 30. With ICRP 2 constants (which are the current basis for DOE and NRC standards) the waste is calculated to have equivalent hazard to the uranium ore at about 300 years. The proposed constants in ICRP 30 increase the time for equivalency to about 400,000 years, although they decrease the absolute hazards. This change is caused predominantly by a large decrease in the dose constant for Ra-226 and a large increase in the constant for Np-327 in ICRP 30. $\mathrm{Ra}-226$ is a daughter product in the Pu-238-U-234 decay chain in HLW and is in secular equilibrium with U-238 and U-234 in uranium ore. $\mathrm{Np}-237$ is a daughter product of the Pu-241-Am-241 decay chain. If the ICRP 30 dose constant for $\mathrm{Np}-237$ is reduced as has been recommended recently, the time for hazard equivalency of HLW and uranium ore is reduced to 30,000 years. Again it should be emphasized, although the hazard of HLW relative to uranium ore is higher and lasts longer with ICRP 30, the absolute hazard is less than with ICRP 2.

1372 (EIR-430) Risk analysis for repositories in north Switzerland. Extent and probability of geologic processes and events. Buergisser, H.M.; Herrnberger, V. (Eidgenoessisches Inst. fuer Reaktorforschung, Wuerenlingen (Switzerland)). Jul 1981. 71 p. (In German). NTIS (US Sales Only), PC A04/MF A01. Order Number DE82903880.

Portions of document are illegible.

The literature study assesses, in the form of expert analysis, geological processes and events for a $1200 \mathrm{~km}^{2}$-area of northern Switzerland, with regard to repositories for medium-and high-active waste (depth 100 to $600 \mathrm{~m}$ and 600 to $2500 \mathrm{~m}$, respectively) over the next $10^{6}$ years. The area, which comprises parts of the Tabular Jura, the folded Jura and the Molasse Basin, the latter two being parts of the Alpine Orogene, has undergone a non-uniform geologic development since the Oligocene. Within the next $10^{4}$ to $10^{5}$ years a maximum earthquake intensity of VIII-IX (MSK-scale) has been predicted. After thic period, particularly in the southern and eastern parts of the area, glaciations will probably occur, with asuciated erosion of possibly 200 to $300 \mathrm{~m}$. Fluvial erosion as a response to an uplift could reach similar values after $10^{5}$ to $10^{6}$ years; however, there are no data on the recent relative vertical crustal movements of the area. The risk of a meteorite impact is considered small as compared to that of these factors. Seismic activity and the position and extent of faults are so poorly known within the area that the faulting probability cannot be derived at present. Flooding by the sea, intrusion of magma, diapirism, metamorphism and volcanic eruptions are not considered to be risk factors for final repositories in northern Switzerland. For the shallow-type repositories, the risk of denudation and landslides have to be judged when locality-bound projects have been proposed.

1373 (INIS-mf-7744, pp vp) Barriers in a final storage for radioactive wastes. Beckmann, $H$. (Technische Univ. Clausthal, Clausthal-Zellerfeld (Germany, F.R.)); Brueggemann, R.; Hossain, S. (Technische Univ. Berlin (Germany, F.R.)); Giesel, W.; Jaritz, W. (Bundesanstalt fuer Geowissenschaften und Rohstoffe, Hannover (Germany, F.R.)); Kienzler, B.; Koester, R.; Korthaus, E. (Kernforschungszentrum Karlsruhe G.m.b.H. (Germany, F.R.)); Altenhein, F.K.; Levi, H.W. Oct 1981. (In German). NTIS (US Sales Only), PC A20/MF A01.

In Project safety studies - nuclear waste management (PSE). Summarizing interim report.

Mining, geological and technical barriers can stop or retard the release of radionuclides of the final storage site (Gorleben). Investigations in the field of salt mechanics, on the leaching behaviour of radionuclides sealed in HAW-glass blocks and MAW-cement products as well as on the transport processes in the pit building filled with alkaline solution serve to assess the release of nuclides.

1374 (Juel-Conf-42-Vol.2, pp 603-620) Criteria for high level waste disposal and characterization of barriers. Pottier, $\mathbf{P}$. (C.E.N., Cadarache, France); Sousselier, Y. Jun 1981. NTIS (US Sales Only), PC A21/MF A01. (CONF-810650-Vol.2).

From International seminar on chemistry and process engineering for high-level waste solidification; Julich, F.R. Germany ( 1 Jun 1981).

This paper discusses the safety analysis, aims and criteria for barriers for the disposal of high-level radioactive wastes in geological formations. The barriers consist of the waste form, the packing (canister and overpack), the geochemical barrier (backfilling) and the host rock. The processes of safety analysis is generally an iterative one taking into account the confinement qualities of the different barriers.

1376 (NUREG/CR-3160) Parameters and variables appearing in radiological assessment codes. Final report. Mills, M.; Vogt, D.; Mann, B. (Teknekron Research, Inc., Bethesda, MD (USA)). Jun 1983. 164p. NTIS, PC A08/MF A01 - GPO $\$ 5.50$. Order Number DE83902454.

This report defines the parameters and variables appearing in radiological assessment codes and presents typical values and ranges of values for each. Areas covered by the report include radionuclide source term calculations, dose to man and health effects, atmospheric transport, and environmental pathway and food chain calculations. The data in this report were compiled to help guide the selection of values of parameters and variables to be used in code benchmarking.

1376 (NUREG/CR-3209) Summary of computer codes for radiological assessment. Mills, M.; Vogt, D. (Teknekron Research, Inc., Bethesda, MD (USA)). Mar 1983. 198p. NTIS, PC A09/MF A01 - GPO \$7.00. Order Number DE83901815. This report is the second in a series of five reports that will provide critical reviews and summaries of computer programs that can be used to analyze the potential performance of a high-level radioactive waste repository. The computer programs identified address the following areas: radionuclide source term, radiation shielding, airborne transport, surface water transport, dose to man, and health effects. The report identifies 242 computer codes for use in analyzing radiological assessment issues and provides a summary description of 17 computer programs. These 17 computer programs are being used by the US Department of Energy, the US Nuclear Regulatory Commission, or the US Environmental Protection Agency to analyze high-level waste management or reactor effluent releases.

1377 (NUREG/CR-3235-Vols.5-6) Technical assistance for regulatory development: review and evaluation of the draft EPA standard 40CFR191 for disposal of high-level waste. Volumes 5 and 6. (Sandia National Labs., Albuquerque, NM 
(USA)). Apr 1983. Contract AC04-76DP00789. 144p. (SAND-82-1557-Vols.5-6). NTIS, PC A07/MF A01 - GPO $\$ 6.50$. Order Number DE83012241.

Portions are illegible in microfiche products.

Simple models are presented for the estimation of individual and population health effects for long-term radionuclide releases to the surface environment. In Volume 5, models based on the use of asymptotic solutions to mixed cell models in conjunction with appropriate usage rates, dose factors, risk factors, and population estimates are demonstrated. These simple models may be useful in evaluating topics such as the potential importance of individual radionuclides, different release patterns or exposure pathways, and the relationship between individual and population exposures. Illustrative model calculations are compared with the calculated population exposures on which the Draft (No. 19) EPA Standard 40CFR191 is based. In Volume 6, simple models are employed to provide insights into the degree of conservatism in the health effects per curie value presented by the draft standard. No attempt is made to encompass all uncertainty in the input parameters use in the calculations, and some of the modeling assumptions used in this analysis are different from those of the EPA. Three sets of calculations of health effects (cancer deaths) per curie release are presented and discussed in terms of their potential implications upon the curie release limits of the EPA draft standard. Individual volumes are indexed.

1378 (NUREG/CR-3235-Vols.5-6, pp 88p, Vol. 5) Health effects associtated with unit radonuclide releases to the environment. Helton, J.C. (Arizona State Univ., Tempe). Apr 1983. NTIS, PC A07/MF A01 - GPO \$6.50.

In Technical assistance for regulatory development: review and evaluation of the draft EPA standard 40CFR191 for disposal of highlevel waste. Volumes 5 and 6.

Simple models are presented for the estimation of individual and population health effects (i.e., latent cancer fatalities) for long-term radionuclide releases to the surface environment. These models were suggested by techniques employed by the Environmental Protection Agency in the development of a proposed standard for the disposal of high-level radioactive waste. The modeling approach is based on the use of asymptotic solutions to mixed-cell models in conjunction with appropriate usage rates, dose factors, risk factors, and population estimates. Although the models are simple, it is felt that they can be used in preliminary investigations of topics in high-level waste disposal such as potential importance of individual radionuclides, relative importance of different release patterns or exposure pathways, and relationships between individual and population exposures. The use of the models is illustrated by calculating the population health effects along various exposure pathways for the radionuclides considered in the proposed Environmental Protection Agency Standard. The results of these calculations are compared with the calculated population exposures on which the proposed Environmental Protection Agency Standard is based.

1379 (NUREG/CR-3235-Vols.5-6, pp 54p, Vol. 6) Calculation of health effects per curie release for comparison with the EPA standard. Runkle, G.E. Apr 1983. NTIS, PC A07/MF A01 - GPO \$6.50.

In Technical assistance for regulatory development: review and evaluation of the draft EPA standard 40CFR191 for disposal of highlevel waste. Volumes 5 and 6.

The Environmental Protection Agency (EPA) is developing a standard for geologic disposal of high-level radioactive wastes (40CFR 191) based on radioactive releases (expressed in curies) that may result in 1000 health effects (i.e., latent cancer fatilities) over a 10,000 year period. Health effects calculations were used by EPA to establish the curie release limits. The Fuel Cycle Risk Analysis Division of Sandia National Laboratories was requested by the Nuclear Regulatory Commission (NRC) High-Level Waste Licensing Management Branch to pertorm calculations, using the methodology developed under the Risk Assessment Methodology Program, to compare with the results from the EPA analysis. The intent was to provide some insights into the degree of conservatism in the health effects per curie values presented by the EPA standard. No attempt was made to encompass all the uncertainty in the input parameters used in the calculations and some of the modeling assumptions used in this analysis are different from those of the EPA. Three sets of calculations of health effects (cancer deaths) per curie release were performed in this analysis. The calculational meth ods, the results of the analysis and the potential implication of these results upon the curie release limits of the EPA are discussed in this report.

1380

(ONWI-126) Review of safety assessments of nuclear waste management. Ensminger, D.A.; Kaplan, M.F.; Koplik C.M. (Battelle Pacific Northwest Labs., Richland, WA (USA); Analytic Sciences Corp., Reading, MA (USA)). Nov 1980 Contract AC06-76RL01830. 176p. NTIS, PC A09/MF A01 Order Number DE82018568.

This report reviews past safety assessment studies for the disposal of nuclear wastes in mined repositories. Both pre-closure operations and long-term performance after the repository is closed are considered. The methods used in pre-closure studies are relatively well established, and past studies have covered all important accident scenarios and containment failure modes. However, the results of different studies exhibit a spread of up to five orders of magnitude. This can be attributed largely to variations in accident probabilities and cesium release fractions. The techniques used to assess long-term dangers are more variegated. There is, however, a considerable degree of agreement in the results, and the feasibility of anfe disposal of high-level wastes in general has been well established. Only a few detailed safety analyses have been conducted for particular sites, however. The most difficult problem in calculating long-term risk is to quantify the probability that different scenarios will occur; approaches to safety assessment which avoid this step tend to be more successful. Comparison of pre-closure and post-closure risks is rendered difficult by a number of factors, in particular the difference in time scale. Those studies which have nevertheless attempted to make such a comparison have been notably unsuccessful in doing so.

1381 (PB-83-171207) Environmental pathway models for estimating population health effects from disposal of high-level radioactive waste in geologic repositories. Draft report. (Office of Radiation Programs, Washington, DC (USA)). 1982. 195p. (EPA-520/5-80-002). NTIS, PC A09/MF A01.

This technical report presents the methodology used to assess the long-term population risks from projected releases of waste from a gco logic repository. It describes the models that the Agency developed specifically expects that population distributions, food chains, and living habits may change dramatically over 10,000 years. Rather than attempt to predict such changes, this methodology uses very general models of environmental pathways that consider present values for the various parameters used in the modcls.

1382

(PB-83-187641) Potential individual doses from disposal of high-level radinactive wastes in geologic repositories. Draft report (final). Goldin, A.S.; Serini, B.L.; Struckmeyer, R.K.; Hung, C.; Smith, C.B. (Office of Radiation Programs, Washington, DC (USA)). Jan 1983. 220p. (EPA-520/1-82026). NTIS, PC A10/MF A01.

The technical report presents the mcthodology used to assess the potential annual individual doses and human exposure and geologic media contamination probabilities from projected releases of radioisotopes from a geologic repository. It describes the models that the Envirnnmental Prntestion Agency employed for this analyais and review the various assumptions which were made. Since this analysis is necessarily generic in nature, the methodology uses very general models of environmental pathways and consider $a$ range of values for the variusus paranteters used in the models.

1383 (PB-83-190116) Potential individual doses from disposal of high-level radioactive wastes in geologic repositories. Draft report. (Office of Radiation Programs, Washington, DC (USA)). Jan 1983. 220p. (EPA-520/1-82-007). NTIS, PC A10/MF A01.

The agency has recently published environmental standards addressing disposal of high-level radioactive wastes (40 CFR Part 191) for public review and comment (47 FR 58196). An important part of this effort is the evaluation of how effective mined geologic repositories are for isolating these wastes from the environment for many thousands of years. This technical report presents the methodology used to assess the potential annual individual doses and human exposure and geologic media contamination probabilities from projected releases of radioisotopes from a geologic repository. It describes the models that the Agency employed for this analysis and reviews the various assumptions which were made. Since this analysis is necessarily generic in nature, 
: methodology uses very general models of environmental pathways $d$ considers a range of values for the various parameters used in the sels.

1384 (PNL-4688) Assessment of single-shell tank residual-liquid issues at Hanford Site, Washington. Murthy, K.S.; Stout, L.A.; Napier, B.A.; Reisenauer, A.E.; Landstrom, D.K. (Pacific Northwest Lab., Richland, WA (USA)). Jun 1983. Contract AC06-76RL01830. 130p. NTIS, PC A07/MF A01. Order Number DE83013909.

This report provides an assessment of the overall effectiveness and implications of jet pumping the interstitial liquids (IL) from singleshell tanks at Hanford. The jet-pumping program, currently in progress at Hanford, involves the planned removal of IL contained in 89 of the 149 single-shell tanks and its transfer to double-shell tanks after volume reduction by evaporation. The purpose of this report is to estimate the public and worker doses associated with (1) terminating pumping immediately, (2) pumping to a 100,000 -gal limit per tank, (3) pumping to a 50,000 -gal limit per tank, and (4) pumping to the maximum practical liquid removal level of $30,000 \mathrm{gal}$. Assessment of the cost-effectiveness of these various levels of pumping in minimizing any undue health and safety risks to the public or worker is also presented.

1385 (SAND-81-2163C) Comparison of ICRP2 and ICRP30 for estimating the dose and adverse health effects from potential radionuclide releases from a geologic waste repository. Runkle, G.E.; Soldat, J.K. (Sandia National Labs., Albuquerque, NM (USA); Pacific Northwest Lab., Richland, WA (USA)). 1982. Contract AC04-76DP00789. 12p. (CONF820303-34). NTIS, PC A02/MF A01. Order Number DE82012224.

From Waste management conference (ANS); Tucson, AZ, USA (8 Mar 1982).

Portions of document are illegible.

This paper presents a comparison of the calculated risk of adverse health effects from the radionuclides considered in the inventory of a high-level waste repository using the ICRP2 and ICRP30 internal dosimetry models. A CRPC (cancer risk per curie) index was calculated to compare the two models. The C.RPC indices for the ICRP30 model are approximately 2 to 20 times higher than the ICRP2 indices for most radionuclides for ingestion intakes. However, the ${ }^{207} \mathrm{~Np}$ index is approximately 200 times greater for the ICRP30 model and the ${ }^{228} \mathbf{R a}$ and ${ }^{226} \mathrm{Ra}$ indices are approximately 30 to 90 times greater for the ICRP2 model. Generally, there is closer agreement of the CRPC indices for the inhalation intakes. A scenario that considers a U-tube effect and withdrawal of water from wells downdip from the repository was analyzed. This analysis, based on a hypothetical waste disposal site, considered groundwater transport and environmental transport with subsequent uptake by the human via ingestion and inhalation. The ICRP30 risks are higher by approximately 20 at 10,000 years post closure for the ingestion pathway. Howcver, the ICRP2 risks are higher by factors of approximately 2 to 10 at times greater than 50,000 years. Differences in the mathematical modeling assumptions, gut uptakes and other metabolic parameters between the two models account for most of the variability in the risk estimates.

1386 (SAND-81-2375) Parametric studies of radiological consequences of basaltic volcanism. Logan, S.E.; Link, R.L.; Ng, H.S.; Rockenbach, F.A.; Hong, K.J. (Sandia National Labs., Albuquerque, NM (USA)). Apr 1982. Contract ACO476DP00789. 230p. NTIS, PC A11/MF A01. Order Number DE82015518.

Portions of document are illegible.

The Nevada Nuclear Waste Storage Investigations are studying the possibility of siting a nuclear waste repository in tuff somewhere in the southwest portion of the Nevada Test Site. Recurrence of basaltic volcanism in this region, though of low probability, might threaten the containment and isolation of radioactive waste stored in a repository. This report documents the results of a parametric analysis of radiologic -onsequences of basaltic volcanism which intercepts a radioactive waste epository. Two repository inventories, one containing unreprocessed pent fuel and the other containing high-level waste from reprocessing, and two eruptions, times 100 years and 10,000 years after repository closure, are considered. The specific site chosen for a hypothetical repository is at Yucca Mountain. Parametric thermal modeling indicates that only extreme and very unlikely cases, such as a vcry wide dike that con- tinued to flow for $\mathbf{3 0}$ days, would cause melting of even a few centimeters of the repository host rock or backfill. The most likely fraction of the inventory to become entrained in magma and transported to the surface is estimated to be $8 \times 10^{-5}$ of the total inventory. Most of the erupted material does not become airborne but forms a cinder cone, scoria sheet, and lava flows. The doses resulting from the nonairborne material are calculated to produce less than 1000 health effects in 10,000 years.A special pathway considered is an individual constructing a home using cinders from the cone as a building material. The total activity transported by volcanism above the original land surface was determined for various eruption occurrence times up to 10,000 years. The expected values for releases over a 10,000-year period, considering the probability of occurrence, are only a few curies.

1387 Occupational exposure in an underground mine repository for radioactive waste. Ehrlich, D. (PhysikalischTechnische Bundesanstalt, Bundesallee 100, Postfach 3345, D-3300 Braunschweig). Transactions of the American Nuclear Society; 44: 74-75(Jun 1983). (CONF-830609-).

From ANS annual meeting; Detroit, MI, USA (12 Jun 1983).

AIR FLOW; DOSE LIMITS; HIGH-LEVEL RADIOACTIVE WASTES; INHALATION; OCCUPATIONAL SAFETY; PERSONNEI. DOSIMETRY; UNDERGROUND DISPOSAL; PERSON. NEL; RADIOACTIVE WASTE DISPOSAL; RADIATION DOSES; RADIATION MONITORING; RADIOACTIVE AEROSOLS; RADIOACTIVE WASTE FACILITIES

1388 Results of the occupational dose assessment in the conceptual reference repository description. Norman, N.A. (Bechtel National, Inc., P.O. Box 3965, San Francisco, CA 94119). Transactions of the American Nuclear Society; 44: 75-76(Jun 1983). (CONF-830609-).

From ANS annual meeting; Detroit, MI, USA (12 Jun 1983).

ALARA; DOSE LIMITS; EMPLACEMENT; HIGH-LEVEL RADIOACTIVE WASTES; MATERIALS HANDLING; MINES; OCCUPATIONAL SAFETY; PERSONNEL DOSIMETRY; RADIATION DETECTORS; RADIATION PROTECTION; RADIOAC. TIVE WASTE FACILITIES; SALT DEPOSITS; SHIELDING; PERSONNEL; DOSE RATES; SPENT FUEL CASKS; VENTILATION

1389 Occupational radiation exposure risk at a nuclear waste repository in basalt. Jones, K.A. (Rockwell Hanford Operations, P.O. Box 800, Richland, WA 99352). Transactions of the American Nuclear Society; 44: 76-77(Jun 1983). (CONF830609-).

From ANS annual meeting; Detroit, MI, USA (12 Jun 1983).

BASALT; DOSE EQUIVALENTS; DOSE LIMITS; DOSE RATES; HIGH-LEVEL RADIOACTIVE WASTES; MATERIALS HANDLING; RADIATION DOSES; RADIATION PROTEC. TION; RADIOACTIVE WASTE FACILITIES; RADIOACTIVE WASTE DISPOSAL; RISK ASSESSMENT; PERSONNEL; OCCUPATIONAL SAFETY

1390 Occupational exposure for nuclear waste handling operations at NNWSI repository. Dennis, A.W. (Sandia National Laboratories, P.O. Box 969, Livermore, CA 94550). Transactions of the American Nuclear Society; 44: 77(Jun 1983). (CONF-830609-).

From ANS annual meeting; Detroit, MI, USA (12 Jun 1983).

ALARA; ECONOMIC ANALYSIS; EMPLACEMENT; HIGH-LEVEL RADIOACTIVE WASTES; NEVADA TEST SITE; RADIATION DOSES; RADIATION PROTECTION; RADIOACTIVE WASTE FACILITIES; RECOMMENDATIONS; REMOTE HANDLING EQUIPMENT; SITE SURVEYS; US DOE; US EPA; PERSONNEL; OPERATION; OCCUPATIONAL SAFETY; RADIOACTIVE WASTE DISPOSAL

1391 Summary of ONWI occupational dose assessment program. Waite, D.A. (Battelle, Office of NWTS Integration, 505 King Avenue, Columbus, OH 43201). Transactions of the American Nuclear Society; 44: 78(Jun 1983). (CONF830609-).

From ANS annual meeting; Detroit, MI, USA (12 Jun 1983). ALARA; HIGH-LEVEL RADIOACTIVE WASTES; LICENSING; RADIATION DOSES; RADIATION PROTEC- 
TION; RADIOACTIVE WASTE FACILITIES; REGULATIONS; RADIOACTIVE WASTE DISPOSAL; PERSONNEL; RISK ASSESSMENT; UNDERGROUND DISPOSAL

1392 Probablistic approach to the assessment of the longterm risk linked to the disposal of radioactive waste in geological repositories. Bertozzi, G.; D'Alessandro, M. (Commission of the European Communities, Ispra (Italy). Joint Research Centre). Radioactive Waste Management and the Nuclear Fuel Cycle; 3: No. 2, 117-136(Dec 1982).

A probabilistic approach is described for the assessment of the risk linked to the underground disposal of radioactive materials. Risk quantification is split into the assessment of radioactivity release probabilities and the corresponding consequences. Both probabilities and consequences may be quantified through the use of models which, because of unavoidable uncertainties in input data, are implemented in a probabilistic way. For any uncertain parameter a probability distribution must be defined, in order that the model results reflect the uncertainties of the input data. The probability distribution of the results can be obtained by using analytical codes as well as with Monte Carlo simulations. A simple application of the methodology is given to show what kind of information can be drawn.

1393 Calculation of the probability of volcanic disruption of a high-level radioactive waste repository within southern Nevada, USA. Crowe, B.M.; Johnson. M.E.; Beckman, R.I. (L.os Alamos National Lab., NM (USA)). Radioactive Waste Management and the Nuclear Fuel Cycle; 3; No. 2, 167-190(Dec 1982).

The Nevada Test Site (NTS) area, currently under investigation for disposal of high-level radioactive waste, is located within a northnortheast trending belt of basaltic volcanism that has been active during Quaternary time. Calculations of the probability of disruption of a repository at the NTS by basaltic volcanic activity have been completed as one of a number of approaches to assessing the risk of volcanism. A mathematical model derived for these calculations is described.

1394 Safety analyses for HLW and MLW storage and solidification facilities. Gruen, M.; Mehling, O.; Puttke, B. pp 207-209 of Jahrestagung Kerntechnik '82 - Reaktortagung 1982. Tagungsberichte. Sektion 3: Sicherheit kerntechnischer Anlagen. Eggenstein-Leopoldshafen, Germany, F.R.; Fachinformationszentrum Energie, Physik, Mathematik (1982). (In German)

From Annual meeting on nuclear technology '82; Mannheim, Germany, F.R. (4 - 6 May 1982).

RADIOACTIVE WASTE FACILITIES; RADIOACTIVE WASTE PROCESSING; RADIOACTIVE WASTE STORAGE; SOLIDIFICATION; LIQUID WASTES; INTERMEDIATELEVEL RADIOACTIVE WASTES; HIGH-LEVEL RADIOACTIVE WASTES; RISK ASSESSMENT

1395 Radiological risk analysis of an operating high-level waste repository. Waite, D.A.; Kaczmarsky, M.M. (Battelle Memorial Inst., Columbus, OH (USA). Office of Nuclear Waste Isolation). pp 511-523 of Health impacts of different sources of energy. Proceedings of an international symposium jointly organized by the WHO, the United Nations Environment Programme and the IAEA and held in Nashville, U.S.A. 22-26 June 1981. Vienna, Austria; IAEA (1982). (CONF810652-; IAEA-SM-254/22).

From International symposium on health impacts of different sources of energy; Nashville, TN, USA (22 Jun 1981).

Many dose assessments of generic facilities have been undertaken since the decision was made to pursue a deep geologic repository as the primary option for the disposal of high-level nuclear waste. As the siting and design efforts continue, it seems appropriate to review the various assessments and identify aspects of the repository operation which appear capable of contributing major portions of the potential doses. Representative samples of dose assessment results are exhibited for the important phases of repository development and operation: construction, operation, transport, decommissioning and retrieval. Operational aspects are further subdivided into routine and accidental considerations. These results are analysed to ascertain: (1) the orders of magnitude of the doses from each phase; (2) the relative importance of occupational versus population doses for each phase; and (3) the major facility operations and systems contributing to the doses. Converting all of thr whole-body doses discussed to 50-year dose commitments in person-ren and ranking from high consequences to low, one obtains the following population and uccupational lists: (a) Population: hoist-drop accident; transport; routine operation; and construction. (b) Occupational: hoistdrop accident; routine operation; transport; and construction. Except for the occupational result from the hoist-drop accident, all the postulated doses represented in the above list are orders of magnitude less than the person-rem received by the same groups from natural radiation in the same time period.

1396 Importance of near-field phenomena for nuclide release from a flooded salt dome repository. Storck, $R$. (Technische Universitaet Berlin, Germany). pp 497-507 of Scientific basis for nuclear waste management V. Lutze, W. (ed.). New York, NY; North-Holland (1982). (CONF-820636-).

From 5 . international symposium on the scientific basis for radioactive waste management; Berlin, F.R. Germany (7 Jun 1982).

This paper deals with the first step in identification of relevant near field phenomena for the scenario penetration of brine into a backfilled HLW-repository, directly after its closure. By use of parameter variation, the relevant phenomena for the release of Tc-99 from the salt dome are identified.

1397 Repository sufety-the salient facts. Hewitt. W.M. (Battelle, Orfice of NWTS Integration, 505 King Avenue, Columbus, $\mathrm{OH}$ 43201). Transactions of the American Nuclear Society; 43: 68(1982). (CONF-821103-).

From American Nuclear Society winter meeting; Washington, DC, USA (14 Nov 1982).

HIGH-LEVEL RADIOACTIVE WASTES; RADIATION PROTECTION; RADIOACTIVE WASTE DISPOSAL; RADIOACTIVE WASTE FACILITIES; SAFETY

1398 Impacts of recommendations on radiological considerations for an operating repository. Waite, D.A. (Battelle Office of NWTS Integration, 505 King Avenue, Columbus, $\mathrm{OH}$ 43201). Transactions of the American Nuclear Society; 43: 68-69(1982). (CONF-821103-).

From American Nuclear Society winter meeting; Washington, DC, USA (14 Nov 1982).

ALARA; HIGH-LEVEL RADIOACTIVE WASTES; ICRP; RADIATION PROTECTION; RADIOACTIVE WASTE DISPOSAL; RADIOACTIVE WASTE FACILITIES; RECOMMENDATIONS; REFERENCE MAN; SALT DEPOSITS

1399 Consequences of basaltic volcanism through a highlevel waste repository. Hunter, R.L.; Logan, S.E. (Sandia National Laboratories, P.O. Box 5800, Albuquerque, NM 87185). Transactions of the Americun Nuclear Society: 43; 76-77(1982). (CONF-82 1 ioj3-').

From American Nuclear Socicty winter meeting; Washington, DC, USA (14 Nov 1982).

HIGH-LEVEL RADIOACTIVE WASTES; TUFF; VOLCANISM; MAGMA; MATHEMATICAL MODELS; NEVADA TEST SITE; RADIATION PROTECTION; RADIOACTIVE WASTE DISPOSAL; UNDERGROUND DISPOSAL; VOLCANIC REGIONS

1400 Effects of ICRP Publication 30, the 1980 BEIR Report, and ORIGEN-2 on hazard assessments of high level waste. Cohen, R.I.. (University of Pittsburgh, PA). Transactions of the American Nuclear Society; 38: 84-85(1981). (CONF-810606-).

From American Nuclear Society's annual meeting; Miami Beach, FL, USA (7 Jun 1981).

HEALTH HAZARDS; HIGH-LEVEL RADIOACTIVE WASTES; ICRP; LIVER; NEOPLASMS; NEPTUNIUM 237; PLUTONIUM; RADIOISOTOPES; RADIUM 226; RISK ASSESSMENT

1401 Water dilution volumes for high-level wastes. Choi, J.; Pigford, T.H. (UC, Berkeley, Berkeley, CA 94720). Transactions of the American Nuclear Society; 39: 176-177(1981). (CONF-811103-). 

1981)

From ANS winter meeting; San Francisco, CA, USA (29 Nov

HEALTH HAZARDS; HIGH-LEVEL RADIOACTIVE WASTES; MILL TAILINGS; PWR TYPE REACTORS; REPRO CESSING; SPENT FUELS; URANIUM ORES; DILUTION; RADIOACTIVE WASTE PROCESSING

1402 Risk comparison for transportation, handling, and storage of high-level waste. Ensminger, D.A. (Analytic Sci Corp, Reading, MA 01867). Transactions of the American Nuclear Society; 35: 104-106(1980). (CONF-801107-).

From ANS international conference; Washington, DC, USA (17 Nov 1980)

HIGH-LEVEL RADIOACTIVE WASTES; RADIOACTIVE WASTE MANAGEMENT; RADIOACTIVE WASTE STORAGE; RISK ASSESSMENT; WASTE TRANSPORTATION

1403 Geologic disposal and the taxpayer: the problem of the experts. Mawson, C.A. pp 109-114 of Management of environment [a felicitation volume presented to Dr A.K. Ganguly]. Patel, B. (ed.) (Bhabha Atomic Research Centre, Bombay (India). Health Physics Div.). New Delhi; Wiley Eastern (1980).

Portions are illegible in microfiche products 0 refs.

In the prevailing atmosphere of antipathy to nuclear power and of alarming view the public takes of the disposal of radioactive wastes, a practical approach to the problem is proposed. According to this approach: (1) consequences of disposal must be expressed in terms of real damage to real people in real time, (2) the probability of consequences should be explained in terms of the probability of real consequences that are familiar to the public, (3) calculation of hazard should be done with realistic numbers and safety factors should be stated, (4) administrators should be presented with several reasonable solutions to a problem, with consequent costs and risks stated, and (5) technical experts should supply facts, as they know them, in their field of expertise and they should not make value judgements.

1404 Safety analysis of tank storage for highly radioactive reprocessing waste. Weber, M.; Becker, A.; Obrowski, W. (NUKEM, Germany). Transactions of the American Nuclear Society; 31: 331(1979). (CONF-790519-).

From European nuclear conference; Hamburg, F.R. Germany (6 May 1979)

EXPLOSIONS; HYDROGEN; OFF-GAS SYSTEMS; REPROCESSING; SAFETY; STORAGE; TANKS; STORAGE FACILITIES; HIGH-LEVEL RADIOACTIVE WASTES

\section{0े550 Regulations}

1405 Licensing of high-level radioactive wastes deposits. Shapar, H.R. (Nuclear Regulatory Commission, Washington, DC (USA)). Direito Nuclear; 2: No. 2, 81-88(Jul-Dec 1980). (In Portuguese).

The main legal aspects concerned to the choice of licensing procedures to high - level radioactive wastes deposits is analysed.

1406 (LA-UR-82-695) Advances in measurement of alphacontaminated wastes. Close, D.A.; Crane, T.W.; Caldwell, J.T.; Kunz, W.E.; Shunk, E.R.; Pratt, J.C.; Franks, L.A.; Kominski, S.M. (Los Alamos National Lab., NM (USA); EG and G, Inc., Goleta, CA (USA)). 1982. Contract W-7405-ENG-36. 11p. (CONF-820424-3). NIIS, PC A02/MF A01. Order Number DE82011988.

From ANS topical meeting on treatment and handling of radioactive wastes; Richland, WA, USA (19 Apr 1982).

Portions of document are illegible.

A comprehensive program is in progress at the Los Alamos National Laboratory for the development of sensitive, practical, nondestructive assay techniques for the quantification of low-level transuranics in bulk solid wastes. The program encompasses a broad range of techniques, including sophisticated active and passive gamma-ray spectroscopy, passive neutron detection systems, pulsed portable neutron generator interrogation systems, and electron accelerator-based techniques. The techniques can be used with either low-level or high-level betagamma wastes in either low-density or high-density matrices.
1407 Study for dry storage within the framework of the Swiss waste disposal concept. Kaegi, U.; Friesen, E. pp 480-483 of Jahrestagung Kerntechnik '83 - Reaktortagung 1983. Tagungsberichte. Sektion 4: Brennstoffkreislauf und Abfallbehandlung. Eggenstein-Leopoldshafen, Germany, F.R.; Fachinformationszentrum Energie, Physik, Mathematik (1983). (In German)(CONF-830636-).

From Annual meeting on nuclear technology '83; Berlin, F.R. Germany (14 Jun 1983).

Published in summary form only.

AWAY-FROM-REACTOR STORAGE; SPENT FUEL STORAGE; HIGH-LEVEL RADIOACTIVE WASTES; RADIOACTIVE WASTE STORAGE; FEASIBILITY STUDIES; SWITZER. LAND; VITRIFICATION; AFTER-HEAT REMOVAL; CRITICALITY; SITE SELECTION; SAFETY

1408 NUMATH: a nuclear material holdup estimator for unit operations and chemical processes. Krichinski, A.M. (Oak Ridge National Laboratory, Oak Ridge, TN 37830). Transactions of the American Nuclear Society; 43: 125-126(1982), (CONF-821103-).

From American Nuclear Society winter meeting; Washington, DC, USA (14 Nov 1982).

CALCULATION METHODS; NUCLEAR MATERIALS MANAGEMENT; INVENTORIES; PROCESS SOLUTIONS; N CODES; URANIUM 233; COMPUTER CODES; HIGH-LEVEL RADIOACTIVE WASTES; MATERIAL BALANCE; ORNL; RADIOACTIVE WASTE PROCESSING

1409 (CONF-820845-, pp 31-34) Legislative and political aspects of waste disposal. Davis, E. (American Nuclear Energy Council, Washington, DC). Dec 1982. NTIS, PC A25/MF A01.

From Alpha-contaminated waste management meeting; Gaithersburg, MD, USA (10 Aug 1982).

The American Nuclear Energy Council, an industry-supported organization, has been advocating a comprehensive federal program for nuclear waste management for some time. Realizing the need of legisla. tion to establish a comprehensive program, the council has been working on this legislation for over 4 years. As a result of an impasse over the defense waste issue, the nuclear waste legislation did not make it through the 96th Congress. When the 97th Congress convened, the Senate managed to push through legislation, which was passed in March. The Senate legislation is comprehensive. It addresses the issues related to permanent repository. It also includes provisions dealing with interim storage of spent fuel, a mechanism to resolve disagreements with states, and provides for private-sector financing of the federal waste management program. Four House Committees have completed consideration of nuclear waste legislation The House of Representatives will have to act on the legislation. Once the House has acted, the legislation will have to go into a Conference Committee with the Senate. The Senate and the House conferees will then have to work out their differences. Once these differences have been resolved, the legislation must go back to both the Senate and the House for endorsement of the conference report.

1410 (CONF-820845-, pp 35-38) Legislative and political aspects of waste disposal. Freiwald, J. (House of Representatives, Washington, DC). Dec 1982. NTIS, PC A25/MF A01. From Alpha-contaminated waste management meeting; Gaithersburg, MD, USA (10 Aug 1982).

In the Senate bill on waste disposal the definition for high-level waste was based on the source of the waste. High-level waste was defined as the liquids and solids resulting from reprocessing. The other terms defined in that bill that are crucial for any legislation dealing with high-level waste are storage and disposal. In the Senate bill, the definition of storage specifically mentioned transuranic (TRU) waste, but it did not include TRU waste in the definition of disposal. In the four House versions of the nuclear waste bill, the definition of high-level waste are addressed more carefully. This paper discusses the following four House committee's versions particularly pointing out how TRU waste is defined and handled: (1) Science Committer bill; (2) Interior Committee bill; (3) Commerce Committee bill; and (4) Armed Service Committee bill. The final language concerning TRU waste will depend on the nexi series of conference between these Committees. After resolving any differences, conferences will be held between the House and 
Senate. Here a concensus bill will be developed and it will go to the Rules Committee and then to the floor.

1411 (CONF-820845-, pp 39-45) Legislative and political aspects of waste disposal. Pestorius, $T$. (Office of Science and Tech. Policy, Washington, DC). Dec 1982. NTIS, PC A25/MF A01.

From Alpha-contaminated waste management meeting; Gaithersburg, MD, USA (10 Aug 1982).

The Office of Science and Technology Policy (OSTP) is a policy office, and usually dose not deal with any single issue in great detail. Nuclear waste, however, is an exception because of the strong commitment to effectively fulfill the federal responsibility for waste disposal. OSTP has tried to frame legislation regarding waste to encourage the efficient use of the uranium resources in the United States. It did this by sending a detailed Presidential message on nuclear waste to Congress. Besides legislation, OSTP has been involved in setting up standards. Two that OSTP has been coordinating are the high-level waste standard and the uranium mill tailings. OSTP has worked with the Environmental Protection Agency, the Department of Energy, the Nuclear Regulatory Commission, and the Office of Management and Budget. The high-level waste standard should be out soon.

1412 (CONF-820845-, pp 47-59) Regulatory policy of the EPA related to alpha-contaminated waste. Sjoblom, G. (Environmental Protection Agency, Washington, DC). Dec 1982. NTIS, PC A25/MF A01.

From Alpha-contaminated waste management meeting; Gaithersburg, MD, USA (10 Aug 1982).

The high-level waste standard and transuranic (TRU) waste standard that the Environmental Protection Agency (EPA) is working on are aimed at protecting maximally exposed individuals, as well as cumulative dose to man over long periods of time, (a 10,000 year period). In working on a standard, EPA considered not only public health, but achievability in engineering and alternatives, and the economics that are involved. In 1979 a value of $100 \mathrm{n} \mathrm{Ci} / \mathrm{g}$ was chosen based on a simple model, which indicated that, if such material were in the upper crust, resuspension of the material might result in exceeding 500 millirem in a year to the general public. The choice of numerical value for TRU waste is important in that it represents the upper limit on alpha-emitting radionuclides that will be considered in a low-level standard. The high-level waste standard will cover the operational part of high-level waste disposal. This numerical value that has been chnsen is not settled. It must be subject to a public review so that EPA will be able to get sulficient technical input either to ratify that number, lower that number, or raise the number.

1413 (DOE/NWTS-30, pp 16-18) NNWSI regulatory status and plans. Neal, J.T.; Bertram, S.G. Dec 1982. NTIS, PC A 10/MF A01. (CONF-821205-Absts.).

From NWTS information meeting on basalt waste isolation project, Las Vegas, INV, IJSA (14 Uec 1982).

RADIOAC'TIVE WASTE DISPOSAL; REGULATIONS; SITE SELECTION; HIGH-LEVEL RADIOACTIVE WASTES; GEOLOGIC DEPOSITS; NEVADA; NEVADA TEST SITE

1414 (NUREG/BR-0025-82/05) Information report on state legislation, volume 8 , number 5. Landau, M.S. (Nuclear Regulatory Commission, Washington, DC (USA). Office of State Programs). May 1982. 15p. NTIS, PC A02/MF A01.

The NRC held a meeting with the State Liaison Officers (SLO's) of Region V, in Walnut Creek, California, March 31-April 1. Topics of discussion at the Region V meeting included emergency proparedness, high and low level radioactive waste, transportation, the Agrecment State program, and NRC's regionalization. All States attended except for Nevada, whose representative was prevented from attending due to inclement weather. Legislative activity has been slower in 1982, as is usually the case in even-numbered years. The States whose legislative sessions have already adjourned are Florida, Georgia, Idaho, Indiana, Minnesota, New Mexico, South Dakota, Utah, Virginia (not carried over to 1983), West Virginia and Wyoming.

1415 (NUREG/CR-3235(Vols.2-3-4)) Technical assistance for regulatory development: review and evaluation of the draft EPA Standard 40CFR191 for disposal of high-level waste. (Sandia National Labs., Albuquerque, NM (USẢ)). Apr 1983.
Contract AC04-76DP00789. 315p. (SAND-82-1557(Vols.2 • 4)). NTIS, PC A14/MF A01 - GPO \$9.00. Order Numt DE83012458.

Volumes 2, 3, and 4 are abstracted and indexed separately.

1416 (NUREG/CR-3235(Vols.2-3-4), pp 121p, Vol. 2) Volume 2. A simplified analysis of a hypothetical repository in a basalt formation. Pepping, R.E.; Chu, M.S.; Siegel, M.D. Apr 1983. NTIS, PC Al4/MF A01 - GPO \$9.00. (SAND-821557(Vols.2-3-4))

In Technical assistance for regulatory development: review and evaluation of the draft EPA Standard 40CFR191 for disposal of highlevel waste.

An analysis of a hypothetical nuclear waste repository in a basalt formation has been performed to demonstrate the application of existing analytical tools to the assessment of compliance of the repository with the draft EPA Standard, 40CFR191 (Draft No. 19). The tools have been developed by Sandia National Laboratories for use by NRC in such analyses. The hypothetical site is based on descriptive and quantitative data for a candidate basalt repository in the early stages of site characterization. The effects of uncertainty in the input data on the assessment of compliance are demonstrated. Other sources of uncertainty resulting from interpretation of the standard and its probabilistic nature are discussed. The results of the calculations presented indicate that compliance with the draft standard muy be achieved depending on how the term release is interpreted; namely, is the release due to a unique (single) event or does it involve all probable scenarios.

1417 (NUREG/CR-3235(Vols.2-3-4), pp 109p, Vol. 3) Volume 3. A simplified analysis of a hypothetical repository in a tuff formation. Siegel, M.D.; Chu, M.S.Y. Apr 1983. NTIS, PC A14/MF A01 - GPO \$9.00. (SAND-82-1 557(Vols.2-3-4)) In Technical assistance for regulatory development: review and evaluation of the draft EPA Standard 40CFR191 for disposal of highlevel waste.

Potential radionuclide releases from a hypothetical tuff repository have been calculated and compared to the limits set by the EPA draft Standard 40CFR191. The areas that were examined included the radionuclide solubilities and sorption, the description of the local hydrogeology and the simulation of containment transport in the presence of fracture flow and matrix diffusion. The uncertainties in geochemical and hydrogeological parameters were represented by assigning realistic ranges and probability distributiuns to these variablés. Groundwater flow was described by Darcy's Law and radionuclide travel time was adjusted using calculated retardation factors. Radionuclide discharges weie calculated using the Distributed Velocity Method. The discharges were integrated over five successive 10,000 year periods. Our calculations suggest the following conclusions: (1) sorption of radionuclides by zeolitized tuff is an effective barrier to the migration of actinides even in the absence of solubility constraints; (2) violations of the EPA Draft standard can still ocsur due to discharge of ${ }^{99} \mathrm{~T} \mathrm{C}$ and ${ }^{14} \mathrm{C}$. Retardation due to matrix diffusion, however, may eliminate discharge of these nuclides for realistic ground-waster flow rates; $(3)$ in the absence of sorption by thick sequences of zeolitized tuff, discharges of $\mathrm{U}$ and $\mathrm{Np}$ under oxidizing conditions might exceed the EPA standard. Under reducing conditions, however, the low solubilities of these elements may effectively control radionuclide release.

1418 (NUREG/CR-3235(Vols.2-3-4), pp 80p, Vol. 4) Volume 4. A simplified analysis of a hypothetical repository in a bedded salt formation. Pepping, R.E.; Chu, M.S.; Siegel, M.D. Apr 1983. NTIS, PC A14/MF A01 - GPO \$9.00. (SAND-821557(Vols.2-3-4)).

In Technical assistance for regulatory development: review and evaluation of the draft EPA Standard 40CFR191 for disposal of highlevel waste.

An analysis of a hypothetical nuclear waste repository in a bedded salt formation has been performed to demonstrate the application of existing analytical tools to the assessment of compliance of the repository with the draft EPA Standard, 40CFR191 (Draft No. 1?' The tools have been developed by Sandia National Laboratories for $u$ by NRC in such analyses. The hypothetical site is based on data th are representative of bedded salt geologies in the continental US. The effects of uncertainty in the input data on the assessment of compliance are demonstrated. Other sources of uncertainty resulting from interpretation of the standard and its probabilistic nature are discussed. The 
results of the calculations presented indicate that compliance with the draft standard may be achieved for the groundwater transport scenarios depending on which source model is used. The penetration scenarios (direct canister hit or brine pocket hit) indicate potentially serious consequences; however, these could be mitigated by proper site selection and institutional controls.

1419 (WIPP-DOE-069-Rev.1) TRU waste-acceptance criteria for the Waste Isolation Plant. (Westinghouse Electric Corp., Pittsburgh, PA (USA)). Sep 1981. Contract AC0478AL05346. 63p. NTIS, PC A04/MF A01. Order Number DE82013965.

Portions of document are illegible.

This document is intended to delineate the criteria by which unclassified waste will be accepted for emplacement at the Waste Isolation Pilot Plant (WIPP) in Southeastern New Mexico and describe the bases upon which these criteria were established. These criteria are not intended to be specifications but rather limits that will allow waste generating and shipping sites to develop their own procedures and specifications for preparation of TRU waste for shipment to WIPP. These criteria will also allow waste generating sites to plan future facilities for waste preparation that will produce TRU waste forms that are compatible with WIPP waste emplacement and isolation requirements. These criteria only apply to contact handled $(\mathrm{CH})$ and remote handled (RH) transuranic (TRU) waste forms and are not intended to apply to betagamma wastes, spent fuel, high level waste (HLW) or experimental forms of radioactive waste. The technical objectives of this document can be summarized in three areas: (1) to provide waste acceptance criteria for use in the design of the WIPP Project; (2) to document the technical justification for TRU waste acceptance criteria for bedded salt as the host medium; (3) to provide quantitative guidelines, in the form of criteria, that can be used by waste form developers in designing TRU waste processing systems that will produce TRU waste forms acceptable for geologic disposal in bedded salt. These criteria are to be used as a supplement to Title 10 and 49 of the Code of Federal Regulations as applicable to the WIPP Project. All applicable state and federal regulations relating to shipments of radioactive or hazardous materials will apply to shipments to WIPP.

1420 Nuclear Regulatory Commission activities related to high-level waste materials issues. Cook, F.R. (Nuclear Regulatory Commission, Washington, DC). pp 753-759 of Scientific basis for nuclear waste management. Topp, S.V. (ed.). New York, NY; Elsevier Science Publishing Company, Inc. (1982). (CONF-811122 Pt.1).

From Annual meeting of the Materials Research Society; Boston, MA, USA (16 Nov 1981).

This paper describes the materials related activities of the Nuclear Regulatory Commission's (NRC's) staff in the area of highlevel waste licensing of a geologic repository for nuclear waste. It frames these activities in the context of existing draft regulations for high-level waste disposal.

142.1 Development of safeguards requirements for highlevel waste repositories. Stoddard, J.A.; Harris, L. (Sci Appl Inc). Nuclear Materials Management; 10: 365-371(1981). (CONF-810706-).

From Institute of Nuclear Materials Management conference; San Francisco, CA, USA (13 Jul 1981)

This paper describes the methodology used and the results obtained in a study performed for the NRC to establish a technical basis for the resolution of safeguards issues for high-level waste repositories. In the study, safeguards requirements werc derivod for future genlngic high-level waste repositories accepting wastes from any one of several commercial light water reactor fuel cycles. The safeguards requirements are based primarily on the potential threat to public health and safety due to dispersal of radioactive material at the repository site. Theft was also considered. 8 refs.

\section{ENVIRONMENTAL SCIENCES, TER- RESTRIAL}

\section{Radioactive Materials Monitoring And Transport}

1422 (LBL-11180) Effect of radon transport in groundwater upon gamma-ray borehole logs. Nelson, P.H.; Rachiele, R.; Smith, A. (Lawrence Berkeley Lab., CA (USA)). Sep 1980. Contract W-7405-ENG-48. 130p. (SAC-30). NTIS, PC A07/MF A01. Order Number DE82012616.

Granitic rock at an experimental waste storage site at Stripa, Sweden, is unusually high in natural radioelements ( $40 \mathrm{ppM}$ uranium) with higher concentrations occurring locally in thin chloritic zones and fractures. Groundwater seeping through fractures into open boreholes is consequently highly anomalous in its radon content, with activity as high as one microcurie per liter. When total count gamma-ray logs are run in boreholes where groundwater inflow is appreciable, the result is quite unusual: the radon daughter activity in the water adds considerably to the contribution from the rock, and in fact often dominates the log response. The total gamma activity increases where radon-charged groundwater enters a borehole, and remains at a high level as the water flows along the hole in response to the hydraulic gradient. As a consequence, the gamma log serves as a flow profile, locating zones of water entry (or loss) by an increase (or decrease) in the total gamma activity. A simple model has been developed for flow through a thin crack emanating radon at a rate $E$ showing that the radon concentration of water entering a hole is $E / \Lambda \mathrm{h}$, where $\Lambda$ is the radon decay rate and $h$ the crack aperture, assuming that the flow rate and crack source area are such that an element of water resides within the source area for several radon half-lives or more. Concentration measurements can provide a measurement of the inflow rate. Data from the $127-\mathrm{mm}$ holes in the time-scale drift behave in this fashion.

1423 (Juel-Spez-134) Investigation of the consequences of leakage incidents of a tank with high-concentrated radionuclide solutions and pentration of the radioactive solution into the soil. Schwarzer, K.; Thelen, J. (Kernforschungsanlage Juelich G.m. b.H. (Germany, F.R.). Inst. fuer Nukleare Sicherheitsforschung). Nov 1981. 57p. (In German). NTIS (US Sales Only), PC A04/MF A01. Order Number DE83750096.

The investigations show that in leakage incidents in tanks the essential parameters are the ground water velocity, the amount of leakage and the transversal dispersion coefficient. Among the inspected radiunuclides $\mathrm{Cs}-137, \mathrm{Sr}-90, \mathrm{Ru}-106, \mathrm{Ce}-144$ and the unabsorbable $\mathrm{H}-3$, Sr-90 represents the critical element. The reason is to be found in the low absorbing capacity of the sandy soil for this element and the long half-life of $28.5 \mathrm{a}$. But the results also prove that despite the unfavourable conditions or assumptions the migration of the radionuclides takes place 30 olowly that there. wnuld be enough time for taking appropriate countermeas res. Unfavourable conditions or assumptions to be mentioned are: sandy soil, due to high hydraulic permeability and low absorbing capacity; the delayed release of radionuclides off the solid phase during desorption process not being taken into account; high nuclide concentrations of the inspected HAW-solution. A constant Ksub(d)-value is obtained only after an according dilution of about 4 magnitudes. Provided that the consumption rate of drinking water is $\mathbf{4 0 0}$ $1 / \mathrm{a}$ and assuming an extraction at $1000 \mathrm{~m}$ from the source at a ground water velocity of $0.01 \mathrm{~m} / \mathrm{d}$, the radiation exposure would be $1.2 \times 10^{-7}$ rem/a for H-3 and approximately $0.15 \mathrm{rem} / \mathrm{a}$ (bone dose) for $\mathrm{Sr}-90$.

1424 Evaluation of radionuclide releases from underground waste repositories using the method of status vectors. Zappe, $D$. (Technische Univ., Dresden (German Democratic Republic). Sektion Physik); Runge, K. (Staatliches Amt fuer Atomsicherheit und Strahlenschutz, Berlin (German Democratic Republic)). Kernenergie; 26: No. 9, 352-356(Sep 1983). (CONF-830395-).

From 15. international symposium on radiation protection physics; Dresden, German D.R. (8 Mar 1983).

For safety analyses of underground repositories for radioactive wastes various possible release scenarios have to be defind and anticipated consequences to be calculated and compared. Normally only the main exposure pathways (i.e. the critical pathways) of the radionuclides disposed of in the repository are calculated using deterministic methods 
and varying the parameters. It is proposed to evaluate all the individual pathways including those differing considerably from the critical pathway by forming weighted averages of their consequences. This offers the possibility of including, without any restriction, in the evaluation of the repository the various possible events and processes that influence the function of barriers for the retention of radionuclides. Various states (scenarios) of a repository in a salt formation, which might occur in the course of time have been used as an example. The consequences related to these states and the probabilities of their occurrence or the scenario weights form the components of 'status vectors'. For low- and intermediate-level wastes the overall consequences obtained from these calculations are negligibly small, for high-level wastes they are about $3 \times 10^{-5}$ Sv $\mathrm{a}^{-1} / \mathrm{GW}$ a. These values are reached if at least a part of the barriers is effective. Variations of the weighting factors for the states and their influence on the overall consequences are given.

\section{Site Resource And Use Studies}

1425 (RHO-BW-CR-122-P) Monitoring land- and wateruse dynamics in the Columbia Plateau using remote-sensing computer analysis and integration techniques. Wukelic. G.F..; Fuute, H.P.; Blatr, S.C.; Begej, C.D. (Rockwell International Corp., Canoga Park, CA (USA). Energy Systems Group). Sep 1981. Contract AC06-80RL10000. 88p. (PNL-4047). NTIS, PC A05/MF A01. Order Number DE83013946.

Pul tiunts are illegihle in mirnfirhe products.

This study successfully utilized advanced, remote-sensing computer-analysis techniques to quantify and map land- and water-use trends potentially relevant to siting, developing, and operating a national high-level nuclear waste repository on the US Department of Energy's (DOE) Hanford Site in eastern Washington State. Specifically, using a variety of digital data bases (primarily multidate Landsat data) and digital analysis programs, the study produced unique numerical data and integrated data reference maps relevant to regional (Columbia Plateau) and localized (Pasco Basin) hydrologic considerations associated with developing such a facility. Accordingly, study results should directly contribute to the preparation of the Basalt Waste Isolation Project site-characterization report currently in progress. Moreover, since all study data developed are in digital form, they can be called upon to contribute to furute reference repository location monitoring and reporting efforts, as well as be utilized in other DOE programmatic areas having technical and/or environmental interest in the Columbia Plateau region. The results obtained indicate that multidate digital Landsat data provide an inexpensive, up-to-date, and accurate data base and reference map of natural and cultural features existıng in any region. These data can be (1) computer enhanced to highlight selected surface features of interest; (2) processed/analyzed to provide regional land-cover/use information and trend data; and (3) combined with other line and point data files to accomodate interactive, correlative analyses and integrated color-graphic displays to aid interpretation and modeling efforts.

\section{ENVIRONMENTAL SCIENCES, AQUATIC}

\section{Radioactive Materials Monitoring And Transport}

1426 (LA-9332-MS) Geochemistry studies pertaining to the $C$.tunnel radionuclide migration feld experiment. Norris, A.E.; Aguilar, R.D.; Bayhurst, B.P. . (Los Alamos National Lab., NM (USA)). Nov 1982. Contract W-7405-ENG-36. 45p. NTIS, PC A03/MF A01. Order Number DF.83007154.

I'his report presents the results of geochemical studies of Tunnel Bed tuff that were performed by Los Alamos National Laboratory or done at its direction as part of the Nevada Test Site G-Tunnel Radionuclide Migration Field Experiment. A tuff-treated water was prepared and used in laboratory-scale measurements of radionuclide sorption onto crushed Tunnel Bed tuff, pulverized fracture-fill material, tuff wafers, and a solid tuff core. Modelling studies were undertaken to determine the effects of matrix diffusion and unsaturated tuff on the proposed fracture-flow cxperiments. The initial results of those studies are presented in this report.

1427 Dose conversion factors used in the current Canadian high level waste disposal assessment study. Johnson, J.R.
(Atomic Energy of Canada Ltd., Chalk River, Ontario. Chal River Nuclear Labs.). Radiation Protection Dosimetry; 3: Nc $1-2,47-50(1982)$.

One of the objectives of the Canadian high level waste disposal assessment study is to estimate annual dose rates to various members of the public if ground water should penetrate the proposed engineered containment in the deep rock vault and transport radioactivity to the biosphere. The dose conversion factors being used in this study, are described; these are the 50 year committed effective dose equivalents for infants and adults, and it is demonstrated that, with reasonable assumptions about age dependent intake rates, they give reasonable and conservative estimates of the annual dose rates.

1428 (Juel-Conf-40, pp 73-95) Radioecology of the deepsea. Feldt, W. Nov 1980. (In German). NTIS (US Sales Only), PC A08/MF A01. (CONF-800187-).

From Meeting on deep-sea burial of radioactive wastes; Juelich, F.R. Germany (29 Jan 1980).

The activity released from sea-dumped containers is absorbed by the organism of the benthos and may therefore reach the ourfaoe very much faster than it should be experter by vertical diffusion or by cu? rents alone. The density of the biomass has a maximum near the surface, but increases again, according to recent assumptions, directly above the sea-floor resulting in the occurrence of a bottle neck in the distribution hetwern $7000 \mathrm{~m}$ and $4000 \mathrm{~m}$ of diplly. The widh of this bollle lleck might essentially contribute to the vertical transport. Our knowledge of the conditions in the deep sea is so sparse that quantitative statements on the vertical transport benthos-surface must be regarded as speculations. The different methods of waste disposal (sea-dumping of low- and intermediate-level wastes, and burying of high-level wastes in the sea ground) have got in common the radiation exposure of the abyssal organisms. Their sensitivity to radiation is unknown, and therefore also the disturbance which may be imposed on the abyssal ecosystem. Thus further international research activities on the subject of deep-sea radioecology are necessary before these methods of waste disposal may be considered as safe.

\section{Site Resource And Use Studies}

1429 (RHO-BWI-C-121) Evaluation of water resource economics within the Pasco Basin, Washington. Leaming, G.F. (Atomics International Div, Richland, WA (USA). Roekwell Hanford Operations; Leaming (George) Associates, Marana, $\Lambda Z$ (USA)). 30 Sep 1981. Contract AC06-77RL01030. 53p. NTIS, PC A04/MF A01. Order Number DE82014183.

Portiuns of document are illegible.

The Columbia River basalt beneath the Hanford Site in southcentral Washington is being consideren for possible use a3 a terminal repository medium for high-lcvcl nuclear waste. Such underground storage would require that the facility be contiguous to at least a portion of the ambient groundwater system of the Pasco Basin. This report attempts to evaluate the economic factors and conditions related to the water resources of the Pasco Basin and the probable economic effects associated with selected hypothetical changes in local water demand and supply as a basis for eventual selection of credible watcr supply alternatives and more detailed analyses of the consequences of such alternative sclection It is most likely that total deniand for water for consumptive uses in the Pasco Basin will increase from nearly 2.0 million acre-feet per year in 1980 to almost 2.8 million acre-feet in 2010 , with total demand slightly more than 3.6 million acre-feet per year in 2080. The Culumbia River and other surface streams constitute the source of more than 99 percent of the water availablc cach year for all uses, both consumptive and non-consumptive, in the Pasco Basin. It is estimated that pumped groundwater accounted for 3 percent of the value of all water supplied to consumers of water in the Pasco Basin in 1980. Groundwater's share of the total cost is proportionately higher than groundwater's share of total use because it is generally more costly to acquire than is surface water and the value of water is considerer equivalent to its cost of acquisition. Because groundwater represent: such a small part of the total water supply and demand within the Paser Basin, it is concluded that if the develnpment of a nuclear waste repository on the Hanford Site were to result in changes in the groundwater supply during the next 100 years, the economic impact on the overall water supply picture for the entire basin would be insignificant. 


\section{GENERAL}

1430 (INFO-0046) Scope and nature of the problem of high level nuclear waste disposal. Jennekens, J. (Atomic Energy Control Board, Ottawa, Ontario (Canada)). Sep 1981. 9p. NTIS (US Sales Only), PC A02/MF A01. Order Number DE82701330.

The disposal of high level nuclear waste poses a challenge to the Canadian technical and scientific communities, but a much greater challenge to government and industry leaders who must convince the public that the so-called 'problem' can be resolved by a pragmatic approach utilizing existing skills and knowledge. This paper outlines the objectives of radioactive waste management, the quantities of high level waste expected to be produced by the Canadian nuclear power program, the regulatory process which will apply and the government initiatives which have been and will be taken to ensure that the health, safety, security, and environmental interests of the public will be protected.

1431 (INIS-mf-7034) Indemnification for nuclear damages - recent developments in the United States. Shapar, H.K. (Nuclear Regulatory Commission, Washington, DC (USA)). (Intcrnational Nuclear Law Association (INLA), Brussels (Belgium)). Oct 1981. 16p. (CONF-8109120-20). NTIS (US Sales Only), PC A02/MF A01. Order Number DE82780778.

From Nuclear International Jura '81; Palma de Mallorca, Spain (27 Sep 1981).

Public liability for nuclear damages in the United States is dealt with by a Federal statutory system. The Price-Anderson Act provides a system of compensation for such damages with an overall limit of $\$ 560$ million. This fund is composed of private insurance, a utility assessment pool, and Federal government indemnity. The 1979 accident at the Three Mile Island nuclear station resulted in a less-than-full-scale test of the system. For the most part, the system has performed as intended although certain problems have been brought to light. Legislative proposal since the accident have focused on increasing the $\$ 560$ million limitation on liability, but so far none of these proposals has been acted upon by the U.S. Congress. In the next several years, the Nuclear Regulatory Commission and possibly Congress will consider application of the Price-Anderson system to high-level waste facilities. The Commission must file with the Congress within two years a comprehensive report on the Price-Anderson Act, including recommendations for its continuation and amendment.

1432 (Juel-Conf-42(Vol.2), pp 917-943) HAW-containers as a barrier In final storage. Smailos, E.; Kienzler, B.; Koester, R. Jun 1981. (In German). NTIS (US Sales Only), PC A21/MF A01. (CONF-810650-Vol.2).

From International seminar on chemistry and process engineering for high-level waste solidification; Julich, F.R. Germany (1 Jun 1981).

Due to the design of the HAW-packing as barrier in the ultimate storage with a service life of some $10^{2}$ years, a considerable decrease of the activity released into the biosphere during an accident can be achieved. The results obtained so far show that with hastelloy $\mathrm{C} 4$ and $\mathrm{Al}_{2} \mathrm{O}_{0}$ materials with high corrosion resistance are available. (Comprehensive tables).

1433 (KFK-3380) Analysis of Am and a recovery for it in the pressurized water reactor fuel cycle. Ganser, B. (Kernforschungszentrum Karlsruhe G.m.b.H. (Germany, F.R.). Inst. fuer Radiochemie). Jul 1982. 93p. (In German). NTIS (US Sales Only), PC A05/MF A01. Order Number DE83750050.

Thesis. Submitted to Karlsruhe Univ., Germany, F.R.

In order to estimate the levels of Am in the nuclear fuel cycle, calculations of the Am-concentrations in pressurized water reactors have been carried out using the computer program KORIGEN and the results compared with experimental values. For the experimental determinations, mass spectrometric isotope dilution analysis were made on selected samples. Dihexyl-N,N-diethylcarbamylmethylenephosphonate (DHDECMP), which is capable of the extraction of Am from high active waste during reprocessing, was compared with six further CMP's having larger aliphatic chains and varying degrees of isomerism. The dependence of the extraction coefficient on the nitric acid strength was determined. The extraction coefficient of a selected lanthanide $(\mathrm{Pr})$ was measured in $3 \mathrm{M} \mathrm{HNO}_{0}$ for each CMP. The dependence of the extrac- tion coefficient on the lanthanide concentration in the aqueous phase was determined for DHDECMP. A pure La-DHDECMP compound was isolated and investigated using IR and NMR spectroscopy. The solubilities of the CMP's in $0.1 \mathrm{M}$ and 3.0 M HNO ${ }_{0}$ were measured. For the first time, two selected CMP's were used to extract high activity waste solutions from mixed-oxide fuels and the distribution coefficients fro $\mathrm{Am}, \mathrm{Cm}$ and $\mathrm{Cs}$ at the extraction and the reextraction from the organic phase were determined. The effect of the radiolysis of the extracting agents by extracted radionuclides was investigated.

1434 Effect of composition and radiation on the fracture of a nuclear waste glass. Routbort, J.L.; Matzke, H. (Commission of the European Communities, Karlsruhe (Germany, F.R.). European Inst. for Transuranium Elements). Materials Science and Engineering; 58: No. 2, 229-237(Apr 1983).

The hertzian indentation technique has been used to determine the surface fracture energy and the fracture toughness Ksub(Ic) of a borosilicate glass (Versuchsglas type $98 / 12$ ) developed to contain high level nuclear waste. Using spherical steel indenters whose radii were varied between 1.5 and $7.0 \mathrm{~mm}$, the critical load Psub(c) for crack formation was determined. Psub(c) is a linear function of the indenter radius which does not pass through the origin. This failure of Auerbach's law may be due to the statistical nature of the flaw distribution. Nevertheless, the data are used to estimate that $\mathrm{Ksub}$ (Ic) $>1.77 \pm 0.15 \mathrm{MN}$ msup(-3/2). Adding selected groups of fission products to the base glass leaves Ksub(Ic) unchanged. The addition of lead to the glass or bombardment with $300 \mathrm{keV}$ lead ions lowers Ksub(Ic) by approximately 20\%. This may be due to replacement of $\mathrm{Si}-\mathrm{O}$ bonds by $\mathrm{Pb}-\mathrm{O}$ bonds which are weaker. Radiation with $77 \mathrm{MeV} \alpha$ particles to a dose of 1.3 $\times 10^{15} \alpha$ particles $\mathrm{cm}^{-2}$ increases Ksub(Ic) by approximately $75 \%$. It is concluded that neither the radiation used here nor the simulated fission products will seriously degrade the fracture properties of the nuclear waste glass investigated.

1436 Nuclear energy. Symonds, J.L.; King, A.; Reynolds, J.O.; Saddler, H.; Warner, R.K.; Wilmshurst, R.E. pp 236-248 of Energy 83: towards an energy policy for Australia. Canberra, Australia; Institution of Engineers, Australia (1983). (CONF8305142-).

From Energy 83 conference: towards an energy policy for Australia; Canberra, Australia (11 May 1983).

Paper copy only, copy does not permit microfiche production6 refs.

The nuclear energy scene as it affects Australia and its mining and uranium treatment industries is reviewed. Since the Institution of Engineers, Australia, presented its 'Recommendations for an Energy Policy for Australia' in 1977, mines and mills have begun to produce their uranium products under strict environmental regulations and yellowcake has been exported under contracts which are carefully monitored by the Commonwealth Government.

1436 Large deformation inelastic analysis of impact for shipping casks. Charman, C.M.; Grenier, R.M. (General Atomic Co., San Diego, CA (USA)); Nickell, R.E. (Applied Science and Technology, Poway, CA (USA)). Computer Methods in Applied Mechanics and Engineering; 33: No. 1-3, 759785(Sep 1982).

From Fenomech '81: 2. international conference on finite elements in nonlinear mechanics; Stuttgart (Germany, F.R.) (25 - 28 Aug 1981).

This paper describes the use of two- and three-dimensional nonlinear finite element computer programs to design a radioative material transportation cask to withstand a drop of 30 feet onto an unyielding surface. Because of recent advancement in the area of non-linear finite element code development, the use of such codes for an iterative design process is becoming practicable. The paper begins with a section dealing with a two-dimensional side drop analysis and is followed by a discussion of the general capabilities of DYNA3D and a brief discussion of the implementation of the code on a computational mainframe unlike any for which the developer had intended. Then, a section on threedimensional models of center-of-gravity over a corner impact follows, which introduces design features such as bolted closures, internal impact limiter, seals and shear rifigs. Figs. showing the deformed model grids are included. Stress and strain results are given in the subsequent section. Finally, we interpret these results in terms of possible rules 
being developed by the American Society of Mechanical Engineers (ASME) Boiler and Pressure Vessel Code committees.

1437 Institutional aspects of radioactive waste management. Metlay, D. (Indiana Univ., Bloomington). Radiation Research; 91: No. 1, 34-44(Jul 1982).

From Radiation Research Society meeting; Minneapolis, MN, USA (31 May 1981).

This paper examines the institutional aspects of managing highlevel radioactive wastes. It provides a brief analysis of three different nontechnical issues: intergenerational distribution of risk, logistical problems in operating a waste disposal facility, and the assessment and mitigation of the socioeconomic impacts of repository construction. Two additional questions are considered in greater detail: the institutional design of the federal government's waste management policymaking system and the role of the states in waste management decision-making. Three critiques of the current institutional design are reviewed and assessed. An argument is advanced that those criticisms, while in many cases quite valid, are misdirected. Altering the institutional structure will not reap the benefits its advocates envision. The evolution of the states' role in waste management decisionmaking is then traced. The point is made that the states have received from the federal government greater formal powers and more intimate involvement than they are entitled to possess from a strictly legal standpoint. The paper concludes by speculating that many institutional problems have arisen, have become salient, and in some instances have threatened problem solving because a strong technical foundation for waste management has only recently been erected.

1438 Australia's role in nuclear energy research and training. Australian Foreign Affairs Record; 53: No. 4, 155-159(Apr 1982).

Most nuclear energy research in Australia is carried out at the Australian Atomic Energy Commission. One research area described is work on high-level waste management through the development of SYNROC. Other organisations involved in nuclear science research and education are A.I.N.S.E. (Australian Institute of Nuclear Science and Engineering) which was set up in 1958 by the AAEC and the Australian Universities and A.S.N.T. (Australian School of Nuclear Technology) which was set up by the AAEC and the University of New South Wales. The School runs a variety of intensive courses for both Australian and overseas students. The relationships between Australia and the IAEA and NEA and the provision, by Australia, of nuclear technical assistance to developing countries are discussed.

1439 Vitrification of high-level wastes: A review of the computer thermal analysis for storage canisters. Wescott, R.I.; Slate, S.C. (Battelle Pacific Northwest Labs., Richland, WA (USA)). Nuclear Engineering and Design; 67: No. 3, 447 458(Feb 1982).

CANIST, a two-dimensional ( $r$ and THETA) computer program that solves the unsteady-state, heat-conduction equation, was used to model the thermal behavior of canisters filled with waste glass. CANIST has been found to be a valuable analytical tool for predicting the temperature profile of a waste storage canister as a function of several variables, including the diameter of the canister, the placement of internal fins, the heat generation rate of the waste glass, and the thermophysical properties of the canister and the waste glass. Thus, temperature-dependent processes that may affect the integrity of the glass/ canister unit, for example cracking, can be investigated using an analytical approach.

1440 Effects of ICRP Publication 30 and the 1980 Beir report on hazard assessments of high-level waste. Cohen, B.L. (Pittsburgh Univ., PA (USA)). Nuclear Engineering and Design; 42: No. 2, 133-143(Feb 1982).

ICRP Publication 30 (ICRP30) gives the radiation dose to various body organs in Sv per $\mathrm{Bq}$, which are readily translated into rad per curie ingested for various radioisotopes; the 1980 BEIR Report (BEIR III) gives the risk of fatal cancer per rad of dose to each body organ; the ORIGEN 2 Code gives the curies per tonne of initial uranium fuel in high level waste and in spent fuel. The product of these, $\mathrm{Ci} /$ tonne $\mathrm{X}$ $\mathrm{rad} / \mathrm{Ci} \mathrm{X}$ risk/rad gives the risk/tonne in terms of fatal cancer doses, if ingested, per tonne of uranium. When the resulting curves are compared with those calculated previously (based on ICRP Publication 2 and the 1972 BEIR Report), the hazard is less for the first century but much more thereafter. The principal sources of this greatly increas hazard is the 100-fold increase in absorption through the gut for ${ }^{207} \mathrm{~A}$ in ICRP 30, plus the high cancer risk from radiation to liver in BEl.. III. Some consequences of these changes and some scientific questions relevant to ascertaining their validity are discussed.

1441 Effects of ICRP Publication 30 and the 1980 BEIR Report in hazard assessments of high-level waste. Cohen, B.L. (University of Pittsburgh, PA). Nuclear Engineering and Design; 42: No. 2, 133-143(Feb 1982).

ICRP Publication 30 (ICRP30) gives the radiation dose to various body organs in Sv per Bq, which are readily translated into rad per curie ingested for various radioisotopes; the 1980 BEIR Report (BEIR III) gives the risk of fatal cancer per rad of dose to each body organ; the ORIGEN 2 Code gives the curies per tonne of initial uranium fuel in high level waste and in spent fuel. The product of these, $\mathrm{Ci} /$ tonne $\mathrm{x}$ $\mathrm{rad} / \mathrm{Ci} \times$ risk/rad gives the risk/tonne in terms of fatal cancer doses, if ingested, per tonne of uranium. When the resulting curves are compared with those calculated previously (based on ICRP Publication 2 and the 1972 BEIR Report), the hazard is less for the first century but much more thereafter. The principal sources of this greatly increased hazard is the 100-fold increase in absorption through the gut for $237 \mathrm{~Np}$ in ICRP 30, plus the high cancer risk from radiation to liver in BEIR III. Some consequences of these changes and some scientific questions relevant to ascertaining their validity are discussed.

1442 Cooldown testing of nuclear fuel casks. Anderson, R.T. (Allied-General Nuclear Services, Barnwell, SC (USA)). Nuclear Engineering and Design; 67: No. 3, 397-405(Feb 1982). Shipment of spent nuclear fuel from operating reactors is an important link in resolving the fuel storage and nuclear waste problems. Certain thermal problems must be considered. The nuclear spent fuel, even after a period of pool storage, has sufficient decay heat to necessitate special handling when being shipped to an off-site location. This paper presents the results of development related to the thermal interaction between dry spent fuel casks and nuclear fuel under operating situations. The tests were performed at the Barnwell Nuclear Fuel Plant (BNFP) using full-sized truck and rail casks and electrically heated dummy fuel assemblies. The safe and practical operation of the equipment developed has been shown.

1443 Coprecipitation of radioactive technetium with ferrous hydroxide. Ito, K.; Kanno, T. (Tohoku Univ., Sendai (Japan). Research Inst. of Mineral Dressing and Metallurgy). Nippon Genshiryoku Gakkaishi; 24: No. 2, 150-154(Feb 1982). (In Japanese).

Coprecipitation of radioactive technetium with hydroxides has been studied for the purpose of the management of the high level waste from the nuclear fuel reprocessing. Pertechnetate ion was reduced to $\mathrm{Tc}(\mathrm{IV})$ by ferrous ion ( $\left.\mathrm{Fe}^{2+}\right)$, and then coprecipitated with $\mathrm{Fe}(\mathrm{OH})_{2}$ (I) as $\mathrm{TcO}_{2}$. The $\mathrm{pH}$ of nitric acid solution containing $\mathrm{TcO}_{4}^{-}$was adjusted to 1.0 with $\mathrm{NaOH}$, and $\mathrm{TcO}_{2}$ was precipitated by reducing coprecipitants i.e. (I), $\mathrm{Fe}(\mathrm{OH})_{2}-\mathrm{Zr}(\mathrm{OH})_{4}$ ( II), $\mathrm{Fe}(\mathrm{OH})_{2} \mathrm{Si}(\mathrm{OH})_{4}$ (III) and $\mathrm{Fe}(\mathrm{OH})_{2}-\mathrm{Zr}(\mathrm{OH})_{4} \mathrm{Si}(\mathrm{OH})_{4}$ (IV). Pertechnetate ion quantitatively coprecipitates under the conditions: $\mathrm{Fe}^{2+} \mathrm{gt} 3.6 \times 10^{-0} \mathrm{M} ; \mathrm{pH}>6-12$ for (I), $\mathrm{pH}>4.2-12$ for (II), $\mathrm{pH}>8-12$ for (III) and $\mathrm{pH}>6-12$ for (IV). The presence of excess $\mathrm{Fe}^{2+}$ prevents the dissolution owing to air-oxidation of $\mathrm{TcO}_{3}$ coprecipitated. Pertes.hnetate inn ran be also removed from the waste solution by both the ferrite precipitation method and the iron powder precipitation method.

14 Exposures resulting from nuclas power production. Annex F. pp 249-331 of lonizing radiation: sources and biological effects. New York, NY; United Nations (1982).

This Annex assesses the releases of radioactive materials from the nuclear fuel cycle and their resulting dose commitments to the public. The nuclear fuel cycle includes the mining and milling of uranium ores, conversion to nuclear fuel materials, fabrication of fuel elements, production of power in the nuclear reactor, reprocessing of irradiated fuel and recycling of fissile and fertile nuclides recovered, and $d$ posal of radioactive wastes. This Annex also reviews reactor accidet which have led to unplanned releases of activity into the environmel.., together with estimates of the resulting collective doses. (KRM)

1445 Regulatory perspective on the cleanup of TMI-2: Snyder, B.J. (U.S. Nuclear Regulatory Commission, Washing- 
ton, DC 20555). Transactions of the American Nuclear Society; 43: 2-3(1982). (CONF-821103-).

From American Nuclear Society winter meeting; Washington, DC, USA (14 Nov 1982).

1446 High-integrity container for high-specific-activity wastes. Chapman, R.L.; Haelsig, R.T. (EG \& G Idaho Inc., P.O. Box 1625, Idaho Falls, ID 83415). Transactions of the American Nuclear Society; 43: 143-146(1982). (CONF-821103-).

From American Nuclear Society winter meeting; Washington, DC, USA (14 Nov 1982).

1447 Irradiation effects in SYNROC. Woolfrey, J.L.; Cassidy, D.J.; Reeve, K.D. (Australian Atomic Energy Commission Research Establishment, Lucas Heights). pp 158-163 of Austceram 82. Melbourne (Australia); Australian Ceramic Society (1982). (CONF-8208144-).

From 10. Australian ceramic conference; Melbourne, Australia (24 Aug 1982).

Accelerated irradiation studies indicate that SYNROC will not be adversely affected by irradiation damage over the periods of time (up to $10^{6}$ years) required for the decay of high level wastes to safe levels. Even though therc are changes in volume and density, only small increases in open porosity are observed. For the greatest expansion recorded $(8.6 \mathrm{vol} \%)$, this increase amounts to only $0.03 \%$ of specimen volume.

1448 Report of the Sub-Committee on Nuclear Power of the Committee on Overall Energy Study, (1). tin. Geological Survey Wyoming; No. 128, 1-29(Jul 1981). (In Japanese).

Japan highly depends on overseas countries for energy resources, particularly petroleum. For the country with the-fragile structure of energy supplies, the development and introduction of the energies substituting for petroleum are urgent, among which nuclear energy plays an important role. The sub-committee has made the studies on the policy and measures for promoting the development of nuclear power using its ad hoc committees since the end of August, 1980. In the promotion of nuclear power, such problcms as nuclear safety, plant siting, nuclear fuel cycle and waste management are involved. The report by the subcommittee is described as follows: the direction of nuclear power development, the promotion of the development of nuclear power generation, the independent nuclear fuel cycle in Japan, radioactive waste management, the new multipurpose utilizations of nuclear energy, the advance of nuclear power industry and its problems, the internationalization of the nuclear power policy.

1449 Sub-committee on Nuclear Power Submits report on energy security. Atoms in Japan; 25: No. 6, 7-11(Jun 1981).

Japan will develop uranium resources with the aim of importing at least 65,000 short tons by the year 2000 . The amount is about a half of the total projected shortage. Japan will nperate a commercial enrichment plant of at least 3,000 tons SWU/year capacity by the same year, and will have stockpiled uranium enough to meet the needs for approximately one year. These are the policy options in the report submitted by the Sub-Committee on Nuclear Power to the Ministry of International Trade and Industry. The contents of the report of the SubCommittce cntitled "for establishment of energy security", are given as follows: the direction of nuclear development, the independent nuclcar fuel cycle in Japan, the need for domestic uranium enrichment, the treatment and disposal of radioactive wastes, the new and multi-purpose utilization of nuclear power, the internationalization of nuclear policy, etc.

1460 Application of computer system to environmental monitoring at PNC Tokai Works. Kitahara, Y.; Narita, O.; Shinohara, K.; Asano, T. (Power Reactor and Nuclear Fuel Development Corp., Tokai, Ibaraki (Japan). Tokai Works). pan) $A$; 16: No. 2, 151-163(Jun 1981). (In Japanese).

In the Tokai Establishment, Power Reactor and Nuclear Fuel Development Corp., the development of technologies concerning uranium enrichment, fuel reprocessing, the production of plutonium fuel, the treatment of high level radinactive wastes and so on are carried out. The safety management division was established as an independent organization in PNC, of which the main task is radiation protection, and the radiation control in working environment, the exposure control of workèrs, the cuntrol of exliaust and waste liquid release, the radiation monitoring in surrounding environment are carried out. In order to make environmental monitoring works efficient, the introduction of computer system has been promoted since 1974, and the collection and processing of data were automated. In this report, the computer systems developed and used for environmental monitoring works, the outline of the works utilizing them, the problems and the subjects of future development are described. The main computer is IBM S370, and a small computer IBM S7 for telemetering is connected. The hardware and software used are explained. The visual display of automatically collected data is possible by the development of DIAMOND system. The improvement of TECS is the center of future development.

1461 Thermal conductivity measurements on granulebased composites. Neumann, W. (Oesterreichisches Forschungszentrum Seibersdorf G.m.b.H., Vienna). pan) $A ; 13$ : No. 6, 687694(1981).

From 7. European thermophysical properties conference; Antwerp (Belgium) (30 Jun - 4 Jul 1980).

The effective thermal conductivity of materials made from granules of steel, glass, or inorganic calcines embedded in a matrix material (epoxy resin, lead, or aluminium-silicon alloy), was measured. Various theoretically derived and empirical formulae for the thermal conductivity of such materials were checked against the experimental values. The results are discussed with reference to their possible implications for the conductivity of solidified forms of high-level radioactive waste.

1452 Classification and safety reserves of radioactive material shipping containers. Schulz-Forberg, B.; Huebner, H.W. Karlsruhe, Germany, F.R.; Fachinformationszentrum Energie, Physik, Mathematik (Mar 1979). vp.p. (In German). Available from Fachinformationszentrum Energie, Physik, Mathematik, Karlsruhe (Germany, F.R.).

Taking into account the experimental results and measured data of this study, the applicability of results obtained in the testing of specimens with a scale factor of $1: 2$ for small-size packagings, and of 1:3 for larger packagings seems to be sufficiently accurately demonstrated so as to be taken as a basis of predicting the behaviour of prototype packagings. This is particularly true for the mechanical forces involved, acceleration and deformation. 


\section{CONTENTS}

NUCLEAR FUELS 1

By-Products 1

Fuels Production and Properties 1

Spent Fuels Repocessing 1

Transport and Storage 2

Marketing and Economics $\quad 7$

Wastc Management 7

Environmental Aspects $\quad 177$

Health and Safety $\quad 188$

Regulations 193

ENVIRONMENTAL SCIENCES, TERRESTRIAL 195

Radioactive Materials Monitoring and Transport 195

Site Resource and Use Studies 196

ENVIRONMENTAL SCIENCES, AQUATIC 196

Radioactive Materials Monitoring and Transport 196

Site Resource and Use Studies 196

$\begin{array}{ll}\text { GENERAL } & 197\end{array}$

CORPORATE AUTHOR INDEX - IC

PERSONAL AUTHOR INDEX 15A

SUBJECT INDEX 41S

CONTRACT NUMBER INDEX $153 \mathrm{CN}$

REPORT NUMBER INDEX 160R

$\begin{array}{ll}\text { ORDER NUMBER CORRELATION } & 182 \mathrm{~N}\end{array}$ 


\section{CORPORATE AUTHOR INDEX}

In the Corporate Index, report literature is indexed using the name of the organization or institution responsible for issuing the report. Headings are provided for all report literature and for published literature for which a corporate approach is especially desirable, e.g., symposium and conference proceedings.

Each entry under a corporate heading gives the document title and the volume and abstract numbers. The document type, e.g., $\mathbf{R}$ for Report; country of publication, e.g., US; and language of the document, e.g., In German; are given in parentheses at the end of the entry. If no language statement appears, the language is English. Tables of document types and country of publication codes appear after the indexes.

DOE Energy Information Data Base: Corporate Author Entries (DOE/TIC-4585) is used in the standardization of corporate entries. DOE/TIC-4585 is available for purchase from the National Technical Information Service (NTIS), U. S. Department of Commerce, Springfield, Virginia 22161.

\section{A}

Agapito (J.F.T.) and Associates, Grand Junction, CO (USA)

Evaluation of geotechnical surveillance techniques for monitoring high-level waste repository performance. Final report 19 Sep 81-31 Dec 82, 738 (R;US)

Akademiet for de Tekniske Videnskaber, Lyngby (Denmark)

Investigations of salt domes for the purpose of high-level radioactive waste disposal, 657 (R;DK;DA)

Analytic Sciences Corp., Reading, MA (USA)

Review of safety assessments of nuclear waste management, 1380 (R;US)

Argonne National Lab., IL (USA)

Alternate strategy for commercial high-level radioactive-waste management, 945 (R;US)

Application of neutron-activation analysis to the determination of leach rates of simulated nuclear-waste forms, $127(R ; U S)$

Benefit-cost-risk analysis of alternatives for greater-confinement disposal of radioactive waste, 559 (R;US)

Effect of composition and radiation on the Hertzian indentation behavior of nuclear waste glasses, 145 (R;US)

Final report of experimental laboratory-scale brittle fracture studies of glasses and ceramics, 129 (R;US)

Fuel cycle programs. Quarterly progress report, April-June 1982, 536 (R;US)

Fuel cycle programs. Quarterly progress report, July-September 1982, 538 (R;US)

Fuel cycle programs. Quarterly progress report, July-September 1981, 50 (R;US)

Fuel cycle programs. Quarterly progress report, October-December 1982, 539 (R;US)

Fuel-cycle programs. Quarterly progress report, April-June 1981, 49 (R;US)

Fuel-cycle programs. Quarterly progress report, January-March 1981; 48 (R;US)

Interaction of groundwater and fresh basalt fissure surfaces and its effect on the migration of actinides, 1303 (R;US)

Measurement of leaching from simulated nuclear-waste glass using radiotracers, 128 (R;US)

Octyl(phenyl)-N,N-diisobutylcarbamoylmethylphosphine oxide as an extractant for actinides from nitric acid waste, 146 (R;US)

Oxidation potential $\left(\mathrm{E}_{\mathrm{h}}\right)$ and $\mathrm{pH}$ control during experimentation, 552 (R;US)

trizona Univ., Tucson (USA)

Groundwater flow and transport characteristics of flood basalts as determined from tracer experiments, 880 (R;US)

Arizona Univ., Tucson (USA). Dept. of Hydrology and Water Resources

Unsaturated flow and transport through fractured rock - related to high-level waste repositorics. Final report. Phase I, 748 (R;US)
Arkansas Univ, Fayetteville (USA). Dept. of Agricultural Economics and Rural Sociology

Possible approaches to community development for nuclear waste isolation, 805 ( $R ;$ US)

Atomic Energy Control Board, Ottawa, Ontario (Canada)

Scope and nature of the problem of high level nuclear waste disposal, $1430(R ; C A)$

Atomic Energy of Canada Ltd., Pinawa, Manitoba. Whiteshell Nuclear Research Establishment

Diffusive transport of strontium-85 in sand-bentonite mixtures, 1300 (R;CA)

High-level waste glass field burial tests at CRNL: the effect of geochemical kinetics on the release and migration of fission products in a sandy aquifer, 1299 (R;CA)

Atomic Industrial Forum, Inc., Washington, DC (USA)

Nuclear waste/nuclear power: their futures are linked, 560 (R;US)

Atomies International Div., Richland, WA (USA). Rockwell Hanford Operations

Evaluation of water resource economics within the Pasco Basin, Washington, 1429 (R;US)

Geochemical controls on radionuclide releases from a nuclear waste repository in basalt: estimated solubilities for selected elements, 1329 (R;US)

Auburn Univ, AL (USA)

Analysis of waste-package behavior for high-level waste, 981 (R;US)

Australian Atomic Energy Commission Research Establishment, Lucas Heights

Assessment of overseas developments in methods for treatment and disposal of high-level radioactive wastes, 532 (R;AU)

Use of thorium as an alternative nuclear fuel, $46(R ; A U)$

Australian National Univ., Canberra. Research School of Earth Sciences

Immobilization of sodium and phosphorus-bearing PW-7a waste in SYNROC. Progress report, 346 (R;US)

\section{B}

Battelle Columbus Labs, OH (USA)

Annotated bibliography for the design of waste packages for geologic disposal of spent fuel and high-level waste, 804 (R;US)

Impacts of transportation on a test and evaluation facility for nuclear waste disposal: a systems analysis, 556 (R;US)

Importance of organic compounds in ground water as radionuclidemobilizing agents, 1324 (R;US)

Long-term performance of materials used for high-level waste packaging. Annual report, March 1982-April 1983. Vol.1, 751 (R;US)

Long-term performance of materials used for high-level waste packaging. Quarterly report, April-June 1983, 752 (R;US)

Preliminary environmental implications of alternatives for decommissioning and future use of the Western New York Nuclear Services Center, 1301 (R;US)

Preliminary risk assessment for nuclear waste disposal in space. Volume I. Executive summary of technical report, 720 (R;US)

Preliminary risk assessment for nuclear waste disposal in space. Volume II. Technical report, 721 (R;US)

Battelle Memorial Inst., Columbus, OH (USA). Ofice of Crystalline Repository Development

National survey of crystalline rocks and recommendations of regions to be explored for high-level radioactive waste repository sites. 540 (R;US)

Battelle Memorial Inst, Columbus, OH (USA). Ofince of National Weste Terminal Storage Integration

Implementation of technical conservatism in the development of nuclear waste repositories, 600 (R;US) 
NWTS program criteria for mined geologic disposal of nuclear waste: program objectives, functional requirements, and system performance criteria, 595 (R;US)

NWTS program criteria for mined geologic disposal of nuclear waste: repository performance and development criteria. Public draft, 596 (R;US)

NWTS program criteria for mined geologic disposal of nuclear waste: functional requirements and performance criteria for waste packages for solidified high-level waste and spent fuel, 597 (R;US)

NWTS waste package program plan. Volume I. Program strategy, description, and schedule, 598 (R;US)

NWTS waste package program plan. Volume II. Program logic networks, 599 (R;US)

Projected costs for mined geologic repositories for dispoal of commercial nuclear wastes, 769 (R;US)

Battelle Memorial Inst, Columbus, OH (USA). Office of Nuclear Waste Isolation

Conceptual test plan for site confirmation testing at an exploratory shaft in salt, 826 (R;US)

Conceptual waste package interim performance specifications for waste forms for geologic isolation in salt repositories, 821 (R;US)

Conceptual waste package interim product specifications and data requirements for disposal of borosilicate glass defense high-level waste forms in sait geologic repositories, 823 (R;US)

Evaluation of area studies of the US Gulf Coast Salt Dome Basins: location recommendation report, 785 (R;US)

Functional design criteria for the exploratory shaft in salt, 820 (R;US)

Geological evaluation of Gulf Coast salt domes: overall assessment of the Gulf Interior Region, 784 (R;US)

Interim performance specifications for conceptual waste-package designs for geologic isolation in salt repositories, 822 (R;US)

Laboratory rock mechanics testing manual. Public draft, 807 (R;US)

LAYFLO: a one-dimensional semianalytical model for the migration of a three-member decay chain in a multilayered geologic medium, 1325 (K;US)

National Waste Terminal Storage Program. Technical progress report for the quarter 1 July-30 September 1982, 780 (R;US)

ONWI library reports list, 770 (R;US)

Performance assessment of a shaft seals system in a HLW repository in the Gibson Dome area, 827 (R;US)

Proceedings of the 1980 National Waste Terminal Storage Program information meeting, 787 (R;US)

Results of repository conditions study for commercial and defense high-level nuclear waste and spent fuel repositories in salt, 824 (R;US)

Technical progress report, 1 April-30 June 1981, 775 (R;US)

Technical progress report for the quarter, 1 April-30 June 1982, 779 (R;US)

Technical progress report for the quarter 1 January-31 March 1982,778 (R;US)

Technical progress report for the quarter, 1 July-30 September 1981, 776 (R;US)

Technical progress report for the quarter, 1 October-31 December, 1981, 777 (R;US)

Technical progress report for the quarter 1 October-31 December 1982, 781 (R;US)

Uncertainties and sensitivities in the performance of geologic nuclear waste isolation systems. Volume I. Summary, 809 (R;US)

Waste package materials testing for a salt repository: 1982 status report, 825 (R;US)

Battelle Pacific Northwest Labs., Richland, WA (USA)

Assessment of power reactor waste immobilization by vitrification. Final report, 206 (R;US)

Defense waste transportation: cost and logistics studies, 27 (R;US)

Radiation damage in crystalline nuclear-waste solids, 86 (R;US)

Radiation damage studies related to nuclear waste forms, 298

(R;US)

Review of safety assessments of nuclear waste management, 1380 (R;US)
Solidification of high level wastes. Part IV. Phosphate melts for fixation of radioactive residues from Purex type wastes: three to fifty percent fission product oxides, 134 (R;US)

Bechtel Group, Inc., San Francisco, CA (USA)

Ecosystem studies, endangered species survey - Gibson Dome and Elk Ridge study areas, Paradox Basin, Utah, 1326 (R;US)

Southeastern Utah nuclear-waste-transportation study, 26 (R;US)

Bechtel National, Inc., San Francisco, CA (USA)

Environmental characterization report for the Gulf Interior Region Louisiana study area, 1321 (R;US)

Environmental characterization report for the Gulf Interior Region Mississippi study area, 1322 (R;US)

Environmental characterization report for the Gulf Interior Region, Texas study area, 1323 (R;US)

Immobilizing defense waste at the Savannah River Project, 196 (R;US)

Bhabha Atomic Research Centre, Bombay (India)

Thermal and radiation stability of simulated high-level vitrified waste products, 51 (R;IN)

Boeing Aerospace Co., Seattle, WA (USA)

Analysis of space systems for the space disposal of nuclear waste follow-on study. Volume 1. Executive summary, 718 (R;US)

Analysis of space systems for the space disposal of nuclear waste follow-on study. Volume 2. Technical report, 719 (R;US)

Bollyky Associates, Stamford, CT (USA)

Ozone mass transfer and kinetics experiments, 334 (R;US)

Brookhaven National Lab., Upton, NY (USA)

Container assessment: corrosion study of HLW container materials. Quarterly progress report, July-September 1981, 728 (R;US)

Container assessment - corrosion study of HLW container materials. Quarterly progress report, April-June 1981, 729 (R;US)

Container assessment: corrosion study of HLW container materials. Quarterly progress report, January-March 1982, 730 (R;US)

Draft technical position on waste-package reliability, $546(R ; U S)$

Draft Technical Position Subtask 1.1: waste package performance after repository closure. Volume 1,749 (R;US)

High-level radioactive wste management: a means to social consensus, 542 (R;US)

High-level-waste containment for a thousand years: unique technical and research problems, 544 (R;US)

Identification of crevice corrosion in the titanium alloy TiCode-12 in simulated rock salt brine at $150^{\circ} \mathrm{C}, 545$ (R;US)

Nuclear waste management technical support in the development of nuelear waste form eriterin for the NRC: Tnak 1 Wnatn pnok age overview, 283 (R;US)

Nuclear waste management technical support in the development of nuclear waste form criteria for the NRC. Task 4. Test development review, 284 (R;US)

Nuclear waste management technical support in the developmnt of nuclear waste form criteria for the NRC. Task 5. National waste package program, 285 (R;US)

Nuclear-waste-management technical suppurt in the development of nuclear-waste-form criteria for the NRC. Task 3. Waste inventory review, 73 (R;US)

Packing material testing required to demonstrate compliance with 1000-year radionuclide containment. Semiannual report on waste package verification tests, 739 (R;US)

Post-emplacement monitoring, Subtask 1.2. Draft Technical Position. Vol. 2, 1319 (R;US)

Radiation induced F-center and colloid formation in synthetic $\mathrm{NaCl}$ and natural rock salt: applications to radioactive waste repositories, 543 (R:US)

Review of DOE waste package program. Subtask 1.1. National waste package program, April-September 1982, 735 (R;US)

Review of DOE waste package program. Subtask 1.1. National waste package program, October 1982-March 1983. Vol. 4, 736 (R;US)

Review of DOE waste package program. Subtask 1.1. National waste package program, 286 (R;US)

Review of DOE waste-package program. Subtask 1.1. National waste-package program, September 1981-March 1982. Volume 2, 732 (R;US)

Savannah River Laboratory long-term waste storage program. Progress report No. 5, March-April 1974, 131 (R;US) 
Savannah River Laboratory long-term waste storage program. Progress report No. 7, July-September 1974, 133 (R;US)

iavannah River Laboratory long-term waste storage program. Progress report No. 6, May-Jun 1974, 132 (R;US)

Uncertainties in the thermodynamics of basalt-oxygen and basaltwater reactions, $547(R ; U S)$

Building Research Station, Watford (England)

Backfilling and sealing a repository for high-level radioactive waste - a review, 839 (R;US)

\section{C}

California Univ., Berkeley (USA). Dept. of Nuclear Engineering

Thermal-gradient migration of brine inclusions in salt, 711 (R;US)

CEA Centre d'Etudes Nucleaires de Saclay, 91 - Gif-sur-Yvette (France)

Elimination of used sources. Taking over and storage; 548

(R;FR;In French)

Encapsulation of tritiated wastes in hot laboratories, 137 (R;FR;In French)

CEA Etablissement de la Vallee du Rhone, 30 - Bagnols-sur-Ceze (France)

Actual point about fission products vitrification, 136 (R;FR;In French)

French status of high level radioactive wastes solidification, 135 (R;FR)

Centre d'Etude de l'Energie Nucleaire, Mol (Belgium)

Preparation and microstructural characterization of silicate and borosilicate glasses containing cerium or plutonium, $130(\mathrm{R} ; \mathrm{BE})$

Colorado School of Mines, Golden (USA)

Formulation of an integrated physicochemical-hydrologic model for predicting waste-nuclide retardation in geologic media, 973 (R;US)

Comitato Nazionale per l'Energia Nucleare, Casaccia (Italy). Dipartimento Radiazioni e Ricerche di Sicurezza e Protezione

Laboratory determination of the thermal properties of large-scale samples of natural clays, 550 (R;IT;IT)

Commission of the European Communities, Brussels (Beigium)

Storage of HLW in engineered structures: air-cooled and watercooled concepts. Final report, $640(R ; X E)$

Commission of the European Communities, Ispra (Italy). Joint Research Centre

Actinide recycling in light water reactors: results of reactor physics calculations, 208 (R;XE)

Actinide recycling in light water reactors: results of reactor physics calculations, 294 (R;US)

Commission of the European Communities, Karlsruhe (Germany, F.R.). European Inst. for Transuranium Elements

Effect of composition and radiation on the Hertzian indentation behavior of nuclear waste glasses, 145 (R;US)

Mechanical stability of a $\mathrm{Cm}$-doped celsian glass-ceramic, 143 (R;XE)

Commission of the European Communities, Luxembourg

Storage of HLW in engineered structures: air-cooled and watercooled concepts. Final report, 833 (R;US)

Cornell Univ., Ithaca, NY (USA). Sibley School of Mechanical and Aerospace Engineering

Transient creep model for salt during stress loading and unloading, 969 (R;US)

\section{D}

D'Appolonia Consulting Engineers, Albuquerque, NM (USA)

Basic data report for deepening of drillhole WIPP 13 (Waste Isolation Pilot Plant-WIPP), 975 (R;US)

Basic data report for Drillhole AEC 7 (Waste Isolation Pilot Plant - WIPP), 924 (R;US)

lasic data report for drillhole AEC 8 (Waste Isolation Pilot Plant WIṔP), 925 ( $R ; U S$ )

lasic data report for Drillhole ERDA 10 (Waste Isolation Pilot

Plant - WIPP), 927 (R;US)

Basic data report for Drillhole WIPP 14 (Waste Isolation Pilot Plant - WIPP), 974 (R;US)
Basic data report for drillhole WIPP 12 (Waste Isolation Pilot Plant-WIPP), 984 (R;US)

D'Appolonia Consulting Engineers, Inc., Pittsburgh, PA (USA)

NWTS Field Testing In-Situ Site-Confirmation Tests Workshop, September 8-9, 1980, 802 (R;US)

Delawiare Geological Survey, Newark (USA)

Geologic aspects of disposal of highly radioactive nuclear waste. Open-File Report No. 15, 722 (R;US)

Department of Energy, Aiken, SC (USA). Savannab River Operations Office

Environmental assessment: waste-form selection for SRP high-level waste, SS (R;US)

Evaluation and selection of candidate high-level waste forms, 160 (R;US)

Department of Energy, Las Vegas, NV (USA). Nerada Operations Ofifice

Annotated bibliography of cultural resources literature for the Nevada Nuclear Waste Storage Investigations, 768 (R;US)

Cultural resources overview for the Nevada Nuclear Waste Storage Investigations, Nevada Test Site, Nye County, Nevada, 767 (R;US)

Nevada nuclear waste storage investigations: briefing book, 572 (R;US)

Nevada nuclear waste-storage investigations. Quarterly report, January-March 1982, 756 (R;US)

Nevada Nuclear-Waste-Storage Investigations. Quarterly report, April-June 1982, 757 (R;US)

Overview of energy and mineral resources for the Nevada nuclearwaste-storage investigations, Nevada Test Site, Nye County, Nevada, 763 (R;US)

Department of Energy, Oak Ridge, TN (USA). Technical Information Center

High-level radioactive wastes, 159 (R;US)

Radioactive waste inventories and projections, 62 (R;US)

Radioactive waste processing and disposal: a bibliography, 158 (R;US)

Technology of high-level nuclear waste disposal. Advances in the science and engineering of the management of high-level nuclear wastes. Volume 1, 607 (R;US)

Department of Energy, Washington, DC (USA)

Treatment and conditioning of wastes from US nuclear fuels reprocessing plants, 191 (R;US)

Department of Energy, Washington, DC (USA). Assistant Secretary for Defense Programs

Defense Waste Processing Facility: Savannah River Plant, Aiken,

SC. Final environmental impact statement, 1306 (R;US)

Environmental assessment: waste-form selection for SRP high-level waste, 55 (R;US)

Department, of Energy, Washington, DC (USA). Assistant Secretary for Nuclear Energy

National plan for siting high-level radioactive waste repositories and environmental assessment. Public draft, 583 (R;US)

Department of Energy, Washington, DC(USA). Office of Inspector General

High-level nuclear-waste-immobilization program, 154 (R;US)

Du Pont de Nemours (E.I.) and Co., Aiken, SC (USA)

Development of borosilicate glass for the vitrification plant PAMELA, 530 (TG;US)

Waste Management Program. Technical progress report, OctoberDecember 1982, 65 (R;US)

Du Pont de Nemours (E.I.) and Co, Aiken, SC (USA), Savanmab River Lab.

Alternatives for definse waste-salt disposal, 629 (R;US)

Alternative-waste-form evaluation for Savannah River Plant highlevel waste, 178 (R;US)

Analyses of nuclear fuel and high-level waste by inductively coupled plasma-emission spectroscopy, 68 (R;US)

Assessment of high-level waste form conformance with proposed regulatory and repository criteria, 198 (R;US)

Assessment of processes, facilities, and costs for alternative solid forms for Immobilization of SRP defense wasto, 169 (R!US)

Assessment of Savannah River borosilicate glass in the repository environment, 172 (R;US)

Characterization of borosilicate glass containing Savannah River Plant radioactive waste, 179 (R;US) 
Chemical denitration of radioactive-waste solutions with formic acid, 531 (TG;US)

Comparative leach testing of high-level waste forms, 626 (R;US)

Comparative risk assessments for the production and interim storage of glass and ceramic waste forms: defense waste processing facility, 1370 (R;US)

Comparative transportation risk assessment for borosilicate-glass and ceramic forms for immobilization of SRP Defense waste, 23 (R;US)

Comparison of properties of borosilicate glass and crystalline ceramic forms for immobilization of Savannah River Plant waste, 171 (R;US)

Comparison of SRP high-level waste disposal costs for borosilicate glass and crystalline ceramic waste forms, 635 (R;US)

Composite quarterly technical report: long-term high-level-waste technology, January-March 1982, 66 (R;US)

Composite quarterly technical report long-term high-level-waste technology, October-December 1981, 164 (R;US)

Coordination meeting of high-level waste technology and national waste terminal storage programs, October 21-22, 1981, 624 (R;US)

Decontaminated salt disposal as saltcrete in a landfill. Technical data summary, 636 (R;US)

Decontamination of Savannah River Plant waste glass canisters. $1 \% 5(R ;$ IIS $)$

Description of a cèramic waste form and canister for Savannah River Plant high-level waste, 170 (R;US)

Development, evaluation, and selection of candidate high-level waste forms, 185 (R;US)

Development of a slurry-fed in-can melter for nuclear defense waste , 176 (R;US)

Disposal of Savannah River Plant waste salt, 625 (R;US)

DWPF process control, 186 (R;US)

Efficient particulate scrubber for glass melter off-gas, 183 (R;US)

Evaluation of glass-contact materials for waste glass melters, 189 (R;US)

Hazards analysis of TNX Large-Melter-Off-Gas System, 197 (R;US)

High-level waste description, inventory and hazard, 632 (R;US)

High-level waste-form-product performance evaluation, 627 (R;US)

High-level-waste immobilization, 181 (R;US)

Large-scale continuous process to vitrify nuclear defense waste: operating éxperience with nonradioactive waste, 177 (R;US)

Long-term high-level waste technology. Composite quarterly technical report. July-September 1981, 163 (R;IIS)

Long-term high-level waste technology. Composite quarterly technical report, July-September 1982, 166 (R;US)

Long-term high-level-waste technology. Composite quarterly technical report, April-June 1982, 165 (R;US)

National long-term high-level waste-technology program, 67 (R;US)

Near-surface leaching studies of Pb-implanted Savannah River waste glass, 140 (R;US)

Physical and chemical characterization of synthetic calcined sludge, 167 (R;US)

Physical modeling of a glass melter designed for vitrification of defense waste, 190 (R;US)

Precipitation process for decontaminating water-soluble SRP radioactive waste, 187 (R;US)

Precipitation process for decontamination of water-soluble SRP radioactive waste, 184 (R;US)

Precipitation process for supernate decontamination, 173 (R;US)

Predicting transport requirements for radioactive-waste slurries, 22 (R;US)

Preliminary report on a glass burial experiment in granite, 631 (R;US)

Prevention of stress corrosion cracking in nuclear waste storage tanks, 630 (R;US)

Processability analysis of candidate waste forms, 174 (R;US)

Relative hazard of DWPF reference glass canister ICRP 2 vs ICRP 30, 1371 (R;US)

Research priorities for isolation of defense high-level and transuranic wastes in the United States, 628 (R;US)

Savannah River Plant uverview, 69 (R;US)

Solidification of ash from incineration of low-level radioactive waste, 193 (R;US)
SRP radioactive glass studies: small-scale process development and product performance, 180 (R;US)

Startup of a Joule-heated glass melter with a graphite slurry, 188 (R;US)

Theoretical predictions for continuous-slurry feeding of a glass melter, 182 (R;US)

Treatment and conditioning of wastes from US nuclear fuels reprocessing plants, 191 (R;US)

Waste Management Program. Technical progress report, OctoberDecember 1980, 162 (R;US)

Waste management program technical progress report, JanuaryMarch 1983, 623 (R;US)

Waste-management program. Technical progress report, JanuaryMarch 1981, 622 (R;US)

Waste-management program. Technical progress report, JulySeptember 1981, 64 (R;US)

Waste-management program technical progress report, JulySeptember 1980, 63 (R;US)

Du Pont de Nemours (E.I.) and Co., Aiken, SC (USA). Savannah River Plant

Contamination of the hot gang valve corridor and first-level clean areas of Building 221-F - September 13, 1960, 194 (R;US)

Defense Waste Processing Facility: a remote process for solidificativin of Sivanual Rlver Plant high level waste, IY, (R;IIS)

Deseription of Defïist Waste Piviessilng Facility reference waste form and canister. Revision 1, 168 (R;US)

Immobilizing defense waste at the Savannah River Project, 196 (R;US)

Sampling and analysis of high level waste tank supernatant: an overview, 634 (R;US)

Savaninah River interim waste management program plan, FY1984, 606 (R;US)

Savannah River Plant waste-tank inspection manual, 633 (R;US)

Savannah River Waste Management Program Plan - FY 1982, 157 (R;US)

Waste Management Program. Technical progress report, OctoberDecember 1980, 162 (R;US)

Waste-management program technical progress report, JulySeptember 1980, 63 (R;US)

Du Pont de Nemours (E.I.) and Co., Newark, DE (USA). Engineering Dept.

Processability analysis of candidate waste forms, 174 (R;US)

Du Pont de Nemours (E.I.) and Co., Wilmington, DE (USA). Engineering Dept.

Electrical service and controls for Joule heating of a defense waste experimental glass melter, 192 (R;US)

\section{E}

EG and G Idaho, Inc., Idaho Falls (USA)

Evaluation of forms for the immobilization of high-level and transuranir: wistes, 200 (R;US)

Preparation and leaching of radioactive INEL waste forms, 201 (R;US)

Radioactive waste management: a summary of state laws and administration. National Low-Level Radioactive Waste Mangement Program, 569 (R;US)

Radioactive waste management: a summary of state laws and administration, 570 (R;US)

Scientific data necessary to predict radionuclide migration within or near a mined nuclear repository, 1312 (R;US)

EG and G, Inc., Goleta, CA (USA)

Advances in measurenient of alpha-contaminated wastes, 1406 (R;US)

Measurement of radioactive contaminated wastes, 278 (R;US)

Surveys for desert tortoise on the proposed site of a high-level nuclear waste repository at the Nevada Test Site, 1302 (R;US)

EG and G, Inc., Goleta, CA (USA). Energy Measurements Group

1982 biotic survey of Yucca Mountain, Nevada Test Site, Nye County, Nevada, 1311 (R;US)

Annotated bibliography for biologic overview for the Nevada Nuclear Waste Storage Investigations, Nevada Test Site, Nye County, Nevada, 1309 (R;US) 
Biologic overview for the Nevada Nuclear Waste Storage Investigations, Nevada Test Site, Nye County, Nevada, 1310 (R;US)

_.dgenoessisches Inst. fuer Reaktorforschung, Wuerenlingen (Switzerland)

Data base for a site-specific analysis of the dispersion of radioactive elements from a final repository, 1366 (TG;US)

Glasses for the solidification of high-level radioactive waste: their behavior in the presence of water, 1052 (TG;US)

Risk analysis for repositories in north Switzerland. Extent and probability of geologic processes and events, 1372 (R;CH;GE)

ELKRAFT A.m.b.A., Hellerup (Denmark)

Disposal of high-level nuclear waste from nuclear power plants in Denmark. Salt dome investigations. V.2. Geology, figures, 649 (R;DK)

Disposal of high-level waste from nuclear power plants in Denmark. Salt dome investigations. V.1. Summary, 647 (R;DK)

Disposal of high-level waste from nuclear power plants in Denmark. V.3. Material testing, 650 (R;DK)

Disposal of high-level waste from nuclear power plants in Denmark. Salt dome investigations. V.2. Geology, text, 648 (R;DK)

Disposal of high-level waste from nuclear power plants in Denmark. Salt dome investigations. V.4. Repository design, 651 (R;DK)

Disposal of high-level waste from nuclear power plants in Denmark. Salt dome investigations. V.5. Safety evaluation, 652 (R;DK)

European Company for the Chemical Processing of Irradiated Fuels, Mol (Belgium)

Separation of mercury from HEWC solutions. Laboratory assay, 207 (R;BE;In French)

Exxon Nuclear Idaho Co., Inc., Idaho Falls (USA)

Alternative fuels and heating methods for calciner process heat, 204 (R;US)

Attrition, elutriation, and growth of particles produced in fluidizedbed waste calciners, 202 (R;US)

Evaluation of stainless steel tank corrosion in ICPP high-level radioactive waste service, 203 (R;US)

Evaluation of the safety aspects of the design and operation of temporary/mobile radioactive-waste solidification systems, 287. (R;US)

Preparation and leaching of radioactive INEL waste forms, 201 (R;US)

\section{$\mathbf{F}$}

Fenix and Scisson, Inc., Mercury, NV (USA)

E-field ratio telluric traverses near Fortymile Wash, Nevada Test Site, Nevada, 1027 (R;US)

Resistivity sounding investigation by the Schlumberger method in the Yucca Mountain and Jackass Fiats area, Nevada Test Site, Nevada, 1028 (R;US)

Florida State Univ., Tallahassee (USA). Dept. of Chemistry

Evaluation of DOE radionuclide solubility data and selected retardation parameters: description of calculational and confirmatory experimental activities, 1304 (R;US)

Florida Univ., Gainesville (USA)

Evaluation of conditioned high-level waste forms, 326 (R;US)

Florida Univ., Gainesville (USA). Coll. of Engineering

Surface properties and performance prediction of alternative waste forms. Annual report, October 1, 1981-September 30, 1982. Volume 1, 753 (R;US)

Florida Univ., Gainesville (USA). Dept. of Materials Science and Engineering

Preliminary report on a glass burial experiment in granite, 631 (R;US)

'oster-MIller Assoclates, Inc., Waltham, MA (USA)

Equipment for the emplacement of heat-producing waste in long horizontal boreholes, 555 (R;US)

Foundation Sciences, Inc., Portland, OR (USA)

Laboratory rock mechanics testing manual. Public draft, 807 (R;US)

\section{G}

GA Technologies, Inc., San Diego, CA (USA)

Comparison of cask and dry well concepts for retrievable storage of high-level waste, 641 (R;US)

Economic evaluation of nuclear waste transportation casks, 14 (R;US)

Monitored retrievable storage of nuclear waste in the US, 642 (R;US)

General Accounting Office, Washington, DC (USA). Energy and Minerals Div.

Department of Energy does not plan to use an abandoned salt mine at Lyons, Kansas, for nuclear high-level waste disposal, 637 (R;US)

Is spent fuel or waste from reprocessed spent fuel simpler to dispose of, 533 (R;US)

General Atomic Co., San Diego, CA (USA)

Guidebook for reliability, availability, and maintainability analysis of NWTS repository equipment. Volume II. Appendices, 808 (R;US)

High-Level Waste Transportation-System Development Program design report for Fiscal Year 1980, 34 (R;US)

System evaluation model for selecting spent nuclear fuel storage concepts, 24 (R;US)

General Electric Co., Richland, WA (USA). Hanford Atomic Products Operation

Fixation of radioactive residues. Quarterly progress report, January-March 1961, 213 (R;US)

Management of high-level radioactive wastes, 218 (R;US)

Progress in radioactive residue process development. A summary of Hanford laboratories achievements in this program under General Electric, 1958-1964, 216 (R;US)

Recovery and purification of multi-kilocurie quantities of fission product strontium by cation exchange, 214 (R;US)

Research and development activities: fixation of radioactive residues. Quarterly progress report, April-June 1960, 210 (R;US)

Research and development activities: fixation of radioactive residues. Quarterly progress report, July-September 1960, 212 (R;US)

Research and development activities [on] solidification of radioactive wastes. Progress report, 215 (R;US)

Solid fixation of high-level radioactive waste by sorption on clinoptilolity-scouting studies, 211 (R;US)

Solidification of high level wastes. Part III. Solubility, melting point, flow temperature and thermal conductivity of some calcined, synthetic purex-type wastes, 209 (R;US)

Solidification of high-level radioactive fuel reprocessing wastes by spray and pot calcination. Hot-cell pilot plant studies, 217 (R;US)

Geological Survey, Albuquerque, NM (USA)

Basic data report for drillhole ERDA 6 (Waste Isolation Pilot Plant - WIPP), 923 (R;US)

Geological Survey, Denver, CO (USA)

Basic data report for drillhole ERDA 9 (Waste Isolation Pilot Plant WIPP), 926 (R;US)

Basic data report for drillhole WIPP 11 (Waste Isolation Pilot Plant - WIPP), 928 (R;US)

E-field ratio telluric traverses near Fortymile Wash, Nevada Test Site, Nevada, 1027 (R;US)

Resistivity sounding investigation by the Schlumberger method in the Yucca Mountain and Jackass Flats area, Nevada Test Site, Nevada, 1028 (R;US)

Three-dimensional modeling of the Nevada Test Site and vicinity from teleseismic p-wave residuals, 1022 (R;US)

Geological Survey, Menlo Park, CA (USA)

Preliminary appraisal of gravity and magnetic data at Syncline Ridge, Western Yucca Flat, Nevada Test Site, Nye County, Nevada, 1025 (R;US)

Preliminary results of gravity investigations at Yucca Mountain and vicinity, Southern Nye County, Nevada, 1024 (R;US)

Principal facts of gravity stations with gravity and magnetic profiles from the southwest Nevada Test Site, Nye County, Nevada, as of January $1982,1026(R ; U S)$ 
Geological Survey, Menlo Park, CA (USA). Office of Earthquake Studies

1981 Geodolite observations near Hanford, Washington, 870 (R;US)

Geological Survey, Reston, VA (USA)

Electrical studies at the proposed Wahmonie and Calico Hills nuclear waste sites, Nevada Test Site, Nye Co., Nevada, 1023 (R;US)

Geohydrologic and drill-hole data for test well USW $H-1$, adjacent to Nevada Test Site, Nye County, Nevada, 1030 (R;US)

Study of surface and subsurface ground motions at Calico Hills, Nevada Test Site, 1029 (R;US)

Geological Survey, Washington, DC (USA)

Interpreted resistivity and IP section line W1: Wahmonie Area, Nevada Test Site, Nevada, 567 (R;US)

US Geological Survey research in radioactive waste disposal, fiscal year 1979, 716 (R;US)

Georgia Inst. of Tech., Atlanta (USA)

Reprocessing of nuclear fuel, 6 (R;US)

Uranium hexafluoride actinide transmutation reactors-preliminary design and analysis, 516 (D;US)

GeoTrans, Inc., Reston, VA (USA)

Benchmark problems for repository siting models, 746 (R;US)

Parameters and variables appearing in repository-siting models, 745 (R;US)

Gesellschaft Deutscher Chemiker, Frankfurt am Main (Germany, F.R.). Fachgruppe Nuklearchemie

Proceedings of the international seminar on chemistry and process engineering for high-level liquid waste solidification, 226 (R;DE; In several languages)

Proceedings of the international seminar on chemistry and process engineering for high-level liquid waste solidification, 263 (R;DE;In German and English)

Gesellschaft fuer Strahlen- und Umweltforschung m.b.H. Muenchen, Neuherberg (Germany, F.R.)

Deep underground storage of [radioactive waste]. Annual report 1981,643 (R;DE;GE)

Gilbert/Commonwealth, Reading, PA (USA)

Summary of repository siting models. Final report, 740 (R;US)

Golder Associates, Inc., Bellevue, WA (USA)

Evaluation of alternative shaft-sinking techniques for high-level nuclear waste (HLW) deep geologic repositories. Final report (Task 3), June 1981-July 1982, 741 (R;US)

In-situ test programs related to design and construction of highlevel nuclear waste (HLW) deep geologic repositories. Final report (Task 2), June 1981-November 1982, 743 (R;US)

In-situ test programs related to design and construction of highlevel nuclear waste (HLW) deep geologic repositories. Appendices. Final report (Task 2), June 1981-November 1982, 744 (R;US)

Relationship of an in-situ test facility to a deep geologic repository for high-level nuclear waste. Final report (Task 4), June 1981Outuber 1982, 742 (R;US)

H

Hahn-Meitner-Institut fuer Kernforschung Berlin G.m.b.H. (Germany, F.R.)

Mechanical stability of a $\mathrm{Cm}$-doped celsian glass-ceramic, 143 (R:XE)

Hahn-Meitner-Institut fuer Kernforschung Berlin G.m.b.H. (Germany, F.R.). Projekt Sicherheitsstudien Entsorgung (PSE)

INTRACOIN, Level 1. Benchmark calculations with code SWIFT, 644 (R;DE)

Idaho National Engineering Lab., Idaho Falls (USA)

Environmental evaluation of alternatives for long-term management of Defense high-level radioactive wastes at the Idaho Chemical Processing Plant, 70 (R;US)

Iron-enriched basalt as a waste form for high-level nuclear wastes, 199 (R;US)
Institute of Geological Sciences, Harwell (UK). Environmental Protection Unit

Distribution coefficient (Kd) concept and its applicability to studies of radionuclide migration in geological media, 1313 (R;GB)

Fracture analysis of crystalline rocks: field measurements ard field geomechanical techniques, 638 (R;GB)

Geochemical factors controlling the nuclide release source-term in granite: dissolution of the waste form, 205 (R;GB)

INTERA Environmental Consultants, Inc., Houston, TX (USA)

WAPPA: a Waste Package Performance Assessment code. Technical report, 819 (R;US)

INTERA Environmental Consultants, Lakewood, CO (USA)

Analysis of waste-package behavior for high-level waste, 981 (R;US)

International Nuclear Law Association (INLA), Brussels (Belgium) Indemnification for nuclear damages - recent developments in the United States, 1431 (K;ES)

\section{J}

Japan Atomic Energy Research Inst., Tokyo

Corrosion resistance of metal materials for HI.W canister, hKG (R;JP;In Japanese)

Development of partitioning method - cold and semihot experimental works with partitioning testing apparatus, 225 (R;JP;In Japanese)

Effects of various waste contents on properties of borosilicate glasses for high-level waste solidification in several countries, 223 (R;JP;In Japanese)

Introduction of studies on high-level radioactive waste immobilization in synthetic minerals. Supercalcine and SYNROC, 224 (R;JP;In Japanese)

Study of $\Gamma$-ray irradiation effects on corrosion resistance of alloys for storage of high-level waste packages, (1), 667 (R;JP;In Japanese)

Jysk-Fynske Elsamarbejde (ELSAM, Fredericia (Denmark)

Disposal of high-level nuclear waste from nuclear power plants in Denmark. Salt dome investigations, V.2. Geology, figures, 649 (R;DK)

Disposal of high-level waste from nuclear power plants in Denmark. Salt dome investigations. V.2. Geology, toxt, 648 (R;DK)

Disposal of high-level waste from nuclear power plants in Denmark. Salt dome investigations. V.5. Safety evaluation, 652 (R;DK)

Disposal of high-level waste from nuclear power plants in Denmark. V.3. Material testing, 650 (R;DK)

Disposal of high-level waste from nuclear power plants in Denmark. Salt dome investigations. V.4. Repository design, 651 (R;DK)

Disposal of high-level waste from nuclear power plants in Denmark. Salt dome investigations. V.1. Summary, 647 (R;DK)

Kansas State Univ, Manhattan (USA)

Nuclear-fuel-cycle education: Module 8. Waste management, 155 (K;US)

Kernforschungsanlage Juelich G.m.b.H. (Germany, F.R.). Inst. fuer Chemische Technologie der Nuklearen Entsorgung

Proceedings of the international seminar on chemistry and process engineering for high-level liquid waste solidification, 226 (R;DE;In several languages)

Proceedings of the international seminar on chemistry and process engineering for high-level liquid waste solidification, 263 (R;DE;In German and English)

Kernforschungsanlage Juelich G.m.b.H. (Germany, F.R.). Inst. fuer Nukleare Sicherheitsforschung

Investigation of the consequences of leakage incidents of a tank with high-concentrated radionuclide solutions and pentration of the radioactive solution into the soil, 1423 (R;DE;In German) 
mforschungszentrum Karlsruhe G.m.b.H. (Germany, F.R.)

Decay calculations on medium-level and actinide-containing wastes from the LWR fuel cycle. Pt. 2, 688 (R;DE;In German)

Deep underground storage of [radioactive waste]. Annual report, 1981,643 (R;DE;GE)

Kernforschungszentrum Karlsruhe G.m.b.H. (Germany, F.R.). Inst. fuer Heisse Chemie

Decay calculations on medium-level and actinide-containing wastes from the LWR fuel cycle. Pt. 1. Basic data cvaluation including activity and thermal decay power, 687 (R;DE; In German)

Kernforschungszentrum Karlsruhe G.m.b.H. (Germany, F.R.). Inst. fuer Nukleare Entsorgungstechnik

Analysis of the growth of brine flow pathes in rock salt, 690 (R;DE;In German)

Corrosion studies and mechanical tests on metallic materials for the design of packagings for vitrified high level wastes (HLW), 689 (R;DE; In German)

Corrosion studies on non-metallic inorganic materials for HLW packaging design, 691 (R;DE;In German)

Effect of composition and radiation on the Hertzian indentation behavior of nuclear waste glasses, 145 (R;US)

Preparation and characterization of an improved borosilicate glass for the solidification of high level radioactive fission product solutions (HLW). Pt. 2, 277 (R;DE)

Results report on research and development work 1980 of the Institut fuer Nukleare Entsorgungstechnik, 276 (R;DE;In German)

Kernforschungszentrum Karlsruhe G.m.b.H. (Germany, F.R.). Inst. fuer Radiochemie

Analysis of Am and a recovery for it in the pressurized water reactor fuel cycle, 1433 (R;DE; In German)

Kernforschungszentrum Karlsruhe G.m.b.H. (Germany, F.R.). Projekt Wiederaufarbeitung und Abfallbehandlung

Decay calculations on medium-level and actinide-containing wastes from the LWR fuel cycle. Pt. 1. Basic data evaluation including activity and thermal decay power, 687 (R;DE;In German)

Preparation and characterization of an improved borosilicate glass for the solidification of high level radioactive fission product solutions (HLW). Pt. 2, 277 (R;DE)

Kernforschungszentrum Karlsruhe G.m.b.H. (Germany, F.R.). Schule fuer Kerntechnik

Underground disposal of high active waste, 665 (R;DE)

Vitrification of high active waste. Vitrification techniques, 222 (R;DE)

\section{L}

Law Engineéring Testing Co., Atlanta, GA (USA)

Geological evaluation of Gulf Coast salt domes: overall assessment of the Gulf Interior Region, 784 (R;US)

Law Engineering Testing Co., Marietta, GA (USA)

Permeability of selected sediments in the vicinity of five salt domes in the Gulf Interior Region, 810 (R;US)

Lawrence Berkeley Isab., CA (USA)

Appraisal of nuclear waste isolation in the vadose zone in arid and semiarid regions (with emphasis on the Nevada Test Site), 714 (R;US)

Effect of radon transport in groundwater upon gamma-ray borehole logs, 1422 (R;US)

Instrument reliability for high-level nuclear-waste-repository applications, 1013 (R;US)

Radioactive waste disposal in granite, 712 (R;US)

Results of instrument reliability study for high-level nuclear-waste repositories, 1017 (R;US)

Selected hydrologic and geochemical issues in site characterization for nuclear waste disposal: flood basalts at the Hanford Reservation, 715 (R;US)

Status of geochemical problems relating to the burial of high-level radioactive waste, 1982, 1318 (R;US)

Thermal impact of waste emplacement and surface cooling associated with geologic disposal of nuclear waste, 707 (R;US)

Thermal-gradient migration of brine inclusions in salt, 711 (R;US)

Thermal-gradient migration of brine inclusions in salt crystals, 713 (R;US)
Thermodynamic properties of chemical species in nuclear waste, 812 (R;US)

Lawrence Livermore National Lab., CA (USA)

Borehole-to-borehole geophysical methods applied to investigations of high level waste repository sites, 1019 (R;US)

Climax Granite, Nevada Test Site, as a host for a rock mechanics test facility related to the geologic disposal of high level nuclear wastes, 1002 (R;US)

Densification studies of Synroc D for high-level defense waste, 348 (R;US)

Design of a nuclear-waste package for emplacement in tuff, 1018 (R;US)

Disposal costs for SRP high-level wastes in borosilicate glass and crystalline ceramic waste forms, 1010 (R;US)

Drilling-induced borehole-wall damage at spent fuel test-climax, 1003 (R;US)

Fluid-bed process for SYNROC production, 349 (R;US)

Formulation of SYNROC-D additives for Savannah River Plant high-level radioactive waste, 347 (R;US)

Immobilization of high-level defense wastes in SYNROC-D: recent research and development results on process scale-up, 351 (R;US)

Incorporation of high-level wastes in SYNROC: results from recent process-engineering studies at Lawrence Livermore National Laboratory, $352(\mathbf{R} ; \mathrm{US})$

Initial specifications for nuclear waste package external dimensions and materials, 1005 (R;US)

Instrument reliability for high-level nuclear-waste-repository applications, 1013 (R;US)

Instrumentation report 1: specification, design, calibration, and installation of instrumentation for an experimental, high-level, nuclear waste storage facility, 37 (R;US)

Leaching study of nuclear melt glass: Part I, 1332 (R;US)

Leaching study of PNL 76-68 glass beads using the LLNL continuous-flow method and the PNL-modified IAEA method. Final report, 344 (R;US)

Modeling of nuclear waste disposal by rock melting, 1016 (R;US)

Nuclear waste-form risk assessment for US Defense waste at Savannah River Plant. Annual report FY 1981, 1007 (R;US)

Nuclear waste-form risk assessment for US defense waste at Savannah River Plant. Annual report FY, 1982, 1008 (R;US)

Nuclear-waste disposal in geologic repositories, 1014 (R;US)

Nuclear-waste-package program for high-level isolation in Nevada tuff, 1015 (R;US)

Plutonium doping of SYNROC.D, 350 (R;US)

Postclosure risks of alternative SRP nuclear waste forms in geologic repositories, 1009 (R;US)

Properties of SYNROC. C nuclear-waste form: a state-of-the-art review, 1011 (R;US)

Results of instrument reliability study for high-level nuclear-waste repositories, 1017 (R;US)

Selection of barrier metals for a waste package in tuff, 1020 (R;US)

Sintering of Synroc D, 343 (R;US)

Status report on dissolution model development, 1021 (R;US)

Swedish nuclear waste efforts, 36 (R;US)

SYNROC powder preparation: preliminary fluid bed tests in a cold-flow unit, 345 (R;US)

SYNROC production using a fluid bed calciner, 353 (R;US)

Thermal and thermomechanical calculations of deep-rock nuclear waste disposal with the enhanced SANGRE code, 1012 (R;US)

Uncertainties and sensitivities in the performance of geologic nuclear waste isolation systems. Volume I. Summary, 809 (R;US)

Waste form/rock interaction leaching study using PNL 76-68 glass beads and Umtanum basalt. Part I, 1004 (R;US)

Leaming (George) Associates, Marana, AZ (USA)

Evaluation of water resource economics within the Pasco Basin, Washington, 1429 (R;US)

Little (Arthur D.), Inc., Cambridge, MA (USA)

Technical support of standards for high-level radioactive waste management. Addendum to volumes $C$ and $D$. Final report, 840 (R;US) 
Los Alamos National Lab., NM (USA)

Advances in measurement of alpha-contaminated wastes, 1406 (R;US)

Analysis of the alleged Kyshtym disaster, 1314 (R;US)

Aspects of possible magmatic disruption of a high-level radioactive waste repository in southern Nevada, 699 (R;US)

Effects of long-term exposure of tuffs to high-level nuclear wasterepository conditions. Preliminary report, 693 (R;US)

Elution of radionuclides through columns of crushed rock from the Nevada Test Site, 1315 (R;US)

Evaluation of research and development for terminal isolation of nuclear wastes, 701 (R;US)

Geochemistry studies pertaining to the G-tunnel radionuclide migration field experiment, 1426 (R;US)

Measurement of radioactive contaminated wastes, 278 (R;US)

Methodology for determining time-dependent mechanical properties of tuff subjected to near-field repository conditions, 697 (R;US)

Nevada Nuclear Waste Storage Investigations: exploratory shaft. Phase I. Conceptual desien report, 694 (R;IIS)

Petrologic studies of drill cores USW-G2 and UE2Sb-1H, Yucca Mountain, Nevada, 696 (R;US)

Preliminary identification of interfaces for certification and trans fer of TRU waste to WIPP. 695 (R;US)

Research and development related to the Nevada Nuclear Waste Storage Investigations. Progress report, July 1-September 30. 1981, 692 (R;US)

Research and development related to the Nevada Nuclear Waste Storage Investigations. Progress report, July 1-September 30, 1982, 703 (R;US)

Research and development related to the Nevada Nuclear Waste Storage Investigations. Progress report, October 1-December 31, 1982, 704 (R;US)

Research and development related to the Nevada Nuclear Waste Storage Investigations. Progress report, January 1-March 31, 1983, 705 (R;US)

Sentinel Gap basalt reacted in a temperature gradient, 702 (R;US)

Some geochemical considerations for a potential repository site in tuff at Yucca Mountain, Nevada, 706 (R;US)

Status of volcanic hazard studies for the Nevada Nuclear Waste Storage Investigations, 698 (R;US)

Summary report on the geochemistry of Yucca Mountain and environs, 700 (R;US)

Waste management bibliography, 1978-1983, 279 (R;US)

Louisiana State Univ., Baton Rouge (USA). Inst. for Environinental Studies

Investigation of the utility of Gulf Coast salt domes for the storage or disposal of radioactive wastes, 571 (R;US)

Quaternary geology of Rayburn's salt dome: North Louisiana Salt Dome Basin, 814 (R;US)

\section{M}

Maine Dept. of Conservation, Augusta (USA). Maine Geological Survey Consultation and concurrence for NWTS in crystalline rocks. Quarterly progress report, October 1, 1982-December 31, 1982. 576 (K;US)

Massachusetts Inst. of Tech., Cambridge (USA)

Groundwater flow and transport characteristics of flood basalts as determined from tracer experiments, 880 (R;US)

Transportation system preconceptual description for subseabed disposal of highlevel waste, 33 (R;US)

Miljustyrelsen, Copehihagen (Uenmark). Arbejdsgroppe om Radioaktivt Affald

Proposal for basic safety requirements regarding the disposal of high-level radioactive waste, 646 (R;DK)

Minnesota Univ., Minneapolis (USA)

Constant temperature and thermal gradient investigations of the near field subseabed environment: chemical and thermodiffusional effects, 971 (R;US)

Mitre Corp., Mclean, VA (USA). METREK Div.

Information base for commercial radioactive-waste management, $56(\mathrm{R} ; \mathrm{US})$
N

National Academy of Public Adninistration, Washington, DC (USA)

Building the institutional capacity for managing commercial highlevel radioactive waste, 58 (R;US)

Building the institutional capacity for managing commercial highlevel radioactive waste, 59 (R;US)

National Academy of Sciences, Washington, DC (USA)

Nuclear waste: what to do with it. Academy lorum, 565 (R;US)

Study of the isolation system for geologic disposal of radioactive wastes, 577 (R;US)

Nevada Univ, Las Vegas (USA), Desert Research Inst.

Nevada nuclear waste storage investigations site-characterization activities: preliminary atmospheric assessment of a nuclear waste repository, 765 (R;US)

New Mexico Health and Environment Dept., Santa Fe (USA). Environmental Improvement Div.

EEG review comments on the geotechnical reports provided by DOE to EEG under the stipulated agreement through March 1 , 1983, 562 (R;US)

Now Mexico Univ., Albuquerque (USA). Dept. of Chemical and Nuclear Engineering

Solutions to radionuclide migration with nonlinear retardation mechanisms in backfill material. Intcrim progress repurt, JuneDecember 1982, 1331 (R;US)

Nordlsk Kontaktorgan for Atomenergispoergsmaal, Risoe (Denmark)

Enlarged Nordic cooperative program on nuclear safety. Nordic study on reactor waste: main report, $72(\mathrm{R} ; \mathrm{SE})$.

Nortb Carolina State Univ., Raleigh (USA)

Subsolidus sintering of SYNROC: I. Initial demonstrations, 280 (R;US)

Subsolidus sintering of SYNROC: II. Materials selections, process improvements, waste form evaluations, 281 (R;US)

Nuciear Regulatory Commission, Washington, DC (USA). Office of Nuclear Material Safety and Safeguards

Draft site characterization analysis of the site characterization report for the Basalt Waste Isolation Project, Hanford, Washington Site. Main report and Appendices A through D, 724 (R;US)

Draft site characterization analysis of the site characterization report for the Basalt Waste Isolation Project, Hanford, Washington site. Appendices E through W, 725 (K;US)

Final technical position on documentation of computer codes for liigh-level waste management, 723 (K;US)

Nuclear Regulatory Commission, Washington. DC (USA). Office of State Programs

Information report on state legislation, volume 8, number 5,1414 (R;US)

NUS Corp., Gaithersburg, MD (USA)

Area environmental characterization report of the Dalhart and Palo Duro basins in the Texas Panhandle. Volume I. Dalhart Basin, 782 (R;US)

Area environmental characterization report of the Dalhart and Palo Duro basins in the Texas Panhandle. Volume II. Palo Duro basin, 783 (R;US)

NUS Corp., Rockville, MD (USA)

Environmental characterization of bedded salt formations and overlying areas of the Permian Basin, 1320 (R;US)

Oak Ridge National Lab., TN (USA)

Analysis of waste disposal practice and control at ORNL, 549 (R;US)

Brine migration in salt and its implications in the geologic disposal of nuclear waste, 829 (R;US)

Cement-based radioactive waste hosts formed under elevated temperatures and pressures (FUETAP concretes) for Savannah River Plant high-level defense waste, 293 (R;US)

Coating crystalline nuclear waste forms to improve inertness, 138 (R;US)

Data base for a sitc-specific analysis of the dispersion of radioactive elements from a final repository, 1366 (TG;US)

Economic evaluation of nuclear waste transportation casks, 14 (R;US) 
Electrical-conductivity measurements of leachates for the rapid assessment of wasteform corrosion resistance, 554 (R;US)

Evaluation of DOE radionuclide solubility data and selected retardation parameters: description of calculational and confirmatory experimental activities, 1304 (R;US)

Expected near-field thermal environments in a sequentially loaded spent-fuel or high-level waste repository in salt, 830 (R;US)

Expected very-near-field thermal environments for advanced spentfuel and defense high-level waste packages, 831 (R;US)

Glasses for the solidification of high-level radioactive waste: their behavior in the presence of water, 1052 (TG;US)

Graphical and tabular summaries of decay characteristics for oncethrough PWR, LMFBR, and FFTF fuel cycle materials, 76 (R;US)

Handling and disposing of radioactive waste, 561 (R;US)

Impacts of transportation on a test and evaluation facility for nuclear waste disposal: a systems analysis, 556 (R;US)

Incorporation of high-level nuclear waste in gel spheres, 139 (R;US)

Incorporation of simulated high-level nuclear waste in gel spheres, 292 (R;US)

Integrated data base for spent fuel and radwaste: inventories, 52 (R;US)

Preliminary analysis of the cost and risk of transporting nuclear waste to potential candidate commercial repository sites, 35 (R;US)

Proceedings of the symposium on uncertainties associated with the regulation of the geologic disposal of high-level radioactive waste, 726 (R;US)

Radwaste inventories and projections: an overview, 77 (R;US)

Regional geological assessment of the Devonian-Mississippian shale sequence of the Appalachian, Illinois, and Michigan basins relative to potential storage/disposal of radioactive wastes, 828 (R;US)

Spent fuel and radioactive waste inventories, projections, and characteristics, 574 (R;US)

Spent fuel and radioactive-waste inventories, projections, and characteristics, 57 (R;US)

Studies of waste-canister compatibility, 832 (R;US)

Technetium behavior in sulfide and ferrous iron solutions, 553 (R;US)

Uncertainties associated with geologic disposal of high-level radioactive waste, 1367 (R;US)

Uncertainties in geologic disposal of high-level wastes: groundwater transport of radionuclides and radiological consequences, 737 (R;US)

Waste treatment and disposal. Progress report, May-October 1963, 291 (R;US)

Waste treatment and disposal. Quarterly progress report, FebruaryApril, 1963, 290 (R;US)

Office of Radiation Programs, Washington, DC (USA)

Draft environmental impact statement for 40 CFR part 191: environmental standards for management and disposal of spent nuclear fuel, high-level and transuranic radioactive wastes. Final report, 838 (R;US)

Draft regulatory impact analysis for 40 CFR 191: environmental standards for management and disposal of spent nuclear fuel, high-level and transuranic radioactive wastes. Draft report, 837 (R;US)

Environmental pathway models for estimating population health effects from disposal of high-lcvel radionctive waste in geologic repositories. Draft report, 1381 (R;US)

Population risks from disposal of high-level radioactive wastes in geologic repositories. Draft report, 836 (R;US)

Potential individual doses from disposal of high-level radioactive wastes in geologic repositories. Draft report (final), 1382 (R;US)

Potential individual doses from disposal of high-level radioactive wastes in geologic repositories. Draft report, 1383 (R;US)

Office of Technology Assessmont (U.S. Congress), Washington, DC.

Managing commercial high-level radioactive waste: summary, 78 (R;US)

Ohio State Univ., Columbus (USA)

Nuclear-fuel-cycle education: Module 8. Waste management, 155 (R;US)
Pacific Northwest Lab, Richland, WA (USA)

Annotated bibliography of radioactive waste management publications at Pacific Northwest Laboratory, January 1978 through July 1982, 84 (R;US)

Assessment of single-shell tank residual-liquid issues at Hanford Site, Washington, 1384 (R;US)

Basalt-radionuclide reactions: FY-1981. Annual report, 868 (R;US)

Cesium and strontium fractionation from HLW for thermal-stress reduction in a geologic repository, 327 (R;US)

Cesium chloride compatibility testing program. Annual report, FY 1982, 855 (R;US)

Characterization and testing of basalt glass and glass ceramic waste forms, 313 (R;US)

Commercial waste treatment $R$ and $D$ needs in the United States, 323 (R;US)

Comparison of cask and dry well concepts for retrievable storage of high-level waste, 641 (R;US)

Comparison of cask and drywell storage concepts for a monitored retrievable storage/interim storage system, 853 (R;US)

Comparison of ICRP2 and ICRP30 for estimating the dose and adverse health effects from potential radionuclide releases from a geologic waste repository, 1385 (R;US)

Consolidated waste forms: glass marbles and ceramic pellets, 299 (R;US)

Corrosion experience with a slurry-fed ceramic melter, 321 (R;US)

Deposition of volatile fission products during commercial high-level waste vitrification, 304 (R;US)

Design and performance of feed-delivery systems for simulated radioactive waste slurries, 306 (R;US)

Development and testing of matrices for the encapsulation of glass and ceramic nuclear waste forms, 297 (R;US)

Economic comparison of crystalline ceramic and glass waste forms for HLW disposal, 863 (R;US)

Effect of gamma radiolysis on waste glass leaching on brines, 862 (R;US)

Engineered components for radioactive waste disposal systems - are they technically justified, 861 (R;US)

Enviromechanical testing of Ti-grade 2 and Ti-grade 12 in basalt ground water, 858 (R;US)

Evaluation of conditioned high-level waste forms, 326 (R;US)

Feasibility of incinerating and vitrifying organic resins in a single step, 318 (R;US)

Feasibility of using a high-level waste canister as an engineered barrier in disposal, 842 (R;US)

Full-scale impact tests of simulated high-level waste canisters, 316 (R;US)

General corrosion, irradiation-corrosion, and environmentalmechanical evaluation of nuclear-waste-package structural-barrier materials. Progress report, 843 (R;US)

Glass leaching performance, 856 (R;US)

High-level waste description, inventory and hazard, 632 (R;US)

High-level waste solidification system for the Western New York Nuclear Service Center, 315 (R;US)

High-temperature leaching of an actinide-bearing, simulated highlevel waste glass, 841 (R;US)

In situ vitrification: application analysis for stabilization of transuranic waste, 307 (R;US)

Initial operations in the remote laboratory-scale waste-treatment facility, 312 (R;US)

International fuel-cycle and waste-management technologyexchange activities sponsored by the United States Department of Energy: FY 1982 evaluation report, 85 (R;US)

Investigation of environmentally assisted fracture of metallic nucle ar-waste-package barrier materials in simulated basalt repository environmenis, 844 (R;US)

Materials Characterization Center. Second workshop on irradiation effects in nuclear waste forms. Summary report, 80 (R;US)

Materials Characterization Center workshop on leaching mechanisms of nuclear waste forms, May 19-21, 1982, Gaithersburg, Maryland. Summary report, 82 (R;US)

Monitored retrievable storage: a waste management option for spent fuel and high-level wastes, 864 (R;US) 
Monitoring land and water uses in the Columbia Plateau using remote-sensing computer analysis and integration techniques, 865 (R;US)

MRS/IS facility co-located with a repository: preconceptual design and life-cycle cost estimates, 857 (R;US)

New look at forecasting, 87 (R;US)

Nuclear waste management. Quarterly progress report, October through December 1980, 296 (R;US)

Nuclear waste management. Semiannual progress report, October 1982-March 1983, 303 (R;US)

Nuclear waste package materials testing report: basaltic and tuffaceous environments, 854 (R;US)

Nuclear-waste management semiannual progress report, April 1982-September 1982, 302 (R;US)

Nuclear-waste-management. Semiannual progress report, October 1981-March 1982, 301 (R;US)

Off-gas characteristics of defense waste vitrification using liquidfed Joule-heated ceramic melters, 310 (R;US)

Off-gas characteristics of liquid-fed joule-heated ceramic melters, 319 (R;US)

Operaiting characteristics of a direct liquid-fed vitrification process, 314 (R;US)

Overview of the Monitored Retrievable Storage (MRS) program, 859 (R;US)

Recovery of noble metals from fission products. 324 (R:US)

Remote design features for the West Valley slurry-fed ceramic melter system, 329 (R;US)

Report on geologic remote sensing of the Columbia Plateau, 871 (R;US)

Risk assessment for the transportation of radioactive zeolite liners, 28 (R;US)

Solubility limits of metal ions and mechanism of leaching nuclearwaste glasses, 320 (R;US)

State-of-the-art in-situ determination of transuranics, 88 (R;US)

Status, direction, and critical issues of waste treatment technology, 330 (R;US)

Summary of non-US national and international fuel cycle and radioactive waste management programs 1982, 83 (R;US)

Summary report for the interlaboratory round robin on the MCC-I static leach test method, 300 (R;US)

Systems study of the feasibility of high-level nuclear-waste fractionation for thermal-stress control in a geologic repository: main report. : $31 \mathrm{IX}(\mathrm{R} ; \mathrm{I} / \mathrm{S})$

Systems study of the feasibility of high-level nuclear waste fractionation for thermal stress control in a geologic repository: appendices, 309 (R;US)

Treatment and conditioning of wastes from US nuclear fuels reprocessing plants, 191 (R;US)

Understanding radioactive waste, 79 (R;US)

Using process instrumentation to obviate destructive examination of canisters of HLW glass, 328 (R;US)

Vitrification of highly-loaded SDS zeolites, 325 (R;US)

Vitrification of simulated West Valley waste using a liquid-fed ceramic melter, 331 (R;US)

Waste glasses: requirements and characteristics, 322 (R;US)

Waste glass/metal intcractions in brines, 860 (R;US)

Waste vitrification: a historical perspective, 317 (R;US)

Waste-management $R$ and $D$ for the nuclear fuel cycle, 311 (R;US)

Waste-Mixes Study for space disposal, 815 (R;US)

Workshop on the leaching mechanisms of nuclear-waste forms, October 27-28, 1981. Summary report, 81 (R;US)

Parsons, Brinckerhoff, Quade and Douglas, Inc., Oakland, CA (USA)

Status report on the full-scale electric heater tests at the Hanford Near-Surface Test Facility, 883 (R;US)

Parsons, Brinckerhoff, Quade and Douglas, Inc., San Francisco, CA (USA)

Status report on the full-scale electric-heater tests at the Hanford Near-Surface Test Facility, 882 (R;US)

Pennsylvania State Univ., University Park (USA). Materials Research Lab.

CsAlSi ${ }_{5} \mathrm{O}_{12}$ : a possible host for ${ }^{107} \mathrm{Cs}$ immobilization, 148 (R;US)

Reaction and devitrification of a prototype nuclear-waste-storage glass with hot magnesium-rich brine, 289 (R;US)
Pennsylrania Univ, Philadelphia (USA)

High-level radioactive wste management: a means to social conser sus, 542 (R;US)

Phillips Petroleum Co., Idabo Falls, ID (USA). Atomic Energy Div.

ICPP waste calcining facility safety analysis report, 219 (R;US)

Physikalisch-Technische Bundesanstalt, Braunschweig (Germany, F.R.)

$R$ and $D$ projects and studies: concerning the interim and terminal storage of radioactive wastes, 1281 (TG;US)

Physikalisch-Technische Bundesanstalt, Braunschweig (Germany, F.R.). Forschungs- und Messreaktor

Radiant energy dissipation during final storage of high-level radioactive waste in rock salt, 867 (R;DE;In German)

SALTMP - a FORTRAN program for fast salt temperature calculations in HRW final storage, 866 (R;DE;In German)

Power Reactor and Nuclear Fuel Development Corp., Tokai, Ibaraki (Japan). Tokai Works

Tokai Works annual progress report, April 1980-March 1981, 3 (R;JP)

Power Reactor and Nuclear Fuel Development Corp., Tokyo (Japan)

Current technics and management strategy for Pu-contaminated wastes at PNC, 295 (R;JP)

Programraadet foer Radioaktivt Avfall, Stockholm (Sweden)

Research programme on the conditioning of nuclear power waste. Progress report June 1981, 332 (R;SE)

Radievyj Inst., Leningrad (USSR)

Thermal expansion as a criterion for the quantity of high-level wastes disposed into geological formations, 921 (R;SU;In Rus,sian)

RAND Corp., Santa Monica, CA (USA)

Monitoring technologies for ocean disposal of radioactive waste, 717 (R;US)

Raymond Kaiser Engineers, Inc., Oakland, CA (USA)

Status report on the full-scale electric heater tests at the Hanford Near-Surface Test. Facility, 883 (R;US)

Status report on the full-scale electric-heater tests at the Hanford Near-Surface Test Facility, 882 (R;US)

RE/SPEC, Inc., Rapid City, SD (USA)

Fvaluation of the thermomechanical behavior about a waste container/sleeve in salt, 803 (R;US)

Expected repository environthents in granite: assessment of rock stability of the ontsiner drill holn region, 541 (R;US)

Forced ventilation analysis of a commercial high-level nuclear repository in salt, 811 (R;US)

Preliminary investigation of the thermal and structural influence of ventilation drifts on repository rooms, 786 (R;US)

Risoe National Lab., Roskilde (Denmark)

Corrosion aspects of high-level waste disposal in salt domes, 922 (R;DK)

Robbins Co., Kent, WA (USA)

Equipment for the emplacement of heat-producing waste in long horizontal boreholes, 555 (R;US)

Rockwell International Corp., Canoga Park, CA (USA). Energy Systems Group

Basalt Waste Isolation Project. Quarterly report, October 1, 1981December 31, 1981,895 (R;US)

Ceramic process and plant design for high-level nuclear waste immobilization, 144 (R;US)

Commercial high-level-waste management: options and economics. A comparative analysis of the ceramic and glass waste forms, 150 (R;US)

CsAlSis $\mathrm{O}_{12}$ : a possible host for ${ }^{107} \mathrm{Cs}$ immobilization, 148 (R;US) .

Monitoring land-and water-use dynamics in the Columbia Plateau using remote-sensing computer analysis and integration techniques, 1425 (R;US)

Preliminary assessment of key process steps for converting Savannah River Plant high-level wastes to tailored ceramics, 147 (R;US)

Process description and plant design for preparing ceramic highlevel waste forms, 149 (R;US)

Stability of ceramic waste forms in potential repository environments: a review, 564 (R;US) 
Uplift and subsidence rates in the central Columbia Plateau and their relation to siting a waste repository at Hanford, Washington, 873 (R;US)

Rockwell International Corp., Golden, CO (USA). Rocky Flats Plant

Commercial and Defense nitrate wastes and processing alternatives, 333 (R;US)

Hydrology and Geology Overview Committee reports and responses from the Basalt Waste Isolation Project, 914 (R;US)

Use of glass-metal compounds and ceramic materials to condition hot wastes, 357 (TG;US)

Rockwell International Corp., Richland, WA (USA). Energy Systems Group

Basalt Waste Isolation Project. Quarterly report, July 1, 1981September 30, 1981, 910 (R;US)

Basalt Waste Isolation Project drilling and testing. Quarterly report, April 1, 1982-June 30, 1982, 897 (R;US)

- Basalt Waste Isolation Project drilling and testing. Quarterly report, July 1, 1982-September 30, 1982, 898 (R;US)

Conceptual design of the nuclear-waste repository in basalt, 888 (R;US)

Groundwater flow and transport characleristics of flood basalts as determined from tracer experiments, 880 (R;US)

Long-Term High-Level Defense Waste technology progress report, January-March 1982, 337 (R;US)

Long-Term High-Level Defense-Waste technology. Progress report, April-June 1982, 338 (R;US)

Monitored Retrievable Storage Demonstration Facility and dry well storage field conceptual design study, 912 (R;US)

Nuclear-waste repository in basalt: seal development studies, 887 (R;US)

Numerical modeling of groundwater flow and solute transport for a nuclear-waste repository in basalt, 1327 (R;US)

Radionuclide sorption on basalt-interbed materials. Annual report FY 1981, 1328 (R;US)

Rock mass deformation properties of closely jointed basalt, 889 (R;US)

Status of tank-assessment studies for continued in-tank storage of Hanford Defense waste, 919 (R;US)

Summary of Near-Surface Test Facility results and their application to repository design, 886 (R;US)

Testing of candidate waste-package backfill and canister materials for basalt, 885 (R;US)

Waste package conceptual designs for a repository located in

- basalt, 884 (R;US)

Rockwell International Corp., Richland, WA (USA). Rockwell Hanford Operations

1981 Geodolite observations near Hanford, Washington, 870 (R;US)

Automated data acquisition and analysis system used in the Basalt Wasto Isolation Project's Near-Surface Test Facility, 906 (R;US)

Basalt Waste Isolation Project. Drilling and testing quarterly report, January 1, 1983-March 31, 1983, 900 (R;US)

Basalt waste isolation project. Quarterly report, April 1, 1981-June 30, 1981, 909 (R;US)

Basalt Waste Isolation Project: drilling and testing. Quarterly report, October 1, 1982-December 31, 1982, 899 (R;US)

Basalt waste-isolation project. Quarterly report, January 1-March 31, 1981,908 (R;US)

Basalt waste-isolation project drilling and testing. Quarterly report, January 1-March 31, 1982, 896 (R;US)

Bibliography and documents issued list through fiscal year 1982 , 892 (R;US)

Characterization of reference materials for the Barrier Materials Test Program, 905 (R;US)

Conceptual design requirements for spent fuel, high-level waste, and transuranic waste packages, 903 (R;US)

Determination of rock mass deformation modulus in closely jointed Columbia River basalt, 881 (R;US)

Effects of Hanford high-level waste components on sorption of cobalt, strontium, neptunium, plutonium, and americium on Hanford sediments, $918(\mathrm{R} ; \mathrm{US})$

Engineered waste package concepts for high-level waste isolation in basalt, 874 (R;US)

Evaluation of sodium bentonite and crushed basalt as waste package backfill materials, $90^{\prime 2}$ (K;US)
Experimental investigation of sodium bentonite stability in Hanford basalt, 879 (R;US)

Exploratory Shaft Test Facility in basalt. Project B-314 preconceptual design report, 913 (R;US)

Field guidebook association of engineering geologists. Chapters $1-10,915$ (R;US)

Geochemical behavior of supercalcine waste form: its stability in a basalt environment, 911 (R;US)

Geohydrologic characterization and qualification of a high-level waste site in basalts, 891 (R;US)

Identifiction of a preferred site for the exploratory shaft within the reference repository location: Hanford Site, 916 (R;US)

Native copper deposits of the Portage Lake volcanics, Michigan: their implications with respect to canister stability for nuclear. waste isolation in the Columbia River basalts beneath the Hanford Site, Washington, 904 (R;US)

Overview of waste-handling requirements for a nuclear-waste repository in basalt, 877 (R;US)

Ozone mass transfer and kinetics experiments, 334 (R;US)

Performance allocation traceable to regulatory criteria as applied to site-characterization work at the Basalt Waste Isolation Project, 890 (R;US)

Preliminary interpretation of the tectonic stability of the reference repository location, Cold Creek syncline, Hanford Site, 901 (R;US)

Radionuclide solubility and transport in basalt, 875 (R;US)

Rockwell Hanford Operations. Defense waste and byproducts management monthly report, 335 (R;US)

Rockwell Hanford Operations defense waste and byproducts management monthly report, 336 (R;US)

Rockwell Hanford Operations monthly report summary, June 1983 , 9 (R;US)

Scientific basis for establishing dry-well-monitoring frequencies, 920 (R;US)

Site characterization report for the basalt waste isolation project. Volume I, 601 (R;US)

Site characterization report for the basalt waste isolation project. Volume II, 602 (R;US)

Site characterization report for the basalt waste isolation project. Volume III, 603 (R;US)

Site-screening process on the Hanford Site: a summary report, 878 (R;US)

Status of geologic and hydrologic characterization of a potential nuclear waste repository site in basalt, 894 (R;US)

Status report on the full-scale electric heater tests at the Hanford Near-Surface Test Facility, 883 (R;US)

Status report on the full-scale electric-heater tests at the Hanford Near-Surface Test Facility, 882 (R;US)

Technical conservatism in the design and analysis of a nuclearwaste repusitury in basalt, 907 (R;US)

Testimony of Dr. Raul A. Deju, Basalt Waste Isolation Project, before the Subcommittee on Energy Research and Production, Committee on Sceince and Technology, United States House of Representatives, March 2, 1983, 893 (R;US)

Thermomechanical simulation of a nuclear-waste canister in a repository in basalt, 876 (R;US)

Waste package heat-transfer analysis: model development and temperature estimates for waste packages in a repository located in basalt, 917 (R;US)

Rockwell International Corp., Thousand, Oaks, CA (USA). Science Center

Development of polyphase ceramics for the immobilization of highlevel Defense nuclear waste, 151 (R;US)

\section{$S$}

Sandia National Labs., Albuquerque, NM (USA)

1982 Subseabed Disposal Program. Annual report, site assessment Octuber 1981 to September 1982, 987 (R;UIS)

Alternate strategy for commercial high-level radioactive-waste management, 945 (R;US)

Analysis of waste-package behavior for high-level waste, 981 (R;US) 
Application of a risk-assessment methodology to a hypothetical high-level waste repository in bedded salt, 937 (R;US)

Approach to resolution of geologic uncertainty in the licensing of a high-lcvcl-waste repository in tuff, 985 (R;US)

Assessment of technical criteria of 10CFR60 for geologic disposal of high-level waste, 952 (R;US)

Assessment of the proposed rule (10CFR60) for disposal of highlevel radioactive wastes in geologic repositories. Volume I, 747 (R;US)

Basic data report for deepening of drillhole WIPP 13 (Waste Isolation Pilot Plant-WIPP), 975 (R;US)

Basic data report for Drillhole AEC 7 (Waste Isolation Pilot Plant - WIPP), 924 (R;US)

Basic data report for drillhole AEC 8 (Waste Isolation Pilot Plant WIPP), 925 (R;US)

Basic data report for drillhole ERDA 9 (Waste Isolation Pilot

Plant WIPP), 926 (R;US)

Basic data report for Drillhole ERDA 10 (Waste Isolation Pilot

Plant - WIPP), 927 (R;US)

Basic data teport for drillhole ERDA 6 (Waste Isolation Pilot

Plant - WIPP), 923 (R;US)

Basic data report for Drillhole WIPP 14 (Waste Isolation Pilot

Plant - WIPP), 974 (R;US)

Basic data report for drillhole WIPP 12 (Waste Isolation Pilot

Plant-WIPP), 984 (R;US)

Basic data report for drillhole WIPP 11 (Waste Isolation Pilot

Plant - WIPP), 928 (R;US)

Code development in support of nuclear waste storage investigations for a repository in tuff, 988 (R;US)

Comparison of brines relevant to nuclear waste experimentation, 994 (R;US)

Comparison of ICRP2 and ICRP30 for estimating the dose and adverse health effects from potential radionuclide releases from a geologic waste repository, 1385 (R;US)

Comparison of risks due to HLW and SURF repositories in bedded salt, 1368 (R;US)

Comparison of waste emplacement configurations for a nuclear waste repository in tuff. II. Ventilation analysis, 995 (R;US)

Comparison of waste emplacement configurations for a nuclear waste respositury in tuff. IV. Thermo-hydrological analysis, 996 (R;US)

Compliance assessments of hypothetical geological nuclear waste isolation systems with the draft EPA standard, 982 (R;US)

Conceptual design of field experiments for welded-tuff rockmechanics program, 936 (R;US)

Conclusions regarding geotechnical acceptability of the WIPP site, 990 (R;US)

Constant temperalure and thermal gradient investigations of the ncar field subseabed environment: chemical and thermodiffusional effects, 971 (R;US)

Design considerations for occupational exposure for a potential repository at Yucca Mountain: high-level-waste handling operations, 991 (R;US)

Economic evaluation of nuclear waste transportation casks, 14 (R;US)

Effect of a random variation of rock salt creep on calculations of storage room closure for the WIPP project, 943 (R;US)

Effects of composition, porosity, bedding-plane orientation, water content and a joint on the thermal conductivity of tuff, 970 (R;US)

Effects of wire rope in mitigating a waste-shaft accident, 980 (R;US)

Eyuipment for the emplacement of heat-producing waste in long horizontal boreholes, SSS (R;US)

Experimental and analytical program to evaluate packaging and target hardness as related to nuclear waste transportation systems, 32 (R;US)

Far-field thermal analysis of a high level waste repository in tuff. Topical report RSI-0137, 942 (R;US)

Forced ventilation analysis of a commercial high-level nuclear waste repository in tuff. Topical report RSI-0175, 941 (R;US)

Formulation of an integrated physicochemical-hydrologic model for predicting waste-nuclide retardation in geologic media, 973 (R;US)

Hydrology of sealing a repository in saturated tuff, 992 (R;US)
Isolation of radioactive wastes in salt repositories, 951 (R;US)

Issues related to field testing in tuff, 947 (R;US)

Materials for high-level waste canister/overpacks in salt formations, 949 (R;US)

Methodology for assessing the risk from the disposal of high-level radioactive wastes in deep geologic formations, 993 (R;US)

Natural convection experiments in a liquid-saturated porous medium bounded by vertical coaxial cylinders, 968 (R;US)

Near-field interactions, 929 (R;US)

Near-surface leaching studies of $\mathbf{P b}$-implanted Savannah River waste glass, 140 (R;US)

NNWSI regulatory status and plans, 978 (R;US)

Nuclear-waste disposal: technical issues and status, 979 (R;US)

NWTS transportation interface technology development priority report, 89 (R;US)

Parametric studies of radiological consequences of basaltic volcanism, 1386 (R;US)

PATH1 self-teaching curriculum: example problems for Pathwaysto-Man Model, 1317 (R;US)

Preliminary analysis of the cost and risk of transporting nuclear waste to potcntial candidate commercial repository sites, 35 (R;US)

Preliminary scenarios for consequence assessments of radioactivewaste repositories at the Nevada Test Site, 948 (R;US)

Preliminary technical constraints for a repository in tuff, 977 (R;US)

Preliminary thermal analyses for a nuclear waste repository in tuff, 931 (R;US)

Proceedings of the 1981 subseabed disposal program. Annual workshop, 939 (R;US)

Processing, microstructure, leaching, and long-term stability studies related to titanate high-level waste forms, 340 (R;US)

Program criteria for subseabed disposal of radioactive waste: site qualification plan, 934 (R;US)

Radiation-damage effects in nuclear-waste glasses, 339 (R;US)

Report of the first interim meeting of the Seabed Working Group Engineering Studies Task Group, 944 (R;US)

Review of the status of transportation activities in various waste management systems, 30 ( $R ; U S$ )

Risk methodology for geologic disposal of radioactive waste: model description and user manual for Pathways model, 1316 (R;US)

Risk-analysis methodology for spent-fuel repositories in bedded salt. Final report, 731 (R;IIS)

Sealing concepts for the Waste Isolation Pilot Plant (WIPP) site, 938 (R;US)

Stresses near waste canisters buried in salt, 950 (R;US)

Subseabed disposal program. Annual report, January-December 1980. Volume 1. Summary, 935 (R;US)

Subseabed Disposal Program. Annual report, January-September 1981. Volume I: summary, 953 (R;US)

Subseabed disposal program annual report, January-Septembor 1981. Volume II. Appendices (principal investigator progress repnrts). Part 1, 954 (R;US)

Subseabed disposal program annual report, January-September 1981. Volume II. Appendices (principal investigator progress reports). Part 2, 962 (R;US)

Summary of research and development for disposal of US Defense wastes in the Waste Isolation Pilot Plant (WIPP), 983 (R;US)

Systems overview of the Subseabed Disposal Program, 976 (R;US)

Technical assistance for regulatory development: review and evaluation of the EPA standard 40 CFR.191 for disposal of high-level waste. Vol. 1, 750 (R;US)

Technical assistance for regulatory development: review and evaluation of the draft EPA standard 40CFR 191 for disposal of highlevel waste. Volumes 5 and 6,1377 (R;US)

Technical assistance for regulatory development: review and evaluation of the draft EPA Standard 40CFR 191 for disposal of highlevel waste, 1415 (R;US)

Thermal analyses for a nuclear-waste repository in tuff using USW-G1 borehole data, 946 (R;US)

Thermal analysis of nuclear waste emplacement in welded tuff, $93 \mathrm{v}$ (R;US)

Thermal conductivity of bentonite/quartz high-level-waste-package backfill, 972 (R;US) 
Thermal response to emplacement of nuclear waste in long, horizontal boreholes, $986(\mathrm{R} ; \mathrm{US})$

Thermo-mechanical scoping calculations for a high level nuclear waste repository in tuff, 933 (R;US)

Three-dimensional thermal analysis for a conceptual high-level waste repository in welded tuff, 932 (R;US)

Transient creep model for salt during stress loading and unloading, 969 (R;US)

Transportation packagings for high-level wastes and unprocessed transuranic wastes, 29 (R;US)

Transportation system preconceptual description for subseabed disposal of highlevel waste, 33 (R;US)

Waste Isolation Pilot Plant (WIPP) research and development program: in situ testing plan, March 1982, 940 (R;US)

WIPP research and development program: providing the technical basis for defense waste disposal, 989 (R;US)

Science Applications, Inc., Albuquerque, NM (USA)

Analysis of waste-package behavior for high-level waste, 981 (R;US)

Comparison of risks due to HLW and SURF repositories in bedded salt, 1368 (R;US)

Science Applications, Inc., La Jolla, CA (USA)

Waste package performance evaluation, 806 (R;US)

Science Applications, Inc., Palo Alto, CA (USA)

Risk-assessment methodology development for waste isolation in geologic media, 727 (R;US)

Select Committee on Ontario Hydro Affairs, Toronto, Ontario (Canada)

Management of nuclear fuel waste. Final report, 71 (R;CA)

Southwest Engineering Associates, El Paso, TX (USA)

Experimental and analytical program to evaluate packaging and target hardness as related to nuclear waste transportation systems, 32 (R;US)

Svensk Kaernbraenslefoersoerjning AB, Stockholm

Chemical interaction of clay buffer materials and concrete, 997 (R;SE)

Effect of $\mathrm{Fe}_{2} \mathrm{O}_{0} / \mathrm{ZnO}$ on two glass compositions for solidification on Swedish nuclear wastes, 341 (R;SE)

KBS annual report 1981. Including summaries of technical reports issued during 1981, 998 (R;SE)

Radiolysis of groundwater from HLW stored in copper canisters, 999 (R;SE)

Stripa project, quarterly report October through December 1981 , 655 (R;SE)

\section{T}

Technische Hochschule Aachen (Germany, F.R.). Fakultaet fuer Maschinenwesen

Numerical calculation of three-dimensional long-time temperature fields using a special finite element method: the final storage of highly radioactive waste in salt rocks, 662 (R;DE;In German)

Rail transport of vitrified high-level radioactive fission products risk analysis of a model, 25 (R;DE;In German)

Technische Hogeschool Delft (Netherlands)

Disposal of high-level nuclear waste in rock-salt. A comparison of temperature increases calculated at ECN (Energieonderzoek Centrum Nederland)-Petten, RWTH (Rheinisch-Westfaelische Technische Hochschule Aachen)-Aachen and THD (Delft University of Technology)-Delft, 835 (R;NL)

Impurities in rock-salt: consequences for the temperature increases at the disposal of high-level nuclear waste, 834 (R;US)

Technische Univ. Berlin (Germany, F.R.). Inst. fuer Luft- und Raumfahrt

Appraisal of a target-relevant evaluation of alternative strategies for global radioactive waste disposal, 645 (R;DE; In German)

Teknekron Research, Inc., Bethesda, MD (USA)

Parameters and variables appearing in radiological assessment codes. Final report, 1375 (R;US)

Summary of computer codes for radiological assessment, 1376 (R;US)

Summary of repository siting models. Final report, 740 (R;US)

Texas Energy and Natural Resources Advisory Council, Austin (USA) High-level radioactive-waste-disposal investigations in Texas, 1001 (R;US)
Nuclear energy in Texas: major issues and policy recommendations, 90 (R;US)

Report of the Low-Level Nuclear Waste Subcommittee, 1000 (R;US)

Texas Univ, Austin (USA). Bureau of Economic Geology

Statistical analysis of lineaments and their relation to fracturing, faulting, and halokinesis in the East Texas Basin. Report of investigations No: 110, 566 (R;US)

\section{$\boldsymbol{U}$}

UKAEA Atomic Energy Research Establishment, Harwell

Nuclear-fuel-cycle education: Module 8. Waste management, 155 (R;US)

UKAEA Atomic Energy Research Establishment, Harwell. Research Reactors Div.

Surface storage of vitrified high-level radioactive waste in reinforced-concrete casks, 534 (R;GB)

UKAEA Atomic Energy Research Establishment, Harwell. Theoretical Physics Div.

Coupled heat and groundwater flow in porous rock, 535 (R;GB)

UKAEA Risley Nuclear Power Development Establishment

Denitration of simulated fast reactor highly active liquor waste, $282(R ; G B)$

USAEC Division of Reactor Development, Washington, DC

Report of working meeting on fixation of radioactivity in stable, solid media at the Johns Hopkins University, June 19-21, 1957, 342 (R;US)

USDOE Albuquerque Operations Ofince, NM. Waste Isolation Pilot Plant Project Office

Waste Isolation Pilot Plant. Project progress report, December 1, 1982-February 28, 1983, 1031 (R;US)

USDOE Assistant Secretary for Defense Programs, Washington, DC

Defense waste management plan, 563 (R;US)

USDOE Assistant Secretary for Defense Programs, Washington, DC. Defense Waste and Byproducts Management Div.

Department of Energy plan for recovery and utilization of nuclear byproducts from defense wastes. Volume 1. Executive summary, 2 (R;US)

USDOE Assistant Secretary for Nuclear Energy, Washington, DC

Program summary. Nuclear waste management and fuel cycle programs, 575 (R;US)

USDOE Assistant Secretary for Nuclear Energy, Washington, DC. Office of Terminal Waste Disposal and Remedial Action

Long-term management of liquid high-level radioactive wastes stored at the Western New York Nuclear Service Center, West Valley. Final environmental impact statement, 1305 (R;US)

USDOE Nevada Operations Office, Las Vegas

Bibliography of the published reports, papers, and articles on the Nevada Nuclear Waste Storage Investigations, 754 (R;US)

Bibliography of the published reports, papers, and articles on the Nevada nuclear waste storage investigations, 755 (R;US)

Nevada nuclear. waste storage investigations. Quarterly report, July-September 1982, 758 (R;US)

Nevada Nuclear Waste Storage Investigations. Quarterly report, October-December 1982, 759 (R;US)

Nevada Nuclear Waste Storage Investigations. Quarterly report, January-March, 1983, 760 (R;US)

Nevada Nuclear Waste Storage Investigations: FY 1981 project plan and FY 1982 forecast, 761 (R;US)

Nevada Nuclear Waste Storage Investigations: FY 1982 project plan, $\overline{7} 6 \dot{2}$ (R;US)

Project management plan for exploratory shaft at Yucca Mountain, 764 (R;US)

Public hearings panel report: a summary of public concerns regarding the characterization of a repository site in Nevada, 766 (R;US)

USDOE Nuclear Waste Policy Act Project Office, Washington, DC

Monitored Retrievable Storage proposal research and development research and develupmiemt report, 605 (R;US)

Report on financing the disposal of commercial spent nuclear fuel and processed high-level radioactive waste, 604 (R;US)

Report on financing the disposal of commercial spent nuclear fuel and processed high-level radioactive waste, 21 (R;US) 
USDOE Ofice of Energy Research, Washington, DC. Energy Research Advisory Board

Research and development needs in the Department of Energy. Interim report, $573(\mathbf{R} ; \mathrm{US})$

USDOE Savannah River Operations Office, Aiken, SC

Strategy document: long-term technology program for defense high-level waste, 156 (R;US)

USDOE, Washington, DC

Method for product performance evaluation of candidate waste forms for immobilization of high-level radioactive wastes. Interface working group on high-level waste form selection factors, 161 (R;US)

Ustav Jaderneho Vyzkumu CSKAE, Rez (Czechoslovakia)

Phosphate system as medium for radioactive waste fixation. Simplex experiment. Part 1, 354 (R;CS;In Czech)

Phosphate system as medium for radioactive waste fixation - simplex experiment. Part II, 355 (R;CS;In Czech)

\section{W}

Waterloo Unir, Ontario (Canada). Dept. of Earth Sciences

Diffusive transport of strontium- $\mathbf{8} 5$ in sand-bentonite mixtures, $1300(\mathrm{R} ; \mathrm{CA})$

Westingbouse Electric Corp., Mercury, NV (USA). Advanced Energy Systems Dir.

Commercial nuclear waste research and development program. Annual report, fiscal year 1982, 579 (R;US)

Commercial Nuclear Waste Research and Development Program. Quarterly report,.October-December 1982, 580 (R;US)

Commercial nuclear waste research and development program. Quarterly report, October-December 1981, 60 (R;US)

Commercial nuclear waste research and development program. Quarterly report, January-March 1982, 61 (R;US)

Commercial nuclear-waste research and development program. Quarterly report, July-September 1982, 578 (R;US)

Westinghouse Electric Corp, Mercury, NV (USA). Waste Technology Services Div.

Commercial nuclear waste research and development program. Quarterly report, January-March 1983, 581 (R;US)

Commercial nuclear waste research and development program. Quarterly report, April-June 1983, 582 (R;US)

Westinghouse Electric Corp., Pittsburgh, PA (USA)

TRU waste-acceptance criteria for the Waste Isolation Plant, 1419 (R;US)

Westinghouse Electric Corp., Pittsburgh, PA (USA), Advanced Energy Systems Dir.

Conceptual waste-package designs for disposal of nuclear waste in tuff, 818 (R;US)

Design of a nuclear-waste package for emplacement in tuff, 1018 (R;US)

Engineered waste-package conceptual design: defense high-level waste (Form 1), commercial high-level waste (Form 1), and spent fuel (Form 2) disposal in salt, 817 (R;US)

Engineered waste-package-system design specification, 816 (R;US)

Remote automatic plasma arc-closure welding of a dry-storage canister for spent nuclear fuel and high-level radioactive waste, 551 (R;US)

Waste package conceptual designs for a nuclear repository in basalt, 872 (R;US)

Wisconsin State Government, Madison (USA)

State of Wisconsin Ad Hoc committee on radioactive waste management. Final report, 568 (R;IIS)

Woodward-Clyde Consultants, San Francisco, CA (USA)

Assessment of the effects of surficial geologic processes in the Pasao Basin, 869 (R;US)

In situ and laboratory geotechnical test results from borehole GD-1 in southeast Utah, 813 (R;US) 


\section{PERSONAL AUTHOR INDEX}

Authors' surnames are indexed in the form appearing in the abstracted publication; given names are reduced to initials. Each author entry gives the publication title and the citation number. The document type e.g., R for Report; country of publication, e.g., US; and language of the document, e.g. In German; are given in parentheses at the end of the entry. If no language statement appears, the language is English. Tables of document types and country of publication codes appear after the indexes. For publications with multiple authors, an entry is pro vided for each author. Entries for the second and succeeding authors give references to the first author.

Because of problems involved in the mechanical preparation of this index, all accent marks are omitted. Changes in spelling introduced as a result of accent omission follow standard conventions.

\section{A}

Abe, T., Method of treating high level radioactive solid waste, 389 (P;JP;In Japanese)

Abelin, $\mathbf{H}$., Migration in a single fracture, 1342 (BA;US)

Ackerman, F.J., Fluid-bed process for SYNROC production, 349 (R;US)

SYNROC production using a fluid bed calciner, 353 (R;US)

See Campbell, J.H., 352

See Peters, P.E., 345

Aden, G.D., See Palmer, R.A., 905

See Wood, M.I., 586, 885, 902

Adl, T., $\mathrm{CsAlSi} 5 \mathrm{O}_{12}$ : a possible host for ${ }^{107} \mathrm{Cs}$ immobilization, 148 (R;US)

$\mathrm{CsAlSi} \mathrm{O}_{12}$ : a possible host for ${ }^{107} \mathrm{Cs}$ immobilization, 404 (J;GB)

Aggson, J.R., See St. Juhı, C.M., 738

Aguilar, R.D., See Norris, A.E., 1426

Ahn, T.M., Container assessment: corrosion study of HLW container materials. Quarterly progress report, JulySeptember 1981, 728 (R;US)

Container assessment - corrosion study of HLW container materials. Quarterly progress report, April-June 1981, 729 (R;US)

Container assessment: corrosion study of HLW container materials. Quarterly progress report, January-March 1982, 730 (R;US)

Corrosion of TiCode-12 in a simulated Waste Isolation Pilot Project (WIPP) brine, $1066(\mathrm{~J} ; \mathrm{US})$

Identification of crevice corrosion in the titanium alloy TiCode- 12 in simulated rock salt brine at $150^{\circ} \mathrm{C}, 545$ (R;US)

Nuelear waste management technical support in the development of nuclear waste form criteria for the NRC. Task 4. Test development review, 284 (R;IIS)
See Dayal, R., 283

Ahner, S., Storage of HLW in engineered structures: air-cooled and water-cooled concepts. Final report, 640 (R;XE)

Storage of HLW in engineered structures: air-cooled and water-cooled concepts. Final report, 833 (R;US)

Akers, S.A., Constitutive properties for undisturbed marine sediments in support of the subseabed disposal program, 963 (RA;US)

Albenesius, E.L., See Roberson, W.A., 193

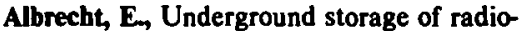
active wastes in salt deposits in the Federal Republic of Germany, 668 (RA;DE;In German)

Alexander, D.H., See Birchard, G.F., 1061

Alger, R.P., See Slaughter, G.M., 810

Allard, B., Actinide sorption on rock minerals, 275 (RA;DE)

Actinide sorption on rock minerals, 677 (RA;DE)

Sorption of actinides in well-defined oxidation states on geologic media, 1351 (BA;US)

See Landstroem, O., 1349

See Torstenfelt, B., 1148, 1175, 1334

Allemann, R.T., Solidification of high-level radioactive fuel reprocessing wastes by spray and pot calcination. Hot-cell pilot plant studies, 217 (R;US)

Allen, C.C., Stability and alteration of naturally occurring low-silica glasses: implications for the long term stability of waste form glasses, 1190 (BA;US)

Allen, G.C., See Sutherland, S.H., 32

Allen, G.C. Jr., Review of the status of transportation activities in various waste management systems, 30 (R;US)

Allen, P.L., Developing technology of microwave vitrification, 392 (J;GB)

Allen, T.L., Startup of a Joule-heated glass melter with a graphite slurry, 188 (R;US)

Allender, J.S., See Bernadzikowski, T.A., 185,627

See Butler, J.L., 170

See Stone, J.A., 171

Aloi, A.S., See Lazarev, L.N., 357

Altenhein, F.K., Long-term radioactivity release from solidified high-level waste. Part I. An approach to evaluating experimental data, 1191 (BA;US)

Long-term radioactivity release from solidified high-level waste. Part II. Parametric study of waste from properties, temperature and time, 1333 (J;US)

See Beckmann, H., 1373

Altenhofen, M.K., Waste package heattransfer analysis: model development and tempcrature estimates for waste packages in a repository located in basalt, 917 (R;US)

See Jacobs, G.K., 1223
Ames, L.L., Basalt-radionuclide reactions: FY-1981. Annual report, 868 (R;US)

Amirijafari, B., See Stevens, C.A., 727

Amos, R., See Crowe, B., 699

Andersen, R., See Blacic, J., 693

See Blacic, J.D., 697

Anderson, A, See Young, K.D., 555

Andersom, D.R., Submarine geologic disposal of nuclear waste, 1253 (J;US)

See Hinga, K.R., 1129

See Hollister, C.D., 1243

See Laine, E.P., 934

Anderson, H.M., Solutions to radionuclide migration with nonlinear retardation mechanisms in backfill material. Interim progress report, JuneDecember 1982, 1331 (R;US)

Anderson, R.T., Cooldown testing of nuclear fuel casks, 1442 (J;NL)

Anderson, R.Y., Deformation-dissolution potential of bedded salt, Waste Isolation Pilot Plant site, Delaware Basin, New Mexico, 1163 (BA;US)

Anderson, W.J., Conceptual design requirements for spent fuel, high-level waste, and transuranic waste packages, 903 (R;US)

Nuclear-waste repository in basalt: seal development studies, 887 (R;US)

See Wood, M.I., 586, 885

Andersson, G., Model for near field migration, 1343 (BA;US)

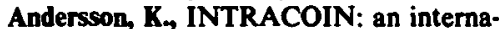
tional nuclide transport code intercomparison study, 1358 (BA;US)

See Allard, B., 1351, 275, 677

See Landstroem, O., 1349

See Torstenfelt, B., 1148, 1175, 1334

Andrews, R.W., See Brandstetter, A., 1034 -

See Sykes, J.F., 1221

Andrews, W.B., Defense waste transportation: cost and logistics studies, 27 (R;US)

Angelini, P., See Lackey, W.J., 245

See Robinson, S.M., 139

See Stinton, D.P., 138, 386, 387

Antonini, M., Point defects and microstructural stability of glasses under irradiation, 1154 (BA;US)

See Lanza, F., 261

See Manara, A., 464

Anzai, T., See Shinohara, T., 488

Apel, U., See Koelle, H.H., 645

Apps, J.A, Geochemistry research planning for underground repositories, 1112 (J;US)

Iron-nickel alloys as canister material for radioactive waste disposal in underground repositories, 708 (RA;US)

Sorptive diffusion in clay gels, 709 (RA;US)

Status of geochemical problems relating to the burial of high-level radioactive waste, $1982,1318^{\circ}$ (R;US)

See Hurrington, R.V., 411

Apted, M.J., Overview of hydrothermal testing of waste package barrier mate- 
rials at the Basalt Waste Isolation Project, 848 (RA;US)

See Holloway, J.R., 911

Araki, K., See Furuya, T., 667

See Murakami, T., 224

Arbital, J.G., See Rickertsen, L.D., 830

Armantrout, G.A., See Rogue, F., 1013

Amett, R.C., See Clifton, P.M., 1327

Amey, B., See Caporuscio, F., 696

Amold, G.W., Near-surface leaching studies of Pb-implanted Savannah River waste glass, 140 (R;US)

Radiation-damage effects in nuclearwaste glasses, 339 (R;US)

Arnold, W.D., Incorporation of simulated high-level nuclear waste in gel spheres, 292 (R;US)

Preparation of gel spheres containing simulated high-level radioactive waste, $381(\mathrm{~J} ; \mathrm{CH})$

Spe Rohinson, S.M , 139

Arondi, W.D., See Lackey, W.J., 245

Arsenault, F.J., NRC regulations for highlevel nuclear waste management, 1092 (J:US)

See. Cnmella, P.A., 1089

Asano, T., See Kitahara, Y., 1450

Aston, S.R., See Baxter, M.S., 1077

Avogadro, A., Relationship between glass leaching mechanism and geochemical transport of radionuclides, 1195 (BA;US)

Underground migration of long-lived radionuclides leached from a borosilicate glass matrix, 1360 (BA;XA)

\section{B}

Babad, H., See Deju, R.A., 890

Baca, R.G., See Clifton, P.M., 1327

Baer, W., Projected occupational exposures during WIPP operations, 1041 (J;US)

Baetsle, L.H., Influence of the reprocessing flow sheet on the HLW solidification technulugy, 239 (RA;DE)

Bahr, W.B., See Kuhn, K.D., 429

Baker, S.M., Satisfying performance assessment data needs, 1222 (J;US)

Balmert, M.E., Development of waste package designs for disposal in a salt repository, 557 (RA;US)

Balzer, R., See Koelle, H.H., 645

Banba, T., See Furuya, T., 223

Bancroft, G.M., See Metson, J.B., 456

Bandyopadhyay, G., Materials studies of simulated interim waste forms for high-level radiactive wastes, 502 (J;US)

Method of preparing nuclear wastes for transportation and interim storage, 391 (P;US)

Barbreau, A., Long-term prediction of the fate of nuclear waste deeply buried in granite. 1186 (BA;GB)

Bard, C.S., See Kocher, D.C., 1367, 737

Barkatt, A., Currelation between dynamic leach test results and geochemical observations, 1059 (J;US)

See Barkatt, A., 1059

See Macebo, P.B., 1192

Barke, G., See Behrens, H., 1352

Barker, J.A., See Black, J.H., 1147

Barnard, W., See Ghovanlou, A.H., 1133

Barnes, M.W., See Roy, D.M., 1156
Barnes, S.M., Corrosion experience with a slurry-fed ceramic melter, 321 (R;US)

Barney, G.S., Radionuclide sorption on basalt-interbed materials. Annual report FY 1981, 1328 (R;US)

See Delegard, C.H., 918

Barr, G.E., See Hunter, R.L., 948

Barthoux, A., Underground storage of radioactive wastes, 1280 (BA;FR;In French)

Barton, C., Paleomagnetic study of areas B1, C1 and E2, 959 (RA;US)

See Laine, E., 958

Barton, G.B., Solidification of high level wastes. Part IV. Phosphate melts for fixation of radioactive residues from Purex type wastes: three to fifty percent fission product oxides, 134 (R;US)

Basham, S.J., Waste package conceptual designs for salt repositories, 593 (RA;US)

Basham, S.J. Jr, Waste packages for highlevel waste repositories, 1043 (J;US)

Basin, S.I., See Stevens, C.A., 727

Batchelor, A.D, See Palmour, H. III., 281

Bates, J.K., Application of neutron. activation analysis to the determination of leach rates of simulated nuclearwaste forms, 127 (R;US)

Measurement of leaching from simulated nuclear-waste glass using radiotracers, 128 (R;US)

See Steindler, M.J., 50, 536, 538, 539

Batist, R. de, See Iseghem, P. van, 274

Baver, C., Fixation of high level solid waste (HLSW) in a $\mathrm{TiO}_{2}$ base ceramic matrix provided with an integrated overpack, 367 (J;US)

Bauhof, F, See Gates, R., 742

See Roberds, W., 743, 744

Baxter, B.J., Combined storage for LWR spent fuel and high-level waste, 16 (RA;US)

Baxter, J.T., Status report on the full-scale electric-heater tests at the Hanford Near-Surface Test Facility, $\mathbf{8 8 2}$ (R;US)

Status report on the full-scale electric heater tests at the Hanford NearSurface Test Facility, 883 (R;US)

Baxter, M.S., Disposal of high-activity nuclear wastes in the oceans, 1050 (J;GB)

Indine-129 in high-activity nuclear wastes: an assessment of the deepocean disposal option, $1077(\mathrm{~J} ; \mathrm{CH})$

Baxter, R.G., Description of Defensc Waste Processing Facility reference waste furiul and canister. Revision 1, 168 (R;US)

Bayhurst, B.P., See Norris, A.E., 1426

Bayhurst, G.K., See Charles, R.W., 702

Bazan, F. Waste form/rock interaction leaching study using PNL 76-68 glass beads and Umtanum basalt. Part I, 1004 (R;US)

See Ackerman, F.J., 349, 353

See Karim, D.P., 417

Beale, H., Storage of high-level radioactive waste, 1079 (J;GB)

Surface storage of vitrified high-level radioactive waste in reinforcedconcrete casks, 534 (R;GB)

Beall, G.W., See Kelly, K.L., 1276

Beary, M.M., See Bollyky, L.J., 334
Bebbington, W.P., Contamination of the hot gang valve corridor and first-level clean areas of Building 221-F - September 13, 1960, 194 (R;US)

Bechthold, W, See Koester, R., 1083

Becker, A., See Weber, M., 1404

Becker, G.W., See Roberson, W.A., 193

Becker, R., See Stieglitz, L., 11

Beckman, R.J., See Crowe, B.M., 1393

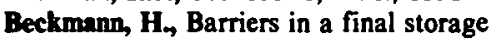
for radioactive wastes, 1373 (RA;DE;In German)

Begej, C.D., See Wukelic, G.E., 1425

Behrens, H., Laboratory tests on the migration behavior of selected fission products in aquifer materials from a potential disposal site in northern Germany, 1352 (BA;US)

Bell. E.J. Overview of energy and mineral resources for the Nevada nucleartialrmtorage inverigatiuns, Nevada Test Site, Nye County, Nevada, 763 (R;US)

Bench, F.D., See Smith. R.R., 452

Benjamin, R.W., Alternatives for definse waste-salt disposal, 629 (R;US)

Benjamin, T.M., See Curtis, D.B., 611

Benny, R.I., Report on financing the disposal of commercial spent nuclear fuel and processed high-level radioactive waste, 21 (R;US)

Report on financing the disposal of commercial spent nuclear fuel and processed high-level radioactive waste, 604 (R;US)

Bensky, M.S., See Deju, R.A., 890

Berger, D.N., Preparation of the high-level waste form for the near-surface test facility, 497 (J;US)

Bergstrom, K.A., Preliminary interpretation of the tectonic stability of the reference repository location, Cold Creek syncline, Hanford Site, 901 (R;US)

Bermadzikowski, T.A., Development, evaluatinn, and selection of candidate highlevel waste forms, 185 (R;US)

High-evel waste-form-product performance evaluation, 627 (R:US)

Berreth, J.R., High level waste management at the Idaho Chemical Processing Plant, 451 (BA;US)

Technology for long-term management of ICPP Defense Waste, 122 (J;US)

See Gombert, D., 378

See Knecht, D.A., 240

Bertozzi, G., Probablistic approach to the assessment of the long-term risk linked to the disposal of radioactive waste in geological repositories, 1392 (J;GB)

Bertrum, S.G., See Neal, J.T., 1413, 978.

Bibler, N.E, Characterization of borosilicate glass containing Savannah River Plant radioactive waste, 179 (R;US)

See Arnold, G.W., 140

Sce Plodinec, M.J., 172, 180

Bida, G., Packing material testing required to demonstrate compliance with 1000 year radionuclide containment. Semiannual report on waste package verification tests, 739 (R;US)

Bidoglio, G., Studies on speciation of americium, technetium and neptunium in simulated vitrified-waste leachates, 1335 (J;US)

See Avogadro, A., 1360 
egg, D.W., Initial specifications for nuclear waste package external dimensions and materials, 1005 (R;US)

See O'Neal, W.C., 1018

Gregory, E.C., Summary of Near-Surface Test Facility results and their application to repository design, 886 (R;US) See Baxter, J.T., 882, 883

Grenier, R.M., See Charman, C.M., 1436 See Osborne, D.M., 558

Grens, J.Z., See Ackerman, F.J., 349, 353 See Campbell, J.H., 352

See Peters, P.E., 345

Grenthe, I., See Biedermann, G., 1353

Gries, W., Handling of nuclear waste in Great Britain, 94 (J;DE;In German)

Griffin, J.R., Repository enginccring design for high-level waste, 1082 (J;GB)

Grissom, M.C., High-level radioactive wastes, 159 (R;US)

Grossi, G., See Donato, A., 2.33

Grover, J.R., High level waste solidification: the future, 227 (RA;DE)

Gruen, M., Safety analyses for HLW and MLW storage and solidification facilities, 1394 (BA;DE;In German)

Gruenewald, W., Status of HAW-CeramicMelting Technology at the Institute of Nuclear Affairs, 235 (RA;DE;GE)

Grundfelt, B., See Andersson, K., 1358

Grunlke, J.M., See Smith, C.B., 836

Guardini, S., Actinide recycling in light water reactors: results of reactor physics calculations, $208(\mathrm{R} ; \mathrm{XE})$

Actinide recycling in light water reactors: results of reactor physics calculations, 294 (R;US)

Guber, W., Borosilicate glass and glass ceramics with real highly radioactive waste, 528 (J;US)

Thermodynamically stable glass ceramic product with radio-nuclides for the removal of radio-active waste and process for its manufacture, 1283 (P;DE;In German)

Guelker, M., INTRACOIN, Level 1. Benchmark calculations with code SWIFT, 644 (R;DE)

Guinan, M.W., See Konynfnburg, R.A., 1053

See Van Konynenburg, R.A., 350

Gulden, T.D., Evaluation of coated particle waste forms, 448 (BA;US)

Gulick, C.W., See Christensen, C.L., 938

Guon, J., Preliminary assessment of key process steps for converting Savannah River Plant high-level wastes to tailored ceramics, 147 (R;US)

See Grantham, L.F., 144, 149

See McKisson, K.L̈., 150

Gureghian, A.B., Analytical solutions of radionuclide migration with onedimensional flow in layered media, 1361 (J;US)

LAYFLO: a one-dimensional semianalytical model for the migration of a three-member decay chain in a multilayered geologic medium, 1325 (R;US)

Performance assessment of a shaft seals system in a HLW repository in the Gibson Dome area, 827 (R;US)

Gurwell, W.E., See Wald, J.W., 297
H

Haaker, R.F., See Ewing, R.C., 1213

See Greegor, R.B., 1215

Hackstein, K.G., See Hrovat, M., 358

Hadermann, J., Data base for a sitespecific analysis of the dispersion of radioactive elements from a final repository, 1366 (TG;US)

Data base for site specific migration analysis of radioactive elements in repositories, 1140 (J;DE;In German)

Study of radionuclide migration from deep-lying repository sites with overlying sedimentary layers, 1348 (BA;US)

See Andersson, K., 1358

Haefele, E.T., See Hill, D., 1071

See Pierce, B., 542

Haelsig, R.T., See Chapman, R.L., 1446

Haferkamp, B., See Mueller, R., 1282

Haideri, A.Q., See Erickson, C.J., 192

Haight, H.G. Jr., See Dunson, J.B. Jr., 169

See Gould, T.H. Jr., 174

Hakl, A.R., Commercial nuclear-waste research and development program. Quarterly report, July-September 1982 , 578 (R;US)

Commercial nuclear waste research and development program. Annual report, fiscal year 1982, 579 (R:US)

Commercial Nuclear Waste Research and Development Program. Quarterly report, October-December 1982, 580 (R;US)

Commercial nuclear waste research and development program. Quarterly report, April-June 1983, 582 (R;US)

Commercial nuclear waste research and development program. Quarterly report, October-December 1981, 60 (R;US)

Commercial nuclear waste research and developirient program. Quarterly report, January-March 1982, 61 (R;US)

Halaszovich, S., Solidification for final storage of high-level radioactive fission product sulutionis from spent fuel elc. ments, 1292 (B;DE;In German)

Solidification for final storage of highlevel radioactive fission product solutions from spent fuel elements, 529 (B;DE;In German)

Use of the drum dryer in radioactive waste solidification, 414 (BA;DE;In German)

Utilization of a drum drier for the conditioning of radioactive wastes, 440 (BA;US)

See Dix, S., 229

See Mirschinka, V., 371

Hall, A.R., Leaching of vitrified high-level radioactive waste, 1194 (BA;US)

Halleck, P., See Blacic, J., 693

Hamacher, M.E., Considerations on the management of wastes expected to be generated by the operation of Brazilian nutleal reactors, 663 (RA;BR;In Portuguese)

See Oliveria, L.F., 102

Hamill, M.L., See Shuri, F.S., 807

Hamilton, E.I., Disposal of high-activity nuclear wastes, $1038(\mathrm{~J} ; \mathrm{GB})$
Hamric, J.P., West Valley Demonstration Project: strategy for an achievable but challenging project, 434 (BA;US)

Hamstra, J., Isolation capability of a salt dome utilized for high-level waste disposal, 1208 (BA;US)

Haney, W.A., See Nelson, J.L., 211

Hannia, W.F., See Ponce, D.A., 1025

Hanson, M.S., Deposition of volatile fission products during commercial high-level waste vitrification, 304 (R;US)

Hardwick, W.H., Vitrification of high level wastes using microwave power, 230 (RA;DE)

Hardy, M.P., See St. John, C.M., 738

Hare, T.M., See Palmour, H. III., 281, 473

See Solomah, A.G., 280

Harker, A.B., Crystalline ceramics as a high-level nuclear waste form, 395 (J;US)

Dissolution mechanisms in high-level nuclear waste consolidation forms, 305 (R'A;US)

Formulation and processing of alumina based ceramic nuclear waste forms, 153 (RA;US)

Formulation and processing of polyphase ceramics for high level nuclear waste, 421 (BA;US)

Improved polyphase ceramic form for high-level defense nuclear waste, 152 (RA;US)

Improved polyphase ceramic for highlevel defense waste, 446 (BA;US)

See Jantzen, C.M., 259, 271

See Morgan, P.E.D., 151

Harmon, K.M., Summary of non-US national and international fuel cycle and radioactive waste management programs 1982, 83 (R;US)

See Lakey, L.T., 85

Harper, T.R., Complex geologic characterization of the repository environment, $1131(\mathrm{~J} ; \mathrm{GB})$

Harrington, R.V., Solidification of highlevel waste, 411 (J;GB)

Harris, L., See Stoddard, J.A., 1421

Harvey, C.O., See Westsik, J.H. Jr., 841

Harvey, K.B., Intercomparison of leachtesting methods and the effects of waste-form composition on test type and duration, 1157 (J;US)

Semi-unified approach to leach testing, 852 (RA;US)

Harvey, T., See Cheung, H., 1009

Harvey, T.F., See Cheung, H.,. 1007, 1008 See Edwards, L.L., 1058

Haseman, G.M., See Moss, M., 970

Hasted, F., Preliminary design of a Danish high level waste disposal facility in the Mors dome, 1142 (J;US)

Technology of systems, 660 (RA;DK;In Danish)

Haug, H.O., Decay calculations on medium-level and actinide-containing wastes from the LWR fuel cycle. Pt. 1. Basic data evaluation including activity and thermal decay power, 687 (R;DE;In German)

Decay calculations on medium-level and aetinide-containing wastes from the LWR fuel cycle. Pt. 2, 688 (R;DE; In German)

Hayden, N.K., See Tyler, L.D., 1224

Hayes, D.E., Analysis of geological and geophysical data from study locations 
liedermann, G., Modelling of the migration of lanthanoids and actinoids in ground water; the medium dependence of equilibrium constants, 1353 (BA;US)

Bielefeld, R.J., Field guidebook association of engineering geologists. Chapters $1-10,915$ (R;US)

Bingham, F.W., See Hunter, R.L., 948

Bingham, G.E., See Smith, R.R., 452

Binnall, E.P., See Rogue, F., 1013, 1017, 93

Birchard, G.F., Natural analogues: a way to increase confidence in predictions of long-term performance of radioactive waste disposal, 1061 (J;US)

Birgersson, I. Diffusion in the matrix of granitic rock: field test in the Stripa mine, 1341 (BA;US)

Bish, D., See Caporuscio, F., 696

Bish, D.L., See Erdal, B.R., 706

Bishop, C.B., See Triplett, M.B., 717

Bishop, H.E., See Myhra, S., 1039

Biurrun, E., Mechanical load on highly radioactive waste containers in final storage, 1141 (BA;DE;In German)

Bixler, N.E., See Mondy, L.A., 996

Bjergbakke, E., See Christensen, H., 1062, 999

Bjorklund, W.J., Operating characteristics of a direct liquid-fed vitrification process, 314 (R;US)

Recent developments in defense waste vitrification, 504 (J;US)

Vitrification of simulated West Valley waste using a liquid-fed ceramic melter, 331 (R;US)

See Jenson, G.A., 324

See McElroy, J.L., 317

See Wheelwright, E.J., 385

Bjornstad, D.J., Issues in the use of payments in lieu of taxes to provide nuclear waste facility siting incentives, $1236(\mathrm{~J} ; \mathrm{CH})$

Blacic, J., Effects of long-term exposure of tuffs to high-level nuclear wasterepository conditions. Preliminary report, 693 (R;US)

Blacic, J.D., Importance of creep failure of hard rock in the near field of a nuclear waste repository, 1270 (BA;XN)

Methodology for determining timedependent mechanical properties of tuff subjected to near-field repository conditions, 697 (R;US)

Black, J.H., Transient hydraulic tests in granite: fissured porous medium analysis and results, 1147 (BA;US)

See Mather, J.D., 1091

Blair, H.T., See McCallum, R.F., 815

See Wald, J.W., 297

Blair, S.C., See Leonhart, L.S., 865

See Wukelic, G.E., 1425

Blanco, R.E., Waste treatment and disposal. Quarterly progress report, February-April, 1963, 290 (R;US)

See Parker, F.L., 291

Blankenship, W.P., See Sprecace, R.P., 551

7lasius, E., Ruthenium nitrosyl complexes in radioactive wacte solutions of reprocessing plants. Pt. I, 12 (J;DE)

Blatts, N., See Saum, C.J., 241

Blesch, C.J., Rapid estimation of the regional thermal impact of a nuclear waste repository, 1260 (J;US)
Blomek, D, Radioactive waste management, 527 (BA;SU;In Russian)

Boatner, L.A., See Kelly, K.L., 1276 See Sales, B.C., 554

Bocoln, W, Laboratory determination of the thermal properties of large-scale samples of natural clays, 550 (R;IT;IT)

Bodde, D.L., Conflicting views on a neutrality criterion for radioactive-waste management, 124 (B;US)

See Cochran, T.B., 95

Boehmer-Christiansen, S.A., Dumping nuclear waste into the sea: international control and the role of science and law, $1055(\mathrm{~J} ; \mathrm{GB})$

Dumping nuclear waste into the sea. International control and the role of science and law, 1057 (J;GB)

Boger, Z., Radioactive waste disposal options for the operation of nuclear power stations, 664 (RA;IL)

Bokelmann, R.F., Economical dry storage of large amounts of spent fuel and vitrified high level radioactive waste, 43 (BA;XN)

Bollyky, LJ., Ozone mass transfer and kinetics experiments, 334 (R;US)

Bond, W.D., See Arnold, W.D., 292, 381

See Lackey, W.J., 245

See Robinson, S.M., 139

Bondietti, E.A., See Lee, S.Y., 553

Bonne, A., See D'Alessandro, M., 1180

Bonner, W.F, Engineering scale multibarrier waste form demonstrations, 499 (J;US)

See Knowlton, D.E., 330

See McElroy, J.L., 317

Bonnet, M., See Barbreau, A., 1186

Bonnevie-Svendsen, M., See Liljenzin, J.O., 243

Bonniaud, $R_{\text {, Actual point about fission }}$ products vitrification, 136 (R;FR;In French)

Boroomand, M.A., See Barkatt, A., 1059

Boss, C.B, See Palmour, H. III., 281

Boult, K.A., See Dalton, J.T., 251

Bourdinaud, P, See Hayet, L., 137

Bowen, J.L., Nevada nuclear waste storage investigations site-charácterization activitics: preliminary atmospheric assessment of a nuclear waste repository, 765 (R;US)

Bower, J.R., See Lakey, L.T., 219

Bowers, D.L., See Steindler, M.J., 50

See Vandegrift, G.F., 1303

Brabers, M., See Casteels, F., 676, 686

Brackmann, A.J., See Newkirk, H.W., 400

Bradbury, M.H., Aqueous phase diffusion in crystalline rock, 1359 (BA;US)

Bradley, n.., Nuclear waste package materials testing report: basaltic and tuffaceous environments, 854 (R;US)

See McVay, G.L., 1307

Bradley, N., Natural draught centralized dry store for irradiated fuel and active waste, 1239 (J;GB)

Braester, C., See Thunvik, R., 1177

Brandshaug, T., Expected repository environments in granite: assessment of rock stability of the container drill hole region, 541 (R:US)

See Klasi, M.L., 942

See Svalstad, D.K., 94 !
Brandstetter, A., Groundwater flow modeling in support of salt site evaluations, 1034 (J;US)

See Wilems, R.E., 1219

Brannen, J.P., See Tyler, L.D., 1224

Branstetter, Lل. Effect of a random variation of rock salt creep on calculations of storage room closure for the WIPP project, 943 (R;US)

Braun, R.L., See Schwartz, L.L., 1296

Bray, LA., Recovery and purification of multi-kilocurie quantities of fission product strontium by cation exchange, 214 (R;US)

See Hodges, F.N, Westsik, J.H. Jr., 1173

Brecher, A., See Pearson, F.J., 1220

Breslin, J.J., See Hof mann, P.L., 607

Brieskorm, D., See Kuelle, H.H., 645

Brinkman, W.H., See Goldmann, K., 18

Brite, D.W., See Wald, J.W., 297

Brodzinski, R.L., State-of-the-art in-situ determination of transuranics, 88 (R;US)

Broker, M.M., See Hoover, D.B., 1023, 1027

Brookins, D.G., Scientific basis for nuclear waste management VI, 366 (B;US)

Uranium-lead radiometric age determinations of naturally occurring U(VI) minerals: application to radwaste storage. 1155 (BA;US)

Brotzen, O., Predictive geology in nuclearwaste management, 1181 (BA;GB)

Brough, W.G., Instrumentation report 1: specification, design, calibration, and installation of instrumentation for an experimental, high-level, nuclear waste storage facility, 37 (R;US)

Brouns, R.A., See Bjorklund, W.J., 314

Brown, D.J., Site-screening process on the Hanford Site: a summary report, 878 (R;US)

Brown, G.A., See Bradley, N., 1239

Brown, H.C., Policy aspects of FederalState relations, 1088 (J;US)

Brown, M.L., Salt repository program: compliance with NEPA, 589 (RA;US)

Brown, R.K., Operational features of the Waste Isolation Pilot Plant (WIPP), 1161 (BA;US)

Browne, L.M., See Wheelwright, E.J., 385

Browning, M.F, See Oma, K.H., 511

Browning, R., See Martin, A., 466

Broxton, D., See Caporuscio, F., 696

Bruckheimer, L., See Rush, F.E., 1030

Brueggemann, R., See Beckmann, H., 1373

Bruehl, H., See Behrens, H., 1352

Bruna, J., Set Biedermann, C., 1353

Brush, L.H., Waste glass - seabed interactions: observations of the Soret effect, 850 (RA;US)

See Klett, R.D., 587, 976

Bryan, G.H., Development and demonstration of a process for vitrification of TMI zeolite, 505 (J;US)

See Siemens, D.H., 325

See Wheelwright, E.J., 385

Bryant, F.C., See Hill, D., 1071

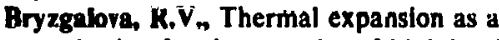
criterion for the quantity of high-level wastes disposed into geological formations, 921 (R;SU;In Russian)

Bucher, J., See Edelstein, N., 812 
Buchheim, B.K., Methodology for a consequence analysis of a nuclear waste disposal facility, $1040(\mathrm{~J} ; \mathrm{US})$

Buckley, S.N., See Antonini, M., 1154

Buckwalter, C.Q., See Wald, J.W., 297

Buddemeier, R.W., Leaching study of PNL 76-68 glass beads using the LLNL continuous-flow method and the PNLmodified IAEA method. Final report, 344 (R;US)

Buelt, J.L., Feasibility of incinerating and vitrifying organic resins in a single step, 318 (R;US)

Incineration/vitrification of simulated low-level institutional wastes in a jouleheated glass melter, 514 (J;US)

See Larson, D.E., 206

Buergisser, H.M., Risk analysis for repositories in north Switzerland. Extent and probability of geologic processes and events, 1372 (R;CH;GE)

Buetow, E., See Guelker, M., 644

Bunnell, L.R., See Wald, J.W., 297

Bunschi, H., See Buchheim, B.K., 1040

Burkholder, H.C., Commercial waste treatment $R$ and $D$ needs in the United States, 323 (R;US)

Commercial waste treatment technology and programs, 359 (J;US)

Engineered components for spent fuel radioactive waste isolation systems: are they technically justified, 1171 (BA;US)

Engineered components for radioactive waste disposal systems - are they technically justified, 861 (R;US)

Burns, B.S., Peak high-level waste package temperature versus annular air gap width, 1046 (J;US)

Burns, F.L., Sec Roy, D.M., 1172

Burns, W.G., Effects of radiation damage and radiolysis on the leaching of vitrified waste, 463 (BA;US)

Burt, K., See Rycrson, F.J., 347

Burton, B.W., Evaluation of research and development for terminal isolation of nuclear wastes, 701 (R;US)

Burton, W.R., Shielded containers for waste could prove beneficial, 1128 (J;GB)

Busch, J.S., Uncoupling thermal problems of terminal storage of spent nuclear fuel assemblies in bedded salt, 1120 (J;NL)

See Julien; H.L., 1121

See Kays, W.M., 1122

Stee Rilchic, J.3., 1119

See Watson, D.L., 1123

Butler, J.L., Description of a ceramic waste form and canister for Savannah River Plant high-level waste, 170 (R;US)

See Dunson, J.B. Jr., 169

Butlin, R.N., Baokfilling and scaling a repository for high-level radioactive waste - a review, 1279 (B;GB)

Backfilling and sealing a repusitory for high-level radioactive waste - a review, 839 (R;US)

Buykx, W.J., Thermophysical properties of SYNROC and its constituent mineral phases, 474 (BA;AU)

See Reeve, K.D., 415, 418, 493, 510

Byerly, D., See Lomenick, T.F., 828

Byers, F., See Caporuscio, F., 696

\section{C}

Caggiano, J.A., See Bergstrom, K.A., 901

Caldwell, J.T., Measurement of radioactive contaminated wastes, 278 (R;US)

See Close, D.A., 1406

Calnan, D.I., See Silva, A.J., 1277

Camagni, P., See Antonini, M., 1154

Camara, B., See Horneber, A., 460

Cameron, D.J., Materials research for the Canadian Nuclear Fuel Waste Management Program, 109 (BA;US)

Cameron, F.X., Human intrusion into geologic repositories for high-level radioactive waste: potential and prevention, $1235(\mathrm{~J} ; \mathrm{CH})$

Cameron, W.A., Evaluation of the AVM process and its applicability to West Valley, 437 (BA;US)

Campbell, J., See Hoenig, C., 348

Camphell, J.E., See Chu, M.S., 747, 981

Campbell, J.H., Immobilization of highlevel defense wastes in SYNROC-D: recent research and development results on process scale-up, 351 (R;US)

Incorporation of high-level wastes in SYNROC: results from recent processengineering studies at Lawrence Livermore National Laboratory, 352 (R;US)

See Ackerman, F.J., 349, 353

See Rozsa, R.B., 1010

Candelieri, T., See Cao, S., 234

Confield, T.R., Stresses near waste canislers buried in salt, 950 (R;US)

Cannon, T.F., See Steindler, M.J., 536, 538,539

Canonico, D.A., See Goldmann, K., 18

Cantale, C., See Donato, A., 233

Cao, S., Prelimianry experience with vitrification inactive pilut plant in Italy, 234 (RA;DE)

Ceporuscio, F., Petrologic studics of drill cores USW-G2 and UE25b-1H, Yucca Mountain, Nevada, 696 (R;US)

Caputo, A.J., See Lackey, W.J., 245

See Stinton, D.P., 138, 386, 387

Carbiener, W.A., Retrievability: the NWTS position, 1264 (J;US)

See Smedes, H.W., 1074

Carnahan, C.L., Selected hydrologic and geochemical issues in site characterization for nuclear wastc disposal: flood basalts at the Hanford Reservation, 715 (R;US)

See Apps, J.A., 1318

Carr, J.A., See Basham, S.J., 593

Curr, W.J., See Crowe, B.M., 6y8

See Snyder, D.B., 1024

Carrell, J.R., High-level waste solidification system for the Western New York Nuclear Service Center, 315 (R;US)

Carson, A.B., See Stahlkopf, K., 1107

Carter, J., See Blacic, J., 693

Carter, J.G., See Hanson, M.S., 304

Carter, L.J., Radwaste paradox, 1054 (J;US)

Carter, W.L., See Notz, K.J., 52

Cashwell, J.W., See Peterson, R.W., 17

See Schneringer, P.E.. 14

See Wilmot, E.L., 35

Cassidy, D.J., See Woolfrey, J.L., 1447

Casteel, K.D., See Murray, J.,

Casteels, F., Corrosion of materials in a clay environment, 676 (RA;DE)
Corrosion of materials in a clay environment, 686 (RA;DE)

See Dresselaers, J., 1150

Cecchetto, E.V., See Hayward, P.J., 455

Chamberlain, H.E., See Dalton, J.T., 251

Chambre, P.L., Solubility-limited fractional dissolution rate of vitrified waste in groundwater, 1228 (J;US)

Solubility-limited dissolution rate in groundwater, 1338 (J;US)

See Ting, D., 1362

See Ting, D.K., 1339

Chapman, C.C., See Siemens, D.H., 329

Chapman, J.F., See Reeve, K.D., 493, 510

Chapman, N.A., Mechanisms of dissolution of radioactive waste storage glasses and cesium migration from a granite repository, 1151 (BA;US)

See Mather, J.D., 1091

See Savage, D., 205

See West, J.M. 1357

Chapman, R.L., High-integrity container for high-specific-activity wastes, 1446 (J;US)

Charles, R.W., Sentinel Gap basalt reacted in a temperature gradient, 702 (R;US)

Charmen, C.M., Large deformation inelastic analysis of impact for shipping casks, 1436 (J;NL)

Chatt, A., See Bidoglio, G., 1335

Chen, C.P., See Hewitt, W.M., 588

Cheung, H., Nuclear waste-form risk assessment for US Defense waste at Savannah River Plant. Annual report FY 1981, 1007 (R;US)

Nuclear waste-form risk assessment for US defense waste at Savannah River Plant. Annual report FY, 1982, 1008 (R;US)

Postclosure risks of alternative SRP nuclear waste forms in geologic repositories, 1009 (R;US)

Chick, LaA, Glass leaching performance, 836 (R;US)

See Lokken, R.O., 313

Chidley, B.E., See Morris, J.B., 242

Chikalla, T.D., Nuclear waste management. Quarterly progress report, October through December 1980, 296 (R;US)

Nuclear waste management. Semiannual progress report, October 1982-March 1983, 303 (R;US)

Nuclear-waste management semiannual progress report, April 1982-September 1982, 302 (R;US)

Nuelcor-waste-manageminfill. Aromianumul progress report, October 1981-March 1982, 301 (R:US)

See Mendel, J.E., 326

Chitombr, G.P. See Hustrulid, W.A., 61 j

Choi, J, Water dilution volumcs for high. level wastes, 1401 (J;US)

Choi, JS., Water-dilution volume for highlevel wastes stored in deep gcologic media, 1232 (J;US)

Chornack, M., See Senterfit, R.M., 1028

Chomack, M.P., See Hoover, D.B., 1023, 1027

Chotin, M.M. Industrial nperating experience at the Marcoule vitrification facility, 432 (BA;US)

Chou, I.M., Migration rates of brine inclusions in single crystals of $\mathrm{NaCl}, 1149$ (BA;US) 
tristenden, R.N., See Blesch, C.J., 1260 uristensen, C.L. Field-test programs of borehole plugs in southeastem New Mexico, 615 (RA;US)

Sealing concepts for the Waste Isolation Pilot Plant (WIPP) site, 938 (R;US)

See Matalucci, R.V., 940

Christensen, $H_{\text {, Radiolysis of groundwater }}$ from HLW stored in copper canisters, 1062 (J;US)

Radiolysis of groundwater from HLW stored in copper canisters, 999 (R;SE)

Christensen, R.N., Criticality safety of high-level waste containing unseparated plutonium, 1259 (J;US)

See Burns, B.S., 1046

Christofferson, J., Host State perspective to high-level waste management criteria and standards for siting, storage, and monitoring, 1096 (J;US)

Chryssostomidis, C., See Miller, M.M., 31 See Shirley, C.G., 20, 33

Chu, M.S., Analysis of waste-package behavior for high-level waste, 981 (R;US)

Assessment of the proposed rule (10CFR60) for disposal of high-level radioactive wastes in geologic repositories. Volume I, 747 (R;US)

See Pepping, R.E., 1416, 1418

See Siegel, M.D., 982

Chu, M.S.Y., Assessment of technical criteria of 10CFR60 for geologic disposal of high-level waste, 952 (R;US)

Comparison of risks due to HLW and SURF repositories in bedded salt, 1368 (R;US)

See Pepping, R.E., 731

See Siegel, M.D., 1417

Claiborne, H.C., See Jenks, G.H., 829

See Rickertsen, L.D., 830, 831

Clark, D.E., Effects of waste composition and loading on the chemical durability of a borosilicate glass, 1188 (BA;US)

Preliminary report on a glass burial experiment in granite, 631 (R;US)

Waste glass/repository interactions, 1193 (BA;US)

See Hench, L.L., 753, 845

See Kircher, J.F., 825

Clark, R.J., See Kelmers, A.D., 1304

Clarke, D.R., See Harker, A.B., 152, 153, 305, 395, 421, 446

See Jantzen, C.M., 259, 271

See Morgan, P.E.D., 151

Clarke, W.M., See Orvis, D.D., 808

Claverie, R.J., See Goldmann, K., 18

Clayton, L.G., See Anderson, W.J., 887

Clelland, D.W., Selection and application of a processing system for the treatment of high level liquid waste in the UK, 430 (BA;US)

Vitrifying Britain's waste, 384 (J;GB)

Clement, J.D., See Wan, P.T., 1048

Clifton, P.M., Numerical modeling of groundwater flow and solute transport for a nuclear-waste repository in basalt, 1327 (R;US)

See Baker, S.M., 1222

ose, D.A., Advances in measurement of alpha-contaminated wastes, 1406 (R;US)

See Caldwell, J.T., 278

Closs, K.D., See Rothemeyer, H., 1275
Cochrain, T.B, Conflicting views on a neutrality criterion for radioactive-waste management, 95 (BA;US)

See Bodde, D.L., 124

Coffinas, F.E, DOE's commercial waste management and fuel cycle programs, 100 (J;US)

DOE's nuclear waste management and fuel cycle programs, 1159 (BA;US)

Colven B.I. Effects of ICRP Publication 30, the 1980 BEIR Report, and ORIGEN-2 on hazard assessments of high level waste, 1400 (J;US)

Effects of ICRP Publication 30 and the 1980 Beir report on hazard assessments of high-level waste, 1440 (J;GB)

Effects of ICRP Publication 30 and the 1980 BEIR Report in hazard assessments of high-level waste, 1441 (J;GB)

High level radioactive waste, 1242 (J;US)

Cohes, J., See Stevens, C.A., 727

Cohen, J.J., Rock-melt concept for nuclear waste disposal, 1254 (J;US)

See Musgrave, B.C., 498

See Schwartz, L.L., 1296

Cole, B.M. See Andrews, W.B., 27

Cole, H.S., Comparison of candidate glasses to vitrify ICPP calcined alumina waste, 469 (J;US)

Coleman, $C J_{n}$ Analyses of nuclear fuel and high-level waste by inductively coupled plasma-emission spectroscopy, 68 (R;US)

Coles, D., See Bazan, F., 1004

Coles, D.G, See Bradley, D.J., 854

See Buddemeier, R.W., 344

See Failor, R.A., 1332

See Karim, D.P., 417

Colligga, J.L., See Odgers, I.L., 612

Collins, E., Annotated bibliography for bio logic overview for the Nevada Nuclear Waste Storage Investigations, Nevada Test Site, Nye Counly, Nevada, 1309 (R;US)

Biologic overview for the Nevada Nuclear Waste Storage Investigations, Nevada Test Site, Nye County, Nevada, 1310 (R;US)

Surveys for desert tortoise on the proposed site of a high-level nuclear waste repository at the Nevada Test Site, 1302 (R;US)

See O'Farrell, T.P., 1311

Coltos, E.L., See Cole, H.S., 469

Comelle, PA., Licensing: technological implications for geologic disposal of high-level radioactive wastes, 1089 (J;US)

See Arsenault, F.J., 1092

Courndt, R., See Scholze, H., 1204

Conwell, F.R. See Frazier, N.A., 1265

Coot, F.R. Nuclear Regulatory Commission activities related to high-level waste materials issues, 1420 (BA;US)

Cook, N.G.W. Thermomechanical studies in granite at Stripa, Sweden, 619 (RA;US)

See Wang, J.S.Y., 1183

Cooley, C.R, DOE implementation of high-level waste criteria and standards, 1093 (J;US)

See Platt, A.M., 216

Cooper, J.D., See Shuri, F.S., 807
Corbet, A.D.W., See Clelland, D.W., 384, 430

Cornman, W.R., Composite quarterly technical report long-term high-level-waste technology, October-December 1981 , 164 (R;US)

Composite quarterly technical report: long-term high-level-waste technology, January-March 1982, 66 (R;US)

Long-term high-level-waste technology. Composite quarterly technical report, April-June 1982, 165 (R;US)

Long-term high-level waste technology. Composite quarterly technical report, July-September 1982, 166 (R;US)

Cornman, W.R. Jr., Long-term high-level waste technology. Composite quarterly technical report, July-September 1981, 163 (R;US)

Cosper, M.B., Large-scale continuous process to vitrify nuclear defense waste: operating experience with nonradioactive waste, 177 (R;US)

Costello, J.M., Assessment of overseas developments in methods for treatment and disposal of high-level radioactive wastes, 532 (R;AU)

Disposal of high-level radioactive wastes, $1116(\mathrm{~J} ; \mathrm{AU})$

Cottam, A.E., See Anderson, W.J., 887

Cotton, T., See Ghovanlou, A.H., 1133

Courtols, G., See Barthoux, A., 1280

Cousens, D., Simulation of radiation damage in HLW glasses accelerated with $(n, \alpha)$ and $(n, f)$ reactions, 654 (RA;AU)

Cousens, D.R., Chemical durability of some HLW glasses: effects of hydrothermal conditions and ionizing radiation, 1203 (BA;US)

Evaluating glasses for high-level radicactive waste immobilization, 1237 (J;US)

Microstructure of Synroc, 454 (BA;US)

Structural chemistry of Synroc, 221 (RA;AU)

Couture, R.A., See Steindler, M.J., 539

Cowan, R., Deadly legacy, 1127 (J;GB)

Cox, N.D., See Schuman, R.P., 200

Craft, T.F, See Eichholz, G.G., 98

See Eicholz, G.G., 1365

Cramer, M:I., See Gregory, E.C., 886

See Kim, K., 889

See Lanigan, D.C., 881

Crandall, J., High-level waste description, inventory and hazard, 632 (R;US)

Crandall, J.L., High-level-waste immobilization, 181 (R;US)

Treatment and conditioning of wastes from US nuclear fuels reprocessing plants, 191 (R;US)

See Gould, T.H. Jr., 178

Crane, T.W., See Caldwell, J.T., 278

See Close, D.A., 1406

Cranmell, R.M. Application of a riskassessment methodology to a hypothetical high-level waste repository in bedded salt, 937 (R;US)

Methodology for assessing the risk from the disposal of high-level radioactive wastes in deep geologic formations, 993 (R;US)

Modeling of waste/near field interactions for a waste repository in bedded salt: the Dynamic Network (DNET) model, 1063 (J;US) 
Crisman, D.P., Native copper deposits of the Portage Lake volcanics, Michigan: their implications with respect to canister stability for nuclear waste isolation in the Columbia River basalts beneath the Hanford Site, Washington, 904 (R;US)

Croff, A.G., Graphical and tabular summaries of decay characteristics for onccthrough PWR, LMFBR, and FFTF fuel cycle materials, 76 (R;US)

Cross, R.W., See Bergstrom, K.A., 901

Crowe, B., Aspects of possible magmatic disruption of a high-level radioactive waste repository in southern Nevada, 699 (R;US)

Crowe, B.M. Calculation of the probability of volcanic disruption of a high-level radioactive waste repository within southern Nevada, USA, 1393 (J;GB)

Status of volcanic hazard studies for the Nevada Nuclear Waste Storage Investigations, 698 (R;US)

See Erdal, B.R., 706

Cunningham, J.P., See Baxter, J.T., 882 , 883

Curtis, D.B., Oklo reactors: natural analogs to nuclear waste repositories, 611 (RA;US)

Cutshall, N.H., See Kelmers, A.D., 1304

Czyscinski, K.S., See Ahn, T.M., 284

\section{D}

D'Alessandro, M., Fault-tree analysis for probabilistic assessment of radioactive. waste segregation: an application to a plastic clay formation at a specific site, 1180 (BA;GB)

See Bertozzi, G., 1392

d'Entremont, P.D., Development of a slurry-fed in-can melter for nuclear defense, waste 176 (R;US)

Daemen, J.J.K., See South, D.L., 1231

Daglish, J., See O'Hara, F.A., 155

Dale, LS., See Lewis, R.A., 375

Dalen, A. van, Ocean-bottom disposal of fission-product krypton-85, 1251 (BA;JP)

Daling, P.M., See McKee, R.W., 863

Dalton, J.T. Influence of metal oxides on the leach rate and crystallisation behaviour of waste glasses, 251 (RA;DE)

Damuth, J.E., See Hayes, D.E., 957

Daniel, J.L., See Johnston, J.W., 300

Daniels, W.K., Kesearch and development related to the Nevada Nuclear Waste Storage Investigations. Progress report, July 1-September 30, 1981, 692 (R;US)

Research and development related to the Nevada Nuclear Waste Storage Investigations. Progress report, July 1-September 30, 1982, 703 (R;US)

Summary report on the geochemistry of Yucca Mountain and environs, 700 (R;US)

See Erdal, B.R., 706

See Ogard, A.E., 704

Daruschy, P., See Guber, W., 1283

Davis, E., Legislative and political aspects of waste disposal, 1409 (RA;US)

Davis, E.J., See Ray, A.K., 1340
Davis, J.C., See Schneider, R.F., 196

Davis, J.D., See Bergstróm, K.A., 901

Davis, J.G., Speech before the Atomic Industrial Forum Conference, 123 (BA;US)

Davis, J.M., See Gimera, R.J., 877

Davis, M.S, Draft Technical Position Subtask 1.1: waste package performance after repository closure. Volume 1,749 (R;US)

High-level-waste containment for a thousand years: unique technical and research problems, $S 44$ (R;US)

Nuclear waste management technical support in the developmnt of nuclear waste form criteria for the NRC. Task S. National waste package program. 285 (R;US)

Review of DOE waste package program. Subtask 1.1. National waste package program, 286 (R;US)

See Schweitzer, D.G., 547

Davis, S.N., Hydrogeology of radioactive waste isolation: the challenge of a rational assessment, 1144 (J;US)

Dawson, P.R., See Munson, D.E., 969

Deyal, $R_{\text {., Nuclear waste management }}$ technical support in the development of nuclear waste form criteria for the NRC. Task 1. Waste package overview, 283 (R;US)

Dayem, N., See Cooley, C.R., 1093

De, A.K., See Lutze, W., 253, 265, 530

De Batist, R., See Van Iseghem, P., 1207. 262

De Korompay, V., See Jakubick, A.T., 1346

De Marsily, G., Application of systems analysis to the disposal of high level waste in deep ocean sediments, 1069 (J;GB)

Predictive geology: with emphasis on nuclear-waste disposal, 96 (J;US)

de Oliveirn, LF.S., See Hamacher, M.E., 663

De Plana, A. See Avogádro, A., 1360

See Bidoglio, G., 1335

De Wames, R.E., See Grantham, L.F., 144

deBakker, P. See Young, K.D., 555

Deckwer, W.D, See Kelm, M., 531

Deeken, P.G. See Steindiler, M.J., 539

DeFigh-Price, $C_{\text {, }}$ Status of tankassessment studies for continued intank storage of Hanford Defense waste, 919 (R;US)

Deichman, J.L., High level waste management and characterization at the Hanford plant site, 1198 (BA;US)

Dejonghe, $\mathrm{P}_{\text {, Discharge of high activity }}$ and long balf-life wastes arising from a nuclear power programme, 373 (J;BE;In French)

Deju, R.A., Basalt waste isolation project. Quarterly report, April 1, 1981-June 30, 1981, 909 (R;US)

Basalt Waste Isolation Project. Quarterly report, July 1, 1981 -September 30 , 1981, 910 (R;US)

Performance allocation traceable to regulatory criteria as applied to sitecharacterization work at the Basalt Waste Isolation Project, 890 (R;US)

Dekois, J.J., See Ahner, S., 640, 833

Delany, J.M. See Carnahan, C.L., 715

Delegard, C.H., Effects of Hanford highlevel waste components on sorption of cobalt, strontium, neptunium, plutonium, and americium on Hanford sediments, 918 (R;US)

Denning, R.S., See Rice, E.E., 720, 721

Dennis, A.W., Design considerations for occupational exposure for a potential repository at Yucca Mountain: highlevel-waste handling operations, 991 (R;US)

Occupational exposure for nuclear waste handling operations at NNWSI repository, 1390 (J;US)

Dese, J.A.E., See Sinclair, R.N., 363

Desroches, J., Elimination of used sources. Taking over and storage, 548 (R;FR;In French)

Detillewx, E., Reprocessing, decontamination and decommissioning waste management at Eurochemic: current and planned activities, 433 (BA;US)

Devaney, H.E. See Moore, J.G., 256, 268

Diamond, H., Alpha spectrum profiling of plutonium in leached simulated highlevel radioactive waste-glass, 1364 (J;US)

Alpha spectrum profiling of plutonium in leached simulated high-level radioactive waste-glass, 517 (J;US)

See Horwitz, E.P., 146

See Karim, D.P., 417

Dickey, B.R., Heat transfer in high-level waste management, 1118 (J;NL)

See Berreth, J.R., 451

Diegle, R.B., See Molecke, M.A., 1101 , 949

Dippel, T., See Kahl, L., 277

Dippold, D.G., See Waddell. J.D., 769

Dith, P., See Napravnik, J., 361

Dix, O.R., Statistical analysis of lineaments and their relation to fracturing, faulting, and halokincsis in the East Texas Basin. Report of investigations No. $110,566(12 ; U S)$

Dix, S., Fissinn product, solidification: FIPS, 229 (RA;DE;GE)

See Halaszovich, S., 414, 440

Dlouby, Z., Disposal of radioactive wastes, 106 (B;NL)

Disposal of radioactive wastes, $\mathbf{4 1 3}$ (B;NL)

Doem, F.E., See Hayward, P.J., 455

Dole, L.R., Cement-based radioactive waste hosts formed under elevated temperatures and pressures (FUETAP concretes) for Savannah River Plant highlevel defense waste, 293 (R;US)

See Moore, J.G., 256, 268

Wolezal, J., Inorganic sorbents in nuclear power plants, 467 (J;CS; In Czech)

Domenica, P.A., See Palciauskas, V.V. 1110

Donath, F.A., Isolation of high-level nuclear wastes in a geologic repository, 1084 (J;US)

See Schwartz, F.W., 1184

Donato, A., Laboratory activity in Italy in the field of vitrification of high-level wastes, 233 (RA;DE)

Dorbandt, J., See Koelle, H.H., 645

Dormuth, K.W., Canadian geoscience research for nuclear fuel waste management, 592 (RA;US)

Dosch, R.G., Processing effects on the behavior of titanate waste forms in aqueous solutions, 1145 (BA;US) 
Processing, microstructure, leaching, and long-term stability studies related to titanate high-level waste forms, 340 (R;US)

Downs, W.F., Scientific data necessary to predict radionuclide migration within or near a mined nuclear repository, 1312 (R;US)

Dran, J.C., Leaching behaviour of ionimplanted simulated HLW glasses and tentative prediction of their alpharecoil aging, 423 (BA;US)

See Petit, J.C., 1205

Draper, E.L. Jr., Nuclear waste management, 119 (J;US)

Dresselaers, J., Corrosion of construction materials in clay environments, 1150 (BA;US)

Drewes, D.R., See Early, T.O., 1329

Drobnik, S., See Kelm, M., 531

Duda, L.E., See Muller, A.B., 973

Duffy, M.A., See Pardue, W.M., 1037

Duguid, J.O., Earth science developments in support of waste isolation, 608 (RA;US)

Dukes, M.D., Disposal of Savannah ${ }^{\circ}$ River Plant waste salt, 625 (R;US)

Duncan, A.G., See Keen, N.J., 1160

Duncan, D.W., See Bergstrom, K.A., 901

Dunson, J.B., Jr., See Gould, T.H. Jr., 174

Dunson, J.B. Jr., Assessment of processes, facilities, and costs for alternative solid forms for immobilization of SRP defense waste, 169 (R;US)

Durham, W.B., See Weed, H.C., 1003

Dytynyshyn, D.J., See Gillham, R.W., 1300

\section{E}

Early, T.O., Geochemical controls on radionuclide releases from a nuclear waste repository in basalt: estimated solubilities for selected elements, 1329 (R;US)

Eastwood, D., See Bida, G., 739

Eaton, R.R., Code development in support of nuclear' waste storage invesligationis for a repository in tuff, 988 (R;US)

See Gartling, D.K., 931

See Sundberg, W.D., 932

Edelstein, N., Thermodynamic properties of chemical species in nuclear waste, 812 (R;US)

Edquist, R., See Smith, C., 567

Edwards, L., See Cheung, H., 1009

Edwards, L.L., Limiting-individual dose from an extended-source high-level waste repository model, 1058 (J;US)

See Cheung, H., 1007, 1008

Egami, R.T., See Bowen, J.L., 765

Egan, D.J. Jr., See Smith, C.B., 836

Eggers, P.E., Storage and transportation of spent fuel and high-level waste using dry storage casks, 15 (RA;US)

Ehlert, $C_{\text {. }}$ Thermomechanical effects of the final storage of high-level radioactlve wastes in salt domes, 1126 (J;DE;In German)

Thermomechanical impact around a borehole in rock salt after HAW disposal, 1294 (J;US)

See Biurrun, E., 1141
Ehrlich, D., Occupational exposure in an underground mine repository for radio active waste, 1387 (J;US)

See Warnecke, E., 671, 681

Eichhol2, G.G., Subsurface migration of radioactive waste materials by particulate transport, 98 (J;US)

Eicholz, G.G., Waste migration by particulate carriers, 1365 (J;US)

Eisenberg, A.M., See Dunson, J.B. Jr., 169 See Gould, T.H. Jr., 174

Eisenbud, M., Status of radioactive waste management: needs for reassessment, 1248 (J;GB)

El Rabaa, A.W., See Hustrulid, W.A., 613

Elder, H.K., See McKee, R.W., 308, 309

Eliasson, T., See Torstenfelt, B., 1334

Ellett, D.M., Effects of wire rope in mitigating a waste-shaft accident, 980 (R;US)

Engel, R.L., See Andrews, W.B., 27

Engelke, H., See Scholze, H., 1204 See Storch, W. 691

Engelmann, C., See Trocellier, P., 1216

Engelmann, H.J., Underground disposal of high active waste, 665 (R;DE)

Ensminger, D.A., Accident scenarios and probabilities for the SDP transportation system, 956 (RA;US)

Review of safety assessments of nuclear waste management, 1380 (R;US)

Risk comparison for transportation, handling, and storage of high-level waste, 1402 (J;US)

Erdal, B.R., Some geochemical considerations for a potential repository site in tuff at Yucca Mountain, Nevada, 706 (R;US)

See Daniels, W.R., 692, 703

Erickson, C.J., Electrical service and controls for Joule heating of a defense waste experimental glass melter; 192 (R;US)

Friksen, T., See Pusch, R., 1174

Erlandson; O.D., See Gulden, T.D., 448

Eschbach, E.A., See Platt, A.M., 509

Ettlinger, L., See Ghovanlou, A.H., 1133

Evans, D.D., Unsaturated flow and transport through fractured rock - related to high-level waste repositories. Final report. Phase I, 748 (R;US)

Evans, J.R., See Monfort, M.E., 1022

Ewing, R.C., Leachability of zircon as a function of alpha dose, 1213 (BA;US)

See Altenhein, F.K., 1191, 1333

See Greegor, R.B., 1215

Eyal, Y., Isotopic fractionation of thorium and uranium upon leaching of monozite: alpha-recoil damage effects, 1214 (BA;US)

\section{$\mathbf{F}$}

Fagan, J.E., See Steindler, M.J., 539

Failor, R., See Bazan, F., 1004

Failor, R.A., Leaching study of nuclear melt glass: Part I, 1332 (R;US)

Farnsworth, R.K., See Oma, K.H., 307

Faussat, A., Low level and Intermedlate level wastes management practice in France, 442 (BA;US)

Feates, F.S., United Kingdom regulatory procedures for radioactive wastes, 369 ( $;$;US)
Fecht, K.R., See Bergstrom, K.A., 901

See Reidel, S.P., 873

Fehringer, D.J., Regulating the development of a waste repository, 1130 (J;GB)

Feige, $Y$., Analysis of waste disposal practice and control at ORNL, 549 (R;US)

Feld, R.H., Quantitative determination of crystalline phases in nuclear waste glasses, 458 (BA;US)

Feldt, W., Radioecology of the deep-sea, 1428 (RA;DE;In German)

Feller, K.G., See Sutcliffe, W.G., 809

Ferri, D., See Biedermann, G., 1353

Feuerstein, D.W., See Koelle, H.H., 645

Finch, W.C., System evaluation model for selecting spent nuclear fuel storage concepts, 24 (R;US)

Findley, D., See Gonano, L., 741

Finley, N.C., See Helton, J.C., 1317

Fisher, J., See Laine, E., 958

Fisk, A., See Young, K.D., 555

Fitzpatrick, F.J., See Buchheim, B.K., 1040

Fleischer, R.L., Alpha-recoil damage and solution effects in minerals: uranium isotopic disequilibrium and radon release, $1073(\mathrm{~J} ; \mathrm{GB})$

Fleming, K.N., See Frank, M.V., 1255

Fetcher, J.F., Monitored retrievable storage: a waste management option for spent fuel and high-level wastes, 864 (R;US)

Flinn, J.E., See Welch, J.M., 199, 416

Flintoff, J., See Harker, A.B., 446

See Jantzen, C.M., 259, 271

Flintof, J.F., See Grantham, L.F., 144, 149,450

See Harker, A.B., 421

Flintoff, J.J., See Harker, A.B., 152, 153

See Morgan, P.E.D., 151

Flood, R.D., See Hayes, D.E., 957

Flynn, K.F., See Bates, J.K., 127

Foote, H.P., See Leonhart, L.S., 865

See Wukelic, G.E., 1425

Forberg, S., Immobilization of reprocessing waste by means of titanate ion exchangers, 248 (RA;DE)

Forch, K., See Mirschinka, V., 371

Forcti, U., See Mirschinka, V., 371

Ford, L.H., Sec Allen, P.L., 392

See Saum, C.J., 241, 282

Forster, Wm. O., Dumping of solid packaged radioactivity in the deep oceans, 1291 (BA;IN)

Fortsch, M., Process improvements in the NWCF, 393 (J;US)

Fowler, G., See Robinson, K.S., 231

Frank, M.V., Three aspects of reliability for a geological nuclear waste repository, 1255 (J;US)

See Orvis, D.D., 808

Franks, L.A., See Close, D.A., 1406

Franz, E.M., See Ahn, T.M., 284

Frazier, N.A., Geological status of NWTS repository siting activities in the paradox basin, 1265 (J;US)

Freebom, W.P., See Komarneni, S., 289

See White, W.B., 851

Frelwald, J., Legislative and pulitical aspects of waste disposal, 1410 (RA;US)

Friant, J., See Young, K.D., 555

Fried, S., See Nash, K., 1247

Fried, S.M., See Vandegrift, G.F., 1303 
Friedlander, A.L., See Rice, E.E., 720, 721

Friedman, A.M., See Diamond, H., 1364, 517

See Karim, D.P., 417

See Nash, K., 1247

Friesen, E., See Kaegi, U., 1407

Fry, C.J., See Robinson, C.H., 246

Fujiwara, K., See Furuya, T., 667

Fukszawa, T., See Uetake, N., 470

Fukumoto, S., See Terai, R., 482

Fukuzuka, T., See Furuya, T., 667

Fullam, H.T., Cesium chloride compatibility testing program. Annual report, FY 1982, 855 (R;US)

Fullwood, R.R., See Stevens, C.A., 727

Furuya, T., Corrosion resistance of metal materials for HLW canister, 666 (R;JP;In Japanese)

Effects of various waste contents on properties of borosilicate glasses for high lovel wasto oolidifieation in sovoral countries, 223 (R;JP;In Japanese)

Study of $\boldsymbol{\gamma}$-ray irradiation effects on corrosion resistance of alloys for storage of high-level waste packages, (1), 667 (R;JP;In Japanese)

Fyfe, W.S., Suitability of natural geomedia for radwaste storage, 1060 (J;US)

See Metson, J.B., 456

\section{G}

Gallucci, R.H.V., Risk assessment for the transportation of radioactive zeolite liners, 42 (BA;US)

Galvin, T.M., See Bandyopadhyay, G., 391

Gancarz, A.J., See Curtis, D.B., 611

Ganley, J.T., Monitored retrievable storage of nuclear waste in the US, 642 (R;US)

See Baxter, B.J., 16

See Morissette. R.P.. 641

Ganser, B., Analysis of Am and a recovery for it in the pressurized water reactor fuel cycle, 1433 (R;DE;In German)

Gartling, D.K., Preliminary thermal analyses for a nuclear waste repository in tuff. 931 (R:US)

Gasper, K.A., See Isaacson, R.E., 920

Gates, R., Rclationship of an in-situ tcst facility to a deep geologic repository for high-level nuclear waste. Final report (Task 4), June 1981-October 1982,742 (R;US)

See Gonano, I.., 741

Gattys, F.J., Process and facility for continuous melting and dosed filling in casks of high-activity waste, 520 (P;DE;In German)

Gauvenet, A., Problem of reprocessing and of radioactive wastes in France, 489 (J;FR;In French)

Gayler, R., See Hardwick, W.H., 230

Gehl, S.M., See Bandyopadhyay, G., 502

Gehler, S., See Behrens, H., 1352

Gelhar, L.W., See Leonhart, L.S., 880

George, M.W., See Beale, H., 534

Gephart, R.E., Geohydrologic characterization and qualification of a high-level waste site in basalts, 891 ( $R$;US)

See Price, S.M., 894

Gera, F., Geologic predictions and radioactive-waste disposal: a time limit for the predictive requirements, 1178 (BA;GB)
Gerding, T.J., See Steindler, M.J., 539

See Vandegrift, G.F., 1303

Ghoranluu, A.H., Selecting a repusitury site, 1133 (J;GB)

Gibson, G.W., See Schuman, R.P., 200

Gibson, P.N., See Antonini, M., 1154

See Manara, A., 464

Gibson, S.M., See Varadarajan, R.V., 556

Gidlund, J., See Abelin, H., 1342

Gies, H., Effects of product properties on the final salt storage medium and the conditions producing it, 673 (RA;DE;GE)

Effects of the product properties upon the ultimate storage medium salt and resulting conditions, 683 (RA;DE;In German)

Mineralogical and geochemical factors influenoing the final diopooal of HLW in the Stassfurt halite, 1164 (BA;US)

Giesel, W., See Duchuaum, II., 1373

Gilbert, T.L., Benefit-cost-risk analysis of alternatives for greater-confinement disposal of radioactive waste, 559 (R;US)

Gillham, R.W., Diffusive transport of strontium-85 in sand-bentonite mixtures, 1300 (R;CA)

Gimera, R.J., Overview of waste-handling requirements for a nuclear-waste repository in basalt, 877 (R;US)

Giovanetti, R.C., See Shirley, C.G., 20, 33

Girolimetti, G., See Bocola, W., 550

Giulianelli, G., See Bocola, W., 550

Class, R.S., Effects of radiation on corrosion of titanium, 961 (RA;US)

Glatz, J.P., See Blasius, E., 12

Glora, M.A., Regulatory implementation considerations for deep geologic disposal of high-level radioactive wastes, 1094 (J;US)

See Stein, R., 1227

Gnirk, P., See Lindblom, U., 1137

Gnirk, P.T., Improving public understanding of nuclear waste management. $1136(\mathrm{~J} ; \mathrm{GB})$

See Johnstone, J.K., 977

See Krause, W.B., 621

Goblet, P., See Barbreau, A., 1186

Gockley, G.B., Supernatant treatment considerations for the neutralized waste at West Valley, 370 (I; I,IS)

Goergen, C.R., Sampling and analysis of high level waste tank supernatant: an overview, 634 (R;US)

Goldin, A.S., Potential individual doses from disposal of high-levcl radioactive wastes in geologic repositories. Draft i epuil (final), 1382 (R,US)

Goldmann, K., Construction and inservice inspection rules for nuclear spent fuel and high-level waste transport packagings, 18 (RA;US)

Goles, R.W., Off-gas characteristics of defense waste vitrification using liquidfed Joule-heated ceramic melters, 310 (R;US)

Off-gas characteristics of liquid-fed joule-heated ceramic melters, 319 (R;US)

See Barnes, S.M., 321

Gombert, D., Microwave energy for postcalcination treatment of high-level nuclear wastes, 378 (J;US)

Gonano, L., Evaluation of alternative shaftsinking techniques for high-level nuclear waste (HLW) deep geologic repositories. Final report (Task 3), June 1981-July 1982, 741 (R;US)

See Gates, R., 742

See Roberds, W., 743, 744

Gonzales, S., Preliminary assessment of shales and other argillaceous rocks in the United States, 609 (RA;US)

See Lomenick, T.F., 828

Gooley, R., See Caporuscio, F., 696

Goossens, W.R.A., See Klein, M., 141

Gordon, D.E., Assessment of high-level waste form conformance with proposed regulatory and repository criteria, 198 (R;US)

Research priorities for isolation of defense high-level and transuranic wastes in the I/nited States, 628 (R;US)

Soo Bornadxikowoki, $T$, A $, 185,627$

See Walton, R.D. Jr., 443

Goss, E., See Bjornstad, D.J., 1236

Gould, T.H. Jr., Alternate waste form evaluation for Savannah River Plant highlevel waste, 1104 (J;US)

Alternative-waste-form evaluation for Savannah River Plant high-level waste, 178 (R;US)

Processability analysis of candidate waste forms, 174 (R;US)

See Bernadzikowski, T.A., 185

See Butler, J.L., 170

See Dunson, J.B. Jr., 169

See Stone, J.A., 171

Gould T.H. Jr., See Bernadzikowski, T.A., 627

Graham, D.L., See Leonhart, L.S., 880

Grambow, B., Solubility limits of metal ions and mechanism of leaching nuclear-waste glasses, 320 (R;US)

Grantham, L.F., Ceramic process and plant design for high-lovel nuclear waste immobilization. 144 (R:US)

Process description and plant design for preparing ceramic high-level waste forms, 149 (R;US)

Spray calcination of SRP waste compositions for ceramic waste forms, 450 (BA;US)

Sec MoKisson, R.L., 150

Grass, F., Radioactive wastes, 382 (J;AT; In German)

Grauer, R., Glasses for the solidification of high-level radioactive waste: their behavior in the presence of water, 1052 (TG;US)

Gray, P.L., National long-term high-level waste-technology program, 67 (R;US)

The national long-term high-level waste teclutulugy prugram, 121 (J;US)

See Gordon, D.E., 198

Gray, W.J., Fission product transmutation effects on high-level radioactive waste forms, 401 (J;GB)

Radiation damage studies related to nuclear waste forms, 298 (R;US)

See Wald, J.W., 297

See Wcbcr, W.J., 1278

Greegor, R.B., Investigation of titanium in metamict Nb-Ta-Ti oxides using the extended $x$-ray absorption fine structure technique, 1215 (BA;US)

Greenwood, P.B., See McKinley, I.G., 1313 
$C_{1}, B_{1}$, and $E_{2}$ in the northwest PAC 1 study region, 957 (RA;US)

Hayet, $L$., Encapsulation of tritiated wastes in hot laboratories, 137 (R;FR;In French)

Hayward, P.J., SIMS depth profiling studies of sphene-based ceramics and glass ceramics leached in synthetic groundwater, 455 (BA;US)

See Metson, J.B., 456

Headley, T.J., See Dosch, R.G., 340

Heath, C.A., DOE's program for high-level radioactive waste disposal, 1266 (BA;US)

Heath, G.R., See Hinga, K.R., 1129

See Hollister, C.D., 1243

Heath, W.O., See Larson, D.E., 206

Heckendorn, F.M. II, DWPF process control, $186(R ; I I S)$

Heiken, G., See Caporuscio, F., 696

IIeimeri, II ${ }_{\text {; }}$ Inactive vitrificalium in Mul. Ceranic unelting, feed and uff gas system, pouring equipment for glassmelting, 237 (RA;DE;GE)

Hoinonen, O.J., Sodium titanate in solidifi cation of radivaclive wastes, 247 (RA;DE)

Heller, A., See Blacic, J., 693

Helton, J.C., Health effects associtated with unit radonuclide releases to the environment, 1378 (RA;US)

PATH1 self-teaching curriculum: example problems for Pathways-to-Man Model, 1317 (R;US)

Risk methodology for geologic disposal of radioactive waste: model description and user manual for Pathways model, 1316 (R;US)

See Cranwell, R.M., 937

Hench, L.L., Burial effects on nuclear waste glass, 1202 (BA;US)

riffects of composition, solutions, and burial on nuclear waste glasses, 845 (R A;IIS)

Surface properties and performance prediction of alternative waste forms. Annual report, October 1, 1981September 30, 1982. Volume 1, 753 (R;US)

See Mendel, J.E., 326

Sce Nogucs, J.L., 1206, 341, 461

See Werme, L., 1200

Henderson, N.C., See Frank, M.V., 1255

Hendricks, J.P., Separation of mercury from HEWC solutions. Laboratory assay, 207 ( $R ; B E$;In French)

Hennelly, E.J., See Crandall, J., 632

Henslee, S.P., See Welch, J.M., 199, 416

IInyyey, F.S., Ses Marriutt, R., 486

Heredia, L., See Guelker, M., 644

Heremans, R., See Casteels, F., 676, 686

Hermans, R., See Dejonghe, P., 373

Herrington, B.E., See Voth, D.E., 8Us

Herrmann, A.G., Composition of rocks criteria for the conditioning of highlevel radioactive wastes, 672 (RA;DE;In German)

Composition of rocks - criteria for the conditioning of high-level radioactive wastes, 682 (RA;DE;In German)

Herrnherger, V., See Buergisser, H.M., 1372

Hetherman, J.R., See Silva, A.J., 1277

Heuze, F.E., Climax Granite, Nevada Test Site, as a host for a rock mechanics test facility related to the geologic dis- posal of high level nuclear wastes, 1002 (R;US)

Modeling of nuclear waste disposal by rock melting, 1016 (R;US)

Thermal and thermomechanical calculations of deep-rock nuclear waste disposal with the enhanced SANGRE code, 1012 (R;US)

Hewitt, W.M., Impact of NRC licensing requirements on salt site selection, 588 (RA;US)

Repository safety-the salient facts, 1397 (J;US)

Hibberson, W., See Ringwood, A.E., 515

Hickox, C.E., Comparison of waste emplacement configurations for a nuclear waste repository in tuff. II. Ventilation analysis, 995 (R;US)

Hild, W., See Letilleux, E., 433

See Guber, W., 1283

Hileman. B. Nuclenr waste dispmsal a rase of benign neglect>, 1108 (J;US)

Hill, D., Management of high-level waste repusitory siting, $1071(\mathrm{~J} ; \mathrm{US})$

See Pierce, B., 542

Hill, M.D., CEC project to assess high level wastc disposal options, 1085 (J;GB)

Radiological impact of disposal of UK solid radioactive wastes, present and future, 1081 (J;GB)

See De Marsily, G., 1069

Hills, D.L., See Butlin, R.N., 1279, 839

Hinga, K.R., Disposal of high-level radioactive wastes by burial in the sea floor, 1129 (J;US)

Subseabed disposal program. Annual report, January-December 1980. Volume 1. Summary, y3s (R;US)

Subseabed Disposal Program. Annual report, January-September 1981 Volume I: summary, 953 (R;IJS)

Subseabed disposal program annual report, January-September 1981. Vulunie II. Appendices (princlpal investigator progress reports). Part 1 , 954 (R;US)

Subseabed dispusal program annual report, January-September 1981. Volume II. Appendices (principal investigator progress reports). Part 2 , 962 (R;US)

Hlava, P.F., See Dosch, R.G., 340

Hochstim, See Marriott, R., 486

Hocking, G., See St. John, C.M., 738

Hocking, W.H., See Hayward, P.J., 455

Hockman, J.N., See O'Neal, W.C., 1018

Iovlges, F.N., Sve Biadley, D.J., 851

Hodges, F.N, Westsik, J.H. Jr., Development of a backfill for containment of high-level nuclear waste, 1173 (BA;US)

Hodgkinson, D., Analytical models for thermal rock mechanics. 1217 (B:GB)

Hoeglund, S., See Torstenfelt, B., 1334

Hoenig, C., Densification studies of Synroc $D$ for high-level defense waste, 348 (R;US)

Hoenig, C.L., See Ackerman, F.J., 349, 353

Ste Camplell, J.H., 351, 352

See Newkirk, H.W., 400

Hoexter, D.F., Hydrogeologic testing of the E.J. Kubat borehole, San Juan County, Utah: utilization of a high pressure instrumented flow control system, 537 (RA;US)

Hoffman, T.L., Evaluation of stainless steel tank corrosion in ICPP high-level radioactive waste service, 203 (R;US)

Hofmann, B., See Mueller, R., 1282

Hofmann, P.L., Technology of high-level nuclear waste disposal. Advances in the science and engineering of the management of high-level nuclear wastes. Volume 1, 607 (R;US)

Hofrichter, E., Geological aspects of the final disposal of radioactive waste, 1072 (J;DE;In German)

Hogg, G.W., See Dickey, B.R., 1118

Hoh, J.C., See Steindler, M.J., 539

Hollister, C.D., Subseabed disposal of nuclear wastes, 1243 (J;US)

See Anderson, D.R., 1253

See Hinga, K.R., 1129

Syq Laiue, E.P., P31

Holloway, J.R., Geochemical behavior of supercalcine waste form: its stability in a basalt environment, 911 ( $R_{i}$ IIS)

Holmes, J.C., See Kolb, C.R., 814

Holton, L.K., Evaluation of process alternatives for solidification of the West Valley high-level liquid wastes, 436 (BA;US)

Evaluation of process alternatives for solidification of existing commercial high-level waste, 501 (J;US)

High-level waste solidification system for the Western New York State Service Center, 507 (J;US)

See Carrell, J.R., 315

See Wheelwright, E.J., 385

Hong, K.J., See Logan, S.E., 1386

Hoop, F.J., See Buchheim, B.K., 1040

Hoover, D.B., E-field ratio telluric traverses near Fortymile Wash, Nevada Test Site, Nevada, 1027 (R;US)

rilectrical studies at the propused Wahmonie and Calico Hills nuclear waste sitcs, Nevada Test Site, Nye Co., Nevada, 1023 (R;US)

See Senterfit, R.M., 1028

Hora, M., See Ninomiya, M., 258, 270

Horneber, A., Investigation on the oxidation state and the behaviur of molybdenum in silicate glass, 460 (BA;US)

Horton, R.M., See Welch, J.M., 416

Horwitz, F.P., Octyl(phenyl)-N,Ndiisobutylcarbamoylmethylphosphine oxide as an extractant for actinides from nitric acid waste, 146 (R;US)

Hossain, S., See Beckmann, H., 1373

Hossainini-1 lashemi, Ii., See Kays, W.M., 1122

Hough, A., See Hall, A.R., 1194

Hrovat, M., Highly dense graphite matrix: a new material for the conditioning of radioactive wastes, 358 (J;US)

Highly dense graphite matrix - a new material for the conditioning of radioactive wastes, 394 (J;US)

Hsieh, D., See Siskind, B., 733

Huang, J.C., Comparative risk assessments for the production and interim storage of glass and ceramic waste forms: defensc wastc processing facility, 1370 (R;US)

Huebner, H.W., See Schulz-Forberg, B., 1452

Huerta, M., See Sutherland, S.H., 32 
'lughes, A.E., See Burns, W.G., 463

Iugony, P., See Chotin, M.M., 432

See Moncouyoux, J.P., 228

Hultgren, A., Research programme on the conditioning of nuclear power waste. Progress report June 1981, 332 (R;SE)

Humblet, L., See Hendricks, J.P., 207

Hung, C., See Goldin, A.S., 1382

See Smith, C.B., 836

Hunter, R.L., Consequences of basaltic volcanism through a high-level waste repository, 1399 (J;US)

Preliminary scenarios for consequence assessments of radioactive-waste repositories at the Nevada Test Site, 948 (R;US)

Hunter, T.O., Nuclear-waste disposal: technical issues and status, 979 (R;US)

Summary of research and development for disposal of US Defense wastes in the Waste Isolation Pilot Plant (WIPP), 983 (R;US)

WIPP research and development program: providing the technical basis for defense waste disposal, 989 (R;US)

See Matalucci, R.V., 940

Huschka, H., See Hrovat, M., 358, 394

Hustrulid, W., Potential host media for a high-level waste repository, 1132 (J;GB)

Hustrulid, W.A., Mining technology development in crystalline rock, 613 (RA;US)

\section{I}

Ikeda, T., See Masuda, S., 1244, 1273

Illi, H., R and D projects and studies: concerning the interim and terminal storage of radioactive wastes, 1281 (TG;US)

See Warnecke, E., 671, 681

Indusi, J.P., See Majumdar, D., 73

Irish, E.K., See Wheelwright, E.J., 385

Isaacson, R.E., Scientific basis for establishing dry-well-monitoring frequencies, 920 (R;US)

Iseghem, $\mathbf{P}$. van, Interaction of vitrified high level waste with clay environment, 274 (RA;DE)

Isherwood, D., Nuclear-waste disposal in geologic repositories, 1014 (R;US)

Ito, $\mathbf{K}$., Coprecipitation of radioactive technetium with ferrous hydroxide, 1443 (J;JP;In Japanese)

Itsukaichi, T., See Kiyose, R., 1245

Ittner, T., See Torstenfelt, B., 1334

Iwata, A., See Abe, T., 389

Izahara, $\mathbf{S}$., Preparation and microstructural characterization of silicate and borosilicate glasses containing cerium or plutonium, 130 (R;BE)

\section{$\mathbf{J}$}

Jackson, D.D., HLW leaching mechanisms - program. Semiannual status report, May 1982, 847 (RA;US)

Status report on dissolution model development, 1021 (R;US)

See Cheung, H., 1007

Jackson, M.P.A., See Dix, O.R., 566

Jackson, R.L., See Leonhart, L.S., 880
Jacobi, R.D., See Hayes, D.E., 957

Jacobs, G.K., Radionuclide solubility and transport in basalt, 875 (R;US)

Waste package performance assessment: the importance of the very near-field physicochemical environment, 1223 (J;US)

See Anderson, W.J., 887

See Crisman, D.P., 904

See Deju, R.A., 890

See Early, T.O., 1329

See Salter, P.F., 1354

Jacobsen, F.K., See Orvis, D.D., 808

Jacobsson, A., See Pusch, R., 1174

Jacquet-Francillon, $N_{\text {., Attempt to assess }}$ the long-term crystallization rate of nuclear waste glasses, 457 (BA;US)

See Morlevat, J.P., 269

Jakubick, A.T., Permeability monitoring technique of the near-field and far-field interface openings, 1346 (BA;US)

Jansen, G., See Gureghian, A.B., 1325 , 1361

Jansma, P.E., Principal facts of gravity stations with gravity and magnetic profiles from the southwest Nevada Test Site, Nye County, Nevada, as of January 1982, 1026 (R;US)

Jantzen, C.M., Ceramic nuclear waste forms, 259 (RA;DE)

Ceramic nuclear waste forms, 271 (RA;DE)

See Harker, A.B., 153

Jaquet-Francillon, N., See Morlevat, J.P., 257

Jardine, L.J., Final report of experimental laboratory-scale brittle fracture studies of glasses and ceramics, 129 (R;US)

Respirable fines produced by impacts of simulated alternative high-level waste materials, 419 (BA;US)

See Bates, J.K., 127, 128

See Mecham, W.J., 420

See Northrup, C.J., 945

Jaritz, W., See Beckmann, H., 1373

Jaskot, G.F., See Johnson, A.J., 333

Javandel, I., Analysis of temperature data from the full-scale heater experiments at Stripa, 710 (RA;US)

Jenkins, D.M., See Holloway, J.R., 911

Jenks, G.H., Brine migration in salt and its implications in the geologic disposal of nuclear waste, 829 (R;US)

Jennekens, J., Scope and nature of the problem of high level nuclear waste disposal, 1430 (R;CA)

Jennings, A.S., See Gordon, D.E., 198

Jensen, B.S., Migration of radionuclides with ground water: a discussion of the relevance of the input parameters used in model calculations, 1350 (BA;US)

Jensen, C.D., See Harvey, K.D., 1157

Jensen, P., See Hasted, F., 1142

Jenson, G.A., Recovery of noble metals from fission products, 324 (R;US)

Jenson, K.E.L., Dissolution rate of salt domes on the basis of interpretation of measured salinity profiles, 1209 (BA;US)

Jimenez, R.F., See Baxter, J.T., 882, 883

Jockwer. N., Gas production and liberation from rock salt samples and potential consequences on the disposal of highlevel radioactive waste in salt domes, 1165 (BA;US)
Jockwer, Norbert, Transport phenomena of water and gas components within rock salt in the temperature field of disposed high level waste, 1272 (BA;XN)

Johnson, A.J., Commercial and Defense nitrate wastes and processing alternatives, 333 (R;US)

Johnson, J.R., Dose conversion factors used in the current Canadian high level waste disposal assessment study, 1427 (J;GB)

Johnson, J.S., See Kelmers, A.D., 1304

Johnson, K.D.B., Disposal of high-level radioactive wastes, 1286 (BA;GB)

Johnson, K.S., See Gonzales, S., 609

See Lomenick, T.F., 828

Johnson, L.H., See Melnyk, T.W., 1299

Johnson, M.E., See Crowe, B.M., 1393

Johnson, P., See Blacic, J., 693

Johnson, R.L., Thermal analyses for a nuclear-waste repository in tuff using USW-G1 borehole data, 946 (R;US)

Thermo-mechanical scoping calculations for a high level nuclear waste repository in tuff, 933 (R;US)

Johnston, J.W., Summary report for the interlaboratory round robin on the MCC-1 static leach test method, 300 (R;US)

Johnston, P.D., See Olivier, J.P., 591

Johnston, R.G., See Palmer, R.A., 905

Johnston, R.J., Stability of ceramic waste forms in potential repository environments: a review, $564(\mathrm{R} ; \mathrm{US})$

Johnstone, J.K., Parametric study of the effects of thermal environment on a waste package for a tuff repository, 1271 (BA;XN)

Preliminary technical constraints for a repository in tuff, 977 (R;US)

System study and performance constraints for repository horizon selection at the Nevada Test Site, 1225 (J;US)

Jones, K.A., Occupational radiation exposure risk at a nuclear waste repository in basalt, 1389 (J;US)

Technical conservatism in the design and analysis of a nuclear-waste repository in basalt, 907 (R;US)

Jones, T.E., See Palmer, R.A., 905

Jordan, S.A., See Silva, A.J., 964

Jos, P.H., See Kearney, R.C., 114

Joshi, A.V., Hydrogeological conditions at Mors salt dome, 659 (RA;DK;In Danish)

Joy, D.S., See Peterson, R.W., 17

See Schneringer, P.E., 14

See Varadarajan, R.V., 556

See Wilmot, E.L., 35

Julien, H.L., Near-field salt temperatures resulting from storage of nuclear fuel assemblies in bedded salt, $1121(\mathrm{~J} ; \mathrm{NL})$

See Busch, J.S., 1120

See Ritchie, J.S., 1119

See Watson, D.L., 1123

Jurgensen, A.R., See Clark, D.E., 1188 


\section{$\mathbf{K}$}

Kare, J.L., See Gulden, T.D., 448

Kabele, T.J., See Koplick, C.M., 955

Kacoyannakis, J.F., See Holloway, J.R., 911

Kaczmarsky, M.M., See Waite, D.A., 1395

Kaegi, U., Study for dry storage within the framework of the Swiss waste disposal concept, 1407 (BA;DE;In German)

Kaestner, P.C., See Helton, J.C., 1316

Kahl, L., Hydrolytic durability of two highlevel waste oxide borosilicate glasses (gp 98/12 and gp 98/26), 524 (J;US)

Preparation and characterization of an improved borosilicate glass for the solidification of high level radioactive fission product solutions (HLW). Pt. 2, 277 (R;DE)

See Malzke, H., 145

See Saidl, J., 255, 267

Kalina, D.G., See Horwitz, E.P., 146

Kanetkar, S.M.. See Metson, J.B., 456

Kanka, J., See Kepak, F., 399

Kanno, T. See İto, K., 1443

Kaplan, L., See Horwitz, E.P., 146

Kaplan, M.F., Marking a nuclear waste repository: an archaelogist's perspective, 1095 (J;US)

See Ensminger, D.A., 1380

Karim, D.P., XPS valence state determination of $\mathrm{Np}$ and $\mathrm{Pu}$ in multicomponent borosilicate glass and application to leached 76-68 waste glass surfaces, 417 (BA;US)

Kawamura, S., See Ninomiya, M., 258, 270

See Wada, M., 481

Kawanlshi, N., See Ouka, K., 480

Kays, W.M., Calculation of media temperatures for nuclear sources in geologic depositories by a finite-length line source superposition model (FLLSSM), $1122(\mathrm{~J} ; \mathrm{NL})$

Kearney, R.C., Nuclear waste, the nation, and South Carolina: an update on the major issues, 114 (B;US)

Keen, N.J., Review of radioactive waste management programs in the United Kingdom, 1160 (BA;US)

Kelley, J.A., Borosilicate glass for immobilization of high-level SRP waste, 1105 (J;US)

Solidification of Savannah River plant high-level waste, 506 (J;US)

Seé Maher, R., 195

Kelly, J.E., Public education and participation, 960 (RA;US)

Kelly, K.L., Valence states of actinides in synthetic monazites, 1276 (BA;US)

Kelm, M., Chemical denitration of radioactive-waste solutions with formic acid. 531 (TG;US)

Kelman, J.A., See Harmon, K.M., 83

Kelmers, A.D., Evaluation of DOE radionuclide solubility data and selected retardation parameters: description of calculational and confirmatory experimental activities, 1304 (R;US)

Kelsey, P.V. Jr., See Schuman, R.P., 200

See Welch, J.M., 416

Kempe, T.F., See Martin, A., 466

Kepak, F., Removal of ${ }^{90} \mathrm{Sr}$, ${ }^{107} \mathrm{Cs}$, and ${ }^{106} \mathrm{Ru}$ from vent gases produced during solidification of high-level radioactive solutions, 399 (J;CS)

Kessler, J.H., See Dole, L.R., 293

See Kelmers, A.D., 1304

See Moore, J.G., 256, 268

Kessler, J.L., See Plodinec, M.J., 180

Kesson, S.E., See Ringwood, A.E., 250, 515

Ketchen, D.W., See Ganley, J.T., 642

See Morissette, R.P., 641

Kibbey, A.H., See Notz, K.J., 52

Kidd, R.W., See Oma, K.H., 511

Kienzler, B., Analysis of the growth of brine flow pathes in rock salt, 690 (R;DE;In German)

See Beckmann, H., 1373

See Smailos, E., 1432

Kierstead, J.A., See Levy, P.W., 543

Kikuchi, M., See Uttake, N., 470

Kildow, J.L., Sociopolitical aspects of a subseabed disposal program for high level nuclear waste, 965 (RA;US)

Kill, H., See Koelle, H.H., 645

Kilpatrick, L.L., See Lce, L.M., 173, 184, 187, 397, 4hX

Kim, K., Rock mass deformation properties of closely jointed basalt, 889 (R;US)

See Gregory, E.C., 886

See Lanigan, D.C., 881

Kimball, C.S., See Sandness, G.A., 871

Kincinas, J.E., See Steindler, M.J., 539

King, A., See Symonds, J.L., 1435

King, K.W., Study of surface and subsurface ground motions at Calico Hills, Nevada Test Site, 1029 (R;US)

King, M.S., See Majer, E.L., 1238, 1269

King, R.H., See Hustrulid, W.A., 613

King, T.A., See Leinen, M., 960

Kings, M.S., See Majer, E.L., 1233

Kinoshita, M., Preparation of glass containing high-level radioactive waste (simulated) - copper composite compacts by a continuous pressure sintering method, 479 (J;JP;In Japanese)

KInsey, D., See Bradbury, M.Il., 1359

Kipatsi, H., See Allard, B., 1351

See Torstenfelt, B., 1148, 1175

Kircher, J.F., Waste package materials testing for a salt repository: 1982 status report. 825 (R:US)

See McVay, G.L., 1307

Kirkwood, C.W., Screening for monitored retrievable storage facility locations, 39 (J;US)

Kirstein, B.E., See Lester, D.H., 806

Kitahara, Y., Application of computer system to environmental monitoring at PNC Tokai Works, 1450 (I; IP; In .Iapanese)

Kiyose, R., Basic study on storage of tritium waste, 1245 (J;JP; In Japanese)

Klafiky, R.W., See Levy, P.W., 610

Klamut, C.J., See Ahn, T.M., 284

Klasi, M.L., Far-field thermal analysis of a high level waste repository in tuff. Topical report RSI-0137, 942 (R;US)

Klein, M., Volatilization and trapping of ruthenium in high temperature processes, 141 (RA;US)

Klett, R.D., Systems overview of the subscabed disposal program, 587 (RA;US)

Systems overview of the Subseabed Disposal Program, 976 (R;US)

Klockars, C.E., See Landstroem, Q, 1349

Klotz, D., See Behrens, H., 1352
Kluk, A.F., Management of the DOE inventory of excess radioactively contaminated facilities, 1288 (BA;US)

Knabenschuh, J.L., Present status of the West Valley Demonstration Project. 435 (BA;US)

Knapp, M.R., See Fehringer, D.J., 1130

Knecht, D.A., Immobilization of Idaho Chemical Processing Plant high-level wastes, 240 (RA;DE)

Knotts, J.B. Jr., Fuel Cycle Conference Atomic Industrial Forum, Incorporated, 1267 (BA;US)

Knowlton, D.E., Status, direction, and critical issues of waste treatment technology, 330 (R;US)

See Siemens, D.H., 325

Knox, C.A., Initial operations in the remote laburatury-scale waste-ireatment facillty, 312 (R;US)

See Siemens, D.H., 325

Ko, S.C., See Kirkwood, C.W., 39

Koch, J.U., See Koelle, H.H., 645

Kocher, D.C., Models and risk from highlevel wasto disposal-the missing pieoes, 1090 (J;US)

Proceedings of the symposium on uncertainties associated with the regulation of the geologic disposal of highlevel radioactive waste, 726 (R;US)

Uncertainties associated with geologic disposal of high-level radioactive waste, 1367 (R;US)

Uncertainties in geologic disposal of high-level wastes: groundwater transpoft of radionuclides and radiological consequences, 737 (R;US)

Kocherhuus, J.G., See Nelson, R.A., 813

Kuelle, H.H., Appraisal of a target-relevant evaluation of alternative strategies for global radioactive waste disposal, 645 (R;DE;In German)

Koester, R., Thermal and thermomechanical effects in the repository storage of high level waste, 1083 (J;DE; In German)

See Beckmann. H., 1373

See Pudewills, A., 1166

See Smailos, E., 1432

See Stichel, W 689

Kohn, H.W., Pacing and logistics of highlevel waste disposal, 1047 (J;US)

Kolb, C.R., Quaternary geology of Rayburn's salt dome: North Louisiana Salt Dome Basin, 814 (R;US)

See Martinez, J.D., 57!

Kolba, V.M., See Steindler, M.J., 539

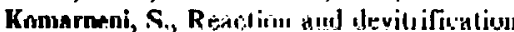
of a prototype nuclear-waste-storage glass with hot magnesium-rich brine, 289 (R;US)

Strontium-basalt reactions under nuclear waste repository conditions, 1152 (BA;US)

See Sasaki, N., 1153

Kominski, S.M., See Close, D.A., 1406

Konynfoburg, R.A., Radiation effects in SYNROC-D, 1053 (J;US)

Koplick, C.M., Subseabed disposal safety analysis, 955 (RA;US)

Koplik, C.M., See Ensminger, D.A., 1380

Korhin, G., See Wollenberg, H.A., 1111 , 714

Korthaus, E., See Beckmann, H., 1373

See Kienzler, B., 690 
See Mueller, R., 1282

See Pudewills, A., 1166

Koski, J.A., See Moss, M., 970

Koster, R., Method for solidifying aqueous radioactive wastes for noncontaminating storage, 379 (P;US)

Koutova, S., See Kepak, F, 399

Kovacs, W.J., See Gulden, T.D., 448

Krause, $K_{\text {., }}$ Final conditioning of high-level fission product solutions, $376(\mathrm{~J} ; \mathrm{DE} ; \mathrm{In}$ German)

Krause, W.B., Domal salt brine migration experiments at Avery Island, Louisiana, 621 (RA;US)

Krejsa, P., Intermediate, long term and final storagc of radioactive substances, 1234 (J;AT;In German)

See Grass, F., 382

Krichinski, A.M., NUMATH: a nuclear material holdup estimator for unit operations and chemical processes, $1408(\mathrm{~J} ; \mathrm{US})$

Krieg, R.D., See Branstetter, L.J., 943

Krivokhatskii, A.S., See Bryzgalova, R.V., 921

Krug, A.D., See Lehnhoff, T.F., 1169

Krumbansl, J.L., Near-field interactions, 929 (R;US)

See Johnstone, J.K., 1271

Kubota, M., Decomposition of oxalic acid with nitric acid, 465 (J;C.H)

Development of partitioning method cold and semihot experimental works with partitioning testing apparatus, 225 (R;JP;In Japanese)

Kuehnel, R.R., See Bokelmann, R.F., 43

Kuhn, K.D., Main activities and achievements of the DWK within the joint development programme, 8 (RA;DE; In German)

Treatment and solidification of high active fission product solutions, 429 (BA:US)

Kuhn, W.L., Using process instrumentation to obviate destructive examination of canisters of HLW glass, 328 (R;US)

See Westsik, J.H. Jr., 841

Kulacki, F.A., See Blessch, C.J., 1260

Kulichenko, V.V., Results of the development of a single-stage glass solidification method, 496 (BA;JP;In Japanese)

Kumar, M.B., See Martinez, J.D., 571

Kumer, L., Relations of equivalence of conditioned radionctive waste, 112 (BA;US)

Kunz, W.E., See Close, D.A., 1406

Kuznetsov, B.S., See Lazarev, L.N., 357

Kuznetsov, Yu.V., See Lazarev, L.N., 357

Kyrs, M., Radioactive waste processing and disposal, 7 (RA;CS; In Czeeh)

\section{L}

Lackey, W.J., Sol-gel-derived waste forms, 245 (RA;DE)

See Stinton, D.P., 138, 386, 387

Lahoda, E.J., Synthesis of alkoxide glass formers for nuclear waste immobilization, 438 (BA;US)

See Gockley, G.B., 370

Laire, E., Site qualificátion activities, 958 (RA;US)
Laine, E.P., Program criteria for subseabed disposal of radioactive waste: site qualification plan, 934 (R;US)

Lakey, L.T., ICPP waste calcining facility safety analysis report, 219 (R;US)

International fuel-cycle and wastemanagement technology-exchange activities sponsored by the United States Department of Energy: FY 1982 evaluation report, 85 (R;US)

Lam, D.J., See Karim, D.P., 417

Lambert, S.J., See Christensen, C.L., 938

Lameille, J.M., See Petit, J.C., 1205

Lamont, N., Environmental impact of nuclear power. Opening address, 1246 (J;GB)

Lamoreaux, G.H., Evaluation of proposed methods of radioactive material container tie-down design for truck transport, 44 (J;US)

Landau, M.S., Information report on state legislation, volume 8, number 5,1414 (R;US)

Landstroem, O., Comparison of in-situ radionuclide migration studies in the Studsvik area and laboratory measurements, 1349 (BA;US)

Landstrom, D.K., See Murthy, K.S., 1384

Lane, D.L., See Palmer, R.A., 905

See Wood, M.I., 902

Lang, H., See Behrens, H., 1352

Langevin, Y., See Dran, J.C., 423 See Petit, J.C., 1205

Langkopf, B.S., Thermal analysis of nuclear waste emplacement in welded tuff, 930 (R;US)

See Tyler, L.D., 1224

Langmuir, D., See Mullcr, A.B., 973

Lanigan, D.C., Determination of rock mass deformation modulus in closely jointed Columbia River basalt, 881 (R;US)

Lanza, F., Critical review of radiation effects on borosilicate glasses, 261 (RA;DE)

Influence of a backfilling material on borosilicate glass leaching, 1197 (BA;US)

See Avogadro, A., 1195

Lappin, A.R., See Johnstone, J.K., 1225

Larker, H., Method to encapsulate radioactive material, 478 (TG;DE;In German)

Larker, H.T., Solidification of radioactive waste forms and waste containment by the H I P O W process, 232 (RA;DE)

Larsen, G., Geolngy of the Mors salt dome, 658 (RA;DK;In Danish)

Larson, D.E., Assessment of power reactor waste immobilization by vitrification. Final report, 206 (R;US)

See Holton, L.K., 436, 501

Larson, L.T., See Bell, F..J., 763

Larsson, S.A., See Landstroem, O., 1349

Laude, F., Fracture appraisal of large scale glass blocks under realistic thermal conditions, 459 (BA;US)

Laughton, A.S., Can the oceans provide acceptable disposal sites for high-level radioactive waste, 1287 (BA;GB)

Iazarev, L.N, Ușe of glass-metal compounds and ceramic materials to condition hot wastes, 357 (TG;US)

Leaming, G.F., Evaluation of water resource economics within the Pasco Basin, Washington, 1429 (R;US)
Lee, B.S., See Ahn, T.M., 1066, 284, 545, 729

See Dayal, R., 283

Lee, L.M., Precipitation process for supernate decontamination, 173 (R;US)

Precipitation process for decontamination of water-soluble SRP radioactive waste, 184 (R;US)

Precipitation process for decontaminating water-soluble SRP radioactive waste, 187 (R;US)

Precipitation process for decontamination of water soluble SRP radioactive waste, 468 (J;US)

Precipitation-adsorption process for the decontamination of nuclear waste supernates, 397 (P;US)

Small-scale processing of actual Savannah River high-level waste, 503 (J;US)

Lee, S.Y., Technetium behavior in sulfide and ferrous iron solutions, 553 (R;US)

Leferre, J., Nuclear waste management in France, 368 (J;US)

Lefevre, J.F., Nuclcar waste management policy in France, 92 (J;US)

Lehnhoff, T.F., Modeling approach to determine short- and long-term thermal and thermomechanical effects of waste emplacement in a repository in basalt, 1169 (BA;US)

Lehnhoff, T.L., See Topcubasi, A.F., 876

Lehto, J., See Heinonen, O.J., 247

Leidinger, B.J.G., See Bokelmann, R.F., 43

Leinen, M., Pacific site assessment, 960 (RA;US)

Leonhart, L.S., Groundwater flow and transport characteristics of flood basalts as determined from tracer experiments, 880 (R;US)

Monitoring land and water uses in the Columbia Plateau using remote-sensing computer analysis and integration techniques, 865 (R;US)

Leopold, D., See Murso, H., 1167

Lester, B.H., See Ross, B., 746

Lester, D.H., Waste package performance assessment, 1274 (BA;XN)

Waste package performance evaluation, 806 (R;US)

Lester, R.K., See Malbrain, C., 1070

Lethco, A.J., See Maher, R., 195

Leukel, R., See Koelle, H.H., 645

Lever, D., See Bradbury, M.H., 1359

Levi, H.W., See Beckmann, H., 1373

Levins, D.M., See Lewis, R.A., 375

See Reeve, K.D., 396, 405, 415, 418 , 472, 493, 510

Levy, P.W., Radiation damage studies on synthetic $\mathrm{NaCl}$ crystals and natural rock salt for radioactive waste disposal applications, 610 (RA;US)

Radiation induced F-center and colloid formation in synthetic $\mathrm{NaCl}$ and natural rock salt: applications to radioactive waste repositories, 543 (R;US)

Levy, W.P., See Silva, A.J., 964

Lewis, A.E., See Schwartz, L.L., 1296

Lewis, J.B., Review of radio-nuclide migration in geological formations, 1337 (J;GB)

Lewis, R.A., Analysis for silicon in solution in high leyel waste glass durability studies, $375(\mathrm{~J} ; \mathrm{GB})$

Glass as a high level waste matrix: a review of the variables, 220 (RA;AU) 
Temperature, pressure and time dependence of dissolution rates of high level radioactive waste glasses, 653 (RA;AU)

See Cousens, D.R., 1203, 1237

Libby, L.M., Evaluation of an international, perpetual, and retrievable facility for storage of vitrified radioactive waste, 97 (J;US)

Liberman, M.S., See Croff, A.G., 76

Lichtner, P.C., See Apps, J.A., 1318

Likar, V.F., See Brown, R.K., 1161

Liljenzin, J.O., Equipment, operation and some results from a hot test of the CTH actinide separation process, 243 (RA;DE)

Lindberg, J.W., See Sandness, G.A., 871

Lindblom, U., Nuclear waste disposal: can we rely on bedrock, 1137 (B;GB)

Link, R.L., See Logan, S.E., 1386

Linker, G., See Matzke, H., 36.5

Lintern, B.C., See Mather, J.D., 1091

Lipkin, J., See Klett, R.D., 587, 976

Lipner, M.II., Nuclear criticality safety analyses for the Waste Isolation Pilot Plant project, 1258 (J;US)

Litovitz, T.A., See Macedo, P.B., 380, 475

Lodding, A., See Hench, L.L., 1202

See Werme, L., 1200

Logan, S.E., Parametric studies of radiological consequences of basaltic volcanism, 1386 (R;US)

See Hunter, R.L., 1399

Loken, M.C., See Tammemagi, H.Y., 1218

Lokken, R.O., Characterization and testing of basalt glass and glass ceramic waste forms, 313 (R;US)

Luman, J.M., See Levy, P.W., 543, 610

Lomenick, T.F., Regional geulogical assessment of the Devonian-Mississippian shale sequence of the Appalachian, Illinois, and Michigan basins relative to potential storage/disposal of radioactive wastes, 828 (R;US)

Long, J.C.S., See Carnahan, C.L., 715

Lorenzo, D.K., Canister arrangement for storing radioactive waste, 1106 (P;US)

Luner, C., See Gilbert, T.L., 559

Lust, L.F., See Bray, L.A., 214

Lutze, W., Development of borosilicate glass for the vitrification facility PAMELA, 253 (RA;DE;GE)

Development of the borosilicate glass for the vitrification plant PAMELA, 265 (RA;DE;In German)

Development of borosilicate glass for the vitrification plant PAMELA, 530 (TG;US)

Scientific basis for nuclear waste management V, 1187 (B;US)

See Altenhein, F.K., 1191, 1333

See Ewing, R.C., 1213

See Horneber, A., 460

Lytle, F.W., See Greegor, R.B., 1215

\section{M}

Macebo, P.B., Flow model for the kinetics of dissolution of nuclear waste forms; a comparison of borosilicate glass, SYNROC and high-silica glass, 1192 (BA;US)

Macedo, P.B., Fixation of radioactive materials in a glass matrix, $380(\mathrm{P} ; \mathrm{AU})$

Fixation of radioactive materials in a glass matrix, 475 (P;CA)

See Barkatt, A., 1059

Machiels, A.J., Functional dependence of leaching on the surface area-to-solution volume ratio, 1067 (J;US)

Madia, W.J., Status of the ONWI salt project, 1226 (J;US)

Madsen, M.M., See Wilmot, E.L., 35

Madsen, N.K., See Sutcliffe, W.G., 809

Maher, R., Defense Waste Processing Facility: a renute provess for solidification of Savannah River Plant higk: level waste, 195 (R;US)

See Kelley, J.A., 506

Majer, E.L., Monitoring an underground repository with modern seismological methods, 1233 (J;US)

Monitoring an underground repository with modern seismological methods, 1238 (J;US)

Monitoring an underground repository with modern seismological methods, 1269 (J;US)

Major, A., See Ringwood, A.E., 515

Majumdar, D., Nuclear-waste-management technical support in the development of nuclear-waste-form criteria for the NRC. Task 3. Waste inventory review, 73 (R;US)

Makino, M., See Masuda, S., 1244, 1273

Malasek, E., Safe disposal of radioactive wastes from nuclear power plants, 656 (RA;CS;In Czech)

Malbrain, C., Impact of thermal constraints on the optimal design of highlevel waste repositories in geologic media, $1070(\mathrm{~J} ; \mathrm{NL})$

Malow, G., Leaching of solidified highly radioactive decay products: overview, 249 (R^;DE;GE)

Mechanisms for hydrothermal leaching of nuclear waste glasses: properties and evaluation of surface layers, 1189 (BA;US)

Mamelle, J., Progress of the vitrification of HLW in France, 525 (J;US)

Manaktala, H.K., See Majumdar, D., 73

Manara, A, Structural effects of radiatioun damage in silica based glasses, 464 (BA;US)

See Antonini, M., 1154

See Lanza, F., 261

Mangold, D.C., See Wang, J.S.Y., 1064, 707

Manion, W.J., Waste management and transportation, 115 (BA;US)

Manm, B., See Mills, M., 1375

Mano, T., See Masuda, S., 1273

Margat, J., See Barbreau, A., 1186

Marine, I.W., Use of radiogenic noble gases for dating groundwater, 617 (RA;US)

Marples, J.A.C., Chemistry in the development of nuclear power, 5.(BA;GB)
See Burns, W.G., 463

See Dalton, J.T., 251

See Hall, A.R., 1194

Marriott, R., Treating nuclear waste, 486 (P;AU)

Marsh, G.P., Materials for high-level waste containment, $1080(\mathrm{~J} ; \mathrm{GB})$

Marshall, W., UK view of the management of high-level waste, $1103(\mathrm{~J} ; \mathrm{XA})$

Marsily, G. de, See Barbreau, A., 1186

Marter, W.L., Relative hazard of DWPF reference glass canister ICRP 2 vs ICRP 30, 1371 (R;US)

Martin, A., Management of vitrified high level radioactive waste, 466 (BA;GB)

Martin, A.B., See Recht, H.L., 513

Martinez, J.D., Investigation of the utility of Gulf Coast salt domes for the storage or disposal of radioactive wastes, 571 (R;US)

Salt domes - the paot and present, keys to the future, 1179 (BA;GB)

Martinez, M.J., See Eaton, R.R., 988

Marx, G., See Murso, H., 1167

Mason, G.W., See Horwitz, E.P., 146

Masuda, S., Assessment of barrier effects on isolation system performance of geologic disposal of high level waste, 1273 (BA;XN)

Disposal of high level wastes, 1244 (J;JP;In Japanese)

See Pigford, T.H., 1249

Mataloni, P., See Cao, S., 234

Matalucci, R.V., Waste Isolation Pilot Plant (WIPP) research and development program: in situ testing plan, March 1982, 940 (R;US)

Mather, J.D., Geological disposal of highlevel radioactive waste - a review of the Institute of Geological Sciences' research programme, 1091 (J;GB)

Matsumoto, K., See Ooka, K., 521

See Sano, A., 471

Matsumulu, T.E., See Ouka, K., 521

Matthews, S.C., See Madia, W.I., 12.26

Mattson, E., Canister materials proposed for final disposal of high level nuclear waste, 685 (RA;DE)

Mattsson, E., Canister materials proposed for final disposal of high level nuclear waste - a review with respect to corrosion resistance, 675 (RA;DE)

Mattsson, H., See Olefjord, I., 1176

Matzke, H., Analysis of thin leaching layers and fracture mechanics of irradiated borosilicate glass for solidification of high-level fission product solutions (HAW), 365 (BA;DE;In German)

Effect of composition and radiation on the Hertzian indentation behavior of nuclear waste glasses, 145 (R;US)

See Rnubort, J.L., 143

See Routbort, J.L., 1434

Maurer, C.A., See Clark, D.E., 1188, 1193

Maurette, M., See Dran, J.C., 423

Mawson, C.A., Geologic disposal and the taxpayer: the problem of the experts, 1403 (BA;IN)

Maxwell, D.E., Heating effects on groundwater flow through a repository fracture system, 120 (J;US)

MeCabe, W.M., See Gregory, E.C., 886

McCallum, R.F., Waste-Mixes Study for space disposal, 815 (R;US)

See McKee, R.W., 308, 309 
McCarthy, G.J., See Komarneni, S., 289

IcClain, W.C., See Gnirk, P.F., 1136

See Klasi, M.L., 942

.McClure, J.D., See Shirley, C.G., 20, 33

McClure, L.W., See McDonald, F.N., 287

McCombie, C., See Hadermann, J., 1348

McCoy, H.E., Concept of a canister for high-level radioactive waste, 1158 (J;US)

Studies of waste-canister compatibility, 832 (R;US)

McCray, J.A., Alternative fuels and heating methods for calciner process heat, 204 (R;US)

McCright, R.D., See Russell, E.W., 1020

McDonald, F.N., Attrition, elutriation, and growth of particles produced in fluidized-bed waste calciners, 202 (R;US)

Evaluation of the safety aspects of the design and operation of temporary/ mobile radioactive-waste solidification systems, 287 (R;US)

McDonell, W.R., Comparison of SRP highlevel waste disposal costs for borosilicate glass and crystalline ceramic waste forms, 635 (R.US)

See Benjamin, R.W., 629

McElroy, J., See Crandall, J.L., 191

McElroy, J.L., Waste vitrification: a historical perspective, 317 (R;US)

Waste-management $R$ and $D$ for the nuclear fuel cycle, 311 (R;US)

See Burkholder, H.C., 359

See Crandall, J., 632

McEvilly, T.V., See Majer, E.L., 1233 , 1238,1269

McEwen, T.J., Fracture analysis of crystalline rocks: field measurements and field geomechanical techniques, 638 (R;GB)

McGarrah, J.E., See Ames, L.L., 868

McGoff, D.J., West Valley high-level waste solidification program, 500 (J;US)

McGuire, P.L., See Nelson, D.C., 694

McIntosh, W.H., See Brown, M.L., 589

McIntyre, N.S., See Alın, T.M., 284

See Dayal, R., 283

McKee, R.W., Cesium and strontium fractionation from HLW for thermal-stress reduction in a geologic repository, 327 (R;US)

Economic comparison of crystalline ceramic and glass waste forms for HLW disposal, 863 (R;US)

Systems study of the feasibility of highlevel nuclear-waste fractionation for thermal-stress control in a geologic repository: main report, 308 (R;US)

Systems study of the feasibility of highlevel nuclear waste fractionation for thermal stress control in a geologic repository: appendices, 309 (R;US)

See McCallum, R.F.; 815

McKenzie, D.E., See Grantham, L.F., 144 , 149

McKinley, I.G., Distribution coefficient (Kd) concept and its applicability to studies of radionuclide migration in geological media, 1313 (R;GB)

Radionuclide retardation during transport through fractured granite, 1355 (BA;US)

See Chapman, N.A., 1151

See West, J.M., 1357

McKisson, R.L., Commercial high-levelwaste management: options and eco- nomics. A comparative analysis of the ceramic and glass waste forms, 150 (R;US)

See Grantham, L.F., 144, 149

McLaren, L.H., Radioactive waste processing and disposal: a bibliography, 158 (R;US)

Radioactive waste inventories and projections, 62 (R;US)

McNatt, F.G., Savannah River Plant waste-tank inspection manual, 633 (R;US)

MeSweeney, T.I., NWTS program waste projection data needs, 1086 (J;US)

See Peterson, R.W., 17

See Waddell, J.D., 769

McVay, G.L., Effects of radiation on the leaching behavior of nuclear waste forms, 1285 (J;US)

Elemental release from glass and spent fuel, 1307 (RA;US)

Review of recent PNL research activities related to glass leaching mechanisms, 846 (RA;US)

See Bradley, D.J., 854

See Karim, D.P. 417

See Pederson, L.R., 862

See Rai, D., 1336

See Shade, J.W., 860

McVey, D.F., See Krumhansl, J.L., 929

Means, J.L., Importance of organic compounds in ground water as radionuclide-mobilizing agents, 1324 (R;US)

Mecham, W.J., Methodology for characterizing brittle fracture of solid waste forms in accidental impacts, 420 (BA;US)

See Jardine, L.J., 129, 419

Mehling, O., See Gruen, M., 1394

Meile, L.J., See Johnson, A.J., 333

Mellen, J.B., See Schneider, R.F., 196

Mellinger, G.B., See Bjorklund, W.J., 331

See Jenson, G.A., 324

Mello, V.E., See Dunson, J.B. Jr., 169

See Gould, T.H. Jr., 174

Melnyk, T.W., High-level waste glass field burial tests at CRNL: the effect of geochemical kinetics on the release and migration of fission products in a sandy aquifer, 1299 (R;CA)

Memmert, G., Evaluation of barriers in a final storage site for radioactive wastes, 674 (RA;DE)

Evaluation of the barriers in an ultimate storage for radioactive waste, 684 (RA;DE;In German)

Mendel, J.E., Characterization of high-level waste forms, 1099 (J;US)

Evaluation of conditioned high-level waste forms, 326 (R;US)

Materials Characterization Center workshop on leaching mechanisms of nuclear waste forms, May 19-21, 1982, Gaithersburg, Maryland. Summary report, 82 (R;US)

Waste glasses: requirements and characteristics, 322 (R;US)

Workshop on the leaching mechanisms of nuclear-waste forms, Ocluber 27-28, 1981. Summary report, 81 (R;US)

Mensing, R.W., See Buddemeier, R.W., 344

Mercer, B.W., See Nelson, J.L., 211
Mercer, J.W., Parameters and variables appearing in repository-siting models, 745 (R;US)

See Ross, B., 746

See Thomas, S.D., 740

Merlin, S., See Cameron, W.A., 437

Merriam, D.F., See De Marsily, G., 96

Merson, T.J., See Nelson, D.C., 694

Merz, E, Overview on product forms, 252 (RA;DE;In German and English)

Overview on product forms, 264

(RA;DE;In German)

Realizing the geological final storage of radioactive wastes, $1125^{\circ}$ (J;DE; In German)

See Halaszovich, S., 414, 440

See Odoj, R., 226, 263

See Schneider, K.A., 1369

Metlay, D., Institutional aspects of radioactive waste management, 1437 (J;US)

Metson, J.B., Leaching of natural and synthetic sphene and perovskite, 456 (BA;US)

Metz, W.C., See Hill, D., 1071

Michel, M.C., See Apps, J.A., 1318

Michelsen, O.B., See Liljenzin, J.O., 243

Miettinen, J.K., See Heinonen, O.J., 247

Miller, M., See Shirley, C.G., 20, 33

Miller, M.M., Subseabed nuclear waste disposal: conceptual ship description, 31 (RA;US)

Miller, N.E., See Stahl, D., 751, 752

See Wurm, K.J., 804

Mills, M., Parameters and variables appearing in radiological assessment codes. Final report, 1375 (R;US)

Summary of computer codes for radiological assessment, 1376 (R;US)

Minner, D.F., See Smock, R.W., 99

Mirschinka, $\mathbf{V}_{\text {. Investigations of suitable }}$ metallic container materials for HAW solidification, 371 (J;US)

See Dix, S., 229

Misplon, M.A., See Rickertsen, L.D., 831

Mitchell, T.H., See Bergstrom, K.A., 901

Miyazaki, K., See Mizuno, F., 483

Mizia, R.E., See Hoffman, T.L., 203

Mizuno, F., Method of solidifying radioactive wastes, 483 (P;JP;In Japanese)

Moak, D.P., See Kircher, J.F., 825

Mobbs, S.F., See De Marsily, G., 1069

Molecke, M.A., Comparison of brines relevant to nuclear waste experimentation, 994 (R;US)

Materials for high-level waste canister/ overpacks in salt formations, 1101 (J;US)

Materials for high-level waste canister/ overpacks in salt formations, 949 (R;US)

See Matalucci, R.V., 940

See Moss, M., 1065, 972

Moncouyoux, J.P., Marcoule vitrification shop: 30 months service, 228 (RA;DE;FR)

Mondy, L.A., Comparison of waste emplacement configurations for a nuclear waste respository in tuff. IV. Thermo-hydrological analysis, 996 (R,US)

Hydrology of sealing a repository in saturated tuff, 992 ( $R ;$ US)

Monfort, M.E., Three-dimensional model ing of the Nevada Test Site and vicin- 
ity from teleseismic p-wave residuals, 1022 (R;US)

Montague, K., Proposal for broader participation in radioactive waste management, $1135(\mathrm{~J} ; \mathrm{GB})$

Montague, P., See Montaguc, K., 1135

Montazer, P.M., See Hustrulid, W.A., 613

Moore, J.G., FUETAP concrete: an alternative radioactive waste host, 256 (RA;DE)

FUETAP concrete - an alternative radioactive waste host, 268 (RA;DE)

See Dole, L.R., 293

Moore, R.L., See Bray, L.A., 214

Morgan, J.M. Jr., Report of working meeting on fixation of radioactivity in stable, solid media at the Johns Hopkins University, June 19-21, 1957, 342 (R;US)

Morgan, M.T., See Dole, L.R., 293

See Moore, J.G., 256, 268

Morgan, P.E.D., Development of polyphase ceramics for the immobilization of high-level Defense nuclear waste, 151 (R;US)

See Harker, A.B., 152, 153, 395, 421, 446

See Jantzen, C.M., 259, 271

Morissette, R.P., Comparison of cask and dry well concepts for retrievable storage of high-level waste, 641 (R;US)

Economics of monitored retrievable storage of nuclear wastes, $\mathbf{4 0}(\mathrm{J} ; \mathrm{US})$

See Finch, W.C., 24

See Ganley, J.T., 642

Morlevat, J.P., Compared quantitative determination of nuclear waste glass crystallization rate by light microscopy and $x$ ray diffraction. 257 (RA;DE)

Compared quantitative determination of nuclear waste glass crystallization rate by light microscopy and $\mathrm{X}$-ray diffraction, 269 (KA;UE)

Momer, N.A, Nuclear power on unstable ground, 1182 (BA;GB)

Morris, F.A., See Smith, R.F., 105

Morris, J.B., Comparison of wet slurry and dry frit (crizzle) feeding of glassformers in pot vitrification, 242 (RA;DE)

Morris, O.M., Savannah River Plant overview, 69 (R;US)

Morrison, G.W., See Croff, A.G., 76

Moser, H., Soe Behrens, H., 1352

Mosley, W.C. Jr., See Slates, R.V., 167

Moss, M., Effects of composition, porosity, bedding plane oriontation, water content and a joint on the thermal conductivity of tuif, 970 (R,US)

Thermal conductivity of bentonite/ quartz high-level waste package backfill, 1065 (J;US)

Thermal conductivity of bentonite/ quartz high-level-waste-package backfill, 972 (R;US)

Motyka, T., Predicting transport requirements for radioactive-waste slurries, 22 (R;US)

Moyer, R.A., Comparative transportation risk assessment for borosilicate-glass and ceramic forms for immobilization of SRP Defense waste, 23 (R;US)

Muehlenweg, U., See Behrens, H., 1352

Mueller, H., See Koelle, H.H., 645

Mueller, R., Method of measurement and borehole probe to measure heat- induced borehole convergence, 1282

(P;DE;In German)

See Pudewills, A., 1166

Mueller, W., See Guber, W., 528

Muller, A.B., Formulation of an integrated physicochemical-hydrologic model for predicting waste-nuclide retardation in geologic media, 973 (R;US)

Munson, D.F., Transient creep model for salt during stress loading and unloading, 969 (R;US)

See Matalucci, R.V., 940

Murakami, T., Introduction of studies on high-level radioactive waste immobilization in synthetic minerals. Supercalcine and SYNROC, 224 (R;JP;In Japanese)

Muraoka, S., See Furuya, T., 666, 667

Murata, T., See Nagaki, H., 1045

See Sano, A., 471

Murphree, B.E., See Tennant, M.H., 407

Murphy, V., See Hardwick, W.H., 230

Murray, C.N., Development of an assessment methodology for the disposal of high-level radioactive waste into deep ocean sediments, 1115 (J;CH)

See Avogadro, A., 1360

See De Marsily, G., 1069

Murray, J., Uranium and nuclear energy: proceedings of the international symposium, 4th, 1979, 1 (B;GB)

Murray, R.L., Understanding radioactive waste, 79 (R;US)

Murso, $H$., Investigation of diffusion of $\mathrm{UO}_{2} \mathrm{Cl}_{2}$ in saturated $\mathrm{NaCl}$ solutions at various temperatures, 1167 (BA;US)

Murthy, K.S., Assessment of single-shell tank residual-liquid issues at Hanford Site, Washington, 1384 (R;US)

Musgrave, B.C., Assessment of rationale for development of improved waste formins, 498 (J;US)

Myer, L.R., See Cook, N.G.W., 619

Myhra, S., Surface analysis features of Synroc B and C, $1039(\mathrm{~J} ; \mathrm{CH})$

See Cousens, D., 654

See Cousens, D.R., 1203, 1237, 454

See Lewis, R.A., 220

See Smart, R.S.C., 1257

\section{$\mathbf{N}$}

Nachmilner, L., Equipment for radioactive waste solidification, 390 (J;CS;In Czech)

Nagai, H., See Okamoto, H., 431

Nagaki, H., Development of CT techuiques for integrity evaluation of vitrified solids, 1045 (J;US)

Nagata, I., See Kinoshita, M., 479

Nair, S., See Ray, A.K., 1170

Nakamura, H., See Kubota, M., 225

Nakramura, S., See Ninomiya, M., 258, 270

Nakamura, Y., See Nagaki, H., 1045

Napier, B.A., See Murthy, K.S., 1384

Napravnik, J., Calcination of liquid radioactive wastes. Part I. Technology of radioactive waste calcination, 361 (J;CS;In Czech)

Narita, O., See Kitahara, Y., 1450

Nash, K., Redox behavior, complexing, and adsorption of hexavalent actinides by humic acid and selected clays, 1247 (J;US)
Neal, J.T., Approach to resolution of geologic uncertainty in the licensing of a high-level-waste repository in tuff, 985 (R;US)

NNWSI regulatory status and plans, 1413 (RA;US)

NNWSI regulatory status and plans, 978 (R;US)

Nelson, D.C., Nevada Nuclear Waste Storage Investigations: expluratury shaft Phase I. Conceptual design report, 694 (R;US)

Nelson, J.L., Solid fixation of high-level radioactive waste by sorption on clinoptilolity-scouting studies, 211 (R;US)

See Westerman, R.E., 1100, 843

Nelson, P.H., Effect of radon transport in groundwater upon gamma-ray boroholo logs, 1422 (R;US)

Nelson, R.A., In situ and laboratory geotechnical test results from borehole GD-1 in southeast Utah, 813 (R;US)

Nelson, R.L., Seo Marples, J،A.C, 5

Nelson, R.S., See Burns, W.G., 463

Nens, B., See Trocellier, P., 1216

Neretnieks, I., Influence of microfissures in crystalline rock on radionuclide migration, 1185 (BA;GB)

Leach rates of high level waste and spent fuel: limiting rates as determined by backfill and bedrock conditions, 1345 (BA;US)

The movement of a redox front downstream from a repository for nuclear waste, 1032 ( $\mathrm{J} ; \mathrm{US})$

See Abelin, H., 1342

See Andersson, G., 1343

See Birgersson, L., 1341

See Rasmuson, A., 1344

See Skagius, K., 1168

Nervick, K.H.. See Houver, D.B., 1023

Nesbitt, H.W., See Metson, J.B., 456

Nesbitt, J.F., Alternative waste forms: pro cess feasibility, 477 (J;US)

See Slate, S.C., 842

See Smith, R.I., 857

Neumann, L., See Napravnik, J., 361

Neumann, W., Process for producing biologically noxious waste, particularly radioactive waste, wich can be stored, 519 (P;DE;In German)

Thermal conductivity measurements on granule-based composites, 1451 (J;GB)

See Blasius, E., 12

Newchurch, E.J., See Martinez, J.D., 571

Nericumh, W.F., Higlılevel waste dispusalitechnical and institutional siting factors remaining to be resolved, 1087 (J;US)

Status of site characterization in the Permian Basin, 590 (R^;US)

Newkirk, H.W., Synroc technningy fnr immobilizing U.S. defense waste, 400 (J;US)

Newton, D.C., See Cooley, C.R., 1093

Ng, H.S., See Logan, S.E., 1386

Nickell, R.E., Design rules for containmen systems of nuclear spent fuel and high level waste transport packagings, 19

(RA;US)

See Charman, C.M., 1436

See Goldmann, K., 18

See Osborne, D.M., 558 
Ninomiya, M., Diopside glass-ceramic material for the immobilization of radioactive wastes, 258 (RA;DE)

Diopside glass-ceramic material for the immobilization of radioactive wastes, 270 (RA;DE)

Nitsche, H., See Edelstein, N., 812

Nogues, J.L., Comparative study of seven glasses for solidification of nuclear wastes, 1206 (BA;US)

Effect of $\mathrm{Fe}_{2} \mathrm{O}_{0} / \mathrm{ZnO}$ on two glass compositions for solidification on Swedish nuclear wastes, 341 (R;SE)

Effect of $\mathrm{Fe}_{2} \mathrm{O}_{0} / \mathrm{ZnO}$ on two glass compositions for solidification of Swedish nuclear wastes, 461 (BA;US)

Noonan, A.F., See Palmer, R.A., 905

Norby, B.C., See Hoffman, T.L., 203

Norman, N.A., Results of the occupational dose assessment in the conceptual reference repository description, 1388 (J;US)

Norris, A.E., Geochemistry studies pertaining to the G-tunnel radionuclide migration field experiment, 1426 (R;US)

Northrup, C.J., Alternate strategy for commercial high-level radioactive-waste management, 945 (R;US)

See Dosch, R.G., 340

Northrup, C.J.M., See Arnold, G.W., 140

Notz, K.J., Integrated data base for spent fuel and radwaste: inventories, 52 (R;US)

Radwaste inventories and projections: an overview, 77 (R;US)

Nowak, E.J., Backfill barriers for nuclear waste repositories in salt, 670 (RA;DE)

Backfill barriers for nuclear waste repositories in salt, 680 (RA;DE)

Diffusion of leached constituents in backfill barriers, 849 (RA;US)

Nunziato, J.W., See Eaton, R.R., 988

Nuttall, H.E., Analysis of diffusional ion transport in ocean sediments: subsea. floor disposal of radioactive waste, 1250 (J;US)

See Ray, A.K., 1170, 1340

See Travis, B.J., 1356

O'Farrell, T.P., 1982 biotic survey of Yucca Mountain, Nevada Test Site, Nye County, Nevada, 1311 (R;US)

See Collins, E., 1302, 1309, 1310

O'Hara, F.A., Nuclear-fuel-cycle education: Module 8. Waste management, 155 (R;US)

O'Neal, W.C., Design of a nuclear-waste package for emplacement in tuff, 1018 (R;US)

See Gregg, D.W., 1005

See Russell, E.W., 1020

Obrowski, W., See Weber, M., 1404

Odgers, I.L., National waste terminal storage conceptual reference repository description, 612 (RA;US)

Odoj, R., On-line measurements of the volatilization of ruthenium in a vitrification process, 422 (BA;US)

Proceedings of the international seminar on chemistry and process engineering for high-level liquid waste solidification, 226 (R;DE;In several languages)
Proceedings of the international seminar on chemistry and process engineering for high-level liquid waste solidification, 263 (R;DE;In German and English)

See Mirschinka, V., 371

Oertel, G. See Crandall, J.L., 191

Oesterle, F.P., Present status of the Gorleben site investigations, 1036 (J;US)

Offermann, P., See Pickering, S., 1196

See Richter, H., 1212

See Roubort, J.L., 143

See Wald, J.W., 1211

Ogard, A.E., Research and development related to the Nevada Nuclear Waste Storage Investigations. Progress report, October 1-December 31, 1982, 704 (R;US)

See Erdal, B.R., 706

See Wolfsberg, K., 705

Oguino, N., Sce Ooka, K., 480, 521

Ohnishi, N., See Okamoto, H., 431

Okamota, H., Development of high-level liquid waste solidification in PNC, 431 (BA;US)

Okamoto, T., See Kiyose, R., 1245

Olefjord, I., Analysis of the corrosion products formed on $\mathrm{Ti}$ and a $\mathrm{Ti}$-Pd alloy during exposure in hot water, 1176 (BA;US)

Oliveria, L.F., Projections of high-level waste production in Brazil, 102 (J;US)

Olivier, J.P., OECD Nuclear Energy Agency activities in high-level waste disposal, 591 (RA;US)

Olofsson, U., See Allard, B., 1351

See Torstenfelt, B., 1148, 1175, 1334

Olsen, C.R., See Kocher, D.C., 1367

Oma, K.H., Alternative waste form development - low-temperature pyrolytic carbon coatings, 511 (J;US)

In situ vitrification: application analysis for stabilization of transuranic waste, 307 (R;US)

See Buelt, J.L., 514

See Treat, R.L., 447

Ondracek, G., Method for producing molded bodies containing highly active radioactive wastes from glass granules embedded in a metallic matrix, 360 (P;US)

Process for producing formed parts containing high-activity waste and consisting of glass granules in a metal matrix, 523 (P;DE; In German)

See Bauer, C., 367

Ondrejcin, R.S., Prevention of stress corrosion cracking in nuclear waste storage tanks, 630 (R;US)

Stress corrosion cracking test with slow strain rate and constant current, 1293 (BA;US)

Ooka, K., Melting of glass by direct induction heating in ceramic container, 480 (J;JP;In Japanese)

Plant for solidification and storage of high-level radioactive waste, 521 (J;JP;In Japanese)

Oosaki, T., See Abe, T., 389

Orlowski, S., European Communities' R and $D$ activities on treatment and liandling of radioactive waste, 426 (BA;US)

Management of radioactive wastes in Europe (EC) history, philosophy and plans for the next five years, 108 (BA;US)

Ortiz, N.R., Technical assistance for regulatory development: review and evaluation of the EPA standard 40 CFR191 for disposal of high-level waste. Vol. 1, 750 (R;US)

See Chu, M.S., 747

See Chu, M.S.Y., 1368, 952

See Cranwell, R.M., 993

See Pepping, R.E., 731

Orvis, D.D., Guidebook for reliability, availability, and maintainability analysis of NWTS repository equipment. Volume II. Appendices, 808 (R;US)

See Frank, M.V., 1255

Osborne, D.M. Two- and three-dimensional dynamic analyses of DHLW casks, 558 (RA;US)

Oser, B, See Kelm, M., 531

Ostenak, C.A., See Whitty, W.J., 695

Oszuszky, F., See Kumer, L., 112

Otto, R., See Hoenig, C., 348

Oversby, V.M., Leaching studies on SYNROC at $95^{\circ} \mathrm{C}$ and $200^{\circ} \mathrm{C}, 1114$ (J;CH)

Properties of SYNROC C nuclear-waste form: a state-of-the-art review, 1011 (R;US)

See Ringwood, A.E., 250, 515

Owen, D.E., See Welch, J.M., 416

Oylear, J.M., See Andrews, W.B., 27

\section{$\mathbf{P}$}

Pacaud, F., See Jacquet-Francillon, N., 457

Palau, G.L., Consequences of radiation effects on zeolites during interim storage, 1230 (J;US)

Palciauskas, V.V., Characterization of drained and undrained response of thermally loaded repository rocks, 1110 (J;US)

Palmer, R.A., Characierization of reference materials for the Barrier Materials Test Program, 905 (R;US)

See Johnston, R.J., 564

Palmour, H. III, Sintering of SYNROC: case history for phase formatinn and densification of complex oxide systems, 473 (BA;NL)

Subsolidus sintering of SYNROC: 11 . Materials selections, process improvements, waste form evaluations, 281 (R;US)

See Solomah, A.G., 280

Panno, S.V., Post-emplacement monitoring, Subtask 1.2. Draft Technical Position. Vol. 2, 1319 (R;US)

Papouchado, L.M., See Kelley, J.A., 1105 See Lee, L.M., 503

Pardue, W.M., Development of a comparative analysis of potential repository salt sites, 1037 (J;US)

Parker, F.L., Waste treatment and disposal. Progress report, May-October 1963, 291 (R;US)

See Blanco, R.E., 290

See Feige, Y., 549

Partain, W.L., See Larson, D.E., 206

See Slate, S.C., 842

Partlett, J.W., See Williams, R.F., 1290

Patrick, W.C., See Brough, W.G., 37 
Pearce, D.W., Fixation of radioactive residues. Quarterly progress report, January-March 1961, 213 (R;US)

Research and development activities: fixation of radioactive residues. Quarterly progress report, April-June 1960, 210 (R;US)

Research and development activities: fixation of radioactive residues. Quarterly progress report, July-September 1960 , 212 (R;US)

Pearson, F.J., WAPPA: an integrated waste package performance assessment code, 1220 (J;US)

Peaudecerf, P, See Barbreau, A., 1186

Pedersen, A., Evaluation of waste disposal safety, 661 (RA;DK;In Danish)

Pederson, LR., Effect of gamma radiolysis on waste glass leaching on brines, 862 (R;US)

See McVay, G.L., 1285

See Shade, J.W., 860

Pendleton, M., Information of fice concept, 594 (RA;US)

Penrose, J., See Cousens, D.R., 221, 454

Pentinghaus, H., SYNROC through melting: thermal analysis, thermogravimetry and crystal chemical characterization of phases, 260 (RA;DE)

Thermal analysis, thermogravimetry and crystal chemical characterization of phases, 273 (RA;DE)

Pepping, R.E., Risk-analysis methodology for spent-fuel repositories in bedded salt. Final report, 731 (R;US)

Volume 2. A simplified analysis of a hypothetical repository in a basalt formation, 1416 (RA:IIS)

Volume 4. A simplified analysis of a hypothetical repository in a bedded salt formation, 1418 (RA;US)

See Chu, M.S., 747

See Chu, M.S.Y., 952

See Sicgcl, M.D., 982

Percival, C.M., See Klett, R.D., 587, 976

Perez, J.M. Jr., Design and performance of feed-delivery systems for simulated radioactive waste slurries, 306 (R;US)

Effects of cracks on glass leaching, 126 (J;US)

Perge, A., Historical overview, 53 (RA;US)

Permar, P.H., Coordination meeting of high-level waste technology and national waste terminal storage programs, October 21-22, 1981, 624 (R;US)

See Gordon, D.E., IU8

Perry, D., See Apps, J.A., 1318

Perry, F., See Crowe, B., 699

Persson, G., See Liljenzin, J.O., 243

Persson, O., See Landstroem, O., 1349

Pescatore, C., See Machiels, A.J., 1067

See Sastre, C., 546

Pestorius, T., Legislative and political aspects of waste disposal, 1411 (RA;US)

Petek, M., See Sales, B.C., 554

Peters, Lo, See Izahara, S., 130

Peters, P.E., SYNROC powder preparation: preliminary fluid bed tests in a cold-flow unit, 345 (R;US)

See Ackerman, F.J., 349

See Campbell, J.H., 352
Peters, R.D., Fracturing of simulated highlevel waste glass in canisters, 1117 (J;NL)

Peters, R.R., Thermal response to emplacement of nuclear waste in long, horizontal boreholes, 986 (R;US)

See Johnstone, J.K., 1225

Peterson, J.M., Radiological risks for the alternatives for the long-term management of high-level wastes at West Valley, 1261 (J;US)

See Gilbert, T.L., 559

Peterson, R.W., Estimated transportation cost and risk for operation of a nuclear waste repository at candidate sites, 17 (RA;US)

See McSweeney, T.I., 1086

See Varadarajan, R.V., 556

Petit, J.C., Leaching behavior of a simple borosilicate glass in a confined environment, 1205 (BA;US)

See Dran, J.C. 423

Petrie, G.M., New look at forecasting, 87 (R;US)

Phillips, S., See Gonano, L., 741

Pickering, S., Leaching of actinides from simulated nuclcar waste glass, 1196 (BA;US)

See Matzke, H., 365

Pickett, J.B., See Dunson, J.B. Jr., 169

Pieraggi, A., See Chotin, M.M., 432

See Moncouyoux, J.P., 228

Pierce, B., High-level radioactive wste management: a means to social consensus, 542 (R;US)

Pierce, B.L., See Hill, D., 1071

Pietz, J., See Anderson, H.M., 1331

Pigford, T., Geological disposal of radioactive waste, 1113 (J;US)

Pigford, T.H., Possibility of high level waste underground disposal, 1249 (J;JP;In Japanese)

Soe Chambre, P.L., 1228, 1338

See Choi. J.. 1401

See Ting, $\overline{\mathrm{D}}$., 1362

See Ting, D.K., 1339

Pillay, K.K.S., See Palau, G.L., 1230

See Whitty, W.J., 695

Pippin, L.C., Cultural resources ovcrvicw for the Nevada Nuclear Waste Storage Investigations, Nevada Test Site, Nye County, Nevada, 767 (R;US)

Pirk, H., See Hrovat, M., 394

Pirk, H.A., See Hrovat, M., 358

Pitman, S.G., Enviromechanical testing of Ti-grade 2 and Ti-grade 12 in basalt ground water, 858 (R;US)

Investigation of environmentally assisted fracture of metallic nuclear-wastepackage barrier materials in simulated hasalt repository environments, 844 (R;US)

See Slate, S.C., 842

See Westerman, R.E., 1100, 843

Platt, A.M., Management of high-level radioactive wastes, 218 (R;US)

Progress in radioactive residue process development. A summary of Hanford laboratories achievements in this program under General Electric, 1958 . 1964, 216 (R;US)

Research and development activities [on] solidification of radioactive wastes. Progress report, 215 (R;US)
Rethinking the management of high-level nuclear waste: the need for fractionation, 509 (J;US)

See Jenson, G.A., 324

Platts, N., See Saum, C.J., 282

Plewinsky, B., See Murso, H., 1167

Plodinec, M.J., Assessment of Savannah River borosilicate glass in the repository environment, 172 (R;US)

SRP radioactive glass studies: small-scale process development and product performance, 180 (R;US)

Ploumen, $\mathbf{P}$, Far field temperature calculations for high-level waste disposal in salt formations, 1295 (J;US)

Numerical calculation of threedimensional long-time temperature fields using a special finite element method: the final storage of highly radioactive waste in salt rocks, 662 (R;DE;In German)

Pobereskin, M., See Pardue, W.M., 1037

Poeppel, R.B., See Bandyopadhyay, G., 502

Pollak, G.D., See Sutcliffe, W.G., 809

Ponce, D.A., Preliminary appraisal of gravity and magnetic data at Syncline Ridge, Western Yucca Flat, Nevada Test Site, Nye County, Nevada, 1025 (R;US)

See Jansma, P.E., 1026

Pope, J.M., See Bjorklund, W.J., 331

See Gockley, G.B., 370

See Lahoda, E.J., 438

Pope, R.B., See Allen, G.C. Jr., 30

Porter, M.A., See Allen, T.L., 188

Postula, F.D., See Finch, W.C., 24

Potter, P.E., See Marples, J.A.C., 5

Pottier, P., Criteria fur high level waste disposal and characterization of barriers, $13 / 4$ (KA;DE)

Criteria fü high level wusto dispusul and characterization of barriers, 678 (RA;DE)

Powell, G.F, See Eichholz, G.G., 98

See Eicholz, G.G., 1365

Powell, J.A., See Chikalla, T.D., 296, 301, 302, 303

Pratt, J.C., See Close, D.A., 1406

Prendergast, J.T. Jr., Decommissioning alternatives for Savannah River Plant high-level waste tank 16, 142 (RA;US)

Price, S.M., Status of geologic and hydrologic characterization of a potential nuclear waste repository site in basalt, 894 (R;US)

See Gephart, R.E., 891

Priebe, S.J., Siee Giombert, D., 378

Priest, C.C., See Rice, E.E., 616

Prince, A., Sep. Sastre, C., 546

Pudewills, A., Thermomechanical in-situ experiments and finite element computations, 1166 (BA;US)

Pusch, R., Chemical interaction of clay buffer materials and concrete, 997 (R;SE)

Ion/water migration phenomena in dense bentonites, 1174 (BA;US)

Puttke, B., See Ahner, S., 640, 833

See Gruen, M., 1394 
Jueille, P., See Jacquet-Francillon, N., 457

\section{$\mathbf{R}$}

Rabe, H., See Schiewer, E., 462

Rachiele, R., See Nelson, P.H., 1422

Radke, C.J., See Apps, J.A., 709

Rae, J., Coupled heat and groundwater flow in porous rock, $535(R ; G B)$

Rai, D., Neptunium concentrations in solutions contacting actinide-doped glass, 1336 (J;US)

See Strickert, R.G., 1146

Raines, G.E., See Gureghian, A.B., 827

Ramirez, A.L., Borehole-to-borehole geophysical methods applied to investigations of high level waste repository sites, 1019 (R;US)

Ramm, E.J., Apparatus and mcthod for immobilizing waste material, 484 (P;AU)

Containing waste material, 1075 (P;AU)

Containing waste material, 402 (P;AU)

Demonstration of SYNROC fabrication scaleup on a non-radioactive basis, 444 (BA;US)

See Reeve, K.D., 396, 405, 415, 418 , $472,493,510$

Ramthun, II., Radiant energy dissipation during final storage of high-level radio active waste in rock salt, $867(\mathrm{R} ; \mathrm{DE}$; In German)

SALTMP - a FORTRAN program for fast salt temperature calculations in HRW final storage, 866 (R;DE;In German)

Randall, C.T., Hazards analysis of TNX Large Melter-Off-Gas System, 197 (R;US)

See Cosper, M.B., 177

See Motyka, T., 22

Rankin, W.N., Decontamination of Savannah River Plant waste glass canisters 175 (R;US)

Evaluation of glass-contact materials for waste glass melters, 189 (R;US)

Rasmuson, A., Model for far neld migration, 1344 (BA;US)

See Andersson, G., 1343

Rasmussen, D.E., Comparison of cask and drywell storage concepts for a monitored retrievable storage/interim storage system, 853 (R;US)

Overview of the Monitored Retrievable Storage (MRS) program, 859 (R;US)

See Morissette, R.P., 641

Rasmusson, P.C., See Osborne, D.M., 558

Rastogi, R.C., Thermal and radiation stability of simulated high-level vitrifled waste products, 51 (R;IN)

Raverts, J.M., See Lipner, M.H., 1258

Ray, A.K., Combined analytical model for preformance assessment of the waste package/geologic medium systems, 1170 (BA;US)

Diffusional transport of radionuclide chains in sub-seabeds, $1340(\mathrm{~J} ; \mathrm{GB})$

See Nuttall, H.E., 1250

Raybold, N.A., See Treher, E.N., 1315

Recht, H.L., Status of development of the tailored ceramic high-level nuclear waste form, 513 (J;US)
See McKisson, R.L., 150

Reda, D.C., Natural convection experiments in a liquid-saturated porous medium bounded by vertical coaxial cylinders, 968 (R;US)

Reedy, G.T., See Jardine, L.J., 129, 419

Reeve, K.D., Development and testing of SYNROC $C$ as a high level nuclear waste form, 415 (J;AU)

Development and testing of SYNROC C as a high level nuclear waste form, 418 (BA;US)

Development and testing of SYNROC for high-level radioactive waste fixation, 493 (J;AU)

Development and testing of SYNROC for high level radioactive waste fixation, 510 (J;US)

Fabication and properties of SYNROC, 472 (BA;AU)

SYNROC for containment of high-level nuclear waste, 396 (J;US)

Synroc programme progresses, 405 (J;GB)

See Ramm, E.J., 444, 484

See Woolfrey, J.L., 1447

Rego, J., See Bazan, F., 1004

See Buddemeier, R.W., 344

Rego, J.A.H., See Failor, R.A., 1332

Reidel, S.P., Uplift and subsidence rates in the central Columbia Plateau and their relation to siting a waste repository at Hanford, Washington, 873 (R;US)

See Bergstrom, K.A., 901

Reinhart, C.G., Preliminary environmental implications of alternatives for decommissioning and future use of the Western New York Nuclear Services Center, 1301 (R;US)

Reisenauer, A.E., See Murthy, K.S., 1384

Relyea, J.F., See Serne, R.J., 1308

Revelli, M., See Cheung, H., 1009

Revelli, M.A., See Cheung, H., 1007

See O'Neal, W.C., 1018

Reynolds, J.O., See Symonds, J.L., 1435

Rhoads, W.A., See Collins, E., 1309, 1310

Rice, E.E., Overview of nuclear waste disposal in space, 616 (RA;US)

Preliminary risk assessment for nuclear waste disposal in space. Volume I. Executive summary of technical report, 720 (R;US)

Preliminary risk assessment for nuclear waste disposal in space. Volume II. Technical report, 721 (R;US)

Richards, H.J., See Feates, F.S., 369

Richardson, G.L., Phantom dissolution leach model, 1268 (J;US)

Richter, H., Characterization of mechanical properties of nuclear waste glasses, 1212 (BA;US)

Rickertsen, L.D., Expected near-field thermal environments in a sequentially loaded spent-fuel or high-level waste repository in salt, 830 (R;US)

Expected very-near-field thermal environments for advanced spent-fuel and defense high-level waste packages, 831 (R;US)

Riege, U., See Koster, K., 379

Ringwood, A.E., Immobilization of sodium and phosphorus-bearing PW-7a waste in SYNROC. Progress report, 346 (R;US)
Immobilization of high-level nuclear reactor wastes in SYNROC: a current appraisal, 515 (J;US)

Process for the treatment of high level nuclear wastes, 398 (P;US)

SYNROC: leaching performance and process technology, 250 (RA;DE)

Synroc solution, 356 (J;ZA)

Treatment of high level nuclear waste, 388 (P;AU)

See Newkirk, H.W., 400

See Oversby, V.M., 1114

See Ramm, E.J., 1075, 402

See Sinclair, W., 518

Risoluti, P., See Cao, S., 234

Ritchie, J.S., Thermal criteria for terminal storage of spent nuclear fuel assemblies in bedded salt, 1119 (J;NL)

See Busch, J.S., 1120

See Julien, H.L., 1121

See Watson, D.L., 1123

Riviere, J.C., See Myhra, S., 1039

Robbins, J.E., See Savage, D., 1201

Roberds, W. In-situ test programs related to design and construction of high-level nuclear waste (HLW) deep geologic repositories. Final report (Task 2), June 1981-November 1982, 743 (R;US)

In-situ test programs related to design and construction of high-level nuclear waste (HLW) deep geologic repositories. Appendices. Final report (Task 2), June 1981-November 1982, 744 (R;US)

Roberson, W.A., Solidification of ash from incineration of low-level radioactive waste, 193 (R;US)

Roberts, F.P., See Allemann, R.T., 217

See Bray, L.A., 214

See Turcotte, R.P., 377, 86

See Weber, W.J., 103

Roberts, L., See O'Hara, F.A., 155

Roberts, L.E.J., See Marples, J.A.C., 5

Robertson, J.B., See Schneider, R., 716

Robertson, T.J.M., See Beale, H., 534

Robin, M.J.I., See Gillham, R.W., 1300

Robinson, C.H., Steam explosions caused by te contact of molten glass and water, 246 (RA;DE)

Robinson, G, Sintering of Synroc D, 343 (R;US)

Robinson, K.S., Design of Harwell Joule Ceramic Melter, 231 (RA;DE)

Robinson, P.C., See Rae, J., 535

Robinson, R.S., See Clark, D.E., 631

Robinson, S.M., Incorporation of high-level nuclear waste in gel spheres, 139 (R;US)

See Arnold, W.D., 292, 381

See Dole, L.R., 293

Kockenbach, F.A., See Logan, S.E., 1386

Rodger, W.A., See Peterson, J.M., 1261

See Williams, R.F., 1290

Roerba, $K_{\text {, }}$ Corrosion aspects of high-level waste disposal in salt domes, 922 (R;DK)

Roesel, F., See Hadermann, J., 1348

Roethemeyer, $H_{\text {, }}$ Concept of final storage in gcologieal formations, 669 (RA;DE;GE)

Concepts for the ultimate storage in geological formations, 679 (RA;DE; In German)

High level waste disposal, 1289 (J;US) 
Rogers, G.C., See Dole, L.R., 293

See Moore, J.G., 256. 268

Rogers, V.L., See Barkatt, A., 1059

Roggendorf, H., See Scholze, H., 1204

Rogozin, Yu.M., See Bryzgalova, R.V., 921

Rogue, F., Instrument reliability for highlevel nuclear-waste-repository applications, 1013 (R;US)

Results of instrument reliability study for high-level nuclear-waste repositories, 1017 (R;US)

Results of instrument reliability study for high-level nuclear waste repositories, 93 (J;US)

Rohay, A.C., See Bergstrom, K.A., 901

Romesberg, L.E., See Wilmot, E.L., 29

Ronsecco, C., See Lanza, F., 1197

Ropp, R.C., Molecular glasses for nuclear waste encapsulation, 1076 (P;US)

Rosasco, P.V., See Hustrulid, W.A., 613

Roseboom, E.H. Jr., See Schneider, R., 716

Rosinger, E.L.J., Nuclear fucl waste disposal - status of the Canadian program, 1263 (J;US)

Ross, B., Benchmark problems for repository siting models, 746 (R;US)

See Mercer, J.W., 745

See Thomas, S.D., 740

Ross, H.P., See Smith, C., 567

Ross-Brown, D.M., See Maxwell, D.E., $12 C$

Rossington, C.S., See Newkirk, H.W., 400

Rothemeyer, H., High-level waste disposal, 1275 (J;US)

Rothfuchs, T., See Gies, H., 673, 683

Rothman, A.J., Nuclear waste package pro gram for high-level isolation in Nevada tuff, 1102 (J;US)

Nuclear-waste-package program for highlevel isolation in Nevada tuff, 1015 (R;US)

See O'Neal, W.C., 1018

See Scully, L.W, 584

Roubort, J.L., Mechanical stability of a Cm-doped celsian glass-ceramic, 143 (R;XE)

Routbort, J.L., Effect of composition and radiation on the fracture of a nuclear waste glass, $1434(\mathrm{~J} ; \mathrm{CH})$

See Matzke, H., 145

Routt, K.R., Physical modeling of a glass melter designed for vitrification of defense waste, 190 (R;US)

Theoretical predictions for continuousslurry feeding of a glass melter, 182 (R:US)

See Allen, T.L., 188

Rowe, M.D., See Hill, D., 1071

Roy, D.M., Geochemical factors in borehole-shaft plug longevity, 614 (RA;US)

Leach characterization of cement encapsulated wastes, 1156 (J;US)

Recent advances in repository seal materials, 1172 (BA;US)

Roy, R., Hydroxylated ceramic waste forms and the absurdity of leach tests, 254 (RA;DE)

Hydroxylated ceramic waste forms and the absurdity of 'leach tests', 266 (RA;DE)

See Sasaki, N., 1153

Rozsa, R., See Ryerson, F.J., 347

Rozsa, R.B., Disposal costs for SRP highlevel wastes in borosilicate glass and crystalline ceramic waste forms, 1010 (R;US)

See Campbell, J.H., 351

Ruiz-Lopez, M.C., See Kahl, L., 277

Rummery, T.E., See Rosinger, E.L.J., 1263

Rundberg, R.S., See Daniels, W.R., 700

See Erdal, B.R., 706

Runge, K., See Zappe, D., 1424

Runkle, G.E., Calculation of health effects per curie release for comparison with the EPA standard, 1379 (RA;US)

Comparison of ICRP2 and ICRP30 for estimating the dose and adverse health effects from potential radionuclide releases from a geologic waste repository, 1385 (R;US)

See Cranwell, R.M., 993

Ruppen, J.A., See Molecke, M.A., 1101, 949

Rush, F.E., Geohydrologic and drill-hole data for test well USW H-1, adjacent to Nevada Test Site, Nye County, Nevada, 1030 (R;US)

Rusin, J.M., Review of multibarrier waste form development, 272 (RA;DE)

See Bonner, W.F., 499

See Oma, K.H., 307, 51

See Treat, R.L., 299

See Turcotte, R.P., 377

See Wald, J.W., 297

Russ, J.C., See Palmour, H. III., 281

Russell, E.W., Selection of barrier metals for a waste package in tuff, 1020 (R;US)

See O'Neal, W.C., 1018

Russell, J.E., See Klasi, M.L., 942

Ryan, R.K., See Reeve, K.D., 493, 510

Rydberg, J., Swedish nuclear waste efforts, 36 (R;US)

See Allard, B., 275, 677

Ryerson, F.J., Formulation of SYNROC-D additives for Savannah River Plant high-level radioactive waste, $34 \%$ (R;UIS)

See Ackerman, F.J., 349, 353

See Newkirk, H.W., 400

\section{$\mathbf{S}$}

Sabatini, R.L., See Ahn, T.M., 1066

Sacharov, V.N., Combustion in the reactor of long-lived transuranium elements as a means of reducing the toxicity of HAW, 522 (J;DE; In German)

Saddler, H., See Symonds, J.L., 1435

Sahim, S., Solar-thermionic generator for nuclear wastes disposal in the space, 1138 (J;DE)

Sahm, A., See Kuhn, K.D., 8

Saidl, J., Capacity of the borosilicate glass VG 98/12 for components of the highlevel radioactive waste, 267 (RA;DE; In German)

Receptivity of borosilicate glass VG 98/12 for incorporating high-level radioactive wastes, 255 (RA;DE;GE)

See Guber, W., 1283, 528

See Kahl, L., 277

See Matzke, H., 145

Saint-Gaudens, M., See Laude, F., 459

Sakane, T., See Ninomiya, M., 258, 270

Salander, C., Policy and development of reprocessing of spent nuclear fuel and high-level radioactive waste manage- ment in the F.R. Germany, $13(\mathrm{~J} ; \mathrm{JP}$; In Japanese)

Sales, B.C., Electrical-conductivity measurements of leachates for the rapid assessment of wasteform corrosion resistance, 554 (R;US)

Salmon, A., Transport of highly radioactive materials, 45 (J;US)

Salter, P.F., Evaluation of radionuclide transport: effect of radionuclide sorption and solubility, 1354 (BA;US)

Salvatore, F., See Biedermann, G., 1353

Sandness, G.A., Report on geologic remote sensing of the Columbia Plateau, 871 (R;US)

Sano, A., Nondestructive techniques for evaluating the integrity of vitrified solids, 471 (J;JP)

See Nagaki, H., 1045

Santarova, M., Phosphate system as medium for radiuaclive waste fixatioun. Simplex experiment. Part I, 354 (R:CS:In Czech)

See Vojtech, 0., 355, 362

Sasaki, N., Backfill-waste interactions in repository simulating tests, 1153 (BA;US)

Sastre, C., Draft technical position on waste-package reliability, 546 (R;US)

Sato, A., See Kubota, M., 225

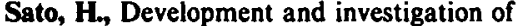
the accepting system for solidified bodies returned from foreign countries after reprocessing, 487 (J;JP;In Japanese)

Sato, T., See Mizuno, F., 483, 483

Sauls, M.L., See Collins, E., 1302

Saum, C.J., Denitration of simulated fast ractor highly active liquor waste, 241 (RA;DE)

Denitration of simulated fast reactor highly active liquor waste, 282 (R;GB)

Saunders, F.A.II., Management of radioactive, wastes, $1 \mathrm{nl}$ ( $\mathrm{B} ; \mathrm{r}, \mathrm{B})$

Savage, D., Geochemical factors controlling the nuclide release source-term in granite: dissolution of the waste form, 205 (R;GB)

Interaction of borosilicate glass and granodiorite at $100^{\circ} \mathrm{C}, 50 \mathrm{MPa}$ : implications for models of radionuclide release, 1201 (BA;US)

See Chapman, N.A., 1151

Schauer, V., See Skokan, A., 441

Scheetz, B.E., See Komarneni, S., 289

See Roy, D.M., 1156

See Sasaki, N., 1153

Schetfller, K., See Koster, K., 3/9

Schiewer, E., Materials balance: scientific fundamentals for the quality assurance of vitrified waste, 462 (BA;US)

See Lutze, W., 253, 265, 530

Schmidt-Hansberg, T., See Hrovat, M., 358,394

Schmierer, K.E., See Sandness, G.A., 871

Schnapp, M.R., See Nelson, R.A., 813

Schneider, A., Reprocessing of nuclear fuel, 6 (R;US)

Schneider, K., Rail transport of vitrified high-level radioactive fission products risk analysis of a model, 25 (R;DE; In German)

Schneider, K.A., Risk analysis of transporting vitrified high level radioactive waste by train, 1369 (RA;US) 
c-hneider, R., US Geological Survey research in radioactive waste disposal, fiscal year 1979, 716 (R;US)

Schneider, R.F., Immobilizing defense waste at the Savannah River Project, 196 (R;US)

Schneringer, P.E., Economic evaluation of nuclear waste transportation casks, 14 (R;US)

Schoen, R., See Ehlert, C., 1294

Scholze, H., Determination of the corrosion mechanisms of high-level waste containing glass, 1204 (BA;US)

Schornhorst, J.R., See O'Neal, W.C., 1018

Schulte, B., See Wiese, H., 1199

Schulz, W.W. See Anderson, W.J., 887

Schulz-Forberg, B., Classification and safety reserves of radioactive material shipping containers, 1452 (B;DE; In German)

Schuman, R.P., Evaluation of forms for the immobilization of high-level and transuranic wastes, 200 (R;US)

Leach testing of INEL waste forms in a gamma field, 1044 (J;US)

Preparation and leaching of radioactive INEL waste forms, 201 (R;US)

See Welch, J.M., 199, 416

Schuyler, R.L. III, See Dunson, J.B. Jr., 169

See Gould, T.H. Jr., 174

Schwartz, F.W., Influence of faulting on groundwater flow and contaminant transport, 1184 (BA;GB)

Schwartz, L.L., High-level radioactive waste isolation by incorporation in silicate rock, 1296 (BA;XA)

Schwarzer, K., Investigation of the consequences of leakage incidents of a tank with high-concentrated radionuclide solutions and pentration of the radioactive solution into the soil, 1423 (R;DE;In German)

Schweingruber, M.R., Evaluation of solubility and speciation of actinides in natural groundwaters, 1347 (BA;US)

Schweitzer, D.G., Uncertainties in the thermodynamics of basalt-oxygen and basalt-water reastions, 547 (R;IIS)

See Davis, M.S., 286, 749

Schwitz, R., See Odoj, R., 422

Scott, L.A., See Gureghian, A.B., 1361, 827

Scully, L.W., Repository and engineering barriers design, 584 (RA;US)

See Young, K.D., 555

Segall, R.L., See Cousens, D.R., 1203, $1237,221,454$

See Lewis, R.A., 220

See Smart, R.S.C., 1257

Seitz, M.G., Oxidation potential $\left(E_{b}\right)$ and pH control during experimentation, 552 (R;US)

Study of the migration of leached radionuclides in a natural fissure of granite rock, $1240(\mathrm{~J} ; \mathrm{CS})$

See Vandegrift, G.F., 1303

Calf, S., See Crowe, B., 699

liga, M., Seee Seitz, M r, 1240

marge, E., See Caporuscio, F., 696

senoo, M., See Furuya, T., 223

Senterfit, R.M., Resistivity sounding investigation by the Schlumberger method in the Yucca Mountain and Jackass
Flats area, Nevada Test Site, Nevada, 1028 (R;US)

Serini, B.L., See Goldin, A.S., 1382

Seme, R.J., Status of radionuclide sorptiondesorption studies performed by the WRIT program, 1308 (RA;US)

Sevigny, G.J., See Barnes, S.M., 321

See Goles, R.W., 310, 319

Seyfried, W:E. Jr., See Thornton, E.C., 971

Shade, J.W., Waste glass/metal interactions in brines, 860 (R;US)

Shafranek, L.F., See Kelley, J.A., 506

See Maher, R., 195

Shankland, T., See Blacic, J., 693

Shao, $J_{\text {, }}$ Uniform- and pitting-corrosion data requirements for $\mathrm{TiCode}-12 \mathrm{high}$ level-waste containers, 734 (RA;US)

Shapar, H.K., Indemnification for nuclear damage's - recent developments in the United States, 1431 (R;ES)

Shapar, H.R., Licensing of high-level radio active wastes deposits, 1405 (J;BR;In Portuguese)

Shashukov, E.A., See Lazarev, L.N., 357

Shaw, T.M., See Harker, A.B., 152

See Morgan, P.E.D., 151

Shemilt, L.W., Overview of nuclear waste management, 1068 (J;GB)

Sheng, G., See Shemilt, L.W., 1068

Shephard, L.E., 1982 Subseabed Disposal Program. Annual report, site assessment October 1981 to September 1982; 987 (R;US)

Shimoda, H., Policy and some problems on the return waste, 118 (J;JP;In Japanese)

Shinohara, K., See Kitahara, Y., 1450

Shinohara, T., Flame-resistant radioactive waste processing method, 488 (P;JP;In Japanese)

Shirley, C.G., NWTS transportation interface technology development priority report, 89 (R;US)

Transportation system preconceptual description for subscabcd disposal of high-level waste, 20 (RA;US)

Transportation system preconceptual description for subseabed disposal of highlevel waste, 33 (R;US)

See Millor, M:M., 31

Shunk, E.R., See Close, D.A., 1406

Shuri, F.S., Laboratory rock mechanics testing manual. Public draft, 807 (R;US)

Sibbitt, W.L., See Nelson, D.C., 694

Siegel, M.D., Compliance asscssments of hypothetical geological nuclear waste isolation systems with the draft EPA standard, 982 (R;US)

Volume 3. A simplified analysis of a hypothetical repository in a tuff formation, 1417 (RA;US)

See Chu, M.S., 981

See Chu, M.S.Y., 952

See Pepping, R.E., 1416, 1418

Siemens, D.H., Remote design features for the West Valley slurry-fed ceramic melter system, 329 (R;US)

Vitrification of highly-loaded SDS zeolites, 32.5 (R;US)

See Bryan, G.H., 505

See Carrell, J.R., 315

See Holton, L.K., 507

See Wheelwright, E.J., 385

Siever, R., See Ghovanlou, A.H., 1133
Sill, C.W., See Welch, J.M., 416

Silling, S.A.; Final technical position on documentation of computer codes for high-level waste management, 723 (R;US)

Silva, A.J., Geotechnical studies for subseabed disposal: high level radioactive wastes, 964 (RA;US)

Low-gradient permeability testing of fine-grained marine sediments, 1277 (J;US)

Silva, R., See Edelstein, N., 812

Silva, R.J., See Apps, J.A., 1318

See Carnahan, C.L., 715

Silviera, D.J., See McCallum, R.F., 815

See McKee, R.W., 308, 309

Simmons, G.R., See Dormuth, K.W., 592

Simmons, J.II., See Macebo, P.B., 1192

See Macedo, P.B., 380, 475

Simon, R., See Orlowski, S., 426

Simon, R.A., See Orlowski, S., 108

Sinclair, R.N. Neutron scattering studies of vitrified radioactive waste, 363 (J;US)

Sinclair, W., Alpha-recoil damage in natural zirconolite and perovskite, 518 (J;JP)

See Ringwood, A.E., 515

Sinitsyns, G.S., See Bryzgalova, R.V., 921

Sinnock, $S_{\text {n, }}$ Results of the area-to-location phase of repository siting in Nevada, 1035 (J;US)

Siskind, B., Near-field repository conditions in basalt and salt, 733 (RA;US)

Sjoblom, G., Regulatory policy of the EPA related to alpha-contaminated waste, 1412 (RA;US)

Sjoreen, A.L., See Kocher, D.C., 1367, 737

Skaba, V., See Nachmilner, L., 390

Skagius, $K_{\text {. }}$ Diffusion in crystalline rocks, 1168 (BA;US)

Skoblar, L.T. Nuclear waste/nuclear power: their futures are linked, 560 (R;US)

Skokan, A., Microstructures and crystallization in simulated waste glasses, 441 (BA;US)

Slate, S.C., Feasibility of using a high-level waste canister as an engineered barrier in disposal, 842 (R;US)

Full-scale impact tests of simulated highlevel waste canisters, 1098 (J;US)

Full-scale impact tests of simulated highlevel waste canisters, 316 (R;US)

See Kuhn, W.L., 328

See Peters, R.D., 1117

See Treat, R.L., 447

See Wescott, R.I., 1439

Slates, R.V., Physical and chemical characterization of synthetic calcined sludge, 167 (R;US)

Slaughter, G.M., Permeability of selected sediments in the vicinity of five salt domes in the Gulf Interior Region, 810 (R;US)

Smailos, Em HAW-containers as a barrier in final storage, 1432 (RA;DE;In German)

See Stichel, W., 689

See Storch, W., 691

Smart, R. St. C., See Cousens, D.R., 221

See Lewis, R.A., 220

Smart, R.S.C. Disposal of high-level radioactive wastes, 1257 (BA;AU)

See Cousens, D.R., 1203 
Smart, R.St.C., See Cousens, D.R., 1237, 454

See Lewis, R.A., 375, 653

Smedes, H., National program for isolating high-level nuclear waste, 410 (J;GB)

Smedes, H.W., National survey of crystalline rocks and recommendations of regions to be explored for high-level radioactive wastc repository sites, 540 (R;US)

United States steps up waste isolation programme, 1074 (J;GB)

Smith, A., See Nelson, P.H., 1422

Smith, B.G.R., See Guardini, S., 208, 294

Smith, C., Interpreted resistivity and IP section line W1: Wahmonie Area, Nevada Test Site, Nevadà, 567 (R;US)

Smith, C.B., Population risks from disposal of high-level radioactive wastes in geologic repositories. Draft report, 836 (IR;US)

See Goldin, A.S., 1382

Smith, C.F., See Musgrave, B.C., 498

Smith, D., See Anderson, H.M., 1331

Smith, F.M., See Bray, L.A., 214

Smith, G.S., See Newkirk, H.W., 400

Smith, M.J., Engineered waste package concepts for high-level waste isolation in basalt, 874 (R;US)

Waste package conceptual designs for a repository located in basalt, 585 (RA;US)

Waste package conceptual designs for a repository located in basalt, 884 (R;US)

Smith, P.K., See Bibler, N.E., 179

Smith, R., See Ackerman. F.J., 349

Smith, R.D., High-level radioactive-wastedisposal investigations in Texas, 1001 (R;US)

Smith, R.F., High-level nuclear waste: who governs, 105 (J;GB)

Smith, R.I., MRS/IS facility co-located with a repository: preconceptual design and life-cycle cost estimates, 857 (R;US)

Smith, R.R., New waste calcining facility at ICPP, 452 (BA;US)

See Fortsch, M., 393

Smock, R.W, Radwaste panel, NRC head highlight 44th American Power Conference program, 99 (J;US)

Snyder, B.J., Regulatory perspective on the cleanup of TMI-2', 1445 (J;US)

Snyder, D.B., Preliminary results of gravity investigations at Yucca Mountain and vicinity, Southern Nye County, Nevada, 1024 (K;US)

See Jansma, P.E., 1026

Soldat, J.K., See Runkle, G.E., 1385

Solomah, A.G., Developments, recommendations and applications of tests for evaluating the chemical stability of high-level radioactive solid waste forms, $1056(\mathrm{~J} ; \mathrm{GB})$

Effect of temperature on the leaching performance of SYNROC-B waste form, 1229 (J;US)

Effects of gamma irradiation on the leaching behavior of a synthetic mineral waste form (SYNROC-B), 1143 (J;US)

Effects of high-level gamma irradiation on radioactivity release from waste disposal forms, 1097 (J;US)
HLW fixation in sintered modified SYNROC-B ceramics: chemical stability evaluation, 526 ( $\mathrm{J}$;US)

Influence of gamma irradiation on the leaching behavior of a modified SYNROC-B ceramic waste form, 424 (BA;US)

Leachability study of modified Synroc-B waste form: effect of gamma irradiation, 412 (J;GB)

Leaching behavior of sintered modified SYNROC-B waste forms: effect of HLW addition, 445 (BA;US)

Model for predicting leaching behavior of high-level radioactive waste forms, 508 (J;US)

Subsolidus sintering of SYNROC: I. Initial demonstrations, 280 (R;US)

See Palmour, H. III., 281

Solomon, K.A., See Triplett, M.B., 717

Sombres, $C$, iffench status of high level radioactive wastes solidification, 135 (R;FR)

Vitrification of high active waste. Vitrification techniques, 222 (R;DE)

Sombret, M., See Mamelle, J., 525

Someno, K., See Mizuno, F; 483

Soo, P., Review of DOE waste-package prugram. Subtask 1.1. National wastepackage program, September 1981 March 1982. Volume 2, 732 .(R;US)

Review of DOE waste package program. Subtask 1.1. National waste package program, April-September 1982, 735 (R;US)

Review of DOE waste package program. Subtask 1.1. National waste package program, October 1982-March 1983. Vol. 4, 736 (R;US)

See Ahn, T.M., 1066, 545, 728, 729, 730

See Dayal, R., 283

See Shao, J., 734

Soper, P.D., See Plodinec, M.J., 180

Sopher, C., See Barton, C., 959

See Lalne, E., 958

Soran, D.M., Analysis of the alleged Kyshtym disaster, 1314 (R;US)

Sousanpour, W., See Barkatt, A., 1059

Sousselier, $Y_{\text {, }}$ National and cooperative program for waste management in France, 427 (BA;US)

See Pottier, P., 1374, 678

South, D.L., Laboratory studies of fluid flow through borehole seals, 1231 (J;US)

Spahiu, K., See Biedermann, G., 1353

Spencer, R.K., See Wang, J.S.Y., 707

Splcuchi, K., See Blacic, J., 693

Spoljaric, N., Geologic aspects of disposal of highly radioactive nuclear waste. Open-File Report No. 15, 722 (R:US)

Sprecace, R.P., Remote automatic plasma arc-closure welding of a dry-storage canister for spent nuclear fuel and high-level radioactive waste, $\mathbf{5 5 1}$ (R;US)

Sprecher, W.M., See Benny, R.I., 21, 604

St. John, C.M., Evaluation of geotechnical surveillance techniques for monitoring high-level waste repository performance. Final report 19 Sep 81-31 Dec 82. 738 (R;US)

Repository design, 1134 (J;GB)

Stahl, D., Long-term performance of materials used for high-level waste packag- ing. Annual report, March 1982-April 1983. Vol.1, 751 (R;US)

Long-term performance of materials used for high-level waste packaging. Quarterly report, April-June 1983, 752 (R;US)

Stahlkopf, K., Geologic disposal of nuclear waste, 1107 (J;US)

Stammler, M., See Feld, R.H., 458

Staner, P., See Ahner, S., 640, 833

Stanford, R.E.L., Role of utility nuclear waste management group, 75 (RA;US)

Stanners, D.A., See Murray, C.N., 1115

Staples, B.A., See Schuman, R.P., 201

Starr, J.L., Automated data acquisition and analysis system used in the Basalt Waste Isolation Project's Near-Surface Test Facility, 906 (R;US)

Stein, R., Licensing considerations for the NWTS program, 1227 (J;US)

Nuclear waste legislation: issues and impacts, 1033 (J;US)

See Vieth, D.L.. 38

Steindler, M.J., Fuel cycle programs. Quar terly progress report, July-September 1981, 50 (R;US)

Fuel cycle programs. Quarterly progress report, April-June 1982 , 536 (R;US)

Fuel cycle programs. Quarterly progress report, July-September 1982, 538 (R;US)

Fuel cycle programs. Quarterly progress report, October-December 1982, 539 (R;US)

Fuel-cycle programs. Quarterly progress report, January-March 1981, 48 (R;US)

Fuel-cycle programs. Quarterly progress report, April-June 1981, 49 (R;US)

See Bates, J.K., 127, 128

See Jardine, L.J., 129

See Mechaıı, W.J., 420

See Northrup, C.J., 945

Stejskal, J., See Santarova, M., 354

See Vojtech, O., 362

Stevens, C.A., Risk-assessment methodology development for waste isolation in geologic media, 727 (R;US)

Stevens, P.R., See Schncider, R., 716

Stichel, W., Corrosion studies and mechanical tests on metallic materials for the design of packagings for vitrified high level wastes (HLW), 689 (R;DE;In German)

Stieglitz, L., Formation of inflammable radiolytic gases in the PUREX-process, 11 (J;DE;In German)

Stlliman, D.B., See Soran, D.M., 1314

Stinton, D.P., Coating crystalline nuclear waste forms to improve inertness, 138 (R;US)

Coating of crystalline nuclear waste forms to improve inertness, 386 (J;US)

Coating of crystallinc nuclear waste forms to improve inertness, 387 (J;US)

See Dole, L.R., 293

See Lackey, W.J., 245

See Robinson, S.M., 139

Stoddard, J.A., Development of safeguards requirements for high-level waste repositories, 1421 (J;US)

Stogner, J.M., Method for subsurface disposal of radioactive waste, 1297 (P;US)

Stone, C.M., See Branstetter, L.J., 943 
ine, J.A., Comparative leach testing of high-level waste forms, 626 (R;US)

Comparison of properties of borosilicate glass and crystalline ceramic forms for immobilization of Savannah River Plant waste, 171 (R;US)

See Bernadzikowski, T.A., 627

See Slates, R.V., 167

Stoneham, A.M., See Burns, W.G., 463

Storch, W., Corrosion studies on nonmetallic inorganic materials for $\mathrm{HLW}$ packaging design, 691 (R;DE;In German)

Storck, R., Importance of near-field phenomena for nuclide release from a flooded salt dome repository, 1396 (BA;US)

Stout, L.A., See Murthy, K.S., 1384

Strait, S.R., See Baker, S.M., 1222

Strickert, R.G., Predicting Pu concentralions in solutions contacting geologic materials, 1146 (BA;US)

See Rai, D., 1336

Strickmann, G., See Ploumen, P., 1295

Struckmeyer, R.K., See Goldin, A.S., 1382

Struxness, E.G., See Feige, Y., 549

Stuckwisch, S.E., See Chu, M.S., 981

Stueger, R., See Grass, F., 382

Stula, R.T., See Lester, D.H., 806

Suessmilch, J., See Santarova, M., 354

See Vojtech, O., 355, 362

Sugier, A., See Lefevre, J., 368

Sullivan, J.C., See Nash, K., 1247

Sumner, W., Alpha-contaminated waste from reprocessing of nuclear fuel, 54 (RA;US)

Sundberg, W.D., Three-dimensional thermal analysis for a conceptual high-level waste repository in welded tuff, 932 (R;US)

See Johnstone, J.K., 1271

Susskind, L., Annual report of the management system design group, 967 (RA;US)

Sutcliffe, W.G., Incertainties and sensitivities in the performance of geologic nuclear waste isolation systems. Volume I. Summary, 809 (R;US)

Sutherland, S.H., Experimental and analytical program to evaluate packaging and target hardness as related to nuclear waste transportation systems, 32 (R;US)

Suzuki, A., See Kiyose, R., 1245

Svalstad, D.K., Forced ventilation analysis of a commercial high-level nuclear repository in salt, 811 (R;US)

Forced ventilation analysis of a commercial high-level nuclear waste repository in tuff. Topical report RSI-0175, 941 (R;US)

Swanson, J.I., See McCallum, R.F., 815

See McKee, R.W., 308, 309

Swyler, K.J., See Ahn, T.M., 284

See Dayal, R., 283

See Levy, P.W., 610

Sykes, J.F., Application of performance assessment models to evaluate uncertainty in site characterization, 1221 (J;US)

ymonds, I.I..., Nuclear energy, 1435

(BA;AU)

Szeless, A., See Kumer, L., 112

Szoke, P., See Barkatt, A., 1059

Szymanski, J.S., See Ilarper, T.R., 1131

\section{$\mathbf{T}$}

Tachimori, S., See Kubota, M., 225

Talbert, D.M., Report of the first interim meeting of the Seabed Working Group Engineering Studies Task Group, 944 (R;US)

See De Marsily, G., 1069

Tallman, R.L., See Welch, J.M., 199, 416

Tammemagi, H.Y., Thermomechanical modeling and the selection of a salt repository site, 1218 (J;US)

Tanaka, S., See Kiyose, R., 1245

Tarutani, K., See Ooka, K., 521

Tas, H., See Casteels, F., 676, 686

See Dresselaers, J, 1150

Tashiro, S, See Furuya, T., 666; 667

See Murakami, T., 224

Tassoni, E, See Bocola, W., 550

Tempest, P.A., Comparison of borosilicate glass and synthetic minerals as media for the inmobilization of high-level radioactive waste, 490 (J;US)

Tennant, M.H., Sensitivity calculations for low-heat generating defense waste repository temperatures, $1124(\mathrm{~J} ; \mathrm{NL})$

Vitrification of nuclear waste with low heat generation, 407 (J;NL)

Terai, R., Change of electrical properties of the borosilicate glasses containing $\mathrm{MoO}_{0}$ by phase separation, 482 (J;JP;In Japanese)

See Kinoshita, M., 479

See Wakabayashi, H., 476

Testa, L., See Bocola, W., 550

Tewes, H.A., See Cohen, J.J., 1254

Tewhey, J.D., See Newkirk, H.W., 400

Thelen, J., See Schwarzer, K., 1423

Thirumalai, K., See Lehnhoff, T.F., 1169

See Topcubasi, A.F., 876

Thomas, R.K.; See Gartling, D.K., 931

Thomas, S.D., Summary of repository siting models. Final report, 740 (R;US)

See Mercer, J.W., 745

See Ross, B., 746

Thompson, G.M., See Leonhart, L.S., 880

Thoms, R.L., See Martinez, J.D., 571

Thordarson, W., See Rush, F.E., 1030

Thornton, E.C., Constant temperature and thermal gradient investigations of the near field subseabed environment: chemical and thermodiffusional effects, 971 (R;US)

Thunvik, R., Hydrothermal conditions around a radioactive waste repository, 1177 (BA;US)

Tiffany, B., See Slates, R.V., 167

Timmermans, W., See Iseghem, P. van, 274

See Van Iseghem, P., 1207, 262

Ting, D., Migration of long actinides chains in geologic media, 1362 ( $\mathrm{J}$;US)

Ting, D.K., Radionuclide migration in a two-dimensional flow fiẹld, 1339 (J;US)

Tittlova, E., See Malasek, E., 656

Tomari, H., See Furuya, T., 667

Topcubasi, A.F., Thermomechanical simulation of a nuclear-waste canister in a repository in basalt, 876 (R;US)

See Baxter, J.T. 882, 883

Topp, S.V., Scientific basis for nuclear waste management, 107 (B;US)

Tormey, T.V., See Moss, M., 970
Torstenfelt, B., Diffusion measurements in compacted bentonite, 1148 (BA;US)

Radionuclide migration into natural fracture surfaces of granitic rock, 1334 (J;US)

Transport of actinides through a bentonite backfill, 1175 (BA;US)

See Allard, B., 1351

See Landstroem, O., 1349

Tosetti, R.J., See Choi, J.S., 1232

Toshima, I., Method and device for solidifying high level liquid wastes from a re-processing plant, 403 (P;JP;In Japanese)

Toyoshima, I., Method of processing offgas condensate containing precipitations resulted upon solidification of high-level liquid wastes issued from a reprocessing plant, 406 (P;JP;In Japanese)

Trauger, D.B., Handling and disposing of radioactive waste, 561 (R;US)

Traverso, G.M., See Cosper, M.B., 177

Travis, B.J., Comparative analysis of fractured and porous medium radionuclide transport, 1356 (BA;US)

Treat, R.L., Consolidated waste forms: glass marbles and ceramic pellets, 299 (R;US)

High level waste forms: glass marbles and thermal spray coatings, 447 (BA;US)

See Bonner, W.F., 499

See Nesbitt, J.F., 477

Treber, E.N., Elution of radionuclides through columns of crushed rock from the Nevada Test Site, 1315 (R;US)

Trent, B.C., See Maxwell, D.E., 120

Triplett, M.B., Monitoring technologies for ocean disposal of radioactive waste, 717 (R;US)

Trocellier, P., Utilization of charged particle backscattering to study the near surface region of glasses. Application to depth profiling of lanthanium, cerium, throium and uranium induced by aqueous leaching, 1216 (BA;US)

Tsang, C.F., See Wang, J.S.Y., 1064, 1183 , 707

Tsang, Y.W., See Witherspoon, P.A., 618

Tsukumo, K., See Abe, T., 389

Tsunoda, N., Present status of techniques for the solidification of high level wastes, 492 (J;JP;In Japanese)

Tullborg, E.L., See Landstroem, O., 1349

Turcotte, R.P., Radiation damage in nuclear waste ceramics, 377 (J;US)

Radiation damage in crystalline nuclearwaste solids, 86 (R;US)

Radiation effects in high-level radioactive waste forms, $116(\mathrm{~J} ; \mathrm{CH})$

See Click, L.A., 856

See Gray, W.J., 298

See Mendel, J.E., 326

See Weber, W.J., 103, 80

Turi, J.A., See McGoff, D.J., 500

Turner, D.A., Conceptual design of the nuclear-waste repository in basalt, 888 (R;US)

Turner, P.S.; See Cousens, D.R., 1203, $1237,221,454$

See Lewis, R.A., 220

See Smart, R.S.C., 1257

Tuthill, E.J., See Hill, D., 1071

Tyce, R.C., See Triplett, M.B., 717 
Tyler, L.D., Performance assessment in the Nevada Nuclear Waste Storage Investigations, 1224 (J;US)

Tympl, M., See Dolezal, J., 467

\section{0}

Ubbes, W.F., Conceptual test plan for site confirmation testing at an exploratory shaft in salt, 826 (R;US)

Uchida, K., See Nagaki, H., 1045

See Sano, A., 471

Uematsu, K., Status of high level and alpha bearing waste management in PNC, 428 (BA;US)

Uerpmann, E.P., Seal for underground ultimate storage sites for radioactive waste, and method of installing the seal, 1284 ( $P$; $\dot{D E} ;$ In Germàn்)

Uetake, N., Immobilization of radioactive liquid waste using water glass, 470 (J;US)

Uher, E., See Kepak, F., 399

Uny, G., See Morlevat, J.P., 257, 269

Upsun, U.L., See Allcmann, R.T., 217

Urakawa, T., Method of processing high level radioactive wastes by glass solidification, 408 (P;JP;In Japanese)

Urwongse, L., See Clark, D.E., 1188

\section{V}

Van As, D., See Forster, Wm. O., 1291

Van Cleve, J.E. Jr, See Lorenzo, D.K., 1106

van den Broek, W.M.G.T., Disposal of high-level nuclear waste in rock-salt. $\Lambda$ culluar isus of limperature inoroaseo calculated at ECN (Energieonderzoek Centrum Nederland)-Petten, RWTH (Rheinisch-Westfaelische Technische Hochschule Aachen)-Aachen and THD (Delft University of Technology)-Delft, R.35 (R;NL)

Impurities in rock-salt: consequences for the temperature increases at the disposal of high-level nuclear waste, 834 (R;US)

van Dorp, F., See De Marsily, G., 1069

van Geel, J., Inactive Pamela vitrification facility in Mol; manufacture and characteristics of Vitromet products, 238 (RA;DE;GE)

See Detilleux, E., 433

See Hendricks, J.P., 207

Van Iseghem, P., Chemical stability of simulated HLW forms in ronntast with clay media, 1207 (BA;US)

Interaction of vitrified high level waste with clay environment, 262 (RA;DE)

Scc Izahara, S, 130

Van Konynenburg, R.A., Plutonium doping of SYNROC-D, 350 (R;US)

van Rütré, F., See Lanza, F., 261

Van Sambeek. L.L., Dome-salt thermomechanical experiments at Avery Island, Louisiana, 620 (RA;US)

Van Tuyl, H.H., See Bray, L.A., 214

Vance, E.R., Radiation and transmutation effects on crystalline radioactive waste forms, 1262 (J;US)

See AdI, T., 148, 404

Vandegrift, G.F., Interaction of groundwater and fresh basalt fissure surfaces and its effect on the migration of actinides, 1303 (R;US)

See Steindler, M.J., 48, 49

Vaniman, D, See Caporuscio, F., 696

See Crowe, B., 699

Vaniman, D.T., See Crowe, B.M., 698

See Daniels, W.R., 692, 703

See Erdal, B.R., 706

See Ogard, A.E., 704

See Wolfsberg, K., 705

Varadarajan, R.V., Impacts of transportation on a test and evaluation facility for nuclear waste disposal: a systems analysis, 556 (R;US)

Varadrajan, R.V., See Peterson, R.W., 17

Vassent, B., See Dran, J.C., 423

Veakis, E., See Dayal, R., 283

Vernaz, E., See Laude, F., 459

Vieth, D.Ln, Background and historical perspectives for monitored retrievable storage, 38 (J;US)

High-level waste disposal, 1042 (J;US)

Vogler, S., See Steindler, M.J., 48, 49

Vogt, D., See Mills, M., 1375, 1376

Vojtoch, $\mathbf{O}$., Phosphate system as medium for radioactive waste fixation - simplex experiment. Part II, 355 (R;CS;In Czech)

Phosphate systems - a medium for high level waste solidification, 362 (J;CS;In Czech)

See Santarova, M., 354

Voldan, J., See Vojtech, O., 355

Voth, D.E., Possible approaches to community development for nuclear waste isolation, 805 (R;US)

\section{W}

Wada, M., Some glass-ceramics for special applications, 481 (J;JP)

Waddell, J.D., Projected costs for mined geologic ropositories for dispoal of commercial nuclear wastes, 769 (R;US)

Wagner, R.A., Preliminary investigation of the thermal and structural influence of ventilation drifts on repository rooms, 786 (R;US)

See Tammemagi, H.Y., 1218

Wahi, K.K., See Chu, M.S., 747, 981

See Chu, M.S.Y., 1368

See Ortiz, N.R., 750

See Pepping, R.E., 731

Wahlig, B.G., See Eichholz, G.G., 98

Waite, D.A., Impacts of recommendations on radiological considerations for an operating repusitury, 1398 (J;US)

Radiological risk analysis of an operating high-level waste repository, 1395 (BA;XA)

Summary of ONWI occupational dose assessment prugram, 1391 (J;US)

Wakabayashi, H., High temperature corrnsion of heat-resisting alloys by borosilicate melts containing simulated high lcvcl nuclear wastes, 476 (J;JP;In Japanese)

See Terai, R., 482

Wakeley, L.D., See Roy, D.M., 1156

Wald, J.W., Development and testing of inatrices for the encapsulation of glass and ceramic nuclear waste forms, 297 (R;US)
Study of radiation effects in curiumdoped $\mathrm{Gd}_{2} \mathrm{Ti}_{2} \mathrm{O}_{7}$ (pyrochlore) and $\mathrm{CaZrTi}_{2} \mathrm{O}_{7}$ (zironolite), 1211 (BA;US)

See Gray, W.J., 298

See Turcotte, R.P. 377

See Weber, W.J., 1278

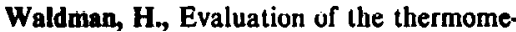
chanical behavior about a waste container/sleeve in salt, 803 (R;US)

Walker, B.A., See Ames, L.L., 868

Walker, C.T., See Pickering, S., 1196

Walmsley, D., See Morris, J.B., 242

Walton, F.B., See Melnyk, T.W., 1299

Walton, R.D. Jr., Department of Energy's selection of high level waste forms, 443 (BA;US)

Wan, P.T., Actinide transmutation with a $\mathrm{UF}_{6}$ reactor, $1048(\mathrm{~J} ; \mathrm{US})$

Uranium hexafluoride actinide transmutation reactors-preliminary design and analysis, 516 (D;US)

Wang, J.S.Y., Long-term thermomechanical and thermohydrological factors controlling the optimal design of a nuclear waste repository, 1064 (J;UIS)

Long-term thermohydrologic behaviour of nuclear-waste repositories, 1183 (BA;GB)

Thermal impact of waste emplacement and surface cooling associated with geologic disposal of nuclear waste, 707 (R;US)

See Wollenberg, H.A., 714

Ware, N., See Ringwood, A.E., 515

Warnecke, E., Requirements for the disposal of high-level radioactive wastes, 671 (RA;DE)

Requirements for the disposal of highlovol radioactive wastes, 681 (RA; ;F)

Warner, R.K., See Symonds, J.L., 1435

Watkins, D.J., See Carnahan, C.L., 715

See Witherspoon, P.A., 618, 712

Watrous, R.A., See Anderson, W.J., 887

Watson, D.L., Uptimization of mine layout for uuclear fuel asscmbly starage, 11,33 (J;NL)

See Busch, J.S., 1120

See Julien, H.L., 1121

See Ritchie, J.S., 1119

Weart, W.D., Conclusions regarding geotechnical acceptability of the WIPP site, 990 (R;US)

Isolation of radioactive wastes in salt repositories, 951 (R;US)

Webb, G.A.M., See De Marsily, G., 1069

See Hill, M.D., 1085

Weber, M., Safety analysis of tank storage fiol lighly radinactive reprocossing waste, 1404 (J;US)

Weber, W.J., Materials Characterization Center. Second workshop on irradiation effects in nuclear waste furms. Summary report, 80 (R;US)

Radiation damage from alpha decay in ceramic nuclear waste forms, 103 (J;CH)

Radiation effects in crystalline high-level nuclear waste solids, 1278 (BA;US)

Review of the current status of radiation effects in solid nuclear waste forms, 372 (J;US)

See McVay, G.L., 1285

See Turcotte, R.P., 86

Wee, C.S., See Shirley, C.G., 20, 33 
-. d, H.C., Drilling-induced borehole-wall damage at spent fuel test-climax, 1003 (R;US)

See Buddemeier, R.W., 344

Wegner, K., Remote aspects of treating and handling radioactive wastes, 449 (BA;US)

Weisenburger, S., Electrodes for the direct heating of highly radioactive borosilicate glasses in HAW-ceramic furnaces, 236 (RA;DE;GE)

High-level waste vitrification technique in a full-scale pilot plant, 439 (BA;US)

Nuclear waste vitrification in a ceramiclined electric glass melter, 409 (J;US)

See Gruenewald, W., 235

See Schiewer, E., 462

Welch, J.M., Irun-enriched basalt as a waste form for high-level nuclear wastes, 199 (R;US)

Iron-enriched basalt for containment of nuclear wastes, 416 (BA; US)

See Schuman, R.P., 201

Weres, O., See Apps, J.A., 1318

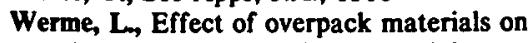
glass leaching in geological burial, 1200 (BA;US)

See Hench, L.L., 1202

Wescott, R.I., Vitrification of high-level wastes: A review of the computer thermal analysis for storage canisters, 1439 (J;NL)

West, J.M., Effect of microbial activity on the containment of radioactive waste in a deep geological repository, 1357 (BA;US)

See Chapman, N.A., 1151

See McKinley, I.G., 1355

Westberry, C.F. III, See Bernadzikowski, T.A., 627

Westerman, R.E., Development of structural barriers for containment of nuclear waste in basalt, 1100 (J;US)

General corrosion, irradiation-corrosion, and environmental-mechanical evaluation of nuclear-waste-package structural-barrier materials. Progress report, 843 (R;US)

See Bradley, D.J., 854

Westermark, T., See Forborg, S., 248

Westsik. J.H. Jr., High-temperature leaching of an actinide-bearing, simulated high-level waste glass, 841 (R;US)

See Perez, J.M. Jr., 126

Weyers, C., See Klein, M., 141

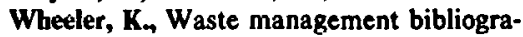
phy, 1978-1983, 279 (R;US)

Wheelwright, EJ., Generation and vitrification of high-level light water reactor liquid waste, 385 (J;US)

See Bray, L.A., 214

Whlpple, C.G., See Libby, L.M., 97

White, A.F., See Apps, J.A., 1318

See Camahan, C.L., 715

White, I.F., IAEA report on actinide partitioning and transmutation, 383 ( $\mathbf{J} ; G B)$

See Hill, M.D., 1081

White, L.A., Regulation of geological disposal of high-level radioactive waste, 1241 (J;US)

ite, R.M., See Slaughter, G.M., 810

White, T.J., Sec Cousens, D.R., 221

White, W.B., Role of oxidation potential, $\mathrm{Eh}$, in the dissolution of nuclear waste materials, 851 (RA;US)
See Komarneni, S., 289

Whitty, W.J., Preliminary identification of interfaces for certification and transfer of TRU waste to WIPP, 695 (R;US)

Wickens, L.M., See Rae, J., 535

Wicks, G.G., Borosilicate glass as a matrix for immobilization of SRP high-level waste, 512 (J;US)

See Clark, D.E., 631

See Plodinec, M.J., 172

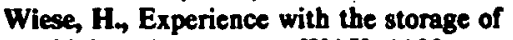
high active waste at WAK, 1199 (B;DE)

Wigley, M.R, See Tammemagi, H.Y., 1218

Wilbur, C.K., See Vandegrift, G.F., 1303

Wilcox, R.E, See Martinez, J.D., 571

Wildanger, W., See Gonano, L., 741

Wilde, R.T., Conceptual design of monitored retrievable storage faclities, 41 (J;US)

Wilems, R.E., Post-closure performance assessment methodology for the Office of Nuclear Waste Isolation, 1219 (J;US)

See Williams, R.F., 1290

Wiles, L.E., See McKee, R.W., 308, 309, 863

Wilke, R.J., See Ahn, T.M., 284

See Dayal, R., 283

Wilkinson, W.I, Chemistry of the nuclear fuel cycle, 4 (BA;GB)

Williams, R., See Stahlkopf, K., 1107

Williams, R.F., EPRI's programs in lowlevel waste management, 74 (RA;US)

Illustrative nuclear waste disposal criteria and their application to higblevel waste repository design, 1290 (J;US)

Williams, W.A, See Smith, C.B., 836

Wilmot, E.L., Preliminary analysis of the cost and risk of transporting nuclear waste to potential candidate commercial repository sites, 35 (R;US)

Transportation packagings for high-level wastes and unprocessed transuranic wastes, 29 (R;US)

See Peterson, R.W., 17

Wilmshurst, R.E, See Symonds, J.L., 1435

Wilson, C.R., See Carnahan, C.L., 715

Wilson, D山., Use of thorium as an alternative nuclear fuel, $46(R ; A U)$

Wilson, E.G., See Costello, J.M., 532

Wilson, R.K., See Eaton, R.R., 988

See Mondy, L.A., 996

Wilson, W.B, See Walton, R.D. Jr., 443

Winske, P., See Ehlert, C., 1294

See Ploumen, P., 1295

Witherspoon, P.A, Radioactive waste dispusal in granite, $712(R ; U S)$

Theoretical and laboratory investigations of flow through fractures in crystalline rock, 618 (RA;US)

See Javandel, I., 710

See Wang, J.S.Y., 1183

Wolf, H.C., See d'Entremont, P.D., 176

Wolfsberg, $K_{\text {, }}$ Research and development related to the Nevada Nuclear Waste Storage Investigations. Progress report, January 1-March 31, 1983, 705 (R;US)

See Daniels, W.R., 692, 700

See Erdal, B.R., 706

Wollenberg, H.A., Appraisal of nuclear waste isolation in the vadose zone in arid and semiarid regions (with empha- sis on the Nevada Test Site), 714

(R;US)

Underground openings for in situ experiments, 1111 (J;US)

Wood, D.E., Risk assessment for storage of high-level waste in single shell tanks, 1256 (J;US)

Wood, M.I., Evaluation of sodium bentonite and crushed basalt as waste package backfill materials, 902 (R;US)

Experimental investigation of sodium bentonite stability in Hanford basalt, 879 (R;US)

Testing of candidate waste package backfill and canister materials for basalt, 586 (RA;US)

Testing of candidate waste-package backfill and canister materials for basalt, 885 (R;US)

Woodward, J., See, Ahn, T.M., Ink6

Woolfrey, J.L., Irradiation effects in SYNROC, 1447 (BA;AU)

See Ramm, E.J., 484

See Reeve, K.D., 396, 405, 415, 418, $472,493,510$

Woolsey, G.B., See Lee, L.M., 503

Wright, A.C., See Sinclair, R.N., 363

Wright, G.T., Efficient particulate scrubber for glass melter off-gas, 183 (R;US)

Wright, W.V., See Huang, J.C., 1370

See Lee, L.M., 187

Wuertz, R., See Stieglitz, L., 11

Wukelic, G.E., Monitoring land- and wateruse dynamics in the Columbia Plateau using remote-sensing computer analysis and integration techniques, 1425 (R;US)

See Leonhart, L.S., 865

Wurm, K.J., Annotated bibliography for the design of waste packages for geo logic disposal of spent fuel and highlevel waste, 804 (R;US)

Wurtele, M.G., See Libby, L.M., 97

\section{$\mathbf{Y}$}

Yagnik, S.K., Thermal-gradient migration of brine inclusions in salt, 711 (R;US)

Thermal-gradient migration of brine inclusions in salt crystals, 713 (R;US)

Yamaguchi, I., See Kubota, M., 225

Yamamota, M., See Okamoto, H., 431

Yamanaka, H., See Terai, R., 482

Yamanake. T. See Ninomiya. M.. 258. 270

Yamashita, K., See Terai, R., 482

Yasufuku, K., See Abe, T., 389

Yates, K.R., See Christensen, R.N., 1259

Yayanos, A.A., Deep sea biophysics, 1330 (RA;US)

Yoshimura, H.R., See Sutherland, S.H., 32

Young, J.P., See Kelly, K.L., 1276

Young, K.D., Equipment for the emplacement of heat-producing waste in long horizontal boreholes, SSS (R;US)

Yuan, Y.C., See Peterson, J.M., 1261 


\section{Z}

Zand, S.M., Dissolution of evaporites and its possible impact on the integrity of the Waste Isolation Pilot Plant (WIPP)

New Mexico, USA, 1162 (BA;US)

Zappe, D., Evaluation of radionuclide releases from underground waste repositories using the method of status vectors, 1424 (J;DD)

Zarzycki, J., See Nogues, J.L., 1206

Zavoshy, S., See Chambre, P.L., 1338

Zavoshy, S.d., See Chambre, P.L., 1228

Zerga, D.L., See Pippin, L.C., 767

Zeyfang, R.W., See Kelley, J.A., 506

See Maher, R., 195

Zhu, B.F., See Clark, D.E., 631

Zimmer, E., Solidification of high-level radioactive wastes and alpha-bearing wastes by a sol-gel process, 244 (RA;DE)

Zimmerman, C.A., See Hoffman, T.L., 203

Zimmerman, R.M., Conceptual design of field experiments for welded-tuff rockmechanics program, 936 (R;US)

Issues related to field testing in tuff, 947 (R;US)

Zumwalt, L.R., See Solomah, A.G., 412 , 526 


\section{SUBJECT INDEX}

The subject index is based on the use of subject descriptors selected from a controlled thesaurus of terms (DOE/TIC-7000). Subject descriptors and qualifiers (subheadings) are selected and presented in the following format:

SUBJECT DESCRIPTOR

Qualifier

Title, (supplementary information), citation number, (R:US)

The notations in the final parentheses indicate the type of document, c.g., $\mathbf{R}$ for Report; the country of publication, e.g., US; and the language if other than English, e.g., In German. Tables of document types and country of publication codes appear after the indexes.

The title may be supplemented with additional words, or a phrase, if it appears additional information would be helpful. In cases for which the title contains little or no information related to the subject entry, it may be replaced entirely by the supplementary information. A qualifier is not always required, and in such cases the title will follow the unqualified subject descriptor.

The descriptors selected for use as subject terms are generally the names of specific materials, things, or processes. To the extent possible, a qualifier is selected to describe the properties of, or processes applied to, the subject term.

Index entries are selected to indicate the important ideas and concepts presented in a document, rather- than words that may appear in the text. Within the available thesaurus terms, the most probable or logical place to look for typical information is selected. "See references" are included to guide users from synonymous terms or phrases to the descriptor selected as a subject heading for the concept. (e.g. Pipeline Quality Gas see HIGH BTU GAS). "See also references" are used to indicate where to find references to subject concepts that are narrower, broader, or related to a particular subject heading. To complete an exhaustive search of a given subject, all such headings should be reviewed. Copies of DOE/TIC-7000, the Subject Thesaurus, are available from the National Technical Information Service (NTIS), U. S. Department of Commerce, Springfield, VA 22161.

\section{A}

AAEC

Program Management

Develnpment and testing of SYNROC for high level radioactive waste fixation, 510 (J;US)

Radioactive Waste Processing

Development and testing of SYNROC C as a high level nuclear waste form, 418 (BA;US)

\section{ABANDONED SITES}

Inventories

Integrated data base for spent fuel and radwaste: inventories, 52 (R;US)

\section{ABSORBED DOSES}

SEe RADIATION DOSES

\section{ACTINIDE BURNER REACTORS}

Comparative Evaluatlons

Uranium hexafluoride actinide transmutation

reactors-preliminary design and analysis, 516 (D;US)

Design

Uranium hexafluoride actinide transmutation

reactors-preliminary design and analysis, 516 (D;US)

\section{ACTINIDES}

See also AMERICIUM

NEPTUNIUM

PLUTONIUM

PROTAC"INIUM

THORIUM

URANIUM

\section{Adsorption}

Actinide sorption on rock minerals, 275 (RA;DE)

Chemisorption

Actinide sorption on rock minerals, 275 (RA;DE)

Diffusion

Modelling of the migration of lanthanoids and actinoids in ground water; the medium dependence of equilibrium constants, 1353 (BA;US)

Environmental Trunsport

Migration of long actinides chains in geologic media, 1362 (J;US)

Ion Exchange

Equipment, operation and some results from a hot test of the CTH actinide separation process, 243 (RA;DE)

Recycling

Actinide recycling in light water reactors: results of reactor physics calculations, 208 (R;XE)

Solubility

Elemental release from glass and spent fuel, 1307 (RA;US)

Solvent Extraction

Equipment, operation and some results from a hot test of the CTH actinide separation process, 243 (RA;DE)

Thermodynamic Properties

Technical progress report for the quarter, 1 April-30 June 1982 , 779 (R;US)

Transmutation

Actinide transmutation with a $\mathrm{UF}_{6}$ reactor, $1048(\mathrm{~J} ; \mathrm{US})$

Uranium hexafluoride actinide transmutation reacturs-preliminary design and analysis, 516 (D;US)

\section{ADSORBENTS}

See also SILICA GEL

Performance Testing

Volatilization and trapping of ruthenium in high temperature processes, 141 (RA;US)

\section{AERE}

Radioactive Waste Processing

Comparison of wet slurry and dry frit (crizzle) feeding of glassformers in pot vitrification, 242 (RA;DE)

\section{AEROSOLS}

Decontamination

Efficient particulate scrubber for glass melter off-gas, 183 (R;US)

AIR

Radiolysis

Influence of gamma irradiation on the leaching behavior of a modified SYNROC-B ceramic waste form, 424 (BA;US)

\section{AIRBORNE PARTICLES}

SoO P.ARTICULATESS

AIRBORNE PARTICULATES

See PARTICULATES

ALABAMA

Geology

Regional geological assessment of the Devonian-Mississippian shale sequence of the Appalachian, Illinois, and Michigan basins relative to potential storage/disposal of radioactive wastes, 828 (R;US)

ALAP

SeE RADIATION PROTECTION

ALLOY 800

See INCOLOY 800

ALLOY-HD-8077

See NICKEL BASE ALLOYS 


\section{ALLOY-MA-754 \\ See NICKEL BASE ALLOYS \\ ALLOY-MA-956 \\ See IRON BASE ALLOYS \\ ALLOY-WAZ-16 \\ SEe NICKEI, RASE ALLOYS \\ ALLUVIAL DEPOSITS \\ Compression Strength}

Thermal impact of waste emplacement and surface cooling associated with geologic disposal of nuclear waste, 707 (R;US)

Permeability

Thermal impact of waste emplacement and surface cooling associated with geologic disposal of nuclear waste, 707 (R;US)

\section{Poisson Ratio}

Thermal impact of waste emplacement and surface cooling associated with geologic disposal of nuclear waste, 707 (R;US)

Porosity

Thermal impact of waste emplacement and surface cooling associated with geologic disposal of nuclear waste, 707 (R;US)

Tensile Properties

Thermal impact of waste emplacement and surface cooling associated with geologic disposal of nuclear waste, 707 (R;US)

Thermal Stresses

Thermal impact of waste emplacement and surface cooling associated with geologic disposal of nuclear waste, 707 (R;US)

\section{Young Modulus}

Thermal impact of waste emplacement and surface cooling associated with geologic disposal of nuclear waste, 707 (R;US)

\section{ALPHA-BEARING WASTES}

After-heat

Decay calculations on medium-level and actinide-containing wastes from the LWR fuel cycle. Pt. 1. Basic data evaluation including activity and thermal decay power, 687 (R;DE;In German)

Certification

Preliminary identification of interfaces for certification and transfer of TRIJ waste in WIPP, $695(R ; U S)$

Decay

Decay calculations on medium-level and actinide-containing wastes from the LWR fuel cycle. Pt. 2, 688 (R;DE;In German)

Decay calculations on medium-level and actinide-containing wastes from the LWR fuel cycle. Pt. 1. Basic data evaluation including activity and thermal decay power, $687(\mathrm{R} ; \overline{\mathrm{D}} \overrightarrow{\mathrm{E}}$; In German)

Forecasting

Radiuactive waste inventories and projections, 62 (R;US)

Spent fuel and radioactive-waste inventories, projections, and oharacteristics, 57 (R;US)

Spent fuel and radioactive waste inventories, projections, and characteristics, 574 (R;US)

Inventories

Integrated data base for spent fuel and radwaste: inventories, $\$ 2$ (R;US)

Nuclear-waste-management technical support in the development of nuclear-waste-form rriteria for the NB.C. Task 3. Waoto inventory review, 73 (R;US)

NWTS program waste projection data needs, 1086 (I;US)

Radioactive waste inventories and projections, 62 (R;US)

Spent fuel and radiuactive-waste inventories, projections, and characteristics, 57 (R;US)

Spent fuel and radioactive waste inventories. projections, and characteristics, $574(\mathrm{R} ; \mathrm{US})$

Leaching

Characterization and testing of basalt glass and glass ceramic waste forms, 313 (R;US)

Measuring Methods

Measurement of radioactive contaminated wastes, 278 (R;US)

Nondestructive Analysis

Advances in measurement of alpha-contaminated wastes, 1406 (R;US)

Measurement of radioactive contaminated wastes, 278 (R;US)

\section{Packaging}

Conceptual design requirements for spent fuel, high-level was and transuranic wastc packages, 903 (R;US)

Nuclear waste management technical support in the development of nuclear waste form criteria for the NRC. Task 1. Waste package overview, 283 (R;US)

Transportation packagings for high-level wastes and unprocessed transuranic wastes, 29 (R;US)

Projection Series

NWTS program waste projection data needs, $1086(\mathrm{~J} ; \mathrm{US})$

Radiation Monitoring

Advances in measurement of alpha-contaminated wastes, 1406 (R;US)

Rail Transport

Preliminary analysis of the cost and risk of transporting nuclear waste to potential candidate commercial repository sites, 35 (R;US)

Road Transport

Preliminary analysis of the cost and risk of transporting nuclear waste to potential candidate commercial repository sites, 35 (R;US)

Specifications

TRU waste-acceptance criteria for the Waste Isolation Plant, 1419 (R;US)

Transport

Defense waste transportation: cost and logistics studies, 27 (R;US)

Waste Transportation

Review of the status of transportation activities in various waste management systems, 30 (R;US)

Transportation packagings for high-level wastes and unprocessed transuranic wastes, 29 (R;US)

ALUMINIA

See ALUMINIUM OXIDES

ALUMINIUM

Corrosion Resistance

Curr siun of muterials in a clay environment, $676(R A ; D E)$

Emission Spectroscopy

Analyses of nuclear fuel and high-level waste by inductively coupled plasma-emission spectroscopy, 68 (R;US)

Leaching

Comparison of properties of borosilicate glass and crystalline ceramic forms for immobilization of Savannah River Plant waste, 171 (R;US)

Effects of composition, solutions, and burial on nuclear waste glasses, 845 (RA;US)

Preparation and leaching of radioactive INEL waste forms, 201 (K;US)

ALUMINIUM ALLOYS

See also INCONEL 617

Compatibility

Studies of waste-canister compatibility, 832 (R;US)

Corrosion

Corrosion of materials in a clay environment, 686 (RA;DE)

Corrrationi Reriulnuce

Corr sion of materials in a clay environment, $676(R A ; D E)$

ALUMINIUM COMPLEXES

Solubility

Importance of organic compounds in ground water as radionuclide-mobilizing agents, 1324 (R;US)

ALIMINIUM OXIDES

See also HOLLANDITE

Corrosion Resistance

Canister materials proposed for final disposal of high level nuclear waste - a review with respect to corrosion resistancr 675 (RA;DE)

Corrosion studies on non-metallic inorganio materials for $\mathrm{HL}^{\mathrm{L}}$ packaging design, 691 (R;DE;In German)

ALUMINIUM SILICATES

See also KAOLINITE

SMECTITE 
Crystallization

CsAlSis $\mathrm{O}_{12}$ : a possible host for ${ }^{107} \mathrm{Cs}$ immobilization, 148 (R;US)

Leaching

$\mathrm{CsAlSi}_{5} \mathrm{O}_{12}$ : a possible host for ${ }^{107} \mathrm{Cs}$ immobilization, 404 (J;GB)

CsAlSis $\mathrm{O}_{12}$ : a possible host for ${ }^{107} \mathrm{Cs}$ immobilization, 148 (R;US)

ALUMINUM

See ALUMINIUM

AMERICIUM

Adsorption

Effects of Hanford high-level waste components on sorption of cobalt, strontium, neptunium, plutonium, and americium on Hanford sediments, 918 (R;US)

Sorption of actinides in well-defined oxidation states on geologic media, 1351 (BA;US)

Status of radionuclide sorption-desorption studies performed by the WRIT program, 1308 (RA;US)

Transport of actinides through a bentonite backfill, 1175 (BA;US)

Chemical State

Studies on speciation of americium, technetium and neptunium in simulated vitrified-wastc leachates, 1335 (J;US)

Desorption

Status of radionuclide sorption-desorption studies performed by the WRIT program, 1308 (RA;US)

Diffusion

Studies on speciation of americium, technetium and neptunium in simulated vitrified-waste leachates, 1335 (J;US)

Transport of actinides through a bentonite backfill, 1175 (BA;US)

Distribution

Studies on speciation of americium, technetium and neptunium in simulated vitrified-waste leachates, 1335 (J;US)

Distribution Functions

Sorption of actinides in well-defined oxidation states on geologic media, 1351 (BA;US)

Status of radionuclide sorption-desorption studies performed by the WRIT program, 1308 (RA;US)

Environmental Transport

Actinide sorption on rock minerals, 677 (RA;DE)

Backfill barriers for nuclear waste repositories in salt, 670 (RA;DE)

Leaching

Leaching of actinides from simulated nuclear waste glass, 1196 (BA;US)

Quantitative Chemical Analysis

Analysis of Am and a recovery for it in the pressurized water reactor fuel cycle, 1433 (R;DE;In German)

Solubility

Geochemical controls on radionuclide relcases from a nuclear waste repository in basalt: estimated solubilities for selected elements, 1329 (R;US)

Solvent Extraction

Octyl(phenyl)-N,N-diisobutylcarbamoylmethylphosphine oxide as an extractant for actinides from nitric acid waste, $146(R ; U S)$

Sorption

Radionuclide sorption on basalt-interbed materials. Annual report FY 1981, 1328 (R;US)

Valence

Valence states of actinides in synthetic monazites, 1276 (BA;US)

\section{AMERICIUM 241}

Adsorption

Immobilization of reprocessing waste by means of titanate ion exchangers, 248 (RA;DE)

Diffusion

Diffusion measurements in compacted bentonite, 1148 (BA;US)

Study of radionuclide migration from deep-lying repository sites with over-lying sedimentary layers, 1348 (BA;US)

Leaching

Preparation and leaching of radioactive INEL waste furms, 201 (R;US)

Underground migration of long-lived radionuclides leached from a borosilicate glass matrix, 1360 (BA;XA)

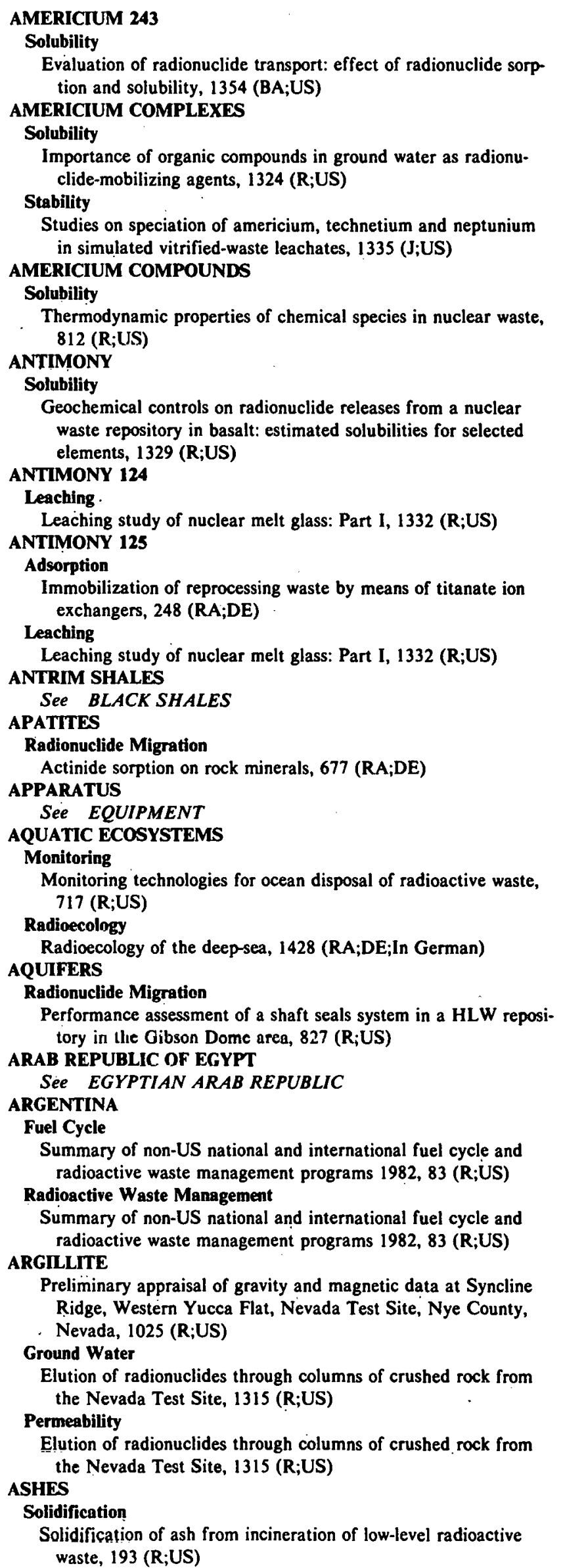

AMERICIUM 243

Solubility

Evaluation of radionuclide transport: effect of radionuclide sorption and solubility, 1354 (BA;US)

mportance of organic compounds in ground water as radionuclide-mobilizing agents, 1324 (R;US)

Studies on speciation of americium, technetium and neptunium in simulated vitrified-waste leachates, 1335 (J;US)

Solubility

Thermodynamic properties of chemical species in nuclear waste, 812 (R;US)

Geochemical controls on radionuclide releases from a nuclear waste repository in basalt: estimated solubilities for selected elements, 1329 (R;US)

Leaching.

Leaching study of nuclear melt glass: Part I, 1332 (R;US)

TIMONY 125

Immobilization of reprocessing waste by means of titanate ion exchangers, 248 (RA;DE)

Leaching study of nuclear melt glass: Part I, 1332 (R;US)

NTRIM SHALES

ATITES

Radionuclide Migration

Actinide sorption on rock minerals, 677 (RA;DE)

Monitoring

Monitoring technologies for ocean disposal of radioactive waste

Radioecology of the deep-sea, 1428 (RA;DE;In German)

UTFES

Performance assessment of a shaft seals system in a HLW reposi-

B REPUBLIC OF EGYPT

SEe EGYPTIAN ARAB REPUBLIC

Summary of non-US national and international fuel cycle and radioactive waste management programs 1982, 83 (R;US)

Summary of non-US national and international fuel cycle and radioactive waste management programs 1982, 83 (R;US)

Preliminary appraisal of gravity and magnetic data at Syncline Ridge, Western Yucca Flat, Nevada Test Site, Nye County,

ound Water

Elution of radionuclides through columns of crushed rock from the Nevada Test Site, 1315 (R;US) the Nevada Test Site, 1315 (R;US)

Solidification waste, 193 (R;US) 


\author{
ATLANTIC OCEAN \\ Oceanography \\ Site qualification activities, 958 (RA;US) \\ Sediments \\ Geotechnical studies for subseabed disposal: high level radioac- \\ tive wastes, 964 (RA;US) \\ Paleomagnetic study of areas B1, Cl and E2, 959 (RA;US) \\ ATOMIC ENERGY \\ See NUCLEAR ENERGY \\ ATOMIC ENERGY RESEARCH ESTABLISHMENT \\ See $A E R E$ \\ ATTAPULGITE \\ Radionuclide Migration \\ Actinide sorption on rock minerals, 677 (RA;DE) \\ AUSTENITIC STEELS \\ Corrosion Resistance \\ Corr sion of materials in a clay environment, $676(R A ; D E)$ \\ AUSTRALIA \\ See also SOUTH AUSTRAIIA \\ Fuel Cycle \\ Summary of non-US national and international fuel cycle and \\ rảdioactıve waste management programs 1982,83 (R;US) \\ Nuclear Energy \\ Australia's role in nuclear energy research and training, 1438 \\ $(\mathrm{J} ; \boldsymbol{\Lambda U})$ \\ Nuclear İndustry \\ Nuclear energy, 1435 (BA:AU) \\ Radioactive Waste Management \\ Radioactive waste management 1980-81. Supplement to AIRAC \\ No. 6, 111 (B;AU) \\ Summary of non-US national and international fuel cycle and \\ radioactive waste management programs 1982, 83 (R;US) \\ AUSTRALIAN ATOMIC ENERGY COMMISSION \\ See AAEC \\ AUSTRAIITES \\ See TEKTITES \\ AUSTRIA \\ Fuel Cycle \\ Summary of non-US national and international fuel cycle and \\ radioactive waste management programs 1982, 83 (R;US) \\ Radioactive Waste Management \\ Summary of non-US national and international fuel cycle and \\ radioactive waste management programs $1982,8.3$ (R;IIS)
}

\section{B}

\section{BACKFII.JING}

Comparative Evaluations

Development of a backfill for containment of high-level nuclear waste, 1173 (BA;US)

Materials Testing

Evaluation of sodium bentonite and crushed basalt as waste package backfill materials, 902 (R;US)

Experimental investigation of sodium bentonite stability in Hanford basalt, 879 (R;US)

Fuel cycle programs. Quarterly progress report, July-September 19R2, 53R (R;US)

Packing material testing required to demonstrate compliance with 1000-year radionuclide containment. Semiannual report on waste package verification tests, 739 (R;US)

Testing of candidate waste package backfill and canister materials for basalt, 586 (RA;US)

Thermal conductivity of bentonite/quartz high-level waste package backfill, 1065 (J;US)

Waste package materials testing for a salt repository: 1982 status report, 825 (R;US)

Performance Testing

Combined analytical model for preformance assessment of the waste package/geologic medium systems, 1170 (BA;US)

Standardization

Nuclear waste management technical support in the developmnt of nuclear waste form criteria for the NRC. Task 5. National waste packagc program, 285 (R;US)
BACTERIA

Population Dynamics

Deep sea biophysics, 1330 (RA;US)

BARIUM

Activation Analysis

Application of neutron-activation analysis to the determination of leach rates of simulated nuclear-waste forms, 127 (R;US)

Adsorption

Geochemistry studies pertaining to the G-tunnel radionuclide migration field experiment, $1426(\mathrm{R} ; \mathrm{US})$

Desorption

Research and development related to the Nevada Nuclear Waste Storage Investigations. Progress report, July 1-September 30, 1981,692 (R;US)

Emission Spectroscopy

Analyses of nuclear fuel and high-level waste by inductively coupled plasma-emission spectroscopy, 68 (R;US)

Leaching

Leaching behavior of sintered modified SYNROC-B waste forms: effect of HLW addition, 445 (BA:US)

Leaching studies on SYNROC at $95^{\circ} \mathrm{C}$ and $200^{\circ} \mathrm{C} .1114(\mathrm{~J} ; \mathrm{CH})$

Processing effects on the behavior of titanate waste forms in aqueous solutions, 1145 (BA;US)

Prncescing, microstruoturo, looching, and long-ter'its stability studies related to titanate high-level wastc forms, 340 (R;US)

Subsolidus sintering of SYNROC: II. Materials selections, process improvements, waste form evaluations, 281 (R;US)

Physical Radiation Effects

Influence of gamma irradiation on the leaching behavior of a modified SYNROC-B ceramic waste form, 424 (BA;US)

Sorption

Research and development related to the Nevada Nuclear Waste Storage Investigations. Progress report, July 1-September 30 , 1981, 692 (R;US)

BARIUM 133

Diffusion

Elution of radionuclides through columns of crushed rock from the Nevada Test Site, 1315 (R;US)

Leaching

Measurement of leaching from simulated nuclear-waste glass using radiotracers, 128 (R;US)

BARNWELL FUEL PROCESSING PLANT

Kadiuactive Waste Manageitiènt

Alpha-contaminated waste from reprocessing of nuclear fuel, 54 (RA;US)

BASALT

Basalt Waste Isolation Project. Quarterly rcport, October 1, 1981-December 31, 1981, 895 (R;US)

Experimental investigation of sodium bentonite stability in Hanford basalt, 879 (R;US)

Hydrology and Geulugy Overview Cummittee reports and responses from the Basalt Waste Isolation Project, 914 (R;US)

Near-field repository conditions in basalt and salt, 733 (RA;US)

Nuclear-waste repository in basalt: seal development studies, 887 (R;US)

Waste package conceptual designs for a repository located in basalt, 884 (R;US)

Bibliographies

Bibliography and documents issued list through fiscal year 1982 (435 citations), 892 (R;US)

Compression Strength

Thermal impact of waste emplacement and surface cooling associated with geologic disposal of nuclear waste, 707 (R;US)

Data Acquisition

Automated data acquisition and analysis system used in the Basalt Waste Isolation Project's Near-Surface Test Facility, 906 (R;US)

Data Analysis

Automated data acquisition and analysis system used in the Basalt Waste Isolation Project's Near-Surface Test Facility, 906 (R:US) 
Deformation

Determination of rock mass deformation modulus in closely jointed Columbia River basalt, 881 (R;US)

Rock mass deformation properties of closely jointed basalt, 889 (R;US)

Summary of Near-Surface Test Facility results and their application to repository design, $886(\mathrm{R} ; \mathrm{US})$

Evaluation

Near-field performance evaluations, 800 (RA;US)

Fracture Properties

Iron-enriched basalt for containment of nuclear wastes, 416 (BA;US)

Geochemistry

Selected hydrologic and geochemical issues in site characterization for nuclear waste disposal: flood basalts at the Hanford Reservation (Pasco Basin basalt), 715 (R;US)

Geology

Basalt waste-isolation project. Quarterly report, January 1-March 31, 1981, 908 (R;US)

Basalt Waste Isolation Project. Drilling and testing quarterly report, January 1, 1983-March 31, 1983, 900 (R;US)

Geohydrologic characterization and qualification of a high-level waste site in basalts, 891 (R;US)

Status of geologic and hydrologic characterization of a potential nuclear waste reporitory site in basalt (Abstract only), 894 (R;US)

Heat Transfer

Waste package heat-transfer analysis: model development and temperature estimates for waste packages in a repository located in basalt, 917 (R;US)

Hydrology

Basalt waste-isolation project drilling and testing. Quarterly report, January 1-March 31, 1982, 896 (R;US)

Basalt Waste Isolation Project drilling and testing. Quarterly report, April 1, 1982-June 30, 1982, 897 (R;US)

Basalt Waste Isolation Project drilling and testing. Quarterly report, July 1, 1982-September 30, 1982, 898 (R;US)

Basalt Waste Isolation Project: drilling and testing. Quarterly report, October 1, 1982-December 31, 1982, 899 (R;US)

Basalt Waste Isolation Project. Drilling and testing quarterly report, January 1, 1983-March 31, 1983, 900 (R;US)

Field guidebook association of engineering geologists. Chapters $1-10,915$ (R;US)

Geohydrologic characterization and qualification of a high-level waste site in basalts, 891 (R;US)

Issues in repository siting, 798 (RA;US)

Selected hydrologic and genchemical issues in site characterization for nuclear waste disposal: flood basalts at the Hanford Rescrvation (Pasco Basin basalt), 715 (R;US)

Status of geologic and hydrologic characterization of a potential nuclear waste repository site in basalt (Abstract only), 894 (R;US)

Leaching

Geochemical behavior of supercalcine waste form: its stability in a basalt environment, 911 (R;US)

Iron-enriched basalt for containment of nuclear wastes, 416 (BA;US)

Summary report for the interlaboratory round robin on the MCC-1 static leach test method, 300 (R;US)

Lithology

Field guidebook association of engineering geologists. Chapters $1-10,915$ (R;US)

Mineralogy

Scientific data necessary to predict radionuclide migration within or near a mined nuclear repository, 1312 (R;US)

Permeability

Thermal impact of waste emplacement and surface cooling associated with geologic disposal of nuclear waste, 707 (R;US)

Pnissnn Ratio

Rock mass deformation properties of closely jointed basalt, 889 (R;US)

Thermal impact of waste emplacement and surface cooling associated will geulugic dispusal of nuclcar waste, 707 (R;US)
Porosity

Thermal impact of waste emplacement and surface cooling associated with geologic disposal of nuclear waste, 707 (R;US)

Radionuclide Migration

Groundwater flow and transport characteristics of flood basalts as determined from tracer experiments, 880 (R;US)

Numerical modeling of groundwater flow and solute transport for a nuclear-waste repository in basalt, 1327 (R;US)

Radionuclide solubility and transport in basalt, 875 (R;US)

Rock Mechanics

Automated data acquisition and analysis system used in the Basalt Waste Isolation Project's Near-Surface Test Facility, 906 (R;US)

Determination of rock mass deformation modulus in closely jointed Columbia River basalt, 881 (R;US)

Field guidebook association of engineering geologists. Chapters $1-10,915$ (R;US)

Repository data base development, 801 (RA;US)

Thermomechanical simulation of a nuclear-waste canister in a repository in basalt, 876 (R;US)

Rock-fluid Interactions

Correlation between dynamic leach test results and geochemical observations, 1059 (J;US)

Earth Sciences, 789 (RA;US)

Evaluation of sodium bentonite and crushed basalt as waste package backfill materials, 902 (R;US)

Evaluation of DOE radionuclide solubility data and selected retardation parameters: description of calculational and confirmatory experimental activities, 1304 (R;US)

Native copper deposits of the Portage Lake volcanics, Michigan: their implications with respect to canister stability for nuclear waste isolation in the Columbia River basalts beneath the Hanford Site, Washington, 904 (R;US)

Sentinel Gap basalt reacted in a temperature gradient, 702 (R;US)

Uncertainties in the thermodynamics of basalt-oxygen and basaltwater reactions, 547 . ( $\dot{\mathbf{R}} ; \mathrm{US})$

Sorptive Properties

Basalt-radionuclide reactions: FY-1981. Annual report, 868 (R;US)

Evaluation of sodium bentonite and crushed basalt as waste package backfill materials, 902 (R;US)

Evaluation of radionuclide transport: effect of radionuclide sorption and solubility, 1354 (BA;US)

Evaluation of DOE radionuclide solubility data and selected retardation parameters: description of calculational and confirmatory experimental activities, 1304 (R;US)

Status of radionuclide sorption-desorption studies performed by the WRIT program, 13 ṇ (RA;IIS)

Strains

Rock mass deformation properties of closely jointed basalt, 889 (R;US)

Stratigraphy

Basalt waste-isolation project drilling and testing. Quarterly repurt, January 1-March 31, 1982, 896 (R;US)

Basalt Waste Isolation Project drilling and testing. Quarterly report, April 1, 1982-June 30, 1982, 897 (R;US)

Basalt Waste Isolation Project drilling and testing. Quarterly report, July 1, 1982-September 30, 1982, 898 (R;US)

Basalt Waste Isolation Project: drilling and testing. Quarterly report, October 1, 1982-December 31, 1982, 899 (R;US)

Selected hydrologic and geochemical issues in site characterization for nuclear waste disposal: flood basalts at the Hanford Reservation (Pasco Basin basalt), 715 (R;US)

Stresses

Rock mass deformation properties of closely jointed basalt, 889 (R;US)

Temperature Distribution

Modeling approach to determine short- and long-term thermal and thermomechunical effects of waste emplacement in a repository in basalt, 1169 (BA;US)

Status report on the full-scale electric heater tests at the Hanford Near-Surface Test Facility, 883 (R;US) 
Temperature Effects

Summary of Near-Surface Test Facility results and their application to repository design, 886 (R;US)

Tensile Properties

Thermal impact of waste emplacement and surface cooling associated with geologic disposal of nuclear waste, 707 (R;US)

Thermal Expansion

Rock mass deformation properties of closely jointed basalt, 889 (R;US)

Thermal Stresses

Cesium and strontium fractionation from HLW for thermal-stress reduction in a geologic repository, 327 (R;US)

Modeling approach to determine short- and long-term thermal and thermomechanical effects of waste emplacement in a repository in basalt, 1169 (BA;US)

Status report on the full-scale electric heater tests at the Hanford Near-Surface Test Facility, 883 (R;US)

Thermal impact of waste emplacement and surface cooling associated with geologic disposal of nuclear waste, 707 (R;US)

Thermomechanical simulation of a nuclear-waste canister in a repository in basalt, 876 (R;US)

Volcanism

Aspects of possible magmatic disruption of a high-level radioactive waste repository in southern Nevada, 699 (R;US)

Waste-rock Interactions

Strontium-basalt reactions under nuclear waste repository conditions, 1152 (BA;US)

Waste form/rock interaction leaching study using PNL 76-68 glass beads and Umtanum basalt. Part I, 1004 (R;US)

Young Modulus

Thermal impact of waste emplacement and surface cooling associated with geologic disposal of nuclear waste, 707 (R;US)

BATTELLE PACIFIC NORTHWEST LABORATORIES

Radioactive Waste Processing

Feasibility of incinerating and vitrifying organic resins in a single step, 318 (R;US)

BELGIUM

Fuel Cycle

Summary of non-US national and international fuel cycle and radioactive waste management programs 1982,83 (R;US)

Radloactive Waste Management

Summary of non-US national and international fuel cycle and radioactive waste management programs 1982,83 (R;US)

Radioactive Waste Processing

Inactive Pamela vitrification facility in Mol; mailufacture and characteristics of Vitromet products (in German), 238 (RA;DE;GE)

Inactive vitrification in Mol. Ceramic melting, feed and off gas system, pouring equipment for glassmelting (In German), 237 (RA;DE;GE)

Influence of the reprocessing flow sheet on the HLW solidification technology, 239 (RA; $\overline{\mathrm{DE}}$ )

BENTONITE

Backfill barriers for nuclear waste repositories in salt. 670 (RA;DE)

Couchomivtry

Nuclear-waste-package program for high-level isolation in Nevuda tuff, 1015 (R;US)

Hydraulic Conductivity

Ion/water migration phenomena in dense bentonites, 1174 (BA;US)

Ieaching

Review of DOE waste package program. Subtask 1.1. National waste package program, October 1982-March 1983. Vol. 4, 736 (R;US)

Permeability

Ion/water migration phenomena in dense bentonites, 1174 (BA;US)

Transport of actinides through a bentonite backfill, 1175 (BA;US)

Physical Properties

Nuclear waste package materials testing report: basaltic and tuffaceous environments, 854 (R;US)
Radiolysis

Radiolysis of groundwater from HLW stored in copper canist 1062 (J;US)

Review of DOE waste package program. Subtask 1.1. National waste package program, October 1982-March 1983. Vol. 4. 736 (R;US)

Radionuclide Migration

Diffusion measurements in compacted bentonite, 1148 (BA;US)

Rock-fluid Interactions

Evaluation of sodium bentonite and crushed basalt as waste package backfill materials, 902 (R;US)

Sorptive Properties

Development of a backfill for containment of high-level nuclear waste, 1173 (BA;US)

Evaluation of sodium bentonite and crushed basalt as waste package backfill materials, 902 (R;US)

Sorption of actinides in well-defined oxidation states on geologic media, 1351 (BA;US)

Transport of actinides through a bentonite backfill, 1175 (BA;US)

Stability

Experimental investigation of sodium bentonite stability in Hanford hasalt, 879 ( $R ; I I S)$

Nuclear waste package materials testing report: basaltio and tuffaceous environments, 854 (R;US)

Thermal Conductivity

Thermal conductivily of bentunite/quartz high-level-wastepackage backfill, 972 (R;US)

Thermal conductivity of bentonite/quartz high-level waste package backfill, 1065 (J;US)

BILLTTONITES

See TEKTITES

BIOLOGICAL MODELS

Aquatic Ecosystems

Deep sea biophysics, 1330 (RA;US)

BIOTITE

Radioniuclide Migration

Actinide sorption on rock minerals, 677 (RA;DE)

BITUMENS

Comparative Evaluntions

Evaluation of forms for the immobilization of high-lcvel and transuranic wastes, 200 (R;US)

\section{BLACK SHALES}

Stratigraphy

Regional geological assessment of the Devonian-Mississippian shale sequence of the Appalachian, Illinois, and Michigan basins relative to potential storage/disposal of radioactive wastes, 828 (R;US)

BLANKETS (BREEDING)

See BREEDING BLANKETS

BLASTS

BNL

See EXPLOSIONS

Radioactive Waste Processing

Savannah River Laboratory long-term waste storage program. Progress report No. 5, March-April 1974, 131 (R;US)

Savannah River Laboratory long-term waste storage program. Progress report No. 6, May-Jun 1974, 132 (R;US)

Savannah River Laboratory long-term waste storage program. Progress report No. 7, July-September 1974, 133 (R;US)

BOILING WATER COOLED AND MODERATED REACTO

See BWR TYPE REACTORS

BOR-60 REACTOR

Fuel Cycle

Radioactive waste processing and disposal, 7 (RA;CS;In Czech)

\section{BOREHOLES}

Brines

Analysis of the growth of brine flow pathes in rock salt, 690 (R;DE;In German)

Caliper Logging

Basic data report for Drillhole WIPP 14 (Waste Isolation Pilot Plant - WIPP), 974 (R;US) 
Closures

Earth science developments in support of waste isolation, 608 (RA;US)

Field-test programs of borehole plugs in southeastern New Mexico, 615 (RA;US)

Geochemical factors in borehole-shaft plug longevity, 614 (RA;US)

Research and development related to the Nevada Nuclear Waste Storage Investigations. Progress report, January 1-March 31, 1983, 705 (R;US)

Thermomechanical in-situ experiments and finite element computations, 1166 (BA;US)

Damage

Drilling-induced borehole-wall damage at spent fuel test-climax, 1003 (R;US)

Drilling

Basalt waste-isolation project drilling and testing. Quarterly report, January 1-March 31, 1982, 896 (R;US)

Basalt Waste Isolation Project drilling and testing. Quarterly report, April 1, 1982-June 30, 1982, 897 (R;US)

Basalt Waste Isolation Project drilling and testing. Quarterly report, July 1, 1982-September 30, 1982, 898 (R;US)

Basalt Waste Isolation Project: drilling and testing. Quarterly report, October 1, 1982-December 31, 1982, 899 (R;US)

Basalt Waste Isolation Project. Drilling and testing quarterly report, January 1, 1983-March 31, 1983, 900 (R;US)

Gamma Logging

Basic data report for Drillhole WIPP 14 (Waste Isolation Pilot Plant - WIPP), 974 (R;US)

Lithology

Basic data report for Drillhole AEC 7 (Waste Isolation Pilot Plant - WIPP) (Lea County, New Mexico), 924 (R;US)

Basic data report for drillhole AEC 8 (Waste Isolation Pilot Plant - WIPP) (Eddy County, New Mexico), 925 (R;US)

Neutron Logging

Basic data report for Drillhole WIPP 14 (Waste Isolation Pilot Plant - WIPP), 974 (R;US)

Plugging

Repository sealing, 794 (RA;US)

Sealing Materials

Geochemical factors in borehole-shaft plug longevity, 614 (RA;US)

Seals

Laboratory studies of fluid flow through borehole seals, 1231 (J;US)

Stratigraphy

Basic data report for Drillhole AEC 7 (Waste Isolation Pilot Plant - WIPP) (Lea County, New Mexico), 924 (R;US)

Basic data report for drillhole AEC 8 (Waste Isolation Pilot

BORON Plant - WIPP) (Eddy County, New Mexico), 925 (R;US)

Adsorption

Waste form/rock interaction leaching study using PNL 76-68 glass beads and Umtanum basalt. Part I, 1004 (R;US)

Emission Spectroscopy

Analyses of nuclear fuel and high-level waste by inductively coupled plasma-emission spectroscopy, 68 (R;US)

Leaching

Waste glass/metal interactions in brines, 860 (R;US)

BOROSILICATE GLASS

See also PYREX

Borosilicate glass and glass ceramics with real highly radioactive waste, 528 ( $\mathrm{J}$;US)

Development of borosilicate glass for the vitrification plant PAMELA, 530 (TG;US)

Development of borosilicate glass for the vitrification facility PAMELA, 253 (RA;DE;GE)

Fixation of radioactive materials in a glass matrix, 380 ( $\mathbf{P}$;AU)

Laboratory activity in Italy in the field of vitrification of highlevel wastes, 233 (RA;DE)

Receptivity of borosilicate glass VG 98/12 for incorporating high-level radioactive wastes (In German), 255 (RA;DE;GE)

Storage of HLW in engineered structures: air-cooled and watercooled concepts. Final report, 640 (R;XE)
Additives

Influence of metal oxides on the leach rate and crystallisation behaviour of waste glasses, 251 (RA;DE)

Chemical Analysis

Analysis for silicon in solution in high level waste glass durability studies, 375 (J;GB)

Chemical Composition

Comparative study of seven glasses for solidification of nuclear wastes, 1206 (BA;US)

Description of Defense Waste Processing Facility reference waste form and canister. Revision 1, 168 (R;US)

Effect of $\mathrm{Fe}_{2} \mathrm{O}_{0} / \mathrm{ZnO}$ on two glass compositions for solidification on Swedish nuclear wastes, 341 (R;SE)

Interaction of vitrified high level waste with clay environment, 262 (RA;DE)

Radiation-damage effects in nuclear-waste glasses, 339 (R;US)

Review of recent PNL research activities related to glass leaching mechanisms, 846 (RA;US)

XPS valence state determination of $\mathrm{Np}$ and $\mathrm{Pu}$ in multicomponent borosilicate glass and application to leached $76-68$ waste glass surfaces, 417 (BA;US)

Chemical Preparation

Preparation and characterization of an improved borosilicate glass for the solidification of high level radioactive fission product solutions (HLW). Pt. 2, 277 (R;DE)

Chemical Radiation Effects

Glasses for the solidification of high-level radioactive waste: their behavior in the presence of water, 1052 (TG;US)

Long-term performance of materials used for high-level waste packaging. Annual report, March 1982-April 1983. Vol.1, 751 (R;US)

Radiation-damage effects in nuclear-waste glasses, 339 (R;US)

Chemical Reactions

Reaction and devitrification of a prototype nuclear-waste-storage glass with hot magnesium-rich brine, 289 (R;US)

Comparative Evaluations

Comparative risk assessments for the production and interim storage of glass and ceramic waste forms: defense waste processing facility, 1370 (R;US)

Comparison of SRP high-level waste disposal costs for borosilicate glass and crystalline ceramic waste forms, 635 (R;US)

Comparison of borosilicate glass and synthetic minerals as media for the immobilization of high-level radioactive waste, 490 (J;US)

Comparison of candidate glasses to vitrify ICPP calcined alumina waste, 469 (J;US)

Description of a ceramic waste form and canister for Savannah River Plant high-level waste, 170 (R;US)

Development, evaluation, and selection of candidate high-level waste forms, 185 ( $R$;US)

Disposal of high-level radioactive wastes, 1257 (BA;AU)

Economic comparison of crystalline ceramic and glass waste forms for HLW disposal, 863 (R;US)

Evaluation and selection of candidate high-level waste forms, 160 (R;US)

Evaluation of conditioned high-level waste forms, 326 (R;US)

Evaluation of forms for the immobilization of high-level and transuranic wastes, 200 (R;US)

Postclosure risks of alternative SRP nuclear waste forms in geologic repositories, 1009 (R;US)

Corrosion

Chemical stability of simulated HLW forms in contact with clay media, 1207 (BA;US)

Comparative study of seven glasses for solidification of nuclear wastes, 1206 (BA;US)

Determination of the corrosion mechanisms of high-level waste containing glass, 1204 (BA;US)

Effect of overpack materials on glass leaching in geological burial, 1200 (BA;US)

Glasses for the solidification of high-level radioactive waste: their behavior in the presence of water, 1052 (TG;US)

Interaction of vitrified high level waste with clay environment, 262 (RA;DE)

Mechanisms for hydrothermal leaching of nuclear waste glasses: properties and evaluation of surface layers, 1189 (BA;US) 
Preliminary report on a glass burial experiment in granite, 631 (R;US)

Review of DOE waste package program. Subtask 1.1. National waste package program, 286 (R;US)

Review of DOE waste package program. Subtask 1.1. National waste package program, October 1982-March 1983. Vol. 4, 736 (R;US)

Corrosion Resistance

Effects of waste composition and loading on the chemical durability of a borosilicate glass, 1188 (BA;US)

Electrical-conductivity measurements of leachates for the rapid assessment of wasteform corrosion resistance, 554 (R;US)

Interaction of vitrified high level waste with clay environment, 274 (RA;DE)

Cost

Comparison of SRP high-level waste disposal costs for borosilicate glass and crystalline ceramic waste forms, 635 (R;US)

Crystal Structure

Neutron scattering studies of vitrified radioactive waste, 363 (J;US)

Crystallization

Attempt to assess the long-term crystallization rate of nuclear waste glissses, 457 (BA;US)

Compared quantitative determination of nuclear waste glass crystailization rate by light microscopy and $\mathrm{x}$ ray diffraction, 257 (RA;DE)

Compared quantitative determination of nuclear waste glass crystallization rate by light microscopy and $\mathrm{X}$-ray diffraction, 269 (RA;DE)

Microstructures and crystallization in simulated waste glasses, 44I (RA;IIS)

Quantitative determination of crystalline phases in nuclear waste glasses, 458 (BA;US)

Dissolution

Chemical durability of some HLW glasses: effects of hydrothermal conditions and ionizing radiation, 1203 (BA;US)

Long-term performance of materials used for high-level waste packaging. Annual report, March 1982-April 1983. Vol.1, 751 (R;US)

Solubility-limited dissolution rate in groundwater, 1338 (J;US)

Solubility-limited fractional dissolution rate of vitrified waste in groundwater, $1228(\mathrm{~J} ; \mathrm{US})$

Status report on dissolution model development, 1021 (R;US)

Economic Analysis

Economic comparison of crystalline ceramic and glass waste forms for HLW disposal, 863 (R;US)

Electric Conductivity

Change of electrical properties of the borosilicate glasses containing $\mathrm{MoO}_{0}$ by phase separation, 482 (J;JP;In Japanese)

Evaluation

Assessment of Savannah River borosilicate glass in the repository environment, 172 ( $\mathbf{K}$;US)

Assessment of high-level waste form conformance with proposed regulatory and repository criteria, 198 (R;US)

Capacity of the borosilicate glass VG $98 / 12$ for components of the high-level radioactive waste, 267 (RA;DE;In German)

Fabrication

Preparation and microstructural characterization of silicate and borosilicate glasses containing cerium or plutonium, 130 (R;BE)

Fracture Properties

Analysis of thin leaching layers and fracture mechanics of irradiated borosilicate glass for solidification of high-level fission product solutions (HAW), 365 (BA;DE;In German)

Characterization of mechanical properties of nuclear waste glasses, 1212 (BA;US)

Effect of composition and radiation on the Hertzian indentation behavior of nuclear waste glasses (77-MeV alpha particles). 145 (R;US)

Fractures

Fracture appraisal of large scale glass blocks under realistic thermal conditions, 459 (BA;US)
Fragmentation

Respirable fines produced by impacts of simulated alternative high-level waste materials, 419 (BA;US)

Heat Transfer

Waste package heat-transfer analysis: model development and temperature estimates for waste packages in a repository located in basalt, 917 (R;US)

Impact Tests

Respirable fines produced by impacts of simulated alternative high-level waste materials, 419 (BA;US)

Ion Implantation

Radiation-damage effects in nuclear-waste glasses, 339 (R;US)

Leachates

Electrical-conductivity measurements of leachates for the rapid assessment of wasteform corrosion resistance, 554 (R;US)

Leaching

Alpha spectrum profiling of plutonium in leached simulated high-level radioactive waste-glass, 517 (J;US)

Burial effects on nuclear waste glass, 1202 (BA;US)

Characterization of borosilicate glass containing Savannah River Plant radioactive waste, 179 (R;US)

Chemical durability of some HLW glasses: effects of hydrothermal conditions and ionizing radiation, 1203 (BA;US)

Comparative leach testıng of high-level wastc forms, 626 (R;US)

Cumparatlve study of seven giasses for solidification of nuclear wastes, 1206 (BA;US)

Comparison of properties of borosilicate glass and crystalline ceramic forms for immobilization of Savannah River Plant waste, 171 (R;US)

Description of Defense Waste Processing Facility reference waste form and canister. Revision 1, 168 (R;US)

Effect of overpack materials on glass leaching in geological burial, 1200 (BA;US)

Effect of $\mathrm{Fe}_{2} \mathrm{O}_{0} / \mathrm{ZnO}$ on two glass compositions for solidification of Swedish nuclear wastes, 461 (BA;US)

Effect of gamma radiolysis on waste glass leaching on brines, $862(R ; U S)$

Effects of cracks on glass leaching, 126 (J;US)

Effects of waste composition and loading on the chemical durability of a borosilicate glass, 1188 (BA;US)

Elemental release from glass and spent fuel, 1307 (RA;US)

Flow model for the kinetics of dissolution of nuclear waste forms; a comparison of borosilicate glass, SYNROC and high-silica glass, 1192 (BA;US)

Fuel cycle programs. Quarterly progress report, April-June 1982 , 536 (R;US)

Fuel cycle programs. Quarterly progress report, July-September 1982, 538 (R;US)

Glacs leaohing performanoe, 856 (R,US)

Glasses for the solidification of high-level radioactive waste: their behavior in the presence of water, 1052 (TG;US)

Hydrolytic durability of two bigh-level waste oxide buroxilicate glasses (gp 98/12 and gp 98/26), 524 (J;US)

Immobilization of high-level nuclear reactor wastes in SYNROC: a current appraisal, 515 (J;US)

Influence of metal oxides on the leach rate and crystallisation behaviour of waste glasses, 251 (RA;DE)

Influence of a backfilling material on borosilicate glass leaching, 1197 (BA;US)

Interaction of vitrified high level waste with clay envirnnment, 262 (RA;DE)

Intercomparison of leach-testing methods and the effects of waste-form composition on test type and duration, 1157 (J;US)

Leach testing of INEL waste forms in a gamma field, 1044 (J;US)

Leaching behavior of a simple borosilicate glass in a confined environment, 1205 (BA;US)

Leaching of vitrified high-level radioactive waste, 1194 (BA;US

I.eaching of actinides from simulated nuclear waste glass, 1196 (BA;US)

Long-term radioactivity release from solidified high-level waste. Part I. An approach to evaluating experimental data, 1191 (BA;US) 
Long-term radioactivity release from solidified high-level waste. Part II. Parametric study of waste from properties, temperature and time, 1333 ( $\mathrm{J} ; \mathrm{US})$

Long-term performance of materials used for high-level waste packaging. Annual report, March 1982-April 1983. Vol.1, 751 (R;US)

Mechanisms of dissolution of radioactive waste storage glasses and cesium migration from a granite repository, 1151 (BA;US)

Mechanisms for hydrothermal leaching of nuclear waste glasses: properties and evaluation of surface layers, 1189 (BA;US)

Neptunium concentrations in solutions contacting actinide-doped glass, 1336 (J;US)

Nuclear waste-form risk assessment for US Defense waste at Savannah River Plant. Annual report FY 1981, 1007 (R;US)

Predicting Pu concentrations in solutions contacting geologic materials, 1146 (BA;US)

Preliminary report on a glass burial experiment in granite, 631 (R;US)

Preparation and leaching of radioactive INEL waste forms, 201 (R;US)

Radiation-damage effects in nuclear-waste glasses, 339 (R;US)

Relationship between glass leaching mechanism and geochemical transport of radionuclides, 1195 (BA;US)

Review of recent PNL research activities related to glass leaching mechanisms, 846 (RA;US)

Review of DOE waste package program. Subtask 1.1. National waste package program, October 1982-March 1983. Vol. 4, 736 (R;US)

Semi-unified approach to leach testing, 852 (RA;US)

Solubility-limited fractional dissolution rate of vitrified waste in groundwater, 1228 (J;US)

SRP radioactive glass studies: small-scale process development and product performance, 180 (R;US)

Study of the migration of leached radionuclides in a natural fissure of granite rock, $1240(\mathrm{~J} ; \mathrm{CS})$

Summary report for the interlaboratory round robin on the MCC-1 static leach test method, 300 (R;US)

Surface properties and performance prediction of alternative waste forms. Annual report, October 1, 1981-September 30, 1982. Volume 1, 753 (R;US)

Underground migration of long-lived radionuclides leached from a borosilicate glass matrix, 1360 (BA;XA)

Utilization of charged particle backscattering to study the near surface region of glasses. Application to depth profiling of lanthanium, cerium, throium and uranium induced by aqueous leaching, 1216 (BA;US)

Waste form/rock interaction leaching study using PNL 76-68 glass beads and Umtanum basalt. Part I, 1004 (R;US)

Waste glass/repository interactions, 1193 (BA;US)

Waste package materials testing for a salt repository: 1982 status report, 825 (R;US)

XPS valence state determination of $\mathrm{Np}$ and $\mathrm{Pu}$ in multicomponent borosilicate glass and application to leached 76-68 waste glass surfaces, 417 (BA;US)

Licensing

Review of DOE waste package program. Subtask 1.1. National waste package program, 286 (R;US)

Material Balance

Materials balance: scientific fundamentals for the quality assurance of vitrificd waste, 462 (BA;US)

Microstructure

Microstructures and crystallization in simulated waste glasses, 441 (BA;US)

Neutron Diffraction

Neutron scattering studies of vitrified radioactive waste, 363 (J;US)

Nucleation

Structural effects of radiation damage in silica based glasses, 464 (BA;US)

Performance Testing

Effects of various waste contents on properties of borosilicate glasses for high-level waste solidification in several countries, 223 (R;JP;In Japanese)
Phase Studies

Change of electrical properties of the borosilicate glasses contain ing $\mathrm{MoO}_{0}$ by phase separation, 482 (J;JP;In Japanese)

Quantitative determination of crystalline phases in nuclear waste glasses, 458 (BA;US)

Phase Transformations

Influence of metal oxides on the leach rate and crystallisation behaviour of waste glasses, 251 (RA;DE)

Physical Radiation Effects

Analysis of thin leaching layers and fracture mechanics of irradiated borosilicate glass for solidification of high-level fission product solutions (HAW), 365 (BA;DE;In German)

Effect of composition and radiation on the Hertzian indentation behavior of nuclear waste glasses (77-MeV alpha particles), 145 (R;US)

Effect of composition and radiation on the fracture of a nuclear waste glass (alpha particles), 1434 (J;CH)

Radiation-damage effects in nuclear-waste glasses, 339 (R;US)

Structural effects of radiation damage in silica based glasses, 464 (BA;US)

\section{Quality Assurance}

Materials balance: scientific fundamentals for the quality assurance of vitrified waste, 462 (BA;US)

Using process instrumentation to obviate destructive examination of canisters of HLW glass, $328(R ; U S)$

Radiation Effects

Chemical durability of some HLW glasses: effects of hydrothermal conditions and ionizing radiation, 1203 (BA;US)

Comparison of properties of borosilicate glass and crystalline ceramic forms for immobilization of Savannah River Plant waste, 171 (R;US)

Glass leaching performance, 856 (R;US)

Review of the current status of radiation effects in solid nuclear waste forms, 372 (J;US)

Radiolysis

Nuclear waste package materials testing report: basaltic and tuffaceous environments, 854 (R;US)

Research Programs

Surface properties and performance prediction of alternative waste forms. Annual report, October 1, 1981-September 30, 1982. Volume 1,753 (R;US)

Sintering

Preparation of glass containing high-level radioactive waste (simulated) - copper composite compacts by a continuous pressure sintering method, 479 (J;JP;In Japanese)

Specifications

Assessment of high-level waste form conformance with proposed regulatory and repository criteria, 198 (R;US)

Conceptual waste package interim product specifications and data requirements for disposal of borosilicate glass defense high-level waste forms in salt geologic repositories, 823 (R;US)

Waste glasses: requirements and characteristics, 322 (R;US)

Stability

Borosilicate glass as a matrix for immobilization of SRP highlevel waste, 512 (J;US)

Structural Chemical Analysis

XPS valence state determination of $\mathrm{Np}$ and $\mathrm{Pu}$ in multicomponent borosilicate glass and application to leached 76-68 waste glass surfaces, 417 (BA;US)

Temperature Effects

Compared quantitative determination of nuclear waste glass crystallization rate by light microscopy and $x$ ray diffraction, 257 (RA;DE)

Comparison of properties of borosilicate glass and crystalline ceramic forms for immobilization of Savannah River Plant waste, 171 (R;US)

Glass leaching performance, 856 (R;US)

Thermal Stresses

Fracture appraisal of large scale glass blocks under realistic thermal conditions, 459 (BA;US)

Waste-rock Interactions

Backfill-waste interactions in repository simulating tests, 1153 (BA;US) 
Interaction of borosilicate glass and granodiorite at $100^{\circ} \mathrm{C}, 50$ MPa: implications for models of radionuclide release, 1201 (BA;US)

Waste glass/repository interactions, 1193 (BA;US)

$\mathrm{X}$-ray Diffraction

Quantitative determination of crystalline phases in nuclear waste glasses, 458 (BA;US)

Young Modulus

Characterization of mechanical properties of nuclear waste glasses, 1212 (BA;US)

BOROSILICATES

See BOROSILICATE GLASS

BRACKISH WATER ECOSYSTEMS

SEe AQUATIC ECOSYSTEMS

BRAZLL

Away-from-reactor Storage

Considerations on the management of wastes expected to be generated by the operation of Brazilian nuclear reactors, 663 (RA;BR;In Portuguese)

Fuel rycle

Summary of non-US national and international fuel cycle and radioactive waste management programs 1982,83 (R;US)

High-level Radioactive Wastes

Projections of high-level waste production in Brazil, 102 (J;US)

Radisactive Wuste Manugemuni

Considerations on the management of wastes expected to be generated by the operation of Brazilian nuclear reactors, 663 (RA;BR;In Portuguese)

Summary of non-US national and international fuel cycle and radioactive waste management programs 1982,83 (R;US)

\section{BREEDING BLANKETS}

Heat .Transfer

Fuel cycle programs. Quarterly progress report, OctoberDecember 1982, 539 (R;US)

Tritium Recovery

Fuel cycle programs. Quarterly progress report, OctoberDecember 1982, 539 (R;US)

\section{BRINFS}

Chemical Composition

Results of repository conditions study for commercial and defense high-level nuclear waste and spent fuel repositories in salt, $82.4(R ;$ US $)$

Chemlcal Reaction

Reaction and devitrification of a prototype nuclear-waste-storage glass with hot magnesium-rich brine, 289 (R;US)

Comparative Evaluations

Comparison of brines relevant to nuclear waste experimentation, 994 (R;US)

Córtosjve Effects

Container assessment: corrosion study of HLW container materials. Quarterly progress report, July-September 1981, 728 (R;US)

Corrosion of TiCode-12 in a simulated Waste Isolation Pilot Proj ect (WIPP) brine, 1066 (J;US)

Identification of crevice corrosion in the titanium alloy TiCode- 12 in simulated rock salt brine at $150^{\circ} \mathrm{C}, 545$ (R;US)

Waste package materials testing for a salt repository: 1982 status report, 825 (R;US)

Diffusion

Brine migration in salt and its implications in the geologic disposal of nuclear waste, 829 (R;US)

Domal salt brine migration experiments at Avery Island, Louisiana, 621 (RA;US)

Migration rates of brine inclusions in single crystals of $\mathrm{NaCl}$, 1149 (BA;US)

Thermal-gradient migration of brine inclusions in salt, 711 (R;US)

Ionic Composition

Comparison of brines relevant to nuclear waste experimentation, 994 (R;US)

Migration

Results of repository conditions study for commercial and defense high-level nuclear waste and spent fuel repositories in salt, 824 (R;US)
Thermal-gradient migration of brine inclusions in salt crystals, 713 (R;US)

Origin

Comparison of brines relevant to nuclear waste experimentation, 994 (R;US)

Radiolysis

Effect of gamma radiolysis on waste glass leaching on brines (Gamma rays), 862 (R;US)

Effects of radiation on corrosion of titanium, 961 (RA;US)

BROOKHAVEN NATIONAL LABORATORY

See BNL.

BUILDING (MANUFACTURING)

See FABRICATION

BWR TYPE REACTORS

Thorium Cycle

Use of thorium as an alternative nuclear fuel, $46(\mathrm{R} ; \mathrm{AU})$

\section{C}

\section{CADMIUM}

Emission Spectroscopy

$\Lambda$ nalyses of nuclear fuel and high-level waste by inductively coupled plasmasmission spert rnsrnny, $6 R$ (R;I IS)

CALCINATION

Off-gas Systems

ICPP waste calcining facility safety analysis report, 219 (R;US)

Process Heat

Alternative fuels and heating methods for calciner process heat, 204 (R;US)

\section{CALCINED WASTES}

Chemical Composition

Physical and chemical characterization of synthetic calcined sludge, 167 (R;US)

Chemical Radiation Effects

Leachability study of modified Synroc-B waste form: effect of gamma irradiation, 412 (J;GB)

Comparative Evaluations

Evaluation of forms for the immobilization of high-level and transuranic wastes, 200 (R;US)

Encapsulation

Process for producing biologically noxious waste, particularly radioactive waste, wich can be stored (German Patent), 519 (P;DE;In German)

Gas Yields

Physical and chemical characterization of synthetic calcined oludge; 167 (R;US)

Leaching

Geochemical behavior of supercalcine waste form: its stability in a basalt environment, 911 (R;US)

Leachability study of modified Synroc-B waste form: effect of gamma irradiation, $412(\mathrm{~J} ; \mathrm{GB})$

Melting Points

Solidification of high level wastes. Part III. Solubility, melting point, flow temperature and thermal conductivity of some cal cined, synthetic purex-type wastes, 209 (R;US)

Microstructure

Geochemical behavior of supercalcine waste form: its stability in a basalt environment, 911 (R;US)

Physical Radiation Effects

Radiation damage from alpha decay in ceramic nuclear waste forms, $103(\mathrm{~J} ; \mathrm{CH})$

Radiation Effects

Radiation damage in nuclear waste ceramics, 377 (J;US)

Solubility

Solidification of high level wastes. Part III. Solubility, melting point, flow temperature and thermal conductivity of some calcined, synthetic purex-type wastes, 209 (R;US)

Structural Chemical Analysis

Physical and chemical characterization of synthetic calcined sludge, 167 (R;US) 
Thermal Conductivity

Fixation of radioactive residues. Quarterly progress report, January-March 1961, 213 (R;US)

Solidification of high level wastes. Part III. Solubility, melting point, flow temperature and thermal conductivity of some calcined, synthetic purex-type wastes, 209 (R;US)

Thermal Gravimetric Analysis

Physical and chemical characterization of synthetic calcined sludge, 167 (R;US)

\section{CALCIUM}

Adsorption Isotherms

Basalt-radionuclide reactions: FY-1981. Annual report, 868 (R;US)

Emission Spectroscopy

Analyses of nuclear fuel and high-level waste by inductively coupled ṕlasma-emission spectroscopy, 68 (R;US)

Leaching

Leaching behavior of sintered modified SYNROC-B waste forms: effect of HLW addition, 445 (BA;US)

Leaching studies on SYNROC at $95^{\circ} \mathrm{C}$ and $200^{\circ} \mathrm{C}, 1114(\mathrm{~J} ; \mathrm{CH})$

Preparation and leaching of radioactive INEL waste forms, 201 (R;US)

Processing effects on the behavior of titanate waste forms in aqueous solutions, 1145 (BA;US)

Processing, microstructure, leaching, and long-term stability studies related to titanate high-level waste forms, 340 (R;US)

Solubility limits of metal ions and mechanism of leaching nuclear-waste glasses, 320 (R;US)

SYNROC: leaching performance and process technology, 250 (RA;DE)

CALCIUM COMPLEXES

Solubility

Importance of organic compounds in ground water as radionuclide-mobilizing agents, 1324 (R;US)

CALCIUM OXIDES

See also PEROVSKITE TYUYAMUNITE ZIRCONOLITE

Leaching

Influence of metal oxides on the leach rate and crystallisation behaviour of waste glasses, 251 (RA;DE)

Physical Radiation Effects

Alpha-recoil damage in natural zirconolite and perovskite, 518 (J;JP)

CANADA

See also ONTARIO

Fuel Cycle

Summary of non-US national and international fuel cycle and radioactive waste management programs 1982, 83 (R:US)

High-level Radioactive Wastes

Materials research for the Canadian Nuclear Fuel Waste Management Program, 109 (BA;US)

Intermediate-level Radioactive Wastes

Materials research for the Canadian Nuclear Fuel Waste Management Program, 109 (BA;US)

Radioactive Waste Disposal

Canadian geoscience research for nuclear fuel waste management, 592 (RA; US)

Materials research for the Canadian Nuclear Fuel Waste Management Program, 109 (BA;US)

Nuclear fuel waste disposal - status of the Canadian program, $1263(\mathrm{~J} ; \mathrm{US})$

Overview of nuclear waste management, $1068(\mathrm{~J} ; \mathrm{GB})$

Radioactive Waste Management

Management of nuclear fuel waste. Final report, 71 (R;CA)

Materials research for the Canadian Nuclear Fuel Waste Management Program, 109 (BA;US)

Overview of nuclear waste management, 1068 (J;GB)

Radioactive waste management and disposal, 639 (RA;CA)

Scope and nature of the problem of high level nuclear waste disposal, 1430 (R;CA)

Summary of non-US national and international fuel cycle and radioactive waste management programs 1982,83 (R;US)
Spent Fuels

Materials research for the Canadian Nuclear Fuel Waste Management Program, 109 (BA;US)

\section{CANCER}

See NEOPLASMS

CANDU TYPE REACTORS

Thorium Cycle

Use of thorium as an alternative nuclear fuel, 46 (R;AU)

\section{CARBON 14}

Environmental Transport

Compliance assessments of hypothetical geological nuclear waste isolation systems with the draft EPA standard, $982(R ; U S)$

Exposures resulting from nuclear power production. Annex $F$, 1444 (BA;US)

\section{CARBON STEELS}

Chemical Composition

Investigations of suitable metallic container materials for HAW solidification, $371(\mathrm{~J} ; \mathrm{US})$

Compatibility

Studies of waste-canister compatibility, 832 (R;US)

Corrosion

Corrosion studies and mechanical tests on metallic materials for the design of packagings for vitrified high level wastes (HLW), 689 (R;DE;In German)

Long-term high-level-waste technology. Composite quarterly technical report, April-June 1982, 165 (R;US)

Long-term performance of materials used for high-level waste packaging. Annual report, March 1982-April 1983. Vol.1, 751 (R;US)

Review of DOE waste package program. Subtask 1.1. National waste package program, October 1982-March 1983. Vol. 4, 736 (R;US)

Waste package conceptual designs for a nuclear repository in basalt, 872 (R;US)

Corrosion Protection

Prevention of stress corrosion cracking in nuclear waste storage tanks, 630 (R;US)

Corrosion Resistance

Corr sion of materials in a clay environment, $676(R A ; D E)$

Selection of barrier metals for a waste package in tuff, 1020 (R;US)

Failures

Review of DOE waste package program. Subtask 1.1. National waste package program, October 1982-March 1983. Vol. 4, 736 (R;US)

Mechanical Properties

Selection of barrier metals for a waste package in tuff, 1020 (R;US)

Plasma Arc Welding

Remote automatic plasma arc-closure welding of a dry-storage canister for spent nuclear fuel and high-level radioactive waste, $551(R ; U S)$

\section{Stress Corrosion}

Prevention of stress corrosion cracking in nuclear waste storage tanks, 630 (R;US)

Stress corrosion cracking test with slow strain rate and constant current, 1293 (BA;US)

Tensile Properties

Corrosion studies and mechanical tests on metallic materials for the design of packagings for vitrified high level wastes (HLW), 689 (R;DE;In German)

Weldability

Selection of barrier metals for a waste package in tuff, 1020 (R;US)

\section{CARBONATE MINFRAIS}

Radiolysis

Gas production and liberation from rock salt samples and potential consequences on the disposal of high-level radioactive waste in salt domes, 1165 (BA;US) 
CARNALLITE

Thermal Conductivity

Impurities in rock-salt: consequences for the temperature increases at the disposal of high-level nuclear waste, 834 (R;US)

CASKS

See also SPENT FUEL CASKS

Design

Economic evaluation of nuclear waste transportation casks, 14 (R;US)

High-Level Waste Transportation-System Development Program design report for Fiscal Year 1980, 34 (R;US)

Transportation packagings for high-level wastes and unprocessed transuranic wastes, 29 (R;US)

\section{Impact Tésts}

Experimental and analytical program to evaluate packaging and target hardness as related to nuclear waste transportation systems, 32 (R;US)

Large deformation inelastic analysis of impact for shipping casks (DYNA3D Code), 1436 (J;NL)

Two and three-dimensional dynamic analyses of DHLW'casks, 558 (RA;US)

Materials

High-Level Waste Transportation-System Development Program design report for Fiscal Year 1980, 34 (R;US)

Materials Testing

Surface storage of vitrified high-level radioactive waste in reinforced-concrete casks, 534 (R;GB)

Performance Testing

Experimental and analytical program to evaluate packaging and target hardness as related to nuclear waste transportation systems, 32 (R;US)

Restraints

Evaluation of proposed methods of radioactive material container tie-down design for truck transport, 44 (J;US)

Static Loads

Mechanical load on highly radioactive waste containers in final storage, 1141 (RA;DE; In German)

Tbermal Analysis

Vitrification of high-level wastes: A review of the computer thermal analysis for storage canisters (CANIST), 1439 (J;NL)

Thermal Stresses

Mechanical load on highly radioactive waste containers in final stornge, 11 11 (BA;DF; In German)

\section{CAST IRON}

\section{Corrosion}

General corrosion, irradiation-corrosion, and environmentalmechanical evaluation of nuclear-waste-package structuralbarrier materials. Progress report, 843 (R;US)

Waste package conceptual designs for a nuclear repository in basali, 872 (R;US)

Corrosion Resistance

Canister materials proposed for final disposal of high level nuclear waste - a review with respect to corrosion resistance, 675 (RA:DE)

Physical Radiation Effects

General corrosion, irradiation-corrosion, and environmentalmechanical evaluation of nuclear-waste-package structuralbarrier materials. Progress report, 843 (R;US)

\section{CELLS (BACTERIAL)}

See BACTERIA

\section{CEMENTS}

Development and testing of matrices for the encapsulation of glass and ceramic nuclear waste forms, 297 (R;US)

Compression Strength

Savannab River Laboratory long-term waste storage program. Progress report No. 5, March-April 1974, 131 (R;US)

Savannah River Laboratory long-term waste storage program. Progress report No. 6, May-Jun 1974, 132 (R;US)

\section{Lèching}

Leach characterization of cement encapsulated wastes, 1156 (J;US)

Savannah River Laboratory long-term waste storage program. Progress report No. 5, March-April 1974, 131 (R;US)
Savannah River Laboratory long-term waste storage program. Progress report No. 6, May-Jun 1974, 132 (R;US)

Savannah River Laboratory long-term waste storage program. Progress report No. 7, July-September 1974, 133 (R;US)

Physical Radiation Effects

Radiation effects in crystalline high-level nuclear waste solids, 1278 (BA;US)

Savannah River Laboratory long-term waste storage program. Progress report No. 6, May-Jun 1974, 132 (R;US)

Temperature Effects

Savannah River Laboratory long-term waste storage program. Progress report No. 6, May-Jun 1974, 132 (R;US)

Thermal Conductivity

Savannah River Laboratory long-term waste storage program. Progress report No. 5, March-April 1974, 131 (R;US)

CERAMIC MELTERS

Theoretical predictions for continuous-slurry feeding of a glass melter, 182 (R;US)

Air Pollution Abatement

Off-gas characteristics of defense waste vitrification using liquidfed Joule-heated ceramic melters, 310 (K;US)

Control Systems

Electrical service and controls for Joule heating of a defense waste experimental glass melter, 192 (R;US)

Design

Design of Harwell Joule Ceramic Melter, 231 (RA;DE)

Electrical service and controls for Joule heating of a defense waste experimental glass melter, 192 (R;US)

High-level waste vitrification technique in a full-scale pilot plant, 439 (BA;US)

Physical modeling of a glass melter designed for vitrification of defense waste, 190 (R;US)

Remote design features for the West Valley slurry-fed ceramic melter system, 329 (R;US)

Status of HAW-Ceramic-Melting Technology at the Institute of Nuclear Affairs (Kernfurschungsizenti uni Kalstulıe; in German), 235 (RA;DE;GE)

Engineered Safety Systems

Hazards analysis of TNX Large Melter-Off-Gas System, 197 (R;ÜS)

Functional Models

Physical modeling of a glass melter designed for vitrification of defense waste, 190 (R;US)

Gaseous Wastes

Off-gas characteristics of defense waste vitrification using liquidfed Joule-heated ceramic melters, 310 (R;US)

Materials Testing

Corrosion experience with a slurry-fed ceramic melter, 321 (R;US)

Evaluation of glass-contact materials for waste glass melters, 189 (R;US)

Off-gas Systems

Efficient particulate scrubber for glass melter off-gas, 183 (R;US)

Hazards analysis of TNX Large Melter-Off-Gas System, 197 (R;US)

Off-gas characteristics of liquid-fed joulc-heatod ceramic melters, 319 (R;US)

Off-gas characteristics of defense waste vitrification using liquidfed Joule-heated ceramic melters, 310 (R;US)

Performance

Physical modeling of a glass melter designed for vitrification of defense waste, 190 (R;US)

Power Supplies

Electrical service and controls for Joule heating of a defense waste experimental glass melter, 192 (R;US)

Start-up

Startup of a Joule-heated glass melter with a graphite slurry, 188 (R;US) 


\section{ERAMICS}

Borosilicate glass and glass ceramics with real highly radioactive waste, 528 (J;US)

Formulation and processing of polyphase ceramics for high level nuclear waste, 421 (BA;US)

Immobilization of Idaho Chemical Processing Plant high-level wastes, 240 (RA;DE)

Bulk Density

Consolidated waste forms: glass marbles and ceramic pellets, 299 (R;US)

Chemical Composition

Diopside glass-ceramic material for the immobilization of radioactive wastes, 258 (RA;DE)

Comparative Evaluations

Comparative risk assessments for the production and interim storage of glass and ceramic waste forms: defense waste processing facility, 1370 (R;US)

Comparison of SRP high:level waste disposal costs for borosilicate glass and crystalline ceramic waste forms, 635 (R;US)

Consolidated waste forms: glass marbles and ceramic pellets, 299 (R;US)

Development and testing of matrices for the encapsulation of glass and ceramic nuclear waste forms, 297 (R;US)

Development, evaluation, and selection of candidate high-level waste forms, 185 (R;US)

Economic comparison of ecrystalline ceramic and glass waste forms for HLW disposal (Titanate-based ceramic), 863 (R;US)

Evaluation and selection of candidate high-level waste forms, 160 (R;US)

Evaluation of conditioned high-level waste forms, 326 (R;US)

Evaluation of forms for the immobilization of high-level and transuranic wastes, 200 '(R;US)

Corrosion Resistance

Canister materials proposed for final disposal of high level nuclear waste - a review with respect to corrosion resistance, 675 (RA;DE)

Corrosion studies on non-metallic inorganic materials for HLW Cost packaging design, 691 (R;DE;In German)

Comparison of SRP high-level waste disposal costs for borosiljcate glass and crystalline ceramic waste forms, 635 (R;US)

Cracks

Dissolution mechanisms in high-level nuclear waste consolidation forms, 305 (RA;US)

Crystallization

Crystalline ceramics as a high-level nuclear waste form, 395 (J;US)

Economic Analysis

Economic comparison of crystalline ceramic and glass waste forms for HLW disposal (Titanate-based ceramic), 863 (R;US)

Evaluation

Assessment of high-level waste form conformance with proposed regulatory and repository criteria, 198 (R;US)

Fractures

Final report of experimental laboratory-scale brittle fracture studies of glasses and ceramics, 129 (R;US)

Fragmentation

Respirable fines produced by impacts of simulated alternative high-level waste materials, 419 (BA;US)

High-level Radioactive Wastes

Dissolution mechanisms in high-level nuclear waste consolidation forms, 305 (RA;US)

Impact Strength

Consolidated waste forms: glass marbles and ceramic pellets, 299 (R;US)

Impact Tests

Final report of experimental laboratory-scale brittle fracture studies of glasses and ceramics, 129 (R;US)

Rospirable fines produced by impaets of simulated alternative high-level waste materials, 419 (BA;US)

Leaching.

Characterization and testing of basalt glass and glass ceramic waste forms, 313 (R;US)

Comparative leach testing of high-level waste forms, 626 (R;US)
Consolidated waste forms: glass marbles and ceramic pellets, 299 (R;ÚS)

Dissolution mechanisms in high-level nuclear waste consolidation forms, 305 (RA;US)

Flow model for the kinetics of dissolution of nuclear waste forms; a comparison of borosilicate glass, SYNROC and high-silica glass, 1192 (BA;US)

Hydroxylated ceramic waste forms and the absurdity of leach tests, 254 (RA;DE)

Leach testing of INEL waste forms in a gamma field, 1044 (J;US)

Leaching of natural and synthetic sphene and perovskite, 456 (BA;US)

Preparation and leaching of radioactive INEL waste forms, 201 (R;US)

SIMS depth profiling studies of sphene-based ceramics and glass ceramics leached in synthetic groundwater, 455 (BA;US)

Manufacturing

Some glass-ceramics for special applications, 481 (J;JP)

Microstructure

Consolidated waste forms: glass marbles and ceramic pellets, 299 (R;US)

Phase Studies

Dissolution mechanisms in high-level nuclear waste consolidation forms, 305 (RA;US)

Physical Radiation Effects

Radiation effects in crystalline high-level nuclear waste solids, 1278 (BA;US)

Porosity

Consolidated waste forms: glass marbles and ceramic pellets, 299 (R;US)

Radiation Effects

Radiation damage in nuclear waste ceramics, 377 (J;US)

Review of the current status of radiation effects in solid nuclear waste forms, 372 (J;US)

Specifications

Assessment of high-level waste form conformance with proposed regulatory and repository criteria, 198 (R;US)

Stability

Waste package, 288 (RA;US)

Surface Coating

-Alternative waste form development - low-temperature pyrolytic carbon coatings, 511 (J;US)

Uses

Some glass-ceramics for special applications, 481 (J;JP)

Waste-rock Interactions

Backfill-waste interactions in repository simulating tests (Glass ceramic, supercalcine ceramic, sintered ceramic waste forms), 1153 (BA;US)

\section{CERIUM}

Absorption Spectroscopy

Subsolidus sintering of SYNROC: I. Initial demonstrations, 280 (R;US)

Activation Analysis

Application of neutron-activation analysis to the determination of leach rates of simulated nuclear-waste forms, 127 (R;US)

Subsolidus sintering of SYNROC: I. Initial demonstrations, 280 (R;US)

\section{Adsorption}

Geochemistry studies pertaining to the G-tunnel radionuclide migration field experiment; 1426 (R;US)

Emission Spectroscopy

Analyses of nuclear fuel and high-level waste by inductively coupled plasma-emission spectroscopy, 68 (R;US)

\section{Leaching}

Comparison of properties of borosilicate glass and crystalline ceramio forms for immobilization of Savannah River Plant waste, 171 ( $\mathbf{k}$; US)

Subsolidus sintering of SYNROC: I. Initial demonstrations, 280 (R;US)

Subsolidus sintering of SYNROC: II. Materials selections, process improvements, waste form evaluations, 281 (R;US) 
Radiation Scattering Analysis

Utilization of charged particle backscattering to study the near surface region of glasses. Application to depth profiling of lanthanium, cerium, throium and uranium induced by aqueous leaching, 1216 (BA;US)

Valence

Valence states of actinides in synthetic monazites, 1276 (BA;US)

\section{CERIUM 139}

Sorption

Distribution coefficient (Kd) concept and its applicability to studies of radionuclide migration in geological media, 1313 (R;GB)

Effect of microbial activity on the containment of radioactive waste in a deep geological repository, 1357 (BA;US)

Radionuclide retardation during transport through fractured granite, 1355 (BA;US)

\section{CERIUM 141}

Leaching

Measurement of leaching from simulated nuclear-waste glass using radiotracers. 128 (R:US)

\section{CERIUM 14A}

Adsorption

Laboratory tests on the migration behavior of selected fission products in aquifer materials from a potential disposal site in nurthern Germany, 1332 (BA;US)

Diffusion

Laboratory tests on the migration behavior of selected fission products in aquifer materials from a potential disposal site in northern Germany, 1352 (BA;US)

Environmental Exposure Pathway

Investigation of the consequences of leakage incidents of a tank with high-concentrated radionuclide solutions and pentration of the radioactive solution into the soil, 1423 (R;DE;In German)

Environmental Transport

Investigation of the consequences of leakage incidents of a tank with high-concentrated radionuclide solutions and pentration of the radioactive solution into the soil, 1423 (R;DE;In German)

Leaching

High-level waste glass field burial tests at CRNL: the effect of geochemical kinetics on the release and migration of fission products in a sandy aquifer, 1299 (R;CA)

Leaching study of nuclear melt glass: Part I, 1332 (R;US)

\section{CERIUM OXIDES}

Leaching

Influence of metal oxides on the leach rate and crystallisation behaviour of waste glasses, 251 (RA;DE)

\section{CERMETS}

Comparative Evaluations

Evaluation of forms for the immobilization of high-level and

\section{CESIUM} transuranic wastes, 200 (R;US)

\section{Absorption Spectroscopy}

Subsolidus sintering of SYNROC: I. Initial demonstrations, 280 (R;US)

\section{Activation Analysis}

Application of neutron-activation analysis to the determination of leach rates of simulated nuclear-waste forms, 127 (R;US)

Subsolidus sintering of SYNROC: 1. Initial demonstrations, 280 (R;US)

\section{Adsorption}

Diffusion in crystalline rocks, 1168 (BA;US)

Geochemistry studies pertaining to the G-tunnel radionuclide migration field experiment, 1426 (R;US)

Importance of organic compounds in ground water as radionuclide-mobilizing agents, 1324 (R;US)

Radionuclide migration into natural fracture surfaces of granitic rock, 1334 (J;US)

Research and development activities: fixation of radioactive residues. Quarterly progress report, April-June 1960, 210 (R;US)

Savannah River Laboratory long-term waste storage program. Progress report No. 7, July-September 1974, 133 (R;US)

Status of radionuclide sorption-desorption studies performed by the WRIT program, 1308 (RA;US)

Transport of actinides through a bentonite backfill, 1175 (BA;US)

\section{Adsorption Isotherms \\ Basalt-radionuclide reactions: FY-1981. Annual report, 868} (R;US)

Desorption

Research and development related to the Nevada Nuclear Waste Storage Investigations. Progress repurt, July 1-September 30, 1981, 692 (R;US)

Status of radionuclide sorption-desorption studies performed by the WRIT program, 1308 (RA;US)

Diffusion

Diffusion in crystalline rocks, 1168 (BA;US)

Transport of actinides through a bentonite backfill, 1175 (BA;US)

Distribution Functions

Radionuclide migration into natural fracture surfaces of granitic rock, 1334 (j;US)

Status of radionuclide sorption-desorption studies performed by the WRIT program, 1308 (RA;US)

Ion Exchange

Research and dẹvelopment activities: fixation of radioactive residucs. Quarterly progreas repart, July.Septemhor 1960, 212 (R;US)

Leaching

Comparison of properties of borosilicate glass and crystalline cer anic fuı ms fur inmubilizalion of Bavainitab River Plant waste, 171 (R;US)

Development and testing of SYNROC $\mathrm{C}$ as a high level nuclear waste form, 418 (BA;US)

FUETAP concrete: an alternative radioactive waste host, 256 (RA;DE)

Hydroxylated ceramic waste forms and the absurdity of leach tests, 254 (RA;DE)

Leaching behavior of sintered modified SYNROC-B waste forms: effect of HLW addition, 445 (BA;US)

Leaching studies on SYNROC at $95^{\circ} \mathrm{C}$ and $200^{\circ} \mathrm{C}, 1114(\mathrm{~J} ; \mathrm{CH})$

Processing effects on the behavior of titanate waste forms in aqueous solutions, 1145 (BA;US)

Savannah River Laboratory long-term waste storage program Progress report No. 7, July-September 1974, 133 (R;US)

Subsolidus sintering of SYNROC: I. Initial demonstrations, 280 (R;US)

Subsolidus sintering of SYNROC: II. Materials selections, pro cess improvements, waste form cvaluations, 281 (K;US)

SYNROC: leaching performance and process technology, 250 (KA;UE)

Waste glass/metal interactions in brines, 860 (R;US)

Physical Radjation Effects

Influence of gamma irradiation on the leaching behavior of a modified SYNROC-B ceramic waste form, 424 (BA;US)

Precipitation

Precipitation-adsorption process for the decontamination of nuclear waste supernates (Patent), 397 (P;US)

Precipitation process for supernate decontamination, 173 (R;US)

Precipitation process for decontamination of water-solublc SRP radioactive waste, 184 (R;US)

Precipitation process for decontaminating water-soluble SRP radioactive waste, 187 (R;US)

Supernatant treatment considerations for the neutralized waste at West Valley, 370 (J;US)

Separation Processes

Cesium and strontium fractionation from HLW for thermal-stress reduction in a geologic repository, 327 (R;US)

Systems study of the feasibility of high-level nuclear-waste fractionation for thermal-stress control in a geologic repository: main report, 308 (R;US)

Systems study of the feasibility of high-level nuclear waste fractionation for thermal stress control in a geologic repository: appendices, 309 (R;US)

Waste-Mixes Study for space disposal, 815 (R;US)

Sorption

Evaluation of sodium bentonite and crushed basalt as waste package backfill materials, 902 (R;US)

Radionuclide sorption on basalt-interbed materials. Annual report FY 1981, 1328 (R;US)

Recent advances in repository seal materials, 1172 (BA;US) 
Research and development related to the Nevada Nuclear Waste Storage Investigations. Progress report, July 1-September 30, 1981,692 (R;US)

Transmutation

Radiation and transmutation effects on crystalline radioactive waste forms, 1262 (J;US)

Volatility

Iron-enriched basalt for containment of nuclear wastes, $\mathbf{4 1 6}$ (BA;US)

\section{CESIUM 134}

Adsorption

Laboratory tests on the migration behavior of selected fission products in aquifer materials from a potential disposal site in northern Germany, 1352 (BA;US)

Diffusion

Laboratory tests on the migration behavior of selected fission products in aquifer materials from a potential disposal site in northern Germany, 1352 (BA;US)

Leaching

Model for predicting leaching behavior of high-level radioactive waste forms, 508 (J;US)

Transmutation

Radiation damage studies related to nuclear waste forms, 298 (R;US)

CESIUM 135

Environmental Transport

Mechanisms of dissolution of radioactive waste storage glasses and cesium migration from a granite repository, 1151 (BA;US)

Leaching

Mechanisms of dissolution of radioactive waste storage glasses and cesium migration from a granite repository, 1151 (BA;US)

\section{CESIUM 137}

Adsorption

Immobilization of reprocessing waste by means of titanate ion exchangers, 248 (RA;DE)

Removal of ${ }^{90} \mathrm{Sr},{ }^{107} \mathrm{Cs}$, and ${ }^{106} \mathrm{Ru}$ from vent gases produced during solidification of high-level radioactive solutions, 399 (J;CS)

Diffusion

Diffusion measurements in compacted bentonite, 1148 (BA;US)

Elution of radionuclides through columns of crushed rock from the Nevada Test Site, 1315 (R;US)

Environmental Exposure Pathway

Investigation of the consequences of leakage incidents of a tank with high-concentrated radionuclide solutions and pentration of the radioactive solution into the soil, 1423 (R;DE;In German)

Environmental Transport

Investigation of the consequences of leakage incidents of a tank with high-concentrated radionuclide solutions and pentration of the radioactive solution into the soil, 1423 (R;DE;In German)

Filtration

Removal of ${ }^{90} \mathrm{Sr},{ }^{107} \mathrm{Cs}$, and ${ }^{106} \mathrm{Ru}$ from vent gases produced during solidification of high-level radioactive solutions, 399 (J;CS)

Ion Exchange

Development of partitioning method - cold and semihot experimental works with partitioning testing apparatus, 225 (R;JP;In Japanese)

Leaching

$\mathrm{CsAlSi}_{5} \mathrm{O}_{12}$ : a possible host for ${ }^{107} \mathrm{Cs}$ immobilization, 148 (R;US)

High-level waste glass field burial tests at CRNL: the effect of geochemical kinetics on the release and migration of fission products in a sandy aquifer, 1299 (R;CA)

Leaching study of nuclear melt glass: Part I, 1332 (R;US)

Measurement of leaching from simulated nuclear-waste glass using radiotracers, 128 (R;US)

Separation Processes

Alternate strategy for commercial high-level radioactive-waste management, 945 (R;US)

orption

Distribution coefficient (Kd) concept and its applicability to studiess of radionuclide migration in geological media, 1313 (R;GB)

Effect of microbial activity on the containment of radioactive waste in a deep geological repository, 1357 (BA;US)
Evaluation of radionuclide transport: effect of radionuclide sorption and solubility, 1354 (BA;US)

Radionuclide retardation during transport through fractured granite, 1355 (BA;US)

\section{CESIUM CHLORIDES}

Compatibility

Cesium chloride compatibility testing program. Annual report, FY 1982, 855 (R;US)

Melting Points

Cesium chloride compatibility testing program. Annual report, FY 1982, 855 (R;US)

Phase Transformations

Cesium chloride compatibility testing program. Annual report, FY 1982, 855 (R;US)

\section{CESIUM SILICATES}

Crystallization

CsAlSis $\mathrm{O}_{12}$ : a possible host for ${ }^{107} \mathrm{Cs}$ immobilization, 148 (R;US)

Leaching

CsAlSis $\mathrm{O}_{12}$ : a possible host for ${ }^{107} \mathrm{Cs}$ immobilization, 404 (J;GB)

CsAlSis $\mathrm{O}_{12}$ : a possible host for ${ }^{107} \mathrm{Cs}$ immobilization, 148 (R;US)

\section{CHALKS}

See LIMESTONE

CHINA

Fuel Cycle

Summary of non-US national and international fuel cycle and radioactive waste management programs 1982,83 (R;US)

Radioactive Waste Management

Summary of non-US national and international fuel cycle and radioactive waste management programs 1982,83 (R;US)

\section{CHROMIUM}

Emission Spectroscopy

Analyses of nuclear fuel and high-level waste by inductively coupled plasma-emission spectroscopy, 68 (R;US)

Leaching

Geochemical behavior of supercalcine waste form: its stability in a basalt environment, 911 (R;US)

Redox Reactions

Investigation on the oxidation state and the behavior of molybdenum in silicate glass, 460 (BA;US)

\section{CHROMIUM OXIDES}

Leaching

Influence of metal oxides on the leach rate and crystallisation behaviour of waste glasses, 251 (RA;DE)

\section{CHROMIUM STEELS}

See also CHROMIUM-MOLYBDENUM STEELS CHROMIUM-NICKEL STEELS

Corrosion Resistance

Corr sion of materials in a clay environment, $676(R A ; D E)$

\section{CHROMIUM-MOLYBDENUM STEELS}

Corrosion

General corrosion, irradiation-corrosion, and environmentalmechanical evaluation of nuclear-waste-package structuralbarrier materials. Progress report, 843 (R;US)

Physical Radiation Effects

General corrosion, irradiation-corrosion, and environmentalmechanical evaluation of nuclear-waste-package structuralbarrier materials. Progress report, 843 (R;US)

\section{CHROMIUM-NICKEL STEELS} STAINLEESS STEEL-.-304

See also STAINLESS STEEL-304L STAINLESS STEEL-309S

STAINLESS STEEL-316

STAINLESS STEEL-321 STAINLESS STEEL-348

\section{Corrosion}

Corrosion studies and mechanical tests on metallic materials for the design of packagings for vitrified high level wastes (HLW), 689 (R;DE;In German)

Tensile Properties

Corrosion studies and mechanical tests on metallic materials for the design of packagings for vitrified high level wastes (HLW), 689 (R;DE;In German) 
CLAYS

\section{See also ATTAPULGITE \\ BENTONITE \\ ILLITE \\ MONTMORILLONITE \\ SMECTITE}

Corrosive Effects

Corrosion of construction materials in clay environments, 1150 (BA;US)

Leaching

Report of working meeting on fixation of radioactivity in stable, solid media at the Johns Hopkins University, June 19-21, 1957, 342 (R;US)

Permeability

Recent advances in repository seal materials, 1172 (BA;US)

Radiolysis

Gas production and liberation from rock salt samples and potential consequences on the disposal of high-level radioactive waste in salt domes, 1165 (BA;US)

Radionuclide Migration

Subsurface migration of radioactive waste materials by particulate transport, 98 (J;US)

Rock-fluid Interactions

Waste glass - seabed interactions: observations of the Soret effect, 850 (RA;US)

Temperature Effects

Laboratory determination of the thermal properties of large-scale samples of natural clays, 550 (R;IT;IT)

Thermal Conductivity

Laboratory determination of the thermal properties of large-scale samples of natural clays, 550 (R;IT;IT)

Thermal conductivity of bentonite/quartz high-level-wastepackage backfill, 972 (R;US)

Thermal Diffusivity

Laboratory determination of the thermal properties of large-scale samples of natural clays, 550 (R;IT;IT)

\section{CLINOPTILOLITE}

Physical Radiation Effects

Research and development activities: fixation of radioactive residues. Quarterly progress report, April-June 1960, 210 (R;US)

Sorptive Propertie:

Solid fixation of high-level radioactive waste by sorption on clinoptilolity-scouting studies, 211 (R;US)

Waste-rock Interactions

Backfill-waste interactions in repository simulating tests, 1153 (BA;US)

\section{CLOSURES}

Field Tests

Field-test programs of borehole plugs in southeastern New Mexico. 615 (RA;US)

Service Life

Geochemical factors in borehole-shaft plug longevity, 614 (RA;US)

\section{COALTEK PROCESS}

See FUEL FEEDING SYSTEMS

COATINGS

Materials Testing

Coating crystalline nuclear waste forms to improve inertness, 138 (R;US)

\section{COBALT}

Activation Analysis

Application of neutron-activation analysis to the determination of leach rates of simulated nuclear-waste forms, 127 (R;US)

\section{Adsorption}

Effects of Hanford high-level waste components on sorption of cobalt, strontium, neptunium, plutonium, and americium on Hanford sediments, 918 (R;US)

Importance of organic compounds in ground water as radionuclide-mobilizing agents, 1324 (R;US)
COBALT 57

Leaching

Leaching study of nuclcar melt glass: Part I, 1332 (R;US)

\section{COBALT 58}

Leaching

Leaching study of nuclear melt glass: Part I, 1332 (R;US)

COBALT 60

Adsorption

Immobilization of reprocessing waste by means of titanate ion exchangers, 248 (RA;DE)

Leaching

Leaching study of nuclear melt glass: Part I, 1332 (R;US)

Sorption

Radionuclide retardation during transport through fractured granite, 1355 (BA;US)

COGEMA MARCOULE

Radioactive Waste Processing

Marcoule vitrification shop: 30 months service (In French), 228 (RA;DE;FR)

COLORADO

Geulugy

Geological status of NWTS repository siting activities in the par: adox basin, 1265 (J;US)

Salt Deposits

Environmental characterization of bedded salt formations and overlying areas of the Permian Basin, 1320 (R;US)

COLUMBIA RIVER BASIN

Geologic Faults

Report on geologic remote sensing of the Columbia Plateau, 871 (R;US)

Geologic History

Uplift and subsidence rates in the central Columbia Plateau and their relation to siting a waste repository at Hanford, Washington, 873 (R;US)

Geology

Assessment of the effects of surficial geologic processes in the Pasco Basin (Pasco Basin portion), 869 (R;US)

Report on geologic remote sensing of the Columbia Plateau, 871 (R;US)

Tectonics

Uplift and subsidence rates in the central Columbia Plateau and their relation to siting a waste repository at Hanfurd, Washington, 873 (R;US)

Water Resources

Evaluation of water resourçe economics within the Pasco Basin, Washington, 1429 (R;US)

COMPOSITE MATERIALS

See also CERMETS

Thermal Conductivity

Thermal conductivity measurements on granule-based composites, 1451 (J;GB)

COMPUTER CODES

A Codes

Formulation of SYNROC-D additives for Savannah River Plant high-level radioactive waste (ADSYN code), 347 (R;US)

Benchmarks

Benchmark problems for repository siting models, 746 (R;US)

C Codes

LAYILU: a one-dimcrisional semianalytical model for the mlgration of a three-member decay chain in a multilayered geologic medium, 1325 (R;US)

Vitrification of high-level wastes: A review of the computer ther$\mathrm{mal}$ analysis for storage canisters, 1439 (J;NL)

D Codes

Large deformation inelastic analysis of impact for shipping casks (DYNA3D Code), 1436 (J;NL)

Ducumentation

Final technical position on documentation of computer codes for high-level waste management, 723 (R;US)

Evaluation

Paramctcrs and variables appearing in radiological assessment codes. Final report, 1375 (R;US)

Summary of repository siting models. Final report (Code typesaturated flow, unsaturated flow, surface water flow, geochemistry, heat transport, solute transport, geomechanical), 740 (R;US) 
F Codes

Calculation of media temperatures for nuclear sources in geologic depositories by a finite-length line source superposition model (FLLSSM), 1122 (J;NL)

P Codes

Status report on dissolution model development, 1021 (R;US)

Radioactive Waste Storage

Calculation of media temperatures for nuclear sources in geologic depositories by a finite-length line source superposition model (FLLSSM), 1122 (J;NL)

S Codes

Code development in support of nuclear waste storage investigations for a repository in tuff (Pore water motion and energy transport in a porous media which may be saturated or partially saturated with a single fluid in liquid state), 988 (R;US)

Thermal and thermomechanical calculations of deep-rock nuclear waste disposal with the enhanced SANGRE code, 1012 (R;US)

W Codes

WAPPA: a Waste Package Performance Assessment code. Technical report, 819 (R;US)

COMPUTER PROGRAMS

SEe COMPUTER CODES

CONCRETES

See also REINFORCED CONCRETE

Chèmical Reactions

Chemical interaction of clay buffer materials and concrete, 997 (R;SE)

Comparative Evaluations

Development, evaluation, and selection of candidate high-level wastc forms, 185 (R;US)

Evaluation of conditioned high-level waste forms, 326 (R;US)

Evaluation of forms for the immobilization of high-level and transuranic wastes, 200 (R;US)

\section{Fractures}

Final report of experimental laboratory-scale brittle fracture studies of glasses and ceramics, 129 (R;US)

Fragmentation

Respirable fines produced by impacts of simulated alternative high-level waste materials, 419 (BA;US)

Impact Strength

Cement-based radioactive waste hosts formed under elevated temperatures and pressures (FUETAP concretes) for Savannah River Plant high-level defense waste, 293 (R;US)

Impact Tests

Final report of experimental laboratory-scale brittle fracture studies of glasses and ceramics, 129 (R;US)

Respirable firtes produced by inpacts uf simulated alternative high-level waste materials, 419 (BA;US)

Leaching

Cement-based radioactive waste hosts formed under elevated temperatures and pressures (FUETAP concretes) for Savannah River Plant high-level defense waste, 293 (R;US)

Dispusal of Savannah River Plant waste salt, 625 (R;US)

FUETAP concrete: an alternative radioactive waste host, 256 (RA;DE)

Materials Testing

Disposal of high-level waste from nuclear power plants in Denmark. V.3. Material testing, 650 (R;DK)

Mechanical Properties

Status of tank-assessment studies for continued in-tank storage of Hanford Defense waste, 919 (R;US)

Performance

Cement-based radioactive waste hosts formed under elevated temperatures and pressures (FUETAP concretes) for Savannah River Plant high-level defense waste, 293 (R;US)

Permeability

Cement-based radioactive waste hosts formed under elevated temperatures and pressures (FUETAP concretes) för Savannah River Plant high-level defense waste, 293 (R;US)

Recent advances in repository seal materials, 1172 (BA;US)

\section{Phase Studies}

Cement-based radioactive waste hosts formed under elevated temperatures and pressures (FUETAP concretes) for Savannah River Plant high-level defense waste, 293 (R;US)

Radiation Effects

Cement-based radioactive waste hosts formed under elevated temperatures and pressures (FUETAP concretes) for Savannah River Plant high-level defense waste, 293 (R;US)

FUETAP concrete - an alternative radioactive waste host, 268 (RA;DE)

Stability

Cement-based radioactive waste hosts formed under elevated temperatures and pressures (FUETAP concretes) for Savannah River Plant high-level defense waste, 293 (R;US)

Temperature Effects

Status of tank-assessment studies for continued in-tank storage of Hanford Defense waste, 919 (R;US)

Thermal Conductivity

Cement-based radioactive waste hosts formed under elevated temperatures and pressures (FUETAP concretes) for Savannah River Plant high-level defense waste, 293 (R;US)

Thermal Degradation

FUETAP concrete - an alternative radioactive waste host, 268 (RA;DE)

CONTAINERS

See also CASKS

$T A N K S$

Closures

Remote automatic plasma arc-closure welding of a dry-storage canister for spent nuclear fuel and high-level radioactive waste, 551 (R;US)

Construction

High-integrity container for high-specific-activity wastes, 1446 (J;US)

Corrosion

Container assessment: corrosion study of HLW container materials. Quarterly progress report, July-September 1981, 728 (R;US)

Corrosion studies and mechanical tests on metallic materials for the design of packagings for vitrified high level wastes (HLW), 689 ( $R$;DE; In German)

Effects of radiation on corrosion of titanium, 961 (RA;US)

Corrosion Resistance

Corrosion resistance of metal materials for HLW canister, 666 Cost (R;JP;In Japanese)

Concept of a canister for high-level radioactive waste, 1158 (J;US)

Crevice Corrosion

Container assessment - corrosion study of HLW container materials. Quarterly progress report, April-June 1981, 729 (R;US)

Decontamination

Decontamination of Savannah River Plant waste glass canisters, 175 (R;US)

Design

Concept of a canister for high-level radioactive waste, 1158 ( $\mathrm{J} ; \mathrm{US})$

Development of waste package designs for disposal in a salt repository, 557 (RA;US)

WAPPA: an integrated waste package performance assessment cuile, 1220 (J;US)

Waste packages for high-level waste repositories, 1043 (J;US)

Impact Tests

Full-scale impact tests of simulated high-level waste canisters, 316 (R;US)

Full-scale impact tests of simulated high-level waste canisters, $1098(\mathrm{~J} ; \mathrm{US})$

Lifetime

High-integrity container for high-specific-activity wastes, 1446 (J;US)

Materials

Container assessment - corrosion study of HLW container materials. Quarterly progress report, April-June 1981, 729 (R;US) 


\section{Materials Testing}

Corrosion of materials in a clay environment, 676 (RA;DE)

Canister materials proposed for final disposal of high level nuclear waste - a review with respect to corrosion resistance, 675 (RA;DE)

Corrosion studies and mechanical tests on metallic materials for the design of packagings for vitrified high level wastes (HLW), 689 (R;DE; In German)

Corrosion of TiCode- 12 in a simulated Waste Isolation Pilot Projcct (WIPP) brine, 1066 (J;US)

Disposal of high-level waste from nuclear power plants in Denmark. V.3. Material testing, 650 (R;DK)

Identification of crevice corrosion in the titanium alloy TiCode-12 in simulated rock salt brine at $150^{\circ} \mathrm{C}$ (Ti-0.3Mo- 0 . $8 \mathrm{Ni}), 545$ (R;US)

Investigations of suitable metallic container materials for HAW solidification, 371 (J;US)

Long-term performance of materials used for high-level waste packaging. Quarterly report, April-June 1983, 752 (R;US)

Studies of waste-canister compatibility, 832 (R;US)

Testing of candidate waste package backfill and canister materials for basalt, 586 (KA;US)

Uniform- and pitting-corrosion data requirements for TiCode-12 high-level-waste containers, 734 (RA;US)

Mathematical Models

WAPPA: an integrated waste package performance assessment code, 1220 (J;US)

Performance

WAPPA: an integrated waste package performance assessment code, 1220 (J;US)

Waste package performance assessment: the importance of the very near-field physicochemical environment, 1223 (J;US)

Performance Testing

Classification and safety reserves of radioactive material shipping cuntainers, 1452 (B;DE; In German)

Waste package conceptual designs for salt repositories, 593 (RA;US)

Safeguard Regulations

High-integrity container for high-specific-activity wastes, 1446 (J;US)

Specincations

High-integrity container for high-specific-activity wastes, 1446 (J;US)

Standardization

Nuclear waste management technical support in the developmnt of nuclear waste form criteria for the NRC. Task 5. National waste package program, 285 (R;US)

Thermal Conductivity

Three-dimensional thermal analysis for a conceptual high-level waste repository in welded tuff, 932 (R;US)

CONTAINMENT

See also CONTAINMENT SYSTEMS

Syñóc Procéśs

SYNROC for containment of high-level nuclear waste, 396 (J;US)

\section{CONTAINMENT SYSTEMS}

Design

Design rules for containment systems of nuclear spent fuel and high-level waste transport packagings, 19 (RA;US)

\section{CONTROL (RADIOACTIVITY)}

SEe. RADIATION MONITORING

\section{COPPER}

Compatibility

Studies of waste-canister compatibility, 832 (R;US)

Corrosion

Container assessment: corrosion study of HLW container materials. Quarterly progress report, July-September 1981, 728 (R;US)

Corrosion Resistance

Corrosion of materials in a clay environment, $676(R A ; D E)$

Canister materials proposed for final disposal of high level nuclear waste - a review with respect to corrosion resistance, 675 (R^;DE)

\section{Crevice Corrosion}

Container assessment - corrosion study of HLW container mi als. Quarterly progress report, April-June 1981, 729 (R;US

Emission Spectroscopy

Analyses of nuclear fuel and high-level waste by inductively coupled plasma-emission spectroscopy, 68 (R;US)

COPPER ALLOYS

See also COPPER BASE ALLOYS INCOLOY 825 MONEL

Corrosion

Corrosion of materials in a clay environment, 686 (RA;DE)

Corrosion Resistance

Corrosion of materials in a clay environment, $676(R A ; D E)$

\section{COPPER BASE ALLOYS}

Compatibility

Studies of waste-canister compatibility, 832 (R;US)

Corrosion

Testing of candidate waste-package backfill and canister materials for basalt (Cupronickel 90/10), 885 (R;US)

COPPER ORES

Native copper deposits of the Portage Lake volcanics, Michigan their implications with respect to canister stability for nuclear waste isolation in the Columbia River basalts beneath the Hanford Site, Washington, 904 (R;US)

\section{CORES (REACTOR)}

See REACTOR CORES

CRYSTALLINE ROCKS

See IGNEOUS ROCKS METAMORPHIC ROCKS

CULTURAL RESOURCES

Cultural resources overview for the Nevada Nuclear Waste Storage Investigations, Nevada Test Site, Nye County, Nevada, 767 (R;US)

Bibliographies

Annotated bibliography of cultural resources literature for the Nevada Nuclear Waste Storage Investigations, 768 (R;US)

\section{CURIUM 245}

Diffusion

Study of radionuclide migration from deep-lying repository sites with over-lying sedimentary layers, 1348 (BA;US)

\section{CURIUM COMPOUNDS}

Hydrolysis

Thermodynamic properties of chemical species in nuclear waste, 812 (R;US)

DEBRIS (NUCLEAR)

SEe FISSION PRODUCTS

\section{DECALSO}

See ION EXCHANGE MATERIALS

\section{DECOMMISSIONING}

Cust

Decommissioning alternatives for Savannah River Plant highlevel waste tank 16, 142 (RA;US)

\section{Planning}

Decommissioning alternatives for Savannah River Plant highlevel waste tank 16, 142 (RA;US)

DECONTAMINATION

Ion Exchange Materials

Fixation of radioactive residues. Quarterly progress report, January-March 1961, 213 (R;US)

Safeguard Regulations

Regulatory perspective on the cleanup of TMI-2', 1445 (J;US)

DECONTAMINATION FACTOR

SeE DECONTAMINATION

DEMINERALIZERS

Vitrification

Development and demonstration of a process for vitrification of TMI zeolite, 505 (J;US) 


\author{
DENMARK \\ Fuel Cycle \\ Summary of non-US national and international fuel cycle and \\ radioactive waste management programs 1982,83 (R;US) \\ Radioactive Waste Disposal \\ Preliminary design of a Danish high level waste disposal facility \\ in the Mors dome, 1142 (J;US) \\ Radioactive Waste Management \\ Summary of non-US national and international fuel cycle and \\ radioactive waste management programs 1982, 83 (R;US) \\ Salt Deposits \\ Geology of the Mors salt dome, 658 (RA;DK;In Danish) \\ Hydrogeological conditions at Mors salt dome, 659 (RA;DK;In \\ Danish) \\ DEPOSITS (GEOLOGICAL) \\ See GEOLOGIC DEPOSITS \\ DEVICES \\ See EQUIPMENT \\ DEVONIAN SHALES \\ See BLACK SHALES \\ DIAMOND DRILLING EQUIPMENT \\ See DRILLING EOUIPMENT \\ DIPHENYLPHOSPHINE OXIDE \\ See ORGANIC PHOSPHORUS COMPOUNDS \\ DISCHARGES (WASTES) \\ See WASTE DISPOSAL \\ DISPOSAL (WASTES) \\ See WASTE DISPOSAL \\ DISSOLUTION \\ See also LEACHING \\ Computerized Simulation \\ Status report on dissolution model development, 1021 (R;US) \\ DPO \\ SEe ORGANIC PHOSPHORUS COMPOUNDS \\ DRILL SHIPS \\ See SHIPS \\ DRILLING EQUIPMENT
}

Equipment for the emplacement of heat-producing waste in long horizontal boreholes (Horizontal vs vertical emplacement), 555 (R;US)

DRY STORAGE

Design

Conceptual design of monitored retrievable storage faclities, 41 ( $\mathrm{J} ; \mathrm{US})$

Feasibility Studies

Economical dry storage of large amounts of spent fuel and vitrified high level radioactive waste, $43(\mathrm{BA} ; \mathrm{XN})$

DRYERS

Utilization of a drum drier for the conditioning of radioactive wastes, 440 (BA;US)

Performance

Use of the drum dryer in radioactive waste solidification, $\mathbf{4 1 4}$ (BA;DE;In German)

DYMAC SYSTEM

See NUCLEAR MATERIALS MANAGEMENT PLUTONIUM

DYNAMIC MATERIALS ACCOUNTABILITY SYSTEM

See NUCLEAR MATERIALS MANAGEMENT PLUTONIUM

\section{$\mathbf{E}$}

EDTA

Oxidation

Ozone mass transfer and kinetics experiments, 334 ( $\left.R_{i} I I S\right)$

Removal

Ozonie mass transfer and kinetics experiments, 334 (R;US)

EFFLUENTS (GASEOUS)

SOO G.ASEOLIS W.ASTES
EFFLUENTS (LIQUID)

See LIQUID WASTES

EGYPTIAN ARAB REPUBLIC

Radioactive Waste Facilities

Evaluation of an international, perpetual, and retrievable facility for storage of vitrified radioactive waste, 97 (J;US)

ELECTRIC POWER RESEARCH INSTITUTE See EPRI

ELECTROLYTE TILES

See MATRIX MATERIALS

ELUTION (SOLUBLE CONSTITUENTS)

See LEACHING

EMPLOYEES

See PERSONNEL

ENERGY POLICY

Japan

Sub-committee on Nuclear Power Submits report on energy security, 1449 (J;JP)

ENGINEERED SAFETY SYSTEMS

See also CONTAINMENT SYSTEMS

Design

Engineered waste package concepts for high-level waste isolation in basalt, 874 (R;US)

NWTS waste package program plan. Volume I. Program strategy, description, and schedule, 598 (R;US)

NWTS waste package program plan. Volume II. Program logic networks, 599 (R;US)

Evaluation

Evaluation of barriers in a final storage site for radioactive wastes, 674 (RA;DE)

Materials Testing

Characterization of reference materials for the Barrier Materials Test Program, 905 (R;US)

Corrosion of construction materials in clay environments, 1150 (BA;US)

Development of structural barriers for containment of nuclear waste in basalt, 1100 (J;US)

Performance

Engineered waste package concepts for high-level waste isolation in basait, $874(R ; U S)$

Performance Testing

Combined analytical model for preformance assessment of the waste package/geologic medium systems, 1170 (BA;US)

Engineered components for spent fuel radioactive waste isolation systems: are they technically justified, 1171 (BA;US)

Seals

Nuclear-waste repository in basalt: seal development studies, 887 (R;US)

ENGLAND

SEe UNITED KINGDOM

ENVIRONMENTAL EXPOSURE PATHWAY

Mathematical Models

PATH1 self-teaching curriculum: example problems for Pathways-to-Man Model, 1317 (R;US)

Risk methodology for geologic disposal of radioactive waste: model description and user manual for Pathways model, 1316 (R;US)

ENVIRONMENTAL PROTECTION AGENCY

See US EPA

ENVIRONMENTAL TRANSPORT

See also RADIONUCLIDE MIGRATION

Mathematical Models

PAT̈HI self-teaching curriculum: example problems for Pathways-to-Man Model, 1317 (R;US)

Risk methodology for geologic disposal of radioactive waste: model description and user manual for Pathways model, 1316 (R;US)

EPA

SeP. IIS EPA 


\section{EPRI}

Radioactive Waste Management

EPRI's programs in low-level waste management, 74 (RA;US)

EQUIPMENT

See also DRILLING EQUIPMENT MATERIALS HANDLING EQUIPMENT

Availability

Guidebook for reliability, availability, and maintainability analysis of NWTS repository equipment. Volume II. Appendices (NWTS repository equipment), 808 (R;US)

Maintenance

Guidebook for reliability, availability, and maintainability analysis of NWTS repository equipment. Volume II. Appendices (NWTS repository equipment), 808 (R;US)

Reliability

Guidebook for reliability, availability, and maintainability analysis of NWTS repository equipment. Volume II. Appendices (NWTS repository equipment), 808 (R;US)

ESTUARINE ECOSYSTEMS

See AQUATIC ECOSYSTEMS

ETHYLENEDIAMINETETRAACETIC ACID

See EDTA

EUROCHEMIC

Fuel Reprocessing Plants

Reprocessing, decontamination and decommissioning waste management at Eurochemic: current and planned activities, 433 (BA;US)

Radioactive Waste Management

Reprocessing, decontamination and decommissioning waste management at Eurochemic: current and planned activities, 433 (BA:IJS)

\section{EUROPEAN COMMUNITIES}

Radioactive Waste Management

European Communities' $\mathbf{R}$ and $\mathrm{D}$ activities on treatment and handling of radioactive waste, 426 (BA;US)

Management of radioactive wastes in Europe (EC) history, philosophy and plans for the next five years, 108 (BA;US)

\section{EUROPIUM}

Activation Analysis

Application of neutron-activation analysis to the determination of leach rates of simulated nuclear-waste forms, 127 (R;US)

Adsorptlon

Geochemistry studies pertaining to the G-tunnel radionuclide migration field experiment, 1426 (R;US)

Desorption

Research and development related to the Nevada Nuclear Waste Storage Investigations. Progress report, July 1-September 30, 1981, 692 (R;US)

Solubility

Geochemical controls on radionuclide releases from a nuclear waste repository in basalt: estimated solubilities for selected elements, 1329 (R;US)

Sorption

Research and development related to the Nevada Nuclear Waste Storage Investigations. Progress report, July 1-September 30 , 1981, 692 (R;US)

\section{EUROPIUM 152}

Leaching

Measurement of leaching from simulated nuclear-waste glass using radiotracers, 128 (R;US)

\section{EUROPIUM 155}

Leaching

Leaching study of nuclear melt glass: Part I, 1332 (R;US)

\section{EVAPORATORS}

Design

Vitrification of high level wastes using microwave power, 230 (RA;DE)

\section{Leaks}

Contamination of the hot gang valve corridor and first-level clean areas of Building 221-F - September 13, 1960, 194 (R;US)

\section{EVAPORITES}

Chènical Radiation Effects

Radiation induced F-center and colloid formation in synthetic $\mathrm{NaCl}$ and natural rock salt: applications to radioactive waste repositories ( 1 to $3 \mathrm{MeV}$ electrons), 543 (R;US)

\section{EXPLOSIONS}

Origin

Steam explosions caused by te contact of molten glass and water, 246 (RA;DE)

EXPOSURE (RADIATION DOSES)

See RADIATION DOSES

EXTENSOMETERS

Failures

Instrument reliability for high-level nuclear-waste-repository applications, 1013 (R;US)

Performance

Instrument reliability for high-level nuclear-waste-repository applications, 1013 (R;US)

Reliability

Instrument reliability fur high-level nuclear-waste-reposltory applicationș, 1013 (Ŗ (R;US)

\section{$F$}

\section{FABRICATION \\ Radiation Hazards \\ Exposures resulting from nuclear power production. Annex $F$ 1444 (BA;US) \\ FACILITIES (NUCLEAR) \\ See NUCLEAR FACILITIES \\ FACILITIES (STORAGE) \\ SPQ STORAGE FACILITIES \\ FACILITIES (TEST) \\ See TEST FACILITIES \\ FAST FLUX TEST FACILITY REACTOR \\ See FFTF REACTOR \\ FEDERAL REGION VI \\ See also LOUISIANA \\ NEW MEXTCO \\ OKLAHOMA \\ TEXAS}

Radioactive Waste Disposal

Environmental characterization report for the Gulf Intcrinr Region Mississippi study area (Lampton, Richton, and Cypress Creek salt domes), 1322 (R;US)

FEDERAL REPUBLIC OF GERMANY

Fuel Cycle

Summary of non-US national and international fuel cycle and radioactive waste management programs 1982,83 (R;US)

Fuel Reprocessing Plants

Policy and development of reprocessing of spent nuclear fuel and high-level radioactive waste management in the F.R. Germany, 13 (J;JP;In Japanese)

High-level Radioactive Wastes

Geological aspects of the final disposal of radioactive waste, 1072 (J;DE; In German)

Underground storage of radioactive wastes in salt deposits in the Federal Republic of Germàny, 668 (KA;UE;In German)

Radioactive Waste Disposal

Geological aspects of the final disposal of radioactive waste, 1072 (J;DE;In German)

Importance of near-field phenomena for nuclide release from a flooded salt dome repository, 1396 (BA;US)

Mineralugical and geochemical factors influencing the final disposal of HLW in the Stassfurt halite, 1164 (BA;US)

Policy and development of reprocessing of spent nuclear fuel and high-level radioactive waste management in the F.R. Germany, 13 (J;JP;In Japanese)

Present status of the Gorleben site investigations, 1036 (J;US)

$R$ and $D$ projects and studies: concerning the interim and termi nal storage of radioactive wastes, 1281 (TG;US)

Requirements for the disposal of high-level radioactive wastes, 671 (RA;DE) 
Radioactive Waste Facilities

$R$ and $D$ projects and studies: concerning the interim and terminal storage of radioactive wastes, 1281 (TG;US)

Radioactive Waste Management

Policy and development of reprocessing of spent nuclear fuel and high-level radioactive waste management in the F.R. Germany, 13 (J;JP;In Japanese)

Summary of non-US national and international fuel cycle and radioactive waste management programs 1982,83 (R;US)

Radioactive Waste Processing

Development of borosilicate glass for the vitrification plant PAMELA, 530 (TG;US)

High-level waste vitrification technique in a full-scale pilot plant, 439 (BA;US)

Nuclear waste vitrification in a ceramic-lined electric glass melter, 409 (J;US)

Policy and development of reprocessing of spent nuclear fuel and high-level radioactive waste management in the F.R. Germany, 13 (J;JP;In Japanese)

SYNROC through melting: thermal analysis, thermogravimetry and crystal chemical characterization of phases, 260 (RA;DE)

Radioactive Waste Storage

Policy and development of reprocessing of spent nuclear fuel and high-level radioactive waste management in the F.R. Germany, 13 (J;JP;In Japanese)

$R$ and $D$ projects and studies: concerning the interim and terminal storage of radioactive wastes, 1281 (TG;US)

Underground storage of radioactive wastes in salt deposits in the Federal Republic of Germany, 668 (RA;DE;In German)

Reprocessing

Policy and development of reprocessing of spent nuclear fuel and high-level radioactive waste management in the F.R. Germany, 13 (J;JP;In Japanese)

Spent Fuel Storage

Policy and development of reprocessing of spent nuclear fuel and high-level radioactive waste management in the F.R. Germany, 13 (J;JP;In Japanese)

\section{FELDSPARS}

Leaching

Alpha-recoil damage and solution effects in minerals: uranium isotopic disequilibrium and radon release, 1073 (J;GB)

FERRITIC STEELS

Corrosion Resistance

Corr sion of materials in a clay environment, $676(R A ; D E)$

FFTF REACTOR

Radioactive Wastes

Graphical and tabular summaries of decay characteristics for once-through PWR, LMFBR, and FFTF fuel cycle materials (Spent fuel, high-level waste fuel can scrap), 76 (R;US)

\section{FINLAND}

Fuel Cycle

Summary of non-US national and international fuel cycle and radioactive waste management programs 1982, 83 (R;US)

Radioactive Waste Management

Summary of non-US national and international fuel cycle and radioactive waste management programs 1982,83 (R;US)

Radioactive Waste Processing

Sodium titanate in solidification of radioactive wastes, 247 (RA;DE)

FISSION PRODUCTS

Decay

Decay calculations on medium-level and actinide-containing wastes from the LWR fuel cycle. Pt. 1. Basic data evaluation including activity and thermal decay power, 687 (R;DE;In German)

Decontamination

Depusitiui of volatile fission products during commercial highlevel waste vitrification, 304 (R;US)

Electron Microprobe Analysis

Deposition of volatile fission products during commercial highlevel waste vitrification, 304 (R;US)
Environmental Transport

Migration of long actinides chains in geologic media, 1362 (J;US)

Rail Transport

Rail transport of vitrified high-level radioactive fission products risk analysis of a model, 25 (R;DE;In German)

Recovery

Disposal of radioactive wastes (Book), 106 (B;NL)

Transmutation

Fission product transmutation effects on high-level radioactive waste forms, 401 (J;GB)

Transportation Systems

Rail transport of vitrified high-level radioactive fission products risk analysis of a model, 25 (R;DE;In German)

FISSIONABLE MATERIALS MANAGEMENT

See NUCLEAR MATERIALS MANAGEMENT

FISSURED FORMATIONS

See FRACTURED RESERVOIRS

FLASKS

See CASKS

FLUIDIZED BED REACTORS

Performance Testing

SYNROC powder preparation: preliminary fluid bed tests in a cold-flow unit, 345 (R;US)

\section{FOOD CHAINS}

Radionuclide Migration

Health effects associtated with unit radonuclide releases to the environment, 1378 (RA;US)

FORMALDEHYDE

Chemical Reactions

Separation of mercury from HEWC solutions. Laboratory assay, 207 (R;BE;In French)

FORMALIN

See FORMALDEHYDE

FORMALITH

See FORMALDEHYDE

FORMIC ACID

Chemical Reactions

Denitration of simulated fast ractor highly active liquor waste, 241 (RA;DE)

FORMIC ALDEHYDE

See FORMALDEHYDE

FORMOL

See FORMALDEHYDE

FORMOSA

See TAIWAN

FOSSILFUEL POWER PLANTS

Comparative Evaluations

High level radioactive waste, 1242 (J;US)

FRACTURED FORMATIONS

See FRACTURED RESERVOIRS

FRACTURED RESERVOIRS

Fluid Flow

Unsaturated flow and transport through fractured rock - related to high-level waste repositories. Final report. Phase I, 748 (R;US)

FRANCE

Fuel Cycle

Summary of non-US national and international fuel cycle and radioactive waste management programs 1982,83 (R;US)

Radioactive Waste Disposal

Long-term prediction of the fate of nuclear waste deeply buried in granite, $1186(\mathrm{BA} ; \mathrm{GB})$

Nuclear waste management in France, 368 (J;US)

Radioactive Waste Facilities

Industrial operating experience at the Marcoule vitrification facility, 432 (BA;US)

Radioactive Waste Management

Low level and intermediate level wastes management practice in France, 442 (BA;US)

National and cooperative program for waste management in

France, 427 (BA;US)

Nuclear waste management in France, 368 (J;US)

Nuclear waste management policy in France, $92(\mathrm{~J} ; \mathrm{US})$ 
Summary of non-US national and international fuel cycle and radioactive waste management programs 1982, 83 (R;US)

Radinactive Waste Processing

Industrial operating experience at the Marcoule vitrification facility, 432 (BA;US)

Nuclear waste management in France, 368 (J;US)

Progress of the vitrification of HLW in France, $525(\mathrm{~J} ; \mathrm{US})$

Radioactive Waste Storage

Problem of reprocessing and of radioactive wastes in France, 489 (J;FR;In French)

FRESH WATER ECOSYSTEMS

See AQUATIC ECOSYSTEMS

FTR REACTOR (RICHLAND)

See FFTF REACTOR

FUEL COOLING INSTALLATIONS

See SPENT FUEL STORAGE

FUEL CYCLE

See also PLUTONIUM RECYCLE THORIUM CYCLE

Program summary. Nuclear waste management and fuel cycle programs, 575 (ík;UŚ)

After-heat

Decay calculations on medium-level and actinide-containing wastes from the LWR fuel cycle. Pt. 1. Basic data evaluation including activity and thermal decay power, 687 (R;DE;In German)

Chemistry

Chemistry of the nuclear fuel cycle, 4 (BA;GB)

Quantitative Chemical Analysis

Analysis of Am and a recovery for it in the pressurized water reactor fuel cycle, 1433 (R;DE;In German)

Radioactive Waste Disposal

Safe disposal of radioactive wastes from nuclear power plants, 656 (RA;CS;In Czech)

Research Programs

Fuel cycle programs. Quarterly progress report, April-June 1982 , 536 (R;US)

Fuel cycle programs. Quarterly progress report, July-September 1982, 538 (R;US)

Fuel cycle programs. Quarterly progress report, OctoberDecember 1982, 539 (R;US)

\section{FUEL FEEDING SYSTEMS}

Design

Design and performance of feed-delivery systems for simulated radioactive waste slurries, 306 (R:US)

Performance

Design and performance of feed-delivery systems for simulated radioactive waste slurries, 306 (R;US)

FUEL REPROCESSING

See REPROCESSING

FUEL REPROCESSING PLANTS

See also BARNWELL FUEL PROCESSING PLANT IDAHO CHEMICAL PROCESSING PLANT WAK

WEST VALLEY PROCESSING PLANT

Cundinamled Reserarch Proyram:

Main activities and achievements of the DWK within the joint development programme, 8 (RA;DE;In Gcrman)

Decommissioning

Reprocessing, decontamination and decommissioning waste management at Eurochemic: current and planned activities, 433 (BA;US)

Decontamination

Reprocessing, decontamination and decommissioning waste management at Eurochemic: current and planned activities, 433 (BA;US)

Environmental Effects

Analysis of the alleged .Kyshtym disaster, 1314 (R;US)

High-level Radioactive Wastes

Compound refining system for separation of gaseous fission products incorporated in a reprocessing pilot plant for spent fuel from neclear power stations, 494 (BA;JP;In Japanese)
Off-gas Systems

Method and device for solidifying high level liquid wastes fror re-processing plant, 403 (P;JP;In Japancsc)

Method of processing off-gas condensate containing precipitations resulted upon solidification of high-level liquid wastes issued from a reprocessing plant, 406 (P;JP;In Japanese)

Planning

Main activities and achievements of the DWK within the joint development programme, 8 (RA;DE;In German)

Report of the Ad Hoc Committee on Reprocessing and Wastes of the Sub-Committee on Nuclear Power of Committee on Overall Energy Study, 485 (J;JP;In Japanese)

Radioactive Waste Management

Reprocessing of nuclear fuel, 6 (R;US)

FUEIS

See also NUCLEAR FUELS

Comparative Evaluations

Nitornative fucls and heating methods for calciner process heal, 204 (R;US)

FIFIS (NIICT.FAR)

See NUCLEAR FUELS

FULVIC ACIDS

Flocculation

- Importance of urganic cumpuunds in ground water as radiunuclide-mobilizing agents, 1324 (R;US)

Solubility

Importance of organic compounds in ground water as radionuclide-mobilizing agents, 1324 (R;US)

FUMIES

See AEROSOLS

\section{G}

\section{GADOLINIUM}

Lesching

Processing effects on the bohavior of titanate waste formo in aqueous solutions, 1145 (BA;US)

Processing, microstructure, leaching, and long-term stability studies related to titanate high-level waste forms, 340 (R;US)

GAGES (STRAIN)

See STRAIN GAGES

GAMMA RADIA'IUN

Higb-level Radioactive Wastes

Borosilicate glass for immobilization of high-level SRP waste, 1105 (J;US)

Solidification

Borosilicate glass for immobilization of high-level SRP waste, 1105 (J;US)

GAS COOLED FAST BREEDER REACTORS

SRe GCFR TYPE REACTORS

GAS COOLED GRAPHITE MODERATED REACTOR

See GCR TYPE REACTORS

GASEOUUS EFFLUENTS

SeE GASEOUS WASTES

GASEOUS WASTES

Chemical Composition

Off-gas characteristics of liquid-fed joule-heated ceramic melters, 319 (R;US)

Corrosive Effects

Off-gas characteristics of liquid-fed joule-heated ceramic meltcrs, 319 (R;US)

Flow Rate

Off-gas characteristics of liquid-fed joule-heated ceramic melters, 319 (R;US)

\section{GCFR TYPE REACTORS}

Thorium Cycle

Use of thorium as an alternative nuclear fuel, 46 (R;AU)

GCR TYPE REACTORS

Thorium Cycle

Use of thorium as an alternative nuclear fuel, 46 (R;AU) 


\section{GELS}

Chemical Preparation

Preparation of gel spheres containing simulated high-level radioactive waste, $381(\mathrm{~J} ; \mathrm{CH})$

\section{GEOLOGIC DEPOSITS}

See also ALLUVIAL DEPOSITS

\section{SALT DEPOSITS}

Suitability of natural geomedia for radwaste storage, 1060 (J;US)

Geology

Hydrogcology of radioactive waste isolation: the challenge of a rational assessment, 1144 (J;US)

Hydrology

Hydrogeology of radioactive waste isolation: the challenge of a rational assessment, 1144 (J;US)

Radionuclide Migration

Earth Sciences, 789 (RA;US)

INTRACOIN, Level 1. Benchmark calculations with code SWIFT, 644 (R;DE)

Technical support of standards for high-level radioactive waste management. Addendum to volumes $\mathrm{C}$ and $\mathrm{D}$. Final report, 840 (R;US)

Rock Mechanics

Composition of rocks - criteria for the conditioning of high-level radioactive wastes. 672 (RA;DE; In German)

Testing

Laboratory rock mechanics testing manual. Public draft, 807 (R;US)

Thermal Stresses

Thermal and thermomechanical calculations of deep-rock nuclear waste disposal with the enhanced SANGRE code, 1012 (R;US)

GERMAN FEDERAL REPUBLIC

See FEDERAL REPUBLIC OF GERMANY

GERMANY (FEDERAL REPUBLIC)

See FEDERAL REPUBLIC OF GERMANY

GLASS

See also BOROSILICATE GLASS

Immobilization of Idaho Chemical Processing Plant high-level wastes, 240 (RA;DE)

Absorption Spectra

Point defects and microstructural stability of glasses under irradiation (Silica glass bombarded by alpha particles, protons and electrons), 1154 (BA;US)

Bulk Density

Consolidated waste forms: glass marbles and ceramic pellets, 299 (R;US)

Chemical Composition

Diopside glass-ceramic material for the immobilization of radioactive wastes, 258 (RA;DE)

Effects of composition, solutions, and burial on nuclear waste glasses, 845 (RA;US)

Investigations of suitable metallic container materials for HAW solidification, 371 (J;US)

Leaching behaviour of ion-implanted simulated HLW glasses and tentative prediction of their alpha-recoil aging, 423 (BA;US)

Measurement of leaching from simulated nuclear-waste glass using radiotracers, 128 (R;US)

Chemical Radiation Effects

Point defects and microstructural stability of glasses under irradiation (Silica glass bombarded by alpha particles, protons and electrons), 1154 (BA;US)

Radiation damage studies related to nuclear waste forms, 298 (R;US)

Comparative Evaluations

Consolidated waste forms: glass marbles and ceramic pellets, 299 (R;US)

Development and testing of matrices for the encapsulation of glass and ceramic nuclear waste forms, 297 (R;US)

Development, evaluation, and selection of candidate high-level waste forms, 185 (R;US)

Evaluation of conditioned high-level waste forms, 326 (R;US)

Evaluation of forms for the immobilization of high-level and transuranic wastes, 200 (R;US)

\section{Corrosion}

Effects of composition, solutions, and burial on nuclear waste glasses, 845 (RA;US)

Geochemical factors controlling the nuclide release source-term in granite: dissolution of the waste form, $205(R ; G B)$

Corrosion Resistance

Corrosion studies on non-metallic inorganic materials for HLW packaging design, 691 (R;DE;In German)

Crystallization

Attempt to assess the long-term crystallization rate of nuclear waste glasses, 457 (BA;US)

Quantitative determination of crystalline phases in nuclear waste glasses, 458 (BA;US)

Cutting

Method of processing high level radioactive wastes by glass solidification, 408 (P;JP;In Japanese)

Density

Radiation damage studies related to nuclear waste forms, 298 (R;US)

Dissolution

Geochemical factors controlling the nuclide release source-term in granite: dissolution of the waste form, 205 (R;GB)

Temperature, pressure and time dependence of dissolution rates of high level radioactive waste glasses, 653 (RA;AU)

Field Tests

Geochemical factors controlling the nuclide release source-term in granite: dissolution of the waste form, 205 (R;GB)

Fracture Properties

Mechanical stability of a Cm-doped celsian glass-ceramic, 143 (R;XE)

Methodology for characterizing brittle fracture of solid waste forms in accidental impacts, 420 (BA;US)

Fractures

Final report of experimental laboratory-scale brittle fracture studies of glasses and ceramics, 129 (R;US)

Fracturing

Fracturing of simulated high-level waste glass in canisters, 1117 (J;NL)

Fragmentation

Respirable fines produced by impacts of simulated alternative high-level waste materials, 419 (BA;US)

Impact Strength

Consolidated waste forms: glass marbles and ceramic pellets, 299 (R;US)

Methodology for characterizing brittle fracture of solid waste forms in accidental impacts, 420 (BA;US)

Impact Tests

Final report of experimental laboratory-scale brittle fracture studies of glasses and ceramics, 129 (R;US)

Full-scale impact tests of simulated high-level waste canisters, 316 (R:US)

Methodology for characterizing brittle fracture of solid waste forms in accidental impacts, 420 (BA;US)

Respirable fines produced by impacts of simulated alternative high-level waste materials, 419 (BA;US)

Ion Implantation

Leaching behaviour of ion-implanted simulated HLW glasses and tentative prediction of their alpha-recoil aging, 423 (BA;US)

Leaching

Alpha spectrum profiling of plutonium in leached simulated high-level radioactive waste-glass, 1364 (J;US)

Application of neutron-activation analysis to the determination of leach rates of simulated nuclear-waste forms, 127 (R;US)

Characterization and testing of basalt glass and glass ceramic waste forms, 313 (R;US)

Coating of crystalline nuclear waste forms to improve inertness, 387 (J;US)

Comparative leach testing of high-level waste forms, $626(R ; U S)$

Consolidated waste forms: glass marbles and ceramic pellets, 299 (R;US)

Development and testing of matrices for the encapsulation of glass and ceramic nuclear waste forms, 297 (R;US)

Effects of cracks on glass leaching, 126 (J;US)

Effects of radiation on the leaching behavior of nuclear waste forms, $1285(\mathrm{~J} ; \mathrm{US})$ 
Effects of composition, solutions, and burial on nuclear waste glasses, 845 (RA;US)

Effects of radiation damage and radiolysis on the leaching of vitrified waste, 463 (BA;US)

Elemental release from glass and spent fuel, 1307 (RA;US)

Flow model for the kinetics of dissolution of nuclear waste forms; a comparison of borosilicate glass, SYNROC and high-silica glass, 1192 (BA;US)

Fuel cycle programs. Quarterly progress report, OctoberDecember 1982, 539 (R;US)

Functional dependence of leaching on the surface area-to-solution volume ratio, 1067 (J;US)

High-level waste glass field burial tests at CRNL: the effect of geochemical kinetics on the release and migration of fission products in a sandy aquifer, 1299 (R;CA)

High-temperature leaching of an actinide-bearing, simulated high-level waste glass, 841 (R;US)

Leaching behaviour of ion-implanted simulated HLW glasses and tentative prediction of their alpha-recoil aging, 423 (BA;US)

Leaching of solidified highly radioactive decay products: overviow (In Gormun), 249 (RA, $\left.A_{1}, G E\right)$

Leaching of natural and synthetic sphene and perovskite, 456 (BA;US)

Leaching study of PNL 76-68 glass beads using the LLNL continuous-flow method and the PNL-modified IAEA method. Finàl réport, 344 ('R;US')

Leaching study of nuclear melt glass: Part I, 1332 (R;US)

Measurement of leaching from simulated nuclear-waste glass using radiotracers, 128 (R;US)

Model for predicting leaching behavior of high-level radioactive waste forms, 508 (J;US)

Near-surface leaching studies of Pb-implanted Savannah River waste glass, 140 (R;US)

Radiation damage studies related to nuclear waste forms, 298 (R;US)

Radiation effects in high-level radioactive waste forms (Actinide radiation), $116(\mathrm{~J} ; \mathrm{CH})$

SIMS depth profiling studies of sphene-based ceramics and glass ceramics leached in synthetic groundwater, 455 (BA;US)

Solubility limits of metal ions and mechanism of leaching nuclear-waste glasses, 320 (R;US)

Stability and alteration of naturally occurring low-silica glasses: implications for the long term stability of waste form glasses, 1190 (BA;US)

Waste glass/metal interactions in brines, 860 ( $R$;US)

Manufacturing

Some glass-ceramics for special applications, 481 (J;JP)

Materials Testing

Evaluating glasses for high-level radioactive waste immobilization, 1237 (J;US)

Melting

Vitrification of high level wastes using microwave power, 230 (RA;DE)

Microstructure

Consolidated waste forms: glass marbles and ceramic pellets, 299 (R;US)

Radiation damage studies related to nuclear waste forms, 298 (R;US)

Nondestructive Testing

Development of CT techniques for integrity evaluation of vitrified solids, 1045 (J;US)

Phase Studies

Quantitative determination of crystalline phases in nuclear waste glasses, 458 (BA;US)

Physical Radiation Effects

Application of neutron-activation analysis to the determination of leach rates of simulated nuclear-waste forms, 127 (R;US)

Critical review of radiation effects on borosilicate glasses, 261 (RA;DE)

Leaching behaviour of ion-implanted simulated HLW glasses and tentative prediction of their alpha-recoil aging, 423 (BA;US)

Mechanical stability of a $\mathrm{Cm}$-doped celsian glass-ceramic, 143 (R;XE)
Point defects and microstructural stability of glasses under irrad:ation (Silica glass bombarded by alpha particles, protons and electrons), 1154 (BA;US)

Radiation effects in crystalline high-level nuclear waste solids, 1278 (BA;US)

Simulation of radiation damage in HLW glasses accelerated with $(n, \alpha)$ and $(n, f)$ reactions, $654(\mathrm{RA} ; \mathrm{AU})$

Porosity

Consolidated waste forms: glass marbles and ceramic pellets, 299 (R;US)

Radiation Effects

Coating of crystalline nuclear waste forms to improve inertness, 387 (J;US)

Effects of radiation on the leaching behavior of nuclear waste forms, 1285 (J;US)

Near-surface leaching studies of $\mathrm{Pb}$-implanted Savannah River waste glass, 140 (R;US)

Scientific basis for nuclear waste management, 107 (B;US)

Stability

Glass as a high level waste matrix: a review of the variables, 220 $(\mathrm{RA} ; \hat{A I J)}$

Stability and alteration of naturally occurring low-silica glasses: implications for the long term stability of waste form glasses, 1190 (BA;US)

Standardization

Nuclear waste management technical support in the developmnt of nuclear waste form criteria for the NRC. Task S. National waste package program, 285 (R;US)

Surface Coating

Alternative waste form development - low-temperature pyrolytic carbon coatings, 511 (J;US)

Temperature Effects

High-temperature leaching of an actinide-bearing, simulated high-level waste glass, 841 (R;US)

Transport

Waste package, 288 (RA;US)

Uses

Some glass-ceramics for special applications, 481 (J;JP)

Waste-rock Interactions

Waste glass - seabed interactions: observations of the Soret cffcct, 850 (RA;US)

$X$-ray Diffraction

Quantitative determination of crystalline phases in nuclear waste glasses, 458 (BA;US)

GLASS MELTERS

See CERAMIC MELTERS

GRANITES

See also GRANODIORITES

Repository, 773 (RA;US)

Compression Strength

Thermal impact of waste emplacement and surface cooling associated with geologic disposal of nuclear waste, 707 (R;US)

Fluid Flow

Fuel cycle programs. Quarterly progress report, April-June 1982 , $536(\mathrm{R} ; \mathrm{US})$

Theoretical and laboratory investigations of flow through fractures in crystalline rock, 618 (RA;US)

Fracture Properties

Radioactive waste disposal in granite (Stripa mine), 712 (R;US)

Fractures

Fracture analysis of crystalline rocks: field ineasurements and field geomechanical techniques, $638(\mathrm{R} ; \mathrm{GB})$

Migration in a single fracture, 1342 (BA;US)

Radionuclide migration into natural fracture surfaces of granitic rock, 1334 (J;US)

Theoretical and laboratory investigations of flow through fractures in crystalline rock, 618 (RA;US)

Gamma Logging

Effect of radon transport in groundwatcr upon gamma-ray borchole logs, 1422 (R;US)

Ground Water

Elution of radionuclides through columns of crushed rock from the Nevada Test Site, 1315 (R;US) 
Hydraulic Conductivity

Transient hydraulic tests in granite: fissured porous medium analysis and results, 1147 (BA;US)

Hydrology

Fuel-cycle programs. Quarterly progress report, January-March 1981, 48 (R;US)

Fuel cycle programs. Quarterly progress report, July-September 1981, 50 (R;US)

Mechanical Properties

Radioactive waste disposal in granite (Stripa mine), 712 (R;US)

Mineralogy

Scientific data necessary to predict radionuclide migration within or near a mined nuclear repository, 1312 (R;US)

\section{Natural Radioactivity}

Effect of radon transport in groundwater upon gamma-ray borehole logs, 1422 (R;US)

Permeability

Aqueous phase diffusion in crystalline rock, 1359 (BA;US)

Diffusion in crystalline rocks, 1168 (BA;US)

Elution of radionuclides through columns of crushed rock from the Nevada Test Site, 1315 (R;US)

Theoretical and laboratory investigations of flow through fractures in crystalline rock, 618 (RA;US)

Thermal impact of waste emplacement and surface cooling associated with geologic disposal of nuclear waste, 707 (R;US)

Poisson Ratio

Thermal impact of waste emplacement and surface cooling associated with geologic disposal of nuclear waste, 707 (R;US)

Thermomechanical studies in granite at Stripa, Sweden, 619 (RA;US)

Porosity

Aqueous phase diffusion in crystalline rock, 1359 (BA;US)

Diffusion in the matrix of granitic rock: field test in the Stripa mine, 1341 (BA;US)

Fuel cycle programs. Quarterly progress report, April-June 1982 $536(\mathrm{R} ; \mathrm{US})$

Thermal impact of waste emplacement and surface cooling associated with geologic disposal of nuclear waste, 707 (R;US)

Radionuclide Migration

Distribution coefficient (Kd) concept and its applicability to studies of radionuclide migration in geological media, 1313 (R;GB)

Mcchanisms of dissolution of radioactive waste storage glasses and cesium migration from a granite repository, 1151 (BA;US)

Study of the migration of leached radionuclides in a natural fissure of granite rock, $1240(\mathrm{~J} ; \mathrm{CS})$

Rock Mechanics

Climax Gidinite, Nevada Test Sitc, as a host for a rook meohan. ics test facility related to the geologic disposal of high level nuclear wastes, 1002 (R;US)

Expected repository environments in granite: assessment of rock stability of the container drill hole region, 541 (R;US)

Fracture analysis of crystalline rocks: field measurements and field geomechanical techniques, 638 (R;GB)

Thermomechanical studies in granite at Stripa, Sweden, 619 (RA;US)

Rock-fluid Interactions

Correlation between dynamic leach test results and geochemical observations, 1059 (J;US)

Site Surveys

Site, 772 (RA;US)

Sorptive Properties

Diffusion in crystalline rocks, 1168 (BA;US)

Distribution coefficient (Kd) concept and its applicability to studies of radionuclide migration in geological media, 1313 (R;GB)

Radionuclide retardation during transport through fractured granite. 1355 (BA;US)

Status of radionuclide sorption-desorption studies performed by the WRIT program, 1308 (RA;US)

Stress Analysis

Expected repository environments in granite: assessment of rock stability of the container drill hole region, 541 (R;US)
Temperature Effects

Analysis of temperature data from the full-scale heater experiments at Stripa, 710 (RA;US)

Radioactive waste disposal in granite (Stripa mine), 712 (R;US)

Tensile Properties

Thermal impact of waste emplacement and surface cooling associated with geologic disposal of nuclear waste, 707 (R;US)

Thermal Expansion

Thermomechanical studies in granite at Stripa, Sweden, 619 (RA;US)

Thermal Stresses

Thermal impact of waste emplacement and surface cooling associated with geologic disposal of nuclear waste, $707(R ; U S)$

Thermomechanical studies in granite at Stripa, Sweden, 619 (RA;US)

Thermodynamic Properties

Analysis of temperature data from the full-scale heater experiments at Stripa, 710 (RA;US)

Thermoelasticity

Expected repository environments in granite: assessment of rock stability of the container drill hole region, 541 (R;US)

Thermomechanical studies in granite at Stripa, Sweden, 619 (RA;US)

Waste-rock Interactions

Modeling of nuclear waste disposal by rock melting, 1016 (R;US)

Young Modulus

Thermal impact of waste emplacement and surface cooling associated with geologic disposal of nuclear waste, 707 (R;US)

Thermomechanical studies in granite at Stripa, Sweden, 619 (RA;US)

\section{GRANODIORITES}

Waste-rock Interactions

Interaction of borosilicate glass and granodiorite at $100^{\circ} \mathrm{C}, 50$ $\mathrm{MPa}$ implications for models of radionuclide release, 1201 (BA;US)

\section{GRAPHTTE}

Comparative Evaluations

Development and testing of matrices for the encapsulation of glass and ceramic nuclear waste forms, 297 (R;US)

Fabrication

Highly dense graphite matrix: a new material for the conditioning of radioactive wastes, 358 (J;US)

Physical Properties

Highly dense graphite matrix - a new material for the conditioning of radioactive wastes, 394 (J;US)

GRAPHITE MODERATOR

See GRAPHITE

GREAT BRITAIN

SEe UNITED KINGDOM

\section{GROUND WATER}

Chemical Composition

Review of recent PNL research activities related to glass leaching mechanisms, 846 (RA;US)

Chemical Reactions

Developments, recommendations and applications of tests for evaluating the chemical stability of high-level radioactive solid waste forms, $1056(\mathrm{~J} ; \mathrm{GB})$

Corrosive Effeetg

Enviromechanical testing of Ti-grade 2 and Ti-grade 12 in basalt ground water, 858 (R;US)

Investigation of environmentally assisted fracture of metallic nuclear-waste-package barrier materials in simulated basalt repository environments, 844 (R;US)

Flow Models

Groundwater flow modeling in support of salt site evaluations, 1034 ( $\mathrm{J} ;$ US)

Hydrothermal conditions around a radioactive waste repository, 1177 (BA;US)

Unsaturated flow and transport through fractured rock - related to high-level waste repositories. Final report. Phase I, 748 (R;US) 


\section{Flow Rate}

Comparison of waste emplacement configurations for a nuclear waste respository in tuff. IV. Thermo-hydrological analysis, 996 (R;US)

Fluid Flow

Earth Sciences, 789 (RA;US)

Geochemistry

Earth Sciences, 789 (RA;US)

Research and development related to the Nevada Nuclear Waste Storage Investigations. Progress report, July 1-September 30, 1982, 703 (R;US)

Summary report on the geochemistry of Yucca Mountain and environs, 700 (R;US)

Hydrology

Earth science developments in support of waste isolation, 608 (RA;US)

Isotope Dating

Status of geochemical problems relating to the burial of highlevel radioactive waste, 1982, 1318 (R;US)

Use of radiogenic noble gases for dating groundwater, 617 (RA;US)

Isotope Ratio

Permeability monitoring technique of the near-field and far-field interfuce opuningy, 1346 (BA;US)

Radiolysis

Radiolysis of groundwater from HLW stored in copper canisters, 1062 (J;US)

Radiolysis of groundwater from HLW stored in copper canisters, 999 (R;SE)

Radionuclide Migration

Application of a risk-assessment methodology to a hypothetical high-level waste repository in bedded salt, 937 (R;US)

Assessment of single-shell tank residual-liquid issues at Hanford Site, Washington, 1384 (R;US)

Basalt-radionuclide reactions: FY-1981. Annual report, 868 (R;US)

Combined analytical model for preformance assessment of the waste package/geologic medium systems, 1170 (BA;US)

Comparative analysis of fractured and porous medium radionuclide transport, 1356 (BA;US)

Comparison of in-situ radionuclide migration studies in the Studsvik area and laboratory measurements, 1349 (BA;US)

Diffusion in the matrix of granitic rock: field test in the Stripa mine, 1341 (BA;US)

Earth Sciences, 789 (RA;US)

Earth science developments in support of waste isolation, 608 (RA;US)

Effect of microbial activity on the containment of radioactive waste in a decp gcological repository, 1357 (BA;US)

Elution of radionuclides through columns of crushed rock from the Nevada Test Site, 1.315 (R;IIS)

Evaluation of solubility and speciation of actinides in natural groundwaters, 1347 (BA;US)

Evaluation of radionuclide transport: effect of radionuclide sorption and solubility, 1354 (BA;US)

Furmulation of an integrated physicochemical-hydrologic model for predicting waste-nuclide retardation in geologic media, 973 (R;US)

Fuel cycle programs. Quarterly progress report, April-June 1982 , 536 (R;US)

Fuel cycle programs. Quarterly progress report, July-September 1982, 538 (R;US)

Fuel cycle programs. Quarterly progress report, October-

December 1982, 539 (R;US)

Geochemical behavior of supercalcine waste form: its stability in a basalt environment, 911 (R;US)

Geochemical controls on radionuclide releases from a nuclear waste repository in basalt: estimated solubilities for selected elements, 1329 (R;US)

Geochemistry studies pertaining to the G-tunnel radionuclide migration field experiment, 1426 (R;US)

Groundwater flow and transport characteristics of flood basalts as determined from tracer experiments, 880 (R;US)
High-level waste glass field burial tests at CRNL: the effect of geochemical kinetics on the release and migration of fission products in a sandy aquifer, 1299 (R;CA)

Hydrothermal conditions around a radioactive waste repository, 1177 (BA;US)

Importance of organic compounds in ground water as radionuclide-mobilizing agents, 1324 (R;US)

Influence of microfissures in crystalline rock on radionuclide migration, 1185 (BA;GB)

Interaction of groundwater and fresh basalt fissure surfaces and its effect on the migration of actinides, 1303 (R;US)

Investigation of the consequences of leakage incidents of a tank with high-concentrated radionuclide solutions and pentration of the radioactive solution into the soil, 1423 (R;DE;In German)

Laboratory tests on the migration behavior of selected fission products in aquifer materials from a potential disposal site in northern Germany, 1352 (BA;US)

LAYFLO: a one-dimensional semianalytical model for the migration of a three-member decay chain in a multilayered geologic medium, 1325 (R;US)

Leach rates of high level waste and spent fuel: limiting rates as determined by backfill and bedrock conditions, 1345 (BA;US)

Leaching study of nuclear melt glass: Part I, 1332 (R;US)

Leaching studic of radioactive glass, 1006 (RA;US)

Mechanisms of dissolution of radioactive waste storage glasses and cesium migration from a granite repository, 1151 (BA;US)

Migration of long actinides chains in geologic media, 1362 (J;US)

Migration in a single fracture, 1342 (BA;US)

Migration of radionuclides with ground water: a discussion of the relevance of the Input parameters used In model calculationsts, 1350 (BA;US)

Model for near field migration, 1343 (BA;US)

Model for far field migration, 1344 (BA;US)

Modelling of the migration of lanthanoids and actinoids in ground water; the medium dependence of equilibrium constants, 1353 (BA;US)

Nuclear waste-form risk assessment for US defense waste at Savannah River Plant. Annual report FY, 1982, 1008 (R;US)

Numerical modeling of groundwater flow and solutc transport for a nuclear-waste repository in basalt, 1327 (R;US)

Possibility of high level wastc underground disposal, 1249 (J;JP;In Japanese)

Radionuclide migration in a two-dimensional flow field, 1339 (J;US)

Radionuclide sorption on basalt-interbed materials. Annual report FY 1981, 1328 (R;US)

Radionuclide retardationi during transpurt through fractured granite, 1355 (BA;US)

Radionuclide migration into natural fraoture surfaces of granitic rock, 1334 (J;US)

Sclected hydrologic and gewchemical issues in site characterization for nuclear waste disposal: flood basalts at the Hanford Reservation, 715 (R;US)

Semi-unified approach to leach testing, 852 (RA;US)

Sorption of actinides in well-defined oxidation states on genlngis. media, 1351 (BA;US)

Status of geochemical problems relating to the burial of highlevel radioactive waste, 1982, 1318 (R;US)

Studies on speciation of americium, technetium and neptunium in simulated vitrified-waste leachates, 1335 (J;US)

Study of radionuclide migration from deep-lying repository sites with over-lying sedimentaty layers, 1348 (BA;US)

Systems. 771 (RA:US)

The movement of a redox front downstream from a repository for nuclear waste, 1032 (J;US)

Uncertainties associated with geologic disposal of high-level radioactive waste, 1367 (R;US)

Uncertainties and sensitivities in the performance of geologic nuclear waste isolation systems. Volume I. Summary, 809 (R;US)

Uncertainties in geologic disposal of high-level wastes: groundwater transport of radionuclides and radiological consequences, 737 (R;US) 
Volume 2. A simplified analysis of a hypothetical repository in a basalt formation, 1416 (RA;US)

Volume 3. A simplified analysis of a hypothetical repository in a tuff formation, 1417 (RA;US)

Volume 4. A simplified analysis of a hypothetical repository in a bedded salt formation, 1418 (RA;US)

Water-dilution volume for high-level wastes stored in deep geologic media, 1232 (J;US)

Rock-fluid Interactions

Earth Sciences, 789 (RA;US)

Native copper deposits of the Portage Lake volcanics, Michigan: their implications with respect to canister stability for nuclear waste isolation in the Columbia River basalts beneath the Hanford Site, Washington, 904 (R;US)

Temperature Effects

Heating effects on groundwater flow through a repository fracture system, $120(\mathrm{~J} ; \mathrm{US})$

GROUND-WATER RESERVES

See AQUIFERS

GROUTING

Permeability

Recent advances in repository seal matcrials, 1172 (B $\Lambda$;US)

GULF COAST

Geology

Geological evaluation of Gulf Coast salt domes: overall assessment of the Gulf Interior Region, 784 (R;US)

Hydrology

Geological evaluation of Gulf Coast salt domes: overall assessment of the Gulf Interior Region, 784 (R;US)

Salt Deposits

Investigation of the utility of Gulf Coast salt domes for the storage or disposal of radioactive wastes, 571 (R;US).

Tectonics

Geological evaluation of Gulf Coast salt domes: overall assessment of the Gulf Interior Region, 784 (R;US)

\section{H}

\section{HAFNIUM}

Activation Analysis

Application of neutron-activation analysis to the determination of leach rates of simulated nuclear-waste forms, 127 (R;US)

HANFORD ATOMIC PRODUCTS OPERATION See HAPO

HANFORD RESERVATION

Geodetic Surveys

1981 Geodolite observations near Hanford, Washington, 870 (R:US)

Geologic Deposits

Preliminary interpretation of the tectonic stability of the reference repository location, Cold Creek syncline, Hanford Site (Pasco Basin), 901 (R;US)

Geology

Basalt waste-isolation project. Quarterly report. January 1-March 31, 1981,908 (R;US)

Draft site characterization analysis of the site characterization report for the Basalt Waste Isolation Project, Hanford, Washington Site. Main report and Appendices A through D, 724 (R;US)

Ficld guidebook ascociation of enginesering genlngists. Chapters 1-10, 915 (R;US)

Hydrology and Geology Overview Committee reports and responses frum the Basalt Waste Isolation Project, 914 (R;US)

Selected hydrologic and geochemical issues in site characterization for nuclear waste disposal: flood basalts at the Hanford Reservation (Pasco Basin basalt), 715 (R;US)

- eophysics

Hydrology and Geology Overview Committee reports and responses from the Basalt Waste Isolation Project, 914 (R;US)

Ground Subsidence

Uplift and subsidence rates in the central Columbia Plateau and their relation to siting a waste repository at Hanford, Washington, 873 (R;US)

\section{Ground Uplift}

Uplift and subsidence rates in the central Columbia Plateau and their relation to siting a waste repository at Hanford, Washington, 873 (R;US)

Hydrology

Basalt waste-iśolation project. Quarterly report, January 1-March 31, 1981,908 (R;US)

Draft site characterization analysis of the site characterization report for the Basalt Waste Isolation Project, Hanford, Washington Site. Main report and Appendices A through D, 724 (R;US)

Groundwater flow and transport characteristics of flood basalts as determined from tracer experiments, 880 (R;US)

Hydrology and Geology Overview Committee reports and responses from the Basalt Waste Isolation Project, 914 (R;US)

Radionuclide migration in a two-dimensional flow field, 1339 (J;US)

Hydrothermal Alteration

Testing of candidate waste package backfill and canister materials for basalt, 586 (RA;US)

Land use

Monitoring land and water uses in the Columbia Plateau using remote-sensing computer analysis and integration techniques, 865 (R;US)

Radioactive Waste Dispósal

Basalt waste-isolation project drilling and testing. Quarterly report, January 1-March 31, 1982, 896 (R;US)

Basalt Waste Isolation Project drilling and testing. Quarterly report, April 1, 1982-June 30, 1982, 897 (R;US)

Basalt Waste Isolation Project drilling and testing. Quarterly report, July 1, 1982-September 30, 1982, 898 (R;US)

Basalt Waste Isolation Project: drilling and testing. Quarterly report, October 1, 1982-December 31, 1982, 899 (R;US)

Basalt Waste Isolation Project. Drilling and testing quarterly report, January 1; 1983-March 31, 1983, 900 (R;US)

Performance allocation traceable to regulatory criteria as applied to site-characterization work at the Basalt Waste Isolation Project, 890 (R;US)

Site characterization report for the basalt waste isolation project. Volume I, 601 (R;US)

Site characterization report for the basalt waste isolation project. Volume II, 602 (R;US)

Site characterization report for the basalt waste isolation project. Volume III, 603 (R;US)

Site-screening process on the Hanford Site: a summary report, 878 (R;US)

Summary of Near-Surface Test Facility results and their application to repository design, 886 (R;US)

Waste package conceptual designs for a repository located in basalt, 884 (R; US)

Radioactive Waste Management

High level waste management and characterization at the Hanford plant site, 1198 (BA;US)

Radiosctive Waste Storage

Assessment of single-shell tank residual-liquid issues at Hanford Site, Washington, 1384 (R;US)

Effects of Hanford high-level waste components on sorption of cobalt, strontium, neptunium, plutonium, and americium on Hanford sediments, 918 (R;US)

Field guidebook association of engineering geologists. Chapters $1-10,915$ (R;US)

Seismic Surveys

Field guidebook association of engineering geologists. Chapters $1-10,915$ (R;US)

Water use

Monitoring land and water uses in the Columbia Plateau using remote-sensing computer analysis and integration techniques, 865 (R;US)

HAPO

Radioactive Waste Management

Rockwell Hanford Operations monthly report summary, June 1983, 9 (R;US)

Rockwell Hanford Operations. Defense waste and byproducts management monthly report, 335 (R;US) 
Rockwell Hanford Operations defense waste and byproducts management monthly report, 336 (R;US)

Strategy document: long-term technology program for defense high-level waste, 156 (R;US)

Radioactive Waste Processing

Long-Term. Highh-Level Defense-Waste technology. Progress report, April-June 1982, 338 (R;US)

Recovery and purification of multi-kilocurie quantities of fission product strontium by cation exchange, 214 (R;US)

Radioactive Waste Storage

Status of tank-assessment studies for continued in-tank storage of Hanford Defense waste, 919 (R;US)

Research Programs

Rockwell Hanford Operations monthly report summary, June 1983, 9 (R;US)

\section{HASTELLOY C}

Corrosion

Corrosion of materials in a clay environment, 686 (RA;DE)

Cörröiion Resistance

Corr sion of materials in a clay environment, $676(\vec{R} A ; \vec{D} \vec{E})$

HASTELLOY C-276

SOO HASTELLOYS

HASTRLLOY C-A

See HASTELLOYS

\section{HASTELLOYS}

See also HASTELLOY C

Corrooion

Corrosion studies and mechanical tests on metallic materials for the design of packagings for vitrified high level wastes (HLW), 689 (R;DE; In German)

Tensile Properties

Corrosion studies and mechanical tests on metallic materials for the design of packagings for vitrified high level wastes (HLW), 689 (R;DE;In German)

\section{HAYNES 188 ALLOY}

Córtiósión

Corrosion experience with a slurry-fed ceramic melter, 321 (R;US)

\section{HAYNES 25 ALLOY}

Corrasion

Corrosion experience with a slurry-fed ceramic melter, 321 (R;US)

HD 8077

See NICKEL BASE ALLOYS

HEALTH PHYSICS

SEe RADIATION PROTECTION

HEAT DISSIPATION

SEQ ENVIRONMENTAL TRANSPORT

HEAT RESISTING ALLOYS

See also HASTELLOYS

INCOLOY ALLOYS

INCONEL 617

STAINLESS STEEL..-304

STAINLESS STEEL-304L

STAINLESS STEEL-316

STAINLESS STEEL-32I

\section{Chemical Composition}

Investigations of suitable metallic container materials for HAW solidification. 371 (J;US)

\section{Corrosion}

Corrosion of materials in a clay environment, 686 (RA;DE)

HECTORITE

See MONTMORILLONITE

HEMATTTE

Thermodynamics

Uncertainties in the thermodynamics of basalt-oxygen and basaltwater reactions, 547 (R;US)

HIGH TEMPERATURE GAS COOLED AND GRAPHITE

See HTGR TYPE REACTORS
HIGH-LEVEL RADIOACTIVE WASTES

High-level waste disposal, 1275 (J;US)

After-heat

Thermal impact of waste emplacement and surface cooling associated with geologic disposal of nuclear waste, 707 (R;US)

Bibliographies

High-level radioactive wastes (812 citations), 159 (R;US)

Biological Radiation Effects

Parameters and variables appearing in radiological assessment codes. Final report, 1375 (R;US)

By-products

Department of Energy plan for recovery and utilization of nuclear byproducts from defense wastes. Volume l. Executive summary, 2 (R;US)

Carcinogenesis

Effects of ICRP Publication 30 and the 1980 Beir report on hazard assessments of high-level waste, $1440(\mathrm{~J} ; \mathrm{GB})$

Carriers

Subsurface migration of radioactive waste materials by particulate transport, 98 (J;US)

Caskis

High-Level Waste Transportation-System Development Program design report for Fiscal Year 1980. 34 (R:US)

Mechanical load on highly radioactive waste containers in fina! storage, 1141 (BA; $\overline{D E}$; In German)

Transportation packagings for high-level wastes and unprocessed transuranic wastes, 29 (R;US)

Chemical Analysis

Anālÿses of nuclear fuel and high-level waste by inductively coupled plasma-emission spectroscopy, 68 (R;US)

Sampling and analysis of high level waste tank supernatant: an overview (Savannah River Plant), 634 (R;US)

State-of-the-art in-situ determination of transuranics, 88 (R;US)

Chemical Reactions

Developments, recommendations and applications of tests for evaluating the chemical stability of high-level radioactive solid waste forms, $1056(\mathrm{~J} ; \mathrm{GB})$

Compatibility

Studies of waste-canister compatibility (Waste forms: Al-Si and Pb-Sn matrix alloys, FUETAP, glass, Synroc D, and waste particles coated with carbon or carbon plus $\mathrm{SiC}), 832(\mathrm{R} ; \mathrm{US})$

Contuiners

Container assessment: corrosion study of HLW container materials. Quarterly progress report, July-September 1981, 728 (R;US)

Corrosion resistance of metal materials for HLW canister. 666 (R;JP;In Japancse)

High-integrity container for high-specific-activity wastes, 1446 (J;US)

Cooling

Thermal impact of waste emplacement and surface cooling associated with geologic disposal of nuclear waste, 707 (R;US)

Corrosive Effects

Prevention of stress corrosion cracking in nuclear waste storage tanks, 630 (R:US)

Criticality

Criticalily safety of ligh-level waste containing unseparated plu tonium, 1259 (J;US)

Diffusion

Sorptive diffusion in clay gels, 709 (RA;US)

Dissolution

Role of oxidation potential, Eh, in the dissolution of nuclear waste materials. 851 (RA:US)

Solubility-limited dissolution rate in groundwater, 1338 (J;US)

Solubility-limited fractional dissolution rate of vitrified waste in groundwater, 1228 (I;US)

Temperature, pressure and time dependence of dissolution rates of high level radioactive waste glasses; 653 (RA;AU)

Emplacement

Equipment for the emplacement of heat-producing wastc in to horizontal boreholes (Horizontal vs vertical emplacement), 555 (R;US)

Thermal response to emplacement of nuclear waste in long, horizontal boreholes, $986(\mathrm{R} ; \mathrm{US})$ 
Encapsulation

Molecular glasses for nuclear waste encapsulation (Patent), 1076 (P;US)

Environmental Effects

Monitoring an underground repository with modern seismological methods, 1269 (J;US)

Environmental Exposure Pathway

Parameters and variables appearing in radiological assessment codes. Final report, 1375 (R;US)

Forecasting

Projections of high-level waste production in Brazil, 102 (J;US)

Radioactive waste inventories and projections, 62 (R;US)

Spent fuel and radioactive-waste inventories, projections, and characteristics, 57 (R;US)

Spent fuel and radioactive waste inventories, projections, and characteristics, 574 (R;US)

\section{Fractionation}

Systems study of the feasibility of high-level nuclear-waste fractionation for thermal-stress control in a geologic repository: main report, 308 (R;US)

Systems study of the feasibility of high-level nuclear waste fractionation for thermal stress control in a geologic repository: appendices, 309 (R;US)

Fracture Properties

Methodology for characterizing brittle fracture of solid waste forms in accidental impacts; 420 (BA;US)

Fragmentation

Respirable fines produced by impacts of simulated alternative high-level waste materials, 419 (BA;US)

Glass

Fracturing of simulated high-level waste glass in canisters, 1117 $(\mathrm{J} ; \mathrm{NL})$

Health Hazards

Effects of ICRP Publication 30, the 1980 BEIR Report, and ORIGEN-2 on hazard assessments of high level waste, 1400 (J;US)

Water dilution volumes for high-level wastes, 1401 (J;US)

Heat Transfer

Near-field interactions, 929 (R;US)

Waste package heat-transfer analysis: model development and temperature estimates for waste packages in a repository located in basalt, 917 (R;US)

Hot Atom Chemistry

Radiation damage studies related to nuclear waste forms, 298 (R;US)

Hydraulic Transport

Predicting transport requirements for radioactive-waste slurries, 22 (R;US)

Impact Strength

Methodology for characterizing brittle fracture of solid waste forms in accidental impacts, 420 (BA;US)

Impact Tests

Methodology for characterizing brittle fracture of solid waste forms in accidental impacts, 420 (BA;US)

Respirable fines produced by impacts of simulated alternative high-level waste materials, 419 (BA;US)

Inventories

High-level waste description. inventory and hazard, 632 (R;US)

Integrated data base for spent fuel and radwaste: inventories, 52 (R;US)

Nuclear-waste-management technical support in the development of nuclear-waste-form criteria for the NRC. Task 3. Waste inventory review, 73 (R;US)

Radioactive waste inventories and projections, 62 (R;US)

Spent fuel and radioactive-waste inventories, projections, and characteristics, 57 (R;US)

Spent fuel and radioactive waste inventories, projections, and characteristics, 574 (R;US)

Land Transport

Risk assessment for the transportation of radioactive zeolite liners, 42 (BA;US)

Southeastern Utah nuclear-waste-transportation study, 26 (R;US)

Transportation system preconceptual description for subseabed disposal of highlevel waste, 33 (R;US)

\section{Leaching}

Alpha spectrum profiling of plutonium in leached simulated high-level radioactive waste-glass, 1364 (J;US)

Alpha spectrum profiling of plutonium in leached simulated high-level radioactive waste-glass, 517 (J;US)

Analysis of waste-package behavior for high-level waste, 981 (R;US)

Characterization and testing of basalt glass and glass ceramic waste forms, 313 (R;US)

Coating of crystalline nuclear waste forms to improve inertness, 386 (J;US)

Coating of crystalline nuclear waste forms to improve inertness, 387 (J;US)

Combined analytical model for preformance assessment of the waste package/geologic medium systems, 1170 (BA;US)

Comparative leach testing of high-level waste forms, 626 (R;US)

Composite quarterly technical report: long-term high-level-waste technology, January-March 1982 (Preparation, fixation, and final handling), 66 (R;US)

Correlation between dynamic leach test results and geochemical observations, 1059 (J;US)

Diffusion of leached constituents in backfill barriers, 849 (RA;US)

Dissolution mechanisms in high-level nuclear waste consolidation forms, 305 (RA;US)

Effect of temperature on the leaching performance of SYNROC-B waste form, 1229 (J;US)

Effects of gamma irradiation on the leaching behavior of a synthetic mineral waste form (SYNROC-B), 1143 (J;US)

Effects of waste composition and loading on the chemical durability of a borosilicate glass, 1188 (BA;US)

Effects of radiation damage and radiolysis on the leaching of vitrified waste, 463 (BA;US)

Glass leaching performance, 856 (R;US)

HLW leaching mechanisms program. Semiannual status report, May 1982 (Numerical model, PROTOCOL, for dissolution reactions of nuclear waste forms in water), 847 (RA;US)

Hydroxylated ceramic waste forms and the absurdity of 'leach tests', 266 (RA;DE)

Immobilization of high-level nuclear reactor wastes in SYNROC: a current appraisal, 515 (J;US)

Leach rates of high level waste and spent fuel: limiting rates as determined by backfill and bedrock conditions, 1345 (BA;US)

Leach testing of INEL waste forms in a gamma field, 1044 (J;US)

Leaching behavior of sintered modified SYNROC-B waste forms: effect of HLW addition, 445 (BA;US)

Leaching studies of radioactive glass, 1006 (RA;US)

Long-term radioactivity release from solidified high-level waste. Part II. Parametric study of waste from properties, temperature and time, 1333 (J;US)

Materials Characterization Center workshop on leaching mechanisms of nuclear waste forms, May 19-21, 1982, Gaithersburg, Maryland. Summary report, 82 (R;US)

Near-surface leaching studies of Pb-implanted Savannah River waste glass, 140 (R;US)

Phantom dissolution leach model, 1268 (J;US)

Processing, microstructure, leaching, and long-term stability studies related to titanate high-level waste forms, 340 (R;US)

Solubility limits of metal ions and mechanism of leaching nuclear-waste glasses, 320 (R;US)

Solubility-limited fractional dissolution rate of vitrified waste in groundwater, 1228 (J;US)

SRP radioactive glass studies: small-scale process development and product performance, 180 (R;US)

Status report on dissolution model development, 1021 (R;US)

Surface properties and performance prediction of alternative waste forms. Annual report, October 1, 1981-September 30, 1982. Volume 1, 753 (R;US)

Workshop on the leaching mechanisms of nuclear-waste forms, October 27-28, 1981. Summary report, 81 (R;US) 
Leaks

Investigation of the consequences of leakage incidents of a tank with high-concentrated radionuclide solutions and pentration of the radioactive solution into the soil, 1423 (R;DE;In German)

Marine Disposal

Development of an assessment methodology for the disposal of high-level radioactive waste into deep ocean sediments, 1115 (J;CH)

Dumping nuclear waste into the sea: international control and the role of science and law, $1055(\mathrm{~J} ; \mathrm{GB})$

Maritime Transport

Subseabed nuclear waste disposal: conceptual ship description, 31 (RA;US)

Transportation system preconceptual description for subseabed disposal of highlevel waste, 33 (R;US)

Materials Handling

NWTS transportation interface technology development priority report, 89 (R;US)

Matcrials Recovery

Department of Energy plan for recovery and utilization of nuclear byproducts from defense wastes. Volume 1. Executive summary, 2 (R;US)

Nondestructive Testing

Nondestructive techniques for evaluating the integrity of vitrified solids, 471 (J;JP)

Packaging

Analysis of waste-package behavior for high-level waste, 981 (R;US)

Basalt waste-isolation project. Quarterly report, January 1-March $31,1981,908$ (R;US)

Conceptual design requirements for spent fuel, high-level waste, and transuranic waste packages, 903 (R;US)

Conceptual waste-package designs for disposal of nuclear waste in tuff, 818 (R;US)

Conceptual waste package interim performance specifications for waste forms for geologic isolation in salt repositories, 821 (R;US)

Construction and inservice inspection rules for nuclear spent fuel and high-level waste transport packagings, 18 (RA;US)

Corrosion studies on non-metallic inorganic materials for HLW packaging design, 691 (R;DE;In German)

Design of a nuclear-waste package for emplacement in tuff, 1018 (R:US)

Design rules for containment systems of nuclear spent fuel and high-level waste transport packagings, 19 (RA;US)

Development of waste package designs for disposal in a salt repository, 557 (RA;US)

Draft Technical Position Subtask 1.1: waste package performance after repository closure. Volume 1,749 (R;US)

Draft technical position on waste-package reliability, 546 (R;US)

Economic evaluation of nuclear waste transportation casks, 14 (R;US)

Engineered waste-package conceptual design: defense high-level waste (Form 1), commercial high-level waste (Form 1), and spent fuel (Form 2) disposal in salt, 817 (R;US)

Geochemical factors controlling the nuclide release source-term in granite: dissolution of the waste form, $205(\mathrm{R} ; \mathrm{GB})$

Impacts of transportation on a test and evaluation facility for nuclear waste disposal: a systems analysis, 556 (R;US)

Initial specifications for nuclear waste package external dimensions and materials, 1005 (R;US)

Nuclear waste management technical support in the developmnt of nuclear waste form criteria for the NRC. Task S. National waste package program, 285 (R;US)

Nuclear waste management technical support in the development of nuclear waste form criteria for the NRC. Task 1 . Waste package overview, 283 (R;US)

NWTS waste package program plan. Volume I. Program strategy, description, and schedule, 598 (R;US)

NWTS waste package program plan. Volume II. Program logic networks, 599 (R;US)

Parametric study of the effects of thermal environment on a waste package for a tuff repository, 1271 (BA;XN)

Review of DOE waste package program. Subtask 1.1. National waste package program, 286 (R;US)
Transportation packagings for high-level wastes and unprocessed transuranic wastes, 29 (R;US)

Waste package heat-transfer analysis: model development and temperature estimates for waste packages in a repository located in basalt, 917 (R;US)

Waste package performance assessment, 1274 (BA;XN)

Waste package conceptual designs for a repository located in basalt, 884 (R;US)

Waste package conceptual designs for a nuclear repository in basalt, 872 (R;US)

Partition

Development of partitioning method - cold and semihot experimental works with partitioning testing apparatus, 225 (R;JP;In Japanese)

Physical Radiation Effects

Radiation and transmutation effects on crystalline radioactive waste forms, 1262 (J;US)

Radiation effects in high-level radioactive waste forms (Actinide radiation), 116 (j;CH)

Simulation of radiation damage in HLW glasses accelerated with $(n, \alpha)$ and $(n, n)$ reactione; $651(R \wedge ; A U)$

Projection Series

NWTS program waste projection data needs, 1086 (J;US)

Radiation Hazards

Effects of ICRP Publication 30 and the 1980 Beir report on hazard assessments of high-level waste, 1440 (J;GB)

Effects of ICRP Publication 30 and the 1980 BEIR Report in hazard assessments of high-level waste, 1441 (J;GB)

High-level waste description, inventory and hazard, 632 (R;US)

Relative hazard of DWPF reference glass canister ICRP 2 vs ICRP 30, 1371 (R;US)

Radioactive Waste Disposal

Environmental impact of nuclear power. Opening address, 1246 (J;GB)

Solar-thermionic generator for nuclear wastes disposal in the space, $1138(\mathrm{~J} ; \mathrm{DE})$

Utilization of charged particle backscattering to study the near surface region of glasses. Application to depth profiling of lanthanium, cerium, throium and uranium induced by aqueous leaching, 1216 (BA;US)

Radioactive Waste Management

High-Level Nuclear-Waste Management. Oversight Hearlng before the Subcommittee on Energy and the Environment of the Committee on Interiur and Insular Affairs, House of Representatives, Ninety-Seventh Congress, Second Session, June $17,1982,110$ (B;US)

Radioactive Waste Processing

Disposal of radioactive wastes, 413 (B;NL)

Plant for solidification and storage of high-level radioactive waste, 521 (J:JP;In Japanese)

Radioactive Waste Storage

Heat transfer in high-level waste management, 1118 (J;NL)

Near-field salt temperatures resulting from storage of nuclear fuel assemblies in bedded salt, $1121(\mathrm{~J} ; \mathrm{NL})$

Nuclear waste, the nation, and Suuth Carulina: an update on the major issues, 114 (B;US)

Numerical calculation of three-dimensional long-time temperature fields using a special finite element method: the final storage of highly radioactive waste in salt rocks, $662(R ; D E ; I n$ German)

Optimization of mine layout for nuclear fuel assembly storage, 1123 (J;NL)

Sensitivity calculations for low-heat generating defense waste repository temperatures, 1124 (J;NL)

Study for dry storage within the framework of the Swiss waste disposal concept, 1407 (BA;DE;In German)

Thermal criteria for terminal storage of spent nuclear fuel assem blies in bedded salt, $1119(\mathrm{~J} ; \mathrm{NL})$

Uncoupling thermal problems of terminal storage of spent nuclear fuel assemblies in bedded salt, $1120(\mathrm{~J} ; \mathrm{NL})$

Radioactive Wastes

Radwaste panel, NRC head highlight 44th American Power Conference program, 99 (J;US) 
adioactivity

Parameters and variables appearing in radiological assessment codes. Final report, 1375 (R;US)

Radiochemistry

Radiation damage studies related to nuclear waste forms, 298 (R;US)

Radionuclide Migration

Parameters and variables appearing in radiological assessment codes. Final report, 1375 (R;US)

Subsurface migration of radioactive waste materials by particulate transport, $98(\mathrm{~J} ; \mathrm{US})$

Rail Transport

High-Level Waste Transportation-System Development Program design report for Fiscal Year 1980, 34 (R;US)

Preliminary analysis of the cost and risk of transporting nuclear waste to potential candidate commercial repository sites, 35 (R;US)

Rail transport of vitrified high-level radioactive fission products risk analysis of a model, 25 (R;DE;In German)

Risk analysis of transporting vitrified high level radioactive waste by train (Borosilicate glass waste furm), 1369 (RA;US)

Southeastern Utah nuclear-waste-transportation study, 26 (R;US)

Redox Potential

Role of oxidation potential, $\mathrm{Eh}$, in the dissolution of nuclear waste materials, 851 (RA;US)

Regulations

NRC regulations for high-level nuclear waste management, 1092 (J;US)

Remote Handling

High-level waste solidification system for the Western New York State Service Center, 507 (J;US)

Research Programs

Policy and some problems on the return waste, 118 (J;JP;In Japanese)

Retrieval Systems

Background and historical perspectives for monitored retrievable storage, 38 (J;US)

Risk Assessment

Effects of ICRP Publication 30, the 1980 BEIR Report, and ORIGEN-2 on hazard assessments of high level waste, 1400 (J;US)

Rail transport of vitrified high-level radioactive fission products risk analysis of a model, 25 (R;DE;In German)

Road Transport

Preliminary analysis of the cost and risk of transporting nuclear waste to potential candidate commercial repository sites, 35 (R;US)

Safety

Criticality safety of high-level waste containing unseparated plutonium, 1259 (J;US)

Sampling

Sampling and analysis of high level waste tank supernatant: an overview (Savannah River Plant), 634 (R;US)

Sealing Materials

Corrosion studies on non-metallic inorganic materials for HLW packaging design, 691 (R;DE;In German)

Seismic Effects

Monitoring an undergrnund repository with modern seismological methods, 1269 (J;US)

Self-irradiation

Fissiull product transmutation effects on high-level radioactive. waste forms, $401(\mathrm{~J} ; \mathrm{GB})$

Simulation

Preparation of gel spheres containing simulated high-level radioactive waste, $381(\mathrm{~J} ; \mathrm{CH})$

Solidification

Apparatus and method for immobilizing waste material (Patent), 484 (P;AU)

Effects of various waste contents on properties of hnrosilicate glasses for high-level waste solidification in several countries, 223 (R;JP;In Japancse)

Evaluation and selection of candidate high-level waste forms, 160 (R;US)
Introduction of studies on high-level radioactive waste immobilization' in synthetic minerals. Supercalcine and SYNROC, 224 (R;JP;In Japanese)

Phosphate system as medium for radioactive waste fixation. Simplex experiment. Part .I, 354 (R;CS;In Czech)

Plant for solidification and storage of high-level radioactive waste, 521 (J;JP;In Japanese)

Thermodynamically stable glass ceramic product with radionuclides for the removal of radio-active waste and process for its manufacture (Patent), 1283 (P;DE;In German)

Solvent Extraction

Octyl(phenyl)-N,N-diisobutylcarbamoylmethylphosphine oxide as an extractant for actinides from nitric acid waste, 146 (R;US)

Stability

Developments, recommendations and applications of tests for evaluating the chemical stability of high-level radioactive solid waste forms, 1056 (J;GB)

High-level waste-form-product performance evaluation (Leaching; waste loading; mechanical stability), 627 (R;US)

Storage Facilities

Safety analysis of tank storage for highly radioactive reprocessing waste, $1404(\mathrm{~J} ; \mathrm{US})$

Surface Coating

Alternative waste form development - low-temperature pyrolytic carbon coatings, 511 (J;US)

Coating of crystalline nuclear waste forms to improve inertness, $386(\mathrm{~J} ;$ US $)$

Transmutation

Actinide recycling in light water reactors: results of reactor physics calculations, 208 (R;XE)

Combustion in the reactor of long-lived transuranium elements as a means of reducing the toxicity of HAW ((high-level radioactive wastes)), 522 (J;DE;In German)

Transport

Defense waste transportation: cost and logistics studies, 27 (R;US)

Storage and transportation of spent fuel and high-level waste using dry storage casks, 15 (RA;US)

Transportation Systems

High-Level Waste Transportation-System Development Program design report for Fiscal Year 1980, 34 (R;US)

Rail transport of vitrified high-level radioactive fission products risk analysis of a model, 25 (R;DE;In German)

Transportation system preconceptual description for subseabed disposal of highlevel waste, 33 (R;US)

Underground Disposal

Comparison of risks due to HLW and SURF repositories in bedded salt, 1368 (R;US)

Geologic disposal of nuclear waste, 1107 (J;US)

Isolation of high-level nuclear wastes in a geologic repository, 1084 (J;US)

Underground openings for in situ experiments, 1111 (J;US)

Vitrification

Development and investigation of the accepting system for solidified bodies returned from foreign countries after reprocessing, 487 (J;JP; In Japanese)

High temperature corrosion of heat-resisting alloys by borosilicate melts containing simulated high level nuclear wastes, 476 (J;JP;In Japanese)

Present status of techniques for the solidification of high lével wastes, 492 (J;JP;In Japanese)

Solidification for final storage of high-level radioactive fission product solutions from spent fuel elements, 1292 (B;DE;In German)

Vitrification of high-level wastes: A review of the computer thermal analysis for storage canisters (CANIST), 1439 (J;NL)

Waste Transportation

Comparative transportation risk assessment for borosilicate-glass and ceramic forms for immobilization of SRP Defense waste, 23 (R:US)

Economic evaluation of nuclear waste transportation casks, i4 ( $\dot{R} ;$ US $)$

Estimated transportation cost and risk for operation of a nuclear waste repository at candidate sites, 17 (RA;US) 
Impacts of transportation on a test and evaluation facility for nuclear waste disposal: a systems analysis, 556 (R;US)

Nuclear waste/nuclear power: their futures are linked, 560 (R;US)

NWTS transportation interface technology development priority report, 89 (R;US)

Radioactive waste management, 527 (BA;SU;In Russian)

Review of the status of transportation activities in various waste management systems, 30 (R;US)

Risk comparison for transportation, handling, and storage of high-level waste, 1402 (J;US),

Solar-thermionic generator for nuclear wastes disposal in the space, 1138 (J;DE)

Transport of highly radioactive materials, 45 (J;US)

Transportation packagings for high-level wastes and unprocessed transuranic wastes, 29 (R;US)

Transportation system preconceptual description for subseabed disposal of high-level waste, 20 (RA;US)

Waste-rock Interactions

Modeling of nuclear waste disposal by rock melting, 1016 (R;US)

Waste form/rock interaction leaching study using PNL 76-68 HOISTS glass beads and Umtanum basalt. Part I, 1004 (R;US)

Ropes

Effects of wire rope in mitigating a waste-shaft accident, $98 \cap$ (R;US')

HOLLANDITE

Phase Studies

Subsolidus sintering of SYNROC: I. Initial demonstrations, 280 (R;US)

Specific Heat

Thermophysical properties of SYNROC and its constituent mineral phases, 474 (BA;AU)

Thermal Conductivity

Thermophysical properties of SYNROC and its constituent mineral phases, 474 (BA;AU)

Thermal Diffusivity

Thermophysical properties of SYNROC and its constituent mineral phases, 474 (RA; $\mathrm{AII})$

HOT WATER

Corrosive Effects

Analysis of the corrosion products formed on $\mathrm{Ti}$ and a Ti-Pd alloy during exposure in hot water, 1176 (BA;US)

IITGR TYPE REACTORS

Thorium Cycle

Use of thorium as an alternative nuclear fuel, 46 (R;AU)

HUGENHOLTZ-PINES THEORY

See HYDROGEN

HUMAN POPULATIONS

Delayed Radiation Effects

Calcuilation of health effects per curie release for comparison with the EPA standard, 1379 (RA;US)

Health effects associtated with unit radonuclide releases to the environment, 1378 (RA,US)

Technical assistance for regulatory development: review and evaluation of the draft EPA standard 40CFR191 for disposal of ligh-level waste. Volumes 5 and 6,1377 (R;US)

Uncertainties in geologic disposal of high-level wạtes: groundwatèr transport of radionuclides and radiological consequences, 737 (R;US)

\section{Dose Kates}

Assessment of single-shell tank residual-liquid issues at Hanford Site, Washington, 1384 (R:US)

\section{Radlation Doses}

Environmental pathway models for estimating population health effects from disposal of high-level radioactive waste in genlogic repositories. Draft report, 1381 (R;US)

Nuclear waste-form risk assessment for US defense waste at Savannah River Plant. Annual report FY, 1982, 1008 (R;US)

Performance assessment of a shaft seals system in a HLW repository in the Gibson Dome area, 827 (R;US)

Potential individual doses from disposal of high-level radioactive wastes in geologic repositories. Draft report (final), 1382 (R;US)
Potential individual doses from disposal of high-level radioactiv wastes in geologic repositories. Draft report, 1383 (R;US)

Radiological risk analysis of an operating high-level waste repos. tury, 1395 (BA;XA)

Risk assessment for the transportation of radioactive zeolite liners, 42 (BA;US)

Uncertainties in geologic disposal of high-level wastes: groundwater transport of radionuclides and radiological consequences, 737 (R;US)

Radiation Hazards

Draft environmental impact statement for 40 CFR part 191: environmental standards for management and disposal of spent nuclear fuel, high-level and transuranic radioactive wastes. Final report, 838 (R;US)

Population risks from disposal of high-level radioactive wastes in geologic repositories. Draft report, 836 (R;US)

HUMANS

SEe HUMAN POPULATIONS

HUMIC ACIDS

Adsorption

Redox bahavior, oomplexing, and adsorption of hexavalent actnides by humic acid and selected clays (Siting, marine disposal of high-level radioactive waste), 1247 (J;US)

Flocculation

Importânec òl ù ganıc cumpuunds In ground water às radıonuclide-mobilizing agents, 1324 (R;US)

Solubility

Importance of organic compounds in ground water as radionuclide-mobilizing agents, 1324 (R;US)

\section{HYDROGEN}

Diffusion

Radiolysis of groundwater from HLW stored in copper canisters, 999 (R;SE)

G Value

Formation of inflammable radiolytic gases in the PUREXprocess, 11 (J;DE;In German)

Radiolysis of groundwater from HLW stored in copper canisters. $1062(\mathrm{~J} ; \mathrm{US})$

HYDROGEN 3

SeE TRITIUM

HYDROGEN HYDROXIDES

See WATER

HYNROGEN PHOSPHATES

See PHOSPHORIC ACID

\section{IDAHU CHEMICAL PRUCESSING PLANT}

Radioactive Waste Facilities

New waste calcining facility at ICPP, 452 (BA;US)

Radloactive Waste Management

Environmental evaluation of alternatives for long-term management of Defense high-level radioactive wastes at the Idaho Chemical Processing Plant, 70 (R;US)

Iligh level waste manayement at the Idaho Chemical l'roecssing Plant, 451 (BA;US)

Strategy document: long-term technology program for defense high-level waste, $156(\mathbf{R} ; \mathrm{US})$

Technology for long-term management of ICPP Defense Waste, $122(\mathrm{~J} ; \mathrm{US})$

Radioactive Waste Processing

Alternative fuels and heating methods for calciner process heat, 204 (R;US)

ICPP waste calcining facility safety analysis report, 219 (R;US:

Immobilization of Idaho Chemical Processing Plant high-level wastes, 240 (RA;DE)

New waste calcining facility at ICPP, 452 (BA;US)

Tanks

Evaluation of stainless steel tank corrosion in ICPP high-level radioactive waste service, 203 (R:US) 


\section{IGNEOUS ROCKS}

Cracks

Drilling-induced borehole-wall damage at spent fuel test-climax (Quartz monzonite; microcracks), 1003 (R;US)

Damage

Drilling-induced borehole-wall damage at spent fuel test-climax (Quartz monzonite; microcracks), 1003 (R;US)

Seismology

Monitoring an underground repository with modern seismological methods, 1238 (J;US)

\section{ILLINOIS}

Geology

Regional geological assessment of the Devonian-Mississippian shale sequence of the Appalachian, Illinois, and Michigan basins relative to potential storage/disposal of radioactive wastes, 828 (R;US)

\section{ILLINOIS BASIN}

Geology

Regional geological assessment of the Devonian-Mississippian shale sequence of the Appalachian, Illinois, and Michigan basins relative to potential storage/disposal of radioactive wastcs, 828 (R;US)

\section{ILLTTE}

Adsorption

Redox behavior, complexing, and adsorption of hexavalent actinides by humic acid and selected clays (Siting, marine disposal of high-level radioactive waste), 1247 (J;US)

Compression Strength

Constitutive properties for undisturbed marine sediments in support of the subseabed disposal program, 963 (RA;US)

Creep

Constitutive properties for undisturbed marine sediments in support of the subseabed disposal program, 963 (RA;US)

Pressure Measurement

Constitutive properties for undisturbed marine sediments in supShear port of the subseabed disposal program, 963 (RA;US)

Constitutive properties for undisturbed marine sediments in support of the subseabed disposal program, 963 (RA;US)

Sorptive Properties

Importance of organic compounds in ground water as radionuclide-mobilizing agents, 1324 (R;US)

\section{INCOLOY 800}

Corrosion

Corrosion studies and mechanical tests on metallic materials for the design of packagings for vitrified high level wastes (HLW), 689 (R;DE;In German)

Tensile Properties

Corrosion studies and mechanical tests on metallic matcrials for the design of packagings for vitrified high level wastes (HLW), 689 (R;DE; In German)

INCOLOY 825

Corrosion

Corrosion studies and mechanical tests on metallic materials for the design of packagings for vitrified high level wastes (HLW), 689 (R;DE;In German)

Corrosion Resistance

Selection of barrier metals for a waste package in tuff, 1020 (R;US)

Mechanical Properties

Selection of barrier metals for a waste package in tuff, 1020 (R;US)

Tensile Properties

Corrosion studies and mechanical tests on metallic materials for the design of packagings for vitrified high level wastes (HLW), 689 (R;DE; In German)

Weldability

Selection of barrier metals for a waste package in tuff, 1020 (R;US)

\section{INCOLOY ALLOYS}

See also INCOLOY 800

INCOLOY 825

Corrosion

High temperature corrosion of heat-resisting alloys by borosilicate melts containing simulated high level nuclear wastes, 476 (J;JP;In Japanese)

Radiation Effects

Study of $\boldsymbol{\gamma}$-ray irradiation effects on corrosion resistance of alloys for storage of high-level waste packages, (1), 667 (R;JP;In Japanese)

Stress Corrosion

Study of $\boldsymbol{\gamma}$-ray irradiation effects on corrosion resistance of alloys for storage of high-level waste packages, (1), 667 (R;JP;In Japanese)

\section{INCOMPRESSIBLE FLOW}

Numerical Solution

Code development in support of nuclear waste storage investigations for a repository in tuff (Pore water motion and energy transport in a porous media which may be saturated or partially saturated with a single fluid in liquid state), 988 (R;US)

INCONEL 600

Chemical Composition

Investigations of suitable metallic container materials for HAW solidification, $371(\mathrm{~J} ; \mathrm{US})$

Compatibility

Studies of waste-canister compatibility, 832 (R;US)

Corrosion

Corrosion experience with a slurry-fed ceramic melter, 321 (R;US)

Radiation Effects

Study of $\gamma$-ray irradiation effects on corrosion resistance of alloys for storage of high-level waste packages, (1), 667 (R;JP;In Japanese)

Stress Corrosion

Study of $\boldsymbol{\gamma}$-ray irradiation effects on corrosion resistance of alloys for storage of high-level waste packages, (1), 667 (R;JP;In Japanese)

INCONEL 617

Corrosion

Corrosion experience with a slurry-fed ceramic melter, 321 (R;US)

INCONEL 625

Compatibility

Studies of waste-canister compatibility, 832 (R;US)

Corrosion

Corrosion experience with a slurry-fed ceramic melter, 321 (R;US)

Corrosion studies and mechanical tests on metallic materials for the design of packagings for vitrified high level wastes (HLW), 689 (R;DE;In German)

Corrosion of materials in a clay environment, 686 (RA;DE)

High temperature corrosion of heat-resisting alloys by borosilicate melts containing simulated high level nuclear wastes, 476 (J;JP;In Japanese)

Corrosion Resistance

Corr sion of materials in a clay environment, $676(R A ; D E)$

Feasibility of using a high-level waste canister as an engineered barrier in disposal, 842 (R;US)

Radiation Effects

Study of $\gamma$-ray irradiation effects on corrosion resistance of alloys for storage of high-level waste packages, (1), 667 (R;JP;In Japanese)

\section{Stress Corrosion}

Study of $\gamma$-ray irradiation effects on corrosion resistance of alloys for storage of high-level waste packages, (1), 667 (R;JP;In Japanese)

Tensile Properties

Corrosion studies and mechanical tests on metallic materials for the design of packagings for vitrified high level wastes (HLW), 689 (R;DE;In German) 


\section{INCONEL 690}

Compatibility

Studies of waste-canister compatibility, 832 (R;US)

\section{Corrosion}

Corrosion experience with a slurry-fed ceramic melter, 321 (R;US)

\section{INDIA}

Fuel Cycle

Summary of non-US national and international fuel cycle and radioactive waste management programs 1982, 83 (R;US)

Radioactive Waste Management

Summary of non-US national and international fuel cycle and radioactive waste management programs 1982,83 (R;US)

\section{INDIANA}

Geology

Regional geological assessment of the Devonian-Mississippian shale sequence of the Appalachian, Illinois, and Michigan basins relative to potential storage/disposal of radioactive wastes, 828 (R;US)

INORGANIC ION EXCHANGERS

See also MONTMORILLONITE TEOLITES

Comparative Evaluations

Evaluation of conditioned high-level waste forms, 326 (R;US)

Uses

Inorganic sorbents in nuclear power plants, 467 (J;CS;In Czech)

INSTRUMENTS (MEASURING)

See MEASURING INSTRUMENTS

INTERMEDIATE-LEVEL RADIOACTIVE WASTES

After-heat

Decay calculations on medium-level and actinide-containing wastes from the LWR fuel cycle. Pt. 1. Basic data evaluation including activity and thermal decay power, 687 (R;DE;In German)

Decay

Decay calculations on medium-level and actinide-containing wastes from the LWR fuel cycle. Pt. 2, 688 (R;DE;In German)

Decay calculations on medium-level and actinide-containing wastes from the LWR fuel cycle. Pt. 1. Basic data evaluation including activity and thermal decay power, 687 (R;DE; In German)

Forecasting

Radioactive waste inventories and projections, 62 (R;US)

Inventories

Radioactive waste inventories and projections, 62 (R;US)

Radioactive Wuste Dlsposa!

Environmental impact of nuclear power. Opening address, 1246 (I;iRA)

IODINE

Activation Analysis

Application of neutron-activation analysis to the determination of leach rates of simulated nuclear-waste forms, 127 (R;US)

Adsorption

Geochemistry studies pertaining to the G-tunnel radionuclide migration field experiment, 1426 (R;US)

Separation Processes

Waste-Mixes Study for space disposal, 815 (R;US)

IODINE 125

Adsorption

Laboratory tests on the migration behavior of selected fission products in aquifer materials from a potential disposal site in northern Germany, 1352 (BA;US)

Diffusion

Laboratory tests on the migration behavior of selected fission products in aquifer materials from a potential disposal site in northern Germany, 1352 (BA;US)

IODINE 129

Environmental Expasare Pathway

lodine-129 in high-activity nuclear wastes: an assessment of the deep-ocean disposal option, $1077(\mathrm{~J} ; \mathrm{CH})$

\author{
IODINE 131 \\ Adsorption \\ Laboratory tests on the migration behavior of selected fission \\ products in aquifer materials from a potential disposal site in \\ northern Germany, 1352 (BA;US) \\ Diffusion \\ Comparison of in-situ radionuclide migration studies in the Stud- \\ svik area and laboratory measurements, 1349 (BA;US) \\ Laboratory tests on the migration behavior of selected fission \\ products in aquifer materials from a potential disposal site in \\ northern Germany, 1352 (B $;$; US) \\ Sorption \\ Comparison of in-situ radionuclide migration studies in the Stud- \\ svik area and laboratory measurements, 1349 (BA;US) \\ IODINE IODIDES \\ See IODINE \\ IODINE ISOTOPES
}

See also IODINE 125

IODINE 129

IODINE 1.3

Environmental Transport

Expoeures raculting from nueloar power production. Allmex $P$. 1444 (BA;US)

ION EXCHANGE MATERIALS

SEe alsn INORFANIT INN FXCHANGERS

Syntheyis

Sodium titanate in solidification of radioactive wastes (Finland), 247 (RA;DE)

\section{ION EXCHANGE MEMBRANES}

See ION EXCHANGE MATERIALS

IRON

Absorption Spectroscopy

Subsolidus sintering of SYNROC: I. Initial demonstrations, 280 (R;US)

Activation Analysis

Subsolidus sintcring of SYNROC: I. Initial demonstrations, 280 (R;US)

Chemical Reactions

Waste glass/metal interactions in brines, 860 (R;US)

Corrosion

Corrosion of construction materials in clay environments, 1150 (BA;US)

Emission Spectroscopy

Analyses of nuclear fuel and high-lcvel waste by inductively cuus pled plasma-emission spectroscnpy, KR (R;I IS)

Leaching

Comparison of properties of borosilicate glass and crystalline ceramic forms for immobilization of Savannah River Plant waste, 171 (R;US)

FUETAP cơncicte. all alternative radioactive waste host, 256 (RA;DE)

Preparation and leaching of radioactive INEL waste forms, 201 (R;US)

Subsolidus sintering of SYNROC: I. Initial demonstrations, 280 (R;US)

Subsolidus sintering of SYNROC: II. Materials selections, process improvements, waste form evaluations, 281 (R;US)

Redox Reactions

Investigation on the oxidation state and the behavior of molybdenum in silicate glass, 460 (BA;US)

\section{IRON ALLOYS}

See also HASTELLOYS

HAYNES 188 ALLOY

HAYNES 25 ALLOY

INCONEL 600

INCONEL 625

IRON BASE ALLOYS

Corrosion Resistance

Waste package materials testing for a salt repository: 1982 sta report, 825 (R;US)

Moliceular Structure

Iron-nickel alloys as canister material for radioactive wastc disposal in underground repositories, 708 (RA;US) 


\section{Thermodynamic Properties}

Iron-nickel alloys as canister material for radioactive waste disposal in underground repositories, 708 (RA;US)

IRON BASE ALLOYS

See also CAST IRON

INCOLOY ALLOYS

STEELS

\section{Corrosion}

Nuclear waste package materials testing report: basaltic and tuffaceous environments, 854 (R;US)

Mechanical Properties

Nuclear waste package materials testing report: basaltic and tuffaceous environments, 854 (R;US)

Physical Radiation Effects

Nuclear waste package materials testing report: basaltic and tuffaceous environments, 854 (R;US)

\section{IRON COMPLEXES}

Solubility

Importance of organic compounds in ground water as radionuclide-mobilizing agents, 1324 (R;US)

\section{IRON HYDROXIDES}

Coprecipitation

Coprecipitation of radioactive technetium with ferrous hydroxide, 1443 (J;JP;In Japanese)

\section{IRON OXIDES}

See also HEMATITE

MAGNETITE ZIRCONOLITE

Leaching

Influence of metal oxides on the leach rate and crystallisation behaviour of waste glasses, 251 (RA;DE)

\section{IRON SULFATES}

Chemical Reactions

Technetium behavior in sulfide and ferrous iron solutions, 553 (R;US)

ISRAEL

Radioactive Waste Disposal

Radioactive waste disposal options for the operation of nuclear

ITALY power stations, 664 (RA;IL)

Fuel Cycle

Summary of non-US national and international fuel cycle and radioactive waste management programs 1982,83 (R;US)

Radioactive Waste Disposal

Laboratory determination of the thermal properties of large-scale samples of natural clays, 550 (R;IT;IT)

Radioactive Waste Management

Summary of non-US national and international fuel cycle and radioactive waste management programs 1982, 83 (R;US)

Radloactive Waste Pruciessiing

Laboratory activity in Italy in the field of vitrification of highlevel wastes, 233 (RA;DE)

Prelimianry experience with vitrification inactive pilot plant in Italy, 234 (RA;DE)

\section{J}

JAPAN

Fuel Cycle

Summary of non-US national and international fuel cycle and radioactive waste management programs 1982, 83 (R;US)

Fuel Reprocessing Plants Report of the Ad Hoc Committee on Reprocessing and Wastes of the Sub-Committee on Nuclear Power of Committee on Overall Energy Study, 485 (J;JP;In Japanese)

High-level Radioactive Wastes

Policy and some problems on the return waste, 118 (J;JP;In Japanese)

Radioactive Waste Uisposal

Report of the Ad Hoc Committee on Reprocessing and Wastes of the Sub-Committee on Nuclear Power of Committee on Overall Energy Study, 485 (J;JP;In Japanese)
Radioactive Waste Management

Status of high level and alpha bearing waste management in PNC, 428 (BA;US)

Summary of non-US national and international fuel cycle and radioactive waste management programs 1982, 83 (R;US)

Radioactive Waste Processing

Development of high-level liquid waste solidification in PNC, 431 (BA;US)

Report of the Ad Hoc Committee on Reprocessing and Wastes of the Sub-Committee on Nuclear Power of Committee on Overall Energy Study, 485 (J;JP;In Japanese)

\section{$\mathbf{K}$}

KANSAS

Salt Deposits

Environmental characterization of bedded salt formations and overlying areas of the Permian Basin, 1320 (R;US)

\section{KAOLINITE}

Sorptive Properties

Importance of organic compounds in ground water as radionuclide-mobilizing agents, 1324 (R;US)

KARLSRUHE NUCLEAR RESEARCH CENTER See KERNFORSCHUNGSZENTRUM KARLSRUHE

KARLSRUHE REPROCESSING PLANT See WAK

KENTUCKY

Geology

Regional geological assessment of the Devonian-Mississippian shale sequence of the Appalachian, Illinois, and Michigan basins relative to potential storage/disposal of radioactive wastes, 828 (R;US)

KERNFORSCHUNGSZENTRUM KARLSRUHE

Radioactive Waste Disposal

Deep underground storage of [radioactive waste]. Annual report, 1981, 643 (R;DE;GE)

Radioactive Waste Management

Results report on research and development work 1980 of the Institut fuer Nukleare Entsorgungstechnik, 276 (R;DE;In German)

Radioactive Waste Processing

Electrodes for the direct heating of highly radioactive borosilicate glasses in HAW-ceramic furnaces, 236 (RA;DE;GE)

Status of HAW-Ceramic-Melting Technology at the Institute of Nuclear Affairs, 235 (RA;DE;GE)

KOREA (SOUTH)

See REPUBLIC OF KOREA

KRYPTON 85

Fnvironmental Transport

Exposures resulting from nuclear power production. Annex F, 1444 (BA;US)

Radioactive Waste Disposal

Ocean-bottom disposal of fission-product krypton-85, 1251 (BA;JP)

\section{LAND USE}

Remote Sensing

Monitoring land and water uses in the Columbia Plateau using remote-sensing computer analysis and integration techniques, 865 (R;US)

LANTHANIDUS

See RARE EARTHS

LANTHANUM

Activation Analysis

Subsolidus sintering of SYNROC: I. Initial demonstrations, 280 (R;US)

Emission Spectroscopy

Analyses of nuclear fuel and high-level waste by inductively coupled plasma-emission spectroscopy, 68 (R;US) 
Leaching

Subsolidus sintering of SYNROC: I. Initial demonstrations, 280 (R;US)

Radiation Scattering Analysis

Utilization of charged particle backscattering to study the near surface region of glasses. Application to depth profiling of lanthanium, cerium, throium and uranium induced by aqueous leaching, 1216 (BA;US)

LANTHANUM CHROMITES

See CHROMIUM OXIDES

LANTHANUM PHOSPHATES

Spectrophotometry

Valence states of actinides in synthetic monazites, 1276 (BA;US)

LEACHATES

Activation Analysis

Application of neutron-activation analysis to the determination of leach rates of simulated nuclear-waste forms, 127 (R;US)

Chemical Analysis

Measurement of leaching from simulated nuclear-waste glass using radiotracers, 128 (R;US)

Electrle Conductivity

Electrical-conductivity measurements of leachates for the rapid assessment of wasteform corrosion resistance, 554 (R;US)

LEACHING

Physical Radiation Effects

Radiation effects in high-level radioactive waste forms (Actinide radiation), 116 (J;CII)

LEAD

Corrosion Resistance

Canister materials proposed for final disposal of high level nuclear waste - a review with respect to corrosion resistance, 675 (RA;DE)

Emission Spectroscopy

Analyses of nuclear fuel and high-level waste by inductively coupled plassma-emission spectroscopy, 68 (R;US)

Solubility

Geochemical controls on radionuclide releases from a nuclear waste repository in basalt: estimated solubilities for selected elements, 1329 (R;US)

LIGHT WATER COOLED REACTORS

See WATER COOLED REACTORS

LIGHT WATER MODERATED REACTORS

SE WATER MODERATED REACTORS

\section{LIMESTONE}

Permeability

Hydrogeologic testing of the E.J. Kubat borehole, San Juan County, Utah: utilization of a high pressure instrumented flow control system, 537 (RA;US)

Sorptive Properties

Status of radionuclide sorption-desorption studies performed by LINERS the WRIT program, 1308 (RA;US)

Thermal Stresses

Evaluation of the thermomechanical behavior about a waste container/slecve in salt, 803 (R;US)

Thermoelasticity

Evaluation of the thermomechanical behavior about a waste conlainer/sleeve in salt, 803 (R;US)

LIOUID EFFLUENTS

SEe LIQUID WASTES

LIQUID METAL TEST FACILITIES

SEE TEST FACILITIES

I.IQIJID WASTES

Radioactive Waste Processing

Melting of glass by direct induction heating in ceramic container, 480 (J;JP;In Japanese)

Plant for solidification and storage of high-level radioactive waste, 521 (J;JP;In Japanese)

Solidification

Plant for solidification and storage of high-level radioactive waste, 521 (J;JP;In Japanese)

Preparation of glass containing high-level radioactive waste (simulated) - copper composite compacts by a continuous pressure sintering method, 479 (J;JP;In Japanese)

\author{
Vitrification \\ Melting of glass by direct induction heating in ceramic containt \\ 480 (J;JP;In Japanese) \\ LIXIVIATION \\ See LEACHING \\ LMFBR TYPE REACTORS \\ See also BOR-60 REACTOR \\ Fuel Cycle \\ Tokai Works annual progress report, April 1980-March 1981, 3 \\ (R;JP) \\ Radioactive Wastes
}

Graphical and tabular summaries of decay characteristics for once-through PWR, LMFBR, and FFTF fuel cycle materials (Spent fuel, high-level waste fuel can scrap), 76 (R;US)

Thorium Cycle

Use of thorium as an alternative nuclear fuel, 46 (R;AU)

LOUISIANA

Hydrology

Technical progress report for the quarter, 1 April-30 June 1982. 779 (R;US)

Radioactive Waste Dispmal

Environmental characterization report for the Gulf Interior Region Louisiana study area (Vacherie and Rayburn salt domes), 1321 (R;US)

Salt Depusits

Dome-salt thermomechanical experiments at Avery Island, Louisiana, 620 (RA;US)

Permeability of selected sediments in the vicinity of five salt domes in the Gulf Interior Region, 810 (R;US)

Quaternary geology of Rayburn's salt dome: North Louisiana Salt Dome Basin, 814 (R;US)

\section{LOW-LEVEL RADIOACTIVE WASTES}

After-beat

Decay calculations on medium-level and actinide-containing wastes from the LWR fuel cycle. Pt. 1. Basic data evaluation including activity and thermal decay power, 687 (R;DE; In German)

Decsy

Decay calculations on medium-level and actinide-containing wastes from the LWR fuel cycle. Pt. 1. Basic data evaluation including activity and thermal decay power, 687 (R;DE; In Germun)

Forecasting

Radioactlve waste inventories and projections, 62 (R;US)

Spent fuel and radioactive-waste inventories, projections, and characteristics, 57 (R;US)

Spent fuel and radioactive waste inventories, projections, and characteristics, 574 (R;US)

Government Policies

Nuclear waste, the nation, and South Carolina: an update on the major issues, 114 (B;US)

Inventories

Integrated data base for spent fuel and radwaste: inventories, 52 (R;US)

Radioactive waste inventories and projections, 62 (R;US)

Spent fuel and radioactive-wastec inventories, projections, and characteristics, 57 (R;US)

Spent fuel and radioactive waste inventories, projections, and characteristics, 574 (R;US)

Radioactive Wastes

Radwaste panel, NRC head highlight 44th American Power Confcrence program, 99 (J;US)

Stack Disposal

Status of radioactive waste management: needs for reassessment, $1248(\mathrm{~J} ; \mathrm{GB})$

Waste Transportation

Nuclear waste/nuclear power: their futures are linked, 560 (R;US)

Review of the status of transportation activities in various waste management systems, 30 (R;US) 


\section{LWBR TYPE REACTORS}

Fuel Assembly Dismantling

Fuel cycle programs. Quarterly progress report, April-June 1982 536 (R;US)

Fuel cycle programs. Quarterly progress report, July-September 1982,538 (R;US)

Fuel Elements

Fuel-cycle programs. Quarterly progress report, April-June 1981, 49 . (R;US)

Fuel Rods

Fuel-cycle programs. Quarterly progress report, January-March 1981,48 (R;US)

Spent Fuel Elements

Fuel cycle programs. Quarterly progress report, July-September 1981,50 (R;US)

Fuel cycle programs. Quarterly progress report, April-June 1982 , 536 (R;US)

Fuel cycle programs. Quarterly progress report, July-September 1982,538 (R;US)

Thorium Cycle

Use of thorium as an alternative nuclear fuel, 46 (R;AU)

LWR TYPE REACTORS

See WATER COOLED REACTORS

\section{M}

MA 754

See NICKEL BASE ALLOYS

MA 956

See IRON BASE ALLOYS

MAGNESIUM

Emission Spectroscopy

Analyses of nuclear fuel and high-level waste by inductively coupled plasma-emission spectroscopy, 68 (R;US)

Leaching

Effects of composition, solutions, and burial on nuclear waste glasses, 845 (RA;US)

Preparation and leaching of radioactive INEL waste forms, 201 (R;US)

\section{MAGNESIUM OXIDES}

Leaching

Influence of metal oxides on the leach rate and crystallisation behaviour of waste glasses, 251 (RA;DE)

\section{MAGNETITE}

Thermodynamics

Uncertainties in the thermodynamics of basalt-oxygen and basaltwater reactions, 547 (R;US)

MAINE

Geology

Consultation and concurrence for NWTS in crystalline rocks. Quarterly progress report, October 1, 1982-December 31, $1982,576(R ; U S)$

Radioactive Waste Disposal

Consultation and concurrence for NWTS in crystalline rocks. Quarterly progress report, October 1, 1982-December 31, 1982, 576 (R;US)

MALIGNANCIES

See NEOPLASMS

MAN

Environmental Exposure Pallıway

PATH1 self-teaching curriculum: example problems for Pathways-to-Man Model, 1317 (R;US)

Summary of computer codes for radiological assessment, 1376 (R;US)

Radiation Doses

Summary of computer codes for radiological assessment, 1376 (R;US)

MANGANESE

Adsorption

Geochemistry studies pertaining to the G-tunnel radionuclide migration field experiment, $1426(\mathrm{R} ; \mathrm{US})$
Emission Spectroscopy

Analyses of nuclear fuel and high-level waste by inductively coupled plasma-emission spectroscopy, 68 (R;US)

MANGANESE 54

Leaching

Leaching study of nuclear melt glass: Part I, 1332 (R;US)

MARINE DISPOSAL

Environmental Impacts

Radioecology of the deep-sea, 1428 (RA;DE;In German)

Feasibility Studies

Submarine geologic disposal of nuclear waste, 1253 (J;US)

Subseabed disposal program annual report, January-September 1981. Volume II. Appendices (principal investigator progress reports). Part 1, 954 (R;US)

International Laws

Dumping nuclear waste into the sea: international control and the role of science and law, $1055(\mathrm{~J} ; \mathrm{GB})$

Meetings

Report of the first interim meeting of the Seabed Working Group Engineering Studies Task Group, 944 (R;US)

Research Programs

Program criteria for subseabed disposal of radioactive waste: site qualification plạ, 934 (R;US)

Subseabed disposal program annual report, January-September 1981. Volume II. Appendices (principal investigator progress reports). Part 1, 954 (R;US)

Risk Assessment

Accident scenarios and probabilities for the SDP transportation system, 956 (RA;US)

Safety

Accident scenarios and probabilities for the SDP transportation system, 956 (RA;US)

Subseabed disposal safety analysis, 955 (RA;US)

Site Selection

Analysis of geological and geophysical data from study locations $C_{1}, B_{1}$, and $E_{2}$ in the northwest PAC 1 study region, 957 (RA;US)

Can the oceans provide acceptable disposal sites for high-level radioactive waste, 1287 (BA;GB)

Geotechnical studies for subseabed disposal: high level radioactive wastes, 964 (RA;US)

Pacific site assessment, 960 (RA;US)

Paleomagnetic study of areas B1, Cl and E2, 959 (RA;US)

Program criteria for subseabed disposal of radioactive waste: site qualification plan, 934 (R;US)

Site qualification activities, 958 (RA;US)

Waste-rock Interactions

Near-field interactions, 929 (R;US)

MARINE ECOSYSTEMS

Sée AQUATIC ECOSY'STEMS

MASURIUM

See TECHNETIUM

MATERIALS (COMPOSITE)

See COMPOSITE MATERIALS

MATERIALS HANDLING EOUIPMENT

See also HOISTS

Equipment for the emplacement of heat-producing waste in long horizontal boreholes (Horizontal vs vertical emplacement), 555 (R;US)

MATERIALS (POROUS)

SPe POROIIS MATERIALS

MATERIALS TESTING

Standardization

Nuclear waste management technical support in the development of nuclear waste form criteria for the NRC. Task 4 . Test development review, 284 (R;US)

MATRIX MATERIALS

Comparative Evaluations

Development and testing of matrices for the encapsulation of glass and ceramic nuclear waste furms (Ceramics, glass, graphite, or mctal), 297 (R;US)

Evaluation of conditioned high-level waste forms, 326 (R;US) 


\section{MEASURING INSTRUMENTS \\ See also STRAIN GAGES THERMOCOUPLES \\ Reliability \\ Results of instrument reliability study for high-level nuclear waste repositories, 93 (J;US) \\ MERCURY \\ Activation Analysis \\ Application of neutron-activation analysis to the determination of leach rates of simulated nuclear-waste forms, 127 (R;US) \\ Reduction \\ Separation of mercury from HEWC solutions. Laboratory assay, 207 (R;BE;In French) \\ Separation Processes \\ Separation of mercury from HEWC solutions. Laboratory assay, \\ METALS 207 (R;BE;In French) \\ See also ACTINIDES \\ ALUMINIUM \\ ANTIMONY \\ CADMIUM \\ $L E A D$ \\ IAERCURY \\ RARE EARTHS \\ TIN \\ ZINC}

Comparative Evaluations

Development and testing of matrices for the encapsulation of glass and ceramic nuclear waste forms, 297 (R;US)

\section{METAMORPHIC ROCKS}

Fractures

Fracture analysis of crystalline rocks: field measurements and field geomechanical techniques, 638 (R;GB)

Rock Mechanics

Fracture analysis of crystalline rocks: field measurements and

MEXICO field geomechanical techniques, 638 (R;GB)

Fuel Cycle

Summary of non-US national and international fuel cycle and radioactive waste management programs 1982, 83 (R;US)

Radionctive Waste Management

Summary of non-US national and international fuel cycle and MICA radioactive waste management programs 1982,83 (R;US)

See also BIOTITE

Leaching

Alpha-recoil damage and solution effects in minerals: uranium isotopic disequilibrium and radon release, 1073 (J;GB)

\section{MICHIGAN}

Copper Ores

Native copper deposits of the Portage Lake volcanics, Michigan: their implications with respect to canister stability for nuclear waste isolation in the Columbia River basalts beneath the Han-

Geology ford Site, Washington, 904 (R;US)

Regional geological assessment of the Devonian-Mississippian ohale défuience of the Appalaehian, Illinois, and Michigail basins relative to potential storage/disposal of radioactive wastes, 828 (R;US)

\section{MICROSPHERES}

Chemical Preparation

Preparation of gel spheres containing simulated high-level radioactive waste, 381 ( $\mathrm{J} ; \mathrm{CH})$

MIGRATION (RADIONUCLIDE)

SEe RADIONUCLIDE MIGRATION

MILL TAILINGS

Forecasting

Radioactive waste inventories and projections, 62 (R;US)

Spent fuel and radioactive-waste inventories, projections, and characteristics, 57 (R;US)

Spent fuel and radioactive waste inventories, projections, and characteristics, 574 (R;US)
Inventories

Integrated data base for spent fuel and radwaste: inventories, (R;US)

Radioactive waste iriventuries and projections, 62 (R;US)

Spent fuel and radioactive-waste inventories, projections, and characteristics, 57 (R;US)

Spent fuel and radioactive waste inventories, projections, and characteristics, 574 (R;US)

Stabilization

Nuclear waste management. Semiannual progress report, October 1982-March 1983, 303 (R;US)

MILLING

Radiation Hazards

Exposures resulting from nuclear power production. Annex $F$, 1444 (BA;US)

MINE-MOUTH GENERATING PLANTS

See FOSSIL-FUEL POWER PLANTS

MINERAL RESOURCES

Resource Assessment

Overview of energy and mineral resources for the Nevada nuclear-waste-storage investigations, Nevada Test Site, Nye County, Nevada, 763 (R;US)

MINERALS

See also CARBONATE MINERALS

FELDSPARS

PYROCHLORE

PYROXENES

SILICATE MINERALS

ZEOLITES

Sorptive Properties

Actinide sorption on rock minerals, 275 (RA;DE)

MINING

Radiation Hazards

Exposures resulting from nuclear power production. Annex F, 1444 (BA;US)

Test Facilities

Mining technology development in crystalline rock, 613 (RA;US)

MISSISSIPPI

Hydrolos

Technical progress report for the quarter, 1 April-30 June 1982. 779 (R;US)

Salt Deposits

Permeability of selected sediments in the vicinity of five salt domes in the Gulf Interior Region. 810 (R:US)

MUDELS (BIOLOGICAL)

See' BIOLOGICAL MODELS

MOISTURE

Corrosive Effects

Corrosion of construction materials in clay environments, 1150 (BA;US)

MOLDAVTTES

Seé TEKTITES

MOLTEN SALT REACTORS

Thorium Cycle

Use of thorium as an alternative nuclear fuel, 46 (R;AU)

MULYBUENUM

Ahsorption Spectroscopy

Subsolidus sintering of SYNROC: I. Initial demonstrations, 280 (R;US)

Activation Analysis

Application of ncutron-activation unalysis to the determination of leach rates of simulated nuclear-waste forms, 127 (R;US)

Subsolidus sintering of SYNROC: I. Initial demonstrations, 280 (R;US)

Adsorption

Waste form/rock interaction leaching study using PNL 76-68 glass beads and Umtanum basalt. Part I, 1004 (R;US)

Centrifugation

Influence of the reprocessing flow sheet on the HLW solidifict tion technology, 239 (RA;DE)

Emission Spectroscopy

Analyses of nuclear fuel and high-level waste by inductively coupled plasma-emission spectroscopy, 68 (R;US) 
Leaching

Geochemical behavior of supercalcine waste form: its stability in a basalt environment, 911 (R;US)

Processing effects on the behavior of titanate waste forms in aqueous solutions, 1145 (BA;US)

Subsolidus sintering of SYNROC: I. Initial demonstrations, 280 (R;US)

Waste glass/metal interactions in brines, 860 (R;US)

Redox Reactions

Investigation on the oxidation state and the behavior of molybdenum in silicate glass, 460 (BA;US)

Valence

Investigation on the oxidation state and the behavior of molybdenum in silicate glass, 460 (BA;US)

MOLYBDENUM ALLOYS

See also HASTELLOYS

INCOLOY 825

INCONEL 617

INCONEL 625

STAINLESS STEEL-316

\section{Corrosion}

Corrosion studies and mechanical tests on metallic materials for the design of packagings for vitrified high level wastes (HLW), 689 (R;DE;In German)

General corrosion, irradiation-corrosion, and environmentalmechanical evaluation of nuclear-waste-package structuralbarrier materials. Progress report, 843 (R;US)

Physical Radiation Effects

General corrosion, irradiation-corrosion, and environmentalmechanical evaluation of nuclear-waste-package structuralbarrier materials. Progress report, 843 (R;US)

Tensile Properties

Corrosion studies and mechanical tests on metallic materials for the design of packagings for vitrified high level wastes (HLW), 689 (R;DE;In German)

\section{MOLYBDENUM OXIDES}

Leaching

Influence of metal oxides on the leach rate and crystallisation behaviour of waste glasses, 251 (RA;DE)

MONAZITES

Repository, 773 (RA;US)

Corrosion Resistance

Electrical-conductivity measurements of leachates for the rapid assessment of wasteform corrusion resistance, 554 (R;US)

Isotope Ratio

Isotopic fractionation of thorium and uranium upon leaching of monozite: alpha-recoil damage effects, 1214 (BA;US)

Leachates

Electrical-conductivity measurements of leachates for the rapld assessment of wasteform corrosion resistance, 554 (R;US)

\section{Leaching}

Isotopic fractionation of thorium and uranium upon leaching of monozite: alpha-recoil damage effects, 1214 (BA;US)

Radiation Effects

Isotopic fractionation of thorium and uranium upon leaching of monozite: alpha-recoil damage effects, 1214 (BA;US)

MONE.I.

Compatibility

Studies of waste-canister compatibility, 832 (R;US)

MONITORING (RADIATION)

See RADIATION MONITORING

\section{MONTMORILLONITE}

Radionuclide Migration

Actinide sorption on rock minerals, 677 (RA;DE)

Sorptive Properties

Importance of organic compounds in ground water as radionuclide-mobilizing agents, 1324 (R;US)

Sorptive diffusion in clay gels, 709 (RA;US)

Waste-rock Interactions

Backfill-waste interactions in repository simulating tests, 1153 (BA;US)

\section{MORTARS}

Permeability

Recent advances in repository seal materials, 1172 (BA;US)

N

\section{NEODYMIUM}

Absorption Spectroscopy

Subsolidus sintering of SYNROC: I. Initial demonstrations, 280 (R;US)

Actiration Analysis

Subsolidus sintering of SYNROC: I. Initial demonstrations, 280 (R;US)

Geochemistry

Oklo reactors: natural analogs to nuclear waste repositories, 611 (RA;US)

Leaching

Leaching studies on SYNROC at $95^{\circ} \mathrm{C}$ and $200^{\circ} \mathrm{C}, 1114(\mathrm{~J} ; \mathrm{CH})$

Subsolidus sintering of SYNROC: I. Initial demonstrations, 280 (R;US)

SYNROC: leaching performance and process technology, 250 (RA;DE)

\section{NEODYMIUM COMPOUNDS}

See also NEODYMIUM OXIDES

Hydrolysis

Thermodynamic properties of chemical species in nuclear waste, 812 (R;US)

Solubility

Thermodynamic properties of chemical species in nuclear waste, 812 (R;US)

\section{NEODYMIUM OXIDES}

Leaching

Influence of metal oxides on the leach rate and crystallisation behaviour of waste glasses, 251 (RA;DE)

NEOPLASMS

Risk Assessment

Effects of ICRP Publication 30 and the 1980 BEIR Report in hazard assessments of high-level waste, 1441 (J;GB)

NEPTUNIUM

Activation Analysis

Application of neutron-activation analysis to the determination of leach rates of simulated nuclear-waste forms, 127 (R;US)

Adsorption

Effects of Hanford high-level waste components on sorption of cobalt, strontium, neptunium, plutonium, and americium on Hanford sediments, 918 (R;US)

Evaluation of DOE radionuclide solubility data and selected retardation parameters: description of calculational and confirmatory experimental activities, 1304 (R;US)

Geochemistry studies pertaining to the G-tunnel radionuclide migration fièld experiment, 1426 (R;US)

Sorption of actinides in well-defined oxidation states on geologic media, 1351 (BA;US)

Transport of actinides through a bentonite backfill, 1175 (BA;US)

Waste form/rock interaction leaching study using PNL 76-68 glass beads and Umtanum basalt. Part I, 1004 (R;US)

Adsorption Isotherms

Basalt-radionuclide reactions: FY-1981. Annual report, 868 (R;US)

Chemical State

Evaluation of solubility and speciation of actinides in natural groundwaters, 1347 (BA;US)

Studies on speciation of americium, technetium and neptunium in simulated vitrified-waste leachates, $1335(\mathrm{~J} ; \mathrm{US})$

Chemisorption

Redox behavior, complexing, and adsorption of hexavalent actinides by humic acid and selected clays (Siting, marine disposal of high-level radioactive waste), 1247 (J;US)

Diffusion

Studies on speciation of americium, technetium and neptunium in simulated vitrified-waste leachates, 1335 (J;US)

Transport of actinides through a bentonite backfill, 1175 (BA;US) 


\section{Distribution}

Studies on speciation of americium, technetium and neptunium in simulated vitrified-waste leachates, 1335 (J;US)

Distribution Functions

Sorption of actinides in well-defined oxidation states on geologic media, 1351 (BA;US)

Environmental Transport

Actinide sorption on rock minerals, 677 (RA;DE)

Compliance assessments of hypothetical geological nuclear waste isolation systems with the draft EPA standard, 982 (R;US)

Leaching

Evaluation of solubility and speciation of actinides in natural ground waters, 1347 (BA;US)

Leaching of actinides from simulated nuclear waste glass, 1196 (BA;US)

Leaching study of PNL 76-68 glass beads using the LLNL continuous-flow method and the PNL-modified IAEA method. Final report, 344 (R;US)

Neptunium concentrations in solutions contacting actinide-doped glass, 1336 (J;US)

Oxidation

Redox behavior, complexing, and adsorption of hexavalent actinides by numic acid and selected clays (Siting, marine disposal of high-level radioactive waste), 1247 (J;US)

Redox Potentinl

Thermodynamic properties of chemical species in nuclear waste, 812 (R;US)

Solubility

Evaluation of solubility and speciation of actinides in natural groundwaters, 1347 (BA;US)

Evaluation of DOE radionuclide solubility data and selected retardation parameters: description of calculational and confirmatory experimental activities, 1304 (R;US)

Geochemical controls on radionuclide releases from a nuclear waste repository in basalt: estimated solubilities for selected elements, 1329 (R;US)

Neptunium concentrations in solutions contacting actinide-doped glass, $1336(\mathrm{~J} ; \mathrm{US})$

Solvent Lxtraction

Chemistry of the nuclear fuel cycle, 4 (BA;GB)

Octyl(phenyl)-N,N-diisobutylcarbamoylmethylphosphine oxide as an extractant for actinides from nitric acid waste, 146 (R;US)

Sorption

Evaluation of sodium bentonite and crushed basalt as waste package backfill materials, 902 (R;US)

Radionuclide sorption on basalt-interbed materials. Annual report FY 1981, 1328 (R;US)

Valence

Valence states of actinides in synthetic monazites, 1276 (BA;US)

XPS valence state determination of $\mathrm{Np}$ and $\mathrm{Pu}$ in multicomponent borosilicate glass and application to leached $76-68$ waste glass surfaces, 417 (BA;US)

NEPTUNIUM 237

Alphs Spectra

Alpha spectrum profiling of plutonium in leached simulated high-level radioactive waste-glass, 517 (J;US)

\section{Diffusion}

Study of radionuclide migration from deep-lying repository sites with over-lying sedimentary layers, 1348 (B $\Lambda$;US)

Leaching

Alpha spectrum profiling of plutonium in leached simulated high-level radioactive waste-glass, 1364 (J;US)

Leach rates of high level waste and spent fuel: limiting rates as determined by backfill and bedrock conditions, 1345 (BA;US)

Underground migration of long-lived radionuclides leached from a borosilicate glass matrix, 1360 (BA;XA)

Waste package performance evaluation, 806 (R;US)

\section{Solubility}

Evaluation of radionuclide transport: effect of radionuclide sorption and solubility, 1354 (BA;US)

Leach rates of high level waste and spent fuel: limiting rates as determined by backfill and bedrock conditions, 1345 (BA;US)

\section{Sorption}

Evaluation of radionuclide transport: effect of radionuclide sorp tion and solubility, 1354 (BA;US)

\section{NEPTUNIUM COMPLEXES}

Solubility

Importance of organic compounds in ground water as radionuclide-mubilizing agents, 1324 (R;US)

\section{NETHERLANDS}

Fuel Cycle

Summary of non-US national and international fuel cyclc and radioactive waste management programs 1982, 83 (R;US)

Radioactive Waste Management

Summary of non-US national and international fuel cycle and radioactive waste management programs 1982, 83 (R;US)

\section{NEVADA TEST SITE}

Baseline Ecology

1982 biotic survey of Yucca Mountain, Nevada Test Site, Nye County, Nevada, 1311 (R;US)

Annotated bibliography for biolügic oveiview fur the Nevada Nuclear Waste Storage Investigations, Nevada Test Site, Nye County, Nevada, 1309 (R;US)

Biologic overview for the Nevada Nuclear Waste Storage Investigations, Nevada Test Site, Nye County, Nevada, 1310 (R;US)

\section{Cullural Resuurces}

Aniutated bibliugrapliy of culiural resources literature for the Nevada Nuclear Waste Storage Investigations, 768 (R;US)

Cultural resources overview for the Nevada Nuclear Waste Storage Investigations, Nevada Test Site, Nye County, Nevada, 767 (R;US)

Electrical Surveys

Electrical studies at the proposed Wahmonie and Calico Hills nuclear waste sitcs, Ncvada Test Site, Nye Co., Nevada, 1023 (R;US)

Geochemistry

Nevada Nuclear Waste Sturage Investigations: FY 1982 project plan, 762 (R;US)

\section{Geology}

Issues in repository sting, 798 (RA;US)

Nevada Nuclear-Waste-Storage Investigations. Quarterly report, April-June 1982, 757 (R;US)

Nevada Nuclear Waste Storage Investigations: FY 1982 project plan, '76? (R:US)

Geophysical Surveys

Geohydrologic and drill-hole data for test well USW $\mathrm{H}-1$, adjacent to Nevada Test Site, Nye County, Nevada (Yucca mountain), 1030 (R;US)

Gravity Surveys

Preliminary results of gravity investigations at Yucca Mountain and vicinity, Southern Nye County, Nevada, 1024 (R:US)

Preliminary appraisal of gravity and magnetic data at Syncline Ridge, Western Yucca Flat, Nevada Test Site, Nye County, Nevada, 1 U25 (K;US)

Principal facts of gravity stations with gravity and magnetic profiles from the southwest Nevada Test Site. Nye County, Nevada, as of January 1982, 1026 (R;US)

Frotiutd Mutiun

Study of surface and subsurface ground motions at Calico Hills, Nevada Test Sitc (Calico Hills), 1029 (R;US)

High-level Radioactive Wastes

Consequences of basaltic volcanism through a high-level waste repository, 1399 (J;US)

System study and performance constraints for repository horizon selection at the Nevada Test Site, 1225 (J;US)

Hydrology

Geohydrologic and drill-hole data for test well USW H-1, adjacent to Nevada Test Site, Nye County, Nevada (Yucca mountain), 1030 (R;US)

Nevada Nuclear-Waste-Storage Investigations. Quarterly report, April-June 1982, 757 (R;US)

Nevada Nuclear Waste Storage Investigations: FY 1982 project plan, $762(R ; U S)$ 
Lithology

Geohydrologic and drill-hole data for test well USW $\mathrm{H}-1$, adjacent to Nevada Test Site, Nye County, Nevada (Yucca mountain), 1030 (R;US)

Magnetic Surveys

Preliminary appraisal of gravity and magnetic data at Syncline Ridge, Western Yucca Flat, Nevada Test Site, Nye County, Nevada, 1025 (R;US)

Principal facts of gravity stations with gravity and magnetic profiles from the southwest Nevada Test Site, Nye County, Nevada, as of January 1982, 1026 (R;US)

Mineral Resources

Overview of energy and mineral resources for the Nevada nuclear-waste-storage investigations, Nevada Test Site, Nye County, Nevada, 763 (R;US)

Radioactive Waste Disposal

Appraisal of nuclcar waste isolation in the vadose zone in arid and semiarid reginns (with emphasis on the Nevada Test Site), 714 (R;US)

Aspects of possible magmatic disruption of a high-level radioactive waste repusitury in southern Nevada, 699 (R;US)

Bibliography of the published reports, papers, and articles on the Nevada Nuclear Waste Storage Investigations, 754 (R;US)

Bibliography of the published reports, papers, and articles on the Nevada nuclear waste storage investigations, 755 (R;US)

Calculation of the probability of volcanic disruption of a highlevel radioactive waste repository within southern Nevada, USA, 1393 (J;GB)

Commercial nuclear waste research and development program. Quarterly report, April-June 1983, 582 (R;US)

Consequences of basaltic volcanism through a high-level waste repository, 1399 (J;US)

Nevada nuclear waste-storage investigations. Quarterly report, January-March 1982 (Studies of Nevada Test Site as repository for high-level radwaste), 756 (R;US)

Nevada Nuclear Waste Storage Investigations. Quarterly report, October-December 1982, 759 (R;US)

Nevada Nuclear Waste Storage Investigations: FY 1981 project plan and FY 1982 forecast, 761 (R;US)

Nevada Nuclear Waste Storage Investigations. Quarterly report, January-March, 1983, 760 (R;US)

Performance assessment in the Nevada Nuclear Waste Storage Investigations, $1224(\mathrm{~J} ; \mathrm{US})$

Preliminary results of gravity investigations at Yucca Mountain and vicinity, Southern Nye County, Nevada, 1024 (R;US)

Research and development related to the Nevada Nuclear Waste Storage Investigations. Progress report, January 1-March 31, 1983,705 (R;US)

Results of the area-to-location phase of repository siting in Nevada, 1035 (J;US)

Summary report on the geochemistry of Yucca Mountain and environs, 700 (R;US)

System study and performance constraints for repository horizon selection at the Nevada Test Site, 1225 (J;US)

Radioactive Waste Facilities

Nevada nuclear waste storage investigations site-characterization activities: preliminary atmospheric assessment of a nuclear waste repository, 765 (R;US)

Nevada Nuclear Waste Storage Investigations: FY 1982 project plan, 762 (R;US)

Radioactive Waste Storage

Far-field thermal analysis of a high level waste repository in tuff Topical report RSI-0137, 942 (R;US)

Issues related to field testing in tuff, 947 (R;US)

Monitoring an underground repository with modern seismological methods, 1233 (J;US)

Nevada Nuclear Waste Storage Investigations: exploratory shaft. Phase I. Conceptual design report, 694 (R;US)

Nuclear-waste-package program for high-level isolation in Nevada tuff, 1015 (R;US)

Parametric studies of radiological consequences of basaltic volcanism, 1386 (R;US)

Preliminary scenarios for consequence assessments of radioactivewaste repositories at the Nevada Test Site, 948 (R:US)
Preliminary appraisal of gravity and magnetic data at Syncline Ridge, Western Yucca Flat, Nevada Test Site, Nye County, Nevada, 1025 (R;US)

Resistivity Surveys

Interpreted resistivity and IP section line W1: Wahmonie Area, Nevada Test Site, Nevada, 567 (R;US)

Resistivity sounding investigation by the Schlumberger method in the Yucca Mountain and Jackass Flats area, Nevada Test Site, Nevada, 1028 (R;US)

Resource Assessment

Overview of energy and mineral resources for the Nevada nuclear-waste-storage investigations, Nevada Test Site, Nye County, Nevada, 763 (R;US)

Seismicity

Status of volcanic hazard studies for the Nevada Nuclear Waste Storage Investigations, 698 (R;US)

\section{Seismology}

Three-dimensional modeling of the Nevada Test Site and vicinity from teleseismic p-wave residuals, 1022 (R;US)

\section{Spent Fuel Storage}

Instrumentation report 1: specification, design, calibration, and installation of instrumentation for an experimental, high-level, nuclear waste storage facility, 37 (R;US)

Nevada nuclear waste storage investigations: briefing book, 572 (R;US)

Tectonics

Issues in repository siting, 798 (RA;US)

Telluric Surveys

E-field ratio telluric traverses near Fortymile Wash, Nevada Test Site, Nevada, 1027 (R;US)

Volcanism

Consequences of basaltic volcanism through a high-level waste repository, 1399 (J;US)

Parametric studies of radiological consequences of basaltic volcanism, 1386 (R;US)

Status of volcanic hazard studies for the Nevada Nuclear Waste Storage Investigations, 698 (R;US)

NEW MEXICO

Geology

Basic data report for drillhole WIPP 11 (Waste Isolation Pilot Plant - WIPP), 928 (R;US)

Basic data report for drillhole ERDA 6 (Waste Isolation Pilot Plant - WIPP), 923 (R;US)

Basic data report for drillhole ERDA 9 (Waste Isolation Pilot Plant WIPP), 926 (R;US)

Higb-level Radioactive Wastes

Field-test programs of borehole plugs in southeastern New Mexico, 615 (RA;US)

Hydrology

Basic data report for Drillhole ERDA 10 (Waste Isolation Pilot Plant - WIPP), 927 (R;US)

\section{Lithology}

Basic data report for deepening of drillhole WIPP 13 (Waste Isolation Pilot Plant-WIPP), 975 (R;US)

Basic data report for drillhole WIPP 12 (Waste Isolation Pilot Plant-WIPP), 984 (R;US)

Basic data report for drillhole ERDA 6 (Waste Isolation Pilot Plant - WIPP), 923 (R;US)

Basic data report for drillhole ERDA 9 (Waste Isolation Pilot Plant WIPP), 926 (R;US)

Radioactive Waste Disposal

Basic data report for drillhole WIPP 11 (Waste Isolation Pilot Plant - WIPP), 928 (R;US)

Basic data report for deepening of drillhole WIPP 13 (Waste Isolation Pilot Plant-WIPP), 975 (R;US)

Basic data report for drillhole WIPP 12 (Waste Isolation Pilot Plant-WIPP), 984 (R;US)

Field-test programs of borehole plugs in southeastern New Mexico, 615 (RA;US)

Operational features of the Waste Isolation Pilot Plant (WIPP), 1161 (BA;US) 


\section{Radioactive Waste Facilities}

Basic data report for deepening of drillhole WIPP 13 (Waste Isolation Pilot Plant-WIPP), 97.5 (R;IUS)

Summary of research and development for disposal of US Defense wastes in the Waste Isolation Pilot Plant (WIPP), 983 (R;US)

Salt Deposits

Basic data report for drillhole ERDA 6 (Waste Isolation Pilot Plant - WIPP), 923 (R;US)

Environmental characterization of bedded salt formations and overlying areas of the Permian Basin, 1320 (R;US)

Seismic Surveys

Basic data report for drillhole WIPP 11 (Waste Isolation Pilot Plant - WIPP), 928 (R;US)

Stratigraphy

Basic data report for Drillhole WIPP 14 (Waste Isolation Pilot Plant - WIPP), 974 (R;US)

Basic data report for deepening of drillhole WIPP 13 (Waste Isolation Pilot Plant-WIPP), 975 (R;US)

Basic data report for drillhole WIPP 12 (Waste Isolation Pilot Plant-WIPP), 984 (R;US)

Uranium Minerals

Uranium-lead radiometric age determinations of naturally occurring U(VI) minerals: application to radwaste storage, 1155 (BÁ;US)

\section{NEW YORK}

Geology

Regional geological assessment of the Devonian-Mississippian shale sequence of the Appalachian, Illinois, and Michigan basins relative to potential storage/disposal of radioactive wastes, 828 (R;US)

NICKEL

Emission Spectroscopy

Analyses of nuclear fuel and high-level waste by inductively coupled plasma-emission spectroscopy, 68 (R;US)

Solubility

Geochemical controls on radionuclide releases from a nuclear waste repository in basalt: estimated solubilities for selected elements, 1329 (R;US)

NICKEL AIJOYS

See also CHROMIUM-NICKEL STEELS

HASTELLOYS

HAYNES 25 ALLOY

INCOLOY ALLOYS NICKEL BASE ALLOYS

Compatibility

Studies of waste-canister compatibility, 832 (R;US)

Corrusion

Corrosion of materials in a clay environment, 686 (KA;DE)

General corrosion, irradiation-corrosion, and environmentalmechanical evaluation of nuclear-waste-package structuralbarrier materials. Progress report, 843 (R;US)

Testing of candidate waste-package backfill and canister materials for basalt (Cupronickel 90/10), 885 (R;US)

Corrosion Resistance

Corr sion of materials in a clay environment, $676(R A ; D E)$

Waste package materials testing for a salt repository: 1982 statu report, 825 (R;US)

Molecular Structure

Iron-nickel alloys as canister material for radioactive waste disposal in underground repositories, 708 (RA;US)

Physical Radiation Effects

General corrosion, irradiation-corrosion, and environmentalmechanical evaluation of nuclear-waste-package structuralbarricr matcrials. Progrcss report, 843 (R;US)

Thermodynamic Properties

Iron-nickel alloys as canister material for radioactive waste disposal in underground repositories, 708 (RA;US)

NICKEL BASE ALLOYS

See also HASTELLOY C

HAYNES 188 ALLOY

MONEL

\section{Corrosion}

Corrosion studies and mechanical tests on metallic materials for the design of packagings for vitrified high level wastes (HI.W), 689 (R;DE;In German)

Corrosion Resistance

Canister materials proposed for final disposal of high level nuclear waste - a review with respect to corrosion resistance, 675 (RA;DE)

Tensile Properties

Corrosion studies and mechanical tests on metallic materials for the design of packagings for vitrified high level wastes (HLW), 689 (R;DE; In German)

\section{NICKEL SULFIDES}

Fabrication

Highly dense graphite matrix: a new material for the conditioning of radioactive wastes, 358 (J;US)

\section{NICKEL-CHROMIUM STEELS}

Carrosinn

Corrosion studies and mechanical tests on metallic materials for the design of packagings for vitrified high level wastes (HLW), 689 (R;DE; In German)

Tensile Properties

Corrosion studies and mechanical tests on metallic materials for the design of packanings for vitrified high level wastes (HLW), 689 (R;DE;In German)

\section{NIOBIUM 95}

Adsorption

Laboratory tests on the migration behavior of selected fission products in aquifer materials from a potential disposal site in northern Germany, 1352 (BA;US)

Diffusion

Laboratory tests on the migration behavior of selected fission products in aquifer materials from a potential disposal site in northern Germany, 1352 (BA;US)

\section{NIOBIUM OXIDES}

Crystal Structure

Investigation of titanium in metamict Nb-Ta-Ti oxides using the extended $x$-ray absorption fine structure technique, 1215 (BA;US)

Radiation Effects

Investigation of titanium in metanich Nb-Ta-Ti uxides using the extended $x$-ray absorption fine structure technique, 1215 (BA;US)

NONRADIOACTIVE WASTE DISPOSAL See WASTE DISPOSAL

NONRADIOACTIVE WASTES

SeP GASEOUS WASTES

LQUID WASTES

SOLID WASTES

NORTHERN IRELAND

SEE UNITED KINCDOM

NORWAY

Radioactive Waste Processing

Equipment, operation and some results from a hot test of the CTH actinide separation process, 243 (RA;DE)

\section{NUCLEAR CONTROVERSY}

SER HUMAN POPULATIONS

NUCLEAR ENERGY

Cost Benefit Analysis

Nuclear energy in Texas: major issues and policy recommendations, 90 (R;US)

Environmental Impacts

Nuclear energy in Texas: major issues and policy recommendations, 90 (R;US)

Research Programs

Australia's role in nuclear energy research and training, 1438 (J;AU)

NUCLEAR FACILTTES

See also FUEL REPROCESSING PLANTS

NUCLEAR POWER PLANTS

RADIOACTIVE WASTE FACILITIES 
Tassification

Management of the DOE inventory of excess radioactively contaminated facilities, 1288 (BA;US)

Decommissioning

Spent fuel and radioactive-waste inventories, projections, and characteristics, 57 (R;US)

Off-gas Systems

Control of semivolatile radionuclides in gaseous effluents at nuclear facilities, 453 (B;XA)

\section{NUCLEAR FUELS}

See also SPENT FUELS

Chemistry

Chemistry in the development of nuclear power, 5 (BA;GB)

Production

Tokai Works annual progress report; April 1980-March 1981, 3 (R;JP)

Transpoirt

Transport of highly radioactive materials, 45 (J;US)

NUCLEAR INDUSTRY

Cost Benefit Analysis

Nuclear energy in Texas: major issues and policy recommendations, 90 (R;US)

Environmental Impacts

Nuclear energy in Texas: major issues and policy recommendations, 90 (R;US)

Radioactive Waste Management

Role of utility nuclear waste management group, 75 (RA;US)

NUCLEAR INSURANCE

Indemnification for nuclear damages - recent developments in the United States, 1431 (R;ES)

\section{NUCLEAR MATERIALS MANAGEMENT}

Inventories

NUMATH: a nuclear material holdup estimator for unit operations and chemical processes, $1408(\mathrm{~J} ; \mathrm{US})$

Safeguards

Development of safeguards requirements for high-level waste repositories, $1421(\mathrm{~J} ; \mathrm{US})$

NUCLEAR POWER

Japan

Report of the Sub-Committee on Nuclear Power of the Committee on Overall Energy Study, (1), 1448 (J;JP;In Japanese)

NUCLEAR POWER PLANTS

Comparative Evaluations

High level radioactive waste, 1242 (J;US)

High-level Radioactive Wastes

Radwaste panel, NRC head highlight 44th American Power Conference program, 99 (J;US)

Low-level Radioactive Wastes

Radwaste panel, NRC head highlight 44th American Power Conference program, 99 (J;US)

Nuclear Insurance

Indemnification for nuclear damages - recent developments in the United States, 1431 (R;ES)

Radioactive Waste Disposal

Disposal of high-level waste from nuclear power plants in Denmark. Salt dome investigations. V.1. Summary, 647 (R;DK)

Radioactive waste disposal options for the operation of nuclear power stations, 664 (RA;IL)

Safe disposal of radioactive wastes from nuclear power plants, 656 (RA;CS; In Czech)

Radioactive Waste Processing

Evaluation of the safety aspects of the design and operation of temporary/mobile radioactive-waste solidification systems, 287 (R;US)

Research programme on the conditioning of nuclear power waste. Prngress repnrt. June 1981, 332 (R;SE)

IUCLEAR POWER STATIONS

See NUCLEAR POWER PLANTS

NUCLEAR SAFETY

SEe RADIATION PROTECTION
NUCLEAR WASTES

SRe RADIOACTIVE WASTES

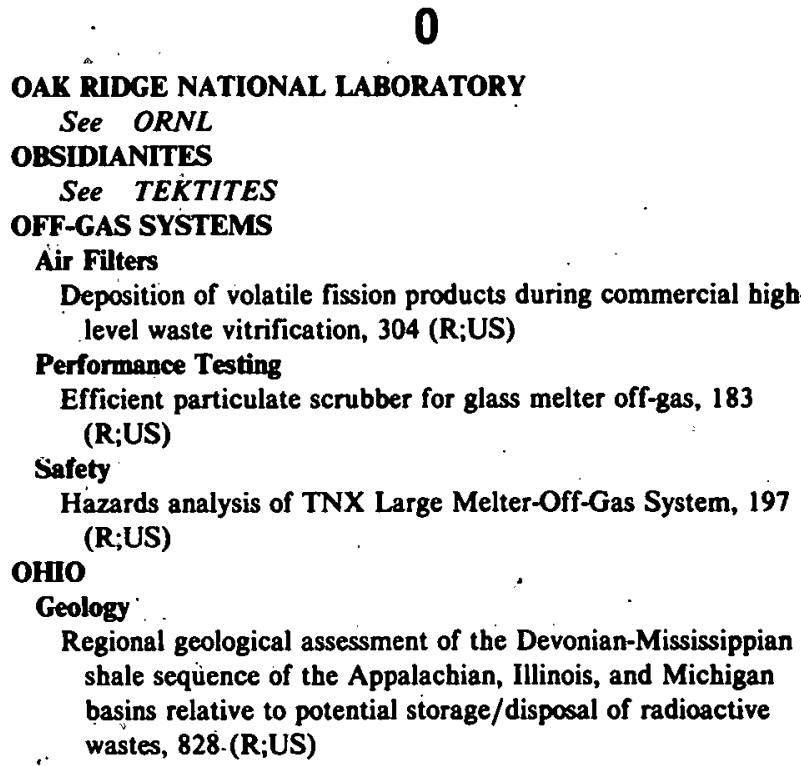

OIiL SAND OIS

See BITUMENS

OIL WELIS

Oil Saturation

Fuel-cycle programs. Quarterly progress report, January-March 1981, 48 (R;US)

Fuel-cycle programs. Quarterly progress report, April-June 1981, 49 (R;US)

OKLAHOMA

Salt Deposits

Environmental characterization of bedded salt formations and overlying areas of the Permian Basin, 1320 (R;US)

OKLO PHENOMENON

Oklo reactors: natural analogs to nuclear waste repositories, 611 (RA;US)

\section{ONTARIO}

Radioactive Waste Management

Management of nuclear fuel waste. Final report, 71 (R;CA)

OPERATION (REACTOR)

SEe REACTOR OPERATION

ORGANIC PIIOSPHORUS COMPOUNDS

Solvent Properties

Octyl(phenyl)-N,N-diisobutylcarbamoylmethylphosphine oxide as an extractant for actinides from nitric acid waste, 146 (R;US)

ORGANIC POLYMERS

Comparative Evaluations

Evaluation of forms for the immobilization of high-level and transuranic wastes, 200 (R;US)

ORNL

Radioactive Waste Disposal

Analysis of waste disposal practice and control at ORNL, 549 (R;US)

Waste treatment and disposal. Quarterly progress report, February-April, 1963, 290 (R;US)

Radioactive Waste Processing

Sol-gel-derived waste forms, 245 (RA;DE)

Waste treatment and disposal. Quarterly progress report, February-April, 1963, 290 (R;US)

\section{OXALIC ACID}

Decomposition

Decomposition of oxalic acid with nitric acid, $465(\mathrm{~J} ; \mathrm{CH})$

OXYGEN HYDRIDES

See WATER 


\author{
OXYMETHYLENE \\ See FORMALDEHYDE \\ OZONE \\ Chemical Reaction Kinetics \\ Ozone mass transfer and kinetics experiments, 334 (R;US) \\ Mass Transfer \\ Ozone mass transfer and kinetics experiments, 334 (R;US)
}

\section{P}

\section{PACIFIC NORTHWEST LABORATORIES} See BATTELLE PACIFIC NORTHWEST LABORATORIES

\section{PACIFIC OCEAN}

Geological Surveys

Analysis of geological and geophysical data from study locations $C_{1}, B_{1}$, and $E_{2}$ in the northwest PAC 1 study region, 957 (RA;US)

Geophysical Surveys

Analysis of geological and geophysical data from study locations $C_{1}, B_{1}$, and $E_{2}$ in the northwest. PAC 1 study reginn, 957 (RA;US)

Sediments

Constitutive properties for undisturbed marine sediments in support of the subseabed disposal program, 963 (RA;US)

Geotechnical studies for subseabed disposal: high level radioactive wastes, 964 (RA;US)

Pacific site assessment, 960 (RA;US)

PACKAGING

Bibliographies

Annotated bibliography for the design of waste packages for geologic disposal of spent fuel and high-level waste, 804 (R;US)

Comparative Evaluations

Review of DOE waste package program. Subtask 1.1. National waste package program, October 1982-March 1983. Vol. 4, 736 (R;US)

Cóst

Conceptual waste-package designs for disposal of nuclear waste in tuff, $818(R ; U S)$

Desigm

Conceptual design requirements for spent fuel, high-level waste, and transuranic waste packages, 903 (R,US)

Conceptual waste-package designs for disposal of nuclear waste in tuff, 818 (R;US)

Design of a nuclear-waste package for emplacement in tuff, 1018 (R;US)

Development of waste package designs for disposal in a salt repository. $\$ 57$ (RA:US)

Engineered waste-package-system design specification, 816 (R;US)

Expected very-near-field thermal environments for advanced spent-fuel and defense high-level waste packages, 831 (R;US)

Initial specifications for nuclear waste package external dimensions and materials, 1005 (R;US)

Package design, 796 (RA;US)

Transportation packagings for high-level wastes and unprocessed transuranic wastes, 29 (R;US)

Waste package conceptual designs for a repository located in basalt, 884 (R;US)

Waste package conceptual designs for salt repositories, 593 (RA;US)

\section{Dimensions}

Initial specifications for nuclear waste package external dimensions and materials, 1005 (R;US)

Evaluation

Near-field performance evaluations, 800 (RA;US)

Review of DOE waste package program. Subtask 1.1. National waste package program, April-September 1982, 735 (R;US)

Inspection

Construction and inservice inspection rules for nuclear spent fuel and high-level waste transport packagings, 18 (RA;US)

\section{Materials Testing}

Long-term performance of materiais used for high-level waste packaging. Annual report, March 1982-April 1983. Vol.1, 75 (R;US)

Long-term performance of materials used for high-level waste packaging. Quarterly report, April-June 1983, 752 (R;US)

Packing material testing required to demonstrate compliance with 1000 -year radionuclide containment. Semiannual report on waste package verification tests, 739 (R;US)

Review of DOE waste-package program. Subtask 1.1. National waste-package program, September 1981-March 1982. Volume 2, 732 (R;US)

Review of DOE waste package program. Subtask 1.1. National waste package program, October 1982-March 1983. Vol. 4, 736 (R;US)

Testing of candidate waste packàge backfill and canister materials for basalt, 586 (RA;US)

Waste package materials testing for a salt repository: 1982 status repoft, 825 ( $\mathbf{R} ; \bar{U} S)$

Performance

NWTS program criteria for mined geologic disposal of nuclear waste: functional requirements and performance criteria for waste packages for solidified high-level waste and spent fuel, 597 (R;US)

Post-emplacement monitoring, Subtask 1.2. Draft Technical Position. Vol. 2, 1319 (R;US)

WAPPA: a Waste Package Performance Assessment code. Technical report, 819 (R;US)

WAPPA: an integrated waste package performance assessment code, 1220 (J;US)

Waste package performance assessment: the importance of the very near-field physicochemical environment, 1223 (J;US)

Performance Testing

Combined analytical model for preformance assessment of the waste package/geologic medium systems, 1170 (BA;US)

Draft Technical Position Subtask 1.1: waste package performance after repository closure. Volume 1,749 (R;US)

Long-tcrm performance of materiäls used fuı high-level waste packaging. Annual report, March 1982-April 1983. Vol.1, 751 (R:US)

Waste package performance evaluation, 806 (R;US)

Reliability

Draft technical position on waste-package reliability, 546 (R;US)

Research Programs

Review of DOE waste-package program. Subtask 1.1. National waste-package program, September 1981-March 1982. Volumc $2,732(\mathrm{R} ; \mathrm{US})$

\section{Specifications}

Conceptual wasle package interim perfurmante specifications for waste forms for genlngir. isnlation in salt repositories, 821 (R;US)

Conceptual waste package interim product specifications and data requirements for disposal of borosilicate glass defense high-level waste forms' in salt geologic repositories, 823 (R;US)

Englneered waste-packàgè-system design specification, 816 (R;US)

Initial specifications for nuclear waste package external dimensions and materials, 1005 (R;US)

Interim performance specifications for conceptual waste-package designs for gcologic isolation in salt repusituries, 822 (R;US)

NWTS program criteria for mined geologic disposal of nuclear waste: functional requirements and performance criteria for waste packages for solidified high-level waste and spent fuel, 597 (R;US)

Standardization

Nuclear waste management technical support in the developm-' of nuclear waste form criteria for the NRC. Task 5 . Nationi waste package program, 285 (R;US)

\section{PAKISTAN}

Fuel Cycle

Summary of non-US national and international fuel cycle and radioactive waste management programs 1982, 83 (R;US) 
Radioactive Waste Management

Summary of non-US national and international fuel cycle and radioactive waste management programs 1982,83 (R;US)

\section{PALlADIUM}

Centrifugation

Influence of the reprocessing flow sheet on the HLW solidification technology, 239 (RA;DE)

Materials Recovery

Recovery of noble metals from fission products, 324 (R;US)

Solubility

Geochemical controls on radionuclide releases from a nuclear waste repository in basalt: estimated solubilities for selected elements, 1329 (R;US)

\section{PALLADIUM ALLOYS}

Corrosion Products

Analysis of the corrosion products formed on Ti and a Ti-Pd alloy during exposure in hot water, 1176 (BA;US)

\section{PARTICULATES}

Environmental Transport

Exposures resulting from nuclear power production. Annex $F$, 1444 (BA;US)

PENNSYLVANIA

Geology

Regional geological assessment of the Devonian-Mississippian shale sequence of the Appalachian, Illinois, and Michigan basins relative to potential storage/disposal of radioactive wastes, 828 (R;US)

PEOPLE

SEe HUMAN POPULATIONS

PEOPLES REPUBLIC OF CHINA

See CHINA

PERMUTIT (INORGANIC)

SEO INORGANIC ION EXCHANGERS

PEROVSKITE

Leaching

Leaching of natural and synthetic sphene and perovskite, 456 (BA;US)

Phase Studies

Subsolidus sintering of SYNROC: I. Initial demonstrations, 280 (R;US)

Physical Radiation Effects

Alpha-recoil damage in natural zirconolite and perovskite, 518 $(\mathrm{J} ; \mathrm{JP})$

Specific Heat

Thermophysical properties of SYNROC and its constituent mineral phases, 474 (BA;AU)

Thermal Conductivity

Thermophysical properties of SYNROC and its constituent mineral phases, 474 (BA;AU)

Thermal Diffusivity

Thermophysical properties of SYNROC and its constituent mineral phases, 474 (BA;AU)

\section{PERSONNEL}

Dose Rates

Results of the occupational dose assessment in the conceptual reference repository description, 1388 (J;US)

Radiation Doses

Occupational exposure in an underground mine repository for radioactive waste, 1387 (J;US)

Occupational radiation exposure risk at a nuclear waste repository in basalt, 1389 (J;US)

Projected occupational exposures during WIPP operations, 1041 (J;US)

Summary of ONWI occupational dose assessment program, 1391 (J;US)

Radiation Protection

Design considerations for nccupational exposure for a potential repository at Yucca Mountain: high-level-waste handling operations, 991 (R;US)

Occupational exposure for nuclear waste handling operations at NNWSI repository, 1390 (J;US)

\section{PERTECHNETATES}

Chemical Reactions

Technetium behavior in sulfide and ferrous iron solutions, 553 (R;US)

Precipitation

Technetium behavior in sulfide and ferrous iron solutions, 553 (R;US)

\section{PHOSPHORIC ACID}

Leaching

Influence of metal oxides on the leach rate and crystallisation behaviour of waste glasses, 251 (RA;DE)

\section{PHYSICAL PROPERTIES}

Caisks

Description of Defense Waste Processing Facility reference waste form and canister. Revision 1, 168 (R;US)

\section{PLASMA ARC WELDING}

Remote automatic plasma arc-closure welding of a dry-storage canister for spent nuclear fuel and high-level radioactive waste, 551 (R;US)

PLUGS

See CLOSURES

PLUTONIUM

Adsorption

Effects of Hanford high-level waste components on sorption of cobalt, strontium, neptunium, plutonium, and americium on Hanford sediments, 918 (R;US)

Geochemistry studies pertaining to the G-tunnel radionuclide migration field experiment, 1426 (R;US)

Immobilization of reprocessing waste by means of titanate ion exchangers, 248 (RA;DE)

Precipitation process for supernate decontamination, 173 (R;US)

Precipitation process for decontamination of water-soluble SRP radioactive waste, $184(\mathrm{R} ; \mathrm{US})$

Precipitation process for decontaminating water-soluble SRP radioactive waste, 187 ( $R ; U S)$

Sorption of actinides in well-defined oxidation states on geologic media, 1351 (BA;US)

Status of radionuclide sorption-desorption studies performed by the WRIT program, 1308 (RA;US)

Transport of actinides through a bentonite backfill, 1175 (BA;US)

Waste form/rock interaction leaching study using PNL 76-68 glass beads and Umtanum basalt. Part I, 1004 (R;US)

Adsorption Isotherms

Basalt-radionuclide reactions: FY-1981. Annual report, 868 (R;US)

Chemical State

Evaluation of solubility and speciation of actinides in natural groundwaters, 1347 (BA;US)

Chemisorption

Redox behavior, complexing, and adsorption of hexavalent actinides by humic acid and selected clays (Siting, marine disposal of high-level radioactive waste), 1247 (J;US)

Desorption

Status of radionuclide sorption-desorption studies performed by the WRIT program, 1308 (RA;US)

Diffusion

Transport of actinides through a bentonite backfill, 1175 (BA;US)

Distribution Functions

Sorption of actinides in well-defined oxidation states on geologic media, 1351 (BA;US)

Status of radionuclide sorption-desorption studies performed by the WRIT program, 1308 (RA;US)

Environmental Transport

Actinide sorption on rock minerals, 677 (RA;DE)

Backfill barriers for nuclear waste repositories in salt, 670 (RA;DE)

Leaching

Evaluation of solubility and speciation of actinides in natural groundwaters, 1347 (BA;US)

FUETAP concrete: an alternative radioactive waste host, 256 (RA;DE)

Leaching of actinides from simulated nuclear waste glass, 1196 (BA;US) 
Leaching study of PNL 76-68 glass beads using the. LLNL continuous-flow method and the PNL-modified IAEA method. Final report, 344 (R;US)

Predicting Pu concentrations in solutions contacting geologic materials, 1146 (BA;US)

Nuclear Reaction Analysis

State-of-the-art in-situ determination of transuranics, 88 (R;US)

\section{Oxidation}

Redox behavior, complexing, and adsorption of hexavalent actinides by humic acid and selected clays (Siting, marine disposal of high-level radioactive waste), 1247 (J;US)

Redox Potential

Thermodynamic properties of chemical species in nuclear waste, 812 (R;US)

Separation Processes

Waste-Mixes Study for space disposal, 815 (R;US)

Solubility

Evaluation of solubility and speciation of actinides in natural groundwaters, 1347 (BA;US)

Geochemical controls on radionuclide releases from a nuclear waste repository in basalt: estimated solubilities for selected elements, 1329 (R;US)

Solvent Extraction

Chemistry of the nuclear fuel cycle, 4 (BA;GB)

Octyl(phenyl)-N,N-diisobutylcarbamoylmethylphosphine oxide as an extractant for actinides from nitric acid waste, 146 (R;US)

Valence

Valence states of actinides in synthetic monazites, 1276 (BA;US)

XPS valence state determination of $\mathrm{Np}$ and $\mathrm{Pu}$ in multicomponent borosilicate glass and application to leached 76-68 waste glass surfaces, 417 (BA;US)

\section{PLUTONIUM 238}

Leaching

Underground migration of long-lived radionuclides leached from a borosilicate glass matrix, 1360 (BA;XA)

\section{PLUTONIUM 239}

Alpha Spectra

Alpha spectrum profiling of plutonium in leached simulated high-level radioactive waste-glass, 517 (J;US)

Leaching

Alpha spectrum profiling of plutonium in leached simulated high-level radioactive wastc-glass, 1364 (J;US)

Leach rates of high level waste and spent fuel: limiting rates as determined by backfill and bedrock conditions, 1345 (BA;US)

Leaching study of nuclear melt glass: Part I, 1332 (R;US)

Solubility

Evaluation of radionuclide transport: effect of radionuclide sorption and solubility, 1354 (BA;US)

Leach rates of high level waste and spent fuel: limiting rates as determined by backfill and bedrock conditions, 1345 (BA;US)

Solvent Extraction

Development of partitioning method - cold and semihot experimental works with partitioning testing apparatus, 225 (R;JP;In Japanese)

\section{PLUTONIUM 240}

Solübility

Evaluation of radionuclide transport: effect of radionuclide sorption and solubility, 1354 (BA;US)

\section{PLUTONIUM 242}

Leaching

Leach rates of high level waste and spent fuel: limiting rates as determined by backfill and bedrock conditions, 1345 (BA;US)

Solubility

Evaluation of radionuclide transport: effect of radionuclide sorption and solubility, 1354 (BA;US)

Leach rates of high level waste and spent fuel: limiting rates as determined by backfill and bedrock conditions, 1345 (BA;US)

\section{PLUTONIUM COMPLEXES}

Solubility

Importance of organic compounds in ground water as radionuclide-mobilizing agents, 1324 (R;US)

\section{PLUTONIUM DIOXIDE}

Phase Studies

Chemistry in the development of nuclear power, 5 (BA;GB)

Solubility

Predicting Pu concentrations in solutions contacting geologic materials, 1146 (BA;US)

Thermodynamic Properties

Chemistry in the development of nuclear power, 5 (BA;GB)

PLUTONIUM HYDROXIDES

Solubility

Predicting Pu concentrations in solutions contacting geologic materials, 1146 (BA;US)

PLUTONIUM RECYCLE

Tokai Works annual progress report, April 1980-March 1981, 3 (R;JP)

PNC

Radiation Monitoring

Applieation of computer system to environmental monitoring at PNC Tokai Works, 1450 (J;JP;In Japanese)

Radioactive Waste Facilities

Current technics and management strategy for Pu-contaminated wastes at PNC (Japan), 295 (R;JP)

PNL

See BATTELLE PACIFIC NORTHWEST LABORATORIES

POLICY

SEe ENERGY POLICY

POLYACRYLONITRILE

See ORGANIC POLYMERS

\section{POLYHALTTE}

Specific Heat

Impurities in rock-salt: consequences for the temperature increases at the disposal of high-level nuclear waste, 834 (R;US)

Thermal Conductivity

Impurities in rock-salt: consequences for the tempcrature increases at the disposal of high-level nuclear waste, 834 (R;US)

POLY(ISOBUTYLENE OXIDE)

SeE ORGANIC POLYMERS

POLYMERS

See also ORGANIC POLYMERS

Corrosion Resistance

Canister materials proposed for final disposal of high level nuclear waste - a review with respect to corrosion resistance, 675 (RA;DE)

POLYTETRAOXANE

SER ORGANIC POLYMEKS

POROUS MATERIALS

Fluid Flow

Aqueous phase diffusion in crystalline rock, 1359 (BA;US)

Heat Flow

Coupled heat and groundwater flow in porous rock. 535 (R;GB)

Incompressible Flow

Crode develupusent in supporn of nuolear wastè storage investigations for a repository in tuff (Pore water motion and energy transport in a porous media which may be saturated or partially saturated with a single fluid in liquid state), 988 (R;US)

Liquid Flow

Coupled heat and groundwater flow in porous rock, 535 (R;GB)

Natural Convection

Natural convection experiments in a liquid-saturated porous medium bounded by vertical coaxial cylinders, 968 (R;US)

Radionuclide Migration

Comparative analysis of fractured and porous medium radionuclide transport, 1356 (BA;US)

\section{POTASSIUM}

Leaching

Preparation and leaching of radioactive INEL waste forms, 201 (R;US) 


\section{ASSIUM CHLORIDES \\ See also CARNALLITE}

-clusions

Thermal-gradient migration of brine inclusions in salt crystals

(Synthetic single crystals of $\mathrm{NaCl}$ and $\mathrm{KCl}$ ), 713 (R;US)

POWER REACTOR NUCLEAR FUEL DEVELOPMENT CO

See PNC

PRESSURIZED WATER COOLED

Moderated ReactoP

See PWR TYPE REACTORS

PRESSURIZED WATER REACTORS

See PWR TYPE REACTORS

PROPERTIES (PHYSICAL)

SE PHYSICAL PROPERTIES

\section{PROTACTINIUM}

Adsorption

Sorption of actinides in well-defined oxidation states on geologic media, 135l (BA;US)

Transport of actinides through a bentonite backfill, 1175 (BA;US)

Diffusion

Transport of actinides through a bentonite backfill, 1175 (BA;US)

Distribution Functions

Sorption of actinides in well-defined oxidation states on geologic media, 1351 (BA;US)

PROTECTION (RADIATION)

SE RADIATION PROTECTION

PUREX PROCESS

Rockwell Hanford Operations monthly report summary, June 1983, 9 (R;US)

High-level Radioactive Wastes

Formation of inflammable radiolytic gases in the PUREXprocess, 11 (J;DE;In German)

PWR TYPE REACTORS

See also THREE MILE ISLAND-2 REACTOR

Fuel Cycle

Analysis of Am and a recovery for it in the pressurized water reactor fuel cycle, 1433 (R;DE; In German)

Radioactive Wastes

Graphical and tabular summaries of decay characteristics for once-through PWR, LMFBR, and FFTF fuel cycle materials (Spent fuel, high-level waste fuel can scrap), $76(R ; U S)$

Reactur Cores

Analysis of Am and a recovery for it in the pressurized water reactor fuel cycle, 1433 (R;DE; In German)

Spent Fuel Elements

Generation and vitrification of high-level light water reactor liquid waste, 385 (J;US)

Thorium Cycle

Use of thorium as an alternative nuclear fuel, $46(R ; A U)$

PYREX

Fracture Properties

Methodology for characterizing brittle fracture of solid waste forms in accidental impacts, 420 (BA;US)

Impact Strength

Methodology for characterizing brittle fracture of solid waste forms in accidental impacts, 420 (BA;US)

Impact Tests

Methodology for characterizing brittle fracture of solid waste forms in accidental impacts, 420 (BA;US)

PYROCARBON

See PYROLYTIC CARBON

PYROCHLORE

Chemical Radiation Effects

Radiation damage studies related to nuclear waste forms, 298 (R;US)

licrostructure

Radiation damage studies related to nuclear waste forms, 298 (R;US)

Radiation Effects

Study of radiation effects in curium-doped $\mathrm{Gd}_{2} \mathrm{Ti}_{2} \mathrm{O}_{7}$ (pyrochlore) and $\mathrm{CaZrTi}_{2} \mathrm{O}_{7}$ (zirunulite), 1211 (BA;US)

\section{Self-irradiation}

Study of radiation effects in curium-doped $\mathrm{Gd}_{2} \mathrm{Ti}_{2} \mathrm{O}_{7}$ (pyrochlore) and $\mathrm{CaZrTi}_{2} \mathrm{O}_{7}$ (zironolite), 1211 (BA;US)

\section{PYROLYTIC CARBON}

Chemical Vapor Deposition

Coating crystalline nuclear waste forms to improve inertness, 138 (R;US)

Evaluation of coated particle waste forms, 448 (BA;US)

Leaching

Coating crystalline nuclear waste forms to improve inertness, 138 (R;US)

\section{PYROXENES}

Leaching

Alpha-recoil damage and solution effects in minerals: uranium PYRRHITE isotopic disequilibrium and radon release, $1073(\mathrm{~J} ; \mathrm{GB})$

\section{See PYROCHLORE}

\section{QUARTZ}

Leaching

Alpha-recoil damage and solution effects in minerals: uranium isotopic disequilibrium and radon release, $1073(\mathrm{~J} ; \mathrm{GB})$

Radionuclide Migration

Actinide sorption on rock minerals, 677 (RA;DE)

Thermal Conductivity

Thermal conductivity of bentonite/quartz high-level-wastepackage backfill, $972 \cdot(R ; U S)$

Thermal conductivity of bentonite/quartz high-level waste package backfill, 1065 (J;US)

\section{$\mathbf{R}$}

RADIATION BURDEN

SEe RADIATION DOSES

RADIATION DECONTAMINATION

SEe DECONTAMINATION

RADIATION DOSES

Mathematical Models

Uncertainties associated with geologic disposal of high-level radioactive waste, 1367 (R;US)

RADIATION EXPOSURE (DOSES)

SEe RADIATION DOSES

RADIATION HYGIENE

SRe RADIATION PROTECTION

RADIATION MONITORING

Data Processing

Application of computer system to environmental monitoring at PNC Tokai Works, 1450 (J;JP;In Japanese)

RADIATION PROTECTION

Repository safety-the salient facts, 1397 (J;US)

RADIATION SAFETY

SRe RADIATION PROTECTION

RADIOACTIVE BIOLOGICAL WASTES

SEe RADIOACTIVE WASTES

RADIOACTIVE GASEOUS WASTES

See GASEOUS WASTES

RADIOACTIVE WASTES

RADIOACTIVE MATERIALS

See also FISSION PRODUCTS

$$
\text { RADIOACTIVE WASTES }
$$

Containers

Classification and safety reserves of radioactive material shipping containers, 1452 (B;DE;In German)

Land Transport

Evaluation of proposed methods of radioactive material container tie-down design for truck transport, 44 (J;US)

Packaging

Commercial nuclear waste research and development program. Quarterly report, January-March 1982, 61 (R;US) 


\section{Regulations}

Information report on state legislation, volume 8, number 5,1414 (R;US)

Transport

Risk assessment for the transportation of radioactive zeolite liners, 28 (R;US)

Transport of highly radioactive materials, 45 (J;US)

RADIOACTIVE WASTE DISPOSAL

Deadly legacy, 1127 (J;GB)

Disposal of radioactive wastes (Book), 106 (B;NL)

Intermediate, long term and final storage of radioactive substances, 1234 (J;AT;In German)

ONWI library reports list, 770 (R;US)

After-heat

SALTMP - a FORTRAN program for fast salt temperature calculations in HRW final storage, 866 (R;DE;In German)

Air Pollution

Nevada nuclear waste storage investigations site-characterization activities: preliminary atmospheric assessment of a nuclear waste repository, 765 (R;US)

Alpha-bearing Wastes

Basic data report for deepening of drillhole WIPP 13 (Waste Isolation Pilot Plant-WIPP), 975 (R;US)

Conceptual design requirements for spent fuel, high-level waste, and transuranic waste packages, 903 (R;US)

Decay calculations on medium-level and actinide-containing wastes from the LWR fuel cycle. Pt. 2, 688 (R;DE; In German)

Defense waste management plan, 563 (R;US)

Evaluation of research and development for terminal isolation of nuclear wastes, 701 (R;US)

Handling and disposing of radioactive waste, 561 (R;US)

Implementation of technical conservatism in the development of nuclear waste repositories, 600 (R;US)

Legislative and political aspects of waste disposal, 1410 (RA;US)

National Waste Terminal Storage Program. Technical progress report for the quarter 1 July-30 September 1982, 780 (R;US)

Nuclear-fuel-cycle education: Module 8. Waste management, 155 (R;US)

NWTS program waste projection data needs, 1086 (J;US)

Operational features of the Waste Isolation Pilot Plant (WIPP), 1161 (BA;US)

Preliminary identification of interfaces for certification and transfer of TRU waste to WIPP, 695 (R;US)

Probablistic approach to the assessment of the long-term risk linked to the disposal of radioactive waste in geological repositories, 1392 (J;GB)

Regulatory policy of the EPA related to alpha-contaminated waste, 1412 (RA;US)

Research priorities for isolation of defense high-level and transuranic wastes in the United States, 628 (R;US)

Site investigations for repositories for solid radioactive wastes in deep continental geological formations, 1139 (B;XA)

Status of high level and alpha bearing waste management in PNC, 428 (BA;US)

Technical progress report for the quarter, 1 July-30 September 1981.776 (R;IIS)

Technical progress report for the quarter, 1 October-31 December, 1981,777 (R;US')

Technical progress report for the quarter, 1 April-30 June 1982, 779 (R;US)

TRU waste-acceptance criteria for the Waste Isolation Plant, 1419 (K;US)

US Geological Survey research in radioactive waste disposal, fiscal year $1979,716(R ; I I S)$

Waste Isolation Pilot Plant (WIPP) research and development program: in situ testing plan, March 1982, 940 (R;US)

Waste-Mixes Study for space disposal, 815 (R;US)

WIPP research and development program: providing the technical basis for defense waste disposal, 989 (R;US)

\section{Backfilling}

Backfill-waste interactions in repository simulating tests, 1153 (BA;US)

Backfilling and sealing a repository for high-level radioactive waste - a review, 839 (R;US)
Combined analytical model for preformance assessment of the waste package/geologic medium systems, 1170 (BA;US)

Development of a backfill for containment of high-level nuclear waste, 1173 (BA;US)

Diffusion measurements in compacted bentonite, 1148 (BA;US)

Fuel cycle programs. Quarterly progress report, July-September 1982, 538 (R;US)

Nuclear waste package program for high-level isolation in Nevada tuff, 1102 (J;US)

Nuclear waste package materials testing report: basaltic and tuffaceous environments, 854 (R;US)

Testing of candidate waste package backfill and canister materials for basalt, 586 (RA;US)

Thermal conductivity of bentonite/quartz high-level waste package backfill, 1065 (J;US)

Basalt

Assessment of the proposed rule (10CFR60) for disposal of highlevel radioactive wastes in geologic repositories. Volume I, 747 (R;US)

Cesium and strontium fractionation from HLW for thermal-stress reduction in a geologic repository, 327 (R;US)

Groundwater flow and transport characteristics of flood basalts as determined from tracer experiments, 880 (R;US)

Near-field repository conditions in basalt and salt, 733 (RA;US)

Site-screening process on the Hanford Site: a summary report, 878 (R;US)

\section{Bibliographies}

Bibliography of the published reports, papers, and articles on the Nevada Nuclear Waste Storage Investigations (387 citations), 754 (R;US)

Bibliography of the published reports, papers, and articles on the Nevada nuclear waste storage investigations (482 citations), 755 (R;US)

Radioactive waste processing and disposal: a bibliography, 158 (R;US)

Boreholes

Laboratory studies of fluid flow through borehole seals, 1231 (J;US)

Calculation Methods

Effect of a random variation of rock salt creep on calculations of storage roxm closure for the WIPP project, $943(\mathrm{R} ; \mathrm{US})$

Carbon 14

Fuel cycle programs. Quarterly progress report, April-June 1982 , 536 (R;US)

Fuel cycle programs. Quarterly progress report, July-September 1982, 538 (R;US)

Fuel cycle programs. Quarterly progress report, October-

Cesium December 1982, 539 (R;US)

Systems study of the feasibility of high-level nuclear-waste fractionation for thormal-3trcs3 control in a gcologic icipusituiy: main report, 308 (R;US)

Systems study of the feasibility of high-level nuclear waste fractlonation for thermal strèśs control in a geologic repository: appendices, 309 ( $R ; \mathbf{I}, \mathbf{S}$ )

Cesium 137

Alternate strategy for commercial high-level radioactive-waste management, 945 (R;US)

Comparative Evalualiuns:

Is spent fuel or waste from reprocessed spent fuel simpler to dispose of, $533(\mathrm{R} ; \mathrm{US})$

Nuclear waste/nuclear power: their futures are linked, 560 (R;US)

Computer Codes

Summary of repository siting models. Final report, 740 (R;US)

Computerized Simulation

Final technical position on documentation of computer codes $\mathrm{f}$ high-level waste management, 723 (R;US)

Concretes

Chemical interaction of clay buffer materials and concrete, 997 (R;SE) 
Configuration

Comparison of waste emplacement configurations for a nuclear waste repository in tuff. II. Ventilation analysis, 995 (R;US)

Comparison of waste emplacement configurations for a nuclear waste respository in tuff. IV. Thermo-hydrological analysis, 996 (R;US)

Constraints

Preliminary technical constraints for a repository in tuff, 977 (R;US)

Containers

Corr sion of materials in a clay environment, $676(R A ; D E)$

Canister materials proposed for final disposal of high level nuclear waste - a review with respect to corrosion resistance, 675 (RA;DE)

Canister materials proposed for final disposal of high level nuclear waste, 685 (RA;DE)

Concept of a canister for high-level radioactive waste, 1158 (J;US)

Container assessment - corrosion study of HLW container materials. Quarterly progress report, April-June 1981, 729 (R;US)

Corrosion studies and mechanical tests on metallic materials for the design of packagings for vitrified high level wastes (HLW), 689 (R;DE;In German)

Corrosion of materials in a clay environment, 686 (RA;DE)

Development of waste package designs for disposal in a salt repository, 557 (RA;US)

Effects of radiation on corrosion of titanium, 961 (RA;US)

Feasibility of using a high-level waste canister as an engineered barrier in disposal, 842 (R;US)

HAW-containers as a barrier in final storage, 1432 (RA;DE; In German)

Identification of crevice corrosion in the titanium alloy TiCode- 12 in simulated rock salt brine at $150^{\circ} \mathrm{C}, 545$ (R;US)

Long-term performance of materials used for high-level waste packaging. Quarterly report, April-June 1983, 752 (R;US)

Ocean-bottom disposal of fission-product krypton-85, 1251 (BA;JP)

Parametric study of the effects of thermal environment on a waste package for a tuff repository, 1271 (BA;XN)

Shielded containers for waste could prove beneficial, 1128 (J;GB)

Studies of waste-canister compatibility, 832 (R;US)

Testing of candidate waste package backfill and canister materials for basalt, 586 (RA;US)

Uniform- and pitting-corrosion data requirements for TiCode-12 high-level-waste containers, 734 (RA;US)

WAPPA: an integrated waste package performance assessment code, $1220(\mathrm{~J} ; \mathrm{US})$

Waste Management Program. Technical progress report, October-December 1980, 162 (R;US)

Waste packages for high-levcl waste repositories, 1043 (J;US)

Coordinated Research Programs

CEC project to assess high level waste disposal options, 1085 (J;GB)

Earth Sciences, 789 (RA;US)

Far-field performance evaluations, 793 (RA;US)

Issues in repository siting, 798 (RA;US)

Near-field performance evaluations, 800 (RA;US)

Overview of the National Waste Terminal Storage Program, 788 (RA;US)

Package design, 796 (RA;US)

Package materials, waste form, 792 (RA;US)

Quality assurance, 799 (RA;US)

Repository design and equipment, 791 (RA;US)

Repository sealing, 794 (RA;US)

Repository data base, field tests, 795 (RA;US)

Repository data base development, 801 (RA;US)

Status of gcologio/environmental characterizations, 797 (RA;US)

Systems, 790 (RA;US)

Corrosion

Corrosion studies on non-metallic inorganic materials for HLW packaging design, 691 (R;DE;In German)
Corrosion Resistance

Corrosion aspects of high-level waste disposal in salt domes, 922 (R;DK)

Interaction of vitrified high level waste with clay environment,

Cost 274 (RA;DE)

Comparison of SRP high-level waste disposal costs for borosilicate glass and crystalline ceramic waste forms, 635 (R;US)

Disposal costs for SRP high-level wastes in borosilicate glass and crystalline ceramic waste forms, 1010 (R;US)

Draft regulatory impact analysis for 40 CFR 191: environmental standards for management and disposal of spent nuclear fuel, high-level and transuranic radioactive wastes. Draft report, 837 (R;US)

Projected costs for mined geologic repositories for dispoal of commercial nuclear wastes, 769 (R;US)

Systems, 771 (RA;US)

\section{Cost Benefit Analysis}

Benefit-cost-risk analysis of alternatives for greater-confinement disposal of radioactive waste, $559(\mathrm{R} ; \mathrm{US})$

Criticality

Nuclear criticality safety analyses for the Waste Isolation Pilot Plant project, 1258 (J;US)

Data Acquisition

Repository data base development, 801 (RA;US)

Decision Making

High-level radioactive wste management: a means to social consensus, 542 (R;US)

Possible approaches to community development for nuclear waste isolation, 805 (R;US)

Economic Impact

Evaluation of water resource economics within the Pasco Basin, Washington, 1429 (R;US)

Economics

Research and development activities: fixation of radioactive residues. Quarterly progress report, April-June 1960, 210 (R;US)

Encapsulation

Corrosion aspects of high-level waste disposal in salt domes, 922 (R;DK)

Engineered Safety Systems

Backfill barriers for nuclear waste repositories in salt, 670 (RA;DE)

Combined analytical model for preformance assessment of the waste package/geologic medium systems, 1170 (BA;US)

Corrosion of construction materials in clay environments, 1150 (BA;US)

Criteria for high level waste disposal and characterization of barriers, 1374 (RA;DE)

Development of structural barriers for containment of nuclear waste in basalt, 1100 (J;US)

Engineered waste package concepts for high-level waste isolation in basalt, 874 (R;US)

Engineered components for spent fuel radioactive waste isolation systems: are they technically justified, 1171 (BA;US)

Engineered components for radioactive waste disposal systems are they technically justified, 861 (R;US)

Evaluation of barriers in a final storage site for radioactive wastes, 674 (RA;DE)

Expected very-near-field thermal environments for advanced spent-fuel and defense high-level waste packages, 831 (R;US)

High-level-waste containment for a thousand years: unique technical and research problems (USA), 544 (R;US)

Management of radioactive wastes in Europe (EC) history, philosophy and plans for the next five years, 108 (BA;US)

Nuclear-waste-management. Semiannual progress report, October 1981-March 1982, 301 (R;US)

Nuclear-waste disposal in geologic repositories, 1014 (R;US)

Nuclear-waste repository in basalt: seal development studies, 887 (R;US)

Nuclear waste management. Quarterly progress report, October through Derember 1980, 296 (R:US)

NWTS Field Testing In-Situ Site-Confirmation Tests Workshop, September 8-9, 1980, 802 (R;US)

NWTS waste package program plan. Volume I. Program strategy, description, and schedule, 598 (R;US) 
NWTS waste package program plan. Volume II. Program logic networks, 599 (R;US)

Overview of hydrothermal testing of waste package barrier materials at the Basalt Waste Isolation Project, 848 (RA;US)

Package design, 796 (RA;US)

Radionuclide solubility and transport in basalt, 875 (R;US)

Scientific basis for nuclear waste management, 107 (B;US)

Waste package heat-transfer analysis: model development and temperature estimates for waste packages in a repository located in basalt, 917 (R;US)

Waste package conceptual designs for a repository located in basalt, 884 (R;US)

Environmental Effects

Analysis of the alleged Kyshtym disaster, 1314 (R;US)

High-level waste disposal, 1042 (J;US)

Environmental Impacts

1982 biotic survey of Yucca Mountain, Nevada Test Site, Nye County, Nevada, 1311 (R;US)

- Alternatives for definse waste-salt disposal, 629 (R;US)

Biologic overview for the Nevada Nuclear Waste Storage Investigations, Nevada Test Site, Nye County, Nevada, 1310 (R;US)

Draft environmental impact statement for 40 CFR part 191: environmental standards for management and disposal of spent nuclear fuel, high-level and transuranic radioactive wastes. Final reprt, 838 (R;US)

Draft regulatory impact analysis for $\mathbf{4 0}$ CFR 191: environmental standards for management and disposal of spent nuclear fuel, high-level and transuranic radioactive wastes. Draft report, 837 (R;US)

Environmental evaluation of alternatives for long-term management of Defense high-level radioactive wastes at the Idaho Chemical Processing Plant, 70 (R;US)

Environmental characterization report for the Gulf Interior Region Louisiana study area (Vacherie and Rayburn salt domes), 1321 (R;US)

Environmental characterization report for the Gulf Interior Region Mississippi study area (Lampton, Richton, and Cypress Creek salt domes), 1322 (R;US)

Environmental characterization report for the Gulf Interior Region, Texas study area (Oakwood, Palestine and Keechi salt domes), 1323 (R;US)

Environmental characterization of bedded salt formations and overlying areas of the Permian Basin, 1320 (R;US)

Evaluation of waste disposal safety, 661 (RA;DK;In Danish)

Monitoring technologies for ocean disposal of radioactive waste, 717 (R;US)

Population risks from disposal of high-level radioactive wastes in geologic repositories. Draft report, 836 (R;US)

Rock-melt concept for nuclear waste disposal, 1254 (J;US)

Evaluation

Evaluation of area studies of the US Gulf Coast Salt Dome Basins: location recommendation report, 785 (R;US)

Evaluation of research and development for terminal isolation of nuclear wastes, 701 (R;US)

Far-field performance evaluations, 793 (RA;US)

Hydrology and Geology Overview Committee reports and responises from the Basalt Waste Isolation Project, 914 (R;US)

Fault Tree Analysis

Fault-tree analycic for probabilictio a00000ment of radioactivewaste segregation: an application to a plastic clay formation at a specific site. $1180(\mathrm{BA} ; \mathrm{GB})$

Feasibility Studies

Hydrogeological conditions at Mors salt dume, 659 (RA;DK;In Danish)

Hydrogeologic testing of the E.J. Kubat borehole, San Juan County, Utah: utilization of a high pressure instrumented flow control system, 537 (RA;US)

Subseabed disposal program annual report, January-September 1981. Volume II. Appendices (principal investigator progress reports). Part 1, 954 (R;US)

\section{Field Tests}

NWTS Field Testing In-Situ Site-Confirmation Tcsts Workshop, September 8-9, 1980, 802 (R;US)

Radioactive waste disposal in granite (Stripa mine), 712 (R;US)

Repository data base, field tests, 795 (RA;US)
Test facilities and excavations, 774 (RA;US)

Financing

Report on financing the disposal of commercial spent nucleat fucl and processed high-level radioactive waste, 21 (R;US)

Report on financing the disposal of commercial spent nuclear fuel and processed high-level radioactive waste, 604 (R;US)

Fission Product Release

Effects of high-level gamma irradiation on radioactivity release from waste disposal forms, 1097 (J;US)

Fault-tree analysis for probabilistic assessment of radioactivewaste segregation: an application to a plastic clay formation at a specific site, 1180 (BA;GB)

\section{Gaseous Wastes}

Fuel cycle programs. Quarterly progress report, April-June 1982 , 536 (R;US)

Fuel cycle programs. Quarterly progress report, July-September 1982,538 (R;US)

\section{Geochemistry}

Geochemistry research planning for underground repositories, 1112 (J;US)

\section{Geologic Deposits}

Actinide sorption on rock minerals, 677 (RA;DE)

Alternate strategy for commercial high-level radioactive-waste management, 945 (R;US)

Analytical models for therinal ruck mechanles, 1217 (B;OB)

Annotated bibliography for the design of waste packages for geo logic disposal of spent fuel and high-level waste, 804 (R;US)

Appraisal of nuclear waste isolation in the vadose zone in arid and semiarid regions (with emphasis on the Nevada Test Site), 714 (R;US)

Approach to resolution of geologic uncertainty in the licensing of a high-level-waste repository in tuff, 985 (R;US)

Assessment of the proposed rule (10CFR60) for disposal of highlevel radioactive wastes in geologic repositories. Volume $I, 747$ (R;US)

Automated data acquisition and analysis system used in the Basalt Waste Isolation Project's Near-Surface Test Facility, 906 (R;US)

Basalt Waste Isolation Project. Quarterly report, October 1, 1981-December 31, 1981, 895 (R;US)

Basalt Waste Isolation Project drilling and testing. Quarterly report, April 1, 1982-June 30, 1982, 897 (R;US)

Basalt Waste Isolation Project drilling and testing. Quarterly report, July 1, 1982-September 30. 1982.898 (R;US)

Basalt Waste Isolation Project: drilling and testing. Quarterly report, October 1, 1982-December 31, 1982, 899 (R;US)

Basalt Waste Isolation Project. Drilling and testing quarterly report, January 1, 1983-March 31, 1983, 900 (R;US)

Basic data report for deepening of drillhole WIPP 13 (Waste Isolation Pilot Plant-WIPP), 975 (R;US)

Basic data report for drillhole WIPP 12 (Waste Isolation Pilot Plant-WJPP), 984 (R;IIS)

Bibliography and documents issued list through fiscal year 1982, $892(R ; U S)$

Canadian geoscience research for nuclear fuel waste management, 592 (RA;US)

CEC project to assess high level waste disposal options, 1085 (J;GB)

Cumilimenl turlydlual mulel for preformance assessment of the waste package/geologic medium systems, 1170 (BA;US)

Commercial high-level-waste management: options and cconomics. A comparative analysis of the ceramic and glass waste forms, 150 (R;US)

Comparative analysis of fractured and porous medium radionuclide transport, 1356 (BA:US)

Complex geologic characterization of the repository environment, 1131 (J;GB)

Composition of rocks - criteria for the conditioning of high-level radioactive wastes, 672 (RA;DE;In German)

Concept of final storage in geological formations, 669 (RA;DE;GE)

Conceptual design of field experiments for welded-tuff rockmechanics program, 936 (R;US)

Conceptual design requirements for spent fuel, high-level waste, and transuranic waste packages, 903 (R;US) 
Coupled heat and groundwater flow in porous rock, 535 (R;GB)

Criteria for high level waste disposal and characterization of barriers, 1374 (RA;DE)

Deep underground storage of [radioactive waste]. Annual report, 1981,643 (R;DE;GE)

Defense waste management plan, 563 (R;US)

Determination of rock mass deformation modulus in closely jointed Columbia River basalt, 881 (R;US)

Development of structural barriers for containment of nuclear waste in basalt, $1100(\mathrm{~J} ; \mathrm{US})$

Development of a backfill for containment of high-level nuclear waste, 1173 (BA;US)

Diffusion in the matrix of granitic rock: field test in the Stripa mine, 1341 (BA;US)

Draft site characterization analysis of the site characterization report for the Basalt Waste Isolation Project, Hanford, Washington Site. Main report and Appendices A through D, 724 (R;US)

Draft site characterization analysis of the site characterization report for the Basalt Waste Isolation Project, Hanford, Washington site. Appendices E through W, 725 (R;US)

Effect of microbial activity on the containment of radioactive waste in a deep geological repository, 1357 (BA;US)

Evaluation of research and development for terminal isolation of nuclear wastes, 701 (R;US)

Evaluation of barriers in a final storage site for radioactive wastes, 674 (RA;DE)

Evaluation of radionuclide transport: effect of radionuclide sorption and solubility, 1354 (BA;US)

Far-field performance evaluations, 793 (RA;US)

Fault-tree analysis for probabilistic assessment of radioactivewaste segregation: an application to a plastic clay formation at a specific site, 1180 (BA;GB)

Formulation of an integrated physicochemical-hydrologic model for predicting waste-nuclide retardation in geologic media, 973 (R;US)

Geochemical controls on radionuclide releases from a nuclear waste repository in basalt: estimated solubilities for selected elements, 1329 (R;US)

Geohydrologic characterization and qualification of a high-level waste site in basalts, 891 (R;US)

Geologic predictions and radioactive-waste disposal: a time limit for the predictive requirements, 1178 (BA;GB)

Geological disposal of high-level radioactive waste - a review of the Institute of Geological Sciences' research programme, 1091 (J;GB)

High-level-waste containment for a thousand years: unique technical and research problems (USA), 544 (R;US)

Hydrogeology of radioactive waste isolation: the challenge of a rational assessment, 1144 (J;US)

Hydrothermal conditions around a radioactive waste repository, 1177 (BA;US)

Identifiction of a preferred site for the exploratory shaft within the reference repository location: Hanford Site (Pasco Basin), 916 (R;US)

Implementation of technical conservatism in the development of nuclear waste repositories, 600 (R;US)

Improving public understanding of nuclear waste management, $1136(\mathrm{~J} ; \mathrm{GB})$

In-situ test programs related to design and construction of highlevel nuclear waste (HLW) deep geologic repositories. Final report (Task 2), June 1981-November 1982, 743 (R;US)

In-situ test programs related to design and construction of highlevel nuclear wastc (HLW) deep geologic repositories. Appendices. Final report (Task 2), June 1981-November 1982, 744 (R;US)

Influence of faulting on groundwater flow and contaminant transport, 1184 (BA;GB)

Influence of microfissures in crystalline rock on radionuclide migration, 1185 (BA;GB)

INTRACOIN: an international nuclide transport code intercomparison study, 1358 (BA;US) !

INTRACOIN, Level 1. Benchmark calculations with code SWITFT, 644 (R;DE)
Investigation of environmentally assisted fracture of metallic nuclear-waste-package barrier materials in simulated basalt repository environments, 844 (R;US)

Ion/water migration phenomena in dense bentonites, 1174 (BA;US)

Licensing: technological implications for geologic disposal of high-level radioactive wastes, 1089 (J;US)

Long-term thermohydrologic behaviour of nuclear-waste repositories, 1183 (BA;GB)

Long-term prediction of the fate of nuclear waste deeply buried in granite, $1186(\mathrm{BA} ; \mathrm{GB})$

Materials research for the Canadian Nuclear Fuel Waste Management Program, 109 (BA;US)

Methodology for assessing the risk from the disposal of high-level radioactive wastes in deep geologic formations, 993 (R;US)

Migration in a single fracture, 1342 (BA;US)

Migration of radionuclides with ground water: a discussion of the relevance of the input parameters used in model calculations, 1350 (BA;US)

Model for near field migration, 1343 (BA;US)

Model for far field migration, 1344 (BA;US)

Modeling approach to determine short- and long-term thermal and thermomechanical effects of waste emplacement in a repository in basalt, 1169 (BA;US)

National program for isolating high-level nuclear waste, 410 (J;GB)

National survey of crystalline rocks and recommendations of regions to be explored for high-level radioactive waste repository sites, 540 (R;US)

Native copper deposits of the Portage Lake volcanics, Michigan: their implications with respect to canister stability for nuclear waste isolation in the Columbia River basalts beneath the Hanford Site, Washington, 904 (R;US)

Nevada Nuclear-Waste-Storage Investigations. Quarterly report, April-June 1982, 757 (R;US)

Nevada nuclear waste storage investigations: briefing book (Yucca Mountain tuff), 572 (R;US)

Nevada Nuclear Waste Storage Investigations. Quarterly report, October-December 1982, 759 (R;US)

Nevada Nuclear Waste Storage Investigations. Quarterly report, January-March, 1983, 760 (R;US)

NNWSI regulatory status and plans, 1413 (RA;US)

Nuclear power on unstable ground, 1182 (BA;GB)

Nuclear-waste disposal in geologic repositories, 1014 (R;US)

Nuclear-waste repository in basalt: seal development studies, 887 (R;US)

Nuclear-waste disposal: technical issues and status, 979 (R;US)

Nuclear waste package program for high-level isolation in Nevada tuff, 1102 (J;US)

Nuclear waste management. Semiannual progress report, October 1982-March 1983, 303 (R;US)

Nuclear waste: what to do with it. Academy forum, 565 (R;US)

NWTS Field Testing In-Situ Site-Confirmation Tests Workshop, September 8-9, 1980, 802 (R;US)

NWTS program criteria for mined geologic disposal of nuclear waste: repository performance and development criteria. Public draft, 596 (R;US)

OECD Nuclear Energy Agency activities in high-level waste disposal, 591 (RA;US)

Overview of the National Waste Terminal Storage Program, 788 (RA;US)

Petrologic studies of drill cores USW-G2 and UE25b-1H, Yucca Mountain, Nevada, 696 (R;US)

Post-emplacement monitoring, Subtask 1.2. Draft Technical Position. Vol. 2, 1319 (R;US)

Potential host media for a high-level waste repository, 1132 (J;GB)

Predictive geology in nuclear-waste management, 1181 (BA;GB)

Probablistic approach to the assessment of the long-term risk linked to the disposal of radioactive waste in geological repositories, 1392 (J;GB)

Proceedings of the international seminar on chemistry and process engineering for high-level liquid waste solidification, 263 (R;DF;In German and English) 
Projected costs for mined geologic repositories for dispoal of commercial nuclear wastes, 769 (R;US)

Promotion of the research and development of high level radioactive waste treatment and disposal, 491 (I;JP;In Japanese)

Radionuclide retardation during transport through fractured granite, 1355 (BA;US)

Regional geological assessment of the Devonian-Mississippian shale sequence of the Appalachian, Illinois, and Michigan basins relative to potential storage/disposal of radioactive wastes, $828(R ; U S)$

Regulatory implementation considerations for deep geologic disposal of high-level radioactive wastes, 1094 (J;US)

Relationship of an in-situ test facility to a deep geologic repository for high-level nuclear waste. Final report (Task 4), June 1981-October 1982, 742 (R;US)

Repository design and equipment, 791 (RA;US)

Repository data base development, 801 (RA;US)

Repository design, 1134 (J;GB)

Repository and engineering barriers design, 584 (RA;US)

Research and development related to the Nevada Nuclear Waste Storage Investigations. Progress report, October 1-December 31, 1982, 704 (R;US)

Research priorities for isolation of defense high-level and transuranic wastes in the United States, 628 (R;US)

Review of DOE waste package program. Subtask 1.1. National waste package program, April-September 1982, 735 (R;US)

Review of radioactive waste management programs in the United Kingdom, 1160 (BA;US)

Risk-assessment methodology development for waste isolation in geologic mcdia, 727 (R;US)

Rock mass deformation properties of closely jointed basalt, 889 (R;US)

Satisfying performance assessment data needs, 1222 (J;US)

Scientific basis for nuclear waste management V, 1187 (B;US)

Selected hydrologic and geochemical issues in site characterization for nuclear waste disposal: flood basalts at the Hanford Reservation, 715 (R;US)

Selecting a repository site, 1133 (J;GB)

Site characterization report for the basalt waste isolation project. Volume I, 601 (R;US)

Site characterization report for the basalt waste isolation project. Volume II, 602 (R;US)

Site characterization report for the basalt waste isolation project. Volume III, 603 (R;US)

Site investigations for repositories for solid radioactive wastes in deep continental geological formations, $1139(\mathbf{B} ; \mathbf{X A})$

Some geochemical considerations for a potential repository site in tuff at Yucca Mountain, Nevada, 706 (R;US)

Status of high level and alpha bearing waste management in PNC, 42.8 (BA;IIS)

Status of geochemical problems relating to the burial of highlevel radioactive waste, 1982,1318 (R;US)

Status of geologic and hydrologic characterization of a potential nuclear waste repository site in basalt (Abstract only), 894 (R;US)

Status report on the full-scale electric heater tests at the Hanford Near-Surface Test Facility, 883 (R;US)

Stripa project, quarterly report October through December 1981, 655 (R;SE)

Study of radionuclide migration from deep-lying repository sites with over-lying sedimentary layers, 1348 (BA;US)

Suitability of natural geomedia for radwaste storage, 1060 (

Technical progress report for the quarter, 1 July-30 September 1981,776 (R;US)

Technical progress report for the quarter, 1 October-31 December, 1981, 777 (R;US)

Technical progress report for the quarter, 1 April-30 June 1982, 779 (R;US)

Technical progress report for the quarter 1 January-31 March 1982,778 (R;US)

Technical conservatism in the design and analysis of a nuclearwaste repository in basalt, 907 (R;US)
Technical assistance for regulatory development: review and evaluation of the draft EPA Standard 40CFR191 for disposal of high-level waste, 1415 (R;US)

Testimony of Dr. Raul A. Deju, Basalt Waste Isulation Project, before the Subcommittee on Energy Research and Production, Committee on Sceince and Technology, United States House of Representatives, March 2, 1983, 893 (R;US)

Thermal impact of waste emplacement and surface cooling associated with geologic disposal of nuclear waste, 707 (R;US)

Transient hydraulic tests in granite: fissured porous medium analysis and results, 1147 (BA;US)

Uncertainties in geologic disposal of high-level wastes: groundwater transport of radionuclides and radiological consequences, 737 (R;US)

Volume 2. A simplified analysis of a hypothetical repository in a basalt formation, 1416 (RA;US)

Volume 3. A simplified analysis of a hypothetical repository in a tuff formation, 1417 (RA;US)

Waste package performance assessment, $1274(\mathrm{BA} ; \mathrm{XN})$

Waste package conceptual designs for a repository located in basalt, 884 (R;US)

Waste package conceptual designs for a nuclear repository in basalt, 872 (R;US)

Waste package performance assessment: the importance of the very near-field physicochemical environment, 1223 (J;US)

Geological Surveys

Predictive geology: with emphasis on nuclear-waste disposal, 96 (J;US)

Status of geologic/environmental characterizations, 797 (RA;US)

\section{Geology}

Geological aspects of the final disposal of radioactive waste (Federal Republic of Germany), 1072 (J;DE;In German)

Hydrogeology of radioactive waste isolation: the challenge of a rational assessment, 1144 (J;US)

Global Aspects

Appraisal of a target-relevant evaluation of alternative strategies for global radioactive waste disposal (1980-2030), 645 (R;DE;In German)

Government Policies

National program for isolating high-level nuclear waste, 410 (J;GB)

Nuclear waste legislation: issues and impacts, 1033 (J;US)

Policy aspects of Federal-State relations, 1088 (J;US)

Quality assurance, 799 (RA;US)

Ground Disposal

Analysis of waste disposal practice and control at ORNL, 549 (R;US)

\section{Health Hazards}

Methodology for assessing the risk from the disposal of high-level radioactive wastes in deep geologic formations, 993 (R;US)

Summary of computer codes for radiological assessment, 1376 (R;US)

\section{Hearings}

Nuclear waste management comprehensive legislation: Bouquard/Lujan Proposal. Hearings before the Subcommittee on Energy Research and Production of the Committee on Science and Technology, US House of Representatives, Ninety-Seventh Congress, First Session, June 17.18; Ootober S, 7, 1981, 1210 (B;US)

Heat Transfer

Long-term thermohydrologic behaviour of nuclear-waste reposito ries, 1183 (BA;GB)

Peak high-level waste package temperature versus annular air gap width, 1046 (J;US)

High-level Radioactive Wastes

"Project Crystal" for ultimate storage of highly radioactive waste, 1049 (J;CH;In German)

1982 Subseabed Disposal Program. Annual report, site assessment October 1981 to September 1982, 987 (R;US)

Accident scenarios and probabilities for the SDP transportatior system, 956 (RA;US)

Actinide sorption on rock minerals, 677 (RA;DE)

Actinide sorption on rock minerals, 275 (RA;DE)

Actinide transmutation with a $U^{-} F_{6}$ reactor, 1048 (J;US) 
Alternate strategy for commercial high-level radioactive-waste management, 945 (R;US)

Analysis of diffusional ion transport in ocean sediments: subseafloor disposal of radioactive waste, 1250 (J;US)

Analysis of space systems for the space disposal of nuclear waste follow-on study. Volume 1. Executive summary, 718 (R;US)

Analysis of space systems for the space disposal of nuclear waste follow-on study. Volume 2. Technical report, 719 (R;US)

Analysis of waste-package behavior for high-level waste, 981 (R;US)

Analytical models for thermal rock mechanics, 1217 (B;GB)

Annotated bibliography for the design of waste packages for geologic disposal of spent fuel and high-level waste, 804 (R;US)

Annual report of the management system design group, 967 (RA;US)

Answers to your questions about high-level nuclear waste isolation, 1298 (B;US)

Application of systems analysis to the disposal of high level waste in deep ocean sediments, $1069(\mathrm{~J} ; \mathrm{GB})$

Appraisal of nuclear waste isolation in the vadose zone in arid and semiarid regions (with cmphasis on the Nevada Test Site), 714 (R;US)

Aspects of possible magmatic disruption of a high-level radiuactive waste repository in southern Nevada, 699 (R;US)

Assessment of the proposed rule (10CFR60) for disposal of highlevel radioactive wastes in geologic repositories. Volume I, 747 (R;US)

Assessment of overseas developments in methods for treatment and disposal of high-level radioactive wastes, $532(\mathrm{R} ; \mathrm{AU})$

Backfill barriers for nuclear waste repositories in salt, 670 (RA;DE)

Backfill barriers for nuclear waste repositories in salt, 680 (RA;DE)

Backfilling and sealing a repository for high-level radioactive waste - a review, 1279 (B;GB)

Barriers in a final storage for radioactive wastes, 1373 (RA;DE;In German)

Basalt waste-isolation project. Quarterly report, January 1-March 31, 1981, 908 (R;US)

Basalt Waste Isolation Project. Quarterly report, October 1, 1981-December 31, 1981, 895 (R;US)

Basalt waste isolation project. Quarterly report, April 1, 1981June 30, 1981, 909 (R;US)

Basalt waste-isolation project drilling and testing. Quarterly report, January 1-March 31, 1982, 896 (R;US)

Basalt Waste Isolation Project drilling and testing. Quarterly report, April 1, 1982-June 30, 1982, 897 (R;US)

Basalt Waste Isolation Project drilling and testing. Quarterly report, July 1, 1982-September 30, 1982, 898 (R;US)

Basalt Waste Isolation Project: drilling and testing. Quarterly report, October 1, 1982-December 31, 1982, 899 (R;US)

Borehole-to-borehole geophysical methods applied to investigations of high level waste repository sites, 1019 (R;US)

Burial effects on nuclear waste glass, 1202 (BA;US)

Calculation of the probability of volcanic disruption of a highlevel radioactive waste repository within southern Nevada, USA, 1393 (J;GB)

Canadian geoscience research for nuclear fuel waste management, 592 (RA;US)

Canister materials proposed for final disposal of high level niviclear wastc, 685 (RA;DE)

CEC project to assess high level waste disposal options, 1085 (J;GB)

Characterization of drained and undrained response of thermally loaded repository rocks, 1110 (J;US)

Chemical durability of some HLW glasses: effects of hydrothermal conditions and ionizing radiation, 1203 (BA;US)

Combined analytical model for preformance assessment of the waste package/geologic me.dium systems. 1170 (BA;US)

Commercial nuclear-waste research and development program. Quarterly report, July-September 1982, 578 (R;US)

Commercial nuclear waste research and development program. Annual report. fiscal year 1982,579 (R;US)
Commercial high-level-waste management: options and economics. A comparative analysis of the ceramic and glass waste forms, 150 (R;US)

Commercial Nuclear Waste Research and Development Program. Quarterly report, October-December 1982, 580 (R;US)

Commercial nuclear waste research and development program. Quarterly report, January-March 1983, 581 (R;US)

Commercial nuclear waste research and development program. Quarterly report, April-June 1983, 582 (R;US)

Comparative analysis of fractured and porous medium radionuclide transport, 1356 (BA;US)

Comparison of waste emplacement configurations for a nuclear waste respository in tuff. IV. Thermo-hydrological analysis, 996 (R;US)

Composition of rocks - criteria for the conditioning of high-level radioactive wastes, 672 (RA;DE;In German)

Concept of final storage in geological formations, 669 (RA;DE;GE)

Concept of a canister for high-level radioactive waste, 1158 ( $\mathrm{J} ; \mathrm{US})$

Conceptual design requirements for spent fuel, high-level waste, and transuranic waste packages, 903 (R;US)

Conceptual waste-package designs for disposal of nuclear waste in tuff, 818 (R;US)

Conceptual waste package interim performance specifications for waste forms for geologic isolation in salt repositories, 821 (R;US)

Conceptual waste package interim product specifications and data requirements for disposal of borosilicate glass defense high-level waste forms in salt geologic repositories, 823 (R;US)

Consequences of radiation effects on zeolites during interim storage, 1230 (J;US)

Container assessment - corrosion study of $H L W$ container materials. Quarterly progress report, April-June 1981, 729 (R;US)

Coordination meeting of high-level waste technology and national waste terminal storage programs, October 21-22, 1981, 624 (R;US)

Corrosion studies and mechanical tests on metallic materials for the design of packagings for vitrified high level wastes (HLW), 689 (R;DE;In German)

Corrosion of materials in a clay environment, 686 (RA;DE)

Corrosion studies on non-metallic inorganic materials for HLW packaging design, 691 (R;DE;In German)

Criteria for high level waste disposal and characterization of barriers, 1374 (RA;DE)

Criteria for high level waste disposal and characterization of barriers, 678 (RA;DE)

Deep underground storage of [radioactive waste]. Annual report, 1981,643 (R;DE;GE)

Defense waste management plan, 563 (R;US)

Deformation-dissolution potential of bedded salt, Waste Isolation Pilot Plant site, Delaware Basin, New Mexico, 1163 (BA;US)

Department of Energy does not plan to use an abandoned salt mine at Lyons, Kansas, for nuclear high-level waste disposal, 637 (R;US)

Design of a nuclear-waste package for emplacement in tuff, 1018 (R;US)

Determination of rock mass deformation modulus in closely jointed Columbia River basalt, 881 (R;US)

Determination of the corrosion mechanisms of high-level waste containing glass, 1204 (BA;US)

Development of an assessment methodology for the disposal of high-level radioactive waste into deep ocean sedimenis, 1115 (J;CH)

Development of structural barriers for containment of nuclear waste in basalt, 1100 (J;US)

Development of a backfill for containment of high-level nuclear waste, 1173 (BA;US)

Developments, recommendations and applications of tests for evaluating the chemical stability of high-level radioactive solid waste forms, $1056(\mathrm{~J} ; G B)$

Development of CT techniques for integrity evaluation of vitrified solids, 1045 (J;US)

Diffusion measurements in compacted bentonite, 1148 (BA;US) 
Diffusion in the matrix of granitic rock: field test in the Stripa mine, 1341 (BA;US)

Discharge of high activity and long half-life wastes arising from a nuclear power programme, 373 (J;BE; In French)

Disposal of high-level radioactive wastes by burial in the sea floor, 1129 (J;US)

Disposal of high-level radioactive wastes, 1257 (BA;AU)

Disposal of high-level radioactive wastes, 1286 (BA;GB)

Disposal of high-level radioactive wastes, $1116(\mathrm{~J} ; \mathrm{AU})$

Disposal of high-level nuclcar waste in rock-salt. A comparison of temperature increases calculated at ECN (Energieonderzoek Centrum Nederland)-Petten, RWTH (Rheinisch-Westfaelische Technische Hochschule Aachen)-Aachen and THD (Delft University of Technology)-Delft, 835 (R;NL)

Disposal of high level wastes, 1244 (J;JP;In Japanese)

Disposal of high-activity nuclear wastes, 1038 (J;GB)

Disposal of high-activity nuclear wastes in the oceans, 1050 (J;GB).

Dissolution of evaporites and its possible impact on the integrity of the Waste Isolation Pilot Plant (WIPP) New Mexico, USA, 1162 (BA;US)

Dissolution rate of salt domes on the basis of interpretation of measured salinity profiles, 1209 (BA;US)

DOE's program for high-level radioactive waste disposal, 1266 (BA;US)

Dose conversion factors used in the current Canadian high level waste disposal assessment study, 1427 (I;GB)

Draft site characterization analysis of the site characterization report for the Basalt Waste Isolation Project, Hanford, Washington Site. Main report and Appendices A through D, 724 (R;US)

Draft site characterization analysis of the site characterization report for the Basalt Waste Isolation Project, Hanford, Washington site. Appendices E through W, 725 (R;US)

Draft Technical Position Subtask 1.1: waste package performance after repository closure. Volume 1, 749 (R;US)

Dumping nuclear waste into the sea. International control and the role of science and law, $1057(\mathrm{~J} ; \mathrm{GB})$

Dumping of solid packaged radioactivity in the deep oceans, 1291 (BA;IN)

Earth Sciences, 789 (RA:US)

Earth science developments in support of waste isolation, 608 (RA;US)

Effect of microbial activity on the containment of radioactive waste in a decp geological repositury, 1357 (BA;US)

Effect of overpack materials on glass leaching in geological burial, 1200 (BA;US)

Effect of temperature un the leaching performance of SYNROC-B waste form, 1229 (J;US)

Effects of long-term exposure of tuffs to high-level nuclear wasterepository conditions. Preliminary report, 693 (R;US)

Effects of radiation on the leaching behavior of nuclear waste forms, 1285 ( $\mathrm{J}$;US $)$

Effects of radiation on corrosion of titanium, 961 (RA;US)

Effects of product properties on the final salt storage medium and the conditions producing it (In German), 673 (RA;DE;GE)

Effects of waste composition and loading on the chemical durabillity of a bofosilicate glass, 1 I 68 (DA;US)

Elution of radionuclides through columns of crushed rock from the Nevada Tcst Site, 1315 (R;US)

Engineered waste package concepts for high-level waste isolation in basalt, 874 ( $R ; \dot{U S})$

Engineered components for spent fuel radioactive waste isolation systems: are they technically justified, 1171 (BA;US)

Engineered waste-package conceptual design: defense high-level waste (Form 1), commercial high-level waste (Form 1), and spent fuel (Form 2) disposal in salt, 817 (R;US)

Engineered components for radioactive waste disposal systems are they technically justified, 861 (R;US)

Environmental evaluation of alternatives for long-term management of Defense high-level radioactive wastes at the Idaho Chemical Processing Plant, 70 (R;US)

Evaluation of research and development for terminal isolation of nuclear wastes, 701 (R;US)
Evaluation of barriers in a final storage site for radioactive wastes, 674 (RA;DE)

Evaluation of radionuclide transport: effect of radionuclide so. tion and solubility, 1354 (BA;US)

Expected near-field thermal environments in a sequentially loaded spent-fuel or high-level waste repository in salt, 830 (R;US)

Expected very-near-field thermal environments for advanced spent-fuel and defense high-level waste packages, 831 (R;US)

Far-field performance evaluations, 793 (RA;US)

Flow model for the kinetics of dissolution of nuclear waste forms; a comparison of borosilicate glass, SYNROC and high-silica glass, 1192 (BA;US)

Forced ventilation analysis of a commercial high-level nuclear repository in salt, 811 (R;US)

Fuel Cycle Conference Atomic Industrial Forum, Incorporated, 1267 (BA;US)

Gas production and liberation from rock salt samples and potenthal consequences on the disposal of high-level radioactive waste in salt domes, 1165 (BA;US)

Geohydrologic characterization and qualification of a high-level waste site in basalts, 891 (R;US)

Geologic aspects of disposal of highly radioactive nuclear waste. Open-File Report No. 15, 722 (R;US)

Oeulugle predictions and radioactive-waste disposal: a time limit for the predictive requirements, 1178 (BA;GB)

Geological status of NWTS repository siting activities in the paradox basin, 1265 (J;US)

Geological disposal of radioactive waste, 1113 (J;US)

Geological disposal of high-level radioactive waste - a review of the Institute of Geological Sciences' research programme, 1091 (J;GB)

Geological disposal of radioactive waste. Research in the OECD area, $1109(\mathrm{~B} ; \mathrm{XN})$

Geology of the Mors salt dome, 658 (RA;DK;In Danish)

Handling and disposing of radioactive waste, 561 (R;US)

HAW-containers as a barrier in final storage, 1432 (RA;DE; In German)

High-level radioactive waste isolation by incorporation in silicate rock, $1296(\mathrm{BA} ; \mathrm{XA})$

High-level-waste containment for a thousand years: unique technical and research problems (USA). 544 (R;US)

High-level waste disposalitechnical and institutional siting factors remaining to be resolved, 1087 (J;US)

High level waste disposal, 1289 (J;US)

High-level radioactive wastes, 159 (R;US)

High-level waste disposal, 1275 (J;US)

High-level radioactive wste management: a means to social consensus, 542 (R;US)

High-level radioactive-wasto-disposal investigations in Texas, 1001 (R;US)

High-level waste disposal, 1042 (J;US)

Hydrogeology of radioactive waste isolation: the challenge of a rational assessment, 1144 (J;US)

Hydrogeological conditions at Mors salt dome, 659 (RA;DK;In Danish)

Hydruthermal conditions around a radioactive waste repository, 1177 (BA;US)

Identifiction of a preferred site for the exploratory shaft within the reference repnsitnry losation: Hanford Site (Pasco Basin), 916 (R;US)

Illustrative nuclear waste disposal criteria and their application to high-level waste repository design, 1290 (J;US)

Impact of NRC licensing requirements on salt site selection, 588 (RA;US)

Implementation of technical conservatism in the development of nuclear waste repositories, 600 (R;US)

Importance of creep failure of hard rock in the near field of a nuclear waste repository, 1270 (BA;XN)

Importance of near-field phenomena for nuclide release from a flooded salt dome repository, 1396 (BA;US)

In situ and laboratory geotechnical test results from borehole GD-1 in southeast Utah, 813 (R;US) 
In-situ test programs related to design and construction of highlevel nuclear waste (HLW) deep geologic repositories. Final report (Task 2), June 1981-November 1982, 743 (R;US)

In-situ test programs related to design and construction of highlevel nuclear waste (HLW) deep geologic repositories. Appendices. Final report (Task 2), June 1981-November 1982, 744 (R;US)

Influence of a backfilling material on borosilicate glass leaching, 1197 (BA;US)

Interaction of borosilicate glass and granodiorite at $100^{\circ} \mathrm{C}, 50$ $\mathrm{MPa}$ : implications for models of radionuclide release, 1201 (BA;US)

Intercomparison of leach-testing methods and the effects of waste-form composition on test type and duration, 1157 (J;US)

INTRACOIN: an international nuclide transport code intercomparison study, 1358 (BA;US)

INTRACOIN, Level 1. Benchmark calculations with code SWIFT, 644 (R;DE)

Investigations of salt domes for the purpose of high-level radioactive waste disposal, 657 (R;DK;DA)

Investigation of environmentally assisted fracture of metallic nuclear-waste-package barrier materials in simulated basalt repository environments, 844 (R;US)

Investigation of diffusion of $\mathrm{UO}_{2} \mathrm{Cl}_{2}$ in saturated $\mathrm{NaCl}$ solutions at various temperatures, 1167 (BA;US)

Iodine-129 in high-activity nuclear wastes: an assessment of the deep-ocean disposal option, $1077(\mathrm{~J} ; \mathrm{CH})$

Ion/water migration phenomena in dense bentonites, 1174 (BA;US)

Isolation capability of a salt dome utilized for high-level waste disposal, 1208 (BA;US)

Isotopic fractionation of thorium and uranium upon leaching of monozite: alpha-recoil damage effects, 1214 (BA;US)

Laboratory determination of the thermal properties of large-scale samples of natural clays, 550 (R;IT;IT)

Laboratory studies of fluid flow through borehole seals, 1231 (J;US)

Leach characterization of cement encapsulated wastes, 1156 (J;US)

Leach testing of INEL waste forms in a gamma field, 1044 ( $\mathrm{J} ; \mathrm{US})$

Leaching behavior of a simple borosilicate glass in a confined environment, 1205 (BA;US)

Leaching of vitrified high-level radioactive waste, 1194 (BA;US)

Leaching of actinides from simulated nuclear waste glass, 1196 (BA;US)

Legislative and political aspects of waste disposal, 1409 (RA;US)

Legislative and political aspects of waste disposal, 1410 (RA;US)

Legislative and political aspects of waste disposal, 1411 (RA;US)

Licensing: ter.hnolngical implications for geologic disposal of high-level radioactive wastes, 1089 (J;US)

Licensing considerations for the NWTS program, 1227 (J;US)

Long-Term High-Level Defense-Waste technology. Progress report, April-June 1982, 338 (R;US)

Long-term radioactivity release from solidified high-level waste. Part I. An approach to evaluating experimental data, 1191 (BA;US)

Long-term thermomechanical and thermohydrological factors controlling the optimal deșign of a nuclear waste repository, 1064 (J;US)

Low-gradient permeability testing of fine-grained marine sediments, 1277 (J;US)

Management of high-level waste repository siting, 1071 (J;US)

Materials for high-level waste canister/overpacks in salt formations, 949 (R;US)

Materials for high-level waste canister/overpacks in salt formations, 1101 (J;US)

Mechanisms for hydrothermal leaching of nuclear waste glasses: properties and evaluation of surface layers, 1189 (BA;US)

Method of measurement and borehole probe to measure heatinduced borehole cunvergence, 1282 (P;DE;In German)

Methodology for assessing the risk from the disposal of high-level radioactive wastes in deep geologic formations, 993 (R;US)

Migration in a single fracture, 1342 (BA;US)
Migration of radionuclides with ground water: a discussion of the relevance of the input parameters used in model calculations, 1350 (BA;US)

Mineralogical and geochemical factors influencing the final disposal of HLW in the Stassfurt halite, 1164 (BA;US)

Model for near field migration, 1343 (BA;US)

Model for far field migration, 1344 (BA;US)

Modeling approach to determine short- and long-term thermal and thermomechanical effects of waste emplacement in a repository in basalt, 1169 (BA;US)

Models and risk from high-level waste disposal-the missing pieces, 1090 (J;US)

Monitoring an underground repository with modern seismological methods, 1238 (J;US)

Monitoring technologies for ocean disposal of radioactive waste, 717 (R;US)

National survey of crystalline rocks and recommendations of regions to be explored for high-level radioactive waste repository sites, 540 (R;US)

National Waste Terminal Storage Program. Technical progress report for the quarter 1 July-30 September 1982,780 (R;US)

National waste terminal storage conceptual reference repository description, 612 (RA;US)

Natural analogues: a way to increase confidence in predictions of long-term performance of radioactive waste disposal, 1061 (J;US)

Near-field repository conditions in basalt and salt, 733 (RA;US)

Nevada Nuclear Waste Storage Investigations. Quarterly report, January-March, 1983, 760 (R;US)

NNWSI regulatory status and plans, 1413 (RA;US)

Nuclear fuel waste disposal - status of the Canadian program, 1263 (J;US)

Nuclear-fuel-cycle education: Module 8. Waste management, 155 (R;US)

Nuclear trash. What do we do, 1078 (J;AU)

Nuclear-waste-management. Semiannual progress report, October 1981-March 1982, 301 (R;US)

Nuclear-waste disposal in geologic repositories, 1014 (R;US)

Nuclear-waste disposal: technical issues and status, 979 (R;US)

Nuclear waste package program for high-level isolation in

Nevada tuff, 1102 (J;US)

Nuclear waste disposal: a case of benign neglect>, 1108 (J;US)

Nuclear waste management. Quarterly progress report, October through December 1980, 296 (R;US)

Nuclear Waste Policy Act of 1982; proposed general guidelines for recommendation of sites for nuclear waste repositories, 1051 (J;US)

Nuclear waste management comprehensive legislation: Bouquard/Lujan Proposal. Hearings before the Subcommittee on Energy Research and Production of the Committee on Science and Technology, US House of Representatives, Ninety-Seventh Congress, First Session, June 17-18; October 5, 7, 1981, 1210 (B;US)

Nuclear waste management. Semiannual progress report, October 1982-March 1983, 303 (R;US)

Nuclear waste-form risk assessment for US defense waste at Savannah River Plant. Annual report FY, 1982, 1008 (R;US)

Nuclear waste legislation: issues and impacts, 1033 (J;US)

Nuclear waste: what to do with it. Academy forum, 565 (R;US)

NWTS Field Testing In-Situ Site-Confirmation Tests Workshop, September 8-9, 1980, 802 (R;US)

NWTS program criteria for mined geologic disposal of nuclear waste: repository performance and development criteria. Public draft, 596 (R;US)

NWTS program waste projection data needs, $1086(\mathrm{~J} ; \mathrm{US})$

NWTS program criteria for mined geologic disposal of nuclear waste: functional requirements and performance criteria for waste packages for solidified high-level waste and spent fuel, 597 (R;US)

NWTS waste package program plan. Volume I. Program strategy, description, and schedule, 598 (R;US)

NWTS waste package program plan. Volume II. Prograrn lugic networks, 599 (R;US)

OECD Nuclear Energy Agency activities in high-level waste disposal, 591 (RA;US) 
Overview of the National Waste Terminal Storage Program, 788 (RA;US)

Overview of nuclear waste disposal in space, 616 (RA;US)

Pacific site assessment, 960 (RA;US)

Pacing and logistics of high-level waste disposal, 1047 (J;US)

Package design, 796 (RA;US)

Package materials, waste form, 792 (RA;US)

Packing material testing required to demonstrate compliance with 1000-year radionuclide containment. Semiannual report on waste package verification tests, 739 (R;US)

Paleomagnetic study of areas B1, C1 and E2, 959 (RA;US)

Parameters and variables appearing in repository-siting models, 745 (R;US)

Parametric study of the effects of thermal environment on a waste package for a tuff repository, $1271(\mathrm{BA} ; \mathrm{YN})$

Peak high-level waste package temperature versus annular air gap width, 1046 (J;US)

Performance allocation traceable to regulatory criteria as applied to site-characterization work at the Basalt Waste Isolation Project, 890 (R,U3)

Performance assessment in the Nevada Nuclear Waste Storage Investigations, 1224 (J;US)

Petrologic studies of drill cores USW-G2 and UE25b-1H, Yucca Mountaiiı, Nevada, 696 (R;US)

Policy aspects of Federal-State relations, 1088 (J;US)

Possibility of high level waste underground disposal, 1249 (J;JP;In Japanese)

Post-closure performance assessment methodology for the Office of Nuclear Waste Isolation, 1219 (J;US)

Post-emplacement monitoring, Subtask 1.2. Draft Technical Position. Vol. 2, 1319 (R;US)

Preliminary results of gravity investigations at Yucca Mountain and vicinity, Southern Nye County, Nevada, 1024 (R;US)

Preliminary environmental implications of alternatives for decommissioning and future use of the Westem New York Nuclear Services Center, 1301 (R;US)

Preliminary risk assessment for nuclear waste disposal in space. Volume I. Executive summary of technical report, 720 (R;US)

Preliminary risk assessment for nuclear waste disposal in space. Volume II. Technical report, 721 (R;US)

Probablistic approach to the assessment of the long-term risk linked to the disposal of radioactive waste in geological repositories, $1392(\mathrm{~J} ; \mathrm{GB})$

Proceedings of the symposium on uncertainties associated with the regulation of the geologic disposal of high-level radioactive waste, 726 (R;US)

Proceedings of the international seminar on chemistry and process engineering for high-level liquid waste solidification, 226 (R;DE;In several languages)

Proceedings of the 1980 National Waste Terminal Storage Program information meeting, 787 (R;US)

Proceedings of the international seminar on chemistry and pro cess engineering for high-level liquid waste solidification, 263 (R;DE;In German and English)

Program summary. Nuclear waste management and fuel cycle programs, 575 (R;US)

Projected costs for mined geologic repositories for dispoal of commercial nuclear wastes, 769 (R;US)

Promotion of the research and development of high level radioactive waste treatment and disposal, 491 (J;JP;In Japanese)

Quality assurance, 799 (RA;US)

$\mathbf{R}$ and $\mathbf{D}$ projects and studies: concerning the interim and terminal storage of radioactive wastes, 1281 (TG;US)

Radiant energy dissipation during final storage of high-level radioactive waste in rock salt, 867 (R;DE;In German)

Radioactive wastes, 382 (J;AT; In German)

Radioactive waste management, 527 (BA;SU;In Russian)

Radioactive waste disposal options for the operation of nuclear power stations, 664 (RA;IL)

Radiological risk analysis of an operating high-level waste repository, 1395 (BA;XA)

Radiological impact of disposal of UK solid radioactive wastes, present and future, $1081(\mathrm{~J} ; \mathrm{GB})$

Radiolysis of ground water from HLW stored in copper canisters, 999 (R:SE)
Radionuclide retardation during transport through fractured granite, 1355 (BA;US)

Radwaste paradox, 1054 (J;US)

Recent advances in repository seal materials, 1172 (BA;US)

Regional geological assessment of the Devonian-Mississippian shale sequence of the Appalachian, Illinois, and Michigan basins relative to potential storage/disposal of radioactive wastes, 828 (R;US)

Regulating the development of a waste repository, $1130(\mathrm{~J} ; \mathrm{GB})$

Regulation of geological disposal of high-level radioactive waste, 1241 (J;US)

Regulatory policy of the EPA related to alpha-contaminated waste, 1412 (RA;US)

Relationship of an in-situ test facility to a deep geologic repository for high-level nuclear waste. Final report (Task 4), June 1981-October 1982, 742 (R;US)

Relationship between glass leaching mechanism and geochemical transport of radionuclides, 1195 (BA;US)

Report on financing the disposal of commercial spent nuclear fuel and processed high-level radioactive wastc, 21 (R;US)

Report on financing the disposal of commercial spent nuclear fuel and processed high-level radioactive waste, 604 (R;US)

Repository design and equipment, 791 (RA;US)

Repository data base, field tests, 795 (RA;US)

Repository and engineering barricrs tesign, 584 (RA;US)

Repository safety-the salient facts, 1397 (J;US)

Requirements for the disposal of high-level radioactive wastes (Federal Republic of Germany), 671 (RA;DE)

Requirements for the disposal of high-level radioactive wastes, 681 (RA;DE)

Research priorities for isolation of defense high-level and transuranic wastes in the United States, 628 (R;US)

Results of repository conditions study for commercial and defense high-level nuclear waste and spent fuel repositories in salt, 824 (R;US)

Review of radio-nuclide migration in geological formations, 1337 (J;GB)

Review of DOE waste package program. Subtask 1.1. National waste package program, April-September 1982, 735 (R;US)

Review of radioactive waste management programs in the United Kingdom, 1160 (BA;US)

Risk-analysis methodology for spent-fuel repositories in bedded salt. Final report, 731 (R;US)

Risk-assessment methodology development for waste isolation in geologic media, 727 (R;US)

Rock-melt concept for nuclear waste disposal, 1254 (J;US)

Role of oxidation potential, $\mathrm{Eh}$, in the dissolution of nuclear waste materials, 851 (RA;US)

Salt domes - the past and present, keys to the future, 1179 (BA;GB)

SALTMP - a FORTRAN prugram fur fast salt temperature calculations in HRW final storage, 866 (R;DE;In German)

Satisfying performance assessment data needs, 1222 (J;US)

Scientific basis for nuclear waste management V, 1187 (B;US)

Scientific data necessary to predict radionuclide migration within or near a mined nuclear repository, 1312 (R;US)

Seal for underground ultimate storagc sites for radioactive waste, and method of installing the seal, 1284 (P;DE;In German)

Second annual report made to the Secretary of State for the Environment, Secretary of State for Scotland, Secretary of State for Wales, 117 (B;GB)

Selected hydrologic and geochemical issues in site characterization for nuclear waste disposal: flood basalts at the Hanford Reservation, 715 (R;US)

Shielded containers for waste could prove beneficial, 1128 (J;GB)

Site characterization report for the basalt waste isolation project. Volume I, 601 (R;US)

Site characterization report for the basalt waste isolation projec Volume II, 602 (R;US)

Site characterization report for the basalt waste isolation projec Volume III, 603 (R;US)

Site investigations for repositories for solid radioactive wastes in deep continental geological formations, 1139 (B;XA)

Site qualification activities, 958 (RA;US) 
Sociopolitical aspects of a subseabed disposal program for high level nuclear waste, 965 (RA;US)

Solubility-limited fractional dissolution rate of vitrified waste in groundwater, 1228 (J;US)

Some geochemical considerations for a potential repository site in tuff at Yucca Mountain, Nevada, 706 (R;US)

Stability and alteration of naturally occurring low-silica glasses: implications for the long term stability of waste form glasses, 1190 (BA;US)

State of Wisconsin Ad Hoc committee on radioactive waste management. Final report, 568 (R;US)

Status of geologic/environmental characterizations, 797 (RA;US)

Status of high level and alpha bearing waste management in PNC, 428 (BA;US)

Status of geochemical problems relating to the burial of highlevel radioactive waste, 1982, 1318 (R;US)

Strategy document: long-term technolugy program for defense high-level waste, 156 (R;US)

Strontium-basalt reactions under nuclear waste repository conditions, 1152 (BA;US)

Studies of waste-canister compatibility, 832 (R;US)

Study of radionuclide migration from deep-lying repository sites with over-lying sedimentary layers, 1348 (BA;US)

Study of the isolation system for geologic disposal of radioactive wastes, 577 (R;US)

Submarine geologic disposal of nuclear waste, 1253 (J;US)

Subseabed disposal program. Annual report, January-December 1980. Volume 1. Summary, 935 (R;US)

Subseabed disposal of nuclear wastes, 1243 (J;US)

Subseabed disposal program annual report, January-September 1981. Volume II. Appendices (principal investigator progress reports). Part 1, 954 (R;US)

Subseabed Disposal Program. Annual report, January-September 1981. Volume I: summary, 953 (R;US)

Summary of research and development for disposal of US Defense wastes in the Waste Isolation Pilot Plant (WIPP), 983 (R;US)

Summary report on the geochemistry of Yucca Mountain and environs, 700 (R;US)

Swedish nuclear waste efforts, 36 (R;US)

Synroc technology for immobilizing U.S. defense waste, 400 (J;US)

Systems, 790 (RA;US)

Systems study of the feasibility of high-level nuclear-waste fractionation for thermal-stress control in a geologic repository: main report, 308 (R;US)

Systems study of the feasibility of high-level nuclear waste fractionation for thermal stress control in a geologic repository: appendices, 309 (R;US)

Technical progress report for the quarter, 1 July-30 September 1981,776 (R;US)

Technical progress report for the quarter, 1 October-31 December, 1981, 777 (R;US)

Technical progress report for the quarter, 1 April-30 June 1982, 779 (R;US)

Technical progress report for the quarter 1 January-31 March 1982, 778 (R;US

Technical assistance for regulatory development: review and cvaluation of the EPA standard 40 CFR191 for disposal of highlevel waste. Vol. 1, 750 (R;US)

Technical conservatism in the design and analysis of a nuclearwaste repository in basalt, 907 (R;US)

Technical assistance for regulatory development: review and evaluation of the draft EPA Standard 40CFR191 for disposal of high-level waste, 1415 (R;US)

Technology of systems, 660 (RA;DK;In Danish)

Technology of high-level nuclear waste disposal. Advances in the science and engineering of the management of high-level nuclear wastes. Volume $1,607(\mathrm{R} ; \mathrm{US})$

Testimony of Dr. Raul A. Deju, Basalt Waste Isolation Project, before the Subcommittee on Energy Research and Production, Committee on Sceince and Technology, United States House of Representatives, March 2, 1983, 893 (R;US)

Thermal analysis of nuclear waste emplacement in welded tuff, 930 (R;US)
Thermal impact of waste emplacement and surface cooling associated with geologic disposal of nuclear waste, 707 (R;US)

Thermal response to emplacement of nuclear waste in long, horizontal boreholes, 986 (R;US)

Thermomechanical in-situ experiments and finite element computations, 1166 (BA;US)

Thermomechanical modeling and the selection of a salt repository site, $1218(\mathrm{~J} ; \mathrm{US})$

Transient hydraulic tests in granite: fissured porous medium analysis and results, 1147 (BA;US)

Transport phenomena of water and gas components within rock salt in the temperature field of disposed high level waste, 1272 (BA; XN)

UK view of the management of high-level waste, 1103 (J;XA)

Uncertainties in geologic disposal of high-level wastes: groundwater transport of radionuclides and radiological consequences, 737 (R;US)

Underground disposal of high active waste, 665 (R;DE)

United States steps up waste isolation programme, 1074 (J;GB)

US Geological Survey research in radioactive waste disposal, fiscal year 1979, 716 (R;US)

Volume 2. A simplified analysis of a hypothetical repository in a basalt formation, 1416 (RA;US)

Volume 3. A simplified analysis of a hypothetical repository in a tuff formation, 1417 (RA;US)

Volume 4. A simplified analysis of a hypothetical repository in a bedded salt formation, 1418 (RA;US)

WAPPA: a Waste Package Performance Assessment code. Technical report, 819 (R;US)

Waste glass - seabed interactions: observations of the Soret effect, 850 (RA;US)

Waste glass/repusitory interactions, 1193 (BA;US)

Waste Isolation Pilot Plant (WIPP) research and development program: in situ testing plan, March 1982, 940 (R;US)

Waste Management Program. Technical progress report, October-December 1980, 162 (R;US)

Waste migration by particulate carriers, 1365 (J;US)

Waste-Mixes Study for space disposal, 815 (R;US)

Waste package performance assessment, 1274 (BA;XN)

Waste package conceptual designs for a repository located in basalt, 884 (R;US)

Waste package conceptual designs for a nuclear repository in basalt, 872 (R;US)

Waste package conceptual designs for a repository located in basalt, 585 (RA;US)

Waste package performance assessment: the importance of the very near-field physicochemical environment, 1223 (J;US)

Waste packages for high-level waste repositories, 1043 (J;US)

Waste treatment and disposal. Progress report, May-October 1963, 291 (R;US)

Water-dilution volume for high-level wastes stored in deep geologic media, 1232 (J;US)

West Valley Demonstration Project: strategy for an achievable but challenging project, 434 (BA;US)

WIPP research and development program: providing the technical basis for defense waste disposal, 989 (R;US)

Hydrology

Disposal of high-level nuclear waste from nuclear power plants in Denmark. Salt dome investigations. V.2. Geology, figures, 649 (R;DK)

Hydrogeology of radioactive waste isolation: the challenge of a rational assessment, 1144 (J;US)

Information Needs

High-Level Nuclear-Waste Management. Oversight Hearing before the Subcommittee on Energy and the Environment of the Committee on Interior and Insular Affairs, House of Representatives, Ninety-Seventh Congress; Second Session, June $17,1982,110$ (B;US)

Institudional Factors

Building the institutional capacity for managing commercial high-level radioactive waste, 59 (R;US)

Proceedings of the 1980 National Waste Terminal Storage Program information meeting, 787 (R;US) 
Intermediate-level Radioactive Wastes

Appraisal of a target-relevant evaluation of alternative strategies for global radioactive waste disposal (1980-2030), 645 (R;DE;In German)

Barriers in a final storage for radioactive wastes, 1373 (RA;DE;In German)

Decay calculations on medium-level and actinide-containing wastes from the LWR fuel cycle. Pt. 2, 688 (R;DE;In German)

Deep underground storage of [radioactive waste]. Annual report, 1981, 643 (R;DE;GE)

Leach characterization of cement encapsulated wastes, 1156 (J;US)

Low level and intermediate level wastes management practice in France, 442 (BA;US)

Policy and development of reprocessing of spent nuclear fuel and high-level radioactive waste management in the F.R. Germany, 13 (J;JP;In Japanese)

Radioactive wastes, 382 (J;AT;In German)

Radiological impact of disposal of UK solid radioactive wastes, present and future, $1081(\mathrm{~J} ; \mathrm{GB})$

Review of radioactive waste management programs in the United Kingdom, 1160 (BA;US)

Rock-melt concept for nuclear waste disposal, 1254 (J;US)

Scientific basis for nuclear waste management V, 1187 (B;US)

International Cooperation

Stripa project, quarterly report October through December 1981, $655(R ; S E)$

Iodine 129

Fuel cycle programs. Quarterly progress report, April-June 1982 .536 (R;US)

Fuel cycle programs. Quarterly progress report, July-September 1982, 538 (R;US)

Fuel cycle programs. Quarterly progress report, OctoberDecember 1982, 539 (R;US)

Krypton 85

Fuel cycle programs. Quarterly progress report, April-June 1982 , 536 (R;US)

Fuel cycle programs. Quarterly progress report, July-September 1982,538 (R;US)

Fuel cycle programs. Quarterly progress report, OctoberDecember 1982, 539 (R;US)

Nuclear-waste management semiannual progress report, April 1982-September 1982, 302. (R; I IS)

Nuclear waste management. Semiannual progress report, Octobef 1982-March 1983, 303 (R;US)

Laws

Radioactive waste management: a summary of state laws and administration. National Low-Level Radioactive Waste Mangement Program, 569 (R;US)

Radioactive waste management: a summary of state laws and administration, 570 (R;US)

Legal Aspects

Dumping nuclear waste into the sea: international control and the role of science and law, 1055 (J;GB)

Legislation

Legislative and political aspects of waste disposal, 1409 (RA;US)

Legislative and political aspects of waste disposal, 1410 (RA;US)

Nuclear waste management comprehensive legislation: Bouquard/Lujan Proposal. Hearings before the Subcommittee on Energy Research and Production of the Committee on Science and Technology, US House of Representatives. Ninety-Seventh Congress, First Session, June 17-18; October 5, 7, 1981, 1210 (B;US)

Nuclear waste legislation: issues and impacts, 1033 (J;US)

Radioactive waste management: a summary of state laws and administration. National Low-Level Radioactive Waste Mangement Program, 569 (R:US)

Radioactive waste management: a summary of state laws and administration, 570 (R;US)

Licensing

Licensing: technological implications for geologic disposal of high-level radioactive wastes, 1089 (J;US)

NNWSI regulatory status and plans, 978 (R;US)

Regulating the development of a waste repository, 1130 (J;GB)
Liquid Wastes

Promotion of the research and development of high level radioactive waste treatment and disposal, 491 (J;JP;In Japanese)

Low-level Radioactive Wastes

Analysis of waste disposal practice and control at ORNL, 549 (R;US)

Appraisal of a target-relevant evaluation of alternative strategies for global radioactive waste disposal (1980-2030), 645 (R;DE;In German)

Benefit-cost-risk analysis of alternatives for greater-confinement disposal of radioactive waste, 559 (R;US)

Decontaminated salt disposal as saltcrete in a landfill. Technical data summary, 636 (R;US)

Deep underground storage of [radioactive waste]. Annual report, 1981, 643 (R;DE;GE)

Disposal of Savannah River Plant waste salt, 625 (R;US)

Handling and disposing of radioactive waste, 561 (R;US)

Long-Term High-Level Defense-Waste technology. Progress report, April-June 1982, 338 (R;US)

Low level and intermediate level wastes management practice in France, 442 (BA;US)

Nuclear-fuel-cycle education: Module 8. Waste management, 155 (R;US)

Nuclear waste disposal: a case of benign neglect $>, 1108$ (J;US)

Policy and development of reprocessing of spent nuclear fuel and high-level radioactive waste management in the F.R. Germany, 13 (J;JP;In Japanese)

Radioactive wastes, 382 (J;AT;In German)

Radioactive waste disposal options for the operation of nuclear power stations, 664 (RA;IL)

Radioactive waste management: a summary of state laws and administration. National Low-Level Radiuactive Waste Mangement Program, 569 (R;US)

Radioactive waste management: a summary of state laws and administration, 570 (R;US)

Radiological impact of disposal of UK solid radioactive wastes, present and future, 1081 (J;GB)

Report of the Low-Level Nuclear Waste Subcommittee, 1000 (R;Ú)

Review of radio-nuclide migration in geological formations, 1337 $(\mathrm{J} ; \mathrm{GB})$

Review of radioactive waste management programs in the United Kingdom, 1160 (BA;US)

Rock-melt concept for nuclear waste dispnsal, 1254 (J;US)

Scientific basis for nuclear waste management V, 1187 (B;US)

State of Wisconsin Ad Hoc committee on radioactive waste management. Final report, 568 (R;US)

Waste treatment and disposal. Progress report, May-October 1963, 291 (R;US)

Marine Disposal

Accident scenarios and probabilities for the SDP transportation system, 956 (RA;US)

Analysis of geological and geophysical data from study locations $C_{1}, B_{1}$, and $E_{2}$ in the northwest PAC 1 study region, 957 (RA;US)

Annual report of the management system design group, 967 (RA;US)

Application of systems analysis to the disposal of high level waste in deep ocean sediments, 1069 (J;GB)

Assessment of overseas developments in methods for treatment and disposal of higb-level radioactive wastes, 532 (R;AU)

Can the oceans provide acceptable disposal sites for high-level radioactive waste, 1287 (BA;GB)

Constant temperature and thermal gradient investigations of the near field subseabed environment: chemical and thermodiffusional effects, 971 (R;US)

Development of an assessment methodology for the disposal of high-level radioactive waste into deep ocean sediments, 1115 $(\mathrm{J} ; \mathrm{CH})$

Diffusional transport of radionuclide chains in sub-seabeds, $134 \mathrm{C}$ (J;GB)

Disposal of high-level radioactive wastes by burial in the sea noor, 1129 (J;US)

Disposal of high-activity nuclear wastes, $1038(\mathrm{~J} ; \mathrm{GB})$ 
Disposal of high-activity nuclear wastes in the oceans, 1050 (J;GB)

Dumping nuclear waste into the sea. International control and the role of science and law, 1057 (J;GB)

Dumping of solid packaged radioactivity in the deep oceans, 1291 (BA;IN)

Effects of radiation on corrosion of titanium, 961 (RA;US)

European Communities' $R$ and $D$ activities on treatment and handling of radioactive waste, 426 (BA;US)

Geotechnical studies for subseabed disposal: high level radioactive wastes, 964 (RA;US)

Iodine-129 in high-activity nuclear wastes: an assessment of the deep-ocean disposal option, $1077(\mathrm{~J} ; \mathrm{CH})$

Low-gradient permeability testing of fine-grained marine sediments, 1277 (J;US)

Monitoring technologies for ocean disposal of radioactive waste, 7.17 (R;US)

National long-term high-level waste-technology program, 67 (R;US)

Near-field interactions, 929 (R;US)

Ocean-bottom disposal of fission-product krypton-85, 1251 (BA;JP)

Pacific site assessment, 960 (RA;US)

Paleomagnetic study of areas B1,C1 and E2, 959 (RA;US)

Proceedings of the 1981 subseabed disposal program. Annual workshop, 939 (R;US)

Program criteria for subseabed disposal of radioactive waste: site qualification plan, 934 (R;US)

Public education and participation, 966 (RA;US)

$R$ and $D$ projects and studies: concerning the interim and terminal storage of radioactive wastes, 1281 (TG;US)

Report of the first interim meeting of the Seabed Working Group Engineering Studies Task Group, 944 (R;US)

Review of radioactive waste management programs in the United Kingdom, 1160 (BA;US)

Site qualification activities, 958 (RA;US)

Sociopolitical aspects of a subseabed disposal program for high level nuclear wastc, 965 (RA;US)

Status of radioactive waste management: needs for reassessment, 1248 (J;GB)

Submarine geologic disposal of nuclear waste, 1253 (J;US)

Subseabed disposal program. Annual report, January-December 1980. Volume 1. Summary, 935 (R;US)

Subseabed disposal of nuclear wastes, 1243 (J;US)

Subseabed disposal program annual report, January-September 1981. Volume II. Appendices (principal investigator progress reports). Part 1, 954 (R;US)

Subseabed disposal safety analysis, 955 (RA;US)

Subseahed disposal program annual report. January-September 1981. Volume II. Appendices (principal investigator progress reports). Part 2, 962 (R;US)

Subseabed Disposal Program. Annual report, January-September 1981. Volume I: summary, 953 (R;US)

Systems overview of the Subseabed Disposal Program, 976 (R;US)

UK view of the management of high-level waste, $1103(\mathrm{~J} ; \mathrm{XA})$

Waste glass - seabed interactions: observations of the Soret effect, 850 (RA;US)

Materials Testing

Analysis of the corrosion products formed on $\mathrm{Ti}$ and a Ti-Pd alloy during exposure in hot water, 1176 (BA;US)

Container assessment: corrosion study of HLW container materials. Quarterly progress report, January-March 1982, 730 (R;US)

Corrosion resistance of metal materials for $\mathrm{HLW}$ canister, 666 (R;JP;In Japanese)

Nuclear waste package materials testing report: basaltic and tuffaceous environments, 854 (R;US)

Selection of barrier metals for a waste package in tuff, 1020 (R;US)

Mathematical Models

Post-closure performance assessment methodology for the Office of Nuclear Waste Isolation, 1219 (I;US)
Matrix Materials

FUETAP concrete - an alternative radioactive waste host, 268 (RA;DE)

Measuring Instruments

Results of instrument reliability study for high-level nuclear waste repositories, 93 (J;US)

Meetings

Coordination meeting of high-level waste technology and national waste terminal storage programs, October 21-22, 1981, 624 (R;US)

Hydrology and Geology Overview Committee reports and responses from the Basalt Waste Isolation Project, 914 (R;US)

International fuel-cycle and waste-management technologyexchange activities sponsored by the United States Department of Energy: FY 1982 evaluation report, 85 (R;US)

NWTS Field Testing In-Situ Site-Confirmation Tests Workshop, September 8-9, 1980, 802 (R;US)

Proceedings of the symposium on uncertainties associated with the regulation of the geologic disposal of high-level radioactive waste, 726 (R;US)

Proceedings of the 1981 subseabed disposal program. Annual workshop, 939 (R;US)

Proceedings of the 1980 National Waste Terminal Storage Program information meeting, 787 (R;US)

Proceedings of the ANS topical meeting on the treatment and handling of radioactive wastes, 425 (B;US)

Scientific basis for nuclear waste management V, 1187 (B;US)

Mill Tailings

Handling and disposing of radioactive waste, 561 (R;US)

Nuclear waste disposal: a case of benign neglect>, 1108 (J;US)

Nuclear waste management. Semiannual progress report, October 1982-March 1983, 303 (R;US)

Program summary. Nuclear waste management and fuel cycle programs, 575 (R;US)

US Geological Survey research in radioactive waste disposal, fiscal year 1979, 716 (R;US)

Monitoring

Post-emplacement monitoring, Subtask 1.2. Draft Technical Position. Vol. 2, 1319 (R;US)

Results of instrument reliability study for high-level nuclear waste repositories, 93 (J;US)

Multi-parameter Analysis

Preliminary thermal analyses for a nuclear waste repository in tuff, 931 (R;US)

Packaging

Annotated bibliography for the design of waste packages for geologic disposal of spent fuel and high-level waste, 804 (R;US)

Combined analytical model for preformance assessment of the waste package/geologic medium systems, 1170 (BA;US)

Conceptual waste-package designs for disposal of nuclear waste in tuff, 818 (R;US)

Coordination meeting of high-level waste technology and national waste terminal storage programs, October 21-22, 1981, 624 (R;US)

Draft Technical Position Subtask 1.1: waste package performance after repository closure. Volume 1,749 (R;US)

Engineered waste-package-system design specification, 816 (R;US)

Expected very-near-field thermal environments for advanced spent-fuel and defense high-level waste packages, 831 (R;US)

Impacts of transportation on a test and evaluation facility for nuclear waste disposal: a systems analysis, 556 (R;US)

Interim performance specifications for conceptual waste-package designs for geologic isolation in salt repositories, 822 (R;US)

Long-term performance of materials used for high-level waste packaging. Quarterly report, April-June 1983, 752 (R;US)

Materials for high-level waste canister/overpacks in salt formations, 949 (R;US)

Materials for high-level waste canister/overpacks in salt formations, 1101 (J;US)

National waste terminal storage conceptual reference repository description, 612 (RA;US)

Near-ficld performance evaluations, 800 (RA;US)

Nuclear waste package program for high-level isolation in Nevada tuff, 1102 (J;US) 
Nuclear waste package materials testing report: basaltic and tuffaceous environments, 854 (R;US)

NWTS Field Testing In-Situ Site-Confirmation Tests Workshop, September 8-9, 1980, 802 (R;US)

NWTS program criteria for mined geologic disposal of nuclear waste: functional requirements and performance criteria for waste packages for solidified high-level waste and spent fuel, 597 (R;US)

Overview of the National Waste Terminal Storage Program, 788 (RA;US)

Package design, 796 (RA;US)

Package materials; waste form, 792 (RA;US)

Proceedings of the 1980 National Waste Terminal Storage Program information meeting, 787 (R;US)

Review of DOE waste package program. Subtask 1.1. National waste package program, April-September 1982, 735 (R;US)

Review of DOE waste-package program. Subtask 1.1. National waste-package program, September 1981-March 1982. Volume 2, 732 (R;US)

Testing of candidate waste package backfill and canister materiuls for buzult, 588 (RA;US)

WAPPA: a Waste Package Performance Assessment code. Technical report, 819 (R;US)

Waste package conceptual designs for a repository located in basalt, 884 (R;US)

Waste package performance evaluation, 806 (R;US)

Waste package conceptual designs for a repository located in basalt, 585 (RA;US)

Waste package conceptual designs for salt repositories, 593 (RA;US)

Waste package materials testing for a salt repository: 1982 status report, 825 (R;US)

Waste packages for high-level waste repositories, 1043 (J;US)

Performince

Post-closure performance assessment methodology for the Office of Nuclear Waste Isolation, $1219(\mathrm{~J} ; \mathrm{US})$

\section{Permit Applications}

DOE's program for high-level radioactive waste disposal, 1266 (BA;US)

\section{Planning}

Pacing and logistics of high-level waste disposal, 1047 (J;US)

Report of the Ad Hoc Committee on Reprocessing and Wastes of the Sub-Committee on Nuclear Power of Committee on Overall Energy Study, 485 (J;JP; In Japanese)

\section{Political Aspects}

Dumping nuclear waste into the sea: international control and the role of science and law, 1055 ( $\mathrm{J} ; \mathrm{GB})$

Legislative and political aspects of waste disposal, 1409 (RA;US)

Legislative and political aspects of waste disposal, 1410 (RA;US)

Legislative and political aspects of waste disposal, 1411 (RA;US)

Radwaste paradox, 1054 (J;US)

Sociopolitical aspects of a subseabed disposal program for high level nuclear waste, 965 (RA;US)

Program Management

Project management plan for exploratory shaft at Yucca Mountain. 764 (R:US)

Public Information

Information office concept, 594 (RA;US)

Proposal for broader participation in radioactive waste management, 1135 (J:GB)

Public education and participation, 966 (RA;US)

Public Opinion

Gcologic disposal and the taxpayer: the problem of the experts, 1403 (BA;IN)

Public Policy

Conflicting views on a neutrality criterion for radioactive-waste management. 95 (BA:US)

Proposal for broader participation in radioactive waste management. 1135 (J:GB)

Public Retations

Proposal for broader participation in radioactive waste management. 1135 (J:GB)
Quality Assurance

Quality assurance, 799 (RA;US)

Radiation Doses

Dose conversion factors used in the current Canadian high level waste disposal assessment study, 1427 (J;GB)

Radiation Effects

Conflicting views on a neutrality criterion for radioactive-waste management, 95 (BA;US)

Radiation Hazards

Calculation of health effects per curie release for comparison with the EPA standard, 1379 (RA;US)

Comparison of ICRP2 and ICRP30 for estimating the dose and adverse health effects from potential radionuclide releases from a geologic waste repository, 1385 (R;US)

Environmental pathway models for estimating population health effects from disposal of high-level radioactive waste in geologic repositories. Draft report, 1381 (R;US)

Exposures resulting from nuclear power production. Annex $F$, 1444 (BA;US)

Health effects associtated with unit radonuclide releases to the environment., 1378 (RA;IIS)

Potential individual doses from disposal of high-level radioactive wastes in geologic repositories. Draft report, 1383 (R;US)

Buirirnary of conitputer cudes for radiulugical assessment, 1370 (R, UIS)

Technical assistance for regulatory development: revicw and cvaluation of the draft EPA standard 40CFR191 for disposal of high-level waste. Volumes $S$ and 6, 1377 (R;US)

Radiation Protection

Impacts of recommendations on radiological considerations for an operating repository, 1398 (J;US)

Radionuclide Migration

Actinide sorption on rock minerals, 677 (RA;DE)

Actinide sorption on rock minerals, 275 (RA;DE)

Analysis of waste-package behavior for high-level waste, 981 (R;US)

Analytical solutions of radionuclide migration with onedimensional flow in layered media, 1361 (J;US)

Application of systems analysis to the disposal of high level waste in deep ocean sediments, $1069(\mathrm{~J} ; \mathrm{GB})$

Assessment of barrier effects on isolation system performance of geologic disposal of high level waste, 1273 (BA:XN)

Comparative analysis of fractured and porous medium radionuclide transport, 1356 (BA;US)

Criteria for high level waste disposal and characterization of barriers, 678 (RA;DE)

Data base for a site-specific analysis of the dispersion of radioactive elements from a final repository, 1366 (TG;US)

Diffusion of leached constituents in backfill barriers, 849 (RA;US)

Earth Sciences, 789 (RA;US)

Effect of microbial activity on the containment of radioactive waste in a dccp gcological repository, 1357 (BA;US)

Effects of composition, solutions, and burial on nuclear waste glasses, 845 (RA;US)

Elution of a adiunuclictes through colunns of crushed rock from the Nevada Test Site, 1315 (R;US)

Fär-field performance evaluations, 793 (RA;US)

Fault-tree analysis for probabilistic assessment of radioactivewaste segregation: an application to a plastic clay formation at a specific site, 1180 (BA;GR)

Formulation of an integrated physicochemical-hydrologic model for predicting waste-nuclide retardation in geologic media, 973 (R;US)

Fuel cycle programs. Quarterly progress report, Aprit-June 1982 , 536 (R;US)

Fuel cycle programs. Quarterly progress report, July-September 1982,538 (R;US)

Geochemical controls on radionuclide releases from a nuclear waste repository in basalt: estimated solubilities for selected elements, 1329 (R;US)

Geological disposal of high-level radioactive waste - a review of the Institute of Geological Sciences' research programme, 1091 $(\mathrm{J} ; \mathrm{GB})$ 
High-level waste glass field burial tests at CRNL: the effect of geochemical kinetics on the release and migration of fission products in a sandy aquifer, 1299 (R;CA)

Importance of near-field phenomena for nuclide release from a flooded salt dome repository, 1396 (BA;US)

Influence of microfissures in crystalline rock on radionuclide migration, 1185 (BA;GB)

Interaction of groundwater and fresh basalt fissure surfaces and its effect on the migration of actinides, 1303 (R;US)

INTRACOIN: an international nuclide transport code intercomparison study, 1358 (BA;US)

Long-term radioactivity release from solidified high-level waste. Part II. Parametric study of waste from properties, temperature and time, 1333 (J;US)

Migration of long actinides chains in geologic media, 1362 (J;US)

Model for predicting leaching behavior of high-level radioactive waste forms, 508 (J;US)

Model for near field migration, 1343 (BA;US)

Models and risk from high-level waste disposal-the missing pieces, 1090 (J;US)

Nuclear waste management. Quarterly prugress report, October through December 1980, 296 (R;US)

Nuclear waste-form risk assessment for US defense waste at Savannah River Plant. Annual report FY, 1982, 1008 (R;US)

Permeability monitoring technique of the near-field and far-field interface openings, 1346 (BA;US)

Possibility of high level waste underground disposal, 1249 (J;JP;In Japanese)

Relationship between glass leaching mechanism and geochemical transport of radionuclides, 1195 (BA;US)

Repository data base, field tests, 795 (RA;US)

Requirements for the disposal of high-level radioactive wastes (Federal Republic of Germany), 671 (RA;DE)

Review of radio-nuclide migration in geological formations, 1337 (J;GB)

Role of oxidation potential, Eh, in the dissolution of nuclear waste materials, 851 (RA;US)

Scientific basis for nuclear waste management V, 1187 (B;US)

Scientific data necessary to predict radionuclide migration within or near a mined nuclear repository, 1312 (R;US)

Semi-unified approach to leach testing, 852 (RA;US)

Solutions to radionuclide migration with nonlinear retardation mechanisms in backfill material. Interim progress report, JuneDecember 1982, 1331 (R;US)

Stability of ceramic waste forms in potential repository environments: a review, $564(\mathrm{R} ; \mathrm{US})$

Technical assistance for regulatory development: review and evaluation of the draft EPA standard 40CFR191 for dispusal of high-level waste. Volumes $S$ and 6,1377 (R;US)

The movement of a redox front downstream from a repository for nuclear waste, 1032 (J;US)

Uncertainties associated with geologic disposal of high-level radioactive waste, 1367 (R;US)

Uncertainties and sensitivities in the performance of geologic nuclear waste isolation systems. Volume I. Summary, 809 (R;US)

Underground migration of long-lived radionuclides leached from a borosilicate glass matrix, 1360 (BA;XA)

Volume 2. A simplified analysis of a hypothetical repository in a basalt formation, 1416 (RA;US)

Volume 3. A simplified analysis of a hypothetical repository in a tuff formation, 1417 (RA;US)

Waste migration by particulate carriers, 1365 .(J;US)

Water-dilution volume for high-level wastes stored in deep geologic media, 1232 (J;US)

Recommendations

Nuclear Waste Policy Act of 1982; proposed general guidelines for recommendation of sites for nuclear waste repositories, 1051 (J;US)

NWTS program criteria for mined geologic disposal of nuclear waste: program objectives, functional requirements, and system performance criteria, 595 (R;US)

\section{Redox Reactions}

The movement of a redox front downstream from a repository for nuclear waste, 1032 (J;US)

\section{Regulations}

Assessment of technical criteria of 10CFR60 for geologic disposal of high-level waste, 952 (R;US)

Assessment of high-level waste form conformance with proposed regulatory and repository criteria, 198 (R;US)

Assessment of the proposed rule (10CFR60) for disposal of highlevel radioactive wastes in geologic repositories. Volume 1,747 (R;US)

Fuel Cycle Conference Atomic Industrial Forum, Incorporated, 1267 (BA;US)

High-Level Nuclear-Waste Management. Oversight Hearing before the Subcommittee on Energy and the Environment of the Committee on Interior and Insular Affairs, House of Representatives, Ninety-Seventh Congress, Second Session, June $17,1982,110$ (B;US)

NNWSI regulatory status and plans, 1413. (RA;US)

NRC regulations for high-level nuclear waste management, 1092 (J;US)

Nuclear Regulatory Commission activities related to high-level waste materials issues; 1420 (BA;US)

Overview of the National Waste Terminal Storage Program, 788 (RA;US)

Regulatory implementation considerations for deep geologic disposal of high-level radioactive wastes, 1094 (J;US)

Regulatory policy of the EPA related to alpha-contaminated waste, 1412 (RA;US)

Technical assistance for regulatory development: review and evaluation of the draft EPA Standard 40CFR191 for disposal of high-level waste, 1415 (R;US)

Reliability

Evaluation of the barriers in an ultimate storage for radioactive waste, 684 (RA;DE;In German)

\section{Research Programs}

Answers to your questions about high-level nuclear waste isolation, 1298 (B;US)

Basalt waste-isolation project. Quarterly report, January 1-March 31, 1981,908 (R;US)

Basalt Waste Isolation Project. Quarterly report, October 1 , 1981-December 31, 1981,895 (R;US)

Basalt waste isolation project. Quarterly report, April 1, 1981June 30, 1981, 909 (R;US)

Basalt Waste Isolation Project. Drilling and testing quarterly report, January 1, 1983-March 31, 1983, 900 (R;US)

Commercial nuclear-waste research and development program. Quarterly report, July-September 1982, 578 (R;US)

Commercial nuclear waste research and development program. Annual report, fiscal year 1982, 579 (R;US)

Commercial Nuclear Waste Research and Development Program. Quarterly report, October-December 1982, 580 (R;US)

Commercial nuclear waste research and development program. Quarterly report, January-March 1983, 581 (R;US)

Commercial nuclear waste research and development program. Quarterly report, April-June 1983, 582 (R;US)

Evaluation of research and development for terminal isolation of nuclear wastes, 701 (R;US)

Geochemistry research planning for underground repositories, 1112 (J;US)

Geologic disposal of nuclear waste, 1107 (J;US)

KBS annual report 1981. Including summaries of technical reports issued during 1981, 998 (R;SE)

Management of radioactive wastes in Europe (EC) history, philosophy and plans for the next five years, 108 (BA;US)

Materials research for the Canadian Nuclear Fuel Waste Management Program, 109 (BA;US)

Nevada Nuclear-Waste-Storage Investigations. Quarterly report, April-June 1982, 757 (R;US)

Nevada Nuclear Waste Storage Investigations. Quarterly report, October-December 1982, 159 (R;US)

Nevada Nuclear Waste Storage Investigations: FY 1981 project plan and FY 1982 forecast, 761 (R;US)

Nevada Nuclear Waste Storage Investigations: FY 1982 project plan, 762 (R;US) 
Nevada Nuclear Waste Storage Investigations. Quarterly report, January-March, 1983, 760 (R;US)

$R$ and $D$ projects and studies: concerning the interim and terminal storage of radioactive wastes, 1281 (TG;US)

Radioactive waste management and disposal, 639 (RA;CA)

Research and development activities: fixation of radioactive residues. Quarterly progress report, April-June 1960, 210 (R;US)

Research and development related to the Nevada Nuclear Waste Storage Investigations. Progress report, October 1-December 31, 1982, 704 (R;US)

Research and development related to the Nevada Nuclear Waste Storage Investigations. Progress report, January 1-March 31, 1983,705 (R;US)

Subseabed disposal program annual report, January-September 1981. Volume II. Appendices (principal investigator progress reports). Part 1, 954 (R;US)

Subseabed disposal program annual report, January-September 1981. Volume II. Appendices (principal investigator progress reports). Part 2, 962 (R:US)

Technical progress report, 1 April-30 June 1981, 775 (R;US)

Technical progress report for the quarter, 1 July-30 September 1981, 776 (R;US)

Technical progress report for the quarter, 1 October-31 December, 1981, 777 (R;US)

Technical progress report for the quarter, 1 April-30 June 1982, 779 (R;US)

Technical progress report for the quarter 1 January-31 March 1982, 778 (K;US)

Technical progress report for the quarter 1 October-31 December 1982, 781 (R;US)

UK view of the management of high-level waste, 1103 (J;XA)

Understanding radioactive waste, 79 (R;US)

Wasste Management Program. 'Technical progress report, October-December 1980, 162 (R;US)

Waste treatment and disposal. Quarterly progress report, February-April, 1963, $290(\mathrm{R} ; \mathrm{US})$

Reviews

Predictive geology: with emphasis on nuclear-waste disposal, 96 (J;US)

\section{Risk Assessment}

Accident scenarios and probabilities for the SDP transportation system, 956 (RA;US)

Application of a risk-assessment methodology to a hypothetical high-lovol waste repository in bedded sall, 937 (R;U3)

Aspects of possible magmatic disruption of a high-level radioactive waste repository in southern Nevada, 699 (R;US)

Assessment of technical criteria of 10CFR60 for geologic disposal of high-level waste, 952 (R;US)

Barriers in a final storage for radioactive wastes, 1373 (RA;DE; In Gorman)

Calculation of the probability of volcanic disruption of a highlevel radioactive waste repository within southern Nevada, USA, 1393 (J;GB)

Comparison of risks due to HLW and SURF repositories in bedded salt, 1368 (R;US)

Criteria for high level waste disposal and characterization of barriers, 1374 (RA;DE)

Development of an assessment methodology for the disposal of high-level radloactive waste into deep ocean sediments, 1115 (J;CH)

Geologic disposal and the taxpayer: the problem of the experts, 1403 (BA;IN)

Geological disposal of radioactive waste, 1113 (J;US)

Hydrogeology of radioactive waste isolation: the challenge of a rational acsessment, 1144 (J;US)

Management of radioactive wastes in Europe (EC) history, philosophy and plans for the next five years, 108 (BA;US)

Methodology for a consequence analysis of a nuclear waste disposal facility, 1040 (J;US)

Models and risk from high-level waste disposal-the missing pieces, 1090 (J;US)

New look at forecasting (Characterizing future geologic events which may alter underground repository behavior), 87 (R;US)

Nuclear waste-form risk assessment for US Defense waste at Savannah River Plant. Annual report FY 1981, 1007 (R;US)
Nuclear waste-form risk assessment for US defense waste at Savannah River Plant. Annual report FY, 1982, 1008 (R;US)

Overview of nuclear waste management, $1068(\mathrm{~J} ; \mathrm{GB})$

Performance assessment in the Nevada Nuclear Waste Storage Investigations, 1224 (J;US)

Population risks from disposal of high-level radioactive wastes in geologic repositories. Draft report, 836 (R;US)

Postclosure risks of alternative SRP nuclear waste forms in gealogic repositories, 1009 (R;US)

Preliminary risk assessment for nuclear waste disposal in space. Volume I. Executive summary of technical report, 720 (R;US)

Preliminary risk assessment for nuclear waste disposal in space. Volume II. Technical report, 721 (R;US)

Probablistic approach to the assessment of the long-term risk linked to the disposal of radioactive waste in geological repositories, $1392(\mathrm{~J} ; \mathrm{GB})$

Proceedings of the international seminar on chemistry and process engineering for high-level liquid waste solidification, 263 (R;DE;In German and English)

Radiological risk analysis of an operating high-level waste repositnry, 1395 (BA;XA)

Requirements for the disposal of high-level radioactive wastes (Federal Republic of Germany), 671 (RA;DE)

Risk analysis for repositories in north Switzerland. Extent and probability of geologic processes and events, 1372 (R;CH;GE)

Risk-analysis methodology for spent-fuel repositories in bedded salt. Final report, 731 (R;US)

Risk-assessment methodology development for waste isolation in geolngic media, 727 (R;US)

Risk methodology for geologic disposal of radioactive waste: model description and user manual for Pathways model, 1316 (R;US)

Satisfying performance assessment data needs, 1222 (J;US)

Technical assistance for regulatory development: review and evaluation of the EPA standard 40 CFR191 for disposal of highlevel waste. Vol. 1, 750 (R;US)

Uncertainties and sensitivities in the performance of geologic nuclear waste isolation systems. Volume I. Summary, 809 (R;US)

Waste Management Program. Technical progress report, October-December 1980, 162 (R;US)

Waste treatment and disposal. Progress report, May-October 1963, $291(\mathrm{R} ; \mathrm{US})$

Rock Mechanics

Analytical models for thermal rock mechanics, 1217 (B;GB)

Climax Granite, Nevada Test Site, as a host for a rock mechanics test facility related to the geologic disposal of high level nuclear wastes, 1002 (R;US)

Composition of rocks - critcria for the conditiuning of hlgh-level radioactive wastes, 682 (RA;DE;In German)

Importance of creep failure of hard rock in the near field of a nuclear waste repository, $1270(\mathrm{BA} ; \mathrm{XN})$

Rock-nuid Interactions

Constant temperature and thermal gradient investigations of the near field subseabed environment: chemical and thermodiffusivinal effects, 971 (R;US)

Long-term radioactivity release from snlidified high-level waste. Part II. Parametric study of waste from properties, temperature and time, 1333 (J:US)

Overview of hydrothermal testing of waste package barrier materials at the Basalt Waste Isolation Project, 848 (R^;US)

Review of recent PNL research activities related to glass leaching mechanisms, 846 (RA;US)

Waste glass - seabed interactions: observations of the Soret

Safety effect, 850 (RA;US)

Accident scenarios and probabilities for the SDP transportation system, 956 (RA;US)

Barriers in a final storage for radioactive wastes, 1373 (RA;DF; In German)

Decay calculations on medium-level and actinide-containing wastes from the LWR fuel cycle. Pt. 2, 688 (R;DE;In German)

Evaluation of waste disposal safety, 661 (RA;DK;In Danish)

High-level-waste immobilization, 181 (R;US) 
Nuclear criticality safety analyses for the Waste Isolation Pilot Plant project, 1258 (J;US)

Requirements for the disposal of high-level radioactive wastes, 681 (RA;DE)

Subseabed disposal safety analysis, 955 (RA;US)

Safety Standards

Proposal for basic safety requirements regarding the disposal of high-level radioactive waste, 646 (R;DK)

Salt Deposits

Assessment of the proposed rule (10CFR60) for disposal of highlevel radioactive wastes in geologic repositories. Volume I, 747 (R;US)

Assessment of overseas developments in methods for treatment and disposal of high-level radioactive wastes, 532 (R;AU)

Backfill barriers for nuclear waste repositories in salt, 670 (RA;DE)

Brine migration in salt and its implications in the geologic disposal of nuclear waste, 829 (R;US)

Conceptual waste package interim performance specifications for waste forms for geologic isolation in salt repositories, 821 (R;US)

Conceptual waste package interim product specifications and data requirements for disposal of borosilicate glass defense high-level waste forms in salt geologic repositories, 823 (R;US)

Conclusions regarding geotechnical acceptability of the WIPP site, 990 (R;US)

Corrosion studies on non-metallic inorganic materials for HLW packaging design, 691 (R;DE;In German)

Deformation-dissolution potential of bedded salt, Waste Isolation Pilot Plant site, Delaware Basin, New Mexico, 1163 (BA;US)

Development of a comparative analysis of potential repository salt sites, 1037 (J;US)

Disposal of high-level nuclear waste in rock-salt. A comparison of temperature increases calculated at ECN (Energieonderzoek Centrum Nederland)-Petten, RWTH (Rheinisch-Westfaelische Technische Hochschule Aachen)-Aachen and THD (Delft University of Technology)-Delft, 835 (R;NL)

Dissolution of evaporites and its possible impact on the integrity of the Waste Isolation Pilot Plant (WIPP) New Mexico, USA, 1162 (BA;US)

Dissolution rate of salt domes on the basis of interpretation of measured salinity profiles, 1209 (BA;US)

EEG review comments on the geotechnical reports provided by DOE to EEG under the stipulated agreement through March 1, 1983, 562 (R;US)

Effect of a random variation of rock salt creep on calculations of storage room closure for the WIPP project, 943 (R;US)

Effects of product properties on the final salt storage medium and the conditions producing it (In German), 673 (RA;DE;GE)

Effects of the product propertics upon the ultimate storage medium salt and resulting conditions, 683 (RA;DE;In German)

Engineered waste-package conceptual design: defense high-level waste (Form 1), commercial high-level waste (Form 1), and spent fuel (Form 2) disposal in salt, 817 (R;US)

Environmental characterization report for the Gulf Interior Region Louisiana study area (Vacherie and Rayburn salt domes), 1321 (R;US)

Environmental characterization report for the Gulf Interior Region Mississippi study area (Lampton, Richton, and Cypress Creek salt domes), 1322 (R;US)

Environmental characterization report for the Gulf Interior Region, Texas study area (Oakwood, Palestine and Keechi salt domes), 1323 (R;US)

Evaluation of waste disposal safety, 661 (RA;DK;In Danish)

Evaluation of the thermomechanical behavior about a waste container/sleeve in salt, 803 (R;US)

Expected near-field thermal environments in a sequentially loaded spent-fuel or high-level waste repository in salt, 830 (R;US)

Expected very-near-field thermal environments for advanced spent-fuel and defense high-level waste packages, 831 (R;US)

Far field temperature calculations for high-level waste disposal in salt formations, 1295 (J;US)

Forced ventilation analysis of a commercial high-level nuclear repository in salt, 811 (R;US)
Gas production and liberation from rock salt samples and potential consequences on the disposal of high-level radioactive waste in salt domes, 1165 (BA;US)

Geological aspects of the final disposal of radioactive waste (Federal Republic of Germany), 1072 (J;DE;In German)

Geology of the Mors salt dome, 658 (RA;DK;In Danish)

Hydrogeological conditions at Mors salt dome, 659 (RA;DK;In Danish)

Impact of NRC licensing requirements on salt site selection, 588 (RA;US)

Importance of near-field phenomena for nuclide release from a flooded salt dome repository, 1396 (BA;US)

Impurities in rock-salt: consequences for the temperature increases at the disposal of high-level nuclear waste, 834 (R;US)

In situ and laboratory geotechnical test results from borehole GD-1 in southeast Utah, 813 (R;US)

In-situ test programs related to design and construction of highlevel nuclear waste (HLW) deep geologic repositories. Final report (Task 2), June 1981-November 1982, 743 (R;US)

In-situ test programs related to design and construction of highlevel nuclear waste (HLW) deep geologic repositories. Appendices. Final report (Task 2), June 1981-November 1982, 744 (R;US)

Investigations of salt domes for the purpose of high-level radioactive waste disposal, 657 (R;DK;DA)

Investigation of diffusion of $\mathrm{UO}_{2} \mathrm{Cl}_{2}$ in saturated $\mathrm{NaCl}$ solutions at various temperatures, 1167 (BA;US)

Isolation capability of a salt dome utilized for high-level waste disposal, 1208 (BA;US)

Materials for high-level waste canister/overpacks in salt formations, 949 (R;US)

Materials for high-level waste canister/overpacks in salt formations, 1101 (J;US)

Method of measurement and borehole probe to measure heatinduced borehole convergence, 1282 (P;DE;In German)

Migration rates of brine inclusions in single crystals of $\mathrm{NaCl}$, 1149 (BA;US)

Modeling of waste/near field interactions for a waste repository in bedded salt: the Dynamic Network (DNET) model, 1063 (J;US)

National waste terminal storage conceptual reference repository description, 612 (RA;US)

Near-field repository conditions in basalt and salt, 733 (RA;US)

Nuclear-waste disposal in geologic repositories, 1014 (R;US)

Peak high-level waste package temperature versus annular air gap width, 1046 (J;US)

Performance assessment of a shaft seals system in a HLW repository in the Gibson Dome area, 827 (R;US)

Policy and development of reprocessing of spent nuclear fuel and high-level radioactive waste management in the F.R. Germany, 13 (J;JP;In Japanese)

Preliminary design of a Danish high level waste disposal facility in the Mors dome, 1142 (J;US)

Present status of the Gorleben site investigations, 1036 (J;US)

Projected costs for mined geologic repositories for dispoal of commercial nuclear wastes, 769 (R;US)

Radiant energy dissipation during final storage of high-level radioactive waste in rock salt, 867 (R;DE;In German)

Regulatory implementation considerations for deep geologic disposal of high-level radioactive wastes, 1094 (J;US)

Requirements for the disposal of high-level radioactive wastes (Federal Republic of Germany), 671 (RA;DE)

Review of DOE waste package program. Subtask 1.1. National waste package program, April-September 1982, 735 (R;US)

Risk-analysis methodology for spent-fuel repositories in bedded salt. Final report, 731 (R;US)

Salt domes - the past and present, keys to the future, 1179 (BA;GB)

Salt repository program: compliance with NEPA, 589 (RA;US)

SALTMP - a FORTRAN program for fast salt temperature calculations in HRW final storage, 866 (R;DE;In German)

Scientific basis for nuclear waste management $V, 1187$ (B;US)

Seal for underground ultimate storage sites for radioactive waste, and method of installing the seal, 1284 (P;DE;In German) 
Status of site characterization in the Permian Basin, 590 (RA;US)

Status of the ONWI salt project, 1226 (J;US)

Summary of research and development for disposal of US Defense wastes in the Waste Isolation Pilot Plant (WIPP), 983 (R;US)

Technical progress report for the quarter, 1 July-30 September 1981, 776 (R;US)

Technical progress report for the quarter, 1 October-31 December, 1981, 777 (R;US)

Technical progress report for the quarter, 1 April-30 June 1982, 779 (R;US)

Technical progress report for the quarter 1 January-31 March 1982, 778 (R;US)

Technical assistance for regulatory development: review and evaluation of the draft EPA Standard 40CFR191 for disposal of high-level waste, 1415 (R;US)

Technical progress report for the quarter 1 October-31 December 1982, 781 (R;US)

Technology of systems, 660 (RA;DK;In Danish)

Thermal expansion as a criterion for the quantity of high-level wastes disposed into geological formations, 921 (R;SU; In Russian)

Thermomechanical impact around a borehole in rock salt after HAW disposal, 1294 (J;US)

Thermomechanical in-situ experiments and finite element computations, 1166 (BA;US)

Thermomechanical modeling and the selection of a salt repository site, $1218(\mathrm{~J} ; \mathrm{US})$

Transient creep model for salt during stress loading and unloading, 969 (R;US)

Transport phenomena of water and gas components within rock salt in the temperature field of disposed high level waste, 1272 (BA;XN)

Volume 4. A simplified analysis of a hypothetical repository in a bedded salt formation, 1418 (RA;US)

Waste Isolation Pilot Plant (WIPP) research and development program: in situ testing plan, March 1982, 940 (R;US)

Waste package conceptual designs for salt repositories, 593 (RA;US)

Waste treatment and disposal. Quarterly progress report, February-April, 1963, 290 (R;US)

WIPP research and development program: providing the technical basis for defense waste disposal, 989 (R;US)

Sea bed

1982 Subseabed Disposal Program. Annual report, site assessment October 1981 to September 1982. 987 (R;US)

Analysis of diffusional ion transport in ocean sediments: subseafloor disposal of radioactive waste, 1250 (J;US)

Systems overview of the subseabed disposal program, 587 (RA;US)

Sealed Sources

Elimination of used sources. Taking over and storage, 548 (R;FR;In Fronoh)

Sealing Materials

Performance assessment of a shaft seals system in a HLW repository in the Gibson Dunie ared, 827 (R;US)

Recent advances in repository seal materials. 1172 (BA;US)

Repository sealing, 794 (RA;US)

Seals

Nuclear-waste repository in basalt: seal development studies, 887 (R;US)

Seismic Detection

Deep underground storage of [radioactive waste]. Annual report, 1981,643 (R;DE;GE)

Seismic Effects

Repository design and equipment, 791 (RA;US)

Shaft Excavations

Evaluation of alternative shaft-sinking techniques for high-level nuclear waste (HLW) deep geologic repositories. Final report (Task 3), June 1981-July 1982, 741 (R:US)

Identifiction of a preferred site for the exploratory shaft within the reference repository location: Hanford Site (Pasco Basin), 916 (R;US)

Technology of systems, 660 (RA;DK;In Danish)

\section{Site Selection}

Analysis of geological and geophysical data from study locatiol $C_{1}, B_{1}$, and $E_{2}$ in the northwest PAC 1 study rcgion, 957 (RA;US)

Area environmental characterization report of the Dalhart and Palo Duro basins in the Texas Panhandle. Volume I. Dalhart Basin, 782 (R;US)

Basalt waste-isolation project drilling and testing. Quarterly report, January 1-March 31, 1982, 896 (R;US)

Basalt Waste Isolation Project drilling and testing. Quarterly report, April 1, 1982-June 30, 1982, 897 (R;US)

Basalt Waste Isolation Project drilling and testing. Quarterly report, July 1, 1982-September 30, 1982, 898 (R;US)

Basalt Waste Isolation Project: drilling and testing. Quarterly report, October 1, 1982-December 31, 1982, 899 (R;US)

Basalt Waste Isolation Project. Drilling and testing quarterly report, January 1, 1983-March 31, 1983, 900 (R;US)

Benchmark problems for repository siting models, 746 (R;US)

Borehole-to-borehole geophysical methods applied to investigations of high level waste repository sites, 1019 (R;US)

Department of Enorgy doos not plan to use an abandoned salt mine at L.yons, Kansas, for nuclear high-level waste disposal, 637 (R;US)

DOE implementation of high-level waste criteria and standards, 1093 (J;US)

DOE's program for high-level radioactive waste disposal, 1266 (BA;US)

E-field ratio telluric traverses near Fortymile Wash, Nevada Test Site, Nevada, 1027 (R;US)

Environmental characterization of bedded salt formations and overlying areas of the Permian Basin, 1320 (R;US)

EPRI's programs in low-level waste management, 74 (RA;US)

Geological status of NWTS repository siting activities in the paradox basin, 1265 (J;US)

Geological evaluation of Gulf Coast salt domes: overall assessment of the Gulf Interior Region, 784 (R;US)

Geotechnical studies for subseabed disposal: high level radioactive wastes, 964 (RA;US)

High-level waste disposalitechnical and institutional siting factors remaining to be resolved, 1087 (J;US)

High-level waste disposal, 1275 (J;US)

Hydrogeologic testing of the E.J. Kubat borehole, San Juan County, Utah: utilization of a high pressure instrumented flow contrnl system, 537 (RA;US)

Issues in repository siting, 798 (RA;US)

Laboratory rock mechanics testing manual. Public draft, 807 (R;US)

Management of high-level waste repository siting, 1071 (J;US)

National survey of crystalline rocks and recommendations of regions to be explored for high-level radioactive waste repository sites, 540 (R;US)

Nevada Nuclear Waste Storage Investigations: FY 1982 project plan, 762 (R;US)

NWTS Field Testing In-Situ Site-Confirmation Tests Workshop, September 8-9, 1980, 802 (R;US)

Overvlew of the National Waste Terminal Siorage Program, 788 (RA:US)

Pacific site assessment, 960 (RA;US)

Paleomagnetic study of areas B1, C1 and E2, 959 (RA;US)

Parameters and variables appearing in repository-siting models, 745 (R;US)

Performance assessment in the Nevada Nuclear Waste Storage Investigations, $1224(\mathrm{~J} ; \mathrm{US})$

Possible approaches to community development for nuclear waste isolation, 805 (R;US)

Preliminary results of gravity investigations at Yucca Mountain and vicinity, Southern Nye County, Nevada, 1024 (R;US)

Preliminary assessment of shales and other argillaceous rocks $i$ the United States, 609 (RA;US)

Present status of the Gorleben site investigations, 1036 (J;US)

Proposal for broader participation in radioactive waste management, $1135(\mathrm{~J} ; \mathrm{GB})$

Radwaste paradox, 1054 (J;US)

Repository design, 1134 (J;GB) 
Resistivity sounding investigation by the Schlumberger method in the Yucca Mountain and Jackass Flats area, Nevada Test Site, Nevada, 1028 (R;US)

Salt repository program: compliance with NEPA, 589 (RA;US)

Satisfying performance assessment data needs, 1222 (J;US)

Selecting a repository site, 1133 (J;GB)

Site, 772 (RA;US)

Site characterization report for the basalt waste isolation project. Volume I, 601 (R;US)

Site characterization report for the basalt waste isolation project. Volume II, 602 (R;US)

Site characterization report for the basalt waste isolation project. Volume III, 603 (R;US)

Site investigations for repositories for solid radioactive wastes in deep continental geological formations, 1139 (B;XA)

Site qualification activities, 958 (RA;US)

Site-screening process on the Hanford Site: a summary report, 878 (R;US)

Socioeconomic evaluations, 47 (RA;US)

Status of geologic/environmental characterizations, 797 (RA;US)

Status of site characterization in the Permian Basin, 590 (RA;US)

Status of the ONWI salt project, 1226 (J;US)

Subseabed disposal program annual report, January-September 1981. Volume II. Appendices (principal investigator progress reports). Part 2, 962 (R;US)

Summary of repository siting models. Final report, 740 (R;US)

Summary of Near-Surface Test Facility results and their application to repository design, 886 (R;US)

Surveys for desert tortoise on the proposed site of a high-level nuclear waste repository at the Nevada Test Site, 1302 (R;US)

US Geological Survey research in radioactive waste disposal, fiscal year 1979, $716(R ; U S)$

Site Surveys

Area environmental characterization report of the Dalhart and Palo Duro basins in the Texas Panhandle. Volume II. Palo Duro basin, 783 (R;US)

Development of a comparative analysis of potential repository salt sites, 1037 (J;US)

Draft site characterization analysis of the site characterization report for the Basalt Waste Isolation Project, Hanford, Washington Site. Main report and Appendices A through D, 724 (R;US)

Draft site characterization analysis of the site characterization report for the Basalt Waste Isolation Project, Hanford, Washington site. Appendices $E$ through W, 725 (R;US)

Ecosystem studies, endangered species survey - Gibson Dome and Elk Ridge study areas, Paradox Basin, Utah, 1326 (R;US)

Nevada nuclear waste-storage investigations. Quarterly report, January-March 1982 (Studies of Nevada Test Site as repository for high-level radwaste), 756 (R;US)

Overview of nuclear waste management, $1068(\mathrm{~J} ; \mathrm{GB})$

Results of the area-to-location phase of repository siting in Nevada, 1035 (J;US)

Site characterization report for the basalt waste isolation project. Volume I, 601 (R;US)

Site characterization report for the basalt waste isolation project. Volume II, 602 (R;US)

Site characterization report for the basalt waste isolation project. Volume III, 603 (R;US)

Status of the ONWI salt project, $1226(\mathrm{~J} ; \mathrm{US})$

Socio-economic Factors

Proceedings of the 1980 National Waste Terminal Storage Program information meeting, 787 (R;US)

Rock-melt concept for nuclear waste disposal, 1254 (J;US)

Socioeconomic evaluations, 47 (RA;US)

Sociopolitical aspects of a subseabed disposal program for high level nuclear waste, 965 (RA;US)

Solid Wastes

Alternatives for definse waste-salt disposal, 629 (R;US)

Radiological impact of disposal of UK solid radioactive wastes, preseint and future, $1081(\mathrm{~J} ; \mathrm{GB})$
Solidification

Disposal of high level wastes, 1244 (J;JP;In Japanese)

Space

Analysis of space systems for the space disposal of nuclear waste follow-on study. Volume 1. Executive summary, 718 (R;US)

Analysis of space systems for the space disposal of nuclear waste follow-on study. Volume 2. Technical report, 719 (R;US)

Overview of nuclear waste disposal in space, 616 (RA;US)

Preliminary risk assessment for nuclear waste disposal in space. Volume I. Executive summary of technical report, 720 (R;US)

Preliminary risk assessment for nuclear waste disposal in space. Volume II. Technical report, 721 (R;US)

Waste-Mixes Study for space disposal, 815 (R;US)

Specifications

NWTS program criteria for mined geologic disposal of nuclear waste: repository performance and development criteria. Public draft, 596 (R;US)

Spent Fuel Casks

Canister materials proposed for final disposal of high level nuclear waste - a review with respect to corrosion resistance, 675 (RA;DE)

Spent Fuel Elements

Highly dense graphite matrix: a new material for the conditioning of radioactive wastes, 358 (J;US)

Nuclear waste disposal: a case of benign neglect>, 1108 (J;US)

Spent Fuels

Annotated bibliography for the design of waste packages for geologic disposal of spent fuel and high-level waste, 804 (R;US)

Conceptual design requirements for spent fuel, high-level waste, and transuranic waste packages, 903 (R;US)

Conceptual waste-package designs for disposal of nuclear waste in tuff, 818 (R;US)

Disposal of high-level radioactive wastes, 1116 (J;AU)

DOE implementation of high-level waste criteria and standards, 1093 (J;US)

Expected very-near-field thermal environments for advanced spent-fuel and defense high-level waste packages, 831 (R;US)

National waste terminal storage conceptual reference repository description, 612 (RA;US)

Nuclear fuel waste disposal - status of the Canadian program, 1263 (J;US)

Package materials, waste form, 792 (RA;US)

Radiolysis of groundwater from HLW stored in copper canisters, 999 (R;SE)

Rapid estimation of the regional thermal impact of a nuclear waste repository, 1260 (J;US)

Repository data base, field tests, 795 (RA;US)

Results of repository conditions study for commercial and defense high-level nuclear waste and spent fuel repositories in salt, 824 (R;US)

Risk-analysis methodology for spent-fuel repositories in bedded salt. Final report, 731 (R;US)

Sociopolitical aspects of a subseabed disposal program for high level nuclear waste, 965 (RA;US)

Thermal analysis of nuclear waste emplacement in welded tuff, 930 (R;US)

Thermal impact of waste emplacement and surface cooling associated with geologic disposal of nuclear waste, 707 (R;US)

Waste package conceptual designs for a repository located in basalt, 884 (R;US)

Standards

DOE implementation of high-level waste criteria and standards, 1093 (J;US)

Draft environmental impact statement for 40 CFR part 191: environmental standards for management and disposal of spent nuclear fuel, high-level and transuranic radioactive wastes. Final report, 838 (R;US)

Draft regulatory impact analysis for 40 CFR 191: environmental standards for management and disposal of spent nuclear fuel, high-level and transuranic radioactive wastes. Draft report, 837 (R;US)

Technical assistance for regulatory development: review and evaluation of the EPA standard 40 CFR 191 for disposal of highlevel waste. Vol. 1, 750 (R;US) 
Strontium

Systems study of the feasibility of high-level nuclear-waste fractionation for thermal-stress control in a geologic repository: main report, 308 (R;US)

Systems study of the feasibility of high-level nuclear waste fractionation for thermal stress control in a geologic repository: appendices, 309 (R;US)

Strontium 90

Alternate strategy for commercial high-level radioactive-waste management, 945 (R;US)

Synthetic Rocks

Nuclear trash. What do we do, 1078 (J;AU)

Synroc technology for immobilizing U.S. defense waste, 400 (J;US)

Systems Analysis

Application of systems analysis to the disposal of high level waste in deep ocean sediments, 1069 (J;GB)

Systems, 771 (RA;US)

Systems, 790 (RA;US)

Waste-Mixes Study for space disposal, 815 (R;US)

Technology Assessment

Engineered components for radioactive waste disposal systems are they technically justified, 861 (R;US)

Licensing: technological implications for geologic disposal of high-level radioactive wastes, 1089 (J:US)

Nuclear-waste disposal: technical issues and status, 979 (R;US)

Overview of nuclear waste management, 1068 (J;GB)

Pacing and logistics of high-level waste disposal, 1047 (J;US)

Study of the isolation system for geologic disposal of radioactive wastes, 577 (R;US)

Technology Transfer

International fuel-cycle and waste-management technologyexchange activities sponsored by the United States Department of Energy: FY 1982 evaluation report, 85 (R;US)

Temperature Effects

Thermal impact of waste emplacement and surface cooling associated with geologic disposal of nuclear waste, 707 (R;US)

Temperature Gradients

Far field temperature calculations for high-level waste disposal in salt formations, 1295 (J;US)

Peak high-level waste package temperature versus annular air gap width, 1046 (J;US)

Rapid estimation of the regional thermal impact of a nuclear waste repository, 1260 (J;US)

Test Facilitips

Exploratory Shaft Test Facility in basalt. Project B-314 preconceptual design report, 913 (R;US)

Summary of Near-Surface Test Facility results and their application to repository design, 886 (R;US)

Testing

Waste Isolation Pilot Plant (WIPP) research and development program: in situ testing plan, March 1982, 940 (R;US)

-Thermal Conduction

SALTMP - a FORTRAN program for fast salt temperature calculations in HRW final storage, 866 (R;DE;In German)

Tritium

Fuel cycle programs. Quarterly progress report, April-June 1982 .536 (R;IIS)

Fuel cycle programs. Quarterly progress report, July-September 1982, 538 (R;US)

Fuel cycle programs. Quarterly progress report, October-

Tuff December 1982, 539 (R;US)

Nevada nuclear waste storage investigations. Quarterly report, Jüly-September 1982,758 (K;US)

NNWSI regulatory status and plans, 978 (R;US)

Parametric study of the effects of thermal environment on a waste package for a tuff repository, 1271 (BA;XN)

Preliminary technical constraints for a repository in tuff, 97 ? (R;US)

Project management plan for exploratory shaft at Yucca Mountain, 764 (R;US)

System study and performance constraints for repository horizon selection at the Nevada Test Site, 1225 (J;US)
Underground Disposal

"Project Crystal" for ultimate storage of highly radioactive waste, 1049 (J;CH;In German)

Actinide sorption on rock minerals, 677 (RA;DE)

Alternatives for definse waste-salt disposal, 629 (R;US)

Application of a risk-assessment methodology to a hypothetical high-level waste repository in bedded salt, 937 (R;US)

Assessment of overseas developments in methods for treatment and disposal of high-level radioactive wastes, 532 (R;AU)

Backfill barriers for nuclear waste repositories in salt, 670 (RA;DE)

Backfilling and sealing a repository for high-level radioactive waste - a review, 839 (R;US)

Backfilling and sealing a repository for high-level radioactive waste - a review, 1279 (B;GB)

Basalt waste-isolation project. Quarterly report, January 1-March 31, 1981, 908 (R;US)

Basalt Waste Isolation Project. Quarterly report, October 1, 1981-December 31, 1981, 895 (R;US)

Basalt waste isolation project. Quarterly report, April 1, 1981June 30, 1981, 909 (R;US)

Basalt waste-isolation project drilling and testing. Quarterly report, January 1-March 31, 1982, 896 (R;US)

Basalt Waste Isolation Project. Quarterly report, July 1, 1981Seplember 30, 1981,910 (R;US)

Benefit-cost-risk analysis of alternatives for greater-confinement disposal of radioactive waste, 559 (R;US)

Characterization of drained and undrained response of thermally loaded repository rocks, 1110 (J;US)

Composition of rocks - criteria for the conditioning of high-level radioactive wastes, 682 (RA;DE; In German).

Concept of final storage in geological formations, 669 (RA;DE;GE)

Conceptual waste-package designs for disposal of nuclear waste in tuff, 818 (R;US)

Coordination mecting of high-level waste technulugy and national waste terminal storage programs, October 21-22, 1981, 624 (R;US)

Coupled heat and groundwater flow in porous rock, 535 (R;GB)

Decay calculations on medium-level and actinide-containing wastes from the LWR fuel cycle. Pt. 2, 688 (R;DE;In German)

Deep underground storage of [radioactive waste]. Annual report, 1981,643 (R;DE;GE)

Developments, recommendations and applications of tests for evaluating the chemical stability of high-level radioactive solid wasle forms, 1056 (J;GB)

Discharge of high activity and long half-life wastes arising from a nuclear power programme, 373 (J;BE;In French)

Disposal of high-level waste from nuclear power plants in Denmark. Salt dome investigations. V.1. Summary, 647 (R;DK)

Disposal of high-level waste from nuclear power plants in Denmark. Salt dome investigations. V.2. Geology, text, 648 (R;DK)

Disposal of high-level nuclear waste from nuclear power plants in Denmark. Salt dome investigations. V.2. Geology, figures, 649 (R;DK)

Disposal of high-level waste from nuclear power plants in Denmark. Salt dome investigations. V.4. Repository design, 651 (R;DK)

Disposal of high-level waste from nuclear power plants in Denmark. Salt dome investigations. V.5. Safcty evaluation, 652 (R;DK)

Dispossal of high-level radioactive wastes, $1116(\mathrm{~J} ; \mathrm{AU})$

Disposal of high level wastes, 1244 (J;JP; In Japanese)

Effects of long-term exposure of tuffs to high-level nuclear wasterepository conditions. Preliminary report, 693 (R;US)

Effects of product properties on the final salt storage medium and the conditions producing it (In German), 673 (RA;DE;GE)

Evaluation of area studies of the US Gulf Coast Salt Dome Basins: location recommendation report, 785 (R;US)

Evaluation of research and development for terminal isolation nuclear wastes, 701 (R;US)

Evaluation of barriers in a final storage site for radioactivc wastes, 674 (RA;DE)

Geologic disposal of nuclear waste, 1107 (J;US) 
Geologic disposal and the taxpayer: the problem of the experts, 1403 (BA;IN)

Geological status of NWTS repository siting activities in the paradox basin, 1265 (J;US)

Geological disposal of radioactive waste, 1113 (J;US)

Geological disposal of radioactive wastc. Research in the OECD area, $1109(\mathrm{~B} ; \mathrm{XN})$

High-level radioactive waste isolation by incorporation in silicate rock, 1296 (BA;XA)

High level waste disposal, 1289 (J;US)

Hydrology and Geology Overview Committee reports and .responses from the Basalt Waste Isolation Project, 914 (R;US)

Improving public understanding of nuclear waste management, $1136(\mathrm{~J} ; \mathrm{GB})$

Influence of faulting on groundwater flow and contaminant transport, 1184 (BA;GB)

Influence of microfissures in crystalline rock on radionuclide migration, 1185 (BA;GB)

INTRACOIN, Level 1. Benchmark calculations with code SWIFT, 644 (R;DE)

Isolation of radioactive wastes in salt repositories, 951 (R;US)

Laboratory determination of the thermal properties of large-scale samples of natural clays, 550 (R;IT;IT)

Long-term thermohydrologic behaviour of nuclear-waste repositories, 1183 (BA;GB)

Long-term prediction of the fate of nuclear waste deeply buried in granite, 1186 (BA;GB)

Low level and intermediate level wastes management practice in France, 442 (BA;US)

Method for subsurface disposal of radioactive waste (Patent), 1297 (P;US)

Modeling of nuclear waste disposal by rock melting, 1016 (R;US)

Monitoring an underground repository with modern seismological methods, 1238 (J;US)

National long-term high-level waste-technology program, 67 (R;US)

Near-field performance evaluations, 800 (RA;US)

Nevada Nuclear-Waste-Storage Investigations. Quarterly report, April-June 1982, 757 (R;US)

Nevada Nuclear Waste Storage Investigations: FY 1981 project plan and FY 1982 forecast, 761 (R;US)

Nevada Nuclear Waste Storage Investigations: FY 1982 project plan, 762 (R;US)

Nuclear power on unstable ground, 1182 (BA;GB)

Nuclear waste disposal: can we rely on bedrock, 1137 (B;GB)

NWTS program criteria for mined geologic disposal of nuclear waste: program objectives, functional requirements, and system performance criteria, 595 (R;US)

NWTS program criteria for mined geologic disposal of nuclear waste: repository perfor mance and development criteria. Publio draft, 596 (R;US)

Petrologic studies of drill cores USW-G2 and UE25b-1H, Yucca Mountain, Nevada, 696 (R;US)

Possibility of high level waste underground disposal, 1249 (J;JP;In Japanese)

Predictive geology in nuclear-waste management, 1181 (BA;GB)

Promotion of the research and development of high level radioactive waste treatment and disposal, 491 (J;JP;In Japanese)

Radioactive waste disposal in granite (Stripa mine), 712 (R;US)

Radiological risk analysis of an operating high-level waste repository, 1395 (BA;XA)

Regulation of geological disposal of high-level radioactive waste, 1241 (J;US)

Regulatory implementation considerations for deep geologic disposal of high-level radioactive wastes, 1094 (J;US)

Repository, 773 (RA;US)

Repository design and equipment, 791 (RA;US)

Repository sealing, 794 (RA;US)

Repository data base, field tests, 795 (RA;US)

Requirements for the disposal of high-level radioactive wastes (Federal Republic of Germany), 671 (RA;DE)

Results of repository conditions study for commercial and defense high-level nuclear waste and spent fuel repositories in salt, 824 (R;US)
Review of safety assessments of nuclear waste management (Preclosure and long-term risks), 1380 (R;US)

Review of radio-nuclide migration in geological formations, 1337 (J;GB)

Review of radioactive waste management programs in the United Kingdom, 1160 (BA;US)

Risk-analysis methodology for spent-fuel repositories in bedded salt. Final report, 731 (R;US)

Rock-melt concept for nuclear waste disposal, 1254 (J;US)

Shielded containers for waste could prove beneficial, 1128 (J;GB)

Site investigations for repositories for solid radioactive wastes in deep continental geological formations, 1139 (B;XA)

Status of radioactive waste management: needs for reassessment, 1248 (J;GB)

Summary of Near-Surface Test Facility results and their application to repository design, $886(\mathrm{R} ; \mathrm{US})$

System study and performance constraints for repository horizon selection at the Nevada Test Site, 1225 (J;US)

Systems, 790 (RA;US)

Systems study of the feasibility of high-level nuclear-waste fractionation for thermal-stress control in a geologic repository: main report, 308 (R;US)

Systems study of the feasibility of high-level nuclear waste fractionation for thermal stress control in a geologic repository: appendices, 309 (R;US)

Technical progress report for the quarter 1 January-31 March 1982, 778 (R;US)

Technical support of standards for high-level radioactive waste management. Addendum to volumes $C$ and $D$. Final report, 840 (R;US)

Thermal analysis of nuclear waste emplacement in welded tuff, 930 (R;US)

Thermal expansion as a criterion for the quantity of high-level wastes disposed into geological formations, 921 (R;SU;In Russian)

Three aspects of reliability for a geological nuclear waste repository, 1255 (J;US)

Transport phenomena of water and gas components within rock salt in the temperature field of disposed high level waste, 1272 (BA; XN)

UK view of the management of high-level waste, 1103 (J;XA)

Underground disposal of high active waste, $665(\mathrm{R} ; \mathrm{DE})$

Underground storage of radioactive wastes, 1280 (BA;FR;In French)

United States steps up waste isolation programme, 1074 (J;GB)

Waste migration by particulate carriers, 1365 (J;US)

Waste-rock Interactions

Backfill-waste interactions in repository simulating tests, 1153 (BA;US)

Effects of product properties on the final salt storage medium and the conditions producing it (In German), 673 (RA;DE;GE)

Geochemical behavior of supercalcine waste form: its stability in a basalt environment, 911 (R;US)

Long-term radioactivity release from solidified high-level waste. Part II. Parametric study of waste from properties, temperature and time, 1333 (J;US)

Materials Characterization Center workshop on leaching mechanisms of nuclear waste forms, May 19-21, 1982, Gaithersburg, Maryland. Summary report, 82 (R;US)

Overview of hydrothermal testing of waste package barrier materials at the Basalt Waste Isolation Project, 848 (RA;US)

Package materials, waste form, 792 (RA;US)

Review of recent PNL research activities related to glass leaching mechanisms, 846 (RA;US)

Sorptive diffusion in clay gels, 709 (RA;US)

Strontium-basalt reactions under nuclear waste repository conditions, 1152 (BA;US)

Waste glass - seabed interactions: observations of the Soret effect, 850 (RA;US)

Water Requirements

Evaluation of water resource economics within the Pasco Basin, Washington, 1429 (R;US) 
Wells

Waste treatment and disposal. Quarterly progress report, February-April, 1963, 290 (R;US)

\section{RADIOACTIVE WASTE FACILITIES}

Actual point about fission products vitrification, 136 (R;FR;In French)

WIPP research and development program: providing the technical basis for defense waste disposal, 989 (R;US)

Accidents

Effects of wire rope in mitigating a waste-shaft accident, 980 (R;US)

Air Pollution

Nevada nuclear waste storage investigations site-characterization activities: preliminary atmospheric assessment of a nuclear waste repository, 765 (R;US)

Availability

Three aspects of reliability for a geological nuclear waste repository, 1255 (J;US)

Chemical Efiluents

Initial operations in the remote laboratory-scale waste-treatment facility, 312 (R;US)

Closures

Sealing concepts for the Waste Isolation Pilot Plant (WIPP) site, 938 (R;US)

Cumpliance

Technical assistance for regulatory development: review and evaluation of the draft EPA Standard 40CFR19i for disposal of high-level waste, 1415 (R;US)

Volume 2. A simplified analysis of a hypothetical repository in a basalt formation, 1416 (RA;US)

Volume 3. A simplified analysis of a hypothetical repository in a tuff formation, 1417 (RA;US)

Volume 4. A simplified analysis of a hypothetical repository in a bedded salt formation, 1418 (RA;US)

Construction

In-situ test programs related to design and construction of highlevel nuclear waste (HLW) deep geologic repositories. Final report (Task 2), June 1981-November 1982, 743 (R;US)

In-situ test programs related to design and construction of highlevel nuclear waste (HLW) deep geologic repositories. Appendices. Final report (Task 2), June 1981-November 1982, 744 (R;US)

Nuclear fuel waste disposal - status of the Canadian program, 1263 ( $I ;$ UIS)

NWTS Field Testing In-Situ Site-Confirmation Tests Workshop, September 8-9, 1980, 802 (R;US)

Cooling

Forced ventilation analysis of a commercial high-level nuclear repository in salt, 811 (R;US)

Forced ventilation analysis of a commercial high-level nuclear

Cost waste repository in tuff. Topical report RSI-0175, 941 (R;US)

MRS/IS facility co-located with a repository: preconceptual design and life-cycle cost estimates, 857 (R;US)

Projected costs for mined geologic repositories for dispoal of commercial nuclear wastes, 769 (R;US)

Critteallty

Nuclear criticality safety analyses for the Waste Isolation Pilot Plant project, 1258 (J;US)

Data Acquisition

Repository data base development, 801 (RA;US)

Decommissioning

Preliminary environmental implications of alternatives for decommissioning and future use of the Western New York Nuclear Services Center, 1301 (R;US)

Decontamination

Preliminary environmental implications of alternatives for decommissioning and future use of the Western New York Nuclear Services Center, 1301 (R;IIS)

Demonstration Plants

New SYNROC plant offers N-waste disposal solution, 374 (J;AU)
Design

Comparison of cask and drywell storage concepts for a monitor retrievable storage/interim storage system, 853 (R;US)

Conceptual design of the nuclear-waste repository in basalt, 888 (R;US)

Conceptual design of monitored retrievable storage faclities, 41 (J;US)

Conceptual waste-package designs for disposal of nuclear waste in tuff, 818 (R;US)

Design considerations for occupational exposure for a potential repository at Yucca Mountain: high-level-waste handling operations, 991 (R;US)

Disposal of high-level waste from nuclear power plants in Denmark. Salt dome investigations. V.4. Repository design, 651 (R;DK)

Expected near-field thermal environments in a sequentially loaded spent-fuel or high-level waste repository in salt, 830 (R;US)

Iligh-level waste solidifieation systein for the Westeıll New Yuik Nuclear Service Center, 315 (R;US)

Illustrative nuclear waste disposal criteria and their application to high-level waste repository design, 1290 (J;US)

In-situ test programs related to design and construction of highlevel nuclear waste (HLW) deep geologic repositories. Final report (Task 2), June 1981-Nuvember 1982, 743 (R;US)

In-situ test programs related to design and construction of highlevel nuclear waste (HLW) deep geologic repositories. Appendices. Final report (Task 2), June 1981-November 1982, 744 (R;US)

Long-term thermomechanical and thermohydrological factors controlling the optimal design of a nuclear waste repository, 1064 (J;US)

Marking a nuclear waste repository: an archaelogist's perspective, 1095 (J;US)

Monitored Retrievable Storage Demonstration Facility and dry well storage field conceptual design study, 912 (R;US)

MRS/IS facility co-located with a repository: preconceptual design and life-cycle cost estimates, 857 (R;US)

National waste terminal storage conceptual reference repository description, 612 (RA;US)

Near-field performance evaluations, 800 (RA;US)

New waste calcining facility at ICPP, 452 (BA;US)

NWTS Field Testing In-Situ Site-Confirmation Tests Workshop. September 8-9, 1980, 802 (R;US)

Overview of waste-handling requirements for a nuclear-waste repository in basalt, 877 (R;US)

Preliminary design of a Danish high level waste disposal facility in the Mors dome, 1142 (J;US)

Process description and plant design for preparing ceramic highlevel waste forms, 149 (R;US)

Repository, 773 (RA;US)

Repository design and equipment, 791 (RA;US)

Repository design, 1134 (J;GB)

Repository engineering design for high-level waste, 1082 (J;GB)

Repository and engineering barriers design, 584 (RA;US)

Techuical couservatism in the design and analysis of a nuclearwaste repository in basalt, 907 (R;US)

Thermal response to emplacement of nuclcar wastc in long, horizontal boreholes, $986(\mathrm{R} ; \mathrm{US})$

Three aspects of reliability for a geological nuclear waste repository, 1255 (J;US)

Waste package conceptual designs for a repository located in basalt, 585 (RA;US)

Economic Amalysis

Ceramic process and plant design for high-level nuclear waste immobilization, 144 (R;US)

Engineered Safety Systems

Testing of candidate waste package backfill and canister mateh als for basalt, 586 (RA;US)

Waste package conceptual designs for a repository located in basalt, 585 (RA;US) 
Engineering

Repository, 773 (RA;US)

Environmental Impact Statements

Defense Waste Processing Facility: Savannah River Plant, Aiken, SC. Final environmental impact statement, 1306 (R;US)

Environmental Impacts

Salt repository program: compliance with NEPA, 589 (RA;US)

Equipment

Guidebook for reliability, availability, and maintainability analysis of NWTS repository equipment. Volume II. Appendices, 808 (R;US)

Financing

Report on financing the disposal of commercial spent nuclear fuel and processed high-level radioactive waste, 604 (R;US)

Geophysical Surveys

Basic data report for Drillhole WIPP 14 (Waste Isolation Pilot Plant - WIPP), 974 (R;US)

Government Policies

National plan for siting high-level radioactive waste repositories and environmental assessment. Public draft, 583 (R;US)

High-level Radioactive Wastes

Approach to resolution of geologic uncertainty in the licensing of a high-level-waste repository in tuff, 985 (R;US)

Hoists

Effects of wire rope in mitigating a waste-shaft accident, 980 (R;US)

Licensing

Approach to resolution of geologic uncertainty in the licensing of a high-level-waste repository in tuff, 985 (R;US)

Impact of NRC licensing requirements on salt site selection, 588 (RA;US)

Licensing considerations for the NWTS program, 1227 (J;US)

NNWSI regulatory status and plans, 978 (R;US)

Nuclear Regulatory Commission activities related to high-level waste materials issues, 1420 (BA;US)

NWTS Field Testing In-Situ Site-Confirmation Tests Workshop, September 8-9, 1980, 802 (R;US)

Maintenance

Commercial nuclcar waste research and development program. Quarterly report, October-December 1981, 60 (R;US)

Mathematical Models

Application of performance assessment models to evaluate uncertainty in site characterization, $1221(\mathrm{~J} ; \mathrm{US})$

Measuring Instruments

Instrument reliability for high-level nuclear-waste-repository applications, 1013 (R;US)

Results of instrument reliability study for high-level nuclear waste repositories, 93 (J;US)

Mining Engineering

Limiting-individual dose from an extended-source high-level waste repository model, 1058 (J;US)

Monitoring

Post-emplacement monitoring, Subtask 1.2. Draft Technical Position. Vol. 2, 1319 (R;US)

Nuclear Insurance

Indemnification for nuclear damages - recent developments in the United States, 1431 (R;ES)

Occupational Safety

Occupational exposure in an underground mine repository for radioáctive waste, 1387 (J;US)

Occupational radiation exposure risk at a nuclear waste repository in basalt, 1389 (J;US)

Occupational exposure for nuclear waste handling operations at NNWSI repository, 1390 (J;US)

Results of the occupational dose assessment in the conceptual reference repository description, 1388 (J;US)

Off-gas Systems

Volatilization and trapping of ruthenium in high temperature processes, 141 (RA;US)

Operation

Design considerations fur uccupatiủial exposurc for a potential repository at Yucca Mountain: high-level-waste handling operations, 991 (R;US)

Industrial operating experience at the Marcoule vitrification facility, 432 (BA;US)
Occupational exposure for nuclear waste handling operations at NNWSI repository, 1390 (J;US)

Performance

Evaluation of research and development for terminal isolation of nuclear wastes, 701 (R;US)

Natural analogues: a way to increase confidence in predictions of long-term performance of radioactive waste disposal, 1061 (J;US)

Parameters and variables appearing in radiological assessment codes. Final report, 1375 (R;US)

Post-closure performance assessment methodology for the Office of Nuclear Waste Isolation, 1219 (J;US)

Satisfying performance assessment data needs, 1222 (J;US)

System study and performance constraints for repository horizon selection at the Nevada Test Site, 1225 (J;US)

Technical conservatism in the design and analysis of a nuclearwaste repository in basalt, 907 (R;US)

Personnel

Occupational exposure in an underground mine repository for radioactive waste, 1387 (J;US)

Occupational radiation exposure risk at a nuclear waste repository in basalt, 1389 (J;US)

Projected occupational exposures during WIPP operations, 1041 (J;US)

Results of the occupational dose assessment in the conceptual reference repository description, 1388 (J;US)

Summary of ONWI occupational dose assessment program, 1391 (J;US)

Personnel Dosimetry

Occupational exposure in an underground mine repository for radioactive waste, 1387 (J;US)

Results of the occupational dose assessment in the conceptual reference repository description, 1388 (J;US)

Physical Protection

Human intrusion into geologic repositories for high-level radioactive waste: potential and prevention, $1235(\mathrm{~J} ; \mathrm{CH})$

Pilot Plants

Basic data report for drillhole WIPP 11 (Waste Isolation Pilot Plant - WIPP), 928 (R;US)

Basic data report for deepening of drillhole WIPP 13 (Waste Isolation Pilot Plant-WIPP), 975 (R;US)

Basic data report for drillhole WIPP 12 (Waste Isolation Pilot Plant-WIPP), 984 (R;US)

Basic data report for drillhole ERDA 6 (Waste Isolation Pilot Plant - WIPP), 923 (R;US)

Basic data report for drillhole ERDA 9 (Waste Isolation Pilot Plant WIPP), 926 (R;US)

Planning

Answers to your questions about high-level nuclear waste isolation, 1298 (B;US)

Defense Waste Processing Facility: a remote process for solidification of Savannah River Plant high level waste, 195 (R;US)

Evaluation of an international, perpetual, and retrievable facility for storage of vitrified radioactive waste, 97 (J;US)

Process Control

DWPF process control, 186 (R;US)

Radiation Doses

Limiting-individual dose from an extended-source high-level waste repository model, 1058 (J;US)

Radiation Hazards

Radiological risk analysis of an operating high-level waste repository, 1395 (BA;XA)

Waste-Mixes Study for space disposal, 815 (R;US)

Radiation Protection

NWTS transportation interface technology development priority report, 89 (R;US)

Occupational radiation exposure risk at a nuclear waste repository in basalt, 1389 (J;US)

Occupational exposure for nuclear waste handling operations at NNWSI repository, '1390 (J;US)

Projected occupational exposures during WIPP operations, 1041 (J;US)

Results of the occupational dose assessment in the conceptual reference repository description, 1388 (J;US) 
Summary of ONWI occupational dose assessment program, 1391 (J;US)

Radioactive Efiluents

Initial operations in the remote laboratory-scale waste-treatment facility, 312 (R;US)

Radionuclide Migration

Volume 4. A simplified analysis of a hypothetical repository in a bedded salt formation, 1418 (RA;US)

Reliability

Three aspects of reliability for a geological nuclear waste repository, 1255 (J;US)

Remote Handling Equipment

Remote aspects of treating and handling radioactive wastes, 449 (BA;US)

Research Programs

Answers to your questions about high-level nuclear waste isolation, 1298 (B;US)

Waste Isolation Pilot Plant. Project progress report, December 1 , 1982-Fehriary 28, 1983, 1031 (R;US)

Risk Assessment

Disposal of high-level waste from nuclear power olants in Denmark. Salt dome investigations. V.5. Safety evaluation, 652 (R;DK)

\section{Safeguands}

Development of safeguards requirements for high-level waste

Sofety repositories, 1421 (J;US)

Repository safety-the salient facts, 1397 (J;US)

Solt Deposits

Isolation of radioactive wastes in salt repositories, 951 (R;US)

Results of the occupational dose assessment in the conceptual reference repository description, 1388 (J;US)

Sealing Materials

Sealing concepts for the Waste Isolation Pilot Plant (WIPP) site, 938 (R;US)

Seals

Hydrology of sealing a repository in saturated tuff, 992 (R;US)

Repository sealing, 794 (RA;US)

Seismic Efiects

Repository design and equipment, 791 (RA;US)

Setsmology

Monitoring an underground repository with modern seismological methods, 1238 (J;US)

Site Selection

Application of performance assessment models to evaluate uncertainty in site characterization, 1221 (J;US)

Evaluation of an international, perpetual, and retrievable facility for storage of vitrified radioactive waste, 97 (J;US)

High-level radioactive-waste-disposal investigations in Texas, 1001 (R;US)

Interpreted resistivity and IP section line W1: Wahmonie Area, Nevada Test Site, Nevada, 567 (R;US)

Isolation of high-level nuclear wastes in a geologic repository, 1084 (J;US)

Issues in repository siting, 798 (RA;US)

Issues related to field testing in tuff, 947 (R;US)

Management of high-level waste repository siting, 1071 (J;US)

National plan for siting high-level radioactive waste repositories and environmental assessment. Public draft, 583 (R;US)

Nuclear fuel waste disposal - status of the Canadian program, 1263 (J;US)

Parameters and variables appearing in repository-siting models, 745 (R;US)

Public hearings panel report: a summary of public concerns regarding the characterization of a repository site in Nevada (Yucca Mountain), 766 (R;US)

Site investigations for repositories for solid radioactive wastes in deep continental geological formations, 1139 (B;XA)

Status of geologic/environmental characterizations, 797 (RA;US)

Summary of Near-Surface Test Facility results and their application to repository design, 886 (R;US)

Summary report on the geochemistry of Yucca Mountain and environs, 700 (R;US)
Unsaturated flow and transport through fractured rock - relat to high-level waste repositories. Final report. Phase I, 748 (R;US)

Site Surveys

Electrical studies at the proposed Wahmonie and Calico Hills nuclear waste sites, Nevada Test Site, Nye Co., Nevada, 1023 (R;US)

Occupational exposure for nuclear waste handling operations at NNWSI repository, 1390 (J;US)

Status of site characterization in the Permian Basin, $\mathbf{5 9 0}$ (RA;US)

Specifications

NWTS program criteria for mined geologic disposal of nuclear waste: repository performance and development criteria. Public draft, 596 (R;US)

Systems Analysis

Impacts of transportation on a test and evaluation facility for nuclear waste disposal: a systems analysis (Packaging facility), 556 (R;US)

Three aspects of reliability for a geological nuclear waste repository, 1255 (J:US)

Taxes

Issues in the use of payments in lieu of taxes to provide nuclear waste fooility oiting ineontivos, 1236 (J;CII)

Temperature Vistribution

Large-scale continuous process to vitrify nuclear defense wastc: operating experience with nonradioactive waste, 177 (R;US)

Temperature Effects

Results of repository conditions study for commercial and defense high-level nuclear waste and spent fuel repositories in salt, 824 (R;US)

Test Facilities

Relationship of an in-situ test facility to a deep geologic repository for high-level nuclear waste. Final report (Task 4), June 1981-October 1982, 742 (R;US)

Test facilities and excavations, 774 (RA;US)

Testing

Waste lsolation Pilot Plant (WIPP) research and development program: in situ testing plan, March 1982, 940 (R;US)

Thermal Analysis

Thermal analyses for a nuclear-waste repository in tuff using USW-G1 borehole data, 946 (R;US)

Thermel Stresces

Long-term thermomechanical and thermohydrolngical fastnrs controlling the optimal design of a nuclear waste repository, 1064 (J;US)

\section{Ventilation}

Forced ventilation analysis of a commercial high-level nuclear waste repository in tuff. Topical report RSI-0175, 941 (R;US)

Results of the occupational dose assessment in the conceptual reference repository description, 1388 (J;US)

Ventilation Systems

Comparison of waste emplacement configurations fó a uutlear waste repository in tuff. II. Ventilation analysis, 995 (R;US)

RADIOACTIVE WASTE MANAGEMENT

Historical overvicw (USA), 53 (RA;US)

Management of radioactive wastes, 101 (B;GB)

ONWI library reports list, 770 (R;US)

Radioactive waste management. Response by the Town and Country Planning Association to the white paper 'Radioactive Waste Management' - Cmnd 8607, 113 (B;GB)

Rockwell Hanford Operations monthly report summary, June 1983, 9 (R;US)

Waste-management prugram. Technical progresś report, JulySeptember 1981, 64 (R;US)

Alpha-bearing Wastes

Alpha-contuminated waste from reprocessing of nuclear fuel, 54 (RA;US)

DOE's nuclear waste management and fuel cycle programs, 1 (BA;US)

International fuel-cycle and waste-management technologyexchange activities sponsored by the United States Department of Energy: FY 1982 evaluation report, 85 (R;US)

Nuclear waste management, 119 (J;US) 
Waste Management Program. Technical progress report, October-December 1980, 162 (R;US)

\section{Bibliographies}

Annotated bibliography of radioactive waste management publications at Pacific Northwest Laboratory, January 1978 through July 1982 (831 abstracts), 84 (R;US)

Waste management bibliography, 1978-1983 (233 Citations), 279 (R;US)

\section{Coordinated Research Programs}

Enlarged Nordic cooperative program on nuclear safety. Nordic Cost study on reactor waste: main report, $72(\mathrm{R} ; \mathrm{SE})$

Draft regulatory impact analysis for 40 CFR 191: environmental standards for management and disposal of spent nuclear fuel, high-level and transuranic radioactive wastes. Draft report, 837 (R;US)

Relations of equivalence of conditioned radioactive waste, 112 (BA;US)

Demonstration Programs

Radiological risks for the alternatives for the long-term management of high-level wastes at West Valley, 1261 (J;US)

Environmental Impact Statements

Long-term management of liquid high-level radioactive wastes stored at the Western New York Nuclear Service Center, West Valley. Final environmental impact statement, 1305 (R;US)

Environmental Impacts

Draft environmental impact statement for 40 CFR part 191: environmental standards for management and disposal of spent nuclear fuel, high-level and transuranic radioactive wastes. Final report, 838 (R;US)

Draft regulatory impact analysis for 40 CFR 191: environmental standards for management and disposal of spent nuclear fuel, high-level and transuranic radioactive wastes. Draft report, 837 (R;US)

Gaseous Wastes

Nuclear-waste management semiannual progress report, April 1982-September 1982, 302 (R;US)

Government Policies

High-level nuclear waste: who governs, 105 (J;GB)

Managing commercial high-level radioactive waste: summary, 78 (R;US)

National program for isolating high-level nuclear waste, 410 " (J;GB)

Nuclear waste management policy in France, 92 (J;US)

Hearings

High-Level Nuclear-Waste Management. Oversight Hearing before the Subcommittee on Energy and the Environment of the Committee on Interior and Insular Affairs, House of Representatives, Ninety-Seventh Congress, Second Session, June $17,1982,110$ (B;US)

Radioactlve waste legislation. Hearings before the Subeommittoo on Energy and the Environment of the Committee on Interior and Insular Affairs, House of Representatives, Ninety-Seventh Congress, First Session on H.R. 1993; H.R. 2800; H.R. 2840; H.R. 2881; H.R. 3809, June 23 and 25; July 9, 1981, 1252 (B;US)

High-level Radioactive Wastes

Alternate strategy for commercial high-level radioactive-waste management, 945 (R;US)

Annual report of the management system design group, 967 (RA;US)

Assessment of overseas developments in methods for treatment and disposal of high-level radiuactive wastes, $532(\mathrm{R} ; \mathrm{AU})$

Building the institutional capacity for managing commercial high-level radioactive waste, $59(\mathrm{R} ; \mathrm{US})$

Composite quarterly technical report: long-term high-level-waste technology, January-March 1982 (Preparation, fixation, and final handling), 66 (R;US)

Considerations on the management of wastes expected to be generated by the operation of Brazilian nuclear reactors, 663 (RA;BR;In Portuguese)

DOE's commercial waste management and fuel cycle programs, $100(\mathrm{~J} ; \mathrm{US})$

Enlarged Nordic cooperative program on nuclear safety. Nordic study on reactor waste; main report, 72 (R;SE)
Environmental evaluation of alternatives for long-term management of Defense high-level radioactive wastes at the Idaho Chemical Processing Plant, 70 (R;US)

Handling of nuclear waste in Great Britain, 94 (J;DE;In German)

High level waste solidification: the future, 227 (RA;DE)

High-level radioactive wastes, 159 (R; US)

High level waste management and characterization at the Hanford plant site, 1198 (BA;US)

Information base for commercial radioactive-waste management, $56(\mathrm{R} ; \mathrm{US})$

International fuel-cycle and waste-management technologyexchange activities sponsored by the United States Department of Energy: FY 1982 evaluation report, 85 (R;US)

Long-term management of liquid high-level radioactive wastes stored at the Western New York Nuclear Service Center, West Valley. Final environmental impact statement, 1305 (R;US)

Management of bigh-level radioactive wastes, 218 (R;US)

Management of high-level waste repository siting, 1071 (J;US)

Management of vitrified high level radioactive waste, 466 (BA;GB)

Managing commercial high-level radioactive waste: summary, 78 (R;US)

Materials Characterization Center workshop on leaching mechanisms of nuclear waste forms, May 19-21, 1982, Gaithersburg, Maryland. Summary report, 82 (R;US)

National long-term high-level waste-technology program, 67 (R;US)

Nuclear Regulatory Commission activities related to high-level waste materials issues, 1420 (BA;US)

Nuclear waste management, 104 (J;US)

Nuclear-waste-management. Semiannual progress report, October 1981-March 1982, 301 (R;US)

Nuclear waste management, 119 (J;US)

Nuclear-waste management semiannual progress report, April 1982-September 1982, 302 (R;US)

Nuclear waste management. Quarterly progress report, October through December 1980, 296 (R;US)

Radioactive waste management 1980-81. Supplement to AIRAC No. 6, $111(\mathrm{~B} ; \mathrm{AU})$

Role of utility nuclear waste management group, 75 (RA;US)

Savannah River Waste Management Program Plan - FY 1982, 157 (R;US)

Second annual report made to the Secretary of State for the Environment, Secretary of State for Scotland, Secretary of State for Wales, 117 (B;GB)

Speech before the Atomic Industrial Forum Conference, 123 (BA;US)

Strategy document: long-term technology program for defense high-level waste, 156 (R;US)

Swedish nuclear waste efforts, 36 (R;US)

Technology for long-term management of ICPP Defense Waste, 122 (J;US)

The national long-term high-level waste technology program, 121 (J;US)

Uranium and nuclear energy: proceedings of the international symposium, 4th, 1979, l (B;GB)

Waste Management Program. Technical progress report, October-December 1980, 162 (R;US)

Waste-management program. Technical progress report, JanuaryMarch 1981, 622 (R;US)

Institutional Factors

Building the institutional capacity for managing commercial high-level radioactive waste, 58 (R;US)

Building the institutional capacity for managing commercial high-level radioactive waste, 59 (R;US)

Institutional aspects of radioactive waste management, 1437 (J;US)

Intermediate-level Radioactive Wastes

Handling of nuclear waste in Great Britain, 94 (J;DE;In German)

Nuclear waste management, i 04 (J;US)

Radioactive waste management $1980-81$. Supplement to AIRAC No. 6, $111(\mathrm{~B} ; \mathrm{AU})$ 
Second annual report made to the Secretary of State for the Environment, Secretary of State for Scotland, Secretary of State for Wales, 117 (B;GB)

Laws

Radioactive waste management: a summary of state laws and administration. National Low-Level Radioactive Waste Mangement Program, 569 (R;US)

Radioactive waste management: a summary of state laws and administration, 570 (R;US)

Legal Aspects

Nuclear Regulatory Commission activities related to high-level waste materials issues, 1420 (BA;US)

Legislation

High-level nuclear waste: who governs, 105 (J;GB)

Radioactive waste management: a summary of state laws and administration. National Low-Level Radioactive Waste Mangement Program, 569 (R;US)

Radioactive waste management: a summary of state laws and administration, $\mathbf{5 7 0}$ (R;US)

Low- levol Radiogetive Wasteo

DOE's nuclear waste management and fuel cycle programs, 1159 (BA;US)

EPRI's programs in low-level waste management, 74 (RA;US)

Handling of nuclear waste in Great Britain, 94 (J;DE;In German)

Information base for commercial radioactive-waste management, 56 (R;US)

International fuel-cycle and waste-management technologyexchange activities sponsored by the United States Department of Energy: FY 1982 evaluation report, 85 (R;US)

Nuclear-waste-management. Semiannual progress report, October 1981-March 1982, 301 (R;US)

Nuclear waste management, 119 (J;US)

Nuclear-waste management semiannual progress report, April 1982-September 1982, 302 (R;US)

Nuclear waste management. Semiannual progress report, Octo ber 1982-March 1983, 303 (R;US)

Radioactive waste management 1980-81. Supplement to AIRAC No. 6, 111 (B;AU)

Radioactive waste management: a summary of state laws and administration. National Low-Level Radioactive Waste Mangement Program, 569 (R;US)

Radioactive waste management: a summary of state laws and administration, 570 (R;US)

Role of utility nuclear waste management group, 75 (RA;US)

Savannah River Waste Management Program Plan - FY 1982, 157 (R;US)

Second annual report made to the Secretary of State for the Environment, Secretary of State for Scotland, Secretary of State for Wales, 117 (B;GB)

Speech before the Atomic Industrial Forum Conference, 123 (BA;US)

Waste Management Program. Technical progress report, October. Decomber 1980, 162 (R;UE)

Waste-management program. Technical progress report, JanuaryMarch 1981, 622 (R;US)

Meetings

International fuel-cycle and waste-management technologyexchange activities sponsored by the United States Department of Energy: FY 1982 evaluation report, 85 (R;US)

Scientific basis for nuclear waste management, 107 (B;US)

Scientific basis for nuclear waste management V, 1187 (B;US)

Scientific basis for nuclear waste management VI, 366 (B;US)

Uranium and nuclear energy: proceedings of the international symposium, 4th, 1979, 1 (B;GB)

Workshop on the leaching mechanisms of nuclear-waste forms, October 27-28, 1981. Summary report, 81 (R;US)

Mill Tallings

Information base for commercial radioactive-waste management, 56 (R;US)

Nuclear wastc management, 119 (J;US)

\section{Political Aspects}

Conflicting views on a neutrality criterion for radioactive-waste management (Monograph), 124 (B;US)

Public Information

Improving public understanding of nuclear waste management, $1136(\mathrm{~J} ; \mathrm{GB})$

Proposal for broader participation in radioactive waste management, 1135 (J;GB)

Public Policy

Conflicting views on a neutrality criterion for radioactive-waste management (Monograph), 124 (B;US)

Improving public understanding of nuclear waste management, $1136(\mathrm{~J} ; \mathrm{GB})$

Proposal for broader participation in radioactive waste management, 1135 (J;GB)

Public Relations

Improving public understanding of nuclear waste management, 1136 (J;GB)

Management of nuelcar fucl waste. Final rejuit, 71 (R,CA)

Proposal for broader participation in radioactive waste management, $1135(\mathrm{~J} ; \mathrm{GB})$

Recommendations

High-level nuclear waste: who governs, 105 (J;GB)

Research Programs

Composite quarterly technical report: longeterm high-level-waste technology, January-March 1982 (Preparation, fixation, and final handling), 66 (R;US)

Management of radioactive wastes in Europe (EC) history, philosophy and plans for the next five years, 108 (BA;US)

Nuclear-waste-management. Semiannual progress report, October 1981-March 1982, 301 (R;US)

Nuclear-waste management semiannual progress report, April 1982-September 1982, 302 (R;US)

Nuclear waste management. Quarterly progress report, October through December 1980, 296 (R;US)

Nuclear waste management. Semiannual progress report, October 1982-March 1983, 303 (R;US)

Results report on research and development work 1980 of the Institut fuer Nukleare Entsorgungstechnik, 276 (R;DE;In German)

Waste-management program technical progress report, JulySeptember 1980, 63 (R;US)

Waste-management $R$ and $D$ for the nuclear fuel cycle, 311 (R;US)

Waste Management Program. Technical progress report, October-December 1982, 65 (R;US)

Waste management program technical progress report, JanuaryMarch 1983, 623 (R;US)

Reviews

Waste management and transportation, 115 (BA;US)

Risk Assessment

Conflicting views on a neutrality criterion for radioactive-waste management, 95 (BA;US)

Enlarged Nordic cooperative program on nuclear safety. Nordic study on reactor waste: main repnrt., 72. (R,SE)

Radiological risks for the alternatives for the long-term management of high-level wastes at West Valloy; 1261 (JiUs)

Review of safety assessments of nuclear waste management (Preclosure and long-term risks), 1380 (R;US)

Spent Fuels

Information base for commercial radioactive-waste management, 56 (R;US)

International fuel-cycle and waste-management technologyexchange activities sponsored by the United States Department of Energy: FY 1982 evaluation report, 85 (R;US)

Managing commercial high-level radioactivc waste: summary, 78 (R;US)

Nuclear waste management, 104 (J;US)

Role of utility nuclear waste management group, 75 (RA;US)

Swedish nuclear waste efforts, 36 (R;US)

Technology Assessment

Conflicting views on a neutrality criterion for radioactive-waste management, 95 (BA;US) 
Technology Transfer

International fuel-cycle and waste-management technologyexchange activities sponsored by the United States Department of Energy: FY 1982 evaluation report, 85 (R;US)

RADIOACTIVE WASTE PROCESSING

See also SYNROC PROCESS

Development of partitioning method - cold and semihot experimental works with partitioning testing apparatus, 225 (R;JP;In Japanese)

ONWI library reports list, 770 (R;US)

Radioactive waste processing and disposal, 7 (RA;CS;In Czech)

Adsorption

Removal of ${ }^{90} \mathrm{Sr},{ }^{107} \mathrm{Cs}$, and ${ }^{106} \mathrm{Ru}$ from vent gases produced during solidification of high-level radioactive solutions, 399 (J;CS)

Alpha-bearing Wastes

Characterization and testing of basalt glass and glass ceramic waste forms, 313 (R;US)

Commercial waste treatment $R$ and $D$ needs in the United States, 323 (R;US)

Commercial waste treatment technology and programs, 359 (J;US)

Equipment, operation and some results from a hot test of the CTH actinide separation process (Norway), 243 (RA;DE)

European Communities' $R$ and $D$ activities on treatment and handling of radioactive waste, 426 (BA;US)

Evaluation of forms for the immobilization of high-level and transuranic wastes, 200 (R;US)

In situ vitrification: application analysis for stabilization of transuranic waste, 307 (R;US)

Iron-enriched basalt for containment of nuclear wastes, $\mathbf{4 1 6}$ (BA;US)

Method for solidifying aqueous radioactive wastes for noncontaminating storage (Patent), 379 (P;US)

Nuclear-fuel-cycle education: Module 8. Waste management, 155 (R;US)

Nuclear-waste-management. Semiannual progress report, October 1981-March 1982, 301 (R;US)

Nuclear waste management. Quarterly progress report, October through December 1980, 296 (R;US)

Nuclear waste management. Semiannual progress report, October 1982-March 1983, 303 (R;US)

Package materials; waste form, 792 (RA;US)

Preparation and leaching of radioactive INEL waste forms, 201 (R;US)

Reprocessing, decontamination and decommissioning waste management at Eurochemic: current and planned activities, $\mathbf{4 3 3}$ (BA;US)

Solidification of high-level radioactive wastes and alpha-bearing wastes by a sol-gel procese (in German), 244 (RA;DE)

Status, direction, and critical issues of waste treatment technology, 330 (R;US)

Status of high level and alpha bearing waste management in PNC, 428 (BA;US)

Treatment and conditioning of wastes from US nuclear fuels reprocessing plants, 191 (R;US)

TRU waste-acceptence criteria for the Waste Isolation Plant, 1419 (R;US)

Waste Management Program. Technical progress report, October-December 1980, 162 (R;US)

Waste-Mixes Study for space disposal, 815 (R;US)

Wastè package, 288 (RA;US)

Bench-scale Experiments

Immobilization of radioactive liquid waste using water glass, 470 (J;US)

Laboratory activity in Italy in the field of vitrification of highlevel wastes, 233 (RA;DE)

Bibliographies

Radioactive waste processing and disposal: a bibliography, 158 (R;US)

Calcination

Attrition, clutriation, and growth of particles produced in fluidized-bed waste calciners, 202 (R;US)

Calcination of liquid radioactive wastes. Part 1 . Technology of radioactive waste calcination, 361 (j;CS;In $C$ zech)
Denitration of simulated fast ractor highly active liquor waste (UKAEH), 241 (RA;DE)

Fixation of radioactive residues. Quarterly progress report, January-March 1961, 213 (R;US)

ICPP waste calcining facility safety analysis report, 219 (R;US)

Physical and chemical characterization of synthetic calcined sludge, 167 (R;US)

Process for producing biologically noxious waste, particularly radioactive waste, wich can be stored (German Patent), 519 (P;DE;In German)

Research and development activities: fixation of radioactive residues. Quarterly progress report, April-June 1960, 210 (R;US)

Research and development activities: fixation of radioactive residues. Quarterly progress report, July-September 1960, 212 (R;US)

Research and development activities [on] solidification of radioactive wastcs. Progress report, 215 (R;US)

Solidification of high level wastes. Part III. Solubility, melting point, flow temperature and thermal conductivity of some calcined, synthetic purex-type wastes, 209 (R;US)

Solidification of high-level radioactive fuel reprocessing wastes by spray and pot calcination. Hot-cell pilot plant studies, 217 (R;US).

Waste treatment and disposal. Quarterly progress report, February-April, 1963, 290 (R;US)

Calcined Wastes

Immobilization of Idaho Chemical Processing Plant high-level wastes, 240 (RA;DE)

Ceramic Melters

Off-gas characteristics of liquid-fed joule-heated ceramic melters, 319 (R;US)

Cesium

Feasibility of incinerating and vitrifying organic resins in a single step, 318 (R;US)

Precipitation-adsorption process for the decontamination of nuclear waste supernates (Patent), 397 (P;US)

Precipitation process for supernate decontamination, 173 (R;US)

Precipitation process for decontamination of water-soluble SRP radioactive waste, 184 (R;US)

Precipitation process for decontaminating water-soluble SRP radioactive waste, 187 (R;US)

Savannah River Laboratory long-term waste storage program. Progress report No. 7, July-September 1974, 133 (R;US)

Supernatant treatment considerations for the neutralized waste at West Valley, 370 (J;US)

Systems study of the feasibility of high-level nuclear-waste fractionation for thermal-stress control in a geologic repository: main report, 308 (R;US)

Systems study of the feasibility of high-level nuclear waste fractionation for thermal stress control in a geologic repository: appendices, 309 (R;US)

Cesium 137

CsAlSis $\mathrm{O}_{12}$ : a possible host for ${ }^{107} \mathrm{Cs}$ immobilization, 404 (J;GB)

Method of solidifying radioactive wastes (Patent), 483 (P;JP;In Japanese)

Contings

Coating crystalline nuclear waste forms to improve inertness, 138 (R;US)

Combustion

Feasibility of incinerating and vitrifying organic resins in a single step, 318 (R;US)

Solidification of ash from incineration of low-level radioactive waste, 193 (R;US)

Status, direction, and critical issues of waste treatment technolo$8 y, 330$ (R;US)

Comminution

Method of treating high level radioactive solid waste, 389 (P;JP;In Japanese)

Comparative Evaluation

Assessment of processes, facilities, and costs for alternative solid forms for immobilization of SRP defense waste (Burusilicate; glass-lead; glass; concrete; ceramic; and coated particles), 169 (R;US)

Assessment of rationale for development of improved waste forms; 198 (J;US) 
Processability analysis of candidate waste forms (For SRP highlevel wastes), 174 (R;US)

Containers

Feasibility of using a hign-level waste canister as an engineered barrier in disposal, 842 (R;US)

Flame-resistant radioactive waste processing method, 488 (P;JP;In Japanese)

Full-scale impact tests of simulated high-level waste canisters, 316 (R;US)

Coordinated Research Programs

Package materials, waste form, 792 (RA;US)

Savannah River Laboratory long-term waste storage program. Progress report No. 5, March-April 1974, 131 (R;US)

Savannah River Laboratory long-term waste storage program. Progress report No. 6, May-Jun 1974, 132 (R;US)

Savannah River Laboratory long-term waste storage program. Cost Progress report No. 7, July-September 1974, 133 (R;US)

Comparison of SRP high-level waste disposal costs for borosilicate glass and crystalline ceramic waste forms, 635 (R;US)

High-level nuclear-waste-immobilization program, 154 (R;US)

Synthesis of alkoxide glass formers for nuclear waste immobilization, 438 (BA;US)

Cost Bonefit Analysis

Cesium and strontium fractionation from HLW for thermal-stress reduction in a geologic repository, 327 (R;US)

Conditioning of radioactive wastes for storage and disposal, 364 (B;XA)

Systems study of the feasibility of high-level nuclear-waste fractionation for thermal-stress control in a geologic repository: main report, 308 (R;US)

Systems study of the feasibility of high-level nuclear waste fractionation for thermal stress control in a geologic repository: appendices, $309^{\circ}$ (R;US)

Decontamination

Development and demonstration of a process for vitrification of TMI zeolite, 505 (J;US)

Demineralizers

Development and demonstration of a process for vitrification of TMI zeolite, 505 (J;US)

Demonstration Programs

Engineering scale multibarrier waste form demonstrations, 499 (J;US)

Denitration

Chemical denitration of radioactive-waste solutions with formic acid, 531 (TG;US)

Denitration of simulated fast ractor highly active liquor waste (UKAEH), 241 (RA;DE)

Denitration of simulated fast reactor highly active liquor waste, $282(R ; G B)$

Fission product solidification: FIPS (In German), 229

(RA;DE;GE)

Method of processing off-gas condensate containing precipitations resulted upon solidification of high-level liquid wastes issued from a reprocessing plant, 406 (P;JP;In Japanese)

On-line measurcments of the volatilization of ruthenium in a vitrification process, 422 (BA;US)

Ruthenium nitrosyl complexes in radioactive waste solutions of reprocessing plants. Pt. 1, 12 (J;DE)

Dilution

Water dilution volumes for high-level wastes, 1401 (J;US)

Dryers

Use of the drum dryer in radioactive waste solidification, 414 (BA;DE;In German)

Drying

Long-Term High-Level Defense Waste technology progress report, January-March 1982, 337 (R;US)

Economic Analysis

In situ vitrification: application analysis for stabilization of transuranic waste, 307 (R:US)

Economics

Commercial high-level-waste management: options and economics. A comparative analysis of the ceramic and glass waste forms, 150 (R:US)
Research and development activities: fixation of radioactive residues. Quarterly progress report, April-June 1960, 210 (R;US)

Encapsulation

Development and testing of matrices for the encapsulation of glass and ceramic nuclear waste forms, 297 (R;US)

Encapsulation of tritiated wastes in hot laboratories, 137 (R;FR;In French)

Engineering scale multibarrier waste form demonstrations, 499 (J;US)

Fixation of radioactive materials in a glass matrix (Patent), 475 (P;CA)

Leach characterization of cement encapsulated wastes, 1156 (J;US)

Method to encapsulate radioactive material, 478 (TG;DE;In German)

Environmental Impact Statements

Defense Waste Processing Facility: Savannah River Plant, Aiken, SC. Final environmental impact statement, 1306 (R;US)

Evaluation

Evaluation of research and development for terminal isolation of . nuclear wastes, 701 (R;US)

Method for product performance evaluation of candidate waste formo for immobilization of higli-level cadluactlve wastes. Intertface working group on high-level waste form selection factors, 161 (R;US)

Review of DOE waste package program. Subtask 1.1. National waste package program, 286 (R;US)

Evaporation

Influence of the reprocessing flow sheet on the HLW solidification technology, 239 (RA;DE)

Explosions

Steam explosions caused by te contact of molten glass and water, 246 (RA;DE)

Fensibility Studies

Feasibility of incinerating and vitrifying organic resins in a single step, 318 (R;US)

Solidification of high level wastes. Part IV. Phosphate melts for fixation of radioactive residues from Purex type wastes: three to fifty percent fission product oxides, 134 (R;US)

\section{Filtration}

Deposition of volatile fission products during commercial lighlevel waste vitrification, 304 (R;US)

Removal of ${ }^{99} \mathrm{Sr}^{107} \mathrm{C}_{4}$, and ${ }^{106} \mathrm{Ru}$ from vent gases producted during solidification of high-level radioactive solutions, 399 (J;CS)

Financial Incentives

Commercial waste treatment technology and programs, 359 (J;US)

Fission Products

Borosilicate glass and glass ceramics with real highly radioactive waste, 528 (J;US)

Fission product solidification: FIPS (In German), 229 (RA;DE;GE)

Influence of the reprocessing flow sheet on the HLW solidification technology, 239 (RA;DE)

Fractionation

Rethinking the management of high-level nuclear waste: the need for fractionation, $509(\mathrm{~J} ; \mathrm{US})$

Fuel Feeding Systems

Design and performance of feed-delivery systems for simulated radioactive waste slurries, 306 (R;US)

Gaseous Wustes

Compound refining system for separation of gaseous fission products incorporated in a reprocessing pilot plant for spent fuel from neclear power stations, 494 (BA;JP;In Japanese)

Control of semivolatile radionuclides in gaseous effluents at nuclear facilities, 453 (B;XA)

Treatment and conditioning of wastes from US nuclear fuels reprocessing plants, 191 (R;US)

Gelation

Preparation of gel spheres containing simulated high-level radioactive waste, $381(\mathrm{~J} ; \mathrm{CH})$ 
- obal Aspects

Waste vitrification: a historical perspective, 317 (R;US)

wernment Policies

National program for isolating high-level nuclear waste, $\mathbf{4 1 0}$ (J;GB)

High-frequency Heating

Melting of glass by direct induction heating in ceramic container, 480 (J;JP;In Japanese)

Higb-level Radioactive Wastes

Actual point about fission products vitrification, 136 (R;FR;In French)

Alpha-recoil damage in natural zirconolite and perovskite, 518 (J;JP)

Alternate strategy for commercial high-level radioactive-waste management, 945 (R;US)

Alternate waste form evaluation for Savannah River Plant highlevel waste, 1104 (J;US)

Alternative fuels and heating methods for calciner process heat, 204 (R;US)

Analysis of thin leaching layers and fracture mechanics of irradiated borosilicate glass for solidification of high-level fission product solutions (HAW), 365 (BA;DE; In German)

Assessment of Savannah River borosilicate glass in the repository environment, 172 (R;US)

Assessment of high-level waste form conformance with proposed regulatory and repository criteria, 198 (R;US)

Assessment of rationale for development of improved waste forms, 498 (J;US)

Assessment of overseas developments in methods for treatment and disposal of high-level radioactive wastes, $532(\mathrm{R} ; \mathrm{AU})$

Borosilicate glass and glass ceramics with real highly radioactive waste, 528 (J;US)

Calcination of liquid radioactive wastes. Part I. Technology of radioactive waste calcination, 361 (J;CS;In Czech)

Capacity of the borosilicate glass VG 98/12 for components of the high-level radioactive waste, 267 (RA;DE;In German)

Cement-based radioactive waste hosts formed under elevated temperatures and pressures (FUETAP concretes) for Savannah River Plant high-level defense waste, 293 (R;US)

Ceramic nuclear waste forms, 259 (RA;DE)

Ceramic nuclear waste forms, 271 (RA;DE)

Ceramic process and plant design for high-level nuclear waste immobilization, 144 (R;US)

Cesium and strontium fractionation from HLW for thermal-stress reduction in a geologic repository, 327 (R;US)

Change of electrical properties of the borosilicate glasses containing $\mathrm{MoO}_{0}$ by phase separation, 482 (J;JP;In Japanese)

Characterization and testing of basalt glass and glass ceramic waste forms, 313 (R;US)

Characterization of borosilicate glass containing Savannah River

Plant radioactive waste, 179 (R;US)

Chemistry of the nuclear fuel cycle, 4 (BA;GB)

Coating of crystalline nuclear waste forms to improve inertness, 386 (J;US)

Commercial waste treatment $R$ and $D$ needs in the United

States, 323 (R;US)

Commercial high-level-waste management: options and economics. A comparative analysis of the ceramic and glass waste forms, 150 (R;US)

Commercial waste treatment technology and programs, 359 (J;US)

Compared quantitative determination of nuclear waste glass crystallization rate by light microscopy and $x$ ray diffraction, 257 (RA;DE)

Compared quantitative determination of nuclear waste glass crystallization rate by light microscopy and X-ray diffraction, 269 (RA;DE)

Comparison of properties of borosilicate glass and crystalline ceramic forms for immobilization of Savannah River Plant waste, 171 (R;US)

Zomparison of borosilicate glass and synthetic minerals as media for the immobilization of high-level radioactive waste, 490 (J;US)

Comparison of wet slurry and dry frit (crizzle) feeding of glassformers in pot vitrification (AERE), 242 (RA;DE)
Compound refining system for separation of gaseous fission products incorporated in a reprocessing pilot plant for spent fuel from neclear power stations, 494 (BA;JP;In Japanese)

Conditioning of radioactive wastes for storage and disposal, 364 (B;XA)

Consolidated waste forms: glass marbles and ceramic pellets, 299

(R;US)

Containing waste material (Patent), 402 (P;AU)

Control of semivolatile radionuclides in gaseous effuents at nuclear facilities, 453 (B;XA)

Coordination meeting of high-level waste technology and national waste terminal storage programs, October 21-22, 1981, 624 (R;US)

Coprecipitation of radioactive technetium with ferrous hydroxide, 1443 (J;JP;In Japanese)

Critical review of radiation effects on borosilicate glasses, 261 (RA;DE)

Crystalline ceramics as a high-level nuclear waste form, 395 (J;US)

Current technics and management strategy for Pu-contaminated wastes at PNC, 295 (R;JP)

Defense Waste Processing Facility: a remote process for solidification of Savannah River Plant high level waste, 195 (R;US)

Denitration of simulated fast ractor highly active liquor waste (UKAEH), 241 (RA;DE)

Denitration of simulated fast reactor highly active liquor waste, 282 (R;GB)

Department of Energy's selection of high level waste forms, 443 (BA;US)

Depasition of volatile fission products during commercial highlevel waste vitrification, 304 (R;US)

Description of a ceramic waste form and canister for Savannah

River Plant high-level waste, 170 (R;US)

Description of Defense Waste Processing Facility reference waste form and canister. Revision 1, 168 (R;US)

Design and performance of feed-delivery systems for simulated radioactive waste slurries, 306 (R;US)

Design of Harwell Joule Ceramic Melter (UKAEA), 231 (RA;DE)

Developing technology of microwave vitrification, 392 (J;GB)

Development and testing of matrices for the encapsulation of glass and ceramic nuclear waste forms, 297 (R;US)

Development of borosilicate glass for the vitrification plant PAMELA, 530 (TG;US)

Development, evaluation, and selection of candidate high-level waste forms, 185 (R;US)

Development and testing of SYNROC for high level radioactive waste fixation, 510 ( $\mathrm{J}$;US)

Development and testing of SYNROC for high-level radioactive waste fixation, 493 (J;AU)

Development of borosilicate glass for the vitrification facility PAMELA, 253 (RA;DE;GE)

Development and testing of SYNROC $\mathrm{C}$ as a high level nuclear waste form (Australian Atomic Energy Commission), 418 (BA;US)

Development of polyphase ceramics for the immobilization of high-level Defense nuclear waste, 151 (R;US)

Development of high-level liquid waste solidification in PNC, 431 (BA;US)

Diopside glass-ceramic material for the immobilization of radioactive wastes, 258 (RA;DE)

Diopside glass-ceramic material for the immobilization of radioactive wastes, 270 (RA;DE)

Disposal of radioactive wastes (Book), 106 (B;NL)

DWPF process control, 186 (R;US)

Electrodes for the direct heating of highly radioactive borosilicate glasses in HAW-ceramic furnaces (in German; Kernforschungszentrum, Karlsruhe), 236 (RA;DE;GE)

Encapsulation of tritiated wastes in hot laboratories, 137 (R;FR;In French)

Engineering scale multibarrier waste form demonstrations, 499 (J;US)

Equipment, operation and some results from a hot test of the CTH actinide separation process (Norway), 243 (RA;DE) 
Equipment for radioactive waste solidification, 390 (J;CS;In Crech)

European Communities' $\mathbf{R}$ and $\mathbf{D}$ activities on treatment and handling of radioactive waste, 426 (BA;US)

Evaluation of process alternatives for solidification of existing commercial high-level waste, 501 (J;US)

Evaluation of process alternatives for solidification of the West Valley high-level liquid wastes, 436 (BA;US)

Evaluation of the AVM process and its applicability to West Valley, 437 (BA;US)

Evaluation of coated particle waste forms, 448 (BA;US)

Evaluation of forms for the immobilization of high-level and transuranic wastes, 200 (R;US)

Final conditioning of high-level fission product solutions, 376 (J;DE;In German)

Fission product solidification: FIPS (In German), 229

(RA;DE;GE)

Fixation of radioactive residues. Quarterly progress report, January-March 1961, 213 (R;US)

Fixation of radioactive materials in a glass matrix (Patent), 475 (PICA)

Fixation of radioactive materials in a glass matrix, 380 (P;AU)

Fixation of high level solid waste (HLSW) in a $\mathrm{TiO}_{2}$ base ceraimic matrix provided with an integrated overpack, 367 (J;US)

Flame-resistant radioactive waste processing method, 488 (P,JP,In Japanese)

Formulation of SYNROC-D additives for Savannah River Plant high-level radioactive waste, 347 (R;US)

Formulation and processing of polyphase ceramics for high level nuclear waste, 421 (BA;US)

Formulation and processing of alumina based ceramic nuclear waste forms, 153 (RA;US)

FUETAP concrete: an alternative radioactive waste host, 256 (RA;DE)

Full-scale impact tests of simulated high-level waste canisters, $316(R ; U S)$

High level waste solidification: the future, 227 (RA;DE)

High-level radioactive wastes, 159 (R;US)

High-level waste solidification system for the Western New York Nuclear Service Center, 315 (R;US)

High-level nuclear-waste-immobilization program, 154 (R;US)

High-level waste vitrification technique in a full-scale pilot plant, 439 (BA;US)

High level waste forms: glass marbles and thermal spray coatings, 447 (BA;US)

High level waste management at the Idaho Chemical Processing Plant, 451 (BA;US)

Highly dense graphite matrix - a new material for the conditioning of radioactive wastes, 394 (J;US)

HLW fixation in sintered modified SYNROC-B ceramics: chemical stabllity evaluation, 326 (J;US)

IAEA report on actinide partitioning and transmutation, 383 (J;GB)

ICPP waste calcining facility safety analysis report, 219 (R;US)

Immobilization of high-level defense wastes in SYNROC-D: recent research and development results on process scale-up, 351 (R;US)

Immobilization of Idaho Chemical Processing Plant high-level wastes, 240 (RA;DE)

Immobilization of reprocessing waste by means of titanate ion exchangers, 248 (RA;DE)

Immobilization of sodium and phosphorus-bearing PW-7a waste

in SYNROC. Progress report, 346 (R;US)

Immobilization of high-level nuclear reactor wastes in SYNROC: a current appraisal, 515 (J;US)

Immobilization of radioactive liquid waste using water glass, 470 (J;US)

Impact of thermal constraints on the optimal design of bigh-level waste .repositories in geologic media, $1070(\mathrm{~J} ; \mathrm{NL})$

Improved polyphase ceramic form for high-level defense nuclear waste, 152 (RA;US)

Inactive Pamela vitrification facility in Mol; manufacture and characteristics of Vitromet produtts (in German), 238 (RA;DE;GE)
Inactive vitrification in Mol. Ceramic melting, feed and off $\mathbf{g}$ system, pouring equipment for glassmelting (In German), 2 (RA;DE;GE)

Incorporation of high-level wastes in SYNROC: results from recent process-engineering studies at Lawrence Livermore National Laboratory, 352 (R;US)

Incorporation of simulated bigh-level nuclear waste in gel spheres, 292 (R;US)

Incorporation of high-level nuclear waste in gel spheres, 139 (R;US)

Industrial operating experience at the Marcoule vitrification facility, 432 (BA;US)

Influence of the reprocessing flow sheet on the HLW solidification technology, 239 (RA;DE)

Initial operations in the remote laboratory-scale waste-treatment facility, 312 (R;US)

Intercomparison of leach-testing methods and the effects of waste-form composition on test type and duration, 1157 (J;US)

Investigations of suitable metallic container materials for HAW solidification, 371 (J;US)

Iron-enriched basalt as a waste form for high-level nuclear wastes, 199 (R;US)

Iron-enriched basalt for containment of nuclear wastes, $\mathbf{4 1 6}$ (BA;US)

Laboratory activity in Italy in the field of vitrification of highlevel wastes, 233 (RA;DE)

Land Baden-Wuerttemberg: public notice of the Ministry of Economics, Medium-size Industry and Transport concerning a permit under the Atomic Energy Law. - 3rd Supplement to the 9th partial licence for the construction of Karlsruhe reprocessing plant - System for storage and evaporation of high-activity liquid waste (LAVA), 10 (J;DE;In German)

Leach characterization of cement encapsulated wastes, 1156 (J;US)

Leachability study of modified Synroc-B waste form: effect of gamma irradiation, 412 (J;GB)

Leaching of solidified highly radioactive decay products: overview (In German), 249 (RA;DE;GE)

Leaching of natural and synthetic sphene and perovskite, 456 (BA;US)

Long-Term High-Level Defense-Waste technology. Progress report, April-June 1982, 338 (R;US)

Lung-Term High-Level Defense Waste technulugy prugress report, January-March 1982, 337 (R;US)

Long-term high-level-waste technology. Composite quarterly technical report, April-June 1982, 165 (R;US)

Long-term high-level waste technology. Composite quarterly technical report, July-September 1982, 166 (R;US)

Management of vitrified high level radioactive waste, 466 (BA;GB)

Marcoule vitrification shop: 30 months service (In French), 228 (RA;DE;FR)

Materials studies of simulated interim waste forms for high-level radiactive wastes, 502 (J;US)

Materials balance: scientific fundamentals for the quality assurance of vitrified waste, 462 (BA;US)

Method and device fur sulidifying high level liquid wastes frum a re-processing plant, 403 (P;JP;In Japanese)

Method for product performance evaluation of candidate waste forms for immobilization of high-level radioactive wastes. Interface working group on high-level waste form selection factors, 161 (R;US)

Method for solidifying aqueous radioactive wastes for noncontaminating storage (Patent), 379 (P;US)

Method for producing molded bodies containing highly active radioactive wastes from glass granules embedded in a metallic matrix, 360 (P;US)

Method of solidifying radioactive wastes (Patent), 483 (P;JP; Japanese)

Method of preparing nuclear wastes for transportation and interim storage, 391 (P;US)

Method of processing high level radioactive wastes by glass solidification, 408 ( $P$;JP;In Japanese) 
Method of processing off-gas condensate containing precipitations resulted upon solidification of high-level liquid wastes issued from a reprocessing plant, 406 (P;JP;In Japanese)

Method of treating high level radioactive solid waste, 389 (P;JP;In Japanese)

Method to encapsulate radioactive material, 478 (TG;DE;In German)

Microstructures and crystallization in simulated waste glasses, 441 (BA;US)

Microstructure of Synroc, 454 (BA;US)

Microwave energy for post-calcination treatment of high-level nuclear wastes, 378 ( $\mathrm{J}$;US)

New waste calcining facility at ICPP, 452 (BA;US)

Nondestructive techniques for evaluating the integrity of vitrified solids, 471 (J;JP)

Nuclear-fuel-cycle education: Module 8 . Waste management, 155 (R;US)

Nuclear-waste-management. Semiannual progress report, October 1981-March 1982, 301 (R;US)

Nuclear waste vitrification in a ceramic-lined electric glass melter (Federal Republic of Germany), 409 (J;US)

Nuclear-waste management semiannual progress report, April 1982-September 1982, 302 (R;US)

Nuclear waste management. Quarterly progress report, October through December 1980, 296 (R;US)

On-line measurements of the volatilization of ruthenium in a vitrification process, 422 (BA;US)

Overview on product forms (In German), 252 (RA;DE;In German and English)

Overview on product forms, 264 (RA;DE; In German)

Ozone mass transfer and kinetics experiments, 334 (R;US)

Package materials, waste form, 792 (RA;US)

Phosphate system as medium for radioactive waste fixation. Simplex experiment. Part I, 354 (R;CS;In Czech)

Phosphate system as medium for radioactive waste fixation - simplex experiment. Part II, 355 (R;CS;In Czech)

Phosphate systems - a medium for high level waste solidification, 362 (J;CS;In Czech)

Policy and development of reprocessing of spent nuclear fuel and high-level radioactive waste management in the F.R. Germany, 13 (J;JP;In Japanese)

Precipitation-adsorption process for the decontamination of nuclear waste supernates (Patent), 397 (P;US)

Precipitation proccss for supernate decontamination, 173 (R;US)

Precipitation process for decontamination of water-soluble SRP radioactive waste, 184 (R;US)

Precipitation process for decontaminating water-soluble SRP radioactive waste, 187 ( $R ; U S$ )

Precipitation process for decontamination of water soluble SRP radioactive waste, 468 (J;US)

Prelimianry experience with vitrification inactive pilot plant in Italy, 234 (RA;DE)

Preliminary assessment of key process steps for converting Savannah River Plant high-level wastes to tailored ceramics, 147 (R;US)

Preparation and leaching of radioactive INEL waste forms, 201 (R;US)

Preparation of the high-level waste form for the near-surface test facility, 497 (J;US)

Preparation and characterization of an improved borosilicate glass for the solidificatiun of high level radionctive fission product solutions (HLW). Pt. 2, 277 (R;DE)

Preparation of gel spheres containing simulated high-level radioactive waste, $381(\mathrm{~J} ; \mathrm{CH})$

Present status of the West Valley Demonstration Project, 435 (BA;US)

Proceedings of the international seminar on chemistry and process engineering for high-level liquid waste solidification, 226 ( $R ; D E ;$ In several languages)

Proceedings of the international strminar on chemistry and proccss engineering for high-level liquid waste solidification, 263 (R;DE;In German and English)

Process and facility for continuous melting and dosed filling in casks of high-activity waste, 520 (P;DE;In German)
Process description and plant design for preparing ceramic highlevel waste forms, 149 (R;US)

Process for the treatment of high level nuclear wastes (Patent), 398 (P;US)

Process for producing biologically noxious waste, particularly radioactive waste, wich can be stored (German Patent), 519 (P;DE;In German)

Process for producing formed parts containing high-activity waste and consisting of glass granules in a metal matrix, 523 (P;DE;In German)

Process improvements in the NWCF, 393 (J;US)

Processing effects on the behavior of titanate waste forms in aqueous solutions, 1145 (BA;US)

Processing, microstructure, leaching, and long-term stability studies related to titanate high-level waste forms, 340 (R;US)

Progress in radioactive residue process development. A summary of Hanford laboratories achievements in this program under General Electric, 1958-1964, 216 (R;US)

Progress of the vitrification of HLW in France, 525 (J;US)

Promotion of the research and development of high level radioactive waste treatment and disposal, 491 (J;JP;In Japanese)

Radiation and transmutation effects on crystalline radioactive waste forms, 1262 (J;US)

Radioactive wastes, 382 (J;AT;In German)

Radioactive waste management, 527 (BA;SU;In Russian)

Recent developments in defense waste vitrification, 504 (J;US)

Receptivity of borosilicate glass VG 98/12 for incorporating high-level radioactive wastes (In German), 255 (RA;DE;GE)

Remote aspects of treating and handling radioactive wastes, 449 (BA;US)

Research and development activities: fixation of radioactive residues. Quarterly progress report, July-September 1960, 212 (R;US)

Research and development activities [on] solidification of radioactive wastes. Progress report, 215 (R;US)

Research in the field of high level waste solidification, 495 (BA;JP;In Japanese)

Rethinking the management of high-level nuclear waste: the need for fractionation, 509 (J;US)

Review of DOE waste package program. Subtask 1.1. National waste package program, 286 (R;US)

Review of multibarrier waste form development, 272 (RA;DE)

Review of radioactive waste management programs in the United Kingdom, 1160 (BA;US)

Ruthenium nitrosyl complexes in radioactive waste solutions of reprocessing plants. Pt. 1, 12 (J;DE)

Safety analyses for HLW and MLW storage and solidification facilities, 1394 (BA;DE;In German)

Savannah River Laboratory long-term waste storage program. Progress report No. 5, March-April 1974, 131 (R;US)

Savannah River Waste Management Program Plan - FY 1982, 157 (R;US)

Seal for underground ultimate storage sites for radioactive waste, and method of installing the seal, 1284 (P;DE;In German)

Selection and application of a processing system for the treatment of high level liquid waste in the UK, 430 (BA;US)

Separation of mercury from HEWC solutions. Laboratory assay, 207 (R;BE;In French)

SIMS depth profiling studies of sphene-based ceramics and glass ceramics leached in synthetic groundwater, 455 (BA;US)

Sintering of Synroc D, 343 (R;US)

Small-scale processing of actual Savannah River high-level waste, $\mathrm{SO} 3$ (J;US)

Sol-gel-derived waste forms (ORNL), 245 (RA;DE)

Solid fixation of high-level radioactive waste by sorption on clinoptilolity-scouting studies, 211 (R;US)

Solidification of high level wastes. Part IV. Phosphate melts for fixation of radioactive residues from Purex type wastes: three to fifty percent fission product oxides, 134 (R;US)

Solidification for final storage of high-level radiuactive fissioni product solutions from spent fuel elements, $S 29$ (B;DE;In German)

Solidification of high-level radioactive wastes and alpha-bearing wastes by a sol-gel process (in German), 244 (RA;DE) 
Solidification of high-level radioactive fuel reprocessing wastes by spray and pot calcination. Hot-cell pilot plant studies, 217 (R;US)

Some glass-ceramics for special applications, 481 (J;JP)

Spray calcination of SRP waste compositions for ceramic waste forms, 450 (BA;US)

SRP radivactive glass studies: small-scale process development and product performance, 180 (R;US)

Status, direction, and critical issues of waste treatment technology, 330 (R;US)

Status of development of the tailored ceramic high-level nuclear waste form, 513 (J;US)

Status of HAW-Ceramic-Melting Technology at the Institute of Nuclear Affairs (Kernforschungszentrum Karlsruhe; in German), 235 (RA;DE;GE)

Status of high level and alpha bearing waste management in PNC, 428 (BA;US)

Strategy document: long-term technology program for defense high-level waste, 156 (R;US)

Subsolidus sintering of SYNROC: I. Initial demonstrations, 280 (R;US)

Subsolidus sintering of SYNROC:- II. Materials selections, process improvements, waste form evaluations, 281 (R;US)

Swedish nuclear waste efforts, 36 (R;US)

SYNROC: leaching performance and process technolngy, 250 (RA;DFi)

Synroc technology for immobilizing U.S. defense waste, $\mathbf{4 0 0}$ (J;US)

SYNROC through melting: thermal analysis, thermogravimetry and crystal chemical characterization of phases (Federal Republic of Germany), 260 (RA;DE)

Synthesis of alkoxide glass formers for nuclear waste immobilization, 438 (BA;US)

Systems study of the feasibility of high-level nuclear-waste fractionation for thermal-stress control in a geologic repository: main report, 308 (R;US)

Systems study of the feasibility of high-level nuclear waste fractionation for thermal stress control in a geologic repository: appendices, 309 (R;US)

Treattlng ñuclear waste (Patent), 486 (P;AU)

Treatment of high level nuclear waste, $388(\mathrm{P} ; \mathrm{AU})$

Treatment and solidification of high active fission product solutions, 429 (BA;US)

Treatment and conditioning of wastes from US̈ nuclear fuels reprocessing plants, 191 (R;US)

UK vlew of the management of high-level waste, $1103(\mathrm{~J} ; \mathrm{XA})$

Use of glass-metal compounds and ceramic materials to condition hot wastes, 357 (TG;US)

Use of the drum dryer in radioactive waste solidification, 414 (BA:DE:In German)

Using process instrumentation to obviate destructive examination of canisters of HLW glass, 328 (R;US)

Utilization of a drum drier for the conditioning of radioactive wastes, 440 (BA:US)

Vitrification of high level wastes using microwave power (United Kingdom), 230 (RA;DE)

Vitrification of highly-loaded SDS zeolites, 325 (R;US)

Vitrification of simulated West Valley waste using a liquid.fed ceramic melter; 331 (R;US)

Vitrification of high active waste. Vitrification techniques, 222 (R;DE)

Vitrifying Britain's waste, 384 (J;GB)

Waste glasses: requirements and characteristics, 322 (R;US)

Waste Management Program. Technical progress report, October-December 1980, 162 (R;US)

Waste-management program. Technical progress report, JanuaryMarch 1981, 622 (R;US)

Waste management program technical progress report, JanuaryMarch 1983, 623 (R;US)

Waste-Mixes Study for space disposal, 815 (R;US)

Waste package, 288 (RA;US)

Waste treatment and disposal. Progress report, May-October 196.3, 291 (R;US)

Waste treatment and disposal. Quarterly progress report, February-April, 1963, 290 (R;US)
Waste vitrification: a historical perspective, 317 (R;US)

West Valley high-level waste solidification program, 500 (J;US)

Workshop on the leaching mechanisms of nuclear-waste forms, October 27-28, 1981. Summary report, 81 (R;US)

Hot Cells

Equipment for radioactive waste solidification, 390 (J;CS;In Czech)

Hot Pressing

Method to encapsulate radioactive material, 478 (TG;DE;In German)

Solidification of radioactive waste forms and waste containment by the H I P O W process (Sweden), 232 (RA;DE)

Induction Furnaces

Melting of glass by direct induction heating in ceramic container, 480 (J;JP;In Japanese)

In-situ Processing

Long-Term High-Level Defense-Waste technology. Progress report, April-June 1982, 338 (R;US)

Intermedlate-level Radloactive Wastes

Conditioning of radioactive wastes for storage and dispnsal, 364 (B;XA)

European Communities' $\mathbf{R}$ and $\mathrm{D}$ activities on treatment and handling of radioactive waste, 426 (BA;US)

Immobilization of sodium and phosphnrus-hearing PW-72 waste in SITJROC. Plogrew ruport, 348 (R; US)

Leach characterization of cement encapsulated wastes, 1156 (J;US)

Low level and intermediate level wastes management practice in France, 442 (BA;US)

Method for solidifying aqueous radioactive wastes for noncontaminating storage (Patent), 379 (P;US)

Progress in radioactive residue process development. A summary of Hanford laboratories achievements in this program under General Electric, 1958-1964, 216 (R;US)

Radinactive wastes, 382 (J;AT;In German)

Remote aspects of treating and handling radioactive wastes, 449 (BA;US)

Reprocessing, decontamination and docommissioning waste ulanagement at Eurochemic: current and planned activities, 433 (BA;US)

Review of radioactive waste management programs in the United Kingdom, 1160 (BA;US)

Ruthenium nitrosyl complexes in radioactive waste solutions of reprocessing plants. Pt. 1, 12 (J;DE)

Safety analyses for HLW and MLW storage and solidification facilities, 1394 (BA;DE;In German)

Utilization of a drum drier for the conditioning of radioactive wastes, 440 (BA;US)

Iodine 129

Nuclear-waste-management. Scmiannual progress report. October 1981-March 1982, 301 (R;US)

Nuclear waste management. Quarterly progress report, Ootober through December 1980, 296 (R;US)

Nuclear waste management. Semiannual progress report, October 1982-March 1983, 303 (R;US)

Ion Exchange

Equipment, operation and some results from a hot test of the CTH actinide separation process (Norway), 243 (RA;DE)

Recovery and purification of multi-kilocurie quantities of fission product strontium by cation exchange, 214 (R;US)

Research and development activities: fixation of radioactive residues. Quarterly progress report, July-September 1960, 212 (R;US)

Waste treatment and disposal. Quarterly progress report, February-April, 1963, 290 (R;US)

Ion Exchange Materials

Sodium titanate in solidification of radioactive wastes (Finland), 247 (RA;DE)

Krypton

Nuclear-waste-management. Semiannual progress report, October 1981-March 1982, 301 (R;US)

Nuclear waste management. Quarterly progress report, October through December 1980, 296 (R;US) 
ןuid Wastes

-alcination of liquid radioactive wastes. Part I. Technology of radioactive waste calcination, 361 (J;CS;In Czech)

Control of semivolatile radionuclides in gaseous effluents at nuclear facilities, 453 (B;XA)

Generation and vitrification of high-level light water reactor liquid waste, 385 ( $\mathrm{J}$;US)

Precipitation process for decontamination of water soluble SRP radioactive waste, 468 (J;US)

Promotion of the research and development of high level radioactive waste treatment and disposal, 491 (J;JP;In Japanese)

Low-level Radioactive Wastes

Assessment of power reactor waste immobilization by vitrification. Final report, 206 (R;US)

Commercial waste treatment technology and programs, 359 ( $\mathrm{J} ; \mathrm{US})$

Conditiuning of radioactive wastes for storage and disposal 364 (B; XA)

Decontaminated salt disposal as saltcrete in a landfill. Technical data summary, 636 (R;US)

Incineration/vitrification of simulated low-level institutional wastes in a joule-heated glass melter, 514 (J;US)

Long-Term High-Level Defense-Waste technology. Progress report, April-June 1982, 338 (R;US)

Low level and intermediate level wastes management practice in France, 442 (BA;US)

Nuclear-fuel-cycle education: Module 8 . Waste management, 155 (R;US)

Radioactive wastes, 382 (J;AT;In German)

Research and development activities: fixation of radioactive residues. Quarterly progress report, July-September 1960, 212 (R;US)

Review of radioactive waste management programs in the United Kingdom, 1160 (BA;US)

Solidification of ash from incineration of low-level radioactive waste, 193 (R;US)

Status, direction, and critical issues of waste treatment technology, 330 (R;US)

Supernatant treatment considerations for the neutralized waste at West Valley, 370 (J;US)

Treatment and conditioning of wastes from US nuclear fuels reprocessing plants, 191 (R;US)

Waste Management Program. Technical progress report, October-December 1980, 162 (R;US)

Waste treatment and disposal. Progress report, May-October 1963, 291 (R;US)

Waste treatment and disposal. Quarterly progress report, February-April, 1963, 290 (R;US)

Materials

Nuclear waste management technical support in the development of nuclear waste form criteria for the NRC. Task 1. Waste package overview, 283 (R;US)

Materials Recovery

Recovery of noble metals from fission products, 324 (R;US)

Materials Testing

Evaluation of conditioned high-level waste forms, 326 (R;US)

Nuclear waste management technical support in the development of nuclear waste form criteria for the NRC. Task 4. Test development review, 284 (R;US)

Matrix Materials

Fixation of high level solid waste (HLSW) in a $\mathrm{TiO}_{2}$ base ceramic matrix provided with an integrated overpack, 367 (J;US)

Highly dense graphite matrix - a new material for the conditioning of radioactive wastes, 394 (J;US)

Meetings

Conditioning of radioactive wastes for storage and disposal, 364 (B;XA)

Coordination meeting of high-level waste technology and national waste terminal storage programs, October 21-22, 1981, 624 (R;US)

International fuel-cycle and waste-management technologyexchange activities sponsored by the United States Department of Energy: FY 1982 evaluation report, 85 (R;US)
Proceedings of the international seminar on chemistry and process engineering for high-level liquid waste solidification, 226 (R;DE;In several languages)

Proceedings of the international seminar on chemistry and process engineering for high-level liquid waste solidification, 263 (R;DE;In German and English)

Proceedings of the ANS topical meeting on the treatment and handling of radioactive wastes, 425 (B;US)

Report of working meeting on fixation of radioactivity in stable, solid media at the Johns Hopkins University, June 19-21, 1957, 342 (R;US)

Mill Tailings

Nuclear-waste-management. Semiannual progress report, October 1981-March 1982, 301 (R;US)

Nuclear waste management. Quarterly progress report, October through December 1980, 296 (R;US)

Nitrates

Commercial and Defense nitrate wastes and processing alternatives, 333 (R;US)

Ofi-gas Systems

Deposition of volatile fission products during commercial highlevel waste vitrification, 304 (R;US)

On-line measurements of the volatilization of ruthenium in a vitrification process, 422 (BA;US)

Operating characteristics of a direct liquid-fed vitrification process, 314 (R;US)

Pilot Plants

Design of Harwell Joule Ceramic Melter (UKAEA), 231 (RA;DE)

High-level waste vitrification technique in a full-scale pilot plant, 439 (BA;US)

Prelimianry experience with vitrification inactive pilot plant in Italy, 234 (RA;DE)

Planning

Report of the Ad Hoc Committee on Reprocessing and Wastes of the Sub-Committee on Nuclear Power of Committee on Overall Energy Study, 485 (J;JP;In Japanese)

Plutonium

Precipitation process for supernate decontamination, 173 (R;US)

Precipitation process for decontamination of water-soluble SRP radioactive waste, 184 ( $R$;US)

Precipitation process for decontaminating water-soluble SRP radioactive waste, 187 (R;US)

Precipitation

Precipitation process for decontamination of water soluble SRP radioactive waste, 468 (J;US)

Quality Control

Nuclear waste management technical support in the development of nuclear waste form criteria for the NRC. Task 4 . Test development revlew, 284 (R;US)

Reduction

Separation of mercury from HEWC solutions. Laboratory assay, 207 (R;BE;In French)

Regulations

Assessment of high-level waste form conformance with proposed regulatory and repository criteria, 198 (R;US)

Waste glasses: requirements and characteristics, 322 (R;US)

Remote Handling Equipment

Remote aspects of treating and handling radioactive wastes, 449 (BA;US)

\section{Research Programs}

Evaluation of research and development for terminal isolation of nuclear wastes, 701 (R;US)

Long-Term High-Level Defense Waste technology progress report, January-March 1982, 337 (R;US)

Long-term high-level-waste technology. Composite quarterly technical report, April-June 1982, 165 (R;US)

Long-term high-level waste technology. Composite quarterly technical report, July-September 1982, 166 (R;US)

Management of high-level radloactive wastes, 218 (R;US)

Research and development activities: fixation of radioactive residues. Quarterly progress report, April-June 1960, 210 (R;US)

Research and development activities [on] solidification of radioactive wastes. Progress report, 215 (R;US) 
Research programme on the conditioning of nuclear power waste. Progress report June 1981, 332 (R;SE)

Rockwell Hanford Operations. Defense waste and byproducts management monthly report, 335 (R;US)

Rockwell Hanford Operations defense waste and byproducts management monthly report, 336 (R;US)

Tokai Works annual progress report, April 1980-March 1981, 3 (R;JP)

Waste Management Program. Technical progress report, October-December 1980, 162 (R;US)

Waste-management program. Technical progress report, JanuaryMarch 1981, 622 (R;US)

Waste treatment and disposal. Quarterly progress report, February-April, 1963, 290 (R;US)

Reviews

Introduction of studies on high-level radioactive waste immobilization in synthetic minerals. Supercalcine and SYNROC, 224 (R;JP;In Japanese)

Risk Ascessment

Comparative risk assessments for the production and interim storage of glass and ceramic waste forms: defense waste processing lacility, 1370 (R;US)

Safety analyses for HLW and MLW storage and solidification facilities. 1394 (BA:DE:In German)

Safcty

Alternative waste forms: process feasibility, 477 (J;US)

Evaluation of the safety aspects of the design and operation of temporary/mobile radioactive-waste solidification systems, 287 (R;US)

Scrubbing

Deposition of volatile fission products during commercial highlevel waste vitrification, 304 (R;US)

Solid Wastes

Method of treating high level radioactive solid waste, 389 (P;JP;In Japanese)

Solidification

Actual point about fission products vitrification, 136 (R;FR;In French)

Alternate waste form evaluation for Savannah River Plant highlevel waste, 1104 (J;US)

Alternative-waste-form evaluation for Savannah River Plant highlevel waste, 178 (R;US)

Alternative waste forms: process feasibility, 477 (J;US)

Alternative waste form development - low-temperature pyrolytic carbon coatings. 511 ( $\mathrm{J} ; \mathrm{US}$ )

Alternative fuels and heating methods for calciner process heat, 204 (R:US)

Analysis of thin leaching layers and fracture mechanics of irradiated borosilicate glass for solidification of high-level fission product solutions (HAW), 365 (BA;DE;In German)

Apparatus and method for immobilizing waste material (Patent), 484 (P;AU)

Assessment of Savannah River borosilicate glass in the repository enviromment, 172 ( $R ; U S$ )

Assessment of rationale for development of improved waste forms, 498 (J;US)

Assessment of overseas developments in methods for treatment and disposal of high-level radioactive wastes, $532(\mathrm{R} ; \mathrm{AIJ})$

Borosilicate glass as a matrix for immobilization of SRP highlevel waste, 512 (J;US)

Borosilicate glass and glass ceramics with real highly radioactive waste, 528 (I; I ISS)

Capacity of the borosilicate glass VG $98 / 12$ for components of the high-level radioactive waste, 267 (RA;DE;In German)

Cement-based radioactive waste hosts formed under elevated temperatures and pressures (FUETAP concretes) for Savannah River Plant high-level defense waste, 293 (R;US)

Ceramic nuclear waste forms, 259 (RA;DE)

Ceramic nuclear waste forms, 271 (RA;DE)

Ceramic process and plant design for high-level nuclear waste immobilization, 144 (R:US)

Change of electrical properties of the borosilicate glasses containing $\mathrm{MoO}_{0}$ by phase separation, 482 (J;JP;In Japanese)

Characterization and testing of basalt glass and glass ceramic waste forms, 313 (R;US)
Characterization of borosilicate glass containing Savannah Riv Plant radioactive waste, 179 (R;US)

Chemistry in the development of nuclear power, 5 (BA;GB)

Coating crystalline nuclear waste forms to improve inertness, 138 (R;US)

Coating of crystalline nuclear waste forms to improve inertness, 386 (J;US)

Commercial high-level-waste management: options and economics. A comparative analysis of the ceramic and glass waste forms, 150 (R;US)

Compared quantitative determination of nuclear waste glass crystallization rate by light microscopy and $\mathrm{x}$ ray diffraction, 257 (RA;DE)

Comparison of borosilicate glass and synthetic minerals as media for the immobilization of high-level radioactive waste, 490 (J;US)

Comparison of wet slurry and dry frit (crizzle) feeding of glassformers in pot vitrification (AERE), 242 (RA;DE)

Comparison of candidate glasses to vitrify ICPP calcined alumina waste, 469 (J;US)

Comiproite quarterly techinlual repurt lung-term high-level-waste technology, October-December 1981, 164 (R;US)

Consolidated waste forms: glass marbles and ceramic pellets, 299 (K;US)

Cóntrool of semıvolatile radionuclides in gaseous effluents at nuclear facilities, 453 (B;XA)

Coordination meeting of high-level waste technology and national waste terminal storage programs, October 21-22, 1981, 624 (R;US)

Crystalline ceramics as a high-level nuclear waste form, 395 (J;US)

CsAlSis $\mathrm{O}_{12}$ : a possible host for ${ }^{107} \mathrm{Cs}$ immobilization, $404(\mathrm{~J} ; \mathrm{GB})$

$\mathrm{CsAlSi}_{5} \mathrm{O}_{12}$ : a possible host for ${ }^{107} \mathrm{Cs}$ immobilization, 148 (R;US)

Decontaminated salt disposal as saltcrete in a landfill. Technical data summary, 636 (R;US)

Defense Waste Processing Facility: a remote process for solidification of Savannah River Plant high level waste, 195 (R;US)

Department of Energy's selection of high level waste forms, 443 (BA;US)

Deposition of volatile fission products during commercial highlevel waste vitrification, 304 (R;US)

Description of a ceramic waste form and canister for Savannah River Plant high-level waste, 170 (R;US)

Descripliun ol Delense Waste Processing Facility reférence wastc form and canister. Revision 1, 168 (R;US)

Design and performance of feed-delivery systems for simulated radioactive waste slurries, 306 (R;US)

Design of Harwell Joule Ceramic Melter (UKAEA), 231 (RA;DE)

Developing technology of microwave vitrification, 392 (J;GB)

Development, evaluation, and selection of candidate high-level waste furms, 185 (R;US)

Development and testing of SYNROC for high level radioactive waste fixation, 510 (J;US)

Development and testing of SYNROC for high-level radioactive waste fixation, 493 (J;AU)

Development of borosilicate glass for the vitrification facility PAMELA, 253 (RA;DE;GE)

Development and testing of SYNROC $\mathrm{C}$ as a high level nuclear waste form (Australian Atnmir. Energy Commission), 418 (BA;US)

Development of polyphase ceramics for the immobilization of high-level Defense nuclear waste, 151 (R;US)

Development of high-level liquid waste solidification in PNC, 431 (BA;US)

Diopside glass-ceramic material for the immobilization of radioactive wastes, 258 (RA;DE)

Diopside glass-ceramic material for the immobilization of radi active wastes, 270 (RA;DE)

Disposal of radioactive wastes (Book), 106 (B;NL)

Dissolution mechanisms in high-level nuclear waste consolidation forms, 305 (RA;US)

DWPF process control, 186 (R;US) 
Electrodes for the direct heating of highly radioactive borosilicate glasses in HAW-ceramic furnaces (in German; Kernforschungszentrum, Karlsruhe), 236 (RA;DE;GE)

Engineering scale multibarrier waste form demonstrations, 499 (J;US)

Environmental assessment: waste-form selection for SRP highlevel waste (Borosilicate glass; crystalline ceramic), 55 (R;US)

Equipment for radioactive waste solidification, 390 (J;CS;In Czech)

European Communities' R and D activities on treatment and handling of radioactive waste, 426 (BA;US)

Evaluation of process alternatives for solidification of existing commercial high-level waste, 501 (J;US)

Evaluation of the safety aspects of the design and operation of temporary/mobile radioactive-waste solidification systems, 287 (R;US)

Evaluation of process alternatives for solidification of the West Valley high-level liquid wastes, 436 (BA;US)

Evaluation of the AVM process and its applicability to West Valley, 437 (BA;US)

Evaluation of forms for the immobilization of high-level and transuranic wastes, 200 (R;US)

Final conditioning of high-level fission product solutions, 376 (J;DE;In German)

Fission product solidification: FIPS (In German), 229 (RA;DE;GE)

Fixation of radioactive materials in a glass matrix, 380 (P;AU)

Fixation of high level solid waste (HLSW) in a $\mathrm{TiO}_{2}$ base ceramic matrix provided with an integrated overpack, 367 ( $\mathrm{J} ; \mathrm{US})$

Formulation and processing of polyphase ceramics for high level nuclear waste, 421 (BA;US)

Formulation and processing of alumina based ceramic nuclear waste forms, 153 (RA;US)

Fuel-cycle programs. Quarterly progress report, January-March 1981,48 (R;US)

Fuel-cycle programs. Quarterly progress report, April-June 1981, 49 (R;US)

Fuel cycle programs. Quarterly progress report, July-Septembcr 1981,50 (R;US)

FUETAP concrete: an alternative radioactive waste host, 256 (RA;DE)

FUETAP concrete - an alternative radioactive waste host, 268 (RA;DE)

Generation and vitrification of high-level light water reactor liquid waste, 385 (J;US)

High-level waste-form-product performance evaluation (Leaching; waste loading; mechanical stability), 627 (R;US)

High-level waste solidification system for the Western New York State Service Center, 507 (J:US)

High level waste solidification: the future, 227 (RA;DE)

High-level waste solidification system for the Western New York Nuclear Service Center, 315 (R;US)

High-level nuclear-waste-immobilization program, 154 (R;US)

High-level waste vitrification technique in a full-scale pilot plant, 439 (BA;US)

High level waste forms: glass marbles and thermal spray coatings, 447 (BA;US)

High level waste management at the Idaho Chemical Processing Plant, 451 (BA;US)

Highly dense graphite matrix - a new material for the conditioning of radioactive wastes, 394 (J;US)

Highly dense graphite matrix: a new material for the conditioning of radioactive wastes, 358 (J;US)

HLW fixation in sintered modified SYNROC.-B ccramics: chemical stability evaluation, $526(\mathrm{~J}$;US)

Hydroxylated ceramic waste forms and the absurdity of leach tests, 254 (RA;DE)

ICPP waste calcining facility safety analysis report, 219 (R;US)

Immohilization of sodium and phosphorus-bearing PW-7a waste in SYNROC. Progress report, 346 (R;US)

Immobilization of radioactive liquid waste using water glass, 470 (J;US)

Immobilizing defense waste at the Savannah River Project, 196 (R;US)
Improved polyphase ceramic form for high-level defense nuclear waste, 152 (RA;US)

Inactive Pamela vitrification facility in Mol; manufacture and characteristics of Vitromet products (in German), 238 (RA;DE;GE)

Inactive vitrification in Mol. Ceramic melting, feed and off gas system, pouring equipment for glassmelting (In German), 237 (RA;DE;GE)

Incorporation of simulated high-level nuclear waste in gel spheres, 292 (R;US)

Industrial operating experience at the Marcoule vitrification facility, 432 (BA;US)

Influence of the reprocessing flow sheet on the HLW solidification technology, 239 (RA;DE)

Initial operations in the remote laboratory-scale waste-treatment facility, 312 (R;US)

Intercomparison of leach-testing methods and the effects of waste-form composition on test type and duration, 1157 (J;US)

Investigations of suitable metallic container materials for HAW solidification, 371 (J;US)

Iron-enriched basalt as a waste form for high-level nuclear wastes, 199 (R;US)

Iron-enriched basalt for containment of nuclear wastes, 416 (BA;US)

Laboratory activity in Italy in the field of vitrification of highlevel wastes, 233 (RA;DE)

Leach characterization of cement encapsulated wastes, 1156 (J;US)

Leaching of solidified highly radioactive decay products: overview (In German), 249 (RA;DE;GE)

Long-term high-level waste technology. Composite quarterly technical report, July-September 1981,163 (R;US)

Long-Term High-Level Defense-Waste technology. Progress report, April-June 1982, 338 (R;US)

Long-Term High-Level Defense Waste technology progress report, January-March 1982, 337 (R;US)

Long-term high-level-waste technology. Composite quarterly technical report, April-June 1982, 165 (R;US)

Long-term high-level waste technology. Composite quarterly technical report, July-September 1982, 166 (R;US)

Low level and intermediate level wastes management practice in France, 442 (BA;US)

Management of high-level radioactive wastes, 218 (R;US)

Management of vitrified high level radioactive waste, 466 (BA;GB)

Marcoule vitrification shop: $\mathbf{3 0}$ months service (In French), 228 (RA;DE;FR)

Materials studies of simulated interim waste forms for high-level radiactive wastes, 502 (J;US)

Materials research for the Canadian Nuclear Fuel Waste Managemenl Pruyram, 109 (BA;US)

Materials balance: scientific fundamentals for the quality assurance of vitrified waste, 462 (BA;US)

Method and device for solidifying high level liquid wastes from a re-processing plant, 403 (P;JP;In Japanese)

Method for product performance evaluation of candidate waste forms for immobilization of high-level radioactive wastes. Interface working group on high-level waste form selection factors, 161 (R;US)

Method for solidifying aqueous radioactive wastes for noncontaminating storage (Patent), 379 (P;US)

Method for producing molded bodies containing highly active radioactive wastes from glass granules embedded in a metallic matrix, 360 (P;US)

Method of solidifying radioactive wastes (Patent), 483 (P;JP;In Japanese)

Method of preparing nuclear wastes for transportation and interim storage, 391 (P;US)

Method of processing high level radioactive wastes by glass solidification, 408 (P;JP;In Japanese)

Method of prooessing off-gas condensate containing precipitations resulted upon solidification of high-level liquid wastes issued from a reproccssing plant, 406 (P;JP;In Japanese)

Microstructures and crystallization in simulated waste glasses, 441 (BA:US) 
Microstructure of Synroc, 454 (BA;US)

Microwave energy for post-calcination treatment of high-level nuclear wastes, 378 (J;US)

Molccular glasses for nuclear waste encapsulation (Patent), 1076 (P;US)

National long-term high-level waste-technology program, 67 (R;US)

New waste calcining facility at ICPP, 452 (BA;US)

Nuclear waste vitrification in a ceramic-lined electric glass melter (Federal Republic of Germany), 409 (J;US)

Nuclear-waste management semiannual progress report, April 1982-September 1982, 302 (R;US)

Nuclear waste management. Quarterly progress report, October through December 1980, 296 (R;US)

Nuclear waste management. Semiannual progress report, October 1982-March 1983, 303 (R;US)

On-line measurements of the volatilization of ruthenium in a vitrification process, 422 (BA;US)

Overview on product forms (In German), 252 (RA;DE;In German and English)

Overview on product forms, 264 (RA;DE;In German)

Package materials, waste form, 792 (RA;US)

Phosphate system as medium for radioactive waste fixation - simplex experiment. Part II, 355 (R;CS;In Czech)

Phosphate systems - a medium for high level waste solidification, 362 (J;CS;In Czech)

Plant for solidification and storage of high-level radioactive waste, 521 (J;JP;In Japanese)

Prelimianry experience with vitrification inactive pilot plant in Italy, 234 (RA;DE)

Preliminary assessment of key process steps for converting Savannah River Plant high-level wastes to tailored ceramics, 147 (R;US)

Preparation and leaching of radioactive INEL waste forms, 201 (R;US)

Preparation of the high-level waste form for the near-surface test facility, 497 (J;US)

Preparation of glass containing high-level radioactive waste (simulated) - copper composite compacts by a continuous pressure sintering method, 479 (J;JP;In Japanese)

Present status of techniques for the solidification of high level wastes, 492 (J;IP;In Japanose)

Present status of the West Valley Demonstration Project, 435 (BA;US)

Proceedings of the international seminar on chemistry and process engineering for high-level liquid waste solidification, 226 (R;DE;In several languages)

Proceedings of the international seminar on chemistry and process engineering for high-level liquid waste solidification, 263 (R;DE;In German and English)

Process description and plant design for preparing ceramic highlevel waste forms, 149 (R;US)

Process for producing formed parts containing high-activity waste and consisting of glass granules in a metal matrix, 523 (P;DE;In German)

Process improvements in the NWCF, 393 (J;US)

Processing effects on the behavior of titanate wastc forms in aqueous solutions, 1145 (BA;US)

Progress in radioactive rćsidue process development. A summary of Hanford laboratories achievements in this program under General Electric, 1958-1964, 216 (R;US)

Progress of the vitrification of HLW in France, 525 (J:US)

Promotion of the research and development of high level radioactive waste treatment and disposal, 491 (J;JP;In Japanese)

Radiation and transmutation effects on crystalline radioactive waste forms, 1262 (J;US)

Radiation damage in nuclear waste ceramics, $377(\mathrm{~J} ; \mathrm{US})$

Receptivity of borosilicate glass VG $98 / 12$ for incorporating high-level radioactive wastes (In German), 255 (RA;DE;GE)

Report of working meeting on fixation of radioactivity in stable, solid mcdia at the Johns Hopkins University, June 19-21, 1957 . 342 (R;US)

Reprocessing, decontamination and decommissioning waste management at Eurochemic: current and planned activities, 433 (BA;US)
Research in the field of high level waste solidification, 495 (BA;JP;In Japanese)

Review of multibarrier waste form development, 272 (RA;D]

Review of radioactive waste management programs in the United Kingdom, 1160 (BA;US)

Safety analyses for HLW and MLW storagc and solidification facilities, 1394 (BA;DE;In German)

Savannah River Laboratory long-term waste storage program. Progress report No. 5, March-April 1974, 131 (R;US)

Savannah River Laboratory long-term waste storage program. Progress report No. 6, May-Jun 1974, 132 (R;US)

Savannah River Laboratory long-term waste storage program. Progress report No. 7, July-September 1974, 133 (R;US)

Savannah River Waste Management Program Plan - FY 1982, 157 (R;US)

Scientific basis for nuclear waste management, 107 (B;US)

Seal for underground ultimate storage sites for radioactive waste, and method of installing the seal, 1284 (P;DE; In German)

Selection and application of a processing system for the treatment of high level liquid waste in the UK, 430 (BA;US)

Sol-gel-derived waste forms (ORNL), 245 (RA;DE)

Solidification of high level wastes. Part IV. Phosphate melts for fixation of radioactive residues from Purex type wastes: three to fifty percent fission product oxides, 134 (R;US)

Solidification of high level wastes. Part III. Solubility, melting point, flow temperature and thermal conductivity of some calcined, synthetic purex-type wastes, 209 (R;US)

Solidification of Savannah River plant high-level waste, 506 (J;US)

Solidification for final storage of high-level radioactive fission product solutions from spent fuel elements, 529 (B;DE; In German)

Solidification of high-level radioactive wastes and alpha-bearing wastes by a sol-gel process (in German), 244 (RA;DE)

Solidification of high-level waste, 411 (J;GB)

Solidification of high-level radioactive fuel reprocessing wastes by spray and pot calcination. Hot-cell pilot plant studies, 217 (R;US)

Solidification of ash from incineration of low-level radioactive waste, 193 (R;US)

Solubility limits of metal iuns and mechanism of leaching nuclear-waste glasses, 320 (R;US)

Some glass-ceramics for special applications, 481 (J;JP)

Spruy calcination of SKP waste compositions for ceramic waste forms, 450 (BA;US)

SRP radioactive glass studies: small-scale process development and product performance, 180 (R;US)

Status of development of the tailored ceramic high-lcvel nuclear waste form, 513 (J;US)

Status of HAW-Ceramic-Melting Technology at the Institute of Nuclear Affairs (Kernforschungszentrum Karlsruhe; in German), 235 (RA;DE;GE)

Status of high level and alpha bearing waste management in PNC, 428 (BA;US)

Subsolidus sintering of SYNROC: I. Initial demonstrations, 280 (R;US)

Subsulidus sintering of SYNROC: II. Materials selections, process improvements, waste form evaluations, 281 (R;US)

Swedish nuclear waste efforts, 36 (R;US)

SYNROC; leaching performance and process technology, 250 (RA;DE)

SYNROC powder preparation: preliminary fluid bed tests in a cold-flow unit, 345 (R;US)

Synroc technology for immobilizing U.S. defense waste, 400 (J;US)

SYNROC through melting: thermal analysis, thermogravimetry and crystal chemical characterization of phases (Federal Republic of Germany), 260 (RA;DE)

Synthesis of alkoxide glass formers for nuclcar waste immubi tion, 438 (BA;US)

Systems study of the feasibility of high-level nuclear-waste fractionation for thermal-stress control in a geologic repository: main report, 308 (R;US) 
Systems study of the feasibility of high-level nuclear waste fractionation for thermal stress control in a geologic repository: appendices, 309 (R;US)

Theoretical predictions for continuous-slurry feeding of a glass melter, 182 (R;US)

Thermal analysis, thermogravimetry and crystal chemical characterization of phases, 273 (RA;DE)

Treatment of high level nuclear waste, 388 (P;AU)

Treatment and solidification of high active fission product solutions, 429 (BA;US)

Use of glass-metal compounds and ceramic materials to condition hot wastes, 357 (TG;US)

Use of the drum dryer in radioactive waste solidification, 414 (BA;DE;In German)

Using process instrumentation to obviate destructive examination of canisters of HLW glass, 328 (R;US)

Utilization of a drum drier for the conditioning of radioactive wastes, 440 (BA;US)

Vitrification of high level wastes using microwave power (United Kingdom), 230 (RA;DE)

Vitrification of highly-loaded SDS zeolites, 325 (R;US)

Vitrification of simulated West Valley waste using a liquid-fed ceramic melter, 331 (R;US)

Waste-management program. Technical progress report, JanuaryMarch 1981, 622 (R;US)

Waste management program technical progress report, JanuaryMarch 1983, 623 (R;US)

Waste treatment and disposal. Progress report, May-October 1963, 291 (R;US)

West Valley high-level waste solidification program, 500 (J;US)

Workshop on the leaching mechanisms of nuclear-waste forms, October 27-28, 1981. Summary report, 81 (R;US)

Solvent Extraction

Equipment, operation and some results from a hot test of the CTH actinide separation process (Norway), 243 (RA;DE)

Ruthenium nitrosyl complexes in radioactive waste solutions of reprocessing plants. $\mathrm{Pt} .1,12(\mathrm{~J} ; \mathrm{DE})$

Sorption

Solid fixation of high-level radioactive waste by sorption on clinoptilolity-scouting studies, 211 (R;US)

Specifications

Nuclear waste management technical support in the developmnt of nuclear waste form criteria for the NRC. Task 5. National waste package program, 285 (R;US)

Nuclear waste management technical support in the development of nuclear waste form criteria for the NRC. Task 1. Waste package overview, 283 (R;US)

Spent Fuels

Waste package, 288 (RA;US)

Strontium

Feasibility of incinerating and vitrifying organic resins in a single step, 318 (R;US)

Precipitation-adsorption process for the decontamination of nuclear waste supernates (Patent), 397 (P;US)

Precipitation process for supernate decontamination, 173 (R;US)

Precipitation process for decontamination of water-soluble SRP radioactive waste, 184 (R;US)

Precipitation process for decontaminating water-soluble SRP radioactive waste, $18^{\prime} /$ (K;US)

Systems study of the feasibility of high-level nuclear-waste fractionation for thermal-stress control in a geologic repository: main report, 308 (R;US)

Systems study of the feasibility of high-level nuclear waste fractionation for thermal stress control in a geologic repository: appendices, 309 (R;US)

Strontium 90

Method of solidifying radioactive wastes (Patent), 483 (P;JP;In Japanese)

Surface Coating

Review of multibarrier waste form development, $2 / 2$ (RA;DE)

Synroc Process

Comparison of properties of borosilicate glass and crystalline ceramic forms for immobilization of Savannah River Plant waste, $171(\mathrm{R} ; \mathrm{US})$
Formulation of SYNROC-D additives for Savannah River Plant high-level radioactive waste, 347 (R;US)

Incorporation of high-level wastes in SYNROC: results from recent process-engineering studies at Lawrence Livermore National Laboratory, 352 (R;US)

Synthetic Rocks

Alpha-recoil damage in natural zirconolite and perovskite, 518 (J;JP)

Apparatus and method for immobilizing waste material (Patent), $484(\mathrm{P} ; \mathrm{AU})$

Development and testing of SYNROC for high-level radioactive waste fixation, $493(\mathrm{~J} ; \mathrm{AU})$

Technology Assessment

Commercial waste treatment $R$ and $D$ needs in the United States, 323 (R;US)

Commercial waste treatment technology and programs, 359 (J;US)

Nuclear waste management technical support in the development of nuclear waste form criteria for the NRC. Task 1. Waste package overview, 283 (R;US)

Waste vitrification: a historical perspective, 317 (R;US)

Technology Transfer

International fuel-cycle and waste-management technologyexchange activities sponsored by the United States Department of Energy: FY 1982 evaluation report, 85 (R;US)

\section{Transmutation}

Actinide recycling in light water reactors: results of reactor physics calculations, 294 (R;US)

Radiation and transmutation effects on crystalline radioactive waste forms, 1262 (J;US)

Treating nuclear waste (Patent), 486 (P;AU)

Uranium hexafluoride actinide transmutation reactors-preliminary design and analysis, 516 (D;US)

Vitrification

Assessment of high-level waste form conformance with proposed regulatory and repository criteria, 198 (R;US)

Assessment of overseas developments in methods for treatment and disposal of high-level radioactive wastes, 532 (R;AU)

Assessment of power reactor waste immobilization by vitrification. Final report, 206 (R;US)

Borosilicate glass as a matrix for immobilization of SRP highlevel waste, 512 (J;US)

Compared quantitative determination of nuclear waste glass crystallization rate by light microscopy and X-ray diffraction, 269 (RA;DE)

Comparison of properties of borosilicate glass and crystalline ceramic forms for immobilization of Savannah River Plant waste, 171 (R;US)

Decontamination of Savannah River Plant waste glass canisters, 175 (R;US)

Development of borosilicate glass for the vitrification plant PAMELA, 530 (TG;US)

Development of a slurry-fed in-can melter for nuclear defense waste , 176 (R;US)

Development and demonstration of a process for vitrification of TMI zeolite, 505 (J;US)

Development of the borosilicate glass for the vitrification plant PAMELA, 265 (RA;DE; In German)

Effect of $\mathrm{Fe}_{2} \mathrm{O}_{0} / \mathrm{ZnO}$ on two glass compositions for solidification un Swedish llucleai wästes, 341 (R;SE)

Evaluating glasses for high-level radioactive waste immobilization, 1237 (J;US)

Feasibility of incinerating and vitrifying organic resins in a single step, 318 (R;US)

French status of high level radioactive wastes solidification, 135 (R;FR)

High temperature corrosion of heat-resisting alloys by borosilicate melts containing simulated high level nuclear wastes, 476 (J;JP;In Japanese)

Immobilization of high-level nuclear reactor wastes in SYNROC: a current appraisal, 515 (J;US)

In situ vitrification: application analysis for stabilization of transuranic waste, 307 (R;US)

Incineration/vitrification of simulated low-level institutional wastes in a joule-heated glass melter, $514(\mathrm{~J} ; \mathrm{US})$ 
Large-scale continuous process to vitrify nuclear defense waste: operating experience with nonradioactive waste, 177 (R;US)

Melting of glass by direct induction heating in ceramic container, 480 (J;JP;In Japanese)

Nondestructive techniques for evaluating the integrity of vitrified solids, 471 (J;JP)

Nuclear waste vitrification in a ceramic-lined electric glass melter (Federal Republic of Germany), 409 (j;US)

Off-gas characteristics of liquid-fed joule-heated ceramic melters, 319 (R;US)

Operating characteristics of a direct liquid-fed vitrification process, 314 (R;US)

Policy and development of reprocessing of spent nuclear fuel and high-level radioactive waste management in the F.R. Germany, 13 (J;JP;In Japanese)

Preparation and microstructural characterization of silicate and borosilicate glasses containing cerium or plutonium, 130 (R;BE)

Preparation and characterization of an improved borosilicate glass for the solidification of high level radioactive fission product solutions (HLW). Pt. 2, 277 (R;DE)

Recent developments in defense waste vitrification, 504 (J:US)

Research and development activities [on] solidification of radioactive wastes. Progress report, 215 (R;US)

Results of the development of a single-stage glass solidification method, 496 (BA;JP;In Japanese)

Small-scale processing of actual Savannah River high-level waste, 503 (J;US)

Status, direction, and critical issues of waste treatment technology, 330 (R;US)

Status of development of the tailored ceramic high-level nuclear waste form, 513 (J;US)

UK view of the management of high-level waste, 1103 (J;XA)

Vitrification of nuclear waste with low heat generation, 407 (J;NL)

Vitrification of high active waste. Vitrification techniques, 222 (R;DE)

Vitrifying Britain's waste, 384 (J;GB)

Waste glasses: requirements and characteristics, 322 (R;US)

Waste vitrification: a historical perspective, 317 (R;US)

Zeolites

Vitrification of highly-loaded SDS zeolites, 325 (R;US)

RADIOACTIVE WASTE STORAGE

Intermediate, long term and final storage of radioactive substances, 1234 (J;A); In German)

Aenustic Fimisginn Trating

Monitoring an underground repository with modern seismological methods, 1233 (J;US)

Alpha-hearing Wastes

Comparison of cask and drywell storage concepts for a monitored retrievable storage/interim storage system, 853 (R;UȘ)

Savannah River interim waste management program plan, FY1904, 606 (R;U3)

Backfriling

Thermal conductivity of bentonite/quartz high-level-wastepackage backfill, 972 (R;US)

Casks

Comparison of cask and dry well concepts for retrievable storage of high-level waste, 641 (R;US)

Surface storage of vitrified high-level radioactive waste in reinforced-concrete casks, 534 (R;GB)

Comparative Evaluations

Comparison of cask and drywell storage concepts for a monitored retrievable storage/interim storage system, 853 (R;US)

Containers

Canister arrangement for storing radioactive waste (Patent), 1106 (P;US)

Materials for high-level waste containment, 1080 (J;GB)

Kemote automatic plasma arc-closure welding of a dry-storage canister for spent nuclear fuel and high-level radioactive waste, 551 (R;US)

Stress corrosion cracking test with slow strain rate and constant current, 1293 (BA;US)

Waste-management program. Technical progress report, JanuaryMarch 1981, 622 (R;US)
Cost

MRS/IS facility co-located with a repository: preconceptual design and life-cycle cost estimates, 857 (R;US)

Dry Storage

Natural draught centralized dry store for irradiated fuel and active waste, 1239 (J;GB)

Remote automatic plasma arc-closure welding of a dry-storage canister for spent nuclear fuel and high-level radioactive waste, 551 (R;US)

Economic Analysis

Economics of monitored retrievable storage of nuclear wastes, 40 (J;US)

Engineered Safety Systems

Nuclear-waste-package program for high-level isolation in Nevada tuff, 1015 (R;US)

Environmental Impacts

Draft environmental impact statement for 40 CFR part 191: environmental standards for management and disposal of spent nuclear fuel, high-level and transuranic radioactive wastes. Final report, 838 (R;US)

Environmental evaluation of alternatives for long-term management of Defenso high-level radiudelipe nastes at the Idaho Chemical Processing Plant, 70 (R;US)

Waste-management program. Technical progress report, JanuaryMarch 1981, 622 (R;US)

Feasibility Studies

Economical dry sturage of large amounts of spent fuel and vitrified high level radioactive waste, 43 (BA;XN)

Study for dry storage within the framework of the Swiss waste disposal concept, 1407 (BA;DE;In German)

Surface storage of vitrified high-level radioactive waste in reinforced-concrete casks, 534 (R;GB)

Gaseous Wastes

Savannah River interim waste management program plan, FY1984, 606 (R;US)

Geologic Depnsits

Far-field thermal analysis of a high level waste repository in tuff. Topical report RSI-0137, 942 (R;US)

Mechanisms of dissolution of radioactive waste storage glasses and cesium migration from a granite repository, 1151 (BA;US)

Monitoring an underground repository with modern seismological methods, 1233 (J;US)

Nuclear-waste-package program for high-level isolation in Nevada tuff, $101 \mathrm{~J}$ (R;US)

Preliminary appraisal of gravity and mngnetis datn at Synclins. Ridge, Western Yucca Flat, Nevada Test Site, Nye County, Nevada, 1025 (R;US)

Glass

Plant for solidification and storage of high-level radioactive waste, 521 (J;JP;In Japanese)

Thermodynamically stable glass ceramic product with radionuelides for the removal of radiuactive waste and process for its manufacture (Patent), 1283 (P;DE;In German)

Government Policies

Problem of reprocessing and of radioactive wastes in France, 489 (J;FR;In French)

Heat Transfer

Heat transfer in high-level waste management، 1118 (J:NL)

High-level Radioactive Wastes

Analysis of waste disposal practice and control at ORNL, 549 (R;US)

Assessment of single-shell tank residual-liquid issues at Hanford Site, Washington, 1384 (R;US)

Background and historical perspectives for monitored retrievable storage, $38(\mathrm{~J} ; \mathrm{US})$

Basic study on storage of tritium waste, 1245 (J;JP;In Japanese)

Combined storage for LWR spent fuel and high-level waste (MOdular DRy EXpandable storage system), 16 (RA;US)

Comparison of cask and drywell storage concepts for a monitol retrievable storage/interim storage system, 853 (R;US)

Comparison of cask and dry well concepts for retrievable stora ${ }_{5}$ of high-level waste, 641 (R;US)

Compliance assessments of hypothetical geological nuclear waste isolation systems with the draft EPA standard, 982 (R;US) 
Concepts for the ultimate storage in geological formations, 679 (RA;DE;In German)

Conceptual design of monitored retrievable storage faclities, 41 (J;US)

Containing waste material (Patent), 1075 (P;AU)

Economical dry storage of large amounts of spent fuel and vitrified high level radioactive waste, 43 (BA;XN)

Economics of monitored retrievable storage of nuclear wastes, 40 (J;US)

Effects of Hanford high-level waste components on sorption of cobalt, strontium, neptunium, plutonium, and americium on Hanford sediments, 918 (R;US)

Environmental evaluation of alternatives for long-term management of Defense high-level radioactive wastes at the Idaho Chemical Processing Plant, 70 (R;US)

Evaluation of an international, perpetual, and retrievable facility for storage of vitrified radioactive waste, $97(\mathrm{~J} ; \mathrm{US})$

Experience with the storage of high active waste at WAK, 1199 (B;DE)

Far-field thermal analysis of a high level waste repository in tuff. Topical report RSI-0137, 942 (R;US)

High level radioactive waste, 1242 (J;US)

High-level radioactive wastes, 159 (R;US)

High level waste management and characterization at the Hanford plant site, 1198 (BA;US)

Host State perspective to high-level waste management criteria and standards for siting, storage, and monitoring, 1096 (J;US)

Impact of thermal constraints on the optimal design of high-level waste repositories in geologic media, $1070(\mathrm{~J} ; \mathrm{NL})$

Issues related to field testing in tuff, 947 (R;US)

Land Baden-Wuerttemberg: public notice of the Ministry of Economics, Medium-size Industry and Transport concerning a permit under the Atomic Energy Law. - 3rd Supplement to the 9th partial licence for the construction of Karlsruhe reprocessing plant - System for storage and evaporation of high-activity liquid waste (LAVA), 10 (J;DE;In German)

Management of vitrified high level radioactive waste, 466 (BA;GB)

Materials for high-level waste containment, $1080(\mathrm{~J} ; \mathrm{GB})$

Mechanisms of dissolution of radioactive waste storage glasses and cesium migration from a granite repository, 1151 (BA;US)

Monitored Retrievable Storage Demonstration Facility and dry well storage field conceptual design study, 912 (R;US)

Monitored retrievable storage of nuclear waste in the US, 642 (R;US)

Monitored Retrievable Storage proposal research and development research and development report, 605 (R;US)

Monitored retrievable storage: a waste management option for spent fuel and high-level wastes, 864 (R;US)

Monitoring an underground repository with modern seismological methods, 1233 (J;US)

MRS/IS facility co-located with a repository: preconceptual design and life-cycle cost estimates, 857 (R;US)

Nuclear-waste-package program for high-level isolation in Nevada tuff, 1015 (R;US)

Overview of the Monitored Retrievable Storage (MRS) program, 859 (R;US)

Parametric studies of radiological consequences of basaltic volcanism, 1386 (R;US)

Policy and development of reprocessing of spent nuclear fuel and high-level radioactive waste management in the F.R. Germany, 13 (J;JP;In Japanese)

Preliminary scenarios for consequence assessments of radioactivewaste repositories at the Nevada Test Site, 948 (R;US)

Prevention of stress corrüsion cracking in nuclear waste storage tanks, 630 (R;US)

Program summary. Nuclear waste management and fuel cycle programs, 575 (R;US)

Promotion of the research and development of high level radioactive waste treatment and disposal, 491 (J;JP;In Japanese)

$R$ and $D$ projects and studies: concerning the interim and terminal storage of radioactive wastes, 1281 (TG;US)

Radioactive waste management, 527 (BA;SU; In Russian)

Realizing the geological final storage of radioactive wastes, 1125 (J;DE;In German)
Remote automatic plasma arc-closure welding of a dry-storage canister for spent nuclear fuel and high-level radioactive waste, 551 (R;US)

Repository engineering design for high-level waste, 1082 (J;GB)

Retrievability: the NWTS position, 1264 (J;US)

Risk assessment for storage of high-level waste in single shell tanks, 1256 (J;US)

Risk comparison for transportation, handling, and storage of high-level waste, 1402 (J;US)

Safety analyses for HLW and MLW storage and solidification facilities, 1394 (BA;DE;In German)

Sampling and analysis of high level waste tank supernatant: an overview (Savannah River Plant), 634 (R;US)

Savannah River Plant waste-tank inspection manual, 633 (R;US)

Savannah River interim waste management program plan, FY1984, 606 (R;US)

Screening for monitored retrievable storage facility locations, 39 (J;US)

Second annual report made to the Secretary of State for the Environment, Secretary of State for Scotland, Secretary of State for Wales, 117 (B;GB)

Statistical analysis of lineaments and their relation to fracturing, faulting, and halokinesis in the East Texas Basin. Report of investigations No. 110, 566 (R;US)

Status of tank-assessment studies for continued in-tank storage of Hanford Defense waste, 919 (R;US)

Storage and transportation of spent fuel and high-level waste using dry storage casks, 15 (RA;US)

Storage of HLW in engineered structures: air-cooled and watercooled concepts. Final report, 640 (R;XE)

Storage of HLW in engineered structures: air-cooled and watercooled concepts. Final report, 833 (R;US)

Storage of high-level radioactive waste, 1079 (J;GB)

Surface storage of vitrified high-level radioactive waste in reinforced-concrete casks, 534 (R;GB)

System evaluation model for selecting spent nuclear fuel storage concepts, 24 (R;US)

Thermal and thermomechanical effects in the repository storage of high level waste, 1083 (J;DE;In German)

Thermomechanical effects of the final storage of high-level radioactive wastes in salt domes, 1126 (J;DE; In German)

Waste Management Program. Technical progress report, October-December 1980, 162 (R;US)

Waste-management program. Technical progress report, JanuaryMarch 1981, 622 (R;US)

West Valley Demonstration Project: strategy for an achievable but challenging project, 434 (BA;US)

Intermediate-level Radioactive Wastes

Safety analyses for HLW and MLW storage and solidification facilities, 1394 (BA;DE;In German)

Leaching

Basic study on storage of tritium waste, 1245 (J;JP;In Japanese)

Leaks

Investigation of the consequences of leakage incidents of a tank with high-concentrated radionuclide solutions and pentration of the radioactive solution into the soil, 1423 (R;DE; In German)

Liquid Wastes

Promotion of the research and development of high level radioactive waste treatment and disposal, 491 (J;JP;In Japanese)

Low-level Radioactive Wastes

Savannah River interim waste management program plan, FY1984, 606 (R;US)

Waste-management program. Technical progress report, JanuaryMarch 1981, 622 (R;US)

Materials Testing

Study of $\gamma$-ray irradiation effects on corrosion resistance of alloys for storage of high-level waste packages, (1), 667 (R;JP;In Japanese)

Testing of candidate waste-package backfill and canistęr materials for basalt, 885 (R;US)

Mill Tailings

Program summary. Nuclear waste management and fuel cycle programs, 575 (R;US) 


\section{Monitoring}

Evaluation of geotechnical surveillance techniques for monitoring high-level waste repository performance. Final report 19 Sep 81-31 Dec 82, 738 (R;US)

Host State perspective to high-level waste management criteria and standards for siting, storage, and monitoring, 1096 ( $\mathrm{J}$;US)

Results of instrument reliability study for high-level nuclearwaste repositories (Geotechnical parameters), 1017 (R;US)

Natural Convection

Natural draught centralized dry store for irradiated fuel and active waste, 1239 (J;GB)

Packaging

Monitored Retrievable Storage Demonstration Facility and dry well storage field conceptual design study, 912 (R;US)

Planning

Overview of the Monitored Retrievable Storage (MRS) program, 859 (R;US)

Radiation Hazards

Exposures resulting from nuclear power production. Annex $F$, 1444 (BA;US)

Radiation Monitoring

Scientific basis for establishing dry-well-monitoring frequencies, 920 (R;US)

Radinartive Woste nispasal

High level radioactive waste, 1242 (J;US)

Radionuclide Migration

Comparative leach testing of high-level waste forms, 626 (R;US)

Compliance assessments of hypothetical geological nuclear waste isolation systems with the draft EPA standard, 982 (R;US)

Data base for site specific migration analysis of radioactive elements in repositories, 1140 (J;DE;In German)

KBS annual report 1981. Including summaries of technical reports issued during 1981, 998 (R;SE)

Mechanisms of dissolution of radioactive waste storage glasses and cesium migration from a granite repository, 1151 (BA;US)

Phantom dissolution leach mudel, 1268 (J;US)

Preliminary scenarios for consequence assessments of radioactivewaste repositories at the Nevada Test Site, 948 (R;US)

Research and development related to the Nevada Nuclear Waste Storage Investigations. Progress report, July 1-September 30, 1981,692 (R;US)

Research Programs

Design considerations for occupational exposure for a potential repository at Yucca Mountain: high-level-waste handling operatinns, 991 (R;US)

KBS annual report 1981. Including summaries of technical reports issued during 1981, 998 (R;SE)

$R$ and $D$ projects and studies: concerning the interim and terminal storage of radioactive wastes, 1281 (TG;US)

Research and development related to the Nevada Nuclear Wastc Storage Investigations. Progress report, July 1-September 30, 1981, 692 (R;US)

Research and development related to the Nevada Nuclear Waste Storage Investigations. Progress report, July 1-September 30, 1982, 703 (R;US)

Rockwell Hanford Operations. Defense waste and byproducts management monthly report, 335 (R;US)

Rockwell Hanford Operations defense, waste and byproducts management monthly report, 336 (R;US)

Waste Management Program. Technical progress report, October-December 1980, 162 (R;US)

Wastc-management program. Technical progress report, JanuaryMarch 1981, 622 (R;US)

Retrieval Systems

Retrievability: the NWTS position, 1264 (J;US)

System evaluation model for selecting spent nuclear fuel storage concepts, 24 (R;US)

Risk Assessment

Comparative risk assessments for the production and interim storage of glass and ceramic waste forms: defensc waste processing facility, 1370 (R;US)

Parametric studies of radiological consequences of basaltic volcanism, 1386 (R;US)

Preliminary scenarios for consequence assessments of radioactivewaste repositories at the Nevada Test Site, 948 (R;US)
Risk assessment for storage of high-level waste in single shell tanks, 1256 (J;US)

Risk comparison for transportation, handling, and storage of high-level waste, 1402 (J;US)

Safety analyses for HLW and MLW storage and solidification facilities, 1394 (BA;DE;In German)

Safety

Realizing the geological final storage of radioactive wastes, 1125 (J;DE;In German)

Salt Deposits

Policy and development of reprocessing of spent nuclear fuel and high-level radioactive waste management in the F.R. Germany, 13 (J;JP;In Japanese)

Report of working meeting on fixation of radioactivity in stable, solid media at the Johns Hopkins University, June 19-21, 1957. 342 (R;US)

Thermal and thermomechanical effects in the repository storage of high level waste, 1083 (J;DE;In German)

Thermomechanical effects of the final storage of high-level radioactive wastes in salt domes, 1126 (J;DE;In German)

Underground storage of radioactive wastes in salt deposits in the Federal Republic of Gcrmany, 668 (RA;DE;In German)

Seismic Surveys

Monitoring àn underground repository with modern seismological methods, 1233 (J;US)

Simulation

Status report on the full-scale electric-heater tests at the Hanford Near-Surface Test Facility, 882 (R;US)

\section{Site Selection}

Field guidebook association of engineering geologists. Chapters $1-10,915$ (R;US)

Host State perspective to high-level waste management criteria and standards for siting, storage, and monitoring, 1096 (J;US)

Issues related to field testing in tuff, 947 (R;US)

Nevada Nuclear Waste Storage Investigations: exploratory shaft. Phase I: Conceptual design report, 694 (R;US)

Preliminary appraisal of gravity and magnetic data at Syncline

Ridge, Western Yucca Flat, Nevada Test Site, Nye County, Nevada, 1025 (R;US)

Screening for monitored retrievable storage facility locations, 39 (J;US)

Statistical analysis of lineaments and their relation to fracturing, falilting, and halokinesis in the East Tcxas Basin. Repurt of investigations No, 110, 566 (R;US)

\section{Solldification}

Promotion of the research and development of high level radioactive waste treatment and disposal, 491 (J;JP;In Japanese)

Spent Fuels

Commercial nuclear waste research and development program. Quarterly report, January-March 1982, 61 (R;US)

Standards

Draft environmental impact statement for 40 CFR part 191: environmental standards for management and disposal of spent nuclear fuel, high-level and transuranic radioactive wastes. Final report, 838 (R;US)

Storage Facilities

Illustrative nuclear waste disposal criteria and their application to high-level waste repository design, 1290 (J;US)

MRS/IS facility co-located with a repository: preconceptual design and life-cycle cost estimates, 857 (R;US)

Storage of HLW in engineered structures: air-cooled and watercooled concepts. Final report, 833 (R;US)

Synthetic Rocks

Containing waste material (Patcnt), 1075 (P;AU)

Systems Analysis

System evaluation model for selecting spent nuclear fuel storage concepts, 24 (R;US)

Tanks

Promotion of the research and development of high level radi tive waste treatment and disposal, 491 (J;JP;In Japanese)

Risk assessment for storage of high-level waste in single shell tanks, 1256 (J;US)

Sampling and analysis of high level waste tank supernatant: an overview (Savannah River Plant), 634 (R;US)

Savannah River Plant waste-tank inspection manual, 633 (R;US) 
Status of tank-assessment studies for continued in-tank storage of Hanford Defense waste, 919 (R;US)

Temperature Control

Calculation of media temperatures for nuclear sources in gealogic depositories by a finite-length line source superposition model (FLLSSM), 1122 (J;NL)

Sensitivity calculations for low-heat generating defense waste repository temperatures, $1124(\mathrm{~J} ; \mathrm{NL})$

Temperature Effects

Heating effects on groundwater flow through a repository fracture system, 120 (J;US)

Test Facilities

Radioactive waste legislation. Hearings before the Subcommittee on Energy and the Environment of the Committee on Interior and Insular Affairs, House of Representatives, Ninety-Seventh Congress, First Session on H.R. 1993; H.R. 2800; H.R. 2840; H.R. 2881; H.R. 3809, June 23 and 25; July 9, 1981, 1252 (B;US)

Thermal Analysis

Far-field thermal analysis of a high level waste repository in tuff. Topical report RSI-0137, 942 (R;US)

Tritium Oxides

$R$ and $D$ projects and studies: concerning the interim and terminal storage of radioactive wastes, 1281 (TG;US)

Underground Disposal

Concepts for the ultimate storage in geological formations, 679 (RA;DE;In German)

Underground Storage

Assessment of single-shell tank residual-liquid issues at Hanford Site, Washington, 1384 (R;US)

Basic study on storage of tritium waste, 1245 (J;JP;In Japanese)

Comparison of cask and dry well concepts for retrievable storage of high-level waste, 641 (R;US)

Data base for site specific migration analysis of radioactive elements in repositories, 1140 (J;DE;In German)

Effects of Hanford high-level waste components on sorption of cobalt, strontium, neptunium, plutonium, and americium on Hanford sediments, 918 (R;US)

Far-field thermal analysis of a high level waste repository in tuff. Topical report RSI-0137, 942 (R;US)

Host State perspective to high-level waste management criteria and standards for siting, storage, and monitoring, 1096 (J;US)

Impact of thermal constraints on the optimal design of high-level waste repositories in geologic media, $1070(\mathrm{~J} ; \mathrm{NL})$

Issues related to field testing in tuff, 947 (R;US)

Monitored Retrievable Storage Demonstration Facility and dry well storage field conceptual design study, 912 (R;US)

Monituring an uiderground repository with modern seismological methods, 1233 (J;US)

Nevada Nuclear Waste Storage Investigations: exploratory shaft. Phase I. Conceptual design report, 694 (R;US)

Nuclear-waste-package program for high-level isolation in Nevada tuff, 1015 (R;US)

Preliminary scenarios for consequence assessments of radioactivewaste repositories at the Nevada Test Site, 948 (R;US)

Realizing the geological final storage of radioactive wastes, 1125 (J;DE;In German)

Repository engineering design for high-level waste, 1082 (J;GB)

Retrievability: the NWTS position, 1264 (J;US)

Savannah River Plant waste-tank inspection manual, 633 (R;US)

Thermomechanical effects of the final storage of high-level radioactive wastes in salt dumes, 1126 (J;DE; In German)

Water Chemistry

Oxidation potential $\left(\mathrm{E}_{\mathrm{h}}\right)$ and $\mathrm{pH}$ control during experimentation, 552 (R;US)

RADIOACTIVE WASTES

See also AIPHA-BEARING WASTES

CALCINED WASTES

HIGH-LEVEL RADIOACTIVE WASTES

INTERMEDIATE-LEVEL RADIOACTIVE WASTES

LOW-LEVEL RADIOACTIVE WASTES

Characterization of high-level waste forms, 1099 (J;US)
Chemical Analysis

Deposition of volatile fission products during commercial highlevel waste vitrification, 304 (R;US)

Chemical Radiation Effects

Materials Characterization Center. Second workshop on irradiation effects in nuclear waste forms. Summary report, 80 (R;US)

Classification

Understanding radioactive waste, 79 (R;US)

Financing

Radwaste panel, NRC head highlight 44th American Power Conference program, 99 (J;US)

Impact Tests

Fuel-cycle programs. Quarterly progress report, January-March 1981,48 (R;US)

Fuel-cycle programs. Quarterly progress report, April-June 1981, 49 (R;US)

Fuel cycle programs. Quarterly progress report, July-September 1981; 50 (R;US)

Inventories

Radwaste inventories and projections: an overview (Integrated Data Base), 77 (R;US)

Leaching

Fuel-cycle programs. Quarterly progress report, January-Marcb 1981,48 (R;US)

Fuel-cycle programs. Quarterly progress report, April-June 1981, 49 (R;US)

Fuel cycle programs. Quarterly progress report, July-September 1981,50 (R;US)

Fuel cycle programs. Quarterly progress report, April-June 1982 , 536 (R;US)

Legislation

Radioactive waste legislation. Hearings before the Subcommittee on Energy and the Environment of the Committee on Interior and Insular Affairs, House of Representatives, Ninety-Seventh Congress, First Session on H.R. 1993; H.R. 2800; H.R. 2840; H.R. 2881; H.R. 3809, June 23 and 25; July 9, 1981, 1252 (B;US)

Materials Handling

Commercial nuclear waste research and development program. Quarterly report, October-December 1981, 60 (R;US)

\section{Meetings}

Materials Characterization Center. Second workshop on irradiation effects in nuclear waste forms. Summary report, 80 (R;US)

Meeting on radioactive wastes, 125 (B;FR;In French)

Radwaste inventories and projections: an overview (Integrated Data Basé), 77 (R;US)

\section{Packaging}

Commercial nuclear waste research and development program. Quarterly report, October-December 1981, 60 (R;US)

Commercial nuclear waste research and development program. Quarterly report, January-March 1982, 61 (R;US)

Physical Radiation Effects

Radiation damage in crystalline nuclear-waste solids, 86 (R;US)

Radiation Efiects

Radiation effects in SYNROC-D, 1053 (J;US)

Radioactivity

Graphical and tabular summaries of decay characteristics for once-through PWR, LMFBR, and FFTF fuel cycle materials (Spent fuel, high-level waste fuel can scrap), 76 (R;US)

Regulations

Information report on state legislation, volume 8 , number $S, 1414$ (R;US)

Reprocessing

Problem of reprocessing and of radioactive wastes in France, 489 (J;FR;In French)

Residual Power

Graphical and tabular summaries of decay characteristics for once-through PWR, LMFBR, and FFTF fuel cycle materials (Spent fuel, high-level waste fuel can scrap), 76 (R;US) 
Stability

Thermal and radiation stability of simulated high-level vitrified waste products, $51\left(R_{;}\right.$IN)

Toxicity

Graphical and tabular summaries of decay characteristics for once-through PWR, LMFBR, and FFTF fuel cycle materials (Spent fuel, high-level waste fuel can scrap), 76 (R;US)

Waste Transportation

Comparative transportation risk assessment for borosilicate-glass and ceramic forms for immobilization of SRP Defense waste, 23 (R;US)

Risk assessment for the transportation of radioactive zeolite liners, 28 (R;US)

RADIOCARBON DATING

See CARBON 14

RADIOISOTOPE MIGRATION

SEe RADIONUCLIDE MIGRATION

\section{RADIOISOTOPES}

Recovery

Disposal of radioactive wastes (Book), 106 (B;NL)

RADIOLOGICAL PROTECTION

SE RADIATION PROTECTION

RADIONUCLDE MIGRATION

Comparative analysis of fractured and porous medium radinnu= clide transport, 1356 (BA;U3)

Analytical Solution

Analytical solutions of radionuclide migration with onedimensional flow in layered media, 1361 (J;US)

Biological Pathways

Radioecology of the deep-sea, 1428 (RA;DE;In German)

Flow Models

Effect of radon transport in groundwater upon gamma-ray borehole logs, 1422 (R;US)

Mathematical Models

Data base for site specific migration analysis of radioactive elements in repositories, 1140 (J;DE;In German)

Formulation of an integrated physicochemical-hydrologic model for predicting waste-nuclide retardation in geologic media, 973 (R;US)

INTRACOIN: an international nuclide transport code intercomparison study, 1358 (BA;US)

LAYFLO: a one-dimensional semianalytical model for the migration of a three-member decay chain in a multilayered geologic medium, 1325 (R;US)

Leach rates of high level waste and spent fuel: limiting rates as determined by backfill and bedrock conditions, 1345 (BA;US)

Model for near field migration, 1343 (BA;US)

Model for far field migration, 1344 (BA;US)

Models and risk from high-level waste disposal-the missing pieces, 1090 (J;US)

Numerical modeling of groundwater flow and solute transport for a nuclear-waste repository in basalt, 1327 (R;US)

Solutions to radionuclide migration with nonlinear retardation mechanisms in backfill material. Interim progress report, JuneDecember 1982, 1331 (R;US)

Uncertainties associated with geologic disposal of high-level radluactive wasto, 1367 (R;USi)

One-dimensional Calculations

LAYFLO: a one-dimensional semianalytical model for the migration of a three-member decay chain in a multilayered geologic medium, 1325 (R:US)

$s$ Codes

INTRACOIN, Level 1. Benchmark calculations with code SWIFT, 644 (R;DE)

Statistical Models

Application of a risk-assessment methodology to a hypothetical high-level waste repository in bedded salt, 937 (R;US)

Time Dependence

Water-dilution volume for high-level wastes stored in deep geologic media, 1232 (J;US)

Two-phase Flow

Radionuclide migration in a two-dimensional flow field, 1339 (J;US)
RADIONUCLIDE TRANSFER (IN ENVIRONMENT)

See RADIONUCLIDE MIGRATION

RADIONUCLIDES

SEe RADIOISOTOPES

RADIOTHORIUM

See THORIUM 228

RADIUM

Adsorption Isotherms

Basalt-radionuclide reactions: FY-1981. Annual report, 868 (R;US)

Environmental Transport

Actinide sorption on rock minerals, 677 (RA;DE)

Sorption

Radionuclide sorption on basalt-interbed materials. Annual report FY 1981, 1328 (R;US)

RADIUM 226

Leaching

Waste package performance evaluation, 806 (R;US)

Sorption

Evaluation of radionuclide transport: effect of radionuclide sorption and solubility, 1354 (BA;US)

RADON

FuVirnimmental Tramsport

Effect of radon transport in groundwater upon gamma-ray borehole logs, 1422 (R;US)

Radintion Monitoring

Effect of radon transport in groundwater upon gamma-ray borehole logs, 1422 (R;US)

\section{RAILWAYS}

Capitalized Cost

Southeastern Utah nuclear-waste-transportation study, 26 (R;US)

Comparative Evaluations

Southeastern Utah nuclear-waste-transportation study, 26 (R;US)

Operating Cost

Southeastern Utah nuclear-waste-transportation study, 26 (R;US)

RARE EARTHS

See also CERIUM

EUROPIUM

GADOLINIUM

LANTHANUM

NEODYMIUMI

SAMARIUM

THULIUM

Difrusion

Modelling of the migration of lanthanoids and actinoids in ground water; the medium dependence of equilibrium constants, 1353 (BA;US)

Ion Exchange

Equipment, operation and some results from a hot test of the CTH actinide separation process, 243 (RA;DE)

Solvent Extraction

Equipment, operation and some results from a hot test of the CTH actinide separation pronsess, 243 (RA;DE)

Octyl(phenyl)-N,N-diisobutylcarbamoylmethylphosphine oxide as an extractant for actinides from nitric acid waste, 146 (R;US)

REACTOR ACCIDENTS

Radiatiun Hazards

Exposurcs resulting from nuclear power production. Annex F, 1444 (BA;US)

REACTOR CORES

Activity Levels

Analysis of Am and a recovery for it in the pressurized water reactor fuel cycle, 1433 (R;DE;In German)

\section{REACTOR FUELS}

See NUCLEAR FUIELS

\section{REACTOR OPERATION}

Radiation Hazards

Exposures resulting from nuclear power production. Annex $F$, 1444 (BA;US) 


\author{
IACTOR SAFETY \\ Meetings \\ Uranium and nuclear energy: proceedings of the international \\ symposium, 4th, 1979, 1 (B;GB) \\ RECYCLE (FUEL) \\ See FUEL CYCLE \\ RECYCLING (FUEL) \\ See REPROCESSING \\ REFRACTORIES \\ Comparative Evaluations \\ Evaluation of glass-contact materials for waste glass melters \\ (Corhart 1215, Corhart 1780, ER-2161), 189 (R;US) \\ Corrosion \\ Corrosion experience with a slurry-fed ceramic melter, 321 \\ (R;US) \\ REFUSE \\ See SOLID WASTES \\ REGION VI \\ SeE FEDERAL REGION VI

\section{REINFORCED CONCRETE}

Surface storage of vitrified high-level radioactive waste in reinforced-concrete casks, 534 (R;GB)

\section{REPROCESSING}

See also PUREX PROCESS

Comparative Evaluations

Is spent fuel or waste from reprocessed spent fuel simpler to dispose of, 533 (R;US)

Government Policies

Policy and development of reprocessing of spent nuclear fuel and high-level radioactive waste management in the F.R. Germany, 13 (J;JP;In Japanese)

High-level Radioactive Wastes

Development and investigation of the accepting system for solidified bodies returned from foreign countries after reprocessing, 487 (J;JP;In Japanese)

Meetings

International fuel-cycle and waste-management technologyexchange activities sponsored by the United States Department of Energy: FY 1982 evaluation report, 85 (R;US)

Radiation Hazards

Exposures resulting from nuclear power production. Annex $\mathbf{F}$, 1444 (BA;US)

Research Programs

Tokai Works annual progress report, April 1980-March 1981, 3 (R;JP)

Technology Transfer

International fuel-cycle and waste-management technologyexchange activities sponsored by the United States Department of Energy: FY 1982 evaluation report, 85 (R;US)

REPUBLIC OF CHINA

See TAIWAN

REPUBLIC OF KOREA

Fuel Cycle

Summary of non-US national and international fuel cycle and radioacrive waste management programs 1982,83 (R;US)

Radioactive Waste Management

Summary of non-US national and international fuel cycle and radioactive waste management programs 1982,83 (R;US)

\section{RESERVOIR ROCK}

Oll Saturation

Fuel cycle programs. Quarterly progress report, July-September 1981, 50 (R;US)

\section{RESIDUAL PETROLEUM}

Measuring Methods

Fuel cycle programs. Quarterly progress report, April-June 1982 , 536 (R;US)

Fucl cycle programs. Quarterly progress report, July-September 1982,538 (R;US)

RESIDUES (RADIOACTIVE)

See RADIOACTIVE WASTES
RETRIEVAL SYSTEMS

Equipment for the emplacement of heat-producing waste in long horizontal boreholes (Horizontal vs vertical emplacement), 555 (R;US)

RHODIUM

Materials Recovery

Recovery of noble metals from fission products, 324 (R;US)

RICHLAND FFTF REACTOR

See FFTF REACTOR

ROCK MECHANICS

Geologic Deposits

Laboratory rock mechanics testing manual. Public draft, 807 (R;US)

Manuals

Laboratory rock mechanics testing manual. Public draft, 807 (R;US)

ROCK SALT

See SALT DEPOSITS

ROCK-FLUID INTERACTIONS

Distribution coefficient (Kd) concept and its applicability to studies of radionuclide migration in geological media, 1313 (R;GB)

Interaction of groundwater and fresh basalt fissure surfaces and its effect on the migration of actinides, 1303 (R;US)

ROCKS

\section{See also IGNEOUS ROCKS \\ METAMORPHIC ROCKS SYNTHETIC ROCKS}

Chemical Composition

Composition of rocks - criteria for the conditioning of high-level radioactive wastes, 682 (RA;DE;In German)

Hydrology

Fuel-cycle programs. Quarterly progress report, January-March 1981, 48 (R;US)

Fuel-cycle programs. Quarterly progress report, April-June 1981, 49 (R;US)

Permeability

Characterization of drained and undrained response of thermally loaded repository rocks, 1110 (J;US)

Porosity

Characterization of drained and undrained response of thermally loaded repository rocks, $1110(\mathrm{~J} ; \mathrm{US})$

Radionuclide Migration

Systems, 771 (RA;US)

Rock Mechanics

Characterization of drained and undrained response of thermally loaded repository rocks, 1110 (J;US)

\section{Stresses}

Monitoring an underground repository with modern seismological methods, 1269 (J;US)

Temperature Effects

Characterization of drained and undrained response of thermally loaded repository rocks, 1110 (J;US)

Thermal Expansion

Thermal expansion as a criterion for the quantity of high-level wastes disposed into geological formations, 921 (R;SU;In Russian)

Thermal Stresses

Thermal and thermomechanical calculations of deep-rock nuclear waste disposal with the enhanced SANGRE codte, 1012 (R;US)

\section{ROOM AND PILLAR MINING}

Temperature Effects

Thermo-mechanical scoping calculations for a high level nuclear waste repository in tuff, 933 (R;US)

\section{ROPES}

Testing

Effects of wire rope in mitigating a waste-shaft accident, 980 (R;US) 


\section{RUBIDIUM \\ Leaching \\ Geochemical behavior of supercalcine waste form: its stability in} a basalt environment, 911 (R;US)

RUTHENIUM

Activation Analysis

Application of neutron-activation analysis to the determination of leach rates of simulated nuclear-waste forms, 127 (R;US)

Subsolidus sintering of SYNROC: I. Initial demonstrations, 280 (R;US)

Adsorption

Process improvements in the NWCF, 393 (J;US)

Volatilization and trapping of ruthenium in high temperature processes, 141 (RA;US)

Centrifugation

Influence of the reprocessing flow sheet on the HLW solidification technology, 239 (RA;DE)

Chemical State

Volatilization and trapping of ruthenium in high temperature processes, 141 (RA;US)

Deposition

Control of semivolatile radionuclides in gaseous effluents at nuclear facilities, 453 (B;XA)

Emission Spectroscopy

Analyses of nuclear fuel and high-level waste by inductively coupled plasma-emission spectroscopy, 68 (R;US)

Evaporation

Control of semivolatile radionuclides in gaseous effluents at nuclear facilities, 453 (B;XA)

Influence of the reprocessing flow sheet on the HLW solidification technology, 239 (RA;DE)

On-line measurements of the volatilization of ruthenium in a vitrification process, 422 (BA;US)

Volatilization and trapping of ruthenium in high temperature processes, 141 (RA;US)

Filtration

Prelimianry experience with vitrification inactive pilot plant in Italy, 234 (KA;DE)

Geochemistry

Oklo reactors: natural analogs to nuclear waste repositories, 611 (RA;US)

Lenching

Subsolidus sintering of SYNROC: I. Initial demonstrations. 280 (R;US)

Materials Recovery

Recovery of noble metals from fission products, 324 (R;US)

Solvent Extraction

Ruthenium nitrosyl complexes in radioactive waste solutions of reprocessing plants. Pt. 1, 12 (J;DE)

Volatility

Denitration of simulated fast ractor highly active liquor waste, 241 (RA;DE)

RUTHENIUM 106

Adsorption

Laboratory tests on the migration behavior of selected fission products in aquifer materials from a potential disposal site in northern Germany, 1352 (BA;US)

Removal of ${ }^{90} \mathrm{Sr},{ }^{107} \mathrm{Cs}$, and ${ }^{106} \mathrm{Ru}$ from vent gases produced during solidification of high-level radioactive solutions, 399 (J;CS)

\section{Diffusion}

Laboratory tests on the migration behavior of selected fission products in aquifer materials from a potential disposal site in northern Germany, 1352 (BA;US)

Environmental Exposure Pathway

Investigation of the consequences of leakage incidents of a tank with high-concentrated radionuclide solutions and pentration of the radioactive solution into the soil, 1423 (R;DE;In German)

Environmental Transport

Investigation of the consequences of leakage incidents of a tank with high-concentrated radionuclide solutions and pentration of the radioactive solution into the soil, 1423 (R;DE;In German)

\section{Filtration}

Removal of ${ }^{90} \mathrm{Sr},{ }^{107} \mathrm{Cs}$, and ${ }^{106} \mathrm{Ru}$ from vent gases produced during solidification of high-level radioactive solutions, 399 (J;CS)

Leaching

High-level waste glass field burial tests at CRNL: the effect of geochemical kinetics on the release and migration of fission products in a sandy aquifer, 1299 (R;CA)

Leaching study of nuclear melt glass: Part I, 1332 (R;US)

Measurement of leaching from simulated nuclear-waste glass using radiotracers, 128 (R;US)

\section{S}

\section{SAFETY (NUCLEAR)}

See RADIATION PROTECTION

SAFETY (REACTOR)

Set REACTOR SAFETY

SALT CAVERNS

Calculation Methods

Effect of a random variation of rock salt creep on calculations of storage room closure for the WIPP project, 943 (R;US)

\section{SALT DEPOSITS}

Isolation capability of a salt dome utilized for high-level waste disposal, 1208 (BA;US)

Near-field repository conditions in basalt and salt, 733 (RA;US)

Repository, 773 (RA;US)

Status of the ONWI salt project, 1226 (J;US)

Boreboles

Method of measurement and borehole probe to measure heatinduced borehole convergence, 1282 (P;DE;In German)

Comparative Evaluations

Development of a comparative analysis of potential repository salt sites, 1037 (J;US)

Compression Strength

In situ and laboratory geotechnical test results from borchole GD-1 in southeast Utah, 813 (R;US)

Thermal impact of waste emplacement and surface cooling associated with geologic disposal of nuclear waste, 707 (R;US)

Cooling

Forced ventilation analysis of a cummercial high-level nuclear reep repository in salt, 811 (R;US)

Effect of a random variation of rock salt creep on calculations of storage room closure for the WIPP project, 943 (R;US)

In situ and laboratory geotechnical test results from borehole GD-1 in southeast Utah, 813 (R;US)

Modeling of waste/near field interactions for a waste repository in bedded salt: the Dynamic Network (DNET) model, 1063 (J;US)

Repository data base development, 801 (RA;US)

Stresses near. waste canisters buried in salt, 950 (R;US)

Technical progress report for the quarter, 1 April-30 June 1982, 779 (R;US)

Transient creep model for salt during stress loading and unloading, 969 (R;US)

Deformation

Conclusions regarding geotechnical acceptability of the WIPP site, 990 (R;US)

Deformation-dissolution polential uf bedded salt, Wastè Isolation Pilot Plant site, Delaware Basin, New Mexico, 1163 (BA;US)

EEG review comments on the geotechnical reports provided by DOE to EEG under the stipulated agreement through March 1, 1983, 562 (R;US)

Preliminary investigation of the thermal and structural influence of ventilation drifts on repository rooms, 786 (R;US)

Dissolution

Basic data report for Drillhole ERDA 10 (Waste Isolation Pilot Plant - WIPP), 927 (R;US)

Conclusions regarding geotechnical acceptability of the WIPP site, 990 (R;US)

Deformation-dissolution potential of bedded salt, Waste Isolation Pilot Plant site, Delaware Basin, New Mexico, 1163 (BA;US) 
Dissolution of evaporites and its possible impact on the integrity of the Waste Isolation Pilot Plant (WIPP) New Mexico, USA, 1162 (BA;US)

EEG review comments on the geotechnical reports provided by DOE to EEG under the stipulated agreement through March 1, 1983, 562 (R;US)

Modeling of waste/near field interactions for a waste repository in bedded salt: the Dynamic Network (DNET) model, 1063 (J;US)

Encapsulation

Corrosion aspects of high-level waste disposal in salt domes, 922 (R;DK)

Evaluation

Evaluation of area studies of the US Gulf Coast Salt Dome Basins: location recommendation report, 785 (R;US)

Near-field performance evaluations; 800 (RA;US)

Fluid Flow

Modeling of waste/near field interactions for a waste repository in bedded salt: the Dynamic Network (DNET) model, 1063 (J;US)

\section{Geochemistry}

Investigation of the utility of Gulf Coast salt domes for the storage or disposal of radioactive wastes, 571 (R;US)

Mineralogical and geochemical factors influencing the final disposal of HLW in the Stassfurt halite, 1164 (BA;US)

Technical progress report for the quarter, 1 April-30 June 1982 , 779 (R;US)

\section{Geologic Faults}

Statistical analysis of lineaments and their relation to fracturing, faulting, and halokinesis in the East Texas Basin. Report of investigations No. 110, 566 (R;US)

Geology

Basic data report for drillhole ERDA 9 (Waste Isolation Pilot

Plant WIPP), 926 (R;US)

Disposal of high-level nuclear waste from nuclear power plants in Denmark. Salt dome investigations. V.2. Geology, figures, 649 (R;DK)

Quaternary geology of Rayburn's salt dome: North Louisiana Salt Dome Basin, 814 (R;US)

Status of site characterization in the Permian Basin, 590 (RA;US)

Geophysical Surveys

Disposal of high-level waste from nuclear power plants in Denmark. Salt dome investigations. V.2. Geology, text, 648 (R;DK)

Heat Transfer

Modeling of waste/near field interactions for a waste repository in bedded salt: the Dynamic Network (DNET) model, 1063 (J;US)

Radiant energy dissipation during final storage of high-level radioactive waste in rock salt (Stored lattice energy), 867 (R:DE;In German)

Hydraulic Conductivity

Groundwater flow modeling in support of salt site evaluations, 1034 (J:US)

Hydrology

Disposal of high-level waste from nuclear power plants in Denmark. Salt dome investigations. V.2. Geology, text, 648 (R:DK)

Dissolution rate of salt domes on the basis of interpretation of measured salinity profiles (Danish salt dome Mors in northern Jutland). 1209 (BA;US)

Hydrogeological conditions at Mors salt dome, 659 (RA;DK;In Danish)

Investigations of sall domes for the purpose of high-level radioactive waste disposal, 657 (R;DK;DA)

Investigation of the utility of Gulf Coast salt domes for the storage or disposal of radioactive wastes, 571 (R;US)

itatus of site characterization in the Permian Basin, 590

(RA;US)

Impurities

Impurities in rock-salt: consequences for the temperature increases at the disposal of high-level nuclear waste, 834 (R;US)
Inclusions

Domal salt brine migration experiments at Avery Island, Louisiana, 621 (RA;US)

Migration rates of brine inclusions in single crystals of $\mathrm{NaCl}$, 1149 (BA;US)

Leaching

Diffusion of leached constituents in backfill barriers, 849 (RA;US)

Lithology

Basic data report for drillhole ERDA 6 (Waste Isolation Pilot Plant - WIPP), 923 (R;US)

Materials Testing

Corrosion aspects of high-level waste disposal in salt domes, 922 (R;DK)

Disposal of high-level waste from nuclear power plants in Denmark. V.3. Material testing, 650 (R;DK)

Mathematical Models

Thermomechanical modeling and the selection of a salt repository site, 1218 (J;US)

Mechanical Properties

Thermomechanical modeling and the selection of a salt repository site, 1218 (J;US)

Mineralogy

Mineralogical and geochemical factors influencing the final disposal of HLW in the Stassfurt halite, 1164 (BA;US)

Scientific data necessary to predict radionuclide migration within or near a mined nuclear repository, 1312 (R;US)

Technical progress report for the quarter, 1 April-30 June 1982, 779 (R;US)

Permeability

Thermal impact of waste emplacement and surface cooling associated with geologic disposal of nuclear waste, 707 (R;US)

Petrology

Investigation of the utility of Gulf Coast salt domes for the storage or disposal of radioactive wastes, 571 (R;US)

Poisson Ratio

Thermal impact of waste emplacement and surface cooling associated with geologic disposal of nuclear waste, 707 (R;US)

Porosity

Thermal impact of waste emplacement and surface cooling associated with geologic disposal of nuclear waste, 707 (R;US)

Radiation Effects

Radiation damage studies on synthetic $\mathrm{NaCl}$ crystals and natural rock salt for radioactive waste disposal applications, 610 (RA;US)

Radiation Heating

Disposal of high-level nuclear waste in rock-salt. A comparison of temperature increases calculated at ECN (Energieonderzoek Centrum Nederland)-Petten, RWTH (Rheinisch-Westfaelische Technische Hochschule Aachen)-Aachen and THD (Delft University of Technology)-Delft, $835(\mathrm{R} ; \mathrm{NL})$

Radioactive Waste Disposal

SALTMP - a FORTRAN program for fast salt temperature calculations in HRW final storage, 866 (R;DE;In German)

Radionuclide Migration

Application of a risk-assessment methodology to a hypothetical high-level waste repository in bedded salt, 937 (R;US)

Compliance assessments of hypothetical geological nuclear waste isolation systems with the draft EPA standard, 982 (R;US)

Diffusion of leached constituents in backfill barriers, 849 (RA;US)

Rock Mechanics

Evaluation of the thermomechanical behavior about a waste container/sleeve in salt, 803 (R;US)

Repository data base development, 801 (RA;US)

Shaft Excavations

Conceptual test plan for site confirmation testing at an exploratory shaft in salt, 826 (R;US)

Functional design criteria for the exploratory shaft in salt, 820 (R;US)

Site Selection

Thermomechanical modeling and the selection of a salt repository sito, $1318\left(\mathrm{~J}_{i} \mathrm{US}\right)$ 
Site Surveys

Site, 772 (RA;US)

Stored Encrgy

Radiant energy dissipation during final storage of high-level radioactive waste in rock salt (Stored lattice energy), 867 (R;DE;In German)

Strains

In situ and laboratory geotechnical test results from borehole GD-1 in southeast Utah, 813 (R;US)

Stratigraphy

Geology of the Mors salt dome, 658 (RA;DK;In Danish)

Groundwater flow modeling in support of salt site evaluations, 1034 (J;US)

Investigations of salt domes for the purpose of high-level radioactive waste disposal; 657 (R;DK;DA)

Mineralogical and geochemical factors influencing the final disposal of HLW in the Stassfurt halite, 1164 (BA;US)

Stresses

In situ and laboratory geotechnical test results from borehole GD-1 in sontheast I.Itah, 813 (R;US)

Stresses near waste canisters buried in salt, 950 (R;US)

System Failure Analysis

Analysis of the growth of brine flow pathes in rock salt, 690 (R;DE:In German)

Tectóniles

Investigation of the utility of Gulf Coast salt domes for the storage or disposal of radioactive wastes, 571 (R;US)

Salt domes - the past and present, keys to the future, 1179 (BA;GB)

Statistical analysis of lineaments and their relation to fracturing, faulting, and halokinesis in the East Texas Basin. Report of investigations No. 110, 566 (R;US)

Temperature Distribution

Thermomechanical in-situ experiments and finite element computations, 1166 (BA;US)

Temperature Effects

Impurities in rock-salt: consequences for the temperature increases at the disposal of high-level nuclear waste, 834 (R;US)

Repository data base development, 801 (RA;US)

Results of repository conditions study for commercial and defense high-level nuclear waste and spent fuel repositories in salt, 824 (R;US)

Thermal and thermomechanical effects in the repository storage of high level waste, 1083 (J;DE;In German)

Thermal-gradient migration of brine inclusions in salt, 711 (R;US)

Thermomechanical modeling and the selection of a salt reposiluiy sile, 1218 (J;US)

Temperature Measurement

Dome-salt thermomechanical experiments at Avery Island, Louisiana, 620 (RA;US)

Tetrisile Properties

Thermal impact of waste emplacement and surface cooling associated with geologic disposal of nuclear waste, 707 (R;US)

Thermal Degradation

Forced ventilation analysis of a commercial high-level nuclear repository in salt, 811 (R;US)

Thermal Expansion

Modeling of waste/near field interactions for a waste repository in bedded salt: the Dynamic Network (DNET) model, 1063 (J:US)

Thermal Stresses

Dome-salt thermomechanical experiments at Avery Island, Loujsiana, $620(R A ; U S)$

Thermal impact of waste emplacement and surface cooling associated with geologic disposal of nuclear waste, 707 (R;US)

Thermomechanical effects of the final storage of high-level radioactive wastes in salt domes, 1126 (J;DE;In German)

Thermoelesticity

Preliminary investigation of the thermal and structural influence of ventilation drifts on repository rooms, 786 (R;US)

Thermomechanical impact around a borehole in rock salt after HAW disposal. 1294 (J;US)
Well Drilling

Present status of the Gorleben site investigations, $1036(\mathrm{~J} ; \mathrm{US})$

Well Logging

Present status of the Gorleben site investigations, 1036 (J;US)

Young Modulus

Thermal impact of waste emplacement and surface cooling associated with geologic disposal of nuclear waste, 707 (R;US)

SALTS

Thermal Conductivity

Impurities in rock-salt: consequences for the temperature increases at the disposal of high-level nuclear waste (Kainite, Kieserite), 834 (R;US)

SAMARIUM

Activation Analysis

Application of neutron-activation analysis to the determination of leach rates of simulated nuclear-waste forms, 127 (R;US)

Solubility

Geochemical controls on radionuclide releases from a nuclear waste repository in basalt: estimated solubilities for selected oloments. 1729 (R,J,9)

SAND

Sorptive Properties

Laboratory tests on the migration behavior of selected fission products in aquifer materials from a potential disposal site in northern Germany, 1352 (BA; $U$ ÜS)

SANDSTONES

Radionuclide Migration

Application of a risk-assessment methodology to a hypothetical high-level waste repository in bedded salt, 937 (R;US)

Sorptive Properties

Radionuclide sorption on basalt-interbed materials. Annual report FY 1981, 1328 (R;US)

\section{SAVANNAH RIVER PLANT}

Accidents

Contamination of the hot gang valve corridor and first-level clean areas of Building 221-F - September 13, 1960, 194 (R;US)

Low-level Radioactive Wastes

Disposal of Savannah River Plant waste salt, 625 (R;US)

Radioactive Waste Disposal

Alternatives for definse waste-salt disposal, 629 (R;US)

Radioactive Waste Facilities

Defense Waste Processing Facility: Savannah River Plant, Aiken, SC. Hinal environmental impact statement, 1306 (R;US)

Disposal of Savannah River Plant waste salt, 625 (R;US)

DWPF process control, 186 (R;US)

Radioactive Waste Management

Savannah River Waste Management Program Plan - FY 1982, 157 (R;US)

Savannah River Plant overview, 69 (R;US)

Savannah River interim waste management program plan, FY. $1984,606(R ; U S)$

Strategy document: Inng-term technology program for defense high-level waste, 156 (R;Ü)

Waste-management program technical progress report, JulySeptember 1980, 63 (R;US)

Waste-management program. Technical progress report, JulySeptember 1981, 64 (R;US)

Waste Management Program. Technical progress report, October-December 1980, 162 (R;US)

Waste-management program. Technical progress report, JanuaryMarch 1981, 622 (R;US)

Waste Management Program. Technical progress report, October-December 1982, 65 (R;US)

Waste management program technical progress report, JanuaryMarch 1983, 623 (R;US)

Radioactive Waste Processing

Alternate waste form evaluation for Savannah River Plant highlevel waste, $1104(\mathrm{~J} ; \mathrm{US})$

Assessment of Savannah Rivcr borosilicate glass in the reposi environmient, 172 (R;US)

Borosilicate glass as a matrix for immobilization of SRP highlevel waste, 512 (J;US)

Borosilicate glass for immobilization of high-level SRP waste, $1105(\mathrm{~J} ; \mathrm{US})$ 
Comparative risk assessments for the production and interim storage of glass and ceramic waste forms: defense waste processing facility, 1370 (R;US)

Comparison of SRP high-level waste disposal costs for borosilicate glass and crystalline ceramic waste forms; 635 (R;US)

Defense Waste Processing Facility: a remote process for solidification of Savannah River Plant high level waste, 195 (R;US)

Immobilizing defense waste at the Savannah River Project, 196 (R;US)

Precipitation process for decontamination of water-soluble SRP radioactive waste, 184 (R;US)

Precipitation process for decontaminating water-soluble SRP radioactive waste, 187 (R;US)

Preliminary assessment of key process steps for converting Savannah River Plant high-level wastes to tailored ceramics, 147 (R;US)

Recent developments in defense waste vitrification, 504 (J;US)

Small-scale processing of actual Savannah River high-level waste, 503 (J;US)

Solidification of Savannah River plant high-level waste, 506 (J;US)

Radioactive Waste Storage

Comparative risk assessments for the production and interim storage of glass and ceramic waste forms: defense waste processing facility, 1370 (R;US)

Sampling and analysis of high level waste tank supernatant: an overview, 634 (R;US)

Solidification

Comparison of SRP high-level waste disposal costs for borosilicate glass and crystalline ceramic waste forms, 635 (R;US)

Storage Facilities

Decommissioning alternatives for Savannah River Plant highlevel waste tank 16, 142 (RA;US)

SCANDINAVIA

See also DENMARK

FINLAND

NORWAY

SWEDEN

Coordinated Research Programs

Enlarged Nordic cooperative program on nuclear safety. Nordic study on reactor waste: main report, 72 (R;SE)

\section{SCANDIUM}

Activation Analysis

Subsolidus sintering of SYNROC: I. Initial demonstrations, 280 (R;US)

Leaching

Subsolidus sintering of SYNROC: I. Initial demonstrations, 280 (R;US)

\section{SEA BED}

Subseabed disposal of nuclear wastes, 1243 (J;US)

Radioecology

Radioecology of the deep-sea, 1428 (RA;DE;In German)

Radionuclide Migration

Analysis of diffusional ion transport in ocean sediments: subseafloor disposal of radioactive waste, 1250 (J;US)

Diffusional transport of radionuclide chains in sub-seabeds, 1340 (J;GB)

Low-gradient permeability testing of fine-grained marine sediments, 1277 (J;US)

Rock-fluid Interactions

Waste glass - seabed interactions: observations of the Soret effect, 850 (RA;US)

Waste-rock Interactions

Waste glass - seabed interactions: observations of the Soret effect, 850 (RA;US)

iA DISPOSAI.

See MARINE DISPOSAL

SEALINC MATERIALS

Performance

Performance assessment of a shaft seals system in a HLW repository in the Gibson Dome area, 827 (R;US)

\section{Permeability}

Recent advances in repository seal materials, 1172 (BA;US)

SEALS

Fluid Flow

Laboratory studies of fluid flow through borehole seals, 1231 (J;US)

Performance Testing

Laboratory studies of fluid flow through borehole seals, 1231 (J;US)

SEAWATER

Rock-fluid Interactions

Constant temperature and thermal gradient investigations of the near field subseabed environment: chemical and thermodiffusional effects, 971 (R;US)

Waste glass - seabed interactions: observations of the Soret effect, 850 (RA;US)

\section{SEDIMENTS}

Chemical Composition

Geotechnical studies for subseabed disposal: high level radioactivc wastes, 964 (R^;US)

Density

Geotechnical studies for subseabed disposal: high level radioactive wastes, 964 (RA;US)

Heat Transfer

Near-field interactions, 929 (R;US)

Leaching

Predicting Pu concentrations in solutions contacting geologic materials, 1146 (BA;US)

Mechanical Properties

Geotechnical studies for subseabed disposal: high level radioactive wastes, 964 (RA;US)

Mineralogy

Pacific site assessment, 960 (RA;US)

Paleomagnetism

Paleomagnetic study of areas B1, C1 and E2, 959 (RA;US)

Permeability

Low-gradient permeability testing of fine-grained marine sediments, 1277 (J;US)

Permeability of selected sediments in the vicinity of five salt domes in the Gulf Interior Region, 810 (R;US)

Physical Properties

Geotechnical studies for subseabed disposal: high level radioactive wastes, 964 (RA;US)

Radionuclide Migration

Effects of Hanford high-level waste components on sorption of cobalt, strontium, neptunium, plutonium, and americium on Hanford sediments, 918 (R;US)

Low-gradient permeability testing of fine-grained marine sediments, 1277 (J;US)

Subseabed disposal program. Annual report, January-December 1980. Volume 1. Summary, 935 (R;US)

Rock-fluid Interactions

Constant temperature and thermal gradient investigations of the near field subseabed environment: chemical and thermodiffusional effects, 971 (R;US)

Sorptive Properties

Effects of Hanford high-level waste components on sorption of cobalt; strontium, neptunium, plutonium, and americium on Hanford sediments, 918 (R;US)

SELENIUM

Adsorption

Geochemistry studies pertaining to the G-tunnel radionuclide migration field experiment, 1426 (R;US)

Adsorption Isotherms

Basalt-radionuclide reactions: FY-1981. Annual report, 868 (R;US)

Solubility

Genchemical controls on radionuclide releases from a nuclear waste repository in basalt: estimated solubilities for selected elements, 1329 (R;US)

Sorption

Radionuclide sorption on basalt-interbed materials. Annual report FY 1981, 1328 (R;US) 


\section{SELENIUM 79 \\ Solubility}

Evaluation of radionuclide transport: effect of radionuclide sorption and solubility, 1354 (BA;US)

SEQUESTRENE

See EDTA

SHAFT EXCAVATIONS

Closures

Geochemical factors in borehole-shaft plug longevity, 614 (RA;US)

Research and development related to the Nevada Nuclear Waste Storage Investigations. Progress report, January 1-March 31, 1983, 705 (R;US)

Comparative Evaluations

Evaluation of alternative shaft-sinking techniques for high-level nuclear waste (HLW) deep geologic repositories. Final report (Task 3), June 1981-July 1982, 741 (R;US)

Cost

Nevada Nuclear Waste Storage Investigations: exploratory shaft. Phase I. Conceptual design report, 694 (R;US)

Design

Functional design criteria for the exploratory shaft in salt, $\mathbf{8 2 0}$ (R;US)

Nevada Nuclear Waste Storage Investigations: exploratory shaft. Phase I. Conceptual design report, 694 (R;US)

Sealing Materials

Geochemical factors in borehole-shaft plug longevity, 614 (RA;US)

Site Selection

Identifiction of a preferred site for the exploratory shaft within the reference repository location: Hanford Site, 916 (R;US)

SHALES

See also ARGILLITE

Compression Strength

Thermal impact of waste emplacement and surface cooling associated with gcologic disposal of nuclear waste, 707 (R;US)

Evaluation

Preliminary assessment of shales and other argillaceous rocks in the United States, 609 (RA;US)

Hydrology

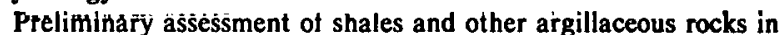
the United States, 609 (RA;US)

Permeability

Thermal impact of waste emplacement and surface cooling associated with geologic disposal of nuclear waste, 707 (R;US)

Poisson Ratio

Thermal impact of waste emplacement and surface cooling associated with geologic disposal of nuclear waste, 707 (R;US)

Porosity

Thermal impact of waste emplacement and surface cooling associated with geologic disposal of nuclear waste, 707 (R;US)

Site Surveys

Site, 772 (RA;US)

Stratigraphy

Regional geological assessment of the Devonian-Mississippian shale sequence of the Appalachian, Illinois, and Michigan basins relative to potential storage/disposal of radioactive wastes, 828 (R;US)

Tensile Properties

Thermal impact of waste emplacement and surface cooling associated with geologic disposal of nuclear waste, 707 (R;US)

Thermal Stresses

Thermal impact of waste emplacement and surface cooling associated with geologic disposal of nuclear waste, 707 (R;US)

Young Modulus

Thermal impact of waste emplacement and surface cooling assoSHIPS ciated with geologic disposal of nuclear waste, 707 (R;US)

Design

Subseabed nuclear waste disposal: conceptual ship description, 31 (RA;US)
SIALON

See ALUMINIUM OXIDES

SILICA

Chemical Reactions

Waste glass/metal interactions in brines, 860 (R;US)

Leaching

Effects of composition, solutions, and burial on nuclear waste glasses, 845 (RA;US)

Physical Radiation Effects

Structural effects of radiation damage in silica based glasses, 464 (BA;US)

SILICA GEL

Sorptive Properties

Process improvements in the NWCF, 393 (J;US)

SILICATE MINERAIS

See also BIOTITE

CLINOPTILOLITE

KAOLINITE

SMECTITE

ZIRCON

Phase Transformations

Selected hydrologic and geochemical issues in site characterization for nuolenr waste disposal: flood basalts at the Hanford Reservation, 715 (R;US)

SILICEOUS ROCK

See SANDSTONES

SILICON

Absorption Spectroscopy

Analysis for silicon in solution in high level waste glass durability studies, 375 (J;GB)

Emission Spectroscopy

Analyses of nuclear fuel and high-level waste by inductively coupled plasma-emission spectroscopy, 68 (K;US)

Analysis for silicon in solution in high level waste glass durability studies, 375 (J;GB)

Leaching

Geochemical behavior of supercalcine waste form: its stability in a basalt environment, 911 (R;US)

Preparation and leaching of radioactive INEL waste forms, 201 (R;US)

Solubility limits of metal ions and mechanism of leaching nucleMr-waste glasses, 320 (K;US)

Waste glass/metal interactions in brines, 860 (R;US)

SILICON CARBIDES

Chemical Vapor Deposition

Coating crystalline nuclear waste forms to improve inertness, 138 (R;US)

Evaluation of coated particle waste forms, 448 (BA;US)

Corrosion Resistance

Flame-resistant radioactive waste processing method, 488 (P;JP;In Japanese)

Leaching

Coating crystailine nuclear waste forms to improve inertness, 138 (R;US)

SILVER

Activation Analysis

Application of neutron-activation analysis to the determination of leach rates of simulated nuclear-waste forms, 127 (R;US)

Subsolidus sintering of SYNROC: I. Initial demonstrations, 280 (R;US)

Leaching

Subsolidus sintering of SYNROC: I. Initial demonstrations, 280 (R;US)

SLUDGES

Gas Yields

Physical and chemical characterization of synthetic calcined sludge, 167 (R;US)

Thermal Gravimetric Analysis

Physical and chemical characterization of synthetic calcined sludge, 167 (R;US) 
'CTITE

sorption

Redox behavior, complexing, and adsorption of hexavalent actinides by humic acid and selected clays (Siting, marine disposal of high-level radioactive waste), 1247 (J;US)

Phase Transformations

Selected hydrologic and geochemical issues in site characterization for nuclear waste disposal: flood basalts at the Hanford Reservation, 715 (R;US)

Sorptive Properties

Sorptive diffusion in clay gels, 709 (RA;US)

\section{SODIUM}

Adsorption

Geochemistry studies pertaining to the G-tunnel radionuclide migration field experiment, 1426 (R;US)

Adsorption Isotherms

Basalt-radionuclide reactions: FY-1981. Annual report, 868 (R;US)

Emission Spectroscopy

Analyses of nuclear fuel and high-level waste by inductively coupled plasma-emission spectroscopy, 68 (R;US)

Leaching

Comparison of properties of borosilicate glass and crystalline ceramic forms for immobilization of Savannah River Plant waste, 171 (R;US)

Geochemical behavior of supercalcine waste form: its stability in a basalt environment, 911 (R;US)

Preparation and leaching of radioactive INEL waste forms, 201 (R;US)

SODIUM 22

Leaching

Leaching study of nuclear melt glass: Part I, 1332 (R;US)

\section{SODIUM CHLORIDES}

Chemical Radiation Effects

Radiation induced F-center and colloid formation in synthetic $\mathrm{NaCl}$ and natural rock salt: applications to radioactive waste repositories ( 1 to $3 \mathrm{MeV}$ electrons), 543 (R;US)

Inclusions

Thermal-gradient migration of brine inclusions in salt crystals (Synthetic single crystals of $\mathrm{NaCl}$ and $\mathrm{KCl}$ ), 713 (R;US)

Radiation Effects

Radiation damage studies on synthetic $\mathrm{NaCl}$ crystals and natural rock salt for radioactive waste disposal applications, 610 (RA;US)

SODIUM COMPLEXES

Solubility

Importance of organic compounds in ground water as radionuclide-mobilizing agents, 1324 (K;US)

SODIUM MINERALS

SOILS

SEe MINERALS

Radionuclide Migration

Assessment of single-shell tank residual-liquid issues at Hanford Site, Washington, 1384 (R;US)

Diffusive transport of strüritium- 85 in sand-bentonite mixtures, 1300 (R;CA)

Health effects associtated with unit radonuclide releases to the environment, 1378 (RA;US)

High-level waste glass field burial tests at CRNL: the effect of geochemical kinetics on the release and migration of fission products in a sandy aquifer, $1299(\mathrm{R} ; \mathrm{CA})$

Investigation of the consequences of leakage incidents of a tank with high-concentrated radionuclide solutions and pentration of the radioactive solution into the soil, 1423 (R;DE;In German)

\section{SOL-GEL PROCESS}

Sol-gel-derived waste forms, 245 (RA;DE)

Sulidification of high-level radionctive wastes and alpha-bearing wastes by a sol-gel process (in German), 244 (RA;DE)

SOLID WASTES

Nondestructive Testing

Nondestructive techniques for evaluating the integrity of vitrified solids, $471(\mathrm{~J} ; \mathrm{JP})$

\section{Physical Radiation Effects}

Radiation damage from alpha decay in ceramic nuclear waste forms, $103(\mathrm{~J} ; \mathrm{CH})$

\section{SOUTH AFRICA}

Fuel Cycle

Summary of non-US national and international fuel cycle and radioactive waste management programs 1982, 83 (R;US)

Radioactive Waste Management

Summary of non-US national and international fuel cycle and radioactive waste management programs 1982, 83 (R;US)

SOUTH AUSTRALIA

Uranium Mines

Report of the Select Committee of the Legislative Council on uranium resources, 1363 (B;AU)

SOUTH CAROLINA

Higb-level Radioactive Wastes

Nuclear waste, the nation, and South Carolina: an update on the major issues, 114 (B;US)

Low-level Radioactive Wastes

Nuclear waste, the nation, and South Carolina: an update on the major issues, 114 (B;US)

\section{SOUTH KOREA}

See REPUBLIC OF KOREA

SOUTHWEST REGION

SEe FEDERAL REGION VI

SOVIET UNION

See USSR

SPACE VEHICLES

Radioactive Waste Disposal

Solar-thermionic generator for nuclear wastes disposal in the space, 1138 (J;DE)

Solar Electric Propulsion

Solar-thermionic generator for nuclear wastes disposal in the space, 1138 (J;DE)

SPACECRAFT POWER SUPPLIES

Thermionic Converters

Solar-thermionic generator for nuclear wastes disposal in the space, 1138 (J;DE)

SPAIN

Fuel Cycle

Summary of non-US national and international fuel cycle and radioactive waste management programs 1982, 83 (R;US)

Radioactive Waste Management

Summary of non-US national and international fuel cycle and radioactive waste management programs 1982,83 (R;US)

SPENT FUEL CASKS

Storage and transportation of spent fuel and high-level waste using dry storage casks, 15 (RA;US)

Closures

Remote automatic plasma arc-closure welding of a dry-storage canister for spent nuclear fuel and high-level radioactive waste, 551 (R;US)

\section{Fabrication}

Highly dense graphite matrix: a new material for the conditioning of radioactive wastes, 358 (J;US)

Materials Testing

Canister materials proposed for final disposal of high level nuclear waste - a review with respect to corrosion resistance, 675 (RA;DE)

Performance Testing

Cooldown testing of nuclear fuel casks, 1442 (J;NL)

SPENT FUEL ELEMENTS

Packaging

Engineered waste-package conceptual design: defense high-level waste (Form 1), commercial high-level waste (Form 1), and spent fuel'(Form 2) disposal in salt, 817 (R;US)

Waste package conceptual designs for a nuclear repository in basalt, 872 (R;US)

Spent Fuel Casks

Cooldown testing of nuclear fuel casks, $1442(\mathrm{~J} ; \mathrm{NL})$ 


\section{SPENT FUEL STORAGE}

\section{See also DRY STORAGE}

Combined storage for LWR spent fuel and high-level waste (MOdular DRy EXpandable storage system), 16 (RA;US)

Handling and disposing of radioactive waste, 561 (R;US)

Monitored retrievable storage of nuclear waste in the US, 642 (R;US)

Monitored Retrievable Storage proposal research and development research and development report, 605 (R;US)

Monitored retrievable storage: a waste management option for spent fuel and high-level wastes, 864 (R;US)

National waste terminal storage conceptual reference repository description, 612 (RA;US)

ONWI library reports list, 770 (R;US)

Parametric studies of radiological consequences of basaltic volcanism, 1386 (R;US)

Radioactive waste management and disposal, 639 (RA;CA)

Away-from-reactor Storage

Study for dry storage within the framework of the Swiss waste disposal concept, 1407 (BA;DE;In German)

Comparative Evaluations

Comparison of cask and drywell storage concepts for a monitored retrievable storage/interim storage system, 853 (R;US)

Is spent fuel or waste from reprocessed spent fuel simpler to dispriso uf, 333 (R;US)

Cost

MRS/IS facility co-located with a repository: preconceptual design and life-cycle cost estimates, 857 (R;US)

\section{Dry Storage}

Natural draught centralized dry store for irradiated fuel and active waste, 1239 (J;GB)

Remote automatic plasma arc-closure welding of a dry-storage canister for spent nuclear fuel and high-level radioactive waste, 551 (R;US)

Economic Anglysis

Economics of monitored retrievable storage of nuclear wastes, 40 (J;US)

Environmental Impacts

Draft environmental impact statement for 40 CFR part 191: environmental standards for management and disposal of spent nuclear fuel, high-level and transuranic radioactive wastes. Final repurt, 838 (R;US)

Fïnancing

Report on financing the disposal of commercial spent nuclear

Mines fuel and processed high-level radioactive waste, 21 (R;US)

Optimization of mine layout for nuclear fuel assembly storage, $1123(\mathrm{~J} ; \mathrm{NL})$

Natural Cunvection

Natural draught centralized dry store for irradiated fuel and active waste, $1239(\mathrm{~J} ; \mathrm{GB})$

Planning

Overview of the Monitored Retrievable Storage (MRS) program, 859 (R;US)

Research Programs

Commercial nuclear-waste research and development program. Quarterly repnrt, July-September 1982, 578 (R:US)

Commercial nuclear waste research and development program. Annual report, fiscal year 1982. 579 (R;US)

Commercial Nuclear Waste Research and Development Program. Quarterly report, October-December 1982, 580 (R;US)

Commercial nuclear waste research and development program. Quarterly report, January-March 1983, 581 (R;US)

Commercial nuclear waste research and development program. Quarterly report, April-June 1983, 582 (R;US)

Retrieval Systems

Background and historical perspectives for monitored retrievable storage, 38 (J;US)

System evaluation model for selecting spent nuclear fuel storagc concepts, 24 (R;US)

Salt Deposits

Expected near-field thermal environments in a sequentially loaded spent-fuel or high-level waste repository in salt, 830 (R;US)
Near-field salt temperatures resulting from storage of nuclear

fuel assemblies in bedded salt, $1121(\mathrm{~J} ; \mathrm{NL})$

Optimization of mine layout for nuclear fuel assembly storage, $1123(\mathrm{~J} ; \mathrm{NL})$

Thermal criteria for terminal storage of spent nuclear fuel assemblies in bedded salt, $1119(\mathrm{~J} ; \mathrm{NL})$

Uncoupling thermal problems of terminal storage of spent nuclear fuel assemblies in bedded salt, $1120(\mathrm{~J} ; \mathrm{NL})$

Site Selection

Screening for monitored retrievable storage facility locations, 39 (J;US)

Spent Fuel Casks

Policy and development of reprocessing of spent nuclear fuel and high-level radioactive waste management in the F.R. Germany, 13 (J;JP;In Japanese)

Remote automatic plasma arc-closure welding of a dry-storage canister for spent nuclear fuel and high-level radioactive waste, SS1 (R;US)

Storage Facilities

Instrumentation report 1: specification, design, calibration, and installation of instrumentation for an experimental, high-level, nuclear waste storage facility, 37 (R;US)

MRS/IS facility co-located with a repository: preconceptual derign and lifo uyulu ceut ostimatcs, U'J/ (K,US)

Systems Analysis

System evaluation model for selecting spent nuclear fuel storage concepts, 24 (R;US)

Temperature Gradients

Rapid estimation of the regional thermal impact of a nuclear waste repository, $1260(\mathrm{~J} ; \mathrm{US})$

Temperature Monitoring

Near-field salt temperatures resulting from storage of nuclear fuel assemblies in bedded salt, $1121(\mathrm{~J} ; \mathrm{NL})$

Thermal Analysis

Uncoupling thermal problems of terminal storage of spent nuclear fuel assemblies in bedded salt, $1120(\mathrm{~J} ; \mathrm{NL})$

Underground Storage

Instrumentation report 1: specification, design, calibration, and installation of instrumentation for an experimental, high-level, nuclear waste storage facility, 37 (R;US)

Swedish nuclear waste efforts, 36 (R;US)

\section{SPENT FUELS}

After-heat

Thermal impact of waste emplacement and surface cooling associated with geologic disposal of nuclear waste, 707 (R;US)

Cooling

Thermal impast of waste emplacement and surface cooling associatod with gcologic dispusal of nuclear waste, 707 (K;US)

Forecasting

Spent fuel and radioactive-waste inventories, projections, and characteristics, 57 (R;US)

Spent fuel and radioactive waste inventories, projections, and characteristics, 574 (R;US)

Heal Transfer

Waste package heat-transfer analysis; model development and temperature estimates for waste packages in a repository located in basalt, 917 (R;US)

Inventories

Integrated data base for spent fuel and radwaste: inventories, 52 (R;US)

Nuclear-waste-management technical support in the development of nuclear-waste-form criteria for the NRC. Task 3. Waste inventory review, 73 (R;US)

NWTS program waste projection data needs, 1086 (J;US)

Radwaste inventories and projections: an overview (Integrated Data Base), 77 (R;US)

Spent fuel and radioactive-waste inventories, projections, and characteristics, 57 (R;US)

Spent fuel and radioactive wastc inventories, projections, and characteristics, 574 (R;US)

Land Transport

Southeastern Utah nuclear-waste-transportation study, 26 (R;US) 


\section{I eaching}

Elemental release from glass and spent fuel, 1307 (RA;US)

Leach rates of high level waste and spent fuel: limiting rates as determined by backfill and bedrock conditions, 1345 (BA;US)

Waste package materials testing for a salt repository: 1982 status report, 825 (R;US)

Packaging

Commercial nuclear waste research and development program. Quarterly report, January-March 1982,61 (R;US)

Conceptual design requirements for spent fuel, high-level waste, and transuranic waste packages, 903 (R;US)

Conceptual waste-package designs for disposal of nuclear waste in tuff, 818 (R;US)

Conceptual waste package interim performance specifications for waste forms for geologic isolation in salt repositories, 821 (R;US)

Construction and inservice inspection rules for nuclear spent fuel and high-level waste transport packagings, 18 (RA;US)

Design rules for containment systems of nuclear spent fuel and high-level waste transport packagings, 19 (RA;US)

Economic evaluation of nuclear waste transportation casks, 14 (R;US)

Monitored Retrievable Storage Demonstration Facility and dry well storage field conceptual design study, 912 (R;US)

Nuclear waste management technical support in the development of nuclear waste form criteria for the NRC. Task 1 . Waste package overview, 283 (R;US)

Waste package heat-transfer analysis: model development and temperature estimates for waste packages in a repository located in basalt, 917 (R;US)

Waste package conceptual designs for a repository located in basalt, 884 (R;US)

Projection Series

NWTS program waste projection data needs, 1086 (J;US)

Radioactivity

Graphical and tabular summaries of decay characteristics for once-through PWR, LMFBR, and FFTF fuel cycle materials (Spent fuel, high-level waste fuel can scrap), 76 (R;US)

Rail Transport

Preliminary analysis of the cost and risk of transporting nuclear waste to potential candidate commercial repository sites, 35 (R;US)

Southeastern Utah nuclear-waste-transportation study, 26 (R;US)

Reprocessing

Assessment of overseas developments in methods for treatment and disposal of high-level radioactive wastes, 532 (R;AU)

Chemistry of the nuclear fuel cycle, 4 (BA;GB)

International fuel-cycle and waste-management technologyexchange activities sponsored by the United States Department of Energy: FY 1982 evaluation report, 85 (R;ÜS)

Program summary. Nuclear waste management and fuel cycle programs, 575 (R;US)

Radioactive waste processing and disposal, 7 (RA;CS;In Czech)

Reprocessing of nuclear fuel, 6 (R;US)

Residual Power

Graphical and tabular summaries of decay characteristics for once-through PWR, LMFBR, and FFTF fuel cycle materials (Spent. fuel, high-level waste fuel çan scrap), 76 (R;US)

Road Transport

Preliminary analysis of the cost and risk of transporting nuclear waste to potential candidate commercial repository sites, 35 (R;US)

Toxicity

Graphical and tabular summaries of decay characteristics for once-through PWR, LMFBR, and FFTF fuel cycle materials (Spent fuel, high-level waste fuel can scrap), 76 (R;US)

Transport

Commercial nuclear waste research and development program. Quartorly report, January-March 1982, 61 (R:IJS)

Review of the status of transportation activities in various waste management systems, 30 (R;US)

Storage and transportation of spent fuel and high-level waste using dry storage casks. 15 (RA:US)
Underground Disposal

Comparison of risks due to HLW and SURF repositories in bedded salt, $1368(R ; U S)$

Waste Transportation

Economic evaluation of nuclear waste transportation casks, 14 (R;US)

SPHENE

Leaching

Leaching of natural and synthetic sphene and perovskite, 456 (BA;US)

SIMS depth profiling studies of sphene-based ceramics and glass ceramics leached in synthetic groundwater, 455 (BA;US)

\section{STAINLESS STEEL-304}

\section{Corrosion}

Consequences of radiation effects on zeolites during interim storage, 1230 (J;US)

High temperature corrosion of heat-resisting alloys by borosilicate melts containing simulated high level nuclear wastes, 476 (J;JP;In Japanese)

Long-term performance of materials used for high-level waste packaging. Annual report, March 1982-April 1983. Vol.1, 751 (R;US)

Hydrogen Embrittlement

Long-term performance of materials used for high-level waste packaging. Annual report, March 1982-April 1983. Vol.1, 751 (R;US)

\section{Radiation Effects}

Study of $\gamma$-ray irradiation effects on corrosion resistance of alloys for storage of high-level waste packages, (1), 667 (R;JP;In Japanese)

Stress Corrosion

Study of $\boldsymbol{\gamma}$-ray irradiation effects on corrosion resistance of alloys for storage of high-level waste packages, (1), 667 (R;JP;In Japanese)

\section{STAINLESS STEEL-304L}

Compatibility

Studies of waste-canister compatibility, 832 (R;US)

Corrosion

Evaluation of stainless steel tank corrosion in ICPP high-level radioactive waste service, 203 (R;US)

Long-term performance of materials used for high-level waste packaging. Annual report, March 1982-April 1983. Vol.1, 75I (R;US)

Long-term performance of materials used for high-level waste packaging. Quarterly report, April-June 1983, 752 (R;US)

Corrosion Resistance

Selection of barrier metals for a waste package in tuff, 1020 (R;US)

Crevice Corrosion

Review of DOE waste package program. Subtask 1.1. National waste package program, April-September 1982, 735 (R;US)

Electrochemical Corrosion

Review of DOE waste package program. Subtask 1.1. National waste package program, April-September 1982, 735 (R;US)

\section{Failures}

Review of DOE waste package program. Subtask 1.1. National waste package program, October 1982-March 1983. Vol. 4, 736 (R;US)

Mechanical Properties

Selection of barrier metals for a waste package in tuff, 1020 (R;US)

Pitting Corrosion

Review of DOE waste package program. Subtask 1.1. National waste package program, April-September 1982, 735 (R;US)

Radiation Effects

Study of $\gamma$-ray irradiation effects on corrosion resistance of alloys for storage of high-level waste packages, (1), 667 (R;JP;In Japanese)

Stress Corrosion

Review of DOE waste package program. Subtask 1.1. National waste package program, April-September 1982, 735 (R;US)

Study of $\gamma$-ray irradiation effects on corrosion resistance of alloys for storage of high-level waste packages, (1), 667 (R;JP;In Japanese) 
Weldability

Selection of barrier metals for a waste package in tuff, 1020 (R;US)

\section{STAINLESS STEEL-309S}

Radiation Effects

Study of $\gamma$-ray irradiation effects on corrosion resistance of alloys for storage of high-level waste packages, (1), 667 (R;JP;In Japanese)

Stress Corrosion

Study of $\boldsymbol{\gamma}$-ray irradiation effects on corrosion resistance of alloys for storage of high-level waste packages, (1), 667 (R;JP;In Japanese)

STAINLESS STEEL-316

Corrosion

Consequences of radiation effects on zeolites during interim storage, 1230 (J;US)

STAINLESS STEEL-316L

Compatibility

Cesium chloride compatibility testing program. Annual report, FY 1982, 855 (K;US)

Corrosion

Cesium chloride compatibility testing program. Annual report, FY 1982, 855 (R;US)

Corrosion Resistance

Selection of barrier mctals for a waste package in tuff, 1020 (R;US)

Mechanical Properties

Selection of barrier metals for a waste package in tuff, 1020 (R;US)

Wetdability

Selection of barrier metals for a waste package in tuff, 1020 (R;US)

STAINLESS STEEL 321

Corrosion Resistance

Selection of barrier metals for a waste package in tuff, 1020 (R;US)

Mechanical Properties

Selection of barrier metals for a waste package in tuff, 1020 (R;US)

Weldability

Selection of barrier metals for a waste package in tuff, 1020 (R;US)

STAINLESS STEEL-348

Corrosion

Evaluation of stainless steel tank corrosion in ICPP high-level radioactive waste service, 203 (R;US)

STAINLESS STEELS

STAINLESS STEEL-304

See also STAINLESS STEEL-304L

STAINLESS STEEL-309S

STAINLESS STEEL-316

STAINLESS STEEL-316L

STAINL.FSS STEEL-32I

STAINLESS STEEL-348

Corrosion

Corrosion of materials in a clay environment, 686 (RA;DE)

Corrosion Resistance

Waste package materials testing for a salt repository: 1982 status report, 825 (R;US)

STEELS

See also AUSTENITIC STEEIS

CARBON STEELS

CHROMIUM STEELS

FERRITIC STEELS

STAINLESS STEELS

Corrosion

Consequences of radiation effects on zeolites during interim storage (ASTM 1020), 1230 (J;US)

Corrosion studies and mechanical tests on metallic materials for the design of packagings for vitrified high level wastes (HLW), 689 (R;DE; In German)

Testing of candidate waste-package backfill and canister materials for basalt, 885 (R;US)
High-level Radioactive Wastes

Corrosion of materials in a clay environment, 686 (RA;DE)

Tensile Properties

Corrosion studies and mechanical tests on metallic materials tor the design of packagings for vitrified high level wastes (HLW), 689 (R;DE;In German)

STORAGE

See also SPENT FUEL STORAGE

Underground Storage

Marking a nuclear wastc repository: an archaelogist's perspective, 1095 (J;US)

\section{STORAGE FACILITIES}

Construction

Storage of HLW in engineered structures: air-cooled and watercooled concepts. Final report, 833 (R;US)

Swedish nuclear waste efforts, 36 (R;US)

Cooling Systems

Storage of HLW in engineered structures: air-cooled and watercorled cuicicols. Final repurt, 833 (R;US)

Cost

MRS/IS facility co-located with a repository: preconceptual design and life-cycle cost estimates, 857 (R;US)

Decommissioning

Decommissioning alternatives for Savannah River Plant high-

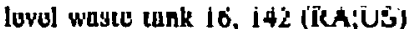

Design

Comparison of cask and drywell storage concepts for a monitored retrievable storage/interim storage system, 853 (R;US)

MRS/IS facillty co-located with a repository: preconceptual design and life-cycle cost estimates, 857 (R;US)

Equipment

Instrumentation report 1: specification, design, calibration, and installation of instrumentation for an experimental, high-level, nuclear waste storage facility, 37 (R;US)

Safety

Safety analysis of tank storage for highly radioactive reprocessing waste, 1404 (J;US)

STORAGE (SPENT' FUEL)

See SPENT FUEL STORAGE

STRAIN GAGES

Failures

Instrument reliability for high-level nuolear-wastc-rcpository applications, 1013 (R;IS)

Performance

Instrument reliability for high-level nuclear-waste-repository applications, 1013 (R;US)

Reliability

Instrument reliability for high-level nuclear-waste-repository applications, 1013 (R;US)

STRONTIUM

Adsorption

Diffusion in crystalline rocks, 1168 (BA;ÜS)

Effects of Hanford high-level waste components on sorption of cobalt, strontium, neptunium, plutonium, and americium on Hanford sediments, 918 (R;US)

Geochemistry studles pertaining to the $\mathrm{G}$-tunnel radionuclide migration field experiment, 1426 (R;US)

Importance of organic compounds in ground water as radionuclide-mobilizing agents, 1324 (R;US)

Precipitation process for supernate decontamination, 173 (R;US)

Precipitation process for decontamination of water-soluble SRP radioactive wastc, 184 (R;US)

Precipitation process for decontaminating water-soluble SRP radioactive waste, 187 (R;US)

Status of radionuclide sorption-desorption studies performed by the WRIT program, 1308 (RA;US)

Transport of actinides through a bentonite backfill, 1175 (BA;US)

Adsorption Isotherms

Basalt-radionuclide reactions: FY-1981. Annual report, 868 (R;US) 


\section{Desorption}

Research and development related to the Nevada Nuclear Waste Storage Investigations. Progress report, July 1-September 30, 1981, 692 (R;US)

Status of radionuclide sorption-desorption studies performed by the WRIT program, 1308 (RA;US)

\section{Diffusion}

Diffusion in crystalline rocks, 1168 (BA;US)

Transport of actinides through a bentonite backfill, 1175 (BA;US)

Distribution Functions

Status of radionuclide sorption-desorption studies performed by the WRIT program, 1308 (RA;US)

Emission Spectroscopy

Analyses of nuclear fuel and high-level waste by inductively coupled plasma-emission spectroscopy, 68 (R;US)

Leaching

Comparison of properties of borosilicate glass and crystalline ceramic forms for immobilization of Savannah River Plant waste, 171 (R;US)

Development and testing of SYNROC $\mathrm{C}$ as a high level nuclear waste form, 418 (BA;US)

FUETAP concrete: an alternative -radioactive waste host, 256 (RA;DE)

Hydroxylated ceramic waste forms and the absurdity of leach tests, 254 (RA;DE)

Leaching studies on SYNROC at $95^{\circ} \mathrm{C}$ and $200^{\circ} \mathrm{C}, 1114(\mathrm{~J} ; \mathrm{CH})$

Processing effects on the behavior of titanate waste forms in aqueous solutions, 1145 (BA;US)

Processing, microstructure, leaching, and long-term stability studies related to titanale high-level waste forms, 340 (R;US)

Savannah River Laboratory long-term waste storage program. Progress report No. 5, March-April 1974, 131 (R;US)

Savannah River Laboratory long-term waste storage program. Progress report No. 6, May-Jun 1974, 132 (R;US)

\section{Precipitation}

Precipitation-adsorption process for the decontamination of nuclear waste supernates (Patent), 397 (P;US)

Separation Processes

Cesium and strontium fractionation from HLW for thermal-stress reduction in a geologic repository, 327 (R;US)

Systems study of the feasibility of high-level nuclear-waste fractionation for thermal-stress control in a geologic repository: main report, 308 (R;US)

Systems study of the feasibility of high-level nuclear waste fractionation for thermal stress control in a geologic repository: appendices, 309 (R;US)

Waste-Mixes Study for space disposal, 815 (R;US)

Sorption

Evaluation of sodium bentonite and crushed basalt as waste package backfill materials, 902 (R;US)

Radionuclide sorption on basalt-interbed materials. Annual report FY 1981, 1328 (R;US)

Recent advances in repository seal materials, 1172 (BA;US)

Research and development related to the Nevada Nuclear Waste Storage Investigations. Progress report, July 1-September 30, 1981,692 (R;US)

Transmutation

Radiatiưr ànd transmutation effects on crystalline radioactive waste forms, 1262 (J;US)

Volatility

Iron-enriched basalt for containment of nuclear wastes, 416 (BA;US)

Waste-rock Interactions

Strontium-basalt reactions under nuclear waste repository conditions, 1152 (BA;US)

\section{STRUNIIUM 85}

Adsorption

Laboratory tests on the migration behavior of selected fission products in aquifer materials from a potential disposal site in northern Germany, 1352 (BA;US)
Diffusion

Comparison of in-situ radionuclide migration studies in the Studsvik area and laboratory measurements, 1349 (BA;US)

Diffusion measurements in compacted bentonite, 1148 (BA;US)

Laboratory tests on the migration behavior of selected fission products in aquifer materials from a potential disposal site in northern Germany, 1352 (BA;US)

Distribution Functions

Diffusive transport of strontium-85 in sand-bentonite mixtures, 1300 (R;CA)

Leaching

Measurement of leaching from simulated nuclear-waste glass using radiotracers, 128 (R;US)

Sorption

Comparison of in-situ radionuclide migration studies in the Studsvik area and laboratory measurements, 1349 (BA;US)

Evaluation of radionuclide transport: effect of radionuclide sorption and solubility, 1354 (BA;US)

Radionuclide retardation during transport through fractured granite, 1355 (BA;US)

\section{STRONTTUM 90}

Adsorption

Immobilization of reprocessing waste by means of titanate ion exchangers, 248 (RA;DE)

Diffusion

Elution of radionuclides through columns of crushed rock from the Nevada Test Site, 1315 (R;US)

Environmental Exposure Pathway

Investigation of the consequences of leakage incidents of a tank with high-concentrated radionuclide solutions and pentration of the radioactive solution into the soil, 1423 (R;DE;In German)

Environmental Transport

Investigation of the consequences of leakage incidents of a tank with high-concentrated radionuclide solutions and pentration of the radioactive solution into the soil, 1423 (R;DE;In German)

\section{Filtration}

Removal of ${ }^{90} \mathrm{Sr},{ }^{107} \mathrm{Cs}$, and ${ }^{106} \mathrm{Ru}$ from vent gases produced during solidification of high-level radioactive solutions, 399 (J;CS)

Ion Exchange

Development of partitioning method - cold and semihot experimental works with partitioning testing apparatus, 225 (R;JP;In Japanese)

Leaching

High-level waste glass field burial tests at CRNL: the effect of geochemical kinetics on the release and migration of fission products in a sandy aquifer, 1299 (R,CA)

Waste package performance evaluation, 806 (R;US)

Purification

Recovery and purification of multi-kilocurie quantities of fission product strontium by cation exchange, 214 (R;US)

Radioactive Waste Processing

Recovery and purification of multi-kilocurie quantities of fission product strontium by cation exchange, 214 (R;US)

Recovery

Recovery and purification of multi-kilocurie quantities of fission product strontium by cation exchange, 214 (R;US)

Separation Processes

Alternate strategy for commercial high-level radioactive-waste management, 945 (R;US)

Sorption

Distribution coefficient (Kd) concept and its applicability to studies of radionuclide migration in geological media, 1313 (R;GB)

\section{SIIIT.FIJR}

Corrosive Effects

Container assessment: corrosion study of HLW container materials. Quarterly progress report, July-September 1981, 728 (R;IIS) 


\section{SULFUR SULFIDES \\ SEe SULFUR \\ SUPERALLOYS \\ See HEAT RESISTING ALLOYS \\ SURFACE WATERS \\ Isotope Ratio}

Permeability monitoring technique of the near-field and far-field interface openings, 1346 (BA;US)

Radionuclide Migration

Health effects associtated with unit radonuclide releases to the environment, 1378 (RA;US)

SURVEILLANCE (RADIOACTIVITY)

SEe RADIATION MONITORING

SURVEY (RADIOACTIVITY)

SEe RADIATION MONITORING

SWEDEN

Fuel Cycle

Summary of non-US national and international fuel cycle and radioactive waste management programs 1982,83 (R;US)

\section{Radioactive Waste Disposal}

Nuclear power on unstable ground, 1182 (BA;GB)

Predictive geology in nuclear-waste management, 1181 (BA;GB)

Stripa project, quarterly report October through December 1981, 655 (R;SE)

Radioactive Waste Managcment

Summary of non-US national and international fuel cycle and radioactive waste management programs 1982,83 (R;US)

Swedish nuclear waste efforts, 36 (R;US)

Radioactive Waste Processing

Solidification of radioactive waste forms and waste containment by the H I P O W process, 232 (RA;DE)

\section{SWITZERLAND}

Fuel Cycle

Summary of non-US national and international fuel cycle and radioactive waste management programs 1982, 83 (R;US)

Radioactive Waste Disposal

Data base for a site-specific analysis of the dispersion of radioactive elements from a final repository, 1366 (TG;US)

Risk analysis for repositories in north Switzerland. Extent and probability of geologic processes and events, $1372(\mathrm{R} ; \mathrm{CH} ; \mathrm{GE})$

Radioactive Waste Management

Summary of non-US national and international fuel cycle and

SYNROC radioactive waste management programs 1982,83 (R;US)

\section{SEQ SYNTHETIC ROCKS}

SYNROC PROCESS

Alternate waste form evaluation for Savannah River Plant highlevel waste, 1104 (J;US)

Comparison of properties of borosilicate glass and crystalline ceramic forms for immobilization of Savannah River Plant waste, 171 (R;US)

Containing waste material (Patent), 402 (P;AU)

Development and testing of SYNROC for high level radioactive waste fixation, 310 (J;US)

HLW fixation in sintered modified SYNROC-B ceramics: chemical stability evaluation, 526 (J;US)

Incorporation of high-level wastes in SYNROC: results from recent process-engineering studies at Lawrence Livermore National Laboratory, 352 (R;US)

Leaching behavior of sintered modified SYNROC-B waste forms: effect of HLW addition, 445 (BA;US)

Properties of SYNROC C nuclear-waste form: a state-of-the-art review, 1011 (R;US)

Sodium titanate in solidification of radioactive wastes, 247 (KA;DE)

SYNROC: leaching performance and process technology, 250 (RA;DE)

SYNROC production using a fluid bed calciner, 353 (R;US)

Synroc technology for immobilizing U.S. defense waste, 400 (J;US)

SYNROC through melting: thermal analysis, thermogravimetry and crystal chemical characterization of phases (Federal Republic of Germany), 260 (RA;DE)

Treatment of high level nuclear waste, 388 (P;AU)
Additives

Formulation of SYNROC-D additives for Savannah River Plan high-level radioactive waste, 347 (R;US)

Comparative Evaluations

Description of a ceramic waste form and canister for Savannah River Plant high-level waste, 170 (R;US)

Evaluation

Immobilization of sodium and phosphorus-bearing PW-7a waste in SYNROC. Progress report (SYNROC C), 346 (R;US)

High-level Radioactive Wastes

Containing waste material (Patent), 1075 (P;AU)

Demonstration of SYNROC fabrication scaleup on a nonradioactive basis, 444 (BA;US)

Effects of gamma irradiation on the leaching behavior of a synthetic mineral waste form (SYNROC-B), 1143 (J;US)

Improved polyphase ceramic for high-level defense waste, $\mathbf{4 4 6}$ (BA;US)

New SYNROC plant offers $\mathrm{N}$-waste disposal solution, 374 (J;AU)

Sintering of SYNROC: case history for phase formation and densification of complex oxide systems, 473 (BA;NI.,)

SYNROC for containment of high-level nuclear waste, 396 (J;US)

Synroc programme progresses, 405 (J;GB)

Synroc solution (Immnhilisation of high-level radiusctive wastes), $356(J ; Z A)$

Modifications

Subsolidus sintering of SYNROC: I. Initial demonstrations, 280 (R;US)

Subsolidus sintering of SYNROC: II. Materials selections, process improvements, waste form evaluations, 281 (R;US)

Research Programs

Development and testing of SYNROC C as a high level nuclear waste form, 415 (J;AU)

Immobilization of high-level defense wastes in SYNROC-D: recent research and development results on process scale-up, 351 (R;US)

Technology Assessment

Development and testing of SYNROC $\mathrm{C}$ as a high level nuclear waste form (Australian Atomic Energy Commission), 418 (BA;US)

SYNTHETIC ROCKS

Development and testing of SYNROC $\mathrm{C}$ as a high level nuclear waste form, 415 (J;AU)

Auger Electron Spectroscopy

Surface analysis features of Synroc B̈ and C, $1039(\mathrm{~J} ; \mathrm{CH})$

Chemical Composition

Immobilization of sodium and phosphorus-bearing PW-7a waste in SYNROC. Progress report (SYNROC C), 346 (R;US)

Influence of gamma irradiation on the leaching behavior of a modified SYNROC-B ceramic waste form, 424 (BA;US)

Properties of SYNROC C nuclear-waste form: a state-of-the-art review, 1011 (R;US)

Sintering of Synrors D, 343 (R;US)

Subsolidus sintering of SYNROC: I. Initial demonstrations, 280 (R;US)

Subsolidus sintering of SYNROC: II. Matcrials selections, process improvements, waste form evaluations, 281 (R;US)

Chemical Radiation Efftels

Leachability study of modified Synroc-B waste form: effect of gamma irradiation, 412 (J;GB)

Radiation damage studies related to nuclear waste forms, 298 (R;US)

Comparative Evaluations

Comparison of borosilicate glacs and synthetic mincrals as media for the immobilization of high-level radioactive waste, 490 (J;US)

Development, evaluation, and selection of candidate high-level waste forms, $185(R ; U S)$

Disposal of high-level radioactive wastes, 1257 (BA;AU)

Evaluation and selection of candidate high-level waste forms, 14 (R;US)

Evaluation of conditioned high-level waste forms, 326 (R;US)

Evaluation of forms for the immobilization of high-level and transuranić wastes, 200 (R;US) 
Postclosure risks of alternative SRP nuclear waste forms in geologic repositories, 1009 (R;US)

-orrosion Resistance

Electrical-conductivity measurements of leachates for the rapid Density assessment of wasteform corrosion resistance, 554 (R;US)

Densification studies of Synroc D for high-level defense waste, 348 (R;US)

Subsolidus sintering of SYNROC: II. Materials selections, process improvements, waste form evaluations, 281 (R;US)

Fabrication

Apparatus and method for immobilizing waste material (Patent), 484 (P;AU)

Demonstration of SYNROC fabrication scaleup on a nonradioactive basis, 444 (BA;US)

Fabication and properties of SYNROC, 472 (BA;AU)

Properties of SYNROC C nuclear-waste form: a state-of-the-art review, 1011 (R;US)

Sintering of Synroc D, 343 (R;US)

Fractures

Final report of experimental laboratory-scale brittle fracture studies of glasses and ceramics, 129 (R;US)

Fragmentation

Respirable fines produced by impacts of simulated alternative high-level waste materials, 419 (BA;US)

Hot Pressing

Densification studies of Synroc D for high-level defense waste, 348 (R;US)

Impact Tests

Final report of experimental laboratory-scale brittle fracture studies of glasses and ceramics, 129 (R;US)

Respirable fines produced by impacts of simulated alternative high-level waste materials, 419 (BA;US)

Leachates

Electrical-conductivity measurements of leachates for the rapid assessment of wasteform corrosion resistance, 554 (R;US)

Leaching

Comparative leach testing of high-level waste forms, 626 (R;US)

Cumparison of properties of borosilicatc glass and crystalline ceramic forms for immobilization of Savannah River Plant waste, 171 (R;US)

Development and testing of SYNROC $\mathrm{C}$ as a high level nuclear waste form, 418 (BA;US)

Effect of temperature on the leaching performance of SYNROC-B waste form, 1229 (J;US)

Effects of gamma irradiation on the leaching behavior of a synthetic mineral waste form (SYNROC-B), 1143 (J;US)

Effects of high-level gamma irradiation on radioactivity release from waste disposal forms, 1097 (J;US)

Flow model for the kinetics of dissolution of nuclear waste forms; a comparison of borosilicate glass, SYNROC and high-silica glass, 1192 (BA;US)

HLW fixation in sintered modified SYNROC-B ceramics: chemical stability evaluation, 526 (J;US)

Immobilization of high-level nuclear reactor wastes in SYNROC: a current appraisal, S15 (J;US)

Improved polyphase ceramic form for high-level defense nuclear waste, 152 (RA;US)

Improved polyphase ceramic for high-level defense waste, 446 (BA;US)

Influence of gamma irradiation on the leaching behavior of a modified SYNROR.-B r.eramic waste form. 424 (BA:US)

Leachability study of modified Synroc-B waste form: effect of gamma irradiation, $412(\mathrm{~J} ; \mathrm{GB})$

Leaching behavior of sintered modified SYNROC-B waste forms: effect of HLW addition, 445 (BA;US)

Leaching studies on SYNROC at $95^{\circ} \mathrm{C}$ and $200^{\circ} \mathrm{C}, 1114(\mathrm{~J} ; \mathrm{CH})$

Model for predicting leaching behavior of high-level radioactive waste forms, 508 (J;US)

Nuclear waste-form risk assessment for US Defense waste at Savannah River Plant. Annual report FY 1981, 1007 (R;US)

Plutonium doping of SYNROC-D, 350 (R;US)

Subsolidus sintering of SYNROC: I. Initial demonstrations, 280 (R;US)
Subsolidus sintering of SYNROC: II. Materials selections, process improvements, waste form evaluations, 281 (R;US)

SYNROC: leaching performance and process technology, 250 (RA;DE)

Synroc technology for immobilizing U.S. defense waste, 400 (J;US)

\section{Licensing}

Review of DOE waste package program. Subtask 1.1. National waste package program, 286 (R;US)

\section{Microstructure}

Microstructure of Synroc (Synroc B and C), 454 (BA;US)

Subsolidus sintering of SYNROC: II. Materials selections, process improvements, waste form evaluations, 281 (R;US)

Optimization

Thermal analysis, thermogravimetry and crystal chemical charac terization of phases, 273 (RA;DE)

Phase Studies

Subsolidus sintering of SYNROC: I. Initial demonstrations, 280 (R;US)

SYNROC through melting: thermal analysis, thermogravimetry and crystal chemical characterization of phases (Federal Republic of Germany), 260 (RA;DE)

Photoelectron Spectroscopy

Surface analysis features of Synroc B and C, $1039(\mathrm{~J} ; \mathrm{CH})$

Physical Properties

Development and testing of SYNROC for high-level radioactive waste fixation, 493 (J;AU)

Properties of SYNROC C nuclear-waste form: a state-of-the-art review, 1011 (R;US)

Physical Radiation Effects

Development and testing of SYNROC for high level radioactive waste fixation, 510 (J;US)

Development and testing of SYNROC for high-level radioactive waste fixation, 493 (J;AU)

Development and testing of SYNROC $\mathrm{C}$ as a high level nuclear waste form, 418 (BA;US)

Effects of high-level gamma irradiation on radioactivity release from waste disposal forms, 1097 (J;US)

Influence of gamma irradiation on the leaching behavior of a modified SYNROC-B ceramic waste form, 424 (BA;US)

Introduction of studies on high-ievel radioactive waste immobilization in synthetic minerals. Supercalcine and SYNROC, 224 (R;JP;In Japanese)

Irradiation effects in SYNROC, 1447 (BA;AU)

Plutonium doping of SYNROC-D, 350 (R;US)

Radiation damage from alpha decay in ceramic nuclear waste forms, 103 (J;CH)

Subsolidus sintering of SYNROC: II. Materials selections, process improvements, waste form evaluations, 281 (R;US)

Production

Fluid-bed process for SYNROC production, 349 (R;US)

SYNROC powder preparation: preliminary fluid bed tests in a cold-flow unit, 345 (R;US)

Radiation Effects

Comparison of properties of borosilicate glass and crystalline ceramic forms for immobilization of Savannah River Plant waste, 171 (R;US)

Effects of gamma irradiation on the leaching behavior of a synthetic mineral waste form (SYNROC-B), 1143 (J;US)

Properties of SYNROC C nuclear-waste form: a state-of-the-art review, 1011 (R;US)

Radiation effects in SYNROC-D, 1053 (J;US)

Review of the current status of radiation effects in solid nuclear waste forms, 372 (J;US)

Scientific basis for nuclear waste management, 107 (B;US)

Research Programs

Development and testing of SYNROC for high level radioactive waste fixation, $\$ 10$ (J;US)

Sintering

Sintering of Synroc D, 343 (R;US)

Sintering of SYNROC: case history for phase formation and densification of complex oxide systems, 473 (BA;NL) 


\section{Specific Heat}

Thermophysical properties of SYNROC and its constituent mineral phases, 474 (BA;AU)

\section{Stability}

Introduction of studies on high-level radioactive waste immobilization in synthetic minerals. Supercalcine and SYNROC, 224 (R;JP;In Japanese)

Structural Chemical Analysis

Structural chemistry of Synroc, 221 (RA;AU)

Temperature Effects

Comparison of properties of borosilicate glass and crystalline ceramic forms for immobilization of Savannah River Plant waste, 171 (R;US)

Thermal Conductivity

Development and testing of SYNROC $\mathrm{C}$ as a high level nuclear waste form, 418 (BA;US)

Thermophysical properties of SYNROC and its constituent mineral phases, 474 (BA;AU)

Thermal Diffusivity

Thermophysical properties of SYNROC and its constituent mineral phases, 474 (BA;AU)

\section{T}

TAIWAN

Fuel Cycle

Summary of non-US national and international fuel cycle and radioactive waste management programs 1982, 83 (R;US)

Radioactive Waste Management

Summary of non-US national and international fuel cycle and radioactive waste management programs 1982, 83 (R;US)

TANK FARMS

See STORAGE FACILITIES

TANKS

\section{Corrosion}

Long-Term High-Level Defense-Waste technology. Progress report, April-June 1982, 338 (R;US)

Corrosion Protection

Prevention of stress corrosion cracking in nuclear waste storage tanks, 630 (R;US)

Sampling and analysis of high level waste tank supernatant: an overview (Savannah River Plant), 634 (R;US)

Inspection

Savannah River Plant waste-tank inspection manual, 633 (R;US)

Leak Testing

Savannah River Plant waste-tank inspection manual, 633 (R;US)

Materials Testing

Evaluation of stainless steel tank corrosion in ICPP high-level radioactive waste service, 203 (R;US)

Performance Testing

Status' of tank-assessment studies for continued in-tank storage of Hanford Defense waste. 919 (R;US)

Stress Corrosion

Prevention of stress corrosion cracking in nuclear waste storage tanks, 630 (R;US)

TANTALUM OXIDES

Crystal Structure

Investigation of titanium in metamict Nb-Ta-Ti oxides using the extended $x$-ray absorption fine structure technique, 1215 (BA;US)

Radiation Efrects

Investigation of titanium in metamict $\mathrm{Nb}-\mathrm{Ta}-\mathrm{Ti}$ oxides using the extended $x$-ray absorption fine structure technique, 1215 (BA;US)

TAR SAND OIL

SEe BITUMENS

TECHNETIUM

Adsorption

Evaluation of DOE radionuclide solubility data and selected retardation parameters: description of calculational and confirmatory experimental activities, 1304 (R;US)

Geochemistry studies pertaining to the G-tunnel radionuclide migration field experiment, 1426 (R;US)
Adsorption Isotherms

Basalt-radionuclide reactions: FY-1981. Annual report, 868 (R;US)

Centrifugation

Influence of the reprocessing flow sheet on the HLW solidification technology, 239 (RA;DE)

Chemical State

Studies on speciation of americium, technetium and neptunium in simulated vitrified-waste leachates, 1335 (J;US)

Coprecipitation

Coprecipitation of radioactive technetium with ferrous hydroxide, 1443 (J;JP;In Japanese)

Diffusion

Studies on speciation of americium, technetium and neptunium in simulated vitrified-waste leachates, 1335 (J;US)

Distribution

Studies on speciation of americium, technetium and neptunium in simulated vitrified-waste leachates, 1335 (J;US)

Geochemistry

Oklo reactors: natural analogs to nuclear waste repositories, 611 (RA;US)

Separation Processes

Waste-Mixes Study for space disposal, 815 (R;US)

Solubility

Evaluation of DOE radionuclide solubility data and selected retardation parameters: description of calculational and confirmatory experimental activities, 1304 (R;US)

Solvent Extraction

Chemistry of the nuclear fuel cycle, 4 (BA;GB)

Sorption

Radionuelido sorption on bazalt-interbed materials. Annual report FY 1981, 1328 (R;US)

\section{TECHNETIUM 95}

Adsorption

Laboratory tests on the migration behavior of selected fission products in aquifer materials from a potential disposal site in northern Germany, 1352 (BA;UȘ)

Diffusion

Elution of radionuclides through columns of crushed rock from the Nevada Test Site, 1315 (R;US)

Laboratory tests on the migration behavior of selected fission products in aquifet materials from a potential disposal site in northern Germany, 1352 (BA;US)

TECHNETIUM 99

Adsorption

Laboratory tests on the migration behavior of selected fission products in aquifer materials from a potential disposal site in northern Germany, 1352 (BA;US)

Diffusion

Diffusion measurements in compacted bentonite, 1148 (BA;US)

Laboratory tests on the migratinn hehaving of selected fission products in aquifer materials from a potential disposal site in northern Germany, 1352 (BA;US)

Environmiental Transport

Compliance assessments of hypothetical geological nuclear waste isolation systems with the draft EYA standard, 982 (R;US)

Leaching

Underground migration of long-lived radionuclides leached from a borosilicate glass matrix, $1360(\mathrm{BA} ; \mathrm{XA})$

Waste package performance evaluation, 806 (R;US)

Solubility

Evaluation of radionuclide transport: effect of radionuclide sorption and solubility, 1354 (BA;US)

\section{TEKTITES}

Leaching

Fuel cycle programs. Quarterly progress report, OctoberDecember 1982, 539 (R;US)

\section{TELLURIUM}

Activation Analysis

Application of neutron-activation analysis to the determination of leach rates of simulated nuclear-waste forms, 127 (R;US) - 


\section{TENNESSEE \\ Geology \\ Regional geological assessment of the Devonian-Mississippian shale sequence of the Appalachian, Illinois, and Michigan basins relative to potential storage/disposal of radioactive wastes, 828 (R;US) \\ TEST FACILITIES \\ Design \\ Exploratory Shaft Test Facility in basalt. Project B-314 pre- conceptual design report, 913 (R;US)}

TESTING (MATERIALS)

EXAS

See MATERIALS TESTING

Aerial Surveying

Statistical analysis of lineaments and their relation to fracturing, faulting, and halokinesis in the East Texas Basin. Report of investigations No. 110, 566 (R;US)

Air Quality

Area environmental characterization report of the Dalhart and Palo Duro basins in the Texas Panhandle. Volume I. Dalhart Basin, 782 (R;US)

Area environmental characterization report of the Dalhart and Palo Duro basins in the Texas Panhandle. Volume II. Palo Duro basin, 783 (R;US)

Aquatic Ecosystems

Area environmental characterization report of the Dalhart and Palo Duro basins in the Texas Panhandle. Volume I. Dalhart Basin, 782 (R;US)

Area environmental characterization report of the Dalhart and Palo Duro basins in the Texas Panhandle. Volume II. Palo Duro basin, 783 (R;US)

Background Radiation

Area environmental characterization report of the Dalhart and Palo Duro basins in the Texas Panhandle. Volume I. Dalhart Basin, 782 (R;US)

Area environmental characterization report of the Dalhart and Palo Duro basins in the Texas Panhandle. Volume II. Palo Duro basin, 783 (R;US)

Demography

Area environmental characterization report of the Dalhart and Palo Duro basins in the Texas Panhandle. Volume I. Dalhart Basin, 782 (R;US)

Area environmental characterization report of the Dalhart and Palo Duro basins in the Texas Panhandle. Volume II. Palo Duro basin, 783 (R;US)

Geologic Structures

Statistical analysis of lineaments and their relation to fracturing, faulting, and halokinesis in the East Texas Basin. Report of investigations No. 110, 566 (R;US)

Geophysical Surveys

Statistical analysis of lineaments and their relation to fracturing, faulting, and halokinesis in the East Texas Basin. Report of investigations No. 110, 566 (R;US)

Land use

Area environmental characterization report of the Dalhart and Palo Duro basins in the Texas Panhandle. Volume I. Dalhart Basin, 782 (R;US)

Area environmental characterization report of the Dalhart and Palo Duro basins in the Texas Panhandle. Volume II. Palo Duro basin, 783 (R;US)

Meteorology

Area environmental characterization report of the Dalhart and Palo Duro basins in the Texas Panhandle. Volume l. Dalhart Basin, 782 (R;US)

Area environmental characterization report of the Dalhart and Palo Duro basins in the Texas Panhandle. Volume II. Palo Duro basin, 783 (R;US) 、

Nuclear Energy

Nuclear energy in Texas: major issues and pulicy recommenda tions, 90 (R;US)

Nuclear Industry

Nuclear energy in Texas: major issues and policy recommendalivis, 90 (R;US)
Radioactive Waste Disposal

Environmental characterization report for the Gulf Interior Region, Texas study area (Oakwood, Palestine and Keechi salt domes), 1323 (R;US)

High-level radioactive-waste-disposal investigations in Texas, 1001 (R;US)

Report of the Low-Level Nuclear Waste Subcommittee, 1000 (R;US)

Salt Deposits

Environmental characterization of bedded salt formations and overlying areas of the Permian Basin, 1320 (R;US)

Permeability of selected sediments in the vicinity of five salt domes in the Gulf Interior Region, 810 (R;US)

Statistical analysis of lineaments and their relation to fracturing, faulting, and halokinesis in the East Texas Basin. Report of investigations No. 110,566 (R;US)

Site Selection

Area environmental characterization report of the Dalhart and Palo Duro basins in the Texas Panhandle. Volume II. Palo Duro basin, 783 (R;US)

Socio-economic Factors

Area environmental characterization report of the Dalhart and Palo Duro basins in the Texas Panhandle. Volume I. Dalhart Basin, 782 (R;US)

Area environmental characterization report of the Dalhart and Palo Duro basins in the Texas Panhandle. Volume II. Palo Duro basin, 783 (R;US)

Terrestrial Ecosystems

Area environmental characterization report of the Dalhart and Palo Duro basins in the Texas Panhandle. Volume I. Dalhart Basin, 782 (R;US)

Area environmental characterization report of the Dalhart and Palo Duro basins in the Texas Panhandle. Volume II. Palo Duro basin, 783 (R;US)

THERMOCOUPLES

Failures

Instrument reliability for high-level nuclear-waste-repository applications, 1013 (R;US)

Performance

Instrument reliability for high-level nuclear-waste-repository applications, 1013 (R;US)

Reliability

Instrument reliability for high-level nuclear-waste-repository applications, 1013 (R;US)

THORIUM

Adsorption

Sorption of actinides in well-defined oxidation states on geologic media, 1351 (BA;US)

Transport of actinides through a bentonite backfill, 1175 (BA;US)

Chemical State

Evaluation of solubility and speciation of actinides in natural groundwaters, 1347 (BA;US)

Diffusion

Transport of actinides through a bentonite backfill, 1175 (BA;US)

Distribution Functions

Sorption of actinides in well-defined oxidation states on geologic media, 1351 (BA;US)

Emission Spectroscopy

Analyses of nuclear fuel and high-level waste by inductively coupled plasma-emission spectroscopy, 68 (R;US)

Environmental Transport

Actinide sorption on rock minerals, 677 (RA;DE)

Leaching

Evaluation of solubility and speciation of actinides in natural groundwaters, 1347 (BA;US)

Radiation Scattering Analysis

Utilization of charged particle backscattering to study the near surface region of glasses. Application to depth profiling of lanthanium, cerium, throium and uranium induced by aqueous leaching. 1216 (BA;US) 
Solubility

Evaluation of solubility and speciation of actinides in natural groundwaters, 1347 (BA;US)

Geochemical controls on radionuclide releases from a nuclear waste repository in basalt: estimated solubilities for selected elements, 1329 (R;US)

THORIUM 228

Dissolution

Isotopic fractionation of thorium and uranium upon leaching of monozite: alpha-rccoil damage effects, 1214 (BA;US)

THORIUM 230

Dissolution

Isotopic fractionation of thorium and uranium upon leaching of monozite: alpha-recoil damage effects, 1214 (BA;US)

Leaching

Leach rates of high level waste and spent fuel: limiting rates as determined by backfill and bedrock conditions, 1345 (BA;US)

Solubility

Leach rates of high level waste and spent fuel: limiting rates as determined by backfill and bedrock conditions, 1345 (BA;US)

\section{THORIUM 232}

Dissolution

Isotopic fractionation of thorium and uranium upon leaching of monozite: alpha-recoil damage effects, 1214 (BA;US)

\section{THORIUM COMPLEXES}

Solubility

Importance of organic compounds in ground water as radionuclide-mobilizing agents, 1324 (R;US)

\section{THORIUM CYCLE}

Economics

Use of thorium as an alternative nuclear fuel, 46 (R;AU)

Safety

Use of thorium as an alternative nuclear fuel, $46(\mathrm{R} ; \mathrm{AU})$

THREE MILE ISLAND-2 REACTOR

Decontamination

Development and demonstration of a process for vitrification of TMI zeolite, 505 (J;US)

Regulatory perspective on the cleanup of TMI-2, 1445 (J;US)

Radioactive Waste Disposal

High-integrity container for high-specific-activity wastes, 1446 (J;US)

THULUM

Activation Analysis

Application of neutron-activation analysis to the determination of leach rates of simulated nuclear-waste forms, $127(\mathrm{R} ; \mathrm{US})$

\section{TIGHT SANDS}

TIN

\section{See SANDSTONES}

\section{Adsorption}

Geochemistry studies pertaining to the G-tunnel radionuclide migration field experiment, 1426 (R;US)

Emission Spectroscopy

Analyses of nuclear fuel and high-level waste by inductively coupled plasma-emission spectroscopy, 68 (R;US)

Solubility

Geochemical controls on radionuclide releases from a nuclear waste repository in basalt: estimated solubilities for selected elements, 1329 (R;US)

\section{TITANATES}

Leaching

Processing effects on the behavior of titanate waste forms in aqueous solutions, 1145 (BA:US)

Synthesls

Sodium titanate in solidification of radioactive wastes, 247 (RA;DE)

\section{TITANIUMM}

Compatibility

Studies of waste-canister compatibility, 832 (R;US)

\section{Corrosion}

Container assessment: corrosion study of HLW container materials. Quarterly progress report, July-September 1981, 728 (R;US)

Corrosion experience with a slurry-fed ceramic melter, 321 (R;US)
Long-term performance of materials used for high-level waste packaging. Quarterly report, April-June 1983, 752 (R;US)

Corrosion Products

Analysis of the corrosion products formed on $\mathrm{Ti}$ and a Ti-Pd alloy during exposure in hot water, 1176 (BA;US)

Corrosion Resistance

Corr sion of materials in a clay environment, $676(R A ; D E)$

Crevice Corrosion

Container assessment - corrosion study of HLW container materials. Quarterly progress report, April-June. 1981, 729 (R;US)

Leaching

Leaching studies on SYNROC at $95^{\circ} \mathrm{C}$ and $200^{\circ} \mathrm{C}, 1114(\mathrm{~J} ; \mathrm{CH})$

Processing effects on the behavior of titanate waste forms in aqueous solutions, 1145 (BA;US)

Processing, microstructure, leaching, and long-term stability studies related to titanate high-level waste forms, 340 (R;US)

SYNROC: leaching performance and process technology, 250 (RA;DE)

Redox Reactions

Investigation on the oxidation state and the behavior of molybdenum in silicate glass, 460 (BA;US)

TITANIUM ALLOYS

See also INCOLOY 825 TITANIUM B.ASE ALLOYS

Compatibilitj

Studies of waste-canister compatibility, 832 (R;US)

Corrosion

Corrosion of materials in a clay environment, 686 (RA;DE)

Enviromechanical testing of Ti-grade 2 and Ti-grade 12 in basalt ground water, 858 (R;US)

Corrosion Resistance

Corr sion of materials in a clay environment, $676(R A ; D E)$

Canister materials proposed for final disposal of high level nuclear waste - a review with respect to corrosion resistance, 675 (RA;DE)

Feasibility of using a high-level waste canister as an engineered barrier in disposal, 842 (R;US)

Fatigue

Enviromechanical testing of Ti-grade 2 and Ti-grade 12 in basalt ground water, 858 (R;US)

Physical Radiation Effects

Enviromechanical testing of Ti-grade 2 and Ti-grade 12 in basalt ground water, 858 (R;US)

\section{Strain Rate}

Enviromechanical testing of Ti-grade 2 and Ti-grade 12 in basalt ground water, 858 (R;US)

\section{TITANIUM BASE ALLOYS}

\section{Corrosion}

Corrosion studies and mechanical tests on metallic materials for the design of packagings for vitrified high level wastes (HLW), 689 (R;DE;In German)

Effects of radiation on corrosion of titanium, 961 (RA;US)

General corrosion, irradiation-corrosion, and environmentalmechanical evaluation of nuclear-waste-package structuralbarrier materials. Progress report, 843 (R;US)

Investigation of environmentally assisted fracture of metallic nuolear-waste-package basrier materials in simulated basalt repository environments, 844 (R;US)

Long-term performance of materials used for high-level waste packaging. Annual report, March 1982-April 1983. Yol.1, 751 (R;US)

Materials for high-level waste canister/overpacks in salt formations, 949 (R;US)

Nuclear waste package materials testing report: basaltic and tuffaceous environments, 854 (R;US)

Uniform- and pitting-corrosion data requirements for TiCode-12 high-level-waste containers, 734 (RA;US)

Corrosion Products

Analysis of the corrosion products formed on $\mathrm{Ti}$ and a $\mathrm{Ti}-\mathrm{Pd}$ alloy during exposure in hot water, 1176 (BA;US)

Corrosion Resistance

Waste package materials testing for a salt repository: 1982 status report, 825 (R;US) 
Crack Propagation

Investigation of environmentally assisted fracture of metallic nuclear-waste-package barrier materials in simulated basalt repository environments, 844 (R;US)

Crevice Corrosion

Container assessment: corrosion study of HLW container materials. Quarterly progress report, January-March 1982, 730 (R;US)

Corrosion of TiCode-12 in a simulated Waste Isolation Pilot Project (WIPP) brine, 1066 (J;US)

Identification of crevice corrosion in the titanium alloy TiCode- 12 in simulated rock salt brine at $150^{\circ} \mathrm{C}$ (Ti-0.3Mo-0. $8 \mathrm{Ni}), 545$ (R;US)

Review of DOE waste package program. Subtask 1.1. National waste package program, April-September 1982, 735 (R;US)

Electrochemical Corrosion

Review of DOE waste package program. Subtask 1.1. National waste package program, April-September 1982, 735 (R;US)

\section{Elongation}

Materials for high-level waste canister/overpacks in salt formations, 949 (R;US)

Failures

Review of DOE waste package program. Subtask 1.1. National waste package program, October 1982-March 1983. Vol. 4, 736 (R;US)

Fatigue

Investigation of environmentally assisted fracture of metallic nuclear-waste-package barrier materials in simulated basalt repository environments, 844 (R;US)

Fracture Properties

Corrosion of TiCode-12 in a simulated Waste Isolation Pilot Project (WIPP) brine, 1066 (J;US)

Hydrogen Embrittlement

Corrosion of TiCode-12 in a simulated Waste Isolation Pilot Project (WIPP) brine, 1066 (J;US)

Mechanical Properties

Nuclear waste package materials testing report: basaltic and tuffaceous environments, 854 (R;US)

Microstructure

Materials for high-level waste canister/overpacks in salt formations, 949 (R;US)

Physical Radiation Effects

General corrosion, irradiation-corrosion, and environmentalmechanical evaluation of nuclear-waste-package structuralbarrier materials. Progress report, 843 (R;US)

Materials for high-level waste canister/overpacks in salt formations, 949 (R;US)

Nuclear waste package materıals testıng report: basaltic and tuffaceous environments, 854 (R;US)

Pitting Corrosion

Review of DOE waste package program. Subtask 1.1. National waste package program, April-September 1982, 735 (R;US)

Strain Rate

Investigation of environmentally assisted fracture of metallic nuclear-waste-paikkagc barricr materials in simulated basalt repository environments, 844 (R;US)

Stress Corrosion

Review of DOE waste package program. Subtask 1.1. National wastc package program, April-Septemher 1987, 739 (R;IIS)

Tensile Properties

Corrosion studies and mechanical tests on metallic materials for the design of packagings for vitrificd high level wastes (HLW), 689 (R;DE;In German)

Materials for high-level waste canister/overpacks in salt formations, 949 (R;US)

Vield Strength

Materials for high-level waste canister/overparks in salt formations, 949 (R;US)

TITANIUM OXIDES

See also HOLLANDITE

PEROVSKITE

ZIRCONOLITE

\section{Crystal Structure}

Investigation of titanium in metamict Nb-Ta-Ti oxides using the extended $x$-ray absorption fine structure technique, 1215 (BA;US)

Leaching

Influence of metal oxides on the leach rate and crystallisation behaviour of waste glasses, 251 (RA;DE)

Physical Radiation Effects

Alpha-recoil damage in natural zirconolite and perovskite, 518 (J;JP)

Radiation Effects

Investigation of titanium in metamict $\mathrm{Nb}-\mathrm{Ta}-\mathrm{Ti}$ oxides using the extended $x$-ray absorption fine structure technique, 1215 (BA;US)

TRANSFER (IN ENVIRONMENT)

SEe RADIONUCLIDE MIGRATION

TRANSPLUTONIUM ELEMENTS

See also AMERICIUM

Combustion

Combustion in the reactor of long-lived transuranium elements as a means of reducing the toxicity of HAW, $522(\mathrm{~J} ; \mathrm{DE} ; \mathrm{In}$ German)

\section{TRANSPORTATION SYSTEMS}

Design

High-Level Waste Transportation-System Development Program design report for Fiscal Year 1980, 34 (R;US)

TRANSURANIUM ELEMENTS

See also NEPTUNIUM

\section{PLUTONIUM}

TRANSPLUTONIUM ELEMENTS

After-heat

Decay calculations on medium-level and actinide-containing wastes from the LWR fuel cycle. Pt. 1. Basic data evaluation including activity and thermal decay power, 687 (R;DE;In German)

Decay

Decay calculations on medium-level and actinide-containing wastes from the LWR fuel cycle. Pt. 1. Basic data evaluation including activity and thermal decay power, 687 (R;DE; In German)

Gamma Spectroscopy

State-of-the-art in-situ determination of transuranics, 88 (R;US)

Geochemistry

Alpha-recoil damage and solution effects in minerals: uranium isotopic disequilibrium and radon release, 1073 (J;GB)

Nuclear Reaction Analysis

State-of-the-art in-situ determination of transuranics, 88 (R;US)

Recovery

Disposal of radioactive wastes (Book), 106 (B;NL)

TRANSURANIUM WASTES

See ALPHA-BEARING WASTES

TRITIUM

Diffusion

Comparison of in-situ radionuclide migration studies in the Studsvik area and laboratory measurements, 1349 (BA;US)

Environmental Exposure Pathway

Investigation of the consequences of leakage incidents of a tank with high-concentrated radionuclide solutions and pentration of the radioactive solution into the soil, 1423 (R;DE;In German)

Favironmental Transport

Exposures resulting from nuclear power production. Annex $F$, 1444 (BA;US)

Investigation of the consequences of leakage incidents of a tank with high-concentrated radionuclide solutions and pentration of the radioactive solution into the soil, 1423 (R;DE; In German)

Sorption

Comparison of in-situ radionuclide migration studies in the Studsvik area and laboratory measurements, 1349 (BA;US)

Underground Storage

Basic study on storage of tritium waste, 1245 (J;JP;In Japanese) 


\section{TRU WASTES}

See ALPHA-BEARING WASTES

\section{TRUCKS}

Comparative Evaluations

Southeastern Utah nuclear-waste-transportation study, 26 (R;US)

Operating Cost

Southeastern Utah nuclear-waste-transportation study, 26 (R;US)

\section{TUFF}

Conceptual waste-package designs for disposal of nuclear waste

in tuff, 818 (R;US)

Nuclear-waste-package program for high-level isolation in Nevada tuff, 1015 (R;US)

Compression Strength

Thermal impact of waste emplacement and surface cooling assoCreep ciated with geologic disposal of nuclear waste, 707 (R;US)

Issues related to field testing in tuff, 947 (R;US)

Methodology for determining time-dependent mechanical properties of tuff subjected to near-field repository conditions, 697 (R,UB)

Evaluation

Near-field performance evaluations, 800 (RA:US)

Fleld Tests

Effects of long-term exposure of tuffs to high-level nuclear wasterepository conditions. Preliminary report. 693 (R;US)

Flow Models

Comparison of waste emplacement configurations for a nuclear waste respository in tuff. IV. Thermo-hydrological analysis, 996 (R;US)

Geochemistry

Geochemistry studies pertaining to the G-tunnel radionuclide migration field experiment, 1426 (R;US)

Research and development related to the Nevada Nuclear Waste Storage Investigations. Progress report, July 1-September 30, 1981, 692 (R;US)

Research and development related to the Nevada Nuclear Waste Storage Investigations. Progress report, October 1-December 31, 1982, 704 (R;US)

Research and development related to the Nevada Nuclear Waste Storage Investigations. Progress report, July 1-September 30, 1982, 703 (R;US)

Research and development related to the Nevada Nuclear Waste Storage Investigations. Progress report, January 1-March 31, 1983, 705 (R;US)

Some geochemical considerations for a potential repository site in tuff at Yucca Mountain, Nevada, 706 (R;US)

Summary report on the geochemistry of Yucca Mountain and environs, 700 (R;US)

Gravity Surveys

Preliminary results of gravity investigations at Yucca Mountain and vicinity, Southern Nye County, Nevada, 1024 (R;US)

Ground Water

Elution of radionuclides through columns of crushed rock from the Nevada Test Site, 1315 (R;US)

Hydrology

Hydrology of sealing a repository in saturated tuff, 992 (R;US)

Incompressible Flow

Code development in support of nuclear waste storage investigations for a repository in tuff (Pore water motion and encrgy transport in a porous media which may be saturated or par-

Joints tially saturated with a single fluid in liquid state), 988 (R;US)

Issues related to field testing in tuff, 947 (R;US)

Mechanical Properties

Effects of long-term exposure of tuffs to high-level nuclear wasterepository conditions. Preliminary report, 693 (R;US)

Methodology for determining time-dependent mechanical properties of tuff subjected to near-field repository conditions, 697 (R:US)

Thermo-mechanical scoping calculations for a high level nuclear wastc repository in tuff, 933 (R;US)
Mechanical Tests

Nevada nuclear waste storage investigations. Quarterly report. July-Septernber 1982, 758 (R;US)

\section{Mineralogy}

Nevada Nuclear-Waste-Storage Investigations. Quarterly report, April-June 1982, 757 (R;US)

Research and development related to the Nevada Nuclear Waste Storage Investigations. Progress report, January I-March 31, 1983, 705 (R;US)

Scientific data necessary to predict radionuclide migration within or near a mined nuclear repository, 1312 (R;US)

Nondestructive Analysis

Nevada nuclear waste storage investigations. Quarterly report, July-September 1982, 758 (R;US)

Packaging

Research and development related to the Nevada Nuclear Waste Storage Investigations. Progress report, July 1-September 30 , 1981,692 (R;US)

Permeability

Elution of radionuclides thrnugh columns of remshed reck from the Nevada Test Site, 1315 (R;US)

Thermal impact of waste emplacement and surface cooling associated with geologic dispusal of nuclear waste, 707 (R;US)

Potrology

Nevada Nuclear-Waste-Storage Investigations. Quarterly report, April-Junc 1982, 757 (R;US)

Petrologic studies of drill cores USW-G2 and UE25b-1 H, Yucca Mountain, Nevada, 696 (R;US)

Research and development related to the Nevada Nuclear Waste Storage Investigations. Progress report, July 1-September 30 , 1981, 692 (R;US)

Research and development related to the Nevada Nuclear Waste Storage Investigations. Progress report, July 1-September 30, 1982, 703 (R;US)

Research and development related to the Nevada Nuclear Waste Storagc Investigations. Progress report, January 1-March 31, 1983, 705 (R:US)

Poisson Ratio

Thermal impact of waste emplacement and surface cooling associated with geologic disposal of nuclear waste, 707 (R;US)

Porosity

Thermal impact of waste emplacement and surface cooling associated with geologic disposal of nuclear waste, 707 (R;US)

Radionuclide Migration

Compliance assessments of hypothetical geological nuclear waste isolation systems with the draft EPA standard, 982 (R;US)

Research and development related to the Nevada Nuclear Waste Storage Investigations. Progress report, January 1-March 31, 1983, 705 (R;US)

Rock Mechanics

Conceptual design of field experiments for welded-tuff rockmechanics program, 936 ( $\mathbf{R} ; U \dot{S})$

Issues related to field testing in tuff, 947 (R;US)

Shaft Excavations

Project management plan for exploratory shaft at Yucca Mounthiil, 764 (R;US)

Sorptive Properties

Geochemistry studies pertaining to the G-tunnel radionuclide migration field experiment, 1426 (R;US)

Radionuclide sorption on basalt-interbed materials. Annual report FY 1981, 1328 (R;US)

Research and development related to the Nevada Nuclear Waste Storage Investigations. Progress report, July 1-September 30, 1981, 692 (R;US)

Status of radionuclide sorption-desorption studies performed by the WRIT program, 1308 (RA;US)

Specific Heat

Three-dimensional thermal analysis for a conceptual high-level waste repository in welded tuff, 932 (R;US)

Strains

Methodology for determining time-dependent mechanical properties of tuff subjected to near-field repository conditions, 697 (R;US) 
*. atigraphy

?etrologic studies of drill cores USW-G2 and UE25b-1H, Yucca Mountain, Nevada, 696 (R;US)

Preliminary thermal analyses for a nuclear waste repository in tuff, 931 (R;US)

Stresses

Methodology for determining time-dependent mechanical properties of tuff subjected to near-field repository conditions, 697 (R;US)

Temperature Distribution

Comparison of waste emplacement configurations for a nuclear waste respository in tuff. IV. Thermo-hydrological analysis, 996 (R;US)

Thermal response to emplacement of nuclear waste in long, horizontal boreholes, 986 (R;US)

Temperature Effects

Comparison of waste emplacement configurations for a nuclear waste respository in tuff. IV. Thermo-hydrological analysis, 996 (R;US)

Effects of long-term exposure of tuffs to high-level nuclear wasterepository conditions. Preliminary report, 693 (R;US)

Issues related to field testing in tuff, 947 (R;US)

Repository data base development, 801 (RA;US)

Thermal analysis of nuclear waste emplacement in welded tuff, 930 (R;US)

Tensile Properties

Thermal impact of waste emplacement and surface cooling associated with geologic disposal of nuclear waste, 707 (R;US)

Thermal Analysis

Far-field thermal analysis of a high level waste repository in tuff. Topical report RSI-0137, 942 (R;US)

Preliminary thermal analyses for a nuclear waste repository in tuff, 931 (R;US)

Thermal analyses for a nuclear-waste repository in tuff using USW-Gl borehole data, 946 (R;US)

Thermal Conductivity

Effects of composition, porosity, bedding-plane orientation, water content and a joint on the thermal conductivity of tuff, 970 (R;US)

Three-dimensional thermal analysis for a conceptual high-level waste repository in welded tuff, 932 (R;US)

Thermal Stresses

Thermal impact of waste emplacement and surface cooling associated with geologic disposal of nuclear waste, 707 (R;US)

Thermo-mechanical scoping calculations for a high level nuclear waste repository in tuff, 933 (R;US)

Young Modulus

Thermal impact of waste emplacement and surface cooling associated with geologic dispnsal of nuclear waste, 707 (R;US)

\section{TUMORS}

See NEOPLASMS

TURTLES

Spatial Distribution

Surveys for desert tortoise on the proposed site of a high-level nuclear wastc repository at the Nevada Test Site, 1302 (R;US)

\section{TYUYAMUNITE}

Stability

Uranium-lead radiometric age determinations of naturally occurring $\mathrm{U}(\mathrm{VI})$ minerals: application to radwaste storage (Grants mineral belt, New Mexico), 1155 (BA;US)

\section{U}

UAR

See EGYPTIAN ARAB REPUBLIC

UK ATOMIC ENERGY AGENCY

\section{iee UKAEA}

EA

iée also $A E R E$

Radioactive Waste Processing

Denitration of simulated fast ractor highly active liquor waste, 241 (RA;DE)

Design of Harwell Joule Ceramic Melter, 231 (RA;DE)

\section{UNDERGROUND DISPOSAL}

Field Tests

Underground openings for in situ experiments, 1111 (J;US)

Radiation Hazards

Decay calculations on medium-level and actinide-containing wastes from the LWR fuel cycle. Pt. 2, 688 (R;DE;In German)

Radionuclide Migration

Disposal of high level wastes, 1244 (J;JP;In Japanese)

Evaluation of radionuclide releases from underground waste repositories using the method of status vectors, 1424 (J;DD)

Reliability

Nuclear waste disposal: can we rely on bedrock, 1137 (B;GB)

Risk Assessment

Decay calculations on medium-level and actinide-containing

Safety wastes from the LWR fuel cycle. Pt. 2, 688 (R;DE;In German)

Evaluation of radionuclide releases from underground waste repositorics using the method of status vectors, 1424 (J;DD)

UNION OF SOVIET SOCIALIST REPUBLICS

Sëe USSR

UNITED ARAB REPUBLIC

See EGYPTIAN ARAB REPUBLIC

UNITED KINGDOM

Fuel Cycle

Summary of non-US national and international fuel cycle and radioactive waste management programs 1982,83 (R;US)

High-level Radioactive Wastes

Management- of vitrified high level radioactive waste, 466 (BA;GB)

Radioactive Waste Disposal

Deadly legacy (Radioactive waste disposal), 1127 (J;GB)

UK view of the management of high-level waste, $1103(\mathrm{~J} ; \mathrm{XA})$

United Kingdom regulatory procedures for radioactive wastes, 369 (J;US)

Radioactive Waste Facilities

Vitrifying Britain's waste, 384 (J;GB)

Radioactive Waste Management

Handling of nuclear waste in Great Britain, 94 (J;DE;In German)

Management of radioactive wastes, 101 (B;GB)

Radioactive waste management. Response by the Town and Country Planning Association to the white paper 'Radioactive Waste Management' - Cmnd 8607, 113 (B;GB)

Review of radioactive waste management programs in the United Kingdom, 1160 (BA;US)

Summary of non-US national and international fuel cycle and radioactive waste management programs 1982,83 (R;US)

United Kingdom regulatory procedures for radioactive wastes, 369 (J;US)

Badinactive Waste Processing

High level waste solidification: the future, 227 (RA;DE)

Selection and application of a processing system for the treatment of high level liquid waste in the UK, 430 (BA;US)

United Kingdom regulatory procedures for radioactive wastes, 369 (J;US)

UNITED STATES OF AMERICA

See USA

URANTUM

Absorption Spectroscopy

Subsolidus sintering of SYNROC: I. Initial demonstrations, 280 (R;US)

Activation Analysis

Subsolidus sintering of SYNROC: I. Initial demonstrations, 280 (R;US)

Adsorption

Geochemistry studies pertaining to the G-tunnel radionuclide migration field experiment, 1426 ( $R$;US)

Importance of organic compounds in ground water as radionuclide-mobilizing agents, 1324 (R;US)

Sorption of actinides in well-defined oxidation states on geologic media, 1351 (BA;US)

Transport of actinides through a bentonite backfill, 1175 (BA;US)

Waste form/rock interaction leaching study using PNL 76-68 glass beads and Umtanum basalt. Part I, 1004 (R;US) 
Adsorption Isotherms

Basalt-radionuclide reactions: FY-1981. Annual report, 868 (R;US)

Chemical Analysis

Analyses of nuclear fuel and high-level waste by inductively coupled plasma-emission spectroscopy, 68 (R;US)

Chemical State

Evaluation of solubility and speciation of actinides in natural groundwaters, 1347 (BA;US)

\section{Chemisorption}

Redox behavior, complexing, and adsorption of hexavalent actiriides by humic acid and selected clays (Siting, marine disposal of high-level radioactive waste), 1247 (J;US)

Diffusion

Transport of actinides through a bentonite backfill, 1175 (BA;US)

Distribution Functions

Sorption of actinides in well-defined oxidation states on geologic mèdia, 1351 (BA;US)

Environmental Transport

Actinide sorption on rock minerals, 677 (RA;DE)

Compliance assessments of hypothetical geological nuclear waste isolation systems with the draft EPA standard, 982 (R;US)

Fabrication

Chemistry of the nuclear fuel cycle, 4 (BA;GB)

Leaching

Evaluation of solubility and speciation of actinides in natural groundwaters, 1347 (BA;US)

Leaching of actinides from simulated nuclear waste glass, 1196 (BA;US)

Leaching studies on SYNROC at $95^{\circ} \mathrm{C}$ and $200^{\circ} \mathrm{C}, 1114(\mathrm{~J} ; \mathrm{CH})$

Processing effects on the behavior of titanate waste forms in aqueous solutions, 1145 (BA;US)

Processing, microstructure, leảching, and long-term stability studies related to titanate high-level waste forms, 340 (R;US)

Subsolidus sintering of SYNROC: I. Initial demonstrations, 280 (R;US)

Subsolidus sintering of SYNROC: II. Materials selections, process improvements, waste form evaluations, 281 (R;US)

SYNROC: leaching performance and process technology, 250 (RA:DE)

Meetings

Uranium and nuclear energy: proceedings of the international symposium, 4th, 1979, 1 (B;GB)

\section{Oxidation}

Redox behavior, complexing, and adsorption of hexavalent actinides by humic acid and selected clays (Siting, marine disposal of high-level radioactive waste), 1247 (J;US)

Radiation Scattering Analysis

Utilization of charged particle backscattering to study the near surface region of glasses. Application to depth profiling of lanthanium, cerium, throium and uranium induced by aqueous leaching. 1216 (BA:US)

Solubility

Evaluation of solubility and speciation of actinides in natural groundwaters, 1347 (BA;US)

Geochemical controls on radionuclide releases from a nuclear waste repository in basalt: estimated solubilities for selected elements, 1329 (R;US)

Sorption

Evaluation of sodium bentonite and crushed basalt as waste package backfill materials, 902 (R;US)

Radionuclide sorption on basalt-interbed materials. Annual report FY 1981, 1328 (R; US)

Supply And Demànd

Uranium and nuclear energy: proceedings of the international symposium, 4th, 1979, 1 (B;GB)

Valence

Vulence states of actinides in synthetic monazites, 1276 (BA;US)
URANIUM 233

Diffusion

Study of radionuclide migration from deep-lying repository sitᄂ. with over-lying sedimentary layers, 1348 (BA;US)

Solubility

Evaluation of radionuclide transport: effect of radionuclide sorption and solubility, 1354 (BA;US)

Sorption

Evaluation of radionuclide transport: effect of radionuclide sorption and solubility, 1354 (BA;US)

URANIUM 234

Dissolution

Isotopic fractionation of thorium and uranium upon leaching of monozite: alpha-recoil damage effects, 1214 (BA;US)

Solubility

Evaluation of radionuclide transport: effect of radionuclide sorption and solubility, 1354 (BA;US)

\section{URANIUM 235}

Solubility

Evaluation of radionuclide transport: effect of radionuclide sorption and solubility, 1354 (BA;US)

\section{URANIUM 236}

Solubility

Evaluation of radionuclide transport: effect of radionuclide sorption and solubility, 1354 (BA;US)

URANIUM 238

Adsorption

Immobilization of reprocessing waste by means of titanate ion exchangers, 248 (RA;DE)

Dissolution

Isotopic fractionation of thorium and uranium upon leaching of monozite: alpha-recoil damage effects, 1214 (BA;US)

Leaching

Leach rates of high level waste and spent fuel: limiting rates as determined by backfill and bedrock conditions, 1345 (BA;US)

Waste package performance evaluation, 806 (R;US)

Solubility

Evaluation of radionuclide transport: effect of radionuclide sorption and solubility, 1354 (BA;US)

Leach rates of high level waste and spent fuel: limiting rates as determined by backfill and bedrock conditions, 1345 (BA;US)

URANIUM COMFLEXES

Solubility

Importance of organic compounds in ground water as radionuclide-mobilizing agents, 1324 (R;US)

\section{URANIUM DIOXIDE}

Fabrication

Chemistry of the nuclear fuel cycle, 4 (BA;GB)

Phase Studies

Chemistry in the development of nuclear power, 5 (BA;GB)

Thermodynamic Properties

Chemistry in the development of nuclear power, 5 (BA;GB)

URANIUM II

SeE URANIUM 234

URANIUM MINERALS

See also TYUYAMUNITE

URANOPHANE

Age Estimation

Uranium-lead radiometric age determinations of naturally occurring U(VI) minerals: application to radwaste storage (Grants mineral belt, New Mexico), 1155 (BA;US)

URANIUM MINES

Recommendations

Report of the Select Committee of the Legislative Council or uranium resources, 1363 (B;AU)

\section{URANOPHANE}

Stability

Uranium-lead radiometric age determinations of naturally occurring $U(V I)$ minerals: application to radwaste storage (Grants mineral belt, New Mexico), 1155 (BA;US) 


\section{RANYL CHLORIDES}

Diffusion

Investigation of diffusion of $\mathrm{UO}_{2} \mathrm{Cl}_{2}$ in saturated $\mathrm{NaCl}$ solutions at various temperatures, 1167 (BA;US)

US AEC

See also BNL

HAPO

IDAHO CHEMICAL PROCESSING PLANT

ORNL

SAVANNAH RIVER PLANT

Radioactive Waste Management

Management of high-level radioactive wastes, 218 (R;US)

US ATOMIC ENERGY COMMISSION

See US AEC

US DOE

See also BATTELLE PACIFIC NORTHWEST LABORATORIES $B N L$

HANFORD RESERVATION

$\bar{H} A P O$

IDAHO CHEMICAL PROCESSING PLANT

NEVADA TEST SITE

ORNL

SAVANNAH RIVER PLANT

Fuel Cycle

DOE's nuclear waste management and fuel cycle programs, 1159 (BA;US)

High-level Radioactive Wastes

DOE's nuclear waste management and fuel cycle programs, 1159 (BA;US)

Radioactive Waste Disposal

Department of Energy does not plan to use an abandoned salt mine at Lyons, Kansas, for nuclear high-level waste disposal, 637 (R;US)

NWTS program criteria for mined geologic disposal of nuclear waste: program objectives, functional requirements, and system performance criteria, 595 (R;US)

Radioactive Waste Management

DOE implementation of high-level waste criteria and standards, 1093 (J;US)

DOE's commercial waste management and fuel cycle programs, 100 (J;US)

DOE's nuclear waste management and fuel cycle programs, 1159 (BA;US)

Information base for commercial radioactive-waste management, 56 (R;US)

Review of the status of transportation activities in various waste management systems (Transportation Technology Center), 30 (R;US)

Reactor Decommissioning

Management of the DOE inventory of excess radioactively contaminated facilities, 1288 (BA;US).

Research Programs

Answers to your questions abuut high-level nuclear waste isolation, 1298 (B;US)

Geochemistry research planning for undergrourid repositories, 1112 (J;US)

Program summary. Nuclear waste management and fuel cycle programs, 575 (R;US)

Research and development needs in the Department of Energy. Interim report, 573 (R;US)

US EPA

Regulations

Assessment of high-level waste form conformance with proposed regulatory and repository criteria, 198 (R;US)

Compliance assessments of hypothetical geological nuclear waste isolation systems with the draft EPA standard, 982 (R;US)

Regulatory policy of the EPA related to alpha-contaminated waste, 1412 (RA;US)

Waste glasses: requirements and characteristics, 322 (R;US)
US NRC

Regulations

Assessment of high-level waste form conformance with proposed regulatory and repository criteria, 198 (R;US)

NRC regulations for high-level nuclear waste management, 1092 (J;US)

Nuclear Regulatory Commission activities related to high-level waste materials issues, 1420 (BA;US) USA

Waste glasses: requirements and characteristics, 322 (R;US)

\section{See also FEDERAL REGION VI GULF COAST}

Hydrology

Earth Sciences, 789 (RA;US)

Igneous Rocks

National survey of crystalline rocks and recommendations of regions to be explored for high-level radioactive waste repository sites, 540 (R;US)

Metamorphic Rocks

National survey of crystalline rocks and recommendations of regions to be explored for high-level radioactive waste repository sites, 540 (R;US)

Nuclear Power Plants

Indemnification for nuclear damages - recent developments in the United States, 1431 (R;ES)

Radioactive Waste Disposal

Defense waste management plan, 563 (R;US)

Earth Sciences, 789 (RA;US)

High-level-waste containment for a thousand years: unique technical and research problems, 544 (R;US)

Managing commercial high-level radioactive waste, 91 (J;GB)

National survey of crystalline rocks and recommendations of regions to be explored for high-level radioactive waste repository sites, 540 (R;US)

Overview of the National Waste Terminal Storage Program, 788 (RA;US)

Repository design and equipment, 791 (RA;US)

Research priorities for isolation of defense high-level and transuranic wastes in the United States, 628 (R;US)

Socioeconomic evaluations, 47 (RA;US)

Systems, 790 (RA;US)

Radloactive Waste Facilities

Indemnification for nuclear damages - recent developments in the United States, 1431 (R;ES)

Radioactive Waste Management

Historical overview, 53 (RA;US)

National long-term high-level waste-technology program, 67 (R;US)

Nuclear waste management, 119 (J;US)

Waste-management $R$ and $D$ for the nuclear fuel cycle, 311 (R;US)

Radioactive Waste Processing

Alternative waste forms: process feasibility, 477 (J;US)

Commercial waste treatment $R$ and $D$ needs in the United States, $323 \cdot(R ; U S)$

Treatment and conditioning of wastes from US nuclear fuels reprocessing plants, 191 (R;US)

Radioactive Waste Storage

Managing commercial high-level radioactive waste, 91 (J;GB)

Shales

Preliminary assessment of shales and other argillaceous rocks in the United States, 609 (RA;US)

USSR

Fuel Cycle

Summary of non-US national and international fuel cycle and radioactive waste management programs 1982,83 (R;US)

\section{Land Pollution}

Analysis of the alleged Kyshtym disaster, 1314 (R;US)

Radioactive Waste Management

Summary of non-US national and international fuel cycle and radioactive waste management programs 1982, 83 (R;US) 


\section{Water Pollution}

UTAH

Analysis of the alleged Kyshtym disaster, 1314 (R;US)

\section{Aquatic Ecosystems}

Ecosystem studies, endangered speciés survey - Gibson Dome and Elk Ridge study areas, Paradox Basin, Utah, 1326 (R;US)

Endangered Species

Ecosystem studies, endangered species survey - Gibson Dome and Elk Ridge study areas, Paradox Basin, Utah, 1326 (R;US)

Geology

Geological status of NWTS repository siting activities in the paradox basin, 1265 (J;US)

Terriestrial Ecosystems

Ecosystem studies, endangered species survey - Gibson Dome and Elk Ridge study areas, Paradox Basin, Utah, 1326 (R;US)

Transportation Systems

Southeastern Utah nuclear-waste-transportation study, 26 (R;US)

\section{$\mathbf{V}$}

\section{VANADIUM}

Emission Spectroscopy

Analyses of nuclear fuel and high-level waste by inductively coupled plasma-emission spectroscopy, 68 (R;US)

VANADIUM MINERALS

See MINERALS

VERSENE

SEE EDTA

VESSELS

See CONTAINERS

VIRGINIA

Geology

Regional geological assessment of the Devonian-Mississippian shale sequence of the Appalachian, Illinois, and Michigan basins relative to potential storage/disposal of radioactive wastes, 828 (R;US)

\section{in}

WAK

Construction Permits

Land Baden-Wuerttemberg: public notice of the Ministry of Economics, Medium-size Industry and Transport concerning a permit under the Atomic Energy Law. - 3rd Supplement to the 9th partial licence for the construction of Karlsruhe reprocessing plant - System for storage and evaporation of high-activity liquid waste (LAVA), 10 (J;DE;In German)

Radioactive Waste Storage

Experience with the storage of high active waste at WAK, 1199 (B:DE)

WASHINGTON

Land use

Monitoring land-and water-use dynamics in the Columbia Plateau using remote-sensing computer analysis and integration lechriques, 1425 (R;US)

Water use

Monitoring land- and water-use dynamics in the Columbia Plateau using remote-sensing computer analysis and integration techniques, 1425 (R;US)

WASTE BURIAL

SEe UNDERGROUND DISPOSAL

WASTE DISPOSAL

See also MARINE DISPOSAL RADIOACTIVE WASTE DISPOSAL UNDERGROUND DISPOSAL

Licensing

Licensing of high-level radioactive wastes deposits, 1405 (J;BR;In Portuguese)

Permit Applications

DOE's program for high-level radioactive waste disposal, 1266 (BA;US)
Site Selection

DOE's program for high-level radioactive waste disposal, 1266 (BA;US)

Licensing of high-level radioactive wastes deposits, 1405 (J;BR;In Portuguese)

\section{WASTE PELLETS}

Drying

Microwave energy for post-calcination treatment of high-level nuclear wastes, 378 (J;US)

WASTE SOLUTIONS

SE LIQUID WASTES

WASTE TRANSPORTATION

Transportation system preconceptual description for subseabed disposal of high-level waste, 20 (RA;US)

Cost

Defense waste transportation: cost and logistics studies, 27

(R;US)

Economic evaluation of nuolear waste transportation casks, 14 (R;US)

Estimated transportation cost and risk for operation of a nuclear waste repository at candidate sites, 17 (RA;US)

Impacts of transportation on a test and evaluation facility for nuclear waste disposal: a systems analysis, 556 (R;US)

Preliminary analysis of the cost and risk of transporting nuclear waste to potential candidate commercial repository sites, 35 (R;US)

Mathematical Models

Defense waste. transmrtation: cost and logirtics ctudios, 27 (R;US)

Risk Assessment

Estimated transportation cost and risk for operation of a nuclear waste repository at candidate sites, 17 (RA;US)

Preliminary analysis of the cost and risk of transporting nuclear waste to potential candidate commercial repository sites, 35 (R;US)

Risk assessment for the transportation of radioactive zeolite liners, 28 (R;US)

WASTE-ROCK INTERACTIONS

Basalt-radionuclide reactions: FY-1981. Annual report, 868 (R;US)

Diffusion in crystalline rocks, 1168 (BA;US)

Interaction of borosilicate glass and granodiorite at $100^{\circ} \mathrm{C}$, 50 $\mathrm{MPa}$ : implications for models of radionuclide release, 1201 (BA;US)

Interaction of groundwater and fresh basalt fissure surfaces and its effect on the migration of actinides, 1303 (R;US)

Status of radionuclide sorption-desorption studies performed by the WRIT program, 1308 (RA;US)

Waste glass/repository interactions, 1193 (BA;US)

Temperature Effects

Analysis of tcmpcrature data from the full-scale heyter experiments at Stripa, 710 (RA;US)

WATER

$\begin{array}{ll}\text { See also } & \text { GROUND WATER } \\ & \text { HOT WATER } \\ \text { SEAWATER }\end{array}$

Radiolysis

Effects of radiation damage and radiolysis on the leaching of vitrified waste, 463 (BA;US)

Influence of gamma irradiation on the leaching behavior of a modified SYNROC-B ceramic waste form, 424 (BA;US)

WATER CONTENT

See MOISTURE

WATER COOLANT

See WATER

WATER COOLED REACTORS

Siee also BWR TYPE REACTORS

LWBR TYPE REACTORS

PWR TYPE REACTORS

Radioactive Waste Processing

Actinide recycling in light water reactors: results of reactor physics calculations, 294 (R;US) 
'actor Physics

Actinide recycling in light water reactors: results of reactor physics calculations, $208(R ; X E)$

\section{WATER MODERATED REACTORS}

See also BWR TYPE REACTORS

\section{LWBR TYPE REACTORS} PWR TYPE REACTORS

Comparative Evaluations

Uranium hexafluoride actinide transmutation

reactors-preliminary design and analysis, $\$ 16$ (D;US)

\section{WATER MODERATOR}

Sëe WATER

WATER USE

Remote Sensing

Monitoring land and water uses in the Columbia Plateau using remote-sensing computer analysis and integration techniques, 865 (R;US)

\section{WATERBORNE PARTICLES}

SEE PARTICULATES

WAZ 16

See NICKEL BASE ALLOYS

WEST GERMANY

See FEDERAL REPUBLIC OF GERMANY

WEST VALLEY PROCESSING PLANT

Decommissioning

Preliminary environmental implications of alternatives for decommissioning and future use of the Western New York Nuclear Services Center, 1301 (R;US)

Decontamination

Preliminary environmental implications of alternatives for decommissioning and future use of the Western New York Nuclear Services Center, 1301 (R;US)

West Valley Demonstration Project: strategy for an achievable but challenging project, 434 (BA;US)

Environmental Impacts

Preliminary environmental implications of alternatives for decommissioning and future use of the Western New York Nuclear Services Center, 1301 (R;US)

Radioactive Waste Management

Long-term management of liquid high-level radioactive wastes stored at the Western New York Nuclear Service Center, West Valley. Final environmental impact statement, 1305 (R;US)

Kadiological fisks for the aliernatives for llie lungaterm management of high-lcvel wastes at West Valley, 1261 (J;US)

Radioactive Waste Processing

High-level waste solidification system for the Western New York State Service Center, 507 (J;US)

\section{WEST VIRGINIA}

Geology

Regional geological assessment of the Devonian-Mississippian shale sequence of the Appalachian, Illinois, and Michigan basins relative to potential storage/disposal of radioactive wastcs, 828 (R;US)

WISCONSIN

\section{Radioactive Waste Management}

State of Wisconsin Ad Hoc committee on radioactive waste management. Final report, 568 (R;US)

\section{WORKERS}

$$
\text { See PERSONNEL }
$$

\section{Z}

\section{ZEOLTES}

See also CLINOPTILOLITE

Chemical Radiation Effects

Consequences of radiation effects on zeolites during interim storage, 1230 (J;US)

Leaching

Review of DOE waste package program. Subtask 1.1. National waste package program, October 1982-March 1983. Vol. 4, 736 (R;US)

Phase Transformations

Selected hydrologic and geochemical issues in site characterization for nuclear waste disposal: flood basalts at the Hanford Reservation, 715 (R;US)

Radiolysis

Review of DOE waste package program. Subtask 1.1. National waste package program, October 1982-March 1983. Vol. 4, 736 (R;US)

Sorptive Properties

Development of a backfill for containment of high-level nuclear waste, 1173 (BA;US)

Development of partitioning method - cold and semihot experimental works with partitioning testing apparatus, 225 (R;JP;In Japanese)

Transport

Risk assessment for the transportation of radioactive zeolite liners, 28 (R;US)

ZINC

Activation Analysis

Application of neutron-activation analysis to the determination of leach rates of simulated nuclear-waste forms, 127 (R;US)

Emission Spectroscopy

Analyses of nuclear fuel and high-level waste by inductively coupled plasma-emission spectroscopy, 68 (R;US)

Leaching

Effects of composition, solutions, and burial on nuclear waste glasses, 845 (RÄ;US)

\section{ZINC OXIDES}

Leaching

Influence of metal oxides on the leach rate and crystallisation behaviour of waste glasses, 251 (RA;DE)

ZIRCON

Leaching

Leachability of zircon as a function of alpha dose, 1213 (BA;US)

Radiation Effects

Leachability of zircon as a function of alpha dose, 1213 (BA;US) ZIRCONIUM

Activation Analysis

Application of neutron-activation analysis to the determination of leach rates of simulated nuclear-waste forms, 127 (R;US)

Emission Spectroscopy

Analyses of nuclear fuel and high-level waste by inductively coupled plasma-emission spectroscopy, 68 (R;US)

\section{Leaching}

Comparison of properties of borosilicate glass and crystalline ceramic forms for immobilization of Savannah River Plant waste, 171 (R;US)

Leaching studies on SYNROC at $95^{\circ} \mathrm{C}$ and $200^{\circ} \mathrm{C}, 1114(\mathrm{~J} ; \mathrm{CH})$

SYNROC: leaching performance and process technology, 250 (RA;DE)

Solubility

Geochemical controls on radionuclide releases from a nuclear waste repository in basalt: estimated solubilities for selected elements, 1329 (R;US)

\section{ZIRCONIUM 95}

Adsorption

Laboratory tests on the migration behavior of selected fission products in aquifer materials from a potential disposal site in northern Germany, 1352 (BA;US) 
Diffusion

Laboratory tests on the migration behavior of selected fission products in aquifer materials from a potential disposal site in northern Germany, 1352 (BA;US)

Leaching

Leaching study of nuclear melt glass: Part I, 1332 (R;US)

ZIRCONIUM ALLOYS

Corrosion Resistance

Canister materials proposed for final disposal of high level nuclear waste - a review with respect to corrosion resistance, 675 (RA;DE)

ZIRCONIUM OXIDES

See also ZIRCONOLITE

Leaching

Influence of metal oxides on the leach rate and crystallisation behaviour of waste glasses, 251 (RA;DE)

Physical Radiation Effects

Alpha-recoil damage in natural zirconolite and perovskite, 518 (.Ji.JP)

\section{ZIRCONOLITE}

Chemical Radiation Effects

Radiation damage studies related to nuclear waste forms, 298 (R;US)

Microstructure

Radiation damage studies related to nuclear waste forms, 298 (K;US)

Phase Studies

Subsolidus sintering of SYNROC: I. Initial demonstrations, 280 (R;US)

Radiation Effects

Study of radiation effects in curium-doped $\mathrm{Gd}_{2} \mathrm{Ti}_{2} \mathrm{O}_{7}$ (pyrochlore) and $\mathrm{CaZrTi}_{2} \mathrm{O}_{7}$ (zironolite), 1211 (BA;US)

Seli-irradiation

Study of radiation effects in curium-doped $\mathrm{Gd}_{2} \mathrm{Ti}_{2} \mathrm{O}_{7}$ (pyrochlore) and $\mathrm{CaZrTi}_{2} \mathrm{O}_{7}$ (zironolite), 1211 (BA:US)

Specific Heat

Thermophysical properties of SYNROC and its constituent mineral phases, 474 (BA;AU)

Thermal Conductivity

Thermophysical properties of SYNROC and its constituent mineral phases, 474 (BA;AU)

Thermal Diffusivity

Thermophysical properties of SYNROC and its constituent mineral phases, 474 (BA;AU) 


\section{CONTRACT NUMBER INDEX}

Numbers assigned to DOE contracts under which documents announced in this publication were prepared are included in this index.

Contract No.

Abstract No. Report No.

AC01-80ET40110

Mitre Corp., Mclean, VA (USA). METREx Div.

56 DOE/ET/40110-1

AC01-80ET 44112 National Academy of Sciences, Washington, DC (USA)

565 DOE/ET/44112-T1

AC01-81NE44146 National Academy of Public Administration, $\begin{array}{ll}\text { Washington, DC (USA) } \\ 58 & \text { DOE/NE/44146-T1 } \\ 59 & \text { DOE/NE/44146-T2 }\end{array}$

AC02-76CH00016 - Brookhaven National Lab., Upton, NY

$\begin{array}{ll}\text { (USA) } & \\ 73 & \text { NUREG/CR-2333-Vol.3 } \\ 131 & \text { BNL-18978 } \\ 132 & \text { BNL-19109 } \\ 133 & \text { BNL-19323 } \\ 283 & \text { NUREG/CR-2333-Vol.1 } \\ 284 & \text { NUREG/CR-2333-Vol.4 } \\ 285 & \text { NUREG/CR-2333-Vol.5 } \\ 286 & \text { NUREG/CR-2482 } \\ 542 & \text { BNL-32669 } \\ 543 & \text { BNL-33451 } \\ 544 & \text { BNL-NUREG-31329 } \\ 545 . & \text { BNL-NUREG-32107 } \\ 546 & \text { BNL-NUREG-33192 } \\ 547 & \text { BNL-NUREG-33579 }\end{array}$

Louisiang State Univ, Baton Rouge (USA). Inst. for Environmental Studies 571 DOE/NBM-3004280

Brookhaven National Lab, Upton, NY (USA)

728

$$
\begin{aligned}
& \text { NUREG/CR-2317-Vol.1- } \\
& \text { No.3 }
\end{aligned}
$$

729 NIIREG/CR-2317-Vol. 1NOS.1-2

730 NUREG/CR-2317-Vol.2No.1

732 NUREG/CR-2482-Vol.2

735 NUREG/CR-2482-Vol.3

736 NURE.G/CR-2482-Vol.4

739 NUREG/CR-2755

749 NUREG/CR-3219-Vol.1

1319 NUREG/CR-3219-Vol.2

AC02-83CH10140 Battelle Memorial Inst., Columbus, OH

(USA), Office of Crystalline Repository Development

$$
540
$$

$$
\text { BMI-OCRD-1 }
$$

Battelle Memorial Inst., Columbus, $\mathrm{OH}$

(USA). Ofrice of Nuclear Waste Isola-

$$
\begin{array}{ll}
\text { tion } & \\
822 & \text { ONWI-463 } \\
827 & \text { ONWI-494 }
\end{array}
$$$$
1325 \text { ONWI-466 }
$$

..003-76SF00098

Lawrence

ONWI-466

$\begin{array}{cc}\text { Lawrence Berkeley Lab., CA (USA) } \\ 707 & \text { LBL-13341 } \\ 711 & \text { LBL-14080 } \\ 712 & \text { LBL-14082 }\end{array}$

Contract No.

AC03-765F00098

AC0476DP00789
Abstract No. Report No.

$\begin{array}{ll}713 & \text { LBL-14752 } \\ 714 & \text { LBL-15010 } \\ 715 & \text { LBL-15029 } \\ 1064 & \text { Materials Research Society } \\ & \text { Symposia Proceedings 15 } \\ & 531-538 \text { (1983). }\end{array}$

Lawrence Berkeley Lab, CA (USA) 1318 NUREG/CR-3062

Oak Ridge National Lab, TN (USA) 20 CONF-830528-Summ. (1983).

Sandia National Labs., Albuquerque, NM

$\begin{array}{cc}\text { (USA) } & \\ 29 & \text { SAND-81-2133 } \\ 30 & \text { SAND-82-0510C } \\ 32 & \text { SAND-82-1994C } \\ 33 & \text { SAND-82-1997C } \\ \text { General Atomic Co., San Diego, CA (USA) } \\ 34 & \text { SAND-82-7202 } \\ \text { Sandia National Labs, Albuquerque, NM }\end{array}$

$\begin{array}{ll}\text { (USA) } & \\ 35 & \text { SAND-83-0867 } \\ 89 & \text { SAND-82-1804 } \\ 140 & \text { CONF-820636-4 } \\ 339 & \text { SAND-82-1045C } \\ 340 & \text { SAND-82-2980 } \\ 555 & \text { CONF-830205-7 } \\ 731 & \text { NUREG/CR-2402 } \\ 747 & \text { NUREG/CR-3111-Vol.1 } \\ 750 & \text { NUREG/CR-3235-Vol.1 }\end{array}$

Pacific Nortbwest Lab, Richland, WA

$$
\text { (USA) }
$$

849 PNL-4382(Aug 1982).

Sandia National Labs., Albuquerque, NM

\begin{tabular}{ll} 
(USA) & \\
923 & SAND-79-0267 \\
924 & SAND-79-0268 \\
925 & SAND-79-0269 \\
926 & SAND-79-0270 \\
927 & SAND-79-0271 \\
928 & SAND-79-0272 \\
929 & SAND-79-0406C \\
930 & SAND-80-2639 \\
931 & SAND-80-2813 \\
932 & SAND-81-0215 \\
933 & SAND-81-0629 \\
934 & SAND-81-0709 \\
935 & SAND-81-1095 \\
936 & SAND-81-1768 \\
937 & SAND-81-2169C \\
938 & SAND-81-2195-Rev. \\
939 & SAND-81-2498 \\
940 & SAND-81-2628 \\
941 & SAND-81-7206 \\
942 & SAND-81-7210 \\
943 & SAND-82-0024 \\
944 & SAND-82-0127 \\
945 & SAND-82-0158C. \\
946 & SAND-82-0170 \\
947 & SAND-82-0312C \\
\hline
\end{tabular}


Contract No.

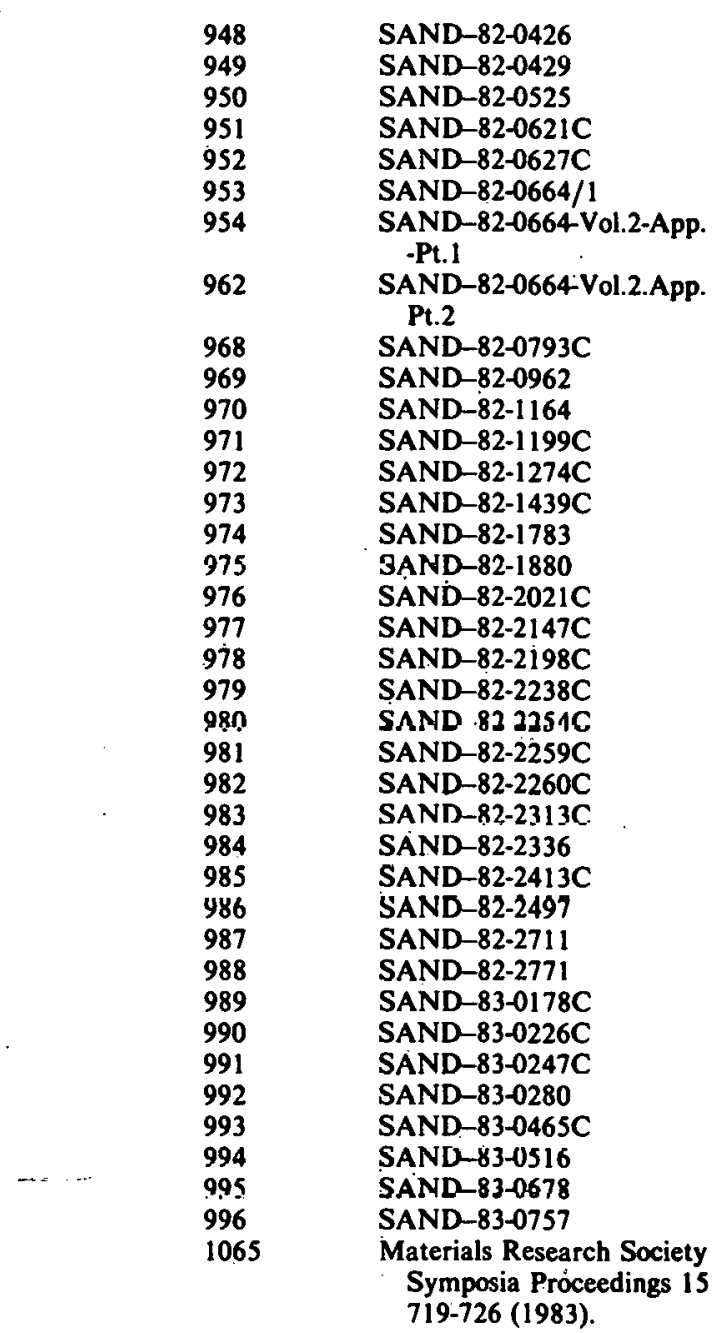

AC04-76DP00789 Sandia National Labs., Albuquerque, NM

$\begin{array}{ll}\text { (USA) } & \\ 1316 & \text { NUREG/CR-1636-Vol.1 } \\ 1317 & \text { NUREG/CR-2394 }\end{array}$

Néw Mexico Univ., Albuquerque (USA).

Dept. of Chemical and Nuclear Engi-

neering
1331

Sandia National Labs., Albuquerque, NM

\begin{tabular}{ll} 
(USA) & \multicolumn{1}{c}{ CONF-830205-3 } \\
1368 & NUREG/CR-3235-Vols. \\
1377 & SAND-81-2163C \\
1385 & SAND-81-2375 \\
1386 & NUREG/CR-3235(Vols.2- \\
1415 & $3-4)$
\end{tabular}

AC04-76DP03533 Rockwell International Corp., Golden, CO

$\begin{array}{ll}\text { (USA). Rocky Flats Plant } \\ 333 & \text { RFP-3282 } \\ 357 & \text { RFP-Trans-405(Jun 1983). }\end{array}$

AC04-78AL05346 Westinghouse Electric Corp., Pittsburgh, PA

(USA)
1419 WIPP-DOE-069-Rev.l

AC04-78AL10752 New Mexico Health and Environment Dept, Santa Fe (USA). Environmental Improvement Div.
Contract No. Abstract No. Report No.

ACO6-76RL01830 Oak Ridge National Lab, TN (USA)

17 CONF-830528-Summ. (1983).

Bechtel Group, Inc., San Francisco, CA $26^{\text {(USA) ONWI-404 }}$

Battelle Pacific Northwest Labs., Richland, WA (USA)

27 PNL-3721

Pacific Northwest Lab, Richland, WA

$\begin{array}{ll}\text { (USA) } & \\ 28 & \text { PNL-4032 } \\ 79 & \text { PNL-3570 } \\ 80 & \text { PNL-4121 } \\ 81 & \text { PNL-4228 } \\ 82 & \text { PNL-4382 } \\ 83 & \text { PNL-4405 } \\ 84 & \text { PNL-4441 } \\ 85 & \text { PNL-4637 }\end{array}$

Dattelle Pacific Nurdlowest Labs., Rlchland, WA (USA)

86 PNLSA-8916

Pacific Nortbwest Lab., Richland, WA

$\begin{array}{ll}\text { (USA) } & \\ 88 & \text { PNL-SA-10124 }\end{array}$

Battelle Pacific Northwest Labs, Richland, 206 WA (USA)

Pennsylvania State Univ, University Park (USA). Materials Research Lab. 289 ONWI-305

Paoifie Northwest Lab., Riehland, WA $\begin{array}{ll}\text { (USA) } & \\ 296 & \text { PNL-3000-8 } \\ 297 & \text { PNL-4098 }\end{array}$

Battelle Pacific Northwest I abs, Bichland, WA (USA)

Pacific Northwest Lab, Richland, WA

$\begin{array}{ll}\text { (USA) } & \\ 299 & \text { PNL-4146 } \\ 300 & \text { PNL-4249 } \\ 301 & \text { PNL-4250-1 } \\ 302 & \text { PNL-4250-2 } \\ 303 & \text { PNL-4250-3 } \\ 304 & \text { PNL-4299 } \\ 306 & \text { PNL-4423 } \\ 307 & \text { PNL-4442 } \\ 308 & \text { PNL-4530-Vol.1 } \\ 309 & \text { PNL-4530-Vol.2 } \\ 310 & \text { PNL-4819 } \\ 311 & \text { PNI-SA-8529 } \\ 312 & \text { PNL-SA-9045 } \\ 313 & \text { PNL-SA 9545 } \\ 314 & \text { PNL-SA-9811 } \\ 315 & \text { PNL-SA-10199 } \\ 316 & \text { PNL-SA-10249 } \\ 317 & \text { PNL-SA-10261 } \\ 318 & \text { PNL-SA-10269 } \\ 319 & \text { PNL-SA-10290 } \\ 320 & \text { PNL-SA-10303 } \\ 321 & \text { PNL-SA-10316 } \\ 322 & \text { PNL-SA-10365 } \\ 323 & \text { PNL-SA-10489 } \\ 324 & \text { PNL-SA-10521 } \\ 325 & \text { PNL-SA-10528 } \\ 326 & \text { PNL-SA-10712 }\end{array}$


Contract No.

Abstract No.

Report No

PNL-SA-10777

PNL-SA-10829

PNL-SA-10934

PNL-SA-11136

PNL-SA-11199

Materials Research Society Symposia Proceedings 15 $407-414$ (1983).

AC06-76RL01830

ACO6-76RL01830

329

331

372

Pacific Northwest Laboratory, Richland, Washington 99352

377 Journal of the American Ceramic Society 65 NO. 12. 589-593 (Dec 1982).
RE/SPEC, Inc., Rapid City, SD (USA) S41 BMI/OCRD-9

Battelle Columbus Labs., OH (USA) 556 CONF-830528-18

Battelle Memorial Inst., Columbus, OH (USA). Office of National Waste Terminal Storage Integration

595 DOE/NWTS-33(1)(Final)

596 DOE/NWTS-33-3

597 DOE/NWTS-33(4a)

598 DOE/NWTS-96-Vol.1

599 - DOE/NWTS-96-Vol.2

600 DOE/NWTS-147(1)

Department of Energy, Oak Ridge, TN

(USA). Technical Information Center

607 DOE/TIC-4621-Vol.I

610 DOE/TIC-4621-Vol. I(1981).

614 DOE/TIC-4621-Vol. I(1981).

Battelle Memorial Inst., Columbus, 0 H

(USA). Office of National Waste Terminal Storage Integration

769 ONI-3

Battelle Memorial Inst., Columbus, $\mathrm{OH}$

(USA). Office of Nuclear Waste Isolation

$770 \quad$ ONWI-2-Suppl.3

775 ONWI-9-81-3

776 ONWI-9(81-4)

777 ONWI-9(82-1)

778 ONWI-9(82-2)

779 ONWI-9(82.3)

780 ONWI-9(82-4)

781 ONWI-9(83-1)

NUS Corp., Gaithersburg, MD (USA)

782 ONWI-102-Vol.1

783 ONWI-102-Vol.2

Battelle Memorial Inst., Columbus, $\mathrm{OH}$

(USA). Office of Nuclear Waste Isola-

$\begin{array}{cc}\text { tion } & \\ 784 & \text { ONWI-106 } \\ 785 & \text { ONWI-109 }\end{array}$

RE/SPEC, Inc, Rapid City, SD (ISA)

786 ONWI-139

Battelle Memorial Inst., Columbus, $\mathrm{OH}$

(USA). Office of Nuclear Waste Isolation

787

ONWI-21 2

D'Appolonia Consulting Engineers, Inc.,

Pittsburgh, PA (USA)

802 UNW̄I-217

RE/SPEC, Inc., Rapid City, SD (USA)

803 ONWI-235

Battelle Columbus Labs., OH (USA)

804 ONWI-251.
Contract No.

Abstract No. Report No.

Arkansas Univ., Fayetteville (USA)

Dept. of Agricultural Economics and

Rural Sociology

805 ONWI-269

Science Applications, Inc., La Jolla, CA (USA)

806

ONWI-302

Battelle Memorial Inst., Columbus, $\mathrm{OH}$ (USA). Office of Nuclear Waste Isolation

807 ONWI-311

General Atomic Co., San Diego, CA (USA)

808 ONWI-334-Vol.2-App.

Law Engineering Testing Co, Marietta, GA (USA)

810

ONWI-356

RE/SPEC, Inc., Rapid City, SD (USA)

811 ONWI-383

Lawrence Berkeley Lab., CA (USA)

812 ONWI-399

Woodward-Clyde Consultants, San Francisco, CA (USA)

813 ONWI-400

Louisiana State Univ, Baton Rouge (USA). Inst. for Environmental Studies

$814 \quad$ ONWI-416

Pacific Northwest Lab., Richland, WA (USA)

815 ONWI-422

Westinghouse Electric Corp., Pittsburgh, PA (USA). Advanced Energy Systems Div.

816 ONWI-423

817 ONWI-438

818 ONWI-439

INTERA Environmental Consultants, Inc., Houston, TX (USA)

819 ONWI-452

Battelle Memorial Inst., Columbus, OH (USA), Office of Nuclear Waste Isolation

$820 \quad$ ONWI-455

821 ONWI-462

823 ONWI-464

824 ONWI-483

825 ONWI-490

826 ONWI- 493

Pacific Northwest Lab., Richland, WA (USA)

841 PNL-3172

$842 \quad$ PNL-4266

$843 \quad$ PNL-4364

$844 \quad$ PNL-4379

$853 \quad$ PNL-4450

$854 \quad$ PNL-4452

$855 \quad$ PNL-4556

$856 \quad$ PNL-4576

857 PNL-4625

858 PNL=SA=10375

859 PNL-SA-10649

860 PNL-SA-10913

861 PNL-SA-11273

862 PNL-SA-11297

863 PNL-SA-11308

864 PNL-SA-11579

865 PNL-SA-11648

NUS Corp., Rockville, MD (USA)

1320 ONWI-27

Bechtel National, Inc., San Francisco, CA (USA)

1321

ONWI -192 
Contract No.

ACQ6-77RL01030

\begin{tabular}{|c|c|}
\hline Abstract No. & Report No. \\
\hline $\begin{array}{l}1322 \\
1323\end{array}$ & $\begin{array}{l}\text { ONWI-193 } \\
\text { ONWI-194 }\end{array}$ \\
\hline $\begin{array}{l}\text { Battelle Colur } \\
1324\end{array}$ & $\begin{array}{l}\text { bus Labs., OH (USA) } \\
\text { ONWI-348 }\end{array}$ \\
\hline $\begin{array}{c}\text { Bechtel Grout } \\
\text { (USA) } \\
1326\end{array}$ & $\begin{array}{l}\text { Inc., San Francisca, CA } \\
\text { ONWI-470 }\end{array}$ \\
\hline $\begin{array}{c}\text { Battelle Pacif } \\
\text { WA (US } \\
1380\end{array}$ & $\begin{array}{l}\text { Northwest Labs., Richland } \\
\text { ONWI-126 }\end{array}$ \\
\hline $\begin{array}{c}\text { Pacific North } \\
\text { (USA) } \\
1384\end{array}$ & st Lab., Richland, WA \\
\hline
\end{tabular}

Rockwell International Corp., Richland, WA (USA), Rockwell Hanford Operations 9 RHO-PB-SR-10-BSUMJUN-83

334 RHO-C -47

335 RHO-PB-SR-10-B-WMJUN-83

336 RHO-PB-SR-10-B-WMSept.-83

Rockwell ln̈tènational Corp., Kichland, WA (USA). Energy Systems Group 337 RHO-WM-SR-82-1-2Q-P 338 RHO-WM-SR-82-1-3QP

Rockwell International Corp., Richland, WA (USA). Rockwell Hanford Operations 601 DOF./RI.-82-3-Vnl.I 602 DOE/RL-82-3-Vol.2 603 DOE/RL-82-3-Vol.3

Pacific Northwest Lab., Richland, WA (USA)

868 RHO-BW-CR-127-P

Woodward-Clyde Consultants, San Francisco, CA (USA)

869 RHO-BW-CR-129P

Geological Survey, Mento Park, CA (USA). Office of Earthquake Studies 870 RHO-BW-CR-132-P

Parifir Northwest Lab., Riclibinl, WA (USA)

871 RHO-BW-CR-133P

Westinghouse Electric Corp., Pittsburgh, PA (USA). Advanced Energy Systems Div. 872 RHO-BW-CR-136-P

Rockwell International Corp., Canoga Park, CA (USA). Energy Systems Group 873 RHO-BW-SA-1 87P

Rockwell International Corp., Richland, ẆA (USA). Rockwell Hanford Operations 874 RHO-BW-SA-203-P 875 RHO-RW-SA-204-P 876 RHO-BW-SA-205A-P 877 RHO-BW-SA-209-P 878 RHO-BW-SA-216P 879 RHO-BW-SA-219-P

Rockwell International Corp, Richland, WA (USA). Energy Systems Group 880 RHO-BW-SA-220P

Rockwell International Corp., Richland, WA (USA). Rockwell Hanford Operations 881 RHO-BW-SA-229P 882 RHO-BW-SA-231A-P 883 RHO-BW-SA-231-P

Rockwell International Corp, Richland, WA

(USA). Energy Systems Group 884 RHO-BW-SA-254-P 885 RHO-BW-SA-255-P
Contract No.

Abstract No. Report No.

$\begin{array}{ll}886 & \text { RHO-BW-SA-256-P } \\ 887 & \text { RHO-BW-SA-258-P } \\ 888 & \text { RHO-BW-SA-259-P } \\ 889 & \text { RHO-BW-SA-267-P }\end{array}$

Rockwell International Corp., Richland, WA (USA). Rockwell Hanford Operations
890 RHO-BW-SA-271P
891 RHO-BW-SA-277P
892 RHO-BW-SA-281-P
893 RHO-BW-SA-293P
894 RHO-BW-SA-298A-P

Rockwell International Corp., Canoga Park, CA (USA). Energy Systems Group 895 RHO-BW-SR-82-1-1Q-P

Rockwell International Corp., Richland, WA (USA). Rockwell Hanford Operations 896 RHO-BW-SR-82-2-1Q-P

Rockwell International Corp., Richland, WA (USA), Energy Systems Group 897 RHO-BW-SR-82-2-2Q-P $898 \quad$ RHIO-DW $=3 R=82=2=9 Q-P$

Ruikmell Interuadional Curp., Richland, WA

$\begin{array}{ll}\text { (USA). Rockwell Hanford Operations } \\ 899 & \text { RHO-BW-SR-82-2-4QP } \\ 900 & \text { RHO-BW-SR-83-1-1Q-P } \\ 901 & \text { RHO-BW-ST-19-P } \\ 902 & \text { RHO-BW-ST-21-P } \\ 903 & \text { RHO-BW-ST-25P } \\ 905 & \text { KHU-BW-ST-27-P } \\ 906 & \text { RHO-BW-ST-33-P } \\ 907 & \text { RHO-BW-ST-36P } \\ 908 & \text { RHO-BWI-81-100-2Q } \\ 909 & \text { RHO-BWI-81-100-3Q }\end{array}$

Rockwell Internationnal Corp., Richland, WA (USA). Energy Systems Group 910 RHO-BWI-81-100-4Q

Rockwell International Corp., Richland, WA (USA). Rockwell Hanford Operations 911 RHR-BWI-S=105

Rockwell International Corp., Rirhland, WA (USA). Energy Systems Group 912 RHO-RWI-C-118

Rockwell International Corp., Richland, WA (USA). Rockwell Hanford Operations 913 RHO-BWI-LD-32

Rockwell International Corp., Golden, CO (USA). Rocky Flats Plant 914 RIO-BWI-LD-50

Rockwiell International Corp., Richland, WA (USA). Rockwell Hainford Operations

$\begin{array}{ll}915 & \text { RHO-BWI-SA-158 } \\ 917 & \text { RHO-BWI-ST-1月 } \\ 918 & \text { RHO-RE-ST-1-P }\end{array}$

Rockwell İntemationál Corp., Richland, WA (USA). Energy Systems Group 919 RHO-RF.ST-4P

Rockwell International Corp., Richland, WA (USA). Rockwell Hanford Operations 920 RHO-ST-34

Rockwèli International Corp., Richland, WA (USA), Energy Systems Group 1327 RHO-BW-SA-188 1328 RHO-BW-ST-3SP

Atomics International Div., Richland, WA (USA). Rockwell Hanford Operations 1329 RHO-BW-ST-39P 1429 RHO-BWI-C-121 
Contract No.

16-80RL10000

AC07-76ID01570

AC07-791D01675

AC07-81NE44139

AC08-81NV10162

AC08-81NV10171

AC08-82NV10250

AC08-83NV10282

AC09.76SRं00001
Abstract No. Report No.

Rockwell Intemational Corp., Canoga Park, CA (USA). Energy Systems Group

1425 RHO-BW-CR-122-P

Idaho National Engineering Lab, Idabo Falis (USA)

199 EGG-FM-5758

EG and G Idabo, Inc., Idabo Falls (USA)

200 EGG-FM-6045

$201 \quad$ EGG-M-07881

569 DOE/LLW-18T-Rev.1

570 DOE/LLW-18T-Rev.2

1312 EGG-FM-6202

Idaho National Engineering Lab., Idaho Falls (USA)

70 IDO-10105

Exxon Nuclear Idaho Co., Inc., Idaho Falls (USA)

$\begin{array}{ll}202 & \text { ENICO-1123 } \\ 203 & \text { ENICO-1131 } \\ 204 & \text { ENICO-1139 } \\ 240 & \text { Juel-Conf-42-Vol.1(Jun } \\ & 1981 \text { ). }\end{array}$

Westinghouse R and D Center, Pittsburgh, PA

$370 \quad$ Materials Research Society Symposia Proceedings 15 663-668 (1983).

Department of Energy, Las Vegas, NV (USA). Nevada Operations Ofitice

$\begin{array}{ll}767 & \text { NVO-266 } \\ 768 & \text { NVO-267 }\end{array}$

Westinghouse Electric Corp., Pittsburgh, PA (USA), Advanced Energy Systems Div. 551 CONF-820544-1

Westinghouse Electric Corp., Mereury, NV (USA). Advanced Energy Systems Div.

$\begin{array}{ll}60 & \text { DOE/NV/10250-1 } \\ 61^{\circ} & \text { DOE/NV/10250-2 } \\ 578 & \text { DOE/NV/10250-4 } \\ 579 & \text { DOE/NV/10250-5 } \\ 580 & \text { DOE/NV/10250-7 }\end{array}$

Westinghouse Electric Corp., Mercury, NV (USA). Waste Technology Services Dir. 581 DOE/NV/10250-8 582 DOE/NV/10250-9

EC and G, Inc., Goleta, CA (LISA)

1302 CONF-830329-1

EG and G, Inc., Goleta, CA (USA), Energy Measurements Group

1309 EGG-1183-2419

1310 EGG-1183-2460

$1311 \quad$ EGG-10282-2004

Du Pont de Nemours (E.I.) and Con Aiken, SC (USA). Savannah River Lab.

$\begin{array}{ll}22 & \text { DP-MS-82-60 } \\ 23 & \text { DPST-82-294 }\end{array}$

Du Pont de Nemours (E.I.) and Co., Aiken, SC (USA). Savannah River Plant 63 DP-80-125-3

Du Pont de Nemours (E.I.) and Co., Alken, SC (USA). Savannah River Lab. 64 DP-81-125-3

Du Pont de Nemours (E.I.) and Co, Aiken, G5 SC (USA) DP-82-125-4

Du Pont de Nemours (E.I.) and Co., Aiken, SC (USA). Sarannah River Lab. $\begin{array}{ll}66 & \text { DP-82-157-1 } \\ 67 & \text { DP-MS-81-57 }\end{array}$
Contract No.

Abstract No. Report No.

$\begin{array}{ll}68 & \text { DP-MS-83-44 } \\ 69 & \text { DP-MS-83-55 }\end{array}$

USDOE Technical Information Center, Oak Ridge, TN

142

CONF-821005-(1982).

Du Pont de Nemours (E.I.) and Co, Alkein SC (USA). Savanmah River Plant $157^{\circ}$ DOE/SR/WM-82-1

Du Pont de Nemours (E.I.) and Co, Alken, SC (USA). Savannah River Lab.

162 : DP-80-125-4

$\therefore 163$ DP-81-157-3

164 DP-81-157-4

165 DP-82-157-2

166 DP-82-157-3

167 DP-1541

Du' Pont de Nemours (E.I.) and Co, Alken, SC (USA). Savannah River Plant 168 DP-1606-Rev.1

Du Pout de Nemours (E.I.) and Co., Allien, SC (USA). Sivannah River Lab.

169 DP-1625

170 DP-1626

171 DP-1627

172 DP-1629

173 DP-1636

174 DP-MS-81-111

175 DP-MS-81-112

176 DP-MS-81-119

177 DP-MS $-81-120$

178 DP.MS-82-2

179 DP-MS-82-8

180 DP-MS-82-26-Rev.

181 DP-MS-82-31

182 DP-MS $-82-40$

183 DP-MS-82-42

184 DP-MS-82-46

185 DP-MS-82-47

186 DP-MS-82-65

187 DP-MS-82-73

188 DP-MS-82-91

189 DP-MS-82-94

190 DP-MS-82.96

191 DP-MS-83-22

Du Pont de Nemours (E.I.) and Co., WImington, DE (USA). Engineering Dept. 192 DP-MS-83-24

Du Pont de Nemours (E.I.) and Co, Allken, SC (USA) Sivannih River Lab. $193^{\circ}$ DP-MS-83-75

Du Pont de Nemours (E...) and Co, Ailken SC (USA) Savaniah River Plant $\begin{array}{ll}194 & \text { DPSPU-60-11-34 } \\ 195 & \text { DPSPU-8i-30-16 }\end{array}$

Bechtel National, Inc., San Francisca, CA (USA)

196 DPSPU-83-30-4-Rev.

Du Pont de Nemours (E.I.) and Con Alken, SC (USA) Savannah River Lab.

$\begin{array}{ll}197 & \text { DPST-81-914 } \\ 198 & \text { DPST-82-410 } \\ 397 & \text { (19 May 1982). }\end{array}$

Du Pont de Nemours (E.I.) and Co, Aiken, SC (USA)

530 DP-tr-20([nd]).

Du Pout de Nemours (E.I.) and Co, Aikea, SC (USA). Savannab River Lab. $53 i$ DP-tr-22([nd]).

Du Pont de Nemours (E.I.) and Con Aiken, SC (USA). Savannah River Plant 
Contract No.

AC09-80ET41902

AC09-81SR10957

AC97-79ET44605

Alub-78EI 44802
606 DOE/SR-WM-84-1

Department of Energy, Oak Ridge, TN

(USA). Technical Information Center 617 DOE/TIC-4621-Vol 1(1981).

Do Pont de Nemours (E.I.) and Co, Aiken, SC (USA). Sarannah River Lab.
$\begin{array}{ll}622 & \text { DP-81-125-1 } \\ 623 & \text { DP-83-125-1 }\end{array}$
$623 \quad$ DP-83-12
625 DP-MS-81-92
626 DP-MS-81-109
627 DP-MS-81-114
628 DP-MS-81-127
629 DP-MS-82-81
630 DP-MS-83-18

Florida Univ, Gainesville (USA). Dept. of Materials Science and Engineering 631 DPे-MS-83-66

Du Pont de Nemours (E.I.) and Co., Aiken, SC (USA). Sàvannah River Lab. 632 DP-MS-83-107

Du Pont de Nemours (E.I.) and Co, Aiken, SC (USA). Savannah River Plant
633
b34

Du Pont de Nemours (E.I.) and Co., Aiken, SC (USA). Sarannah River Lab.

$\begin{array}{ll}635 & \text { DPST-82-346 } \\ 636 & \text { DPSTD-82-65-Rev.1 } \\ 1370 & \text { DPST-82-327 } \\ 1371 & \text { DPST-82-475 }\end{array}$

1371 DPST-82-475

Rockwell International Corp. Canoga Part, CA (ISA). Fnergy Systems Group 147 DOE/ET/41900-12 148 DOE/ET/41900-14 149 DOE/ET/41900-16 150 DÖF/ET/41900-17

Rockwell International Corp, Thousand Gaks, CA (USA). Sclence Center 151 DOE/ET/41900-18

Rockwell International Corp., Canoga Park, CA (USA). Energy Systems Group S64. DOE/ET/41900-13

North Carolina State Univ, Rạleigh (USA) 280 NCSU-80-4

North Carolina State Univ, Raleigh (USA) 281 NCSU-81-3

Texas Univ., Austịn (USA). Bureau of Economic Geotogy 566 DOE/ET/44605-T1

Geological Survey, Washington, DC (USA) 567 DOE/ET/44802-T3

Geological Survey, Denver, Co (USA) 1022 USGS-OFR-82-409

Geological Survey, Reston, VA (USA) 1023 USGS-OFR-82-466

Geological Survey, Menlo Park, CA (USA)

1024 USGS-OFR-82-701

1025 USGS=OFR-82-931

1026 USGS-OFR-82-1041

Geological Survey, Denver, CO (USA)

1027 USGS-OFR-82-1042

1028 USGS-OFR-82-1043

Geological Survey, Reston, VA (USA)

1029 USGS-OFR-82-1044

1030 USGS-OFR-83-141
Contract No.

A197-79ET46603

AM06-76RL02225

AS09-76SR00952

AT(10-1)-205

AT(45-1)-1350

AT01-80NE93031

AT03-81SF11528

AT03-81SF11572

FG012-81ET46634

FG02-81NEA6640

W-31-109-ENG-38

W-740S-ENG-26
Abstract No. Report No.

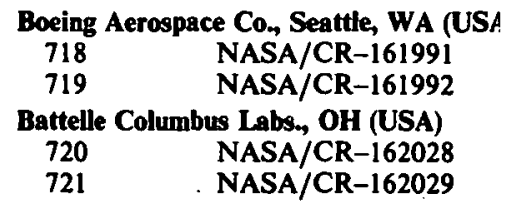

Rockmell Intemational Corp., Richland, WA (USA). Rockwell Hanford Operations 904 RHO-BW-ST-26-P

Georgia Inst. of Tech., Atlanta (USA)

6 DOE/NE/00952-7

Ohio State Univ., Columbus (USA)

$$
155 \text { DOE/SR/00952-T7 }
$$

Phillips Petroleum Co., Idaho Falls, ID (USA) Atomic Energy Div.

$$
219 \text { IDO-14620 }
$$

General Electric Co., Richland, WA (USA). Hanford Atomic Products Operation

$$
215 \text { HW-81829 }
$$

National Academy of Sciences, Washington, DC (USA)

577 DOE/NE/93031-T1

General Atomic Co., San Diega, CA (USA)

24 GA-A-16631

GA Technologies, Inc., San Diego, CA (USA)

041 GA-A-1697i

642 GA-A-17053

Rockwell International Corp. Canoga Park, CA (USA). Energy Systems Group 144 . CONF-830205-33

Wisconsin State Government, Madison - (USA)

568 DOE/ET/46634-1

Maine Dept. of Conservation, Augusta (USA). Maine Geological Survey 576 DOE/NE/46640-7

Argonne National Lab., IL (USA)

48 ANL-81-35
49
50

50 ANI $-81-82$

127 ANL-81-34

128 ANL-82-10

129 ANL-82-39

Commission of the European Communities, Karlsruhe (Germany, F.R.). European Inst. for Transuranium Elements 143 CONF-821107-50 145 CONF-830451-18

Argonne National Lab, í̄ (USA) 146 CONF-830808-5

391 (1 Jun 1982).

$536 \quad$ ANL-82-58

$538 \quad$ NNL-82-78

539 ANL-83:19

552 CONF-821053-2

559 CONF-831047-49

$1303 \quad C O N F=830375-1$

GA Technologies, Inc., San Diego, CA (USA) 14 CONF-830528-13

Oak Ridge National Lab., TN (USA)

52 CONF-820609-46

57 DOE/NF-0017-1

$76 \quad$ ORNL/TM-8061

77 ORNL/TM-8322

138 CONF-810528-16

139 CONF-811088-7

245 Juel-Conf-42-Vol.1(Jun - 1981).

256 Juel-Conf-42-Vol.2(Jun 1981). 
Contract No.

W-7405-ENG-36

W-7405-ENG-48
Abstract No.

Report No.

$$
\begin{aligned}
& 290 \\
& 291 \\
& 292 \\
& 293 \\
& 386
\end{aligned}
$$

ORNL-TM-603

ORNL-TM-757

ORNL/TM-8124

ORNL/TM-8579

American Ceramic Society

Bulletin 65 NO. 8. 394

398 (Aug 1982).

W-7405-ENG-26

Oak Ridge National Lab, TN (USA)

$\begin{array}{ll}549 & \text { CF-60-8-72 } \\ 553 & \text { CONF-821107-32 } \\ 554 & \text { CONF-821107-33 } \\ 561 & \text { CONF-8308111-2 } \\ 574 & \text { DOE/NE-0017/2 } \\ 726 & \text { NUREG/CP-0022 } \\ 737 & \text { NUREG/CR-2506 } \\ 828 & \text { ORNL-5703 } \\ 829 & \text { ORNL-5818 } \\ 830 & \text { ORNL/TM-8083 } \\ 831 & \text { ORNL/TM-8109 } \\ 832 & \text { ORNL/TM-8491 }\end{array}$

Eidgenoessisches Inst. fuer Realtorforschumg, Woerenlinges (Switzertand) 1052 ORNL-tr-4980(Feb 1983).

Oak Ridge National Lab, TN (USA) 1304 CONF-8308126-1-Draft 1366 ORNL-tr-4994([nd]). 1367 CONF-820303-27

Los Abmos National Lab.; NM (USA)

$\begin{array}{ll}278 & \text { LA-UR-83-307 } \\ 279 & \text { LALP-81-59-Rev } \\ 692 & \text { LA-9095-PR } \\ 693 & \text { LA-9174-PR } \\ 694 & \text { LA-9179-MS } \\ 695 & \text { LA-9207-MS } \\ 696 & \text { LA-9255-MS } \\ 697 & \text { LA-9322-MS } \\ 698 & \text { LA-9325-MS } \\ 699 & \text { LA-9326-MS } \\ 700 & \text { LA-9328-MS } \\ 701 & \text { LA-9396-MS } \\ 702 & \text { LA-9481-MS } \\ 703 & \text { LA-9577-PR } \\ 704 & \text { LA-9666-PR } \\ 705 & \text { LA-9793-PR } \\ 706 & \text { LA-UR-83-1304 } \\ 1314 & \text { LA-9217-MS } \\ 1315 & \text { LA-9329-MS } \\ 1406 & \text { LA-UR-82-695 } \\ 1426 & \text { LA-9332-MS }\end{array}$

Lawrence Livermore National Lab., CA

$\begin{array}{ll}\text { (USA) } & \\ 36 & \text { UCRL-53154 } \\ 37 & \text { UCRL-53248 } \\ 343 & \text { UCID-19404 } \\ 344 & \text { UCID-19492 } \\ 345 & \text { UCID-19598 }\end{array}$

Australian National Unily, Canberra. Research School of Earth Sclences 346 UCRL-15480

Lawrence Lịvermore National Lab, CA

$\begin{array}{ll}\text { (USA) } & \\ 347 & \text { UCRL-53237 } \\ 348 & \text { UCRL-53392 } \\ 349 & \text { UCRL-53405 } \\ 350 & \text { UCRL-53425 } \\ 351 & \text { UCRL=86558 } \\ 352 & \text { UCRL-87065 } \\ 353 & \text { UCRL-87742 }\end{array}$

Department of Energy, Oal Ridge, TN (USA). Technical Information Center
Contract No.

$\begin{array}{cl}\text { Abstract No. } & \text { Report No. } \\ 618 & \text { DOE/TIC-4621-Vol. } \\ & 1(1981) . \\ 619 & \text { DOE/TIC-4621-Vol. } \\ & 1(1981) .\end{array}$

Battelle Memorial Inst, Columbus, OH (USA). Office of Nuclear Waste Isolation

809 ONWI-352-Vol.1-Summ

Lawrence Livermore National Iab, CA (USA)

1002 UCID-18946

1003 UCID-19672

1004 UCID-19764

1005 UCID-19926

1007 UCRL-53188-81

1008 UCRL-53188-82

1009 UCRL-53269

1010 UCRL-53315

1011 UCRL-53326

1012 UCRL-53394

1013 UCRL-86225

1014 UCRL-86582

1015 UCRL-87091

1016 UCRL-87510

1017 UCRL-87808

1018 UCRL-88192

$1019 \quad$ UCRL-88648

1020 UCRL-89404

1021 UCRL-89656

1238 International Journal of

Rock Mechanics and

Mining Sciences and

Geomechanics Abstracts

W-7405-ENG-48 18

Lawrence Livermore National Lab, CA (USA) . UCID-19729
1332

Lawrence Berkeley Lab, CA (USA) 1422 LBL-11180

W-7405-ENG-92-110 Battelle Columbus Labs, OH (USA) 1301 BMI-X-698-Rev. 


\section{REPORT NUMBER INDEX}

The numbers assigned to reports cited in this publication appear in the first column of the index. Cross reference information and/or availability are included as appropriate. Abbreviations used in the availability column are elaborated at the end of the index. To expedite the filling of orders for reports from NTIS and TIC, an order number in the form, DE82000001 or TI82000001, appears in column 3. Order numbers with DE prefixes may be used in ordering from both NTIS and TIC. Numbers with TI prefixes are valid at TIC only. Requesters are urged to use this order number where provided. In column 4, one or more DOE distribution categories are indicated, e.8. , STD-10, MN-25. STD indicates distribution was made in full-size copy; MN indicates microcopy distribution; ND indicates no distribution was made. These category numbers are included to assist DOE librarians in responding to requests for reports in their collections. The UC (Unclassified Distribution Categories) are defined in DOE/TIC-4500, available from TIC. The citation numbers are given in column 5. NOTE: DOE attempts to disseminate its information as broadly as possible. Even though a report may contain some degree of illegibility, it is believed that the report may still make a contribution. The presence of a 1,2 , or 3 following NTIS in the availability column denotes the extent to which a report may be illegible and the form in which it is available: $1=1$ to $5 \%$ illegibility, available PC and MF; 2 - 6 to $10 \%$ illegibility, available MF only; $3=11 \%$ or more illegibility, available PC only. For the users of DOE/RECON, report ordering may be accomplished more expeditiously via the on-line system. Employees of MNF ni MnF. mntrartore gre urged to order from the Toohnioal Information Center (TIC); all others should order from the National Technical Information Service (NTIS). Procedures for on-line ordering are detailed in the DOE/ RECON User's Manual (DOE/TIC-4586).

Report No.

$\underset{526}{\text { AASC/E }}$

550

AD-A-

$106573 / 9$

AFCI.

6836

6838

AERE-R-

10222

10341

10439

A STR

944

AESD-TME3142

ANL

$81-34$

81-35

81-53

81-82

82.10

82-39

82-58

82-78

83-19

BARC-

1136

BuG-

546

BMI-

2105-Vol.1

BMI-OCRD

1

9

BMI-X.

698-Rev.

BNL

18978

19109

19323

32669

33451
Availability

NTIS (US Sales Only), PC A07/MF A01 NTIS (US Sales Only), PC A04/MF A01

NTIS, PC A04/MF A01

Atomic Energy of Canada Ltd., Whiteshell Nuclear Research Establishment, Pinawa, Manitoba ROE 1LO, Canada

Atomic Energy of Canada Ltd., Whiteshell Nuclear Research Establishment, Pinawa, Manitoba ROE 1LO, Canada

NTIS (US Sales Only), PC A03/MF A01

H.M. Stationery Office, Price Pound 6.00

Nucl. Energy (Inst. Nucl. Eng.), 21: No. 4, 253-265(Aug 1982)

N1IS (US Sales Only), PC A03/MF A01

See RHO-BW-CR-136-P

NTIS, PC A03/MF A01

NTIS, PC A06/MF A01

NTIS, PC A06/MF A01

NTIS, PC A07/MF A0i

NTIS, PC A04/MF A01

NTIS, PC A07/MF A01

NTIS, PC A07/MF A01

NTIS, PC. AnR/MF AO1; 1

NTIS, PC A09/MF A01

NTIS (US Sales Only), PC A03/MF A01

NTIS (US Sales Only), PC A03/MF A01

See NUREG/CR-3405-Vol.1

NTIS, PC A06/MF A01; 1

NTIS, PC A03/MF A01

NTIS, PC A12/MF A01

NTIS, PC A02/MF A01

NTIS, PC A02/MF A0I

NTIS, PC A02/MF A01

NTIS, PC A02/MF A01

NTIS, PC A03/MF A01
DE82703266 MN -86 46

DE83703468 MN -70 532

ND -70

1299

ND -70

1300

DE83700874 MN -70 534

1217

1080

DE83700876 MN -70 535

$\begin{array}{lll}\text { DE83006520 MN -70 } & 872\end{array}$

DE82008709 STD -70 127

DE82014373 STD -70 48

DE82014374 STD -70 49

DE83000921 STD -70 50

DE83003340 STD -70 128

DE83005939 STD -70 129

DE83011014 STD -70 536

DE83011920 STD -70 338

DE84000191 STD $-70 \quad 539$

DE82904604 MN -70 51

DE82700674 MN -70 130

DE83902820 MN -71 751

DE83010252 MN -70 $\quad 540$

DE84002553 STD -70 541

DE83004726 MN +70A $\quad 1301$

DE.82014639 MN -70 131

DE82014642 MN -70 132

DE82014637 MN -70 133

DE83008592 MN -70 542

DE83016732 MN -70 543 


eport No.
NL-NUREG-
31329
32107
33192
33579
51449
$51449-$ Vol.1-No.3
$51449-$ Vol.2-No.1
51458
$51458-$ Vol.1
$51458-$ Vol.3
$51458-$ Vol.4
51494 Vol.1
51494 Vol.2
51494 Vol.3
$51494-$ Vol.4
51544
$51658-$ Vol.1
$51658-$ Vol.2
BNWL-
80
CEA-CONF-
6223
6241
6278
6307
CF-
$60-8-72$
CNEN-RT/ING-
$81-12$
CONF-790284-

CONF-790519-

CONF-7906121-

CONF-7909192

CONF-800609.

1.9

CONF-800943-

21

CONF-8009314-

CONF-801107.
Availability

NTIS, PC A02/MF A01

NTIS, PC A02/MF A01

NTIS, PC A04/MF A01

NTIS, PC A02/MF A01 - GPO

See NUREG/CR-2317-Vol.1NOS.1-2

See NUREG/CR-2317-Vol.1-No.3

See NUREG/CR-2317-Vol.2-No.1

See NUREG/CR-2333-Vol.5

See NUREG/CR-2333-Vol.1

See NUREG/CR-2333-Vol.3

See NUREG/CR-2333-Vol.4

See NUREG/CR-2482

See NUREG/CR-2482-Vol.2

See NUREG/CR-2482-Vol.3

See NUREG/CR-2482-Vol.4

See NUREG/CR-2755

See NUREG/CR-3219-Vol.1

See NUREG/CR-3219-Vol.2

NTIS

NTIS (US Sales Only), PC A02/MF A01

NTIS (US Sales Only), PC A02/MF A01 NTIS (US Sales Only), PC A02/MF A01 NTIS (US Sales Only), PC A03/MF A01

\section{NTIS}

NTIS (US Sales Only), PC A04/MF A01

(Symposium on recent trends in hydrogeology, Berkeley, CA, USA, 8-9 Feb 1979).

Geol. Soc. Am., Spec. Pap., No. 189, 389 396(1982)

(European nuclear conference, Hamburg, F.R. Germany, 6-11 May 1979)

Trans. Am. Nucl. Soc., 31: 528-530(Nov 1979)

Trans. Am. Nucl. Soc., 31: \$41-543(1979)

Trans. Am. Nucl. Soc., 31: S39-541(1979)

Trans. Am. Nucl. Soc., 31: 331(1979)

(Permiability and groundwater containment transport symposium, Philadelphia, PA, USA, 17-23 Jun 1979)

Am. Soc. Test. Mater., Spec. Tech. Publ., 121-136(1981)

(4. international symposium on uranium and nuclear energy, London, UK, 10-12 Sep 1979) London, Engl.; Min J Books, Ltd (1980).

(10. international symposium on effects of radiation on materials, Savannah, GA, USA, 3-5 Jun 1980)

See PNL-SA-8916

(National topical meeting of fuel cycles for the 80's, Gatlinburg, TN, USA, 29 Sep-2 Oct 1980)

See PNL-SA-8529

(Nuclear Waste Terminai Storage (NWTS)

field testing in situ site confirmation tests workshop, Columbus, OH, USA, 8-9 Sep 1980)

See ONWI-217

(ANS international conference, Washington, DC, USA, 17-21 Nov 1980)

Trans. Am. Nucl. Soc., 35: 8(1980)

Trans. Am. Nucl. Soc., 35: 68-69(1980)

Trans. Am. Nucl. Soc., 35: 191-193(1980)

Trans. Am. Nucl. Soc., 37: 165(1981)

Trans. Am. Nucl. Soc., 35: 9(1980)

Trans. Am. Nucl. Soc., 35: 63-65(1980)

Trans. Am. Nucl. Soc., 35: 66-67(1980)

Trans. Am. Nucl. Sn, 35: 1n4-1n6(198n)

See PNL-SA-9045
Order No. Distribution Category

Abstract No.

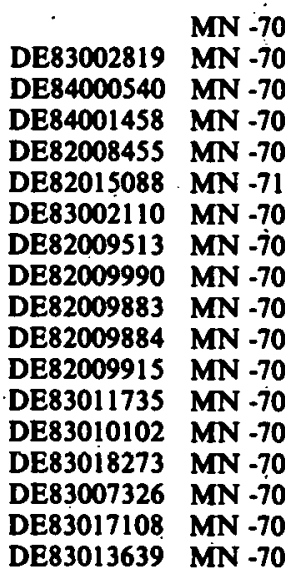

544

545

546

547

729

728

730

285

283

73

284

286

732

735

736

739

749

1319

134

DE83700877 MN -70

DE82703060 MN -70

DE83701825 MN -70

DE83701042 MN -70

548

135

136

137

549

DE82902983 MN -70

550

1144

528

1294

1295

1404

1277

1.

DE82010732 MN -70

86

DE83002679 MN -70

DE83012618 MN -70

802

45

525

526

1275

1289

1290

1365

ian?.

DE83002702 MN -70 312 
Report No.

CONF-801209-

CONF-810217.

CONF-810273-

CONF-810372

CONF-810528-

16

CONF-810606-

CONF-810650-

Vol.1

Vol.1

CONF-810706-

CONF-8109120-

20

CONF-811025.

28-Draft

CONF-811088-
Availability

Order No.

Distribution Category

Abstract No.

(2. annual National Waste Terminal Storage (NWTS) information meeting, Columbus,

OH, USA, 9-11 Dec 1980)

See ONWI-212

DE83001317 MN -70

787

(ANS waste management conference, Tucson, AZ, USA, 23-26 Feb 1981)

Proc. Symp. Waste Manage., 1: 365373(1981)

Proc. Symp. Waste Manage., 1: 249 266(1981)

Proc. Symp. Waste Manage., 1: 277 292(1981)

Proc. Symp. Waste Manage., 1: 321335(1981)

Proc. Symp. Waste Manage., 2: 825-

836(1981)

Proc. Symp. Waste Manage., 1: 87-108(1981)

Proc. Symp. Waste Manage., 1: 487.

495(1981)

Proc. Symp. Waste Manage., 2: 1135 -

1145(1981)

(Meeting on radioactive wastes, Cadarache,

France, 2-6 Feb 1981)

Fontenay-aux-Roses, France; Societe Fran-

caise de Radioprotection (1981).

(Symposium on uncertainties associated with the regulation of the geologic disposal of highlevel radioactive waste, Gatlinburg, $T N$,

USA, 9-15 Mar 1981)

See NUREG/CP-0022

(83. American Ceramic Society symposium on nucleation and crystallization in glasses,

Washington, DC, USA, 3-6 May 1981)

NTIS, PC A03/MF A01

(American Nuclear Society's annual meeting, Miami Diacli, FL, USA, 7:12 Jun 1981)

Trans. Am. Nucl. Soc., 38: 21-22(1981)

Trans. Am. Nucl. Soc., 38: 130-13i(1981)

Trans. Am. Nucl. Soc., 38: 131(1981)

Trans: Am. Nucl. Soc., 38: 132-133(1981)

Trans. Am. Nucl. Soc., 38: 133-134(1981)

Tram. All. Nucl. Suv., 38: 134(1981)

Trans. Am. Nucl. Soc., 38: 170-171(1981)

Trans. Am. Nucl. Soc., 38: 715-716(1981)

Trans. Am. Nucl. Soc., 38: 87-88(1981)

Trans. Am. Nucl. Soc., 38: 89-90(1981)

Trans. Am. Nucl. Soc., 38: 167-168(1981)

Trans. Am. Nucl. Soc., 38: 106-107(1981)

Trans. Am. Nucl. Soc., 38: 362-363(1981)

Trans. Am. Nucl. Soc., 38: 363-364(1981)

Trains. Am. Nucl. SOc., 38: 163-164(1981)

Trans. Am. Nucl. Soc., 38: 84-85(1981)

(International seminar on chemistry and process engineering for high-level waste solidification. Julioh. F,R. Germany, 1-5 Jun 1281)

See Juel-Conf-42-Vol.1

See Juel-Conf-42-Vol.2

(Institute of Nuclear Materials Management conference, San Prancisco, CA, USA, 13-16 Jul 1981)

Nucl. Mater. Managé., 10: 365-371(1981)

(Nuclear Internationàl Jura '81, Palma de

Mallorca, Spain, 27 Sep-1 Oct 1981)

See INIS-mf-7034

(25. conference on analytical chemistry and nuclear technology, Gatlinburg, TN, USA, 6 Oct 1981)

See DPSPU-81-30-7

(American Ceramic Society conference, Newport Beach, CA, USA, 25-28 Oct 1981)

See PNL-SA-9545

NTIS, PC A02; 3
DE82008883 MN -70

DE82015675 MN -70 
- ort No.

Availability

Order No

Distribution Category

Abstract No

NF-8110162-

1

CONF-811103-

120

CONF-811122.

Pt.1

CONF-820154

1

CONF-820270-

3

CONF-820303-

7

22

27

28

29

34

39

CONF-820359-

1

CONF-820420-

CONF-820422-

1

2

CONF-820424

3

4

13

17

21

22

23

26

31-Rev.

34

35

38

CONF-820544
(52. annual executive conference; Palm

Springs, CA, USA, 19-21 Oct 1981)

NTIS, PC A02/MF A01

(ANS winter meeting, San Francisco, CA, USA, 29 Nov-4 Dec 1981)

Trans. Am. Nucl. Soc., 39: 192(1981)

Trans. Am. Nucl. Soc., 39: 180-182(1981)

Trans. Am. Nucl. Soc., 39: 224-225(1981)

Trans. Am. Nucl. Soc., 39: 225-226(1981)

Trans. Am. Nucl. Soc., 39: 171(1981)

Trans. Am. Nucl. Soc., 39: 173-174(1981)

Trans. Am. Nucl. Soc., 39: 174-176(1981)

Trans. Am. Nucl. Soc., 39: 228(1981)

Trans. Am. Nucl. Soc., 39: 354-355(1981)

Trans. Am. Nucl. Soc., 39: 177-179(1981)

Trans. Am. Nucl. Soc., 39: 179-180(1981)

Trans. Am. Nucl. Soc., 39: 353-354(1981)

Trans. Am. Nucl. Soc., 39: 176-177(1981)

See DP-MS-81-57

(Annual meeting of the Materials Research

Society, Boston, MA, USA, 16-19 Nov 1981)

New York, NY; Elsevier Science Publishing

Company, Inc. (1982).

-

phia, PA, USA, 19 Jan 1982)

See UCRL-86582

(SFRP meeting on radioactive sources, Paris,

France, 16 Feb 1982)

See CEA-CONF-6223

(Waste management conference (ANS),

Tucson, AZ; USA, 8-11 Mar 1982)

See SAND-82-0510C

See DP-MS-81-92

NTIS, PC A02/MF A01

See SAND-81-2169C

See SAND-82-0627C

See SAND-81-2163C

See EGG-M-07881

(Western regional American Nuclear Society student conference, Corvallis, OR, USA,

28-30 Mar 1982)

See LBL-14080

(International ENS/ANS conference on

nuclear energy with emphasis on fuel cycles, Brussels, Belgium, 26-30 Apr 1982)

Trans. Am. Nucl. Soc., 40: 83-86(1982)

Trans. Am. Nucl. Soć., 40! 99-101(1982)

(American Society of Civil Engineers confer-

ence, Las Vegas, NV, USA, 26-30 Apr 1982)

See SAND-82-0621C

See LBL-14082

(ANS topical meeting on treatment and handling of radioactive wastes, Richland, WA,

USA, 19-22 Apr 1982)

NTIS, PC A99/MF A01

See LA-UR-82-695

See UCRL-86558

See PNL-SA-9811

See PNLSA-10316

See DP-MS-82-31

See PNL_SA-10261

See PNL-SA-10269

See PNL-SA-10365

See DPSPU-81-30-16

See DP-MS-82-26-Rev.

See CEA-CONF-6241

See RHO-BW-SA-209-P

See PNL-SA-10ig9

(International conference on welding technology for energy applications, Gatlinburg, TN, USA. 16-19 May 1982)

NTIS, PC A03/MF A01
(Society of Civil Engineers conference on
DE82902176 MN -70 560

DE82008186 MN -70

44
120
121
122
503
504
505
506
508
1260
1261
1262
1401
67

107

DE82020176 MN -70

1014

DE83700877 MN -70

548

DE82011469 MN -70

30

DE82008917 MN -70

DE82008810 MN -70

DE82011947 MN -70

DE82011948 MN -70

DE82012224 MN -41

DE82016342 MN -70

DE82013007 MN -70

711

1142

1143

DE82008191 MN -70

951

DE82008873 MN -70

712

DE83007269

DE82011988 MN -70

DE82012263 MN -70

DE82012854 MN -70

DE82013725 MN -70

DE82014172 MN -70

DE82014847 MN -70

DE82014856 MN -70

DE82014855 MN -70

DE82017676 MN -70

DE82019158 MN -70

DE82703060 MN -70

DE82021716 MN -70

DE83002723 MN -7U

314

321

181

317

318

322

195

180

135

877

315 
Report No.

CONF-820547-

1
2
5
7
8

CONF-820550-

1

5

CONF-820570-

3

CUNF-6205132-

CONF-8205164

1

CONF-820609-

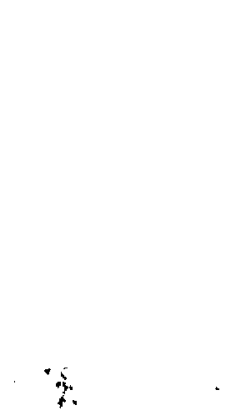

\footnotetext{
17

20

46

56

58

60

CONT-820615-

2

CONF-820636-
}

Availability

Order No. Distribution Category

Abstract No.

(84. annual meeting of the American Ceramic Society, Cincinnati, OH, USA, 2-5 May

1982)

See DP-MS-81-109

See DP-MS-81-114

See DP-MS-81-111

See DP-MS-81-119

Sée DP-MS-81-120

(Geological Society of America Rocky Mountain section meeting, Bozeman, MT, USA, 7-8 May.1982)

See RHO-BW-SA-188

See RHO-BW-SA-187P

(Materials Characterization Center workshop on leaching, Gaithersburg, MD, USA, 19-21 May 1982.)

See SAND-82-1199C

(Annual meeting of the German Society of Metallurgical and Mining Engineers (Gesellschaft Deutscher Metallhuetten- und Bergleute), Freiburg/Breisgau, F.R. Germany, 12-15 May 1982)

Erzmetall, 35: No. 11, 570-575(Nov 1982)

(French Nuclear Energy Society meeting on nuclear waste, Paris, France, 13 May 1982) See CEA-CONF-6278

(American Nuclear Society annual meeting, Los Angeles, CA, USA, 6-11 Jun 1982)

Trans. Am. Nucl. Soc., 41: 126(Jun 1982)

Trans. Am. Nucl. Soc., 41: 126-127(Jun 1982)

Trans. Am. Nucl. Soc., 41: 127-129(Jun 1982)

Trans. Am. Nucl. Soc., 41: 129-130(Jun 1982)

Trans. Am. Nucl. Soc., 41: 226(Jun 1982)

Tran̉s. Am. Nucl. Soc., 41 : 88(Jun 1982)

Trans. Am. Nucl. Soc., 41: 87(Jun 1982)

Trans. Am. Nucl. Soc., 41: 157-158(Jun 1982)

Trans. Ain. Nucl. Soc., 41: 279-280(Jun 1982)

Trans. Am. Nucl. Soc., 41: 278(Jun 1982)

Trans. Am. Nucl. Soc., 41: 85(Jun 1982)

Trans. Am. Nucl. Soc., 41: 229(Jun 1982)

Trans. Am. Nucl. Soc., 41: 230(Jun 1982)

Trans. Am. Nucl. Soc., 41: 231(Juñ 1982)

Trans. Am. Nucl. Soc., 41: 232(Jun 1982)

Trans. Am. Nucl. Soc., 41: 94(Jun 1982)

Trans. Am. Nucl. Soc., 41: 95(Jun 1982)

Trans. Am. Nucl. Soc., 41: 95(Jun 1982)

Trạns. Am. Nucl. Soc., 41: 96(Jun 1982)

Trans. Ám. Nucl. Soc., 41: 98(Jun 1982)

Trans. Am. Nucl. Soc., 41: 154-155(Jun 1982)

Trans. Am. Nucl. Soc., 41: 160(Jun 1982)

Trans. Am. Nucl. Soc., 41: 280(Jun 1982)

Trans. Am. Nucl. Soc., 41: 283(Jun 1982)

Trans. Am. Nucl. Soc., 41: 284-285(Jun 1982)

Trans. Am. Nucl. Soc., 41: 285்(Jun 1982)

Tràns. Am. Nucl. Soc., 41: 276-277(Jun 1982)

Tràns. Am. Nucl. Soc., 41: 277-278(Jun 1982)

Trans. Am. Nucl. Soc., 41: 153-154(Jun 1982)

Trans. Am. Nucl. Soc., 41: 156-157(Jun 1982)

See RHO-BW.SA-203-P

See RHO-BW-SA-204-P

NTIS, PC A02/MF A01

See DP-MS-82-2

See PNL-SA-10249

See PNL-SA-10184S

See UCRL-8709]

(26. American Association of Cost Engineers annual meeting, Houston, TX, USA, 27-30 Jun 1982)

See GA-A-16631

(5. international symposium on the scientific basis for radioactive waste management, Berlin, F.R. Germany, 7-10 Jun 1982)

$\begin{array}{lll}\text { DE82014176 } & \text { MN -70 } & 626 \\ \text { DE82013897 } & \text { MN -70 } & 627 \\ \text { DE82014442 } & \text { MN -70 } & 174 \\ \text { DE82015511 } & \text { MN -70 } & 176 \\ \text { DE82015512 } & \text { MN -70 } & 177\end{array}$

DE82014486 MN -70 1327

DE82016062 MN -70 873

DE82018589 MN -70

971

1072

$\begin{array}{lll}\text { DE83701825 MN -70 } & 136\end{array}$

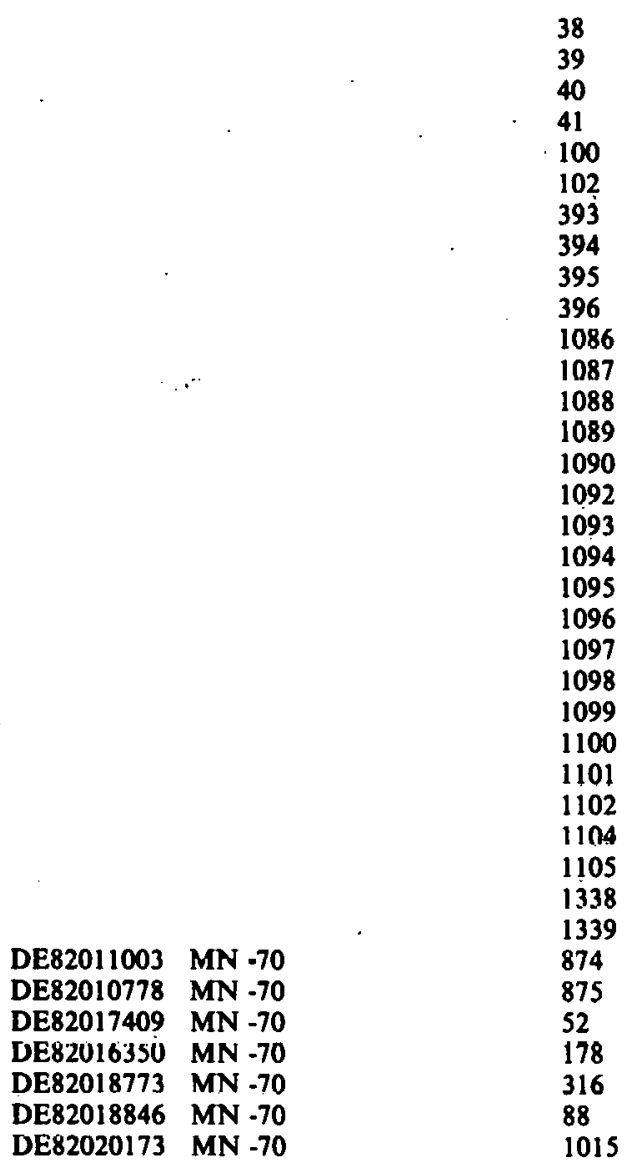

DE82017574 MN -70 
Damart No.

Availability

Onder No

Distribution Category

Abstract No.

Elsevier Science Publishing Co., Inc., 52 ,Van derbilt Ave., New York, NY 10017

See UCRL-87065

NTIS, PC A02/MF A01

$\therefore \quad$ See DP-MS-81-127

$\therefore$

CONF-820651-

See PNLSA-10489

(International symposium on the conditioning of radioactive wastes for storage and disposal, Utrecht, Netherlands, 21-25 Jun 1982)

Vienna, Austria; International Atomic Energy

Agency (1983).

See SAND-82-0158C

CONF-820678-

2

CONF-8206106-

CONF-8206149-

3

CONF-820705-

i

CONF-820803-

3

15

CONF-820833-

15

CONF-820864

3

CONF-820891-

5

CONF-820903-

3

CONF-820943-

CONF-820955-

2

CONF-820964

2

CONF-8209134-

18

19

CONF-821011-

(International symposium on bydrometerology. Denver, CO, USA, 13-17 Jun 1982)

See PNLSA-10124

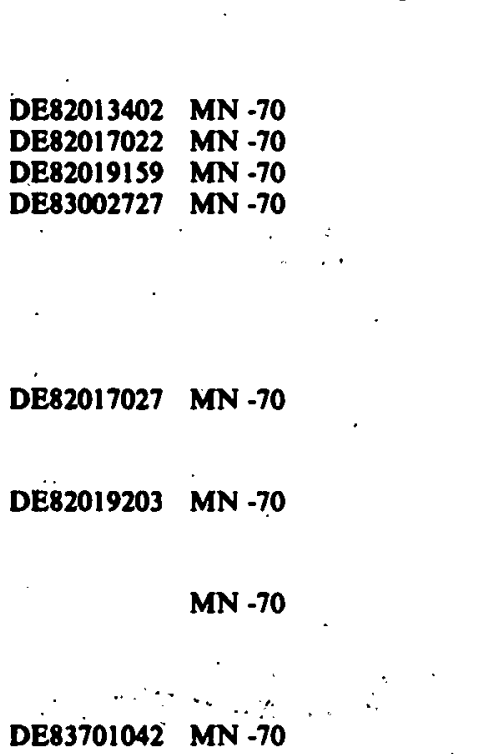

(14. conference on nuclear safety, Tokyo, Japan, 4 Jun 1982)

See BNL-NUREG-31329

(21. Euratom workshop on hot laboratories and remote handling, Mol, Belgium, 10-11 Jun 1982)

See CEA-CONF-6307

(2. joint ASME/ANS nuclear engineering conference, Portland, OR, USA, 25-28 Jul 1982)

See SAND-81-2133

(23. US symposium on rock mechanies, Berkeley, CA, USA, 25-27 Aug 1982)

See UCRL-87510

See SAND-82-0312C

Sée RHO-BW-SA-231-P

(17. DOE nuclear air cleaning conference, Denver, CO, USA, 1-6 Aug 1982)

See PNL-SA-10290

(4. europhysical topical conference on lattice defects in ionic crystals, Dublin, Ireland, 30 Aug-3 Sep 1982)

See SAND-82-1045C

(18. ceramic science conference on advances in materials characterization, Alfred. NY, USA, 16-18 Aug 1982)

See DP-MS-82-8

(ANS topical meeting, Niagara Falls, NY, USA, 22-24 Sep 1982)

See DP-MS-81-112

(ANS topical meeting - spent fuel storage,

Savannah, GA, USA, 26-29 Sep 1982)

\section{See PNL-SA-10649}

(ASTM conference on titanium and zirconium in industrial applications, New Orleans, LA, USA, 21-23 Sep 1982)

See BNL-NUREG-32107

See PNLSA'10375

(Joint Pacific Northwest American Geophysical Uniiuii ánd Amerièun Assuciatioun für the Advancement of Science meeting, Fairbanks, AK, USA, 14-18 Sep 1982)

See KHO-BW-SA-220P

See RHO-BW-SA-229P

DE83000718 MN -70

880

DE82021719 MN -70

881

(IAEA interregional training course on waste management in nuclear facilities, Karlsruhe, F.R. Germany, 6 Sep-1 Oct 1982)

Karlsruhe, Germany; Fachinformationszentrum fuer Energie, Physik, Mathematik (1982).

See INIS-mf-8524

DE83002819 MN -70 
Report No.

CONF-821052-

2

CONF-821053-

2

CONF-821090

1

CONF-821103-

33

89

92

CONF-821107.

4
6
11
32
33
38
40
42
44
50
52
Pt.1
Pt.1
Pt.1
Pt.1
Pt.1
Pt.1
Pt.1
Availability

Order No.

Distribution Category

Abstract No.

(4. international workshop on immunedeficient animals in experimental research, Chexbres, Switzerland, 31 Oct-3 Nov 1982) See LA-UR-83-1304

DE83012682 MN -70

706

(Workshop on standardization of methods for measuring migration of radionuclides in geomedia, Berlin, F.R. Germany, 25-29 Oct 1982)

NTIS, PC A02/MF A01

DE83014287 MN -70

552

Institute for Nuclear Materials Management seminar on spent fuel management and waste disposal, Washington, DC, USA, 20-22 Oct 1982)

See SAND-82-2238C

DE83002185 MN -70

(American Nuclear Society winter meeting, Washington, DC, USA, 14-19 Nov 1982)

Trans. Am. Nucl. Soc., 43: 124-125(1982)

Trans. Am. Nucl. Soc., 43: 109(1982)

Trans. Am. Nucl. Soc., 43: 110-111(1982)

Trans. Am. Nucl. Soc., 43: 69-70(1982)

Trans. Am. Nucl. Soc., 43: 70-71(1982)

Trans. Am. Nucl. Soc., 43: 71-72(1982)

Trans. Am. Nucl. Soc., 43: 72(1982)

Trans. Am. Nucl. Soc., 43: 73(1982)

Trans. Am. Nucl. Soc., 43: 73-75(1982)

Trans. Am. Nucl. Soc., 43: 75(1982)

Trans. Am. Nucl. Soc., 43: 75-76(1982)

Trans. Am. Nucl. Soc., 43: 86(1982)

Trans. Am. Nucl. Soc., 43: 87(1982)

Trans. Am. Nucl. Soc., 43: $111-112$ (1982)

Trans. Am. Nucl. Soc., 43: 112-113(1982)

Trans. Am. Nucl. Soc., 43: 113-114(1982)

Trans. Am. Nucl. Soc., 43: 114-115(1982)

Trans. Am. Nucl. Soc., 43: 122-124(1982)

Trans. Am. Nucl. Soc., 43: 73(1982)

Trans. Am. Nucl. Soc., 43: 68(1982)

Trans. Am. Nucl. Soc., 43: 68-69(1982)

Trans. Am. Nucl. Soc., 43: 76-77(1982)

Trans. Am. Nucl. Soc., 43: 125-126(1982)

Trans. Am. Nucl. Suc., 43: 2-3(1982)

Trans. Am. Nucl. Soc., 43: 143-146(1982)

See PNL-SA-10521

Seg DP-MS-82-46

See PNL-SA-10528

(6. international symposium on the scientific basis for radioactive waste management (Materials Research Society), Boston, MA, USA, 14 Nov 1982)

See SAND-82-1274C

See SAND-82-2260C

See UCRL-87742

NTIS, PC A02/MF A01

NTIS, PC A02/MF A01

See SAND-82-1439C

See SAND-82-2259C

See DP-MS-82-4n

See DP-MS-82-42

NTIS, PC A02; 3

See RHO-BW-SA-219-P

Elsevier Science Publishing Co., Ins., 52 Vanderbilt $\Lambda$ venue, New York, NY 10017 Mater. Res. Soc. Symp. Proc., 15: 7176(1983)

Mater. Res. Soc. Symp. Proc., 15: 391397(1983)

Mater. Res. Suc. Symp. Proc., 15: 399405(1983)

Mater. Res. Soc. Symp. Proc., 15: 663668(1983)

Mater. Res. Soc. Symp. Proc., 15: 695702(1983)

Mater. Res. Soc. Symp. Proc., 15: 407-

414(1983)

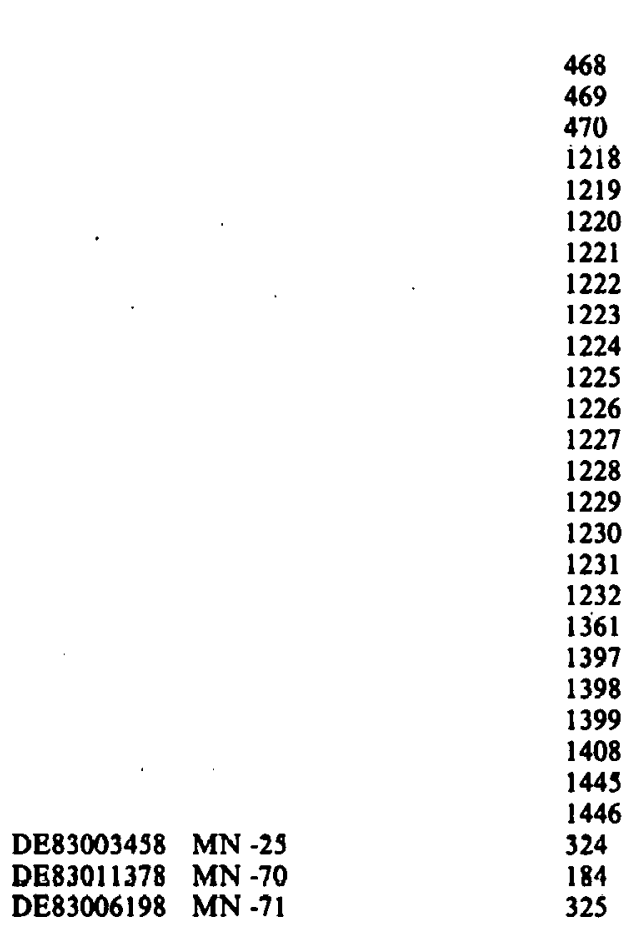

DE82022160 MN -70 972

DE83001170 MN -70 982

DE83001181 MN -70 353

DE83003418 MN -70 . 553

DE83003419 MN -70 : 554

DE83005006 MN -70 973

DE83005008 MN -70

DF.83004928 MN -70

DE83002687 MN $-70 \quad 183$

DE83007862 ND -70 143

$\begin{array}{lll}\text { DE83008328 } & \text { MN -70 } & 879\end{array}$

366

367

368

369

370

371

372 
Danart No. $\because \quad$ Availability

t.1

Pt.1

Pt.1

Pt.1

Pt.1

Pt.1

Pt.1

Pt.1

Pt.1

Pt.1

Pt.1

Pt.1

CONF-821205-

23

CONF-830205-

1
2
3
4
5
6
7
8
10
11
15
20
24
26
28
32
33

CONF-830301-

1

CONF-830315-

CONF-830329-

CUNF-830347234(198.3) 289(1983) 329(1983) 436(1983) 514(1983) 538(1983) 726(1983) 767(1983) 216(1983) 280(1983) 346(1983) 382(1983) Dec 1982) 1983)
Mater. Res. Soc. Symp. Proc., 15: $227-$

Mater. Res. Soc. Symp. Proc., 15: 281 .

Mater. Res. Soc. Symp. Proc., 15: 323-

Mater. Res. Soc. Symp. Proc., 15: 429-

Mater. Res. Soc. Symp. Proc., 15: 507 -

Mater. Res. Soc. Symp. Proc., 15: 531-

Mater. Res. Soc. Symp. Proc., 15: 719 -

Mater. Res. Soc. Symp. Proc., 15: 761-

Mater. Res. Soc. Symp. Proc., 15: 209-

Mater. Res. Soc. Symp. Proc., 15: 269-

Mater. Res. Soc. Symp. Proc., 15: 339-

Mater. Res. Soc. Symp. Proc., 15: 373-

(NWTS information meeting on basalt waste isolation project, Las Vegas, NV, USA, 14-16

See RHO-BW-SA-216P

See SAND-82-2147C

See SAND-82-2021C

See SAND-82-2198C

See RHO-BW-SA-254-P

See RHO-BW-SA-258-P

See RHO-BW-SA-256-P

See RHO-BW-SA-255-P

See RHO-BW-SA-259-P

(Waste management conference, Tucson, $A Z$, USA, 27 Feb-3 Mar 1983)

See SAND-82-2254C

See UCRL-86225

NTIS, PC A02/MF A01;

See UCRL-88648

See SAND-83-0226C

See SAND-83-0178C

NTIS, PC A02/MF A01

See UCRL-88192

Set OA-A-16971

See LA-UR-83-307

See PNL-SA-10777

See PNL-SA-10829

See RHO-BW-SA-277P

See DP-MS-82-65

See DP-MS-82-81

See RHO-BW-SA-271P

NTIS, PC A02/MF A01; 1

(ASME-JSME thermal engineering joint conference, Honolulu, HI, USA, 20-24 Mar

See SAND-82-0793C

DE82017024 MN -38

968

(GSA symposium on geological licensing topics in high- and low-level nuclear waste, College Station, TX, USA, 3-4 Mar 1983) See SAND-82-2413C

DE83006204 MN -70 985

(Desert Tortoise Council symposium, Lake Havasu, AZ, USA, 26-28 Mar 1983) NTIS, PC A02/MF A01; 1

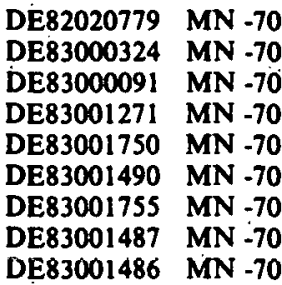

878

977

976

978

884

887

886

885

888

DE83005830 MN -70

DE83006281 MN -70 1013

DE83006807 MN -11 1368

DE83006410 MN -70 1019

DE3006483 MN -70

DE83006480 MN -70

DE83006399 MN -70 $\quad 555$

DE83006428 MN -70 1018

DE83006802 MN -70 641

DE83007541 MN -70

DE83008238 MN -70 327

DE83007876 MN -70 328

DE83009655 MN -70 . 891

DE83011381 MN -70 186

DE83011608 MN -70 $\quad 629$

DE83015079 MN -70 $\quad 890$

DE83016080 MN -70 $\quad 144$

(Atomic industrial forum fuel cycle conference, Kansas City, MO, USA, 20-23 Mar 1983)

See DPSPU-83-30-4-Rev. 
Report No.

CONF-830350-

1

CONF-830375-

1

CONF-830395-

CONF-830446-

1

CONF-830451-

2
3
5
8
10
14
18
19
22
23

CONF-830467.

1

CONF-830523.

2
4
5
15
17
20

CONF-830528-

13
18
28
29
54
CONF-830575-

5

CONF-830609-
Availability

Order No.

Distribution Category

Abstract No.

(8. international technical conference on slurry transport, San Francisco, CA, USA, 15-18 Mar 1983)

See DP-MS-82-60

(Conference on geochemical behavior of disposed radioactive waste, Seattle, WA, USA, 20-25 Mar 1983)

NTIS, PC A02/MF A01

(15. international symposium on radiation protection physics, Dresden, German D.R., 8-11 Mar 1983)

Kernenergie, 26: No. 9, 352-356(Sep 1983)

(American Power conference, Chicago, IL, USA, 18-20 Apr 1983)

See GA-A-17053

(85. American Ceramic Society annual meeting, Chicago, IL, USA, 25-28 Apr 1983)

See DP-MS-82-94

See DP-MS-82-96

See PNL-SA-10934

See DP-MS-82-91

Bei PPJL-OA-1119?

Soo PNL.SA-11308

NTIS, PC A02/MF A01

See PNL-SA-11297

See PNLSA-10913

See DP-MS-83-66

(Multivariable regression symposium, Nashville, TN, USA, 27-28 Apr 1983)

See DP-MS-83-18

(International conference on radioactive waste management, Seattle, WA, USA, 16-20 May 1983)

See SAND-82-2313C

See BNL-32669

See SAND-83-0465C

See PNL-SÁ-11273

See PNL-SA-10712

See DP-MS-83-22

(7. international symposium on packaging and transportation of radioactive materials, New Orleans, LA, USA, 15-20 May 1983)

NTIS, PC A02/MF A01

NTIS, PC A02/MF A01

See SAND-82-1994C

See SAND-82-1997C

See SAND-83-0867

(Spring meeting of the Japan Society of Corrosion Engineers, Tokyo, Japan, 18-20 May 1983)

See BNL-33451

(ANS annual meeting, Detroit, MI, USA, 12-17 Jun 1983)

Trans. Am. Nucl. Soc., 44: 100-101(Jun 1983)

Trans. Am. Nucl. Soc., 44: 58(Jun 1983)

Trans. Am. Nucl. Soc., 14: 58-59(Jun 1983)

Trans. Am. Nucl. Soc., 44: 60-61(Jun 1983)

Trans. Am. Nucl. Soc., 44: 61(Jun 1983)

Trans. Am. Nucl. Soc., 44: 61-62(Jun 1983)

Trans. Am. Nucl. Soc., 44: '18(Jun 1983)

Trans. Am. Nucl. Soc., 44: 101-102(Jun 1983)

Trans. Am. Nucl. Soc., 44: 102-103(Jun 1983)

Trans. Am. Nucl. Soc., 44: 119-120(Jun 1983)

Trans. Am. Nucl. Soc., 44: 120(Jun 1983)

Trans. Am. Nucl. Soc., 44: 121-122(Jun 1983)

Trans. Am. Nucl. Soc., 44: 122(Jun 1983)

Trans. Am. Nucl. Soc., 44: 123-124(Jun 1983)

Trans. Am. Nucl. Soc., 44: 74-75(Jun 1983)

Trans. Am. Nucl. Soc., 44: 75-76(Jun 1983)

Trans. Am. Nucl. Soc., 44: 76-77(Jun 1983)

Trans. Am. Nucl. Soc., 44: 77(Jun 1983)

Trans. Am. Nucl. Soc., 44: 78(Jun 1983)
DE83011377 MN -70

DE83014348 MN -70

642

DE83011452 MN -70

DE83011445 MN -70

DE83011950 MN -70

DE83011451 MN -70

DEB3012514 MT -70

DE 83013251 MN -70

DE83014346 MN -70

DE83016177 MN -70

DE83016786 MN.70

DE83017426 MN -70

DE83012006 MN -70

630

$\begin{array}{lll}\text { DE83007840 } & \text { MN -70 } & 983 \\ \text { DE83008592 } & \text { MN -70 } & 542 \\ \text { DE83009164 } & \text { MN -70 } & 993 \\ \text { DE83013259 } & \text { MN -70 } & 861 \\ \text { DE83013368 } & \text { MN -70 } & 326 \\ \text { DE83016834 } & \text { MN -86 } & 191\end{array}$

DE83012650 MN -71

DE83012651 MN -70

DE83013471. MN -71

DE83013684 MN -71

DE83016348 MN -70

189

190

329

188

331

863

145

862

860

631

983

542

861

191

DE83016732 MN -70

543

359

1033

1034

1035

1036

1037

1041

1042

1043

1044

1045

1046

1047

1048

1387

1388

1389

1390

1391

DE83013472 MN -41 


\section{Deport No. \\ JNF-830633- \\ 1-Summ. \\ CONF-830650- \\ 1 \\ CONF-8306118- \\ 1 \\ CONF-830808- \\ 5 \\ CONF-830812-}

58

CONF-830847-

4

CONF-8308111-

2

CONF-8308126-

1-Draft

2

CONF-831025-

10

CONF-831047-

49

CONF-831063-

5

CONF-831070-

1

CONF-8310186-

1

CONF-8310192

1

CONF-831174

. 3

CP.

$6 / 82$

DOE/AL/10752-

22

DOE/DP0013-Vol.1 0015

DF./FA151

0179

DOE/EIS0081 0082-FINAL
Availability

Order No.

Abstract No.

(3. symposium on separation science and technology for energy applications, Gatlinburg,

TN, USA, 27 Jun-1 Jul 1983)

See DP-MS-82-73

DE83011446 MN -70

187

(FRG/US workshop on size reduction and decontamination facilities, Karlsruhe, F.R.

Germany, 27-30 Jun 1983)

See DP-MS-83-55

DE83013340 MN -2

69

(4. semiannual workshop on the leaching mechanisms of defense high-level waste forms, State College, PA, USA, 20-22 Jun 1983) See UCRL-89656

DE83016194 MN -70

(International solvent extraction conference, Denver, CO, USA, 26 Aug-2 Sep 1983) NTIS, PC A02/MF A01

(18. intersociety energy conversion engineering conference, Orlando, FL, USA, 21-26 Aug 1983)

See PNL-SA-11136

(Energy resource management '83, San Francisco, CA, USA, 23-27 Aug 1983)

See PNLSA-11648

-

146

DE83010774 MN 4

(Conference on the future of nuclear power, Nara, Japan, 22-24 Aug 1983)

NTIS, PC A02/MF A01

(NRC research annual review meeting of nuclear waste management research on geochemistry of HLW disposal, Reston, VA,

USA, 30 Aug-1 Sep 1983)

NTIS, PC A02/MF A01

See BNL-NUREG-33579

(26. ORNL-DOE conference on analytical chemistry in energy technology, Knoxville, TN, USA, 11.13 Oct 1983)

See DP-MS-83-44

DE84001342 MN -70

DE84001415 MN -70

865

DE83017221 MN -70

561

(American Nuclear Society winter meeting, San Francisco, CA, USA, 30 Oct-4 Nov 1983)

NTIS, PC A02/MF A01

(American Institute of Chemical Engineers fall annual meeting, Washington, DC, USA, 30 Oct-3 Nov 1983)

See DP-MS-83-107

(IEEE/IAS glass industry committee meeting, Mexico City, Mexico, 3-7 Oct 1983)

See DP-MS-83-24

(Symposium on the GEM experience - an American education success story, Laurel, MD, USA, 18-19 Oct 1983)

See DP-MS-83-75

DE84000731 MN -70

DE84001458 MN -70

1304

547

DE84001592 MN -10

68

DE83014694 MN -70B

559

DE84002429 MN -70

632

DE83012171 MN -70

192

DE84001530 MN -70B

193

(Geological Society of America annual meeting, Indianapolis, IN, USA, 31 Oct-3 Nov 1983)

See RHO-BW-SA-298A-P

DE84001113 MN -70

894

(Materials Research Society annual mepting, Boston, MA, USA, 14-17 Nov 1983) See UCRL-89404

DE83018182 MN -70

1020

See PB-83-184184

NTIS, PC A13/MF A01; 1

DE83011783 MN -70

562

NTIS, PC A02/MF A01; 1

NTIS, PC A03/MF A01

DE83017049 STD -70

DE83013816 MN -70

2

563

See DOE/NWTS-4

DE82009290 \$TU -70

583

DE82019365 STD -70

55

DE82017565 STD -70

NTIS, PC A23/MF AO1 
Report No.

Availability

NTIS, PC A13/MF A01

DOE/FT/401 10-

1

DOE/ET/41900-

12

13

14

16

17

18

DOE/ET/44112-

T1

DOE/ET/44605T1

DOE/ET/44802 T3

DOE/ET/46617TI

DOE/ET/46634

DOE/IG-

0187

Dö: $/ \mathbf{L} \mathbf{L}$ I8'F-Rev.1 18T-Rev.2

DOE/NBM3004280 3011069 3012262

DOE/NE-

0017-1

$0017 / 2$

0039

DOE/NE/00952

DOE/NE/44146T1

T2

DOE/NE/466407

TOOE/NE/93031T1

DOE/NV/10250.

1
2
4
5
7
8
9

DOE/NWTS-

4

33(1)(Final)

33-3

33(4a)

96-Vol.1

96-Vol.2

147(1)

DOE/RL

82-3-Vol.1

82-3-Vol.2

82-3-Yol.3

DOE/S

0020 .

$0020 / 1$

0021

DOE/SR/00952. T7

DOE/SR-WM79-3-Rev. $2 / 83$ 82-1

84-1
NTIS, PC A04/MF A01 NTIS, PC A03/MF A01 NTIS, PC A02/MF A01 NTIS, PC A04/MF A01; 1 NTIS, PC A07/MF A01 NTIS, PC A06/MF A01

NTIS, PC A03/MF A01

NTIS, PC A03/MF A01

NTIS, PC A02/MF A01

See DUE/ET/44605-T1

NTIS, PC A08/MF A01; 1

TIC

NTIS, PC A21/MF A01 NTIS, PC A21/MF A01

NTIS, PC A25/MF A01 NTIS, PC A02/MF A01 NTIS, PC A06/MF A01

NTIS, PC A15/MF A01 NTIS, PC Al4/MF A01; 1 NTIS, PC A05/MF A01

NTIS, PC A05/MF A01; 1

NTIS, PC A04/MF A01 NTIS, PC A04/MF A01

NTIS, PC A02/MF A01

NTIS, PC A16/MF A01; 1

NTIS, PC A02/MF A01 NTIS, PC A03/MF A01 NTIS, PC A04/MF A01 NTIS, PC A03/MF A01 NTIS, PC A03/MF A01 NTIS, PC A03/MF A01

NTIS, PC A10/MF A01 NTIS, PC A04/MF A01 NTIS, PC A03/MF A01 NTIS, PC A03/MF A01 NTIS, PC A11/MP A01 NTIS, PC A04/MF A01 NTIS, PC A02/MF A01

NTIS, PC A99/MF A01; 1 NTIS, PC A25/MF A01; 1 NTIS, PC A99/MF A01; 1

NTIS, PC A04/MF A01 NTIS, PC A04/MF A01 NTIS, PC A02/MF A01

NTIS, PC. A06/MF ADI; 1

NTIS, PC A03/MF A01 NTIS, PC A07/MF A01 NTIS, PC A09/MF A01; 1 NTIS, PC A02/MF-A01
Order No. Distribution Category

Abstract No.

$\begin{array}{clc}\text { DE83001240 } & \text { MN -70 } & 56 \\ \text { DE82012506 } & \text { STD -70 } & \\ \text { DE82013288 } & \text { STD -70 } & 147 \\ \text { DE82013246 } & \text { STD -70 } & 564 \\ \text { DE83007885 } & \text { STD -70 } & 148 \\ \text { DE83006235 } & \text { STD -70 } & 149 \\ \text { DE83007892 } & \text { STD -70 } & 150 \\ & & 151 \\ \text { DE84001181 } & \text { MN -70 } & 565\end{array}$

$\begin{array}{lll}\text { DE82013791 MN -70 } & 566\end{array}$

$\begin{array}{lll}\text { DE82008518 STD -70 } & 567\end{array}$

DE82013791 : MN -70 $\quad 566$

$\begin{array}{lll}\text { DE83008373 MN -70 } & 568\end{array}$

DE83006930 ND -70 154

DE83013155 STD -70B $\quad 569$

DE83017655 STD -70B $\quad 570$

DE83004280 MN -70

DE83011069 MN -70 572

DE83012262 MN -13 573

DE83001800 STD -70 57

DE84001618 STD -70A 574

DE82021692 STD -70 575

DE83012394 MN -83 . 6

DE82019468 MN -7U

DE83001125 MN -70 59

DE83007142 MN -70 $\quad 576$

DE84001084 MN -70 $\quad 577$

DE82016026 MN -70 60

DE82016041 MN -70 61

DE83004754 MN -70 578

DE83004770 MN -70 579

DE83009732 MN -70

DE83014384 MN -70 . . $\quad 581$

DE83017654 MN -70 582

DE82009290 STD -70

DE82014259 STD -70 595

DE82021021 STD -70

DE83004323 MN -70 597

DE82010888 MN $-70 \quad 598$

DE82010862 MN -70 599

DE83011111 MN -70 600

DE83017084 MN -70 $\quad 601$

DE83017044. MN -70 . 602

DE83017077 MN -70 . 603

DE83014080 MN $-70 \quad 21$

DE83014886 MN -70 604

DE83014567 MN -70

$\begin{array}{lll}\text { DE83015187 MN -83 } & 155\end{array}$

DE83010816 STD -70 156

DE82006070 MN -70 157

DE84000579 MN -70 606 


\begin{tabular}{|c|c|c|}
\hline No. & & ilability \\
\hline 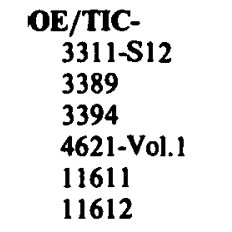 & & $\begin{array}{l}\text { NTIS } \\
\text { NTIS\$16.25 } \\
\text { NTIS } \$ 7.00 \\
\text { NTIS\$18.00 } \\
\text { NTIS, PC A06/MF A01 } \\
\text { NTIS, PC A04/MF A01 }\end{array}$ \\
\hline $\begin{array}{l}\text { DOE-tr- } \\
246\end{array}$ & & NTIS, PC A14/MF A01 \\
\hline $\begin{array}{l}\text { DP- } \\
80-125-3 \\
80-125-4 \\
81-125-1 \\
81-125-3 \\
81-157-3 \\
81-157-4 \\
82-125-4 \\
82-157-1 \\
82-157-2 \\
82-157-3 \\
83-125-1 \\
1541 \\
1606-\text { Rev.1 } \\
1625 \\
1626 \\
1627 \\
1629 \\
1631 \\
1636\end{array}$ & & $\begin{array}{l}\text { NTIS, PC A10/MF A01 } \\
\text { NTIS MF A01 } \\
\text { NTIS, PC A10/MF A01 } \\
\text { NTIS, PC A06/MF } 101 \\
\text { NTIS, PC A06/MF A01 } \\
\text { NTIS, PC A05/MF A01 } \\
\text { NTIS, PC A10/MF A01; } 1 \\
\text { NTIS, PC A04/MF A01 } \\
\text { NTIS, PC A04/MF A01 } \\
\text { NTIS, PC A02/MF A01 } \\
\text { NTIS, PC A08/MF A01; } 1 \\
\text { NTIS, PC A04/MF A01 } \\
\text { NTIS, PC A04/MF A01; } 1 \\
\text { NTIS, PC A11/MF A01 } \\
\text { NTIS, PC A03/MF A01 } \\
\text { NTIS, PC A07/MF A01 } \\
\text { NTIS, PC A05/MF A01 } \\
\text { NTIS, PC A08/MF A01 } \\
\text { NTIS, PC A03/MF A01 }\end{array}$ \\
\hline $\begin{array}{l}\text { P-MS- } \\
81-57 \\
81-92 \\
81-109 \\
81-111 \\
81-112 \\
81-114 \\
81-119 \\
81-120 \\
81-127 \\
82-2 \\
82-8 \\
82-26-\text { Rev. } \\
82-31 \\
82-40 \\
82-42 \\
82-46 \\
82-47 \\
82-60 \\
82-65 \\
82-73 \\
82-81 \\
82-91 \\
82-94 \\
82-96 \\
83-18 \\
83-22 \\
83-24 \\
83-44 \\
83-55 \\
83-66 \\
83-75 \\
83-107\end{array}$ & & $\begin{array}{l}\text { NTIS, PC A02/MF A01 } \\
\text { NTIS, PC A02/MF A01 } \\
\text { NTIS, PC A02/MF A01 } \\
\text { NTIS, PC A02/MF A01 } \\
\text { NTIS, PC A02/MF A01 } \\
\text { NTIS, PC A02/MF A01 } \\
\text { NTIS, PC A02/MF A01 } \\
\text { NTIS, PC A03/MF A01 } \\
\text { NTIS, PC A02/MF A01 } \\
\text { NTIS, PC A03/MF A01 } \\
\text { NTIS, PC A02/MF A01 } \\
\text { NTIS, PC A03/MF A01 } \\
\text { NTIS, PC A02/MF A01 } \\
\text { NTIS, PC A02/MF A01 } \\
\text { NTIS, PC A02/MF A01 } \\
\text { NTIS, PC A02/MF A01. } \\
\text { NTIS, PC A03/MF A01 } \\
\text { NTIS, PC A03/MF A01; } 1 \\
\text { NTIS, PC A02/MF A01; 1 } \\
\text { NTIS, PC A02/MF A01 } \\
\text { NTIS, PC A03/MF A01 } \\
\text { NTIS, PC A02/MF A01 } \\
\text { NTIS, PC A02/MF A01 } \\
\text { NTIS, PC A03/MF A01; } 1 \\
\text { NTIS, PC A03/MF A01; 1 } \\
\text { NTIS, PC A03/MF A01; 1 } \\
\text { NTIS, PC A02/MF A01 } \\
\text { NTIS, PC A02/MF A01 } \\
\text { NTIS, PC A02/MF A01; 1 } \\
\text { NTIS, PC A02/MF A01 } \\
\text { NTIS, PC A02/MF A01 } \\
\text { NTIS, PC A03/MF A01 }\end{array}$ \\
\hline $\begin{array}{r}\text { P-tr- } \\
20 \\
22\end{array}$ & & $\begin{array}{l}\text { NTIS, PC A02/MF A01 } \\
\text { NTIS, PC A02/MF A01 }\end{array}$ \\
\hline $\begin{array}{l}\text { DPSP- } \\
\text { 79-1003(Del.) }\end{array}$ & & NTIS, PC A05/MF A01 \\
\hline $\begin{array}{l}\text { DPSPU- } \\
\begin{array}{l}60-11-34 \\
81-30-7 \\
81-30-16 \\
83-30-4-\text { Rev. }\end{array}\end{array}$ & & $\begin{array}{l}\text { NTIS MF A01; } 2 \\
\text { NTIS, PC A02/MF A01 } \\
\text { NTIS, PC A02/MF A01 } \\
\text { NTIS, PC A02/MF A01 }\end{array}$ \\
\hline
\end{tabular}

Order No. Distribution Category Abstract No.

DE83007280 STD -70

DE82012272 STD -70 159

DE82012267 STD $-70 \quad 62$

DE82009594 STD -70 607

DE82014134 STD -70 160

DE82014916 STD -70 161

DE82001061 MN -70 1281

DE82008953 STD -70

DE82017564 STD -70 162

DE82018213 STD -70 622

DE82019745. STD $-70 \quad 64$

DE82012374 STD -70 163

DE82016240 STD $-70 \quad 164$

DE83017050 STD -70 65

DE83000531 STD .70 66

DE83003830 STD -70 165

DE83010987 STD -70 166

DE84001210 STD -70

DE82012373 STD $-4 \quad 167$

DE84001627 STD -70 168

DE82014221 MN -70 169

DE82014350 STD -70 170

DE82014133 STD -70 171

DE82014913 STD -70 172

DE83001657 STD -70 $\quad 624$

DE83010815 STD -70 173

DE82008186 MN -70 $\quad 67$

DE82008917 MN -70 ․ 625

DE82014176 MN -70

DE82014442 MN -70 174

DE82015510 MN -70 175

DE82013897 MN -70 627

DE82015511 MN -70 176

DE82015512 MN -70 177

$\begin{array}{lll}\text { DE82019159 } & \text { MN }-70 & 628\end{array}$

DE82016350 MN -70 178

DE83004931 MN -70 179

DE82019158 MN -70. 180

DE82014172 MN -70 . . 181

DE83004928 MN -70 182

DE83002687 MN -70 183

DE83011378 MN -70 $\quad 184$

DE82020155 MN $-70 \quad 185$

DE830113\% MN - 70

DE83011381 MN -70 186

DE83011446 MN -70 187

DE83011608 MN -70 629

DE83011451 MN -70 188

DE83011452 MN -70 189

DE83011445 MN -70 190

DE83012006 MN -70

DE83016834 MN -86 191

DE83012171 MN -70 192

DE84001592 MN -10 68

DE83013340 MN -2 69

DE8301\%426 MN -70 ' 631

DE84001530 MN -70B 193

DE84002429 MN -70 632

DE82000531 MN -70 $\quad 530$

DE82018764 MN -70 531

DE83004821 MN -70 633

DE83016832 MN -86 194

DE83000680. MN -70 634

DE82017676 MN -70 195

DE83012866 MN -70 196 
Report No.

DPST-

81-914

$82-294$

$82-327$

$82-346$

$82-410$

$82-475$

DPSTD82-65-Rev.1

EGG-

1183-2419

$1183-2460$

10282-2004

EGG-FM-

5758

6045

6202

EGG-M-

EIR-

07881

430

EMD-

82-64

ENICO-

1123

1131

1139

ENPU-

80-7

80-11

$80-12$

EPA-

520/1-82-007

$520 / 1-82-026$

520/5-80-002

EPRI-NP-

3225

ESC-DOE

13360

13364

13369

13391

13397

13400

ETR310

EUR-

7340

7340-EN

7426

GA-A-

16124

16631

16971

17053

GSF-T-

133

HMI-PSE-

HW$82 / 4-B$

66091

66571

66796
Availability

NTIS, PC A04/MF A01; 1

NTIS, PC A03/MF A01

NTIS, PC A07/MF A01

NTIS, PC A03/MF A01

NTIS, PC A03/MF A01

NTIS, PC A02/MF A01

NTIS, PC A03/MF A01; 1

NTIS, PC A03/MF A01

NTIS, PC A04/MF A01; 1

NTIS, PC A03/MF A01

NTIS, PC A03/MF A01

NTIS, PC A05/MF A01

NTIS, PC A04/MF A01

NTIS MF A01

NTIS (US Sales Only); PC A04/MF A01

General Accounting Office, Washington, DC 20548

NTIS, PC A04/MF A01

NTIS, PC A03/MF A01

NTTIS, PC A03/MF A01

Environmental Protection Unit, Institure of Geological Sciences, Building 151, Harwell Laboratory, Oxfordshire OXII ORA.

England:

Environmental Protection Unit, Institure of Geological Sciences, Building 151, Harwell Laboratory, Oxfordshire OXI1 ORA.

England

Environmental Protection Unit, Institure of Geological Sciences, Building 151, Harweli Laboratory, Oxfordshire OXII ORA.

England

See PB-83-190116

See PB-83-187641

See PB-83-171207

NTIS, PC A08/MF A 01

See DOE/ET/41900-12

See DOE/ET/41900-13

See DOE/ET/41900-14

See DOE/ET/41900-17

See DOE/ET/41900-16

See DOE/ET/41900-18

NTIS (US Sạles Ọnly), PC A03/MF AO1

NTIS (US Sales Only), PC A07/MF A01 See PB-82-190950

NTIS (US Sales Only), PC. AnS/MF A01

See SAND-82-7202

NTIS; PC A02/MF A01

NTIS, PC A02/MF AO1

NTIS, PC A02/MF AO1

NTIS (US Sales Oṇly), PC A03/MF A01; 1

NTIS (US Sales Only), PC A0S/MF A01

NTIS

NTIS

NTIS
Order No. Distribution Category

Abstract No.

DE83012206 MN -70.

DE82014168 MN -71 23

DE82014169 MN -70 1370

DE82015011 MN -70 635

DE82015753 MN -70 198

DE83012457 MN $-41 \quad 1371$

DE83012204 MN -70 636

DE84000864 MN -70 $\quad 1309$

DE84000863 MN -70 1310

DE84000865 MN -70 1311

DE83005698 MN -70 199

DE84002613 MN -70 200

DE83012102 MN -70 1312

DE82016342 MN -70 201

$\begin{array}{lll}\text { DE82903880 MN }-70 & 1372\end{array}$

MN $-70 \quad 637$

DE83002731 STD -70 202

DE83011033 STD -70 203

DE83̣0173̣93 MN -70 204

ND -70

ND -70

638

ND -70

205

1383

1382

1381

DE84001893 MN -70B 206

DE82012506 STD -70 147

DE82013288 STD -70 $\quad$ S64

DE82013246 STD -70 148

DE83006235 STD -70 150

DE83007885 STD -70 149

- DE83007892 STD -70 151

DE83701312 MN -86 207

DE82701148 MN -70 $\quad 640$

$\begin{array}{lll}\text { DE82701948 MN }-70 & 833 \\ & & 208\end{array}$

DE83002965 STD -70 . 34

DE82017574 MN -70 24

DE83006802 MN -70 641

DE83010809. MN -70 642

DE83902467 MN -70 643

DE83750873 MN -70 644 


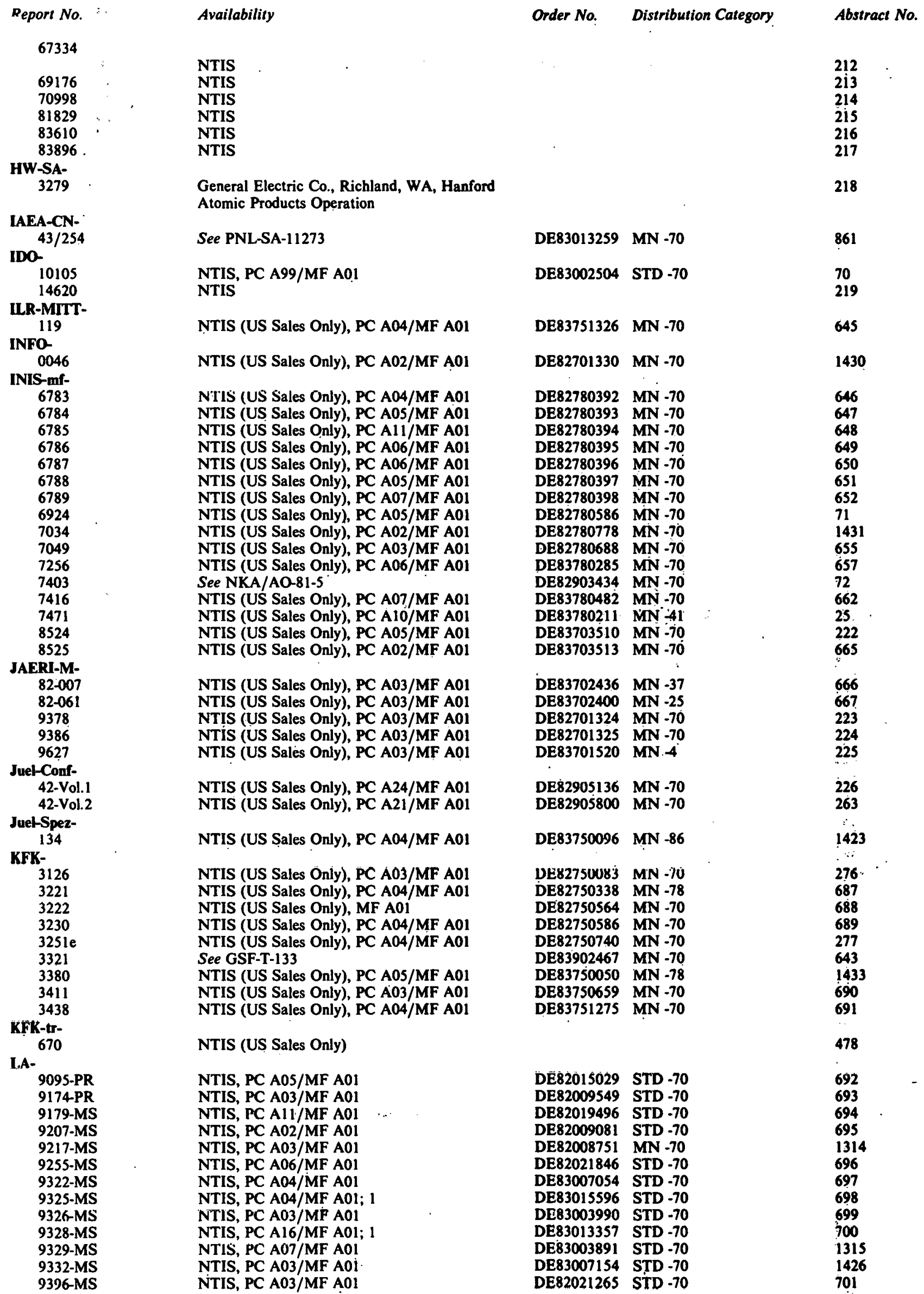


Report No.

9481-MS

9577-PR

9666-PR

9793-PR

LA-UR-

82-695

83-307

83-1304

LALP.

81-59-Rev.

LBL

11180

13341

14080

14082

14325

14752

15010

I SU2Y

15103

N-

8229145

8229146

NASA/CR-

161991

161992

162028

162029

NCSU-

$80-4$

81-3

ND-R-

658(S)

NKA/AO-

81-5

NP-

3901071

NUREG-

0856

0960-Vul.1

0960-Vol.2

NUREG/BR-

C025-82/05

NUREG/CP-

0022

NI IRFG/GR-

1636-Vol.1

1672-Vol.4

2317-Vol.1-No.3

2317-Vol.1NOS

$1 \cdot 2$

2317-Vol.2-No.1

2333-Vol.1

2333-Vol. 3

2333-Vol.4

2333-Vol.5

2394

2402

2482

2482-Vol. 2

2482-Vol.3

2482-Vol.4

2506

2547

2731

2755

2782

2854-813-1164D

2910

2959-813-1165

3062
Availability

Order No.

Distribution Category

Abstract No.

NTIS, PC A03/MF A01

NTIS, PC A05/MF A01; 1

NTIS, PC A04/MF A01

NTIS, PC A04/MF A01

NTIS, PC A02/MF A01

NTIS, PC A02/MF A01; 1

NTIS, PC A02/MF A01; 1

NTIS, PC A02/MF A01

NTIS, PC A07/MF AD1

NTIS, PC A11/MF A01

NTIS, PC A02/MF A01

NTIS, PC A02/MF A01

Sce ONWI-399

NTIS, PC A07/MF A01

NTTIS, PC A0T/MF A01; 1

NIIS, PC A09/MF A01; 1

See NUREG/CR-3062

NTIS, PC A05/MF A01

NTIS, PC A06/MF A01

NTIS, PC A02/MF A01; 1

NTIS, PC A09/MF A01; 1

NTIS, PC A03/MF A01; 1

NTIS, PC A12/MF A01; 1

NTIS, PC A02/MF A01

NTIS, PC A05/MF A01

NTIS (US Sales Only), PC A02/MF A01

NTIS (US Sales Only), PC A06/MF A01

NTIS, PC A03/MF A01

NTIS, PC A02/MF A01 - GPO \$3.00

NTIS, PC A18/MF A01; 1 - GPO

NTIS, PC A19/MF A01; 1 - GPO

NTIS, PC A02/MF A01

NTIS, PC A21/MF A01

NTIS, PC A08/MF A01 - GPO

NTIS, PC A04/MF A01 - GPO $\$ 5.00$

NTIS, PC A03/MF A01

NTIS, PC A02/MF A01

NTIS, PC A03/MF A01 - GPO

NTIS, PC A19/MF A01

NTIS, Pr. A 12/MF An1

NTIS, PC A09/MF A01

NTIS, PC A04/MF A01

NTIS PC E06/MF \$4.75; 1 - GPO \$7.00

NTIS, PC A06/MF A01; 1 - GPO

NTIS, PC A07/MF A0I

NTIS, PC A07/MF A01 - GPO $\$ 6.00$

NTIS, PC A13/MF A01; 1 - GPO $\$ 8.00$

NTIS, PC A09/MF A01; 1 - GPO $\$ 6.00$

NTIS, PC A 10/MF A01 - GPO

NTIS, PC A17/MF A01

NTIS- GPO $\$ 4.75$

NTIS, PC A03/MF A01 - GPO $\$ 4.50$

GPO*\$7.50

NTIS, PC A09/MF A01; 1 - GPO $\$ 7.00$

See LBL-13341

NTIS, PC A04/MF A01; 1 - GPO \$5:00

NTIS, PC A17/MF A01; 1 - GPO $\$ 9.50$ $\begin{array}{ll}\text { DE83008281 } & \text { STD -70 } \\ \text { DE83016352 } & \text { STD -70 } \\ \text { DE83014805 } & \text { STD -70 } \\ \text { DE84002271 } & \text { STD -70 } \\ & \\ \text { DE82011988 } & \text { MN -70 } \\ \text { DE83007541 } & \text { MN -70 } \\ \text { DE83012682 } & \text { MN -70 } \\ & \\ \text { DE83016007 } & \text { MN -70 } \\ & \\ \text { DE82012616 } & \text { STD -70 } \\ \text { DE83007314 } & \text { MN -70 } \\ \text { DE82013007 } & \text { MN -70 } \\ \text { DE82008873 } & \text { MN -70 } \\ \text { DE83005249 } & \text { MN -70 } \\ \text { DE83004180 } & \text { MN -34 } \\ \text { DE84001066 } & \text { MN -70 } \\ \text { DE83007204 } & \text { MN -70 } \\ \text { DE83010251 } & \text { MN -70 } \\ \text { DE83025 } & \end{array}$

702

703

704

705

1406

278

706

279

1422

707

711

712

812

713

1114

715

1318

716

717

DE83006640 MN -70 $\quad 718$

$\begin{array}{lll}\text { DE83006639 } & \text { MN -70 } & 719 \\ \text { DE83006637 } & \text { MN -70 } & 720\end{array}$

DE83006638 MN -70 721

DE82019466 MN -70 280

DE82019467 MN $-70 \quad 281$

$\begin{array}{lll}\text { DE83702200 MN -70 } & 282\end{array}$

DE82903434 MN -70 7 .

DE83901071 MN -70 $\quad 722$

DE83902637 MN -70 723

LE83Y்U160̈ MN - $/ 10$

DE83901642 MN -70 725

1414

$\begin{array}{lll}\text { DE82008883 MN }-70 & 726\end{array}$

$\begin{array}{lll} & \text { MN -70 } & 1316 \\ \text { DE83901048 } & \text { MN -70 } & 727\end{array}$

DE82015088 MN -71 728

DE82008455 MN -70 729

DE83002110 MN $-70 \quad 730$

DE82009990 MN -70 283

DFR2Mn9883 MN -70

DE82009884 MN -70 284

DE82009513 MN -70 285

DE83007228 MN -70 1317

DE83016132 MN -4 731

DE82009915 MN -70 286

DE83011735 MN -70

DE83014484 MN -70

738

287

739

740

741

707

742

1318
DE83010102 MN -70 735

DE83018273 MN -70 • 736 
Report No.

Availability

Order No.

Distribution Category

Abstract No.

3065-813-1163-

Vol.1

3065-813-1163-

Vol.2

3066

3097

3111-Vol.1

3160

3206

3209

3219.Vol.1

3219-Vol.2

3235-Vol.1

3235(Vols. 2-3-4)

3235-Vols.5-6

3405-Vol.1

3427-Vol.1

3472-Vol.1

NVO-

196-24-Rev. 2

196-24(Rev.3)

196-30

196-31

196-32

196-33

196-34

196-36

196-37

250

255

258

263

266

267

ONI-

3

ONWI

2-Suppl.3

9-81-3

9(81-4)

9(82-1)

9(82-2)

9(82-3)

9(82-4)

9(83-1)

27

102-Vol.1

102-Vol. 2

106

109

126

139

192

193

194

212

217

235

251

269

302

305

311

334-Vol.2-App.

348

352-Vol.1-Summ

356

383

399

400

404

416
NTIS, PC A10/MF A01; - GPO \$7.50

NTIS, PC A99/MF A01; 1 - GPO \$13.00

NTIS, PC A12/MF A01 - GPO $\$ 8.00$

NTIS, PC A07/MF A01 - GPO \$6.50; 1

NTIS, PC A09/MF A01 - GPO

NTIS, PC A08/MF A01 - GPO \$5.50

NTIS, PC A11/MF A01; 1 - GPO $\$ 7.50$

NTIS, PC A09/MF A01 - GPO $\$ 7.00$

NTIS, PC A04/MF A01 - GPO

NTIS, PC A06/MF A01; 1 - GPO $\$ 5.00$

NTIS, PC A02/MF A01; 1

NTIS, PC A14/MF A01 - GPO $\$ 9.00$

NTIS, PC A07/MF A01; 1 : GPO 96.50

NTIS, PC A09/MF A01 - GPO $\$ 6.00$

NTIS, PC A04/MF A01 - GPO \$4.25

NTIS, PC A06/MF A01 - GPO \$4.75

NTIS, PC A04/MF A01

NTIS, PC A05/MF A01

NTIS, PC A04/MF A01

NTIS, PC A05/MF A01

NTIS, PC A05/MF A01

NTIS, PC A04/MF A01

NTIS, PC A04/MF A01

NTIS, PC .A05/MF A01

NTIS, PC A05/MF A01

NTIS, PC A04/MF A01

NTIS, PC A05/MF A01; 1

NTIS, PC A03/MF A01

NTIS, PC A09/MF A01

NTIS, PC A06/MF A01

NTIS, PC A04/MF A01

NTIS, PC A04/MF A01; 1

NTIS, PC A06/MF A01

NTIS, PC A12/MF A01

NTIS, PC A11/MF A01

NTIS, PC A09/MF A01

NTIS, PC.A09/MF A01

NTIS, PC A10/MF A01; 1

NTIS, PC A09/MF A01; 1

NTIS, PC A06/MF A01;1

NTIS, PC A19/MF A01; 1

NTIS, PC A09/MF A01; 1

NTIS, PC A14/MF A01; 1

NTIS, PC A08/MF A01

NTIS, PC A10/MF A01

NTIS, PC A09/MF A01

NTIS, PC A03/MF A01

NTIS, PC A12/MF A01; 1

NTIS MF A01; 2

NTIS, PC A11/MF A01; 1

NTIS, PC A17/MF A01

NTIS, PC A09/MF A01; 1

NTIS, PC A05/MF A01

NTIS, PC A16/MF AO

NTIS, PC A04/MF AO

NTIS PC E08/MF A01; 1

NTIS, PC A04/MF A01

NTIS, PC A14/MF AO

NTIS, PC A11/MF A01

NTIS, PC A04/MF A01

NTIS, PC A03/MF A01

NTIS, PC A05/MF A01; 1

NTIS, PC A06/MF A01; 1

NTIS, PC A04/MF A01

NTIS, PC A10/MF A0

NTIS, PC A05/MF A01

NTIS, PC A09/MF A01; 1

$\begin{array}{lll}\text { DE83901897 } & \text { MN -70 } & 743 \\ \text { DE83901895 } & \text { MN -70 } & 744 \\ & & \\ \text { DE83900701 } & \text { MN -70 } & 745 \\ \text { DE83900956 } & \text { MN -70 } & 746 \\ \text { DE83016862 } & \text { MN -70 } & 747 \\ \text { DE83902454 } & \text { MN -41 } & 1375 \\ \text { DE83901845 } & \text { MN -70 } & 748 \\ \text { DE83901815 } & \text { MN -70 } & 1376 \\ \text { DE83017108 } & \text { MN -70 } & 749 \\ \text { DE83013639 } & \text { MN -70 } & 1319 \\ \text { DE83011884 } & \text { MN -70 } & 750 \\ \text { DE83012458 } & \text { MN -70 } & 1415 \\ \text { DE83012241 } & \text { MN -70 } & 1377 \\ \text { DE83902820 } & \text { MN -71 } & 751 \\ \text { DE84900307 } & \text { MN -70 } & 752 \\ \text { DE84900049 } & \text { MN -70 } & 753\end{array}$

DE82019280 STD -70

DE83012511 STD -70

DE82016400 STD $-70 \quad 756$

DE82021099 STD -70

DE83017610 STD $-70 \quad 758$

DE830176i1 STD -70

DE84001834 STD -70 760

DE83016456 MN -70

DE83016187 MN -70 $\quad 762$

DE83001418 STD -70

DE83017146 MN -70 764

DE83011476 STD -70 * 765

DE84002240 STD -70 766

DE84002824 STD -70 $\because \quad 767$

DE84002825 STD -70 * $\quad 768$

$\begin{array}{lll}\text { DE83004872 MN }-70 & 769\end{array}$

DE82022248 MN -70 770

DE82015780 MN -70 775

DE83005479 MN $-70 \quad 776$

DE83005478 MN -70 777

DE83008477 MN -70 778

DE83007304 MN -70 . $\quad 779$

DE830i.2117 MN -70 . $\quad 780$

DE84001687. MN $-70 . \quad \therefore \cdots, 781$

DE83018271 MN -70 $\quad \cdots \quad \cdots, \quad 1320$

DE83007219 MN -70 782

DE83007215 MN -70 $\quad 783$

DE82015755 MN -70 784

DE82013192 STD $-70 \quad 785$

DE82018568 MN $-70 \quad 1380$

DE83016917 MN -70 786

DE83010526 MN -70

DE83010525 MN -70 1322

DE83010220 MN -70

DE83001317 MN -70

DE83012618 MN -70 802

DE83011133 - MN -70 $\quad 803$

DE83004362 MN $70 \quad 804$

DE83005334 MN-70 $\because \quad 805$

DE83011935 MN -70 $\quad 806$

DE83002639 MN .70 $\quad 289$

DE82015763 MN.70 . $\quad 807$

DE83005213 MN -70

DE83005335 MN -70 1324

DE82017176 STD -70 809

DE83008126 MN -70 $\quad 810$

DE83016098 MN -70

DE83005249 MN -70 812

DE83004904 MN -70

DE83002763 MN -70 26

DE83007232 MN .70 814 


Report No.
422
423
423
438
439
452
455
462
463
464
466
470
483
490
493
494
ORN1
5703
5818
5838
ORNLTM-
603
757
8061
8083
8109
8124
8322
8491
8579
ORNL-tr-
4980
4994
OTA-O-
172

PATENTS-AU-
A $80 / 64353 / A /$ A 80/65,176/A/ A $81 / 73,825 / \mathrm{B} /$ A
$1978 / 34,388 / B /$ A
$1979 / 48,708 / B$
A
$1981 / 72,825 / \mathrm{A} /$.
PATENTS-CA-

$1,110,051 / \mathrm{A} /$

PATENTS-DE

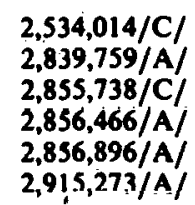

PATENTS-JP.

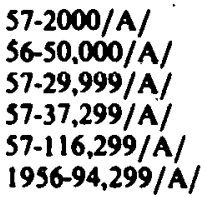

Availability

Order No.

Distribution Category

Abstract No.

NTIS, PC A10/MF A01; 1

NTIS, PC A04/MF A01; 1

NTIS, PC A21/MF A01; 1

NTIS, PC A20/MF A01; 1

NTIS (US Sales Only), PC E13/MF A01; 1

NTIS, PC A03/MF A0i

NTIS, PC A03/MF A01

NTIS, PC A03/MF A01

NTIS, PC A03/MF A01

NTIS, PC A05/MF A01; 1

NTIS, PC A04/MF A01; 1

NTIS, PC A03/MF A01

NTIS, PC A07/MF A01

NTIS, PC A03/MF A01

NTIS, PC A05/MF A01; 1

NTIS, PC A08/MF A01

NTIS, PC A05/MF A01

See NUREG/CR-2506

Oak Ridge National Lab., TN

NTIS

NTIS MF A01

NTIS, PC AO4/MF A01

NTIS, PC A04/MF A01

NTIS, PC A03/MF A01

NTIS, PC A03/MF A01

NTIS, PC A05/MF A01

NTIS, PC A05/MF A01

NTIS, PC A07/MF A01; 1

NTIS, PC A03/MF A01

Office of Technology Assessment (US Congresș), Washington. DC 20510

(Australian: Commissioner of Patents, Patents Office, Woden, A.C.T. 2606, Australia, 50 cents per copy)

\begin{tabular}{|c|c|c|}
\hline $\begin{array}{l}\text { DE83006935 } \\
\text { DE83014061 } \\
\text { DE83012767 } \\
\text { DE83013112 } \\
\text { DE83011665 } \\
\text { DE84001218 } \\
\text { DE83013814 } \\
\text { DE83014372 } \\
\text { DE83013902 } \\
\text { DE83013890 } \\
\text { DE83011126 } \\
\text { DE83016536 } \\
\text { DE83016811 } \\
\text { DE84001008 } \\
\text { DE84000527 }\end{array}$ & $\begin{array}{l}\text { MN -70 } \\
\text { MN -70 } \\
\text { MN -70 } \\
\text { MN -70 } \\
\text { MN -70 } \\
\text { STD -70 } \\
\text { MN -70 } \\
\text { MN -70 } \\
\text { MN -70 } \\
\text { MN -70 } \\
\text { MN -70 } \\
\text { MN -70 } \\
\text { MN -70 } \\
\text { MN -70 } \\
\text { MN -70 }\end{array}$ & $\begin{array}{l}815 \\
816 \\
817 \\
818 \\
819 \\
820 \\
821 \\
822 \\
823 \\
1325 \\
1326 \\
824 \\
825 \\
826 \\
827\end{array}$ \\
\hline $\begin{array}{l}\text { DE83005178 } \\
\text { DE82006119 } \\
\text { DE83014484 }\end{array}$ & $\begin{array}{l}\text { MN -70 } \\
\text { STD -70 } \\
\text { MN -70 }\end{array}$ & $\begin{array}{l}828 \\
829 \\
737\end{array}$ \\
\hline $\begin{array}{l}\text { DE82007128 } \\
\text { DE82007129 } \\
\text { DE82009607 } \\
\text { DE83004171 } \\
\text { DE82018208 } \\
\text { DE83005917 } \\
\text { DE83009878 }\end{array}$ & $\begin{array}{l}\text { STD }-70 \\
\text { STD }-70 \\
\text { STD }-70 \\
\text { STD }-70 \\
\text { STD }-70 \\
\text { STD }-70 \\
\text { STD }-70\end{array}$ & $\begin{array}{l}290 \\
291 \\
76 \\
830 \\
831 \\
292 \\
77 \\
832 \\
293\end{array}$ \\
\hline $\begin{array}{l}\text { DE83013402 } \\
\text { DE83015040 }\end{array}$ & $\begin{array}{l}\text { MN -70 } \\
\text { MN -70 }\end{array}$ & $\begin{array}{l}1052 \\
1366\end{array}$ \\
\hline & & 78 \\
\hline
\end{tabular}

(Canadian: Commissioner of Patents, Ottawa, Canada, KIA OEI; $\$ 1.00$ per copy)

(German (F.R.): Deutsches Patentamt, Schriftenvertricb, Gitschizer Str. 97:103, 1000 Berlin 60, F.R. Germany, DM 4.50- per copy, plus pustage)

(Japanese: Hatsumei-Kyokai (Invention Association), 17 Shiba, Nishikubo-Akefune Cho, Minato-Ku, Tokyo, Japan, $\$ .20$ per page, plus postage) 


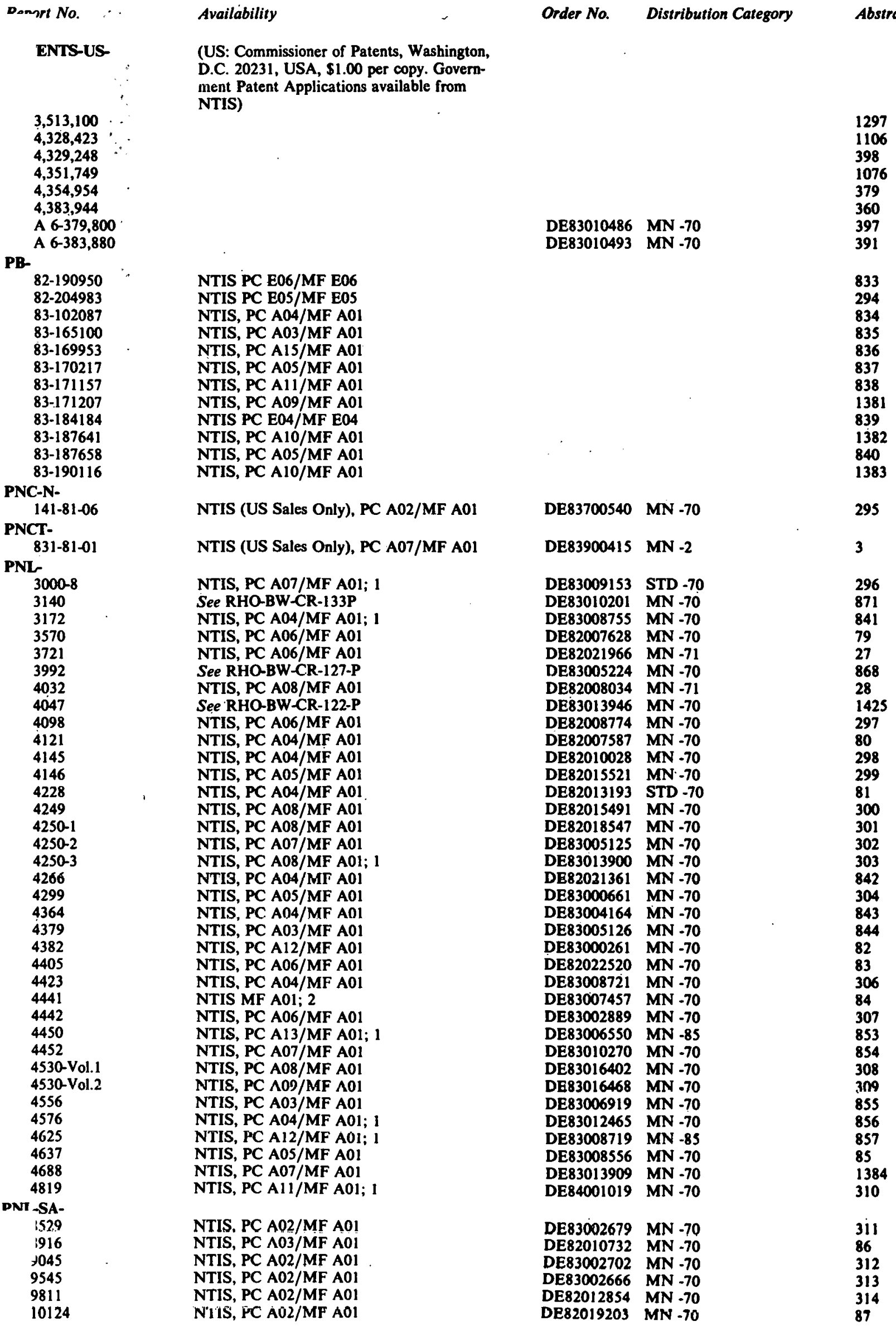


Report No.

$10184 S$

10199

10249

10261

10269

10290

10303

10316

10365

10375

10489

10521

10528

10649

10712

10777

10829

10913

10934

11136

11199

11273

11297

11308

11579

11648

PRAV.

3-28

PTB-FMRB-

86

88

RFP-

3282

RFP-Trans

405

RHO BW-CR-

122-P

127-P

$129 \mathrm{P}$

132-P

$133 P$

$136 \mathrm{P}$

RHO-BW-SA-

$187 P$

188

203-P

204P

20SA-P

209-P

216P

219-P

220P

229P

231A-P

231.P

254-P

255-P

256-P

258-P

259-P

267-P

271P

277P

281-P

293P

298A-P

RIL-BW-SR82-1-1Q-P 82-2-10-P 82-2-2Q-P 82-2-30-P B2-2-4QP
Availability

Order No.

Distribution Category

Abstract No.

NTIS, PC A02/MF A01

NTIS, PC A02/MF A01

NTIS, PC A02/MF A01

NTIS MF A01

NTIS MF AOI

NTIS, PC A03/MF A01

NTIS MF A01

NTIS, PC A02/MF A01

NTIS, PC A02/MF A01

NTIS, PC A03/MF A01

NTIS, PC A02/MF A01

NTIS, PC A02/MF A01

NTIS, PC A02/MF A01

NTIS, PC A02/MF A01

NTIS, PC A02/MF A01

NTIS, PC A02/MF A01

NTIS, PC A02/MF A01

NTIS, PC A02/MF A01

NTIS, PC A02/MF A01

NTIS, PC A02/MF AO1

NTIS, PC A02/MF A01

NTIS, PC A02/MF A01

NTIS, PC A02/MF A01

NTIS, PC A02/MP AO1

NTIS, PC A02/MF A01

NTIS, PC A02/MF A01; 1

NTIS (US Sales Only), PC A03/MF A01

NTIS (US Sales Only), MF A01

NTIS (US Sales Only), MF A01

NTIS, PC A02/MF A01

NTIS, PC A02/MF A01; 1

NTIS, PC A0S/MF A01; 1

NTIS, PC A07/MF A01

NTIS, PC A06/MF A01

NTIS MF A01; 2

NTIS, PC A16/MF A01; 1

NTIS, PC A09/MF A0I

NTIS, PC A02/MF A01

NTIS, PC A03/MF A01

NTIS, PC A02/MF A01

NTIS, PC A02/MF AO1

NTIS, PC A02/MF A01

NTIS, PC A02/MF A01

NTIS, PC A03/MF A01

NTIS, PC A02/MF AO1

NTIS, PC A02/MF AO1

NTIS, PC A02/MF A01; 1

NTIS, PC A03/MF A01

NTIS, PC A03/MF A01

NTIS, PC A02/MF A01

NTIS, PC A02/MF AO1

NTIS, PC A02/MF A01

NTIS, PC A02/MF A01

NTIS, PC A02/MF A01

NTIS, PC A02/MF A01; I

NTIS MF A01; 2

NTIS, PC A02/MF A01; 1

NTIS, PC A04/MF A01

NTIS, PC A02/MF A01

NTIS, PC A02/MF A01

NTIS; PC A05/MF A01

NTIS, PC A03/MF A01

NTIS, PC A03/MF A01

NTIS. PC A04/MF A0!

NTIS, PC A03/MF AOI
DE82018846 MN -70

DE83002723 MN -70 315

DE82018773 MN -70 316

DE82014847 MN -70 $\quad 317$

DE82014856 MN -70

DE83002072 MN -70 319

DE82014851 MN -70 320

DE82013725 MN -70

DE82014855 MN -70. 322

DE83006202 MN -70 . $\quad 858$

DE83002727 MN -70 323

DE83003458 MN -25 324

DE83006198 MN -71 325

DE83002738 MN -85

DE83013368 MN -70 326

DER3MR2.38 MN -70 327

DE83007876 MN -70 328

DE83016786 MN -70 $\quad 860$

DE83011950 MN -70 . 329

DE84001342 MN -70 . 330

DE83012514 MN -70 331

DE83013259 MN -70

DE83016177 MN -70

DE83013251 MN -70 . . . 863

DE83018286 MN -70 $\quad \cdots \quad 864$

DE84001415 MN -70 $\quad 865$

$\begin{array}{lll}\text { DE82701328 MN -70 } & 332\end{array}$

DE82750525 MN -70 $\quad 866$

DE82750523 MN -70 867

DE83000027 STD -70 333

DE83013308 MN -70 $\quad 357$

DE83013946 MN $-70 \quad 1425$

DE83005224 MN -70 $\quad 868$

DE83005495 MN -70 869

DE83005227 MN -70 $\quad 870$

DE83010201 MN -70 871

DE83006520 MN -70 872

DE82016062 MN -70

DE82014486 MN -70 1327

DE82011003 MN -70

DE82010778 MN -70 875

DE83010608 MN -70 876

DE82021.716 MN -70 877

DE82020779 MN -70 878

DE83008328 MN -70 $\quad 879$

DE83000718 MN -70 $\quad 880$

DE82021719 MN -70 . 881

DE82019256 MN -70 : 882

DE83003716 MN -70 883

DE83001750 MN -70 884

DE83001487 MN -70 $\quad 885$

DE83001755 MN -70 $\quad 886$

DE83001490 MN -70 $\quad 887$

DE83001486 MN -70

DE83006084 MN -4 $\quad 889$

DE83015079 MN -70 890

DE83009655 MN -70 891

DE83004853 MN -70 892

DE84001113 MN -70 894

DE82018197 MN -70

DE83002810 MN -70 896

DE83007947 MN -70 $\quad 897$

DE83008195 MN -70 898

DE83010644 MN -70 899
DE83009580 MN -70 893 


Renort No.
83-1-1Q-P
RHO-BW-ST-
$19-\mathrm{P}$
$21-\mathrm{P}$
$25 \mathrm{P}$
$26-\mathrm{P}$
$27-\mathrm{P}$
33-P
$35 \mathrm{P}$
$36 \mathrm{P}$
$39 \mathrm{P}$

RHO-BWI81-100-2Q 81-100-3Q 81-100-4Q

RHO-BWI-C105 118 121

RHO-BWI-LD 32
50

RHO-BWI-SA158

RHO-BWI-ST16
18

RHO-C47.

RHO-PB-SR10-B-SUMJUN-83 10-B-WM-JUN-83 10-B-WM-Sept.-83

RHO-RE-ST1-P 4-P

RHO-ST. 34

RHO-WM-SR82-1-2Q-P

RI82-1-3QP 125

RISO-I63

SAC30

SAI324-82-PA-Vol.4 SAND-

78-1711-Vol.1

79-0267

79-0268

79-0269

79-0270

79-0271

79-0272

79-04กkC.

80-2639

80-2813

$81-0215$

81-0629

81-0709

81-1095

81-1768

31-2133

$31-2163 \mathrm{C}$

31-2169C

81-2195-Rev.

81-2375

81-2377
Availability

$-\cdots$

NTIS, PC A03/MF A01

NTIS, PC A11/MF A01; 1

NTIS, PC A03/MF A01

NTIS, PC A04/MF A01

NTIS, PC A05/MF A01; 1

NTIS, PC A06/MF A01

NTIS, PC A04/MF A0I

NTIS, PC A04/MF A01

NTIS, PC A04/MF A01; 1

NTIS, PC A04/MF A01

NTIS, PC A07/MF A01

NTIS, PC A06/MF A0I

NTIS, PC A05/MF A01; 1

NTIS, PC A03/MF A01

NTIS MF A01

NTIS, PC A04/MF A01

NTIS, PC À15/MF A01

NTIS, PC A10/MF A01

NTIS, PC A04/MF A01

NTIS, PC A03/MF A01; 1

NTIS, PC A04/MF A01

NTIS, PC A04/MF A01

NTIS, PC A03/MF A01; 1

NTIS, PC A06/MF A01; 1

NTIS, PC A06/MF A01; 1

NTIS, PC A04/MF A01

NTIS, PC A04/MF A01

NTIS, PC A03/MF A01

NTIS, PC A03/MF A01

NTIS, PC A02/MF AOI

NTIS (US Sales Only), PC A02/MF A01

NTIS (US Sales Only), PC A04/MF A01

See LBL-11180

See NUREG/CR-1672-Vol.4

See NUREG/CR-1636-Vol.1

NTIS, PC A06/MF A01; 1

NTIS, PC A07/MF A01; 1

NTIS, PC A08/MF A01; 1

NTIS, PC A06/MF A01; 1

NTIS, PC A09/MF A01; 1

NTIS, PC A05/MF A0I

NTIS, PC. A02/MF A01

NTIS, PC A03/MF A01

NTIS, PC A08/MF A01

NTIS, PC A03/MF A01

NTIS, PC A05/MF A01

NTIS, PC A02/MF A01

NTIS, PC E04/MF E01

NTIS, PC A02/MF A01

NTIS, PC A02/MF A01

NTIS, PC A02/MF A0I

NTIS, PC A02/MF A01

NTIS, PC A02/MF A01; 1

NTIS, PC A11/MF A01

See NUREG/CR-2394
Order No. Distribution Category

Abstract No.

$\begin{array}{lll}\text { DE83017483 MN -70 } & 900\end{array}$

DE83018233 . STD -70 901

DE83007029 MN -70 902

DE83007458 MN -70 . 903

DE83003128 MN -70 . 904

DE83011092 STD -70 905

DE83007340 MN -70 $\quad 906$

DE83008062 MN -70 1328

DE83011928 MN -70 907

DE83013983 . MN -70 1329

DE82012541 MN -70 908

DE82012355 MN -70 $\quad 909$

DE87012031 MN -70 910

DE82006849 MN -70 911

DE82008779 MN -70 912

DE82014183 - MN -70 1429

DE82022289 MN -70 913

DE82007071 MN -70 $\quad 914$

DE82009847 MN -70 915

DE83000151 MN -70 916

DE82014722 STD -70 917

DE82007158 MN -70 334

DE83016171 MN -2 9

DE83016173 MN -70 335

DE84002697 MN -70 336

DE83013777 MN -70 918

$\begin{array}{lll}\text { DE83001141 MN -70 } & 919\end{array}$

DE82014218 STD -11 920

DE82020954 MN -70 337

DE83000375 MN -70 338

DE82701149 MN -70 921

DE82701848 MN -70 922

$\begin{array}{lll}\text { DE82012616 STD }-70 & 1422\end{array}$

$\begin{array}{lll}\text { DE83901048 MN -70 } & 727\end{array}$

MN -70 1316

DE83006392 MN -70

DE83005889 MN -70 924

DE83008481 MN -70 925

DE83006393 MN -70 926

DE83010296 MN -70

DE82012053 MN -70 928

DER2011303 MN -70 929

DE82009353 STD -70 930

DE82008799 MN -70 931

DE82015503 STD -70

DE82007550 MN -70

DE82019846 MN -70 934

DE82012378 STD -70

DE83004252 MN -70

DE82005771 MN -70 29

DE82012224 MN -41 1385

DE82011947 MN -70

DE83013136 MN -70 938

DE82015518 STD -70 1386

DE83007228 MN -70 1317 


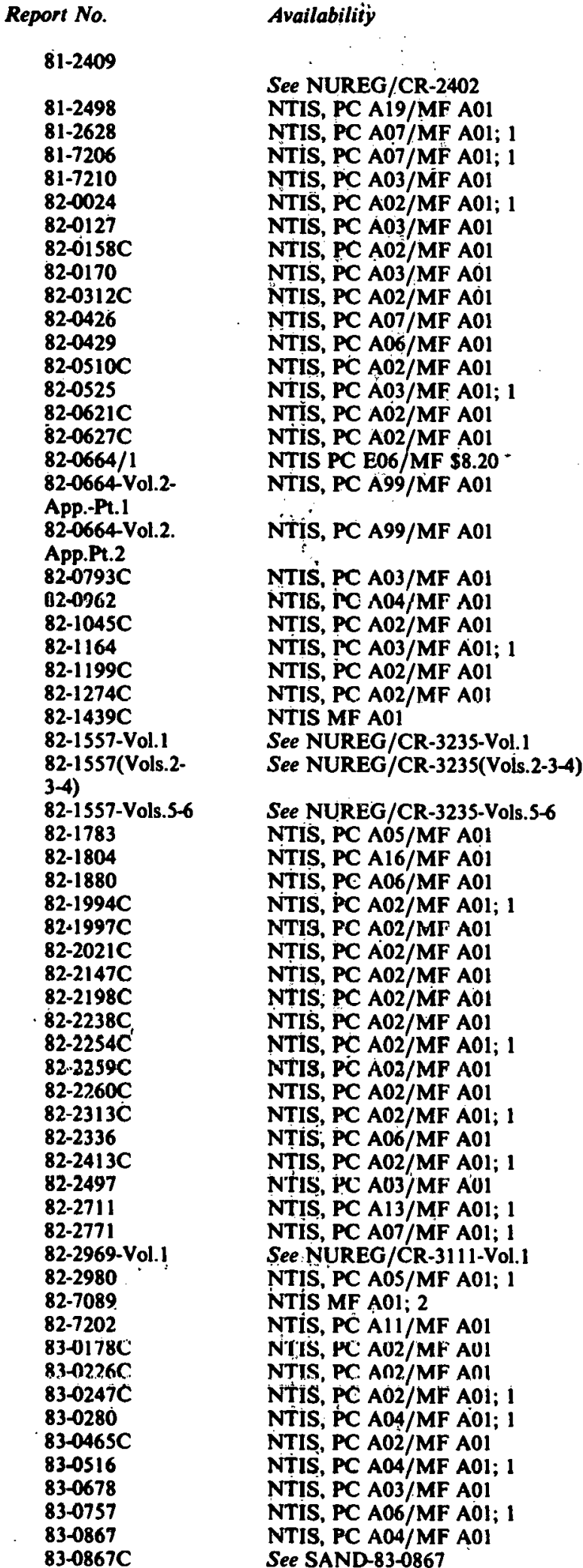

SKBF-KBS-SFR82-01
Order No. Distribution Category Abstract No.

$\begin{array}{lll}\text { DE83016132 } & \text { MN -4 } & 73 \\ \text { DE82011689 } & \text { MN -70 } & 939 \\ \text { DE83005905 } & \text { MN -70 } & 940 \\ \text { DE83014855 } & \text { MN -70 } & 941 \\ \text { DE82019803 } & \text { STD -70 } & 942 \\ \text { DE83005814 } & \text { MN -70 } & 943 \\ \text { DE82009356 } & \text { STD -70 } & 944 \\ \text { DE82017027 } & \text { MN -70 } & 945 \\ \text { DE83002648 } & \text { MN -70 } & 946 \\ \text { DE82013636 } & \text { MN -70 } & 947 \\ \text { DE82018252 } & \text { STD -70 } & 948 \\ \text { DE83006438 } & \text { MN -70 } & 949 \\ \text { DE82011469 } & \text { MN -70 } & 30 \\ \text { DE83014793 } & \text { MN -70 } & 950 \\ \text { DE82008191 } & \text { MN -70 } & 951 \\ \text { DE82011948 } & \text { MN -70 } & 952 \\ \text { DE83005119 } & \text { STD -70 } & 953 \\ \text { DE82020855 } & \text { MN -70 } & 954 \\ \text { DE82020854 } & \text { MN -70 } & 962\end{array}$

DE82017024 MN - $38 \quad 968$

DE83002137 MN -70

DE82021726 MN -70 339

DE83005888 MN -70

DE82018589 MN -70 971

DE82022160 MN -70

DE83005006 MN -70

DE83011884 MN -70

DE83012458 MN -70 1415

DE83012241 MN -70 1377

DE82022241 MN $-70 \quad 974$

DE83017411 STD -71

DE83003782 MN -70 975

DE83013471 MN -71 32

DE83013684 MN 471

DE83000091 MN -70

DE83000324 MN -70 977

DE83001271 MN -70 978

DE83002185 MN -70 979

DE83005830 MN -70 980

DE83003008 MN -70 981

DE83001170 MN -70

DE83007840 MN -70

DE83003833 MN - 70

DE83006204 MN -70 985

DE83011870 SID -70 986

DE84000996 MN -70

$\begin{array}{lll}\text { DE83013140 MN -70 } & 988\end{array}$

DE83016862 MN -70

DE83015637 MN -70 $\quad 340$

DE83017647 MN -70 1331

DE83002965 STD -70 34

DE83006480 MN - $10 \quad 989$

DE83006483 MN $-7 n$ 99n

DE83013472 MN -41

DE83015633 MN -70

DE83009164 MN -70 993

DE83012968 MN .70

DE84000976 MN -70 995

DE84000968 MN -70

DE83016348 MN -70 35

DE83016348 MN -70

DE83700169 MN -70

DE82702957 MN -70

DE83702345 MN -70

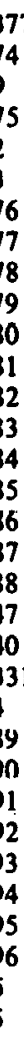

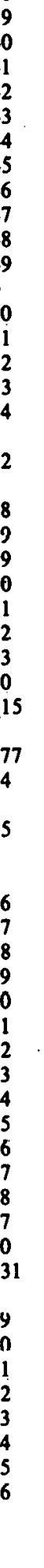

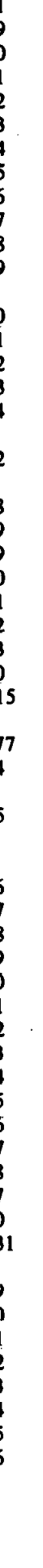

997

$81-15$

$81-17$

82-02
NTIS (US Sales Only), PC A03/MF A01 NTIS (US Sales Only), PC A07/MF A01 NTIS (US Sales Only), PC A03/MF AO 
Report No.

Availability

Order No. Distribution Category

Abstract No.

JDSVIK-NW-

82-27.3

I ENRAC/ADV

79-003

$80-002$

TENRAC/EP-

TID-

83-004 NTIS, PC A04/MF A01; 1

NTIS, PC AOS/MF AOI

NTIS, PC A05/MF A01

NTIS, PC A05/MF A01; 1

NTIS

See PNL-3721

See SAND-82-7202

See SAND-81-2133

UCID-

0202

18946

19404

19492

19598

19672

19729

19764

19926

UCRL

15480

53142-Vol.1-

Summ.

53154

53188-81

53188-82

53237

53248

53269

53315

53326

53392

53394

53405

53425

86225

86558

86582

87065

87091

87510

87742

87808

88192

88648

89404

89656

UJV-

$5734 \mathrm{Ch}$

5927-CH

USGS-CIRC847

USGSOFR:

81-1350

$82-409$

$82-466$

82-701

$82-931$

82-1041

82-1042

82-1043

82-1044

83-141

WIPP-DOE

069-Rev.1

154
NTIS, PC A03/MF A01; 1

NTIS, PC A03/MF AOI

NTIS, PC A12/MF AOI

NTIS, PC A03/MF A01

NTIS, PC A02/MF A01

NTIS, PC A05/MF A01; 1

NTIS, PC A09/MF A01; 1

NTIS, PC A03/MF A01; 1

NTIS, PC A02/MF A01

See ONWI-352-Vol.1-Summ.

NTIS, PC A07/MF A01

NTIS, PC A05/MF A01

NTIS, PC A06/MF A01

NTIS, PC A03/MF A01

NTIS, PC A06/MF A01

NTIS, PC $\Lambda 03 / \mathrm{MF} \Lambda 01$

NTIS, PC A02/MF A01

NTIS, PC A04/MF A01

NTIS, PC A03/MF A01

NTIS, PC A03/MF A01; 1

NTIS, PC A02/MF AOI

NTIS, PC A02/MF A01; 1

NTIS, PC A02/MF A01

NTIS, PC A02/MF A01

NTIS, PC A02/MF A01

NTIS, PC A02/MF A01

NTIS, PC A02/MF A01

NTIS, PC A02/MF A01

NTIS, PC A02/MF A01; 1

NTIS, PC AO2/MF A01; 1

NTIS, PC A02/MF A01

NTIS, PC $\Lambda 02 / M F ~ A 01$

NTIS (US Sales Only), PC A02/MF A01

NTIS (US Sales Only), PC A02/MF A01

See N-8229145

See DOE/ET/44802-T3

NTIS, PC A04/MF A01; 1

NTIS, PC. AnS/MF AnI

NTIS, PC A04/MF A01

NTIS, PC A03/MF A01

NTIS, PC A04/MF A0I; 1

NTIS, PC A02/MF A01; 1

NTIS, PC A03/MF A01; 1

NTIS, PC A02/MF A01; 1

NTIS, PC A03/MF A01; 1

NTIS, PC A04/MF A01

NTIS, PC A02/MF A01
NTIS, PC A03/MF A01

NTIS, PC A02/MF A01
DE83702345 MN -70 999

DE83902076 MN -2 90

DE83902062 MN -70B 1000

$\begin{array}{lll}\text { DE83902061 MN -70 } & 1001\end{array}$

342

DE82021966 MN -71 27

$\begin{array}{lll}\text { DE83002965 } & \text { STD -70 } & 34 \\ \text { DE82005771 } & \text { MN -70 } & 29\end{array}$

$\begin{array}{lll}\text { DE83013648 MN -70 } & 1002\end{array}$

DF.82017118 MN -70 343

DE82021539 MN -70 344

DE83003150 MN -70 345

DE83006417 MN -70 1003

DE83008312 MN -70 1332

DE83012449 MN -70 1004

DE84002110 MN -70 1005

DE83000734 MN -70 346

DE82017176 STD -70 809

DE82016139 STD -70 36

DE82020575 STD -70 1007

DE83017548 STD $-70 \quad 1008$

DE82012495 STD -70 347

DE82016313 STD -70 37

DE82017234 STD -70 1009

DE83003105 STD -70 1010

DE83002464 STD -70 1011

DE83011564 MN -70 348

DE83011054 MN -70 1012

DE83016873 STD -70 349

DF.84000987 STD $-70 \quad 350$

DE83006281 MN -70 1013

DE82012263 MN -70 351

DE82020176 MN -70 1014

DE82013402 MN -70 352

DE82020173 MN -70 1015

DE82012798 MN -70 1016

DE83001181 MN -70 353

DE83001540 MN -70 1017

DE83006428 MN -70 1018

DE83006410 MN -70 1019

DE83018182 MN -70 1020

DE83016194 MN -70 1021

DE82700735 MN -70 354

DE82702759 MN -70 355

716

DE82008518 STD -70 $\quad 567$

$\begin{array}{lll}\text { DE83006666 STD }-70 & 1022\end{array}$

DF.820193nR STD -70 1023

DE83001725 STD -70 1024

DE83001806 STD -70 1025

DE83010300 STD -70 1026

DE83006230 STD -70 1027

DE83006231 STD -70 1028

DE83005245 STD -70 1029

DE83013550 MN -70 1030

DE82013965 MN -70 1419

DE83009949 MN -70 1031 


\section{ORDER NUMBER CORRELATION}

This correlation list is provided for those organizations where document storage is alphanumeric by report number and request may be received by order number. For other information about a document (abstract number, distribution category, availability, and price), please refer to the Report Number Index.

\begin{tabular}{|c|c|c|c|c|c|}
\hline Order No. & Report No. & Order No. & Report No. & Order No. & Report No. \\
\hline DE82000531 & DP-tr-20 & DE82011988 & LA-UR-82-695 & DE82015491 & PNL-4249 \\
\hline DE82001061 & DOE-tr-246 & DE82012031 & RHO-BWI-81-100-4Q & DE82015503 & SAND-81-0215 \\
\hline DE82005771 & SAND-81-2133 & DE82012053 & SAND-79-0272 & DE82015510 & DP-MS-81-112 \\
\hline DE82006070 & DOE/SR/WM-82-1 & DE82012224 & SAND-81-2163C & DE82015511 & DP-MS-81-119 \\
\hline DE82006119 & ORNL-5818 & DE82012263 & UCRL-86.5.58 & DE82015512 & DP-MS-81-120 \\
\hline DE82006849 & RHO-BWI-C-105 & DE82012267 & DOE/TIC-3394 & DE82015518 & SAND-81-2375 \\
\hline DEB2007071 & RIIO-BWI-LD-50 & DE02012272 & DOL/TIC-3389 & I)F.X2.11515521 & PNL-4146 \\
\hline DE82007128 & ORNL/TM-8061 & DE82012355 & RHO-BWI-81-100-3Q & DE82015675 & CONF-810528-16 \\
\hline DE82007129 & ORNL/TM-8083 & DE82012373 & DP-1541 & DE82015753 & DPST-82-410 \\
\hline DE82007158 & RHO-C -47 & DE8201 2374 & DP-81-157-3 & DE82015755 & ONWI-106 \\
\hline DE82007550 & SAND-81-0629 & DE82012378 & SAND-81-1095 & DE 82015763 & ONWI 311 \\
\hline DE8200758\% & PNL-4121 & DE82012495 & UCRL-\$3237 & DE82015780 & ONWI-9-81.3 \\
\hline DE82007628 & PNL-3570 & DE82012506 & DOE/ET/41900-12 & DE82016026 & DOE/NV/10250-1 \\
\hline DE82008034 & PNL-4032 & DE82012541 & RHO-BWI-81-100-2Q & DE82016041 & DOE/NV/10250-2 \\
\hline DE82008157 & DOE/EIS-0082-FINAL & DE82012616 & LBL-11180 & DE82016062 & RHO-BW-SA-187P \\
\hline DE82008186 & DP-MS-81-57 & DE82012798 & UCRL-87510 & DE82016139 & UCRL-53154 \\
\hline DE82008191 & SAND-82-0621C & DE82012854 & PNL-SA-9811 & DE82016240 & DP-81-157-4 \\
\hline \multirow[t]{2}{*}{ DE82008455 } & NUREG/CR-2317-Vol. & DE82013007 & LBL-14080 & DE82016313 & UCRL-53248 \\
\hline & INOS.1-2 & DE82013192 & ONWI-109 & DE82016342 & EGG-M-07881 \\
\hline DE82008518 & $\mathrm{DOE} / \mathrm{ET} / 44802-\mathrm{T} 3$ & DE82013193 & PNL-4228 & DE82016350 & DP-MS-82.2 \\
\hline DE82008709 & ANL-81-34 & DE82013246 & DOE/ET/41900-14 & DE82016400 & NVO-196-30 \\
\hline DE82008751 & LA-9217-MS & DE82013288 & DOE/ET/41900-13 & DE82017022 & CONF-820636-4 \\
\hline DE82008774 & PNL-4098 & DE82013402 & UCRL-87065 & DE82017024 & SAND $82.0793 C$ \\
\hline DE82008779 & RHO-BWI-C-118 & DE82013636 & SAND-82-0312C & DE82017027 & SAND-82-0158C \\
\hline DE82008799 & SAND-80-2813 & DE82013725 & PNL-SA-10316 & DE82017118 & UCID-19404 \\
\hline DE82008810 & CONF-820303-27 & DE82013791 & DOE/ET/44605-T1 & DE 82017176 & ONWI-352-Vol.1- \\
\hline DE82008873 & I.BL.-14082 & DE82013897 & DP-MS-81-114 & & SurituI. \\
\hline DE82008883 & NUREG/CP-0022 & DE82013963 & CONF-820544-1 & DE82017234 & UCRL_-5.3269 \\
\hline DF.82.กn8917 & DP.MS-81-92 & DE82013965 & WIPP-DOE-069-Rev.I & DE82017409 & CONF-820609-46 \\
\hline DE82008953 & DP-80-125-3 & DE82014133 & DP-1627 & DE82017.564 & DP-80.1254 \\
\hline DE82009081 & LA-9207-MS & DE82014134 & DUE/TIC-1161i & DE82017565 & DOE/EIS- 0081 \\
\hline DE82009290 & DOE/NWTS-4 & DE82014168 & DPST-82-294 & DE.87.017574 & GA-A-16631 \\
\hline DE82009353 & SAND-80-2639 & DE82014169 & DPST-82-327 & DE.82017676 & DPSPU-81-30-16 \\
\hline DE82009356 & SAND-82-0127 & DE82014172 & DP-MS-82-31 & DE82018197 & RHO-BW-SR-82-1- \\
\hline \multirow[t]{2}{*}{ DE82009513 } & NUREG/CR-2333- & $\begin{array}{l}\text { DE82014176 } \\
\text { DE82014183 }\end{array}$ & $\begin{array}{l}\text { DP-MS-81-109 } \\
\text { RHO-BWI-C-121 }\end{array}$ & & 1Q-P \\
\hline & Vol.5 & & RHO-ST-34 & DE82018208 & ORNL/TM-8322 \\
\hline DE82009549 & LA-9174-PR & DE82014221 & DP-1625 & DE82018213 & DP-81-125-1 \\
\hline DE82009594 & DOE/TIC-4621-Vol.1 & DE82014259 & DOE/NWTS- & DE820182.52 & SAND-82-0426 \\
\hline DE82009607 & ORNL/TM-8109 & & 33(1)(Final) & DE82018547 & PNL-4250-1 \\
\hline DE82009847 & RHO-BWI-SA-1 58 & DE82014350 & DP-1626 & DE82018568 & ONWI-126 \\
\hline \multirow[t]{2}{*}{ DE82009883 } & NUREG/CR-2333- & DFR?014373 & ANL. 81.35 & DE82018589 & SAND-82-1199C. \\
\hline & Vol.3 & DE82014374 & ANL-81-53 & DE82018764 & DP-tr-22 \\
\hline \multirow[t]{2}{*}{ DE82009884 } & NUREG/CR-2333- & DE82014442 & DP-MS-81.111 & DE82018773 & PNL SA-10219 \\
\hline & Vol.4 & DE82014486 & RHO-BW-SA-188 & DE82018846 & PNL-SA-10184S \\
\hline DE82009915 & NUREG/CR-2482 & DE82014637 & BNL-19323 & DE82019158 & DP-MS-82-26-Rev. \\
\hline \multirow[t]{2}{*}{ DE82009990 } & NUREG/CR-2333- & DE82014639 & BNL-18978 & DE82019159 & DP-MS-81-127 \\
\hline & Vul.1 & DE82014642 & BNL-19109 & DE82019203 & PNL-SA-10124 \\
\hline DE82010028 & PNL-4145 & DE82014722 & RHO-BWI-ST-18 & DE82019256 & RIIO-BW-SA-231A-P \\
\hline DE82010732 & PNL-SA-8916 & DE.82014847 & PNL-SA-10261 & DF.82019280 & NVO-196-24-Rev.2 \\
\hline DE82010778 & RHO-BW-SA-204-P & DE82014851 & PNL-SA-10303 & DE82019308 & USGS-OFR-82-466 \\
\hline DE82010862 & DOE/NWTS-96-Vol.2 & DE82014855 & PNL-SA-10365 & DE82019365 & DOE/EA-0179 \\
\hline DE82010888 & DOE/NWTS-96-Vol.1 & DE82014856 & PNL-SA-10269 & DE82019466 & NCSU-80-4 \\
\hline DE82011003 & RHO-BW-SA-203-P & DE82014913 & DP-1629 & DE82019467 & NCSU-81-3 \\
\hline DE82011303 & SAND-79-0406C & DE82014916 & DOE/TIC-11612 & DE82019468 & $\mathrm{DOE} / \mathrm{NE} / 44146-\mathrm{T} 1$ \\
\hline DE82011469 & SAND-82-0510C & DE82015011 & DPSI-82-346 & DE82019496 & LA-9179-MS \\
\hline DE82011689 & SAND-81.2498 & DE82015029 & LA-9095-PR & DE82019745 & DP-81-125-3 \\
\hline DE82011947 & SAND-81-2169C & DE82015088 & NUREG/CR-2317-Vol. & DE82019803 & SAND-81-7210 \\
\hline DE82011948 & SAND-82-0627C & & $1-$ No.3 & DE82019846 & SAND-81-0709 \\
\hline
\end{tabular}




\section{ler No.}

עट82020155

DE82020173

DE82020176

DE82020575

DE82020779

DE82020854

DE82020855

DE82020954

DE82021021

DE82021099

DE82021265

DE82021361

DE82021539

DE82021692

DE82021716

DE82021719

DE82021726

DE82021846

DE82021966

DE82022160

DE82022241

DE82022248

DE82022271

DE82022289

DE82022520

DE82700674

DE82700735

DE82701148

DE82701149

DE82701324

DE82701325

DE82701328

DE82701330

DE82701848

DE82701948

DE82702759

DE82702957

DE82703060

DE82703266

DE82750083

DE82750338

DE82750523

DE82750525

DF.8275กร64

DE82750586

DE82750740

DE82780392

DE82780393

DE82780394

DE82780395

DE82780396

DE82780397

DE82780398

DE82780586

DE82780688

DE82780778

DE82902176

DE82902983

DE82903434

DE82903880

DE82904604

DE82905136

NFR 2905800

3000027

3000091

uco3000151

DE83000261

DE83000324

DE83000375
Report No.

DP-MS-82-47

UCRL-87091

UCRL-86582

UCRL-53188-81

RHO-BW-SA-216P

SAND-82-0664-Vol.2.

App.Pt.2

SAND-82-0664-Vol.2-

App.-Pt.1

RHO-WM-SR-82-12Q-P

DOE/NWTS-33-3

NVO-196-31

LA-9396-MS

PNL-4266

UCID-19492

DOE/NE-0039

RHO-BW-SA-209-P

RHO-BW-SA-229P

SAND-82-1045C

LA-9255-MS

PNL-3721

SAND-82-1274C

SAND-82-1783

ONWI-2-Suppl.3

CONF-811088-7

RHO-BWI-LD-32

PNL-4405

BLG-546

UJV-5734-Ch

EUR-7340

RI-125

JAERI-M-9378

JAERI-M-9386

PRAV-3-28

INFO-0046

RISO-I-63

EUR-7426

UJV-5927-CH

SKBF/KBS-TR-81-15

CEA-CONF-6241

AAEC/E- 526

KFK-3126

KFK-3221

PTB-FMRB-88

PTB-FMRB-86

KFK-3222

KFK-3230

KFK-3251e

INIS-mf-6783

INIS-mf-6784

INIS-mf-6785

INIS-mf -6786

INIS-mf-6787

INIS-mf-6788

INIS-mf-6789

INIS-mf-6924

INIS-mf-7049

INIS-mf-7034

CONF-8110162-1

CNEN-RT/ING-81-12

NKA/AO-81-5

EIR-430

BARC-1136

Juel-Conf-42-Vol.1

Juel-Conf-42-Vol.2

RFP-3282

SAND-82-2021C

RHO-BWI-ST-16

PNL-4382

SAND-82-2147C

RHO-WM-SR-82-13QP

\section{Order No.}

DE83000531

DE83000661

DE83000680

DE83000718

DE83000734

DE83000921

DE83001125

DE83001141

DE83001170

DE83001181

DE83001240

DE83001271

DE83001317

DE83001418

DE83001486

DE83001487

DE83001490

DE83001540

DF.83001657

DE83001725

DE83001750

DE83001755

DE83001800

DE83001806

DE83002072

DE83002110

DE83002137

DE83002185

DE83002464

DE83002504

DE83002639

DE83002648

DE83002666

DE83002679

DE83002687

DE83002702

DE83002723

DE83002727

DE83002731

DE83002738

DE83002763

DE83002810

DE83002819

DE83002889

DE83002965

DE83003105

DE83003128

DE83003150

DE83003340

DE83003418

DE83003419

DE83003458

DE83003716

DE83003782

DE83003830

DE83003833

DE83003891

DE83003990

DE83004164

DE83004171

DE83004180

DE83004252

DE83004280

DE83004323

DE83004362

DE83004726

DE83004754

DE83004770

DE83004821

DE83004853

Report No.

DP-82-157-1

PNL-4299

DPSPU-81-30-7

RHO-BW-SA-220P

UCRL-15480

ANL-81-82

DOE/NE/44146-T2

RHO-RE-ST-4-P

SAND-82-2260C

UCRL-87742

DOE/ET/40110-1

SAND-82-2198C

ONWI-212

NVO-250

RHO-BW-SA-259-P

RHO-BW-SA-255-P

RHO-BW-SA-258-P

UCRL-87808

DP-1631

USGS-OFR-82-701

RHO-BW-SA-254-P

RHO-BW-SA-256-P

DOE/NE-0017-1

USGS-OFR-82-931

PNL-SA-10290

NUREG/CR-2317-Vol.

2-No.1

SAND-82-0962

SAND-82-2238C

UCRL-53326

IDO-10105

ONWI-305

SAND-82-0170

PNL-SA-9545

PNL-SA-8529

DP-MS-82-42

PNL-SA-9045

PNL-SA-10199

PNL-SA-10489

ENICO-1123

PNL-SA-10649

ONWI-404

RHO-BW-SR-82-2-

1Q-P

BNL-NUREG-32107

PNL -4442

SANU-82-1202

UCRL-53315

RIIO-BW-ST-26-P

UCID-19598

ANL-82-10

CONF-821107-32

CONF-821107-33

PNL-SA-10521

RHO-BW-SA-231-P

SAND-82-1880

DP-82-157-2

SAND-82-2336

LA-9329-MS

LA-9326-MS

PNL 4364

ORNL/TM-8124

LBL-14752

SAND-81-1768

DOE/NBM-3004280

DOE/NWTS-33(4a)

ONWI-251

BMI-X-698-Rev.

DOE/NV/10250-4

DOE/NV/10250-5

DPSP-79-1003(Del.)

RHO-BW-SA-281-P 


\section{Order No.}

DE83007458

DE83007541

DE83007840

DE83007862

DE83007876

DE83007885

DE83007892

DE83007947

DE83008062

DE83008126

DE83008195

DE83008238

DE83008281

DE8300831?

DE83008328

DE83008373

DE03008477

DE83008481

DE83008556

DE83008592

DE83008710

DE83008721

DE83008755

DE83008996

DE83009153

DE83009164

DE83009580

DE83009655

DE83009732

DE83009878

DE83009949

DE83010102

DE83010201

DE83010220

DE83010251

DE83010252

DE83010270

DE83010296

DE83010300

DE83010525

DE83010526

DE83010608

DE83010644

DE83010774

DE83010809

DE83010815

DE83010816

DE83010987

DE83011014

DE83011033

DE83011054

DEB301 1069

DE83011092

DE83011111

DE83011126

DE83011133

DE83011377

DE. 3011378

DE83011381

DE83011445

DE83011446

DE83011451

DE83011452

DE83011476

DE83011564

DE83011608

DE83011665

DE83011735
Report No.

RHO-BW-ST-25P

LA-UR-83-307

SAND-82-2313C

CONF-821107-50

PNL-SA-10829

DOE/ET/41900-16

DOE/ET/41900-18

RHO-BW-SR-82-22Q-P

RHO-BW-ST-35P

ONWI-356

RHO-BW-SR-82-23Q-P

PNL-SA-10777

LA-9481-MS

UCID-19729

RHO-BW-SA-219-P

DOE/ET/46631-1

OPJWI-9(B2-2)

SAND-79-0269

PNL-4637

BNL-32669

PNL-4625

PNL-4423

PNL-3172

CONF-830329-1

PNL-3000-8

SAND-83-0465C

RHO-BW-SA-293P

RHO-BW-SA-277P

DOE/NV/10250-7

ORNL/TM-8579

WIPP-DOE-1 54

NUREG/CR-2482-

Vol.3

RHO-BW-CR-133P

ONWI-194

NUREG/CR-3062

BMI-OCRD-1

PNL-4452

SAND-79-0271

USGS-OFR-82-1041

ONWI-193

ONWI-192

RHO-BW-SA-205A-P

RHO-BW-SR-82-2-4QP

CONF-830808-5

GA-A-17053

DP-1636

DOE/SR-WM-79-3-

RPu ?/83

DP-82-157-3

ANL-82-58

ENICO-1131

UCRL-53394

DOE/NBM-3011069

RHO-BW-ST-27-P

DOE/NWTS-147(1)

ONWI-470

ONWI-235

DP-MS-82-60

DP-MS-8?-46

DP-MS-82-65

DP-MS-82-96

DP-MS-82.73

DP-MS-82-91

DP-MS-82-94

NVO-258

UCRL-53392

DP-MS-82-81

ONWI-452

NUREG/CR-2482

Vol.2
Order No.

DE83011783

DE83011870

DE83011884

DE83011920

DE83011928

DE83011935

DE83011950

DE83012006

DE83012102

DE83012117

DE83012171

DE83012204

DE83012206

DE83012241

DE83012262

DE8301.2394

DE8301'2449

DE83012457

DE83012458

DE83012465

DE83012511

DE83012514

DE83012618

DE83012650

DE83012651

DE83012682

DE83012767

DE83012866

DE83012968

DE83013112

DE83013136

DE83013140

DE83013135

DE83013251

DE83013259

DE83013308

DE83013340

DE83013357

DE83013368

DE83013402

DE83013471

DE83013472

DE83013550

DE83013639

DE83013648

DE83013684

DE83013777

DE83013814

DE83013816

DE83013890

DE83013900

DE83013902

DE83013909

DE83013946

DE83013983

DE83014061

DE.83014080

DE83014287

DE83014346

DE83014348

DE83014372

DE83014384

DE83014484

DE83014567

DE83014694

DE83014793

DE83014805

DE83014855
Report No.

DOE/AL/10752-22

SAND-82-2497

NUREG/CR-3235-

Vol.1

ANL-82-78

RHO-BW-ST-36P

ONWI-302

PNL-SA-10934

DP-MS-83-18

EGG-FM-6202

ONWI-9(82-4)

DP-MS-83-24

DPSTD-82-65-Rev.1

DPST-81-914

NUREG/CR-3235-

Vols.5-6

DOE/NBM-3012262

DOE/INE/00952-7

UCID-19764

DPST-82-475

NUREG/CR-

3235(Yols. 2-3-4)

PNL-4576

NVO-196-24(Rev.3)

PNL-SA-11199

ONWI-217

CONF-830528-13

CONF-830528-18

LA-UR-83-1304

ONWI-438

DPSPU-83-30-4-Rev.

SAND-83-0516

ONWI-439

SAND-81-2195-Rev.

SAND-82-2771

DOE/LLW-18T-ReV.I

PNL-SA-11308

PNL-SA-11273

RFP-Trans-405

DP-MS-83-55

LA-9328-MS

PNL-SA-10712

ORNL-tr-4980

SAND-82-1994C

SAND-83-0247C

USGS-OFR-83-141

NUREG/CR-3219.

Vol.2

UCID-18946

SAND-82-1997C

RHO-RE-ST-1-P

ONWI-462

DOF./DP-O015

ONWI-466

PNL-4250 3

ONWI-464

PNL -4688

RHO-BW-CR-122-P

RHO-BW-ST-39P

ONWI 423

DOF./S-ก020

CONF-821053-2

CONF-830451-18

CONF-830375-1

ONWI-463

DOE/NV/10250-8

NUREG/CR-2506

DOE/S-0021

CONF-831047-49

SAND-82-0525

LA-9666-PR

SAND-81-7206

Order No. 


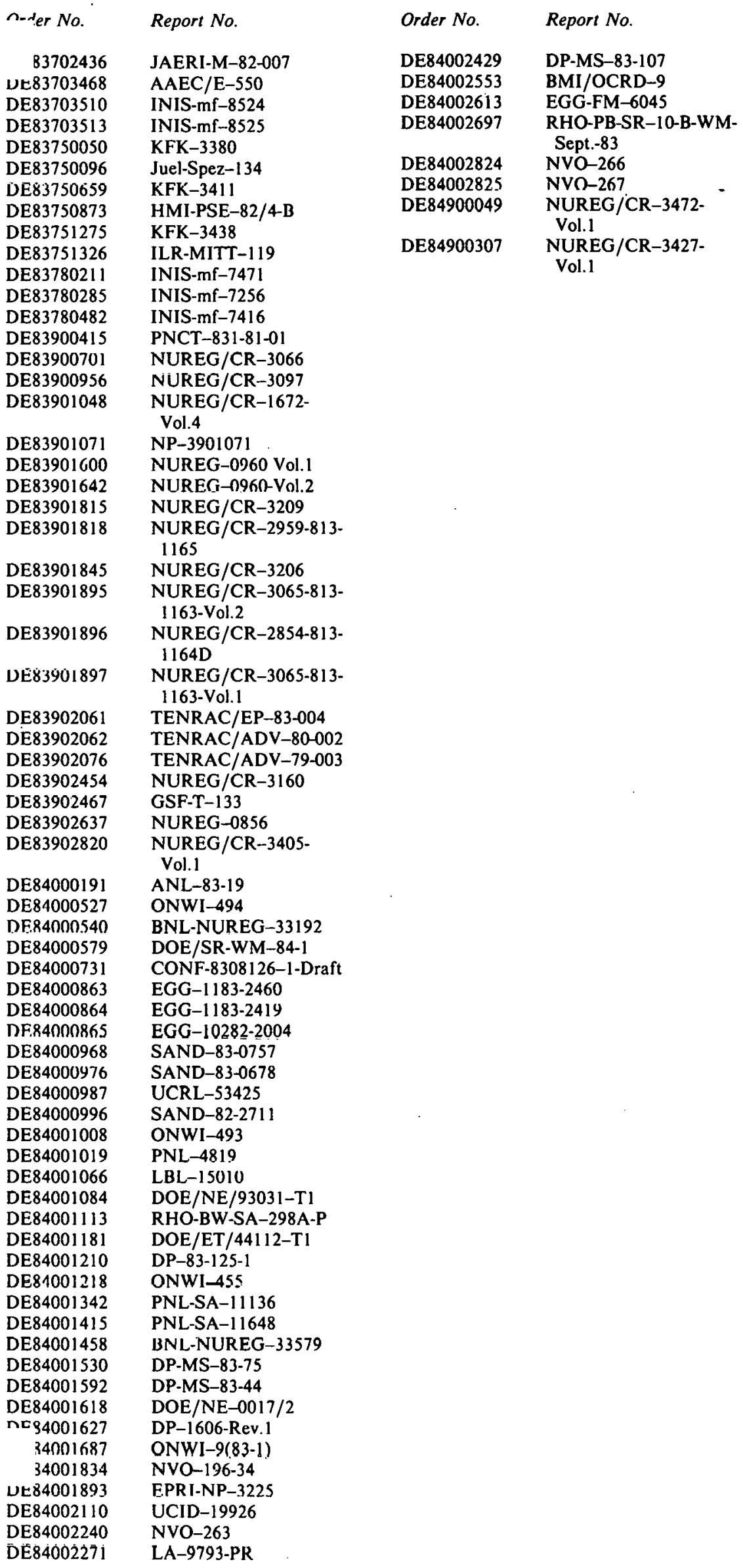

n-der No.

Report No.

83702436

UE83703468

DE83703510

DE83703513

DE83750050

DE83750096

DE 83750659

DE83750873

DE83751275

DE83751326

DE83780211

DE83780285

DE83780482

DE83900415

DE83900701

DE83900956

DE83901048

DE83901071

DE83901600

DE83901642

DE83901815

DE83901818

DE83901845

DE83901895

DE83901896

DE83901897

DE83902061

DE83902062

DE83902076

DE83902454

DE83902467

DE83902637

DE83902820

DE84000191

DE84000527

DER4000540

DE84000579

DE84000731

DE84000863

DE84000864

DF.84000865

DE84000968

DE84000976

DE84000987

DE84000996

DE84001008

DE84001019

DE84001066

DE84001084

DE84001113

DE84001181

DE84001210

DE84001218

DE84001342

DE84001415

DE84001458

DE84001530

DE84001592

DE84001618

nE94001627 34001687

34001834

Ut84001893

DE84002110

DE84002240

DEE84002271

$\begin{array}{ll}\text { Order No. } & \text { Report No. } \\ & \\ \text { DE84002429 } & \text { DP-MS-83-107 } \\ \text { DE84002553 } & \text { BMI/OCRD-9 } \\ \text { DE84002613 } & \text { EGG-FM-6045 } \\ \text { DE84002697 } & \text { RHO-PB-SR-10-B-WM- } \\ & \text { Sept.-83 } \\ \text { DE84002824 } & \text { NVO-266 } \\ \text { DE84002825 } & \text { NVO-267 } \\ \text { DE84900049 } & \text { NUREG/CR-3472- } \\ & \text { Vol.1 } \\ \text { DE84900307 } & \text { NUREG/CR-3427- } \\ & \text { Vol.1 }\end{array}$

I-M-82-007

AEC/E-550

INIS-mf-8524

INIS-mf-8525

KFK-3380

Juel-Spez-1 34

KFK-3411

HMI-PSE-82/4-B

KFK-3438

ILR-MITT-119

INIS-mf-7471

INIS-mf-7256

INIS-mf-7416

PNCT-831-81-01

NUREG/CR-3066

NUREG/CR-3097

NUREG/CR-1672-

Vol.4

NP-3901071

NUREG-0960 Vol.1

NUREG-0960-Vol.2

NUREG/CR-3209

NUREG/CR-2959-813-

1165

NUREG/CR-3206

NUREG/CR-3065-813-

1163-Vol.2

NUREG/CR-2854-813-

1164D

NUREG/CR-3065-813-

1163-Vol.1

TENRAC/EP-83-004

TENRAC/ADV-80-002

TENRAC/ADV-79-003

NUREG/CR-3160

GSF-T-133

NUREG-0856

NUREG/CR-3405-

Vol.1

ANL-83-19

ONWI-494

BNL-NUREG-33192

DOE/SR-WM-84-1

CONF-8308126-1-Draft

EGG-1183-2460

EGG-1183-2419

EGG-10282-2004

SAND-83-0757

SAND-83-0678

UCRL-53425

SAND-82-2711

ONWI-493

PNL-4819

LBL-15010

DOE/NE/93031-T

RHO-BW-SA-298A-P

DOE/ET/44112-T

DP-83-125-1

ONWI-455

PNL-SA-11136

PNL-SA-1 1648

BNL-NUREG-33579

DP-MS-83-75

DP-MS-83-44

DOE/NE-0017/2

DP-1606-Rev.1

ONWI-9(83-1)

NVO-196-34

E.PRI-NP-3225

UCID-19926

NVO-263

LA-9793-PR 


\section{REPORTS}

A document cited in this publication is considered a "report" when it carries a unique identifying number (e.g., ANL-3130, BNL-4000, DP-150, etc.) as the first element of the citation. Usually, one or more of the following abbreviations will appear at or near the end of the citation, indicating the organization from which the document is available. If none of these abbreviations appears, the report should be requested from either the cited publisher or corporate source.

GPO Available from the Superintendent of Documents, U. S. Government Printing Office, Washington, DC 20402.

GPO Dep. Available for inspection or interlibrary loan at GPO depository libraries. Unlimited DOE reports are being sent to the GPO depository libraries. See inside back cover for list of the libraries. These reports are identified by report number, Supt. of Docs. Class Number (E 1.99:) and TIC accession/order number, e.g., DE84003384.

NTIS For sale by the National Technical Information Service (NTIS), 5285 Port Royal Road, Springfield, VA 22161. Paper copy (PC) price codes given are for domestic users; prices for foreign purchasers are in most instances twice the domestic price. There is only one microfiche (MF) price code, MF A01 (\$4.50).

TIC Available from the Office of Scientific and Technical Information, Technical Information Center, P. O. Box 62, Oak Ridge, TN 37831, Attn: Technical Information Division.

NOTE: DOE offices and contractors should order reports from TIC; all others should order from NTIS. For additional information, see the introductory paragraph to the Report Number Index.

\section{NTIS NORTH AMERICAN PRICE CODES}

Most NTIS products and services are now announced by price codes and, therefore, without specific prices in NTIS journals, newsletters and indexes.

The current dollar equivalent for each code is shown in the schedule below. Orders must list the accession number(s) and be accompanied by the total dollar amount from the current schedule.

\begin{tabular}{|c|c|c|}
\hline \multicolumn{3}{|c|}{ NORTH AMERICAN CONTINENT PRICE SCHEDULE } \\
\hline \multicolumn{3}{|c|}{$\begin{array}{l}\text { Customers in Canada, United States, and } \\
\text { Mexico please use this price schedule; other } \\
\text { addressees, write for Folder PR }-360-4 \text {. }\end{array}$} \\
\hline \multirow{3}{*}{$\begin{array}{l}\text { MICROAICHI } \\
\text { AOI ........... } \$ 4.50 \\
\text { PAPER COPY }\end{array}$} & .56 .50 & $101 \ldots \ldots 125.00$ \\
\hline & $502 \ldots \ldots \ldots-1.50$ & $102 \ldots \ldots . . .140 .00$ \\
\hline & {$[03 \ldots \ldots \ldots . . . .50$} & T03................. 240.00 \\
\hline \multirow{2}{*}{$\begin{array}{l}A 02 \ldots \ldots . .1 .00 \\
A 03 \ldots \ldots . . .8 .50\end{array}$} & $604 \ldots \ldots \ldots .11 .50$ & T04 ............. 320.00 \\
\hline & $605 \ldots \ldots \ldots . . .13 .50$ & TOS ..............400.00 \\
\hline $404 \ldots \ldots . . .10 .00$ & $606 \ldots \ldots \ldots \ldots$ & $T 06 \ldots \ldots \ldots . . .465 .00$ \\
\hline A0S ................50 & $607 \ldots \ldots \ldots \ldots . .17 .50$ & $10 \% \ldots \ldots \ldots . . . . . .530 .00$ \\
\hline A06 .......... 13.00 & $608 \ldots \ldots \ldots . . .19 .50$ & T08_............595.00 \\
\hline $407 \ldots . . . . .14 .50$ & $509 \ldots \ldots \ldots . .21 .50$ & $709 \ldots \ldots \ldots 0.00$ \\
\hline$A 08 \ldots \ldots . . . .16 .00$ & E10 _..........23.50 & $T 10 \ldots \ldots . . . . . . . .725 .00$ \\
\hline$A 09 \ldots \ldots \ldots$ & $811 \ldots \ldots . . .25 .50$ & $T 11 \ldots \ldots \ldots . . . . . . .190 .00$ \\
\hline$A 10 \ldots \ldots . . .19 .00$ & $E 12 \ldots \ldots \ldots . . .28 .50$ & $T 12 \ldots \ldots \ldots . . . . .855 .00$ \\
\hline Ail ..........20.50 & $E 13 \ldots \ldots \ldots \ldots .31 .50$ & $T 13 \ldots \ldots \ldots . . . . .920 .00$ \\
\hline$A 12 \ldots \ldots . . .22 .00$ & E14 $\ldots \ldots \ldots . .34 .50$ & $T 14 \ldots \ldots \ldots . . . . . .985 .00$ \\
\hline A13 …........23.50 & EIS _ $\quad 37.50$ & T15 ....... 1,050.00 \\
\hline A14..........25.00 & $E 16 \ldots \ldots \ldots . . .40 .50$ & $T 16 \ldots \ldots \ldots .1,115.00$ \\
\hline A15 ........ 26.50 & {$[17 \ldots \ldots \ldots \ldots$} & {$[17 \ldots \ldots \ldots . .1,180.00$} \\
\hline A16 ............28.00 & E18 ..............46.50 & $\lceil 18 \ldots \ldots \ldots . . . .1,245.00$ \\
\hline$A 17 \ldots \ldots . .29 .50$ & $619 \ldots \ldots \ldots .51 .50$ & $119 \ldots \ldots . .1,310.00$ \\
\hline A18 ...........31.00 & $E 20 \ldots \ldots \ldots \ldots .61 .50$ & 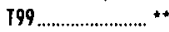 \\
\hline A19 ............32.50 & E99 $\ldots \ldots \ldots \ldots \ldots \ldots \ldots \ldots$ & \\
\hline \multicolumn{3}{|l|}{ A20 ................ } \\
\hline & $\mathrm{NDI} \ldots \ldots \ldots . . . . .35 .00$ & \\
\hline$A 22 \ldots \ldots \ldots .37 .00$ & $\mathrm{NO2 \ldots \ldots \ldots \ldots .....40.00}$ & \\
\hline A22 $\ldots . . .37 .00$ & & \\
\hline & *Add $\$ 1.50$ for eoc & litionol 25- \\
\hline $425 \ldots \ldots \ldots . . .41 .50$ & poge increment, 0 & tion thereof. \\
\hline A99 .................. & **Contoct NTIS for $p$ & \\
\hline \multicolumn{3}{|c|}{ PRICES EFFECTIVE JANUARY I, 1983} \\
\hline
\end{tabular}

U.S. DEPARTMENT OF COMMERCE

National Technical Information Service

5285 Port Royal Road, Springfield, Virginia 22161

\section{NON-REPORT DOCUMENTS}

\section{BOOKS}

Most books cited are available from commercial publishers. Order from the publisher or other source as given, not from TIC.

\section{CONFERENCE PAPERS}

Individual conference papers (or preprints) not available as reports should be requested from the originator or other source as cited.

\section{CONFERENCE PROCEEUINGS}

Many proceedings are available from commercial publishers; in other cases, request from the society, organization, institution, etc., cited as the source.

\section{DISSERTATIONS (THESES)}

Available from University Microfilms International, Dissertation Copies, P. O. Box 1764, Ann Arbor, MI 48106. Request by order number cited, e.g., DA83-22350.

\section{JOURNAL ARTICLES}

Users must use their own resources to obtain (or scan) articles cited-local, state, or university libraries or publishers, reprint services, etc.

\section{MONOGRAPHS}

Request from the organization, institution, society, etc., responsible for the monograph.

\section{PATENTS}

Although not cataloged as reports, patents cited from each country are listed in the Report Number Index under PATENTS-AU, PATENTS-FR, PATENTS-US, etc. U. S. Patent Applications (e.g., A 293,415) are available from NTIS. 


\section{CODES USED ON INDEX LINES}

Following the citation numbers in the Corporate Author, Personal Author, and Subject Indexes, codes are used to designate the type of document, country of publication, and language of the document, e.g., (R:SU:In Russian). The language designator does not appear if the document is published in English.

\section{DOCUMENT TYPES}

\section{J Journal article}

TJ Translation of a journal article

R Report

RA Report analytic

B Book

BA Book analytic

P Patent

E Engineering materials

D Thesis or dissertation

TG Translation (general)

TA Translation (analytic)

\section{COUNTRY CODES}

$\begin{array}{ll}\text { AD } & \text { Andorra } \\ \text { AE } & \text { United Arab Emirates } \\ \text { AF } & \text { Afghanistan } \\ \text { AL } & \text { Albania } \\ \text { AR } & \text { Argentina } \\ \text { AT } & \text { Austria } \\ \text { AU } & \text { Australia } \\ & \\ \text { BB } & \text { Barbados } \\ \text { BD } & \text { Bangladesh } \\ \text { BE } & \text { Belgium } \\ \text { BG } & \text { Bulgaria } \\ \text { BH } & \text { Bahrain } \\ \text { BI } & \text { Burundi } \\ \text { BM } & \text { Bermuda } \\ \text { BN } & \text { Benin } \\ \text { BO } & \text { Bolivia } \\ \text { BR } & \text { Brazil } \\ \text { BS } & \text { Bahamas } \\ \text { BT } & \text { Bhutan } \\ \text { BU } & \text { Burma }\end{array}$

BW Botswana

BY Byelorussian Soviet

Socialist Republic

(Byelorussian SSR)

\section{CA Canada \\ CB Khmer Republic \\ CE Ceylon \\ CF Central African Republic \\ CG Congo \\ $\mathrm{CH}$ Switzerland \\ CI Ivory Coast \\ CL Chile \\ CM United Republic of Cameroon \\ $\mathrm{CN}$ China \\ CO Colombia \\ CR Costa Rica \\ CS Czechoslovakia \\ CU Cuba \\ CY Cyprus}

DD German Democratic Republic

DE Germany, Fcderal Rcpublic of (F.R. Germany)

DK Denmark

DO Dominican Republic

DY Dahomey

DZ Algeria

$\begin{array}{ll}\text { EC } & \text { Ecuador } \\ \text { EG } & \text { Egypt } \\ \text { ES } & \text { Spain } \\ \text { ET } & \text { Ethiopia }\end{array}$

FI Finland

FJ Fiji

FR France
GA Gabon

GB United Kingdom of Great Britain and Northern Ireland (UK)

GD Grenada

GH Ghana

GM Gambia

GN Guinea

GQ Equatorial Guinea

GR Greece

GT Guatemala

GW Guinea-Bissau

GY Guyana

HK Hong Kong

HN Honduras

HT Haiti

HU Hungary

HV Upper Volta

ID Indonesia

IE Ireland

IL Israel

IN India

IQ Iraq

IR Iran

IS Iceland

IT Itàly

JM Jamaica

JO Jordan

JP Japan

KE Kenya

KH Cambodia

KP Democratic People's Republic of Korea

KR Korea, Republic of

KW Kuwait

LA Laos

LB Lebanon 


$\begin{array}{ll}\text { LI } & \text { Liechtenstein } \\ \text { LK } & \text { Sri Lanka } \\ \text { LR } & \text { Liberia } \\ \text { LS } & \text { Lesotho } \\ \text { LU } & \text { Luxembourg } \\ \text { LY } & \text { Libyan Arab Republic } \\ \text { MA } & \text { Morocco } \\ \text { MC } & \text { Monaco } \\ \text { MG } & \text { Madagascar } \\ \text { ML } & \text { Mali } \\ \text { MN } & \text { Mongolia } \\ \text { MR } & \text { Mauritania } \\ \text { MT } & \text { Malta } \\ \text { MU } & \text { Mauritius } \\ \text { MV } & \text { Maldives } \\ \text { MW } & \text { Malawi } \\ \text { MX } & \text { Mexico } \\ \text { MY } & \text { Malaysia } \\ & \\ \text { NE } & \text { Niger } \\ \text { NG } & \text { Nigeria } \\ \text { NI } & \text { Nicaragua } \\ \text { NL } & \text { Netherlands } \\ \text { NU Norway } \\ \text { NP } & \text { Nepal } \\ \text { NR } & \text { Nauru } \\ \text { NZ New Zealand } & \\ \text { OM } & \text { Oman } \\ \text { PA } & \text { Panama } \\ \text { PE } & \text { Peru } \\ \end{array}$

PG Papua New Guinea

PH Philippines

PK Pakistan

PL Poland

PR Puerto Rico

PT Portugal

PY Paraguay

QA Qatar

RH Southern Rhodesia

RO Romania

RW Rwanda

SA Saudi Arabia

SD Sudan

SE Sweden

SG Singapore

SK Sikkim

SL Sicrra Leone

SM Ban Marinu

SN Senegal

SO Somalia

SU Union of Soviet Socialist Republics (USSR)

SV El Salvador

SY Syria

SZ Swaziland

TC Trucial Oman

TD Chad

TG Togo
TH Thailand

TN Tunisia

TO Tonga

TR Turkey

TT Trinidad and Tobago

TW Taiwan

TZ United Republic of Tanzania

UA Ukrainian Soviet Socialist Republic (Ukrainian SSR)

UG Uganda

US United States of America (USA)

UY Uruguay

VA Holy See

VD Democratic Republic of Vie.tnam

VE Ventzuela

VS Republic of Vietnam

WS Wcstern Samoa

YD Democratic Yemen

YE Yemen

YU Yugoslavia

ZA South Africa

ZM Zumbia

ZR Zaire 
PEP AND YOUR LIBRARY CAN HELP YOU OBTAIN THE INFORMATION YOU NEED

Personal Energy Profile (PEP) is a customized computer search service provided to DOE and contractor personnel on DOE/RECON, " the Department of Energy's interactive, on-line information retrieval system managed by the Office of Scientific and Technical Information, Technical Information Center, Oak Ridge, TN. For others, these selective dissemination-of-information services are available on commercial on-line systems. PEP automatically supplies the latest information in your particular field of interest by searching any of the regularly updated data bases on DOE/RECON twice a month, printing out the new bibliographic entries in your search profile, and mailing the printout to you.

A search strategy is custom designed for each PEP subscriber so that his or her unique information needs are met. Your library and the scientific specialists at the Technical Information Center will work with you to design a suit- able search. Once a profile is established, a search is automatically executed twice each month, and the results - in computer printout form-are sent to you.

PEP searches are designed to sort information into the categories most convenient for you-by subject, source or date of publication, author. country of origin, distribution category, etc., or by any combination of these factors. For example, searches can identify:

\section{$\square$ All reports on biomass com-} bustion

$\square$ All reports that have a UC70 distribution category

$\square$ All Japanese contributions to the International Nuclear Information System since 1981

$\square$ All DOE reports from Sandia National Laboratories which deal with transportation of radioactive materials

Using PEP and the

DOE/RECON system, you can design your own personal announcement journal, which will keep you informed of new publications in your field as the DOE/RECON data bases are updated.
Current charges for PEP service are $\$ 2.00$ per update, plus the offprint charge.

For more information on the PEP search service, contact the library at your facility or, if you do not have a library, contact

\section{David Bost, Director}

Science and Technology Division Office of Scientific and

Technical Information

Technical Information Center P. O. Box 62

Qak Ridge, TN 37831

*DOE/RECON was developed to provide rapid and easy access to the energy-related data bases produced or obtained by the Center. The largest of these is the Energy Data Base (EDB) - a collection of international information which grows at a rate of over 180,000 citations per year.

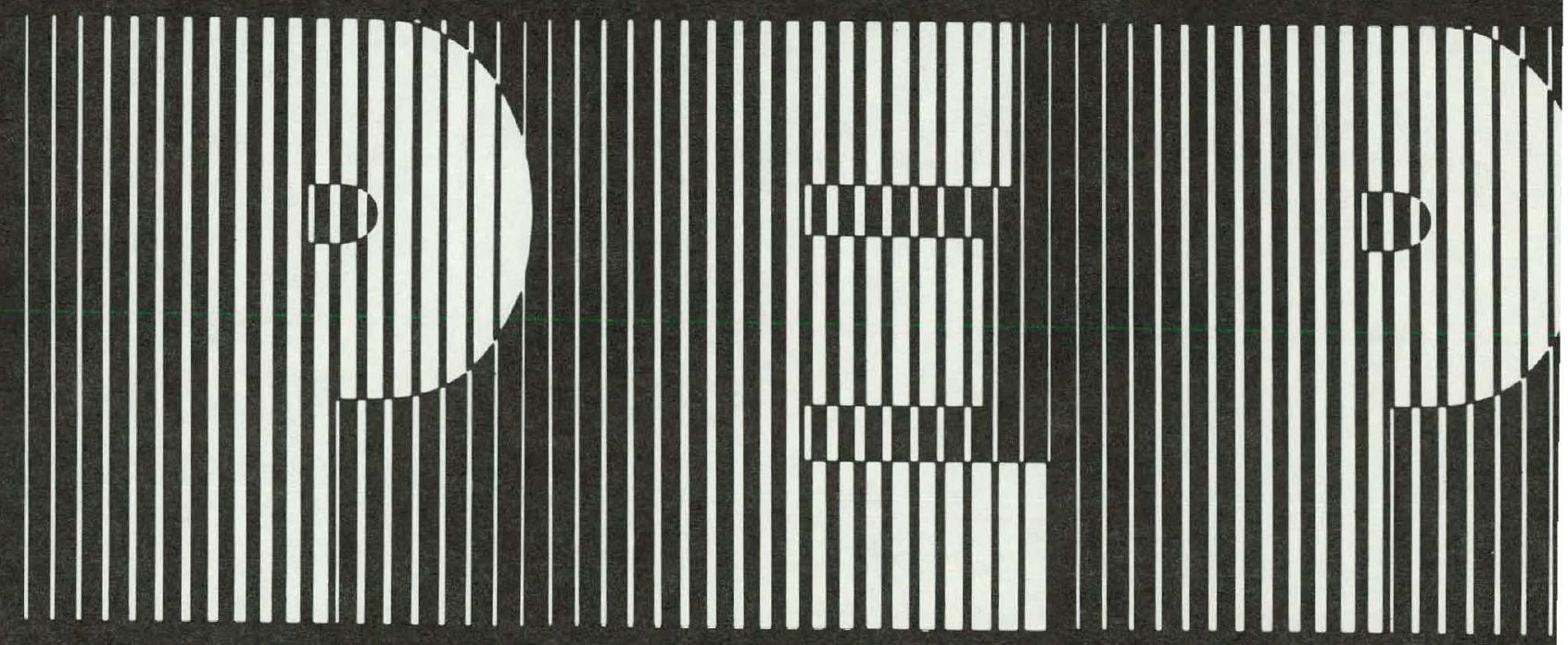




\section{FEDERAL DEPOSITORY LIBRARY PROGRAM}

As a result of an agreement between the U. S. Government Printing Office (GPO) and the Department of Energy, beginning in 1984 DOE reports with unlimited distribution are now included in the Federal Depository Library Program. The Regional Depository Libraries listed below receive and retain at least one copy of nearly every Federal Government publication, either in printed or microfilm form, for use by the general public. These libraries provide reference services and interlibrary loans; however, they are not sales outlets. You may wish to ask your local library to contact a Regional Depository to help you locate specific publications, or you may contact the Regional Depository yourself. Do not send checks or orders to these libraries.

ARKANSAS STATE LIBRARY

One Capitol Mall

Little Ror.k, AR 72201

(501) $371-2326$

AUBURN UNIV. AT MONTGOMERY LIBRARY

Documents Department

Montgomery, AL 36193

(205) $279-9110$, ext. 253

UNIV. OF ALABAMA LIBRARY

Documents Dept.-Box S

University, AL 35486

(205) 348-7369

DEPT. OF LIBRARY, ARCHIVES AND PUBLIC RECORDS

Third Floor-State Cap.

1700 West Washington

Phoenix, AZ 85007

(602) 255-4121

UNIVERSITY OF ARIZONA LIB.

Governments Documents Dept.

Tucson, AZ 85721

(602) 626-5233

CALIFORNIA STATE LIBRARY

Govt. Publications Section

P.O. Box 2037

Sacramento, CA 95809

(916) 322-4572

UNIV. OF COLORADO LIB.

Government Pub. Division

Campus Box 184

Boulder, CO 80309

(303) 492-8834

DENVER PUBLIC LIBRARY

Govt. Pub. Department

1357 Broadway

Denver, CO 80203

(303) 571-2131

CONNECTICUT STATE LIBRARY

Government Documents Unit

231 Capitol Avenue

Hartford, CT 06106

(203) 566-4971

UNIV. OF FLORIDA LIBRARIES

Library West

Documents Department

Gainesville, FL 32611

(904) 392-0367

UNIV. OF GEORGIA LIBRARIES

Government Reference Dept.

Athens, GA 30602

(404) 542-8951

UNIV. OF HAWAII LIBRARY

Govt. Documents Collection

2550 The Mall

H HI 96822

(8) $\quad 1-8230$

U _... : IDAHO LIBRARY

Documents Section

Moscow, ID 83843

(208) 885-6344
ILLINOIS STATE LIBRARY

Information Services Branch

Centennial Building

Springfield, IL 62706

(217) 782-5185

INDIANA STATE LIBRARY

Serials Documents Section

140 North Senate Avenue

Indianapolis, IN 46204

(317) 232-3686

UNIV. OF IOWA LIBRARIES

Govt. Documents Department

lowa City, IA 52242

(319) 353-3318

UNIVERSITY OF KANSAS

Doc. Collect. -Spencer Lib.

Lawrence, KS 66045

(913) 864-4662

UNIV. OF KENTUCKY LIBRARIES

Govt. Pub. Department

Lexington, KY 40506

(606) 257-3139

LOUISIANA STATE UNIVERSITY

Middleton Library

Govt. Docs. Dept.

Baton Rouge, LA 70803

(504) 388-2570

LOUISIANA TECHNICAL UNIV. LIBRARY

Documents Department

Ruston, LA 71272

(318) 257-4962

UNIVERSITY OF MAINE

Raymond $\mathrm{H}$. Fogler Library

Tri-State Regional Documents Depository

Orono, ME 04469

(207) 581-1680

UNIVERSITY OF MARYLAND

McKeldin Lib. -Doc. Div.

College Park, MD 20742

(301) 454-3034

BOSTON PUBLIC LIBRARY

Government Docs. Dept.

Boston, MA 02117

(617) 536-5400 ext. 226

DETROIT PUBLIC LIBRARY

Sociology Department

b201 Woodward Avenue

Detroit, MI 48202

(313) 833-1409

MICHIGAN STATE LIBRARY

P.O. Box 30007

Lansing, Ml 48909

(517) 373-0640

UNIVERSITY OF MINNESOTA

Government Pubs. Division

409 Wilson Library

309 19th Avenue South

Minneapolis, MN 55455

(612) 373-7813
UNIV, OF MISSISSIPPI LIB.

Documents Department

University, MS 38677

(601) 232-5857

UNIV. OF MONTANA

Mansfield Library

Documents Division

Missoula, MT 59812

(406) 243-6700

NEBRASKA LIBRARY COMM.

Federal Documents

1420 P Street

Lincoln, NE 68508

(402) 471-2045

(in cooperation with University

of Nebraska-Lincoln)

UNIVERSITY OF NEVADA LIB.

Govt. Pub. Department

Reno, NV 89557

(702) 784-6579

NEWARK PUBLIC LIBRARY

5 Washington Street

Newark, NJ 07101

(201) 733-7812

UNIVERSITY OF NEW MEXICO

Zimmerman Library

Government Pub. Dept.

Albuquerque, NM 87131

(505) 277-5441

NEW MEXICO STATE LIBRARY

Reference Department

325 Don Gaspar Avenue

Santa $\mathrm{Fe}, \mathrm{NM} 87501$

(505) 827-2033, ext. 22

NEW YORK STATE LIBRARY

Empire State Plaza

Albany, NY 12230

(518) 474-5563

\section{UNIVERSITY OF NORTH}

CAROLINA AT CHAPEL HILL

Wilson Library

BA/SS Documents Division

Chapel Hill, NC 27515

(919) 962-1321

UNIVERSITY OF NORTH DAKOTA

Chester Fritz Library

Documents Department

Grand Forks, ND 58202

(701) 777-2617, ext. 27

(in cooperation with North

Dakota State Univ. Library)

STATE LIBRARY OF OHIO

Documents Department

65 South Front Street

Columbus, $\mathrm{OH} 43215$

(614) 462-7051
OKLAHOMA DEPT. OF LIBRARIES

Government Documents

200 NE 18th Street

Oklahoma City, OK 73105

(405) 521-2502

OKLAHOMA STATE UNIV. LIB.

Documents Department

Stillwater, OK 74078

(405) 624-6546

PORTLAND STATE UNIV. LIB.

Documents Department

P.O. Box 1151

Portland, OR 97207

(503) 229-3673

STATE LIBRARY OF PENN.

Government Pub. Section

P.O. Box 1601

Harrisburg, PA 17105

1717) 787-3752

TEXAS STATE LIBRARY

Public Services Department

P.O. Box 12927-Cap. Sta.

Austin, TX 78753

(512) 471-2996

TEXAS TECH. UNIV. LIBRARY

Govt. Documents Department

Lubbock, TX 79409

(806) $742-2268$

UTAH STATE UNIVERSITY

Merrill Library, U.M.C. 30

Logan, UT 84322

(801) 7502682

UNIVERSITY OF VIRGINIA

Alderman Lib. - Public Doc.

Charlottesville, VA 22901

(804) 924-3133

WASHINGTON STATE LIBRARY

Documents Section

Olympia, WA 98504

(206) 753-4027

WEST VIRGINIA UNIV. LIB.

Documents Department

Morgantown, WV 26506

(304) 293-3640

MILWAUKEE PUBLIC LIBRARY

814 West Wisconsin Avenue

Milwaukee, WI 53233

(414) 278-3000

ST. HIST. LIB. OF WISCONSIN

Government Pub. Section

816 State Street

Madison, WI 53706

(608) 262-4347

WYOMING STATE LIRRARY

Supreme Ct. \& Library Bld.

Cheyenne, WY 82002

(307) 777-6344 
